Prepared in cooperation with the

KANSAS DEPARTMENT OF HEALTH AND ENVIRONMENT and the

KANSAS DEPARTMENT OF TRANSPORTATION

\title{
Estimates of Flow Duration, Mean Flow, and Peak- Discharge Frequency Values for Kansas Stream Locations
}

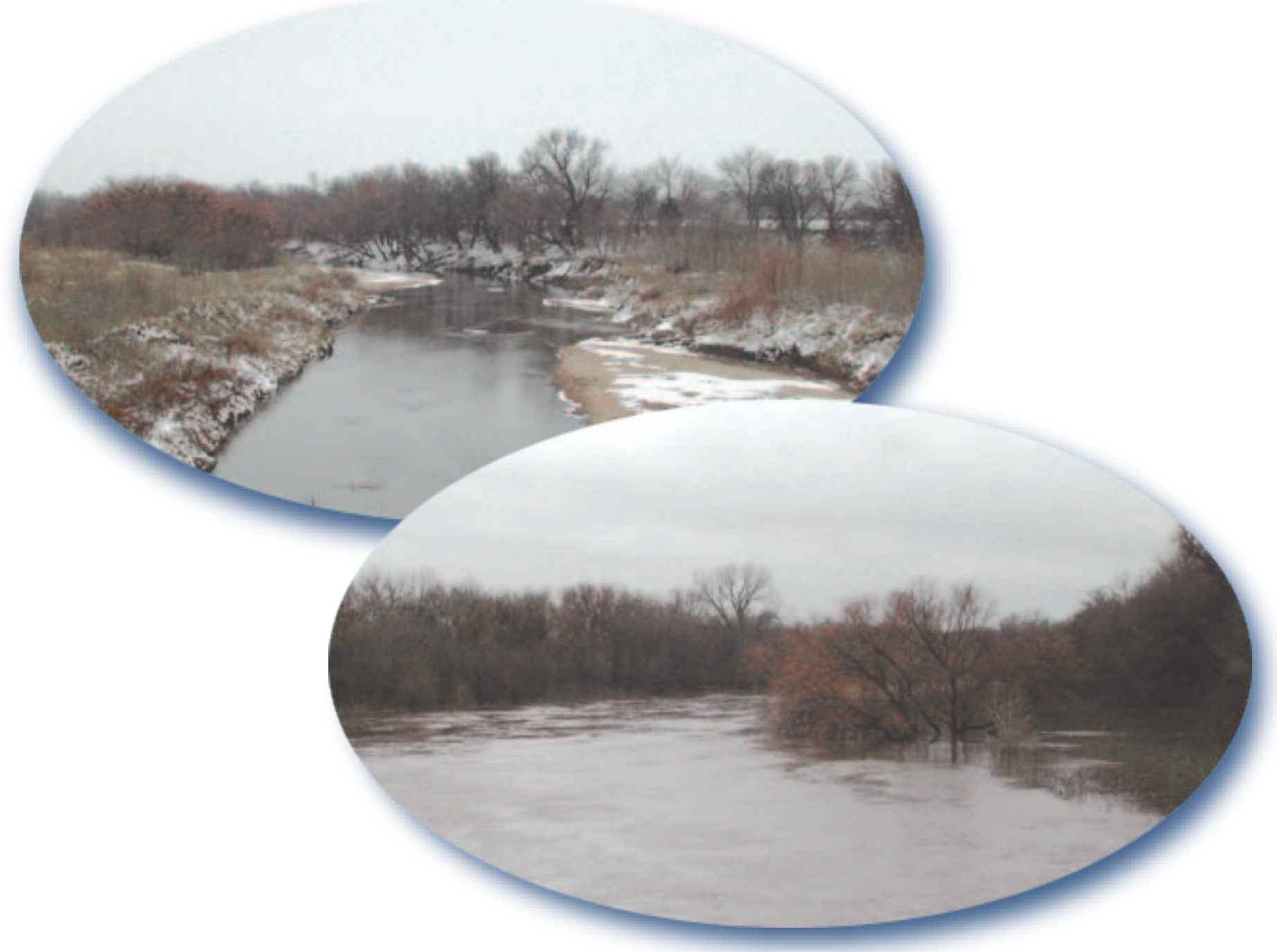

Scientific Investigations Report 2004-5033 
Cover photographs_-Taken by Trudy Bennett, U.S. Geological Survey, Wichita, Kansas. Top left: Little Arkansas River near Sedgwick, Kansas, low flow on February 27, 2003. Bottom right: Little Arkansas River near Sedgwick, Kansas, high flow on March 20, 2003. 


\section{Estimates of Flow Duration, Mean Flow, and Peak-Discharge Frequency Values for Kansas Stream Locations}

By Charles A. Perry, David M. Wolock, and Joshua C. Artman

Prepared in cooperation with the

Kansas Department of Health and Environment

and the Kansas Department of Transportation

Scientific Investigations Report 2004-5033

U.S. Department of the Interior

U.S. Geological Survey 


\title{
U.S. Department of the Interior Gale A. Norton, Secretary
}

\author{
U.S. Geological Survey \\ Charles G. Groat, Director
}

\section{U.S. Geological Survey, Reston, Virginia: 2004}

\author{
For sale by U.S. Geological Survey, Information Services \\ Box 25286, Denver Federal Center \\ Denver, CO 80225 \\ For more information about the USGS and its products: \\ Telephone: 1-888-ASK-USGS \\ World Wide Web: http://www.usgs.gov/
}

Any use of trade, product, or firm names in this publication is for descriptive purposes only and does not imply endorsement by the U.S. Government.

Although this report is in the public domain, permission must be secured from the individual copyright owners to reproduce any copyrighted materials contained within this report.

Suggested citation:

Perry, C.A., Wolock, D.M., and Artman, J.C., 2004, Estimates of flow duration, mean flow, and peak-discharge frequency values for Kansas stream locations: U.S. Geological Survey Scientific Investigations Report 2004-5033, $651 \mathrm{p}$. 


\section{Contents}

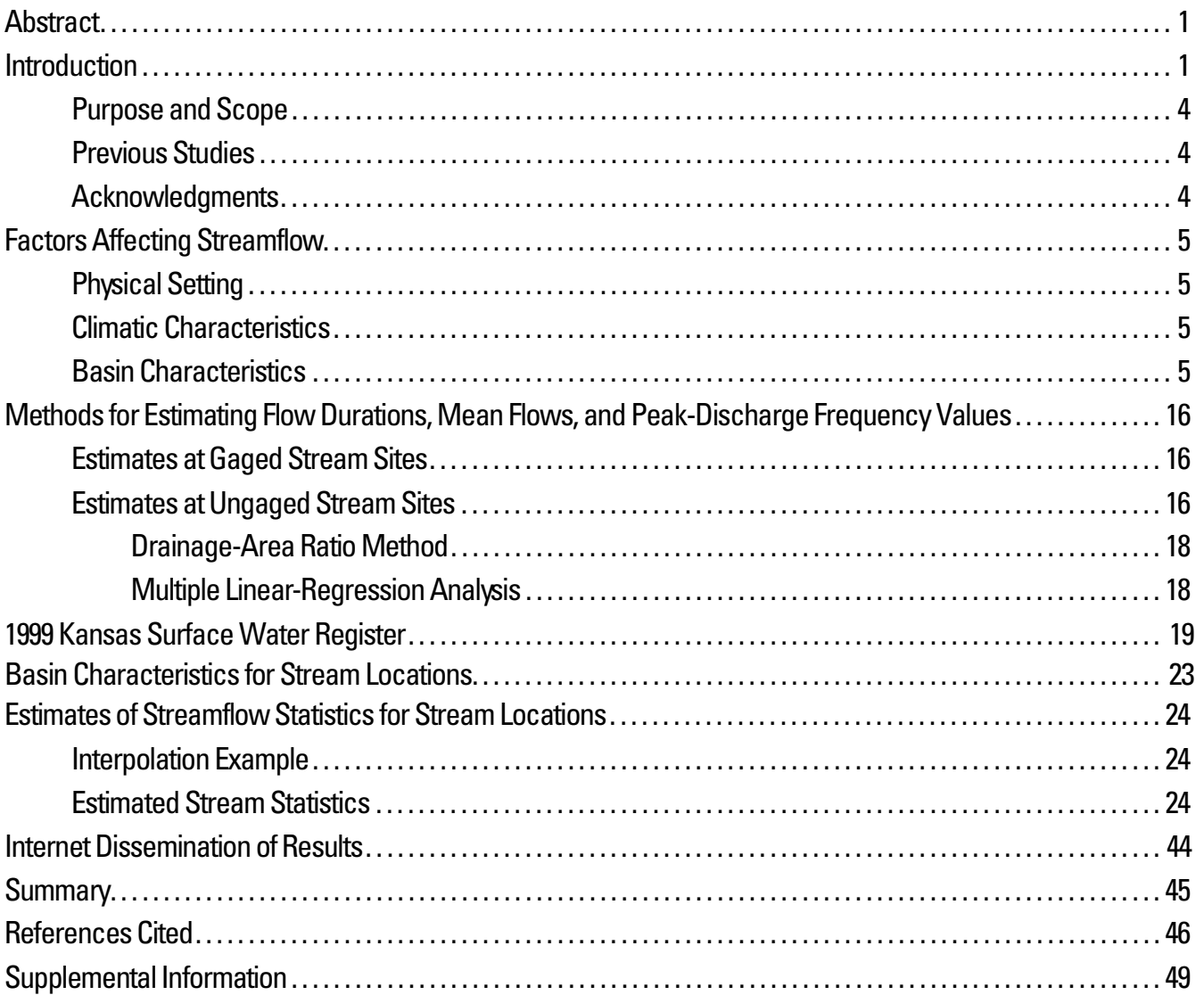

\section{Figures}

\section{1-6. Maps showing:}

1. Location of U.S. Geological Survey streamflow-gaging stations in Kansas and parts of surrounding States with 10 or more years of record that were available for computing flow durations and mean flows.

2. Location of U.S. Geological Survey streamflow-gaging stations with peak-discharge frequency analysis in Kansas and parts of surrounding States with 10 or more years of

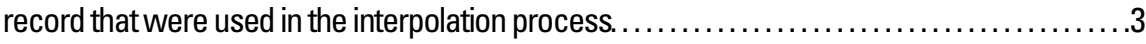

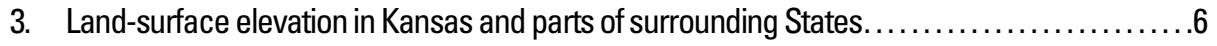

4. Average land-surface slope in Kansas and parts of surrounding States..................

5. Areas of equal average soil permeability in Kansas and parts of surrounding States .........8

6. Mean annual precipitation in Kansas and parts of surrounding States . . . . . . . . . . . . . . 9

7. Graph showing flow-duration curve for Soldier Creek near Topeka, Kansas ................. 17

8. Graphs showing results of regression with Tobit analysis for flow durations of $90,75,50,25$, and

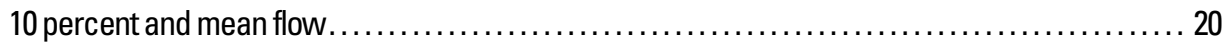

9. Map showing 50-percent flow values for stream locations in central Kansas estimated from regression equations and observed median obtained from streamflow-gaging-station data before interpolation. 
10. Map showing estimated 50-percent flow values for stream locations in central Kansas after using interpolation procedures outlined in table 4 and observed median obtained from streamflow-gaging-station data

11-115. Map showing location of streamflow-statistics determination sites, associated identification numbers, and U.S. Geological Survey streamflow-gaging stations used in the flow-duration and peakdischarge frequency analyses for:

\begin{tabular}{c|c} 
11. & Allen County $\ldots \ldots \ldots \ldots$ \\
12. & Anderson County $\ldots \ldots \ldots \ldots \ldots$
\end{tabular}$\ldots$




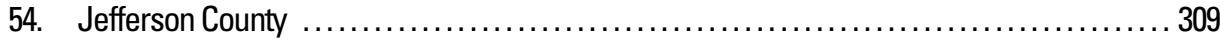

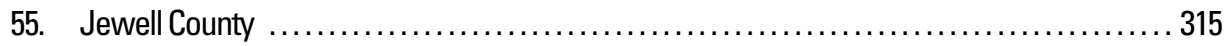

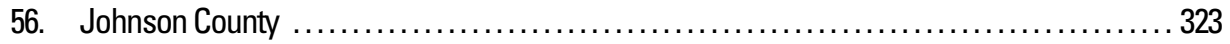

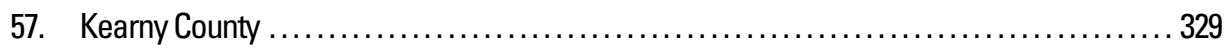

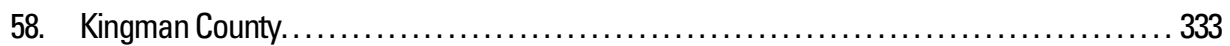

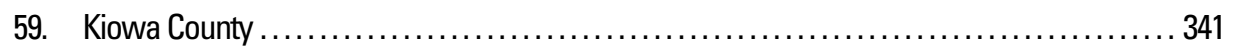

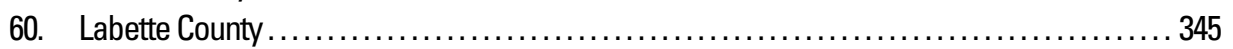

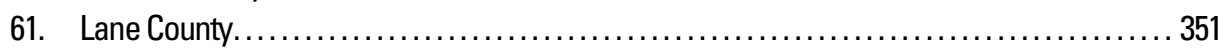

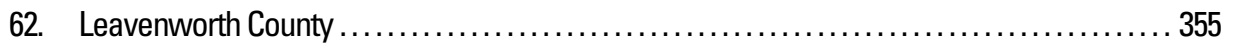

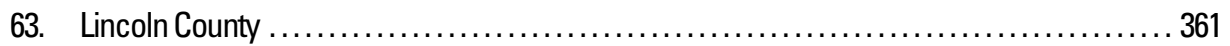

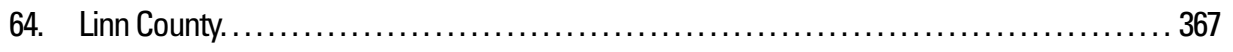

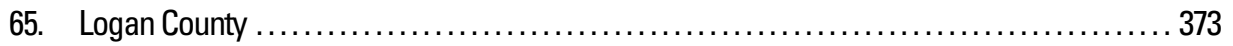

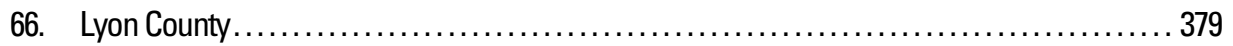

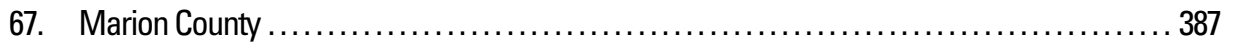

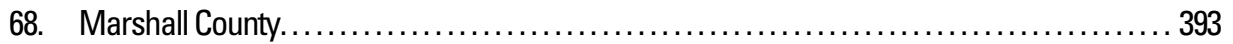

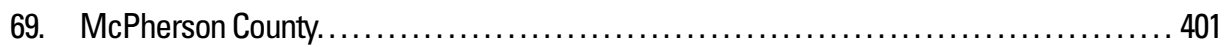

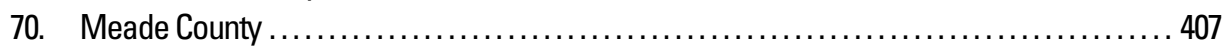

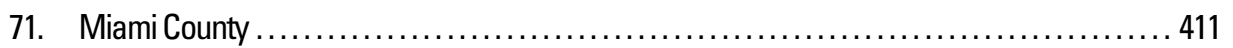

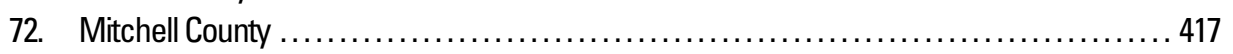

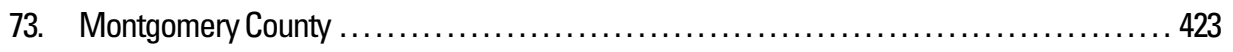

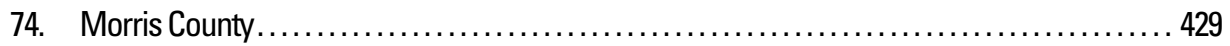

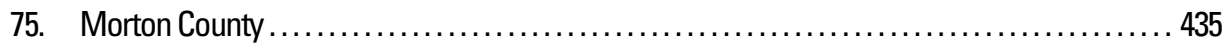

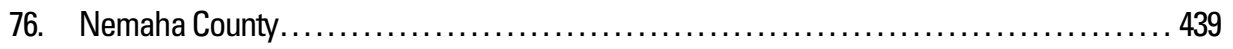

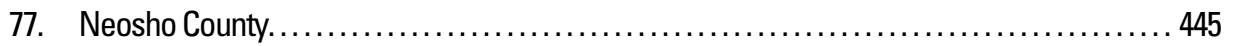

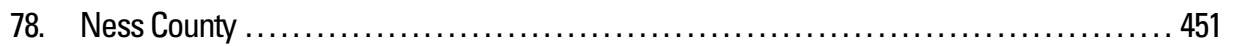

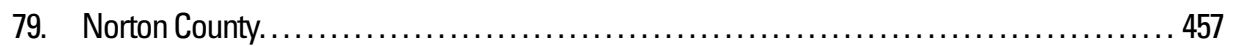

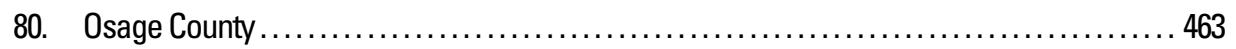

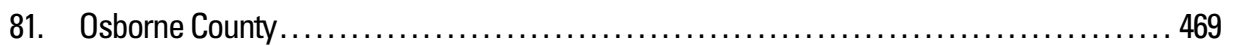

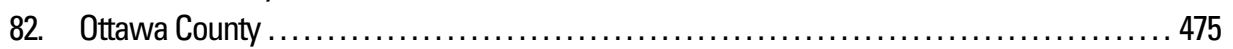

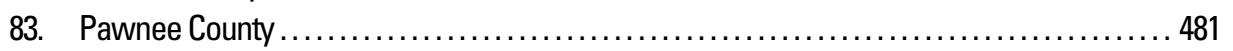

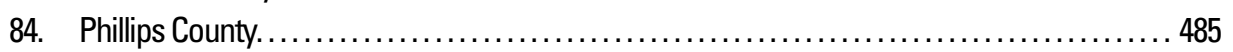

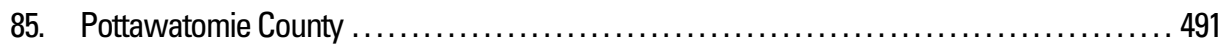

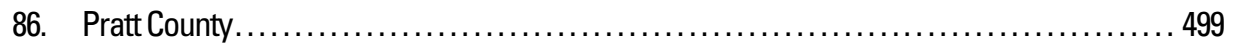

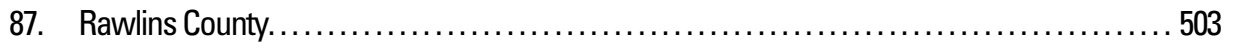

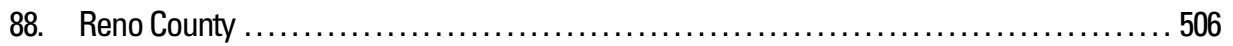

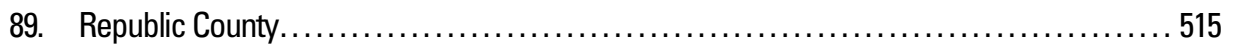

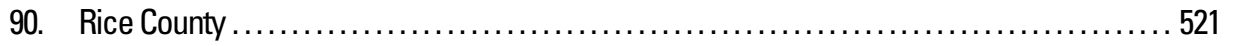

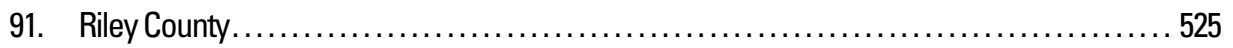

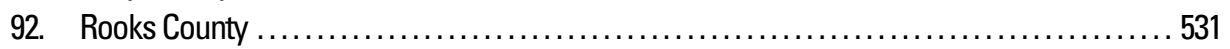

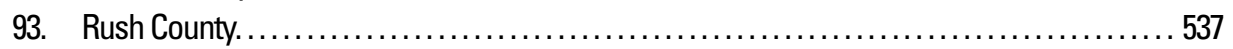

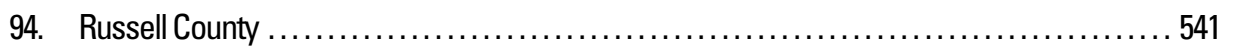

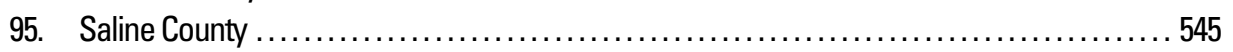

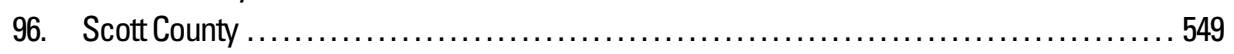

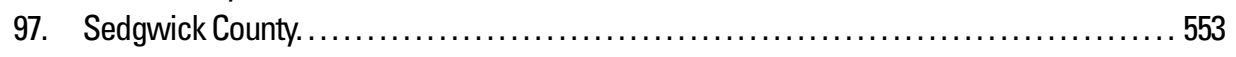

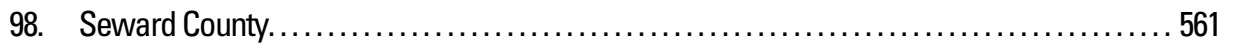

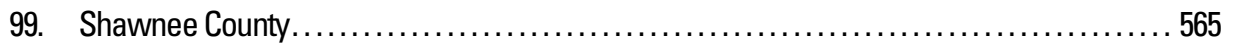

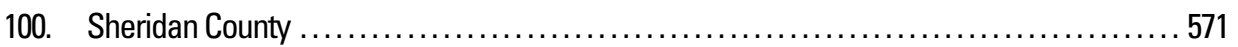

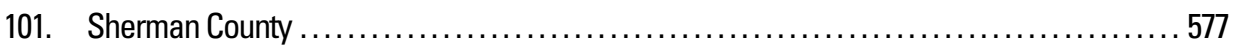

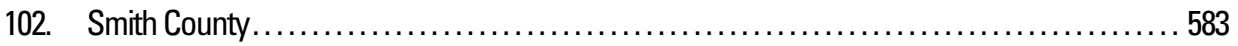




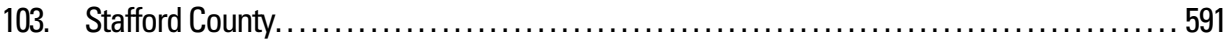

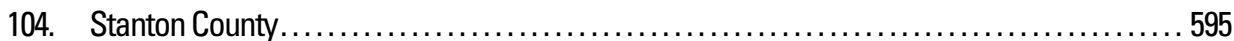

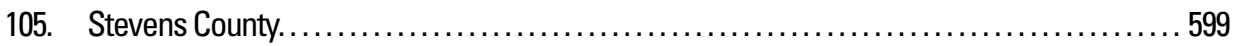

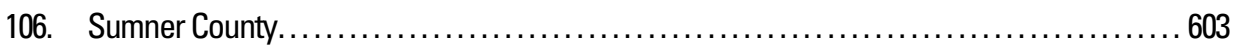

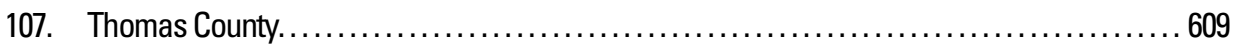

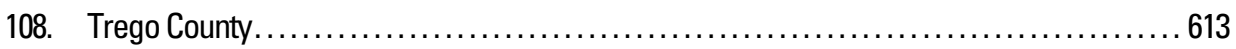

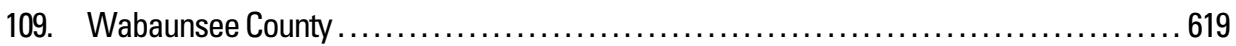

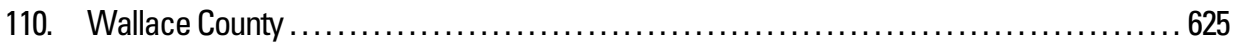

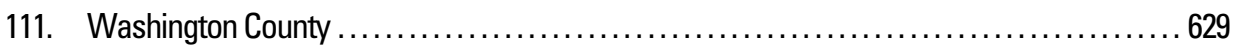

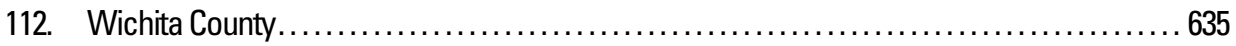

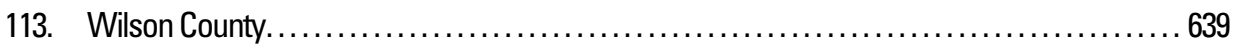

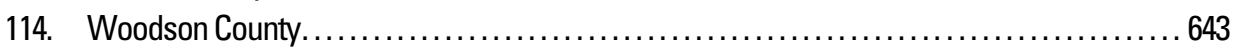

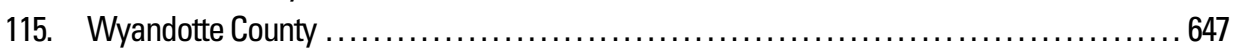

\section{Tables}

1. Streamflow-gaging stations and computed flow-duration values, mean discharge for period of uncontrolled flow record, and climatic and basin characteristics used in regression analyses of uncontrolled flow stream locations in Kansas .................................. 10

2. Regression equations used to estimate $90-, 75-, 50-, 25-$, and 10-percent flow durations and mean flow for uncontrolled flow stream locations on the 1999 Kansas Surface Water Register......... 22

3. Generalized least-squares regression equations for estimating 2- to 100-year peakstreamflow discharges for unregulated, rural streams in Kansas........................ 23

4. Summary of interpolation procedures used to estimate flow duration, mean flow, and peakdischarge flow information for streamflow-statistics determination sites on the 1999 Kansas Surface Water Register

5. Streamflow-gaging stations in Kansas and parts of surrounding States used in the interpolation of flow durations and mean flows at controlled and uncontrolled flow stream locations on the 1999 Kansas Surface Water Register.

6. Streamflow-gaging stations in Kansas and parts of surrounding States used in the interpolation of peak-discharge frequency values at controlled and uncontrolled flow stream locations on the 1999 Kansas Surface Water Register.

7-111. Estimated flow-duration values, mean flow values, and peak-discharge frequency values for controlled and uncontrolled flow stream segments on the 1999 Kansas Surface Water Register for:

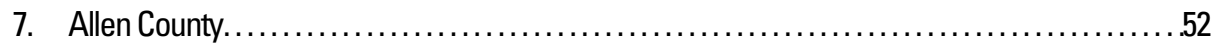

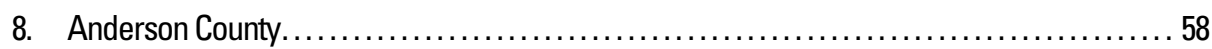

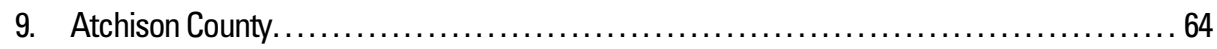

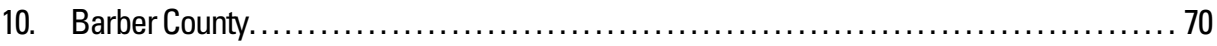

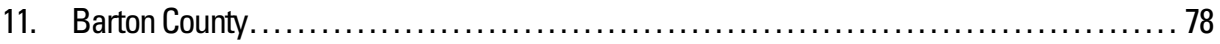

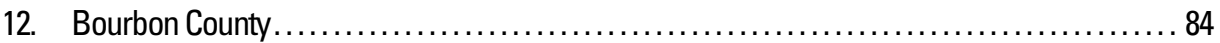

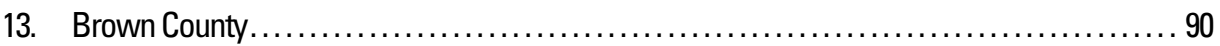

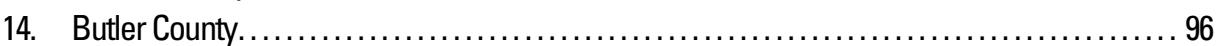

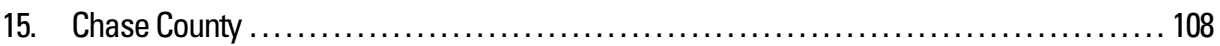

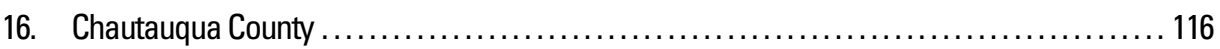

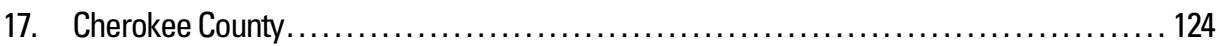

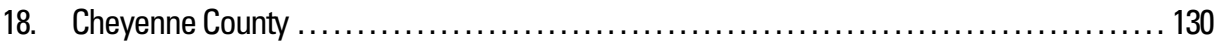

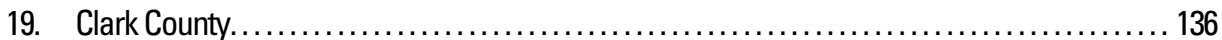

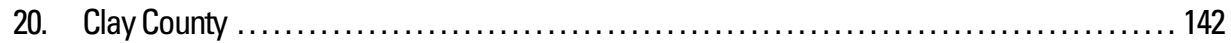




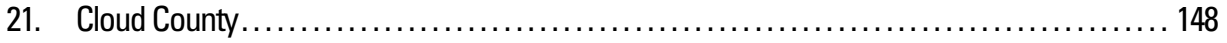

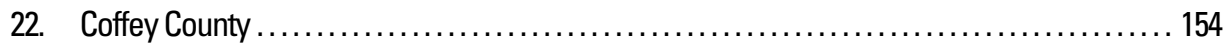

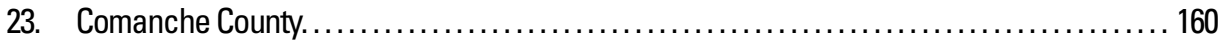

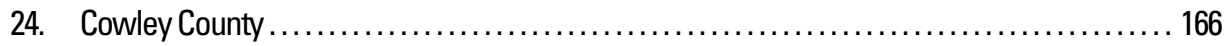

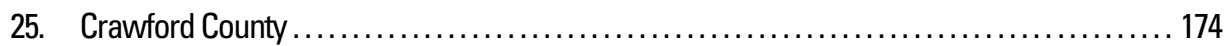

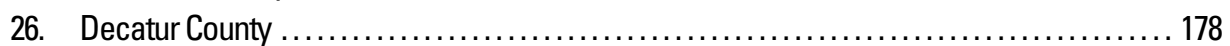

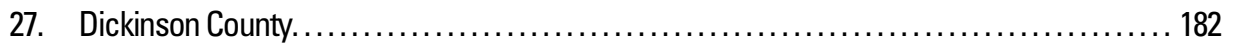

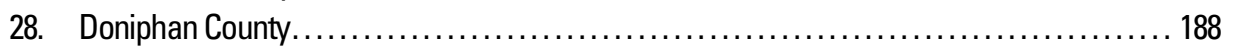

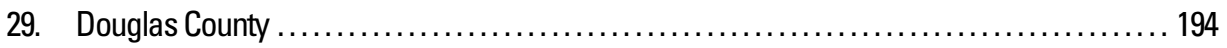

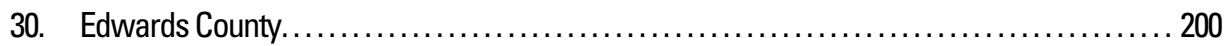

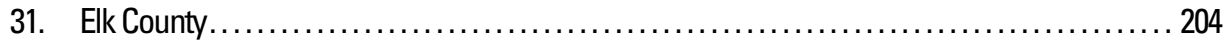

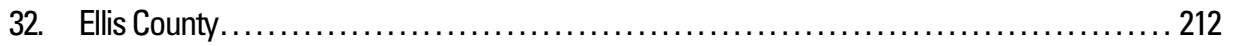

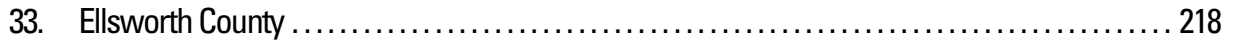

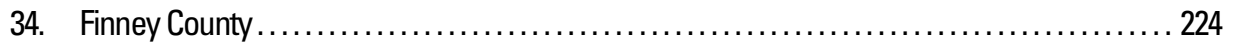

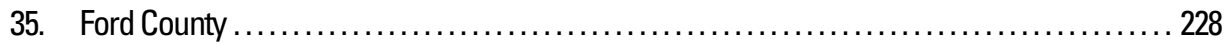

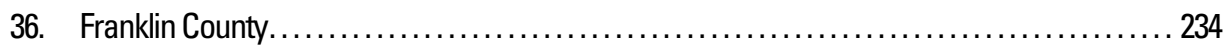

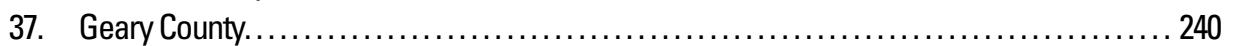

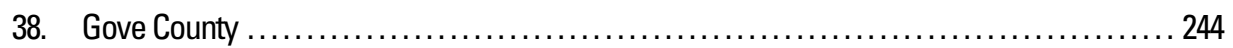

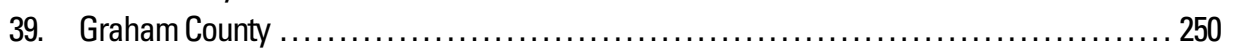

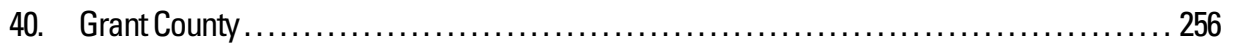

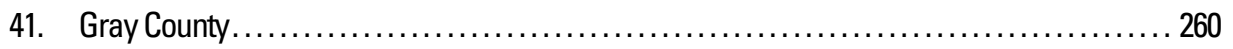

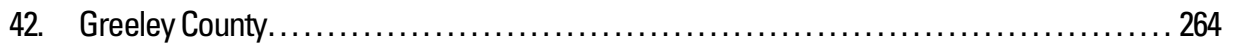

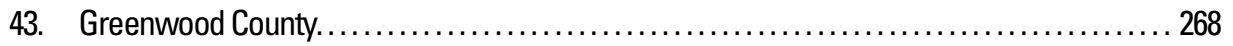

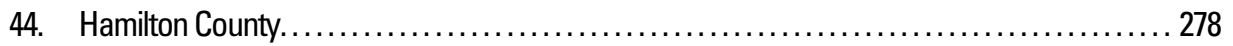

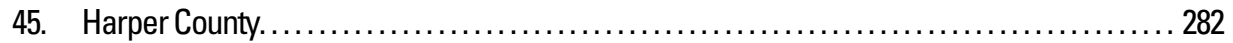

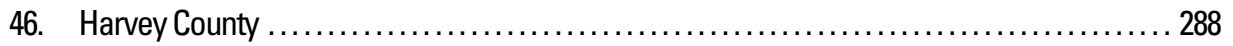

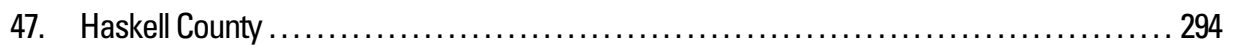

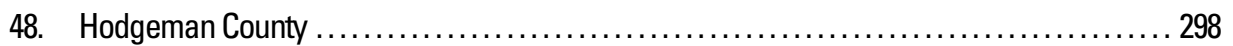

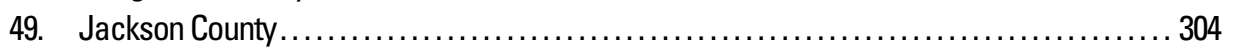

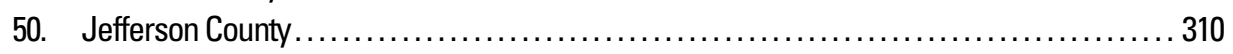

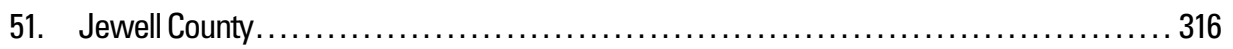

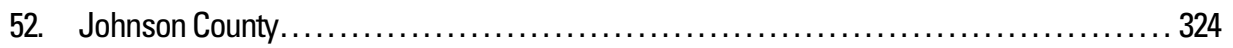

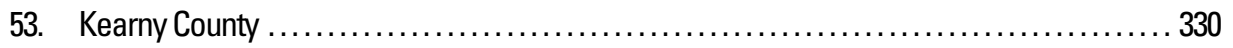

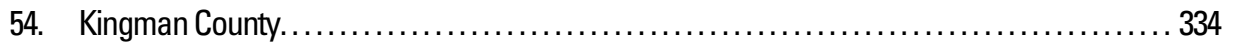

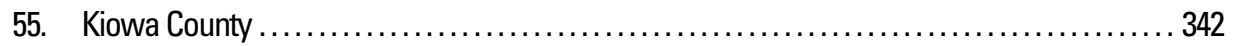

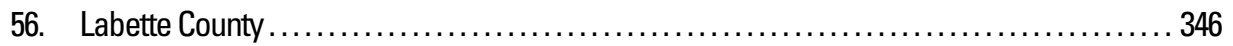

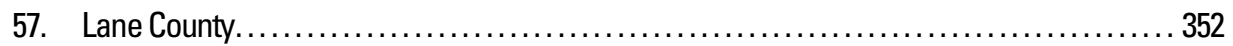

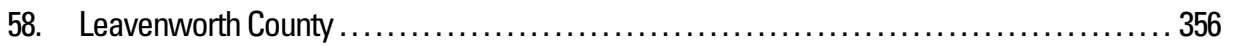

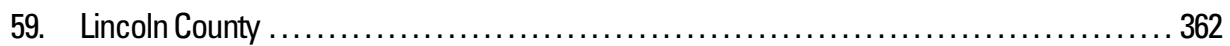

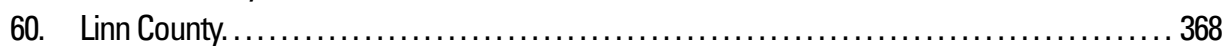

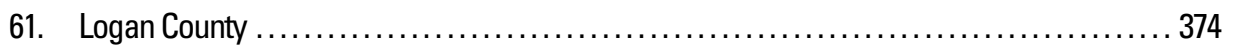

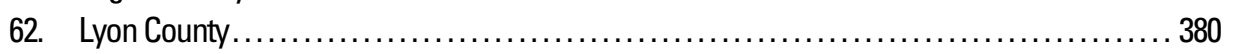

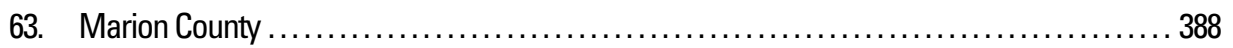

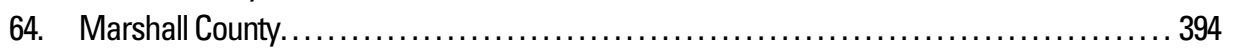

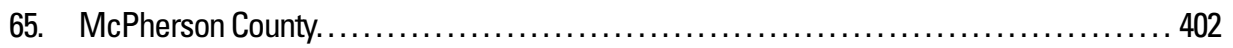

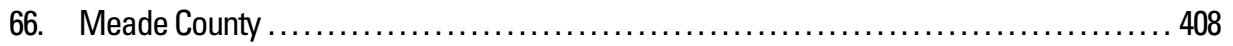

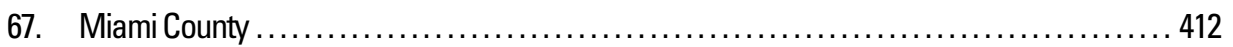

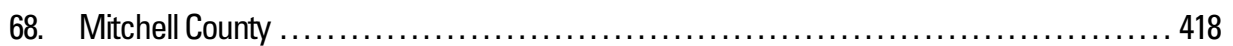

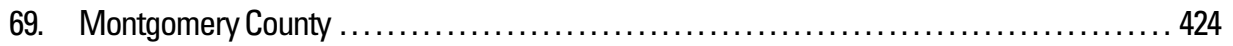




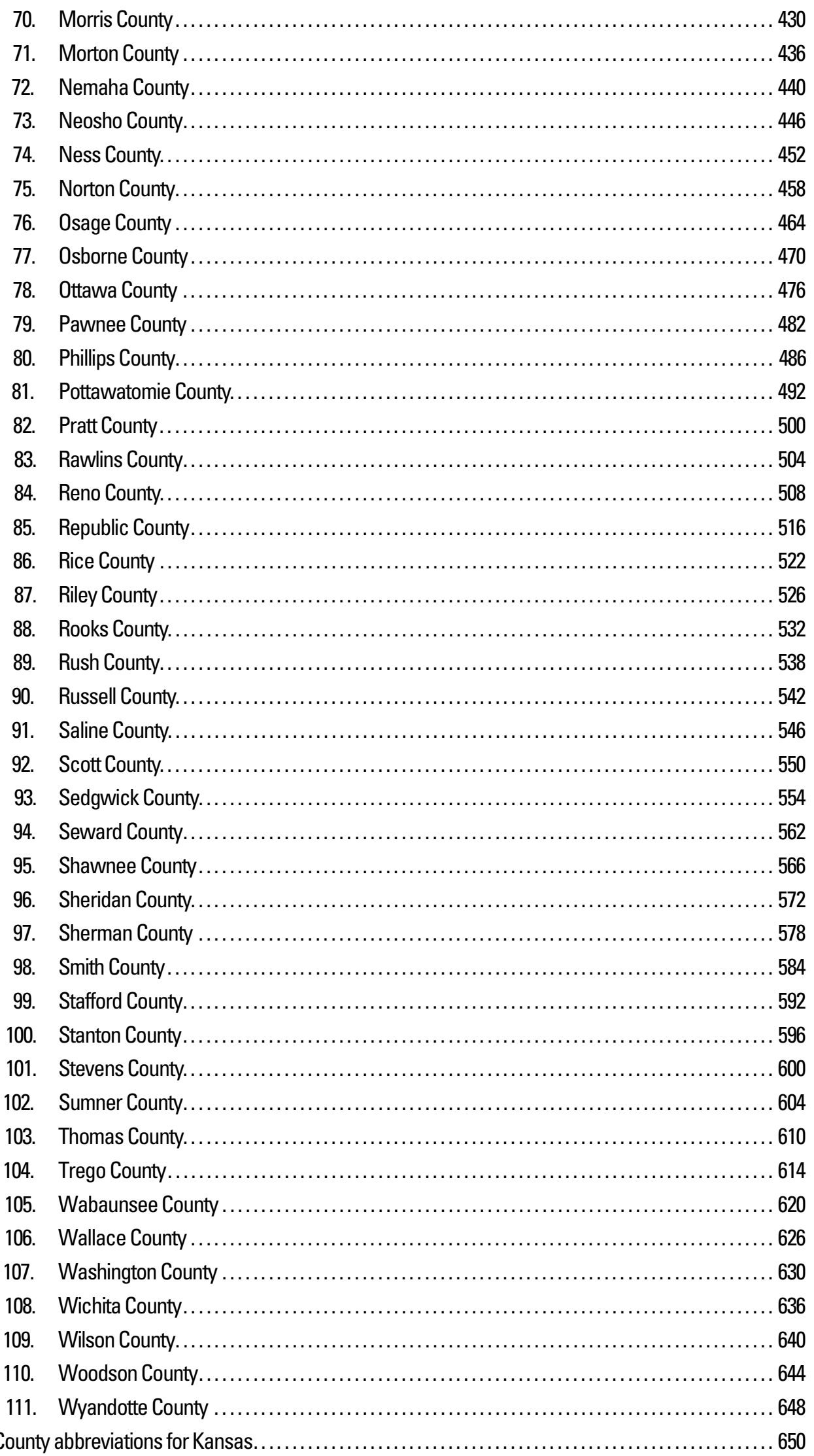




\section{Conversion Factors, Abbreviations, Datum, and Definitions}

\begin{tabular}{lll}
\hline Multiply & By & To obtain \\
\hline cubic foot per second $\left(\mathrm{ft}^{3} / \mathrm{s}\right)$ & 0.02832 & cubic meter per second $\left(\mathrm{m}^{3} / \mathrm{s}\right)$ \\
foot $(\mathrm{ft})$ & 0.3048 & meter $(\mathrm{m})$ \\
foot per mile $(\mathrm{ft} / \mathrm{mi})$ & 0.1894 & meter per kilometer $(\mathrm{m} / \mathrm{km})$ \\
inch (in.) & 2.54 & centimeter $(\mathrm{cm})$ \\
inch per hour $(\mathrm{in} / \mathrm{h})$ & 2.54 & centimeter per hour $(\mathrm{cm} / \mathrm{h})$ \\
meter $(\mathrm{m})$ & 3.281 & foot $(\mathrm{ft})$ \\
mile $(\mathrm{mi})$ & 1.609 & kilometer $(\mathrm{km})$ \\
square mile $\left(\mathrm{mi}^{2}\right)$ & 2.590 & square kilometer $\left(\mathrm{km}^{2}\right)$ \\
\hline
\end{tabular}

Horizontal coordinate information in this report is referenced to the North American Datum of 1983 (NAD 83).

Vertical coordinate information in this report is referenced to the North American Vertical Datum of 1988 (NAVD 88).

Water year in this report is the 12-month period being October 1 and ending September 30 . The water year is designated by the calendar year in which it ends. For example, the period October 1, 1999, through September 30, 2000, is called the "2000 water year." 


\title{
Estimates of Flow Duration, Mean Flow, and Peak- Discharge Frequency Values for Kansas Stream Locations
}

\author{
By Charles A. Perry, David M. Wolock, and Joshua C. Artman
}

\section{Abstract}

Streamflow statistics of flow duration and peak-discharge frequency were estimated for 4,771 individual locations on streams listed on the 1999 Kansas Surface Water Register. These statistics included the flow-duration values of 90, 75, 50, 25 , and 10 percent, as well as the mean flow value. Peak-discharge frequency values were estimated for the 2-, 5-, 10-, 25-, 50-, and 100-year floods.

Least-squares multiple regression techniques were used, along with Tobit analyses, to develop equations for estimating flow-duration values of $90,75,50,25$, and 10 percent and the mean flow for uncontrolled flow stream locations. The contributing-drainage areas of 149 U.S. Geological Survey streamflow-gaging stations in Kansas and parts of surrounding States that had flow uncontrolled by Federal reservoirs and used in the regression analyses ranged from 2.06 to 12,004 square miles. Logarithmic transformations of climatic and basin data were performed to yield the best linear relation for developing equations to compute flow durations and mean flow.

In the regression analyses, the significant climatic and basin characteristics, in order of importance, were contributingdrainage area, mean annual precipitation, mean basin permeability, and mean basin slope. The analyses yielded a model standard error of prediction range of 0.43 logarithmic units for the 90 -percent duration analysis to 0.15 logarithmic units for the 10 -percent duration analysis. The model standard error of prediction was 0.14 logarithmic units for the mean flow. Regression equations used to estimate peak-discharge frequency values were obtained from a previous report, and estimates for the 2-, 5-, 10-, 25-, 50-, and 100-year floods were determined for this report.

The regression equations and an interpolation procedure were used to compute flow durations, mean flow, and estimates of peak-discharge frequency for locations along uncontrolled flow streams on the 1999 Kansas Surface Water Register. Flow durations, mean flow, and peak-discharge frequency values determined at available gaging stations were used to interpolate the regression-estimated flows for the stream locations where available. Streamflow statistics for locations that had uncontrolled flow were interpolated using data from gaging stations weighted according to the drainage area and the bias between the regression-estimated and gaged flow information. On controlled reaches of Kansas streams, the streamflow statistics were interpolated between gaging stations using only gaged data weighted by drainage area.

\section{Introduction}

The expected amount and historical range of flow in Kansas streams are important considerations for the classification, evaluation, and regulation of water supplies, recreation, aquatic-life habitat, pollution control, and the design of hydrologic structures within the State. Water-quality regulations in Kansas apply numeric water-quality criteria to classified stream segments. These 2,232 stream segments are listed on the 1999 Kansas Surface Water Register. This register is maintained by the Kansas Department of Health and Environment (KDHE) and is used to identify designated uses of stream segments.

Flow-duration statistics for specific locations throughout Kansas and parts of surrounding States are available for 216 continuous-record streamflow-gaging stations where daily flow data have been collected by the U.S. Geological Survey (USGS) for 10 or more years (fig. 1). Peak-discharge frequency statistics for 253 peak streamflow-gaging stations in Kansas were used to develop regression equations to estimate peak streamflow at unregulated rural streams (Rasmussen and Perry, 2000). Two hundred and thirty-seven streamflow-gaging stations were used in the interpolation process described in a later section of this report (fig. 2). The current and historical streamflow information collected by the USGS provides a resource for estimating the expected amount and range of streamflow throughout the State. The measured streamflow record can be used to define statistics that summarize historical streamflow amounts at each stream gage. These statistics then can be related to the physical characteristics of the drainage basins that contribute to measured flow at the gage. Furthermore, statistical models that are based on these relations can be used to estimate streamflow statistics for ungaged locations.

To address the need for streamflow statistics, a study of flow durations, mean flow, and peak-discharge frequency information for Kansas streams was conducted by the USGS in cooperation with KDHE and the Kansas Department of Transportation. Streamflow data used in this study were collected by the USGS (Putnam and others, 2001) through other cooperative 


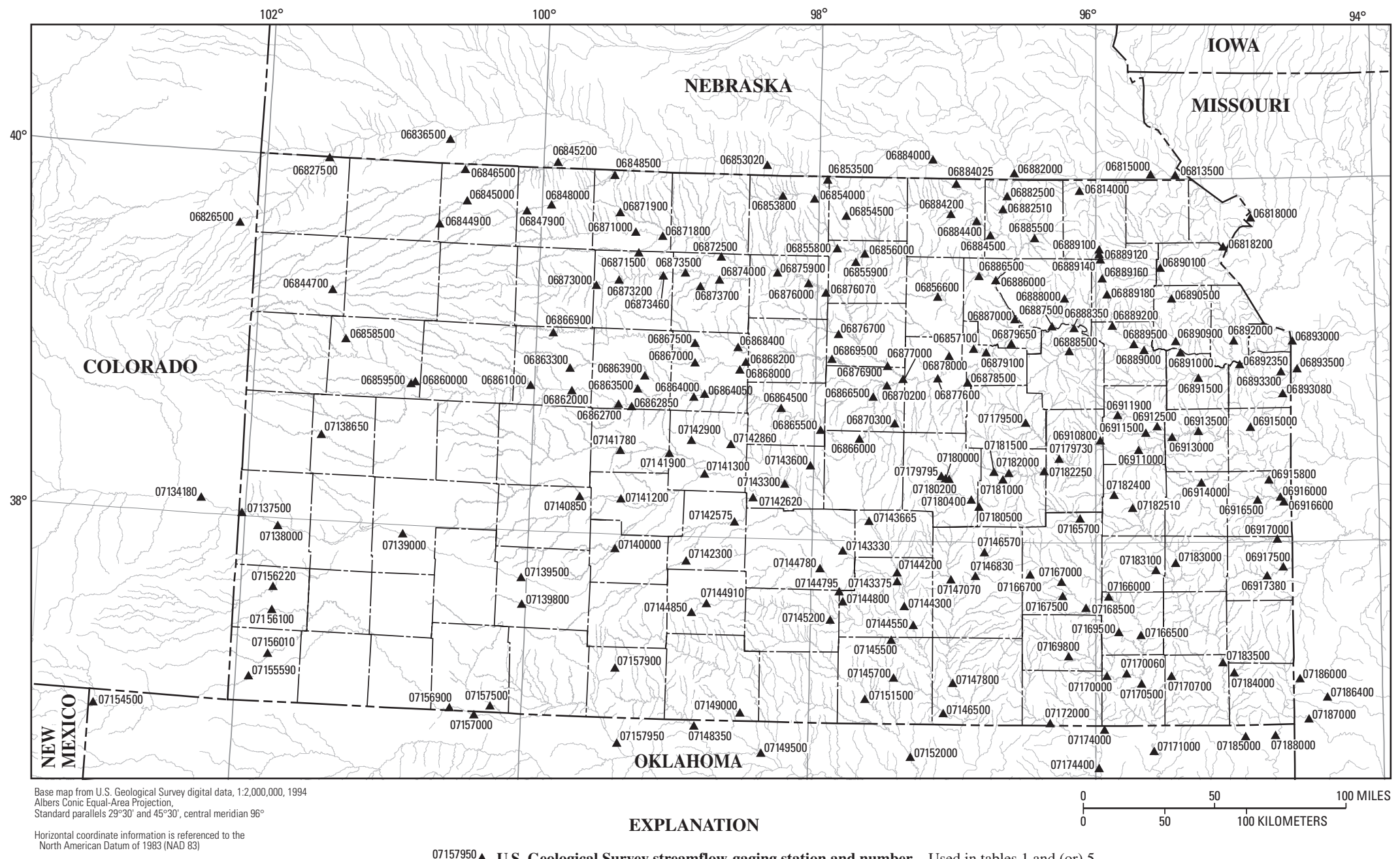

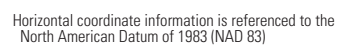

07157950_ U.S. Geological Survey streamflow-gaging station and number-Used in tables 1 and (or) 5

Figure 1. Location of U.S. Geological Survey streamflow-gaging stations in Kansas and parts of surrounding States with 10 or more years of record that were available for computing flow durations and mean flows. 


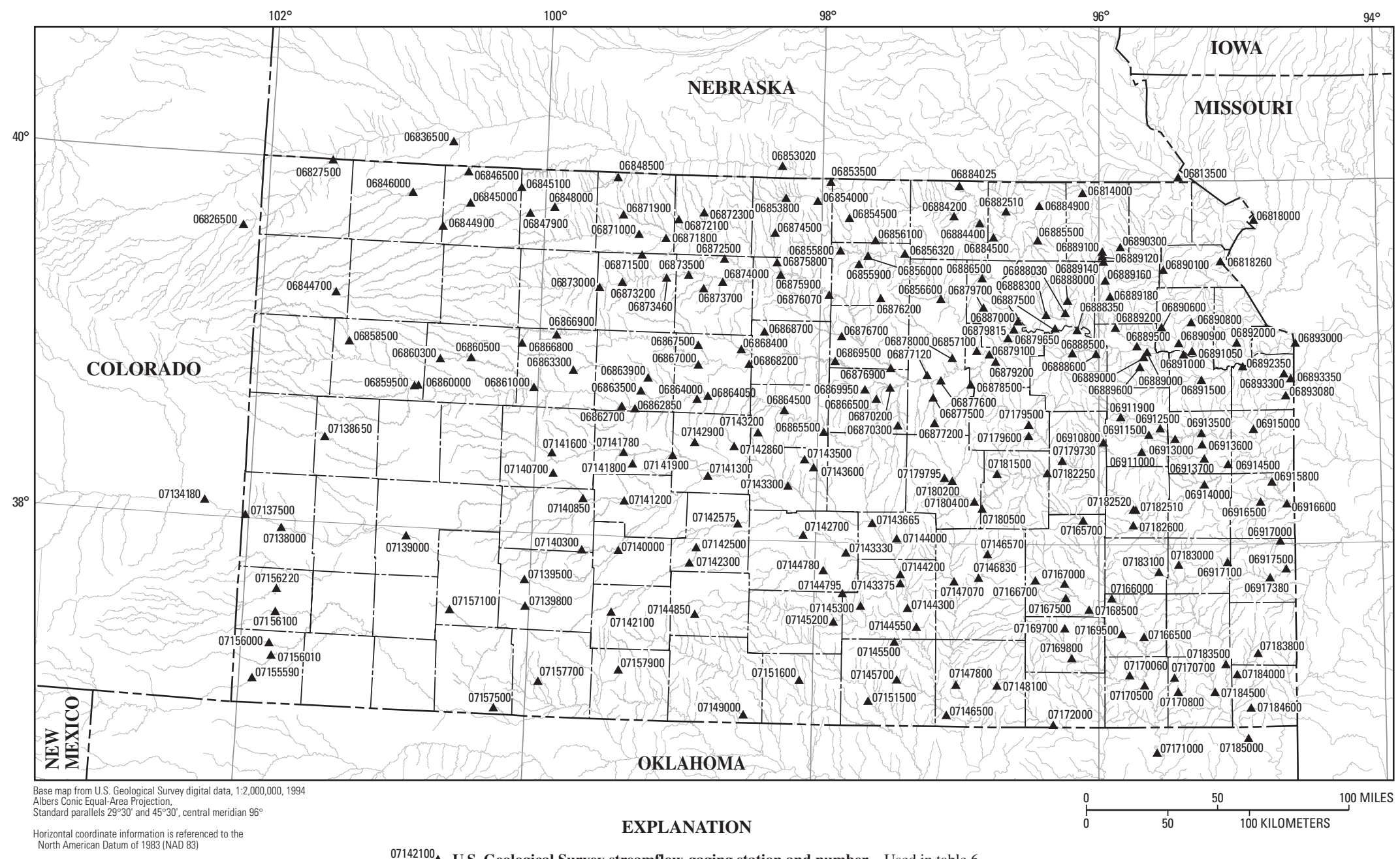

$07142100 \triangle$ U.S. Geological Survey streamflow-gaging station and number-Used in table 6

Figure 2. Location of U.S. Geological Survey streamflow-gaging stations with peak-discharge frequency analysis in Kansas and parts of surrounding States with 10 or more years of record that were used in the interpolation process. 


\section{Estimates of Flow Duration, Mean Flow, and Peak-Discharge Frequency Values for Kansas Stream Locations}

studies with various government agencies. This report includes analyses of flow durations of 90, 75, 50, 25, and 10 percent, mean flows, and the peak-discharge frequency values for the 2-, 5-, 10-, 25-, 50-, and 100-year floods.

\section{Purpose and Scope}

The purpose of this report is to document the methods and results of a study designed to estimate the flow durations, mean flow, and the peak-discharge frequency values for locations on streams on the 1999 Kansas Surface Water Register (KSWR). In this report, 5,427 locations along the KSWR stream coverage were identified as determination sites for which flow statistics were computed from upstream climatic and basin conditions. Flow statistics were not reported for 656 of these determination sites because they were located on reservoirs or irrigation ditches. Flow statistics for each determination site either were computed from gaged-location streamflow records, estimated from statewide regression models, or interpolated between gaged locations or gaged and regression-estimated locations.

This report documents the development of regression models to estimate flow durations and mean flow from climatic and basin characteristics. Regression equations for flow durations were developed using Tobit statistical analysis of uncontrolled flow durations determined at 149 gaging stations.

"Uncontrolled flow" is defined as that streamflow that is unaffected by Federal reservoir releases. The entire period of record for each uncontrolled flow streamflow-gaging station was used in the analyses of flow durations. Regression equations for the estimation of peak discharge for unregulated rural streams in Kansas are available in (Rasmussen and Perry, 2000) and are used in this report. The equations were based on 253 streamflow-gaging stations in Kansas.

This report describes application of the drainage-area ratio method and the regression model method of estimating the flow statistics for stream locations on the 1999 KSWR, the interpolation of estimates for ungaged locations, and the Internet dissemination of results and a geographic-information-system (GIS) database. The information contained in this report can be used by State agencies and others to help in the effective management of Kansas surface-water resources. Optimal reservoir operations, legally distributed in-stream withdrawals, and water-quality concerns are issues directly linked to flow durations. Peak-discharge frequency information is used in the cost effective construction of hydraulic structures such as bridges and culverts and for flood-plain management. The methods described herein can be applied nationwide using USGS streamflow data that are available throughout the United States.

\section{Previous Studies}

A study by Perry and others (2004) determined the median flow (50-percent duration) for the 2,232 complete stream segments on the 1999 KSWR. The methods of analysis for the flow durations and mean flow in this report are similar to the study by Perry and others (2004).

Previous low-flow and flow-duration studies for Kansas include an investigation by Furness (1959) who developed a method for estimating flow-duration curves for ungaged sites that was based on regionalized flow-duration data from 122 continuous-record, streamflow-gaging stations with drainage areas of between 100 and 3,000 $\mathrm{mi}^{2}$ for the period 1921-56. Maps were developed showing a variety of statewide low and mean streamflow. Furness (1959) also noted that the low-flow parts of the flow-duration curves could be verified or improved by relating base-flow measurements at the ungaged site to baseflow measurements at a nearby, index streamflowgaging station.

Jordan (1983) updated the maps developed by Furness (1959) by including additional streamflow-gaging stations and data for the period 1957-76. Jordan's study included a map that depicted the areas of Kansas where the median streamflow for a $500-\mathrm{mi}^{2}$ basin was greater than $0.1 \mathrm{ft}^{3} / \mathrm{s}$. Two studies by Studley $(2000,2001)$ evaluated the application of the Furness method to ungaged stream sites in Kansas using nearby streamflow-gaging stations as index stations. The results of these two recent studies indicated that the Furness method continues to be a useful tool for estimating flow-duration curves for ungaged sites and that the method could be used for sites with drainage areas less than $100 \mathrm{mi}^{2}$.

Many studies have been conducted to evaluate low flow and peak flows from regression equations that relate low flow and peak flow to basin characteristics. In a recent USGS study (Ries and Friesz, 2000), basin characteristics were determined from digital map data, and flow statistics were computed for individual stream segments in Massachusetts using GIS techniques. Ries and Friesz (2000) used the drainage-area ratio method to compute streamflow characteristics for stream segments in Massachusetts that had between 0.5 and 1.5 times the drainage area of streamflow-gaging stations on the same stream. Many other States have used regression analysis to regionalize low-flow frequency statistics including New Hampshire, Rhode Island, and Vermont (Johnson, 1970); New York (Ku and others, 1975); Montana (Parrett and Hull, 1985); Indiana (Arihood and Glatfelter, 1991); and central New England (Wandle and Randall, 1994). All the States have conducted regression analyses to regionalize peak-discharge frequency values, and these studies have been collected into a national report by Ries and Crouse (2002).

\section{Acknowledgments}

The authors would like to thank Aldo "Skip" Vecchia, Timothy Cohn, and Gregory Schwarz of the USGS who helped with the Tobit analyses and with the review of the statistical methods used in this report. The authors also would like to acknowledge the efforts of Michael Butler from KDHE who provided the initial stream coverage and reviewed much of the data presented. 


\section{Factors Affecting Streamflow}

\section{Physical Setting}

Physiographically, Kansas is located almost entirely within the Interior Plains as described by Schoewe (1949). A description of the hydrologic characteristics of the physiographic provinces within the Interior Plains is beyond the scope of this report, but the fact that there are significant variations denotes the complex nature of and difficulty in attempting to define flow characteristics across Kansas.

The topography of the western two-thirds of the State is typical of the High Plains region (Rasmussen and Perry, 2000) and is characterized by flat or gently sloping surfaces with little relief. The topography of the eastern one-third of the State is more variable, with alternating hills and lowlands. Land-surface elevations within the State range from about $700 \mathrm{ft}$ above the North American Vertical Datum of 1988 (NAVD 88) at the Kansas-Oklahoma State line in southeast Kansas to about $4,135 \mathrm{ft}$ above the NAVD 88 at a point near the KansasColorado State line in western Kansas-a vertical difference of about 3,435 ft (fig. 3). The average land-surface slope for Kansas (fig. 4) using 30-m grid elevation data (U.S. Geological Survey, 1998) is about 1.9 degrees.

Other physical characteristics affecting the flow characteristics of watersheds are the types of soils and land-use and treatment practices within the watershed. For example, with all other factors being equal the low-flow potential from watersheds with soils of low permeability (fig. 5) is less than that from watersheds where highly permeable soils tend to allow greater infiltration and a greater ground-water contribution to base flow of the stream (Thomas, 1966). The western two-thirds of the State typically has soils of moderate to high permeability, whereas the eastern one-third has soils of lower permeability. Landtreatment practices, such as contour farming and construction of water-retention structures, can increase the amount of infiltration of runoff to ground water, which ultimately can return to stream channels as base flow. However, land-treatment practices are difficult to assess and apply to the various types of basins statewide.

\section{Climatic Characteristics}

The climate of Kansas is affected by the movement of various air masses of tropical and continental origin over the open, inland plains, and seasonal precipitation extremes are common. About 75 percent of the mean annual precipitation falls from April through September. Precipitation during early spring and late fall occurs in association with frontal air masses that produce low-intensity rainfall of regional coverage. During the summer months, the weather is dominated by warm, moist air from the Gulf of Mexico or by hot, dry air from the Southwest. Summer precipitation generally occurs as high-intensity thunderstorms (Paulson and others, 1991).
Watersheds in Kansas exhibit a wide range of climatic characteristics that affect streamflow. Generally, precipitation varies in an east-west direction, with little north-south variation. The general climate of the western part of Kansas is semiarid with hot, dry summer months and cold, windy winter months. The eastern part of the State tends to be more humid, with sultry summer months and cold, damp winter months. Mean annual precipitation, the major climatic factor affecting streamflow in the State, varies from about 16 in. in extreme western Kansas to about 42 in. in southeastern Kansas (Daly and others, 1997) (fig. 6). Mean annual precipitation values for 149 streamflowgaging station basins used in the regression analyses of flow durations and mean flow for uncontrolled flow stream locations on the KWSR are given in table 1.

\section{Basin Characteristics}

Basin characteristics used in the regression analyses were selected on the basis of their theoretical relation to differences in flow magnitudes of streams, results of previous studies in similar hydrologic environments, and on the ability to measure the characteristics. The basin characteristics considered in this report included contributing-drainage area, in square miles; mean basin elevation, in feet above NAVD 88; mean basin permeability, in inches per hour; mean basin slope, in degrees; a base-flow index (Wahl and Wahl, 1995); mean annual runoff for hydrologic basins in the United States, in cubic feet per second (Gebert and others, 1987); and runoff from a water-balance model (Wolock and McCabe, 1999) (parameter-elevation regressions on independent slope model), in cubic feet per second, using the mean annual precipitation grid for the United States developed by Daly and others (1994). The mean annual runoff reflects the difference between precipitation and evapotranspiration. Selected basin characteristics for the 149 streamflow-gaging stations used in the final regression analyses for uncontrolled flow stream locations are provided in table 1 .

All basin characteristics were measured from digital-map data using automated GIS procedures. The automated procedure was created using the AML programming language of the ARC/INFO GIS software (Environmental Systems Research Institute, Inc., 1991). The automated procedure determined the drainage-basin boundary at the gaging station or for the downstream end of a stream segment and created a digital data layer of the basin boundary, then overlaid the boundary on the other digital data layers to determine the other basin characteristics for the station or location. The grid values then were averaged for the area within the contributing-drainage basin. Basin slope was determined from the 30-m elevation grid using the GIS command SLOPE (Burrough, 1986). The slope in degrees is essentially an average of the slope between the center grid cell and its eight surrounding cells.

The peak-discharge frequency regression equations developed by Rasmussen and Perry (2000) require channel slope as one of their variables. Channel slope is defined as the difference 


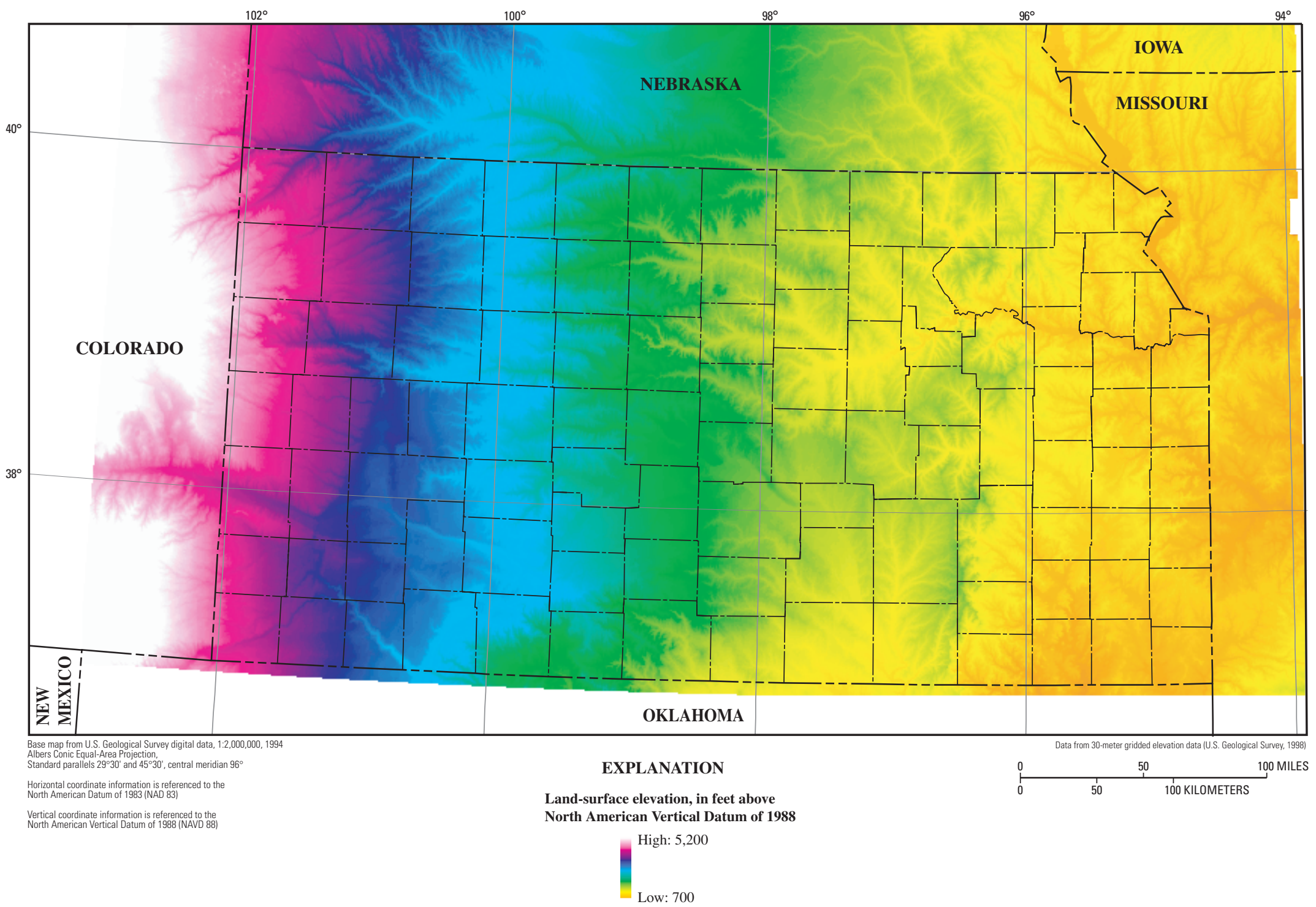

Figure 3. Land-surface elevation in Kansas and parts of surrounding States. 


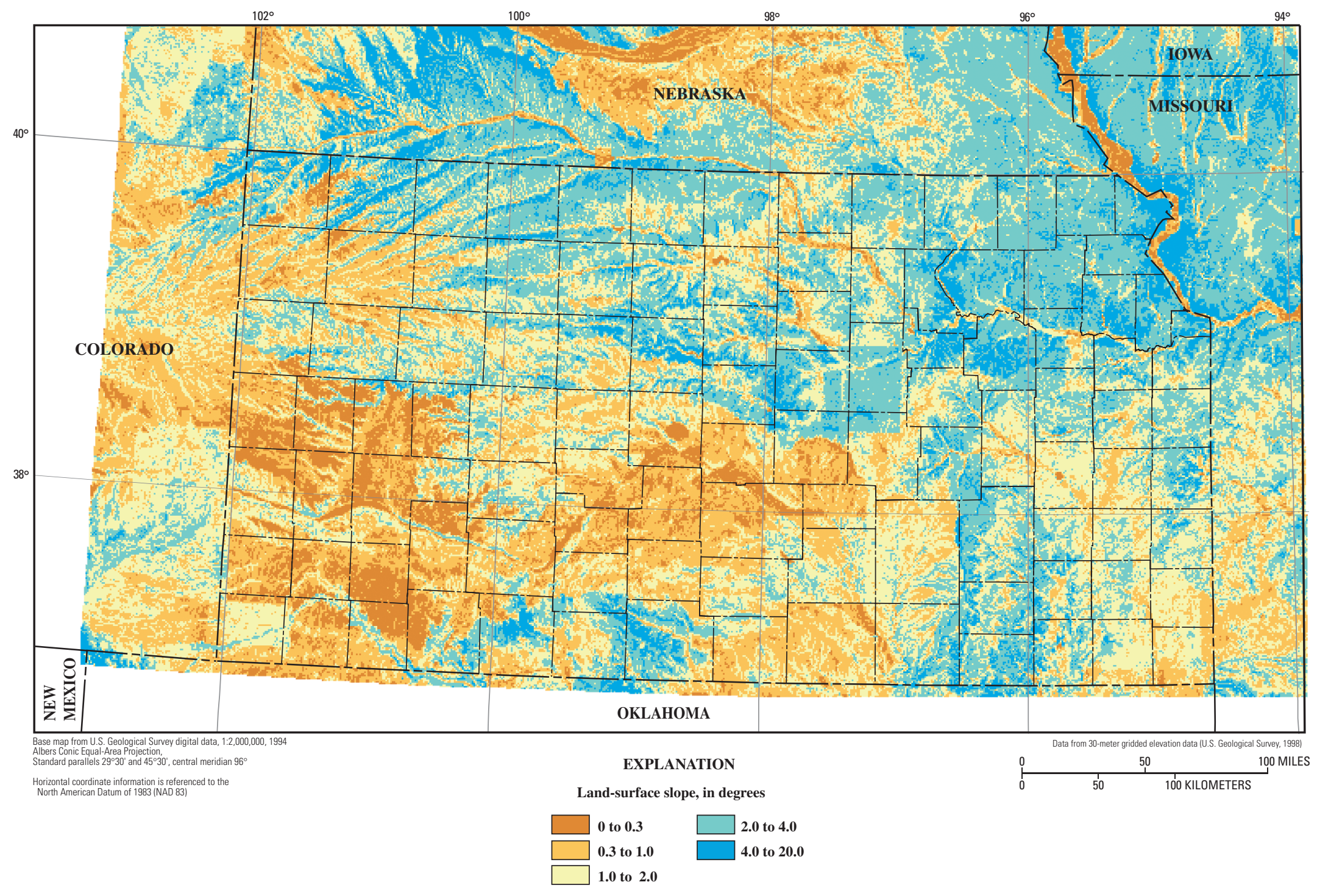

Figure 4. Average land-surface slope in Kansas and parts of surrounding States. 


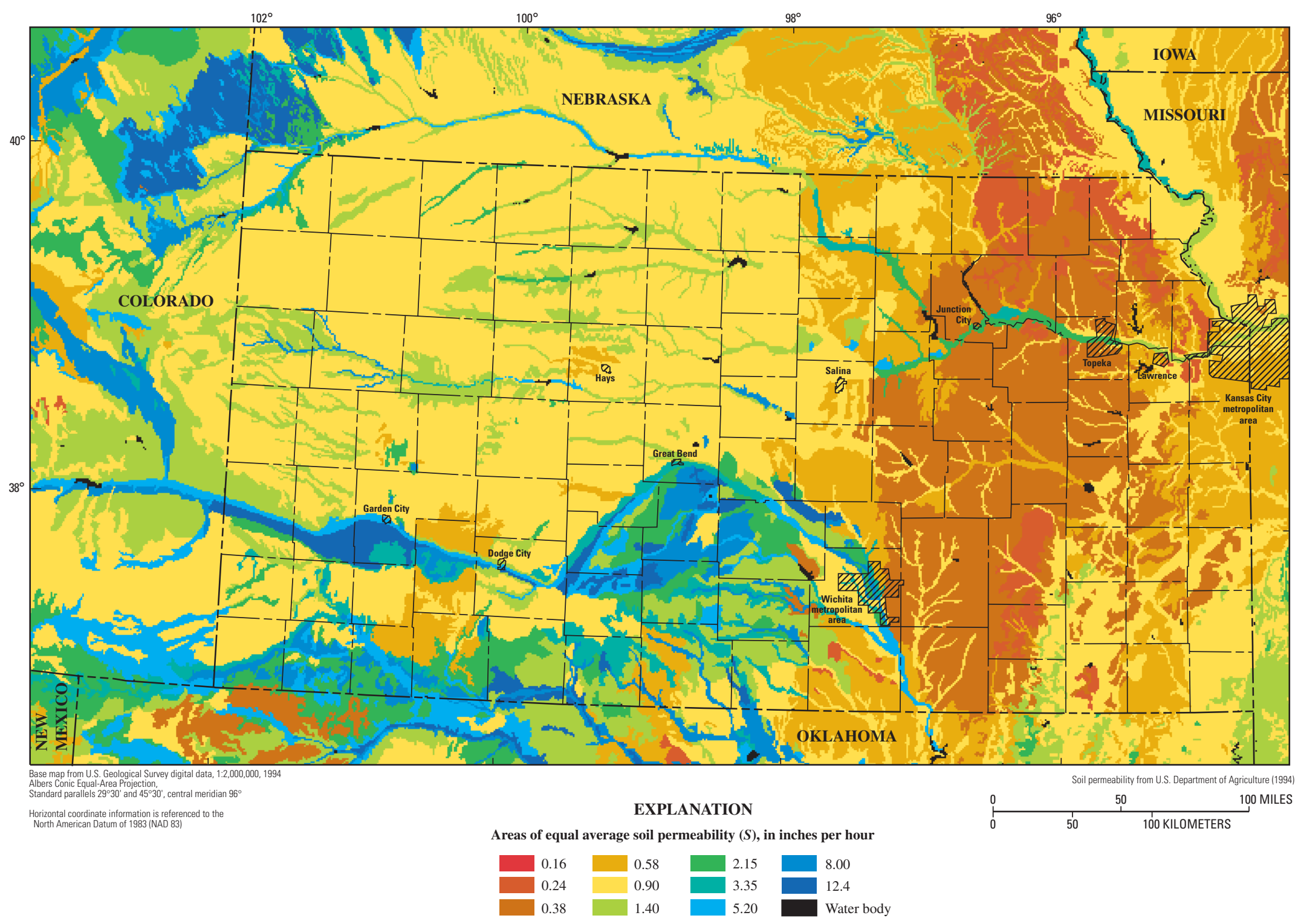

Figure 5. Areas of equal average soil permeability in Kansas and parts of surrounding States. 


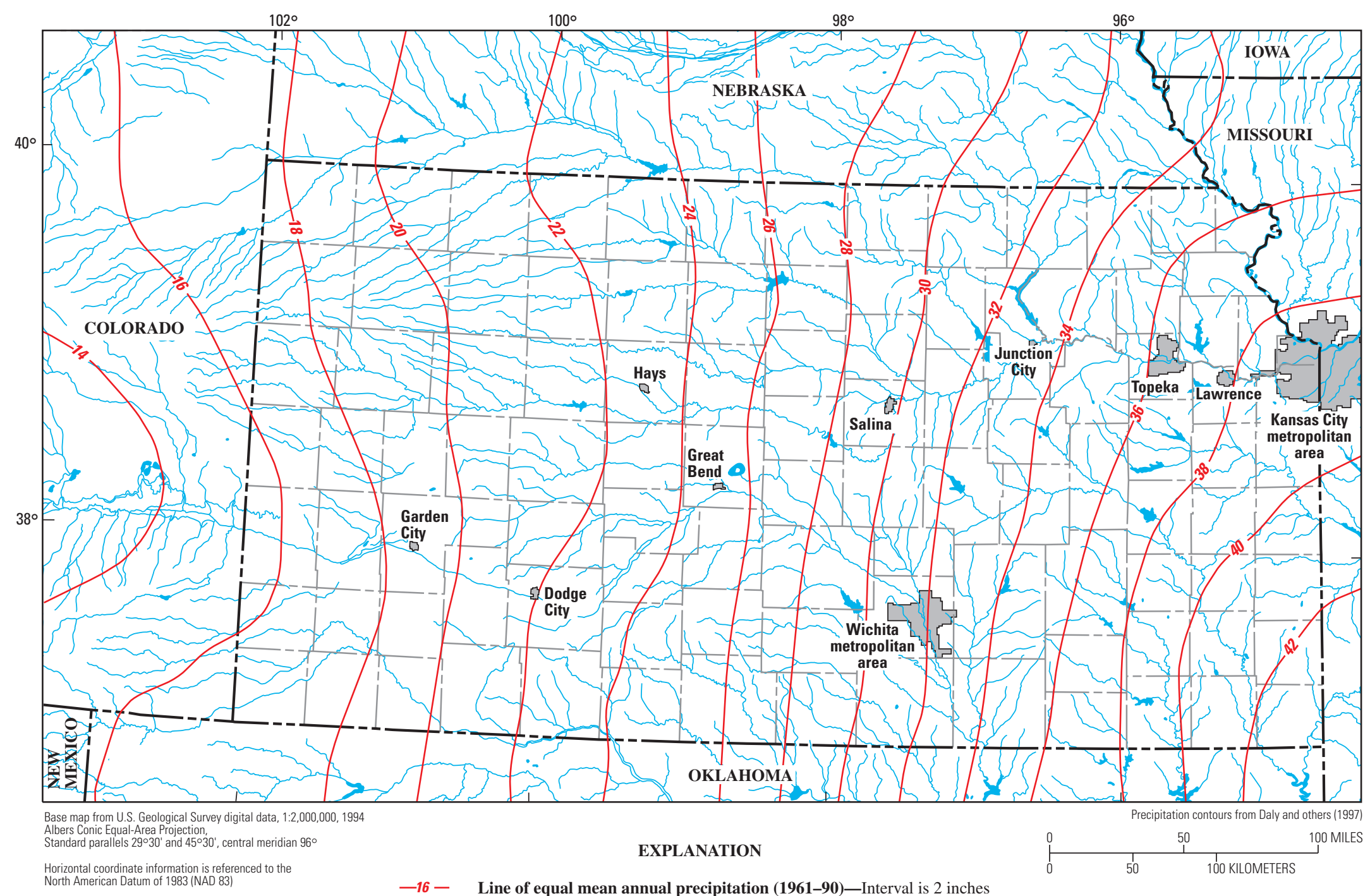

Figure 6. Mean annual precipitation in Kansas and parts of surrounding States (Daly and others, 1997). 
Table 1. Streamflow-gaging stations and computed flow-duration values, mean discharge for period of uncontrolled flow record, and climatic and basin characteristics used in regression analyses of uncontrolled flow stream locations in Kansas.

$\left[\mathrm{ft}^{3} / \mathrm{s}\right.$, cubic feet per second; $\mathrm{mi}^{2}$, square miles; in/h, inches per hour]

\begin{tabular}{|c|c|c|c|c|c|c|c|c|c|c|c|c|c|}
\hline \multirow[b]{2}{*}{$\begin{array}{c}\text { Station } \\
\text { number } \\
\text { (fig. 1) }\end{array}$} & \multirow[b]{2}{*}{ Station name } & \multirow[b]{2}{*}{$\begin{array}{c}\text { Period of } \\
\text { record } \\
\text { (water years) }\end{array}$} & \multirow[b]{2}{*}{$\begin{array}{l}\text { Years } \\
\text { of } \\
\text { record }\end{array}$} & \multicolumn{5}{|c|}{$\begin{array}{l}\text { Flow-duration values }\left(\mathrm{ft}^{3} / \mathrm{s}\right) \text { for indicated } \\
\text { percentage of time flow equaled or exceeded }\end{array}$} & \multirow{2}{*}{$\begin{array}{c}\text { Mean } \\
\text { discharge } \\
\text { for period } \\
\text { of uncon- } \\
\text { trolled } \\
\text { record } \\
\left(\mathrm{ft}^{3} / \mathbf{s}\right)\end{array}$} & \multirow{2}{*}{$\begin{array}{c}\text { Contribut- } \\
\text { ing- } \\
\text { drainage } \\
\text { area } \\
\left(\mathrm{mi}^{2}\right)\end{array}$} & \multirow{2}{*}{$\begin{array}{c}\text { Mean } \\
\text { annual } \\
\text { precipita- } \\
\text { tion, } \\
\text { 1961-901 } \\
\text { (inches) }\end{array}$} & \multirow{2}{*}{$\begin{array}{c}\text { Mean } \\
\text { basin } \\
\text { perme- } \\
\text { ability }{ }^{2} \\
\text { (in/h) }\end{array}$} & \multirow{2}{*}{$\begin{array}{c}\text { Mean } \\
\text { basin } \\
\text { slope }^{3} \\
\text { (degrees) }\end{array}$} \\
\hline & & & & $\begin{array}{c}90- \\
\text { percent }\end{array}$ & $\begin{array}{c}75- \\
\text { percent }\end{array}$ & $\begin{array}{c}50- \\
\text { percent }\end{array}$ & $\begin{array}{c}25- \\
\text { percent }\end{array}$ & $\begin{array}{c}10- \\
\text { percent }\end{array}$ & & & & & \\
\hline$\overline{06814000}$ & Turkey Creek near Seneca, KS & $1950-2000$ & 51 & 2.1 & 7.4 & 22 & 69 & 203 & 129 & 276 & 32.35 & 0.467 & 3.1 \\
\hline 06815000 & Big Nemaha River at Falls City, NE & 1944-2000 & 56 & 45 & 80 & 159 & 390 & 1,050 & 631 & 1,339 & 32.55 & .510 & 2.8 \\
\hline 06818200 & Doniphan Creek at Doniphan, KS & $1961-70$ & 10 & .24 & .50 & .87 & 1.6 & 3.8 & 3.0 & 4.15 & 36.71 & 1.08 & 4.8 \\
\hline 06836500 & Driftwood Creek near McCook, NE & $1977-86$ & 10 & .30 & 2.1 & 4.8 & 6.7 & 11 & 9.5 & 361 & 20.94 & 1.30 & 2.9 \\
\hline 06844700 & South Fork Sappa Creek near Brewster, KS & $1968-87$ & 20 & 0 & 0 & 0 & 0 & 0 & .23 & 71.3 & 18.40 & 1.30 & .83 \\
\hline 06844900 & South Fork Sappa Creek near Achilles, KS & 1960-2000 & 41 & 0 & 0 & 0 & .32 & 2.3 & 3.4 & 412 & 19.20 & 1.30 & 1.4 \\
\hline 06845000 & Sappa Creek near Oberlin, KS & 1930-2000 & 71 & 0 & .10 & .70 & .54 & 18 & 16 & 1,086 & 19.82 & 1.32 & 1.5 \\
\hline 06845200 & Sappa Creek near Beaver City, NE & $1938-72$ & 35 & 0 & 1.1 & 5.0 & 17 & 60 & 38 & 1,500 & 20.57 & 1.36 & 1.9 \\
\hline 06847900 & Prairie Dog Creek above Keith Sebelius Lake, KS & $1963-2000$ & 38 & 0 & .16 & 2.1 & 5.8 & 11 & 9.0 & 590 & 20.65 & 1.36 & 1.7 \\
\hline 06848000 & Prairie Dog Creek at Norton, KS & $1945-63$ & 19 & .70 & 3.0 & 7.5 & 15 & 42 & 39 & 684 & 20.96 & 1.36 & 1.9 \\
\hline 06848500 & Prairie Dog Creek near Woodruff, KS & $1930-63$ & 34 & 0 & 3.7 & 10 & 23 & 71 & 57 & 1,007 & 21.56 & 1.37 & 2.1 \\
\hline 06853800 & White Rock Creek near Burr Oak, KS & $1958-2000$ & 43 & .47 & 1.8 & 6.0 & 17 & 40 & 29 & 227 & 26.49 & 1.30 & 2.5 \\
\hline 06854000 & White Rock Creek at Lovewell, KS & $1947-56$ & 10 & 0 & 1.6 & 5.7 & 20 & 80 & 68 & 354 & 27.07 & 1.31 & 2.6 \\
\hline 06855800 & Buffalo Creek near Jamestown, KS & $1960-89$ & 30 & .95 & 3.1 & 11 & 41 & 133 & 72 & 330 & 27.94 & 1.10 & 1.9 \\
\hline 06855900 & Wolf Creek near Concordia, KS & 1963-81 & 19 & 0 & .10 & 1.0 & 4.3 & 17 & 11 & 56 & 28.76 & 1.01 & 2.5 \\
\hline 06858500 & North Fork Smoky Hill River near McAllaster, KS & $1948-84$ & 27 & 0 & 0 & 0 & 1.0 & 3.0 & 3.7 & 752 & 17.12 & 1.53 & 1.3 \\
\hline 06859500 & Ladder Creek below Chalk Creek near Scott City, KS & $1952-79$ & 28 & 0 & .39 & 1.9 & 4.0 & 10 & 8.0 & 1,432 & 17.65 & 1.40 & 1.0 \\
\hline 06860000 & Smoky Hill River at Elkader, KS & 1940-2000 & 61 & 0 & .11 & 1.5 & 6.0 & 22 & 24 & 3,555 & 17.67 & 1.53 & 1.3 \\
\hline 06861000 & Smoky Hill River near Arnold, KS & $1951-2000$ & 50 & .01 & .17 & 2.3 & 15 & 47 & 44 & 5,220 & 18.44 & 1.52 & 1.4 \\
\hline 06863300 & Big Creek near Ogallah, KS & $1956-68$ & 13 & .20 & 1.2 & 2.9 & 6.6 & 16 & 22 & 297 & 21.43 & 1.28 & 1.1 \\
\hline 06863500 & Big Creek near Hays, KS & $1947-2000$ & 54 & 1.9 & 3.5 & 7.9 & 19 & 38 & 33 & 594 & 21.80 & 1.18 & 1.4 \\
\hline 06863900 & North Fork Big Creek near Victoria, KS & $1963-86$ & 24 & 0 & 0 & 0 & 0 & .90 & 3.1 & 90.3 & 22.61 & 1.20 & 1.7 \\
\hline 06864000 & Smoky Hill River near Russell, KS & $1940-49$ & 10 & 5.0 & 15 & 40 & 104 & 356 & 184 & 6,965 & 19.48 & 1.46 & 1.4 \\
\hline 06866000 & Smoky Hill River near Lindsborg, KS & $1906-47$ & 42 & 18 & 30 & 57 & 148 & 498 & 244 & 8,110 & 20.60 & 1.46 & 1.5 \\
\hline 06866500 & Smoky Hill River near Mentor, KS & $1925-47$ & 23 & 35 & 60 & 116 & 218 & 560 & 329 & 8,358 & 20.84 & 1.45 & 1.6 \\
\hline 06866900 & Saline River near Wakeeney, KS & 1956-2000 & 45 & 0 & .03 & 2.6 & 12 & 24 & 21 & 696 & 20.60 & 1.37 & 1.5 \\
\hline
\end{tabular}


Table 1. Streamflow-gaging stations and computed flow-duration values, mean discharge for period of uncontrolled flow record, and climatic and basin characteristics used in regression analyses of uncontrolled flow stream locations in Kansas. - Continued

$\left[\mathrm{ft}^{3} / \mathrm{s}\right.$, cubic feet per second; $\mathrm{mi}^{2}$, square miles; in/h, inches per hour]

\begin{tabular}{|c|c|c|c|c|c|c|c|c|c|c|c|c|c|}
\hline \multirow[b]{2}{*}{$\begin{array}{c}\text { Station } \\
\text { number } \\
\text { (fig. 1) }\end{array}$} & \multirow[b]{2}{*}{ Station name } & \multirow[b]{2}{*}{$\begin{array}{c}\text { Period of } \\
\text { record } \\
\text { (water years) }\end{array}$} & \multirow[b]{2}{*}{$\begin{array}{l}\text { Years } \\
\text { of } \\
\text { record }\end{array}$} & \multicolumn{5}{|c|}{$\begin{array}{c}\text { Flow-duration values }\left(\mathrm{ft}^{3} / \mathrm{s}\right) \text { for indicated } \\
\text { percentage of time flow equaled or exceeded }\end{array}$} & \multirow{2}{*}{$\begin{array}{c}\text { Mean } \\
\text { discharge } \\
\text { for period } \\
\text { of uncon- } \\
\text { trolled } \\
\text { record } \\
\left(\mathrm{ft}^{3} / \mathrm{s}\right)\end{array}$} & \multirow{2}{*}{$\begin{array}{l}\text { Contribut- } \\
\text { ing- } \\
\text { drainage } \\
\text { area } \\
\left(\mathrm{mi}^{2}\right)\end{array}$} & \multirow{2}{*}{$\begin{array}{c}\text { Mean } \\
\text { annual } \\
\text { precipita- } \\
\text { tion, } \\
\text { 1961-90 } 1 \\
\text { (inches) }\end{array}$} & \multirow{2}{*}{$\begin{array}{l}\text { Mean } \\
\text { basin } \\
\text { perme- } \\
\text { ability }{ }^{2} \\
\text { (in/h) }\end{array}$} & \multirow{2}{*}{$\begin{array}{c}\text { Mean } \\
\text { basin } \\
\text { slope }^{3} \\
\text { (degrees) }\end{array}$} \\
\hline & & & & $\begin{array}{c}90- \\
\text { percent }\end{array}$ & $\begin{array}{c}\text { 75- } \\
\text { percent }\end{array}$ & $\begin{array}{c}50- \\
\text { percent }\end{array}$ & $\begin{array}{c}25- \\
\text { percent }\end{array}$ & $\begin{array}{c}10- \\
\text { percent }\end{array}$ & & & & & \\
\hline 06867000 & Saline River near Russell, KS & $1946-2000$ & 55 & 4.8 & 12 & 31 & 73 & 165 & 98 & 1,502 & 21.68 & 1.39 & 2.2 \\
\hline 06868000 & Saline River near Wilson, KS & $1930-63$ & 34 & 11 & 21 & 46 & 107 & 274 & 166 & 1,900 & 22.36 & 1.36 & 2.4 \\
\hline 06868400 & Wolf Creek near Lucas, KS & 1960-71 & 12 & .70 & 1.1 & 2.2 & 6.8 & 18 & 16 & 163 & 25.02 & 1.20 & 2.6 \\
\hline 06869500 & Saline River at Tescott, KS & $1920-63$ & 44 & 15 & 34 & 70 & 157 & 418 & 247 & 2,820 & 23.69 & 1.31 & 2.5 \\
\hline 06870300 & Gypsum Creek near Gypsum, KS & $1955-2000$ & 46 & 0 & 1.0 & 7.0 & 15 & 34 & 26 & 120 & 30.88 & .882 & 2.9 \\
\hline 06871000 & North Fork Solomon River at Glade, KS & 1953-2000 & 48 & 0 & .20 & 8.4 & 22 & 48 & 28 & 849 & 21.31 & 1.34 & 2.5 \\
\hline 06871500 & Bow Creek near Stockton, KS & $1952-2000$ & 49 & .44 & 2.6 & 5.6 & 10 & 18 & 14 & 341 & 21.57 & 1.45 & 1.8 \\
\hline 06871800 & North Fork Solomon River at Kirwin, KS & $1920-54$ & 35 & 4.4 & 15 & 31 & 52 & 120 & 87 & 1,367 & 21.47 & 1.38 & 2.3 \\
\hline 06871900 & Deer Creek near Phillipsburg, KS & $1967-81$ & 15 & 0 & 0 & .69 & 1.5 & 2.7 & 4.0 & 65 & 23.00 & 1.37 & 3.0 \\
\hline 06873000 & $\begin{array}{l}\text { South Fork Solomon River above Webster } \\
\text { Reservoir, KS }\end{array}$ & 1946-2000 & 55 & .03 & 1.0 & 14 & 37 & 77 & 54 & 1,040 & 20.87 & 1.46 & 2.1 \\
\hline 06873500 & South Fork Solomon River at Alton, KS & $1920-57$ & 38 & 2.2 & 9.0 & 30 & 62 & 145 & 104 & 1,720 & 21.64 & 1.41 & 2.1 \\
\hline 06873700 & Kill Creek near Bloomington, KS & $1964-81$ & 18 & 0 & 0 & 0 & 0 & 1.8 & 2.1 & 49.4 & 24.57 & 1.25 & 2.6 \\
\hline 06876000 & Solomon River at Beloit, KS & $1930-54$ & 25 & 10 & 40 & 93 & 224 & 743 & 457 & 5,530 & 23.06 & 1.33 & 2.2 \\
\hline 06876700 & Salt Creek near Ada, KS & 1960-2000 & 41 & 1.6 & 4.0 & 12 & 30 & 106 & 70 & 384 & 26.98 & 1.11 & 2.6 \\
\hline 06877000 & Smoky Hill River at Solomon, KS & 1919-34 & 16 & 126 & 202 & 404 & 750 & 1,880 & 931 & 8,830 & 22.1 & 1.39 & 1.9 \\
\hline 06878000 & Chapman Creek near Chapman, KS & $1955-2000$ & 46 & 7.7 & 13 & 24 & 49 & 126 & 93 & 300 & 30.89 & 1.02 & 2.2 \\
\hline 06878500 & Lyon Creek near Woodbine, KS & $1955-74$ & 20 & 3.4 & 17 & 33 & 65 & 135 & 108 & 230 & 34.13 & .533 & 2.4 \\
\hline 06879650 & Kings Creek near Manhattan, KS & $1980-2000$ & 20 & 0 & 0 & .19 & 2.0 & 5.7 & 2.6 & 4.09 & 33.00 & .458 & 5.9 \\
\hline 06882000 & Big Blue River near Barnestown, NE & 1933-2000 & 78 & 103 & 167 & 280 & 616 & 1,800 & 867 & 4,447 & 28.54 & .820 & 1.3 \\
\hline 06882500 & Big Blue River near Hull, KS & $1931-40$ & 10 & 88 & 130 & 217 & 365 & 895 & 470 & 4,685 & 28.66 & .810 & 1.4 \\
\hline 06882510 & Big Blue River at Marysville, KS & $1985-2000$ & 16 & 200 & 286 & 467 & 924 & 2,590 & 1,190 & 4,777 & 28.67 & .809 & 1.4 \\
\hline 06884000 & Little Blue River near Fairbury, NE & $1911-2000$ & 90 & 92 & 119 & 160 & 251 & 581 & 383 & 2,350 & 27.34 & 1.43 & 1.4 \\
\hline 06884025 & Little Blue River at Hollenberg, KS & 1975-2000 & 26 & 109 & 141 & 211 & 377 & 885 & 535 & 2,752 & 27.64 & 1.37 & 1.6 \\
\hline 06884200 & Mill Creek at Washington, KS & 1960-2000 & 41 & 3.2 & 7.5 & 19 & 56 & 181 & 108 & 344 & 30.62 & .908 & 2.4 \\
\hline 06884400 & Little Blue River near Barnes, KS & 1959-2000 & 42 & 128 & 170 & 268 & 525 & 1,340 & 704 & 3,324 & 28.23 & 1.28 & 1.7 \\
\hline
\end{tabular}


Table 1. Streamflow-gaging stations and computed flow-duration values, mean discharge for period of uncontrolled flow record, and climatic and basin characteristics used in regression analyses of uncontrolled flow stream locations in Kansas. - Continued

$\left[\mathrm{ft}^{3} / \mathrm{s}\right.$, cubic feet per second; $\mathrm{mi}^{2}$, square miles; in/h, inches per hour]

\begin{tabular}{|c|c|c|c|c|c|c|c|c|c|c|c|c|c|}
\hline \multirow[b]{2}{*}{$\begin{array}{c}\text { Station } \\
\text { number } \\
\text { (fig. 1) }\end{array}$} & \multirow[b]{2}{*}{ Station name } & \multirow[b]{2}{*}{$\begin{array}{c}\text { Period of } \\
\text { record } \\
\text { (water years) }\end{array}$} & \multirow[b]{2}{*}{$\begin{array}{l}\text { Years } \\
\text { of } \\
\text { record }\end{array}$} & \multicolumn{5}{|c|}{$\begin{array}{c}\text { Flow-duration values }\left(\mathrm{ft}^{3} / \mathrm{s}\right) \text { for indicated } \\
\text { percentage of time flow equaled or exceeded }\end{array}$} & \multirow{2}{*}{$\begin{array}{c}\text { Mean } \\
\text { discharge } \\
\text { for period } \\
\text { of uncon- } \\
\text { trolled } \\
\text { record } \\
\left(\mathrm{ft}^{3} / \mathrm{s}\right)\end{array}$} & \multirow{2}{*}{$\begin{array}{l}\text { Contribut- } \\
\text { ing- } \\
\text { drainage } \\
\text { area } \\
\left(\mathrm{mi}^{2}\right)\end{array}$} & \multirow{2}{*}{$\begin{array}{c}\text { Mean } \\
\text { annual } \\
\text { precipita- } \\
\text { tion, } \\
\text { 1961-90 } 1 \\
\text { (inches) }\end{array}$} & \multirow{2}{*}{$\begin{array}{c}\text { Mean } \\
\text { basin } \\
\text { perme- } \\
\text { ability }{ }^{2} \\
\text { (in/h) }\end{array}$} & \multirow{2}{*}{$\begin{array}{c}\text { Mean } \\
\text { basin } \\
\text { slope }^{3} \\
\text { (degrees) }^{3}\end{array}$} \\
\hline & & & & $\begin{array}{c}90- \\
\text { percent }\end{array}$ & $\begin{array}{c}\text { 75- } \\
\text { percent }\end{array}$ & $\begin{array}{c}\text { 50- } \\
\text { percent }\end{array}$ & $\begin{array}{c}25- \\
\text { percent }\end{array}$ & $\begin{array}{c}10- \\
\text { percent }\end{array}$ & & & & & \\
\hline 06884500 & Little Blue River at Waterville, KS & $1923-57$ & 35 & 104 & 144 & 200 & 385 & 1,110 & 619 & 3,509 & 28.23 & 1.28 & 1.7 \\
\hline 06885500 & Black Vermillion River near Frankfort, KS & $1954-2000$ & 47 & 4.0 & 11 & 29 & 79 & 246 & 174 & 410 & 33.31 & .359 & 2.4 \\
\hline 06886000 & Big Blue River at Randolph, KS & $1919-60$ & 42 & 270 & 380 & 600 & 1,240 & 3,410 & 1,690 & 9,100 & 28.94 & .946 & 1.7 \\
\hline 06886500 & Fancy Creek at Winkler, KS & $1955-71$ & 17 & 0 & 2.0 & 11 & 23 & 59 & 47 & 174 & 30.98 & .731 & 2.5 \\
\hline 06888000 & Vermillion Creek near Wamego, KS & $1937-71$ & 35 & .40 & 2.5 & 17 & 53 & 143 & 87 & 243 & 34.94 & .427 & 3.4 \\
\hline 06888500 & Mill Creek near Paxico, KS & $1955-2000$ & 46 & 4.8 & 19 & 56 & 156 & 339 & 194 & 316 & 34.67 & .505 & 4.2 \\
\hline 06889100 & Soldier Creek near Goff, KS & $1965-86$ & 22 & 0 & 0 & .08 & .30 & 1.0 & 1.4 & 2.06 & 35.54 & .318 & 2.6 \\
\hline 06889120 & Soldier Creek near Bancroft, KS & $1965-87$ & 23 & .01 & .15 & .55 & 2.0 & 6.0 & 6.9 & 10.5 & 35.16 & .344 & 2.6 \\
\hline 06889140 & Soldier Creek near Soldier, KS & 1965-98 & 34 & .21 & .48 & 1.2 & 3.5 & 10 & 11 & 16.9 & 35.16 & .359 & 2.7 \\
\hline 06889160 & Soldier Creek near Circleville, KS & $1965-2000$ & 36 & .80 & 2.0 & 4.6 & 13 & 39 & 32 & 49.3 & 35.82 & .381 & 2.8 \\
\hline 06889180 & Soldier Creek near St. Clere, KS & $1965-80$ & 16 & 2.3 & 5.0 & 10 & 28 & 75 & 51 & 80 & 35.53 & .434 & 3.0 \\
\hline 06889200 & Soldier Creek near Delia, KS & 1959-2000 & 42 & 2.9 & 8.4 & 21 & 58 & 153 & 99 & 157 & 35.63 & .476 & 3.2 \\
\hline 06889500 & Soldier Creek near Topeka, KS & $1930-2000$ & 71 & 2.2 & 9.4 & 30 & 89 & 243 & 158 & 290 & 35.73 & .557 & 3.3 \\
\hline 06890100 & Delaware River near Muscotah, KS & $1970-2000$ & 31 & 6.2 & 18 & 51 & 146 & 450 & 280 & 431 & 35.97 & .398 & 3.1 \\
\hline 06890500 & Delaware River at Valley Falls, KS & $1923-67$ & 45 & 6.0 & 18 & 65 & 189 & 600 & 388 & 922 & 36.28 & .432 & 3.1 \\
\hline 06891500 & Wakarusa River near Lawrence, KS & $1930-76$ & 47 & 0 & 2.0 & 23 & 105 & 333 & 195 & 425 & 36.61 & .617 & 2.6 \\
\hline 06892000 & Stranger Creek near Tonganoxie, KS & 1930-2000 & 71 & 2.0 & 9.0 & 40 & 135 & 433 & 247 & 406 & 37.89 & .503 & 3.2 \\
\hline 06893080 & Blue River near Stanley, KS & $1975-2000$ & 26 & .07 & .69 & 5.0 & 19 & 57 & 36 & 46 & 39.35 & .609 & 2.1 \\
\hline 06910800 & Marais des Cygnes River near Reading, KS & 1970-2000 & 31 & .18 & 2.3 & 15 & 56 & 174 & 113 & 177 & 35.80 & .399 & 2.2 \\
\hline 06911000 & Marais des Cygnes River at Melvern, KS & $1940-64$ & 25 & 0 & 3.0 & 23 & 86 & 300 & 196 & 351 & 36.61 & .421 & 2.2 \\
\hline 06911500 & Salt Creek near Lyndon, KS & 1940-99 & 60 & 0 & .54 & 5.1 & 24 & 82 & 66 & 111 & 36.60 & .461 & 2.1 \\
\hline 06911900 & Dragoon Creek near Burlingame, KS & $1961-2000$ & 40 & 0 & 1.0 & 8.0 & 32 & 93 & 68 & 114 & 36.07 & .443 & 2.7 \\
\hline 06912500 & Hundred and Ten Mile Creek near Quenemo, KS & $1940-62$ & 23 & 0 & 1.2 & 17 & 79 & 246 & 181 & 322 & 36.21 & .465 & 2.3 \\
\hline 06913000 & Marais des Cygnes River near Pomona, KS & $1923-62$ & 40 & 1.0 & 3.0 & 26 & 126 & 417 & 303 & 1,040 & 36.54 & .478 & 2.2 \\
\hline 06913500 & Marais des Cygnes River near Ottawa, KS & 1903-62 & 60 & 1.5 & 10 & 70 & 306 & 1,040 & 627 & 1,250 & 36.70 & .520 & 2.2 \\
\hline 06914000 & Pottawatomie Creek near Garnett, KS & 1940-2000 & 61 & .10 & 2.0 & 21 & 90 & 361 & 235 & 334 & 38.31 & .545 & 1.3 \\
\hline
\end{tabular}


Table 1. Streamflow-gaging stations and computed flow-duration values, mean discharge for period of uncontrolled flow record, and climatic and basin characteristics used in regression analyses of uncontrolled flow stream locations in Kansas. — Continued

$\left[\mathrm{ft}^{3} / \mathrm{s}\right.$, cubic feet per second; $\mathrm{mi}^{2}$, square miles; in/h, inches per hour]

\begin{tabular}{|c|c|c|c|c|c|c|c|c|c|c|c|c|c|}
\hline \multirow[b]{2}{*}{$\begin{array}{l}\text { Station } \\
\text { number } \\
\text { (fig. 1) }\end{array}$} & \multirow[b]{2}{*}{ Station name } & \multirow[b]{2}{*}{$\begin{array}{c}\text { Period of } \\
\text { record } \\
\text { (water years) }\end{array}$} & \multirow[b]{2}{*}{$\begin{array}{l}\text { Years } \\
\text { of } \\
\text { record }\end{array}$} & \multicolumn{5}{|c|}{$\begin{array}{l}\text { Flow-duration values }\left(\mathrm{ft}^{3} / \mathrm{s}\right) \text { for indicated } \\
\text { percentage of time flow equaled or exceeded }\end{array}$} & \multirow{2}{*}{ 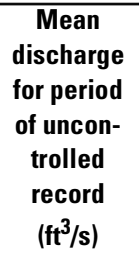 } & \multirow{2}{*}{$\begin{array}{c}\text { Contribut- } \\
\text { ing- } \\
\text { drainage } \\
\text { area } \\
\left(\mathbf{m i}^{2}\right)\end{array}$} & \multirow{2}{*}{$\begin{array}{c}\text { Mean } \\
\text { annual } \\
\text { precipita- } \\
\text { tion, } \\
\text { 1961-901 } \\
\text { (inches) }\end{array}$} & \multirow{2}{*}{$\begin{array}{c}\text { Mean } \\
\text { basin } \\
\text { perme- } \\
\text { ability }{ }^{2} \\
\text { (in/h) }\end{array}$} & \multirow{2}{*}{$\begin{array}{c}\text { Mean } \\
\text { basin } \\
\text { slope }^{3} \\
\text { (degrees) }\end{array}$} \\
\hline & & & & $\begin{array}{c}90- \\
\text { percent }\end{array}$ & $\begin{array}{c}75- \\
\text { percent }\end{array}$ & $\begin{array}{c}\text { 50- } \\
\text { percent }\end{array}$ & $\begin{array}{c}25- \\
\text { percent }\end{array}$ & $\begin{array}{c}10- \\
\text { percent }\end{array}$ & & & & & \\
\hline$\overline{06915000}$ & Big Bull Creek near Hillsdale, KS & $1959-80$ & 22 & 0.34 & 4.0 & 14 & 50 & 228 & 104 & 147 & 39.25 & 0.660 & 2.1 \\
\hline 06916000 & Marais des Cygnes River at Trading Post, KS & $1929-58$ & 30 & 2.9 & 23 & 209 & 884 & 4,208 & 1,690 & 2,880 & 38.14 & .595 & 2.0 \\
\hline 06916500 & Big Sugar Creek at Farlinville, KS & $1930-70$ & 41 & 0 & 1.2 & 11 & 61 & 185 & 127 & 198 & 40.18 & .657 & 2.2 \\
\hline 06917500 & Marmaton River at Fort Scott, KS & $1922-71$ & 50 & 1.0 & 2.7 & 31 & 130 & 446 & 288 & 408 & 41.47 & .827 & 1.9 \\
\hline 07138650 & White Woman Creek near Leoti, KS & $1967-85$ & 19 & 0 & 0 & 0 & 0 & 0 & 1.0 & 758 & 15.74 & 1.20 & .57 \\
\hline 07139800 & Mulberry Creek near Dodge City, KS & 1969-90 & 22 & 0 & 0 & 0 & 0 & 0 & .64 & 217 & 21.70 & 1.23 & .83 \\
\hline 07140850 & Pawnee River near Burdett, KS & 1982-2000 & 19 & 0 & 0 & 0 & 2.4 & 10 & 11 & 1,252 & 20.49 & 1.11 & 1.1 \\
\hline 07141200 & Pawnee River at Rozel, KS & $1925-2000$ & 76 & 0 & 0 & 3.7 & 15 & 59 & 63 & 2,148 & 20.98 & 1.12 & 1.1 \\
\hline 07142575 & Rattlesnake Creek near Zenith, KS & 1974-2000 & 27 & 4.6 & 12 & 29 & 50 & 82 & 50 & 1,047 & 24.41 & 5.90 & .68 \\
\hline 07142620 & Rattlesnake Creek near Raymond, KS & $1961-98$ & 38 & 2.2 & 4.2 & 24 & 59 & 104 & 49 & 1,167 & 24.41 & 5.90 & .68 \\
\hline 07142860 & Cow Creek near Claflin, KS & $1967-81$ & 15 & 0 & 0 & .13 & .80 & 4.0 & 7.0 & 43 & 25.85 & 1.04 & 1.3 \\
\hline 07142900 & Blood Creek near Boyd, KS & $1963-80$ & 18 & 0 & .10 & .48 & 1.2 & 4.0 & 7.1 & 61 & 24.45 & 1.07 & 1.5 \\
\hline 07143300 & Cow Creek near Lyons, KS & $1939-2000$ & 62 & 3.2 & 6.5 & 12 & 30 & 133 & 80 & 728 & 26.15 & 1.30 & .87 \\
\hline 07143600 & Little Arkansas River near Little River, KS & 1960-70 & 11 & 0 & .20 & .80 & 1.9 & 6.9 & 9.5 & 71 & 27.67 & .856 & 1.3 \\
\hline 07143665 & Little Arkansas River at Alta Mills, KS & 1974-2000 & 27 & 4.9 & 9.2 & 22 & 62 & 325 & 229 & 736 & 29.49 & 2.07 & .759 \\
\hline 07144200 & Little Arkansas River at Valley Center, KS & 1923-2000 & 78 & 21 & 33 & 60 & 127 & 488 & 312 & 1,327 & 30.34 & 2.02 & .754 \\
\hline 07144780 & $\begin{array}{l}\text { North Fork Ninnescah River above Cheney Reservoir, } \\
\text { KS }\end{array}$ & $1966-2000$ & 35 & 24 & 47 & 76 & 126 & 222 & 147 & 787 & 26.87 & 5.48 & .69 \\
\hline 07144800 & North Fork Ninnescah River near Cheney, KS & 1951-64 & 14 & 9.3 & 38 & 85 & 148 & 262 & 160 & 930 & 27.47 & 4.96 & .83 \\
\hline
\end{tabular}


Table 1. Streamflow-gaging stations and computed flow-duration values, mean discharge for period of uncontrolled flow record, and climatic and basin characteristics used in regression analyses of uncontrolled flow stream locations in Kansas. -Continued

$\left[\mathrm{ft}^{3} / \mathrm{s}\right.$, cubic feet per second; $\mathrm{mi}^{2}$, square miles; in/h, inches per hour]

\begin{tabular}{|c|c|c|c|c|c|c|c|c|c|c|c|c|c|}
\hline \multirow[b]{2}{*}{$\begin{array}{l}\text { Station } \\
\text { number } \\
\text { (fig. 1) }\end{array}$} & \multirow[b]{2}{*}{ Station name } & \multirow[b]{2}{*}{$\begin{array}{c}\text { Period of } \\
\text { record } \\
\text { (water years) }\end{array}$} & \multirow[b]{2}{*}{$\begin{array}{l}\text { Years } \\
\text { of } \\
\text { record }\end{array}$} & \multicolumn{5}{|c|}{$\begin{array}{c}\text { Flow-duration values }\left(\mathrm{ft}^{3} / \mathrm{s}\right) \text { for indicated } \\
\text { percentage of time flow equaled or exceeded }\end{array}$} & \multirow{2}{*}{$\begin{array}{c}\text { Mean } \\
\text { discharge } \\
\text { for period } \\
\text { of uncon- } \\
\text { trolled } \\
\text { record } \\
\left(\mathrm{ft}^{3} / \mathrm{s}\right)\end{array}$} & \multirow{2}{*}{$\begin{array}{l}\text { Contribut- } \\
\text { ing- } \\
\text { drainage } \\
\text { area } \\
\left(\mathrm{mi}^{2}\right)\end{array}$} & \multirow{2}{*}{$\begin{array}{c}\text { Mean } \\
\text { annual } \\
\text { precipita- } \\
\text { tion, } \\
\text { 1961-90 } 1 \\
\text { (inches) }\end{array}$} & \multirow{2}{*}{$\begin{array}{c}\text { Mean } \\
\text { basin } \\
\text { perme- } \\
\text { ability }{ }^{2} \\
\text { (in/h) }\end{array}$} & \multirow{2}{*}{$\begin{array}{c}\text { Mean } \\
\text { basin }^{2} \\
\text { slope }^{3} \\
\text { (degrees) }\end{array}$} \\
\hline & & & & $\begin{array}{c}90- \\
\text { percent }\end{array}$ & $\begin{array}{c}75- \\
\text { percent }\end{array}$ & $\begin{array}{c}50- \\
\text { percent }\end{array}$ & $\begin{array}{c}25- \\
\text { percent }\end{array}$ & $\begin{array}{c}10- \\
\text { percent }\end{array}$ & & & & & \\
\hline 07145200 & South Fork Ninnescah River near Murdock, KS & $1951-2000$ & 50 & 66 & 97 & 135 & 194 & 304 & 209 & 650 & 27.25 & 3.08 & 1.3 \\
\hline 07145500 & Ninnescah River near Peck, KS & $1939-63$ & 25 & 78 & 156 & 272 & 450 & 920 & 550 & 2,129 & 28.06 & 3.78 & 1.1 \\
\hline 07145700 & Slate Creek at Wellington, KS & 1970-2000 & 31 & .91 & 3.3 & 8.0 & 21 & 73 & 73 & 154 & 30.73 & .876 & .81 \\
\hline 07146570 & Cole Creek near DeGraff, KS & $1962-79$ & 18 & 0 & .07 & 1.8 & 4.8 & 13 & 17 & 30 & 33.68 & .448 & 1.1 \\
\hline 07147070 & Whitewater River at Towanda, KS & $1962-2000$ & 39 & 7.6 & 17 & 36 & 85 & 242 & 206 & 426 & 33.05 & .468 & 1.2 \\
\hline 07147800 & Walnut River at Winfield, KS & $1922-80$ & 58 & 18 & 48 & 152 & 430 & 1,300 & 793 & 1,880 & 34.31 & .488 & 1.4 \\
\hline 07148350 & Salt Fork Arkansas near Winchester, OK & 1960-93 & 24 & .35 & 7.4 & 34 & 75 & 160 & 96 & 856 & 24.58 & 2.81 & 2.6 \\
\hline 07149000 & Medicine Lodge River near Kiowa, KS & $1939-2000$ & 62 & 12 & 47 & 85 & 141 & 271 & 154 & 903 & 25.47 & 2.56 & 2.7 \\
\hline 07149500 & Salt Fork Arkansas River near Cherokee, OK & $1941-50$ & 10 & 2.0 & 50 & 125 & 246 & 731 & 393 & 2,439 & 25.44 & 2.80 & 2.5 \\
\hline 07151500 & Chikaskia River near Corbin, KS & $1951-2000$ & 50 & 19 & 47 & 94 & 190 & 430 & 250 & 794 & 28.69 & 2.65 & 1.1 \\
\hline 07152000 & Chikaskia River near Blackwell, OK & $1937-2000$ & 64 & 23 & 66 & 144 & 324 & 895 & 585 & 1,859 & 32.94 & .800 & .95 \\
\hline 07154500 & Cimarron River near Kenton, OK & $1951-2000$ & 50 & 0 & .06 & .91 & 2.8 & 7.5 & 17 & 1,106 & 16.29 & 2.07 & 1.0 \\
\hline 07155590 & Cimarron River near Elkhart, KS & $1972-2000$ & 29 & 0 & 0 & 0 & 0 & 1.5 & 11 & 3,410 & 16.33 & 3.04 & 1.8 \\
\hline 07156010 & North Fork Cimarron River at Richfield, KS & $1972-85$ & 14 & 0 & 0 & 0 & 0 & $\mathbb{2}$ & 5.6 & 492 & 16.12 & 3.27 & .97 \\
\hline 07156100 & Sand Arroyo Creek near Johnson, KS & $1972-85$ & 14 & 0 & 0 & 0 & 0 & 0 & .25 & 751 & 15.90 & 3.12 & .93 \\
\hline 07156220 & Bear Creek near Johnson, KS & 1967-98 & 32 & 0 & 0 & 0 & 0 & 0 & 3.4 & 1,093 & 15.85 & 1.27 & 1.2 \\
\hline 07156900 & Cimarron River near Forgan, OK & $1966-2000$ & 35 & 27 & 35 & 45 & 62 & 82 & 58 & 8,536 & 16.85 & 3.16 & 1.1 \\
\hline 07157000 & Cimarron River near Mocane, OK & $1943-65$ & 13 & 27 & 42 & 60 & 85 & 137 & 100 & 8,670 & 17.07 & 3.32 & 1.2 \\
\hline 07157500 & Crooked Creek near Englewood, KS & $1943-2000$ & 58 & 2.4 & 7.1 & 12 & 18 & 33 & 31 & 1,157 & 20.51 & 1.67 & .72 \\
\hline 07157900 & Cavalry Creek at Coldwater, KS & $1967-81$ & 15 & .65 & 1.0 & 1.5 & 2.0 & 2.9 & 3.4 & 39 & 24.81 & 2.73 & 1.1 \\
\hline 07157950 & Cimarron River near Buffalo, OK & 1961-94 & 34 & .03 & 10 & 56 & 123 & 232 & 128 & 12,004 & 19.53 & 3.19 & 1.3 \\
\hline 07165700 & Verdigris River near Madison, KS & $1956-76$ & 21 & .50 & 4.9 & 28 & 78 & 204 & 123 & 181 & 36.14 & .486 & 2.8 \\
\hline 07166000 & Verdigris River near Coyville, KS & $1940-59$ & 20 & 0.10 & 5.2 & 45 & 189 & 670 & 465 & 747 & 36.75 & .541 & 2.4 \\
\hline 07166500 & Verdigris River near Altoona, KS & $1940-59$ & 20 & 0 & 10 & 71 & 295 & 1,230 & 691 & 1,138 & 37.51 & .671 & 2.4 \\
\hline 07167000 & Fall River near Eureka, KS & $1947-76$ & 30 & .70 & 5.2 & 40 & 134 & 324 & 190 & 307 & 35.32 & .515 & 3.1 \\
\hline
\end{tabular}


Table 1. Streamflow-gaging stations and computed flow-duration values, mean discharge for period of uncontrolled flow record, and climatic and basin characteristics used in regression analyses of uncontrolled flow stream locations in Kansas. - Continued

$\left[\mathrm{ft}^{3} / \mathrm{s}\right.$, cubic feet per second; $\mathrm{mi}^{2}$, square miles; in/h, inches per hour]

\begin{tabular}{|c|c|c|c|c|c|c|c|c|c|c|c|c|c|}
\hline \multirow[b]{2}{*}{$\begin{array}{l}\text { Station } \\
\text { number } \\
\text { (fig. 1) }\end{array}$} & \multirow[b]{2}{*}{ Station name } & \multirow[b]{2}{*}{$\begin{array}{l}\text { Period of } \\
\text { record } \\
\text { (water years) }\end{array}$} & \multirow[b]{2}{*}{$\begin{array}{l}\text { Years } \\
\text { of } \\
\text { record }\end{array}$} & \multicolumn{5}{|c|}{$\begin{array}{c}\text { Flow-duration values }\left(\mathrm{ft}^{3} / \mathrm{s}\right) \text { for indicated } \\
\text { percentage of time flow equaled or exceeded }\end{array}$} & \multirow{2}{*}{$\begin{array}{c}\text { Mean } \\
\text { discharge } \\
\text { for period } \\
\text { of uncon- } \\
\text { trolled } \\
\text { record } \\
\left(\mathrm{ft}^{3} / \mathrm{s}\right)\end{array}$} & \multirow{2}{*}{$\begin{array}{l}\text { Contribut- } \\
\text { ing- } \\
\text { drainage } \\
\text { area } \\
\left(\mathrm{mi}^{2}\right)\end{array}$} & \multirow{2}{*}{$\begin{array}{c}\text { Mean } \\
\text { annual } \\
\text { precipita- } \\
\text { tion, } \\
\text { 1961-90 } \\
\text { (inches) }\end{array}$} & \multirow{2}{*}{$\begin{array}{c}\text { Mean } \\
\text { basin } \\
\text { perme- } \\
\text { ability }{ }^{2} \\
\text { (in/h) }\end{array}$} & \multirow{2}{*}{$\begin{array}{c}\text { Mean } \\
\text { basin } \\
\text { slope }^{3} \\
\text { (degrees) }\end{array}$} \\
\hline & & & & $\begin{array}{c}\text { 90- } \\
\text { percent }\end{array}$ & $\begin{array}{c}75- \\
\text { percent }\end{array}$ & $\begin{array}{c}50- \\
\text { percent }\end{array}$ & $\begin{array}{c}25- \\
\text { percent }\end{array}$ & $\begin{array}{c}10- \\
\text { percent }\end{array}$ & & & & & \\
\hline 07167500 & Otter Creek at Climax, KS & $1947-2000$ & 54 & 0 & 1.0 & 10 & 44 & 121 & 82 & 129 & 36.19 & 0.461 & 2.8 \\
\hline 07168500 & Fall River near Fall River, KS & $1905-48$ & 44 & 1.0 & 10 & 53 & 184 & 550 & 331 & 585 & 35.84 & .518 & 2.8 \\
\hline 07169500 & Fall River at Fredonia, KS & $1939-48$ & 10 & 2.4 & 12 & 75 & 272 & 848 & 506 & 827 & 36.26 & 616 & 2.7 \\
\hline 07169800 & Elk River at Elk Falls, KS & $1967-2000$ & 33 & .64 & 2.9 & 22 & 91 & 271 & 154 & 220 & 36.46 & .447 & 2.5 \\
\hline 07170000 & Elk River near Elk City, KS & 1939-69 & 31 & 0 & 2.6 & 26 & 119 & 412 & 308 & 575 & 37.41 & .740 & 2.6 \\
\hline 07170700 & Big Hill Creek near Cherryvale, KS & $1958-2000$ & 43 & 0 & .03 & 1.3 & 10 & 48 & 27 & 37 & 41.31 & .834 & 2.3 \\
\hline 07172000 & Caney River near Elgin, KS & 1940-2000 & 61 & .07 & 5.0 & 40 & 172 & 528 & 273 & 445 & 35.53 & .556 & 3.2 \\
\hline 07174000 & Little Caney River near Copan, OK & $1944-58$ & 15 & .10 & .50 & 9.5 & 64 & 323 & 237 & 424 & 37.51 & 1.01 & 2.9 \\
\hline 07174400 & Caney River above Coon Creek near Bartlesville, OK & 1986-2000 & 15 & 26 & 36 & 173 & 1,820 & 4,420 & 1,290 & 1,392 & 36.75 & .970 & 3.1 \\
\hline 07179500 & Neosho River at Council Grove, KS & $1939-63$ & 25 & 0 & 1.0 & 16 & 48 & 134 & 123 & 250 & 33.77 & .433 & 1.8 \\
\hline 07180000 & Cottonwood River near Marion, KS & 1939-68 & 30 & 3.0 & 8.0 & 18 & 43 & 108 & 112 & 329 & 32.37 & 662 & 1.5 \\
\hline 07180500 & Cedar Creek near Cedar Point, KS & 1939-2000 & 62 & 1.8 & 6.0 & 16 & 36 & 76 & 58 & 110 & 33.33 & .518 & 1.6 \\
\hline 07181000 & Cottonwood River at Elmdale, KS & $1923-32$ & 10 & 20 & 42 & 88 & 220 & 691 & 357 & 1,045 & 32.92 & .566 & 1.6 \\
\hline 07181500 & Middle Creek near Elmdale, KS & $1939-50$ & 12 & 0 & 2.4 & 7.0 & 22 & 59 & 45 & 92 & 34.05 & .456 & 2.2 \\
\hline 07182000 & Cottonwood River at Cottonwood Falls, KS & 1933-67 & 35 & 10 & 34 & 108 & 302 & 785 & 511 & 1,740 & 33.09 & .545 & 1.9 \\
\hline 07182400 & Neosho River at Strawn, KS & 1949-62 & 14 & 5.0 & 54 & 285 & 920 & 3,040 & 1,390 & 2,933 & 34.11 & .508 & 1.9 \\
\hline 07183100 & Owl Creek near Piqua, KS & $1960-70$ & 11 & 0 & .57 & 4.0 & 21 & 115 & 122 & 177 & 40.36 & 609 & 1.7 \\
\hline 07183500 & Neosho River near Parsons, KS & $1922-63$ & 42 & 29 & 133 & 472 & 1,600 & 5,820 & 2,450 & 4,905 & 36.39 & .579 & 1.7 \\
\hline 07184000 & Lightning Creek near McCune, KS & 1939-2000 & 62 & 0 & 1.4 & 12 & 52 & 270 & 169 & 197 & 42.25 & 1.02 & 1.2 \\
\hline 07186000 & Spring River at Waco, MO & $1925-2000$ & 76 & 66 & 124 & 301 & 730 & 1,816 & 947 & 1,164 & 43.38 & 1.41 & 1.2 \\
\hline 07186400 & Center Creek near Carterville, MO & 1963-91 & 29 & 32 & 47 & 97 & 206 & 399 & 204 & 232 & 42.92 & 1.46 & 1.6 \\
\hline 07187000 & Shoal Creek above Joplin, MO & $1942-2000$ & 59 & 88 & 130 & 237 & 448 & 879 & 427 & 427 & 43.21 & 1.48 & 2.7 \\
\hline 07188000 & Spring River near Quapaw, OK & $1940-2000$ & 61 & 211 & 376 & 850 & 1,950 & 4,400 & 2,200 & 2,510 & 43.18 & 1.43 & 1.4 \\
\hline
\end{tabular}

${ }^{1}$ Mean annual precipitation for each gaging station from Daly and others (1997).

${ }^{2}$ Mean basin permeability for each gaging station from U.S. Department of Agriculture (1994).

${ }^{3}$ Mean basin slope for each gaging staiton from U.S. Geological Survey (1998). 
in elevation between 10 - and 85 -percent lengths of the main channel from the point of the stream statistic computation. In the GIS, the coverage for the streams was overlain on the elevation coverage. A program was developed to determine the longest continuous stream length upstream from the point of statistic computation. The 10-percent and 85-percent distance elevations were obtained, and along with the distance between the two points, a main-stream channel slope for each stream location was derived.

\section{Methods for Estimating Flow Durations, Mean Flows, and Peak-Discharge Frequency Values}

Climatic and basin characteristics were used in the analyses of flow durations and mean flows at gaged and ungaged sites on uncontrolled flow streams. For this study, ARC/INFO GIS software was used to estimate climatic and basin characteristics. Many spatial data sets were available for this task, including: (1) 30-year (1961-90) mean annual precipitation data (Daly and others, 1997), (2) 30-m gridded elevation data (U.S. Geological Survey, 1998) for determining contributingdrainage area, mean basin slope, and mean basin elevation, and (3) STATSGO soil-permeability data (U.S. Department of Agriculture, 1994).

The flow-duration information was computed for the 216 gaging stations in Kansas and the surrounding States with at least 10 years of streamflow record. Streamflow at 149 of these stations, which were on uncontrolled flow stream locations (table 1), were included in the regression analyses to develop predictive equations for streamflow. The flows of uncontrolled flow stream locations are unaffected by storage and release from Federal upstream reservoirs. One hundred thirty-one streamflow-gaging stations in Kansas and 18 in surrounding States (3 in Missouri, 5 in Nebraska, and 10 in Oklahoma) measured uncontrolled flow. All available records through water year (October through September) 2000 were used to compute the flow duration and mean flow for these gaging stations.

Three gaging stations in Kansas that measured uncontrolled flow and had at least 10 years of record were not included in the regression analyses. One station, Indian Creek at Overland Park, Kansas (station 06893300), was not used because it is affected by extensive urbanization. Two other stations, Beaver Creek at Cedar Bluffs, Kansas (station 06846500), and Paradise Creek near Paradise, Kansas (station 06867500), were not used because streamflow statistics developed for these stations were not consistent with statistics for other nearby stations. These two gaging stations were located on stream reaches that were losing surface flow to ground water (losing streams).

\section{Estimates at Gaged Stream Sites}

The USGS has established standard methods for estimating flow duration (Searcy, 1959) for streamflow-gaging stations. The computer software programs IOWDM, ANNIE, and SWSTAT were used to format input data, manage and display data, and complete the flow-duration statistical analyses (Lumb and others, 1990; Flynn and others, 1995). These programs are available on the World Wide Web (URL http:// water.usgs.gov/ software/surface_water.html). For this study a computer software program was developed by the USGS (Xiaodong Jian, written commun., 2003) and was used to compute flow durations and mean flow using the data from the streamflow-gaging stations. This software program is on file at the USGS office in Lawrence, Kansas.

Daily mean flows for all complete water years of record were used to determine flow-duration statistics for continuousrecord, streamflow-gaging stations. The water year begins on October 1 and ends on September 30 of the following year. Daily mean flows for USGS streamflow-gaging stations in Kansas are available on the World Wide Web (URL http://waterdata.usgs.gov/ks/nwis/).

A flow-duration curve (fig. 7) is a graphical representation of the percentage of time that streamflow for a given time step (usually daily) is equaled or exceeded during a specified period (usually the complete period of record) at a stream site. Flowduration curves usually are constructed by first ranking all of the daily mean discharges for the period of record at a gaging station from largest to smallest, next computing the probability for each value being equaled or exceeded, then plotting the discharges against their associated exceedance probabilities (Loaiciga, 1989, p. 82). The daily mean discharges are not fit to an assumed distribution. Flow-duration analysis can be done by use of the USGS software described previously or by use of commercially available statistical software.

Flow-duration statistics are points along a flow-duration curve. For example, the 99-percent duration streamflow is equaled or exceeded 99 percent of the time, whereas the 50 -percent duration streamflow (median) is equaled or exceeded 50 percent of the time. Strictly interpreted, flowduration statistics reflect only the period for which they are calculated; however, when the period of record used to compute the statistics is sufficiently long, the statistics often are used as an indicator of probable future conditions (Searcy, 1959).

Estimates of peak discharge for selected frequencies can be computed by using observed annual peak-discharge data for streamflow-gaging stations. Log-Pearson Type-III distributions are fitted to the observed annual peak-discharge data for each streamflow-gaging station by using techniques recommended by the Interagency Committee on Water Data (1981).

\section{Estimates at Ungaged Stream Sites}

Estimates of streamflow statistics often are needed for sites on streams where no gaged data are available. The two 


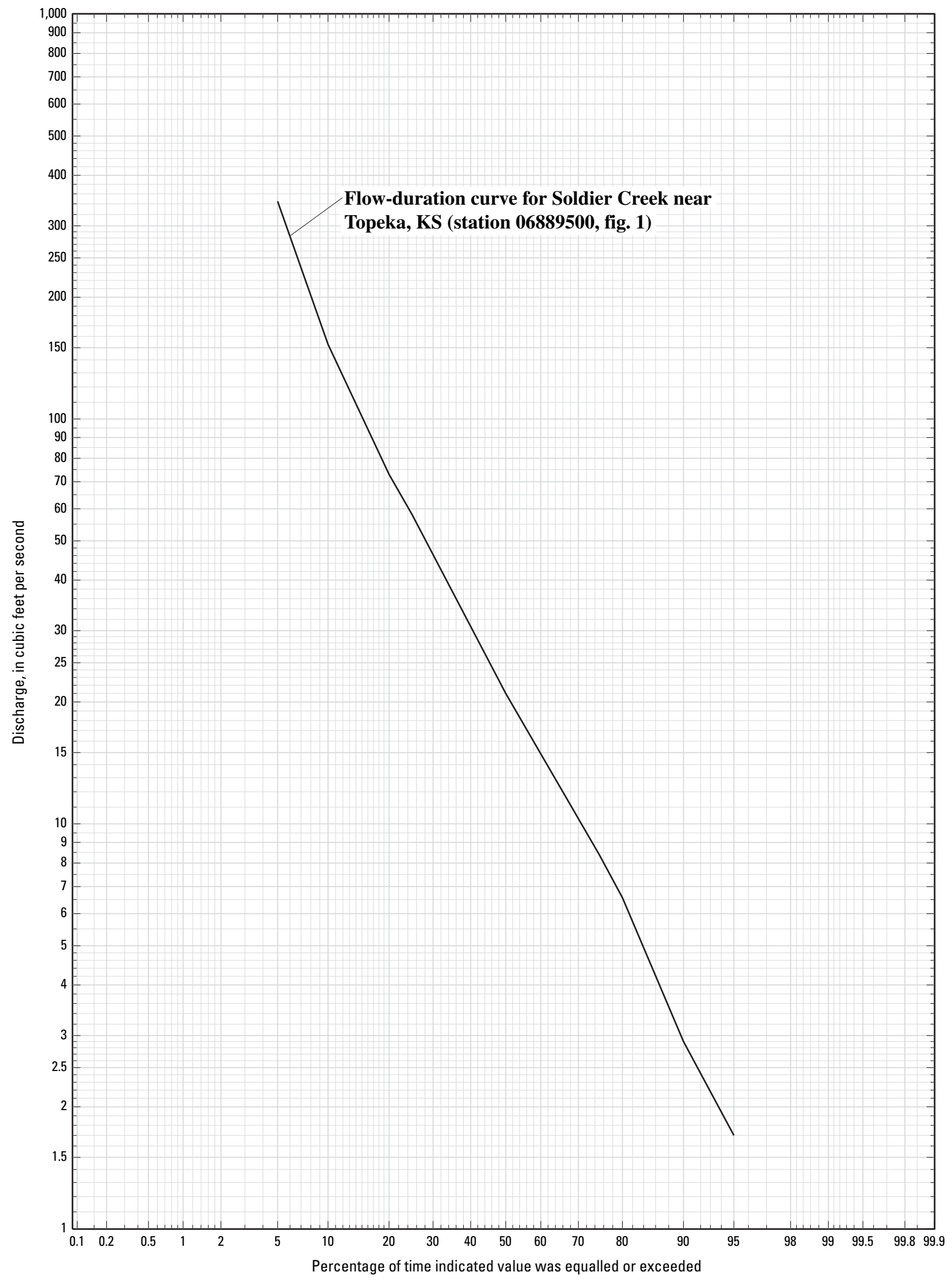

Figure 7. Flow-duration curve for Soldier Creek near Topeka, Kansas (station 06889500, fig. 1). 


\section{Estimates of Flow Duration, Mean Flow, and Peak-Discharge Frequency Values for Kansas Stream Locations}

methods most commonly used to estimate statistics for ungaged sites are the drainage-area ratio method and multiple linearregression analysis. The drainage-area ratio method is most appropriate for use when the ungaged site is near a streamflowgaging station on the same stream. Multiple linear-regression analysis is used to obtain estimates for most other ungaged sites.

\section{Drainage-Area Ratio Method}

The drainage-area ratio method assumes that the streamflow at an ungaged site for the same stream is the same per unit area or at least responds in the same fashion as that at a nearby, hydrologically similar streamflow-gaging station used as an index. Drainage areas for the ungaged site and the index station are determined from topographic maps, digital elevation maps (DEMs), or by other GIS methods. Streamflow statistics are computed for the index station, then the statistics are divided by the drainage area to determine streamflow per unit area at the index station. These values are multiplied by the drainage area at the ungaged site to obtain estimated statistics for the site. This method is most commonly applied when the index gaging station is on the same stream as the ungaged site because the accuracy of the method depends on the proximity of the two sites and on similarities in drainage area and on other climatic and basin characteristics of the respective drainage basins.

Several researchers have provided guidelines as to how large the difference in drainage areas can be before use of multiple linear-regression analysis is preferred over use of the drainage-area ratio method. Guidelines have been provided for estimating peak-discharge statistics, and usually the preferred guideline has been that the drainage area for the ungaged site should be within 0.5 and 1.5 times the drainage area of the index station (Sauer, 1974; Choquette, 1988, p. 41; Koltun and Roberts, 1990, p. 6; Lumia, 1991, p. 34; Bisese, 1995, p. 13). One report (Koltun and Schwartz, 1986, p. 32) selected a range of 0.85 to 1.15 times the drainage area of the index station for estimating low flows at ungaged sites in Ohio. None of these researchers provided any scientific basis for use of these guidelines (R.E. Thompson, Jr., U.S. Geological Survey, written commun., 1999).

In this report, streamflow statistics at controlled, ungaged sites were determined by the drainage-area ratio method with no limit on the ratios between the gaged-location drainage area and the ungaged-location drainage area. Flow statistics for uncontrolled flow, ungaged sites were determined by an interpolation procedure that utilized a drainage-area weighted ratio of gaged site information and regression-equation estimates at ungaged sites. This procedure is explained in greater detail in a following section, "Estimates of Streamflow Statistics for Stream Locations."

\section{Multiple Linear-Regression Analysis}

Multiple linear-regression analysis (regression analysis) has been used by the USGS and other researchers throughout the United States and elsewhere to develop equations for estimating streamflow statistics at ungaged sites. In regression analysis, a streamflow statistic (the dependent variable) for a group of gaging stations is related statistically to the climatic or basin characteristics of the drainage basins for the stations (the independent variables). This results in an equation that can be used to estimate the statistic for sites where no streamflow data are available.

Equations can be developed by use of several different regression analysis algorithms. The various algorithms use different methods to minimize the differences between the values of the dependent variable for the stations used in the analysis (the observed values) and the corresponding values provided by the resulting regression equation (the estimated or fitted values). Choice of one algorithm over another depends on the characteristics of the data used in the analysis and on the underlying assumptions for use of the algorithm. The multiple linearregression equation takes the general form:

$$
\Upsilon_{i}=b_{0}+b_{1} X_{1}+b_{2} X_{2}+\ldots+b_{n} X_{n}+\varepsilon_{i}
$$

where $Y_{i}$ is the value of the dependent variable for site $i, X_{1}$ to $X_{n}$ are the $n$ independent variables, $b_{0}$ to $b_{n}$ are the $n+1$ regression-model coefficients, and $\varepsilon_{i}$ is the error (difference between the observed and estimated values of the dependent variable) for site $i$. Assumptions for use of regression analysis are (1) equation 1 adequately describes the relation between the dependent and the independent variables, (2) the variance of the $\varepsilon_{i}$ is constant and independent of the values of $X_{n}$, (3) the $\varepsilon_{i}$ are normally distributed for a Tobit analysis, and (4) the $\varepsilon_{i}$ are independent of each other (Inman and Conover, 1983, p. 367). Tobit analysis is discussed in the following paragraph. Regression analysis results need to be evaluated to assure that these assumptions are met. Streamflow and basin characteristics used in hydrologic regression usually are log-normally distributed; therefore, transformation of the variables to logarithms is usually necessary to satisfy regression assumption 3 . Transformation results in a model of the form:

$$
\log Y_{i}=b_{0}+b_{1} \log X_{1}+b_{2} \log X_{2}+\ldots+b_{n} X_{n}+\varepsilon_{i} .
$$

The algebraically equivalent form when logarithms-base 10 $\left(\log _{10}\right)$ are used in the transformations, and the equation retransformed to original units is:

$$
\begin{gathered}
Y_{i}=10^{b_{o}}\left(X_{1}^{b_{1}}\right)\left(X_{2}^{b_{2}}\right) \ldots\left(X_{n}^{b_{n}}\right) 10 ; \\
Y_{i}=10^{\left[b_{0}+b_{1} \log X_{1}+b_{2} \log X_{2}+\ldots b_{n} \log X_{n}+\varepsilon_{i}\right]} .
\end{gathered}
$$

To include zero values in a logarithmic transformation analysis, the Tobit analysis was used. Tobit analysis is a widely accepted method for estimating a regression-like model when there are adjusted data (Tobin, 1958; Judge and others, 
1985; Cohn, 1988). Adjusted data are data that either are censored or have had a discrete value of delta $(\delta)$ added to them. Censored data are values less than a threshold value and are increased to the censoring value (for example, all values less than 0.7 are increased to 0.7$)$. Discrete values of delta $(\delta)$ are added to all data before transformation and then subtracted from the final regression model value. By applying these techniques, zero values of data can be transformed logarithmically. The Tobit procedure uses a maximum likelihood estimator (Cohn, 1988). The Survival Regression Procedure in the S-Plus 2000 software package (MathSoft, 1999) was used in this study to fit the Tobit model.

A Tobit analysis was conducted for each of the flow durations and for the mean flow data sets, and the resulting plots of observed and regression-estimated values are shown in figures $8 A$ through $8 F$. The graphs show the observed specific flow plotted with the regression-estimated flow. All observed and regression-estimated flow duration have the delta value added.

The equations for regression-estimated flow durations and mean flow and uncertainty measures are listed in table 2. Only the 149 gaging stations on streams with uncontrolled flow with at least 10 years of record (table 1) were used in the regression analyses. The drainage area of these gaging stations ranged from 2.06 to $12,004 \mathrm{mi}^{2}$. The $\mathrm{Chi}^{2}$ is a measure of the fit of the Tobit analysis model. The delta value is varied until the $\mathrm{Chi}^{2}$ is maximized. For these analyses the contributing-drainage area $(C D A)$ was divided by 1,000 , the 30 -year mean precipitation (PREC) was divided by 28 , and the mean basin slope (SLOPE) was divided by 2 before the log transformation was made so that the log values of each of these parameters were balanced between greater than and less than zero. This eliminated the multi-colinearity problems that occur when using squared values (A.V. Vecchia, USGS, written commun., 2002). The addition of the squares of log contributing-drainage area and log mean annual precipitation to the regression equation improved the models substantially.

Regression equations for peak-discharge frequency estimates for Kansas are provided by Rasmussen and Perry (2000). Peak discharges were estimated at recurrence intervals (frequencies) ranging from 2 to 100 years using log-Pearson TypeIII (distributions for 253 streamflow-gaging stations in Kansas (see Rasmussen and Perry, 2000, table 5). The annual peakdischarge data, through the 1997 water year, were from streamflow-gaging stations with uncontrolled flow in mostly rural basins. A weighted least-squares regression model was used to generalize the coefficients of station skewness. The resulting generalized skewness equation provides more reliable skewness estimates for the log-Pearson Type-III analyses than the previously developed equation for Kansas (Clement, 1987).

Rasmussen and Perry (2000) used a generalized leastsquares regression model to develop equations for estimating peak streamflow for sites without stream gages for selected frequencies from selected physical and climatic basin characteristics for sites with stream gages. The equations can be used to estimate peak streamflow for selected frequencies using contributing-drainage area, mean annual precipitation, soil permeability, and slope of the main channel for ungaged sites in Kansas with contributing-drainage areas greater than 0.17 and less than $9,100 \mathrm{mi}^{2}$. The equations and their errors are provided in table 3.

\section{Kansas Surface Water Register}

In 1994, KDHE adopted the Reach File Version 2 (RF2) stream-segment coverage within the State of Kansas as the basic coverage for stream classification. RF2 was completed in the late 1980s by the U.S. Environmental Protection Agency (USEPA) using the Feature File of the USGS Geographic Names Information System (GNIS) to add one new level of reach segments to the Reach File Version 1 (RF1) coverage (U.S. Environmental Protection Agency, 1996). The source of RF1 (completed in 1982) was the USGS's 1:250,000-scale hydrography that was photographically reduced to a scale of 1:500,000 by the National Oceanic and Atmospheric Administration (NOAA).

In addition to the RF2 segments, other segments have been added to the KSWR by KDHE primarily for the protection of aquatic life and other water-quality issues. The 1999 KSWR has 2,232 stream segments. Each segment on the 1999 KSWR is identified by a unique CUSEGA number (tables 7-111, at the end of this report). CUSEGA stands for catalog unit segment number alpha. Individual CUSEGA segments may have one or more subsegments associated with it. The KSWR of June 1, 1999 , is a public document and can be obtained from the World Wide Web (URL http://www.kdhe.state.ks.us/pdf/befs/ register99.pdf).

The original RF2 coverage had almost 30,000 subsegments in Kansas. By combining subsegments and by ending the stream segments at hydrologic breaks such as at the confluence of tributaries or at lakes, the total number of segments within Kansas was reduced to 5,427 for this report. Streamflow statistics were determined at the downstream end of these segments (called streamflow-statistics determination sites in this report). Of the 5,427 determination sites, 656 are located in lakes or along irrigation ditches where statistical computations are not reported. Therefore, stream statistics are reported at 4,771 sites and their associated stream segments.

Because many of the stream basins in Kansas extend into the surrounding States, the data used for developing the 1999 KSWR stream network, which is based on the more detailed $\mathrm{RF} 2$, were joined with the national RF1 coverage that is available for Colorado, Missouri, Nebraska, and Oklahoma. This process was done in a GIS procedure by clipping the Kansas extent of the original RF1 stream coverage and replacing it with the more detailed version of the RF2 stream coverage. The two coverages were joined at the State boundaries for continuity. The line topology was reconfigured so that spatial relations between connecting stream segments (from and to nodes) were updated. Then the updated stream coverage was rechecked to 
A.
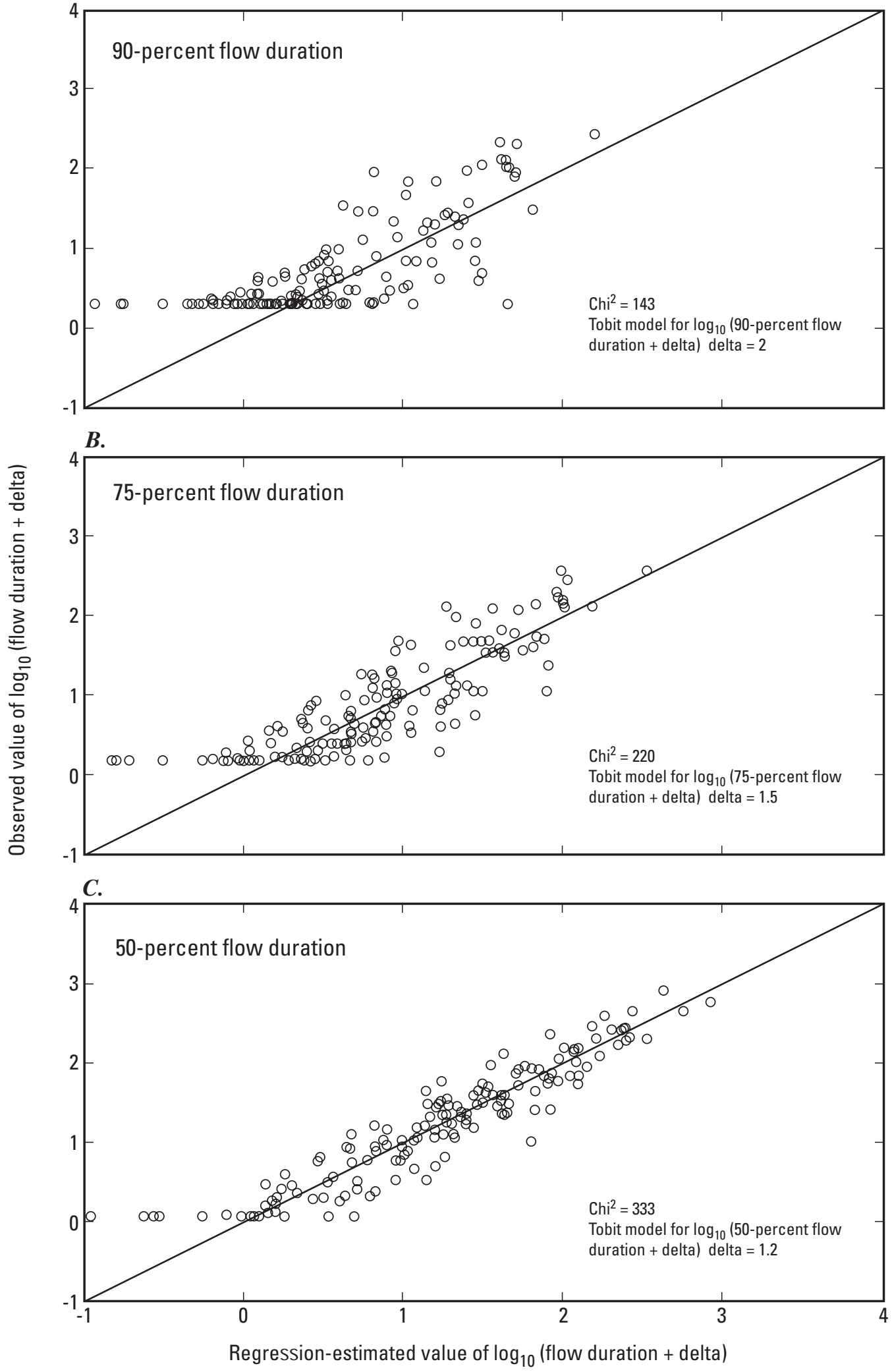

Figure 8. Results of regression with Tobit analysis for flow durations of $(A) 90,(B) 75,(C) 50,(D) 25$, and (E) 10 percent and (F) mean flow. 
$D$.

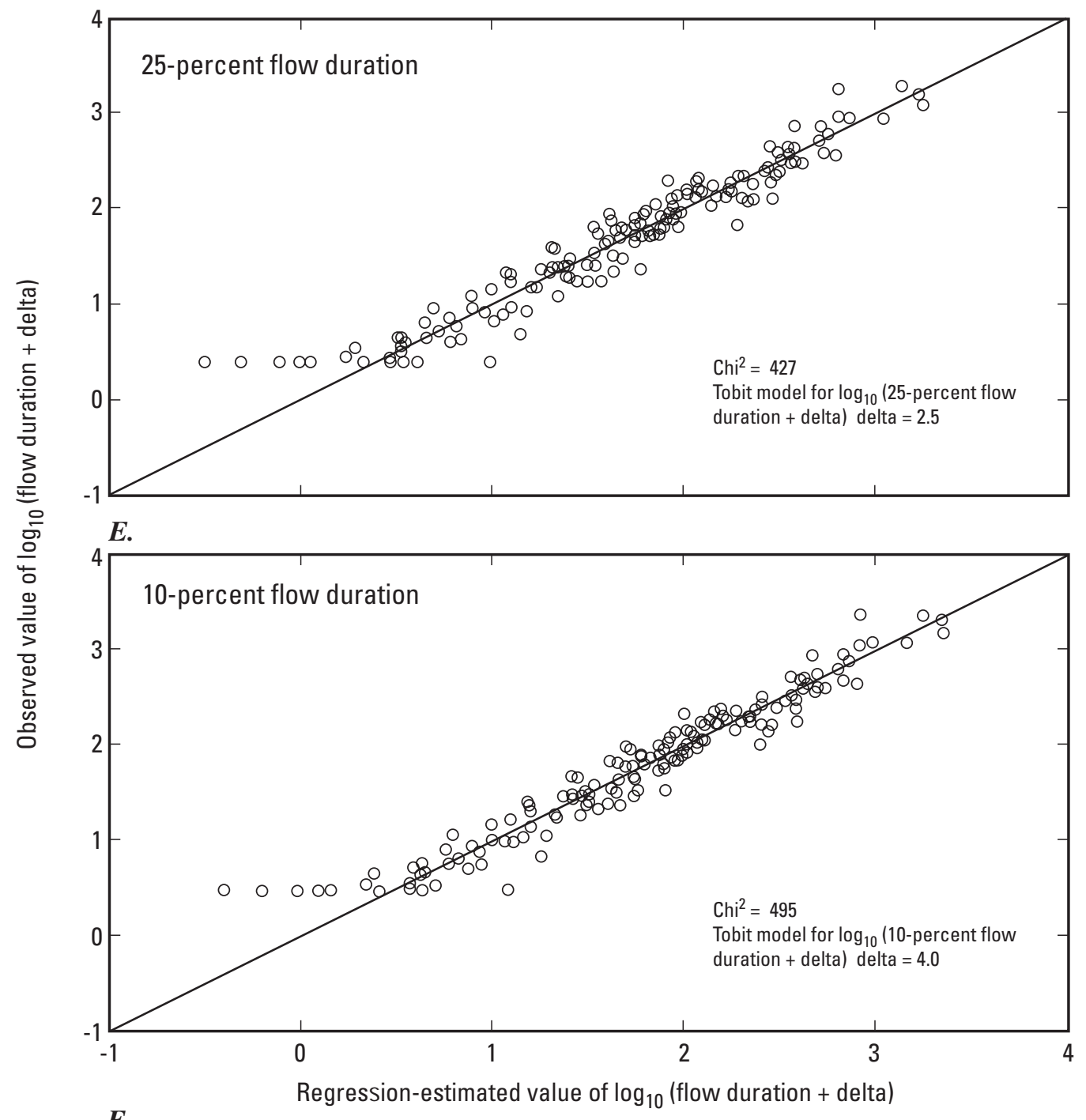

F.

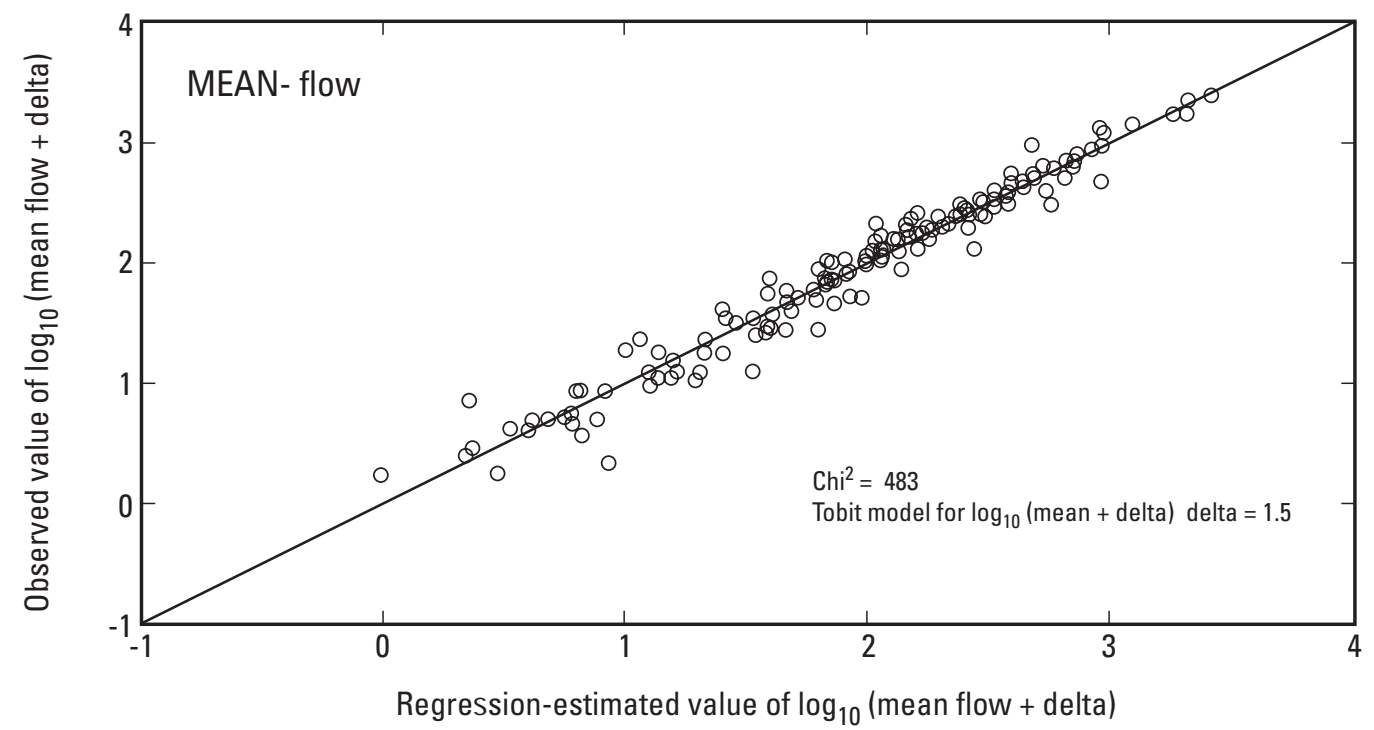

Figure 8. Results of regression with Tobit analysis for flow durations of $(A) 90,(B) 75,(C) 50,(D) 25$, and $(E) 10$ percent and (F) mean flow.-Continued 
Water Register ${ }^{1}$

$\left[\mathrm{Chi}^{2}\right.$, Chi square statistical distribution; $\mathrm{n}$, number of stations used in the analysis; $C D A$, contributing-drainage area, in square miles; PREC, precipitation, in inches; PERM, soil permeability, in inches per hour; SLOPE, land-surface slope, in degrees; $\log d a=\log _{10}\left(\frac{C D A}{1,000}\right) ; \log$ prec $=\log _{10}\left(\frac{\text { PREC }}{28}\right) ; \log$ perm $=\log _{10}$ PERM; $\log$ slope $\left.=\log _{10}\left(\frac{S L O P E}{2}\right)\right]$

\begin{tabular}{|c|c|c|c|c|c|}
\hline Flow value & Equation & $\mathrm{Chi}^{2}$ & $\begin{array}{l}\text { Standard } \\
\text { error } \\
(\log \\
\text { units) }\end{array}$ & $\begin{array}{c}\text { De- } \\
\text { grees } \\
\text { of } \\
\text { free- } \\
\text { dom }\end{array}$ & $\mathbf{n}$ \\
\hline $\begin{array}{c}\text { 90-percent } \\
\text { duration }\end{array}$ & $\mathrm{Q}_{90}=\left[10^{\left[0.902+1.030 \log d a+4.046 \log \text { prec }+0.732 \log \text { perm }+0.008 \log \text { slope }+0.292(\operatorname{logda})^{2}+\left(-17.89(\log \text { prec })^{2}\right)\right]}\right]-2.0$ & 143 & 0.434 & 6 & 149 \\
\hline $\begin{array}{l}\text { 75-percent } \\
\text { duration }\end{array}$ & $\mathrm{Q}_{75}=\left[10^{\left[1.268+1.073 \log d a+4.310 \log p r e c+0.755 \log p e r m+0.339 \log \text { slope }+0.222(\log \mathrm{da})^{2}+\left(-17.63(\log p r e c)^{2}\right)\right]}\right]-1.5$ & 220 & .344 & 6 & 149 \\
\hline $\begin{array}{l}\text { 50-percent } \\
\text { duration }\end{array}$ & $\mathrm{Q}_{50}=\left[10^{\left[1.685+1.124 \log d a+5.283 \log \text { prec }+0.638 \log \text { perm }+0.517 \log \text { slope }+0.152(\operatorname{logda})^{2}+\left(-15.38(\log \text { prec })^{2}\right)\right]}\right]-1.2$ & 333 & .250 & 6 & 149 \\
\hline $\begin{array}{l}\text { 25-percent } \\
\text { duration }\end{array}$ & $\mathrm{Q}_{25}=\left[10^{\left[2.082+1.079 \log d a+5.365 \log \text { prec }+0.393 \log \text { perm }+0.490 \log \text { slope }+0.104(\operatorname{logda})^{2}+\left(-11.10(\log \text { prec })^{2}\right)\right]}\right]-2.5$ & 427 & .177 & 6 & 149 \\
\hline $\begin{array}{l}\text { 10-percent } \\
\text { duration }\end{array}$ & $\mathrm{Q}_{10}=\left[10^{\left[2.502+1.109 \log d a+5.638 \log \text { prec }+0.218 \log \text { perm }+0.434 \log \text { slope }+0.085(\log \mathrm{da})^{2}+\left(-9.984(\log \text { prec })^{2}\right)\right]}\right]-4.0$ & 495 & .154 & 6 & 149 \\
\hline Mean flow & $\mathrm{Q}_{\text {mean }}=\left[10^{\left[2.286+0.978 \log d a+4.884 \log \text { prec }+0.084 \log \text { perm }+0.279 \log \text { slope }+0.040(\operatorname{logda})^{2}+\left(-7.331(\log \text { prec })^{2}\right)\right]}\right]-1.5$ & 483 & .143 & 6 & 149 \\
\hline
\end{tabular}

\footnotetext{
${ }^{1}$ The Kansas Surface Water Register is maintained by the Kansas Department of Health and Environment (Topeka).
} 
Table 3. Generalized least-squares regression equations for estimating 2- to 100-year peakstreamflow discharges for unregulated, rural streams in Kansas (modified from Rasmussen and Perry, 2000).

$\left[\mathrm{Q}_{t}\right.$, estimated peak discharge, in cubic feet per second, for a $t$-year recurrence interval; $C D A$, contributingdrainage area for the site, in square miles; $P$, average mean annual precipitation for the entire basin, in inches; $S l$, slope of the main channel, in feet per mile; $S$, average soil permeability for the entire basin, in inches per hour]

\begin{tabular}{ccc}
\hline $\mathrm{Q}_{\mathrm{t}}$ & \multicolumn{1}{c}{ Regression equations } & $\begin{array}{c}\text { Model standard } \\
\text { error of } \\
\text { prediction } \\
\text { (log } 10 \text { units) }\end{array}$ \\
\hline \multicolumn{3}{c}{ For contributing-drainage areas ranging from 30 to 9,100 square miles } \\
$\mathrm{Q}_{2}$ & $0.00001477(C D A)^{0.646}(P)^{4.307}(S l)^{0.5266}(S)^{-0.1736}$ & 0.155 \\
$\mathrm{Q}_{5}$ & $0.001336(C D A)^{0.590}(P)^{3.373}(S l)^{0.4235}(S)^{-0.2231}$ & .133 \\
$\mathrm{Q}_{10}$ & $0.01085(C D A)^{0.568}(P)^{2.945}(S l)^{0.374}(S)^{-0.248}$ & .131 \\
$\mathrm{Q}_{25}$ & $0.0829(C D A)^{0.549}(P)^{2.532}(S l)^{0.326}(S)^{-0.275}$ & .136 \\
$\mathrm{Q}_{50}$ & $0.283(C D A)^{0.539}(P)^{2.283}(S l)^{0.298}(S)^{-0.293}$ & .144 \\
$\mathrm{Q}_{100}$ & $0.810(C D A)^{0.532}(P)^{2.070}(S l)^{0.272}(S)^{-0.309}$ & .153 \\
& For contributing-drainage areas ranging from 0.17 to less than 30 square miles \\
$\mathrm{Q}_{2}$ & $0.0126(C D A)^{0.579}(P)^{2.824}$ & .216 \\
$\mathrm{Q}_{5}$ & $0.300(C D A)^{0.600}(P)^{2.138}$ & .184 \\
$\mathrm{Q}_{10}$ & $1.224(C D A)^{0.611}(P)^{1.844}$ & .183 \\
$\mathrm{Q}_{25}$ & $4.673(C D A)^{0.622}(P)^{1.572}$ & .198 \\
$\mathrm{Q}_{50}$ & $10.26(C D A)^{0.628}(P)^{1.415}$ & .214 \\
$\mathrm{Q}_{100}$ & $19.80(C D A)^{0.634}(P)^{1.288}$ & .232 \\
\hline & &
\end{tabular}

correct any remaining digitizing errors including cycles, overshoots, and undershoots (that is, an arc that does not extend far enough to intersect another arc). Finally, the topology was checked for consistency (that is, all segments point downstream). All GIS analyses were performed using the Environmental Systems Research Institute (ESRI) ArcGIS and ArcInfo software.

A GIS database was used to manage and display the basin characteristics and estimated streamflow statistics for stream locations on the 1999 KSWR. The relational database design facilitates identification and analysis of data unique to individual stream locations. The stream coverage and associated data are available on the World Wide Web (URL

http://ks.water.usgs.gov/Kansas/studies/strmstats/index.shtml).

\section{Basin Characteristics for Stream Locations}

Drainage basins for each stream location on the 1999 KSWR were determined in the GIS by converting the vector stream-location coverage into a raster-grid network with a raster size of 492 by $492 \mathrm{ft}$ ( 150 by $150 \mathrm{~m}$ ). Euclidean allocation was performed on the rasterized stream network to calculate for each cell the identity of the closest source or stream cell using the Euclidean distance. Euclidean distance is defined as the shortest length between two points in two-dimensional space. GIS Euclidean allocation zones may not coincide with actual topographic basin divides. Therefore, basin contributing-drainage areas obtained by the GIS method used herein may differ from those obtained using conventional topographic planimetering methods.

Mean values for climatic and basin characteristics were calculated for stream-location drainage basins using zonal statistics on basin-characteristic grids with Euclidean allocation zones. Zonal statistics were recorded in an attribute table and included the area and mean of the values of all cells in the basincharacteristic grids that belong to the same Euclidean zone. The climatic and basin characteristics computed included contributing-drainage area, mean annual precipitation, mean basin elevation, mean basin permeability, mean basin slope, a base-flow index (BFI), and mean annual runoff (Gebert and others, 1987) and water-balance model runoff (Wolock and McCabe, 1999) flow values. Output zonal statistics tables were relationally joined back to the original vector streams coverage so that each stream location had an estimated value for each climatic and basin characteristic for the entire basin upstream from each stream location.

One parameter that needed a separate GIS computation was the main channel slope for each of the determination sites. 
The slope of the main channel is measured by dividing the difference in elevation at points in the channel at 10 and 85 percent of the distance upstream along the main channel length by the intervening main channel length. A computer software program was written (D.M.Wolock, USGS, written commun., 2003) that searches upstream from each determination site, finds the longest stream distance, computes the 10- and 85-percent distance locations, and uses the elevation at those locations and distance along the stream channel to determine the main channel slope.

\section{Estimates of Streamflow Statistics for Stream Locations}

Initially, streamflow statistics were computed for each flow determination site. Flow durations and peak-discharge frequencies were checked at each site for a logical progression of flow values. At some determination sites with small drainage areas of less than $5 \mathrm{mi}^{2}$, the quadratic part of the equations for the lower (10- and 25-percent) flow durations resulted in flow values that decreased with increasing durations. If the drainage area resulted in a more negative logarithm, the square of that value could dominate the other factors in the equation, and an anomalously high value for flow would result. All determination sites were checked for decreasing flow with increasing flow duration. Of the few sites that were affected, the higher durations $(50,75$, and 90 percent) were graphically extrapolated to recompute the 10- and 25-percent duration flows.

Next, different interpolation procedures were used to refine the estimate of flow durations, mean flow, and peak-discharge frequency values for each stream location depending on whether the location had controlled or uncontrolled flow and whether or not there was a streamflow-gaging station located either upstream or downstream from the location. These interpolation procedures utilize the previously defined drainage-area ratio method and multiple linear-regression equations and are summarized in table 4 . The interpolation procedures outlined in table 4 for an ungaged location between two gaged locations selects the upstream gage location (if there is more than one) that has the largest drainage area. These procedures were applied to flow statistics developed for each stream location.

Flow-duration and mean flow value computations for controlled and uncontrolled flow streamflow-gaging stations used in the interpolation of the flow duration and mean flow are listed in table 5. Flow durations and mean flows at gages representing controlled stream locations (those with Federal reservoirs upstream) were computed from the controlled period of record only. These records had to be at least 10 years in length for the period 1961 to 2000. Use of the 1961-to-2000 time period maintains a degree of consistency for comparison and interpolation of flow durations and mean flow between gaging stations at controlled locations and is reasonably representative of long-term average precipitation.

Peak-discharge frequency values at controlled and uncontrolled flow streamflow-gaging stations (fig. 2) and used in the interpolation of peak-discharge-frequency values are listed in table 6. Peak-discharge frequency values at gaging stations representing uncontrolled flow stream locations were from Rasmussen and Perry (2000, see table 5). Peak-discharge-frequency values at gaging stations representing controlled stream locations were available from several sources. The U.S. Army Corps of Engineers (USCOE), Kansas City District (oral commun., 2003) was used as the primary source of peak-discharge frequency values for controlled stream locations. If no frequency values for a particular stream location were available from USCOE, then published Federal Emergency Management Agency's Flood Insurance Studies for communities in Kansas (Federal Emergency Management Agency, various years) served as a secondary source. The third and final source of peak-discharge frequency values was a log-Pearson Type-III analyses of the controlled period of record. This final source was a Bulletin 17B estimate (Interagency Advisory Committee on Water Data, 1981) using either the systematic record skew values or the regional skew values to generate the estimated peak-discharge frequency values. When systematic skew values were extremely negative (less than -1.2), the regional skew value was used.

\section{Interpolation Example}

Figure 9 shows part of a stream network and some streamflow-gaging stations in central Kansas. The large numbers next to the gaging stations are the 50-percent (median) flow-duration values observed for those stations. The regression equations used are described in the section on "Multiple Linear-

Regression Analysis." The smaller numbers are the 50-percent flow-duration values estimated from those regression equations. A comparison of the regression-estimated 50-percent flow-duration values with the observed gaging-station 50-percent flow-duration values shows substantial "local" differences between the estimated and the observed values.

Figure 10 shows the effect of interpolating the observed gaging-station 50-percent (median) flow-duration values with the regression-estimated values to develop the best-estimated flow for each stream location. The local differences in estimated 50-percent flow-duration values noted in figure 9 (regression estimates) are not as large in figure 10 (estimates derived in this report) because of the effect of incorporating local gaging-station data. As a result, the interpolation procedure used in this report to develop stream-statistic estimates appears to provide more accurate estimates than those that result from using only the regression equations.

\section{Estimated Stream Statistics}

The interpolation procedure was performed on all the flow durations, the mean flow, and all the peak-discharge values for all uncontrolled and controlled flow determination sites. The 
Table 4. Summary of interpolation procedures used to estimate flow duration, mean flow, and peak-discharge flow information for streamflow-statistics determination sites on the 1999 Kansas Surface Water Register (KSWR)

$[Q$, flow statistic; $D A$, drainage area; $B$, bias equals measured gaging station $Q$ minus regression equation $Q$; Subscripts: $r$, regression-equation estimate of flow; $s$, segment (ungaged); $b$, with bias added; $u$, upstream gaging station; $d$, downstream gaging station; $g$, at streamflow gage

\begin{tabular}{|c|c|c|c|}
\hline $\begin{array}{c}\text { Case } \\
\text { number }\end{array}$ & Case & Controlled segment interpolation procedure & Uncontrolled segment interpolation procedure \\
\hline 1 & No gage on stream. & Never occurs. & Use regression estimate (no adjustment). \\
\hline 2 & Gage on determination site segment . & Use gaged value. & Use gaged value. \\
\hline \multirow[t]{3}{*}{3} & $\begin{array}{l}\text { Gage on stream only upstream or } \\
\text { downstream from that determination } \\
\text { site segment. }\end{array}$ & $\begin{array}{l}\text { Estimate flow from gage data using ratio of gage drain- } \\
\text { age area to the determination site drainage area. Ignore } \\
\text { regression equations. }\end{array}$ & $\begin{array}{l}\text { Use regression estimate adjusted by weighting the bias between the } \\
\text { gaged value and the estimated value at the gaged site by the ratio of } \\
\text { the gaged drainage area to the determination site drainage area. }\end{array}$ \\
\hline & & $Q_{s}=\frac{Q_{u}}{D A_{u}} \times D A_{s} \quad$ or & $B_{s}=\frac{B_{u}}{D A_{u}} \times D A_{s} \times \frac{D A_{u}}{D A_{s}} ;$ or $B_{s}=\frac{B_{d}}{D A_{d}} \times D A_{s} \times \frac{D A_{s}}{D A_{d}}$ \\
\hline & & $Q_{s}=\frac{Q_{d}}{D A_{d}} \times D A_{s}$ & $Q_{s b}=Q_{s r}+B_{s}$ \\
\hline \multirow[t]{3}{*}{4} & $\begin{array}{l}\text { Gage on stream both upstream and } \\
\text { downstream from that flow determina- } \\
\text { tion site segment. }\end{array}$ & $\begin{array}{l}\text { Estimate the flow from upstream and downstream gage } \\
\text { data using weighted average ratios of gage drainage } \\
\text { areas to the determination site drainage area. Ignore } \\
\text { regression equations. }\end{array}$ & $\begin{array}{l}\text { Use regression estimate adjusted by a weighted average bias between } \\
\text { the two gages using drainage areas of gages and the determination } \\
\text { site. }\end{array}$ \\
\hline & & $Q_{s}=\frac{Q_{u}\left(D A_{d}-D A_{s}\right)+Q_{d}\left(D A_{s}-D A_{u}\right)}{D A_{d}-D A_{u}}$ & $B_{s}=\frac{B_{u}\left(D A_{d}-D A_{s}\right)+B_{a}\left(D A_{s}-D A_{u}\right)}{D A_{d}-D A_{u}}$ \\
\hline & & & $Q_{s b}=Q_{s r}+B_{s}$ \\
\hline
\end{tabular}


Table 5. Streamflow-gaging stations in Kansas and parts of suro
stream locations on the 1999 Kansas Surface Water Register.

\begin{tabular}{|c|c|c|c|c|c|c|c|}
\hline \multirow{2}{*}{$\begin{array}{l}\text { Station } \\
\text { number } \\
\text { (fig. 1) }\end{array}$} & \multirow{2}{*}{ Stream name } & \multicolumn{5}{|c|}{$\begin{array}{l}\text { Computed flow duration, in cubic feet per second, for indicated percentage of time flow } \\
\text { equaled or exceeded }\end{array}$} & \multirow{2}{*}{$\begin{array}{l}\text { Mean flow for } \\
\text { period of record } \\
\text { (cubic feet per } \\
\text { second) }\end{array}$} \\
\hline & & 90 percent & 75 percent & 50 percent & 25 percent & 10 percent & \\
\hline 06813500 & Missouri River at Rulo, NE & 22,000 & 33,500 & 40,800 & 53,900 & 69,000 & 44,800 \\
\hline 06814000 & Turkey Creek near Seneca, KS & 2.10 & 7.40 & 22.0 & 69.0 & 203 & 129 \\
\hline 06815000 & Big Nemaha River at Falls City, NE & 45.0 & 80.0 & 160 & 390 & 1,050 & 627 \\
\hline 06818000 & Missouri River at St. Joseph, MO & 23,600 & 34,500 & 43,300 & 57,600 & 75,000 & 48,100 \\
\hline 06826500 & South Fork Republican River near Hale, CO & 5.20 & 5.80 & 6.20 & 7.60 & 39.0 & 16.6 \\
\hline 06827500 & South Fork Republican River near Benkleman, NE & 0 & .52 & 16.0 & 31.0 & 61.0 & 26.9 \\
\hline 06836500 & Driftwood Creek near McCook, NE & .30 & 2.10 & 4.80 & 6.70 & 11.0 & 9.50 \\
\hline 06844700 & South Fork Sappa Creek near Brewster, KS & 0 & 0 & 0 & 0 & 0 & .23 \\
\hline 06844900 & South Fork Sappa Creek near Achilles, KS & 0 & 0 & 0 & .32 & 2.30 & 3.42 \\
\hline 06845000 & Sappa Creek near Oberlin, KS & 0 & .10 & .70 & 5.40 & 18.0 & 16.3 \\
\hline 06845200 & Sappa Creek near Beaver City, NE & 0 & 1.10 & 5.00 & 17.0 & 60.0 & 38.2 \\
\hline 06846500 & Beaver Creek at Cedar Bluffs, KS & 0 & 0 & .02 & 5.65 & 23.0 & 13.6 \\
\hline 06847900 & Prairie Dog Creek above Keith Sebelius Lake, KS & 0 & .16 & 2.10 & 5.80 & 11.0 & 9.05 \\
\hline 06848000 & Prairie Dog Creek at Norton, KS & 0 & .02 & .10 & .47 & 3.10 & 5.50 \\
\hline 06848500 & Prairie Dog Creek near Woodruff, KS & 0 & 0 & 2.00 & 7.50 & 16.0 & 11.1 \\
\hline 06853020 & Republican River at Guide Rock, NE & 6.80 & 55.0 & 111 & 170 & 448 & 212 \\
\hline 06853500 & Republican River near Hardy, NE & 63.0 & 110 & 167 & 307 & 728 & 333 \\
\hline 06853800 & White Rock Creek near Burr Oak, KS & .47 & 1.80 & 6.00 & 17.0 & 40.0 & 28.6 \\
\hline 06854000 & White Rock Creek at Lovewell, KS & .06 & .10 & .20 & .57 & 55.0 & 33.2 \\
\hline 06854500 & Republican River at Scandia, KS & 120 & 155 & 244 & 537 & 1,100 & 484 \\
\hline 06855800 & Buffalo Creek near Jamestown, KS & .95 & 3.10 & 11.0 & 41.0 & 133 & 71.7 \\
\hline 06855900 & Wolf Creek near Concordia, KS & 0 & .10 & 1.00 & 4.30 & 17.0 & 11.0 \\
\hline 06856000 & Republican River at Concordia, KS & 113 & 170 & 284 & 577 & 1,250 & 577 \\
\hline 06856600 & Republican River at Clay Center, KS & 140 & 220 & 396 & 837 & 1,830 & 872 \\
\hline 06857100 & Republican River below Milford Dam, KS & 57.0 & 126 & 357 & 1,020 & 2,400 & 960 \\
\hline 06858500 & North Fork Smoky Hill River near McAllaster, KS & 0 & 0 & 0 & 1.00 & 3.00 & 3.68 \\
\hline 06859500 & Ladder Creek below Chalk Creek near Scott City, KS & 0 & .39 & 1.90 & 4.00 & 10.0 & 8.00 \\
\hline
\end{tabular}


Table 5. Streamflow-gaging stations in Kansas and parts of surrounding States used in the interpolation of flow durations and mean flows at controlled and uncontrolled flow stream locations on the 1999 Kansas Surface Water Register. —Continued

\begin{tabular}{|c|c|c|c|c|c|c|c|}
\hline \multirow{2}{*}{$\begin{array}{l}\text { Station } \\
\text { number } \\
\text { (fig. 1) }\end{array}$} & \multirow{2}{*}{ Stream name } & \multicolumn{5}{|c|}{$\begin{array}{l}\text { Computed flow duration, in cubic feet per second, for indicated percentage of time flow } \\
\text { equaled or exceeded }\end{array}$} & \multirow{2}{*}{$\begin{array}{c}\text { Mean flow for } \\
\text { period of record } \\
\text { (cubic feet per } \\
\text { second) }\end{array}$} \\
\hline & & 90 percent & 75 percent & 50 percent & 25 percent & 10 percent & \\
\hline 06860000 & Smoky Hill River at Elkader, KS & 0 & 0.11 & 1.50 & 6.00 & 22.0 & 23.9 \\
\hline 06861000 & Smoky Hill River near Arnold, KS & .01 & .17 & 2.30 & 15.0 & 47.0 & 44.0 \\
\hline 06862000 & Smoky Hill River at Cedar Bluffs Dam, KS & 0 & 0 & .28 & .90 & 6.50 & 7.98 \\
\hline 06862700 & Smoky Hill River near Schoenchen, KS & .37 & 2.70 & 11.0 & 18.0 & 29.0 & 23.7 \\
\hline 06862850 & Smoky Hill River below Schoenchen, KS & 0 & 0 & 1.80 & 11.0 & 25.0 & 19.6 \\
\hline 06863300 & Big Creek near Ogallah, KS & .20 & 1.20 & 2.90 & 6.60 & 16.0 & 21.7 \\
\hline 06863500 & Big Creek near Hays, KS & 1.90 & 3.50 & 7.90 & 19.0 & 38.0 & 33.3 \\
\hline 06863900 & North Fork Big Creek near Victoria, KS & 0 & 0 & 0 & 0 & .90 & 3.10 \\
\hline 06864000 & Smoky Hill River near Russell, KS & 16.0 & 22.0 & 35.0 & 75.0 & 206 & 148 \\
\hline 06864050 & Smoky Hill River near Bunker Hill, KS & 8.50 & 17.0 & 34.0 & 77.0 & 198 & 128 \\
\hline 06864500 & Smoky Hill River at Ellsworth, KS & 19.0 & 34.0 & 65.0 & 138 & 357 & 223 \\
\hline 06865500 & Smoky Hill River near Langley, KS & 20.0 & 40.0 & 78.0 & 194 & 672 & 267 \\
\hline 06866500 & Smoky Hill River near Mentor, KS & 41.0 & 72.0 & 135 & 315 & 948 & 381 \\
\hline 06866900 & Saline River near Wakeeney, KS & 0 & .03 & 2.60 & 12.0 & 24.0 & 21.3 \\
\hline 06867000 & Saline River near Russell, KS & 4.80 & 12.0 & 31.0 & 73.0 & 165 & 98.4 \\
\hline 06867500 & Paradise Creek near Paradise, KS & 0 & 0 & .13 & 4.00 & 26.0 & 19.4 \\
\hline 06868200 & Saline River at Wilson Dam, KS & 5.10 & 8.70 & 16.0 & 21.0 & 191 & 91.6 \\
\hline 06868400 & Wolf Creek near Lucas, KS & .70 & 1.10 & 2.20 & 6.80 & 18.0 & 16.2 \\
\hline 06869500 & Saline River at Tescott, KS & 15.0 & 24.0 & 45.0 & 126 & 490 & 212 \\
\hline 06870200 & Smoky Hill River at New Cambria, KS & 70.5 & 116 & 224 & 557 & 1,870 & 688 \\
\hline 06870300 & Gypsum Creek near Gypsum, KS & 0 & 1.00 & 7.00 & 15.0 & 34.3 & 25.9 \\
\hline 06871000 & North Fork Solomon River at Glade, KS & 0 & .20 & 8.40 & 22.0 & 48.0 & 27.9 \\
\hline 06871500 & Bow Creek near Stockton, KS & .44 & 2.60 & 5.60 & 10.0 & 18.0 & 13.7 \\
\hline 06871800 & North Fork Solomon River at Kirwin, KS & 0 & 0 & .03 & .12 & .50 & 8.76 \\
\hline 06871900 & Deer Creek near Phillipsburg, KS & 0 & 0 & .69 & 1.50 & 2.70 & 4.04 \\
\hline 06872500 & North Fork Solomon River at Portis, KS & 12.0 & 20.0 & 34.0 & 75.0 & 162 & 98.4 \\
\hline 06873000 & South Fork Solomon River above Webster Reservoir, KS & .03 & 1.00 & 14.0 & 37.0 & 77.0 & 53.8 \\
\hline 06873200 & South Fork Solomon River below Webster Reservoir, KS & 0 & 0 & 0 & 22.0 & 123 & 33.9 \\
\hline
\end{tabular}


Table 5. Streamflow-gaging stations in Kansas and parts of surrounding States used in the interpolation of flow durations and mean flows at controlled and uncontrolled flow stream locations on the 1999 Kansas Surface Water Register. - Continued

\begin{tabular}{|c|c|c|c|c|c|c|c|}
\hline \multirow{2}{*}{$\begin{array}{l}\text { Station } \\
\text { number } \\
\text { (fig. 1) }\end{array}$} & \multirow{2}{*}{ Stream name } & \multicolumn{5}{|c|}{$\begin{array}{l}\text { Computed flow duration, in cubic feet per second, for indicated percentage of time flow } \\
\text { equaled or exceeded }\end{array}$} & \multirow{2}{*}{$\begin{array}{l}\text { Mean flow for } \\
\text { period of record } \\
\text { (cubic feet per } \\
\text { second) }\end{array}$} \\
\hline & & 90 percent & 75 percent & 50 percent & 25 percent & 10 percent & \\
\hline 06873460 & South Fork Solomon River at Woodston, KS & 0.43 & 1.30 & 6.50 & 29.0 & 99.0 & 51.5 \\
\hline 06873700 & Kill Creek near Bloomington, KS & 0 & 0 & 0 & 0 & 1.80 & 2.09 \\
\hline 06874000 & South Fork Solomon River at Osborne, KS & 6.00 & 11.0 & 21.0 & 58.0 & 174 & 91.2 \\
\hline 06875900 & Solomon River near Glen Elder, KS & 14.0 & 21.0 & 54.0 & 186 & 700 & 254 \\
\hline 06876070 & Solomon River near Simpson, KS & 30.0 & 63.0 & 187 & 531 & 1,940 & 561 \\
\hline 06876700 & Salt Creek near Ada, KS & 1.60 & 4.00 & 12.0 & 30.0 & 106 & 70.4 \\
\hline 06876900 & Solomon River at Niles, KS & 57.0 & 85.0 & 174 & 457 & 1,400 & 562 \\
\hline 06877600 & Smoky Hill River at Enterprise, KS & 190 & 299 & 595 & 1,470 & 3,930 & 1,570 \\
\hline 06878000 & Chapman Creek near Chapman, KS & 7.70 & 13.0 & 24.0 & 49.0 & 126 & 93.3 \\
\hline 06878500 & Lyon Creek near Woodbine, KS & 3.40 & 17.0 & 33.0 & 65.0 & 135 & 108 \\
\hline 06879100 & Kansas River at Fort Riley, KS & 408 & 637 & 1,350 & 3,310 & 7,450 & 3,010 \\
\hline 06882000 & Big Blue River near Barnestown, NE & 103 & 167 & 280 & 616 & 1,800 & 868 \\
\hline 06882500 & Big Blue River near Hull, KS & 88.0 & 130 & 217 & 365 & 895 & 470 \\
\hline 06882510 & Big Blue River at Marysville, KS & 200 & 286 & 467 & 924 & 2,590 & 1,190 \\
\hline 06884000 & Little Blue River near Fairbury, NE & 92.0 & 119 & 160 & 251 & 581 & 383 \\
\hline 06884025 & Little Blue River at Hollenberg, KS & 109 & 141 & 211 & 377 & 885 & 535 \\
\hline 06884200 & Mill Creek at Washington, KS & 3.20 & 7.50 & 19.0 & 56.0 & 181 & 108 \\
\hline 06884400 & Little Blue River near Barnes, KS & 128 & 170 & 268 & 525 & 1,340 & 704 \\
\hline 06885500 & Black Vermillion River near Frankfort, KS & 4.00 & 11.0 & 29.0 & 79.0 & 246 & 174 \\
\hline 06886500 & Fancy Creek at Winkler, KS & 0 & 2.00 & 11.0 & 23.0 & 59.0 & 47.4 \\
\hline 06887000 & Big Blue River near Manhattan, KS & 178 & 444 & 974 & 2,450 & 6,500 & 2,490 \\
\hline 06887500 & Kansas River at Wamego, KS & 846 & 1,360 & 2,720 & 6,340 & 13,600 & 5,650 \\
\hline 06888000 & Vermillion Creek near Wamego, KS & .40 & 2.50 & 17.0 & 53.0 & 143 & 87.4 \\
\hline 06888350 & Kansas River near Belvue, KS & 960 & 1,790 & 3,480 & 7,800 & 18,700 & 7,180 \\
\hline 06888500 & Mill Creek near Paxico, KS & 4.80 & 19.0 & 56.0 & 156 & 339 & 194 \\
\hline 06889000 & Kansas River at Topeka, KS & 974 & 1,630 & 3,020 & 7,060 & 15,800 & 6,400 \\
\hline 06889100 & Soldier Creek near Goff, KS & 0 & 0 & .08 & .30 & 1.10 & 1.38 \\
\hline 06889120 & Soldier Creek near Bancroft, KS & .01 & .15 & .55 & 2.00 & 6.60 & 6.94 \\
\hline
\end{tabular}

06889120 Soldier Creek near Bancroft, KS 
Table 5. Streamflow-gaging stations in Kansas and parts of surrounding States used in the interpolation of flow durations and mean flows at controlled and uncontrolled flow stream locations on the 1999 Kansas Surface Water Register. - Continued

\begin{tabular}{|c|c|c|c|c|c|c|c|}
\hline \multirow{2}{*}{$\begin{array}{l}\text { Station } \\
\text { number } \\
\text { (fig. 1) }\end{array}$} & \multirow{2}{*}{ Stream name } & \multicolumn{5}{|c|}{$\begin{array}{l}\text { Computed flow duration, in cubic feet per second, for indicated percentage of time flow } \\
\text { equaled or exceeded }\end{array}$} & \multirow{2}{*}{$\begin{array}{l}\text { Mean flow for } \\
\text { period of record } \\
\text { (cubic feet per } \\
\text { second) }\end{array}$} \\
\hline & & 90 percent & 75 percent & 50 percent & 25 percent & 10 percent & \\
\hline 06889140 & Soldier Creek near Soldier, KS & 0.21 & 0.48 & 1.20 & 3.50 & 10.0 & 10.7 \\
\hline 06889160 & Soldier Creek near Circleville, KS & .80 & 2.00 & 4.60 & 13.0 & 39.0 & 32.4 \\
\hline 06889180 & Soldier Creek near St. Clere, KS & 2.30 & 5.00 & 10.0 & 28.0 & 75.0 & 51.2 \\
\hline 06889200 & Soldier Creek near Delia, KS & 2.90 & 8.40 & 21.0 & 58.0 & 153 & 99.4 \\
\hline 06889500 & Soldier Creek near Topeka, KS & 2.20 & 9.40 & 30.0 & 89.0 & 243 & 158 \\
\hline 06890100 & Delaware River near Muscotah, KS & 6.20 & 18.0 & 51.0 & 146 & 450 & 280 \\
\hline 06890900 & Delaware River below Perry Dam, KS & 25.0 & 25.0 & 100 & 562 & 2,040 & 728 \\
\hline 06891000 & Kansas River at Lecompton, KS & 1,140 & 1,900 & 3,580 & 8,350 & 19,000 & 7,550 \\
\hline 06891500 & Wakarusa River near Lawrence, KS & 5.60 & 14.0 & 29.0 & 260 & 893 & 265 \\
\hline 06892000 & Stranger Creek near Tonganoxie, KS & 2.00 & 9.00 & 40.0 & 135 & 433 & 247 \\
\hline 06892350 & Kansas River at DeSoto, KS & 1,250 & 2,100 & 4,000 & 9,605 & 21,600 & 8,460 \\
\hline 06893000 & Missouri River at Kansas City, MO & 26,600 & 38,500 & 50,200 & 69,600 & 96,400 & 57,900 \\
\hline 06893080 & Big Blue River near Stanley, KS & .07 & .69 & 5.00 & 19.0 & 57.0 & 36.0 \\
\hline 06893300 & Indian Creek at Overland Park, KS & 1.30 & 4.70 & 13.0 & 22.0 & 56.0 & 34.6 \\
\hline 06893500 & Blue River near Kansas City, MO & 6.30 & 20.0 & 46.0 & 114 & 279 & 166 \\
\hline 06910800 & Marais des Cygnes River near Reading, KS & .18 & 2.30 & 15.0 & 56.0 & 174 & 113 \\
\hline 06911000 & Marais des Cygnes River at Melvern, KS & 2.50 & 14.0 & 33.0 & 99.0 & 382 & 208 \\
\hline 06911500 & Salt Creek near Lyndon, KS & 0 & .54 & 5.10 & 24.0 & 82.0 & 66.0 \\
\hline 06911900 & Dragoon Creek near Burlingame, KS & 0 & 1.00 & 8.00 & 32.0 & 93.0 & 68.4 \\
\hline 06912500 & Hundred and Ten Mile Creek near Quenemo, KS & 14.0 & 16.0 & 22.0 & 103 & 515 & 193 \\
\hline 06913000 & Marais des Cygnes River near Pomona, KS & 38.0 & 48.0 & 106 & 712 & 2,350 & 738 \\
\hline 06913500 & Marias des Cygnes River near Ottawa, KS & 40.0 & 51.0 & 146 & 791 & 2,573 & 850 \\
\hline 06914000 & Pottawatomie Creek near Garnett, KS & .10 & 2.00 & 21.0 & 90.0 & 361 & 235 \\
\hline 06915000 & Big Bull Creek near Hillsdale, KS & 4.30 & 6.50 & 20.0 & 71.0 & 375 & 116 \\
\hline 06915800 & Marais des Cygnes River at La Cygne, KS & 64.9 & 117 & 526 & 2,180 & 6,190 & 2,270 \\
\hline 06916500 & Big Sugar Creek at Farlinville, KS & 0 & 1.20 & 11.0 & 61.0 & 185 & 127 \\
\hline 06916600 & Marais des Cygnes River near Kansas-Missouri State line, KS & 41.0 & 109 & 579 & 2,310 & 6,440 & 2,380 \\
\hline 06917000 & Little Osage River at Fulton, KS & .20 & 2.90 & 32.0 & 129 & 396 & 238 \\
\hline
\end{tabular}


Table 5. Streamflow-gaging stations in Kansas and parts of surrounding States used in the interpolation of flow durations and mean flows at controlled and uncontrolled flow stream locations on the 1999 Kansas Surface Water Register. - Continued

\begin{tabular}{|c|c|c|c|c|c|c|c|}
\hline \multirow{2}{*}{$\begin{array}{l}\text { Station } \\
\text { number } \\
\text { (fig. 1) }\end{array}$} & \multirow{2}{*}{ Stream name } & \multicolumn{5}{|c|}{$\begin{array}{l}\text { Computed flow duration, in cubic feet per second, for indicated percentage of time flow } \\
\text { equaled or exceeded }\end{array}$} & \multirow{2}{*}{$\begin{array}{l}\text { Mean flow for } \\
\text { period of record } \\
\text { (cubic feet per } \\
\text { second) }\end{array}$} \\
\hline & & 90 percent & 75 percent & 50 percent & 25 percent & 10 percent & \\
\hline 06917380 & Marmaton River near Marmaton, KS & 0.46 & 4.08 & 43.0 & 159 & 470 & 302 \\
\hline 06917500 & Marmaton River near Fort Scott, KS & 1.00 & 2.70 & 31.0 & 130 & 446 & 288 \\
\hline 07134180 & Arkansas River near Granado, CO & 7.00 & 43.0 & 112 & 198 & 579 & 236 \\
\hline 07137500 & Arkansas River near Coolidge, KS & 9.30 & 47.0 & 129 & 242 & 482 & 237 \\
\hline 07138000 & Arkansas River at Syracuse, KS & 6.50 & 50.0 & 133 & 250 & 474 & 233 \\
\hline 07138650 & White Woman Creek near Leoti, KS & 0 & 0 & 0 & 0 & 0 & 1.00 \\
\hline 07139000 & Arkansas River at Garden City, KS & 0 & 1.10 & 32.0 & 161 & 314 & 163 \\
\hline 07139500 & Arkansas River at Dodge City, KS & 0 & 0 & 8.70 & 71.0 & 180 & 86.5 \\
\hline 07139800 & Mulberry Creek near Dodge City, KS & 0 & 0 & 0 & 0 & 0 & .64 \\
\hline 07140000 & Arkansas River near Kinsley, KS & .71 & 2.60 & 32.0 & 104 & 203 & 101 \\
\hline 07140850 & Pawnee River near Burdett, KS & 0 & 0 & 0 & 2.40 & 10.0 & 11.1 \\
\hline 07141200 & Pawnee River near Larned, KS & 0 & 0 & 3.70 & 15.0 & 59.0 & 63.2 \\
\hline 07141300 & Arkansas River at Great Bend, KS & 2.60 & 6.90 & 41.0 & 150 & 356 & 172 \\
\hline 07141780 & Walnut Creek near Rush Center, KS & 0 & 0 & 1.00 & 13.0 & 30.0 & 24.8 \\
\hline 07141900 & Walnut Creek at Albert, KS & 0 & 0 & 2.30 & 23.0 & 61.4 & 48.9 \\
\hline 07142300 & Rattlesnake Creek near Macksville, KS & 1.20 & 6.50 & 15.0 & 28.0 & 40.0 & 25.9 \\
\hline 07142575 & Rattlesnake Creek near Zenith, KS & 4.60 & 12.0 & 29.0 & 50.0 & 82.3 & 50.5 \\
\hline 07142620 & Rattlesnake Creek near Raymond, KS & 2.20 & 4.20 & 24.0 & 59.0 & 104 & 49.1 \\
\hline 07142860 & Cow Creek near Claflin, KS & 0 & 0 & .13 & .80 & 4.40 & 7.02 \\
\hline 07142900 & Blood Creek near Boyd, KS & 0 & .10 & .48 & 1.20 & 4.40 & 7.09 \\
\hline 07143300 & Cow Creek near Lyons, KS & 3.20 & 6.50 & 12.0 & 30.0 & 133 & 79.6 \\
\hline 07143330 & Arkansas River near Hutchinson, KS & 95.0 & 153 & 275 & 512 & 1,120 & 541 \\
\hline 07143375 & Arkansas River near Maize, KS & 64.0 & 134 & 303 & 600 & 1,260 & 730 \\
\hline 07143600 & Little Arkansas River near Little River, KS & 0 & .20 & .80 & 1.90 & 6.90 & 9.46 \\
\hline 07143665 & Little Arkansas River at Alta Mills, KS & 4.90 & 9.20 & 22.0 & 62.0 & 325 & 229 \\
\hline 07144200 & Little Arkansas River at Valley Center, KS & 21.0 & 33.0 & 60.0 & 127 & 488 & 312 \\
\hline 07144300 & Arkansas River at Wichita, KS & 126 & 226 & 433 & 858 & 2,220 & 1,050 \\
\hline 07144550 & Arkansas River at Derby, KS & 192 & 304 & 541 & 1,110 & 2,590 & 1,210 \\
\hline
\end{tabular}

07144550 Arkansas River at Derby, KS 
Table 5. Streamflow-gaging stations in Kansas and parts of surrounding States used in the interpolation of flow durations and mean flows at controlled and uncontrolled flow stream locations on the 1999 Kansas Surface Water Register. —Continued

\begin{tabular}{|c|c|c|c|c|c|c|c|}
\hline \multirow{2}{*}{$\begin{array}{l}\text { Station } \\
\text { number } \\
\text { (fig. 1) }\end{array}$} & \multirow{2}{*}{ Stream name } & \multicolumn{5}{|c|}{$\begin{array}{l}\text { Computed flow duration, in cubic feet per second, for indicated percentage of time flow } \\
\text { equaled or exceeded }\end{array}$} & \multirow{2}{*}{$\begin{array}{l}\text { Mean flow for } \\
\text { period of record } \\
\text { (cubic feet per } \\
\text { second) }\end{array}$} \\
\hline & & 90 percent & 75 percent & 50 percent & 25 percent & 10 percent & \\
\hline 07144780 & North Fork Ninnescah River above Cheney Reservoir, KS & 24.0 & 47.0 & 76.0 & 126 & 222 & 147 \\
\hline 07144795 & North Fork Ninnescah River at Cheney Dam, KS & .16 & .24 & .48 & 75.0 & 381 & 121 \\
\hline 07144850 & South Fork South Fork Ninnescah River near Pratt, KS & 0 & 0 & 0 & 0 & 0 & 2.63 \\
\hline 07144910 & South Fork Ninnescah River near Pratt, KS & 6.00 & 8.60 & 11.0 & 14.0 & 20.0 & 19.3 \\
\hline 07145200 & South Fork Ninnescah River near Murdock, KS & 66.0 & 97.0 & 135 & 194 & 304 & 209 \\
\hline 07145500 & Ninnescah River near Peck, KS & 76.4 & 125 & 212 & 483 & 1,126 & 511 \\
\hline 07145700 & Slate Creek at Wellington, KS & .91 & 3.30 & 8.00 & 21.0 & 73.2 & 73.1 \\
\hline 07146500 & Arkansas River at Arkansas City, KS & 373 & 558 & 1,030 & 2,000 & 4,440 & 2,090 \\
\hline 07146570 & Cole Creek near DeGraff, KS & 0 & .07 & 1.80 & 4.80 & 13.0 & 16.5 \\
\hline 07146830 & Walnut River at Highway 54 east of El Dorado, KS & 11.0 & 15.0 & 24.0 & 68.3 & 519 & 161 \\
\hline 07147070 & Whitewater River at Towanda, KS & 7.60 & 17.0 & 36.0 & 85.0 & 242 & 206 \\
\hline 07147800 & Walnut River at Winfield, KS & 60.0 & 104 & 262 & 860 & 2,655 & 1,140 \\
\hline 07148350 & Salt Fork Arkansas River near Winchester, OK & .35 & 7.40 & 34.0 & 75.0 & 161 & 96.0 \\
\hline 07149000 & Medicine Lodge River near Kiowa, KS & 12.0 & 47.0 & 85.0 & 141 & 271 & 154 \\
\hline 07149500 & Salt Fork Arkansas River near Cherokee, OK & 2.00 & 50.0 & 125 & 246 & 731 & 393 \\
\hline 07151500 & Chikaskia River near Corbin, KS & 19.0 & 47.0 & 94.0 & 190 & 430 & 250 \\
\hline 07152000 & Chikaskia River near Blackwell, OK & 23.0 & 66.0 & 144 & 324 & 895 & 589 \\
\hline 07154500 & Cimarron River near Kenton, OK & 0 & .06 & .91 & 2.80 & 7.50 & 17.2 \\
\hline 07155590 & Cimarron River near Elkhart, KS & 0 & 0 & 0 & 0 & 1.50 & 10.7 \\
\hline 07156010 & North Fork Cimarron River at Richfield, KS & 0 & 0 & 0 & 0 & .12 & 5.60 \\
\hline 07156100 & Sand Arroyo Creek near Johnson, KS & 0 & 0 & 0 & 0 & 0 & .25 \\
\hline 07156220 & Bear Creek near Johnson, KS & 0 & 0 & 0 & 0 & 0 & 3.35 \\
\hline 07156900 & Cimarron River near Forgan, OK & 27.0 & 35.0 & 45.0 & 62.0 & 82.0 & 58.4 \\
\hline 07157500 & Crooked Creek near Nye, KS & 2.40 & 7.10 & 12.0 & 18.0 & 33.0 & 30.5 \\
\hline 07157900 & Cavalry Creek at Coldwater, KS & .65 & 1.00 & 1.50 & 2.00 & 2.90 & 3.45 \\
\hline 07157950 & Cimarron River near Buffalo, OK & .03 & 10.0 & 56.0 & 123 & 232 & 128 \\
\hline 07165700 & Verdigris River near Madison, KS & .50 & 4.90 & 28.0 & 78.0 & 204 & 123 \\
\hline 07166000 & Verdigris River near Coyville, KS & 6.20 & 12.0 & 74.0 & 470 & 1,760 & 520 \\
\hline
\end{tabular}


Table 5. Streamflow-gaging stations in Kansas and parts of surrounding States used in the interpolation of flow durations and mean flows at controlled and uncontrolled flow stream locations on the 1999 Kansas Surface Water Register. - Continued

\begin{tabular}{|c|c|c|c|c|c|c|c|}
\hline \multirow{2}{*}{$\begin{array}{l}\text { Station } \\
\text { number } \\
\text { (fig. 1) }\end{array}$} & \multirow{2}{*}{ Stream name } & \multicolumn{5}{|c|}{$\begin{array}{l}\text { Computed flow duration, in cubic feet per second, for indicated percentage of time flow } \\
\text { equaled or exceeded }\end{array}$} & \multirow{2}{*}{$\begin{array}{l}\text { Mean flow for } \\
\text { period of record } \\
\text { (cubic feet per } \\
\text { second) }\end{array}$} \\
\hline & & 90 percent & 75 percent & 50 percent & 25 percent & 10 percent & \\
\hline 07166500 & Verdigris River near Altoona, KS & 10.0 & 24.0 & 138 & 800 & 2,590 & 808 \\
\hline 07167000 & Fall River near Eureka, KS & .70 & 5.20 & 40.0 & 134 & 324 & 190 \\
\hline 07167500 & Otter Creek at Climax, KS & 0 & 1.00 & 10.0 & 44.0 & 121 & 82.0 \\
\hline 07168500 & Fall River near Fall River, KS & 5.90 & 13.0 & 53.0 & 270 & 1,061 & 361 \\
\hline 07169500 & Fall River at Fredonia, KS & 11.0 & 20.0 & 92.0 & 492 & 1,740 & 550 \\
\hline 07169800 & Elk River at Elk Falls, KS & .64 & 2.90 & 22.0 & 91.0 & 271 & 154 \\
\hline 07170060 & Elk River below Elk City Lake, KS & 3.00 & 7.10 & 21.0 & 324 & 1,530 & 460 \\
\hline 07170500 & Verdigris River at Independence, KS & 30.0 & 75.0 & 401 & 2,130 & 6,780 & 2,080 \\
\hline 07170700 & Big Hill Creek near Cherryvale, KS & 0 & .02 & .88 & 18.0 & 69.0 & 29.2 \\
\hline 07171000 & Verdigris River near Lenopah, OK & 35.0 & 104 & 570 & 2,690 & 8,425 & 2,580 \\
\hline 07172000 & Caney River near Elgin, KS & .07 & 5.00 & 40.0 & 172 & 528 & 273 \\
\hline 07174000 & Little Caney River near Copan, OK & .10 & .50 & 9.5 & 64.0 & 323 & 238 \\
\hline 07174400 & Caney River above Coon Creek near Bartlesville, OK & 26.0 & 36.0 & 173 & 1,820 & 4,420 & 1,290 \\
\hline 07179500 & Neosho River at Council Grove, KS & 3.20 & 6.30 & 13.0 & 73.0 & 358 & 132 \\
\hline 07179730 & Neosho River near Americus, KS & 12.0 & 21.0 & 70.0 & 240 & 937 & 341 \\
\hline 07179795 & North Cottonwood River below Marion Lake, KS & 1.90 & 3.90 & 7.65 & 14.0 & 110 & 77.8 \\
\hline 07180200 & Cottonwood River at Marion, KS & 8.70 & 13.0 & 32.0 & 107 & 576 & 214 \\
\hline 07180400 & Cottonwood River near Florence, KS & 29.0 & 48.0 & 88.0 & 217 & 738 & 348 \\
\hline 07180500 & Cedar Creek near Cedar Point, KS & 1.80 & 6.00 & 16.0 & 36.0 & 76.0 & 57.6 \\
\hline 07181500 & Middle Creek near Elmdale, KS & 0 & 2.40 & 7.00 & 22.0 & 59.0 & 44.7 \\
\hline 07182250 & Cottonwood River near Plymouth, KS & 48.0 & 105 & 298 & 794 & 2,080 & 950 \\
\hline 07182510 & Neosho River at Burlington, KS & 28.0 & 67.0 & 397 & 1,620 & 5,420 & 1,660 \\
\hline 07183000 & Neosho River near Iola, KS & 42.0 & 119 & 581 & 2,410 & 7,300 & 2,220 \\
\hline 07183100 & Owl Creek near Piqua, KS & 0 & .57 & 4.00 & 21.0 & 115 & 122 \\
\hline 07183500 & Neosho River near Parsons, KS & 54.0 & 182 & 852 & 3,560 & 9,760 & 3,120 \\
\hline 07184000 & Lightning Creek near McCune, KS & 0 & 1.40 & 12.0 & 52.0 & 270 & 169 \\
\hline 07185000 & Neosho River near Commerce, OK & 77.0 & 281 & 1,140 & 4,450 & 11,700 & 4,020 \\
\hline 07186000 & Spring River at Waco, MO & 66.0 & 124 & 301 & 730 & 1,816 & 942 \\
\hline
\end{tabular}

07186000 Spring River at Waco, MO

66.0

124

301

1,816

942 
Table 5. Streamflow-gaging stations in Kansas and parts of surrounding States used in the interpolation of flow durations and mean flows at controlled and uncontrolled flow stream locations on the 1999 Kansas Surface Water Register. - Continued

\begin{tabular}{|c|c|c|c|c|c|c|c|}
\hline \multirow{2}{*}{$\begin{array}{l}\text { Station } \\
\text { number } \\
\text { (fig. 1) }\end{array}$} & \multirow{2}{*}{ Stream name } & \multicolumn{5}{|c|}{$\begin{array}{l}\text { Computed flow duration, in cubic feet per second, for indicated percentage of time flow } \\
\text { equaled or exceeded }\end{array}$} & \multirow{2}{*}{$\begin{array}{l}\text { Mean flow for } \\
\text { period of record } \\
\text { (cubic feet per } \\
\text { second) }\end{array}$} \\
\hline & & 90 percent & 75 percent & 50 percent & 25 percent & 10 percent & \\
\hline 07186400 & Center Creek near Carterville, MO & 32.0 & 47.0 & 97.0 & 206 & 399 & 205 \\
\hline 07187000 & Shoal Creek above Joplin, MO & 88.0 & 130 & 237 & 448 & 879 & 427 \\
\hline 07188000 & Spring River near Quapaw, OK & 211 & 376 & 850 & 1,950 & 4,400 & 2,200 \\
\hline
\end{tabular}


Table 6. Streamflow-gaging stations in Kansas and parts of surrounding States used in the interpolation of peak-discharge frequency values at controlled and uncontrolled flow stream locations on the 1999 Kansas Surface Water Register.

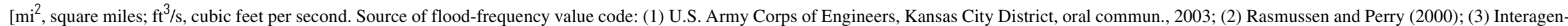

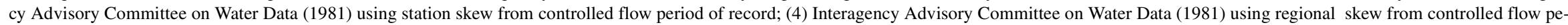
riod of record; and (5) Federal Emergency Management Agency (various years)]

\begin{tabular}{|c|c|c|c|c|c|c|c|c|c|c|}
\hline \multirow{2}{*}{$\begin{array}{l}\text { Station } \\
\text { number } \\
\text { (fig. 2) }\end{array}$} & \multirow{2}{*}{ Stream name } & \multirow{2}{*}{$\begin{array}{l}\text { Contri- } \\
\text { buting- } \\
\text { drainage } \\
\text { area } \\
\left(\mathrm{mi}^{2}\right)\end{array}$} & \multirow{2}{*}{$\begin{array}{c}\text { Con- } \\
\text { trolled } \\
\text { flow } \\
\text { (yes/no) }\end{array}$} & \multicolumn{6}{|c|}{ Peak-discharge estimate $\left(\mathrm{ft}^{3} / \mathrm{s}\right)$ for indicated flood frequency } & \multirow{2}{*}{$\begin{array}{c}\text { Source of } \\
\text { flood- } \\
\text { frequency } \\
\text { value code }\end{array}$} \\
\hline & & & & 2-year & 5 -year & 10-year & 25-year & 50-year & 100-year & \\
\hline 06813500 & Missouri River at Rulo, NE & 418,859 & yes & 96,100 & 132,000 & 158,000 & 184,000 & 220,000 & 250,000 & $(1)$ \\
\hline 06814000 & Turkey Creek near Seneca, KS & 276 & no & 5,820 & 12,500 & 18,400 & 27,700 & 36,000 & 45,300 & $(2)$ \\
\hline 06818000 & Missouri River at St. Joseph, MO & 424,000 & yes & 109,000 & 147,000 & 174,000 & 199,000 & 233,000 & 261,000 & $(1)$ \\
\hline 06818260 & White Clay Creek at Atchison, KS & 13.1 & no & 1,100 & 2,180 & 3,120 & 4,610 & 5,950 & 7,490 & $(2)$ \\
\hline 06826500 & South Fork Republican River near Hale, CO & 1,825 & yes & 113 & 249 & 440 & 920 & 1,590 & 2,735 & (3) \\
\hline 06827500 & South Fork Republican River near Benkleman, NE & 2,740 & yes & 1,000 & 3,700 & 7,180 & 14,400 & 22,300 & 32,900 & (3) \\
\hline 06844700 & South Fork Sappa Creek near Brewster, KS & 74 & no & 52 & 382 & 996 & 2,600 & 4,670 & 7,740 & $(2)$ \\
\hline 06844900 & South Fork Sappa Creek near Achilles, KS & 446 & no & 342 & 1,210 & 2,290 & 4,430 & 6,730 & 9,730 & $(2)$ \\
\hline 06845000 & Sappa Creek near Oberlin, KS & 1,063 & no & 827 & 2,570 & 4,570 & 8,320 & 12,200 & 17,000 & $(2)$ \\
\hline 06845100 & Long Branch Draw near Norcatur, KS & 31.7 & no & 294 & 693 & 1,070 & 1,680 & 2,230 & 2,870 & (2) \\
\hline 06846000 & Beaver Creek at Ludell, KS & 1,460 & no & 440 & 1,110 & 1,780 & 2,920 & 4,000 & 5,300 & $(2)$ \\
\hline 06846500 & Beaver Creek at Cedar Bluffs, KS & 1,620 & no & 413 & 1,060 & 1,730 & 2,960 & 4,190 & 5,740 & (2) \\
\hline 06847900 & Prairie Dog Creek above Keith Sebelius Lake, KS & 590 & no & 599 & 1,680 & 2,820 & 4,820 & 6,760 & 9,100 & $(2)$ \\
\hline 06848000 & Prairie Dog Creek at Norton, KS & 684 & yes & 84 & 155 & 180 & 194 & 199 & 201 & (3) \\
\hline 06848500 & Prairie Dog Creek near Woodruff, KS & 1,007 & yes & 652 & 1,610 & 2,330 & 3,220 & 3,830 & 4,380 & (3) \\
\hline 06853020 & Republican River at Guide Rock, NE & 22,100 & yes & 3,870 & 5,970 & 7,380 & 9,140 & 10,400 & 11,700 & (3) \\
\hline 06853500 & Republican River near Hardy, NE & 22,401 & yes & 4,940 & 8,500 & 11,000 & 14,000 & 16,300 & 18,490 & (3) \\
\hline 06853800 & White Rock Creek near Burr Oak, KS & 227 & no & 1,520 & 3,040 & 4,430 & 6,680 & 8,760 & 11,200 & $(2)$ \\
\hline 06854000 & White Rock Creek at Lovewell, KS & 345 & yes & 460 & 1,300 & 2,260 & 3,760 & 5,080 & 6,380 & (4) \\
\hline 06854500 & Republican River at Scandia, KS & 23,560 & yes & 6,450 & 10,700 & 24,800 & 36,000 & 47,200 & 59,000 & (5) \\
\hline 06855800 & Buffalo Creek near Jamestown, KS & 330 & no & 1,670 & 3,890 & 6,140 & 10,100 & 14,000 & 18,900 & $(2)$ \\
\hline 06855900 & Wolf Creek near Concordia, KS & 56 & no & 910 & 1,770 & 2,490 & 3,570 & 4,490 & 5,520 & $(2)$ \\
\hline 06856000 & Republican River at Concordia, KS & 23,560 & yes & 6,720 & 11,700 & 16,300 & 24,000 & 31,400 & 40,400 & (3) \\
\hline 06856100 & West Creek near Talmo, KS & 42 & no & 676 & 1,870 & 3,250 & 5,920 & 8,780 & 12,600 & $(2)$ \\
\hline 06856320 & Elk Creek at Clyde, KS & 73 & no & 546 & 1,350 & 2,170 & 3,640 & 5,100 & 5,910 & (2) \\
\hline
\end{tabular}


Table 6. Streamflow-gaging stations in Kansas and parts of surrounding States used in the interpolation of peak-discharge frequency values at controlled and uncontrolled flow stream locations on the 1999 Kansas Surface Water Register.-Continued

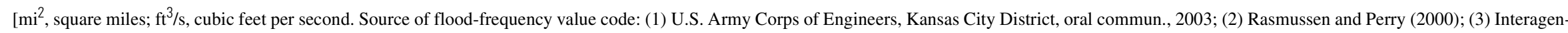

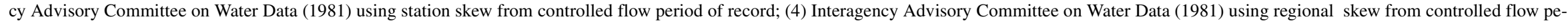
riod of record; and (5) Federal Emergency Management Agency (various years)]

\begin{tabular}{|c|c|c|c|c|c|c|c|c|c|c|}
\hline \multirow{2}{*}{$\begin{array}{l}\text { Station } \\
\text { number } \\
\text { (fig. 2) }\end{array}$} & \multirow{2}{*}{ Stream name } & \multirow{2}{*}{$\begin{array}{l}\text { Contri- } \\
\text { buting- } \\
\text { drainage } \\
\text { area } \\
\left(\mathrm{mi}^{2}\right)\end{array}$} & \multirow{2}{*}{$\begin{array}{l}\text { Con- } \\
\text { trolled } \\
\text { flow } \\
\text { (yes/no) }\end{array}$} & \multicolumn{6}{|c|}{ Peak-discharge estimate $\left(\mathrm{ft}^{3} / \mathrm{s}\right)$ for indicated flood frequency } & \multirow{2}{*}{$\begin{array}{l}\text { Source of } \\
\text { flood- } \\
\text { frequency } \\
\text { value code }\end{array}$} \\
\hline & & & & 2-year & 5-year & 10-year & 25-year & 50-year & 100-year & \\
\hline 06856600 & Republican River at Clay Center, KS & 24,542 & yes & 11,500 & 19,900 & 26,400 & 35,500 & 42,800 & 50,700 & (3) \\
\hline 06857100 & Republican River below Milford Dam, KS & 24,880 & yes & 5,130 & 9,360 & 15,000 & 18,000 & 24,000 & 37,500 & (5) \\
\hline 06858500 & North Fork Smoky Hill River near McAllaster, KS & 670 & no & 327 & 1,930 & 4,580 & 11,000 & 18,900 & 30,100 & $(2)$ \\
\hline 06859500 & Ladder Creek below Chalk Creek near Scott City, KS & 1,460 & no & 646 & 2,590 & 5,270 & 11,100 & 17,900 & 27,500 & (2) \\
\hline 06860000 & Smoky Hill River at Elkader, KS & 3,560 & no & 1,510 & 6,400 & 13,000 & 26,900 & 42,200 & 62,500 & (2) \\
\hline 06860300 & South Branch Hackberry Creek near Orion, KS & 49.6 & no & 397 & 1,190 & 2,050 & 3,590 & 5,110 & 6,970 & (2) \\
\hline 06860500 & Hackberry Creek near Gove, KS & 426 & no & 508 & 2,340 & 5,100 & 11,500 & 19,300 & 30,600 & (2) \\
\hline 06861000 & Smoky Hill River near Arnold, KS & 5,220 & no & 2,230 & 7,660 & 14,400 & 27,800 & 42,300 & 61,400 & (2) \\
\hline 06862700 & Smoky Hill River near Schoenchen, KS & 5,750 & yes & 992 & 4,700 & 9,170 & 16,900 & 23,900 & 31,500 & (3) \\
\hline 06862850 & Smoky Hill River below Schoenchen, KS & 5,810 & yes & 992 & 4,700 & 9,170 & 16,900 & 23,900 & 31,500 & (3) \\
\hline 06863300 & Big Creek near Ogallah, KS & 297 & no & 1,340 & 4,430 & 8,090 & 15,100 & 22,500 & 31,900 & (2) \\
\hline 06863500 & Big Creek near Hays, KS & 594 & no & 1,280 & 3,210 & 5,050 & 7,990 & 10,600 & 13,600 & (2) \\
\hline 06863900 & North Fork Big Creek near Victoria, KS & 54 & no & 263 & 1,250 & 2,640 & 5,570 & 8,770 & 13,000 & (2) \\
\hline 06864000 & Smoky Hill River near Russell, KS & 6,965 & yes & 7,340 & 14,400 & 19,900 & 27,900 & 34,300 & 41,100 & (3) \\
\hline 06864050 & Smoky Hill River near Bunker Hill, KS & 7,075 & yes & 4,010 & 9,850 & 14,800 & 22,100 & 27,900 & 33,900 & (3) \\
\hline 06864500 & Smoky Hill River at Ellsworth, KS & 7,580 & yes & 8,850 & 16,300 & 24,800 & 33,000 & 41,300 & 48,200 & (5) \\
\hline 06865500 & Smoky Hill River near Langley, KS & 7,857 & yes & 2,120 & 4,760 & 7,230 & 11,200 & 14,900 & 19,200 & (3) \\
\hline 06866500 & Smoky Hill River near Mentor, KS & 9,358 & yes & 4,360 & 7,890 & 8,800 & 15,000 & 22,000 & 32,000 & (5) \\
\hline 06866800 & Saline River tributary at Collyer, KS & 3.13 & no & 161 & 565 & 1,050 & 1,980 & 2,930 & 4,130 & $(2)$ \\
\hline 06866900 & Saline River near Wakeeney, KS & 696 & no & 2,720 & 8,200 & 14,200 & 25,100 & 35,900 & 49,100 & (2) \\
\hline 06867000 & Saline River near Russell, KS & 1,500 & no & 2,270 & 6,630 & 11,500 & 20,500 & 29,700 & 41,400 & (2) \\
\hline 06867500 & Paradise Creek near Paradise, KS & 212 & no & 947 & 3,160 & 5,780 & 10,800 & 15,900 & 22,400 & (2) \\
\hline 06868200 & Saline River at Wilson Dam, KS & 1,917 & yes & 744 & 1,610 & 2,190 & 2,840 & 3,250 & 3,610 & (3) \\
\hline 06868400 & Wolf Creek near Lucas, KS & 163 & no & 1,570 & 3,660 & 5,600 & 8,730 & 11,600 & 14,800 & (2) \\
\hline 06868700 & North Branch Spillman Creek near Ash Grove, KS & 26.1 & no & 342 & 1,100 & 1,960 & 3,600 & 5,260 & 7,360 & (2) \\
\hline
\end{tabular}


Table 6. Streamflow-gaging stations in Kansas and parts of surrounding States used in the interpolation of peak-discharge frequency values at controlled and uncontrolled flow stream locations on the 1999 Kansas Surface Water Register.-Continued

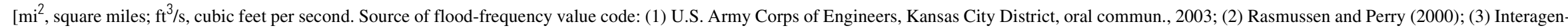

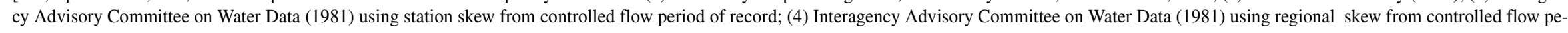
riod of record; and (5) Federal Emergency Management Agency (various years)]

\begin{tabular}{|c|c|c|c|c|c|c|c|c|c|c|}
\hline \multirow{2}{*}{$\begin{array}{c}\text { Station } \\
\text { number } \\
\text { (fig. 2) }\end{array}$} & \multirow{2}{*}{ Stream name } & \multirow{2}{*}{$\begin{array}{l}\text { Contri- } \\
\text { buting- } \\
\text { drainage } \\
\text { area } \\
\left(\mathrm{mi}^{2}\right)\end{array}$} & \multirow{2}{*}{$\begin{array}{c}\text { Con- } \\
\text { trolled } \\
\text { flow } \\
\text { (yes/no) }\end{array}$} & \multicolumn{6}{|c|}{ Peak-discharge estimate $\left(\mathrm{ft}^{3} / \mathrm{s}\right)$ for indicated flood frequency } & \multirow{2}{*}{$\begin{array}{l}\text { Source of } \\
\text { flood- } \\
\text { frequency } \\
\text { value code }\end{array}$} \\
\hline & & & & 2-year & 5-year & 10-year & 25-year & 50-year & 100-year & \\
\hline 06869500 & Saline River at Tescott, KS & 2,820 & yes & 2,530 & 5,030 & 6,790 & 8,970 & 10,500 & 12,000 & (3) \\
\hline 06869950 & Mulberry Creek near Salina, KS & 250 & no & 2,310 & 4,530 & 6,380 & 9,100 & 11,400 & 13,900 & (2) \\
\hline 06870200 & Smoky Hill River at New Cambria, KS & 11,730 & yes & 6,050 & 11,600 & 15,500 & 24,000 & 34,000 & 45,500 & (5) \\
\hline 06870300 & Gypsum Creek near Gypsum, KS & 120 & no & 2,290 & 4,310 & 5,960 & 8,400 & 10,500 & 12,700 & (2) \\
\hline 06871000 & North Fork Solomon River at Glade, KS & 849 & no & 1,580 & 4,790 & 8,320 & 14,700 & 21,000 & 28,800 & (2) \\
\hline 06871500 & Bow Creek near Stockton, KS & 341 & no & 926 & 2,960 & 5,320 & 9,790 & 14,400 & 20,200 & (2) \\
\hline 06871800 & North Fork Solomon River at Kirwin, KS & 1,367 & yes & 18 & 188 & 606 & 2,010 & 4,240 & 8,180 & (3) \\
\hline 06871900 & Deer Creek near Phillipsburg, KS & 65.0 & no & 1,210 & 3,430 & 5,760 & 9,790 & 13,600 & 18,300 & (2) \\
\hline 06872100 & Middle Cedar Creek at Kensington, KS & 58.9 & no & 577 & 1,350 & 2,100 & 3,390 & 4,610 & 6,100 & (2) \\
\hline 06872300 & Middle Beaver Creek near Smith Center, KS & 71.0 & no & 765 & 1,390 & 1,880 & 2,590 & 3,170 & 3,790 & (2) \\
\hline 06872500 & North Fork Solomon River at Portis, KS & 2,315 & yes & 2,860 & 6,380 & 9,620 & 14,800 & 19,500 & 24,900 & (3) \\
\hline 06873000 & South Fork Solomon River above Webster Reservoir, KS & 1,040 & no & 2,800 & 8,330 & 14,600 & 26,300 & 38,300 & 53,600 & (2) \\
\hline 06873200 & South Fork Solomon River below Webster Reservoir, KS & 1,150 & yes & 231 & 677 & 1,080 & 1,640 & 2,090 & 2,530 & (4) \\
\hline 06873460 & South Fork Solomon River at Woodston, KS & 1,502 & yes & 554 & 2,000 & 3,610 & 6,370 & 8,900 & 11,800 & (3) \\
\hline 06873500 & South Fork Solomon River at Alton, KS & 1,720 & no & 3,600 & 12,300 & 23,400 & 46,300 & 72,000 & 107,000 & (2) \\
\hline 06873700 & Kill Creek near Bloomington, KS & 52.0 & no & 182 & 1,150 & 2,890 & 7,480 & 13,600 & 23,100 & (2) \\
\hline 06874000 & South Fork Solomon River at Osborne, KS & 2,012 & yes & 1,400 & 4,850 & 9,310 & 18,700 & 29,500 & 44,300 & (3) \\
\hline 06874500 & East Limestone Creek near Ionia, KS & 25.6 & no & 608 & 1,320 & 1,940 & 2,880 & 3,680 & 4,580 & (2) \\
\hline 06875800 & Limestone Creek near Glen Elder, KS & 210 & no & 1,060 & 1,910 & 2,600 & 3,590 & 4,430 & 5,340 & (2) \\
\hline 06875900 & Solomon River near Glen Elder, KS & 5,340 & yes & 1,720 & 3,630 & 5,170 & 7,350 & 9,080 & 10,900 & (3) \\
\hline 06876070 & Solomon River near Simpson, KS & 5,538 & yes & 3,100 & 6,050 & 8,000 & 10,300 & 11,800 & 13,100 & (3) \\
\hline 06876200 & Middle Pipe Creek near Miltonvale, KS & 10.2 & no & 532 & 1,270 & 2,000 & 3,220 & 4,380 & 5,760 & (2) \\
\hline 06876700 & Salt Creek near Ada, KS & 384 & no & 1,430 & 4,030 & 6,800 & 11,700 & 16,600 & 22,500 & (2) \\
\hline 06876900 & Solomon River at Niles, KS & 6,770 & yes & 5,190 & 10,500 & 18,300 & 28,000 & 38,600 & 50,700 & (3) \\
\hline 06877120 & Mud Creek at Abilene, KS & 87.0 & no & 2,570 & 4,670 & 6,330 & 8,710 & 10,700 & 12,800 & (2) \\
\hline
\end{tabular}

06877120 Mud Creek at Abilene, KS 
Table 6. Streamflow-gaging stations in Kansas and parts of surrounding States used in the interpolation of peak-discharge frequency values at controlled and uncontrolled flow stream locations on the 1999 Kansas Surface Water Register.-Continued

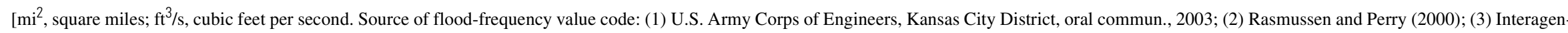

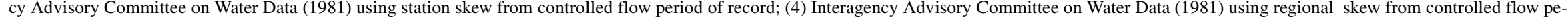
riod of record; and (5) Federal Emergency Management Agency (various years)]

\begin{tabular}{|c|c|c|c|c|c|c|c|c|c|c|}
\hline \multirow{2}{*}{$\begin{array}{l}\text { Station } \\
\text { number } \\
\text { (fig. 2) }\end{array}$} & \multirow[b]{2}{*}{ Stream name } & \multirow{2}{*}{$\begin{array}{l}\text { Contri- } \\
\text { buting- } \\
\text { drainage } \\
\text { area } \\
\left(\mathrm{mi}^{2}\right)\end{array}$} & \multirow{2}{*}{$\begin{array}{l}\text { Con- } \\
\text { trolled } \\
\text { flow } \\
\text { (yes/no) }\end{array}$} & \multicolumn{6}{|c|}{ Peak-discharge estimate $\left(\mathrm{ft}^{3} / \mathrm{s}\right)$ for indicated flood frequency } & \multirow{2}{*}{$\begin{array}{l}\text { Source of } \\
\text { flood- } \\
\text { frequency } \\
\text { value code }\end{array}$} \\
\hline & & & & 2-year & 5-year & 10-year & 25-year & 50-year & 100-year & \\
\hline 06877200 & West Turkey Creek near Elmo, KS & 26.6 & no & 1,200 & 2,220 & 3,000 & 4,080 & 4,940 & 5,850 & $(2)$ \\
\hline 06877500 & Turkey Creek near Abilene, KS & 143 & no & 2,960 & 6,550 & 9,870 & 15,300 & 20,200 & 25,900 & (2) \\
\hline 06877600 & Smoky Hill River at Enterprise, KS & 19,260 & yes & 12,400 & 26,000 & 35,600 & 51,000 & 68,100 & 85,300 & (5) \\
\hline 06878000 & Chapman Creek near Chapman, KS & 300 & no & 3,630 & 7,130 & 10,300 & 15,300 & 20,000 & 25,400 & (2) \\
\hline 06878500 & Lyon Creek near Woodbine, KS & 230 & no & 6,410 & 18,000 & 30,300 & 52,200 & 73,600 & 99,800 & (2) \\
\hline 06879100 & Kansas River at Fort Riley, KS & 44,870 & yes & 19,100 & 31,100 & 44,000 & 73,000 & 102,000 & 140,000 & (5) \\
\hline 06879200 & Clark Creek near Junction City, KS & 200 & no & 4,320 & 8,970 & 13,100 & 19,400 & 25,000 & 31,3000 & (2) \\
\hline 06879650 & Kings Creek near Manhattan, KS & 4.09 & no & 428 & 1,930 & 4,210 & 9,590 & 16,300 & 26,100 & (2) \\
\hline 06879700 & Wildcat Creek at Riley, KS & 14 & no & 936 & 2,020 & 2,980 & 4,450 & 5,740 & 7,190 & (2) \\
\hline 06879815 & Wildcat Creek at Manhattan, KS & 74 & no & 2,470 & 4,380 & 5,880 & 8,030 & 9,800 & 11,700 & (2) \\
\hline 06882510 & Big Blue River at Marysville, KS & 4,780 & no & 19,100 & 32,800 & 42,900 & 56,700 & 67,500 & 78,800 & (2) \\
\hline 06884025 & Little Blue River at Hollenberg, KS & 2,750 & no & 11,200 & 21,000 & 29,300 & 42,100 & 53,400 & 66,300 & (2) \\
\hline 06884200 & Mill Creek at Washington, KS & 344 & no & 4,830 & 8,160 & 10,600 & 13,900 & 16,500 & 19,200 & (2) \\
\hline 06884400 & Little Blue River near Barnes, KS & 3,320 & no & 13,100 & 21,200 & 27,200 & 35,400 & 41,900 & 48,700 & (2) \\
\hline 06884500 & Little Blue River at Waterville, KS & 3,510 & no & 11,600 & 24,000 & 35,400 & 53,700 & 70,600 & 90,300 & (2) \\
\hline 06884900 & Robidoux Creek at Beattie, KS & 40 & no & 1,850 & 3,930 & 5,780 & 8,700 & 11,300 & 14,300 & (2) \\
\hline 06885500 & Black Vermillion River near Frankfort, KS & 410 & no & 7,030 & 15,700 & 24,100 & 38,000 & 51,200 & 66,900 & (2) \\
\hline 06886500 & Fancy Creek at Winkler, KS & 174 & no & 5,690 & 10,600 & 14,600 & 20,500 & 25,400 & 30,700 & (2) \\
\hline 06887000 & Big Blue River near Manhattan, KS & 9,640 & yes & 16,600 & 25,500 & 36,300 & 42,000 & 46,800 & 49,600 & (5) \\
\hline 06887500 & Kansas River at Wamego, KS & 55,280 & yes & 28,600 & 54,500 & 78,400 & 108,000 & 156,000 & 202,000 & (1) \\
\hline 06888000 & Vermillion Creek near Wamego, KS & 243 & no & 6,190 & 12,600 & 17,900 & 25,800 & 32,400 & 39,700 & (2) \\
\hline 06888030 & Vermillion Creek near Louisville, KS & 297 & no & 6,610 & 9,650 & 11,800 & 14,600 & 16,800 & 19,100 & (2) \\
\hline 06888300 & Rock Creek near Louisville, KS & 128 & no & 5,880 & 10,100 & 13,400 & 18,100 & 21,900 & 26,100 & (2) \\
\hline 06888350 & Kansas River near Belvue, KS & 55,870 & yes & 33,800 & 59,800 & 82,100 & 117,000 & 148,000 & 183,000 & (3) \\
\hline 06888500 & Mill Creek near Paxico, KS & 316 & no & 11,600 & 23,000 & 32,400 & 46,300 & 58,000 & 70,800 & (2) \\
\hline
\end{tabular}


Table 6. Streamflow-gaging stations in Kansas and parts of surrounding States used in the interpolation of peak-discharge frequency values at controlled and uncontrolled flow stream locations on the 1999 Kansas Surface Water Register.-Continued

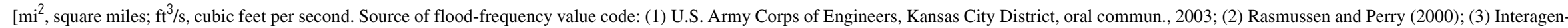

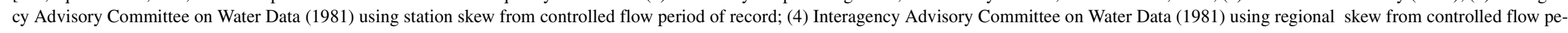
riod of record; and (5) Federal Emergency Management Agency (various years)]

\begin{tabular}{|c|c|c|c|c|c|c|c|c|c|c|}
\hline \multirow{2}{*}{$\begin{array}{c}\text { Station } \\
\text { number } \\
\text { (fig. 2) }\end{array}$} & \multirow[b]{2}{*}{ Stream name } & \multirow{2}{*}{$\begin{array}{l}\text { Contri- } \\
\text { buting- } \\
\text { drainage } \\
\text { area } \\
\left(\mathrm{mi}^{2}\right)\end{array}$} & \multirow{2}{*}{$\begin{array}{c}\text { Con- } \\
\text { trolled } \\
\text { flow } \\
\text { (yes/no) }\end{array}$} & \multicolumn{6}{|c|}{ Peak-discharge estimate $\left(\mathrm{ft}^{3} / \mathrm{s}\right)$ for indicated flood frequency } & \multirow{2}{*}{$\begin{array}{c}\text { Source of } \\
\text { flood- } \\
\text { frequency } \\
\text { value code }\end{array}$} \\
\hline & & & & 2-year & 5-year & 10-year & 25-year & 50-year & 100-year & \\
\hline 06888600 & Dry Creek near Maple Hill, KS & 15.6 & no & 1,670 & 3,080 & 4,280 & 6,110 & 7,730 & 9,560 & $(2)$ \\
\hline 06889000 & Kansas River at Topeka, KS & 56,720 & yes & 36,600 & 67,000 & 93,600 & 123,000 & 173,000 & 217,000 & (1) \\
\hline 06889100 & Soldier Creek near Goff, KS & 2.06 & no & 402 & 880 & 1,340 & 2,120 & 2,870 & 3,770 & (2) \\
\hline 06889120 & Soldier Creek near Bancroft, KS & 10.5 & no & 1,220 & 2,200 & 3,030 & 4,280 & 5,380 & 6,640 & (2) \\
\hline 06889140 & Soldier Creek near Soldier, KS & 16.9 & no & 1,850 & 3,360 & 4,640 & 6,580 & 8,270 & 10,200 & (2) \\
\hline 06889160 & Soldier Creek near Circleville, KS & 49.3 & no & 4,030 & 6,850 & 9,140 & 12,500 & 15,400 & 18,700 & $(2)$ \\
\hline 06889180 & Soldier Creek near St. Clere, KS & 80.0 & no & 4,440 & 7,220 & 9,250 & 12,000 & 14,200 & 16,400 & (2) \\
\hline 06889200 & Soldier Creek near Delia, KS & 157 & no & 4,510 & 7,650 & 10,200 & 13,900 & 17,100 & 20,600 & $(2)$ \\
\hline 06889500 & Soldier Creek near Topeka, KS & 290 & no & 5,970 & 11,700 & 16,400 & 23,300 & 29,000 & 35,200 & (2) \\
\hline 06889600 & South Branch Shunganunga Creek near Pauline, KS & 3.84 & no & 758 & 1,450 & 2,040 & 2,950 & 3,740 & 4,640 & (2) \\
\hline 06889630 & Shunganunga Creek at Topeka, KS & 33.5 & no & 2,150 & 2,910 & 3,390 & 3,970 & 4,390 & 4,790 & $(2)$ \\
\hline 06890100 & Delaware River near Muscotah, KS & 431 & no & 12,500 & 18,700 & 23,000 & 28,600 & 32,900 & 37,400 & (2) \\
\hline 06890300 & Spring Creek near Wetmore, KS & 21.0 & no & 1,600 & 3,680 & 5,750 & 9,360 & 12,900 & 17,200 & (2) \\
\hline 06890600 & Rock Creek near Meriden, KS & 22.0 & no & 2,090 & 3,220 & 4,040 & 5,170 & 6,060 & 7,010 & (2) \\
\hline 06890800 & Slough Creek near Oskaloosa, KS & 31.0 & no & 3,670 & 5,510 & 6,850 & 8,670 & 10,100 & 11,700 & $(2)$ \\
\hline 06890900 & Delaware River below Perry Dam, KS & 1,117 & yes & 6,290 & 9,960 & 17,600 & 24,000 & 29,900 & 32,000 & $(5)$ \\
\hline 06891000 & Kansas River at Lecompton, KS & 58,460 & yes & 44,600 & 80,000 & 109,000 & 138,000 & 190,000 & 231,000 & (1) \\
\hline 06891050 & Stone House Creek at Williamstown, KS & 12.9 & no & 1,720 & 3,720 & 5,470 & 8,160 & 10,500 & 13,100 & (2) \\
\hline 06891500 & Wakarusa River near Lawrence, KS & 425 & yes & 3,580 & 5,590 & 6,540 & 8,200 & 10,180 & 11,910 & $(5)$ \\
\hline 06892000 & Stranger Creek near Tonganoxie, KS & 406 & no & 6,170 & 11,300 & 15,500 & 21,700 & 27,000 & 32,900 & (2) \\
\hline 06892350 & Kansas River at DeSoto, KS & 59,756 & yes & 50,100 & 88,900 & 119,000 & 148,000 & 200,000 & 240,000 & $(1)$ \\
\hline 06893000 & Missouri River at Kansas City, MO & 485,200 & yes & 142,000 & 201,000 & 245,000 & 289,000 & 351,000 & 401,000 & $(1)$ \\
\hline 06893080 & Big Blue River near Stanley, KS & 46.0 & no & 4,820 & 8,610 & 11,700 & 16,400 & 20,500 & 25,000 & (2) \\
\hline 06893300 & Indian Creek at Overland Park, KS & 26.6 & no & 4,060 & 6,210 & 7,770 & 9,890 & 11,600 & 13,300 & $(2)$ \\
\hline 06893350 & Tomahawk Creek near Overland Park, KS & 23.9 & no & 2,630 & 4,890 & 6,760 & $9,, 520$ & 11,900 & 14,500 & (2) \\
\hline
\end{tabular}


Table 6. Streamflow-gaging stations in Kansas and parts of surrounding States used in the interpolation of peak-discharge frequency values at controlled and uncontrolled flow stream locations on the 1999 Kansas Surface Water Register.-Continued

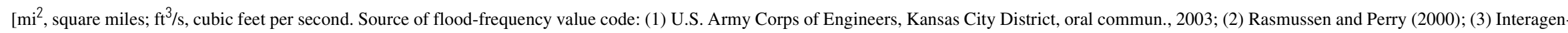

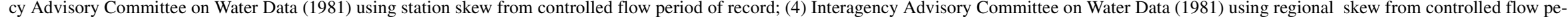
riod of record; and (5) Federal Emergency Management Agency (various years)]

\begin{tabular}{|c|c|c|c|c|c|c|c|c|c|c|}
\hline \multirow{2}{*}{$\begin{array}{l}\text { Station } \\
\text { number } \\
\text { (fig. 2) }\end{array}$} & \multirow{2}{*}{ Stream name } & \multirow{2}{*}{$\begin{array}{l}\text { Contri- } \\
\text { buting- } \\
\text { drainage } \\
\text { area } \\
\left(\mathrm{mi}^{2}\right)\end{array}$} & \multirow{2}{*}{$\begin{array}{c}\text { Con- } \\
\text { trolled } \\
\text { flow } \\
\text { (yes/no) }\end{array}$} & \multicolumn{6}{|c|}{ Peak-discharge estimate $\left(\mathrm{ft}^{3} / \mathrm{s}\right)$ for indicated flood frequency } & \multirow{2}{*}{$\begin{array}{l}\text { Source of } \\
\text { flood- } \\
\text { frequency } \\
\text { value code }\end{array}$} \\
\hline & & & & 2-year & 5-year & 10-year & 25-year & 50-year & 100-year & \\
\hline 06910800 & Marais des Cygnes River near Reading, KS & 177 & no & 7,860 & 18,200 & 28,100 & 44,200 & 59,100 & 76,500 & $(2)$ \\
\hline 06911000 & Marais des Cygnes River at Melvern, KS & 351 & no & 7,050 & 17,300 & 27,300 & 43,800 & 59,000 & 76,900 & (2) \\
\hline 06911500 & Salt Creek near Lyndon, KS & 111 & no & 4,270 & 9,170 & 13,400 & 19,800 & 25,200 & 31,200 & (2) \\
\hline 06911900 & Dragoon Creek near Burlingame, KS & 114 & no & 4,780 & 8,780 & 12,000 & 16,600 & 20,500 & 24,700 & (2) \\
\hline 06912500 & Hundred and Ten Mile Creek near Quenemo, KS & 322 & yes & 1,920 & 3,250 & 4,060 & 4,980 & 5,570 & 6,100 & (4) \\
\hline 06913000 & Marais des Cygnes River near Pomona, KS & 1,040 & yes & 10,700 & 17,800 & 22,900 & 29,400 & 34,300 & 39,200 & (3) \\
\hline 06913500 & Marias des Cygnes River near Ottawa, KS & 1,250 & yes & 12,400 & 19,600 & 24,000 & 28,900 & 32,100 & 35,000 & (3) \\
\hline 06913600 & Rock Creek near Ottawa, KS & 10.2 & no & 597 & 1,300 & 1,960 & 3,060 & 4,090 & 5,310 & (2) \\
\hline 06913700 & Middle Creek near Princeton, KS & 52.0 & no & 3,300 & 5,310 & 6,840 & 8,970 & 10,700 & 12,600 & (2) \\
\hline 06914000 & Pottawatomie Creek near Garnett, KS & 334 & no & 11,300 & 20,100 & 27,100 & 37,200 & 45,700 & 54,900 & (2) \\
\hline 06914500 & Pottawatomie Creek at Lane, KS & 513 & no & 13,400 & 25,500 & 36,000 & 52,100 & 66,500 & 82,800 & $(2)$ \\
\hline 06915000 & Big Bull Creek near Hillsdale, KS & 147 & yes & 986 & 2,140 & 3,040 & 4,230 & 5,130 & 6,020 & (4) \\
\hline 06915800 & Marais des Cygnes River at La Cygne, KS & 2,669 & yes & 24,000 & 30,000 & 37,000 & 59,000 & 78,000 & 100,000 & (5) \\
\hline 06916500 & Big Sugar Creek at Farlinville, KS & 198 & no & 6,490 & 13,100 & 19,200 & 29,000 & 38,100 & 48,800 & (2) \\
\hline 06916600 & Marais des Cygnes River near Kansas-Missouri State line, KS & 3,230 & yes & 23,900 & 36,100 & 44,200 & 54,300 & 61,700 & 69,000 & (3) \\
\hline 06917000 & Little Osage River at Fulton, KS & 295 & no & 8,560 & 14,800 & 19,900 & 27,500 & 34,000 & 41,400 & (2) \\
\hline 06917100 & Marmaton River tributary near Bronson, KS & .88 & no & 204 & 349 & 455 & 577 & 707 & 820 & (2) \\
\hline 06917380 & Marmaton River near Marmaton, KS & 292 & no & 16,600 & 27,900 & 36,900 & 50,000 & 61,100 & 73,200 & (2) \\
\hline 06917500 & Marmaton River near Fort Scott, KS & 408 & no & 11,800 & 22,900 & 32,100 & 45,600 & 56,900 & 69,300 & (2) \\
\hline 07134180 & Arkansas River near Granado, CO & 23,707 & yes & 1,440 & 2,510 & 3,440 & 4,900 & 6,220 & 7,750 & (3) \\
\hline 07137500 & Arkansas River near Coolidge, KS & 25,410 & yes & 2,380 & 6,750 & 13,200 & 29,800 & 53,500 & 94,000 & (3) \\
\hline 07138000 & Arkansas River at Syracuse, KS & 25,763 & yes & 2,140 & 6,210 & 18,200 & 38,000 & 59,400 & 92,800 & (5) \\
\hline 07138650 & White Woman Creek near Leoti, KS & 750 & no & 232 & 1,400 & 3,310 & 7,870 & 13,400 & 21,100 & (2) \\
\hline 07139000 & Arkansas River at Garden City, KS & 27,071 & yes & 590 & 2,783 & 6,500 & 17,000 & 30,000 & 53,000 & (5) \\
\hline 07139500 & Arkansas River at Dodge City, KS & 30,600 & yes & 759 & 3,260 & 11,300 & 22,000 & 34,200 & 49,900 & (5) \\
\hline
\end{tabular}


Table 6. Streamflow-gaging stations in Kansas and parts of surrounding States used in the interpolation of peak-discharge frequency values at controlled and uncontrolled flow stream locations on the 1999 Kansas Surface Water Register.-Continued

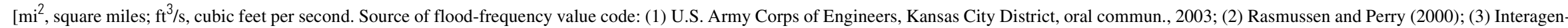

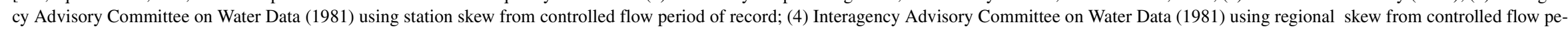
riod of record; and (5) Federal Emergency Management Agency (various years)]

\begin{tabular}{|c|c|c|c|c|c|c|c|c|c|c|}
\hline \multirow{2}{*}{$\begin{array}{c}\text { Station } \\
\text { number } \\
\text { (fig. 2) }\end{array}$} & \multirow[b]{2}{*}{ Stream name } & \multirow{2}{*}{$\begin{array}{l}\text { Contri- } \\
\text { buting- } \\
\text { drainage } \\
\text { area } \\
\left(\mathrm{mi}^{2}\right)\end{array}$} & \multirow{2}{*}{$\begin{array}{c}\text { Con- } \\
\text { trolled } \\
\text { flow } \\
\text { (yes/no) }\end{array}$} & \multicolumn{6}{|c|}{ Peak-discharge estimate $\left(\mathrm{ft}^{3} / \mathrm{s}\right)$ for indicated flood frequency } & \multirow{2}{*}{$\begin{array}{c}\text { Source of } \\
\text { flood- } \\
\text { frequency } \\
\text { value code }\end{array}$} \\
\hline & & & & 2-year & 5-year & 10-year & 25-year & 50-year & 100-year & \\
\hline 07139800 & Mulberry Creek near Dodge City, KS & 73.8 & no & 249 & 675 & 1,090 & 1,770 & 2,380 & 3,080 & $(2)$ \\
\hline 07140000 & Arkansas River near Kinsley, KS & 33,066 & yes & 606 & 3,090 & 8,500 & 16,000 & 25,500 & 39,000 & $(5)$ \\
\hline 07140300 & Whitewoman Creek near Bellefont, KS & 14.0 & no & 179 & 713 & 1,420 & 2,870 & 4,470 & 6,590 & (2) \\
\hline 07140700 & Guzzlers Gulch near Ness City, KS & 582 & no & 425 & 1,260 & 2,130 & 3,650 & 5,080 & 6,780 & (2) \\
\hline 07140850 & Pawnee River near Burdett, KS & 1,090 & no & 469 & 1,430 & 2,460 & 4,300 & 6,070 & 8,210 & (2) \\
\hline 07141200 & Pawnee River near Larned, KS & 2,150 & no & 2,250 & 4,530 & 6,530 & 9,620 & 12,300 & 15,400 & (2) \\
\hline 07141300 & Arkansas River at Great Bend, KS & 34,356 & yes & 2,960 & 7,570 & 15,000 & 22,500 & 29,500 & 36,900 & $(5)$ \\
\hline 07141600 & Long Branch Creek near Ness City, KS & 28 & no & 75 & 433 & 1,000 & 2,310 & 3,840 & 5,950 & $(2)$ \\
\hline 07141780 & Walnut Creek near Rush Center, KS & 1,260 & no & 1,000 & 2,390 & 3,700 & 5,790 & 7,670 & 9,830 & (2) \\
\hline 07141800 & Otter Creek near Rush Center, KS & 17 & no & 394 & 955 & 1,470 & 2,280 & 2,990 & 3,790 & (2) \\
\hline 07141900 & Walnut Creek at Albert, KS & 1,410 & no & 1,310 & 2,850 & 4,200 & 6,250 & 8,010 & 9,970 & $(2)$ \\
\hline 07142100 & Rattlesnake Creek tributary near Mullinville, KS & 10.3 & no & 416 & 1,090 & 1,730 & 2,750 & 3,650 & 4,670 & (2) \\
\hline 07142300 & Rattlesnake Creek near Macksville, KS & 784 & no & 400 & 1,340 & 2,490 & 4,770 & 7,230 & 10,500 & (2) \\
\hline 07142500 & Spring Creek near Dillwyn, KS & 14.3 & no & 305 & 1,170 & 2,260 & 4,400 & 6,650 & 9,530 & (2) \\
\hline 07142575 & Rattlesnake Creek near Zenith, KS & 1,050 & no & 501 & 1,580 & 2,950 & 5,850 & 9,190 & 13,900 & (2) \\
\hline 07142700 & Salt Creek near Partridge, KS & 85 & no & 1,150 & 2,160 & 2,950 & 4,070 & 4,980 & 5,950 & (2) \\
\hline 07142860 & Cow Creek near Claflin, KS & 43 & no & 556 & 1,580 & 2,680 & 4,610 & 6,480 & 8,760 & $(2)$ \\
\hline 07142900 & Blood Creek near Boyd, KS & 61 & no & 955 & 2,320 & 3,570 & 5,540 & 7,270 & 9,220 & (2) \\
\hline 07143200 & Plum Creek near Holyrood, KS & 19 & no & 657 & 1,230 & 1,690 & 2,370 & 2,950 & 3,580 & (2) \\
\hline 07143300 & Cow Creek near Lyons, KS & 728 & no & 1,940 & 4,690 & 7,420 & 12,000 & 16,400 & 21,700 & (2) \\
\hline 07143330 & Arkansas River near Hutchinson, KS & 38,910 & yes & 4,350 & 8,740 & 15,500 & 22,000 & 28,500 & 35,500 & (5) \\
\hline 07143375 & Arkansas River near Maize, KS & 39,110 & yes & 8,270 & 20,800 & 24,000 & 34,500 & 44,800 & 55,200 & $(5)$ \\
\hline 07143500 & Little Arkansas River near Geneseo, KS & 25.0 & no & 927 & 1,360 & 1,630 & 1,970 & 2,210 & 2,440 & (2) \\
\hline 07143600 & Little Arkansas River near Little River, KS & 71.0 & no & 1,200 & 2,360 & 3,360 & 4,900 & 6,240 & 7,760 & $(2)$ \\
\hline 07143665 & Little Arkansas River at Alta Mills, KS & 736 & no & 5,000 & 12,200 & 19,200 & 30,800 & 41,500 & 54,100 & (2) \\
\hline
\end{tabular}


Table 6. Streamflow-gaging stations in Kansas and parts of surrounding States used in the interpolation of peak-discharge frequency values at controlled and uncontrolled flow stream locations on the 1999 Kansas Surface Water Register.-Continued

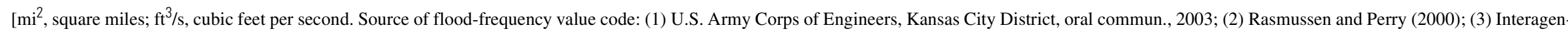

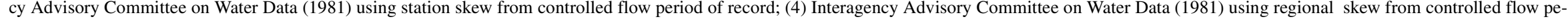
riod of record; and (5) Federal Emergency Management Agency (various years)]

\begin{tabular}{|c|c|c|c|c|c|c|c|c|c|c|}
\hline \multirow{2}{*}{$\begin{array}{c}\text { Station } \\
\text { number } \\
\text { (fig. 2) }\end{array}$} & \multirow[b]{2}{*}{ Stream name } & \multirow{2}{*}{$\begin{array}{l}\text { Contri- } \\
\text { buting- } \\
\text { drainage } \\
\text { area } \\
\left(\mathrm{mi}^{2}\right)\end{array}$} & \multirow{2}{*}{$\begin{array}{c}\text { Con- } \\
\text { trolled } \\
\text { flow } \\
\text { (yes/no) }\end{array}$} & \multicolumn{6}{|c|}{ Peak-discharge estimate $\left(\mathrm{ft}^{3} / \mathrm{s}\right)$ for indicated flood frequency } & \multirow{2}{*}{$\begin{array}{l}\text { Source of } \\
\text { flood- } \\
\text { frequency } \\
\text { value code }\end{array}$} \\
\hline & & & & 2-year & 5-year & 10-year & 25-year & 50-year & 100-year & \\
\hline 07144000 & East Emma Creek near Halstead, KS & 58.0 & no & 3,400 & 8,990 & 14,600 & 24,200 & 33,200 & 43,800 & $(2)$ \\
\hline 07144200 & Little Arkansas River at Valley Center, KS & 1,330 & no & 6,290 & 13,900 & 20,400 & 30,100 & 38,300 & 47,200 & $(2)$ \\
\hline 07144300 & Arkansas River at Wichita, KS & 40,490 & yes & 9,740 & 18,600 & 24,000 & 34,500 & 44,800 & 55,200 & $(5)$ \\
\hline 07144550 & Arkansas River at Derby, KS & 40,830 & yes & 14,200 & 24,000 & 27,200 & 37,000 & 46,200 & 55,200 & (5) \\
\hline 07144780 & North Fork Ninnescah River above Cheney Reservoir, KS & 787 & no & 3,990 & 12,300 & 21,900 & 40,400 & 59,900 & 85,200 & (2) \\
\hline 07144795 & North Fork Ninnescah River at Cheney Dam, KS & 901 & yes & 1,080 & 2,080 & 2,750 & 3,560 & 4,110 & 4,620 & (4) \\
\hline 07144850 & South Fork South Fork Ninnescah River near Pratt, KS & 21 & no & 712 & 1,500 & 2,190 & 3,240 & 4,150 & 5,160 & (2) \\
\hline 07145200 & South Fork Ninnescah River near Murdock, KS & 650 & no & 5,850 & 12,600 & 18,300 & 26,700 & 33,800 & 41,500 & (2) \\
\hline 07145300 & Clear Creek near Garden Plain, KS & 5.03 & no & 612 & 1,060 & 1,380 & 1,800 & 2,120 & 2,430 & (2) \\
\hline 07145500 & Ninnescah River near Peck, KS & 2,129 & yes & 11,500 & 21,700 & 26,900 & 34,000 & 41,300 & 47,300 & (5) \\
\hline 07145700 & Slate Creek at Wellington, KS & 154 & no & 3,620 & 7,650 & 11,000 & 15,800 & 19,800 & 24,100 & (2) \\
\hline 07146500 & Arkansas River at Arkansas City, KS & 43,713 & yes & 20,600 & 40,500 & 44,500 & 63,000 & 80,000 & 99,000 & (5) \\
\hline 07146570 & Cole Creek near DeGraff, KS & 30 & no & 1,950 & 4,420 & 6,700 & 10,400 & 13,700 & 17,500 & (2) \\
\hline 07146830 & Walnut River at Highway 54 east of El Dorado, KS & 350 & yes & 3,630 & 5,970 & 9,500 & 14,000 & 18,000 & 23,000 & $(5)$ \\
\hline 07147070 & Whitewater River at Towanda, KS & 426 & no & 7,490 & 17,300 & 26,200 & 40,500 & 53,300 & 67,900 & $(2)$ \\
\hline 07147800 & Walnut River at Winfield, KS & 1,880 & yes & 18,560 & 36,100 & 44,800 & 66,000 & 86,500 & 109,000 & $(5)$ \\
\hline 07148100 & Grouse Creek near Dexter, KS & 170 & no & 8,250 & 17,000 & 24,500 & 35,800 & 45,500 & 56,100 & (2) \\
\hline 07149000 & Medicine Lodge River near Kiowa, KS & 903 & no & 4,030 & 7,450 & 10,000 & 13,500 & 16,300 & 19,100 & (2) \\
\hline 07151500 & Chikaskia River near Corbin, KS & 794 & no & 8,530 & 18,700 & 27,400 & 40,300 & 51,200 & 63,000 & (2) \\
\hline 07151600 & Rush Creek near Harper, KS & 12.0 & no & 1,190 & 2,290 & 3,140 & 4,310 & 5,250 & 6,220 & (2) \\
\hline 07155590 & Cimarron River near Elkhart, KS & 2,900 & no & 1,330 & 4,000 & 6,800 & 11,600 & 16,000 & 21,300 & (2) \\
\hline 07156000 & North Fork Cimarron River tributary near Richfield, KS & 103 & no & 791 & 2,420 & 4,100 & 6,930 & 9,510 & 12,500 & (2) \\
\hline 07156010 & North Fork Cimarron River at Richfield, KS & 463 & no & 870 & 3,650 & 7,320 & 14,800 & 22,800 & 33,200 & (2) \\
\hline 07156100 & Sand Arroyo Creek near Johnson, KS & 619 & no & 146 & 541 & 1,000 & 1,850 & 2,680 & 3,680 & (2) \\
\hline 07156220 & Bear Creek near Johnson, KS & 835 & no & 724 & 3,020 & 5,940 & 11,600 & 17,300 & 24,400 & (2) \\
\hline
\end{tabular}


Table 6. Streamflow-gaging stations in Kansas and parts of surrounding States used in the interpolation of peak-discharge frequency values at controlled and uncontrolled flow stream locations on the 1999 Kansas Surface Water Register.-Continued

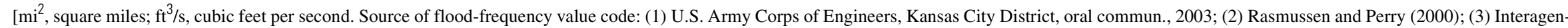

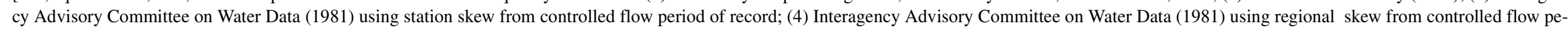
riod of record; and (5) Federal Emergency Management Agency (various years)]

\begin{tabular}{|c|c|c|c|c|c|c|c|c|c|c|}
\hline \multirow{2}{*}{$\begin{array}{c}\text { Station } \\
\text { number } \\
\text { (fig. 2) }\end{array}$} & \multirow{2}{*}{ Stream name } & \multirow{2}{*}{$\begin{array}{l}\text { Contri- } \\
\text { buting- } \\
\text { drainage } \\
\text { area } \\
\left(\mathrm{mi}^{2}\right)\end{array}$} & \multirow{2}{*}{$\begin{array}{c}\text { Con- } \\
\text { trolled } \\
\text { flow } \\
\text { (yes/no) }\end{array}$} & \multicolumn{6}{|c|}{ Peak-discharge estimate $\left(\mathrm{ft}^{3} / \mathrm{s}\right)$ for indicated flood frequency } & \multirow{2}{*}{$\begin{array}{l}\text { Source of } \\
\text { flood- } \\
\text { frequency } \\
\text { value code }\end{array}$} \\
\hline & & & & 2-year & 5 -year & 10-year & 25-year & 50-year & 100-year & \\
\hline 07157100 & Crooked Creek near Copeland, KS & 44 & no & 488 & 1,630 & 2,860 & 4,960 & 6,900 & 9,120 & (2) \\
\hline 07157500 & Crooked Creek near Nye, KS & 1,160 & no & 992 & 3,600 & 6,650 & 12,200 & 17,800 & 24,400 & (2) \\
\hline 07157700 & Kiger Creek near Ashland, KS & 34 & no & 368 & 689 & 929 & 1,250 & 1,500 & 1,750 & (2) \\
\hline 07157900 & Cavalry Creek at Coldwater, KS & 39 & no & 492 & 1,290 & 2,050 & 3,290 & 4,410 & 5,680 & (2) \\
\hline 07165700 & Verdigris River near Madison, KS & 181 & no & 8,120 & 18,700 & 28,400 & 43,800 & 57,600 & 73,400 & $(2)$ \\
\hline 07166000 & Verdigris River near Coyville, KS & 747 & yes & 5,290 & 7,490 & 8,500 & 9,400 & 9,860 & 10,200 & (3) \\
\hline 07166500 & Verdigris River near Altoona, KS & 1,138 & yes & 11,900 & 19,500 & 24,000 & 29,000 & 32,100 & 34,800 & (3) \\
\hline 07166700 & Burnt Creek at Reece, KS & 8.85 & no & 1,630 & 4,100 & 6,570 & 10,800 & 14,800 & 19,600 & (2) \\
\hline 07167000 & Fall River near Eureka, KS & 307 & no & 11,700 & 29,900 & 48,100 & 79,100 & 108,000 & 143,000 & $(2)$ \\
\hline 07167500 & Otter Creek at Climax, KS & 129 & no & 7,480 & 18,000 & 27,400 & 41,800 & 54,200 & 67,600 & (2) \\
\hline 07168500 & Fall River near Fall River, KS & 585 & yes & 3,980 & 6,910 & 8,730 & 10,800 & 12,100 & 13,300 & (4) \\
\hline 07169500 & Fall River at Fredonia, KS & 827 & yes & 9,750 & 17,000 & 21,800 & 27,500 & 31,500 & 35,200 & (4) \\
\hline 07169700 & Snake Creek near Howard, KS & 1.84 & no & 497 & 969 & 1,360 & 1,920 & 2,390 & 2,890 & (2) \\
\hline 07169800 & Elk River at Elk Falls, KS & 220 & no & 9,000 & 21,300 & 33,300 & 53,400 & 72,200 & 94,600 & $(2)$ \\
\hline 07170060 & Elk River below Elk City Lake, KS & 634 & yes & 4,740 & 7,170 & 8,600 & 10,200 & 11,200 & 12,100 & (4) \\
\hline 07170500 & Verdigris River at Independence, KS & 2,892 & yes & 22,900 & 28,000 & 35,500 & 51,000 & 64,000 & 72,000 & $(5)$ \\
\hline 07170600 & Cherry Creek near Cherryvale, KS & 15.0 & no & 2,530 & 4,580 & 6,230 & 8,640 & 10,700 & 12,900 & (2) \\
\hline 07170700 & Big Hill Creek near Cherryvale, KS & 37.0 & yes & 605 & 1,550 & 2,340 & 3,410 & 4,230 & 5,040 & (4) \\
\hline 07170800 & Mud Creek near Mound Valley, KS & 4.22 & no & 1,270 & 2,180 & 2,870 & 3,840 & 4,630 & 5,460 & (2) \\
\hline 07171000 & Verdigris River near Lenopah, OK & 3,639 & yes & 29,700 & 48,000 & 60,900 & 77,700 & 90,400 & 103,000 & (3) \\
\hline 07172000 & Caney River near Elgin, KS & 445 & no & 14,900 & 30,100 & 42,200 & 59,400 & 73,100 & 87,600 & (2) \\
\hline 07179500 & Neosho River at Council Grove, KS & 250 & yes & 1,870 & 3,060 & 3,820 & 4,740 & 5,370 & 5,960 & (4) \\
\hline 07179600 & Four Mile Creek near Council Grove, KS & 55.0 & no & 5,370 & 11,300 & 16,700 & 25,400 & 33,400 & 42,600 & $(2)$ \\
\hline 07179730 & Neosho River near Americus, KS & 622 & yes & 7,400 & 11,600 & 27,500 & 45,000 & 62,500 & 83,500 & $(5)$ \\
\hline 07179795 & North Cottonwood River below Marion Lake, KS & 200 & yes & 1,090 & 2,320 & 3,140 & 4,070 & 4,660 & 5,170 & (3) \\
\hline
\end{tabular}

07179730 Neosho River near Americus, KS

200 yes 
Table 6. Streamflow-gaging stations in Kansas and parts of surrounding States used in the interpolation of peak-discharge frequency values at controlled and uncontrolled flow stream locations on the 1999 Kansas Surface Water Register.-Continued

$\left[\mathrm{mi}^{2}\right.$, square miles; $\mathrm{ft}^{3} / \mathrm{s}$, cubic feet per second. Source of flood-frequency value code: (1) U.S. Army Corps of Engineers, Kansas City District, oral commun., 2003; (2) Rasmussen and Perry (2000); (3) Interagency Advisory Committee on Water Data (1981) using station skew from controlled flow period of record; (4) Interagency Advisory Committee on Water Data (1981) using regional skew from controlled flow period of record; and (5) Federal Emergency Management Agency (various years)]

\begin{tabular}{|c|c|c|c|c|c|c|c|c|c|c|}
\hline \multirow{2}{*}{$\begin{array}{l}\text { Station } \\
\text { number } \\
\text { (fig. 2) }\end{array}$} & \multirow[b]{2}{*}{ Stream name } & \multirow{2}{*}{$\begin{array}{l}\text { Contri- } \\
\text { buting- } \\
\text { drainage } \\
\text { area } \\
\left(\mathrm{mi}^{2}\right)\end{array}$} & \multirow{2}{*}{$\begin{array}{c}\text { Con- } \\
\text { trolled } \\
\text { flow } \\
\text { (yes } / \text { no) }\end{array}$} & \multicolumn{6}{|c|}{ Peak-discharge estimate $\left(\mathrm{ft}^{3} / \mathrm{s}\right)$ for indicated flood frequency } & \multirow{2}{*}{$\begin{array}{c}\text { Source of } \\
\text { flood- } \\
\text { frequency } \\
\text { value code }\end{array}$} \\
\hline & & & & 2-year & 5 -year & 10-year & 25-year & 50-year & 100-year & \\
\hline 07180200 & Cottonwood River at Marion, KS & 502 & yes & 7,180 & 15,300 & 24,000 & 37,500 & 52,000 & 70,000 & $(5)$ \\
\hline 07180400 & Cottonwood River near Florence, KS & 754 & yes & 8,610 & 18,300 & 28,500 & 47,600 & 67,700 & 94,400 & (3) \\
\hline 07180500 & Cedar Creek near Cedar Point, KS & 110 & no & 5,740 & 10,800 & 14,700 & 20,100 & 24,500 & 28,900 & (2) \\
\hline 07181500 & Middle Creek near Elmdale, KS & 92.0 & no & 6,960 & 15,000 & 22,100 & 33,100 & 42,700 & 53,500 & (2) \\
\hline 07182250 & Cottonwood River near Plymouth, KS & 1,740 & yes & 14,500 & 25,400 & 33,500 & 53,000 & 75,000 & 105,000 & $(5)$ \\
\hline 07182510 & Neosho River at Burlington, KS & 3,042 & yes & 10,900 & 14,100 & 16,000 & 40,000 & 71,000 & 125,000 & $(5)$ \\
\hline 07182520 & Rock Creek at Burlington, KS & 8.27 & no & 1,020 & 2,370 & 3,660 & 5,760 & 7,690 & 9,960 & (2) \\
\hline 07182600 & North Big Creek near Burlington, KS & 46 & no & 3,210 & 4,850 & 6,000 & 7,500 & 8,660 & 9,830 & (2) \\
\hline 07183000 & Neosho River near Iola, KS & 3,818 & yes & 22,000 & 31,300 & 33,800 & 59,500 & 86,000 & 122,300 & $(5)$ \\
\hline 07183100 & Owl Creek near Piqua, KS & 177 & no & 6,940 & 14,400 & 20,900 & 31,200 & 40,200 & 50,500 & $(2)$ \\
\hline 07183500 & Neosho River near Parsons, KS & 4,905 & yes & 29,400 & 42,100 & 50,410 & 60,000 & 86,000 & 121,000 & $(5)$ \\
\hline 07183800 & Limestone Creek near Beulah, KS & 12.0 & no & 3,140 & 6,540 & 9,400 & 13,600 & 17,200 & 21,100 & $(2)$ \\
\hline 07184000 & Lightning Creek near McCune, KS & 197 & no & 7,250 & 16,800 & 26,300 & 42,600 & 58,300 & 77,600 & (2) \\
\hline 07184500 & Labette Creek near Oswego, KS & 211 & no & 8,330 & 13,200 & 16,600 & 21,200 & 24,700 & 28,300 & $(2)$ \\
\hline 07184600 & Fly Creek near Faulkner, KS & 27.0 & no & 4,190 & 11,000 & 17,900 & 29,700 & 40,900 & 54,200 & $(2)$ \\
\hline 07185000 & Neosho River near Commerce, OK & 5,876 & yes & 37,700 & 58,300 & 69,600 & 105,000 & 139,100 & 175,000 & (3) \\
\hline
\end{tabular}




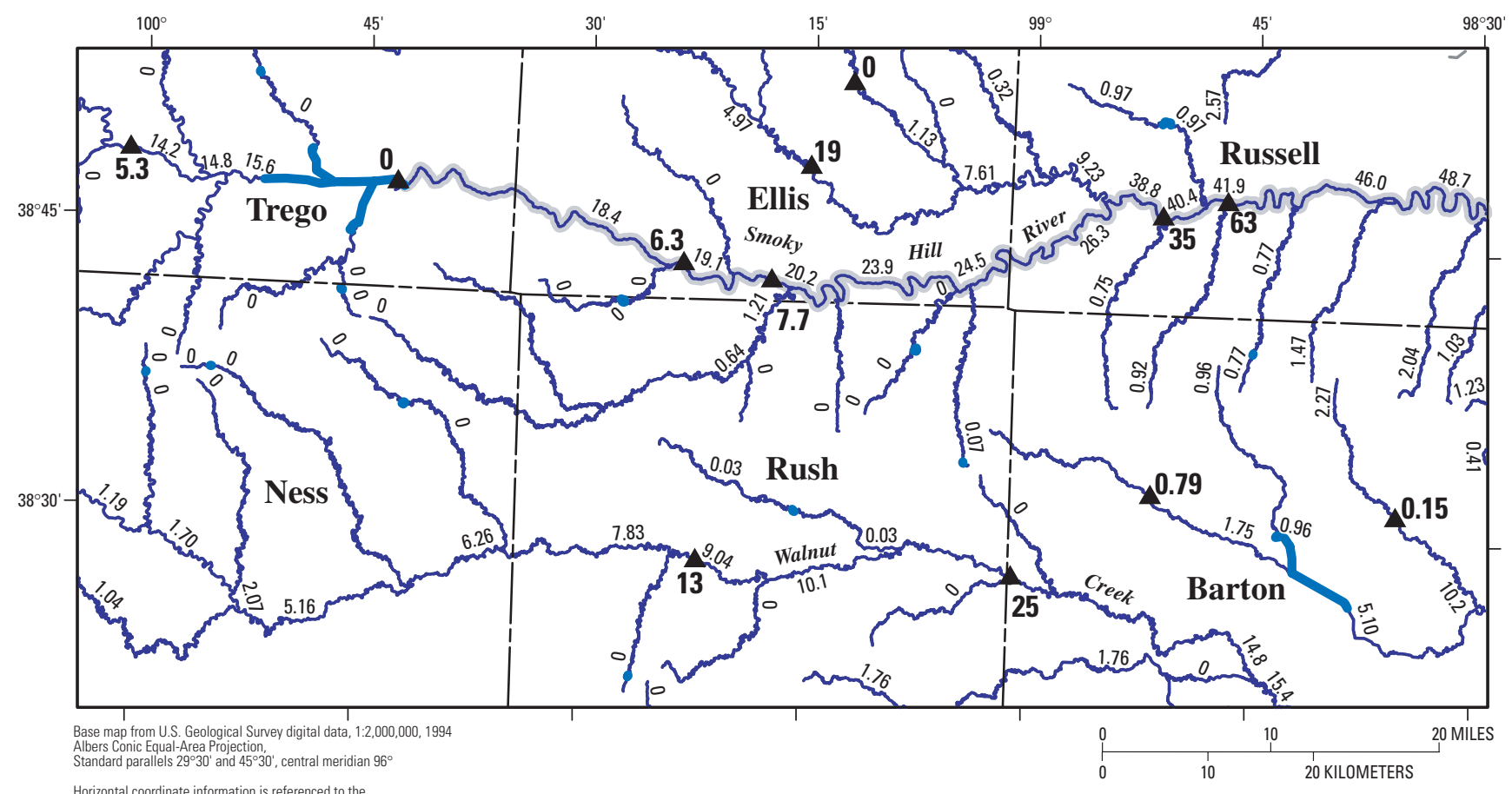
Horizontal coordinate information is referenced to the
North American Datum of 1983 (NAD 83)

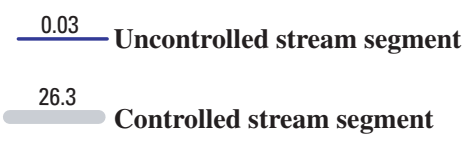

EXPLANATION

Note: Smaller numbers along the stream segments are flow values, in cubic feet per second, estimated from regression equations for 50-percent (median) flow duration.

Figure 9. 50-percent (median) flow values for stream locations in central Kansas estimated from regression equations and observed median obtained from streamflow-gaging-station data before interpolation (modified from Perry and others, 2004).

final streamflow statistics for each determination site for each county are listed in tables $7-111$ located at the end of this report. A map of each county is provided to locate streams of interest (figs. 11-115, also at the end of this report). These figures show the location of the streamflow-statistics determination sites, the determination-site identification number, location of the gaging stations used in the interpolation process, and the names of the streams. The flow statistics in tables 7-111 are linked to the streamflow-statistics determination sites in figures 11-115 by the determination-site identification number. Although the streamflow-statistics determination sites are located in a unique county, the stream segment can occur in as many as four different counties. These other counties are included under the headings of second, third, and fourth in tables 7-111.

The uncertainty of each specific streamflow statistic varies depending on the analysis used to determine the estimate for that location. The greatest uncertainties exist for streams where no gaging-station information was available and only the regression estimates were used. For these locations, the uncertainty of the estimate is the model standard error of prediction. The uncertainty values are given with the various regression equations (tables 2 and 3). Smaller uncertainty values occur at determination sites where streamflow-gaging stations are avail- able. At those locations the uncertainty is a function of the period of record of the gaging station (sample size) and the type and level of flow analysis conducted (90-, 75-, 50-, 25-, 10-percent flow durations, mean flow, or 5-, 10-, 25-, 50-, or 100-year flood frequency).

\section{Intemet Dissemination of Results}

This report and its associated figures and tables and the GIS database are available and can be downloaded from the World Wide Web (URL http://ks.water.usgs.gov/streamstats). This Web page is maintained by the USGS and has links to the GIS database described in this report to display the streamflow statistics by county for the State of Kansas. The county-map format includes county boundaries, State and Federal highways, and the stream locations for spatial reference. The estimated streamflow statistics, indexed with their respective determination-site identification number, are displayed in a pop-up window as the cursor is placed over a stream location. 


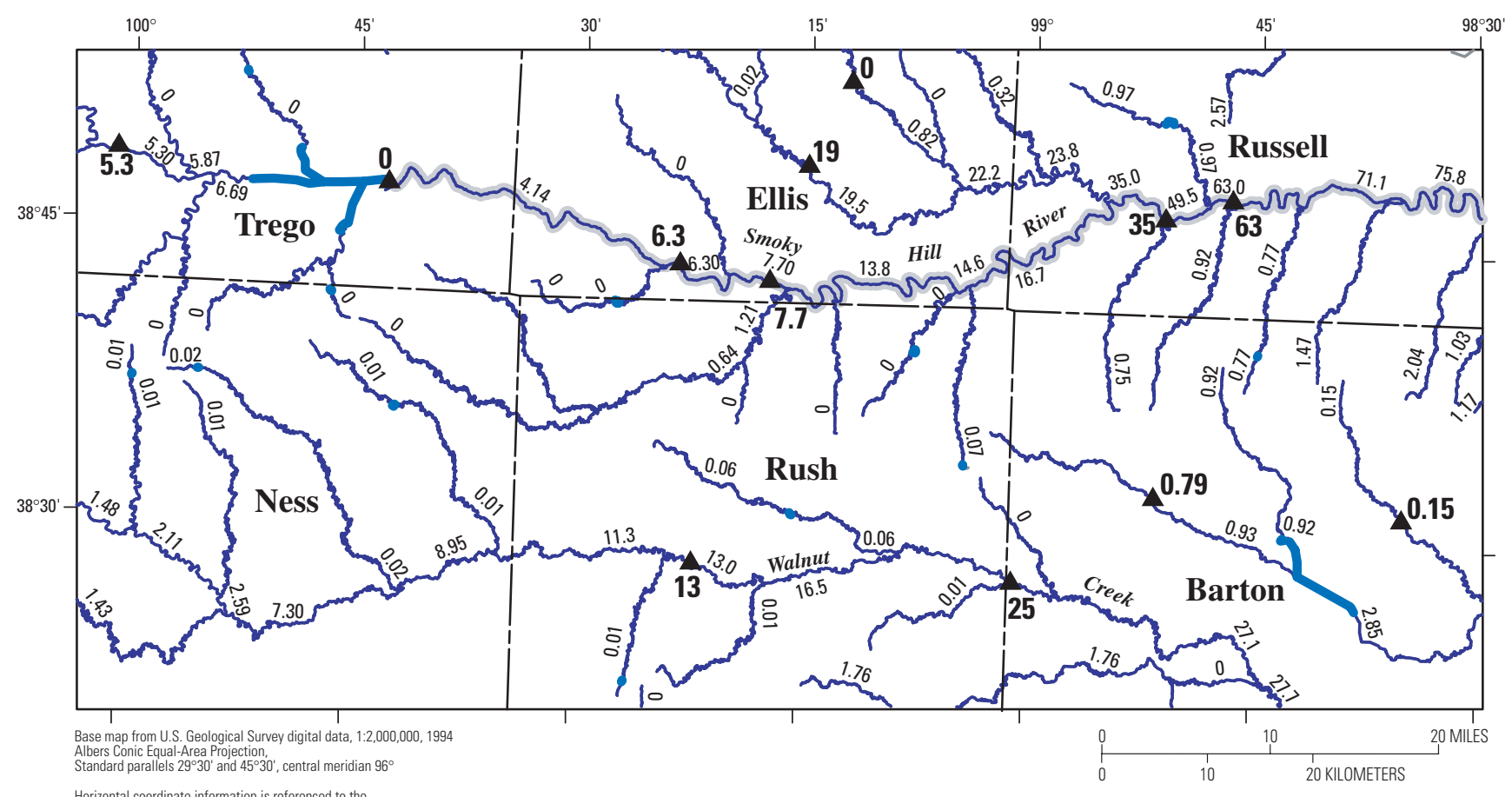

Horizontal coordinate information is referenced to the
North American Datum of 1983 (NAD 83)

EXPLANATION
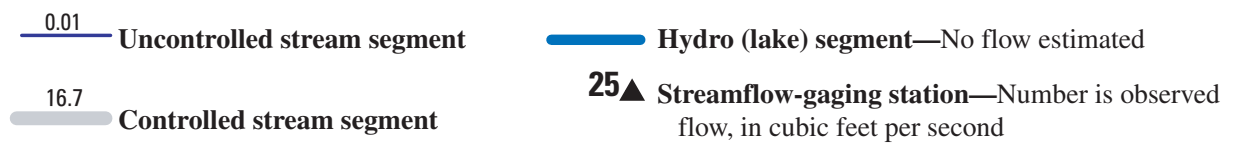

Note: Smaller numbers along the stream segments are 50-percent (median) flow-duration values, in cubic feet per second, estimated using procedures outlined in table 4.

Figure 10. Estimated 50-percent (median) flow values for stream locations in central Kansas after using interpolation procedures outlined in table 4 and observed median obtained from streamflow-gaging-station data (modified from Perry and others, 2004).

\section{Summary}

Streamflow statistics of flow duration and peak-discharge frequency were estimated for 4,771 individual locations on streams listed on the 1999 Kansas Surface Water Register. These statistics included the flow-duration values of 90, 75, 50, 25 , and 10 percent, as well as the mean flow value. Peak-discharge frequency values were estimated for the 2-, 5-, 10-, 25-, 50-, and 100-year floods.

Least-squares multiple-regression techniques, along with Tobit analyses, were used to develop equations for estimating flow durations and mean flows (dependent variables) for ungaged, uncontrolled flow stream locations. These streamflow statistics were determined from streamflow-gaging-station data using the entire period of record. Independent variables in the regression equations were the climatic and basin characteristics for streams flowing through Kansas. In the development of the regression equations, the significant climatic and basin characteristics, in order of importance, were contributing-drainage area, mean annual precipitation, mean basin permeability, and mean basin slope. Only the 149 gaging stations on uncontrolled flow streams (Kansas and parts of surrounding States) with at least 10 years of streamflow record were used in the regression analyses. The contributing-drainage areas of these gages ranged from 2.06 to $12,004 \mathrm{mi}^{2}$.

A logarithmic transformation of the basin characteristics was needed to develop a linear relation for computing flow durations and mean flow. Because there were numerous zero values for flow durations and mean flow in the gaging-station data, the Tobit analysis was used to include those zero values in the regression. The resulting regression equations were used to estimate flow durations and mean flow for the uncontrolled flow stream locations on the 1999 Kansas Surface Water Register.

Estimates of peak-discharge frequency for uncontrolled flow stream locations in Kansas were obtained using equations previously published in U.S. Geological Survey WaterResources Investigations Report 00-4079 (Rasmussen and Perry, 2000), "Estimation of Peak Streamflows for Unregulated Rural Streams in Kansas." This report produced two sets of equations - one for basins between 0.17 and $30 \mathrm{mi}^{2}$ and one for basins between 30 and 9,100 $\mathrm{mi}^{2}$. For streams with a basin size of between 0.17 and $30 \mathrm{mi}^{2}$, peak-discharge frequency values were computed from regression equations with contributingdrainage area and average mean annual precipitation for the basin as the predictor variables. For streams with basin sizes 
between 30 and $9,100 \mathrm{mi}^{2}$, the predictor variables were contributing-drainage area, average mean annual precipitation, mean soil permeability, and the slope of the main channel.

Streamflow-gaging-station data were used to improve the quality of the streamflow-statistic estimates along the streams that had gages. Streamflow statistics for the locations that had uncontrolled flow were interpolated using gaged data weighted according to the drainage area and the bias between the regression estimate and gaged flow information. On controlled reaches of Kansas streams, the streamflow-statistic information was interpolated between gaging stations by using only gaged data weighted by drainage area.

\section{References Cited}

Arihood, L.D., and Glatfelter, D.R., 1991, Method for estimating low-flow characteristics of ungaged streams in Indiana: U.S. Geological Survey Water-Supply Paper 2372, 18 p.

Bisese, J.A., 1995, Methods for estimating the magnitude and frequency of peak discharges of rural, unregulated streams in Virginia: U.S. Geological Survey Water-Resources Investigations Report 94-4148, 70 p., 1 pl.

Burrough, P.A., 1986, Principles of geographical information systems for land resources assessment: New York, Oxford University Press, $50 \mathrm{p}$.

Choquette, A.F., 1988, Regionalization of peak discharges for streams in Kentucky: U.S. Geological Survey WaterResources Investigations Report 88-4209, 105 p., 1 pl.

Clement, R.W., 1987, Floods in Kansas and techniques for estimating their magnitude and frequency on unregulated streams: U.S. Geological Survey Water-Resources Investigations Report 87-4008, $50 \mathrm{p}$.

Cohn, T.A., 1988, Adjusted maximum likelihood estimation of the moments of log-normal population of Type I censored samples: U.S. Geological Survey Open-File Report 88-350, $34 \mathrm{p}$.

Daly, C., Neilson, R.P., and Phillips, D.L., 1994, A statisticaltopographic model for mapping climatological precipitation over mountainous terrain: Journal of Applied Meteorology, v. 33 , no. 2 , p. $140-158$.

Daly, C., Taylor, C.H., and Gibson, W.P., 1997, The PRISM approach to mapping precipitation and temperature, in Reprints of 10th Conference on Applied Climatology, Reno, Nevada: American Meteorological Society, p. 10-12.

Environmental Systems Research Institute, Inc., 1991, Understanding GIS- the ARC/INFO method: Redlands, California, various pagination.

Federal Emergency Management Agency, various years, Flood insurance study for cities of Arkansas City, Kansas, 1985; Burlington, Kansas, 1996; Derby, Kansas, 1996; Dodge City, Kansas, 1995; Ellsworth, Kansas, 1989; Enterprise, Kansas, 1988; Garden City, Kansas, 1997; Great Bend, Kansas, 1996; Hutchinson, Kansas, 2000; Independence, Kansas, 1995; Iola, Kansas, 1990; Junction City, Kansas, 1987; Kin- sley, Kansas, 1978; La Cygne, Kansas, 1975; Maize, Kansas, 1986; Manhattan, Kansas, 2003; Perry, Kansas, 1999; Scandia, Kansas, 1979; Syracruse, Kansas, 2003; Wichita, Kansas, 1986; and Winfield, Kansas, 2002; and the counties of Butler County, Kansas, 2001; Douglas County, Kansas, 2001; Lyon County, Kansas, 1982; Marion County, Kansas, 1978; Saline County, Kansas, 1986; and Sedgwick County, Kansas, 1986: Washington, D.C., various pagination.

Flynn, K.M., Hummel, P.R., Lumb, A.M., and Kittle, J.L., Jr., 1995, User's manual for ANNIE, version 2, a computer program for interactive hydrologic data management: U.S. Geological Survey Water-Resources Investigations Report 954085, $211 \mathrm{p}$.

Furness, L.W., 1959, Kansas streamflow characteristicspart 1, Flow duration: Kansas Water Resources Board Technical Report No. 1, 213 p.

Gebert, W.A., Graczyk, D.J., and Krug, W.R., 1987, Average annual runoff in the United States, 1951-80: U.S.

Geological Survey Hydrologic Investigations Atlas HA-710, 1 sheet, scale 1:7,500,000.

Inman, R.L., and Conover, W.J., 1983, A modern approach to statistics: New York, John Wiley, 497 p.

Interagency Advisory Committee on Water Data, 1981, Guidelines for determining flood flow frequency: Washington, D.C., Bulletin 17B of the Hydrology Committee, 28 p.

Johnson, C.G., 1970, A proposed streamflow data program for central New England: Boston, Massachusetts, U.S. Geological Survey open-file report, $38 \mathrm{p}$.

Jordan, P.R., 1983, Kansas streamflow characteristics-magnitude and frequency of low flows of unregulated streams in Kansas, and estimation of flow-duration curves for ungaged sites: Kansas Water Office Technical Report No. 17, 55 p.

Judge, G.G., Griffiths, W.E., Hill, R.C., Lutkepohl, H., and Lee, T.C., 1985, Qualitative and limited dependent variable models, chapter 18, in The theory and practice of econometrics (2d ed.): New York, Wiley, 1064 p.

Koltun, G.F., and Roberts, J.W., 1990, Techniques for estimating flood-peak discharges of rural, unregulated streams in Ohio: U.S. Geological Survey Water- Resources Investigations Report 89-4126, 68 p., 1 pl.

Koltun, G.F., and Schwartz, R.R., 1986, Multiple-regression equations for estimating low flows at ungaged stream sites in Ohio: U.S. Geological Survey Water-Resources Investigations Report 86-4354, 39 p., 6 pl.

Ku, H.F., Randall, A.D., and MacNish, R.D., 1975, Streamflow in the New York part of the Susquehanna River Basin: New York State Department of Environmental Conservation Bulletin 71, $130 \mathrm{p}$.

Loaiciga, H.A., 1989, Variability of empirical flow quantiles: Journal of Hydraulic Engineering, American Society of Civil Engineers, v. 115, no. 1, p. 82-100.

Lumb, A.M., Kittle, J.L., Jr., and Flynn, K.M., 1990, Users manual for ANNIE, a computer program for interactivelhydrologic analyses and data management: U.S. Geological Survey Water-Resources Investigations Report 89-4080, $236 \mathrm{p}$. 
Lumia, Richard, 1991, Regionalization of flood discharges for rural, unregulated streams in New York, excluding Long Island: U.S. Geological Survey Water-Resources Investigations Report 90-4197, 119 p., 2 pl.

MathSoft, 1999, S-PLUS 2000 guide to statistics: Seattle, Washington, volumes I, II, and III, various pagination.

Parrett, Charles, and Hull, J.A., 1985, Streamflow characteristics of mountain streams in western Montana: U.S. Geological Survey Water-Supply Paper 2260, 58 p.

Paulson, R.W., Chase, E.B., Roberts, R.S., and Moody, D.W., compilers, 1991, National water summary 1988-89-hydrologic events and floods and droughts: U.S. Geological Survey Water-Supply Paper 2375, 591 p.

Perry, C.A., Wolock, D.M., and Artman, J.C., 2004, Estimates of median flows for streams on the 1999 Kansas Surface Water Register: U.S. Geological Survey Scientific Investigations Report 2004-5032, 227 p.

Putnam, J.E., Lacock, D.L., Schneider, D.R., and Carlson, M.D., 2001, Water resources data, Kansas, water year 2000: U.S. Geological Survey Water-Data Report KS-00-1, 505 p.

Rasmussen, P.P., and Perry, C.A., 2000, Estimation of peak streamflows for unregulated rural streams in Kansas: U.S. Geological Survey Water-Resources Investigations Report 00-4079, 33 p.

Ries, K.G., and Crouse, M.Y., 2002, The national flood frequency program, version 3-a computer program for estimating magnitude and frequency of floods for ungaged sites: U.S. Geological Survey Water-Resources Investigations Report 02-4168, 45 p.

Ries, K.G., and Friesz, P.I., 2000, Methods for estimating lowflow statistics for Massachusetts streams: U.S. Geological Survey Water-Resources Investigations Report 00-4135, $81 \mathrm{p}$.

Sauer, V.B., 1974, Techniques for calculating magnitude and frequency of floods in Oklahoma, with compilations of flood data through 1971: U.S. Geological Survey Water-Resources Investigations Report 52-73, 24 p.

Schoewe, W.H., 1949, The geography of Kansas-part 2, Physical geography: Transactions of the Kansas Academy of Science, v. 52, no. 3, p. 261-333.
Searcy, J.K., 1959, Flow-duration curves, manual of hydrology_part 2. Low-flow techniques: U.S. Geological Survey Water-Supply Paper 1542-A, p. 1-33.

Studley, S.E., 2000, Estimated flow-duration curves for selected ungaged sites in the Cimarron and lower Arkansas River Basins in Kansas: U.S. Geological Survey WaterResources Investigations Report 00-4113, 43 p.

Studley, S.E., 2001, Estimated flow-duration curves for selected ungaged sites in Kansas: U.S. Geological Survey Water-Resources Investigations Report 01-4142, 90 p.

Thomas, M.P., 1966, Effect of glacial geology upon the time distribution of streamflow in eastern and southern Connecticut, in Geological Survey Research 1966: U.S. Geological Survey Professional Paper 0550-B, p. B209-B212.

Tobin, J., 1958, Estimation of relationships for limited dependent variables: Econometrica, v. 26, p. 24-36.

U.S. Department of Agriculture, 1994, State soil geographic (STATSGO) data base: Soil Conservation Miscellaneous Publication 1462, 37 p.

U.S. Environmental Protection Agency, 1996, The USEPA reach file, version 1.0 (RF1) for the conterminous United States: Washington, D.C., information available on the World Wide Web, accessed January 14, 2004, at URL http://www.epa.gov/nsdi/projects/rf1.htm

U.S. Geological Survey, 1998, National elevation data base: Sioux Falls, South Dakota, National Mapping Division EROS Data Center, information available on the World Wide Web, accessed June 6, 1998, at URL http://edcwww2.cr.usgs.gov/ned/ned.html

Wahl, K.L., and Wahl, T. L., 1995, Determining the flow of Comal Springs at New Braunfels, Texas, in Texas Water '95: American Society of Civil Engineers, August 16-17, 1995, San Antonio, Texas, p. 77-86.

Wandle, S.W., Jr., and Randall, A.D., 1994, Effects of surficial geology, lakes and swamps, and annual water availability on low flows of streams in central New England, and their use in low-flow estimation: U.S. Geological Survey WaterResources Investigations Report 93-4092, 57 p.

Wolock, D.M., and McCabe, G.J., 1999, Explaining spatial variability in mean annual runoff in the conterminous United States: Journal of Climate Research, v. 11, p. 149-159. 


\section{Supplemental Information}




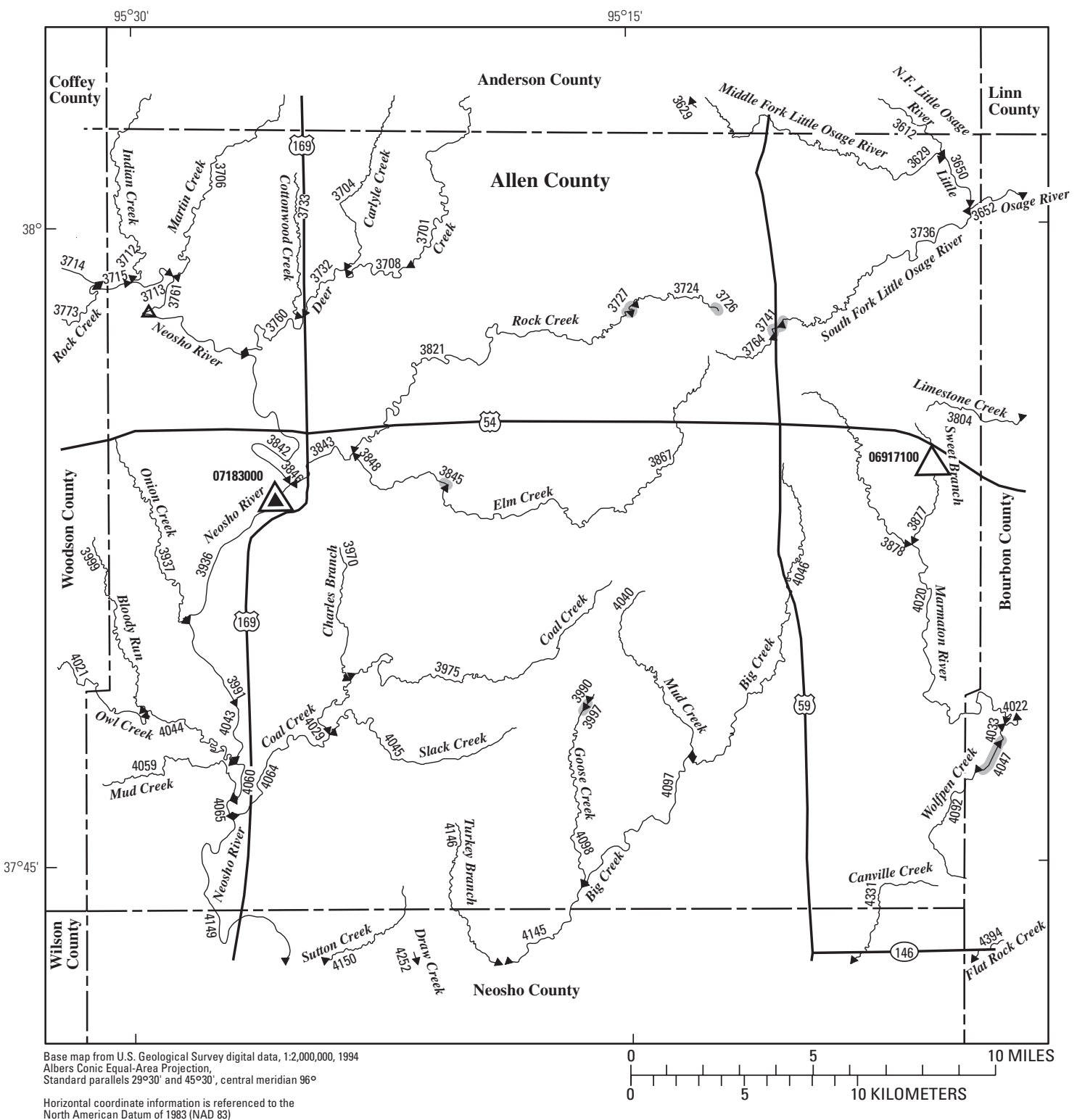

\section{EXPLANATION}

$\$ 6127$ Location of streamflow-statistics determination site (small triangle) and associated identification number-small triangle points in downstream direction

${ }^{06890100} \Delta$ U.S. Geological Survey streamflow-gaging station and number used for estimates of flow duration

${ }^{07183000} \triangle$ U.S. Geological Survey streamflow-gaging station and number used for estimates of peak-discharge frequency values

KANSAS

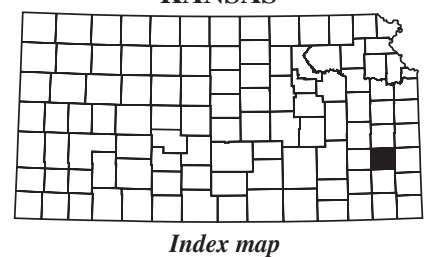

3741

Lake and determination site identification number

Figure 11. Location of streamflow-statistics determination sites, associated identification numbers, and U.S. Geological Survey streamflow-gaging stations used in the flow-duration and peak-discharge frequency analyses for Allen County. 
Table 7. Estimated flow-duration values, mean flow values, and peak-discharge frequency values for controlled and uncontrolled flow stream segments on the 1999 Kansas Surface Water Register for Allen County.

[KSWR, Kansas Surface Water Register; CUSEGA, catalog unit segment number alpha; $\mathrm{mi}^{2}$, square miles; $\mathrm{ft}^{3} / \mathrm{s}$, cubic feet per second; HYDRO, lake or other hydrologic structure; NA, not applicable; NRDitch, irrigation ditch; NRTribal, tribal stream]

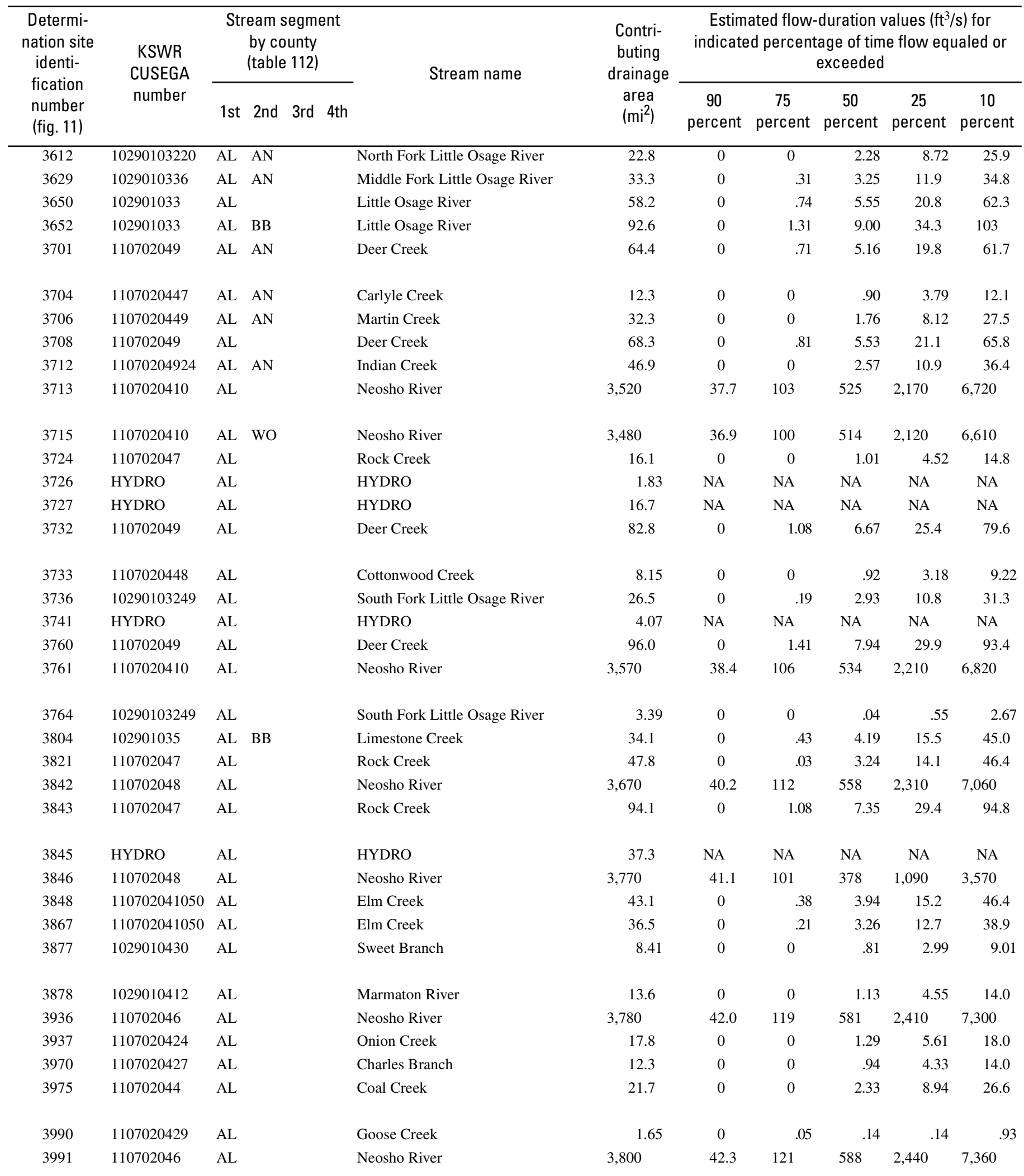


Table 7. Estimated flow-duration values, mean flow values, and peak-discharge frequency values for controlled and uncontrolled flow stream segments on the 1999 Kansas Surface Water Register for Allen County.-Continued

[KSWR, Kansas Surface Water Register; CUSEGA, catalog unit segment number alpha; $\mathrm{mi}^{2}$, square miles; $\mathrm{ft}^{3} / \mathrm{s}$, cubic feet per second; HYDRO, lake or other hydrologic structure; NA, not applicable; NRDitch, irrigation ditch; NRTribal, tribal stream]

\begin{tabular}{|c|c|c|c|c|c|c|c|}
\hline \multirow{2}{*}{$\begin{array}{l}\text { Determi- } \\
\text { nation site } \\
\text { identi- } \\
\text { fication } \\
\text { number } \\
\text { (fig. 11) }\end{array}$} & \multirow{2}{*}{$\begin{array}{c}\text { Estimated mean } \\
\text { flow } \\
\left(\mathrm{ft}^{3} / \mathrm{s}\right)\end{array}$} & \multicolumn{6}{|c|}{ Estimated peak discharge $\left(\mathrm{ft}^{3} / \mathrm{s}\right)$ for indicated peak-discharge frequency } \\
\hline & & 2-year & 5-year & 10-year & 25-year & 50-year & 100-year \\
\hline 3612 & 20.2 & 2,590 & 5,230 & 7,460 & 10,800 & 13,500 & 16,600 \\
\hline 3629 & 27.0 & 3,930 & 7,420 & 10,300 & 14,500 & 17,900 & 21,500 \\
\hline 3650 & 46.2 & 5,420 & 10,000 & 13,800 & 19,300 & 23,900 & 28,700 \\
\hline 3652 & 73.0 & 7,410 & 13,200 & 18,000 & 24,800 & 30,500 & 36,500 \\
\hline 3701 & 47.8 & 5,040 & 9,710 & 13,700 & 19,500 & 24,400 & 29,600 \\
\hline 3704 & 10.4 & 1,770 & 3,560 & 5,050 & 7,280 & 9,100 & 11,200 \\
\hline 3706 & 23.8 & 4,470 & 8,650 & 12,200 & 17,300 & 21,600 & 26,200 \\
\hline 3708 & 50.6 & 5,070 & 9,790 & 13,800 & 19,700 & 24,700 & 30,000 \\
\hline 3712 & 31.3 & 4,760 & 9,230 & 13,000 & 18,500 & 23,200 & 28,200 \\
\hline 3713 & 2,050 & 18,600 & 26,000 & 28,300 & 53,500 & 81,400 & 123,000 \\
\hline 3715 & 2,010 & 17,900 & 25,000 & 27,300 & 52,400 & 80,500 & 123,000 \\
\hline 3724 & 13.1 & 2,150 & 4,300 & 6,100 & 8,780 & 11,000 & 13,500 \\
\hline 3726 & NA & NA & NA & NA & NA & NA & NA \\
\hline 3727 & NA & NA & NA & NA & NA & NA & NA \\
\hline 3732 & 60.4 & 5,510 & 10,600 & 15,000 & 21,300 & 26,700 & 32,500 \\
\hline 3733 & 7.60 & 1,400 & 2,780 & 3,930 & 5,640 & 7,040 & 8,610 \\
\hline 3736 & 23.7 & 2,870 & 5,780 & 8,240 & 11,900 & 14,900 & 18,400 \\
\hline 3741 & NA & NA & NA & NA & NA & NA & NA \\
\hline 3760 & 69.8 & 5,730 & 11,000 & 15,600 & 22,300 & 27,900 & 34,000 \\
\hline 3761 & 2,070 & 19,200 & 26,900 & 29,200 & 54,500 & 82,100 & 123,000 \\
\hline 3764 & 2.98 & 875 & 1,690 & 2,360 & 3,340 & 4,130 & 5,020 \\
\hline 3804 & 32.5 & 6,040 & 10,600 & 14,300 & 19,400 & 23,500 & 27,800 \\
\hline 3821 & 37.5 & 4,710 & 9,250 & 13,100 & 18,900 & 23,700 & 28,900 \\
\hline 3842 & 2,150 & 20,600 & 29,100 & 31,500 & 57,000 & 84,100 & 123,000 \\
\hline 3843 & 71.4 & 6,590 & 12,500 & 17,500 & 24,900 & 31,100 & 37,800 \\
\hline 3845 & NA & NA & NA & NA & NA & NA & NA \\
\hline 3846 & 1,750 & 23,200 & 44,900 & 64,000 & 93,100 & 118,000 & 147,000 \\
\hline 3848 & 35.7 & 4,030 & 7,890 & 11,200 & 16,000 & 20,000 & 24,300 \\
\hline 3867 & 30.4 & 3,530 & 7,000 & 9,980 & 14,400 & 18,100 & 22,000 \\
\hline 3877 & 7.73 & 204 & 349 & 455 & 597 & 707 & 820 \\
\hline 3878 & 11.9 & 1,970 & 3,910 & 5,540 & 7,950 & 9,920 & 12,200 \\
\hline 3936 & 2,220 & 22,000 & 31,300 & 33,800 & 59,500 & 86,000 & 122,000 \\
\hline 3937 & 15.1 & 2,220 & 4,480 & 6,380 & 9,220 & 11,500 & 14,200 \\
\hline 3970 & 11.6 & 1,820 & 3,630 & 5,140 & 7,390 & 9,230 & 11,300 \\
\hline 3975 & 20.4 & 2,570 & 5,170 & 7,360 & 10,600 & 13,300 & 16,300 \\
\hline 3990 & 1.35 & 586 & 1,110 & 1,540 & 2,150 & 2,650 & 3,210 \\
\hline 3991 & 2,240 & 22,200 & 31,600 & 34,200 & 59,500 & 86,000 & 122,000 \\
\hline
\end{tabular}


Table 7. Estimated flow-duration values, mean flow values, and peak-discharge frequency values for controlled and uncontrolled flow stream segments on the 1999 Kansas Surface Water Register for Allen County.-Continued

[KSWR, Kansas Surface Water Register; CUSEGA, catalog unit segment number alpha; $\mathrm{mi}^{2}$, square miles; $\mathrm{ft}^{3} / \mathrm{s}$, cubic feet per second; HYDRO, lake or other hydrologic structure; NA, not applicable; NRDitch, irrigation ditch; NRTribal, tribal stream]

\begin{tabular}{|c|c|c|c|c|c|c|c|c|c|c|c|}
\hline $\begin{array}{l}\text { Determi- } \\
\text { nation site } \\
\text { identi- } \\
\text { fication } \\
\text { number } \\
\text { (fig. 11) }\end{array}$ & $\begin{array}{l}\text { KSWR } \\
\text { CUSEGA } \\
\text { number }\end{array}$ & \multicolumn{3}{|c|}{$\begin{array}{l}\text { Stream segment } \\
\text { by county } \\
\text { (table 112) }\end{array}$} & Stream name & $\begin{array}{l}\text { Contri- } \\
\text { buting } \\
\text { drainage } \\
\text { area } \\
\left(\mathrm{mi}^{2}\right)\end{array}$ & \multicolumn{5}{|c|}{$\begin{array}{c}\text { Estimated flow-duration values }\left(\mathrm{ft}^{3} / \mathrm{s}\right) \text { for } \\
\text { indicated percentage of time flow equaled or } \\
\text { exceeded }\end{array}$} \\
\hline 3997 & HYDRO & $\mathrm{AL}$ & & & HYDRO & 2.21 & NA & NA & NA & NA & NA \\
\hline 4021 & 1107020419 & $\mathrm{AL}$ & WO & & Owl Creek & 178 & 0 & .57 & 4.00 & 21.0 & 115 \\
\hline 4029 & 110702044 & $\mathrm{AL}$ & & & Coal Creek & 36.3 & 0 & .24 & 3.57 & 13.9 & 42.4 \\
\hline 4040 & 1107020431 & $\mathrm{AL}$ & & & Mud Creek & 14.0 & 0 & 0 & 1.41 & 5.19 & 15.3 \\
\hline 4043 & 110702045 & $\mathrm{AL}$ & & & Neosho River & 3,800 & 42.3 & 121 & 588 & 2,440 & 7,360 \\
\hline 4059 & 1107020426 & $\mathrm{AL}$ & & & Mud Creek & 15.2 & 0 & 0 & .80 & 4.24 & 14.7 \\
\hline 4060 & 110702045 & $\mathrm{AL}$ & & & Neosho River & 4,000 & 44.6 & 133 & 641 & 2,660 & 7,840 \\
\hline 4064 & 110702044 & $\mathrm{AL}$ & & & Coal Creek & 59.0 & 0 & .90 & 6.09 & 22.9 & 69.1 \\
\hline 4065 & 110702045 & $\mathrm{AL}$ & & & Neosho River & 4,010 & 44.8 & 134 & 645 & 2,680 & 7,880 \\
\hline 4092 & 1029010437 & $\mathrm{AL}$ & BB & & Wolfpen Creek & 11.8 & 0 & 0 & 1.63 & 5.71 & 16.0 \\
\hline 4097 & 110702042 & $\mathrm{AL}$ & & & Big Creek & 68.5 & 0 & .80 & 5.49 & 21.2 & 66.5 \\
\hline 4098 & 1107020429 & $\mathrm{AL}$ & & & Goose Creek & 12.8 & 0 & .30 & 2.35 & 7.38 & 19.3 \\
\hline 4145 & 110702042 & $\mathrm{AL}$ & NO & & Big Creek & 92.5 & 0 & 1.47 & 8.30 & 31.5 & 97.7 \\
\hline 4146 & 1107020428 & $\mathrm{AL}$ & NO & & Turkey Branch & 11.9 & 0 & .24 & 2.18 & 6.91 & 18.1 \\
\hline
\end{tabular}


Table 7. Estimated flow-duration values, mean flow values, and peak-discharge frequency values for controlled and uncontrolled flow stream segments on the 1999 Kansas Surface Water Register for Allen County.-Continued

[KSWR, Kansas Surface Water Register; CUSEGA, catalog unit segment number alpha; $\mathrm{mi}^{2}$, square miles; $\mathrm{ft}^{3} / \mathrm{s}$, cubic feet per second; HYDRO, lake or other hydrologic structure; NA, not applicable; NRDitch, irrigation ditch; NRTribal, tribal stream]

\begin{tabular}{|c|c|c|c|c|c|c|c|}
\hline \multirow{2}{*}{$\begin{array}{l}\text { Determi- } \\
\text { nation site } \\
\text { identi- } \\
\text { fication } \\
\text { number } \\
\text { (fig. 11) }\end{array}$} & \multirow{2}{*}{$\begin{array}{c}\text { Estimated mean } \\
\text { flow } \\
\left(\mathrm{ft}^{3} / \mathrm{s}\right)\end{array}$} & \multicolumn{6}{|c|}{ Estimated peak discharge $\left(\mathrm{ft}^{3} / \mathrm{s}\right)$ for indicated peak-discharge frequency } \\
\hline & & 2-year & 5-year & 10-year & 25-year & 50-year & 100-year \\
\hline 3997 & NA & NA & NA & $\mathrm{NA}$ & NA & NA & NA \\
\hline 3999 & 11.0 & 1,820 & 3,620 & 5,130 & 7,370 & 9,200 & 11,300 \\
\hline 4020 & 42.0 & 3,750 & 6,750 & 9,190 & 12,700 & 15,700 & 18,700 \\
\hline 4021 & 122 & 11,100 & 21,500 & 30,400 & 44,200 & 56,100 & 69,600 \\
\hline 4029 & 32.1 & 4,230 & 8,160 & 11,500 & 16,300 & 20,300 & 24,600 \\
\hline 4040 & 12.5 & 2,020 & 4,020 & 5,680 & 8,150 & 10,200 & 12,500 \\
\hline 4043 & 2,240 & 22,200 & 31,600 & 34,200 & 59,500 & 86,000 & 122,000 \\
\hline 4044 & 132 & 10,900 & 21,300 & 30,300 & 44,200 & 56,200 & 69,800 \\
\hline 4045 & 13.1 & 1,980 & 3,930 & 5,550 & 7,960 & 9,930 & 12,200 \\
\hline 4046 & 29.2 & 4,110 & 7,830 & 10,900 & 15,400 & 19,100 & 23,100 \\
\hline 4059 & 13.1 & 2,140 & 4,240 & 6,000 & 8,610 & 10,700 & 13,200 \\
\hline 4060 & 2,410 & 23,600 & 33,700 & 37,500 & 59,600 & 86,000 & 122,000 \\
\hline 4064 & 50.5 & 5,010 & 9,550 & 13,400 & 19,000 & 23,600 & 28,600 \\
\hline 4065 & 2,430 & 23,700 & 33,900 & 37,700 & 59,600 & 86,000 & 122,000 \\
\hline 4092 & 12.3 & 1,900 & 3,720 & 5,230 & 7,470 & 9,290 & 11,300 \\
\hline 4097 & 51.8 & 6,000 & 11,100 & 15,400 & 21,500 & 26,500 & 32,000 \\
\hline 4098 & 13.8 & 1,940 & 3,830 & 5,410 & 7,740 & 9,650 & 11,800 \\
\hline 4145 & 72.2 & 6,880 & 12,600 & 17,400 & 24,300 & 30,100 & 36,200 \\
\hline 4146 & 13.0 & 1,880 & 3,710 & 5,220 & 7,460 & 9,280 & 11,300 \\
\hline 4149 & 2,500 & 24,300 & 34,700 & 39,000 & 59,700 & 86,000 & 122,000 \\
\hline 4150 & 12.0 & 1,830 & 3,600 & 5,060 & 7,220 & 8,980 & 11,000 \\
\hline 4331 & 43.1 & 5,250 & 9,760 & 13,500 & 18,900 & 23,400 & 28,100 \\
\hline
\end{tabular}




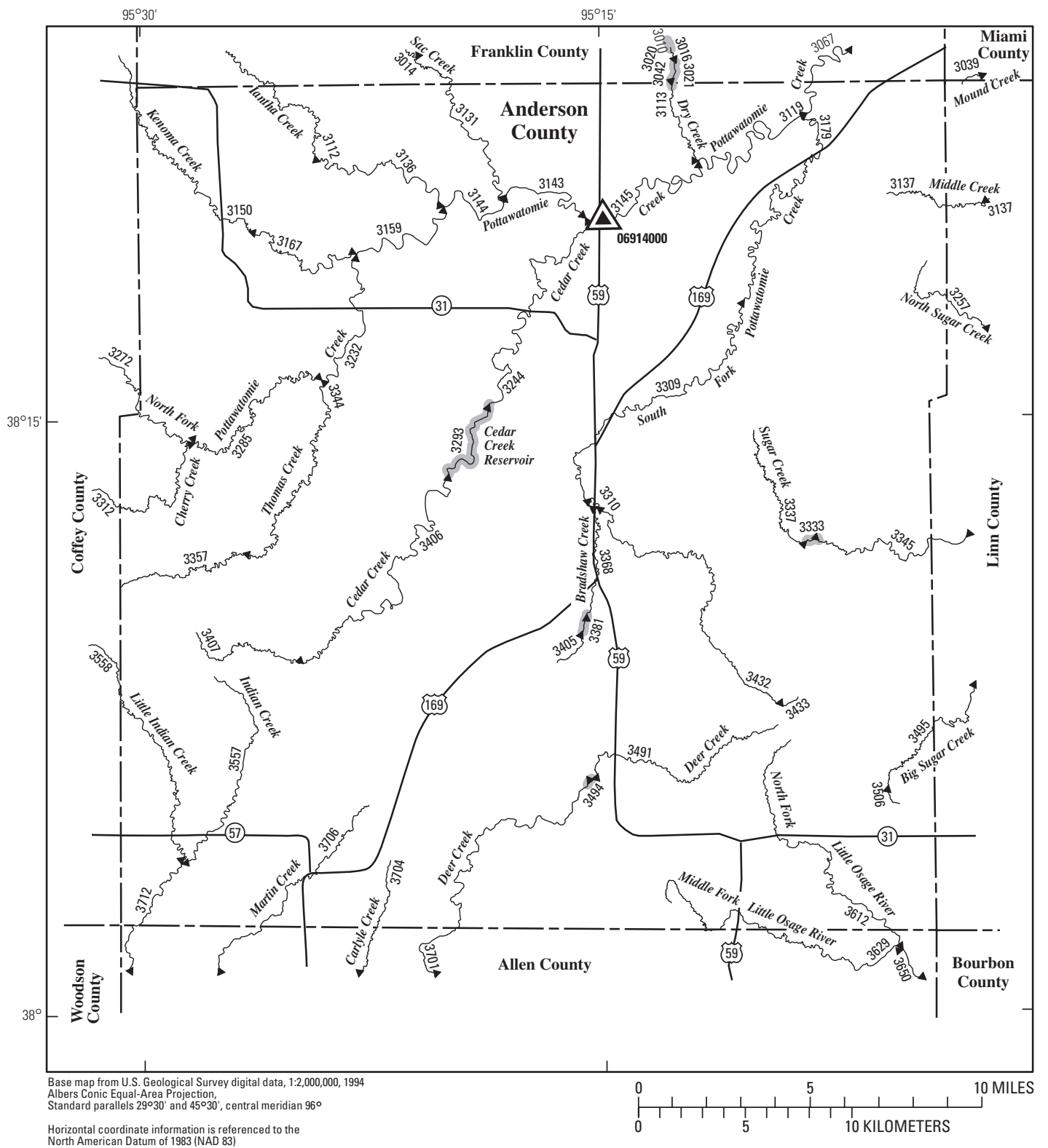

\section{EXPLANATION}

\$706 Location of streamflow-statistics determination site (small triangle) and associated identification number-small triangle points in downstream direction

06914000 U.S. Geological Survey streamflow-gaging station and number used for estimates of flow duration

${ }^{06914000} \triangle$ U.S. Geological Survey streamflow-gaging station and number used for estimates of peak-discharge frequency values

KANSAS

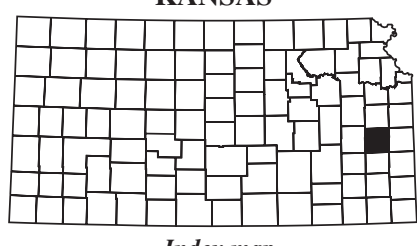

Index map

Lake and determination site identification number

Figure 12. Location of streamflow-statistics determination sites, associated identification numbers, and U.S. Geological Survey streamflow-gaging stations used in the flow-duration and peak-discharge frequency analyses for Anderson County. 


\section{Estimates of Flow Duration, Mean Flow, and Peak-Discharge Frequency Values for Kansas Stream Locations}

Table 8. Estimated flow-duration values, mean flow values, and peak-discharge frequency values for controlled and uncontrolled flow stream segments on the 1999 Kansas Surface Water Register for Anderson County.-Continued

[KSWR, Kansas Surface Water Register; CUSEGA, catalog unit segment number alpha; $\mathrm{mi}^{2}$, square miles; $\mathrm{ft}^{3} / \mathrm{s}$, cubic feet per second; HYDRO, lake or other hydrologic structure; NA, not applicable; NRDitch, irrigation ditch; NRTribal, tribal stream]

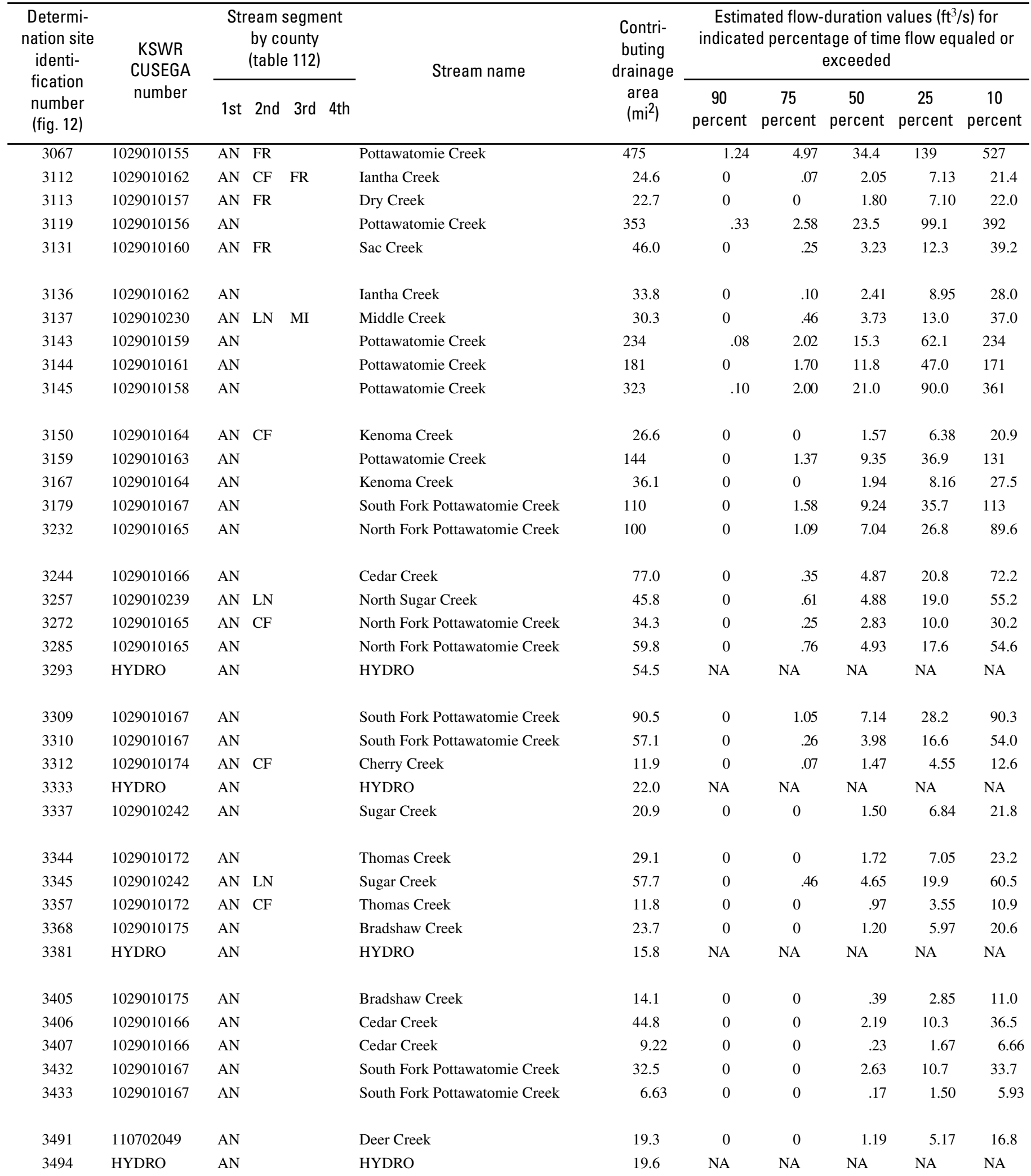


Table 8. Estimated flow-duration values, mean flow values, and peak-discharge frequency values for controlled and uncontrolled flow stream segments on the 1999 Kansas Surface Water Register for Anderson County.-Continued

[KSWR, Kansas Surface Water Register; CUSEGA, catalog unit segment number alpha; $\mathrm{mi}^{2}$, square miles; $\mathrm{ft}^{3} / \mathrm{s}$, cubic feet per second; HYDRO, lake or other hydrologic structure; NA, not applicable; NRDitch, irrigation ditch; NRTribal, tribal stream]

\begin{tabular}{|c|c|c|c|c|c|c|c|}
\hline \multirow{2}{*}{$\begin{array}{l}\text { Determi- } \\
\text { nation site } \\
\text { identi- } \\
\text { fication } \\
\text { number } \\
\text { (fig. 12) }\end{array}$} & \multirow{2}{*}{$\begin{array}{c}\text { Estimated mean } \\
\text { flow } \\
\left(\mathrm{ft}^{3} / \mathrm{s}\right)\end{array}$} & \multicolumn{6}{|c|}{ Estimated peak discharge $\left(\mathrm{ft}^{3} / \mathrm{s}\right)$ for indicated peak-discharge frequency } \\
\hline & & 2-year & 5 -year & 10-year & 25-year & 50-year & 100-year \\
\hline 3067 & 335 & 13,100 & 24,600 & 34,600 & 49,700 & 63,100 & 78,200 \\
\hline 3112 & 17.2 & 2,260 & 4,780 & 6,950 & 10,200 & 13,000 & 16,100 \\
\hline 3113 & 18.0 & 2,410 & 4,960 & 7,130 & 10,400 & 13,100 & 16,100 \\
\hline 3119 & 254 & 11,100 & 20,100 & 27,400 & 38,200 & 47,500 & 57,700 \\
\hline 3131 & 31.2 & 3,430 & 6,990 & 10,100 & 14,700 & 18,600 & 22,900 \\
\hline 3136 & 22.7 & 2,550 & 5,380 & 7,880 & 11,600 & 14,900 & 18,400 \\
\hline 3137 & 27.0 & 4,350 & 8,120 & 11,200 & 15,700 & 19,300 & 23,200 \\
\hline 3143 & 159 & 8,810 & 16,300 & 22,400 & 31,500 & 39,100 & 47,400 \\
\hline 3144 & 120 & 7,150 & 13,600 & 19,100 & 27,200 & 34,100 & 41,700 \\
\hline 3145 & 235 & 11,300 & 20,100 & 27,100 & 37,200 & 45,700 & 54,900 \\
\hline 3150 & 17.6 & 2,320 & 4,930 & 7,180 & 10,600 & 13,500 & 16,700 \\
\hline 3159 & 94.3 & 6,170 & 12,000 & 17,000 & 24,400 & 30,700 & 37,700 \\
\hline 3167 & 23.2 & 3,080 & 6,450 & 9,430 & 13,900 & 17,800 & 22,000 \\
\hline 3179 & 82.8 & 6,700 & 12,800 & 18,100 & 25,700 & 32,300 & 39,400 \\
\hline 3232 & 66.4 & 4,960 & 9,820 & 14,000 & 20,200 & 25,600 & 31,400 \\
\hline 3244 & 55.9 & 4,500 & 9,130 & 13,200 & 19,300 & 24,500 & 30,300 \\
\hline 3257 & 39.8 & 5,580 & 10,300 & 14,200 & 19,800 & 24,400 & 29,400 \\
\hline 3272 & 23.6 & 3,330 & 6,680 & 9,550 & 13,800 & 17,400 & 21,200 \\
\hline 3285 & 40.9 & 3,870 & 7,770 & 11,100 & 16,100 & 20,400 & 25,000 \\
\hline 3293 & NA & NA & NA & NA & NA & NA & NA \\
\hline 3309 & 67.8 & 7,170 & 13,400 & 18,700 & 26,300 & 32,700 & 39,600 \\
\hline 3310 & 43.0 & 6,660 & 12,300 & 17,100 & 23,900 & 29,500 & 35,600 \\
\hline 3312 & 9.89 & 1,560 & 3,210 & 4,610 & 6,710 & 8,430 & 10,400 \\
\hline 3333 & NA & NA & NA & NA & NA & NA & NA \\
\hline 3337 & 17.9 & 2,370 & 4,840 & 6,930 & 10,100 & 12,700 & 15,600 \\
\hline 3344 & 19.9 & 2,740 & 5,680 & 8,190 & 12,000 & 15,100 & 18,700 \\
\hline 3345 & 45.6 & 5,730 & 10,800 & 15,000 & 21,200 & 26,400 & 32,000 \\
\hline 3357 & 9.31 & 1,610 & 3,280 & 4,690 & 6,800 & 8,540 & 10,500 \\
\hline 3368 & 18.2 & 2,570 & 5,250 & 7,510 & 10,900 & 13,700 & 16,900 \\
\hline 3381 & NA & NA & NA & NA & NA & NA & NA \\
\hline 3405 & 10.7 & 1,900 & 3,830 & 5,460 & 7,880 & 9,870 & 12,100 \\
\hline 3406 & 31.1 & 3,080 & 6,570 & 9,700 & 14,500 & 18,600 & 23,200 \\
\hline 3407 & 6.75 & 1,430 & 2,880 & 4,100 & 5,910 & 7,400 & 9,080 \\
\hline 3432 & 26.8 & 4,710 & 8,910 & 12,400 & 17,400 & 21,600 & 26,100 \\
\hline 3433 & 5.80 & 1,260 & 2,480 & 3,500 & 5,000 & 6,220 & 7,600 \\
\hline 3491 & 14.8 & 2,310 & 4,670 & 6,660 & 9,640 & 12,100 & 14,900 \\
\hline 3494 & NA & NA & NA & NA & NA & NA & NA \\
\hline
\end{tabular}




\section{Estimates of Flow Duration, Mean Flow, and Peak-Discharge Frequency Values for Kansas Stream Locations}

Table 8. Estimated flow-duration values, mean flow values, and peak-discharge frequency values for controlled and uncontrolled flow stream segments on the 1999 Kansas Surface Water Register for Anderson County.-Continued

[KSWR, Kansas Surface Water Register; CUSEGA, catalog unit segment number alpha; $\mathrm{mi}^{2}$, square miles; $\mathrm{ft}^{3} / \mathrm{s}$, cubic feet per second; HYDRO, lake or other hydrologic structure; NA, not applicable; NRDitch, irrigation ditch; NRTribal, tribal stream]

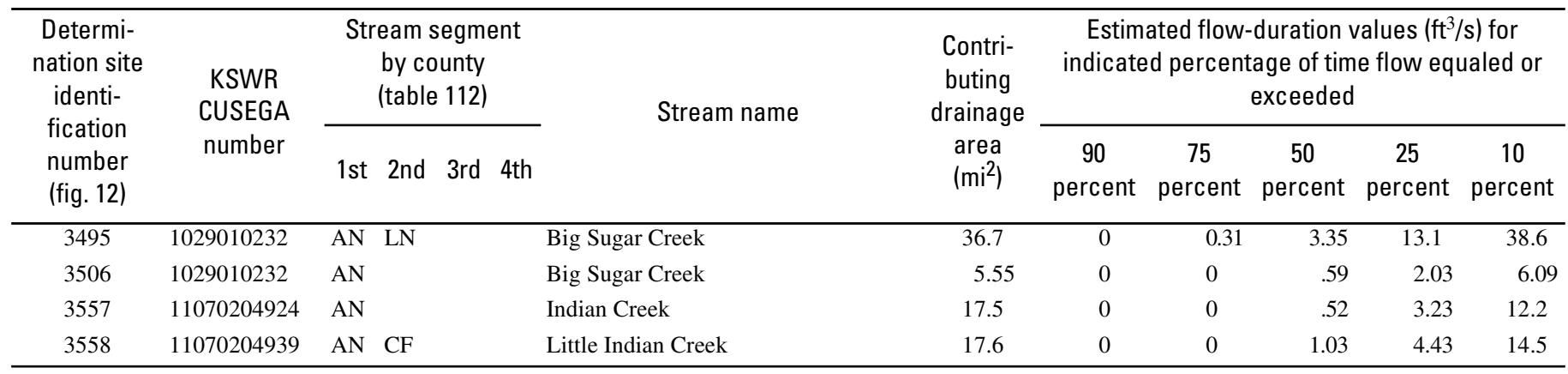


Table 8. Estimated flow-duration values, mean flow values, and peak-discharge frequency values for controlled and uncontrolled flow stream segments on the 1999 Kansas Surface Water Register for Anderson County.-Continued

[KSWR, Kansas Surface Water Register; CUSEGA, catalog unit segment number alpha; $\mathrm{mi}^{2}$, square miles; $\mathrm{ft}^{3} / \mathrm{s}$, cubic feet per second; HYDRO, lake or other hydrologic structure; NA, not applicable; NRDitch, irrigation ditch; NRTribal, tribal stream]

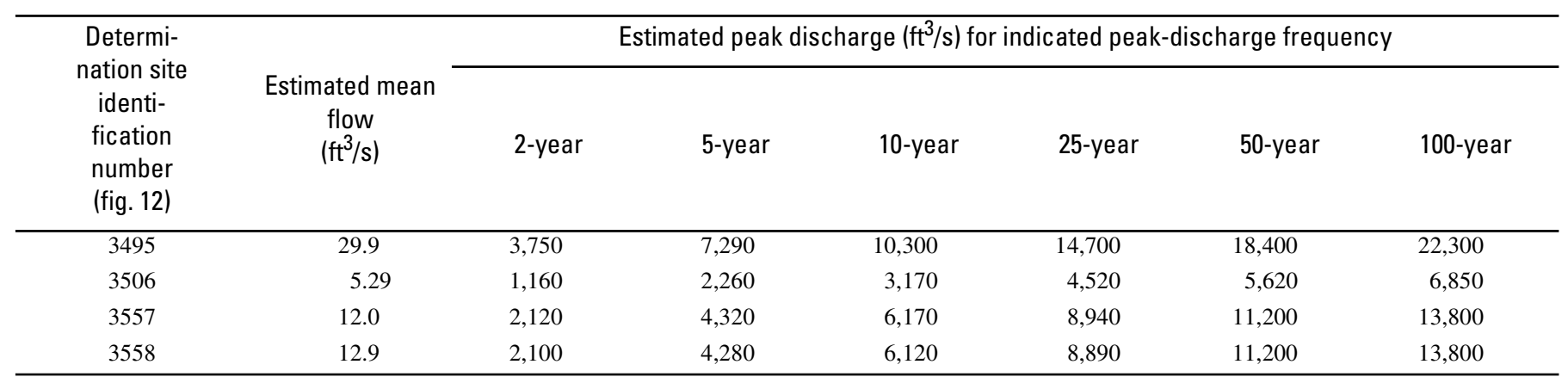




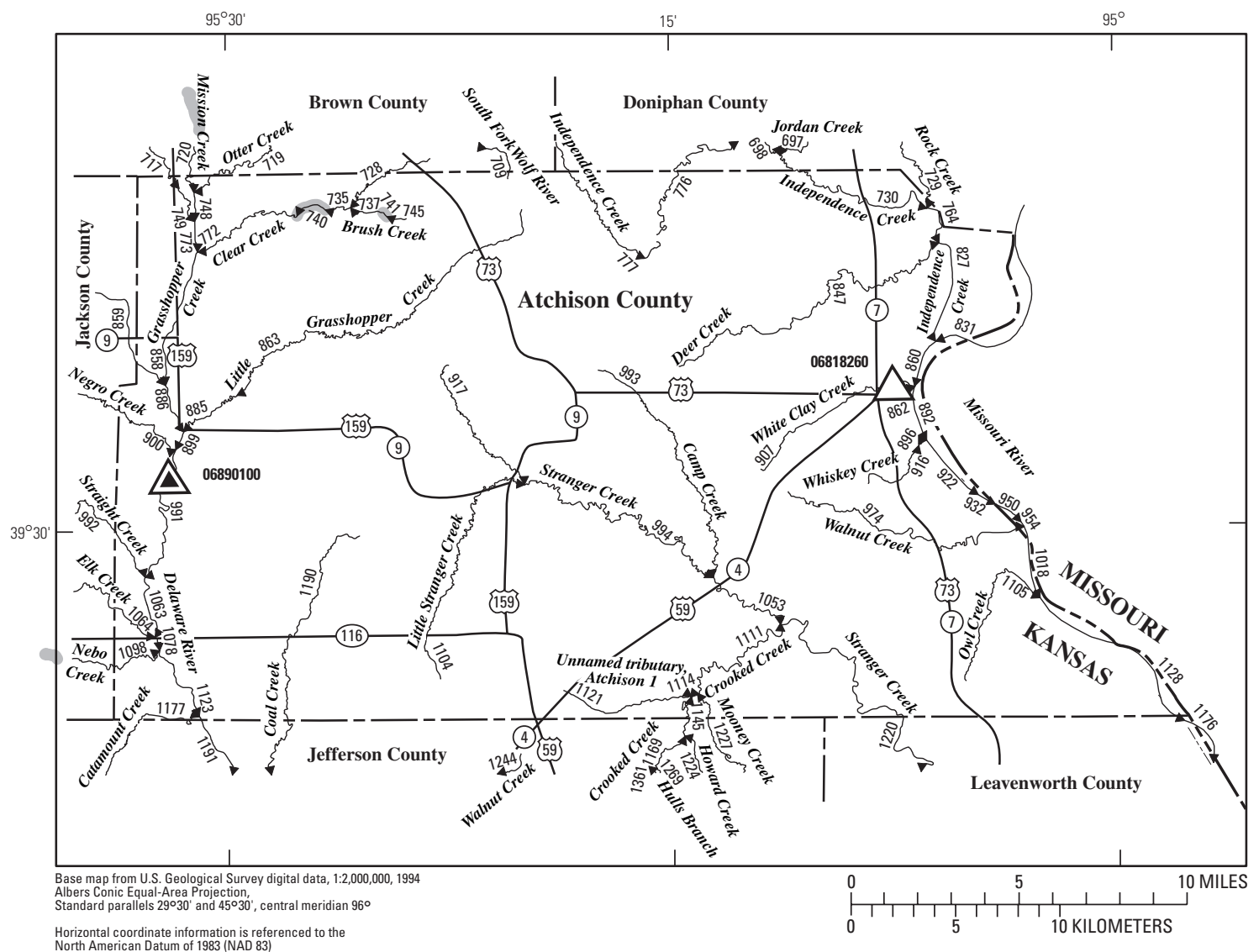

EXPLANATION

1244 Location of streamflow-statistics determination site (small triangle) and associated identification number-small triangle points in downstream direction

${ }^{06890100}$ U.S. Geological Survey streamflow-gaging station and number used for estimates of flow duration

${ }^{06818260} \triangle$ U.S. Geological Survey streamflow-gaging station and number used for estimates of peak-discharge frequency values

KANSAS

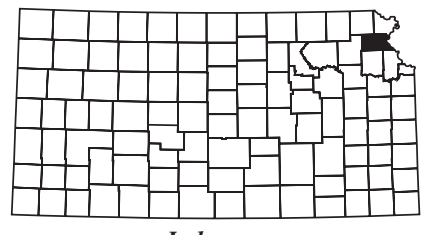

Index map

740 Lake and determination site identification number

Figure 13. Location of streamflow-statistics determination sites, associated identification numbers, and U.S. Geological Survey streamflow-gaging stations used in the flow-duration and peak-discharge frequency analyses for Atchison County. 
Table 9. Estimated flow-duration values, mean flow values, and peak-discharge frequency values for controlled and uncontrolled flow stream segments on the 1999 Kansas Surface Water Register for Atchison County.

[KSWR, Kansas Surface Water Register; CUSEGA, catalog unit segment number alpha; $\mathrm{mi}^{2}$, square miles; $\mathrm{ft}^{3} / \mathrm{s}$, cubic feet per second; HYDRO, lake or other hydrologic structure; NA, not applicable; NRDitch, irrigation ditch; NRTribal, tribal stream]

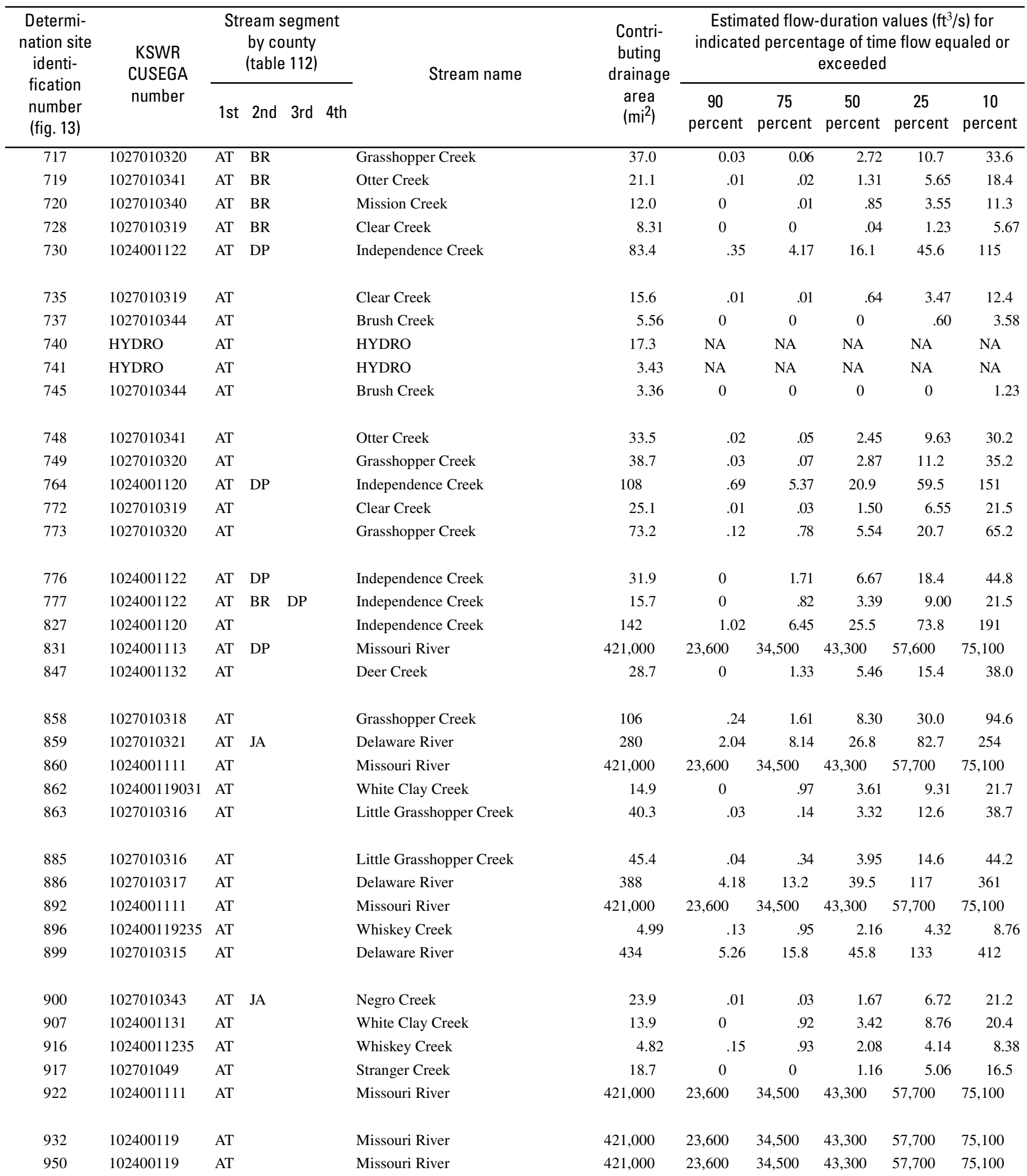


Table 9. Estimated flow-duration values, mean flow values, and peak-discharge frequency values for controlled and uncontrolled flow stream segments on the 1999 Kansas Surface Water Register for Atchison County.-Continued

[KSWR, Kansas Surface Water Register; CUSEGA, catalog unit segment number alpha; $\mathrm{mi}^{2}$, square miles; $\mathrm{ft}^{3} / \mathrm{s}$, cubic feet per second; HYDRO, lake or other hydrologic structure; NA, not applicable; NRDitch, irrigation ditch; NRTribal, tribal stream]

\begin{tabular}{|c|c|c|c|c|c|c|c|}
\hline \multirow{2}{*}{$\begin{array}{l}\text { Determi- } \\
\text { nation site } \\
\text { identi- } \\
\text { fication } \\
\text { number } \\
\text { (fig. 13) }\end{array}$} & \multirow{2}{*}{$\begin{array}{c}\text { Estimated mean } \\
\text { flow } \\
\left(\mathrm{ft}^{3} / \mathrm{s}\right)\end{array}$} & \multicolumn{6}{|c|}{ Estimated peak discharge $\left(\mathrm{ft}^{3} / \mathrm{s}\right)$ for indicated peak-discharge frequency } \\
\hline & & 2-year & 5 -year & 10-year & 25-year & 50-year & 100-year \\
\hline 717 & 26.0 & 3,190 & 6,830 & 10,100 & 15,000 & 19,300 & 24,000 \\
\hline 728 & 5.73 & 1,100 & 2,330 & 3,380 & 4,960 & 6,270 & 7,760 \\
\hline 730 & 68.1 & 4,650 & 8,960 & 12,600 & 17,900 & 22,400 & 27,200 \\
\hline 735 & 10.9 & 1,580 & 3,380 & 4,940 & 7,300 & 9,270 & 11,500 \\
\hline 737 & 3.91 & 867 & 1,820 & 2,630 & 3,850 & 4,860 & 6,000 \\
\hline 748 & 23.6 & 3,520 & 7,320 & 10,700 & 15,700 & 20,000 & 24,700 \\
\hline 749 & 27.2 & 3,350 & 7,110 & 10,500 & 15,500 & 20,000 & 24,800 \\
\hline 764 & 87.5 & 5,380 & 10,200 & 14,400 & 20,300 & 25,300 & 30,700 \\
\hline 772 & 17.6 & 2,080 & 4,490 & 6,590 & 9,800 & 12,500 & 15,500 \\
\hline 773 & 48.5 & 5,000 & 10,200 & 14,700 & 21,500 & 27,400 & 33,900 \\
\hline 776 & 28.0 & 3,090 & 6,080 & 8,610 & 12,300 & 15,400 & 18,600 \\
\hline 777 & 14.1 & 1,610 & 3,440 & 5,020 & 7,420 & 9,410 & 11,700 \\
\hline 827 & 111 & 6,150 & 11,700 & 16,300 & 23,100 & 28,800 & 34,900 \\
\hline 831 & 48,100 & 109,000 & 147,000 & 174,000 & 199,000 & 233,000 & 261,000 \\
\hline 885 & 32.5 & 3,280 & 7,020 & 10,400 & 15,500 & 20,000 & 24,900 \\
\hline 886 & 229 & 11,400 & 18,400 & 23,800 & 31,300 & 37,400 & 44,000 \\
\hline 892 & 48,200 & 109,000 & 147,000 & 174,000 & 200,000 & 234,000 & 262,000 \\
\hline 896 & 5.38 & 852 & 1,760 & 2,540 & 3,690 & 4,650 & 5,720 \\
\hline 899 & 258 & 12,300 & 19,000 & 24,000 & 30,700 & 36,000 & 41,700 \\
\hline 900 & 17.0 & 2,040 & 4,390 & 6,430 & 9,540 & 12,100 & 15,100 \\
\hline 907 & 13.1 & 1,100 & 2,200 & 3,150 & 4,650 & 5,990 & 7,520 \\
\hline 916 & 5.17 & 835 & 1,730 & 2,480 & 3,620 & 4,550 & 5,600 \\
\hline 917 & 13.4 & 1,710 & 3,700 & 5,430 & 8,070 & 10,300 & 12,800 \\
\hline 922 & 48,200 & 109,000 & 147,000 & 174,000 & 200,000 & 234,000 & 262,000 \\
\hline 932 & 48,200 & 109,000 & 147,000 & 174,000 & 200,000 & 234,000 & 262,000 \\
\hline 950 & 48,200 & 109,000 & 147,000 & 174,000 & 200,000 & 234,000 & 262,000 \\
\hline
\end{tabular}


Table 9. Estimated flow-duration values, mean flow values, and peak-discharge frequency values for controlled and uncontrolled flow stream segments on the 1999 Kansas Surface Water Register for Atchison County.-Continued

[KSWR, Kansas Surface Water Register; CUSEGA, catalog unit segment number alpha; $\mathrm{mi}^{2}$, square miles; $\mathrm{ft}^{3} / \mathrm{s}$, cubic feet per second; HYDRO, lake or other hydrologic structure; NA, not applicable; NRDitch, irrigation ditch; NRTribal, tribal stream]

\begin{tabular}{|c|c|c|c|c|c|c|c|c|c|c|c|}
\hline $\begin{array}{l}\text { Determi- } \\
\text { nation site } \\
\text { identi- } \\
\text { fication } \\
\text { number } \\
\text { (fig. 13) }\end{array}$ & $\begin{array}{l}\text { KSWR } \\
\text { CUSEGA } \\
\text { number }\end{array}$ & \multicolumn{3}{|c|}{$\begin{array}{l}\text { Stream segment } \\
\text { by county } \\
\text { (table 112) }\end{array}$} & Stream name & $\begin{array}{l}\text { Contri- } \\
\text { buting } \\
\text { drainage } \\
\text { area } \\
\left(\mathrm{mi}^{2}\right)\end{array}$ & \multicolumn{5}{|c|}{$\begin{array}{c}\text { Estimated flow-duration values }\left(\mathrm{ft}^{3} / \mathrm{s}\right) \text { for } \\
\text { indicated percentage of time flow equaled or } \\
\text { exceeded }\end{array}$} \\
\hline 954 & 102400117 & AT & & & Missouri River & 421,000 & 23,600 & 34,500 & 43,300 & 57,700 & 75,100 \\
\hline 992 & 1027010328 & AT & $\mathrm{JA}$ & & Straight Creek & 113 & .02 & 1.99 & 10.3 & 35.6 & 106 \\
\hline 993 & 1027010441 & AT & & & Camp Creek & 24.8 & 0 & .33 & 2.96 & 9.58 & 26.4 \\
\hline 994 & 102701049 & AT & & & Stranger Creek & 71.3 & .01 & .62 & 5.26 & 19.9 & 62.3 \\
\hline 1018 & 102400117 & AT & & & Missouri River & 421,000 & 23,600 & 34,500 & 43,400 & 57,700 & 75,200 \\
\hline 1078 & 1027010313 & AT & & & Delaware River & 722 & 6.02 & 17.8 & 57.8 & 167 & 523 \\
\hline 1098 & 1027010348 & AT & JA & & Nebo Creek & 15.4 & 0 & 0 & 1.12 & 4.58 & 14.4 \\
\hline 1104 & 10270104959 & AT & & & Little Stranger Creek & 30.6 & 0 & 0 & 1.89 & 7.93 & 25.6 \\
\hline 1105 & 1024001133 & AT & & & Owl Creek & 16.0 & 0 & 1.23 & 4.72 & 12.5 & 28.9 \\
\hline 1111 & 1027010410 & AT & & & Crooked Creek & 75.3 & .01 & .57 & 5.36 & 21.3 & 68.8 \\
\hline 1114 & 1027010410 & AT & & & Crooked Creek & 57.3 & .01 & .14 & 3.74 & 15.5 & 50.9 \\
\hline 1121 & 1027010411 & AT & & & Unnamed tributary, Atchison 1 & 17.5 & 0 & 0 & 1.14 & 5.02 & 16.3 \\
\hline 1123 & 1027010313 & AT & & & Delaware River & 742 & 6.01 & 17.8 & 58.3 & 169 & 529 \\
\hline 1128 & 102400115 & AT & & & Missouri River & 421,000 & 23,600 & 34,500 & 43,400 & 57,700 & 75,200 \\
\hline
\end{tabular}


Table 9. Estimated flow-duration values, mean flow values, and peak-discharge frequency values for controlled and uncontrolled flow stream segments on the 1999 Kansas Surface Water Register for Atchison County.-Continued

[KSWR, Kansas Surface Water Register; CUSEGA, catalog unit segment number alpha; $\mathrm{mi}^{2}$, square miles; $\mathrm{ft}^{3} / \mathrm{s}$, cubic feet per second; HYDRO, lake or other hydrologic structure; NA, not applicable; NRDitch, irrigation ditch; NRTribal, tribal stream]

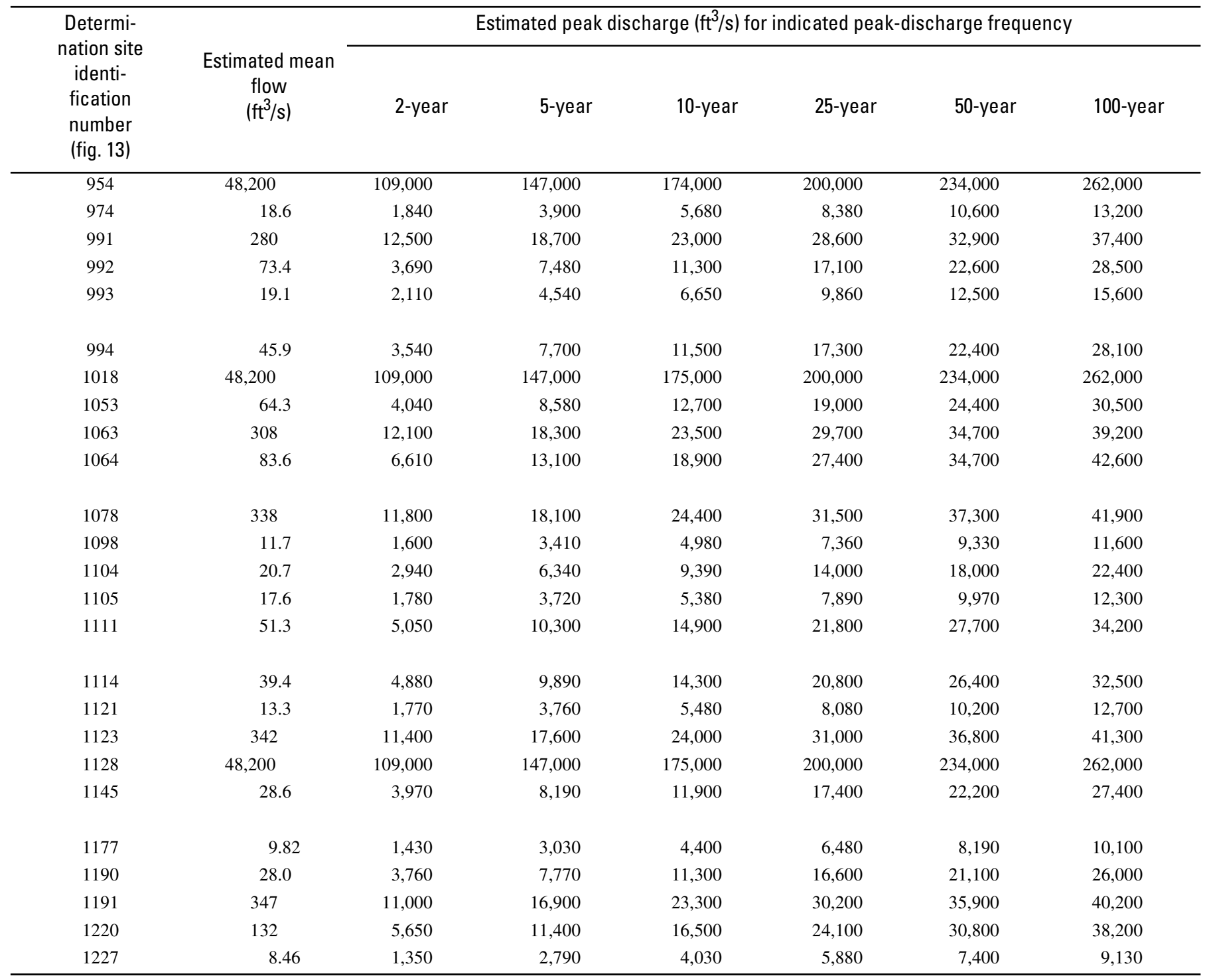




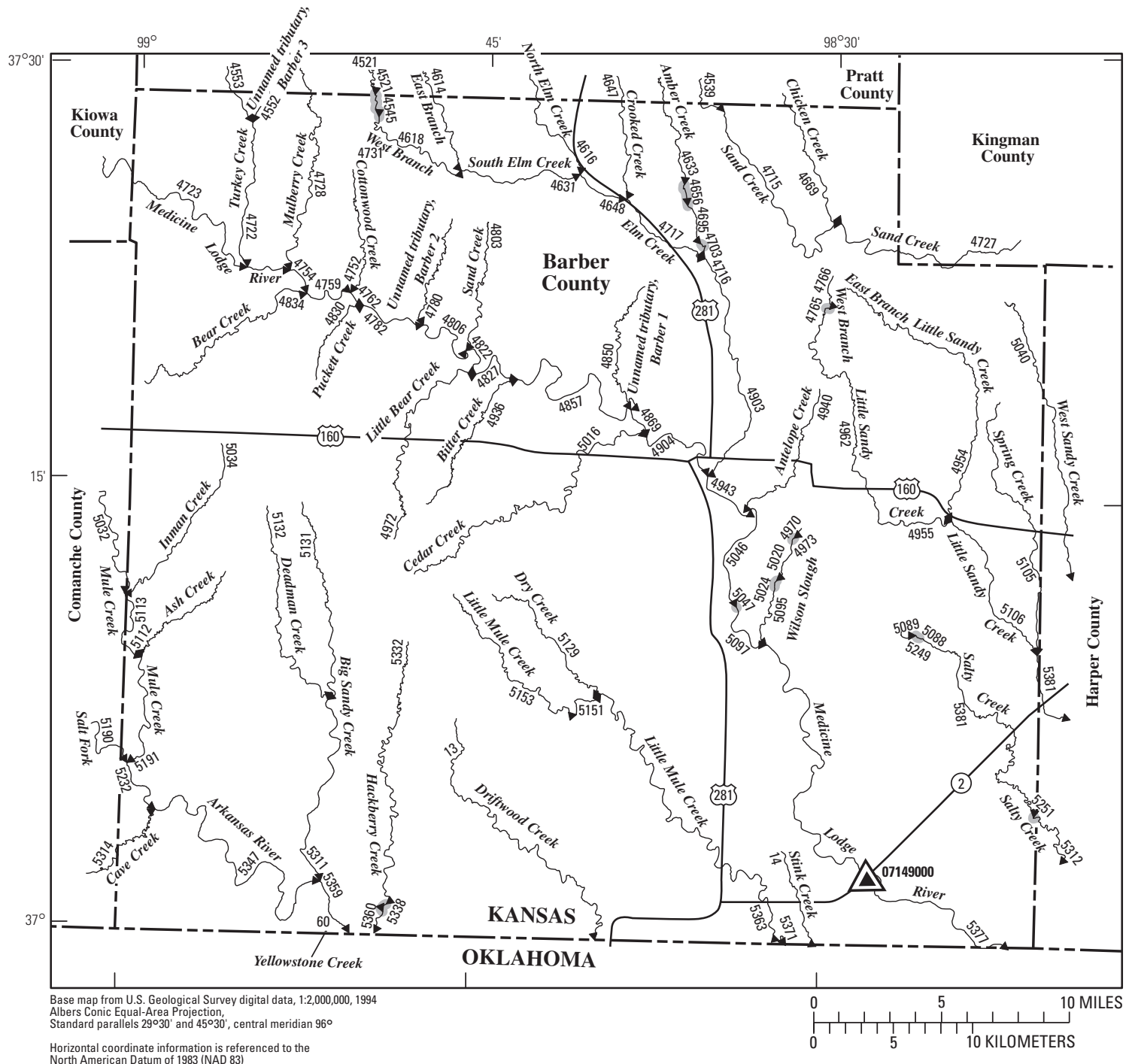

\section{EXPLANATION}

4347 Location of streamflow-statistics determination site (small triangle) and associated identification number-small triangle points in downstream direction

07149000

U.S. Geological Survey streamflow-gaging station and number used for estimates of flow duration

07149000 U.S. Geological Survey streamflow-gaging station and number used for estimates of peak-discharge frequency values

KANSAS

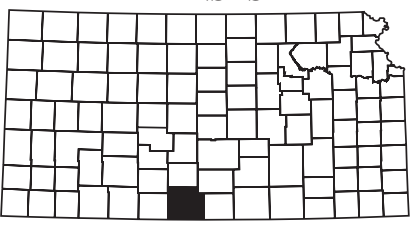

Index map 5338

Lake and determination site identification number

Figure 14. Location of streamflow-statistics determination sites, associated identification numbers, and U.S. Geological Survey streamflow-gaging stations used in the flow-duration and peak-discharge frequency analyses for Barber County. 


\section{Estimates of Flow Duration, Mean Flow, and Peak-Discharge Frequency Values for Kansas Stream Locations}

Table 10. Estimated flow-duration values, mean flow values, and peak-discharge frequency values for controlled and uncontrolled flow stream segments on the 1999 Kansas Surface Water Register for Barber County.

[KSWR, Kansas Surface Water Register; CUSEGA, catalog unit segment number alpha; $\mathrm{mi}^{2}$, square miles; $\mathrm{ft}^{3} / \mathrm{s}$, cubic feet per second; HYDRO, lake or other hydrologic structure; NA, not applicable; NRDitch, irrigation ditch; NRTribal, tribal stream]

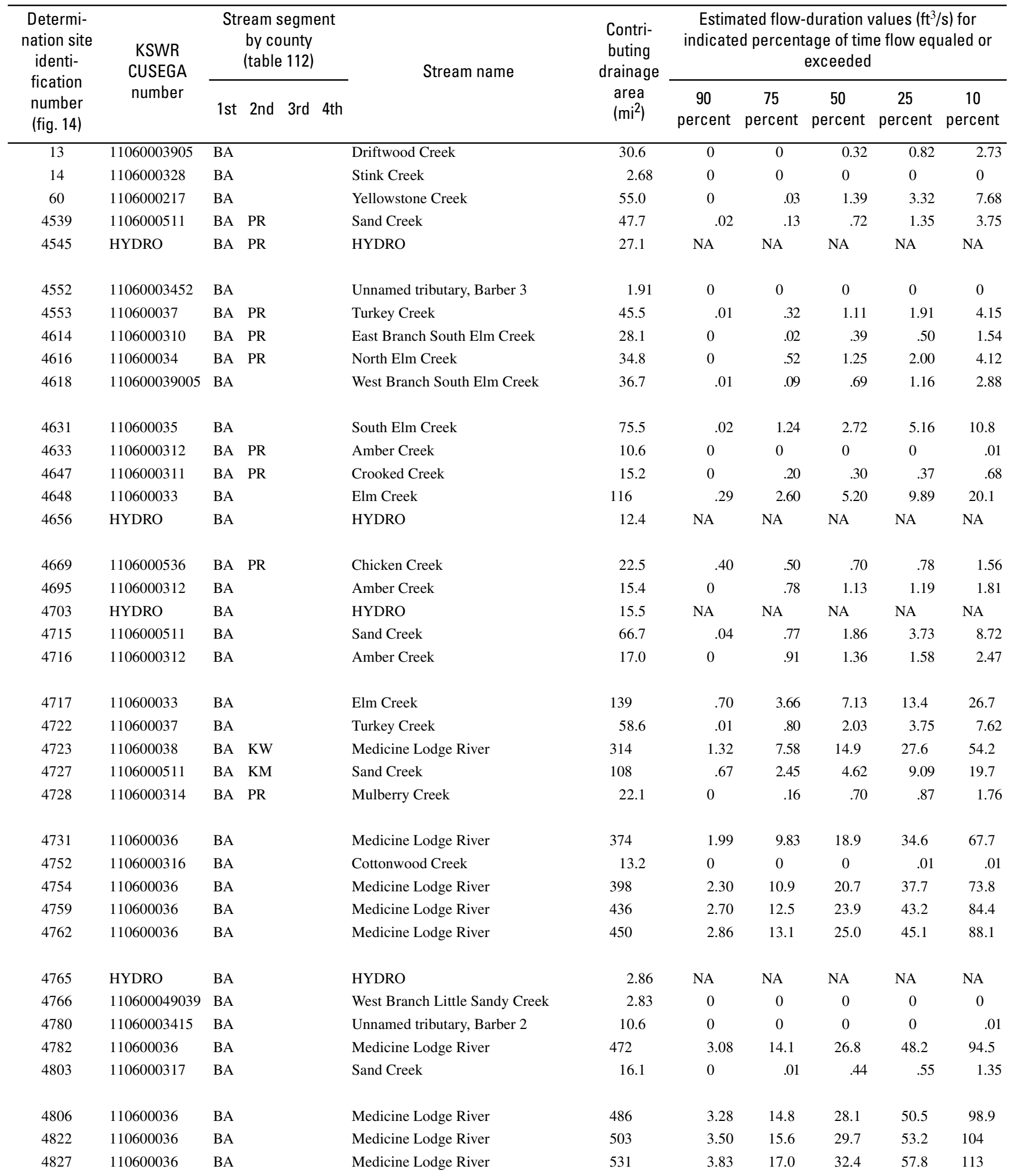


Table 10. Estimated flow-duration values, mean flow values, and peak-discharge frequency values for controlled and uncontrolled flow stream segments on the 1999 Kansas Surface Water Register for Barber County.-Continued

[KSWR, Kansas Surface Water Register; CUSEGA, catalog unit segment number alpha; $\mathrm{mi}^{2}$, square miles; $\mathrm{ft}^{3} / \mathrm{s}$, cubic feet per second; HYDRO, lake or other hydrologic structure; NA, not applicable; NRDitch, irrigation ditch; NRTribal, tribal stream]

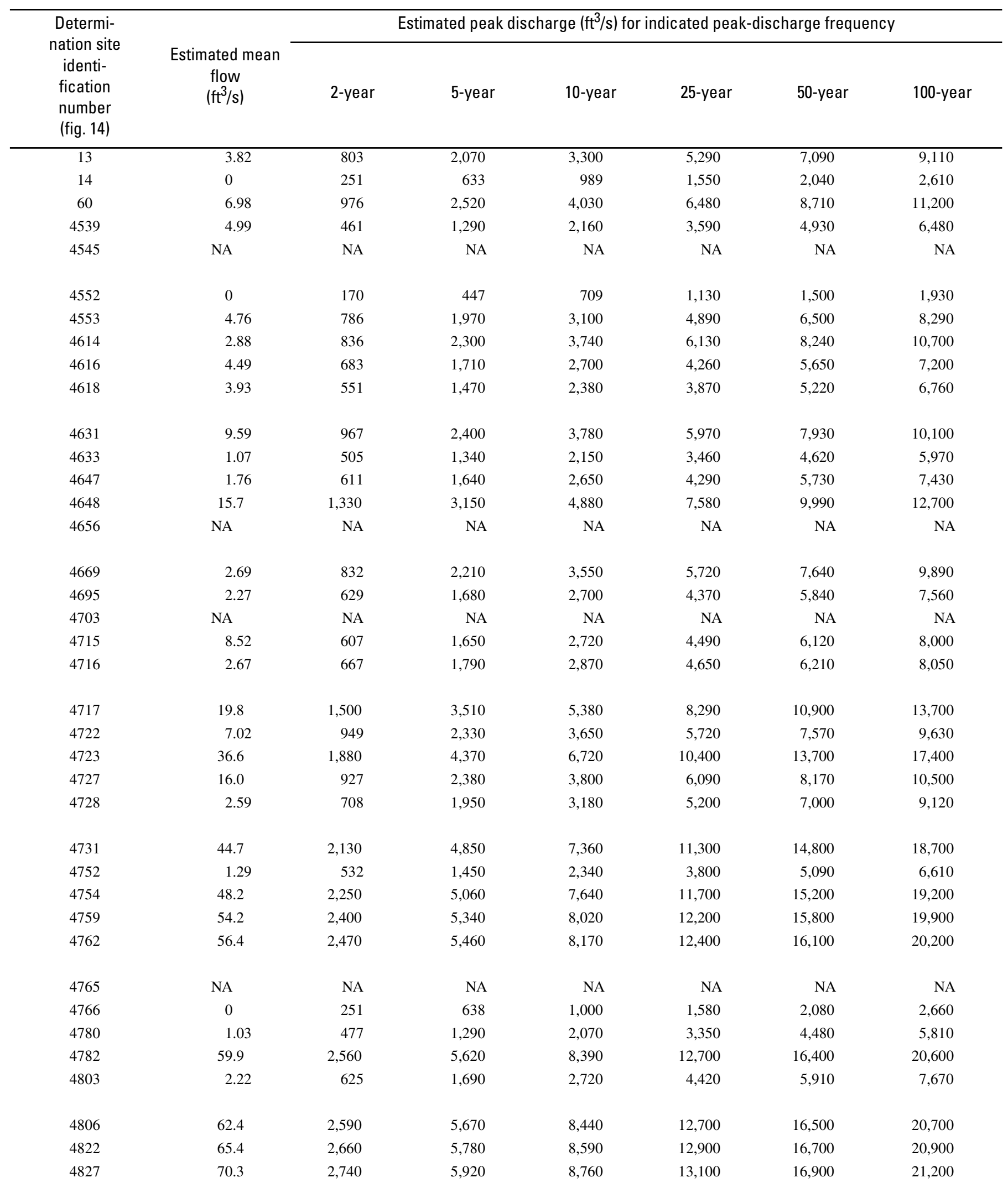




\section{Estimates of Flow Duration, Mean Flow, and Peak-Discharge Frequency Values for Kansas Stream Locations}

Table 10. Estimated flow-duration values, mean flow values, and peak-discharge frequency values for controlled and uncontrolled flow stream segments on the 1999 Kansas Surface Water Register for Barber County.-Continued

[KSWR, Kansas Surface Water Register; CUSEGA, catalog unit segment number alpha; $\mathrm{mi}^{2}$, square miles; $\mathrm{ft}^{3} / \mathrm{s}$, cubic feet per second; HYDRO, lake or other hydrologic structure; NA, not applicable; NRDitch, irrigation ditch; NRTribal, tribal stream]

\begin{tabular}{|c|c|c|c|c|c|c|c|c|c|c|c|}
\hline $\begin{array}{l}\text { Determi- } \\
\text { nation site } \\
\text { identi- } \\
\text { fication } \\
\text { number } \\
\text { (fig. 14) }\end{array}$ & $\begin{array}{l}\text { KSWR } \\
\text { CUSEGA } \\
\text { number }\end{array}$ & \multicolumn{3}{|c|}{$\begin{array}{l}\text { Stream segment } \\
\text { by county } \\
\text { (table 112) }\end{array}$} & Stream name & $\begin{array}{l}\text { Contri- } \\
\text { buting } \\
\text { drainage } \\
\text { area } \\
\left(\mathrm{mi}^{2}\right)\end{array}$ & \multicolumn{5}{|c|}{$\begin{array}{c}\text { Estimated flow-duration values }\left(\mathrm{ft}^{3} / \mathrm{s}\right) \text { for } \\
\text { indicated percentage of time flow equaled or } \\
\text { exceeded }\end{array}$} \\
\hline 4830 & 1106000315 & $\overline{\mathrm{BA}}$ & & & Puckett Creek & 16.0 & 0 & 0.01 & 0.01 & 0.01 & $\overline{0.52}$ \\
\hline 4857 & 110600036 & BA & & & Medicine Lodge River & 560 & 4.31 & 18.7 & 35.6 & 63.2 & 123 \\
\hline 4869 & 110600036 & BA & & & Medicine Lodge River & 582 & 4.64 & 20.0 & 37.9 & 67.1 & 131 \\
\hline 4903 & 110600033 & BA & & & Elm Creek & 183 & 1.42 & 5.71 & 11.1 & 20.4 & 40.2 \\
\hline 4904 & 110600036 & BA & & & Medicine Lodge River & 632 & 5.34 & 22.9 & 43.3 & 76.3 & 149 \\
\hline 4954 & 1106000465 & BA & & & East Branch Little Sandy Creek & 31.0 & .21 & 1.61 & 2.73 & 4.35 & 7.92 \\
\hline 4955 & 1106000439 & BA & & & East Branch Little Sandy Creek & 31.0 & .21 & 1.61 & 2.73 & 4.35 & 7.92 \\
\hline 4962 & 110600049039 & BA & & & West Branch Little Sandy Creek & 41.5 & 0 & 1.23 & 2.42 & 4.23 & 8.41 \\
\hline 4970 & 1106000323 & BA & & & Wilson Slough & 3.82 & 0 & 0 & 0 & 0 & 0 \\
\hline 4972 & 1106000319 & BA & & & Little Bear Creek & 25.3 & 0 & .02 & .77 & 1.52 & 3.49 \\
\hline 4973 & HYDRO & BA & & & HYDRO & 4.19 & NA & NA & NA & NA & NA \\
\hline 5016 & 1106000320 & BA & & & Cedar Creek & 39.8 & .01 & .49 & 1.98 & 4.06 & 8.53 \\
\hline 5020 & 1106000323 & BA & & & Wilson Slough & 9.96 & 0 & 0 & 0 & 0 & 0 \\
\hline 5024 & HYDRO & BA & & & HYDRO & 10.5 & NA & NA & NA & NA & NA \\
\hline 5089 & 1106000440 & BA & & & Salty Creek & 12.9 & 0 & 0 & .53 & .76 & 1.85 \\
\hline 5095 & 1106000323 & BA & & & Wilson Slough & 15.7 & 0 & .01 & .10 & .17 & .94 \\
\hline 5097 & 110600032 & BA & & & Medicine Lodge River & 861 & 9.60 & 38.5 & 70.3 & 118 & 228 \\
\hline 5105 & 1106000466 & BA & HP & & Spring Creek & 18.2 & .21 & .59 & .83 & .93 & 2.00 \\
\hline 5106 & 1106000439 & BA & & & Little Sandy Creek & 86.3 & 1.08 & 3.61 & 6.63 & 11.9 & 23.0 \\
\hline 5112 & 1106000220 & BA & & & Ash Creek & 14.3 & 0 & 0 & .01 & .02 & .04 \\
\hline 5113 & 110600027 & BA & $\mathrm{CM}$ & & Mule Creek & 187 & .19 & 3.54 & 9.02 & 18.6 & 38.2 \\
\hline 5129 & 1106000321 & BA & & & Dry Creek & 23.4 & 0 & 0 & .56 & 1.23 & 3.25 \\
\hline 5131 & 110600025 & BA & & & Big Sandy Creek & 24.9 & 0 & .01 & .70 & 1.22 & 2.67 \\
\hline 5132 & 1106000222 & BA & & & Deadman Creek & 25.8 & 0 & .01 & .38 & .70 & 1.89 \\
\hline 5151 & 110600039 & BA & & & Little Mule Creek & 23.7 & 0 & .01 & .54 & 1.05 & 2.69 \\
\hline 5153 & 110600039 & BA & & & Little Mule Creek & 22.6 & 0 & 0 & .50 & .94 & 2.46 \\
\hline 5190 & 110600028 & BA & $\mathrm{CM}$ & & Salt Fork Arkansas River & 378 & 2.04 & 8.85 & 22.4 & 45.8 & 94.6 \\
\hline
\end{tabular}


Table 10. Estimated flow-duration values, mean flow values, and peak-discharge frequency values for controlled and uncontrolled flow stream segments on the 1999 Kansas Surface Water Register for Barber County.-Continued

[KSWR, Kansas Surface Water Register; CUSEGA, catalog unit segment number alpha; $\mathrm{mi}^{2}$, square miles; $\mathrm{ft}^{3} / \mathrm{s}$, cubic feet per second; HYDRO, lake or other hydrologic structure; NA, not applicable; NRDitch, irrigation ditch; NRTribal, tribal stream]

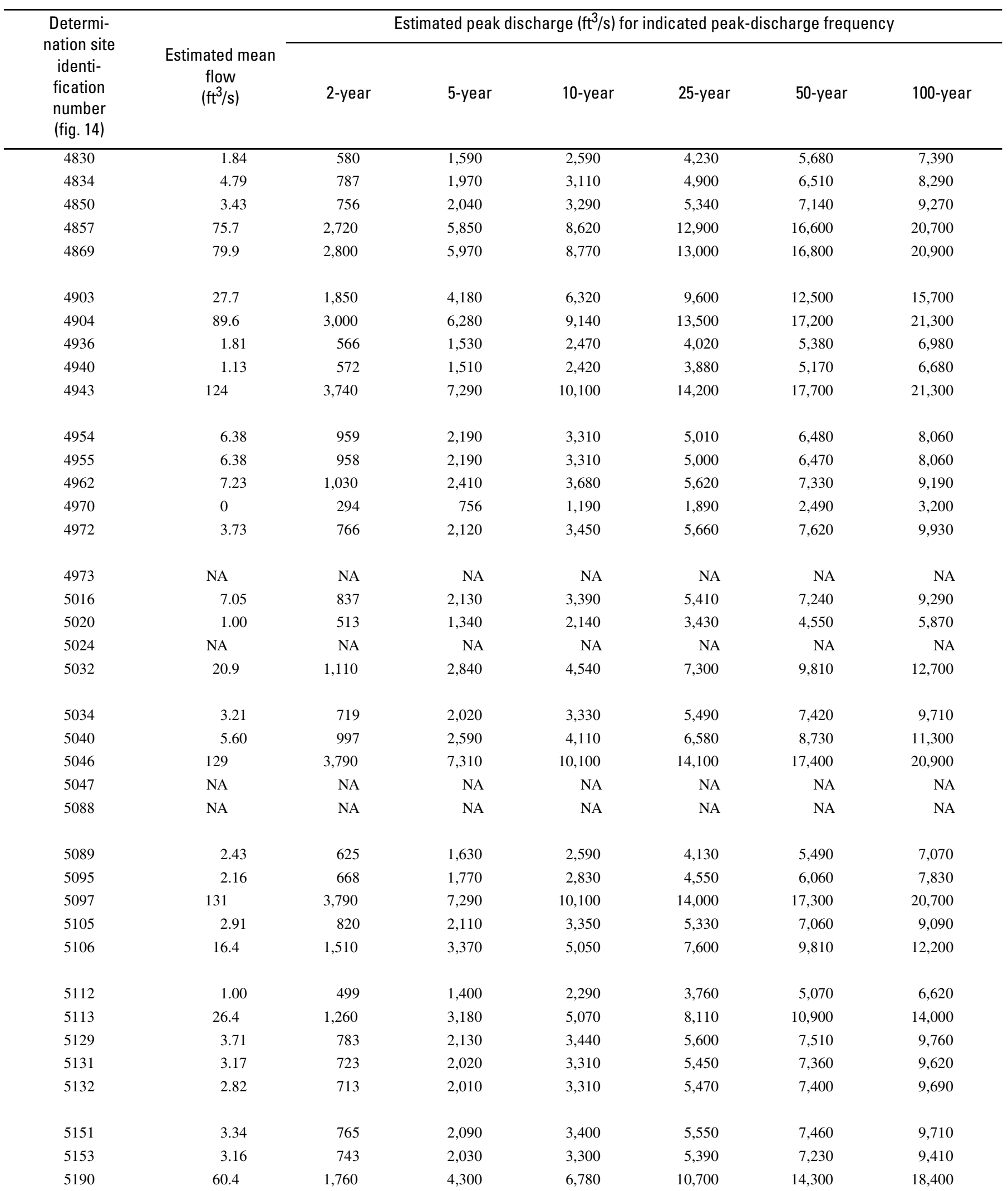




\section{Estimates of Flow Duration, Mean Flow, and Peak-Discharge Frequency Values for Kansas Stream Locations}

Table 10. Estimated flow-duration values, mean flow values, and peak-discharge frequency values for controlled and uncontrolled flow stream segments on the 1999 Kansas Surface Water Register for Barber County.-Continued

[KSWR, Kansas Surface Water Register; CUSEGA, catalog unit segment number alpha; $\mathrm{mi}^{2}$, square miles; $\mathrm{ft}^{3} / \mathrm{s}$, cubic feet per second; HYDRO, lake or other hydrologic structure; NA, not applicable; NRDitch, irrigation ditch; NRTribal, tribal stream]

\begin{tabular}{|c|c|c|c|c|c|c|c|c|c|c|c|}
\hline $\begin{array}{l}\text { Determi- } \\
\text { nation site } \\
\text { identi- } \\
\text { fication } \\
\text { number } \\
\text { (fig. 14) }\end{array}$ & $\begin{array}{l}\text { KSWR } \\
\text { CUSEGA } \\
\text { number }\end{array}$ & \multicolumn{3}{|c|}{$\begin{array}{l}\text { Stream segment } \\
\text { by county } \\
\text { (table 112) }\end{array}$} & Stream name & $\begin{array}{l}\text { Contri- } \\
\text { buting } \\
\text { drainage } \\
\text { area } \\
\left(\mathrm{mi}^{2}\right)\end{array}$ & \multicolumn{5}{|c|}{$\begin{array}{c}\text { Estimated flow-duration values }\left(\mathrm{ft}^{3} / \mathrm{s}\right) \text { for } \\
\text { indicated percentage of time flow equaled or } \\
\text { exceeded }\end{array}$} \\
\hline 5191 & 110600027 & $\mathrm{BA}$ & & & Mule Creek & 215 & 0.41 & 4.24 & 10.8 & 22.3 & 45.9 \\
\hline 5251 & HYDRO & BA & & & HYDRO & 52.7 & NA & NA & NA & NA & NA \\
\hline 5311 & 110600025 & BA & & & Big Sandy Creek & 72.6 & 0 & .71 & 2.69 & 5.79 & 12.3 \\
\hline 5312 & 1106000440 & BA & HP & & Salty Creek & 63.8 & 0 & .60 & 2.23 & 5.36 & 13.3 \\
\hline 5314 & 1106000228 & BA & $\mathrm{CM}$ & & Cave Creek & 24.4 & 0 & .01 & .09 & .22 & 1.11 \\
\hline 5359 & 110600024 & BA & & & Salt Fork Arkansas River & 729 & 4.87 & 20.2 & 57.8 & 120 & 254 \\
\hline 5360 & 1106000223 & BA & & & Hackberry Creek & 40.7 & 0 & .02 & .66 & 1.68 & 4.48 \\
\hline 5363 & 110600039 & BA & & & Little Mule Creek & 105 & 0 & 1.26 & 4.13 & 9.86 & 23.4 \\
\hline 5371 & 110600039 & BA & & & Little Mule Creek & 105 & 0 & 1.26 & 4.15 & 9.90 & 23.5 \\
\hline 5377 & 110600032 & BA & & & Medicine Lodge River & 965 & 12.0 & 47.0 & 85.0 & 141 & 271 \\
\hline 5381 & 1106000439 & BA & HP & & Little Sandy Creek & 141 & 3.21 & 7.25 & 12.4 & 21.3 & 40.3 \\
\hline
\end{tabular}


Table 10. Estimated flow-duration values, mean flow values, and peak-discharge frequency values for controlled and uncontrolled flow stream segments on the 1999 Kansas Surface Water Register for Barber County.-Continued

[KSWR, Kansas Surface Water Register; CUSEGA, catalog unit segment number alpha; $\mathrm{mi}^{2}$, square miles; $\mathrm{ft}^{3} / \mathrm{s}$, cubic feet per second; HYDRO, lake or other hydrologic structure; NA, not applicable; NRDitch, irrigation ditch; NRTribal, tribal stream]

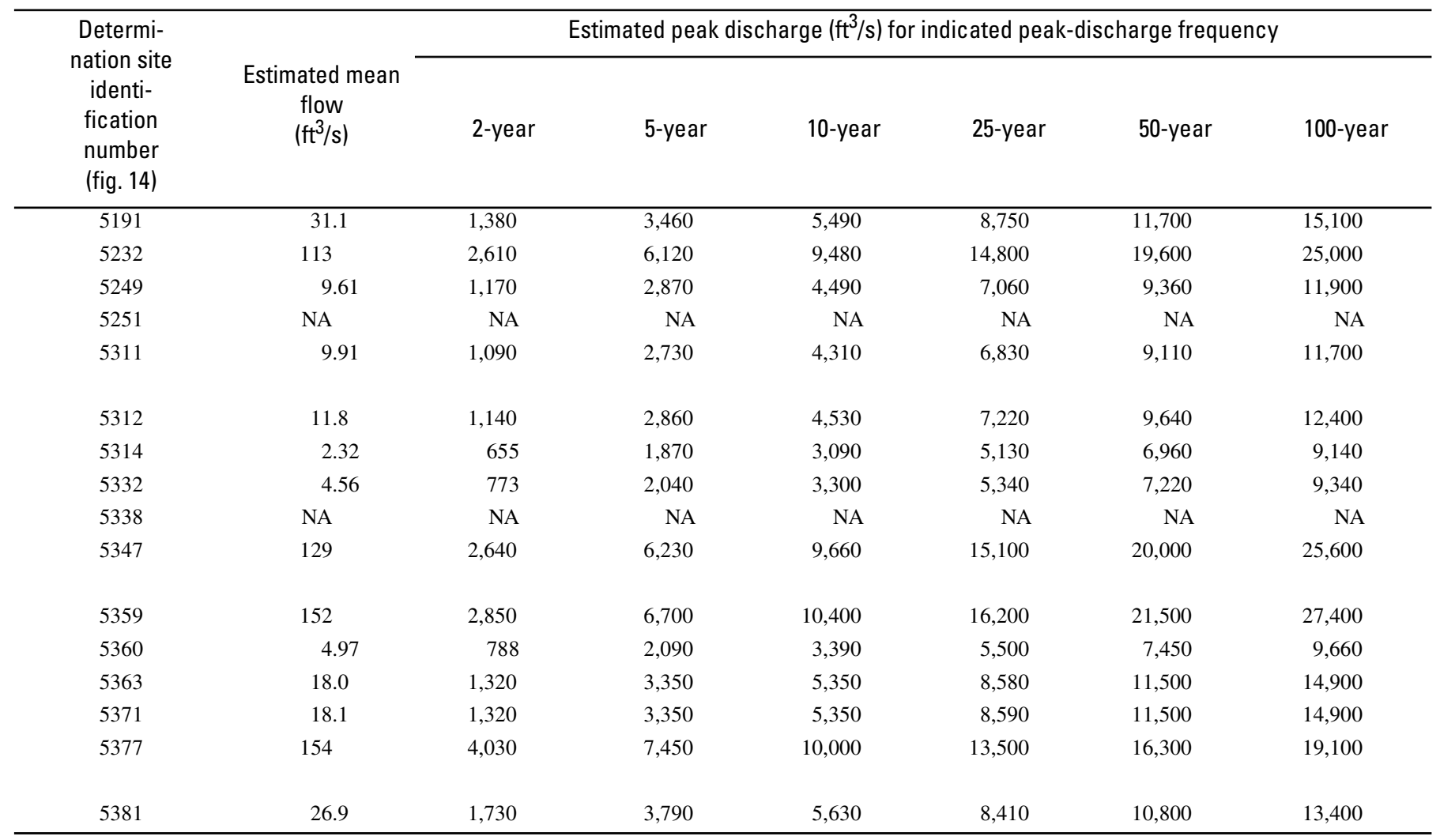




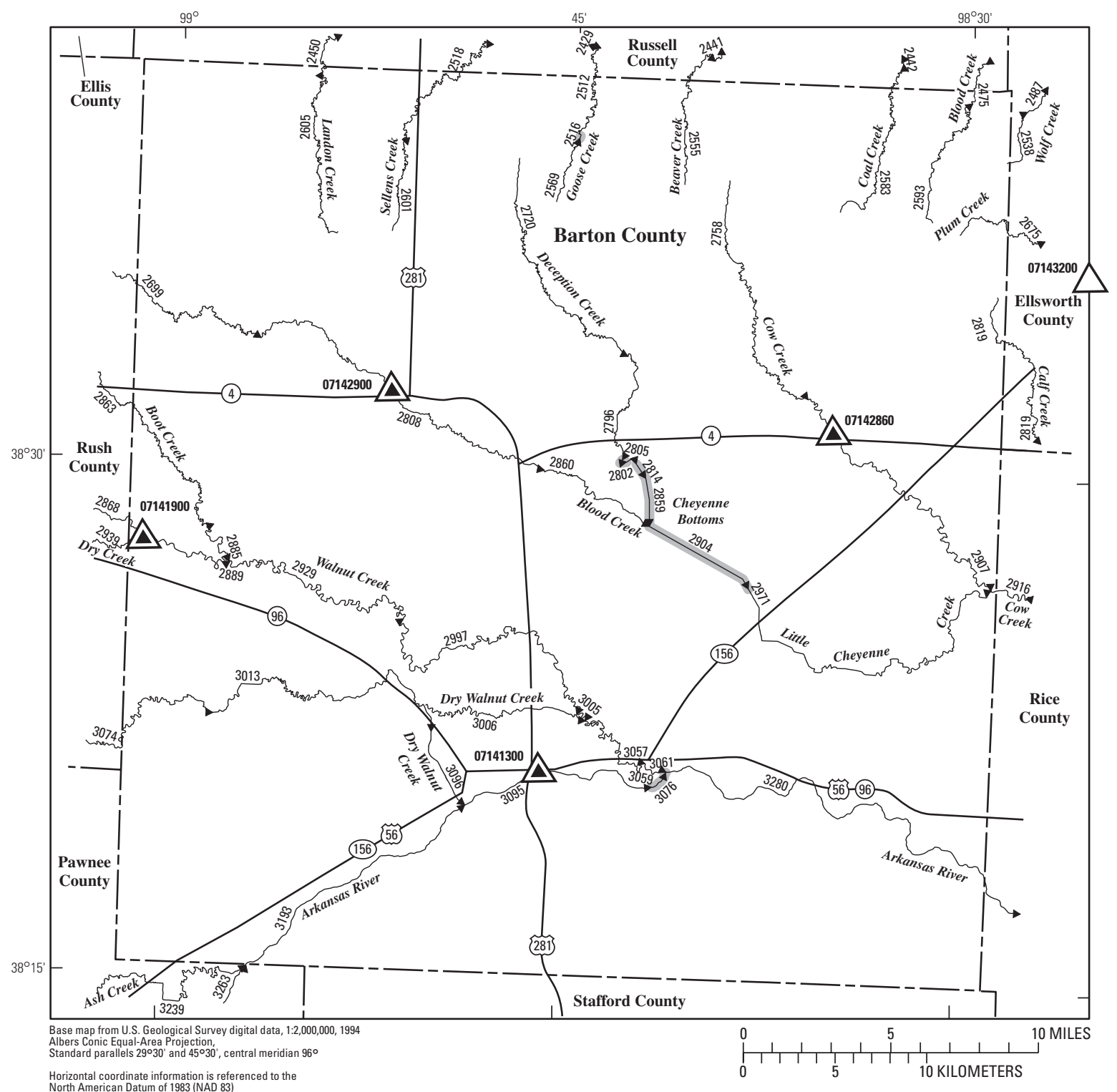

EXPLANATION

3193 Location of streamflow-statistics determination site (small triangle) and associated identification number-small triangle points in downstream direction

$07141300 \Delta$ U.S. Geological Survey streamflow-gaging station and number used for estimates of flow duration

07143200 人

U.S. Geological Survey streamflow-gaging station and number used for estimates of peak-discharge frequency values

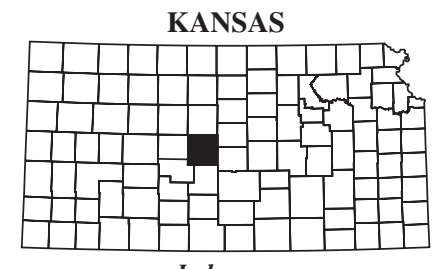

Index map

3076 Lake and determination site identification number

Figure 15. Location of streamflow-statistics determination sites, associated identification numbers, and U.S. Geological Survey streamflow-gaging stations used in the flow-duration and peak-discharge frequency analyses for Barton County. 


\section{Estimates of Flow Duration, Mean Flow, and Peak-Discharge Frequency Values for Kansas Stream Locations}

Table 11. Estimated flow-duration values, mean flow values, and peak-discharge frequency values for controlled and uncontrolled flow stream segments on the 1999 Kansas Surface Water Register for Barton County.

[KSWR, Kansas Surface Water Register; CUSEGA, catalog unit segment number alpha; $\mathrm{mi}^{2}$, square miles; $\mathrm{ft}^{3} / \mathrm{s}$, cubic feet per second; HYDRO, lake or other hydrologic structure; NA, not applicable; NRDitch, irrigation ditch; NRTribal, tribal stream]

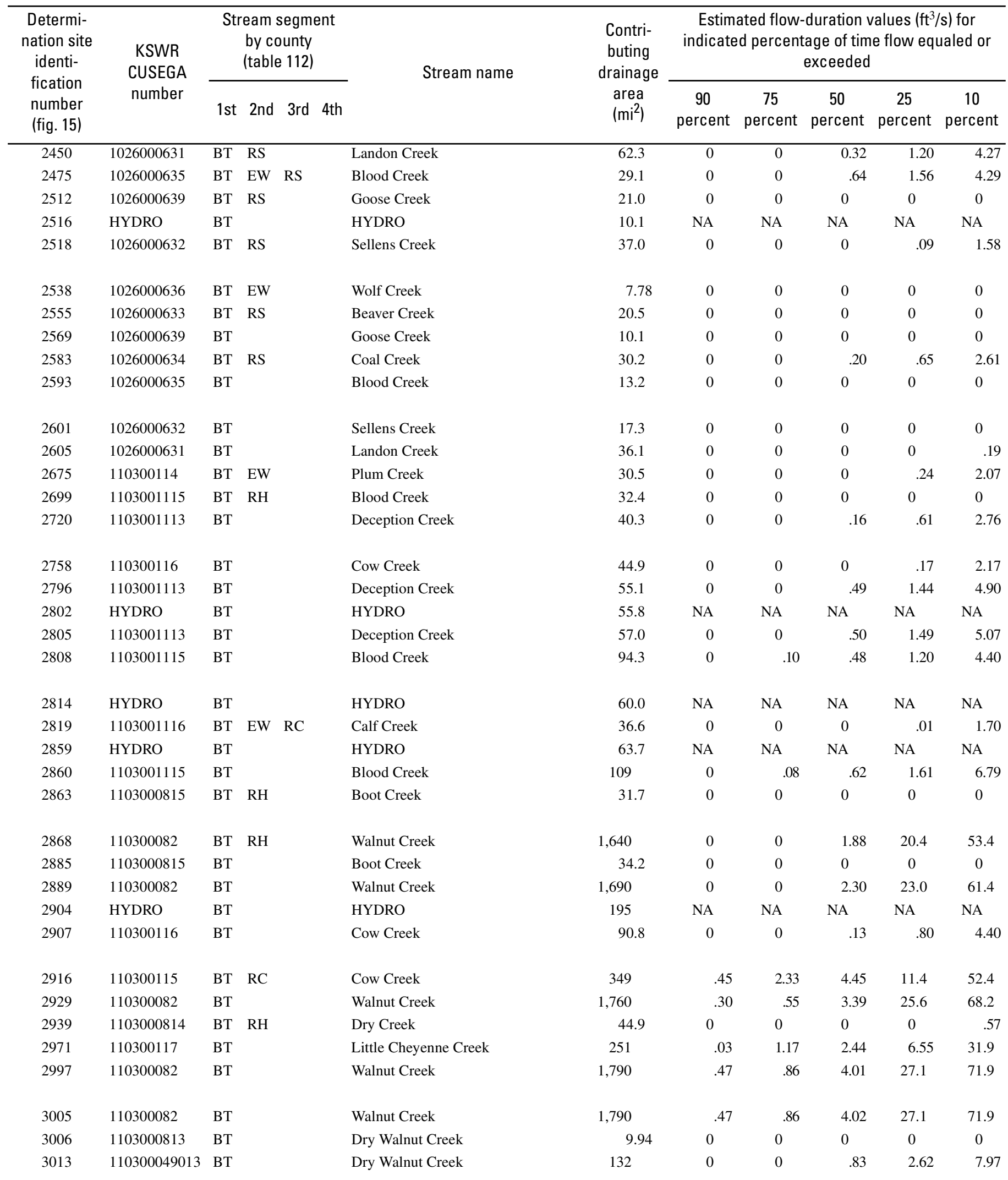


Table 11. Estimated flow-duration values, mean flow values, and peak-discharge frequency values for controlled and uncontrolled flow stream segments on the 1999 Kansas Surface Water Register for Barton County.-Continued

[KSWR, Kansas Surface Water Register; CUSEGA, catalog unit segment number alpha; $\mathrm{mi}^{2}$, square miles; $\mathrm{ft}^{3} / \mathrm{s}$, cubic feet per second; HYDRO, lake or other hydrologic structure; NA, not applicable; NRDitch, irrigation ditch; NRTribal, tribal stream]

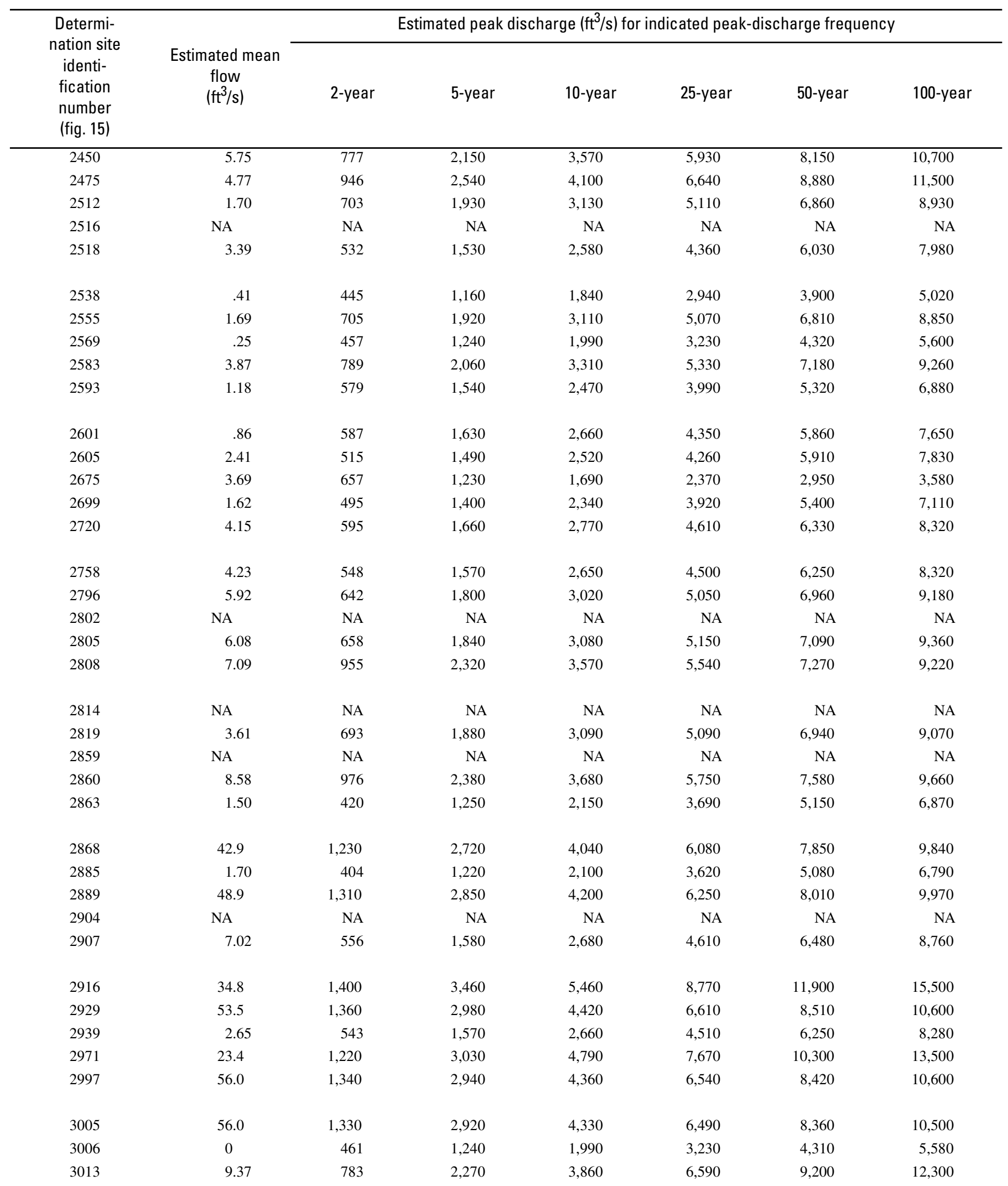




\section{Estimates of Flow Duration, Mean Flow, and Peak-Discharge Frequency Values for Kansas Stream Locations}

Table 11. Estimated flow-duration values, mean flow values, and peak-discharge frequency values for controlled and uncontrolled flow stream segments on the 1999 Kansas Surface Water Register for Barton County.-Continued

[KSWR, Kansas Surface Water Register; CUSEGA, catalog unit segment number alpha; $\mathrm{mi}^{2}$, square miles; $\mathrm{ft}^{3} / \mathrm{s}$, cubic feet per second; HYDRO, lake or other hydrologic structure; NA, not applicable; NRDitch, irrigation ditch; NRTribal, tribal stream]

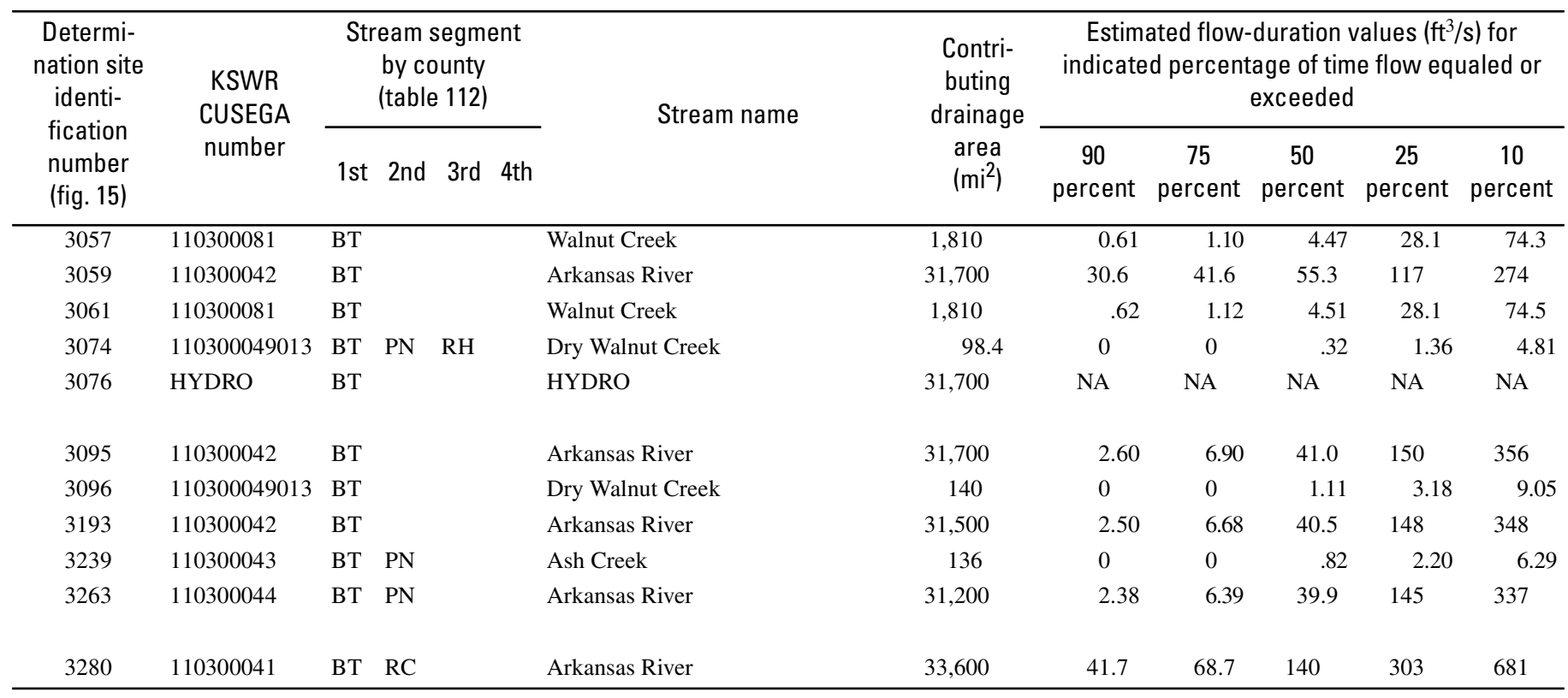


Table 11. Estimated flow-duration values, mean flow values, and peak-discharge frequency values for controlled and uncontrolled flow stream segments on the 1999 Kansas Surface Water Register for Barton County.-Continued

[KSWR, Kansas Surface Water Register; CUSEGA, catalog unit segment number alpha; $\mathrm{mi}^{2}$, square miles; $\mathrm{ft}^{3} / \mathrm{s}$, cubic feet per second; HYDRO, lake or other hydrologic structure; NA, not applicable; NRDitch, irrigation ditch; NRTribal, tribal stream]

\begin{tabular}{|c|c|c|c|c|c|c|c|}
\hline \multirow{2}{*}{$\begin{array}{l}\text { Determi- } \\
\text { nation site } \\
\text { identi- } \\
\text { fication } \\
\text { number } \\
\text { (fig. 15) }\end{array}$} & \multirow{2}{*}{$\begin{array}{c}\text { Estimated mean } \\
\text { flow } \\
\left(\mathrm{ft}^{3} / \mathrm{s}\right)\end{array}$} & \multicolumn{6}{|c|}{ Estimated peak discharge $\left(\mathrm{ft}^{3} / \mathrm{s}\right)$ for indicated peak-discharge frequency } \\
\hline & & 2-year & 5-year & 10 -year & 25-year & 50-year & 100 -year \\
\hline 3057 & 57.6 & 1,330 & 2,920 & 4,320 & 6,470 & 8,340 & 10,400 \\
\hline 3074 & 6.57 & 721 & 2,090 & 3,550 & 6,060 & 8,440 & 11,300 \\
\hline 3076 & NA & NA & NA & NA & NA & NA & NA \\
\hline 3095 & 172 & 2,960 & 7,570 & 15,000 & 22,500 & 29,500 & 36,900 \\
\hline 3096 & 10.1 & 774 & 2,230 & 3,790 & 6,450 & 8,990 & 12,000 \\
\hline 3280 & 328 & 3,550 & 8,060 & 15,200 & 22,300 & 29,100 & 36,300 \\
\hline
\end{tabular}




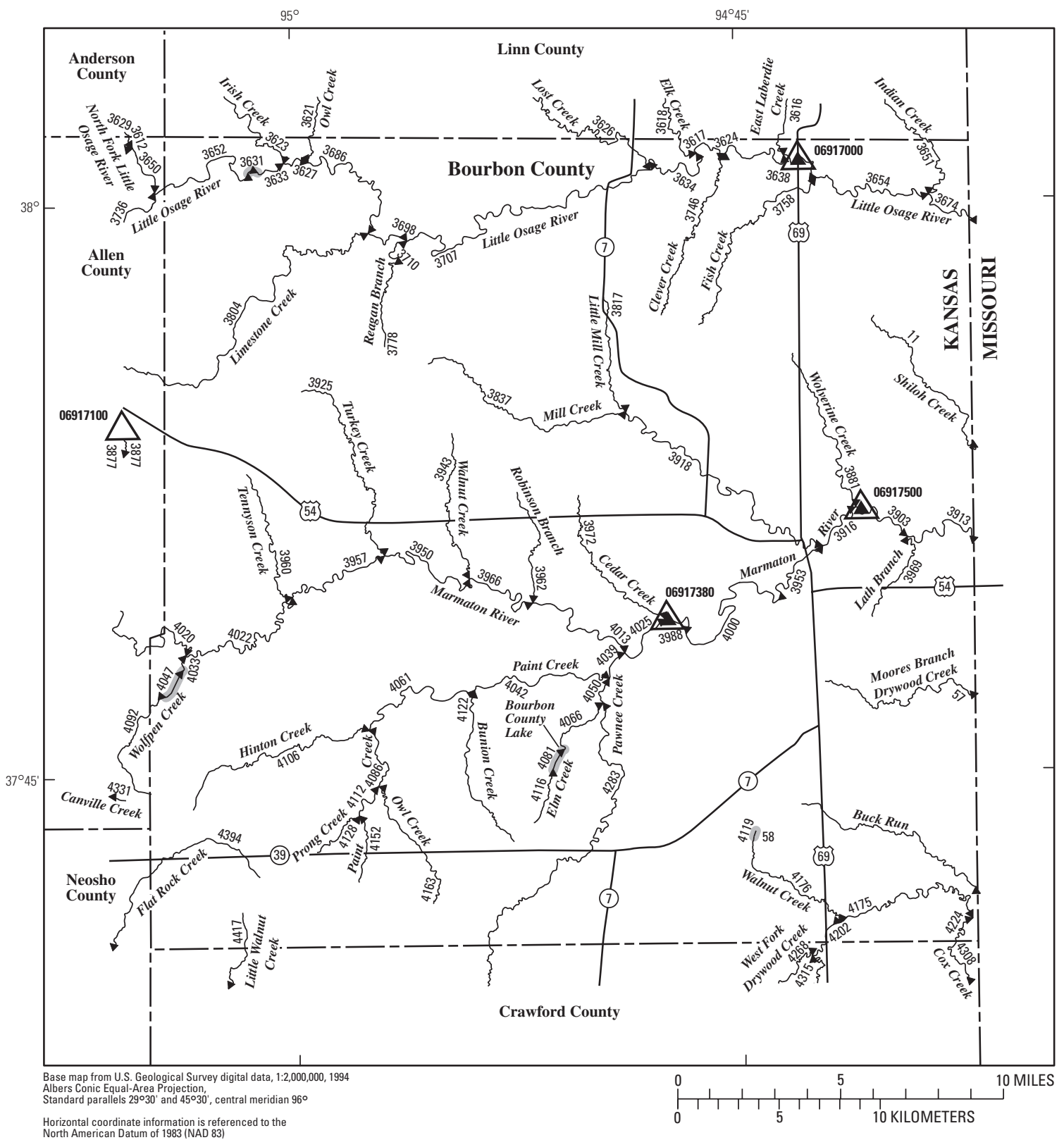

EXPLANATION

0691750

07143200

Location of streamflow-statistics determination site (small triangle) and associated identification number-small triangle points in downstream direction

U.S. Geological Survey streamflow-gaging station and number used for estimates of flow duration

U.S. Geological Survey streamflow-gaging station and number used for estimates of peak-discharge frequency values

KANSAS

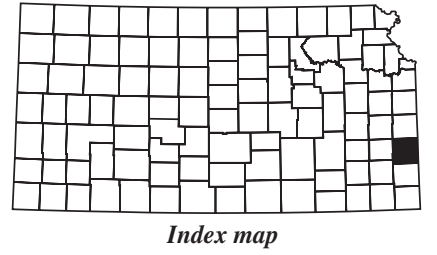

4047

Lake and determination site identification number

Figure 16. Location of streamflow-statistics determination sites, associated identification numbers, and U.S. Geological Survey streamflow-gaging stations used in the flow-duration and peak-discharge frequency analyses for Bourbon County. 


\section{Estimates of Flow Duration, Mean Flow, and Peak-Discharge Frequency Values for Kansas Stream Locations}

Table 12. Estimated flow-duration values, mean flow values, and peak-discharge frequency values for controlled and uncontrolled flow stream segments on the 1999 Kansas Surface Water Register for Bourbon County.

[KSWR, Kansas Surface Water Register; CUSEGA, catalog unit segment number alpha; $\mathrm{mi}^{2}$, square miles; $\mathrm{ft}^{3} / \mathrm{s}$, cubic feet per second; HYDRO, lake or other hydrologic structure; NA, not applicable; NRDitch, irrigation ditch; NRTribal, tribal stream]

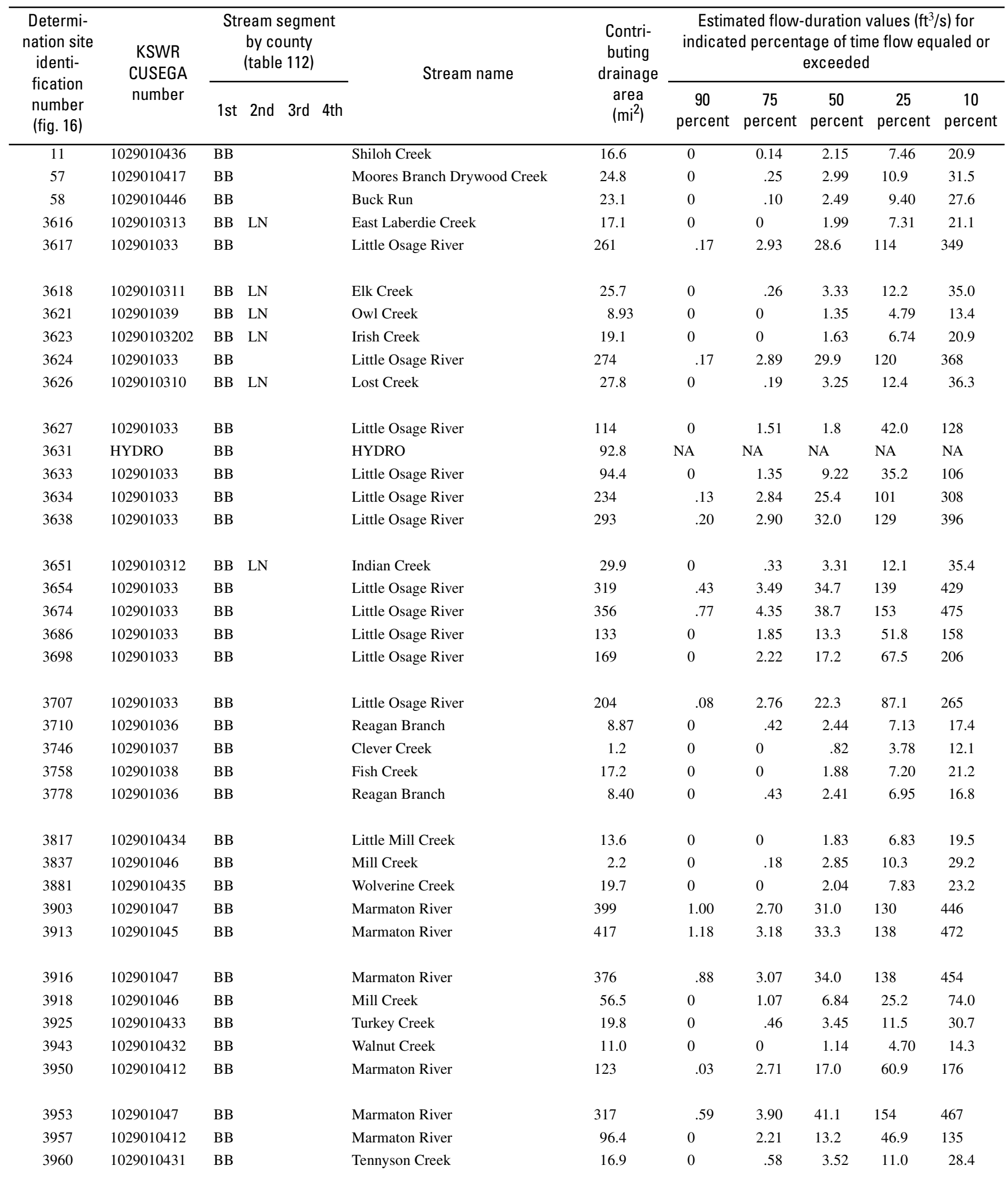


Table 12. Estimated flow-duration values, mean flow values, and peak-discharge frequency values for controlled and uncontrolled flow stream segments on the 1999 Kansas Surface Water Register for Bourbon County.-Continued

[KSWR, Kansas Surface Water Register; CUSEGA, catalog unit segment number alpha; $\mathrm{mi}^{2}$, square miles; $\mathrm{ft}^{3} / \mathrm{s}$, cubic feet per second; HYDRO, lake or other hydrologic structure; NA, not applicable; NRDitch, irrigation ditch; NRTribal, tribal stream]

\begin{tabular}{|c|c|c|c|c|c|c|c|}
\hline \multirow{2}{*}{$\begin{array}{l}\text { Determi- } \\
\text { nation site } \\
\text { identi- } \\
\text { fication } \\
\text { number } \\
\text { (fig. 16) }\end{array}$} & \multirow{2}{*}{$\begin{array}{c}\text { Estimated mean } \\
\text { flow } \\
\left(\mathrm{ft}^{3} / \mathrm{s}\right)\end{array}$} & \multicolumn{6}{|c|}{ Estimated peak discharge $\left(\mathrm{ft}^{3} / \mathrm{s}\right)$ for indicated peak-discharge frequency } \\
\hline & & 2-year & 5-year & 10-year & 25-year & 50-year & 100-year \\
\hline 11 & 16.2 & 2,390 & 4,680 & 6,580 & 9,400 & 11,700 & 14,300 \\
\hline 3616 & 16.4 & 2,330 & 4,600 & 6,500 & 9,330 & 11,600 & 14,200 \\
\hline 3617 & 213 & 8,860 & 15,400 & 20,800 & 28,700 & 35,400 & 42,900 \\
\hline 3618 & 25.6 & 2,920 & 5,840 & 8,290 & 12,000 & 14,900 & 18,400 \\
\hline 3621 & 9.97 & 1,560 & 3,060 & 4,300 & 6,150 & 7,650 & 9,340 \\
\hline 3627 & 89.0 & 8,590 & 15,100 & 20,400 & 28,000 & 34,200 & 40,900 \\
\hline 3631 & NA & NA & NA & NA & NA & NA & NA \\
\hline 3633 & 74.7 & 7,600 & 13,500 & 18,400 & 25,300 & 31,000 & 37,100 \\
\hline 3634 & 191 & 8,690 & 15,300 & 20,700 & 28,600 & 35,400 & 42,800 \\
\hline 3638 & 238 & 8,560 & 14,800 & 19,900 & 27,500 & 34,000 & 41,400 \\
\hline 3651 & 27.0 & 3,250 & 6,500 & 9,240 & 13,300 & 16,700 & 20,500 \\
\hline 3654 & 257 & 8,870 & 15,400 & 20,700 & 28,600 & 35,400 & 43,100 \\
\hline 3674 & 284 & 9,610 & 16,600 & 22,300 & 30,700 & 38,000 & 46,200 \\
\hline 3686 & 106 & 8,660 & 15,300 & 20,700 & 28,500 & 35,000 & 41,900 \\
\hline 3817 & 14.7 & 2,070 & 4,070 & 5,730 & 8,180 & 10,200 & 12,400 \\
\hline 3837 & 21.3 & 2,600 & 5,160 & 7,290 & 10,500 & 13,100 & 16,000 \\
\hline 3881 & 18.5 & 2,610 & 5,150 & 7,270 & 10,400 & 13,000 & 15,900 \\
\hline 3903 & 288 & 11,800 & 22,900 & 32,100 & 45,600 & 56,900 & 69,300 \\
\hline 3913 & 302 & 11,900 & 23,200 & 32,500 & 46,100 & 57,600 & 70,100 \\
\hline 3916 & 292 & 12,800 & 23,900 & 33,100 & 46,500 & 57,800 & 70,200 \\
\hline 3918 & 52.4 & 5,200 & 9,680 & 13,400 & 18,700 & 23,100 & 27,800 \\
\hline 3925 & 21.5 & 2,560 & 5,080 & 7,180 & 10,300 & 12,900 & 15,800 \\
\hline 3943 & 11.5 & 1,840 & 3,600 & 5,050 & 7,200 & 8,940 & 10,900 \\
\hline 3950 & 117 & 8,760 & 15,100 & 20,100 & 27,400 & 33,500 & 40,100 \\
\hline 3953 & 300 & 14,900 & 26,000 & 35,000 & 48,100 & 59,200 & 71,300 \\
\hline 3957 & 90.9 & 7,360 & 12,700 & 17,000 & 23,100 & 28,300 & 33,800 \\
\hline 3960 & 19.3 & 2,320 & 4,600 & 6,490 & 9,320 & 11,600 & 14,200 \\
\hline
\end{tabular}




\section{Estimates of Flow Duration, Mean Flow, and Peak-Discharge Frequency Values for Kansas Stream Locations}

Table 12. Estimated flow-duration values, mean flow values, and peak-discharge frequency values for controlled and uncontrolled flow stream segments on the 1999 Kansas Surface Water Register for Bourbon County.-Continued

[KSWR, Kansas Surface Water Register; CUSEGA, catalog unit segment number alpha; $\mathrm{mi}^{2}$, square miles; $\mathrm{ft}^{3} / \mathrm{s}$, cubic feet per second; HYDRO, lake or other hydrologic structure; NA, not applicable; NRDitch, irrigation ditch; NRTribal, tribal stream]

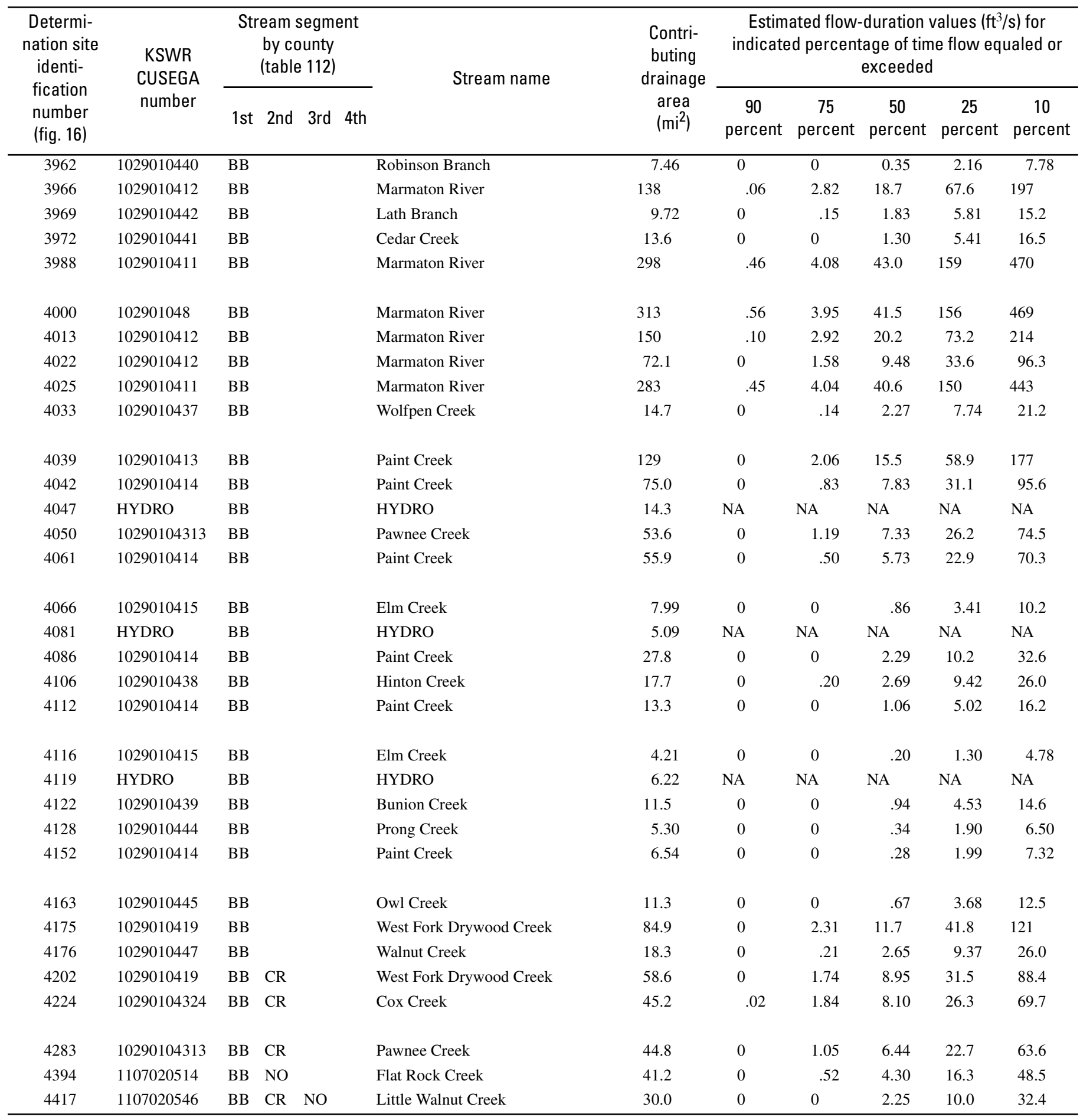


Table 12. Estimated flow-duration values, mean flow values, and peak-discharge frequency values for controlled and uncontrolled flow stream segments on the 1999 Kansas Surface Water Register for Bourbon County.-Continued

[KSWR, Kansas Surface Water Register; CUSEGA, catalog unit segment number alpha; $\mathrm{mi}^{2}$, square miles; $\mathrm{ft}^{3} / \mathrm{s}$, cubic feet per second; HYDRO, lake or other hydrologic structure; NA, not applicable; NRDitch, irrigation ditch; NRTribal, tribal stream]

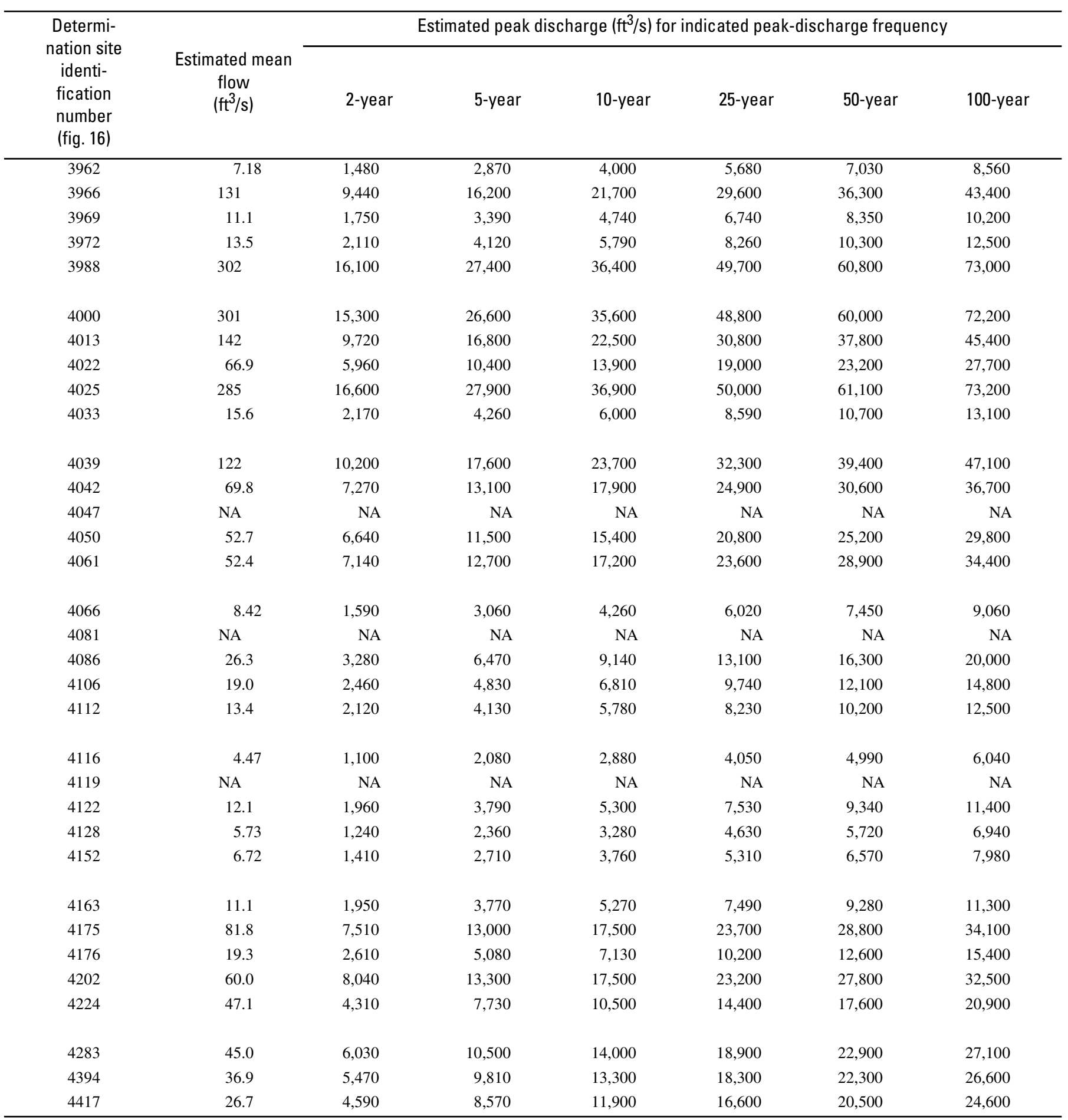




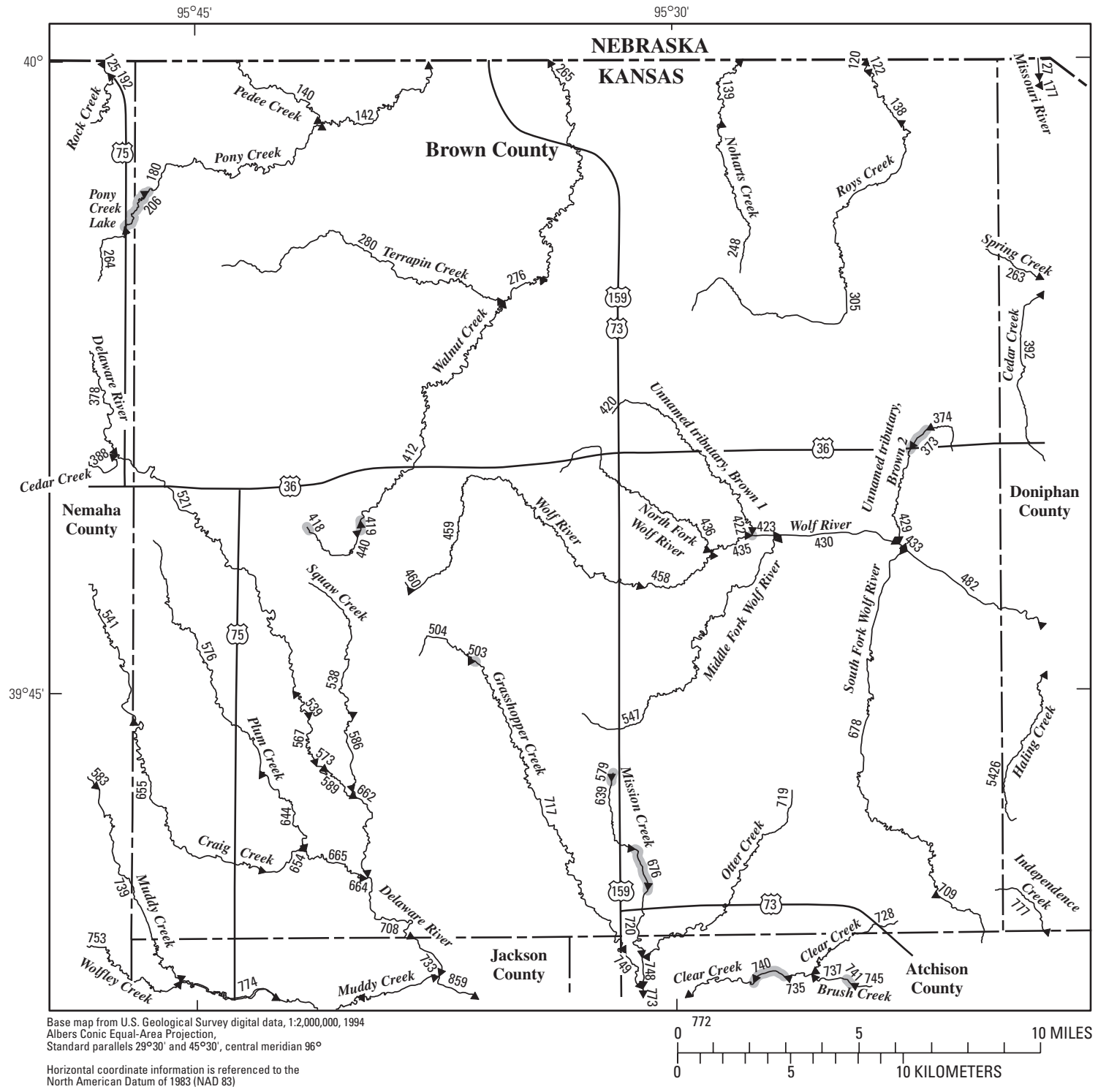

EXPLANATION

774 Location of streamflow-statistics determination site (small triangle) and associated identification number-small triangle points in downstream direction

07141300 U.S. Geological Survey streamflow-gaging station and number used for estimates of flow duration

07143200

\footnotetext{
U.S. Geological Survey streamflow-gaging station and number used for estimates of peak-discharge frequency values

676 Lake and determination site identification number
}

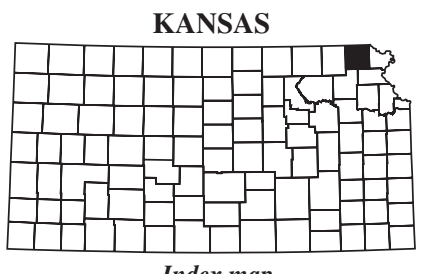

Index map

Figure 17. Location of streamflow-statistics determination sites, associated identification numbers, and U.S. Geological Survey streamflow-gaging stations used in the flow-duration and peak-discharge frequency analyses for Brown County. 


\section{Estimates of Flow Duration, Mean Flow, and Peak-Discharge Frequency Values for Kansas Stream Locations}

Table 13. Estimated flow-duration values, mean flow values, and peak-discharge frequency values for controlled and uncontrolled flow stream segments on the 1999 Kansas Surface Water Register for Brown County.

[KSWR, Kansas Surface Water Register; CUSEGA, catalog unit segment number alpha; $\mathrm{mi}^{2}$, square miles; $\mathrm{ft}^{3} / \mathrm{s}$, cubic feet per second; HYDRO, lake or other hydrologic structure; NA, not applicable; NRDitch, irrigation ditch; NRTribal, tribal stream]

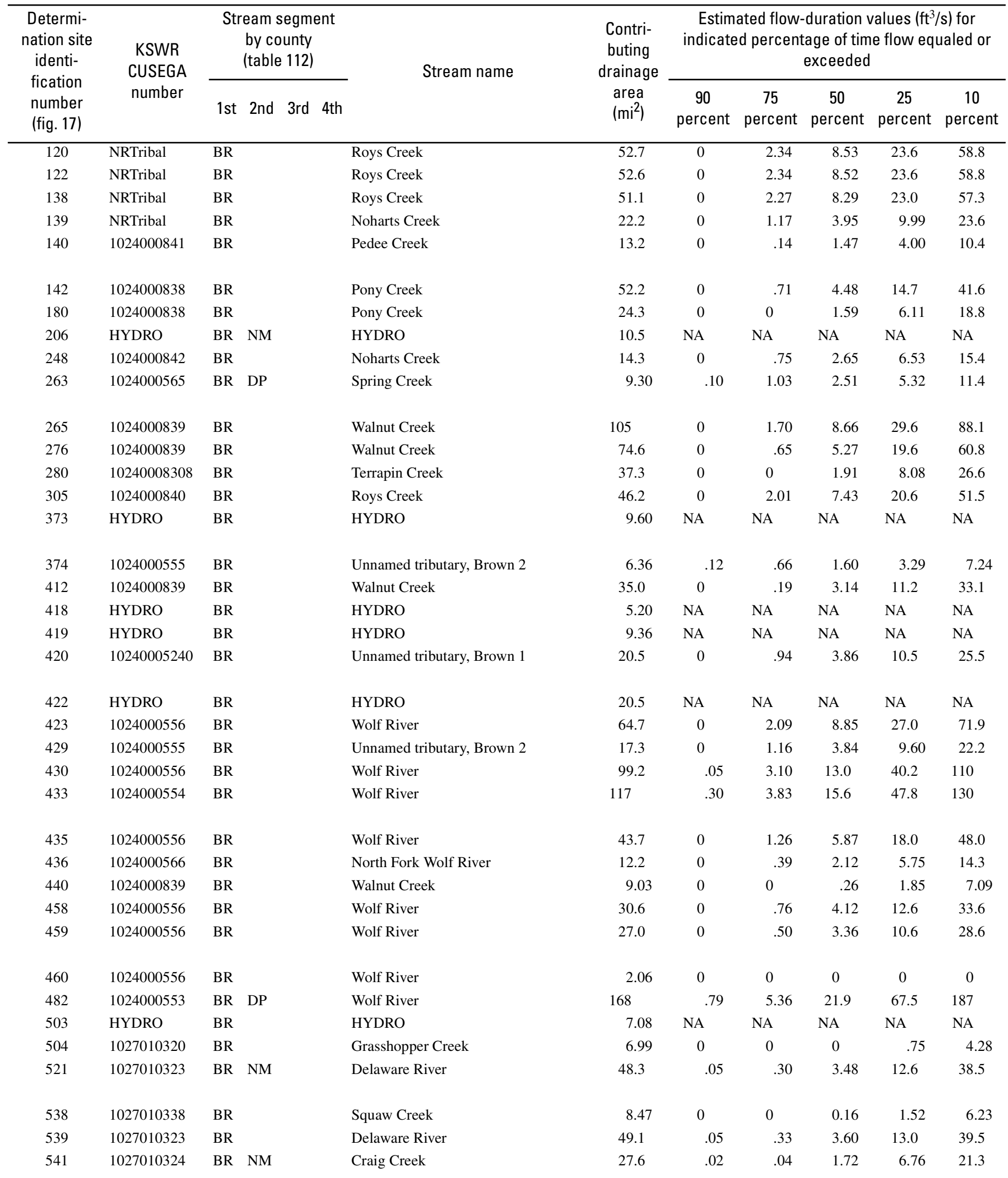


Table 13. Estimated flow-duration values, mean flow values, and peak-discharge frequency values for controlled and uncontrolled flow stream segments on the 1999 Kansas Surface Water Register for Brown County.-Continued

[KSWR, Kansas Surface Water Register; CUSEGA, catalog unit segment number alpha; $\mathrm{mi}^{2}$, square miles; $\mathrm{ft}^{3} / \mathrm{s}$, cubic feet per second; HYDRO, lake or other hydrologic structure; NA, not applicable; NRDitch, irrigation ditch;NRTribal, tribal stream]

\begin{tabular}{|c|c|c|c|c|c|c|c|}
\hline \multirow{2}{*}{$\begin{array}{l}\text { Determi- } \\
\text { nation site } \\
\text { identi- } \\
\text { fication } \\
\text { number } \\
\text { (fig. 17) }\end{array}$} & \multirow{2}{*}{$\begin{array}{c}\text { Estimated mean } \\
\text { flow } \\
\left(\mathrm{ft}^{3} / \mathrm{s}\right)\end{array}$} & \multicolumn{6}{|c|}{ Estimated peak discharge $\left(\mathrm{ft}^{3} / \mathrm{s}\right)$ for indicated peak-discharge frequency } \\
\hline & & 2-year & 5-year & 10 -year & 25-year & 50-year & 100 -year \\
\hline 120 & 37.9 & 3,440 & 6,840 & 9,750 & 14,000 & 17,600 & 21,500 \\
\hline 139 & 16.0 & 1,790 & 3,940 & 5,830 & 8,730 & 11,200 & 14,000 \\
\hline 140 & 8.16 & 1,220 & 2,700 & 4,010 & 6,030 & 7,720 & 9,650 \\
\hline 142 & 30.4 & 3,800 & 7,840 & 11,400 & 16,700 & 21,200 & 26,100 \\
\hline 180 & 15.0 & 1,770 & 3,960 & 5,910 & 8,920 & 11,400 & 14,400 \\
\hline 265 & 62.0 & 4,080 & 8,740 & 13,000 & 19,400 & 25,100 & 31,300 \\
\hline 276 & 45.2 & 4,430 & 9,320 & 13,700 & 20,300 & 26,100 & 32,400 \\
\hline 280 & 21.9 & 4,460 & 9,090 & 13,100 & 19,100 & 24,200 & 29,700 \\
\hline 305 & 33.6 & 3,380 & 6,710 & 9,530 & 13,700 & 17,200 & 20,900 \\
\hline 373 & NA & NA & NA & NA & NA & NA & NA \\
\hline 374 & 5.22 & 935 & 1,970 & 2,850 & 4,180 & 5,280 & 6,530 \\
\hline 412 & 24.5 & 2,940 & 6,290 & 9,280 & 13,800 & 17,700 & 22,000 \\
\hline 418 & NA & NA & NA & NA & NA & NA & NA \\
\hline 419 & NA & NA & NA & NA & NA & NA & NA \\
\hline 430 & 70.5 & 5,310 & 10,300 & 14,600 & 21,000 & 26,300 & 32,100 \\
\hline 433 & 82.0 & 5,860 & 11,300 & 15,900 & 22,600 & 28,300 & 34,400 \\
\hline 435 & 32.7 & 3,340 & 6,750 & 9,690 & 14,000 & 17,700 & 21,700 \\
\hline 436 & 10.2 & 1,380 & 2,940 & 4,290 & 6,320 & 8,010 & 9,940 \\
\hline 440 & 6.49 & 1,110 & 2,370 & 3,460 & 5,110 & 6,480 & 8,040 \\
\hline 458 & 23.4 & 2,740 & 5,640 & 8,150 & 11,900 & 15,100 & 18,500 \\
\hline 459 & 20.5 & 2,190 & 4,740 & 6,970 & 10,400 & 13,200 & 16,500 \\
\hline 460 & .74 & 484 & 997 & 1,430 & 2,070 & 2,600 & 3,190 \\
\hline 482 & 115 & 6,880 & 13,200 & 18,500 & 26,400 & 33,100 & 40,300 \\
\hline 503 & NA & NA & NA & NA & NA & NA & NA \\
\hline 504 & 4.69 & 998 & 2,100 & 3,040 & 4,460 & 5,630 & 6,960 \\
\hline 521 & 29.5 & 3,580 & 7,560 & 11,100 & 16,500 & 21,100 & 26,200 \\
\hline 538 & 5.97 & 1,100 & 2,320 & 3,380 & 4,970 & 6,290 & 7,800 \\
\hline 539 & 30.1 & 3,500 & 7,420 & 10,900 & 16,200 & 20,800 & 25,900 \\
\hline 541 & 17.2 & 1,990 & 4,400 & 6,530 & 9,810 & 12,600 & 15,700 \\
\hline
\end{tabular}




\section{Estimates of Flow Duration, Mean Flow, and Peak-Discharge Frequency Values for Kansas Stream Locations}

Table 13. Estimated flow-duration values, mean flow values, and peak-discharge frequency values for controlled and uncontrolled flow stream segments on the 1999 Kansas Surface Water Register for Brown County.-Continued

[KSWR, Kansas Surface Water Register; CUSEGA, catalog unit segment number alpha; $\mathrm{mi}^{2}$, square miles; $\mathrm{ft}^{3} / \mathrm{s}$, cubic feet per second; HYDRO, lake or other hydrologic structure; NA, not applicable; NRDitch, irrigation ditch; NRTribal, tribal stream]

\begin{tabular}{|c|c|c|c|c|c|c|c|c|c|c|c|}
\hline \multirow{2}{*}{$\begin{array}{l}\text { Determi- } \\
\text { nation site } \\
\text { identi- } \\
\text { fication } \\
\text { number } \\
\text { (fig. 17) }\end{array}$} & \multirow{2}{*}{$\begin{array}{l}\text { KSWR } \\
\text { CUSEGA } \\
\text { number }\end{array}$} & \multicolumn{3}{|c|}{$\begin{array}{l}\text { Stream segment } \\
\text { by county } \\
\text { (table 112) }\end{array}$} & \multirow{2}{*}{ Stream name } & \multirow{2}{*}{$\begin{array}{l}\text { Contri- } \\
\text { buting } \\
\text { drainage } \\
\text { area } \\
\left(\mathrm{mi}^{2}\right)\end{array}$} & \multicolumn{5}{|c|}{$\begin{array}{c}\text { Estimated flow-duration values }\left(\mathrm{ft}^{3} / \mathrm{s}\right) \text { for } \\
\text { indicated percentage of time flow equaled or } \\
\text { exceeded }\end{array}$} \\
\hline & & $1 \mathrm{st}$ & 2nd & $3 r d$ & & & $\begin{array}{c}90 \\
\text { percent }\end{array}$ & $\begin{array}{c}75 \\
\text { percent }\end{array}$ & $\begin{array}{c}50 \\
\text { percent }\end{array}$ & $\begin{array}{c}25 \\
\text { percent }\end{array}$ & $\begin{array}{c}10 \\
\text { percent }\end{array}$ \\
\hline 547 & 1024000567 & $\overline{B R}$ & & & Middle Fork Wolf River & 27.4 & 0 & 0.65 & 3.82 & 11.8 & 31.4 \\
\hline 567 & NRTribal & $\mathrm{BR}$ & & & Delaware River & 50.7 & .06 & .41 & 3.82 & 13.6 & 41.2 \\
\hline 573 & NRTribal & $\mathrm{BR}$ & & & Delaware River & 51.0 & .06 & .42 & 3.86 & 13.7 & 41.5 \\
\hline 576 & 1027010336 & $\mathrm{BR}$ & & & Plum Creek & 16.8 & .01 & .01 & 1.11 & 4.48 & 14.2 \\
\hline 579 & HYDRO & $\mathrm{BR}$ & & & HYDRO & 2.49 & NA & NA & NA & NA & NA \\
\hline 586 & NRTribal & $\mathrm{BR}$ & & & Squaw Creek & 12.8 & 0 & .01 & .83 & 3.55 & 11.5 \\
\hline 589 & NRTribal & $\mathrm{BR}$ & & & Delaware River & 52.4 & .06 & .49 & 4.05 & 14.3 & 43.1 \\
\hline 639 & 1027010340 & $\mathrm{BR}$ & & & Mission Creek & 6.24 & 0 & 0 & .07 & 1.15 & 5.00 \\
\hline 644 & NRTribal & $\mathrm{BR}$ & & & Plum Creek & 2.7 & .01 & .02 & 1.67 & 6.16 & 18.5 \\
\hline 654 & NRTribal & $\mathrm{BR}$ & & & Craig Creek & 45.3 & .04 & .25 & 3.44 & 12.6 & 38.4 \\
\hline 655 & 1027010324 & $\mathrm{BR}$ & & & Craig Creek & 42.7 & .04 & .16 & 3.12 & 11.6 & 35.6 \\
\hline 662 & NRTribal & BR & & & Delaware River & 71.9 & .11 & .99 & 5.82 & 20.3 & 61.2 \\
\hline 664 & 1027010324 & $\mathrm{BR}$ & & & Craig Creek & 68.6 & .10 & .86 & 5.49 & 19.4 & 59.1 \\
\hline 665 & NRTribal & BR & & & Plum Creek & 68.6 & .10 & .86 & 5.49 & 19.4 & 59.1 \\
\hline 676 & HYDRO & $\mathrm{BR}$ & & & HYDRO & 10.2 & NA & NA & NA & NA & NA \\
\hline 678 & 1024000557 & $\mathrm{BR}$ & & & South Fork Wolf River & 36.8 & 0 & .97 & 5.06 & 15.7 & 41.9 \\
\hline 708 & NRTribal & $\mathrm{BR}$ & & & Delaware River & 149 & .48 & 3.14 & 12.7 & 42.2 & 129 \\
\hline 709 & 1024000557 & BR & & & South Fork Wolf River & 4.63 & 0 & 0 & 0 & .27 & 2.67 \\
\hline 733 & 1027010322 & $\mathrm{BR}$ & JA & & Delaware River & 153 & .51 & 3.30 & 13.2 & 43.5 & 133 \\
\hline 739 & 1027010326 & BR & JA & NM & Muddy Creek & 42.7 & .04 & .24 & 3.33 & 12.1 & 36.4 \\
\hline
\end{tabular}


Table 13. Estimated flow-duration values, mean flow values, and peak-discharge frequency values for controlled and uncontrolled flow stream segments on the 1999 Kansas Surface Water Register for Brown County.-Continued

[KSWR, Kansas Surface Water Register; CUSEGA, catalog unit segment number alpha; $\mathrm{mi}^{2}$, square miles; $\mathrm{ft}^{3} / \mathrm{s}$, cubic feet per second; HYDRO, lake or other hydrologic structure; NA, not applicable; NRDitch, irrigation ditch;NRTribal, tribal stream]

\begin{tabular}{|c|c|c|c|c|c|c|c|}
\hline \multirow{2}{*}{$\begin{array}{c}\text { Determi- } \\
\text { nation site } \\
\text { identi- } \\
\text { fication } \\
\text { number } \\
\text { (fig. 17) }\end{array}$} & \multirow{2}{*}{$\begin{array}{c}\text { Estimated mean } \\
\text { flow } \\
\left(\mathrm{ft}^{3} / \mathrm{s}\right)\end{array}$} & \multicolumn{6}{|c|}{ Estimated peak discharge $\left(\mathrm{ft}^{3} / \mathrm{s}\right)$ for indicated peak-discharge frequency } \\
\hline & & 2-year & 5-year & 10-year & 25-year & 50-year & 100-year \\
\hline 547 & 21.8 & 2,230 & 4,810 & 7,070 & 10,500 & 13,400 & 16,700 \\
\hline 567 & 31.2 & 3,450 & 7,320 & 10,800 & 16,100 & 20,600 & 25,700 \\
\hline 573 & 31.4 & 3,460 & 7,350 & 10,800 & 16,100 & 20,700 & 25,700 \\
\hline 576 & 11.7 & 1,590 & 3,440 & 5,050 & 7,500 & 9,550 & 11,900 \\
\hline 579 & NA & NA & NA & NA & NA & NA & NA \\
\hline 586 & 9.55 & 1,400 & 2,990 & 4,360 & 6,440 & 8,170 & 10,100 \\
\hline 589 & 32.4 & 3,470 & 7,360 & 10,800 & 16,100 & 20,700 & 25,800 \\
\hline 639 & 4.81 & 940 & 1,970 & 2,850 & 4,170 & 5,260 & 6,500 \\
\hline 644 & 14.6 & 1,800 & 3,910 & 5,740 & 8,540 & 10,900 & 13,600 \\
\hline 654 & 29.1 & 3,210 & 6,910 & 10,200 & 15,300 & 19,800 & 24,600 \\
\hline 655 & 27.2 & 3,130 & 6,760 & 10,000 & 15,000 & 19,400 & 24,200 \\
\hline 662 & 44.8 & 4,180 & 8,690 & 12,700 & 18,700 & 24,000 & 29,700 \\
\hline 664 & 43.3 & 4,280 & 8,870 & 12,900 & 19,100 & 24,400 & 30,200 \\
\hline 665 & 43.3 & 4,280 & 8,860 & 12,900 & 19,100 & 24,400 & 30,200 \\
\hline 676 & NA & NA & NA & NA & NA & NA & NA \\
\hline 678 & 28.6 & 3,020 & 6,200 & 8,960 & 13,100 & 16,500 & 20,300 \\
\hline 708 & 89.5 & 6,550 & 12,800 & 18,100 & 26,200 & 33,100 & 40,600 \\
\hline 709 & 3.21 & 788 & 1,640 & 2,370 & 3,460 & 4,360 & 5,380 \\
\hline 733 & 91.7 & 6,590 & 12,800 & 18,200 & 26,200 & 33,000 & 40,600 \\
\hline 739 & 27.4 & 3,170 & 6,810 & 10,100 & 15,100 & 19,400 & 24,200 \\
\hline
\end{tabular}




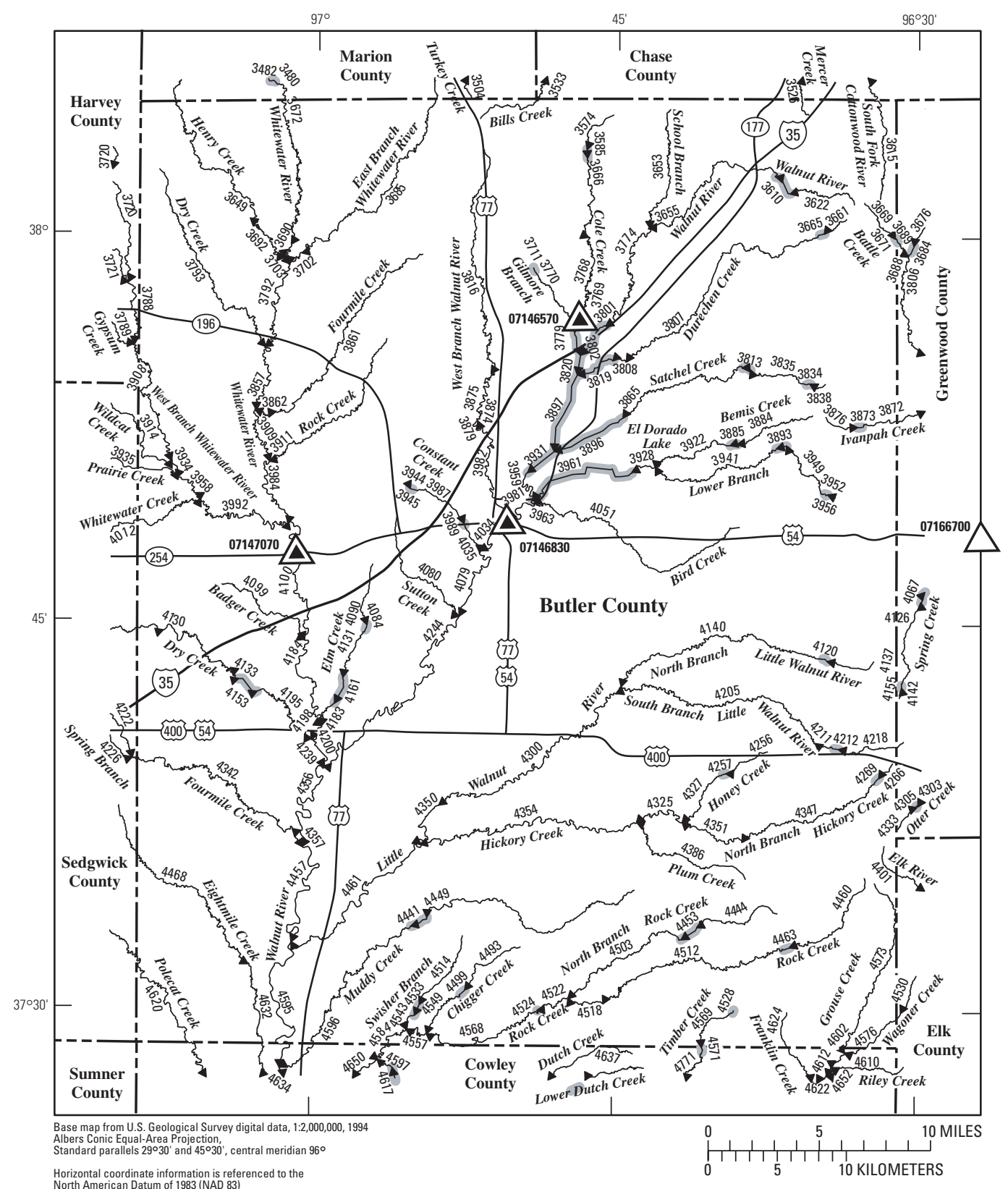

\section{EXPLANATION}

4620 Location of streamflow-statistics determination site (small triangle) and associated identification number-small triangle points in downstream direction

$07146830 \Delta$ U.S. Geological Survey streamflow-gaging station and number used for estimates of flow duration

07166700 入

U.S. Geological Survey streamflow-gaging station and number used for estimates of peak-discharge frequency values

KANSAS

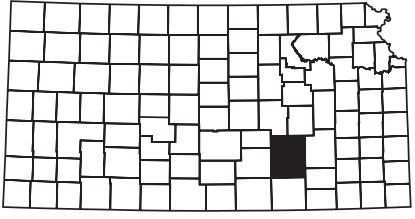

Index map

4617 Lake and determination site identification number

Figure 18. Location of streamflow-statistics determination sites, associated identification numbers, and U.S. Geological Survey streamflow-gaging stations used in the flow-duration and peak-discharge frequency analyses for Butler County. 


\section{Estimates of Flow Duration, Mean Flow, and Peak-Discharge Frequency Values for Kansas Stream Locations}

Table 14. Estimated flow-duration values, mean flow values, and peak-discharge frequency values for controlled and uncontrolled flow stream segments on the 1999 Kansas Surface Water Register for Butler County.

[KSWR, Kansas Surface Water Register; CUSEGA, catalog unit segment number alpha; $\mathrm{mi}^{2}$, square miles; $\mathrm{ft}^{3} / \mathrm{s}$, cubic feet per second; HYDRO, lake or other hydrologic structure; NA, not applicable; NRDitch, irrigation ditch; NRTribal, tribal stream]

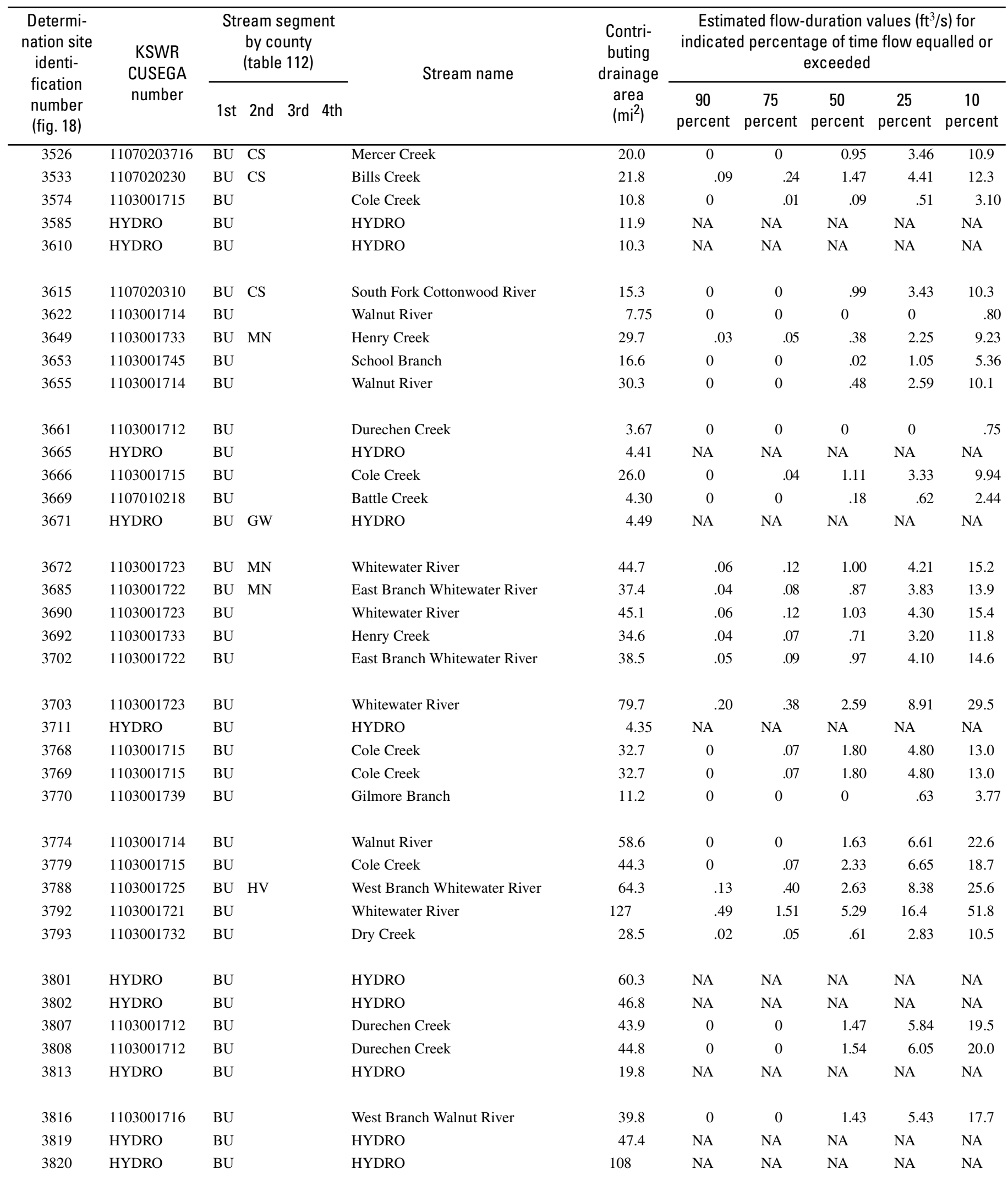


Table 14. Estimated flow-duration values, mean flow values, and peak-discharge frequency values for controlled and uncontrolled flow stream segments on the 1999 Kansas Surface Water Register for Butler County.-Continued

[KSWR, Kansas Surface Water Register; CUSEGA, catalog unit segment number alpha; $\mathrm{mi}^{2}$, square miles; $\mathrm{ft}^{3} / \mathrm{s}$, cubic feet per second; HYDRO, lake or other hydrologic structure; NA, not applicable; NRDitch, irrigation ditch; NRTribal, tribal stream]

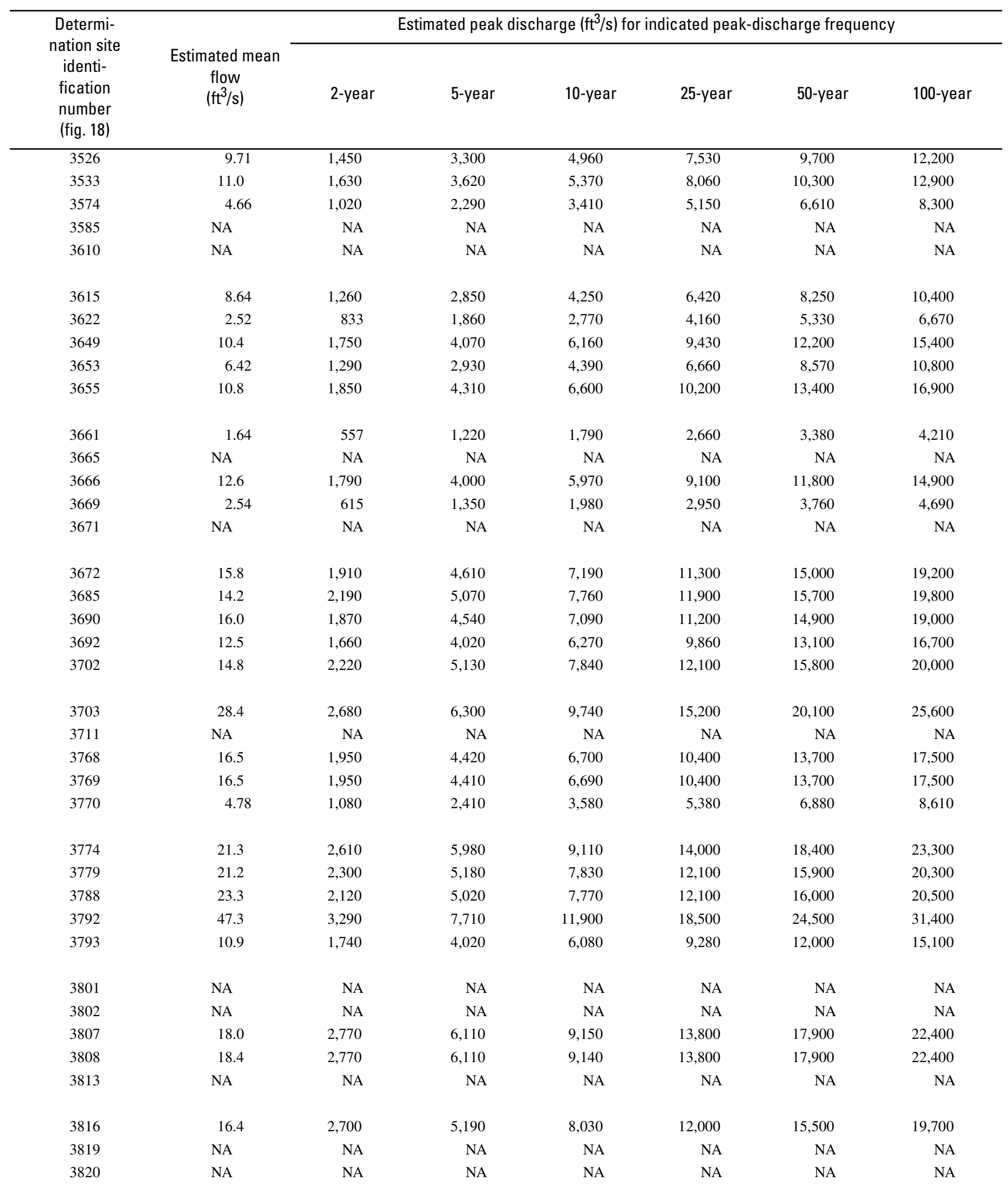




\section{Estimates of Flow Duration, Mean Flow, and Peak-Discharge Frequency Values for Kansas Stream Locations}

Table 14. Estimated flow-duration values, mean flow values, and peak-discharge frequency values for controlled and uncontrolled flow stream segments on the 1999 Kansas Surface Water Register for Butler County._-Continued

[KSWR, Kansas Surface Water Register; CUSEGA, catalog unit segment number alpha; $\mathrm{mi}^{2}$, square miles; $\mathrm{ft}^{3} / \mathrm{s}$, cubic feet per second; HYDRO, lake or other hydrologic structure; NA, not applicable; NRDitch, irrigation ditch; NRTribal, tribal stream]

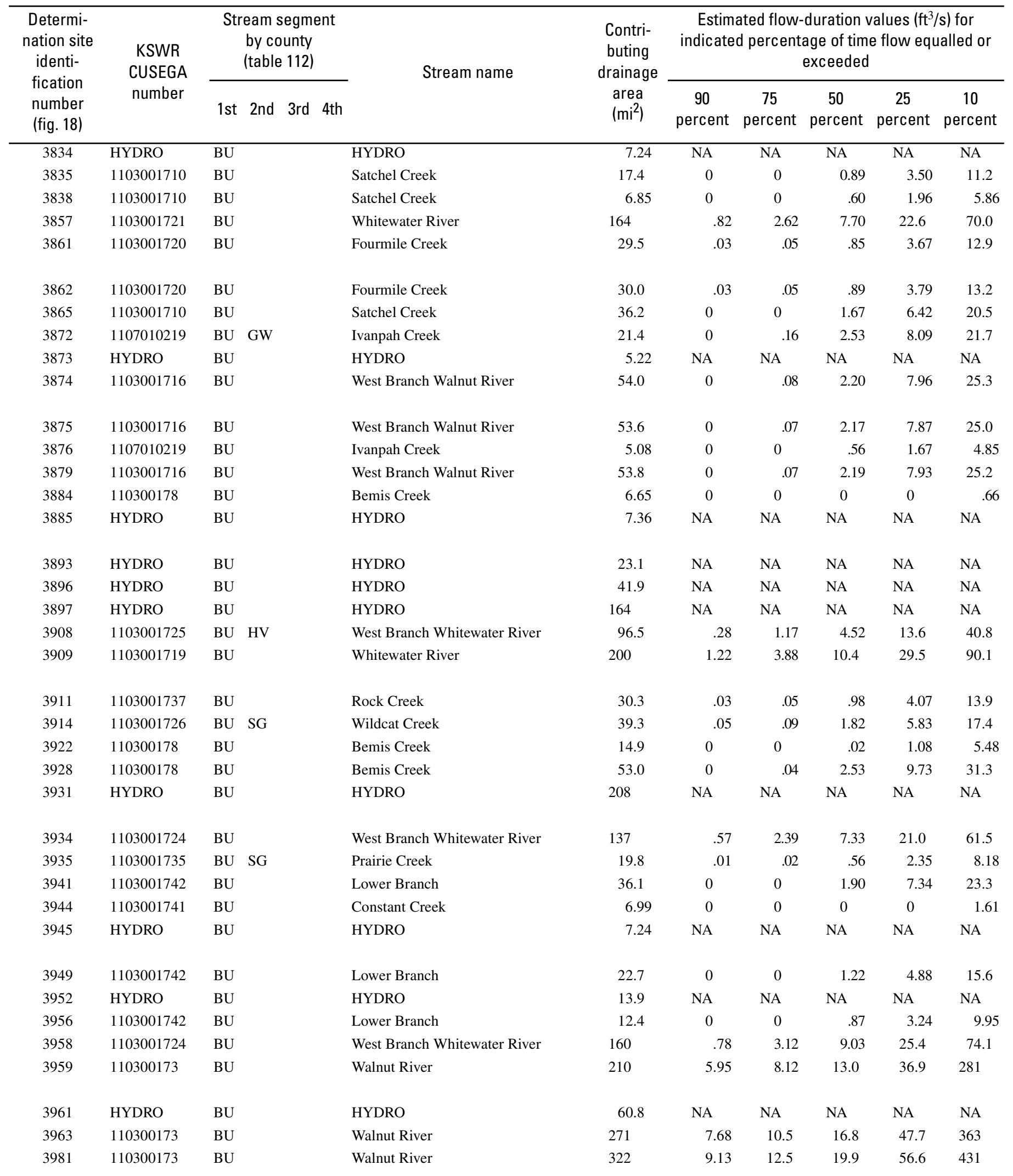


Table 14. Estimated flow-duration values, mean flow values, and peak-discharge frequency values for controlled and uncontrolled flow stream segments on the 1999 Kansas Surface Water Register for Butler County.-Continued

[KSWR, Kansas Surface Water Register; CUSEGA, catalog unit segment number alpha; $\mathrm{mi}^{2}$, square miles; $\mathrm{ft}^{3} / \mathrm{s}$, cubic feet per second; HYDRO, lake or other hydrologic structure; NA, not applicable; NRDitch, irrigation ditch; NRTribal, tribal stream]

\begin{tabular}{|c|c|c|c|c|c|c|c|}
\hline \multirow{2}{*}{$\begin{array}{l}\text { Determi- } \\
\text { nation site } \\
\text { identi- } \\
\text { fication } \\
\text { number } \\
\text { (fig. 18) }\end{array}$} & \multirow{2}{*}{$\begin{array}{c}\text { Estimated mean } \\
\text { flow } \\
\left(\mathrm{ft}^{3} / \mathrm{s}\right)\end{array}$} & \multicolumn{6}{|c|}{ Estimated peak discharge $\left(\mathrm{ft}^{3} / \mathrm{s}\right)$ for indicated peak-discharge frequency } \\
\hline & & 2-year & 5-year & 10-year & 25-year & 50-year & 100-year \\
\hline 3834 & NA & NA & NA & NA & NA & NA & NA \\
\hline 3857 & 62.6 & 3,800 & 8,850 & 13,600 & 21,200 & 28,000 & 35,800 \\
\hline 3861 & 12.6 & 1,840 & 4,220 & 6,360 & 9,680 & 12,500 & 15,700 \\
\hline 3862 & 12.8 & 2,090 & 4,780 & 7,270 & 11,100 & 14,500 & 18,300 \\
\hline 3865 & 17.8 & 2,620 & 5,770 & 8,610 & 13,000 & 16,800 & 21,000 \\
\hline 3875 & 22.1 & 3,260 & 5,730 & 9,020 & 13,400 & 17,300 & 22,000 \\
\hline 3876 & 3.92 & 738 & 1,590 & 2,320 & 3,440 & 4,370 & 5,420 \\
\hline 3879 & 22.2 & 3,250 & 5,710 & 8,990 & 13,400 & 17,200 & 22,000 \\
\hline 3884 & 2.44 & 852 & 1,850 & 2,710 & 4,030 & 5,120 & 6,370 \\
\hline 3885 & NA & NA & NA & NA & NA & NA & NA \\
\hline 3893 & NA & NA & NA & NA & NA & NA & NA \\
\hline 3896 & NA & NA & NA & NA & NA & NA & NA \\
\hline 3897 & NA & NA & NA & NA & NA & NA & NA \\
\hline 3908 & 35.7 & 2,640 & 6,200 & 9,550 & 14,900 & 19,600 & 25,100 \\
\hline 3934 & 52.1 & 3,290 & 7,610 & 11,700 & 18,100 & 23,800 & 30,300 \\
\hline 3935 & 8.18 & 1,350 & 3,130 & 4,730 & 7,230 & 9,350 & 11,800 \\
\hline 3941 & 19.6 & 3,380 & 7,090 & 10,300 & 15,200 & 19,500 & 24,000 \\
\hline 3944 & 2.91 & 841 & 1,850 & 2,720 & 4,060 & 5,170 & 6,450 \\
\hline 3945 & NA & NA & NA & NA & NA & NA & NA \\
\hline 3949 & 13.3 & 1,780 & 3,940 & 5,850 & 8,780 & 11,200 & 14,000 \\
\hline 3952 & NA & NA & NA & NA & NA & NA & NA \\
\hline 3956 & 8.31 & 1,260 & 2,750 & 4,050 & 6,040 & 7,700 & 9,590 \\
\hline 3958 & 62.2 & 3,590 & 8,300 & 12,700 & 19,700 & 25,900 & 33,000 \\
\hline 3959 & 86.9 & 2,050 & 3,980 & 4,940 & 7,280 & 9,540 & 12,000 \\
\hline 3961 & NA & NA & NA & NA & NA & NA & NA \\
\hline 3963 & 112 & 2,640 & 5,140 & 6,380 & 9,390 & 12,300 & 15,500 \\
\hline 3981 & 133 & 3,140 & 6,110 & 7,580 & 11,200 & 14,600 & 18,400 \\
\hline
\end{tabular}


Table 14. Estimated flow-duration values, mean flow values, and peak-discharge frequency values for controlled and uncontrolled flow stream segments on the 1999 Kansas Surface Water Register for Butler County.-Continued

[KSWR, Kansas Surface Water Register; CUSEGA, catalog unit segment number alpha; $\mathrm{mi}^{2}$, square miles; $\mathrm{ft}^{3} / \mathrm{s}$, cubic feet per second; HYDRO, lake or other hydrologic structure; NA, not applicable; NRDitch, irrigation ditch; NRTribal, tribal stream]

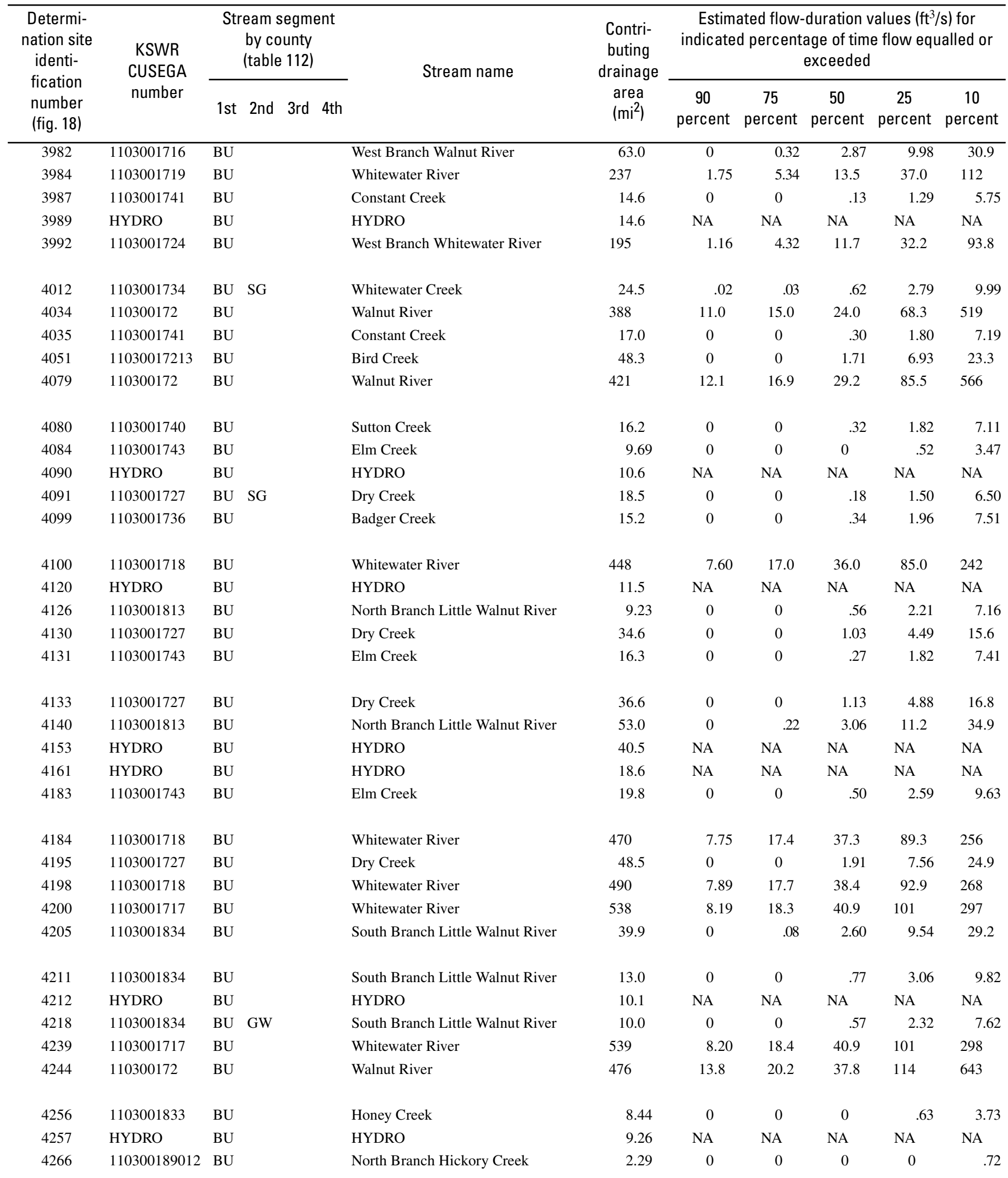


Table 14. Estimated flow-duration values, mean flow values, and peak-discharge frequency values for controlled and uncontrolled flow stream segments on the 1999 Kansas Surface Water Register for Butler County.-Continued

[KSWR, Kansas Surface Water Register; CUSEGA, catalog unit segment number alpha; $\mathrm{mi}^{2}$, square miles; $\mathrm{ft}^{3} / \mathrm{s}$, cubic feet per second; HYDRO, lake or other hydrologic structure; NA, not applicable; NRDitch, irrigation ditch; NRTribal, tribal stream]

\begin{tabular}{|c|c|c|c|c|c|c|c|}
\hline \multirow{2}{*}{$\begin{array}{l}\text { Determi- } \\
\text { nation site } \\
\text { identi- } \\
\text { fication } \\
\text { number } \\
\text { (fig. 18) }\end{array}$} & \multirow{2}{*}{$\begin{array}{c}\text { Estimated mean } \\
\text { flow } \\
\left(\mathrm{ft}^{3} / \mathrm{s}\right)\end{array}$} & \multicolumn{6}{|c|}{ Estimated peak discharge $\left(\mathrm{ft}^{3} / \mathrm{s}\right)$ for indicated peak-discharge frequency } \\
\hline & & 2-year & 5-year & 10-year & 25-year & 50-year & 100 -year \\
\hline 3982 & 26.4 & 3,630 & 5,970 & 9,500 & 14,000 & 18,000 & 23,000 \\
\hline 3989 & NA & NA & NA & NA & NA & NA & NA \\
\hline 3992 & 78.1 & 3,950 & 9,180 & 14,100 & 21,800 & 28,800 & 36,700 \\
\hline 4012 & 10.1 & 1,590 & 3,660 & 5,520 & 8,420 & 10,900 & 13,700 \\
\hline 4034 & 161 & 3,780 & 7,360 & 9,130 & 13,400 & 17,600 & 22,200 \\
\hline 4080 & 7.53 & 1,410 & 3,130 & 4,640 & 6,970 & 8,910 & 11,100 \\
\hline 4084 & 4.49 & 1,060 & 2,320 & 3,420 & 5,100 & 6,500 & 8,100 \\
\hline 4090 & NA & NA & NA & NA & NA & NA & NA \\
\hline 4091 & 7.28 & 1,360 & 3,110 & 4,670 & 7,100 & 9,150 & 11,500 \\
\hline 4099 & 7.59 & 1,340 & 2,970 & 4,420 & 6,630 & 8,490 & 10,600 \\
\hline 4100 & 206 & 7,490 & 17,300 & 26,200 & 40,500 & 53,300 & 67,900 \\
\hline 4120 & NA & NA & NA & NA & NA & NA & NA \\
\hline 4126 & 6.27 & 1,080 & 2,340 & 3,420 & 5,070 & 6,450 & 8,020 \\
\hline 4130 & 14.7 & 1,880 & 4,450 & 6,870 & 10,700 & 14,100 & 17,900 \\
\hline 4184 & 215 & 7,460 & 17,300 & 26,200 & 40,600 & 53,500 & 68,300 \\
\hline 4195 & 21.9 & 2,520 & 5,740 & 8,720 & 13,400 & 17,500 & 22,100 \\
\hline 4198 & 223 & 7,650 & 17,700 & 26,800 & 41,400 & 54,600 & 69,600 \\
\hline 4200 & 242 & 8,170 & 18,700 & 28,300 & 43,600 & 57,300 & 73,000 \\
\hline 4205 & 23.3 & 3,260 & 6,900 & 10,100 & 15,000 & 19,200 & 23,700 \\
\hline 4211 & 8.51 & 1,330 & 2,890 & 4,250 & 6,320 & 8,040 & 10,000 \\
\hline 4212 & NA & NA & NA & NA & NA & NA & NA \\
\hline 4218 & 6.74 & 1,150 & 2,480 & 3,630 & 5,380 & 6,840 & 8,500 \\
\hline 4239 & 242 & 8,140 & 18,600 & 28,200 & 43,500 & 57,200 & 72,900 \\
\hline 4244 & 218 & 4,640 & 9,030 & 11,200 & 16,500 & 21,600 & 27,300 \\
\hline 4256 & 4.49 & 1,020 & 2,210 & 3,230 & 4,790 & 6,080 & 7,560 \\
\hline 4257 & NA & NA & NA & NA & NA & NA & NA \\
\hline 4266 & 1.33 & 490 & 1,020 & 1,470 & 2,150 & 2,710 & 3,330 \\
\hline
\end{tabular}


Table 14. Estimated flow-duration values, mean flow values, and peak-discharge frequency values for controlled and uncontrolled flow stream segments on the 1999 Kansas Surface Water Register for Butler County._Continued

[KSWR, Kansas Surface Water Register; CUSEGA, catalog unit segment number alpha; $\mathrm{mi}^{2}$, square miles; $\mathrm{ft}^{3} / \mathrm{s}$, cubic feet per second; HYDRO, lake or other hydrologic structure; NA, not applicable; NRDitch, irrigation ditch; NRTribal, tribal stream]

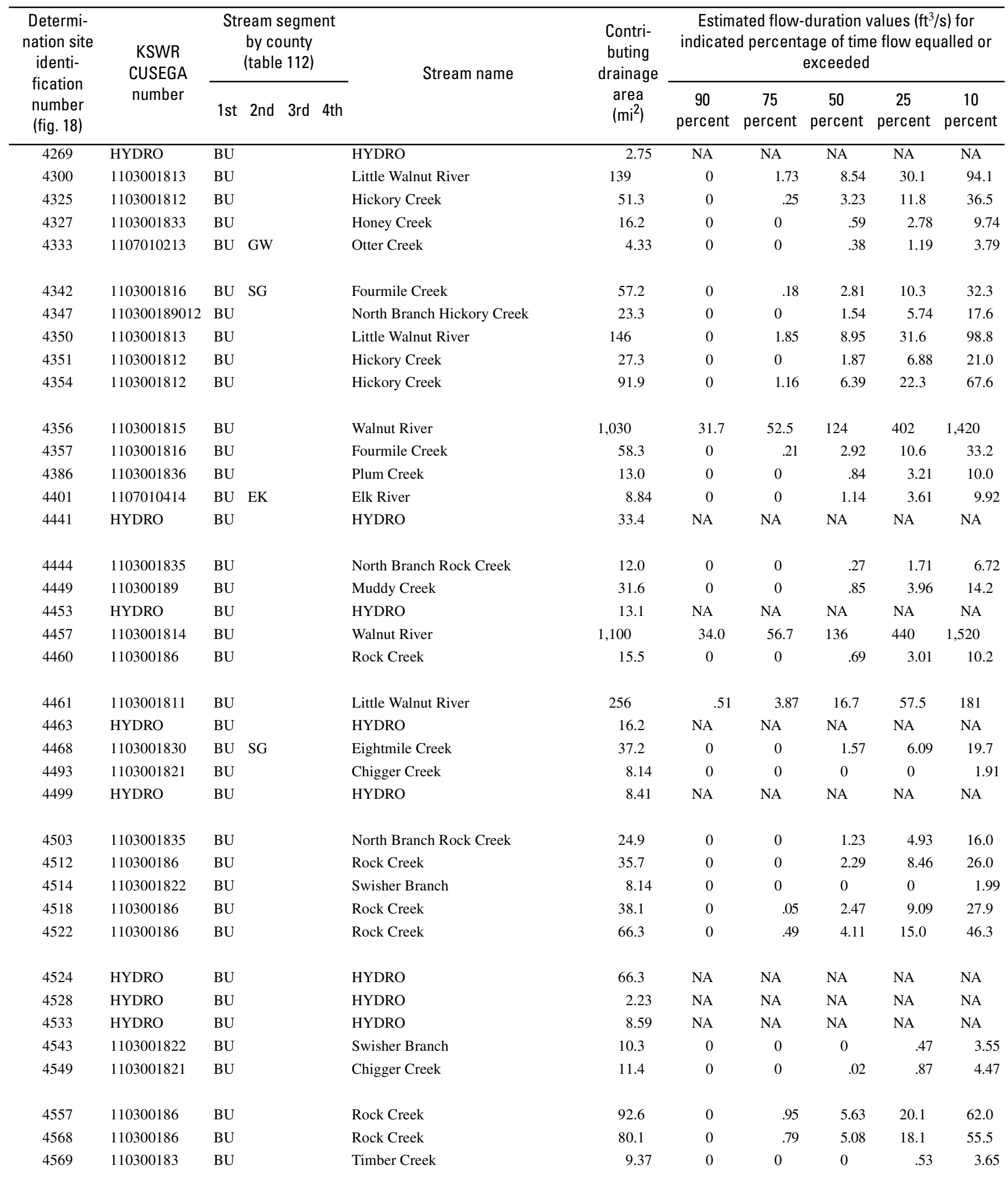


Table 14. Estimated flow-duration values, mean flow values, and peak-discharge frequency values for controlled and uncontrolled flow stream segments on the 1999 Kansas Surface Water Register for Butler County.-Continued

[KSWR, Kansas Surface Water Register; CUSEGA, catalog unit segment number alpha; $\mathrm{mi}^{2}$, square miles; $\mathrm{ft}^{3} / \mathrm{s}$, cubic feet per second; HYDRO, lake or other hydrologic structure; NA, not applicable; NRDitch, irrigation ditch; NRTribal, tribal stream]

\begin{tabular}{|c|c|c|c|c|c|c|c|}
\hline \multirow{2}{*}{$\begin{array}{l}\text { Determi- } \\
\text { nation site } \\
\text { identi- } \\
\text { fication } \\
\text { number } \\
\text { (fig. 18) }\end{array}$} & \multirow{2}{*}{$\begin{array}{c}\text { Estimated mean } \\
\text { flow } \\
\left(\mathrm{ft}^{3} / \mathrm{s}\right)\end{array}$} & \multicolumn{6}{|c|}{ Estimated peak discharge $\left(\mathrm{ft}^{3} / \mathrm{s}\right)$ for indicated peak-discharge frequency } \\
\hline & & 2-year & 5-year & 10 -year & 25-year & 50-year & 100 -year \\
\hline 4269 & NA & NA & NA & NA & NA & NA & NA \\
\hline 4327 & 9.15 & 1,490 & 3,260 & 4,810 & 7,180 & 9,160 & 11,400 \\
\hline 4333 & 3.33 & 718 & 1,520 & 2,200 & 3,230 & 4,090 & 5,050 \\
\hline 4342 & 26.8 & 2,700 & 6,080 & 9,210 & 14,100 & 18,300 & 23,100 \\
\hline 4347 & 14.5 & 1,870 & 4,100 & 6,060 & 9,070 & 11,600 & 14,500 \\
\hline 4356 & 575 & 10,000 & 19,500 & 24,200 & 35,600 & 46,700 & 58,800 \\
\hline 4357 & 27.5 & 2,690 & 6,070 & 9,190 & 14,000 & 18,300 & 23,100 \\
\hline 4386 & 8.54 & 1,310 & 2,860 & 4,210 & 6,270 & 7,980 & 9,950 \\
\hline 4401 & 7.38 & 1,090 & 2,340 & 3,420 & 5,060 & 6,420 & 7,980 \\
\hline 4441 & NA & NA & NA & NA & NA & NA & NA \\
\hline 4444 & 6.82 & 1,270 & 2,760 & 4,050 & 6,020 & 7,650 & 9,530 \\
\hline 4449 & 14.1 & 2,830 & 6,040 & 8,880 & 13,200 & 16,900 & 20,900 \\
\hline 4453 & NA & NA & NA & NA & NA & NA & NA \\
\hline 4457 & 622 & 10,700 & 20,800 & 25,900 & 38,100 & 49,900 & 62,900 \\
\hline 4503 & 14.0 & 1,910 & 4,210 & 6,240 & 9,360 & 12,000 & 15,000 \\
\hline 4512 & 21.0 & 3,080 & 6,530 & 9,580 & 14,200 & 18,200 & 22,500 \\
\hline 4514 & 3.46 & 971 & 2,110 & 3,100 & 4,600 & 5,860 & 7,290 \\
\hline 4518 & 22.4 & 3,150 & 6,680 & 9,800 & 14,500 & 18,600 & 23,000 \\
\hline 4522 & 36.2 & 4,430 & 9,130 & 13,300 & 19,400 & 24,800 & 30,600 \\
\hline 4524 & NA & NA & NA & NA & NA & NA & NA \\
\hline 4528 & NA & NA & NA & NA & NA & NA & NA \\
\hline 4533 & NA & NA & NA & NA & NA & NA & NA \\
\hline 4543 & 4.71 & 1,110 & 2,430 & 3,570 & 5,320 & 6,790 & 8,460 \\
\hline 4549 & 5.35 & 1,170 & 2,570 & 3,790 & 5,650 & 7,210 & 8,990 \\
\hline 4557 & 47.8 & 4,840 & 10,000 & 14,600 & 21,400 & 27,400 & 33,900 \\
\hline 4568 & 42.7 & 4,500 & 9,320 & 13,600 & 20,000 & 25,600 & 31,700 \\
\hline 4569 & 4.63 & 1,070 & 2,330 & 3,420 & 5,070 & 6,450 & 8,030 \\
\hline
\end{tabular}


Table 14. Estimated flow-duration values, mean flow values, and peak-discharge frequency values for controlled and uncontrolled flow stream segments on the 1999 Kansas Surface Water Register for Butler County.-Continued

[KSWR, Kansas Surface Water Register; CUSEGA, catalog unit segment number alpha; $\mathrm{mi}^{2}$, square miles; $\mathrm{ft}^{3} / \mathrm{s}$, cubic feet per second; HYDRO, lake or other hydrologic structure; NA, not applicable; NRDitch, irrigation ditch; NRTribal, tribal stream]

\begin{tabular}{|c|c|c|c|c|c|c|c|c|c|c|c|}
\hline $\begin{array}{l}\text { Determi- } \\
\text { nation site } \\
\text { identi- } \\
\text { fication } \\
\text { number } \\
\text { (fig. 18) }\end{array}$ & $\begin{array}{l}\text { KSWR } \\
\text { CUSEGA } \\
\text { number }\end{array}$ & \multicolumn{3}{|c|}{$\begin{array}{l}\text { Stream segment } \\
\text { by county } \\
\text { (table 112) }\end{array}$} & Stream name & $\begin{array}{l}\text { Contri- } \\
\text { buting } \\
\text { drainage } \\
\text { area } \\
\left(\mathrm{mi}^{2}\right)\end{array}$ & \multicolumn{5}{|c|}{$\begin{array}{c}\text { Estimated flow-duration values }\left(\mathrm{ft}^{3} / \mathrm{s}\right) \text { for } \\
\text { indicated percentage of time flow equalled or } \\
\text { exceeded }\end{array}$} \\
\hline 4571 & HYDRO & $\overline{B U}$ & & & HYDRO & 10.0 & NA & NA & NA & NA & NA \\
\hline 4584 & 110300186 & $\mathrm{BU}$ & $\mathrm{CL}$ & & Rock Creek & 107 & 0 & 1.13 & 6.25 & 22.3 & 69.5 \\
\hline 4595 & 1103001810 & $\mathrm{BU}$ & CL & & Walnut River & 1,360 & 42.6 & 72.4 & 177 & 579 & 1,900 \\
\hline 4596 & 110300189 & $\mathrm{BU}$ & CL & & Muddy Creek & 54.3 & 0 & .22 & 2.74 & 9.88 & 30.9 \\
\hline 4602 & 1106000116 & $\mathrm{BU}$ & $\mathrm{CL}$ & & Grouse Creek & 27.5 & 0 & 0 & 2.07 & 7.53 & 22.6 \\
\hline 4637 & 110300184 & BU & CL & & Dutch Creek & 10.9 & 0 & 0 & 0 & .56 & 3.90 \\
\hline 4771 & 110300183 & $\mathrm{BU}$ & $\mathrm{CL}$ & & Timber Creek & 37.8 & 0 & 0 & 1.93 & 7.32 & 23.2 \\
\hline
\end{tabular}


Table 14. Estimated flow-duration values, mean flow values, and peak-discharge frequency values for controlled and uncontrolled flow stream segments on the 1999 Kansas Surface Water Register for Butler County.-Continued

[KSWR, Kansas Surface Water Register; CUSEGA, catalog unit segment number alpha; $\mathrm{mi}^{2}$, square miles; $\mathrm{ft}^{3} / \mathrm{s}$, cubic feet per second; HYDRO, lake or other hydrologic structure; NA, not applicable; NRDitch, irrigation ditch; NRTribal, tribal stream]

\begin{tabular}{|c|c|c|c|c|c|c|c|}
\hline $\begin{array}{l}\text { Determi- } \\
\text { nation site } \\
\text { identi- } \\
\text { fication } \\
\text { number } \\
\text { (fig. 18) }\end{array}$ & $\begin{array}{c}\text { Estimated mean } \\
\text { flow } \\
\left(\mathrm{ft}^{3} / \mathrm{s}\right)\end{array}$ & \multicolumn{6}{|c|}{ Estimated peak discharge $\left(\mathrm{ft}^{3} / \mathrm{s}\right)$ for indicated peak-discharge frequency } \\
\hline 4571 & NA & NA & NA & NA & $\mathrm{NA}$ & NA & NA \\
\hline 4584 & 53.5 & 5,160 & 10,600 & 15,500 & 22,800 & 29,100 & 36,100 \\
\hline 4595 & 795 & 13,300 & 25,900 & 32,100 & 47,300 & 62,000 & 78,100 \\
\hline 4596 & 26.1 & 3,350 & 7,110 & 10,500 & 15,500 & 19,900 & 24,700 \\
\hline 4602 & 17.9 & 2,120 & 4,660 & 6,880 & 10,300 & 13,100 & 16,400 \\
\hline 4637 & 5.01 & 1,140 & 2,490 & 3,670 & 5,480 & 6,990 & 8,710 \\
\hline 4771 & 19.6 & 2,950 & 6,340 & 9,370 & 14,000 & 18,000 & 22,300 \\
\hline
\end{tabular}




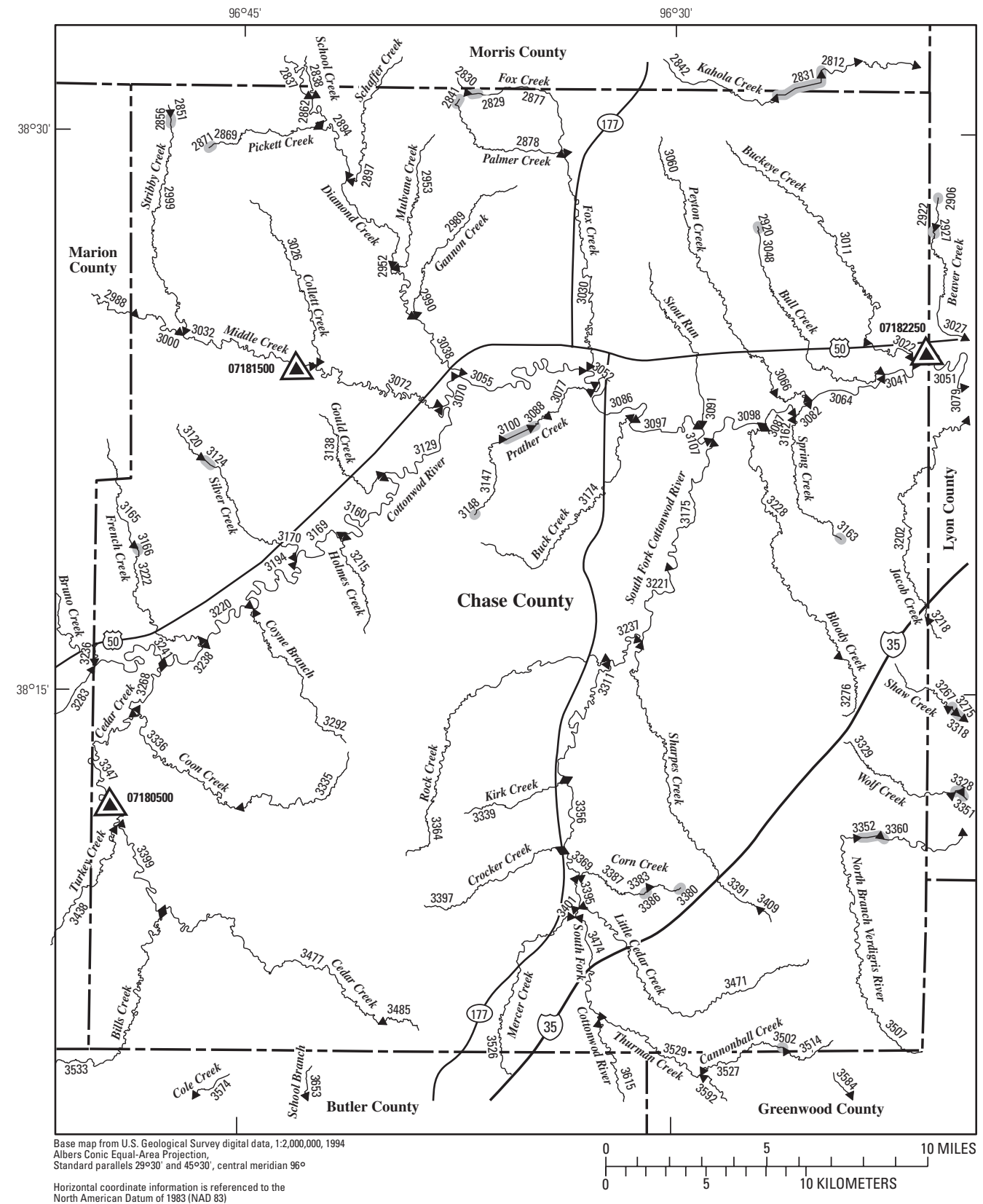

EXPLANATION

\footnotetext{
4773 Location of streamflow-statistics determination site (small triangle) and associated identification number-small triangle points in downstream direction

07182250 U.S. Geological Survey streamflow-gaging station and number used for estimates of flow duration

${ }^{07181500} \triangle$ U.S. Geological Survey streamflow-gaging station and number used for estimates of peak-discharge frequency values

3502 Lake and determination site identification number
}

KANSAS

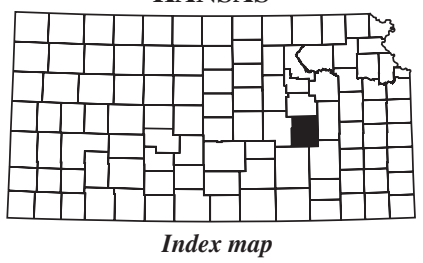

Figure 19. Location of streamflow-statistics determination sites, associated identification numbers, and U.S. Geological Survey streamflow-gaging stations used in the flow-duration and peak-discharge frequency analyses for Chase County. 
Table 15. Estimated flow-duration values, mean flow values, and peak-discharge frequency values for controlled and uncontrolled flow stream segments on the 1999 Kansas Surface Water Register for Chase County.

[KSWR, Kansas Surface Water Register; CUSEGA, catalog unit segment number alpha; $\mathrm{mi}^{2}$, square miles; $\mathrm{ft}^{3} / \mathrm{s}$, cubic feet per second; HYDRO, lake or other hydrologic structure; NA, not applicable; NRDitch, irrigation ditch; NRTribal, tribal stream]

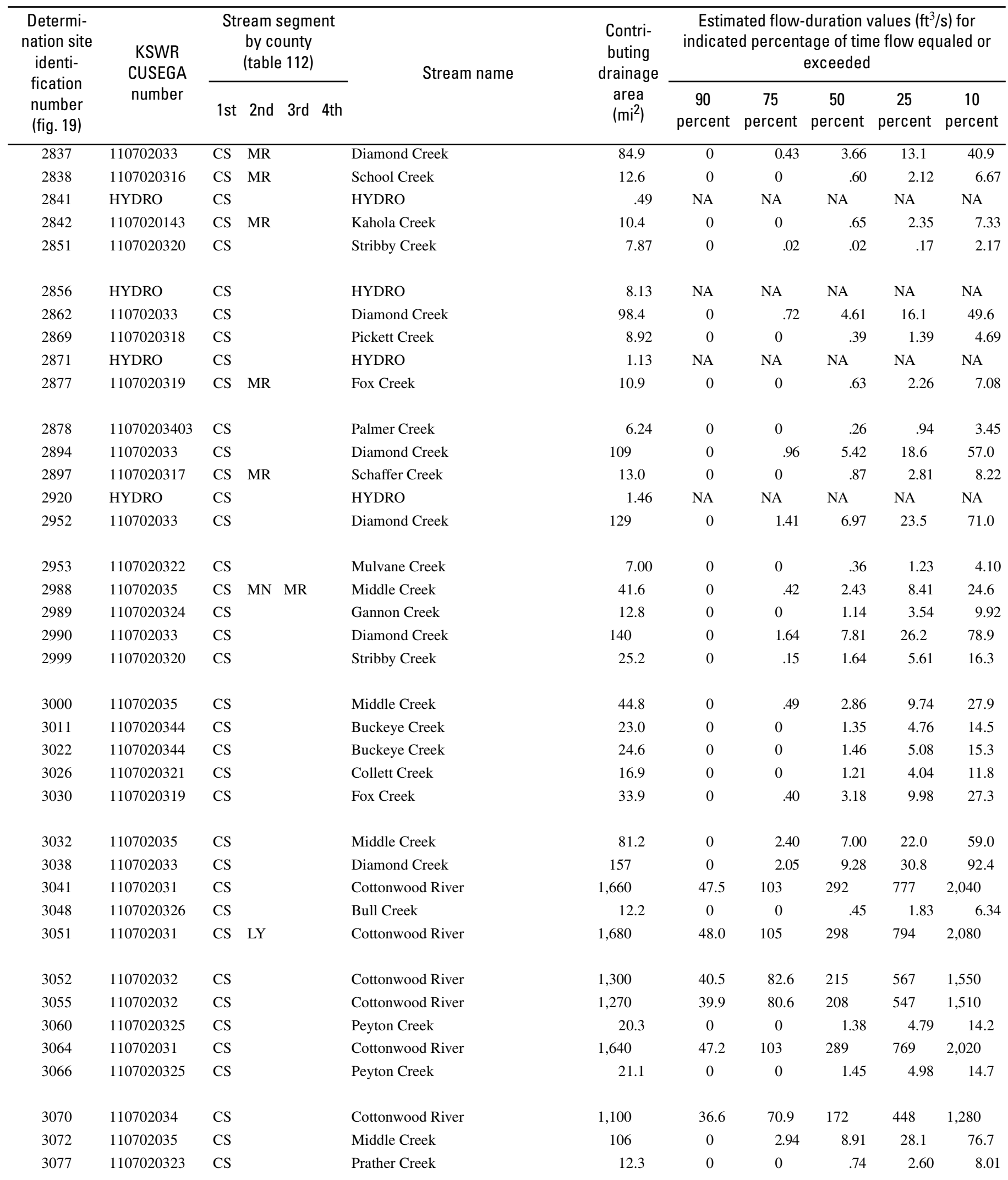


Table 15. Estimated flow-duration values, mean flow values, and peak-discharge frequency values for controlled and uncontrolled flow stream segments on the 1999 Kansas Surface Water Register for Chase County.-Continued

[KSWR, Kansas Surface Water Register; CUSEGA, catalog unit segment number alpha; $\mathrm{mi}^{2}$, square miles; $\mathrm{ft}^{3} / \mathrm{s}$, cubic feet per second; HYDRO, lake or other hydrologic structure; NA, not applicable; NRDitch, irrigation ditch; NRTribal, tribal stream]

\begin{tabular}{|c|c|c|c|c|c|c|c|}
\hline \multirow{2}{*}{$\begin{array}{l}\text { Determi- } \\
\text { nation site } \\
\text { identi- } \\
\text { fication } \\
\text { number } \\
\text { (fig. 19) }\end{array}$} & \multirow{2}{*}{$\begin{array}{c}\text { Estimated mean } \\
\text { flow } \\
\left(\mathrm{ft}^{3} / \mathrm{s}\right)\end{array}$} & \multicolumn{6}{|c|}{ Estimated peak discharge $\left(\mathrm{ft}^{3} / \mathrm{s}\right)$ for indicated peak-discharge frequency } \\
\hline & & 2-year & 5-year & 10 -year & 25-year & 50-year & 100 -year \\
\hline 2837 & 33.6 & 3,570 & 7,890 & 11,900 & 18,000 & 23,400 & 29,400 \\
\hline 2842 & 6.36 & 1,060 & 2,340 & 3,470 & 5,190 & 6,640 & 8,300 \\
\hline 2851 & 3.20 & 841 & 1,900 & 2,830 & 4,260 & 5,480 & 6,860 \\
\hline 2856 & NA & NA & NA & NA & NA & NA & NA \\
\hline 2862 & 39.5 & 3,750 & 8,280 & 12,400 & 18,900 & 24,600 & 30,900 \\
\hline 2878 & 3.47 & 745 & 1,650 & 2,450 & 3,670 & 4,690 & 5,850 \\
\hline 2894 & 44.3 & 3,860 & 8,510 & 12,800 & 19,400 & 25,300 & 31,800 \\
\hline 2897 & 6.94 & 1,080 & 2,460 & 3,690 & 5,600 & 7,220 & 9,070 \\
\hline 2920 & NA & NA & NA & NA & NA & NA & NA \\
\hline 2952 & 53.3 & 4,180 & 9,160 & 13,700 & 20,800 & 27,000 & 34,000 \\
\hline 2953 & 3.89 & 779 & 1,740 & 2,590 & 3,890 & 4,980 & 6,230 \\
\hline 2988 & 20.8 & 3,620 & 7,920 & 11,800 & 17,700 & 22,900 & 28,700 \\
\hline 2989 & 7.88 & 1,150 & 2,570 & 3,830 & 5,770 & 7,410 & 9,280 \\
\hline 2990 & 58.5 & 4,330 & 9,470 & 14,200 & 21,500 & 27,900 & 35,100 \\
\hline 3032 & 44.7 & 6,960 & 15,000 & 22,100 & 33,100 & 42,700 & 53,500 \\
\hline 3038 & 67.0 & 4,550 & 9,910 & 14,800 & 22,400 & 29,200 & 36,700 \\
\hline 3041 & 933 & 14,300 & 25,200 & 33,400 & 52,900 & 74,800 & 105,000 \\
\hline 3048 & 6.24 & 1,160 & 2,580 & 3,820 & 5,730 & 7,330 & 9,170 \\
\hline 3051 & 950 & 14,500 & 25,400 & 33,500 & 53,000 & 75,000 & 105,000 \\
\hline 3052 & 713 & 12,200 & 22,600 & 31,500 & 50,900 & 72,100 & 101,000 \\
\hline 3055 & 692 & 12,000 & 22,400 & 31,400 & 50,700 & 71,900 & 100,000 \\
\hline 3060 & 11.6 & 1,540 & 3,470 & 5,180 & 7,830 & 10,100 & 12,600 \\
\hline 3064 & 924 & 14,300 & 25,100 & 33,300 & 52,800 & 74,700 & 105,000 \\
\hline 3066 & 12.0 & 1,580 & 3,550 & 5,310 & 8,020 & 10,300 & 12,900 \\
\hline 3070 & 590 & 11,000 & 21,100 & 30,500 & 49,800 & 70,600 & 98,700 \\
\hline 3072 & 56.4 & 7,190 & 15,500 & 22,900 & 34,400 & 44,400 & 55,800 \\
\hline 3077 & 6.99 & 1,140 & 2,540 & 3,770 & 5,670 & 7,270 & 9,100 \\
\hline
\end{tabular}


Table 15. Estimated flow-duration values, mean flow values, and peak-discharge frequency values for controlled and uncontrolled flow stream segments on the 1999 Kansas Surface Water Register for Chase County.-Continued

[KSWR, Kansas Surface Water Register; CUSEGA, catalog unit segment number alpha; $\mathrm{mi}^{2}$, square miles; $\mathrm{ft}^{3} / \mathrm{s}$, cubic feet per second; HYDRO, lake or other hydrologic structure; NA, not applicable; NRDitch, irrigation ditch; NRTribal, tribal stream]

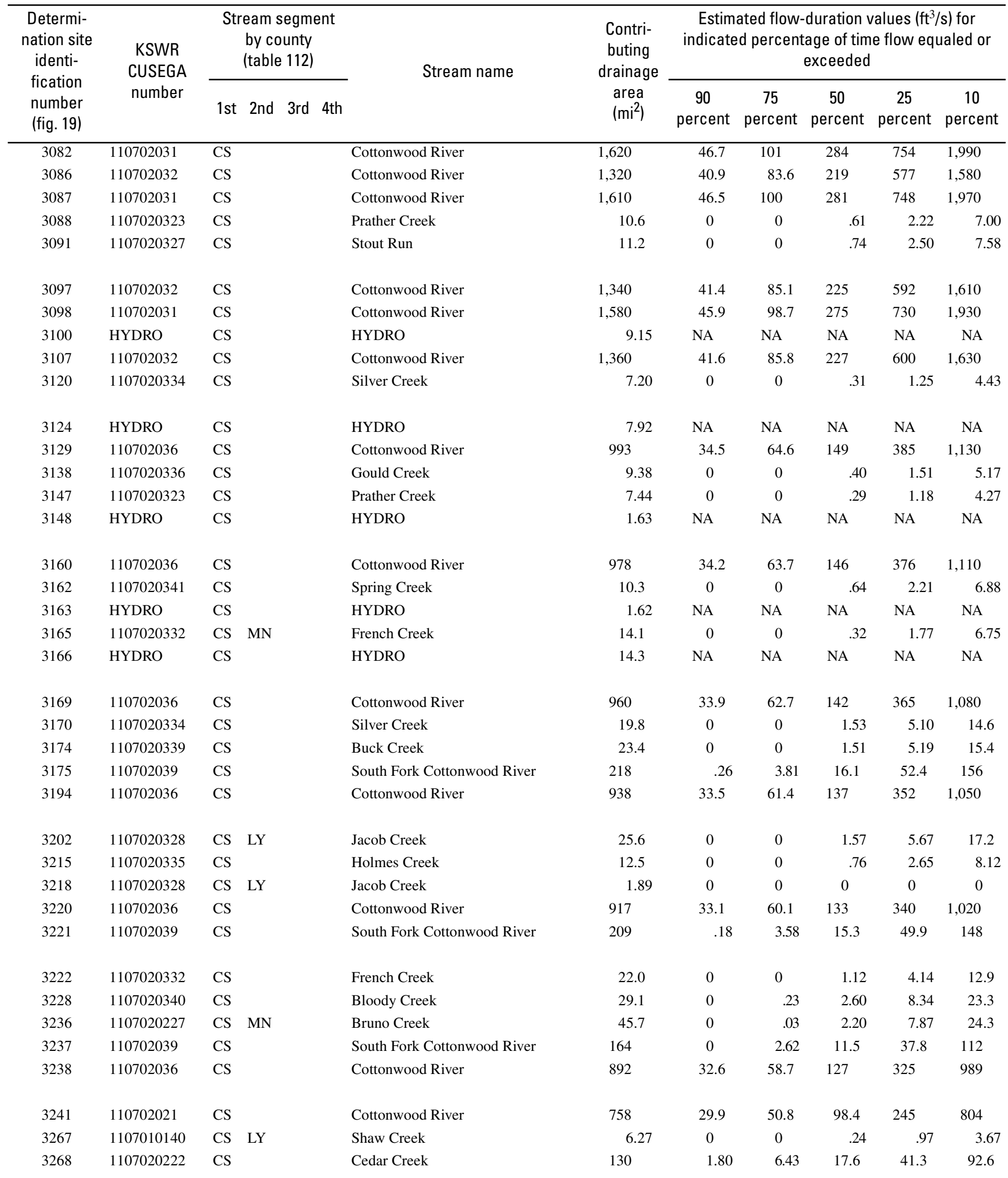


Table 15. Estimated flow-duration values, mean flow values, and peak-discharge frequency values for controlled and uncontrolled flow stream segments on the 1999 Kansas Surface Water Register for Chase County.-Continued

[KSWR, Kansas Surface Water Register; CUSEGA, catalog unit segment number alpha; $\mathrm{mi}^{2}$, square miles; $\mathrm{ft}^{3} / \mathrm{s}$, cubic feet per second; HYDRO, lake or other hydrologic structure; NA, not applicable; NRDitch, irrigation ditch; NRTribal, tribal stream]

\begin{tabular}{|c|c|c|c|c|c|c|c|}
\hline \multirow{2}{*}{$\begin{array}{l}\text { Determi- } \\
\text { nation site } \\
\text { identi- } \\
\text { fication } \\
\text { number } \\
\text { (fig. 19) }\end{array}$} & \multirow{2}{*}{$\begin{array}{c}\text { Estimated mean } \\
\text { flow } \\
\left(\mathrm{ft}^{3} / \mathrm{s}\right)\end{array}$} & \multicolumn{6}{|c|}{ Estimated peak discharge $\left(\mathrm{ft}^{3} / \mathrm{s}\right)$ for indicated peak-discharge frequency } \\
\hline & & 2-year & 5-year & 10 -year & 25-year & 50-year & 100 -year \\
\hline 3082 & 909 & 14,100 & 24,900 & 33,200 & 52,600 & 74,500 & 104,000 \\
\hline 3088 & 6.18 & 1,040 & 2,320 & 3,450 & 5,180 & 6,630 & 8,300 \\
\hline 3091 & 6.57 & 1,090 & 2,420 & 3,590 & 5,400 & 6,910 & 8,640 \\
\hline 3097 & 740 & 12,400 & 22,900 & 31,800 & 51,100 & 72,500 & 101,000 \\
\hline 3098 & 883 & 13,900 & 24,600 & 32,900 & 52,400 & 74,200 & 104,000 \\
\hline 3124 & NA & NA & NA & NA & NA & NA & NA \\
\hline 3129 & 524 & 10,300 & 20,400 & 30,000 & 49,200 & 69,800 & 97,500 \\
\hline 3138 & 5.00 & 958 & 2,140 & 3,170 & 4,760 & 6,100 & 7,630 \\
\hline 3147 & 4.20 & 848 & 1,880 & 2,780 & 4,150 & 5,310 & 6,620 \\
\hline 3148 & NA & NA & NA & NA & NA & NA & NA \\
\hline 3160 & 514 & 10,200 & 20,300 & 29,900 & 49,100 & 69,700 & 97,300 \\
\hline 3162 & 6.11 & 1,060 & 2,340 & 3,460 & 5,180 & 6,620 & 8,270 \\
\hline 3163 & NA & NA & NA & NA & NA & NA & NA \\
\hline 3165 & 6.83 & 1,230 & 2,750 & 4,100 & 6,170 & 7,920 & 9,920 \\
\hline 3202 & 14.3 & 1,840 & 4,110 & 6,130 & 9,250 & 11,900 & 14,900 \\
\hline 3215 & 7.03 & 1,120 & 2,520 & 3,760 & 5,670 & 7,270 & 9,120 \\
\hline 3218 & .48 & 416 & 876 & 1,270 & 1,850 & 2,340 & 2,880 \\
\hline 3220 & 477 & 9,860 & 19,800 & 29,600 & 48,800 & 69,300 & 96,700 \\
\hline 3221 & 100 & 6,310 & 13,000 & 19,100 & 28,200 & 36,100 & 45,000 \\
\hline 3222 & 11.2 & 1,560 & 3,550 & 5,330 & 8,080 & 10,400 & 13,100 \\
\hline 3228 & 17.5 & 1,940 & 4,380 & 6,550 & 9,910 & 12,700 & 16,000 \\
\hline 3236 & 20.4 & 3,320 & 7,080 & 10,400 & 15,400 & 19,800 & 24,600 \\
\hline 3237 & 78.1 & 5,590 & 11,600 & 17,100 & 25,300 & 32,400 & 40,300 \\
\hline 3238 & 461 & 9,710 & 19,600 & 29,400 & 48,600 & 69,100 & 96,400 \\
\hline 3241 & 378 & 8,900 & 18,700 & 28,700 & 47,900 & 68,100 & 94,900 \\
\hline 3267 & 3.75 & 832 & 1,800 & 2,640 & 3,920 & 4,980 & 6,190 \\
\hline 3268 & 69.5 & 6,310 & 12,000 & 16,500 & 22,700 & 27,900 & 33,200 \\
\hline
\end{tabular}


Table 15. Estimated flow-duration values, mean flow values, and peak-discharge frequency values for controlled and uncontrolled flow stream segments on the 1999 Kansas Surface Water Register for Chase County.-Continued

[KSWR, Kansas Surface Water Register; CUSEGA, catalog unit segment number alpha; $\mathrm{mi}^{2}$, square miles; $\mathrm{ft}^{3} / \mathrm{s}$, cubic feet per second; HYDRO, lake or other hydrologic structure; NA, not applicable; NRDitch, irrigation ditch; NRTribal, tribal stream]

\begin{tabular}{|c|c|c|c|c|c|c|c|c|c|c|c|}
\hline $\begin{array}{l}\text { Determi- } \\
\text { nation site } \\
\text { identi- } \\
\text { fication } \\
\text { number } \\
\text { (fig. 19) }\end{array}$ & $\begin{array}{l}\text { KSWR } \\
\text { CUSEGA } \\
\text { number }\end{array}$ & \multicolumn{3}{|c|}{$\begin{array}{l}\text { Stream segment } \\
\text { by county } \\
\text { (table 112) }\end{array}$} & Stream name & $\begin{array}{l}\text { Contri- } \\
\text { buting } \\
\text { drainage } \\
\text { area } \\
\left(\mathrm{mi}^{2}\right)\end{array}$ & \multicolumn{5}{|c|}{$\begin{array}{c}\text { Estimated flow-duration values }\left(\mathrm{ft}^{3} / \mathrm{s}\right) \text { for } \\
\text { indicated percentage of time flow equaled or } \\
\text { exceeded }\end{array}$} \\
\hline 3276 & 1107020340 & $\mathrm{CS}$ & & & Bloody Creek & 8.53 & 0 & 0 & 0.79 & 2.46 & 7.10 \\
\hline 3311 & 110702039 & $\mathrm{CS}$ & & & South Fork Cottonwood River & 129 & 0 & 2.04 & 9.39 & 30.8 & 90.4 \\
\hline 3329 & 1107010141 & $\mathrm{CS}$ & LY & & Wolf Creek & 13.1 & 0 & .01 & 1.09 & 3.62 & 10.5 \\
\hline 3335 & 1107020232 & $\mathrm{CS}$ & & & Coon Creek & 16.6 & 0 & 0 & .25 & 1.58 & 6.42 \\
\hline 3336 & 1107020232 & $\mathrm{CS}$ & & & Coon Creek & 28.7 & 0 & 0 & 1.13 & 4.37 & 14.1 \\
\hline 3356 & 110702039 & CS & & & South Fork Cottonwood River & 114 & 0 & 1.69 & 8.15 & 27.0 & 79.3 \\
\hline 3360 & 1107010115 & CS & LY & & North Branch Verdigris River & 38.6 & .02 & .61 & 4.54 & 14.2 & 38.3 \\
\hline 3364 & 1107020337 & $\mathrm{CS}$ & & & Rock Creek & 33.1 & 0 & .01 & 1.97 & 6.71 & 19.9 \\
\hline 3369 & 110702039 & CS & & & South Fork Cottonwood River & 94.7 & 0 & 1.37 & 6.97 & 23.1 & 67.2 \\
\hline 3380 & HYDRO & $\mathrm{CS}$ & & & HYDRO & .74 & NA & NA & NA & NA & NA \\
\hline 3383 & 1107020347 & CS & & & Corn Creek & 3.96 & 0 & 0 & 0 & .17 & 1.52 \\
\hline 3386 & HYDRO & $\mathrm{CS}$ & & & HYDRO & 4.39 & NA & NA & NA & NA & NA \\
\hline 3387 & 1107020347 & $\mathrm{CS}$ & & & Corn Creek & 7.80 & 0 & 0 & .39 & 1.43 & 4.82 \\
\hline 3391 & 1107020338 & $\mathrm{CS}$ & & & Sharpes Creek & 35.6 & 0 & .41 & 3.36 & 10.7 & 29.6 \\
\hline 3471 & 1107020345 & CS & & & Little Cedar Creek & 18.6 & 0 & 0 & 1.40 & 4.76 & 13.9 \\
\hline 3474 & 110702039 & $\mathrm{CS}$ & & & South Fork Cottonwood River & 46.3 & 0 & .54 & 3.84 & 12.5 & 35.3 \\
\hline 3477 & 1107020222 & $\mathrm{CS}$ & & & Cedar Creek & 38.1 & .27 & .72 & 2.82 & 7.60 & 19.7 \\
\hline 3485 & 1107020222 & $\mathrm{CS}$ & & & Cedar Creek & 8.46 & .01 & .03 & .07 & .12 & 1.23 \\
\hline 3502 & HYDRO & CS & & & HYDRO & 5.04 & NA & NA & NA & NA & NA \\
\hline 3507 & 1107010115 & CS & & & North Branch Verdigris River & 26.5 & .01 & .31 & 3.18 & 9.97 & 26.8 \\
\hline 3514 & 11070203745 & $\mathrm{CS}$ & & & Cannonball Creek & 4.55 & 0 & 0 & .13 & .60 & 2.61 \\
\hline 3527 & 11070203745 & $\mathrm{CS}$ & GW & & Cannonball Creek & 9.30 & 0 & 0 & .60 & 2.14 & 6.72 \\
\hline 3529 & 1107020311 & $\mathrm{CS}$ & GW & & Thurman Creek & 25.9 & 0 & .09 & 2.22 & 7.27 & 20.5 \\
\hline
\end{tabular}


Table 15. Estimated flow-duration values, mean flow values, and peak-discharge frequency values for controlled and uncontrolled flow stream segments on the 1999 Kansas Surface Water Register for Chase County.-Continued

[KSWR, Kansas Surface Water Register; CUSEGA, catalog unit segment number alpha; $\mathrm{mi}^{2}$, square miles; $\mathrm{ft}^{3} / \mathrm{s}$, cubic feet per second; HYDRO, lake or other hydrologic structure; NA, not applicable; NRDitch, irrigation ditch; NRTribal, tribal stream]

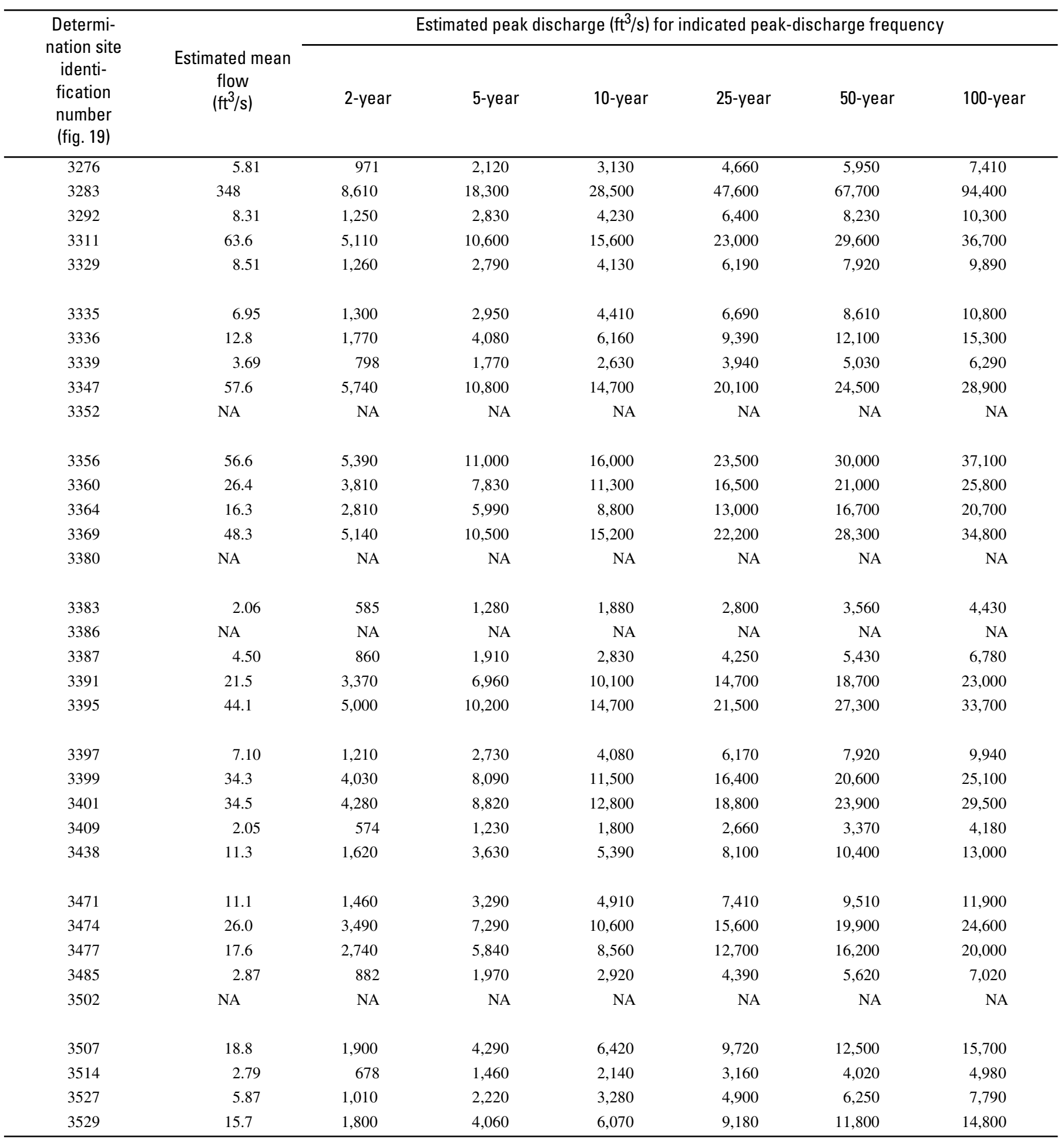


114 Estimates of Flow Duration, Mean Flow, and Peak-Discharge Frequency Values for Kansas Stream Locations 


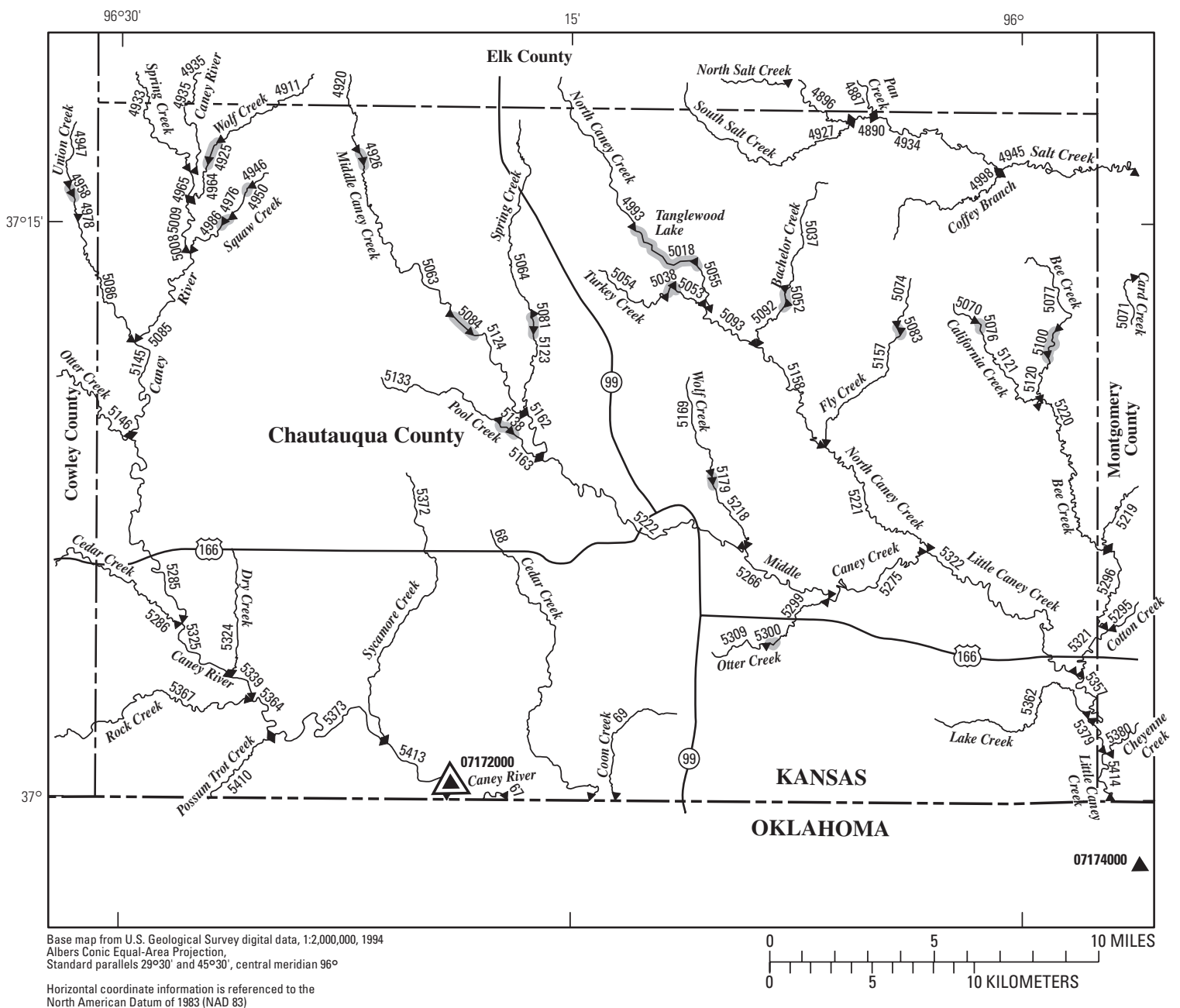

EXPLANATION

5410 Location of streamflow-statistics determination site (small triangle) and associated identification number-small triangle points in downstream direction

$07174000 \Delta$ U.S. Geological Survey streamflow-gaging station and number used for estimates of flow duration

${ }^{07172000} \triangle$ U.S. Geological Survey streamflow-gaging station and number used for estimates of peak-discharge frequency values

KANSAS

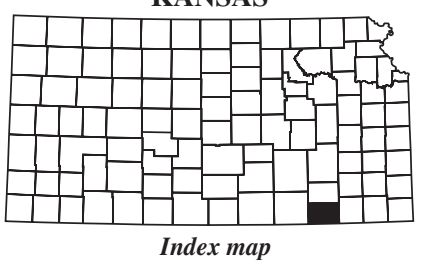

Index map 5179

Lake and determination site identification number

Figure 20. Location of streamflow-statistics determination sites, associated identification numbers, and U.S. Geological Survey streamflow-gaging stations used in the flow-duration and peak-discharge frequency analyses for Chautauqua County. 
Table 16. Estimated flow-duration values, mean flow values, and peak-discharge frequency values for controlled and uncontrolled flow stream segments on the 1999 Kansas Surface Water Register for Chautauqua County.

[KSWR, Kansas Surface Water Register; CUSEGA, catalog unit segment number alpha; $\mathrm{mi}^{2}$, square miles; $\mathrm{ft}^{3} / \mathrm{s}$, cubic feet per second; HYDRO, lake or other hydrologic structure; NA, not applicable; NRDitch, irrigation ditch; NRTribal, tribal stream]

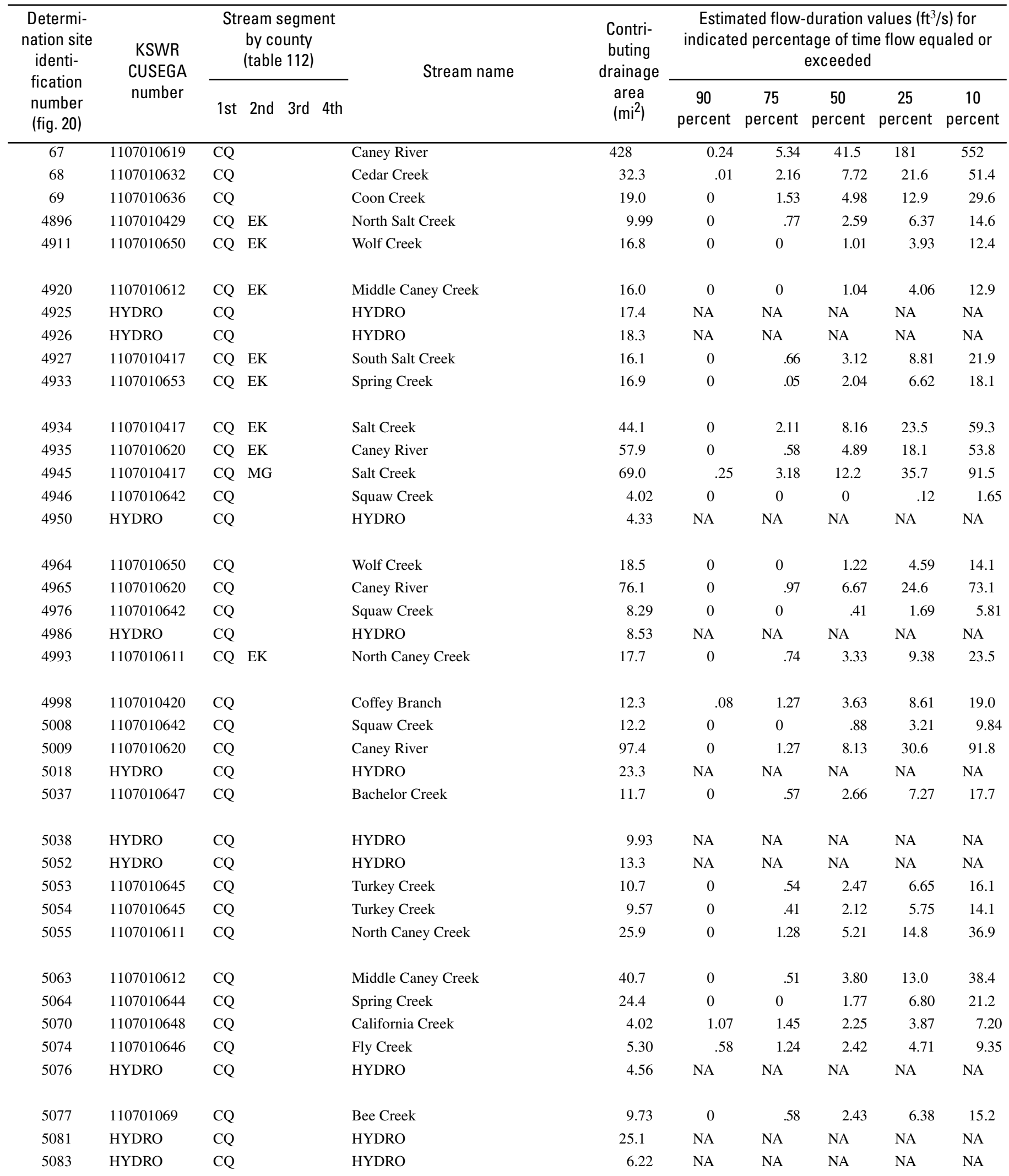


Table 16. Estimated flow-duration values, mean flow values, and peak-discharge frequency values for controlled and uncontrolled flow stream segments on the 1999 Kansas Surface Water Register for Chautauqua County.-Continued

[KSWR, Kansas Surface Water Register; CUSEGA, catalog unit segment number alpha; $\mathrm{mi}^{2}$, square miles; $\mathrm{ft}^{3} / \mathrm{s}$, cubic feet per second; HYDRO, lake or other hydrologic structure; NA, not applicable; NRDitch, irrigation ditch; NRTribal, tribal stream]

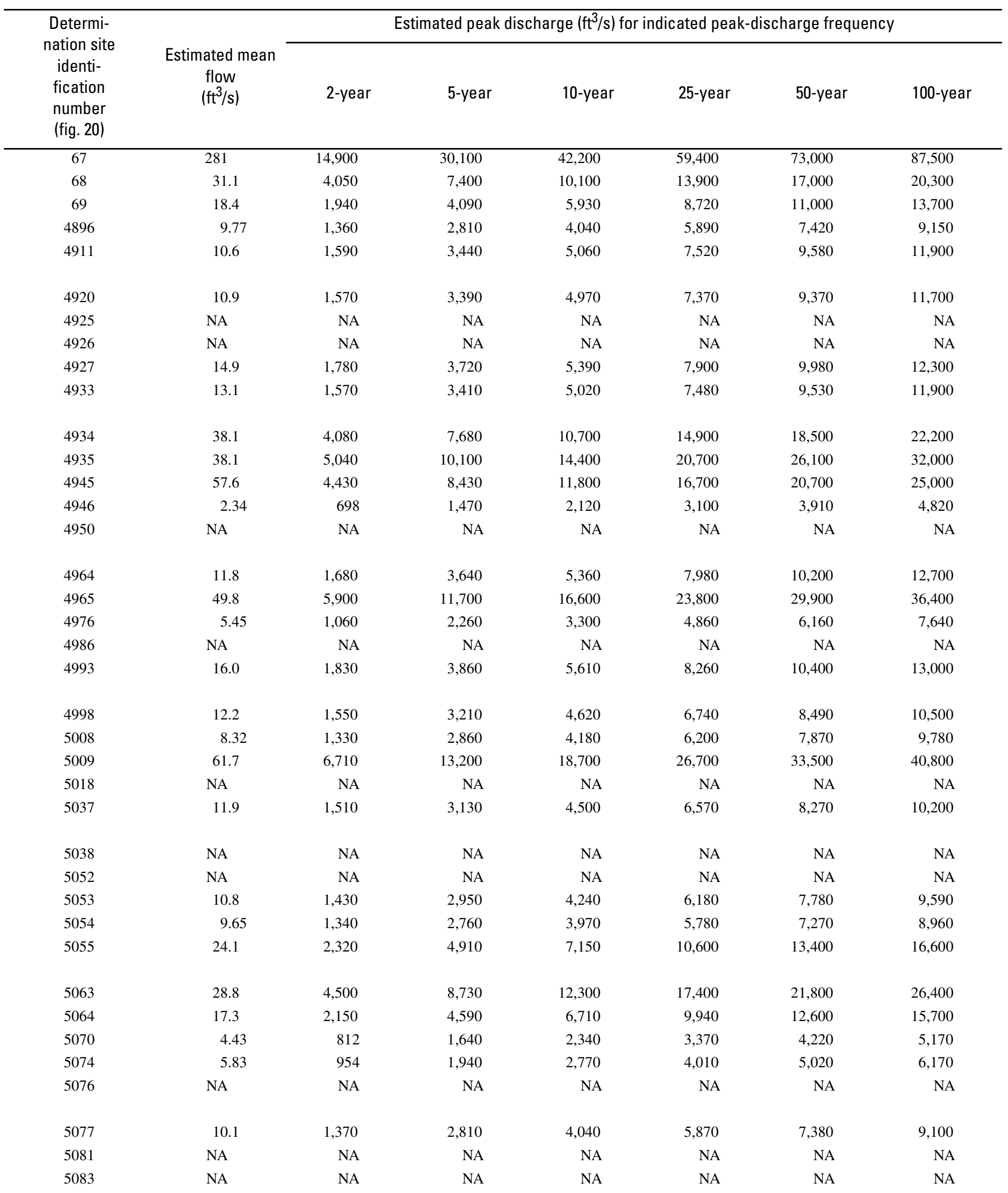


Table 16. Estimated flow-duration values, mean flow values, and peak-discharge frequency values for controlled and uncontrolled flow stream segments on the 1999 Kansas Surface Water Register for Chautauqua County.-Continued

[KSWR, Kansas Surface Water Register; CUSEGA, catalog unit segment number alpha; $\mathrm{mi}^{2}$, square miles; $\mathrm{ft}^{3} / \mathrm{s}$, cubic feet per second; HYDRO, lake or other hydrologic structure; NA, not applicable; NRDitch, irrigation ditch; NRTribal, tribal stream]

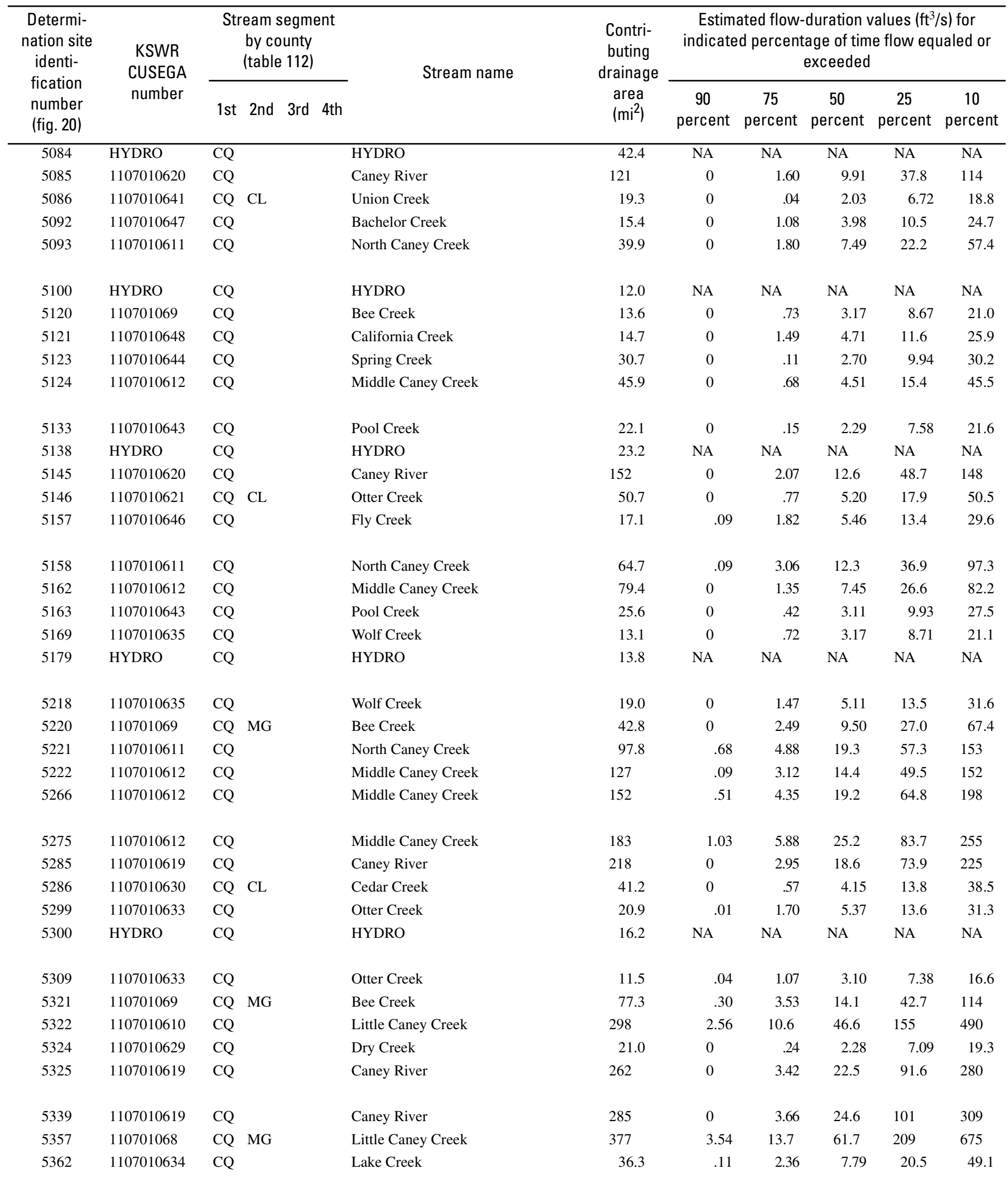


Table 16. Estimated flow-duration values, mean flow values, and peak-discharge frequency values for controlled and uncontrolled flow stream segments on the 1999 Kansas Surface Water Register for Chautauqua County.-Continued

[KSWR, Kansas Surface Water Register; CUSEGA, catalog unit segment number alpha; $\mathrm{mi}^{2}$, square miles; $\mathrm{ft}^{3} / \mathrm{s}$, cubic feet per second; HYDRO, lake or other hydrologic structure; NA, not applicable; NRDitch, irrigation ditch; NRTribal, tribal stream]

\begin{tabular}{|c|c|c|c|c|c|c|c|}
\hline \multirow{2}{*}{$\begin{array}{l}\text { Determi- } \\
\text { nation site } \\
\text { identi- } \\
\text { fication } \\
\text { number } \\
\text { (fig. 20) }\end{array}$} & \multirow{2}{*}{$\begin{array}{c}\text { Estimated mean } \\
\text { flow } \\
\left(\mathrm{ft}^{3} / \mathrm{s}\right)\end{array}$} & \multicolumn{6}{|c|}{ Estimated peak discharge $\left(\mathrm{ft}^{3} / \mathrm{s}\right)$ for indicated peak-discharge frequency } \\
\hline & & 2-year & 5-year & 10-year & 25-year & 50-year & 100 -year \\
\hline 5084 & NA & NA & NA & NA & NA & NA & NA \\
\hline 5092 & 15.8 & 1,770 & 3,690 & 5,330 & 7,790 & 9,830 & 12,100 \\
\hline 5093 & 37.0 & 4,850 & 8,860 & 12,100 & 16,700 & 20,500 & 24,500 \\
\hline 5100 & NA & NA & NA & NA & NA & NA & NA \\
\hline 5120 & 13.9 & 1,660 & 3,440 & 4,950 & 7,230 & 9,110 & 11,200 \\
\hline 5133 & 16.4 & 1,930 & 4,170 & 6,130 & 9,110 & 11,600 & 14,400 \\
\hline 5138 & NA & NA & NA & NA & NA & NA & NA \\
\hline 5145 & 93.7 & 8,020 & 15,800 & 22,400 & 32,100 & 40,300 & 49,000 \\
\hline 5146 & 34.5 & 5,140 & 10,000 & 14,200 & 20,200 & 25,300 & 30,700 \\
\hline 5157 & 18.0 & 1,880 & 3,920 & 5,660 & 8,300 & 10,500 & 13,000 \\
\hline 5158 & 60.8 & 5,130 & 9,510 & 13,100 & 18,300 & 22,600 & 27,100 \\
\hline 5162 & 59.0 & 6,350 & 12,100 & 16,900 & 23,900 & 29,800 & 36,200 \\
\hline 5163 & 20.0 & 2,130 & 4,610 & 6,770 & 10,100 & 12,800 & 16,000 \\
\hline 5169 & 13.8 & 1,660 & 3,410 & 4,900 & 7,140 & 8,980 & 11,100 \\
\hline 5275 & 163 & 7,560 & 14,200 & 19,800 & 28,000 & 34,900 & 42,400 \\
\hline 5285 & 134 & 9,210 & 18,400 & 26,200 & 37,500 & 47,000 & 57,300 \\
\hline 5286 & 26.9 & 4,590 & 9,000 & 12,700 & 18,100 & 22,700 & 27,600 \\
\hline 5299 & 19.9 & 2,040 & 4,310 & 6,260 & 9,220 & 11,700 & 14,500 \\
\hline 5300 & NA & NA & NA & NA & NA & NA & NA \\
\hline 5309 & 11.0 & 1,470 & 3,050 & 4,400 & 6,430 & 8,100 & 10,000 \\
\hline 5321 & 71.8 & 4,320 & 8,340 & 11,800 & 16,700 & 20,900 & 25,400 \\
\hline 5322 & 303 & 9,470 & 17,400 & 24,200 & 34,000 & 42,200 & 51,100 \\
\hline 5324 & 14.4 & 1,780 & 3,900 & 5,750 & 8,590 & 11,000 & 13,700 \\
\hline 5325 & 161 & 10,400 & 20,800 & 29,500 & 42,200 & 52,600 & 63,900 \\
\hline 5339 & 175 & 11,100 & 22,200 & 31,400 & 44,700 & 55,600 & 67,400 \\
\hline 5357 & 414 & 10,700 & 19,600 & 27,100 & 37,900 & 47,000 & 56,900 \\
\hline 5362 & 31.0 & 3,710 & 6,940 & 9,580 & 13,300 & 16,400 & 19,700 \\
\hline
\end{tabular}


Table 16. Estimated flow-duration values, mean flow values, and peak-discharge frequency values for controlled and uncontrolled flow stream segments on the 1999 Kansas Surface Water Register for Chautauqua County.-Continued

[KSWR, Kansas Surface Water Register; CUSEGA, catalog unit segment number alpha; $\mathrm{mi}^{2}$, square miles; $\mathrm{ft}^{3} / \mathrm{s}$, cubic feet per second; HYDRO, lake or other hydrologic structure; NA, not applicable; NRDitch, irrigation ditch; NRTribal, tribal stream]

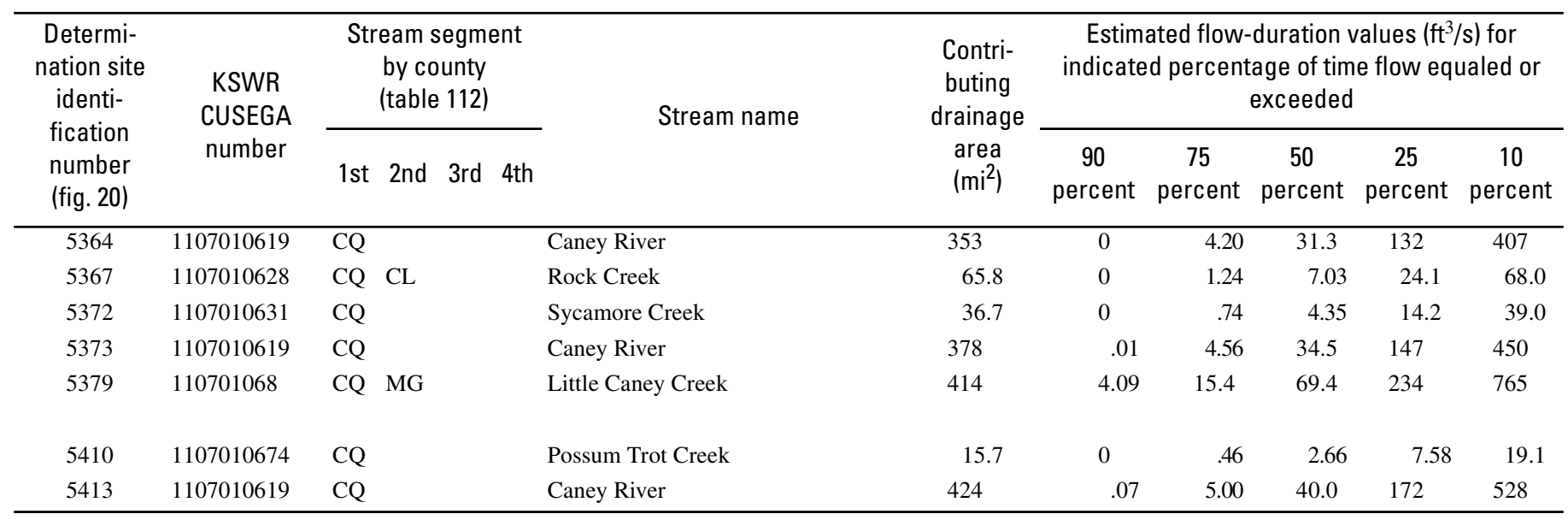


Table 16. Estimated flow-duration values, mean flow values, and peak-discharge frequency values for controlled and uncontrolled flow stream segments on the 1999 Kansas Surface Water Register for Chautauqua County.-Continued

[KSWR, Kansas Surface Water Register; CUSEGA, catalog unit segment number alpha; $\mathrm{mi}^{2}$, square miles; $\mathrm{ft}^{3} / \mathrm{s}$, cubic feet per second; HYDRO, lake or other hydrologic structure; NA, not applicable; NRDitch, irrigation ditch; NRTribal, tribal stream]

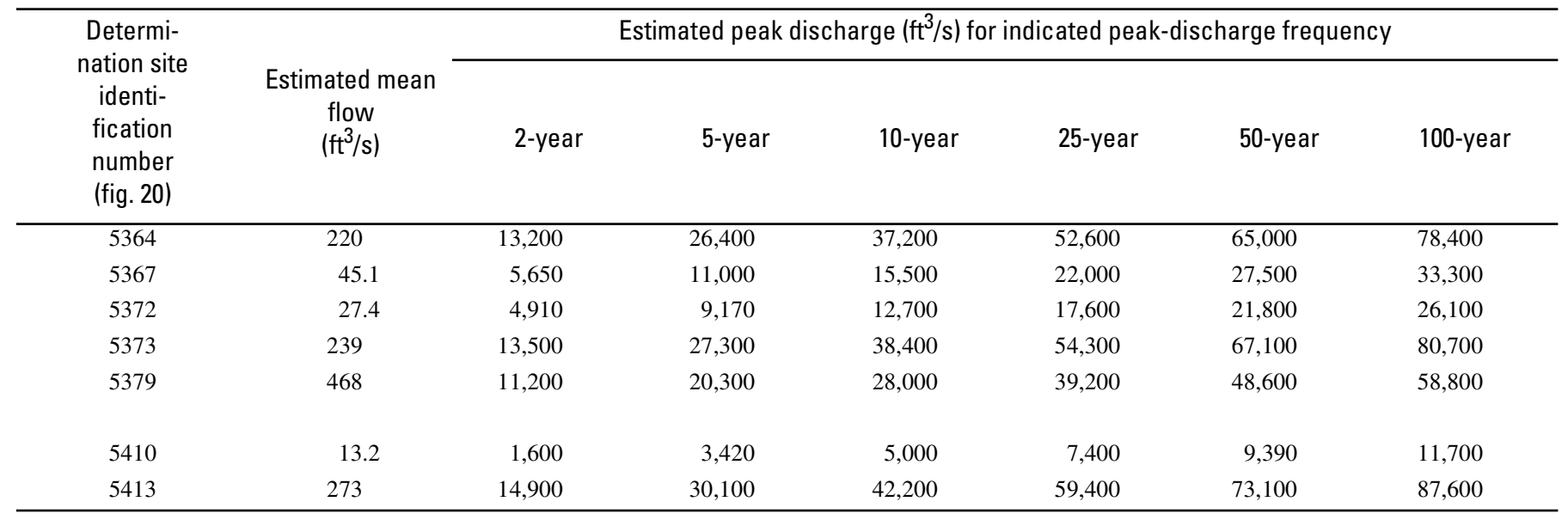




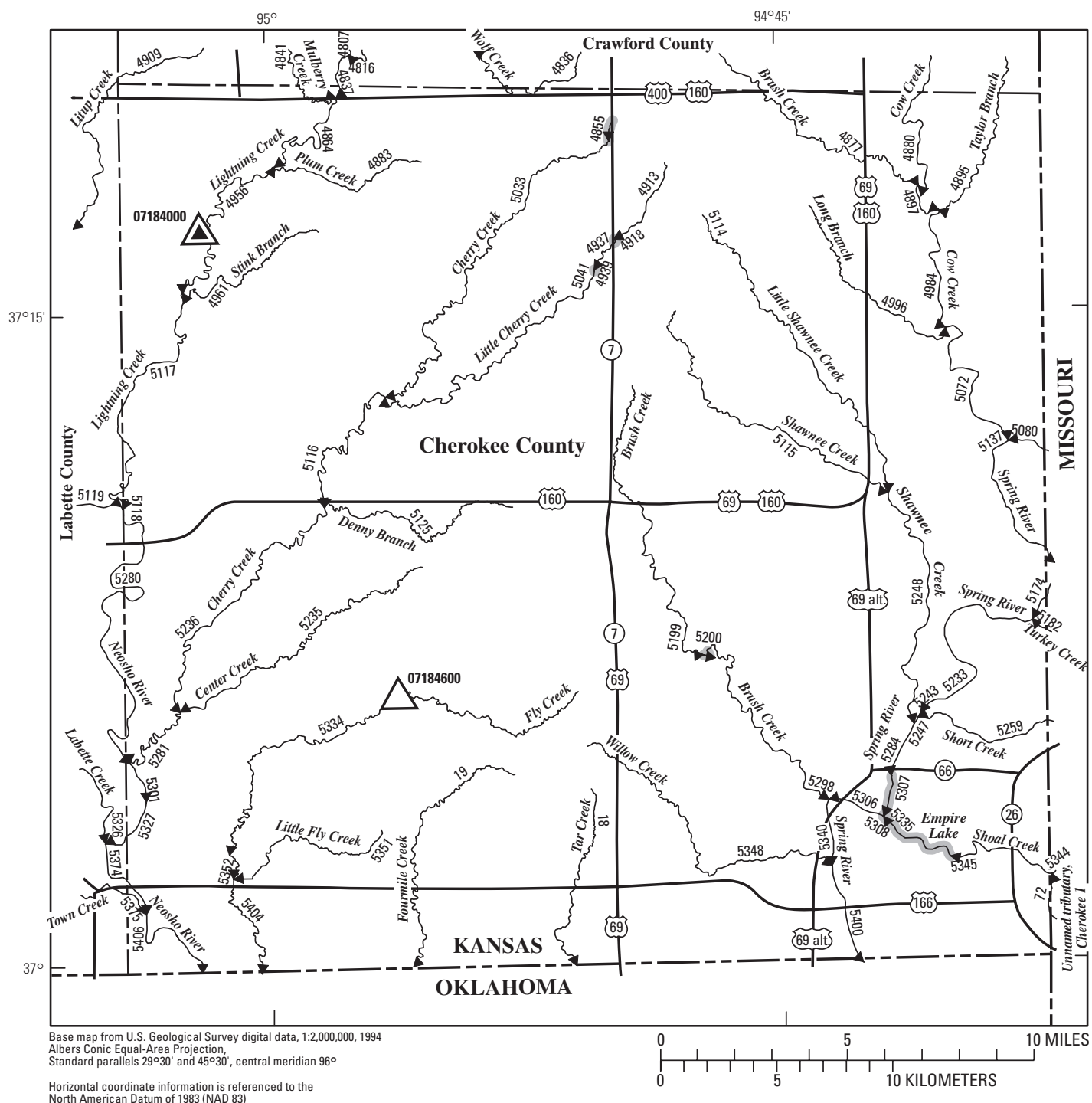

EXPLANATION

4504 Location of streamflow-statistics determination site (small triangle) and associated identification number-small triangle points in downstream direction

07184000

U.S. Geological Survey streamflow-gaging station and number used for estimates of flow duration

${ }^{07184600} \triangle$ U.S. Geological Survey streamflow-gaging station and number used for estimates of peak-discharge frequency values

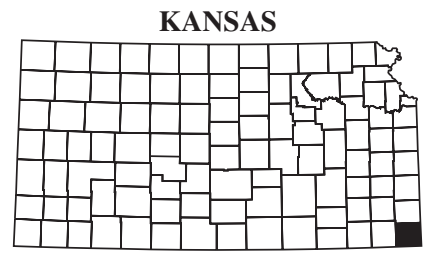

Index map 5335

Lake and determination site identification number

Figure 21. Location of streamflow-statistics determination sites, associated identification numbers, and U.S. Geological Survey streamflow-gaging stations used in the flow-duration and peak-discharge frequency analyses for Cherokee County. 
Table 17. Estimated flow-duration values, mean flow values, and peak-discharge frequency values for controlled and uncontrolled flow stream segments on the 1999 Kansas Surface Water Register for Cherokee County.

[KSWR, Kansas Surface Water Register; CUSEGA, catalog unit segment number alpha; $\mathrm{mi}^{2}$, square miles; $\mathrm{ft}^{3} / \mathrm{s}$, cubic feet per second; HYDRO, lake or other hydrologic structure; NA, not applicable; NRDitch, irrigation ditch; NRTribal, tribal stream]

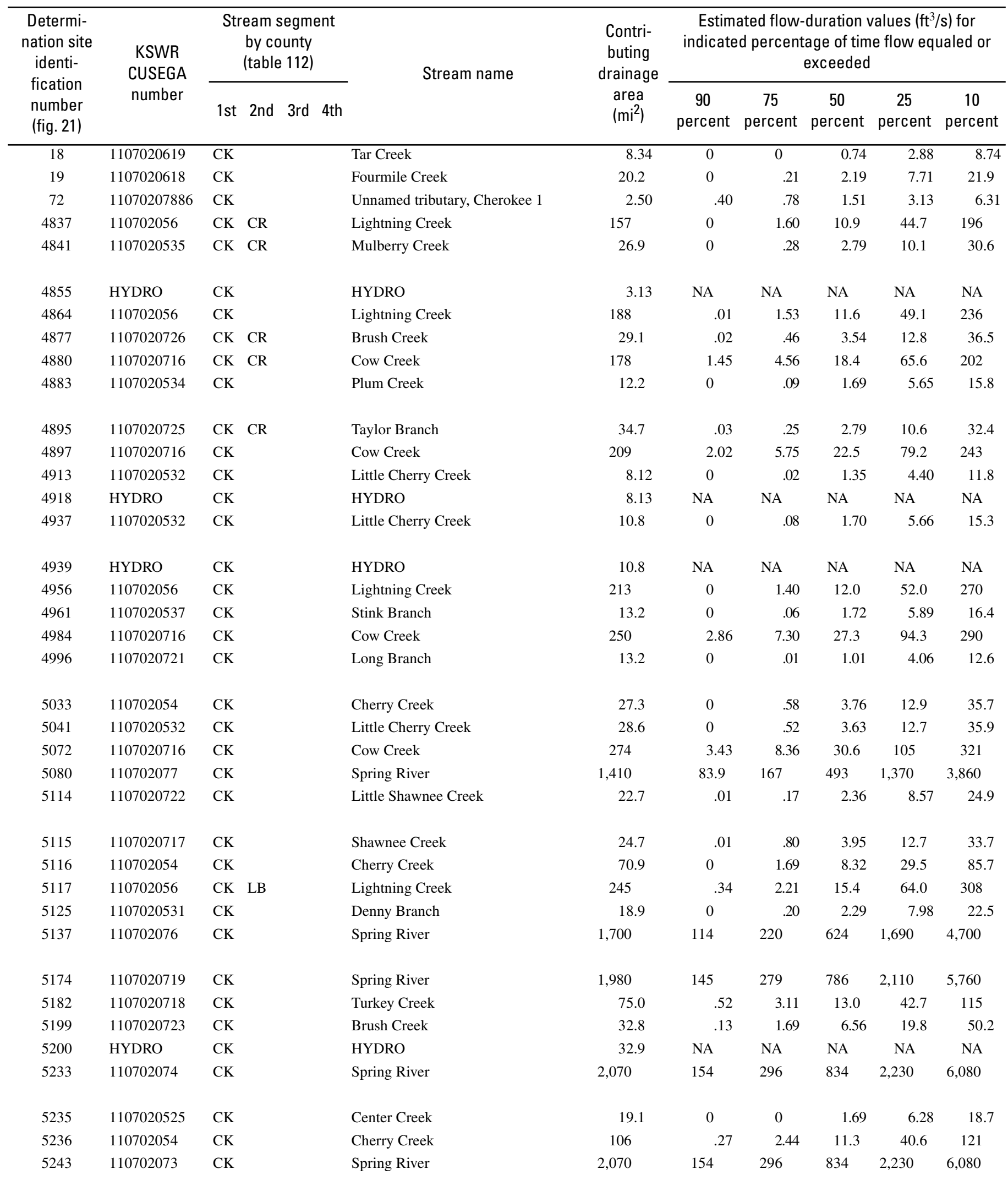


Table 17. Estimated flow-duration values, mean flow values, and peak-discharge frequency values for controlled and uncontrolled flow stream segments on the 1999 Kansas Surface Water Register for Cherokee County.-Continued

[KSWR, Kansas Surface Water Register; CUSEGA, catalog unit segment number alpha; $\mathrm{mi}^{2}$, square miles; $\mathrm{ft}^{3} / \mathrm{s}$, cubic feet per second; HYDRO, lake or other hydrologic structure; NA, not applicable; NRDitch, irrigation ditch; NRTribal, tribal stream]

\begin{tabular}{|c|c|c|c|c|c|c|c|}
\hline \multirow{2}{*}{$\begin{array}{l}\text { Determi- } \\
\text { nation site } \\
\text { identi- } \\
\text { fication } \\
\text { number } \\
\text { (fig. 21) }\end{array}$} & \multirow{2}{*}{$\begin{array}{c}\text { Estimated mean } \\
\text { flow } \\
\left(\mathrm{ft}^{3} / \mathrm{s}\right)\end{array}$} & \multicolumn{6}{|c|}{ Estimated peak discharge $\left(\mathrm{ft}^{3} / \mathrm{s}\right)$ for indicated peak-discharge frequency } \\
\hline & & 2-year & 5-year & 10-year & 25-year & 50-year & 100-year \\
\hline 18 & 7.93 & 1,790 & 3,370 & 4,650 & 6,520 & 8,030 & 9,730 \\
\hline 4837 & 129 & 7,520 & 16,000 & 24,100 & 37,500 & 50,100 & 65,200 \\
\hline 4841 & 23.7 & 3,160 & 6,310 & 8,960 & 13,000 & 16,200 & 20,000 \\
\hline 4855 & NA & NA & NA & $\mathrm{NA}$ & $\mathrm{NA}$ & NA & $\mathrm{NA}$ \\
\hline 4864 & 151 & 7,440 & 16,600 & 25,500 & 40,500 & 54,900 & 72,400 \\
\hline 4895 & 27.4 & 4,400 & 7,920 & 10,800 & 14,800 & 18,100 & 21,500 \\
\hline 4897 & 170 & 10,500 & 18,200 & 24,400 & 33,400 & 40,700 & 48,500 \\
\hline 4913 & 9.10 & 1,650 & 3,150 & 4,370 & 6,170 & 7,630 & 9,260 \\
\hline 4918 & $\mathrm{NA}$ & NA & NA & NA & NA & NA & NA \\
\hline 4937 & 11.7 & 1,940 & 3,720 & 5,190 & 7,350 & 9,100 & 11,100 \\
\hline 4939 & NA & NA & NA & NA & NA & NA & NA \\
\hline 4956 & 169 & 7,250 & 16,800 & 26,300 & 42,600 & 58,300 & 77,600 \\
\hline 4961 & 12.9 & 2,100 & 4,080 & 5,730 & 8,160 & 10,100 & 12,400 \\
\hline 4984 & 200 & 11,300 & 19,500 & 26,200 & 35,800 & 43,600 & 52,100 \\
\hline 5115 & 24.5 & 3,120 & 6,110 & 8,600 & 12,300 & 15,300 & 18,700 \\
\hline 5116 & 61.7 & 4,250 & 8,120 & 11,400 & 16,200 & 20,100 & 24,400 \\
\hline 5117 & 193 & 7,530 & 17,400 & 27,100 & 43,800 & 59,900 & 79,600 \\
\hline 5125 & 17.7 & 2,640 & 5,160 & 7,240 & 10,300 & 12,900 & 15,700 \\
\hline 5137 & 2,460 & 32,000 & 50,400 & 65,500 & 86,700 & 104,000 & 123,000 \\
\hline 5174 & 2,940 & 35,000 & 54,600 & 70,800 & 93,500 & 112,000 & 132,000 \\
\hline 5182 & 76.1 & 10,300 & 16,100 & 20,500 & 26,500 & 31,200 & 36,000 \\
\hline 5199 & 34.0 & 4,020 & 7,100 & 9,570 & 13,000 & 15,700 & 18,600 \\
\hline 5200 & NA & NA & NA & NA & NA & NA & NA \\
\hline 5233 & 3,080 & 34,800 & 54,600 & 70,900 & 93,800 & 113,000 & 133,000 \\
\hline 5235 & 16.0 & 2,680 & 5,220 & 7,330 & 10,500 & 13,000 & 15,900 \\
\hline 5236 & 86.4 & 5,610 & 10,400 & 14,500 & 20,300 & 25,200 & 30,400 \\
\hline 5243 & 3,080 & 34,800 & 54,600 & 70,900 & 93,800 & 113,000 & 133,000 \\
\hline
\end{tabular}


Table 17. Estimated flow-duration values, mean flow values, and peak-discharge frequency values for controlled and uncontrolled flow stream segments on the 1999 Kansas Surface Water Register for Cherokee County.-Continued

[KSWR, Kansas Surface Water Register; CUSEGA, catalog unit segment number alpha; $\mathrm{mi}^{2}$, square miles; $\mathrm{ft}^{3} / \mathrm{s}$, cubic feet per second; HYDRO, lake or other hydrologic structure; NA, not applicable; NRDitch, irrigation ditch; NRTribal, tribal stream]

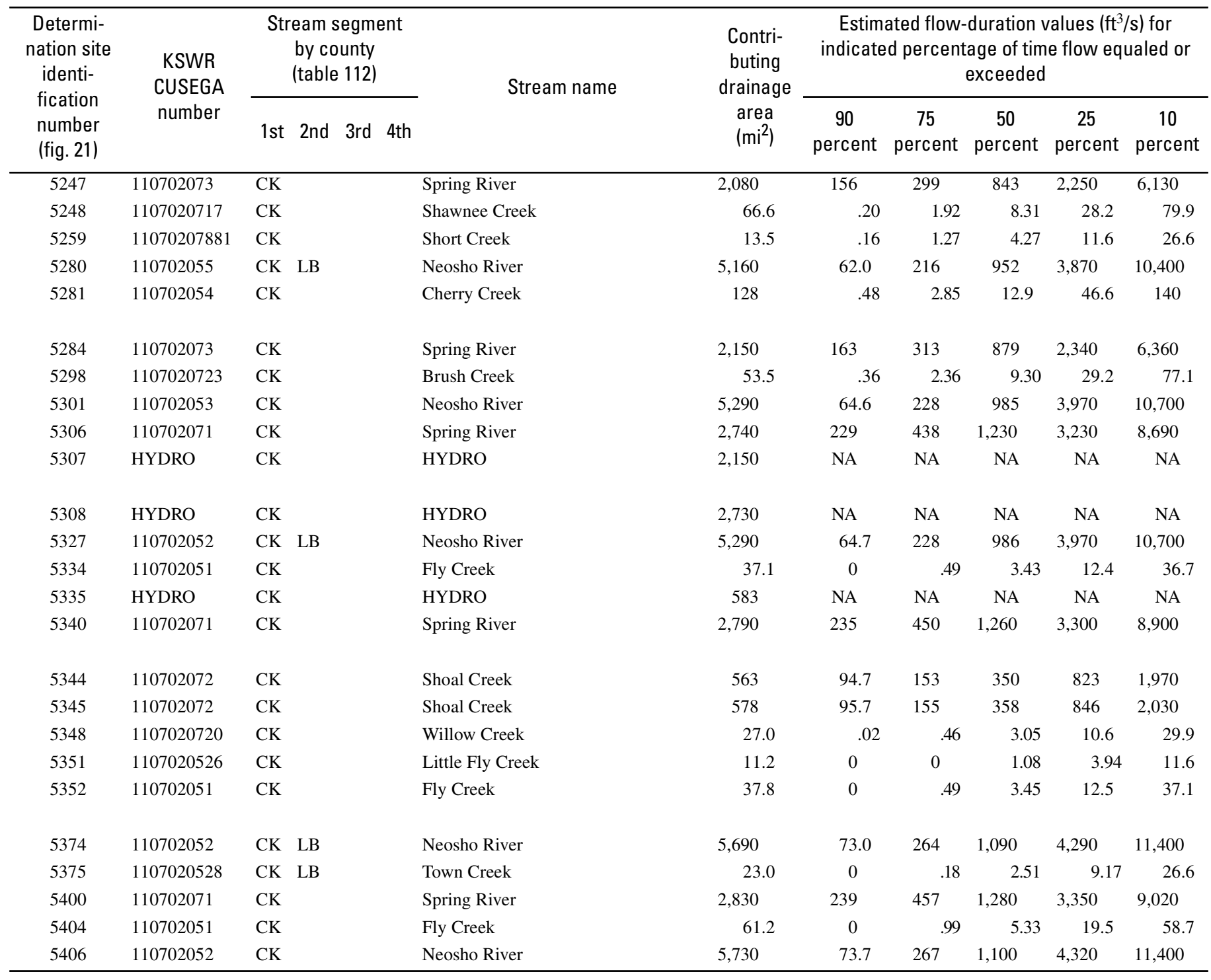


Table 17. Estimated flow-duration values, mean flow values, and peak-discharge frequency values for controlled and uncontrolled flow stream segments on the 1999 Kansas Surface Water Register for Cherokee County.-Continued

[KSWR, Kansas Surface Water Register; CUSEGA, catalog unit segment number alpha; $\mathrm{mi}^{2}$, square miles; $\mathrm{ft}^{3} / \mathrm{s}$, cubic feet per second; HYDRO, lake or other hydrologic structure; NA, not applicable; NRDitch, irrigation ditch; NRTribal, tribal stream]

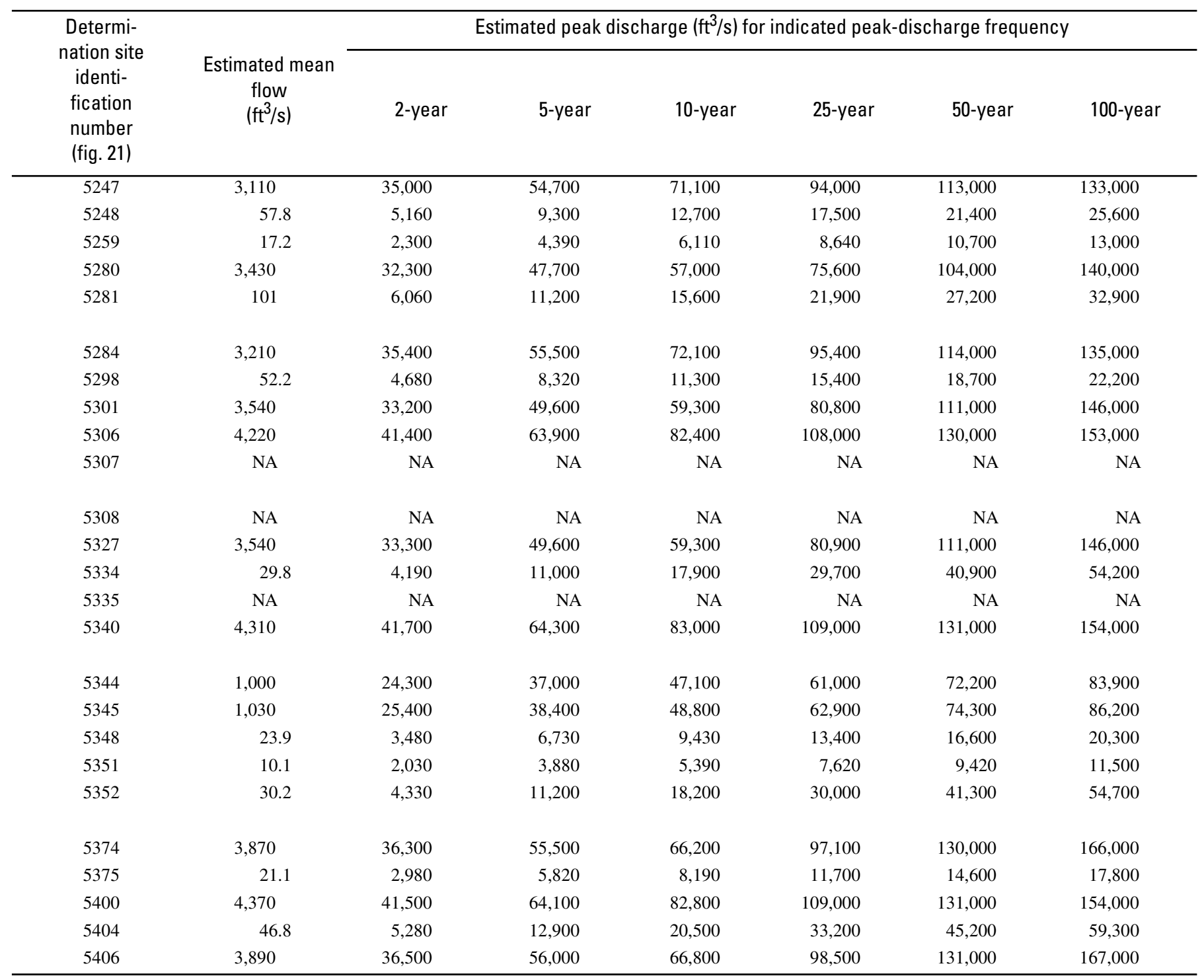




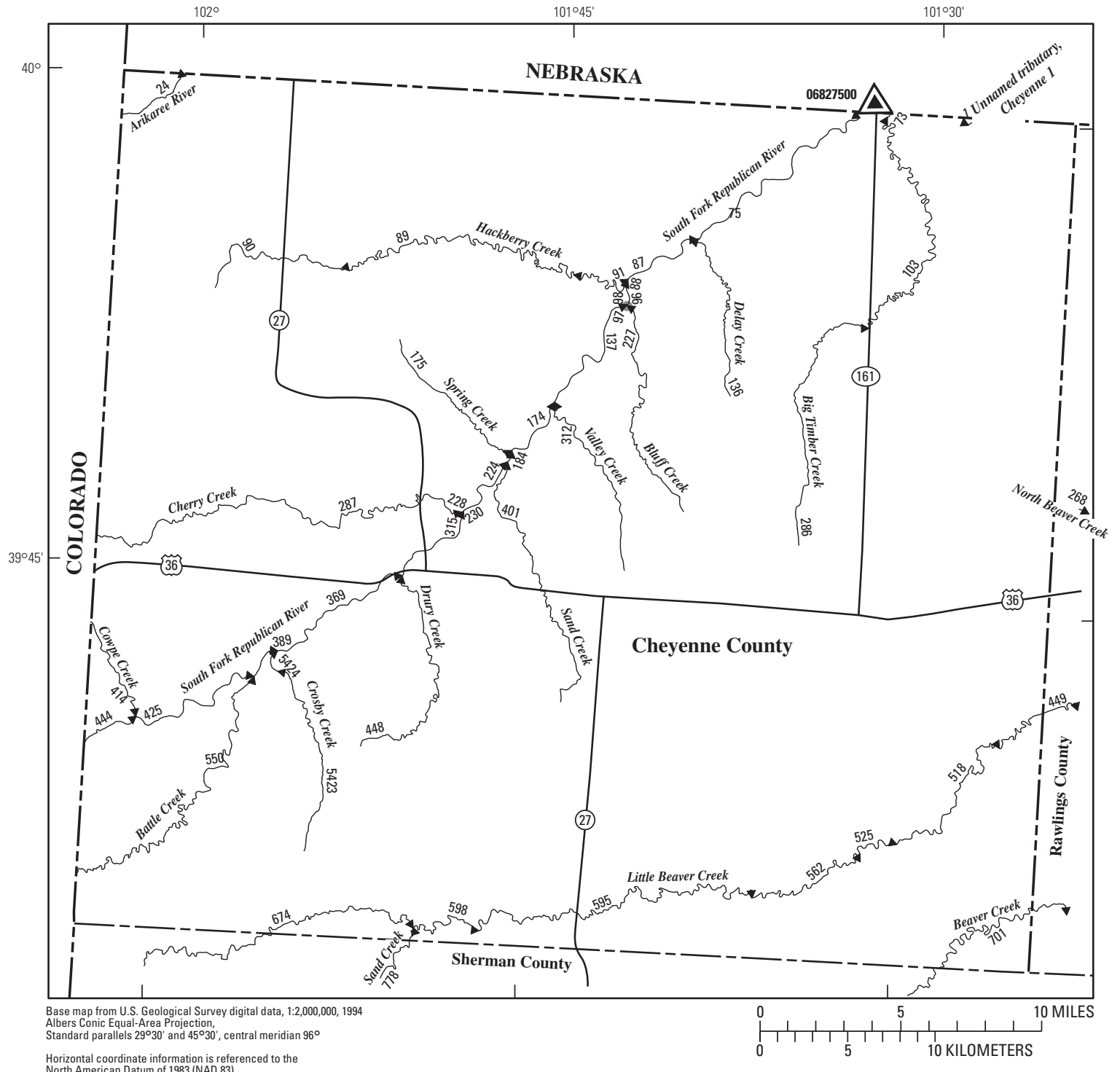

EXPLANATION

778 Location of streamflow-statistics determination site (small triangle) and associated identification number-small triangle points in downstream direction

06827500

A U.S. Geological Survey streamflow-gaging station and number used for estimates of flow duration

06827500

U.S. Geological Survey streamflow-gaging station and number used for estimates of peak-discharge frequency values

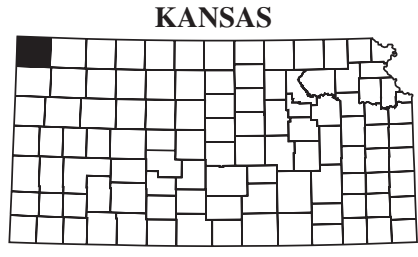

Index map 5335

Lake and determination site identification number

Figure 22. Location of streamflow-statistics determination sites, associated identification numbers, and U.S. Geological Survey streamflow-gaging stations used in the flow-duration and peak-discharge frequency analyses for Cheyenne County. 
Table 18. Estimated flow-duration values, mean flow values, and peak-discharge frequency values for controlled and uncontrolled flow stream segments on the 1999 Kansas Surface Water Register for Cheyenne County.

[KSWR, Kansas Surface Water Register; CUSEGA, catalog unit segment number alpha; $\mathrm{mi}^{2}$, square miles; $\mathrm{ft}^{3} / \mathrm{s}$, cubic feet per second; HYDRO, lake or other hydrologic structure; NA, not applicable; NRDitch, irrigation ditch; NRTribal, tribal stream]

\begin{tabular}{|c|c|c|c|c|c|c|c|c|c|c|c|}
\hline \multirow{2}{*}{$\begin{array}{l}\text { Determi- } \\
\text { nation site } \\
\text { identi- } \\
\text { fication } \\
\text { number } \\
\text { (fig. 22) }\end{array}$} & \multirow{2}{*}{$\begin{array}{l}\text { KSWR } \\
\text { CUSEGA } \\
\text { number }\end{array}$} & \multicolumn{3}{|c|}{$\begin{array}{l}\text { Stream segment } \\
\text { by county } \\
\text { (table 112) }\end{array}$} & \multirow{2}{*}{ Stream name } & \multirow{2}{*}{$\begin{array}{l}\text { Contri- } \\
\text { buting } \\
\text { drainage } \\
\text { area } \\
\left(\mathrm{mi}^{2}\right)\end{array}$} & \multicolumn{5}{|c|}{$\begin{array}{l}\text { Estimated flow-duration values }\left(\mathrm{ft}^{3} / \mathrm{s}\right) \text { for } \\
\text { indicated percentage of time flow equaled or } \\
\text { exceeded }\end{array}$} \\
\hline & & $1 \mathrm{st}$ & 2nd $3 r d$ & 4th & & & $\begin{array}{c}90 \\
\text { percent }\end{array}$ & $\begin{array}{c}75 \\
\text { percent }\end{array}$ & $\begin{array}{c}50 \\
\text { percent }\end{array}$ & $\begin{array}{c}25 \\
\text { percent }\end{array}$ & $\begin{array}{c}10 \\
\text { percent }\end{array}$ \\
\hline 1 & 1025000450 & $\mathrm{CN}$ & & & Unnamed tributary, Cheyenne 1 & 11.0 & 0 & 0 & 0 & 0 & 0 \\
\hline 24 & 102500011 & $\mathrm{CN}$ & & & Arikaree River & 1,960 & 0 & 0 & .67 & 2.78 & 7.05 \\
\hline 73 & 1025000361 & $\mathrm{CN}$ & & & Big Timber Creek & 150 & 0 & 0 & 0 & 0 & 0 \\
\hline 75 & 102500032 & $\mathrm{CN}$ & & & South Fork Republican River & 2,700 & .01 & .53 & 16.0 & 31.0 & 61.0 \\
\hline 87 & 102500032 & $\mathrm{CN}$ & & & South Fork Republican River & 2,650 & .31 & .84 & 15.4 & 29.6 & 59.7 \\
\hline 88 & 102500032 & $\mathrm{CN}$ & & & South Fork Republican River & 2,530 & 0 & 0 & .85 & 3.81 & 10.5 \\
\hline 89 & 102500033 & $\mathrm{CN}$ & & & Hackberry Creek & 105 & 0 & 0 & 0 & 0 & 0 \\
\hline 90 & 102500033 & $\mathrm{CN}$ & & & Hackberry Creek & 49.7 & 0 & 0 & 0 & 0 & 0 \\
\hline 91 & 102500033 & $\mathrm{CN}$ & & & Hackberry Creek & 109 & 0 & 0 & 0 & 0 & 0 \\
\hline 96 & 102500034 & $\mathrm{CN}$ & & & South Fork Republican River & 2,530 & 1.02 & 1.55 & 14.1 & 26.4 & 56.7 \\
\hline 97 & HYDRO & $\mathrm{CN}$ & & & HYDRO & 28.4 & NA & NA & NA & NA & NA \\
\hline 98 & 1025000370 & $\mathrm{CN}$ & & & Bluff Creek & 28.4 & 0 & 0 & 0 & 0 & 0 \\
\hline 103 & 1025000361 & $\mathrm{CN}$ & & & Big Timber Creek & 150 & 0 & 0 & 0 & 0 & 0 \\
\hline 136 & 1025000366 & $\mathrm{CN}$ & & & Delay Creek & 22.1 & 0 & 0 & 0 & 0 & 0 \\
\hline 137 & 102500034 & $\mathrm{CN}$ & & & South Fork Republican River & 2,500 & 1.19 & 1.72 & 13.8 & 25.7 & 56.0 \\
\hline 174 & 102500034 & $\mathrm{CN}$ & & & South Fork Republican River & 2,460 & 1.41 & 1.95 & 13.4 & 24.7 & 55.1 \\
\hline 175 & 1025000367 & $\mathrm{CN}$ & & & Spring Creek & 31.7 & 0 & 0 & 0 & 0 & 0 \\
\hline 184 & 102500034 & $\mathrm{CN}$ & & & South Fork Republican River & 2,430 & 1.62 & 2.16 & 13.0 & 23.7 & 54.2 \\
\hline 224 & 102500034 & $\mathrm{CN}$ & & & South Fork Republican River & 2,370 & 1.96 & 2.51 & 12.3 & 22.2 & 52.7 \\
\hline 227 & 1025000370 & $\mathrm{CN}$ & & & Bluff Creek & 28.4 & 0 & 0 & 0 & 0 & 0 \\
\hline 228 & 102500035 & $\mathrm{CN}$ & & & Cherry Creek & 73.8 & 0 & 0 & 0 & 0 & 0 \\
\hline 230 & 102500035 & $\mathrm{CN}$ & & & Cherry Creek & 73.8 & 0 & 0 & 0 & 0 & 0 \\
\hline 286 & 1025000361 & $\mathrm{CN}$ & & & Big Timber Creek & 90.3 & 0 & 0 & 0 & 0 & 0 \\
\hline 287 & 102500035 & $\mathrm{CN}$ & & & Cherry Creek & 73.8 & 0 & 0 & 0 & 0 & 0 \\
\hline 312 & 1025000369 & $\mathrm{CN}$ & & & Valley Creek & 26.4 & 0 & 0 & 0 & 0 & 0 \\
\hline 315 & 102500036 & $\mathrm{CN}$ & & & South Fork Republican River & 2,290 & 2.41 & 2.97 & 11.5 & 20.2 & 50.8 \\
\hline 369 & 102500037 & $\mathrm{CN}$ & & & South Fork Republican River & 2,240 & 2.67 & 3.23 & 11.0 & 19.0 & 49.7 \\
\hline 389 & 102500037 & $\mathrm{CN}$ & & & South Fork Republican River & 2,200 & 2.92 & 3.48 & 10.5 & 17.9 & 48.7 \\
\hline 401 & 1025000368 & $\mathrm{CN}$ & & & Sand Creek & 59.7 & 0 & 0 & 0 & 0 & 0 \\
\hline 414 & 102500038 & $\mathrm{CN}$ & & & Cowpe Creek & 56.5 & 0 & 0 & 0 & 0 & 0 \\
\hline 425 & 102500037 & $\mathrm{CN}$ & & & South Fork Republican River & 2,160 & 3.17 & 3.74 & 10.0 & 16.7 & 47.6 \\
\hline 444 & 102500039 & $\mathrm{CN}$ & & & South Fork Republican River & 2,090 & 3.58 & 4.15 & 9.25 & 14.9 & 45.9 \\
\hline 448 & 1025000360 & $\mathrm{CN}$ & & & Drury Creek & 39.3 & 0 & 0 & 0 & 0 & 0 \\
\hline 449 & 102500133 & $\mathrm{CN}$ & RA & & Little Beaver Creek & 477 & 0 & 0 & 0 & 0 & 0 \\
\hline 518 & 102500133 & $\mathrm{CN}$ & & & Little Beaver Creek & 420 & 0 & 0 & 0 & 0 & 0 \\
\hline 525 & 102500133 & $\mathrm{CN}$ & & & Little Beaver Creek & 382 & 0 & 0 & 0 & 0 & 0 \\
\hline 550 & 1025000371 & $\mathrm{CN}$ & & & Battle Creek & 39.2 & 0 & 0 & 0 & 0 & 0 \\
\hline 562 & 102500133 & $\mathrm{CN}$ & & & Little Beaver Creek & 366 & 0 & 0 & 0 & 0 & 0 \\
\hline
\end{tabular}


Table 18. Estimated flow-duration values, mean flow values, and peak-discharge frequency values for controlled and uncontrolled flow stream segments on the 1999 Kansas Surface Water Register for Cheyenne County.-Continued

[KSWR, Kansas Surface Water Register; CUSEGA, catalog unit segment number alpha; $\mathrm{mi}^{2}$, square miles; $\mathrm{ft}^{3} / \mathrm{s}$, cubic feet per second; HYDRO, lake or other hydrologic structure; NA, not applicable; NRDitch, irrigation ditch; NRTribal, tribal stream]

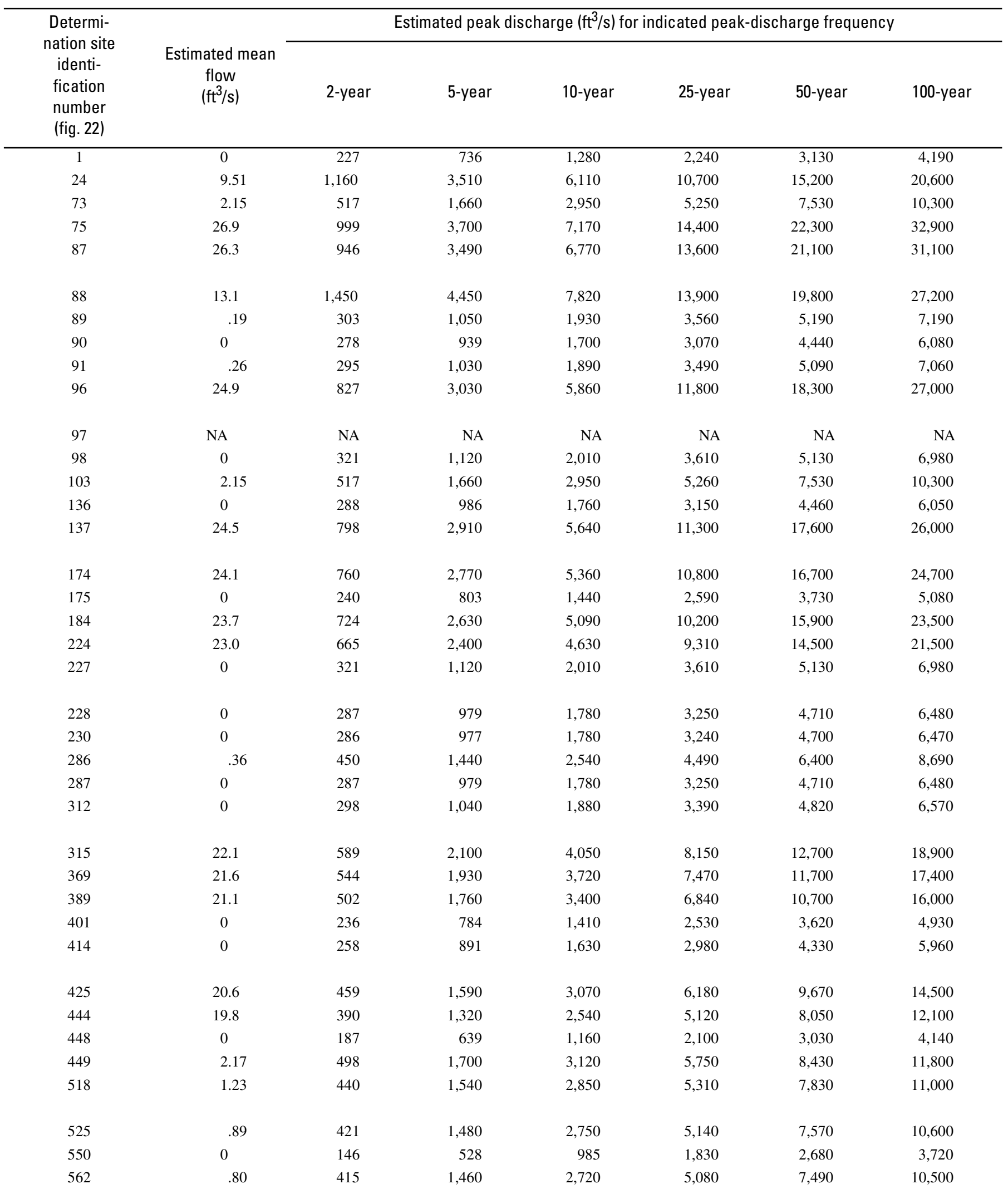




\section{Estimates of Flow Duration, Mean Flow, and Peak-Discharge Frequency Values for Kansas Stream Locations}

Table 18. Estimated flow-duration values, mean flow values, and peak-discharge frequency values for controlled and uncontrolled flow stream segments on the 1999 Kansas Surface Water Register for Cheyenne County.-Continued

[KSWR, Kansas Surface Water Register; CUSEGA, catalog unit segment number alpha; $\mathrm{mi}^{2}$, square miles; $\mathrm{ft}^{3} / \mathrm{s}$, cubic feet per second; HYDRO, lake or other hydrologic structure; NA, not applicable; NRDitch, irrigation ditch; NRTribal, tribal stream]

\begin{tabular}{|c|c|c|c|c|c|c|c|c|c|c|c|c|}
\hline $\begin{array}{l}\text { Determi- } \\
\text { nation site } \\
\text { identi- } \\
\text { fication } \\
\text { number } \\
\text { (fig. 22) }\end{array}$ & $\begin{array}{l}\text { KSWR } \\
\text { CUSEGA } \\
\text { number }\end{array}$ & \multicolumn{4}{|c|}{$\begin{array}{l}\text { Stream segment } \\
\text { by county } \\
\text { (table 112) }\end{array}$} & Stream name & $\begin{array}{l}\text { Contri- } \\
\text { buting } \\
\text { drainage } \\
\text { area } \\
\left(\mathrm{mi}^{2}\right)\end{array}$ & \multicolumn{5}{|c|}{$\begin{array}{l}\text { Estimated flow-duration values }\left(\mathrm{ft}^{3} / \mathrm{s}\right) \text { for } \\
\text { indicated percentage of time flow equaled or } \\
\text { exceeded }\end{array}$} \\
\hline 595 & 102500133 & $\mathrm{CN}$ & & & & Little Beaver Creek & 340 & 0 & 0 & 0 & 0 & 0 \\
\hline 701 & 102500121 & $\mathrm{CN}$ & RA & SH & & Beaver Creek & 571 & 0 & 0 & 0 & 0 & 0 \\
\hline 778 & 102500137 & $\mathrm{CN}$ & SH & & & Sand Creek & 141 & 0 & 0 & 0 & 0 & 0 \\
\hline 5423 & 1025000372 & $\mathrm{CN}$ & & & & Crosby Creek & 25.6 & 0 & 0 & 0 & 0 & 0 \\
\hline 5424 & 1025000372 & $\mathrm{CN}$ & & & & Crosby Creek & 26.0 & 0 & 0 & 0 & 0 & 0 \\
\hline
\end{tabular}


Table 18. Estimated flow-duration values, mean flow values, and peak-discharge frequency values for controlled and uncontrolled flow stream segments on the 1999 Kansas Surface Water Register for Cheyenne County.-Continued

[KSWR, Kansas Surface Water Register; CUSEGA, catalog unit segment number alpha; $\mathrm{mi}^{2}$, square miles; $\mathrm{ft}^{3} / \mathrm{s}$, cubic feet per second; HYDRO, lake or other hydrologic structure; NA, not applicable; NRDitch, irrigation ditch; NRTribal, tribal stream]

\begin{tabular}{|c|c|c|c|c|c|c|c|}
\hline \multirow{2}{*}{$\begin{array}{l}\text { Determi- } \\
\text { nation site } \\
\text { identi- } \\
\text { fication } \\
\text { number } \\
\text { (fig. 22) }\end{array}$} & \multirow{2}{*}{$\begin{array}{c}\text { Estimated mean } \\
\text { flow } \\
\left(\mathrm{ft}^{3} / \mathrm{s}\right)\end{array}$} & \multicolumn{6}{|c|}{ Estimated peak discharge $\left(\mathrm{ft}^{3} / \mathrm{s}\right)$ for indicated peak-discharge frequency } \\
\hline & & 2-year & 5-year & 10-year & 25-year & 50-year & 100-year \\
\hline 595 & 0.66 & 409 & 1,440 & 2,680 & 5,000 & 7,380 & 10,300 \\
\hline 701 & 1.39 & 455 & 1,600 & 2,980 & 5,570 & 8,240 & 11,600 \\
\hline 778 & 0 & 254 & 941 & 1,790 & 3,400 & 5,060 & 7,140 \\
\hline 5423 & 0 & 284 & 999 & 1,810 & 3,270 & 4,650 & 6,340 \\
\hline 5424 & 0 & 286 & 1,010 & 1,830 & 3,300 & 4,700 & 6,410 \\
\hline
\end{tabular}




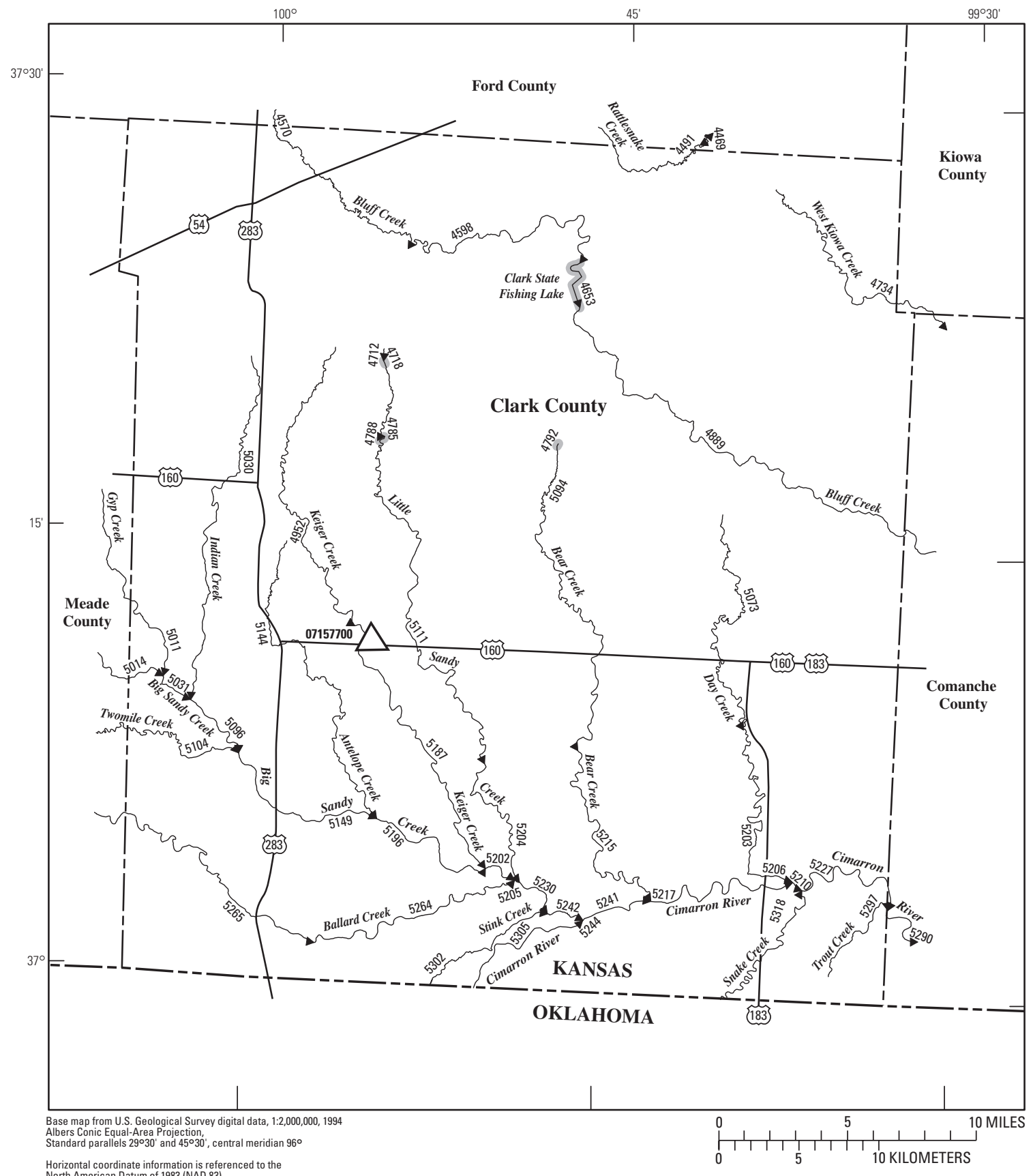

EXPLANATION

$\stackrel{5265}{ }$ Location of streamflow-statistics determination site (small triangle) and associated identification number-small triangle points in downstream direction

07184000 U.S. Geological Survey streamflow-gaging station and number used for estimates of flow duration

07157700

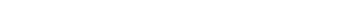

U.S. Geological Survey streamflow-gaging station and number used for estimates of peak-discharge frequency values

KANSAS 4653 Lake and determination site identification number

Figure 23. Location of streamflow-statistics determination sites, associated identification numbers, and U.S. Geological Survey streamflow-gaging stations used in the flow-duration and peak-discharge frequency analyses for Clark County. 
Table 19. Estimated flow-duration values, mean flow values, and peak-discharge frequency values for controlled and uncontrolled flow stream segments on the 1999 Kansas Surface Water Register for Clark County.

[KSWR, Kansas Surface Water Register; CUSEGA, catalog unit segment number alpha; $\mathrm{mi}^{2}$, square miles; $\mathrm{ft}^{3} / \mathrm{s}$, cubic feet per second; HYDRO, lake or other hydrologic structure; NA, not applicable; NRDitch, irrigation ditch; NRTribal, tribal stream]

\begin{tabular}{|c|c|c|c|c|c|c|c|c|c|c|c|}
\hline $\begin{array}{l}\text { Determi- } \\
\text { nation site } \\
\text { identi- } \\
\text { fication } \\
\text { number } \\
\text { (fig. 23) }\end{array}$ & $\begin{array}{l}\text { KSWR } \\
\text { CUSEGA } \\
\text { number }\end{array}$ & \multicolumn{3}{|c|}{$\begin{array}{l}\text { Stream segment } \\
\text { by county } \\
\text { (table 112) }\end{array}$} & Stream name & $\begin{array}{l}\text { Contri- } \\
\text { buting } \\
\text { drainage } \\
\text { area } \\
\left(\mathrm{mi}^{2}\right)\end{array}$ & \multicolumn{5}{|c|}{$\begin{array}{l}\text { Estimated flow-duration values }\left(\mathrm{ft}^{3} / \mathrm{s}\right) \text { for } \\
\text { indicated percentage of time flow equaled or } \\
\text { exceeded }\end{array}$} \\
\hline 4491 & 110300094 & $\mathrm{CA}$ & FO & & Rattlesnake Creek & 47.1 & 0 & 0 & 0 & 0 & 0.61 \\
\hline 4653 & HYDRO & $\mathrm{CA}$ & & & HYDRO & 153 & NA & NA & NA & NA & NA \\
\hline 4712 & 11040008652 & $\mathrm{CA}$ & & & Little Sandy Creek & 8.05 & 0 & 0 & 0 & 0 & 0 \\
\hline 4718 & HYDRO & $\mathrm{CA}$ & & & HYDRO & 8.07 & NA & NA & NA & NA & NA \\
\hline 4734 & 110400081180 & $\mathrm{CA}$ & $\mathrm{CM}$ & KW & West Kiowa Creek & 81.3 & 0 & .60 & 2.03 & 4.13 & 8.64 \\
\hline 4889 & 1104000813 & $\mathrm{CA}$ & $\mathrm{CM}$ & & Bluff Creek & 261 & 0 & .73 & 2.99 & 7.65 & 18.3 \\
\hline 4952 & 110400088 & $\mathrm{CA}$ & & & Keiger Creek & 38.2 & 0 & 0 & 0 & 0 & 0 \\
\hline 5011 & 1104000825 & $\mathrm{CA}$ & ME & & Gyp Creek & 92.7 & 0 & 0 & .06 & .30 & 1.33 \\
\hline 5014 & 110400089 & $\mathrm{CA}$ & ME & & Big Sandy Creek & 77.5 & 0 & 0 & 0 & .04 & 1.03 \\
\hline 5030 & 1104000814 & CA & & & Indian Creek & 56.0 & 0 & 0 & 0 & 0 & .01 \\
\hline 5031 & 110400089 & $\mathrm{CA}$ & & & Big Sandy Creek & 172 & 0 & 0 & 1.02 & 2.63 & 6.32 \\
\hline 5073 & 1104000820 & $\mathrm{CA}$ & & & Day Creek & 60.7 & 0 & 0 & .14 & .49 & 1.88 \\
\hline 5094 & 1104000818 & $\mathrm{CA}$ & & & Bear Creek & 81.3 & 0 & 0 & .47 & 1.31 & 3.45 \\
\hline 5096 & 110400089 & $\mathrm{CA}$ & & & Big Sandy Creek & 235 & 0 & .45 & 2.07 & 4.86 & 10.8 \\
\hline 5202 & 110400087 & $\mathrm{CA}$ & & & Big Sandy Creek & 385 & .02 & 2.71 & 5.97 & 12.2 & 24.5 \\
\hline 5203 & 1104000820 & $\mathrm{CA}$ & & & Day Creek & 91.0 & 0 & 0 & .94 & 2.15 & 5.07 \\
\hline 5204 & 11040008652 & $\mathrm{CA}$ & & & Little Sandy Creek & 91.4 & 0 & 0 & .92 & 2.19 & 5.03 \\
\hline 5205 & 110400086 & $\mathrm{CA}$ & & & Big Sandy Creek & 484 & .80 & 4.39 & 8.79 & 17.2 & 33.6 \\
\hline 5206 & 1104000818 & CA & & & Day Creek & 91.0 & 0 & 0 & .94 & 2.15 & 5.07 \\
\hline 5210 & 110400085 & $\mathrm{CA}$ & & & Cimarron River & 9,420 & 19.2 & 40.6 & 91.7 & 186 & 371 \\
\hline 5215 & 1104000818 & $\mathrm{CA}$ & & & Bear Creek & 105 & 0 & .13 & 1.31 & 2.78 & 5.94 \\
\hline 5217 & 110400085 & $\mathrm{CA}$ & & & Cimarron River & 9,330 & 19.4 & 40.5 & 90.2 & 182 & 361 \\
\hline 5227 & 110400085 & $\mathrm{CA}$ & $\mathrm{CM}$ & & Cimarron River & 9,490 & 19.2 & 41.0 & 93.3 & 190 & 379 \\
\hline 5230 & 110400086 & $\mathrm{CA}$ & & & Big Sandy Creek & 578 & 1.18 & 5.52 & 11.3 & 22.3 & 44.2 \\
\hline 5241 & 110400085 & $\mathrm{CA}$ & & & Cimarron River & 9,210 & 19.6 & 40.3 & 88.1 & 177 & 349 \\
\hline 5242 & 110400086 & $\mathrm{CA}$ & & & Big Sandy Creek & 588 & 1.32 & 5.80 & 11.7 & 23.0 & 45.3 \\
\hline 5244 & 110400085 & $\mathrm{CA}$ & & & Cimarron River & 8,610 & 21.3 & 40.4 & 79.9 & 152 & 295 \\
\hline
\end{tabular}


Table 19. Estimated flow-duration values, mean flow values, and peak-discharge frequency values for controlled and uncontrolled flow stream segments on the 1999 Kansas Surface Water Register for Clark County.-Continued

[KSWR, Kansas Surface Water Register; CUSEGA, catalog unit segment number alpha; $\mathrm{mi}^{2}$, square miles; $\mathrm{ft}^{3} / \mathrm{s}$, cubic feet per second; HYDRO, lake or other hydrologic structure; NA, not applicable; NRDitch, irrigation ditch; NRTribal, tribal stream]

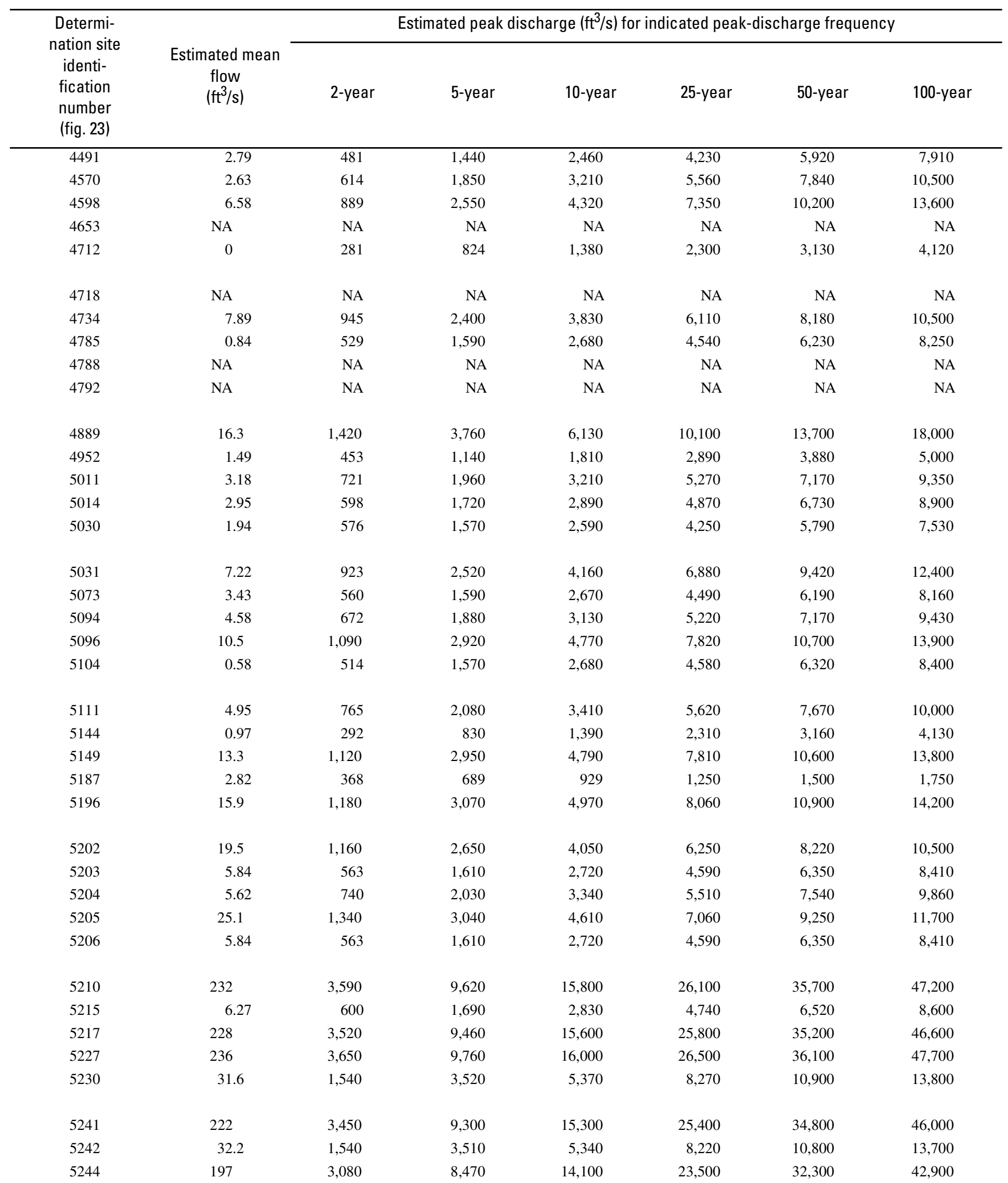




\section{Estimates of Flow Duration, Mean Flow, and Peak-Discharge Frequency Values for Kansas Stream Locations}

Table 19. Estimated flow-duration values, mean flow values, and peak-discharge frequency values for controlled and uncontrolled flow stream segments on the 1999 Kansas Surface Water Register for Clark County.-Continued

[KSWR, Kansas Surface Water Register; CUSEGA, catalog unit segment number alpha; $\mathrm{mi}^{2}$, square miles; $\mathrm{ft}^{3} / \mathrm{s}$, cubic feet per second; HYDRO, lake or other hydrologic structure; NA, not applicable; NRDitch, irrigation ditch; NRTribal, tribal stream]

\begin{tabular}{|c|c|c|c|c|c|c|c|c|c|c|c|}
\hline $\begin{array}{l}\text { Determi- } \\
\text { nation site } \\
\text { identi- } \\
\text { fication } \\
\text { number } \\
\text { (fig. 23) }\end{array}$ & $\begin{array}{l}\text { KSWR } \\
\text { CUSEGA } \\
\text { number }\end{array}$ & \multicolumn{3}{|c|}{$\begin{array}{l}\text { Stream segment } \\
\text { by county } \\
\text { (table 112) }\end{array}$} & Stream name & $\begin{array}{l}\text { Contri- } \\
\text { buting } \\
\text { drainage } \\
\text { area } \\
\left(\mathrm{mi}^{2}\right)\end{array}$ & \multicolumn{5}{|c|}{$\begin{array}{l}\text { Estimated flow-duration values }\left(\mathrm{ft}^{3} / \mathrm{s}\right) \text { for } \\
\text { indicated percentage of time flow equaled or } \\
\text { exceeded }\end{array}$} \\
\hline 5264 & 1104000810 & $\mathrm{CA}$ & & & Ballard Creek & 99.2 & 0 & 0.93 & 1.75 & 2.59 & 4.34 \\
\hline 5297 & 1104000819 & $\mathrm{CA}$ & $\mathrm{CM}$ & & Trout Creek & 30.2 & 0 & .50 & .97 & 1.21 & 2.26 \\
\hline 5302 & 1104000817 & $\mathrm{CA}$ & & & Stink Creek & 7.92 & 0 & 0 & 0 & 0 & 0 \\
\hline 5305 & 1104000811 & $\mathrm{CA}$ & & & Cimarron River & 8,610 & 21.3 & 40.4 & 79.9 & 152 & 295 \\
\hline 5318 & 1104000821 & $\mathrm{CA}$ & & & Snake Creek & 54.3 & 0 & .91 & 1.93 & 3.25 & 6.26 \\
\hline
\end{tabular}


Table 19. Estimated flow-duration values, mean flow values, and peak-discharge frequency values for controlled and uncontrolled flow stream segments on the 1999 Kansas Surface Water Register for Clark County.-Continued

[KSWR, Kansas Surface Water Register; CUSEGA, catalog unit segment number alpha; $\mathrm{mi}^{2}$, square miles; $\mathrm{ft}^{3} / \mathrm{s}$, cubic feet per second; HYDRO, lake or other hydrologic structure; NA, not applicable; NRDitch, irrigation ditch; NRTribal, tribal stream]

\begin{tabular}{|c|c|c|c|c|c|c|c|}
\hline \multirow{2}{*}{$\begin{array}{l}\text { Determi- } \\
\text { nation site } \\
\text { identi- } \\
\text { fication } \\
\text { number } \\
\text { (fig. 23) }\end{array}$} & \multirow{2}{*}{$\begin{array}{c}\text { Estimated mean } \\
\text { flow } \\
\left(\mathrm{ft}^{3} / \mathrm{s}\right)\end{array}$} & \multicolumn{6}{|c|}{ Estimated peak discharge $\left(\mathrm{ft}^{3} / \mathrm{s}\right)$ for indicated peak-discharge frequency } \\
\hline & & 2-year & 5-year & 10 -year & 25-year & 50-year & 100-year \\
\hline 5264 & 4.87 & 496 & 1,340 & 2,190 & 3,570 & 4,830 & 6,280 \\
\hline 5297 & 3.09 & 655 & 1,600 & 2,490 & 3,880 & 5,100 & 6,440 \\
\hline 5302 & 0 & 292 & 846 & 1,400 & 2,340 & 3,180 & 4,170 \\
\hline 5305 & 197 & 3,080 & 8,470 & 14,100 & 23,500 & 32,300 & 42,900 \\
\hline 5318 & 5.96 & 523 & 1,410 & 2,290 & 3,750 & 5,070 & 6,590 \\
\hline
\end{tabular}


140 Estimates of Flow Duration, Mean Flow, and Peak-Discharge Frequency Values for Kansas Stream Locations 


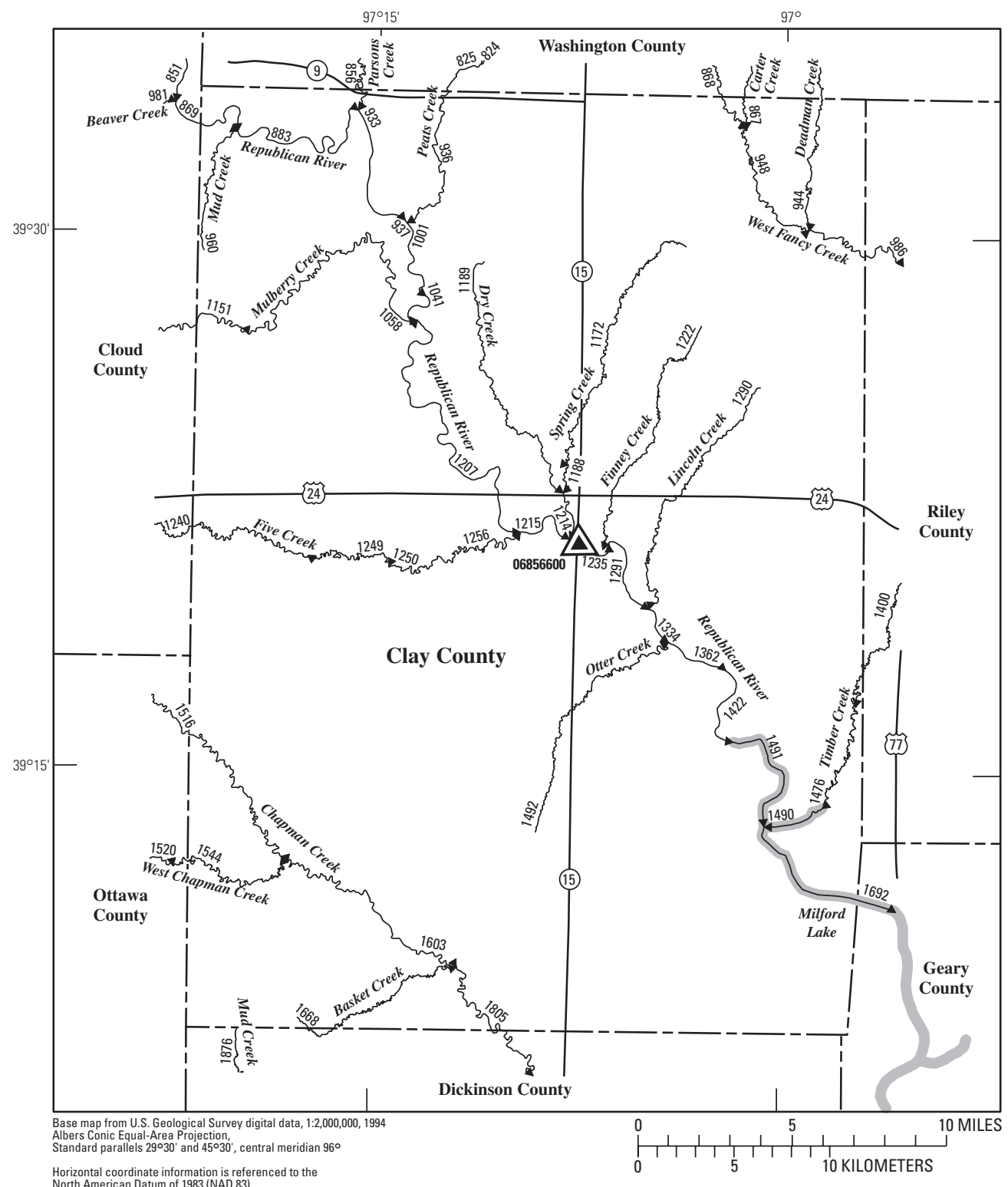

EXPLANATION

«1876 Location of streamflow-statistics determination site (small triangle) and associated identification number-small triangle points in downstream direction

$06856600 \Delta$ U.S. Geological Survey streamflow-gaging station and number used for estimates of flow duration

${ }^{06856600} \triangle$ U.S. Geological Survey streamflow-gaging station and number used for estimates of peak-discharge frequency values

KANSAS

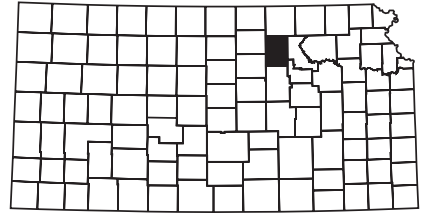

Index map 1692

Lake and determination site identification number

Figure 24. Location of streamflow-statistics determination sites, associated identification numbers, and U.S. Geological Survey streamflow-gaging stations used in the flow-duration and peak-discharge frequency analyses for Clay County. 
Table 20. Estimated flow-duration values, mean flow values, and peak-discharge frequency values for controlled and uncontrolled flow stream segments on the 1999 Kansas Surface Water Register for Clay County.

[KSWR, Kansas Surface Water Register; CUSEGA, catalog unit segment number alpha; $\mathrm{mi}^{2}$, square miles; $\mathrm{ft}^{3} / \mathrm{s}$, cubic feet per second; HYDRO, lake or other hydrologic structure; NA, not applicable; NRDitch, irrigation ditch; NRTribal, tribal stream]

\begin{tabular}{|c|c|c|c|c|c|c|c|c|c|c|c|}
\hline \multirow{2}{*}{$\begin{array}{l}\text { Determi- } \\
\text { nation site } \\
\text { identi- } \\
\text { fication } \\
\text { number } \\
\text { (fig. 24) }\end{array}$} & \multirow{2}{*}{$\begin{array}{l}\text { KSWR } \\
\text { CUSEGA } \\
\text { number }\end{array}$} & \multicolumn{3}{|c|}{$\begin{array}{l}\text { Stream segment } \\
\text { by county } \\
\text { (table 112) }\end{array}$} & \multirow{2}{*}{ Stream name } & \multirow{2}{*}{$\begin{array}{l}\text { Contri- } \\
\text { buting } \\
\text { drainage } \\
\text { area } \\
\left(\mathrm{mi}^{2}\right)\end{array}$} & \multicolumn{5}{|c|}{$\begin{array}{l}\text { Estimated flow-duration values }\left(\mathrm{ft}^{3} / \mathrm{s}\right) \text { for } \\
\text { indicated percentage of time flow equaled or } \\
\text { exceeded }\end{array}$} \\
\hline & & $1 s t$ & 2nd & 3rd 4th & & & $\begin{array}{c}90 \\
\text { percent }\end{array}$ & $\begin{array}{c}75 \\
\text { percent }\end{array}$ & $\begin{array}{c}50 \\
\text { percent }\end{array}$ & $\begin{array}{c}25 \\
\text { percent }\end{array}$ & $\begin{array}{c}10 \\
\text { percent }\end{array}$ \\
\hline 856 & 1025001712 & $\mathrm{CY}$ & WS & & Parsons Creek & 97.7 & $\overline{0}$ & 1.67 & 5.74 & 15.5 & 40.1 \\
\hline 867 & 1027020559 & $\mathrm{CY}$ & WS & & Carter Creek & 17.3 & 0 & 0 & .92 & 2.24 & 6.09 \\
\hline 868 & 1027020529 & $\mathrm{CY}$ & WS & & West Fancy Creek & 31.5 & 0 & .06 & 1.40 & 3.64 & 9.96 \\
\hline 869 & 1025001713 & $\mathrm{CY}$ & $\mathrm{CD}$ & & Republican River & 22,300 & 127 & 196 & 341 & 710 & 1,550 \\
\hline 883 & 1025001713 & CY & & & Republican River & 22,300 & 128 & 197 & 344 & 717 & 1,560 \\
\hline 933 & 1025001711 & $\mathrm{CY}$ & & & Republican River & 22,400 & 130 & 202 & 356 & 743 & 1,620 \\
\hline 936 & 1025001710 & $\mathrm{CY}$ & WS & & Peats Creek & 104 & 0 & 1.94 & 6.47 & 17.4 & 45.0 \\
\hline 937 & 102500179 & $\mathrm{CY}$ & & & Republican River & 22,400 & 183 & 363 & 601 & 956 & 2,010 \\
\hline 944 & 1027020560 & $\mathrm{CY}$ & WS & & Deadman Creek & 21.6 & 0 & 0 & 1.18 & 3.04 & 8.15 \\
\hline 948 & 1027020529 & $\mathrm{CY}$ & & & West Fancy Creek & 62.2 & 0 & .66 & 3.36 & 8.28 & 21.5 \\
\hline 960 & 1025001763 & CY & & & Mud Creek & 13.8 & 0 & 0 & .43 & 1.12 & 3.59 \\
\hline 986 & 1027020529 & $\mathrm{CY}$ & RL & & West Fancy Creek & 104 & 0 & 1.34 & 6.14 & 14.3 & 36.6 \\
\hline 1001 & 102500179 & CY & & & Republican River & 22,500 & 133 & 207 & 367 & 770 & 1,680 \\
\hline 1041 & 102500178 & CY & & & Republican River & 22,500 & 133 & 207 & 368 & 771 & 1,680 \\
\hline 1058 & 1025001740 & CY & & & Mulberry Creek & 72.1 & 0 & 1.12 & 4.09 & 10.9 & 28.3 \\
\hline 1151 & 1025001740 & $\mathrm{CY}$ & $\mathrm{CD}$ & & Mulberry Creek & 44.7 & 0 & .46 & 2.45 & 6.67 & 17.5 \\
\hline 1172 & 102500171354 & CY & & & Spring Creek & 38.2 & 0 & .25 & 1.87 & 5.20 & 14.1 \\
\hline 1188 & 102500171354 & $\mathrm{CY}$ & & & Spring Creek & 39.0 & 0 & .29 & 1.96 & 5.40 & 14.5 \\
\hline 1189 & 102500171369 & $\mathrm{CY}$ & & & Dry Creek & 26.4 & 0 & .64 & 1.72 & 3.61 & 8.64 \\
\hline 1207 & 102500178 & $\mathrm{CY}$ & & & Republican River & 22,600 & 136 & 212 & 378 & 796 & 1,740 \\
\hline 1214 & 102500179354 & $\mathrm{CY}$ & & & Huntress Creek & 66.7 & 0 & 1.34 & 4.10 & 10.3 & 25.9 \\
\hline 1215 & 102500178 & $\mathrm{CY}$ & & & Republican River & 22,700 & 138 & 217 & 388 & 819 & 1,790 \\
\hline 1222 & 1025001764 & $\mathrm{CY}$ & & & Finney Creek & 21.1 & 0 & .13 & 1.29 & 3.21 & 8.36 \\
\hline 1235 & 102500178 & $\mathrm{CY}$ & & & Republican River & 22,800 & 140 & 220 & 396 & 837 & 1,830 \\
\hline 1240 & 10250017413 & $\mathrm{CY}$ & $\mathrm{CD}$ & & Five Creek & 41.0 & 0 & .63 & 2.76 & 7.17 & 18.1 \\
\hline 1249 & 10250017413 & $\mathrm{CY}$ & & & Five Creek & 59.5 & 0 & 1.12 & 4.11 & 10.8 & 27.0 \\
\hline 1250 & 102500113 & $\mathrm{CY}$ & & & Five Creek & 62.8 & 0 & 1.23 & 4.41 & 11.5 & 28.8 \\
\hline 1256 & 10250017413 & $\mathrm{CY}$ & & & Five Creek & 88.0 & 0 & 1.75 & 5.86 & 15.5 & 39.4 \\
\hline 1290 & 1025001765 & CY & & & Lincoln Creek & 40.0 & 0 & .55 & 2.58 & 6.90 & 17.9 \\
\hline 1291 & 102500178 & $\mathrm{CY}$ & & & Republican River & 22,800 & 134 & 213 & 393 & 850 & 1,870 \\
\hline 1334 & 102500178 & $\mathrm{CY}$ & & & Republican River & 22,800 & 124 & 202 & 388 & 873 & 1,940 \\
\hline 1362 & 102500178 & $\mathrm{CY}$ & & & Republican River & 22,900 & 106 & 182 & 380 & 911 & 2,060 \\
\hline 1400 & 102500176 & $\mathrm{CY}$ & RL & & Timber Creek & 41.2 & 0 & .10 & 2.10 & 6.72 & 19.4 \\
\hline 1422 & 102500177 & CY & & & Republican River & 22,900 & 103 & 178 & 378 & 919 & 2,090 \\
\hline 1476 & 102500176 & $\mathrm{CY}$ & & & Timber Creek & 58.7 & 0 & .47 & 3.28 & 10.4 & 29.5 \\
\hline 1490 & HYDRO & $\mathrm{CY}$ & & & HYDRO & 61.3 & NA & NA & NA & NA & NA \\
\hline 1491 & HYDRO & CY & & & HYDRO & 22,900 & NA & NA & NA & NA & NA \\
\hline 1492 & 1025001766 & $\mathrm{CY}$ & & & Otter Creek & 65.0 & 0 & .89 & 3.70 & 10.4 & 28.1 \\
\hline
\end{tabular}


Table 20. Estimated flow-duration values, mean flow values, and peak-discharge frequency values for controlled and uncontrolled flow stream segments on the 1999 Kansas Surface Water Register for Clay County.-Continued

[KSWR, Kansas Surface Water Register; CUSEGA, catalog unit segment number alpha; $\mathrm{mi}^{2}$, square miles; $\mathrm{ft}^{3} / \mathrm{s}$, cubic feet per second; HYDRO, lake or other hydrologic structure; NA, not applicable; NRDitch, irrigation ditch; NRTribal, tribal stream]

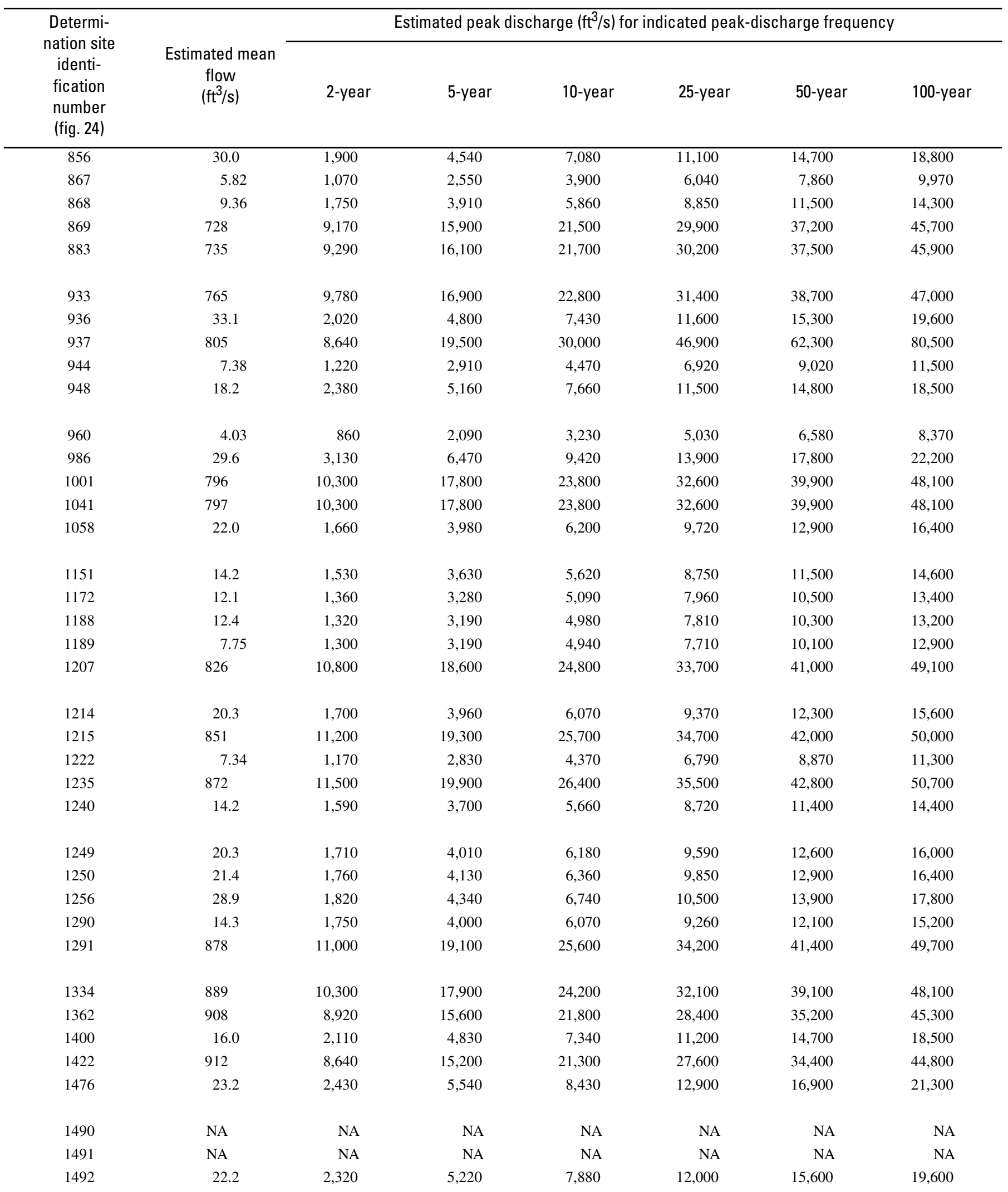


Table 20. Estimated flow-duration values, mean flow values, and peak-discharge frequency values for controlled and uncontrolled flow stream segments on the 1999 Kansas Surface Water Register for Clay County.-Continued

[KSWR, Kansas Surface Water Register; CUSEGA, catalog unit segment number alpha; $\mathrm{mi}^{2}$, square miles; $\mathrm{ft}^{3} / \mathrm{s}$, cubic feet per second; HYDRO, lake or other hydrologic structure; NA, not applicable; NRDitch, irrigation ditch; NRTribal, tribal stream]

\begin{tabular}{|c|c|c|c|c|c|c|c|c|c|c|c|}
\hline $\begin{array}{l}\text { Determi- } \\
\text { nation site } \\
\text { identi- } \\
\text { fication } \\
\text { number } \\
\text { (fig. 24) }\end{array}$ & $\begin{array}{l}\text { KSWR } \\
\text { CUSEGA } \\
\text { number }\end{array}$ & \multicolumn{3}{|c|}{$\begin{array}{l}\text { Stream segment } \\
\text { by county } \\
\text { (table 112) }\end{array}$} & Stream name & $\begin{array}{l}\text { Contri- } \\
\text { buting } \\
\text { drainage } \\
\text { area } \\
\left(\mathrm{mi}^{2}\right)\end{array}$ & \multicolumn{5}{|c|}{$\begin{array}{l}\text { Estimated flow-duration values }\left(\mathrm{ft}^{3} / \mathrm{s}\right) \text { for } \\
\text { indicated percentage of time flow equaled or } \\
\text { exceeded }\end{array}$} \\
\hline 1516 & 102600084 & $\mathrm{CY}$ & OT & & Chapman Creek & 80.6 & 0.38 & 2.33 & 6.31 & 15.1 & 36.6 \\
\hline 1668 & 1026000840 & $\mathrm{CY}$ & DK & & Basket Creek & 24.6 & .04 & .12 & 1.24 & 3.10 & 8.29 \\
\hline 1692 & HYDRO & $\mathrm{CY}$ & GE & & HYDRO & 23,100 & NA & NA & $\mathrm{NA}$ & NA & NA \\
\hline 1805 & 102600083 & $\mathrm{CY}$ & DK & & Chapman Creek & 269 & 5.76 & 10.4 & 19.9 & 41.8 & 107 \\
\hline 1876 & 102600088 & $\mathrm{CY}$ & DK & & Mud Creek & 66.8 & 0 & 1.08 & 4.04 & 10.8 & 27.8 \\
\hline
\end{tabular}


Table 20. Estimated flow-duration values, mean flow values, and peak-discharge frequency values for controlled and uncontrolled flow stream segments on the 1999 Kansas Surface Water Register for Clay County.-Continued

[KSWR, Kansas Surface Water Register; CUSEGA, catalog unit segment number alpha; $\mathrm{mi}^{2}$, square miles; $\mathrm{ft}^{3} / \mathrm{s}$, cubic feet per second; HYDRO, lake or other hydrologic structure; NA, not applicable; NRDitch, irrigation ditch; NRTribal, tribal stream]

\begin{tabular}{|c|c|c|c|c|c|c|c|}
\hline \multirow{2}{*}{$\begin{array}{l}\text { Determi- } \\
\text { nation site } \\
\text { identi- } \\
\text { fication } \\
\text { number } \\
\text { (fig. 24) }\end{array}$} & \multirow{2}{*}{$\begin{array}{c}\text { Estimated mean } \\
\text { flow } \\
\left(\mathrm{ft}^{3} / \mathrm{s}\right)\end{array}$} & \multicolumn{6}{|c|}{ Estimated peak discharge $\left(\mathrm{ft}^{3} / \mathrm{s}\right)$ for indicated peak-discharge frequency } \\
\hline & & 2-year & 5-year & 10-year & 25-year & 50-year & 100-year \\
\hline 1516 & 27.0 & 1,800 & 4,160 & 6,390 & 9,880 & 13,000 & 16,500 \\
\hline 1668 & 7.67 & 1,240 & 3,040 & 4,700 & 7,330 & 9,600 & 12,200 \\
\hline 1692 & NA & NA & NA & NA & NA & NA & NA \\
\hline 1805 & 79.4 & 3,400 & 6,910 & 10,100 & 15,200 & 19,800 & 25,200 \\
\hline 1876 & 21.3 & 1,870 & 4,080 & 6,090 & 9,190 & 11,900 & 15,000 \\
\hline
\end{tabular}




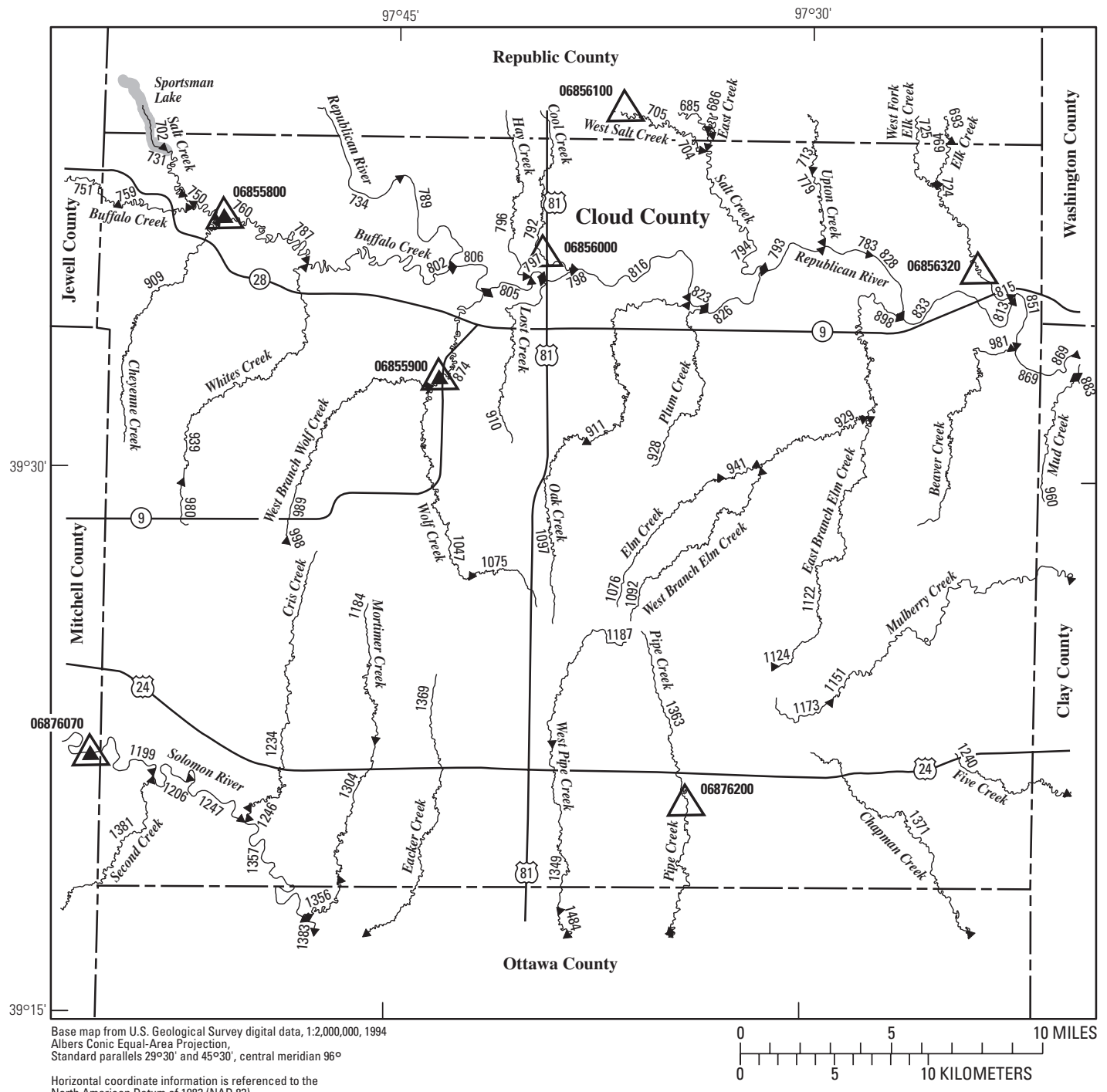

\section{EXPLANATION}

$\stackrel{1357}{\longleftarrow}$ Location of streamflow-statistics determination site (small triangle) and associated identification number-small triangle points in downstream direction

$06876070 \Delta$ U.S. Geological Survey streamflow-gaging station and number used for estimates of flow duration

$06876200 \bigwedge$ U.S. Geological Survey streamflow-gaging station and number used for estimates of peak-discharge frequency values

KANSAS

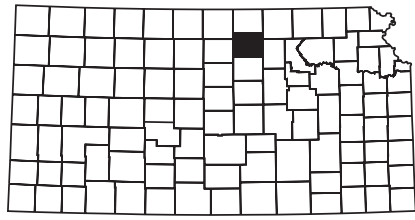

Index map

1365 Lake and determination site identification number

Figure 25. Location of streamflow-statistics determination sites, associated identification numbers, and U.S. Geological Survey streamflow-gaging stations used in the flow-duration and peak-discharge frequency analyses for Cloud County. 
Table 21. Estimated flow-duration values, mean flow values, and peak-discharge frequency values for controlled and uncontrolled flow stream segments on the 1999 Kansas Surface Water Register for Cloud County.

[KSWR, Kansas Surface Water Register; CUSEGA, catalog unit segment number alpha; $\mathrm{mi}^{2}$, square miles; $\mathrm{ft}^{3} / \mathrm{s}$, cubic feet per second; HYDRO, lake or other hydrologic structure; NA, not applicable; NRDitch, irrigation ditch; NRTribal, tribal stream]

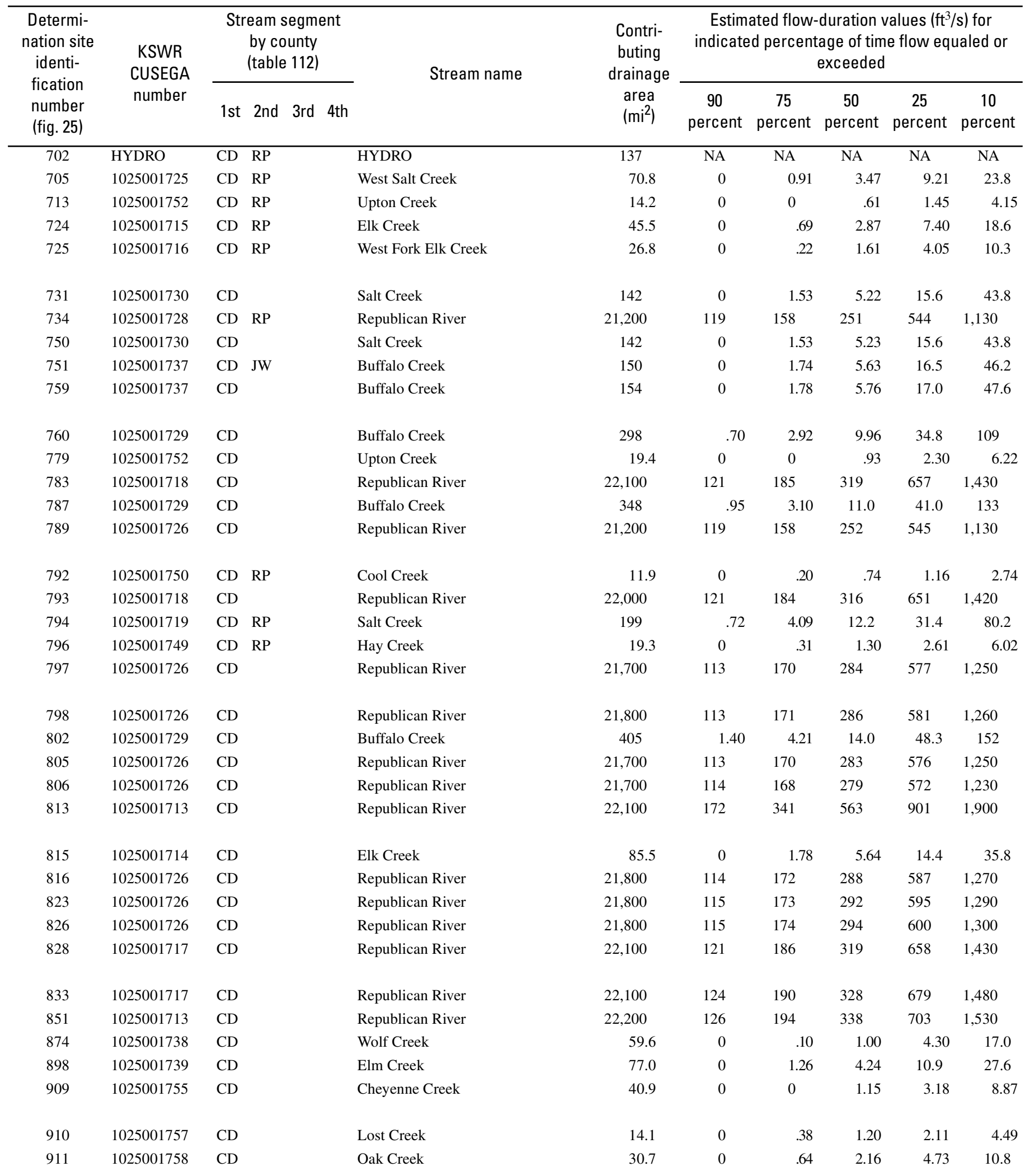


Table 21. Estimated flow-duration values, mean flow values, and peak-discharge frequency values for controlled and uncontrolled flow stream segments on the 1999 Kansas Surface Water Register for Cloud County.-Continued

[KSWR, Kansas Surface Water Register; CUSEGA, catalog unit segment number alpha; $\mathrm{mi}^{2}$, square miles; $\mathrm{ft}^{3} / \mathrm{s}$, cubic feet per second; HYDRO, lake or other hydrologic structure; NA, not applicable; NRDitch, irrigation ditch; NRTribal, tribal stream]

\begin{tabular}{|c|c|c|c|c|c|c|c|}
\hline \multirow{2}{*}{$\begin{array}{l}\text { Determi- } \\
\text { nation site } \\
\text { identi- } \\
\text { fication } \\
\text { number } \\
\text { (fig. 25) }\end{array}$} & \multirow{2}{*}{$\begin{array}{c}\text { Estimated mean } \\
\text { flow } \\
\left(\mathrm{ft}^{3} / \mathrm{s}\right)\end{array}$} & \multicolumn{6}{|c|}{ Estimated peak discharge $\left(\mathrm{ft}^{3} / \mathrm{s}\right)$ for indicated peak-discharge frequency } \\
\hline & & 2-year & 5-year & 10-year & 25-year & 50-year & 100 -year \\
\hline 702 & NA & NA & NA & $\mathrm{NA}$ & $\mathrm{NA}$ & NA & NA \\
\hline 724 & 14.6 & 1,130 & 2,690 & 4,190 & 6,580 & 8,740 & 11,200 \\
\hline 725 & 8.70 & 1,140 & 2,840 & 4,420 & 6,970 & 9,160 & 11,800 \\
\hline 731 & 29.6 & 1,590 & 3,920 & 6,210 & 9,940 & 13,400 & 17,400 \\
\hline 734 & 501 & 6,500 & 10,900 & 23,200 & 33,800 & 44,300 & 55,600 \\
\hline 760 & 61.5 & 1,970 & 4,570 & 7,130 & 11,500 & 15,600 & 20,600 \\
\hline 779 & 5.87 & 1,030 & 2,530 & 3,930 & 6,160 & 8,070 & 10,300 \\
\hline 783 & 668 & 8,200 & 14,200 & 19,400 & 27,500 & 34,900 & 43,600 \\
\hline 787 & 71.7 & 1,670 & 3,890 & 6,140 & 10,100 & 14,000 & 18,900 \\
\hline 789 & 502 & 6,500 & 10,900 & 23,100 & 33,600 & 44,000 & 55,300 \\
\hline 792 & 3.05 & 720 & 1,790 & 2,790 & 4,370 & 5,730 & 7,320 \\
\hline 793 & 661 & 8,090 & 14,000 & 19,200 & 27,300 & 34,700 & 43,300 \\
\hline 794 & 55.6 & 1,910 & 4,530 & 7,220 & 11,900 & 16,500 & 22,400 \\
\hline 796 & 5.37 & 943 & 2,370 & 3,720 & 5,860 & 7,720 & 9,890 \\
\hline 815 & 26.3 & 546 & 1,350 & 2,170 & 3,640 & 5,100 & 6,910 \\
\hline 816 & 588 & 6,900 & 12,000 & 16,700 & 24,400 & 31,800 & 40,800 \\
\hline 823 & 597 & 7,050 & 12,300 & 17,000 & 24,800 & 32,200 & 41,100 \\
\hline 826 & 603 & 7,140 & 12,400 & 17,200 & 25,000 & 32,400 & 41,300 \\
\hline 828 & 669 & 8,210 & 14,300 & 19,400 & 27,600 & 35,000 & 43,600 \\
\hline 833 & 693 & 8,600 & 14,900 & 20,300 & 28,500 & 35,900 & 44,500 \\
\hline 851 & 720 & 9,040 & 15,700 & 21,200 & 29,600 & 36,900 & 45,400 \\
\hline 874 & 11.0 & 910 & 1,770 & 2,490 & 3,570 & 4,490 & 5,520 \\
\hline 898 & 21.4 & 1,610 & 3,880 & 6,050 & 9,490 & 12,600 & 16,100 \\
\hline 909 & 8.06 & 1,030 & 2,610 & 4,140 & 6,600 & 8,830 & 11,300 \\
\hline 910 & 4.02 & 775 & 1,940 & 3,040 & 4,780 & 6,290 & 8,050 \\
\hline 911 & 8.67 & 942 & 2,320 & 3,640 & 5,720 & 7,590 & 9,660 \\
\hline
\end{tabular}


Table 21. Estimated flow-duration values, mean flow values, and peak-discharge frequency values for controlled and uncontrolled flow stream segments on the 1999 Kansas Surface Water Register for Cloud County.-Continued

[KSWR, Kansas Surface Water Register; CUSEGA, catalog unit segment number alpha; $\mathrm{mi}^{2}$, square miles; $\mathrm{ft}^{3} / \mathrm{s}$, cubic feet per second; HYDRO, lake or other hydrologic structure; NA, not applicable; NRDitch, irrigation ditch; NRTribal, tribal stream]

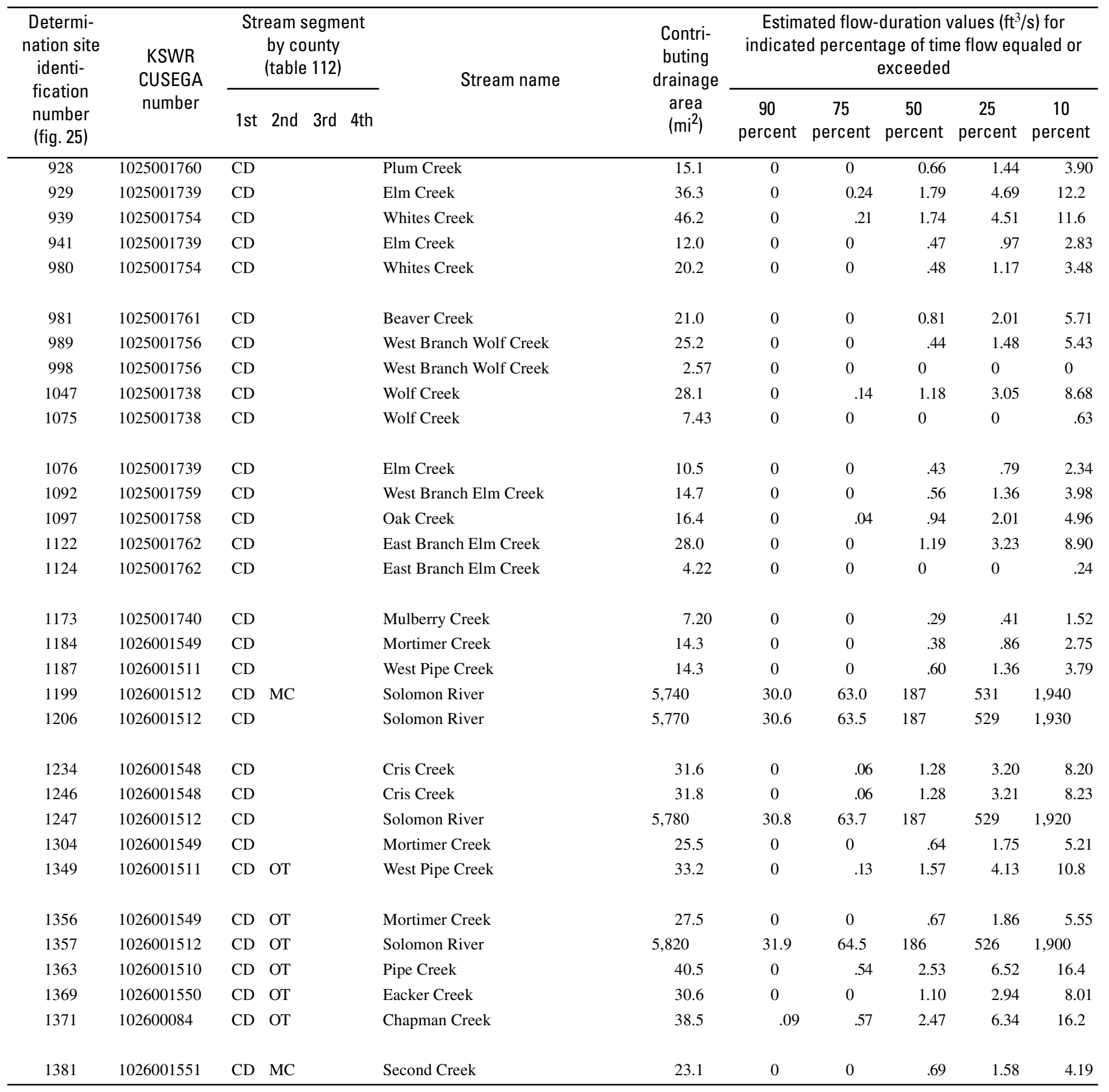


Table 21. Estimated flow-duration values, mean flow values, and peak-discharge frequency values for controlled and uncontrolled flow stream segments on the 1999 Kansas Surface Water Register for Cloud County.-Continued

[KSWR, Kansas Surface Water Register; CUSEGA, catalog unit segment number alpha; $\mathrm{mi}^{2}$, square miles; $\mathrm{ft}^{3} / \mathrm{s}$, cubic feet per second; HYDRO, lake or other hydrologic structure; NA, not applicable; NRDitch, irrigation ditch; NRTribal, tribal stream]

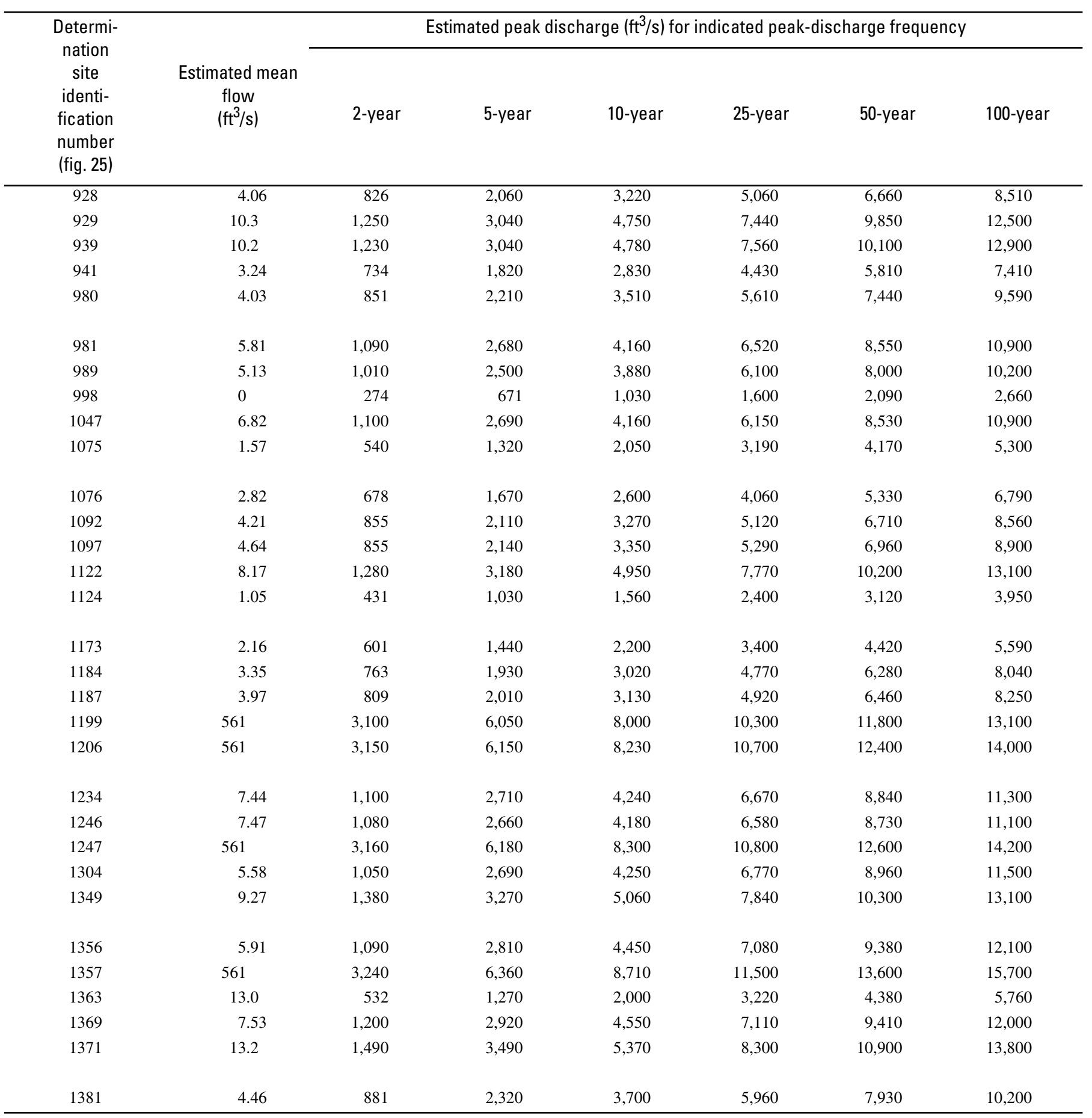




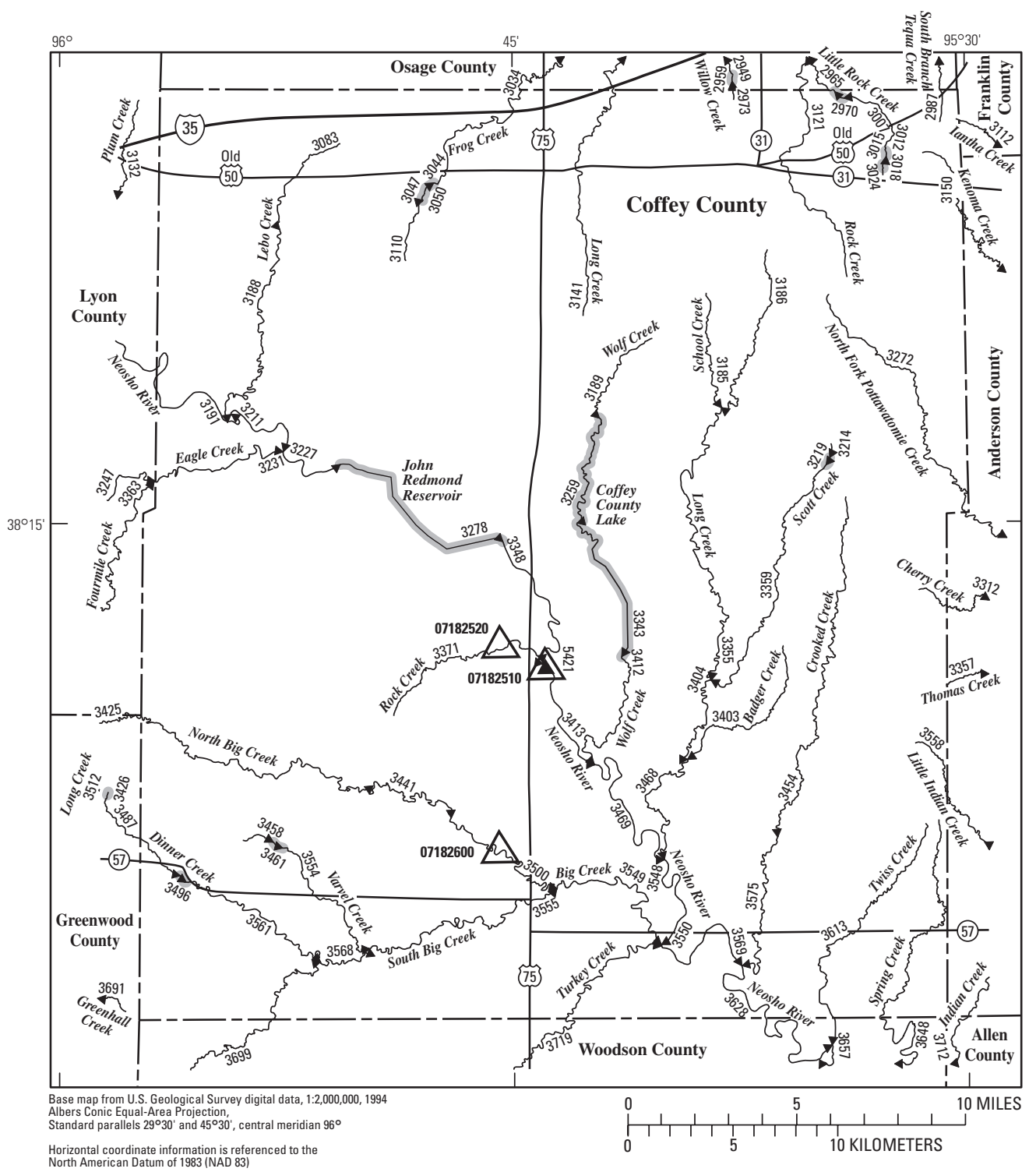

Horizontal coordinate information is referenced to the
North American Datum of 1983 (NAD 83)

\section{EXPLANATION}

3699 Location of streamflow-statistics determination site (small triangle) and associated identification number-small triangle points in downstream direction

$07182510 \Delta$ U.S. Geological Survey streamflow-gaging station and number used for estimates of flow duration

$07182600 \bigwedge$ U.S. Geological Survey streamflow-gaging station and number used for estimates of peak-discharge frequency values

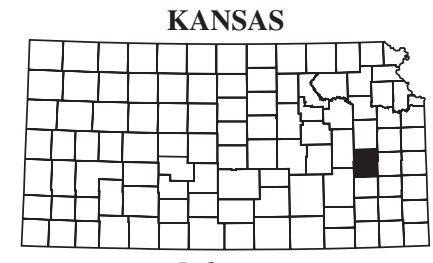

Index map 3343

Lake and determination site identification number

Figure 26. Location of streamflow-statistics determination sites, associated identification numbers, and U.S. Geological Survey streamflow-gaging stations used in the flow-duration and peak-discharge frequency analyses for Coffey County. 
Table 22. Estimated flow-duration values, mean flow values, and peak-discharge frequency values for controlled and uncontrolled flow stream segments on the 1999 Kansas Surface Water Register for Coffey County.

[KSWR, Kansas Surface Water Register; CUSEGA, catalog unit segment number alpha; $\mathrm{mi}^{2}$, square miles; $\mathrm{ft}^{3} / \mathrm{s}$, cubic feet per second; HYDRO, lake or other hydrologic structure; NA, not applicable; NRDitch, irrigation ditch; NRTribal, tribal stream]

\begin{tabular}{|c|c|c|c|c|c|c|c|c|c|c|c|c|}
\hline \multirow{2}{*}{$\begin{array}{l}\text { Determi- } \\
\text { nation site } \\
\text { identi- } \\
\text { fication } \\
\text { number } \\
\text { (fig. 26) }\end{array}$} & \multirow{2}{*}{$\begin{array}{l}\text { KSWR } \\
\text { CUSEGA } \\
\text { number }\end{array}$} & \multicolumn{4}{|c|}{$\begin{array}{l}\text { Stream segment } \\
\text { by county } \\
\text { (table 112) }\end{array}$} & \multirow{2}{*}{ Stream name } & \multirow{2}{*}{$\begin{array}{l}\text { Contri- } \\
\text { buting } \\
\text { drainage } \\
\text { area } \\
\left(\mathrm{mi}^{2}\right)\end{array}$} & \multicolumn{5}{|c|}{$\begin{array}{l}\text { Estimated flow-duration values }\left(\mathrm{ft}^{3} / \mathrm{s}\right) \text { for } \\
\text { indicated percentage of time flow equaled or } \\
\text { exceeded }\end{array}$} \\
\hline & & $1 \mathrm{st}$ & 2nd & 3rd & 4th & & & $\begin{array}{c}90 \\
\text { percent }\end{array}$ & $\begin{array}{c}75 \\
\text { percent }\end{array}$ & $\begin{array}{c}50 \\
\text { percent }\end{array}$ & $\begin{array}{c}25 \\
\text { percent }\end{array}$ & $\begin{array}{c}10 \\
\text { percent }\end{array}$ \\
\hline 2973 & 1029010194 & $\mathrm{CF}$ & OS & & & Willow Creek & 5.60 & $\overline{0}$ & 0 & 0.10 & 0.78 & 3.53 \\
\hline 2987 & 1029010145 & $\mathrm{CF}$ & OS & & & South Branch Tequa Creek & 11.6 & 0 & 0 & .74 & 2.97 & 9.51 \\
\hline 3007 & 1029010173 & $\mathrm{CF}$ & OS & & & Little Rock Creek & 8.96 & 0 & 0 & .13 & 1.15 & 4.99 \\
\hline 3012 & HYDRO & $\mathrm{CF}$ & & & & HYDRO & 3.59 & NA & NA & NA & NA & NA \\
\hline 3015 & 1029010173 & $\mathrm{CF}$ & & & & Little Rock Creek & 3.42 & 0 & 0 & 0 & 0 & .51 \\
\hline 3018 & HYDRO & $\mathrm{CF}$ & & & & HYDRO & 2.74 & NA & NA & NA & NA & NA \\
\hline 3024 & 1029010173 & $\mathrm{CF}$ & & & & Little Rock Creek & 2.48 & 0 & 0 & 0 & 0 & 0 \\
\hline 3034 & 1029010142 & $\mathrm{CF}$ & OS & & & Frog Creek & 51.7 & 0 & .23 & 3.31 & 12.9 & 40.8 \\
\hline 3044 & HYDRO & $\mathrm{CF}$ & & & & HYDRO & 22.2 & NA & NA & NA & NA & NA \\
\hline 3047 & 1029010142 & $\mathrm{CF}$ & & & & Frog Creek & 21.1 & 0 & 0 & 1.04 & 4.61 & 15.5 \\
\hline 3050 & HYDRO & $\mathrm{CF}$ & & & & HYDRO & 20.5 & NA & NA & NA & NA & NA \\
\hline 3083 & 1107020151 & $\mathrm{CF}$ & & & & Lebo Creek & 23.9 & 0 & 0 & .79 & 4.45 & 16.5 \\
\hline 3110 & 1029010142 & $\mathrm{CF}$ & & & & Frog Creek & 20.4 & 0 & 0 & .99 & 4.44 & 15.0 \\
\hline 3121 & 1029010143 & $\mathrm{CF}$ & OS & & & Rock Creek & 20.4 & 0 & 0 & 1.06 & 4.35 & 14.2 \\
\hline 3141 & 102901011531 & $\mathrm{CF}$ & OS & & & Long Creek & 38.7 & 0 & .18 & 2.87 & 10.6 & 32.1 \\
\hline 3185 & 1107020438 & $\mathrm{CF}$ & & & & School Creek & 10.3 & 0 & 0 & .84 & 2.66 & 7.88 \\
\hline 3186 & 1107020412 & $\mathrm{CF}$ & & & & Long Creek & 15.1 & 0 & 0 & 1.29 & 4.10 & 11.8 \\
\hline 3188 & 1107020151 & $\mathrm{CF}$ & & & & Lebo Creek & 45.0 & 0 & 0 & 2.25 & 9.83 & 33.3 \\
\hline 3189 & 1107020437 & $\mathrm{CF}$ & & & & Wolf Creek & 11.9 & 0 & 0 & .81 & 2.76 & 8.48 \\
\hline 3191 & 1107020126 & $\mathrm{CF}$ & LY & & & Neosho River & 2,740 & 31.4 & 73.4 & 380 & 1,480 & 4,850 \\
\hline 3211 & 1107020126 & $\mathrm{CF}$ & & & & Neosho River & 2,790 & 30.6 & 71.9 & 384 & 1,510 & 4,990 \\
\hline 3214 & 1107020440 & $\mathrm{CF}$ & & & & Scott Creek & 4.78 & 0 & 0 & .24 & .79 & 3.01 \\
\hline 3219 & HYDRO & $\mathrm{CF}$ & & & & HYDRO & 5.01 & NA & NA & NA & NA & NA \\
\hline 3227 & 110702011 & $\mathrm{CF}$ & & & & Neosho River & 2,920 & 28.6 & 68.2 & 394 & 1,590 & 5,310 \\
\hline 3231 & 1107020125 & $\mathrm{CF}$ & LY & & & Eagle Creek & 115 & 0 & .88 & 6.14 & 24.4 & 81.8 \\
\hline 3259 & HYDRO & $\mathrm{CF}$ & & & & HYDRO & 24.5 & NA & NA & NA & NA & NA \\
\hline 3278 & HYDRO & $\mathrm{CF}$ & & & & HYDRO & 2,950 & NA & NA & NA & NA & NA \\
\hline 3343 & HYDRO & $\mathrm{CF}$ & & & & HYDRO & 35.4 & NA & NA & NA & NA & NA \\
\hline 3348 & 1107020413 & $\mathrm{CF}$ & & & & Neosho River & 2,960 & 28.0 & 67.0 & 397 & 1,620 & 5,420 \\
\hline 3355 & 1107020412 & $\mathrm{CF}$ & & & & Long Creek & 45.9 & 0 & .82 & 4.06 & 13.0 & 36.6 \\
\hline 3359 & 1107020440 & $\mathrm{CF}$ & & & & Scott Creek & 18.9 & 0 & 0 & 1.56 & 5.47 & 16.2 \\
\hline 3371 & 1107020415 & $\mathrm{CF}$ & & & & Rock Creek & 24.9 & 0 & 0 & 1.15 & 4.94 & 16.5 \\
\hline 3403 & 1107020442 & $\mathrm{CF}$ & & & & Badger Creek & 7.73 & 0 & 0 & 0 & .68 & 4.34 \\
\hline 3404 & 1107020412 & $\mathrm{CF}$ & & & & Long Creek & 68.2 & 0 & 1.25 & 5.80 & 19.1 & 55.2 \\
\hline 3412 & 1107020437 & $\mathrm{CF}$ & & & & Wolf Creek & 41.7 & 0 & .67 & 3.54 & 11.2 & 31.6 \\
\hline 3413 & 1107020413 & $\mathrm{CF}$ & & & & Neosho River & 2,990 & 28.6 & 69.2 & 405 & 1,650 & 5,500 \\
\hline 3425 & 1107020416 & $\mathrm{CF}$ & GW & LY & & North Big Creek & 41.8 & 0 & 0 & 2.43 & 9.89 & 32.1 \\
\hline 3441 & 1107020416 & $\mathrm{CF}$ & & & & North Big Creek & 52.0 & 0 & .19 & 3.23 & 12.7 & 40.6 \\
\hline
\end{tabular}


Table 22. Estimated flow-duration values, mean flow values, and peak-discharge frequency values for controlled and uncontrolled flow stream segments on the 1999 Kansas Surface Water Register for Coffey County.-Continued

[KSWR, Kansas Surface Water Register; CUSEGA, catalog unit segment number alpha; $\mathrm{mi}^{2}$, square miles; $\mathrm{ft}^{3} / \mathrm{s}$, cubic feet per second; HYDRO, lake or other hydrologic structure; NA, not applicable; NRDitch, irrigation ditch; NRTribal, tribal stream]

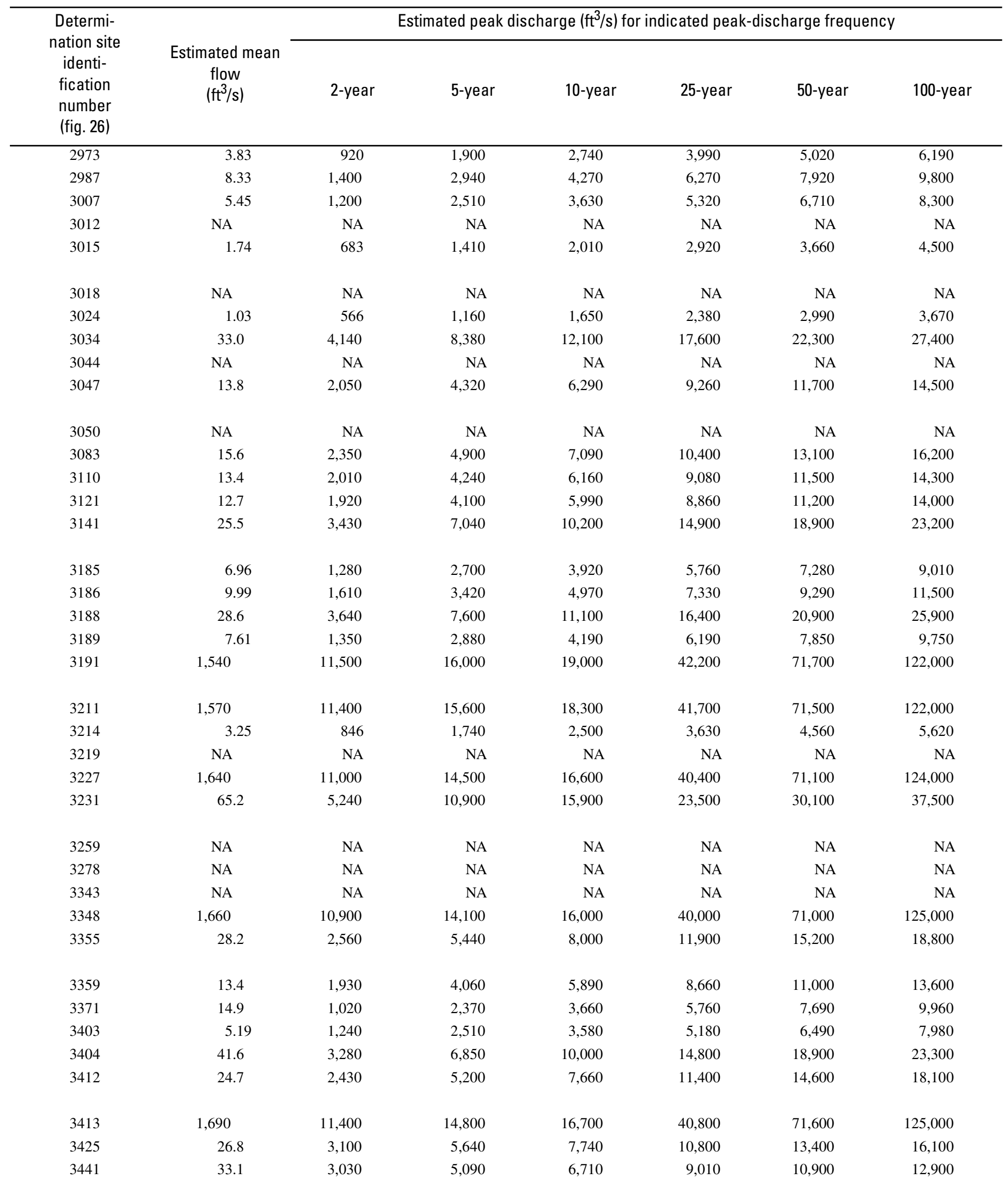


Table 22. Estimated flow-duration values, mean flow values, and peak-discharge frequency values for controlled and uncontrolled flow stream segments on the 1999 Kansas Surface Water Register for Coffey County.-Continued

[KSWR, Kansas Surface Water Register; CUSEGA, catalog unit segment number alpha; $\mathrm{mi}^{2}$, square miles; $\mathrm{ft}^{3} / \mathrm{s}$, cubic feet per second; HYDRO, lake or other hydrologic structure; NA, not applicable; NRDitch, irrigation ditch; NRTribal, tribal stream]

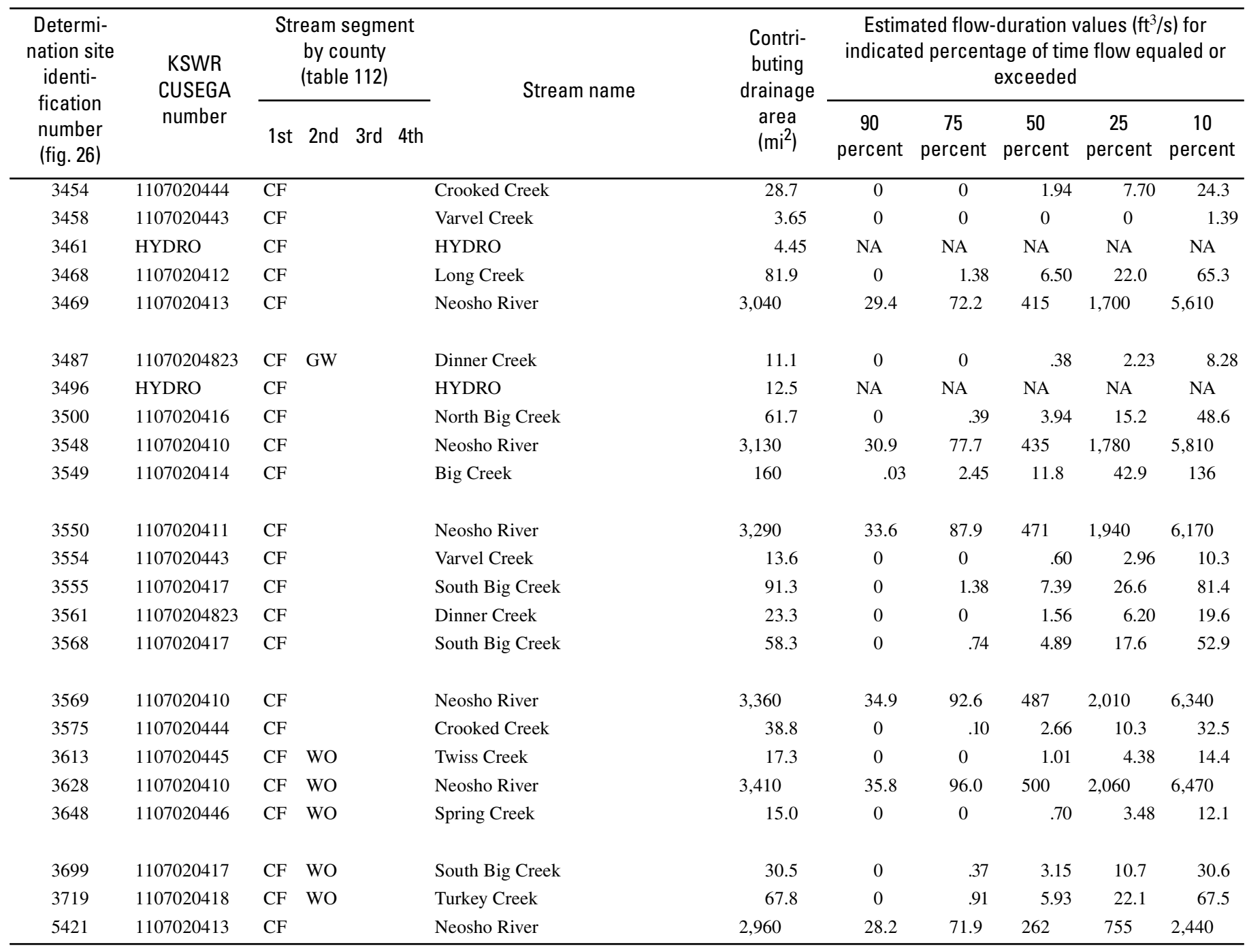


Table 22. Estimated flow-duration values, mean flow values, and peak-discharge frequency values for controlled and uncontrolled flow stream segments on the 1999 Kansas Surface Water Register for Coffey County.-Continued

[KSWR, Kansas Surface Water Register; CUSEGA, catalog unit segment number alpha; $\mathrm{mi}^{2}$, square miles; $\mathrm{ft}^{3} / \mathrm{s}$, cubic feet per second; HYDRO, lake or other hydrologic structure; NA, not applicable; NRDitch, irrigation ditch; NRTribal, tribal stream]

\begin{tabular}{|c|c|c|c|c|c|c|c|}
\hline \multirow{2}{*}{$\begin{array}{l}\text { Determi- } \\
\text { nation site } \\
\text { identi- } \\
\text { fication } \\
\text { number } \\
\text { (fig. 26) }\end{array}$} & \multirow{2}{*}{$\begin{array}{c}\text { Estimated mean } \\
\text { flow } \\
\left(\mathrm{ft}^{3} / \mathrm{s}\right)\end{array}$} & \multicolumn{6}{|c|}{ Estimated peak discharge $\left(\mathrm{ft}^{3} / \mathrm{s}\right)$ for indicated peak-discharge frequency } \\
\hline & & 2-year & 5-year & 10-year & 25-year & 50-year & 100-year \\
\hline 3454 & 20.3 & 2,650 & 5,530 & 8,000 & 11,700 & 14,800 & 18,300 \\
\hline 3458 & 2.34 & 756 & 1,530 & 2,180 & 3,150 & 3,940 & 4,830 \\
\hline 3461 & NA & NA & NA & NA & NA & NA & NA \\
\hline 3468 & 49.5 & 3,390 & 7,150 & 10,500 & 15,600 & 20,000 & 24,900 \\
\hline 3469 & 1,720 & 12,000 & 15,800 & 17,800 & 42,000 & 72,500 & 125,000 \\
\hline 3487 & 7.91 & 1,450 & 3,000 & 4,320 & 6,300 & 7,940 & 9,800 \\
\hline 3496 & NA & NA & NA & NA & NA & NA & NA \\
\hline 3500 & 39.0 & 3,210 & 4,850 & 6,000 & 7,500 & 8,660 & 9,830 \\
\hline 3548 & 1,780 & 13,200 & 17,600 & 19,700 & 44,000 & 74,100 & 124,000 \\
\hline 3549 & 98.6 & 5,750 & 9,550 & 12,500 & 16,800 & 20,200 & 23,900 \\
\hline 3550 & 1,880 & 15,400 & 21,000 & 23,100 & 47,800 & 77,000 & 124,000 \\
\hline 3554 & 9.55 & 1,630 & 3,390 & 4,890 & 7,150 & 9,020 & 11,100 \\
\hline 3555 & 60.3 & 5,250 & 10,400 & 14,800 & 21,400 & 27,000 & 33,100 \\
\hline 3561 & 16.4 & 2,220 & 4,670 & 6,780 & 9,980 & 12,600 & 15,700 \\
\hline 3568 & 40.1 & 4,470 & 8,860 & 12,600 & 18,200 & 22,900 & 28,000 \\
\hline 3569 & 1,930 & 16,400 & 22,600 & 24,700 & 49,600 & 78,400 & 124,000 \\
\hline 3575 & 26.8 & 3,440 & 6,950 & 9,980 & 14,500 & 18,300 & 22,400 \\
\hline 3613 & 12.9 & 2,140 & 4,340 & 6,190 & 8,960 & 11,200 & 13,800 \\
\hline 3628 & 1,970 & 17,100 & 23,700 & 25,900 & 50,900 & 79,400 & 123,000 \\
\hline 3648 & 11.3 & 1,980 & 3,990 & 5,680 & 8,210 & 10,300 & 12,600 \\
\hline 3699 & 23.1 & 3,210 & 6,410 & 9,140 & 13,200 & 16,500 & 20,200 \\
\hline 3719 & 50.4 & 4,210 & 8,500 & 12,200 & 17,800 & 22,600 & 27,800 \\
\hline 5421 & 1,250 & 19,000 & 37,700 & 54,300 & 79,500 & 102,000 & 127,000 \\
\hline
\end{tabular}




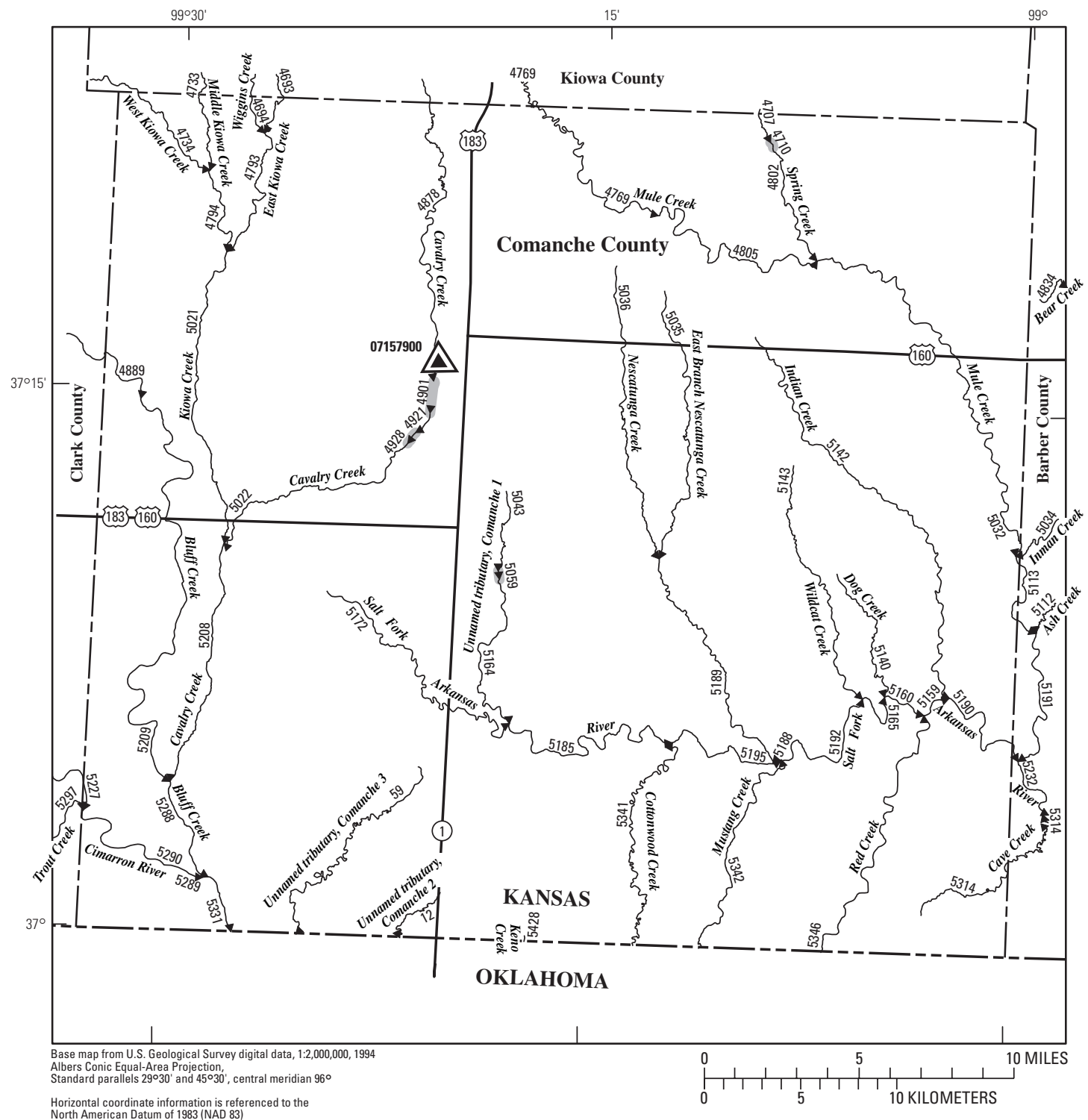

EXPLANATION

\$331 Location of streamflow-statistics determination site (small triangle) and associated identification number-small triangle points in downstream direction

07157900

U.S. Geological Survey streamflow-gaging station and number used for estimates of flow duration

07157900 人

U.S. Geological Survey streamflow-gaging station and number used for estimates of peak-discharge frequency values

KANSAS

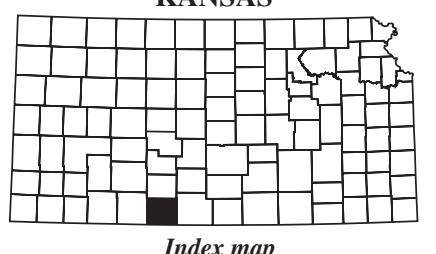

Index map 4903

Lake and determination site identification number

Figure 27. Location of streamflow-statistics determination sites, associated identification numbers, and U.S. Geological Survey streamflow-gaging stations used in the flow-duration and peak-discharge frequency analyses for Comanche County. 
Table 23. Estimated flow-duration values, mean flow values, and peak-discharge frequency values for controlled and uncontrolled flow stream segments on the 1999 Kansas Surface Water Register for Comanche County.

[KSWR, Kansas Surface Water Register; CUSEGA, catalog unit segment number alpha; $\mathrm{mi}^{2}$, square miles; $\mathrm{ft}^{3} / \mathrm{s}$, cubic feet per second; HYDRO, lake or other hydrologic structure; NA, not applicable; NRDitch, irrigation ditch; NRTribal, tribal stream]

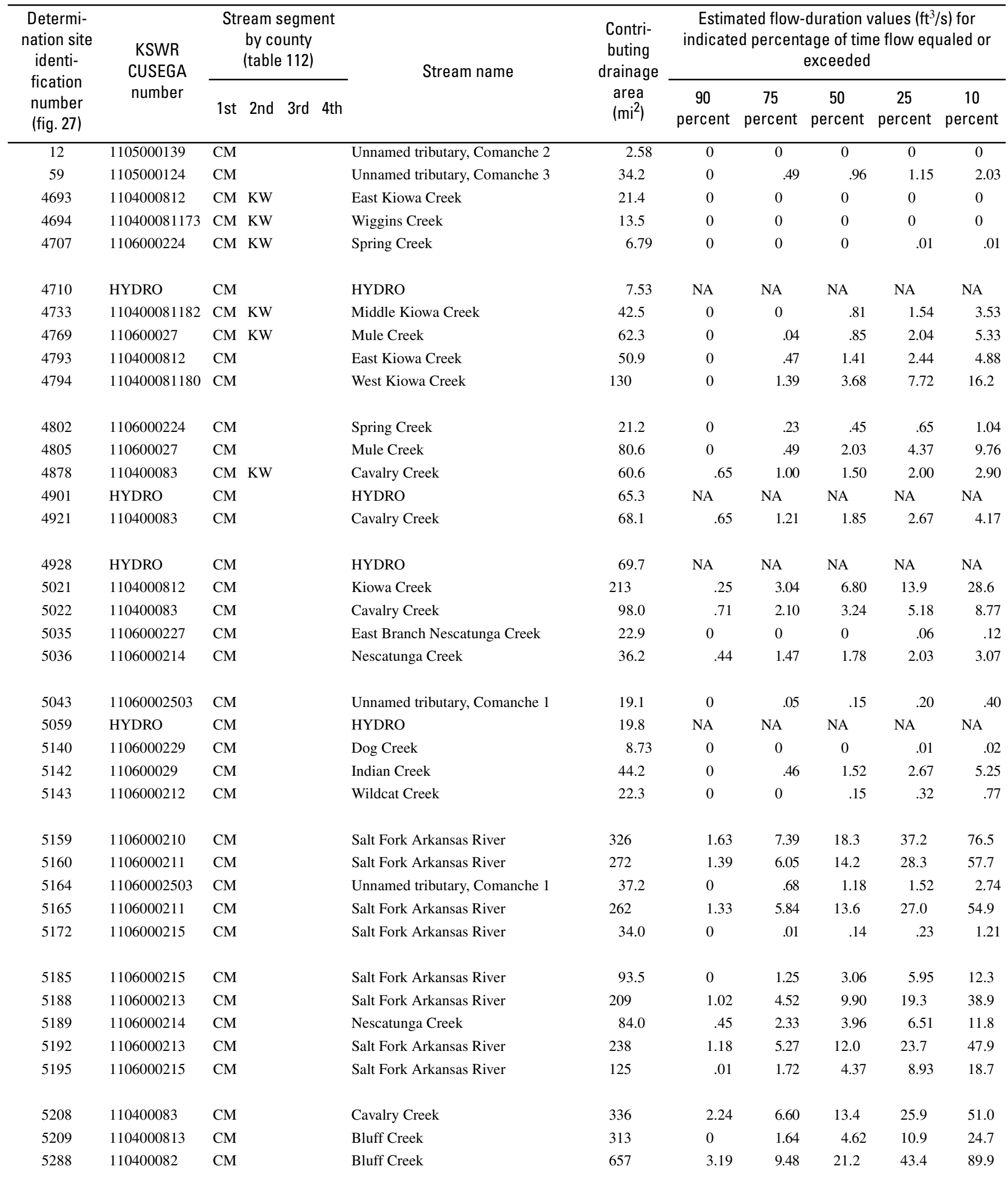


Table 23. Estimated flow-duration values, mean flow values, and peak-discharge frequency values for controlled and uncontrolled flow stream segments on the 1999 Kansas Surface Water Register for Comanche County.-Continued

[KSWR, Kansas Surface Water Register; CUSEGA, catalog unit segment number alpha; $\mathrm{mi}^{2}$, square miles; $\mathrm{ft}^{3} / \mathrm{s}$, cubic feet per second; HYDRO, lake or other hydrologic structure; NA, not applicable; NRDitch, irrigation ditch; NRTribal, tribal stream]

\begin{tabular}{|c|c|c|c|c|c|c|c|}
\hline \multirow{2}{*}{$\begin{array}{l}\text { Determi- } \\
\text { nation site } \\
\text { identi- } \\
\text { fication } \\
\text { number } \\
\text { (fig. } 27 \text { ) }\end{array}$} & \multirow{2}{*}{$\begin{array}{c}\text { Estimated mean } \\
\text { flow } \\
\left(\mathrm{ft}^{3} / \mathrm{s}\right)\end{array}$} & \multicolumn{6}{|c|}{ Estimated peak discharge $\left(\mathrm{ft}^{3} / \mathrm{s}\right)$ for indicated peak-discharge frequency } \\
\hline & & 2-year & 5-year & 10-year & 25-year & 50-year & 100-year \\
\hline 12 & 0 & 185 & 498 & 801 & 1,290 & 1,730 & 2,230 \\
\hline 4694 & .59 & 468 & 1,320 & 2,160 & 3,560 & 4,810 & 6,290 \\
\hline 4707 & .01 & 319 & 882 & 1,430 & 2,340 & 3,150 & 4,100 \\
\hline 4710 & NA & NA & NA & NA & NA & NA & NA \\
\hline 4733 & 4.18 & 725 & 1,870 & 3,000 & 4,810 & 6,450 & 8,300 \\
\hline 4802 & 1.98 & 622 & 1,760 & 2,890 & 4,780 & 6,460 & 8,460 \\
\hline 4805 & 8.86 & 698 & 1,900 & 3,130 & 5,170 & 7,060 & 9,240 \\
\hline 4878 & 3.45 & 492 & 1,290 & 2,050 & 3,290 & 4,410 & 5,680 \\
\hline 4901 & NA & NA & NA & NA & NA & NA & NA \\
\hline 4921 & 4.35 & 538 & 1,390 & 2,200 & 3,520 & 4,710 & 6,060 \\
\hline 4928 & NA & NA & NA & NA & NA & NA & NA \\
\hline 5021 & 21.4 & 1,640 & 3,940 & 6,150 & 9,640 & 12,800 & 16,300 \\
\hline 5022 & 7.51 & 762 & 1,860 & 2,880 & 4,490 & 5,920 & 7,520 \\
\hline 5035 & 1.61 & 661 & 1,860 & 3,060 & 5,060 & 6,840 & 8,960 \\
\hline 5159 & 49.7 & 1,660 & 4,050 & 6,380 & 10,100 & 13,500 & 17,300 \\
\hline 5160 & 38.7 & 1,450 & 3,570 & 5,630 & 8,920 & 11,900 & 15,300 \\
\hline 5164 & 3.45 & 570 & 1,430 & 2,250 & 3,540 & 4,690 & 5,960 \\
\hline 5165 & 37.0 & 1,420 & 3,490 & 5,510 & 8,740 & 11,700 & 15,000 \\
\hline 5172 & 2.72 & 392 & 1,130 & 1,900 & 3,210 & 4,430 & 5,840 \\
\hline 5185 & 10.4 & 739 & 1,950 & 3,160 & 5,140 & 6,950 & 9,010 \\
\hline 5188 & 27.4 & 1,190 & 2,970 & 4,710 & 7,490 & 10,000 & 12,900 \\
\hline 5189 & 9.55 & 957 & 2,270 & 3,500 & 5,400 & 7,070 & 8,910 \\
\hline 5192 & 32.7 & 1,330 & 3,290 & 5,190 & 8,240 & 11,000 & 14,100 \\
\hline 5195 & 14.8 & 895 & 2,340 & 3,790 & 6,150 & 8,310 & 10,800 \\
\hline 5208 & 34.3 & 1,870 & 4,350 & 6,650 & 10,200 & 13,400 & 17,000 \\
\hline 5209 & 20.6 & 1,500 & 3,910 & 6,330 & 10,300 & 14,000 & 18,300 \\
\hline 5288 & 58.8 & 2,420 & 5,750 & 8,920 & 14,000 & 18,600 & 23,800 \\
\hline
\end{tabular}


Table 23. Estimated flow-duration values, mean flow values, and peak-discharge frequency values for controlled and uncontrolled flow stream segments on the 1999 Kansas Surface Water Register for Comanche County._-Continued

[KSWR, Kansas Surface Water Register; CUSEGA, catalog unit segment number alpha; $\mathrm{mi}^{2}$, square miles; $\mathrm{ft}^{3} / \mathrm{s}$, cubic feet per second; HYDRO, lake or other hydrologic structure; NA, not applicable; NRDitch, irrigation ditch; NRTribal, tribal stream]

\begin{tabular}{|c|c|c|c|c|c|c|c|c|c|}
\hline $\begin{array}{l}\text { Determi- } \\
\text { nation site } \\
\text { identi- } \\
\text { fication } \\
\text { number } \\
\text { (fig. } 27 \text { ) }\end{array}$ & $\begin{array}{l}\text { KSWR } \\
\text { CUSEGA } \\
\text { number }\end{array}$ & $\begin{array}{l}\text { Stream segment } \\
\text { by county } \\
\text { (table 112) }\end{array}$ & Stream name & $\begin{array}{l}\text { Contri- } \\
\text { buting } \\
\text { drainage } \\
\text { area } \\
\left(\mathrm{mi}^{2}\right)\end{array}$ & \multicolumn{5}{|c|}{$\begin{array}{l}\text { Estimated flow-duration values }\left(\mathrm{ft}^{3} / \mathrm{s}\right) \text { for } \\
\text { indicated percentage of time flow equaled or } \\
\text { exceeded }\end{array}$} \\
\hline 5289 & 110400081 & $\mathrm{CM}$ & Cimarron River & 9,540 & 19.3 & 41.3 & 94.3 & 193 & 385 \\
\hline 5342 & 1106000231 & $\mathrm{CM}$ & Mustang Creek & 20.6 & 0 & 0 & .17 & .18 & .76 \\
\hline 5346 & 1106000216 & $\mathrm{CM}$ & Red Creek & 53.0 & 0 & .31 & 1.78 & 3.72 & 7.78 \\
\hline 5428 & 1105000122 & $\mathrm{CM}$ & Keno Creek & .25 & 0 & 0 & 0 & 0 & 0 \\
\hline
\end{tabular}


Table 23. Estimated flow-duration values, mean flow values, and peak-discharge frequency values for controlled and uncontrolled flow stream segments on the 1999 Kansas Surface Water Register for Comanche County.-Continued

[KSWR, Kansas Surface Water Register; CUSEGA, catalog unit segment number alpha; $\mathrm{mi}^{2}$, square miles; $\mathrm{ft}^{3} / \mathrm{s}$, cubic feet per second; HYDRO, lake or other hydrologic structure; NA, not applicable; NRDitch, irrigation ditch; NRTribal, tribal stream]

\begin{tabular}{|c|c|c|c|c|c|c|c|}
\hline \multirow{2}{*}{$\begin{array}{l}\text { Determi- } \\
\text { nation site } \\
\text { identi- } \\
\text { fication } \\
\text { number } \\
\text { (fig. 27) }\end{array}$} & \multirow{2}{*}{$\begin{array}{l}\text { Estimated mean } \\
\text { flow } \\
\left(\mathrm{ft}^{3} / \mathrm{s}\right)\end{array}$} & \multicolumn{6}{|c|}{ Estimated peak discharge $\left(\mathrm{ft}^{3} / \mathrm{s}\right)$ for indicated peak-discharge frequency } \\
\hline & & 2-year & 5-year & 10-year & 25-year & 50-year & 100-year \\
\hline 5289 & 239 & 3,690 & 9,830 & 16,100 & 26,600 & 36,300 & 47,900 \\
\hline 5342 & 1.94 & 607 & 1,720 & 2,830 & 4,680 & 6,340 & 8,300 \\
\hline 5346 & 6.75 & 925 & 2,340 & 3,710 & 5,900 & 7,880 & 10,100 \\
\hline 5428 & 0 & 47 & 121 & 191 & 301 & 393 & 506 \\
\hline
\end{tabular}




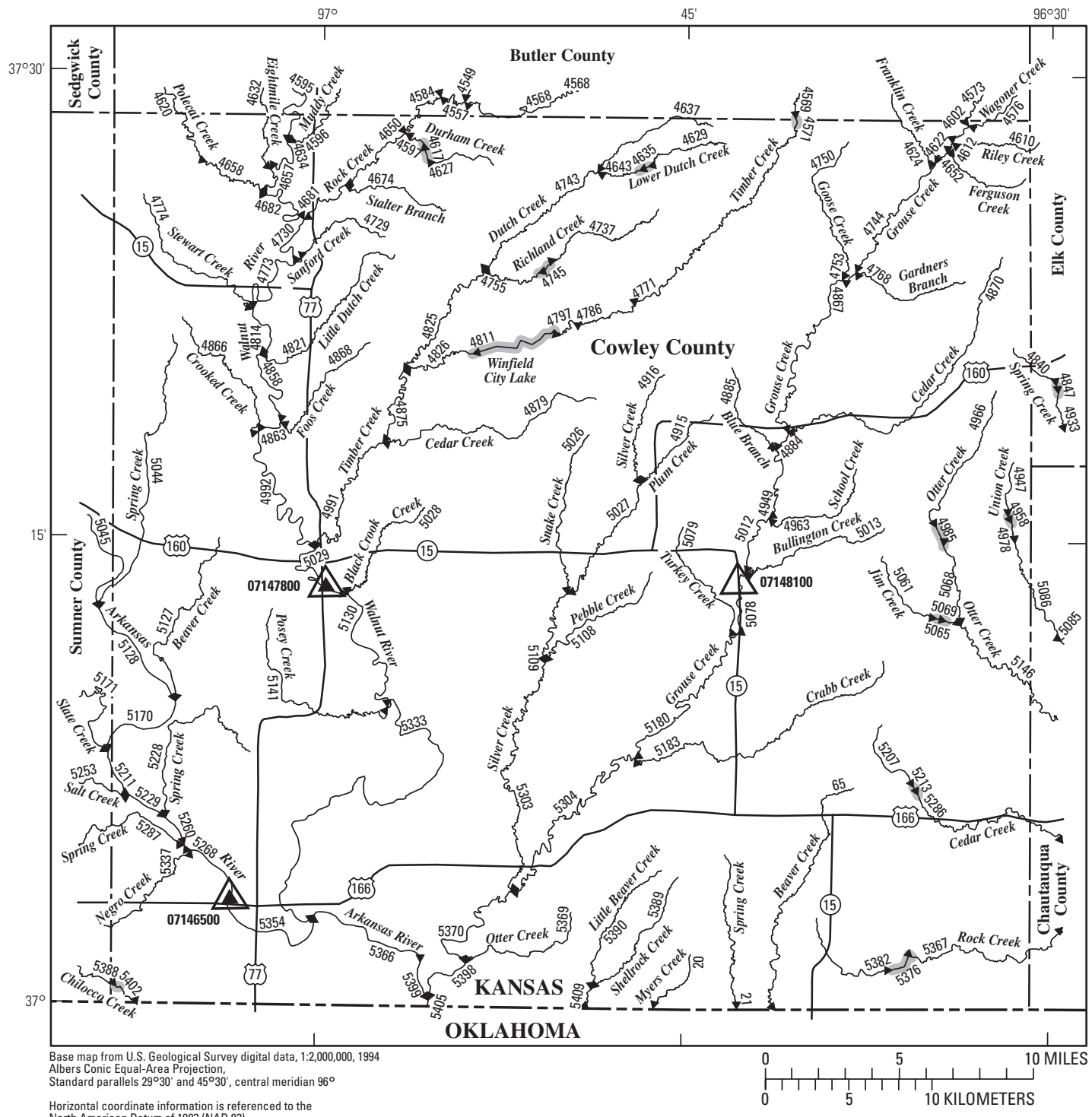

EXPLANATION

$\stackrel{5388}{\longleftarrow}$ Location of streamflow-statistics determination site (small triangle) and associated identification number-small triangle points in downstream direction

${ }^{07146500} \Delta$ U.S. Geological Survey streamflow-gaging station and number used for estimates of flow duration

${ }^{07148100} \triangle$ U.S. Geological Survey streamflow-gaging station and number used for estimates of peak-discharge frequency values

KANSAS

Lake and determination site identification number

Figure 28. Location of streamflow-statistics determination sites, associated identification numbers, and U.S. Geological Survey streamflow-gaging stations used in the flow-duration and peak-discharge frequency analyses for Cowley County. 
Table 24. Estimated flow-duration values, mean flow values, and peak-discharge frequency values for controlled and uncontrolled flow stream segments on the 1999 Kansas Surface Water Register for Cowley County.

[KSWR, Kansas Surface Water Register; CUSEGA, catalog unit segment number alpha; $\mathrm{mi}^{2}$, square miles; $\mathrm{ft}^{3} / \mathrm{s}$, cubic feet per second; HYDRO, lake or other hydrologic structure; NA, not applicable; NRDitch, irrigation ditch; NRTribal, tribal stream]

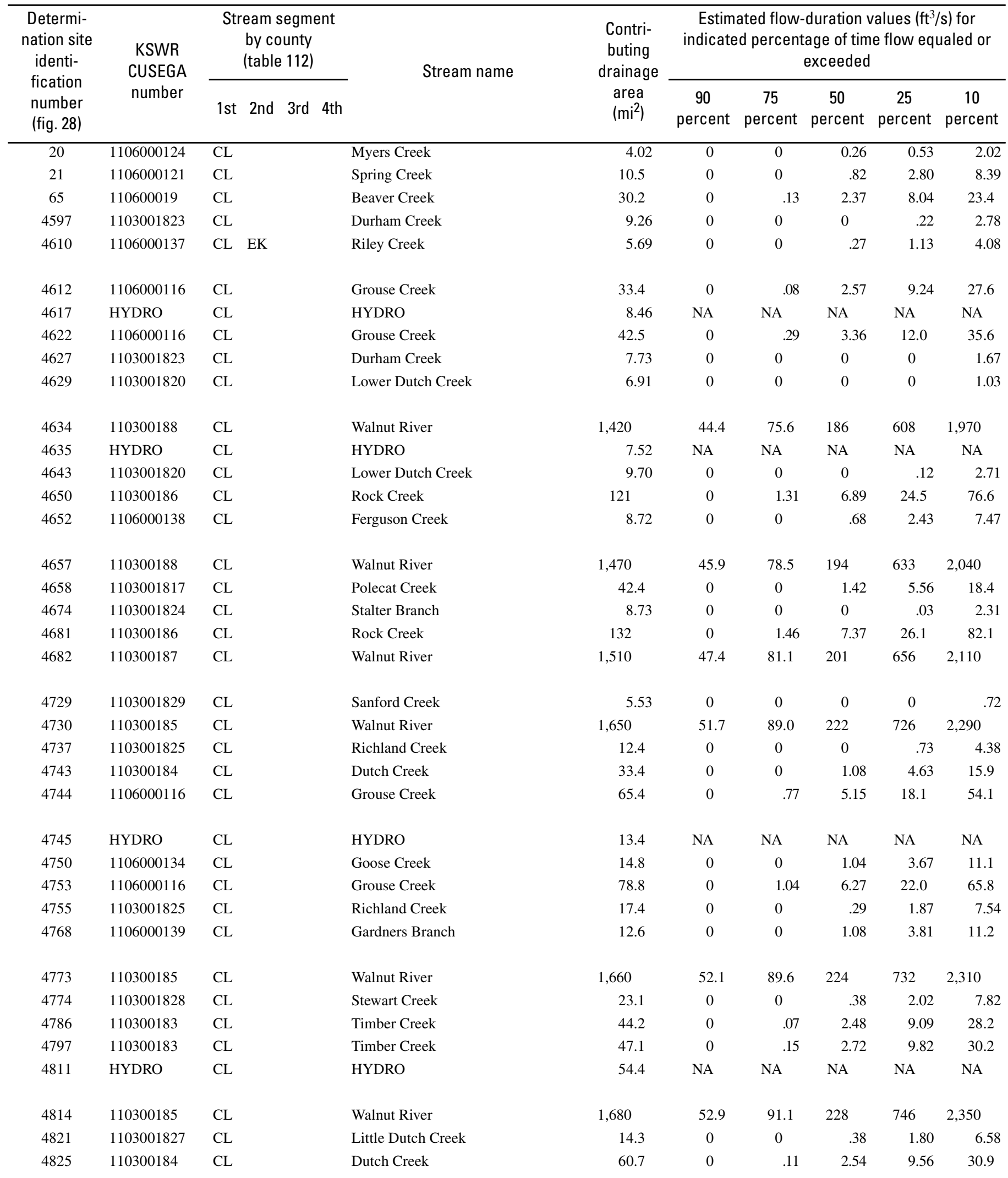


Table 24. Estimated flow-duration values, mean flow values, and peak-discharge frequency values for controlled and uncontrolled flow stream segments on the 1999 Kansas Surface Water Register for Cowley County.-Continued

[KSWR, Kansas Surface Water Register; CUSEGA, catalog unit segment number alpha; $\mathrm{mi}^{2}$, square miles; $\mathrm{ft}^{3} / \mathrm{s}$, cubic feet per second; HYDRO, lake or other hydrologic structure; NA, not applicable; NRDitch, irrigation ditch; NRTribal, tribal stream]

\begin{tabular}{|c|c|c|c|c|c|c|c|}
\hline \multirow{2}{*}{$\begin{array}{l}\text { Determi- } \\
\text { nation site } \\
\text { identi- } \\
\text { fication } \\
\text { number } \\
\text { (fig. 28) }\end{array}$} & \multirow{2}{*}{$\begin{array}{c}\text { Estimated mean } \\
\text { flow } \\
\left(\mathrm{ft}^{3} / \mathrm{s}\right)\end{array}$} & \multicolumn{6}{|c|}{ Estimated peak discharge $\left(\mathrm{ft}^{3} / \mathrm{s}\right)$ for indicated peak-discharge frequency } \\
\hline & & 2-year & 5-year & 10-year & 25-year & 50-year & 100-year \\
\hline 20 & 2.34 & 660 & 1,400 & 2,040 & 3,000 & 3,800 & 4,700 \\
\hline 4597 & 4.01 & 1,000 & 2,210 & 3,260 & 4,870 & 6,210 & 7,750 \\
\hline 4610 & 3.92 & 841 & 1,790 & 2,600 & 3,820 & 4,830 & 5,980 \\
\hline 4612 & 21.5 & 3,840 & 7,750 & 11,100 & 16,000 & 20,300 & 24,700 \\
\hline 4617 & NA & NA & NA & NA & NA & NA & NA \\
\hline 4634 & 831 & 13,900 & 26,900 & 33,400 & 49,300 & 64,600 & 81,300 \\
\hline 4635 & NA & NA & NA & NA & NA & NA & NA \\
\hline 4643 & 4.13 & 1,060 & 2,310 & 3,410 & 5,080 & 6,480 & 8,080 \\
\hline 4650 & 58.8 & 5,410 & 11,100 & 16,200 & 23,900 & 30,500 & 37,900 \\
\hline 4652 & 6.29 & 1,070 & 2,290 & 3,350 & 4,950 & 6,290 & 7,810 \\
\hline 4657 & 862 & 14,300 & 27,900 & 34,600 & 50,900 & 66,700 & 84,100 \\
\hline 4658 & 16.8 & 2,190 & 5,060 & 7,740 & 11,900 & 15,700 & 19,800 \\
\hline 4674 & 3.62 & 940 & 2,080 & 3,080 & 4,610 & 5,900 & 7,360 \\
\hline 4681 & 62.9 & 5,600 & 11,500 & 16,800 & 24,800 & 31,700 & 39,300 \\
\hline 4745 & NA & NA & NA & NA & NA & NA & NA \\
\hline 4750 & 9.31 & 1,400 & 3,070 & 4,530 & 6,760 & 8,620 & 10,800 \\
\hline 4753 & 47.8 & 5,590 & 11,200 & 16,100 & 23,300 & 29,400 & 36,100 \\
\hline 4755 & 7.99 & 1,450 & 3,240 & 4,810 & 7,230 & 9,260 & 11,600 \\
\hline 4768 & 9.00 & 1,300 & 2,840 & 4,170 & 6,200 & 7,890 & 9,830 \\
\hline 4773 & 986 & 16,200 & 31,500 & 39,000 & 57,500 & 75,400 & 95,000 \\
\hline 4774 & 8.37 & 1,460 & 3,400 & 5,160 & 7,890 & 10,200 & 12,900 \\
\hline 4786 & 23.1 & 3,120 & 6,690 & 9,890 & 14,700 & 18,900 & 23,600 \\
\hline 4797 & 24.6 & 3,170 & 6,800 & 10,000 & 15,000 & 19,300 & 23,900 \\
\hline 4811 & NA & NA & NA & NA & NA & NA & NA \\
\hline 4814 & 1,000 & 16,400 & 31,900 & 39,600 & 58,400 & 76,600 & 96,500 \\
\hline 4821 & 6.64 & 1,200 & 2,710 & 4,050 & 6,120 & 7,870 & 9,870 \\
\hline 4825 & 26.8 & 3,860 & 8,120 & 11,900 & 17,600 & 22,600 & 28,000 \\
\hline
\end{tabular}


Table 24. Estimated flow-duration values, mean flow values, and peak-discharge frequency values for controlled and uncontrolled flow stream segments on the 1999 Kansas Surface Water Register for Cowley County.-Continued

[KSWR, Kansas Surface Water Register; CUSEGA, catalog unit segment number alpha; $\mathrm{mi}^{2}$, square miles; $\mathrm{ft}^{3} / \mathrm{s}$, cubic feet per second; HYDRO, lake or other hydrologic structure; NA, not applicable; NRDitch, irrigation ditch; NRTribal, tribal stream]

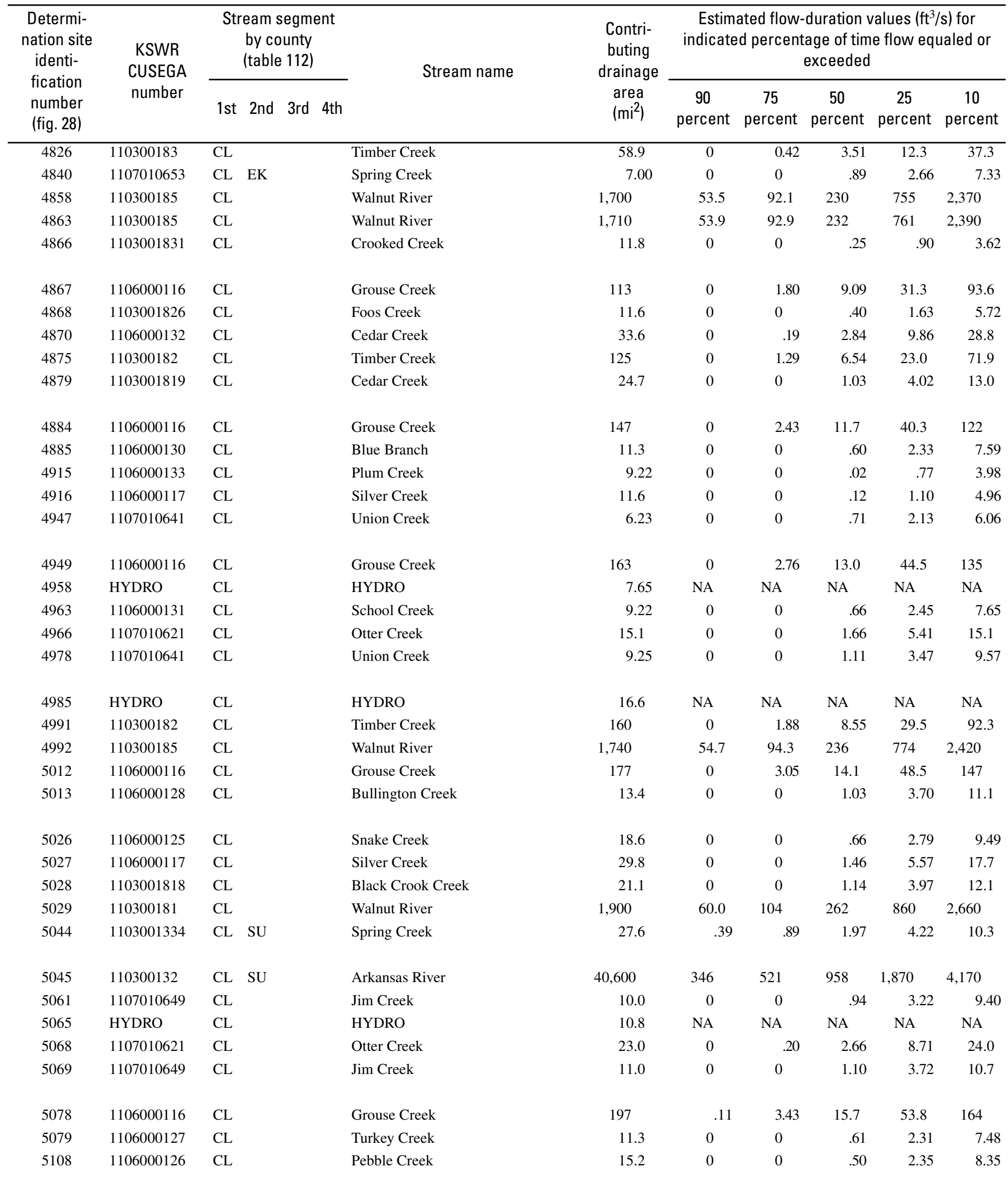


Table 24. Estimated flow-duration values, mean flow values, and peak-discharge frequency values for controlled and uncontrolled flow stream segments on the 1999 Kansas Surface Water Register for Cowley County.-Continued

[KSWR, Kansas Surface Water Register; CUSEGA, catalog unit segment number alpha; $\mathrm{mi}^{2}$, square miles; $\mathrm{ft}^{3} / \mathrm{s}$, cubic feet per second; HYDRO, lake or other hydrologic structure; NA, not applicable; NRDitch, irrigation ditch; NRTribal, tribal stream]

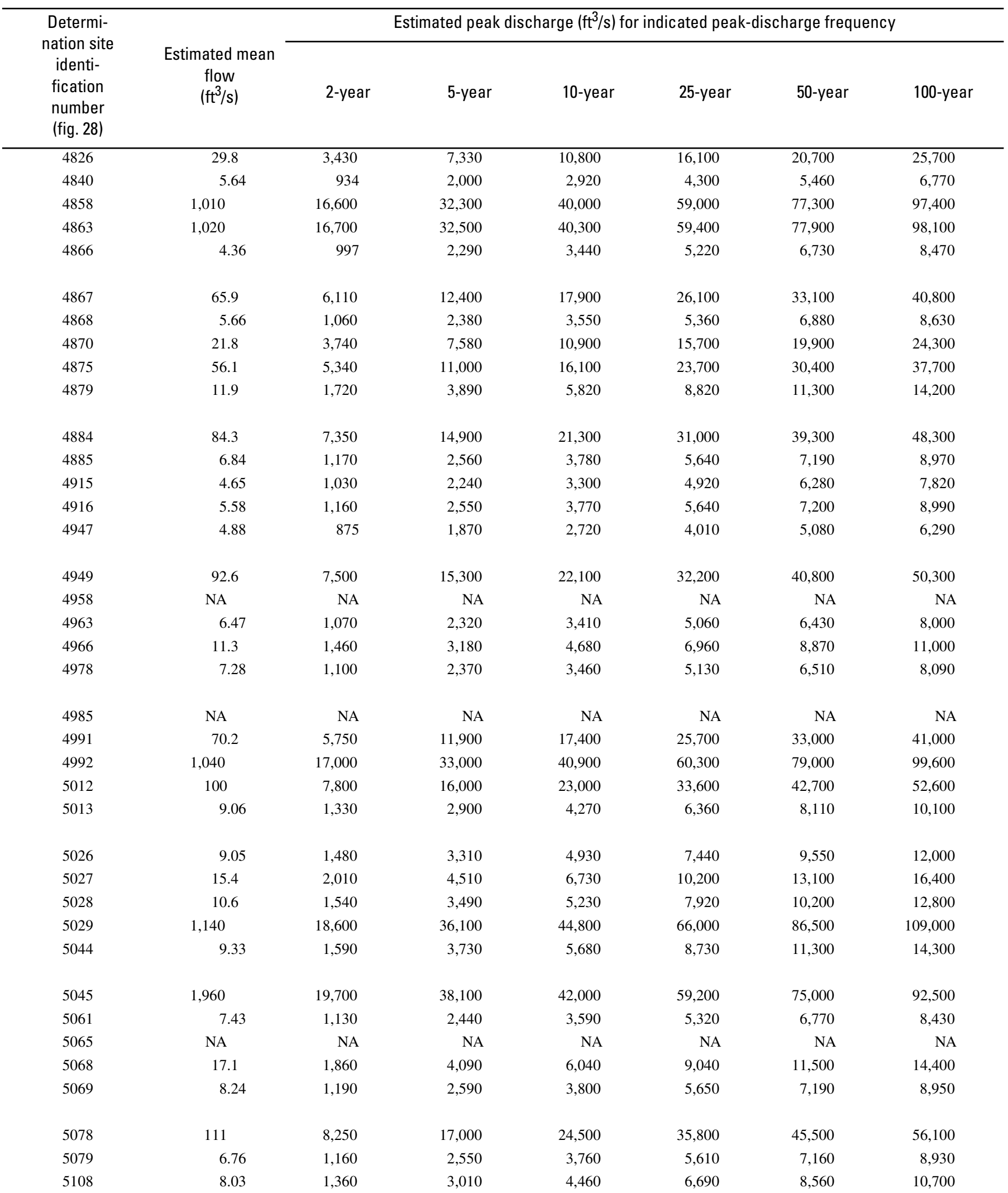


Table 24. Estimated flow-duration values, mean flow values, and peak-discharge frequency values for controlled and uncontrolled flow stream segments on the 1999 Kansas Surface Water Register for Cowley County.-Continued

[KSWR, Kansas Surface Water Register; CUSEGA, catalog unit segment number alpha; $\mathrm{mi}^{2}$, square miles; $\mathrm{ft}^{3} / \mathrm{s}$, cubic feet per second; HYDRO, lake or other hydrologic structure; NA, not applicable; NRDitch, irrigation ditch; NRTribal, tribal stream]

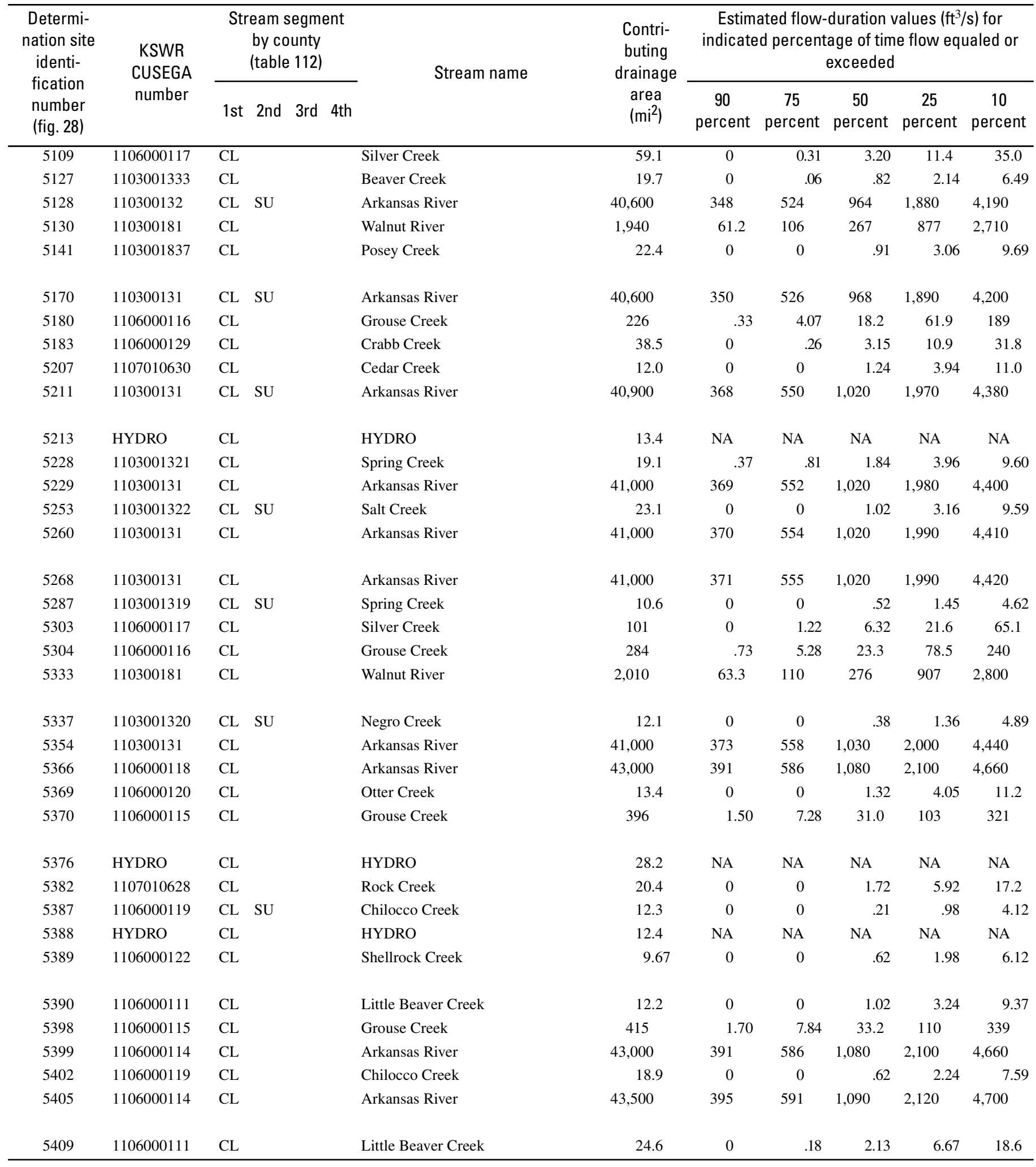


Table 24. Estimated flow-duration values, mean flow values, and peak-discharge frequency values for controlled and uncontrolled flow stream segments on the 1999 Kansas Surface Water Register for Cowley County.-Continued

[KSWR, Kansas Surface Water Register; CUSEGA, catalog unit segment number alpha; $\mathrm{mi}^{2}$, square miles; $\mathrm{ft}^{3} / \mathrm{s}$, cubic feet per second; HYDRO, lake or other hydrologic structure; NA, not applicable; NRDitch, irrigation ditch; NRTribal, tribal stream]

\begin{tabular}{|c|c|c|c|c|c|c|c|}
\hline \multirow{2}{*}{$\begin{array}{l}\text { Determi- } \\
\text { nation site } \\
\text { identi- } \\
\text { fication } \\
\text { number } \\
\text { (fig. 28) }\end{array}$} & \multirow{2}{*}{$\begin{array}{c}\text { Estimated mean } \\
\text { flow } \\
\left(\mathrm{ft}^{3} / \mathrm{s}\right)\end{array}$} & \multicolumn{6}{|c|}{ Estimated peak discharge $\left(\mathrm{ft}^{3} / \mathrm{s}\right)$ for indicated peak-discharge frequency } \\
\hline & & 2-year & 5-year & 10-year & 25-year & 50-year & 100-year \\
\hline 5109 & 28.5 & 3,430 & 7,350 & 10,900 & 16,200 & 20,900 & 26,000 \\
\hline 5130 & 1,170 & 18,900 & 36,800 & 45,700 & 67,300 & 88,200 & 111,000 \\
\hline 5141 & 9.39 & 1,590 & 3,610 & 5,410 & 8,200 & 10,600 & 13,300 \\
\hline 5170 & 1,970 & 19,800 & 38,400 & 42,300 & 59,700 & 75,700 & 93,400 \\
\hline 5180 & 126 & 8,250 & 17,100 & 24,800 & 36,300 & 46,300 & 57,300 \\
\hline 5213 & NA & $\mathrm{NA}$ & NA & $\mathrm{NA}$ & $\mathrm{NA}$ & $\mathrm{NA}$ & $\mathrm{NA}$ \\
\hline 5228 & 8.36 & 1,460 & 3,300 & 4,940 & 7,460 & 9,590 & 12,000 \\
\hline 5229 & 2,070 & 20,500 & 40,100 & 44,100 & 62,400 & 79,200 & 98,000 \\
\hline 5253 & 9.14 & 1,520 & 3,510 & 5,300 & 8,080 & 10,400 & 13,200 \\
\hline 5260 & 2,070 & 20,500 & 40,200 & 44,200 & 62,600 & 79,500 & 98,300 \\
\hline 5268 & 2,080 & 20,500 & 40,300 & 44,300 & 62,700 & 79,600 & 98,500 \\
\hline 5287 & 4.80 & 1,030 & 2,290 & 3,410 & 5,130 & 6,580 & 8,230 \\
\hline 5303 & 49.2 & 4,240 & 9,020 & 13,300 & 19,900 & 25,600 & 31,900 \\
\hline 5304 & 156 & 8,670 & 18,000 & 26,200 & 38,500 & 49,200 & 61,000 \\
\hline 5376 & NA & NA & NA & NA & NA & NA & NA \\
\hline 5382 & 13.5 & 1,760 & 3,850 & 5,670 & 8,460 & 10,800 & 13,500 \\
\hline 5387 & 4.94 & 1,120 & 2,510 & 3,750 & 5,640 & 7,230 & 9,060 \\
\hline 5388 & NA & NA & NA & NA & NA & NA & NA \\
\hline 5389 & 5.67 & 1,080 & 2,350 & 3,460 & 5,140 & 6,540 & 8,150 \\
\hline 5390 & 7.77 & 1,220 & 2,670 & 3,950 & 5,890 & 7,520 & 9,380 \\
\hline 5398 & 214 & 10,000 & 20,700 & 30,000 & 44,100 & 56,300 & 69,800 \\
\hline 5399 & 2,190 & 21,600 & 42,500 & 46,700 & 66,100 & 84,000 & 104,000 \\
\hline 5402 & 7.81 & 1,450 & 3,270 & 4,900 & 7,400 & 9,510 & 11,900 \\
\hline 5405 & 2,210 & 21,800 & 42,900 & 47,100 & 66,700 & 84,800 & 105,000 \\
\hline 5409 & 14.7 & 1,840 & 4,090 & 6,070 & 9,140 & 11,700 & 14,700 \\
\hline
\end{tabular}


172 Estimates of Flow Duration, Mean Flow, and Peak-Discharge Frequency Values for Kansas Stream Locations 


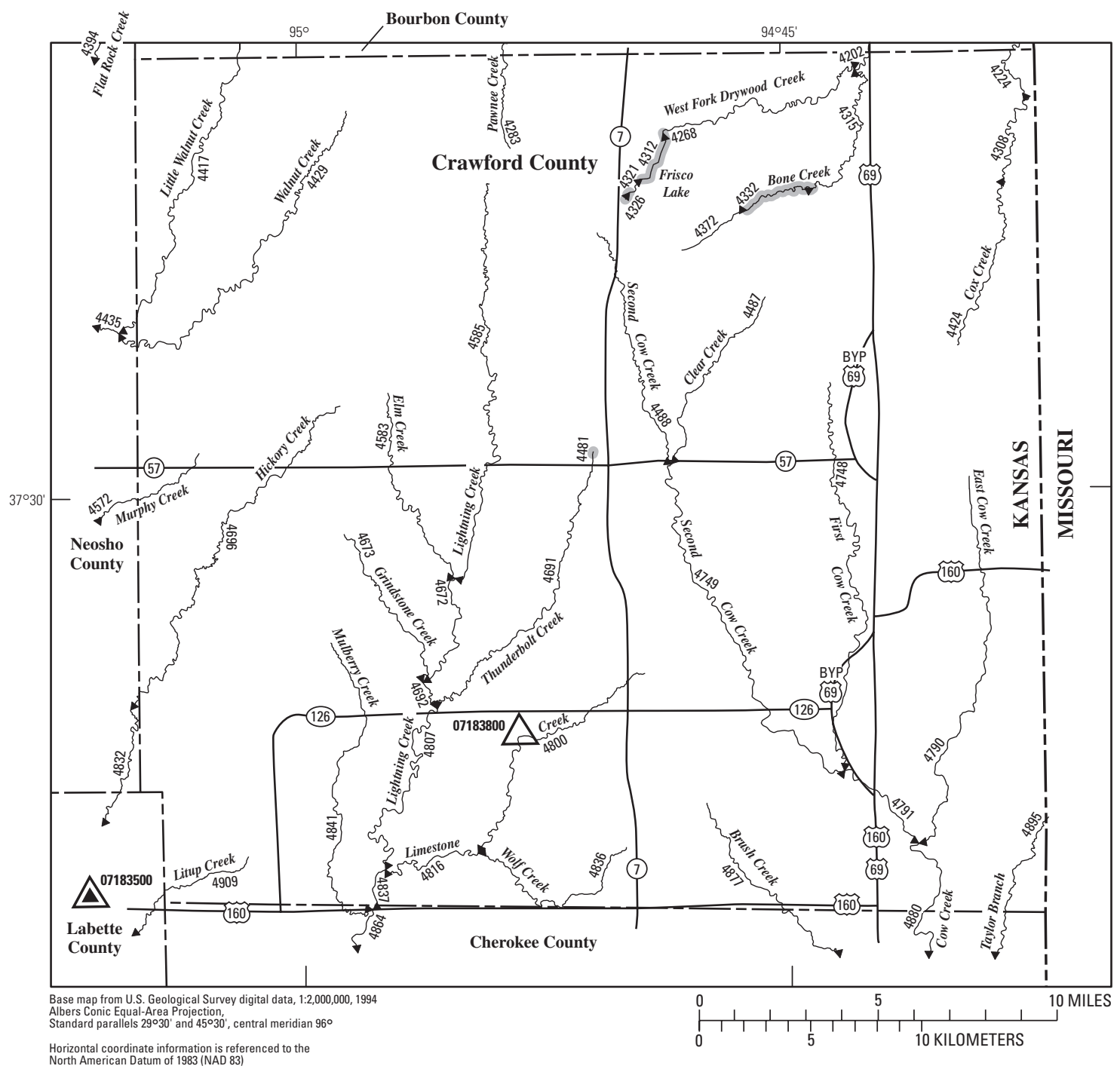

EXPLANATION

4909 Location of streamflow-statistics determination site (small triangle) and associated identification number-small triangle points in downstream direction

${ }^{07183500} \Delta$ U.S. Geological Survey streamflow-gaging station and number used for estimates of flow duration

$\triangle$ U.S. Geological Survey streamflow-gaging station and number used for estimates of peak-discharge frequency values

4855

Lake and determination site identification number

KANSAS

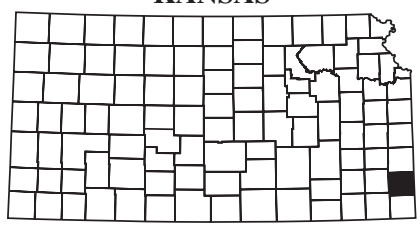

Index map

Figure 29. Location of streamflow-statistics determination sites, associated identification numbers, and U.S. Geological Survey streamflow-gaging stations used in the flow-duration and peak-discharge frequency analyses for Crawford County. 
Table 25. Estimated flow-duration values, mean flow values, and peak-discharge frequency values for controlled and uncontrolled flow stream segments on the 1999 Kansas Surface Water Register for Crawford County.

[KSWR, Kansas Surface Water Register; CUSEGA, catalog unit segment number alpha; $\mathrm{mi}^{2}$, square miles; $\mathrm{ft}^{3} / \mathrm{s}$, cubic feet per second; HYDRO, lake or other hydrologic structure; NA, not applicable; NRDitch, irrigation ditch; NRTribal, tribal stream]

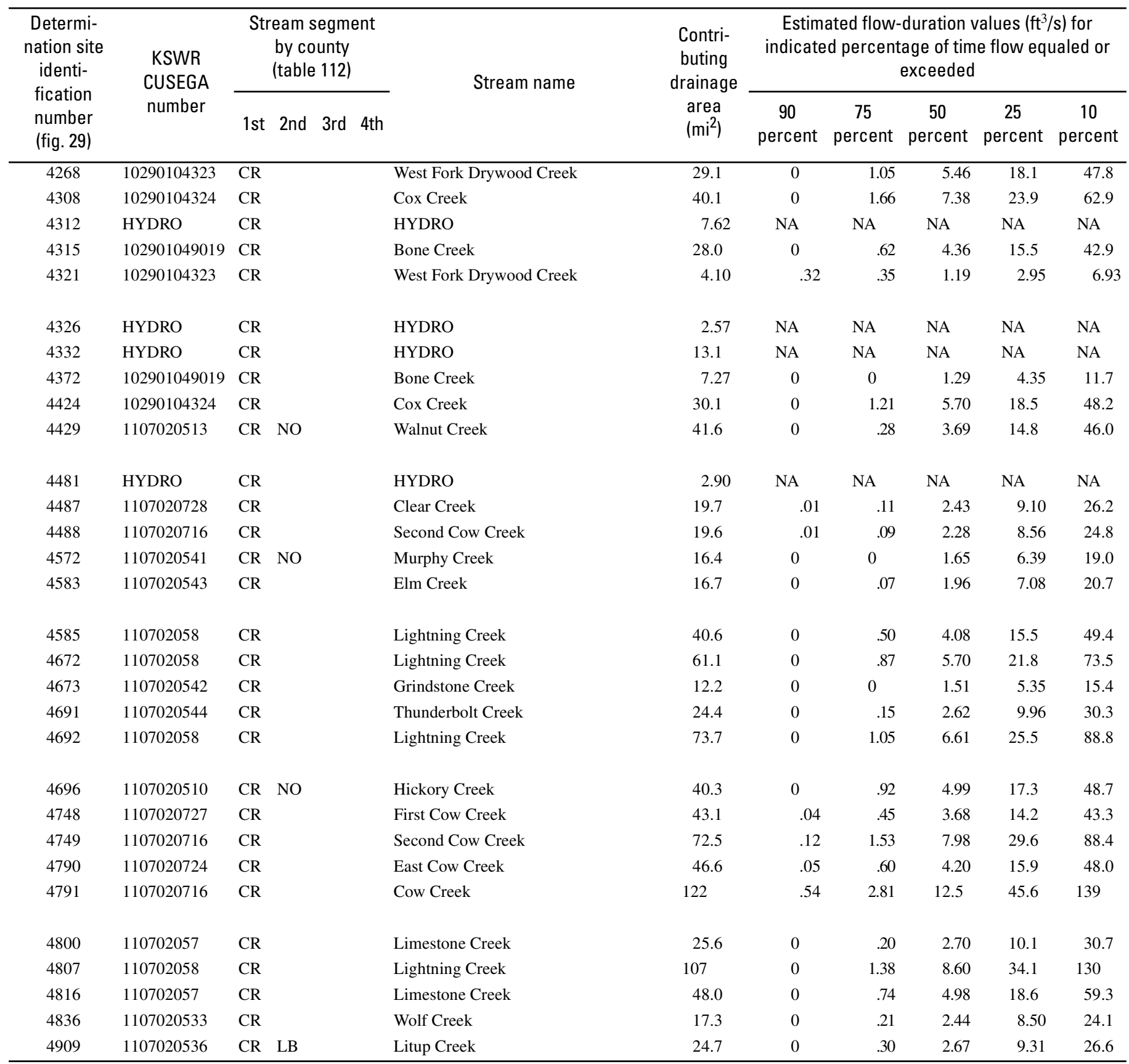


Table 25. Estimated flow-duration values, mean flow values, and peak-discharge frequency values for controlled and uncontrolled flow stream segments on the 1999 Kansas Surface Water Register for Crawford County.-Continued

[KSWR, Kansas Surface Water Register; CUSEGA, catalog unit segment number alpha; $\mathrm{mi}^{2}$, square miles; $\mathrm{ft}^{3} / \mathrm{s}$, cubic feet per second; HYDRO, lake or other hydrologic structure; NA, not applicable; NRDitch, irrigation ditch; NRTribal, tribal stream]

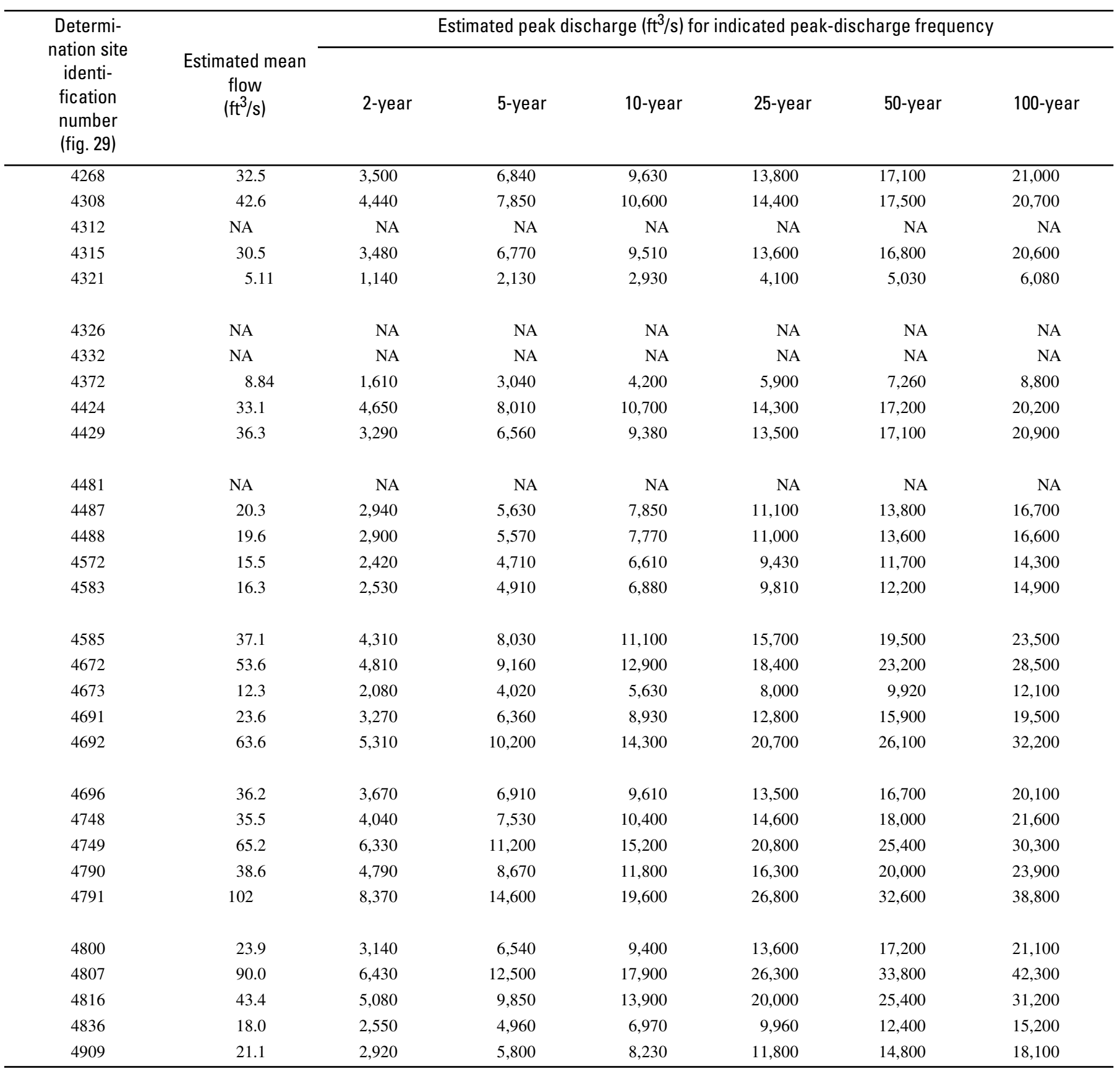


176 Estimates of Flow Duration, Mean Flow, and Peak-Discharge Frequency Values for Kansas Stream Locations 


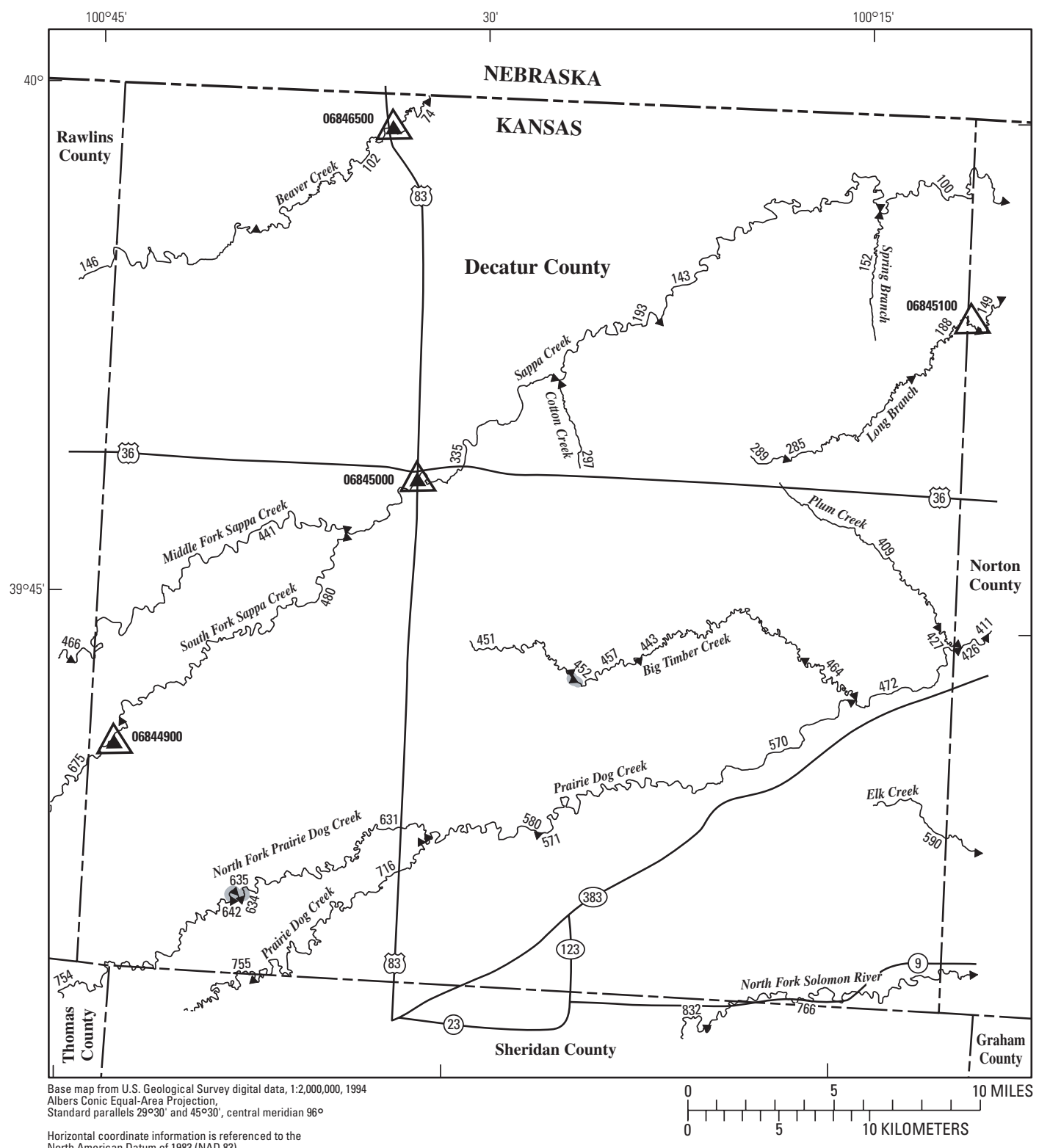

Horizontal coordinate information is referenced to the

EXPLANATION

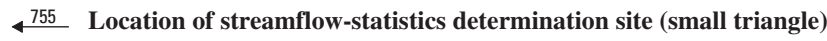
and associated identification number-small triangle points in downstream direction

06844900 U.S. Geological Survey streamflow-gaging station and number used for estimates of flow duration

${ }^{06845100} \bigwedge$ U.S. Geological Survey streamflow-gaging station and number used for estimates of peak-discharge frequency values

KANSAS

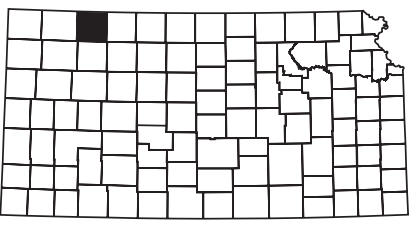

Index map

Lake and determination site identification number

Figure 30. Location of streamflow-statistics determination sites, associated identification numbers, and U.S. Geological Survey streamflow-gaging stations used in the flow-duration and peak-discharge frequency analyses for Decatur County. 
Table 26. Estimated flow-duration values, mean flow values, and peak-discharge frequency values for controlled and uncontrolled flow stream segments on the 1999 Kansas Surface Water Register for Decatur County.

[KSWR, Kansas Surface Water Register; CUSEGA, catalog unit segment number alpha; $\mathrm{mi}^{2}$, square miles; $\mathrm{ft}^{3} / \mathrm{s}$, cubic feet per second; HYDRO, lake or other hydrologic structure; NA, not applicable; NRDitch, irrigation ditch; NRTribal, tribal stream]

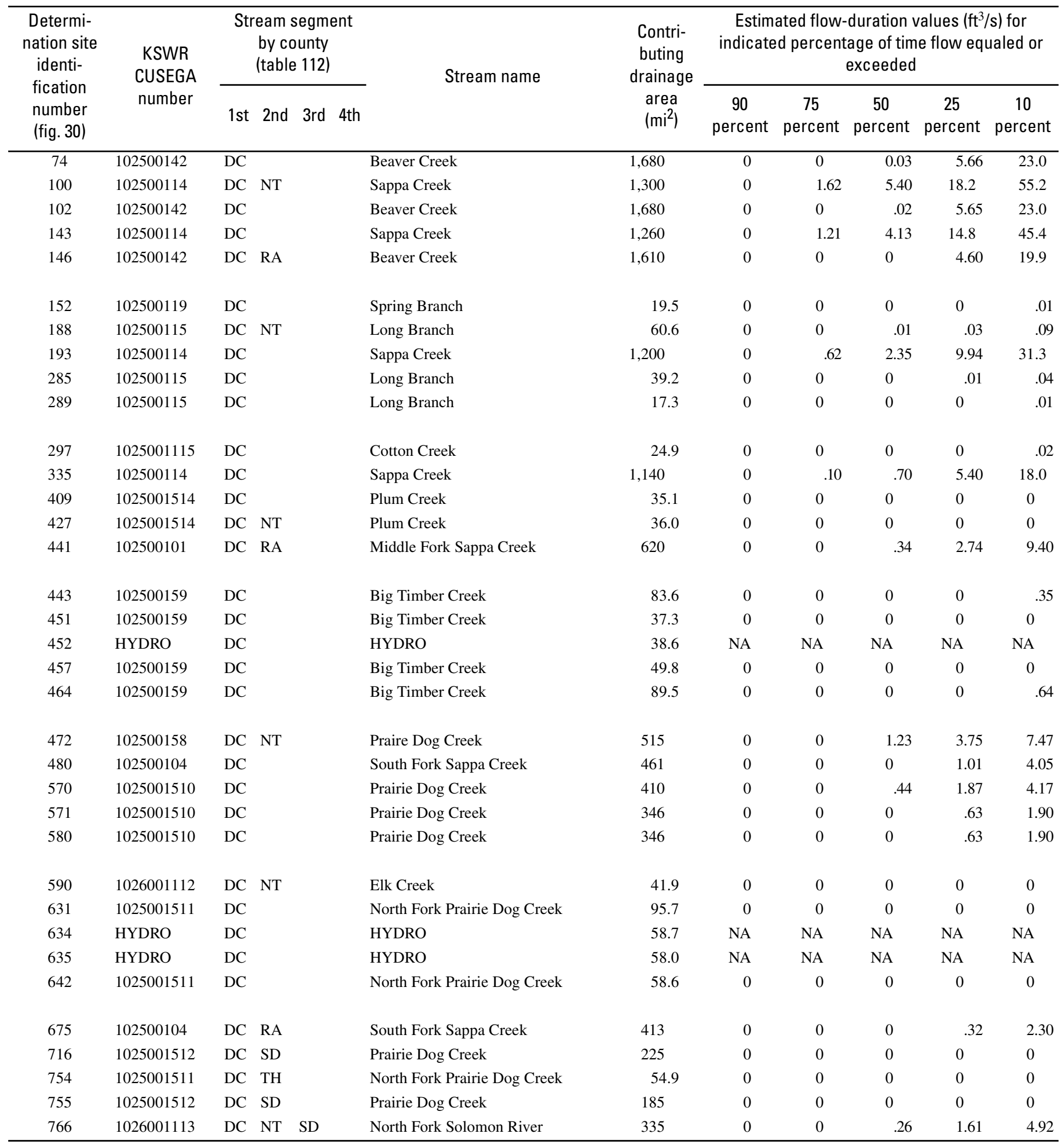


Table 26. Estimated flow-duration values, mean flow values, and peak-discharge frequency values for controlled and uncontrolled flow stream segments on the 1999 Kansas Surface Water Register for Decatur County.-Continued

[KSWR, Kansas Surface Water Register; CUSEGA, catalog unit segment number alpha; $\mathrm{mi}^{2}$, square miles; $\mathrm{ft}^{3} / \mathrm{s}$, cubic feet per second; HYDRO, lake or other hydrologic structure; NA, not applicable; NRDitch, irrigation ditch; NRTribal, tribal stream]

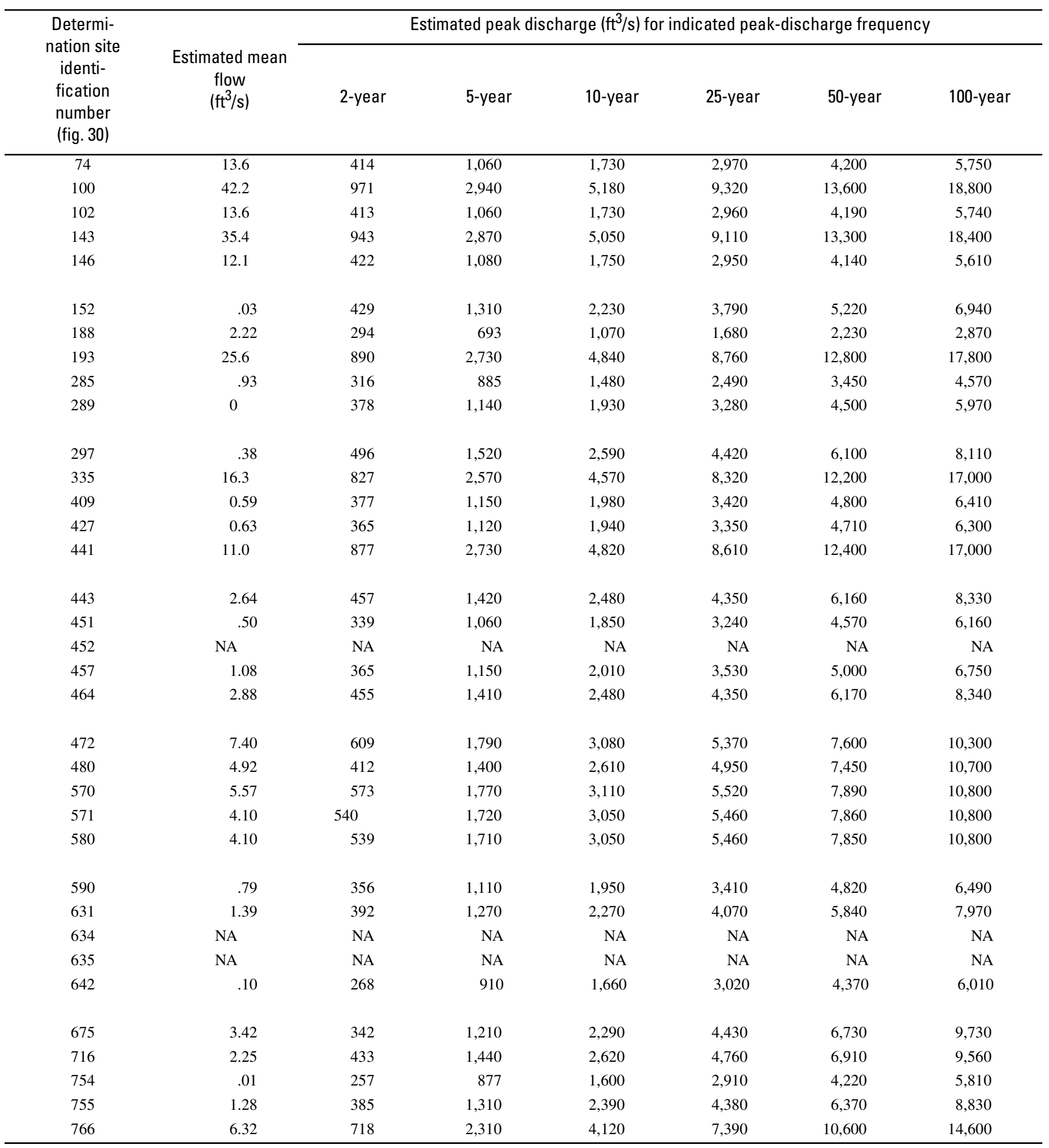




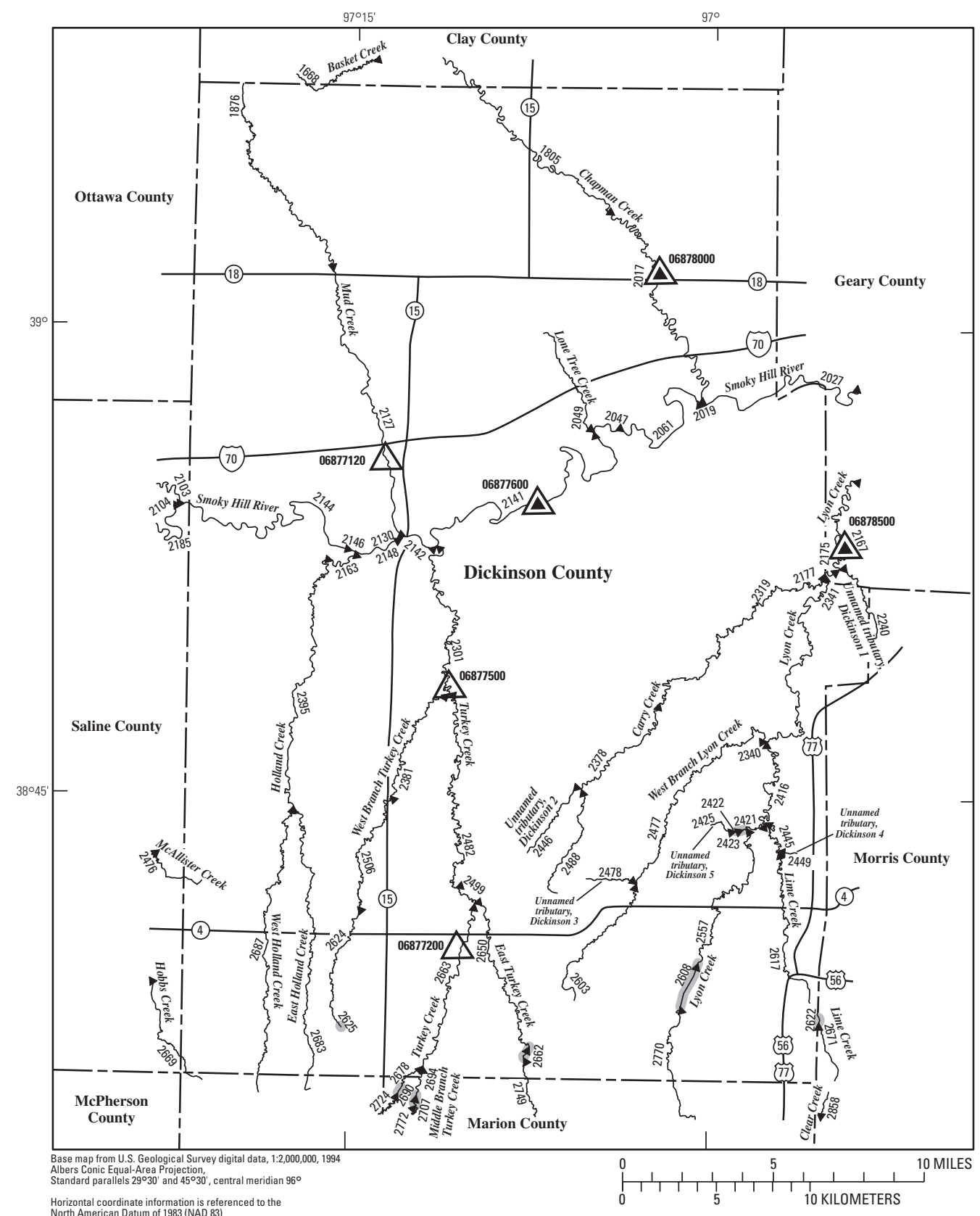

EXPLANATION

\footnotetext{
2669 Location of streamflow-statistics determination site (small triangle) and associated identification number-small triangle points in downstream direction

${ }^{06878500} \Delta$ U.S. Geological Survey streamflow-gaging station and number used for estimates of flow duration

${ }^{06877200} \triangle$ U.S. Geological Survey streamflow-gaging station and number used for estimates of peak-discharge frequency values

2662 Lake and determination site identification number
}

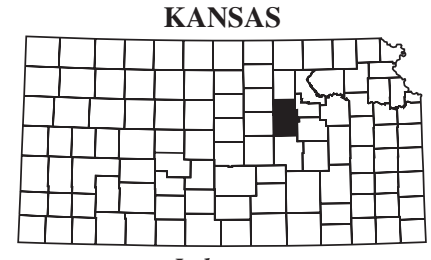

Index map

Figure 31. Location of streamflow-statistics determination sites, associated identification numbers, and U.S. Geological Survey streamflow-gaging stations used in the flow-duration and peak-discharge frequency analyses for Dickinson County. 
Table 27. Estimated flow-duration values, mean flow values, and peak-discharge frequency values for controlled and uncontrolled flow stream segments on the 1999 Kansas Surface Water Register for Dickinson County.

[KSWR, Kansas Surface Water Register; CUSEGA, catalog unit segment number alpha; $\mathrm{mi}^{2}$, square miles; $\mathrm{ft}^{3} / \mathrm{s}$, cubic feet per second; HYDRO, lake or other hydrologic structure; NA, not applicable; NRDitch, irrigation ditch; NRTribal, tribal stream]

\begin{tabular}{|c|c|c|c|c|c|c|c|c|c|c|c|}
\hline \multirow{2}{*}{$\begin{array}{l}\text { Determi- } \\
\text { nation site } \\
\text { identi- } \\
\text { fication } \\
\text { number } \\
\text { (fig. 31) }\end{array}$} & \multirow{2}{*}{$\begin{array}{l}\text { KSWR } \\
\text { CUSEGA } \\
\text { number }\end{array}$} & \multicolumn{3}{|c|}{$\begin{array}{l}\text { Stream segment } \\
\text { by county } \\
\text { (table 112) }\end{array}$} & \multirow{2}{*}{ Stream name } & \multirow{2}{*}{$\begin{array}{l}\text { Contri- } \\
\text { buting } \\
\text { drainage } \\
\text { area } \\
\left(\mathrm{mi}^{2}\right)\end{array}$} & \multicolumn{5}{|c|}{$\begin{array}{c}\text { Estimated flow-duration values }\left(\mathrm{ft}^{3} / \mathrm{s}\right) \text { for } \\
\text { indicated percentage of time flow equaled or } \\
\text { exceeded }\end{array}$} \\
\hline & & $1 s t$ & 2nd & 3rd 4th & & & $\begin{array}{c}90 \\
\text { percent }\end{array}$ & $\begin{array}{c}75 \\
\text { percent }\end{array}$ & $\begin{array}{c}50 \\
\text { percent }\end{array}$ & $\begin{array}{c}25 \\
\text { percent }\end{array}$ & $\begin{array}{c}10 \\
\text { percent }\end{array}$ \\
\hline 2017 & 102600083 & $\overline{\mathrm{DK}}$ & & & Chapman Creek & 313 & 7.70 & 13.0 & 24.0 & 49.0 & 126 \\
\hline 2019 & 102600082 & DK & & & Smoky Hill River & 19,600 & 214 & 414 & 807 & 1,430 & 3,510 \\
\hline 2027 & 102600082 & DK & GE & & Smoky Hill River & 20,000 & 193 & 305 & 608 & 1,500 & 3,990 \\
\hline 2047 & 102600086 & DK & & & Smoky Hill River & 19,600 & 190 & 299 & 596 & 1,470 & 3,940 \\
\hline 2049 & 1026000841 & DK & & & Lone Tree Creek & 32.6 & 0 & .88 & 3.39 & 8.83 & 21.9 \\
\hline 2061 & 102600086 & DK & & & Smoky Hill River & 19,600 & 190 & 300 & 597 & 1,470 & 3,940 \\
\hline 2127 & 102600088 & DK & & & Mud Creek & 130 & .77 & 4.12 & 11.6 & 28.4 & 68.2 \\
\hline 2130 & 102600086 & DK & & & Smoky Hill River & 19,200 & 184 & 290 & 578 & 1,430 & 3,830 \\
\hline 2141 & 102600086 & DK & & & Smoky Hill River & 19,600 & 190 & 299 & 595 & 1,470 & 3,930 \\
\hline 2142 & 102600086 & DK & & & Smoky Hill River & 19,400 & 186 & 294 & 584 & 1,440 & 3,870 \\
\hline 2144 & 1026000810 & DK & SA & & Smoky Hill River & 19,100 & 182 & 288 & 573 & 1,410 & 3,810 \\
\hline 2146 & 102600089 & DK & & & Smoky Hill River & 19,100 & 182 & 288 & 573 & 1,410 & 3,810 \\
\hline 2148 & 102600089 & DK & & & Smoky Hill River & 19,200 & 184 & 290 & 578 & 1,430 & 3,830 \\
\hline 2163 & 1026000825 & DK & & & Holland Creek & 103 & 0 & 2.12 & 8.24 & 23.8 & 63.6 \\
\hline 2175 & 1026000834 & DK & & & Lyon Creek & 171 & .92 & 6.91 & 16.2 & 38.6 & 94.2 \\
\hline 2177 & 1026000831 & DK & GE & & Lyon Creek & 253 & 2.52 & 13.4 & 27.2 & 56.6 & 124 \\
\hline 2240 & 10260008638 & DK & GE & MR & Unnamed tributary, Dickinson 1 & 13.8 & .01 & .03 & .86 & 2.77 & 8.22 \\
\hline 2301 & 1026000828 & DK & & & Turkey Creek & 181 & 0 & 2.90 & 12.4 & 39.7 & 116 \\
\hline 2319 & 1026000835 & DK & & & Carry Creek & 80.9 & .21 & 2.06 & 6.82 & 18.9 & 49.4 \\
\hline 2340 & 1026000834 & DK & & & West Branch Lyon Creek & 152 & .73 & 5.62 & 13.8 & 34.0 & 85.0 \\
\hline 2341 & 1026000831 & DK & GE & & Lyon Creek & 171 & .92 & 6.91 & 16.2 & 38.6 & 94.2 \\
\hline 2378 & 1026000835 & DK & & & Carry Creek & 38.2 & .05 & .39 & 2.86 & 8.82 & 24.5 \\
\hline 2381 & 1026000829 & DK & & & West Branch Turkey Creek & 40.3 & 0 & .23 & 2.67 & 8.55 & 24.2 \\
\hline 2395 & 1026000825 & DK & & & Holland Creek & 101 & 0 & 2.07 & 8.06 & 23.3 & 62.1 \\
\hline 2416 & 1026000831 & DK & & & Lyon Creek & 109 & .37 & 3.19 & 9.01 & 24.0 & 63.1 \\
\hline 2421 & 10260008540 & DK & & & Lyon Creek & 67.0 & .14 & 1.34 & 5.14 & 15.1 & 41.1 \\
\hline 2422 & 10260008542 & DK & & & Unnamed tributary, Dickinson 5 & 3.92 & 0 & 0 & 0 & 0 & .84 \\
\hline 2423 & HYDRO & DK & & & HYDRO & 3.87 & NA & NA & NA & NA & NA \\
\hline 2425 & 10260008542 & DK & & & Unnamed tributary, Dickinson 5 & 3.72 & 0 & 0 & 0 & 0 & .65 \\
\hline 2445 & 1026000851 & DK & & & Lime Creek & 34.8 & .04 & .28 & 2.39 & 7.54 & 21.6 \\
\hline 2446 & 1026000832 & DK & & & Unnamed tributary, Dickinson 2 & 10.6 & 0 & .02 & .47 & 1.72 & 5.68 \\
\hline 2449 & 10260008618 & DK & & & Unnamed tributary, Dickinson 4 & 3.67 & 0 & 0 & 0 & 0 & .93 \\
\hline 2476 & 1026000849 & DK & SA & & McAllister Creek & 18.4 & 0 & 0 & 1.07 & 3.43 & 9.89 \\
\hline 2477 & 1026000834 & DK & & & West Branch Lyon Creek & 42.5 & .06 & .64 & 3.55 & 10.7 & 29.3 \\
\hline 2478 & 10260008515 & DK & & & Unnamed tributary, Dickinson 3 & 3.63 & 0 & 0 & 0 & 0 & .98 \\
\hline 2482 & 1026000830 & DK & & & Turkey Creek & 114 & 0 & 1.48 & 7.38 & 24.4 & 71.5 \\
\hline 2488 & 1026000835 & DK & & & Carry Creek & 9.64 & 0 & .01 & .48 & 1.73 & 5.64 \\
\hline 2499 & 1026000830 & DK & & & Turkey Creek & 86.7 & 0 & 1.01 & 5.66 & 18.7 & 54.6 \\
\hline
\end{tabular}


Table 27. Estimated flow-duration values, mean flow values, and peak-discharge frequency values for controlled and uncontrolled flow stream segments on the 1999 Kansas Surface Water Register for Dickinson County.-Continued

[KSWR, Kansas Surface Water Register; CUSEGA, catalog unit segment number alpha; $\mathrm{mi}^{2}$, square miles; $\mathrm{ft}^{3} / \mathrm{s}$, cubic feet per second; HYDRO, lake or other hydrologic structure; NA, not applicable; NRDitch, irrigation ditch; NRTribal, tribal stream]

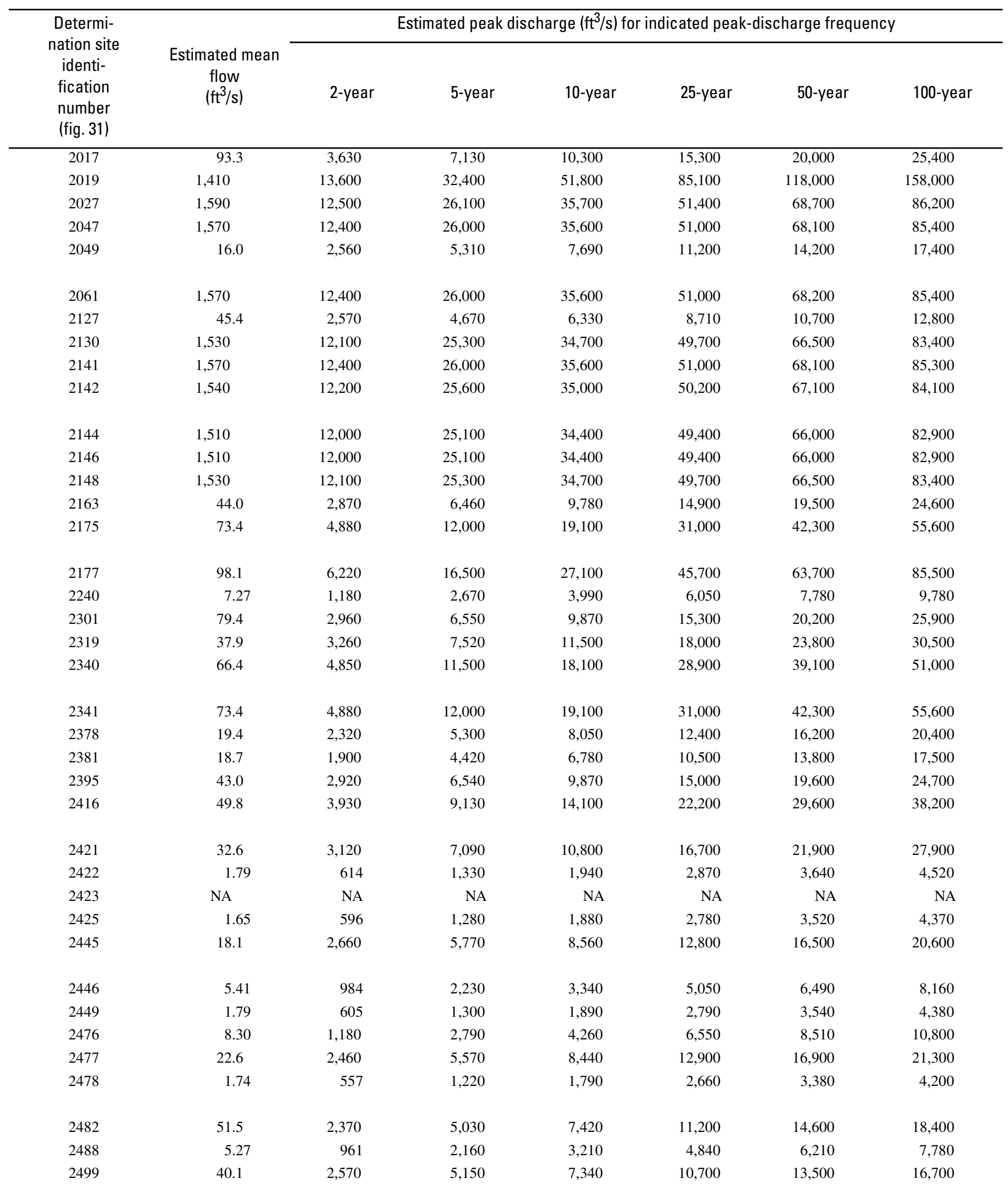


Table 27. Estimated flow-duration values, mean flow values, and peak-discharge frequency values for controlled and uncontrolled flow stream segments on the 1999 Kansas Surface Water Register for Dickinson County.-Continued

[KSWR, Kansas Surface Water Register; CUSEGA, catalog unit segment number alpha; $\mathrm{mi}^{2}$, square miles; $\mathrm{ft}^{3} / \mathrm{s}$, cubic feet per second; HYDRO, lake or other hydrologic structure; NA, not applicable; NRDitch, irrigation ditch; NRTribal, tribal stream]

\begin{tabular}{|c|c|c|c|c|c|c|c|c|c|c|c|}
\hline $\begin{array}{l}\text { Determi- } \\
\text { nation site } \\
\text { identi- } \\
\text { fication } \\
\text { number } \\
\text { (fig. } 31 \text { ) }\end{array}$ & $\begin{array}{l}\text { KSWR } \\
\text { CUSEGA } \\
\text { number }\end{array}$ & \multicolumn{3}{|c|}{$\begin{array}{l}\text { Stream segment } \\
\text { by county } \\
\text { (table 112) }\end{array}$} & Stream name & $\begin{array}{l}\text { Contri- } \\
\text { buting } \\
\text { drainage } \\
\text { area } \\
\left(\mathrm{mi}^{2}\right)\end{array}$ & \multicolumn{5}{|c|}{$\begin{array}{l}\text { Estimated flow-duration values }\left(\mathrm{ft}^{3} / \mathrm{s}\right) \text { for } \\
\text { indicated percentage of time flow equaled or } \\
\text { exceeded }\end{array}$} \\
\hline 2506 & 1026000829 & $\overline{D K}$ & & & West Branch Turkey Creek & 27.2 & 0 & 0 & 1.82 & 5.79 & 16.3 \\
\hline 2608 & HYDRO & DK & & & HYDRO & 49.1 & NA & NA & NA & NA & NA \\
\hline 2617 & 1026000851 & DK & MR & & Lime Creek & 29.4 & .03 & .12 & 1.88 & 6.10 & 17.8 \\
\hline 2624 & 1026000829 & DK & & & West Branch Turkey Creek & 14.1 & 0 & 0 & 1.08 & 3.18 & 8.78 \\
\hline 2625 & HYDRO & DK & & & HYDRO & 2.09 & NA & NA & NA & NA & NA \\
\hline 2669 & 1026000848 & DK & $\mathrm{MN}$ & SA & Hobbs Creek & 30.3 & 0 & .43 & 2.58 & 7.09 & 18.2 \\
\hline 2678 & 1026000830 & DK & $\mathrm{MN}$ & & Turkey Creek & 13.2 & 0 & 0 & 1.13 & 3.20 & 8.64 \\
\hline 2683 & 1026000827 & DK & $\mathrm{MN}$ & & East Holland Creek & 20.5 & 0 & .23 & 2.00 & 5.50 & 14.1 \\
\hline 2687 & 1026000826 & DK & $\mathrm{MN}$ & & West Holland Creek & 32.3 & 0 & .49 & 2.89 & 8.14 & 21.0 \\
\hline 2694 & 1026000858 & DK & $\mathrm{MN}$ & & Middle Branch Turkey Creek & 15.4 & 0 & 0 & .86 & 3.02 & 9.15 \\
\hline 2749 & 1026000850 & DK & $\mathrm{MN}$ & & East Turkey Creek & 23.7 & 0 & 0 & 1.24 & 4.61 & 14.3 \\
\hline 2770 & 10260008540 & DK & $\mathrm{MN}$ & & Lyon Creek & 43.3 & .06 & .38 & 2.85 & 9.00 & 25.7 \\
\hline
\end{tabular}


Table 27. Estimated flow-duration values, mean flow values, and peak-discharge frequency values for controlled and uncontrolled flow stream segments on the 1999 Kansas Surface Water Register for Dickinson County.-Continued

[KSWR, Kansas Surface Water Register; CUSEGA, catalog unit segment number alpha; $\mathrm{mi}^{2}$, square miles; $\mathrm{ft}^{3} / \mathrm{s}$, cubic feet per second; HYDRO, lake or other hydrologic structure; NA, not applicable; NRDitch, irrigation ditch; NRTribal, tribal stream]

\begin{tabular}{|c|c|c|c|c|c|c|c|}
\hline \multirow{2}{*}{$\begin{array}{l}\text { Determi- } \\
\text { nation site } \\
\text { identi- } \\
\text { fication } \\
\text { number } \\
\text { (fig. 31) }\end{array}$} & \multirow[b]{2}{*}{$\begin{array}{c}\text { Estimated mean } \\
\text { flow } \\
\left(\mathrm{ft}^{3} / \mathrm{s}\right)\end{array}$} & \multicolumn{6}{|c|}{ Estimated peak discharge $\left(\mathrm{ft}^{3} / \mathrm{s}\right)$ for indicated peak-discharge frequency } \\
\hline & & 2-year & 5 -year & 10-year & 25 -year & 50 -year & 100-year \\
\hline 2506 & 13.0 & 1,570 & 3,680 & 5,590 & 8,590 & $\begin{array}{ll}11,100 \\
\end{array}$ & 14,100 \\
\hline 2557 & 30.6 & 3,050 & 6,920 & 10,500 & 16,200 & 21,300 & 27,100 \\
\hline 2603 & 11.0 & 1,490 & 3,400 & 5,100 & 7,740 & 9,970 & 12,600 \\
\hline 2608 & NA & NA & NA & NA & NA & NA & NA \\
\hline 2617 & 15.3 & 2,010 & 4,540 & 6,810 & 10,400 & 13,400 & 16,800 \\
\hline 2624 & 7.21 & 1,080 & 2,500 & 3,770 & 5,740 & 7,410 & 9,340 \\
\hline 2625 & NA & NA & NA & NA & NA & NA & NA \\
\hline 2650 & 19.3 & 2,350 & 5,300 & 8,010 & 12,200 & 15,900 & 20,000 \\
\hline 2662 & NA & NA & NA & NA & NA & NA & NA \\
\hline 2663 & 22.3 & 1,200 & 2,220 & 3,000 & 4,080 & 4,940 & 5,850 \\
\hline 2669 & 13.7 & 1,730 & 3,940 & 5,970 & 9,090 & 11,800 & 14,800 \\
\hline 2678 & 7.00 & 969 & 2,190 & 3,270 & 4,930 & 6,320 & 7,920 \\
\hline 2683 & 10.7 & 1,340 & 3,130 & 4,740 & 7,270 & 9,410 & 11,900 \\
\hline 2687 & 15.5 & 1,900 & 4,280 & 6,460 & 9,800 & 12,700 & 15,900 \\
\hline 2694 & 7.84 & 1,050 & 2,360 & 3,520 & 5,300 & 6,780 & 8,500 \\
\hline 2749 & 12.1 & 1,570 & 3,600 & 5,420 & 8,240 & 10,600 & 13,400 \\
\hline 2770 & 21.2 & 3,120 & 6,800 & 10,100 & 15,300 & 19,800 & 24,700 \\
\hline
\end{tabular}




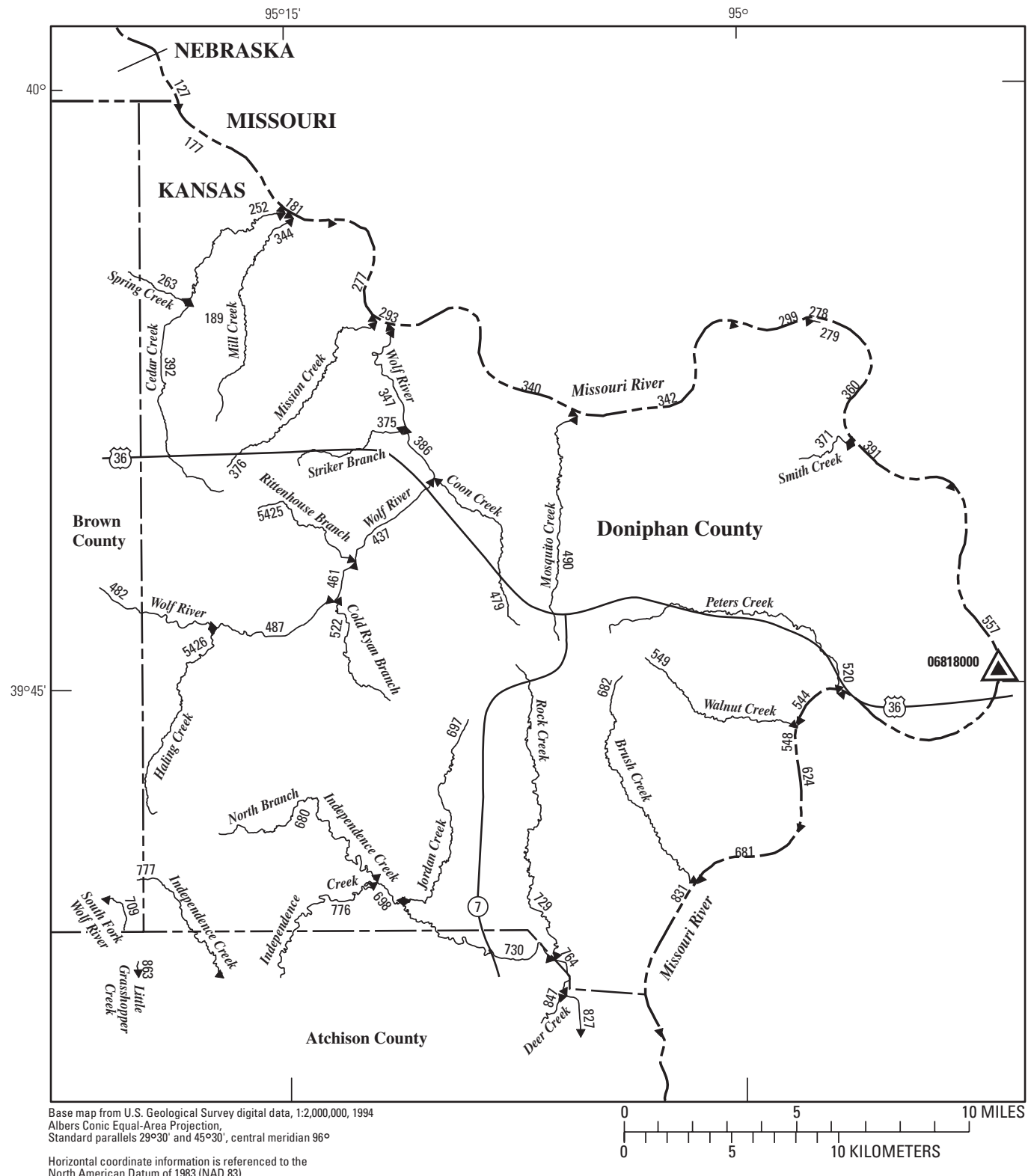

EXPLANATION

863 Location of streamflow-statistics determination site (small triangle) and associated identification number-small triangle points in downstream direction

06818000

U.S. Geological Survey streamflow-gaging station and number used for estimates of flow duration

06818000

U.S. Geological Survey streamflow-gaging station and number used for estimates of peak-discharge frequency values

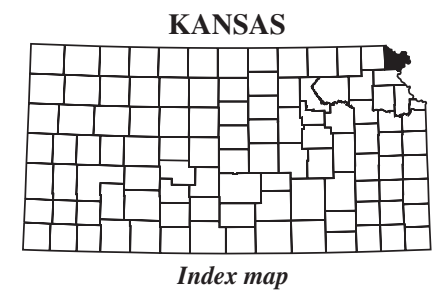

776 Lake and determination site identification number

Figure 32. Location of streamflow-statistics determination sites, associated identification numbers, and U.S. Geological Survey streamflow-gaging stations used in the flow-duration and peak-discharge frequency analyses for Doniphan County. 
Table 28. Estimated flow-duration values, mean flow values, and peak-discharge frequency values for controlled and uncontrolled flow stream segments on the 1999 Kansas Surface Water Register for Doniphan County.

[KSWR, Kansas Surface Water Register; CUSEGA, catalog unit segment number alpha; $\mathrm{mi}^{2}$, square miles; $\mathrm{ft}^{3} / \mathrm{s}$, cubic feet per second; HYDRO, lake or other hydrologic structure; NA, not applicable; NRDitch, irrigation ditch; NRTribal, tribal stream]

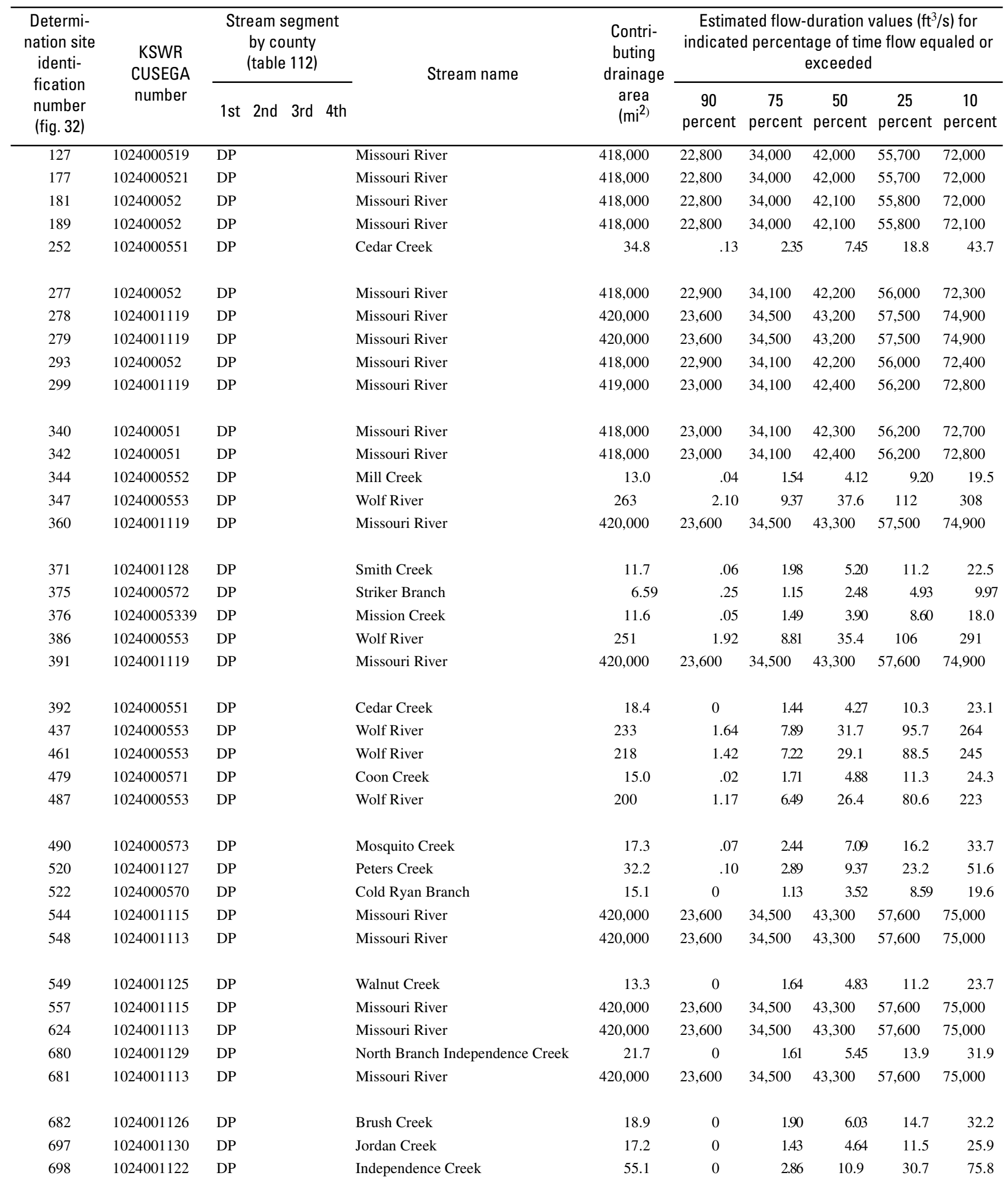


Table 28. Estimated flow-duration values, mean flow values, and peak-discharge frequency values for controlled and uncontrolled flow stream segments on the 1999 Kansas Surface Water Register for Doniphan County.-Continued

[KSWR, Kansas Surface Water Register; CUSEGA, catalog unit segment number alpha; $\mathrm{mi}^{2}$, square miles; $\mathrm{ft}^{3} / \mathrm{s}$, cubic feet per second; HYDRO, lake or other hydrologic structure; NA, not applicable; NRDitch, irrigation ditch; NRTribal, tribal stream]

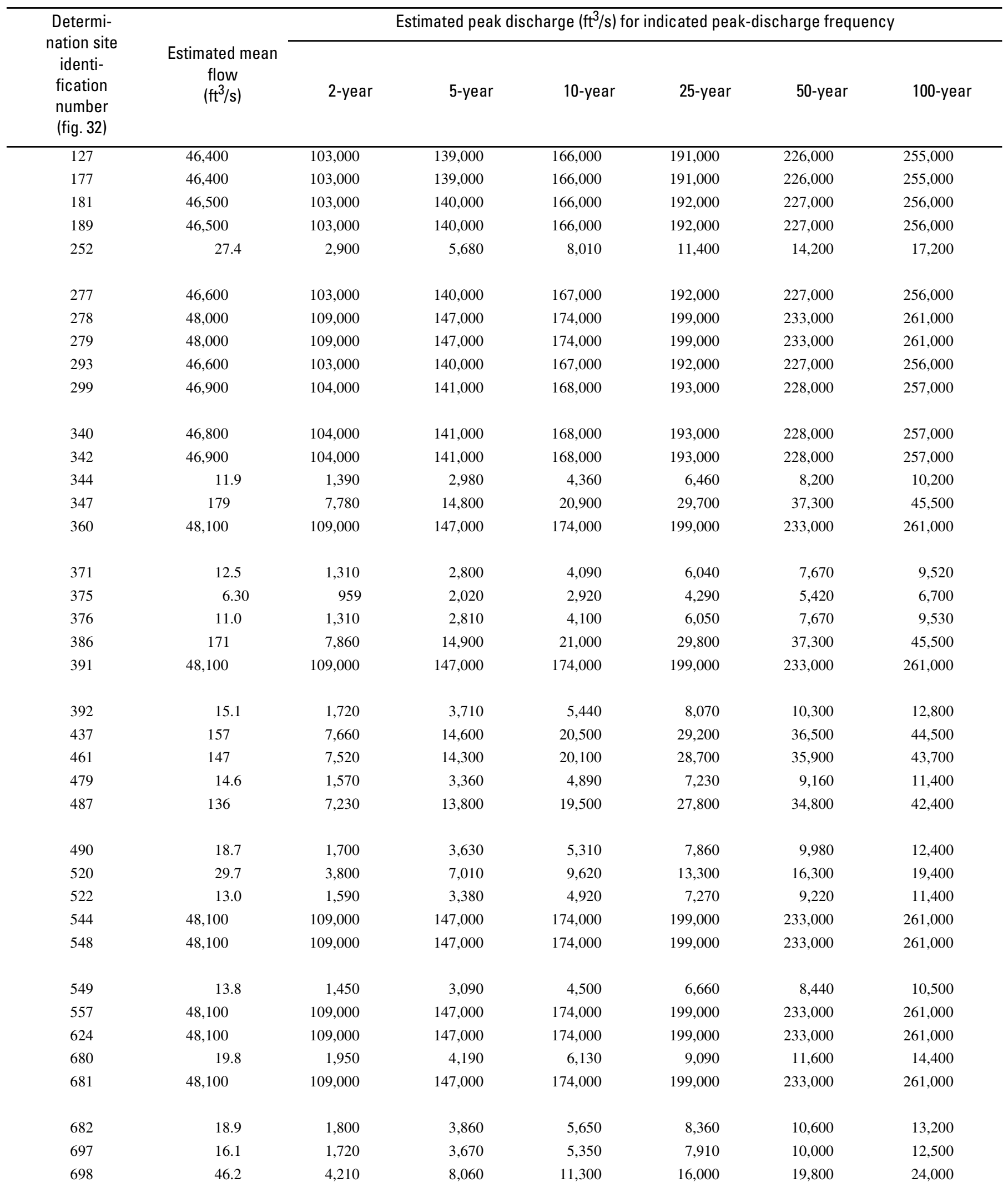


Table 28. Estimated flow-duration values, mean flow values, and peak-discharge frequency values for controlled and uncontrolled flow stream segments on the 1999 Kansas Surface Water Register for Doniphan County.-Continued

[KSWR, Kansas Surface Water Register; CUSEGA, catalog unit segment number alpha; $\mathrm{mi}^{2}$, square miles; $\mathrm{ft}^{3} / \mathrm{s}$, cubic feet per second; HYDRO, lake or other hydrologic structure; NA, not applicable; NRDitch, irrigation ditch; NRTribal, tribal stream]

\begin{tabular}{|c|c|c|c|c|c|c|c|c|c|}
\hline $\begin{array}{l}\text { Determi- } \\
\text { nation site } \\
\text { identi- } \\
\text { fication } \\
\text { number } \\
\text { (fig. 32) }\end{array}$ & $\begin{array}{l}\text { KSWR } \\
\text { CUSEGA } \\
\text { number }\end{array}$ & $\begin{array}{l}\text { Stream segment } \\
\text { by county } \\
\text { (table 112) }\end{array}$ & Stream name & $\begin{array}{l}\text { Contri- } \\
\text { buting } \\
\text { drainage } \\
\text { area } \\
\left(\mathrm{mi}^{2}\right)\end{array}$ & \multicolumn{5}{|c|}{$\begin{array}{c}\text { Estimated flow-duration values }\left(\mathrm{ft}^{3} / \mathrm{s}\right) \text { for } \\
\text { indicated percentage of time flow equaled or } \\
\text { exceeded }\end{array}$} \\
\hline 729 & 1024001121 & $\overline{D P}$ & Rock Creek & 23.1 & 0 & 2.02 & 6.74 & 17.0 & 38.1 \\
\hline
\end{tabular}


Table 28. Estimated flow-duration values, mean flow values, and peak-discharge frequency values for controlled and uncontrolled flow stream segments on the 1999 Kansas Surface Water Register for Doniphan County.-Continued

[KSWR, Kansas Surface Water Register; CUSEGA, catalog unit segment number alpha; $\mathrm{mi}^{2}$, square miles; $\mathrm{ft}^{3} / \mathrm{s}$, cubic feet per second; HYDRO, lake or other hydrologic structure; NA, not applicable; NRDitch, irrigation ditch; NRTribal, tribal stream]

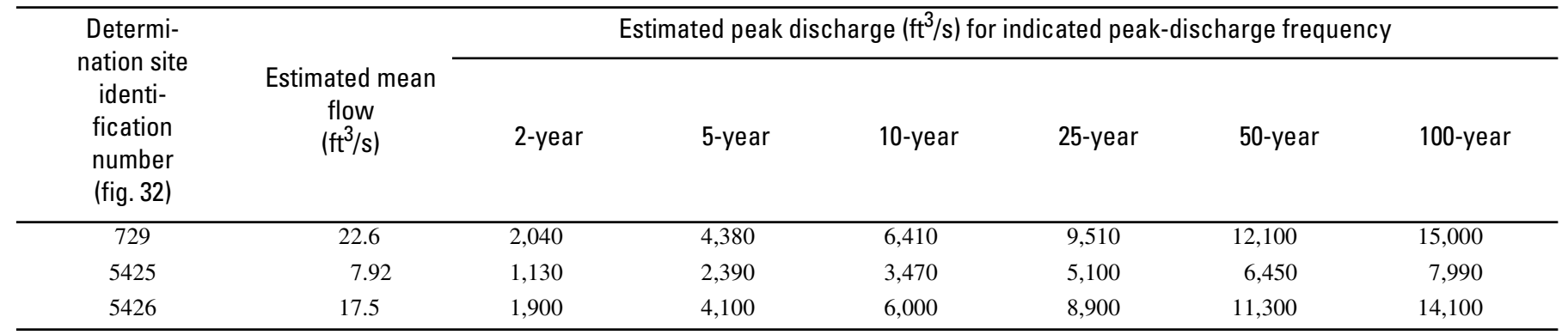




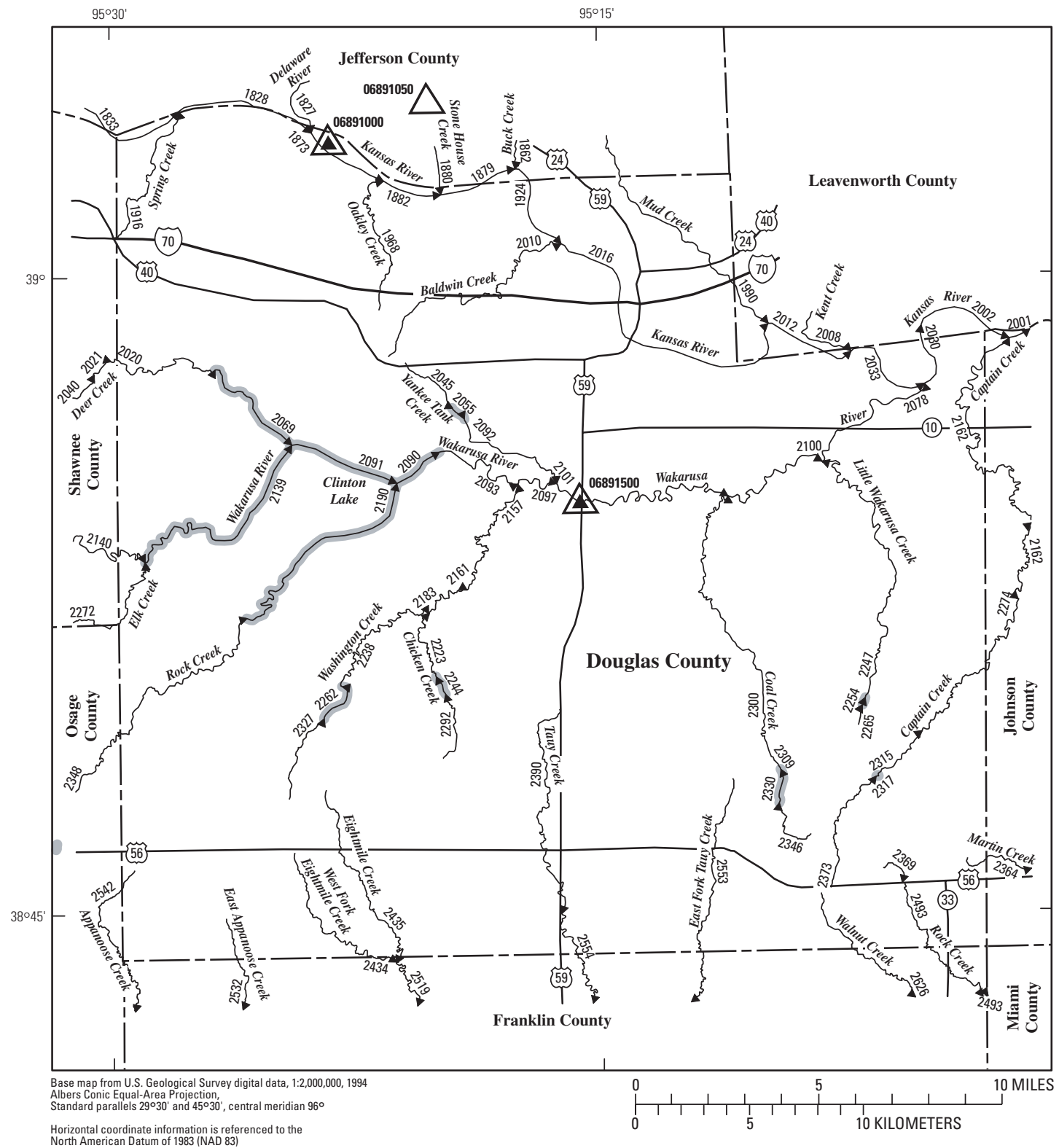

EXPLANATION

\&2532 Location of streamflow-statistics determination site (small triangle) and associated identification number-small triangle points in downstream direction

${ }^{06891050} \Delta$ U.S. Geological Survey streamflow-gaging station and number used for estimates of flow duration

${ }^{06891500} \triangle$ U.S. Geological Survey streamflow-gaging station and number used for estimates of peak-discharge frequency values

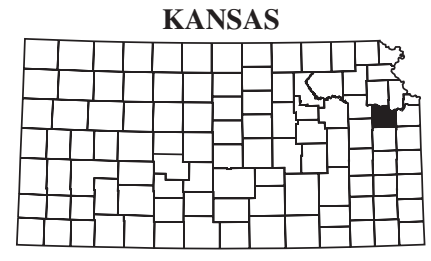

Index map 2330

Lake and determination site identification number

Figure 33. Location of streamflow-statistics determination sites, associated identification numbers, and U.S. Geological Survey streamflow-gaging stations used in the flow-duration and peak-discharge frequency analyses for Douglas County. 
Table 29. Estimated flow-duration values, mean flow values, and peak-discharge frequency values for controlled and uncontrolled flow stream segments on the 1999 Kansas Surface Water Register for Douglas County.

[KSWR, Kansas Surface Water Register; CUSEGA, catalog unit segment number alpha; $\mathrm{mi}^{2}$, square miles; $\mathrm{ft}^{3} / \mathrm{s}$, cubic feet per second; HYDRO, lake or other hydrologic structure; NA, not applicable; NRDitch, irrigation ditch; NRTribal, tribal stream]

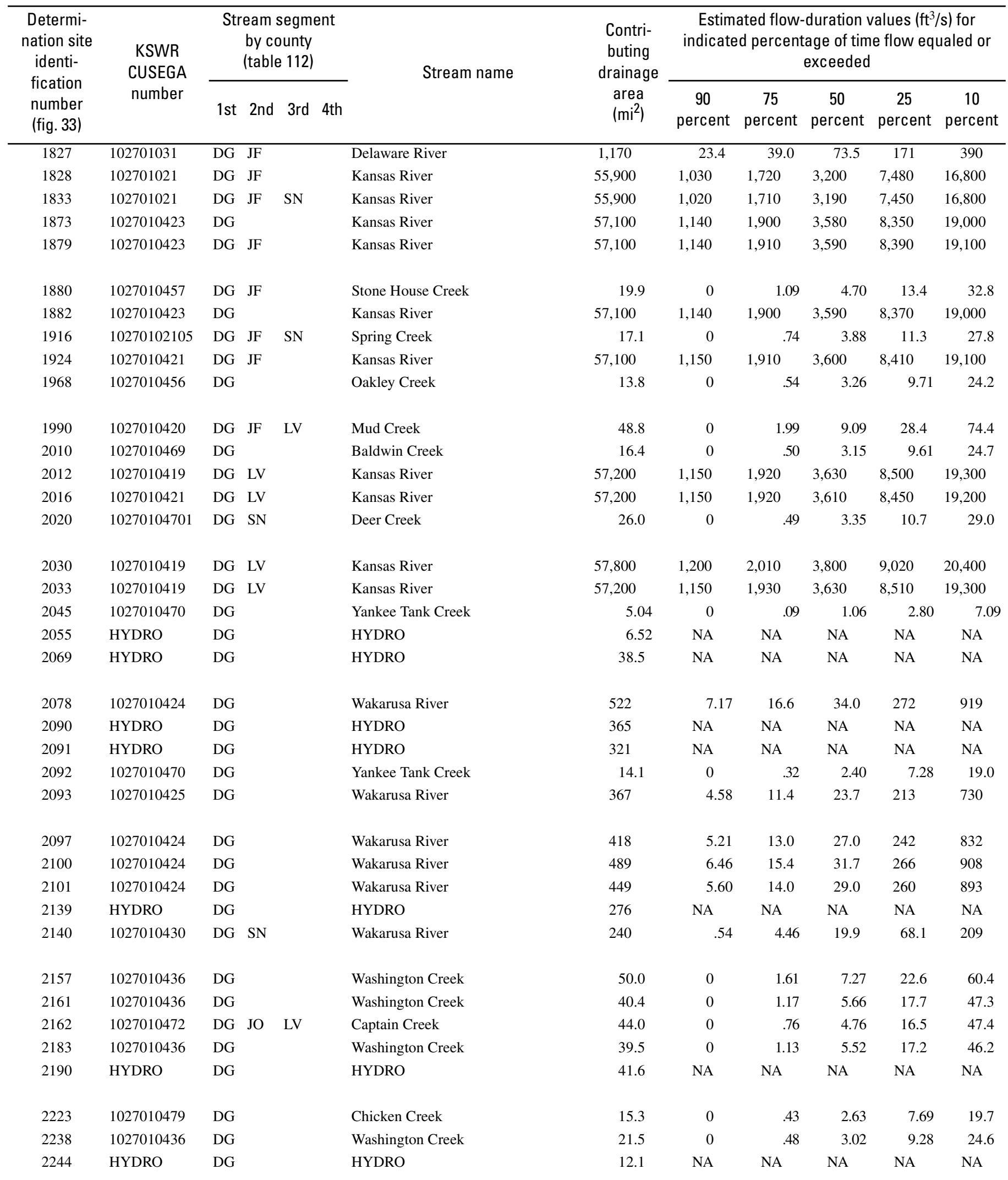


Table 29. Estimated flow-duration values, mean flow values, and peak-discharge frequency values for controlled and uncontrolled flow stream segments on the 1999 Kansas Surface Water Register for Douglas County.-Continued

[KSWR, Kansas Surface Water Register; CUSEGA, catalog unit segment number alpha; $\mathrm{mi}^{2}$, square miles; $\mathrm{ft}^{3} / \mathrm{s}$, cubic feet per second; HYDRO, lake or other hydrologic structure; NA, not applicable; NRDitch, irrigation ditch; NRTribal, tribal stream]

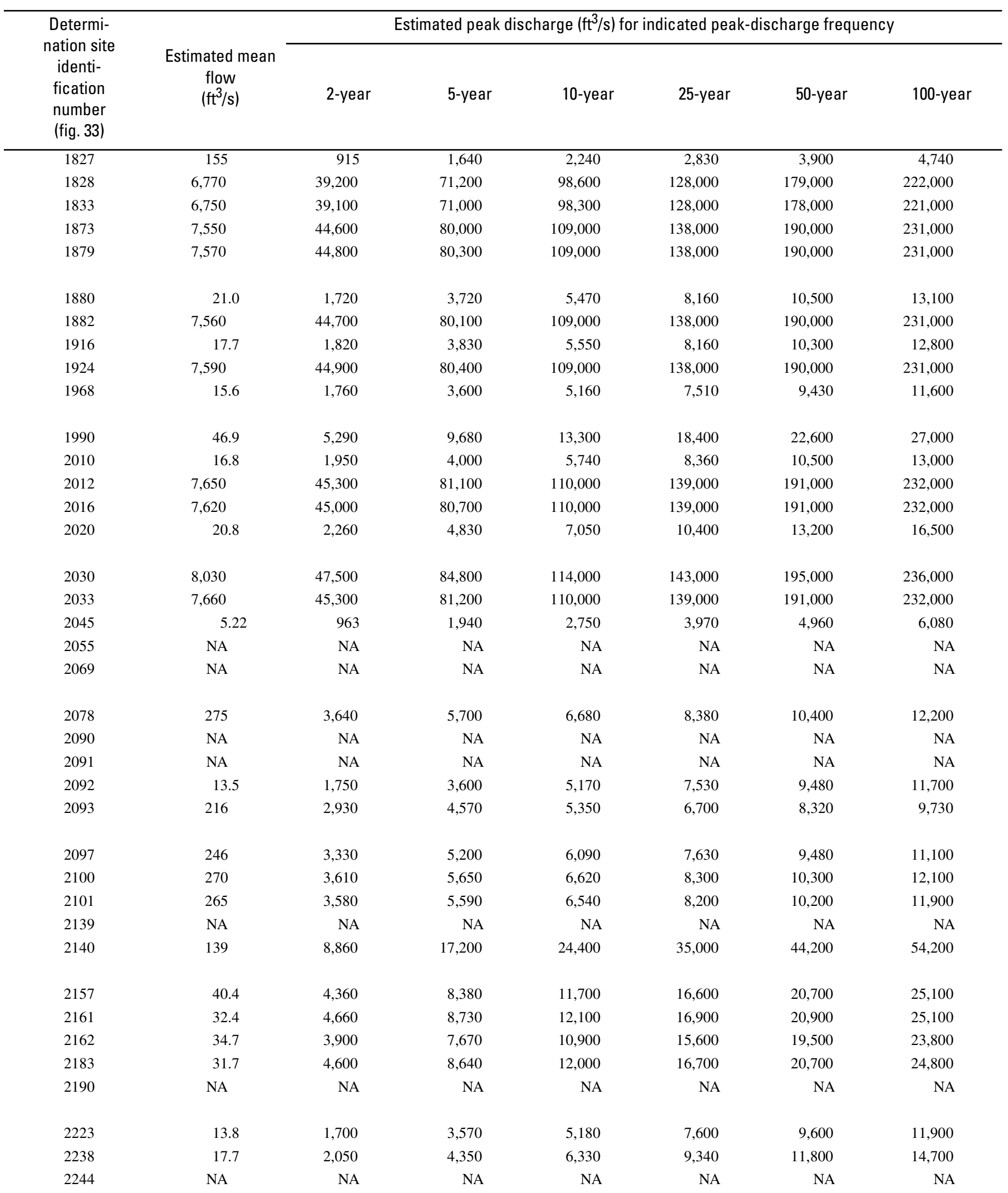


Table 29. Estimated flow-duration values, mean flow values, and peak-discharge frequency values for controlled and uncontrolled flow stream segments on the 1999 Kansas Surface Water Register for Douglas County.-Continued

[KSWR, Kansas Surface Water Register; CUSEGA, catalog unit segment number alpha; $\mathrm{mi}^{2}$, square miles; $\mathrm{ft}^{3} / \mathrm{s}$, cubic feet per second; HYDRO, lake or other hydrologic structure; NA, not applicable; NRDitch, irrigation ditch; NRTribal, tribal stream]

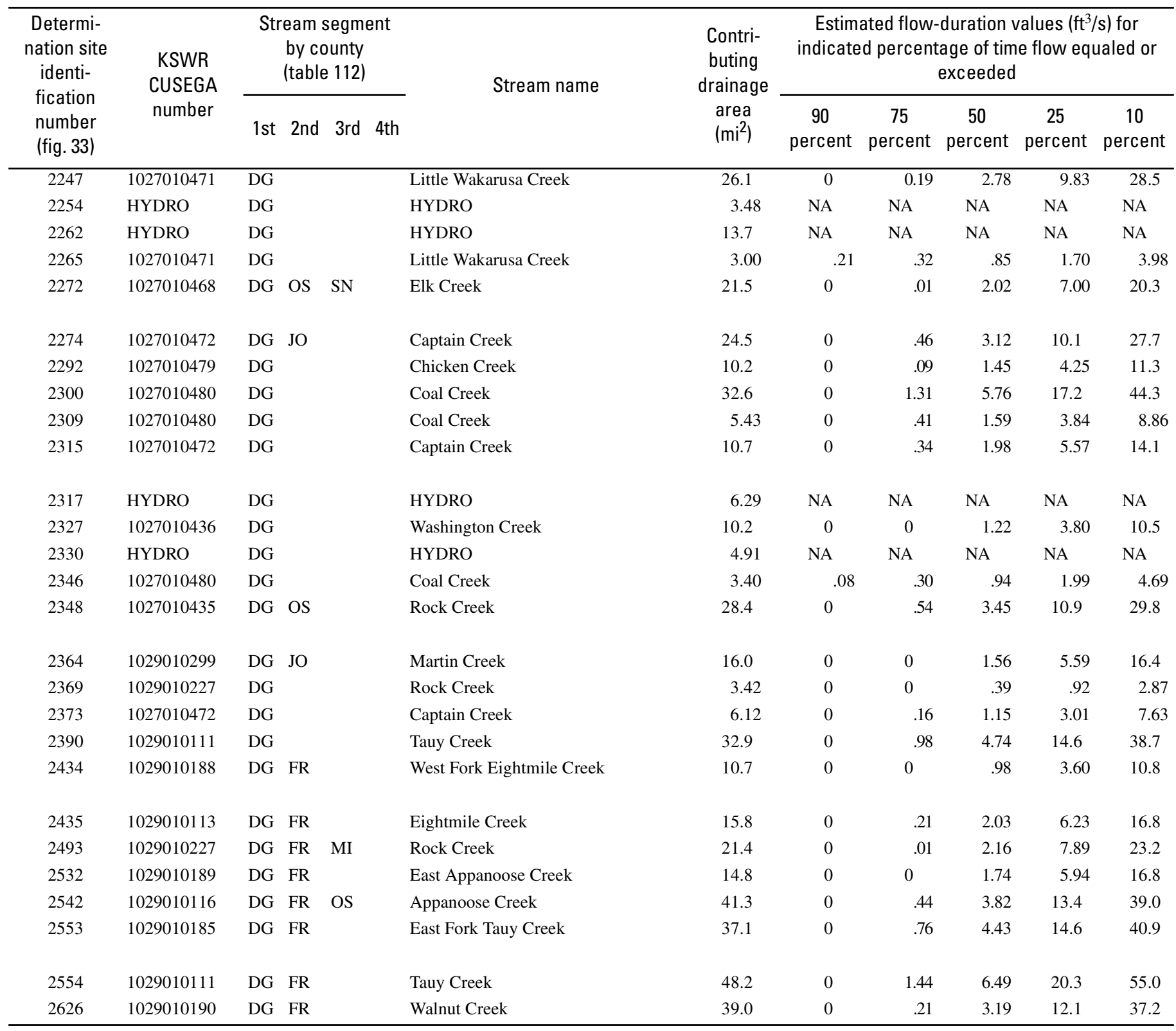


Table 29. Estimated flow-duration values, mean flow values, and peak-discharge frequency values for controlled and uncontrolled flow stream segments on the 1999 Kansas Surface Water Register for Douglas County.-Continued

[KSWR, Kansas Surface Water Register; CUSEGA, catalog unit segment number alpha; $\mathrm{mi}^{2}$, square miles; $\mathrm{ft}^{3} / \mathrm{s}$, cubic feet per second; HYDRO, lake or other hydrologic structure; NA, not applicable; NRDitch, irrigation ditch; NRTribal, tribal stream]

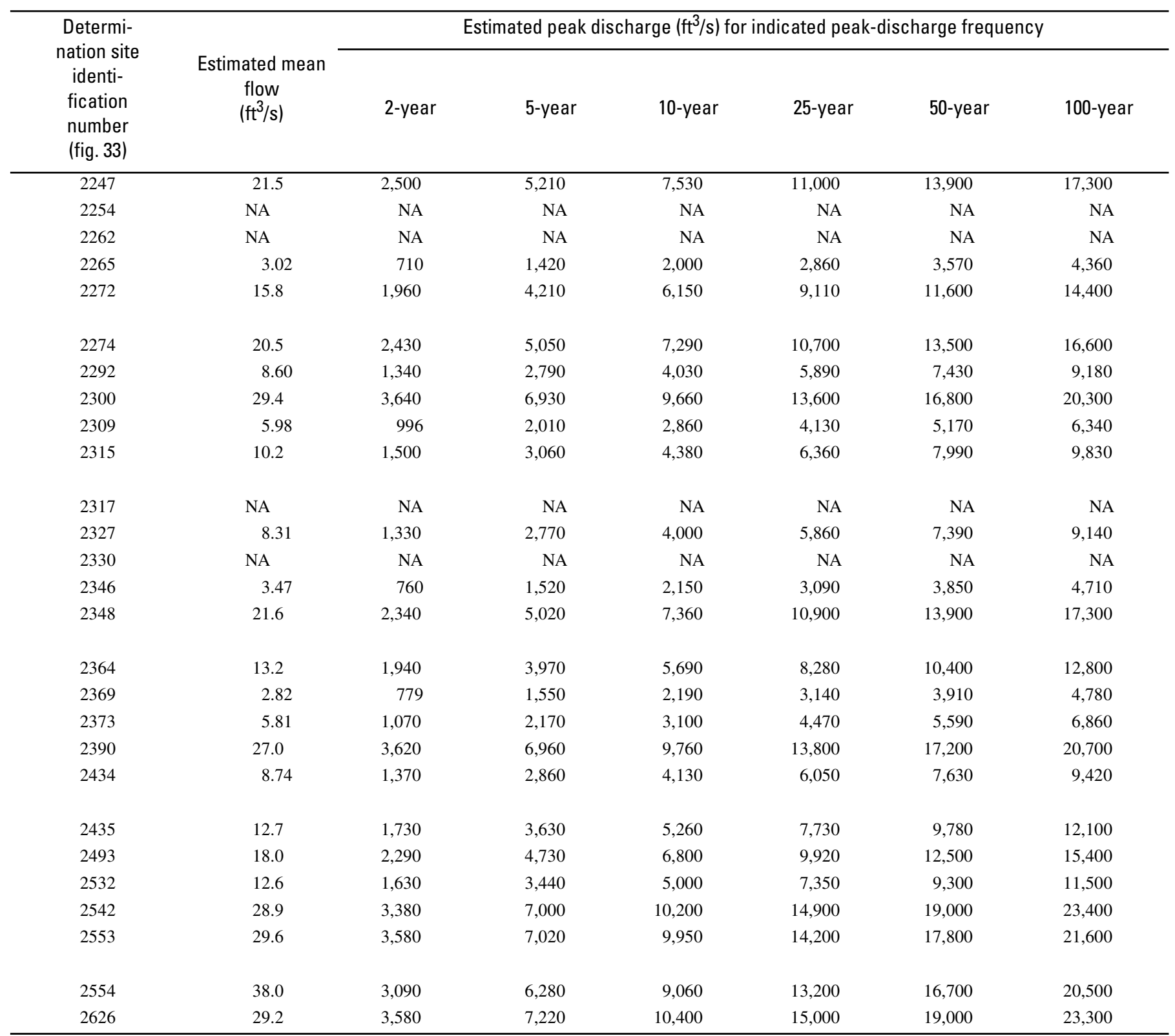




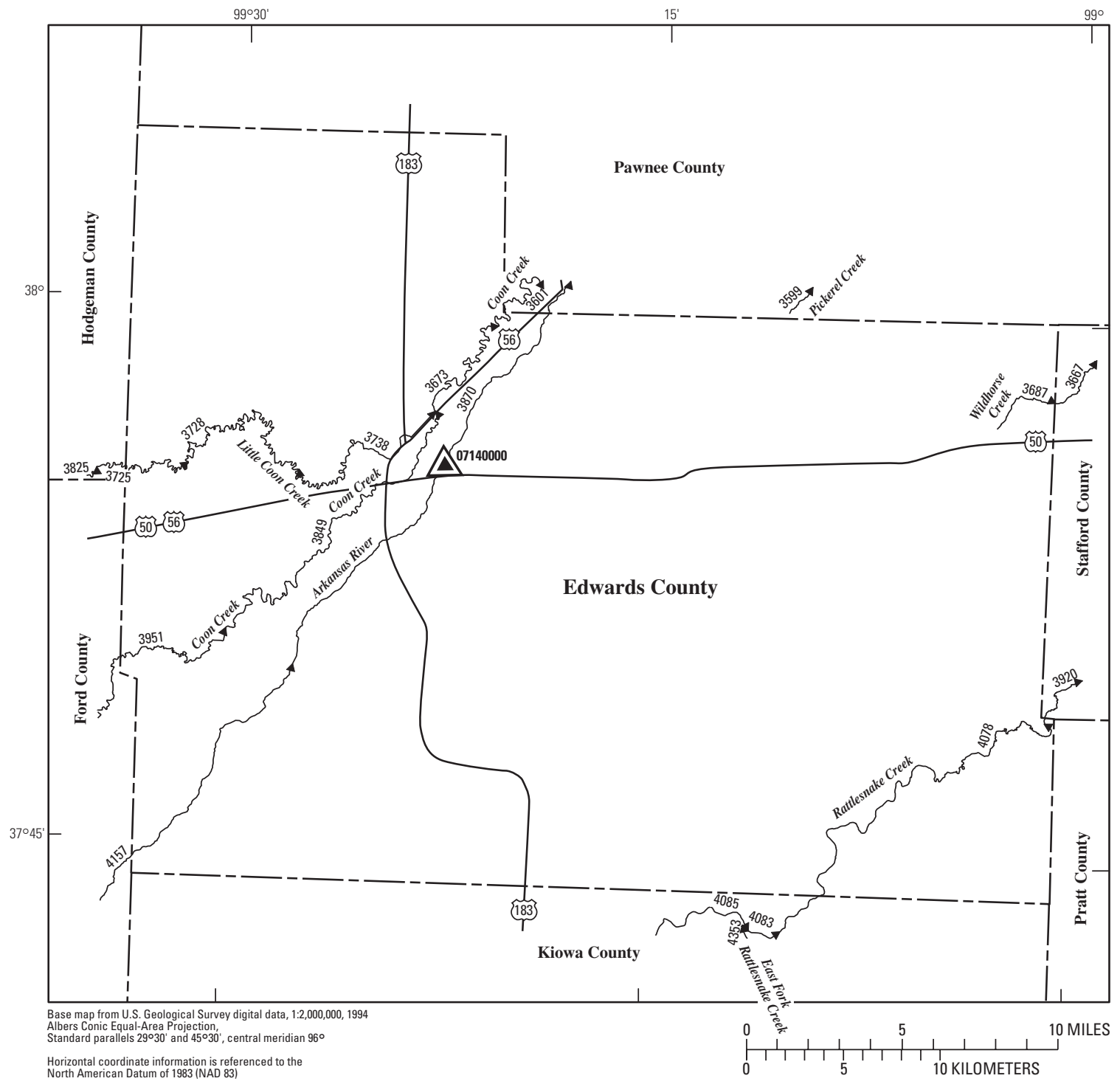

\section{EXPLANATION}

4157 Location of streamflow-statistics determination site (small triangle) and associated identification number-small triangle points in downstream direction

$07140000 \Delta$ U.S. Geological Survey streamflow-gaging station and number used for estimates of flow duration

${ }^{07140000} \triangle$ U.S. Geological Survey streamflow-gaging station and number used for estimates of peak-discharge frequency values

KANSAS

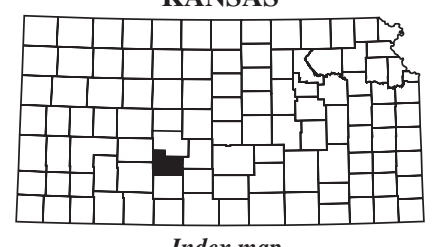

Index map 2330

Lake and determination site identification number

Figure 34. Location of streamflow-statistics determination sites, associated identification numbers, and U.S. Geological Survey streamflow-gaging stations used in the flow-duration and peak-discharge frequency analyses for Edwards County. 
Table 30. Estimated flow-duration values, mean flow values, and peak-discharge frequency values for controlled and uncontrolled flow stream segments on the 1999 Kansas Surface Water Register for Edwards County.

[KSWR, Kansas Surface Water Register; CUSEGA, catalog unit segment number alpha; $\mathrm{mi}^{2}$, square miles; $\mathrm{ft}^{3} / \mathrm{s}$, cubic feet per second; HYDRO, lake or other hydrologic structure; NA, not applicable; NRDitch, irrigation ditch; NRTribal, tribal stream]

\begin{tabular}{|c|c|c|c|c|c|c|c|c|c|c|c|}
\hline $\begin{array}{l}\text { Determi- } \\
\text { nation site } \\
\text { identi- } \\
\text { fication } \\
\text { number } \\
\text { (fig. 34) }\end{array}$ & $\begin{array}{l}\text { KSWR } \\
\text { CUSEGA } \\
\text { number }\end{array}$ & \multicolumn{3}{|c|}{$\begin{array}{l}\text { Stream segment } \\
\text { by county } \\
\text { (table 112) }\end{array}$} & Stream name & $\begin{array}{l}\text { Contri- } \\
\text { buting } \\
\text { drainage } \\
\text { area } \\
\left(\mathrm{mi}^{2}\right)\end{array}$ & \multicolumn{5}{|c|}{$\begin{array}{c}\text { Estimated flow-duration values }\left(\mathrm{ft}^{3} / \mathrm{s}\right) \text { for } \\
\text { indicated percentage of time flow equaled or } \\
\text { exceeded }\end{array}$} \\
\hline 3601 & 110300047 & ED & $\mathrm{PN}$ & & Coon Creek & 436 & 0.18 & 1.66 & 3.85 & 9.40 & 24.0 \\
\hline 3725 & 110300048 & ED & HG & & Little Coon Creek & 117 & 0 & 0 & 0 & .08 & 2.23 \\
\hline 3728 & 110300048 & ED & & & Little Coon Creek & 161 & 0 & 0 & .17 & 1.18 & 5.04 \\
\hline 3738 & 110300048 & ED & & & Little Coon Creek & 175 & 0 & 0 & .48 & 1.81 & 6.32 \\
\hline 3849 & 110300049 & ED & & & Coon Creek & 188 & 0 & .21 & 1.15 & 2.82 & 7.60 \\
\hline 4078 & 110300093 & ED & $\mathrm{KW}$ & & Rattlesnake Creek & 622 & 1.00 & 5.60 & 12.9 & 24.7 & 37.5 \\
\hline 4157 & 1103000410 & ED & FO & & Arkansas River & 28,000 & .58 & 2.11 & 27.6 & 97.8 & 198 \\
\hline
\end{tabular}


Table 30. Estimated flow-duration values, mean flow values, and peak-discharge frequency values for controlled and uncontrolled flow stream segments on the 1999 Kansas Surface Water Register for Edwards County.-Continued

[KSWR, Kansas Surface Water Register; CUSEGA, catalog unit segment number alpha; $\mathrm{mi}^{2}$, square miles; $\mathrm{ft}^{3} / \mathrm{s}$, cubic feet per second; HYDRO, lake or other hydrologic structure; NA, not applicable; NRDitch, irrigation ditch; NRTribal, tribal stream]

\begin{tabular}{|c|c|c|c|c|c|c|c|}
\hline \multirow{2}{*}{$\begin{array}{l}\text { Determi- } \\
\text { nation site } \\
\text { identi- } \\
\text { fication } \\
\text { number } \\
\text { (fig. 34) }\end{array}$} & \multirow{2}{*}{$\begin{array}{c}\text { Estimated mean } \\
\text { flow } \\
\left(\mathrm{ft}^{3} / \mathrm{s}\right)\end{array}$} & \multicolumn{6}{|c|}{ Estimated peak discharge $\left(\mathrm{ft}^{3} / \mathrm{s}\right)$ for indicated peak-discharge frequency } \\
\hline & & 2-year & 5-year & 10-year & 25-year & 50-year & 100-year \\
\hline 3601 & 23.0 & 761 & 2,270 & 3,980 & 7,140 & 10,300 & 14,400 \\
\hline 3725 & 5.05 & 368 & 1,240 & 2,300 & 4,350 & 6,520 & 9,330 \\
\hline 3728 & 7.71 & 404 & 1,370 & 2,550 & 4,820 & 7,230 & 10,300 \\
\hline 3738 & 8.71 & 376 & 1,280 & 2,380 & 4,530 & 6,810 & 9,760 \\
\hline 3849 & 9.28 & 641 & 1,880 & 3,210 & 5,510 & 7,690 & 10,300 \\
\hline 4078 & 25.6 & 438 & 1,430 & 2,600 & 4,880 & 7,290 & 10,500 \\
\hline 4157 & 97.9 & 635 & 3,120 & 9,030 & 17,100 & 27,100 & 41,100 \\
\hline
\end{tabular}




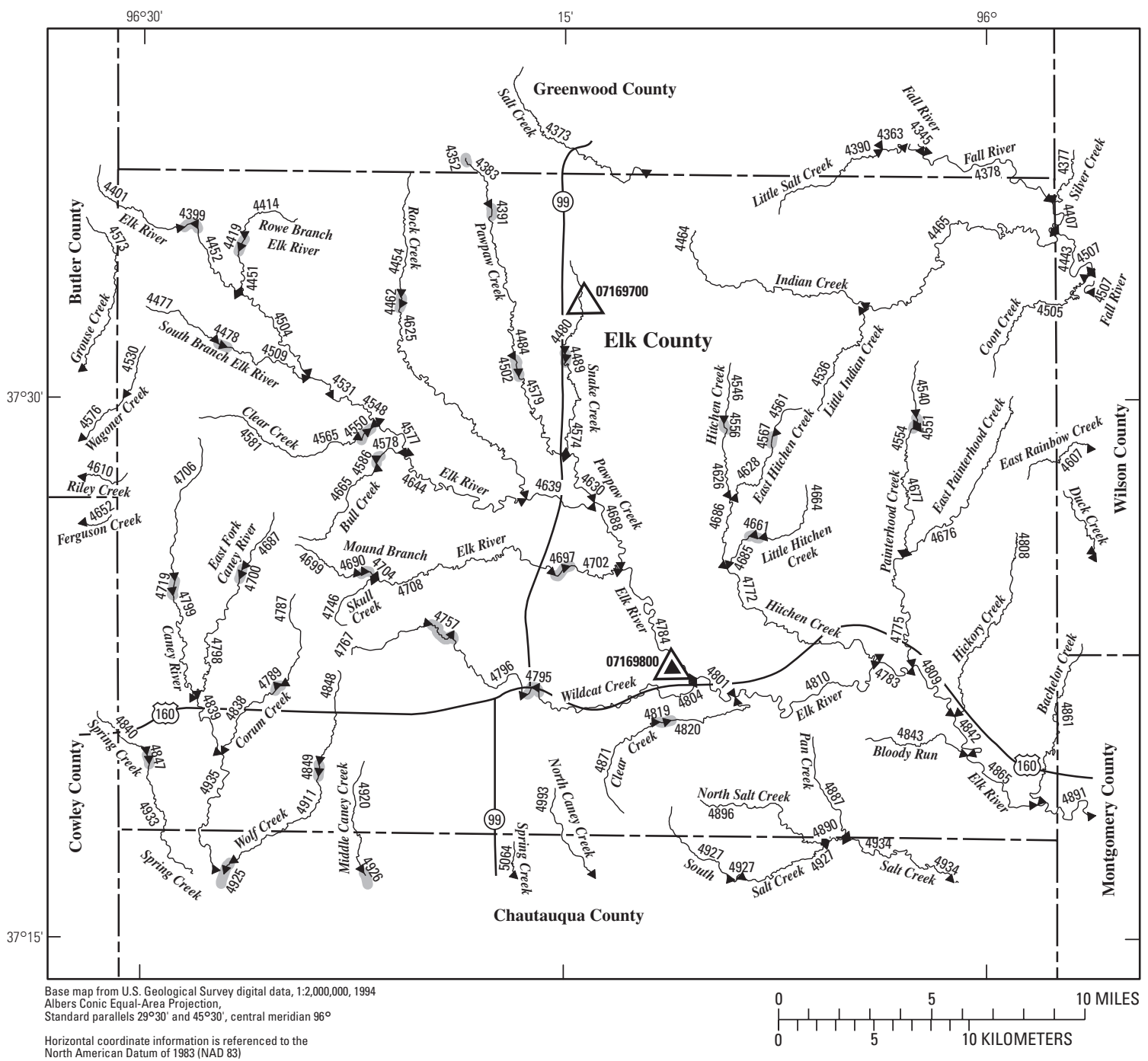

EXPLANATION

4933 Location of streamflow-statistics determination site (small triangle) and associated identification number-small triangle points in downstream direction

07169800 U.S. Geological Survey streamflow-gaging station and number used for estimates of flow duration

${ }^{07169700} \triangle$ U.S. Geological Survey streamflow-gaging station and number used for estimates of peak-discharge frequency values

KANSAS

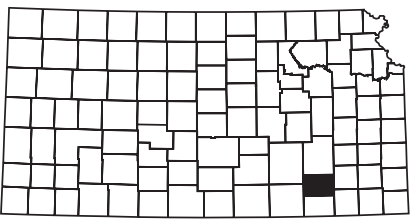

Index map 4925

Lake and determination site identification number

Figure 35. Location of streamflow-statistics determination sites, associated identification numbers, and U.S. Geological Survey streamflow-gaging stations used in the flow-duration and peak-discharge frequency analyses for Elk County. 
Table 31. Estimated flow-duration values, mean flow values, and peak-discharge frequency values for controlled and uncontrolled flow stream segments on the 1999 Kansas Surface Water Register for Elk County.

[KSWR, Kansas Surface Water Register; CUSEGA, catalog unit segment number alpha; $\mathrm{mi}^{2}$, square miles; $\mathrm{ft}^{3} / \mathrm{s}$, cubic feet per second; HYDRO, lake or other hydrologic structure; NA, not applicable; NRDitch, irrigation ditch; NRTribal, tribal stream]

\begin{tabular}{|c|c|c|c|c|c|c|c|c|c|c|c|}
\hline \multirow{2}{*}{$\begin{array}{l}\text { Determi- } \\
\text { nation site } \\
\text { identi- } \\
\text { fication } \\
\text { number } \\
\text { (fig. } 35 \text { ) }\end{array}$} & \multirow{2}{*}{$\begin{array}{l}\text { KSWR } \\
\text { CUSEGA } \\
\text { number }\end{array}$} & \multicolumn{3}{|c|}{$\begin{array}{l}\text { Stream segment } \\
\text { by county } \\
\text { (table 112) }\end{array}$} & \multirow{2}{*}{ Stream name } & \multirow{2}{*}{$\begin{array}{l}\text { Contri- } \\
\text { buting } \\
\text { drainage } \\
\text { area } \\
\left(\mathrm{mi}^{2}\right)\end{array}$} & \multicolumn{5}{|c|}{$\begin{array}{l}\text { Estimated flow-duration values }\left(\mathrm{ft}^{3} / \mathrm{s}\right) \text { for } \\
\text { indicated percentage of time flow equaled or } \\
\text { exceeded }\end{array}$} \\
\hline & & $1 \mathrm{st}$ & 2nd 3rd & 4th & & & $\begin{array}{c}90 \\
\text { percent }\end{array}$ & $\begin{array}{c}75 \\
\text { percent }\end{array}$ & $\begin{array}{c}50 \\
\text { percent }\end{array}$ & $\begin{array}{c}25 \\
\text { percent }\end{array}$ & $\begin{array}{c}10 \\
\text { percent }\end{array}$ \\
\hline 4377 & 1107010233 & EK & GW WL & & Silver Creek & 19.0 & 36 & 2.22 & 6.00 & 13.9 & 29.8 \\
\hline 4378 & 110701022 & EK & GW & & Fall River & 590 & 7.80 & 15.6 & 67.5 & 353 & 1,310 \\
\hline 4383 & 1107010411 & EK & GW & & Pawpaw Creek & 8.32 & 0 & 0 & .49 & 2.01 & 6.66 \\
\hline 4390 & 1107010235 & EK & GW & & Little Salt Creek & 12.2 & 0 & .15 & 1.59 & 4.53 & 11.9 \\
\hline 4391 & HYDRO & EK & & & HYDRO & 8.67 & NA & NA & NA & NA & NA \\
\hline 4399 & HYDRO & EK & & & HYDRO & 11.5 & NA & NA & NA & NA & NA \\
\hline 4407 & 110701022 & EK & WL & & Fall River & 610 & 8.19 & 16.1 & 70.5 & 370 & 1,370 \\
\hline 4414 & 1107010439 & EK & & & Rowe Branch Elk River & 10.6 & 0 & 0 & .94 & 3.52 & 10.6 \\
\hline 4419 & HYDRO & EK & & & HYDRO & 11.0 & SNA & NA & NA & NA & NA \\
\hline 4451 & 1107010439 & EK & & & Rowe Branch Elk River & 12.9 & 0 & 0 & 1.22 & 4.47 & 13.2 \\
\hline 4452 & 1107010414 & EK & & & Elk River & 15.2 & 0 & .02 & 1.96 & 6.36 & 17.3 \\
\hline 4454 & 1107010413 & EK & GW & & Rock Creek & 15.8 & 0 & 0 & .75 & 3.40 & 11.4 \\
\hline 4462 & HYDRO & EK & & & HYDRO & 17.0 & NA & NA & NA & NA & NA \\
\hline 4464 & 1107010215 & EK & & & Indian Creek & 25.2 & 0 & .06 & 2.12 & 7.35 & 21.5 \\
\hline 4465 & 1107010215 & EK & WL & & Indian Creek & 53.9 & 0 & 1.64 & 6.93 & 21.1 & 56.3 \\
\hline 4477 & 1107010438 & EK & & & South Branch Elk River & 6.14 & 0 & 0 & .63 & 2.01 & 5.93 \\
\hline 4478 & HYDRO & EK & & & HYDRO & 6.86 & NA & NA & NA & NA & NA \\
\hline 4480 & 1107010434 & EK & & & Snake Creek & 14.5 & 0 & 0 & .43 & 2.78 & 10.5 \\
\hline 4484 & 1107010411 & EK & & & Pawpaw Creek & 21.9 & 0 & 0 & 1.47 & 6.00 & 18.8 \\
\hline 4489 & HYDRO & EK & & & HYDRO & 14.7 & NA & NA & NA & NA & NA \\
\hline 4502 & HYDRO & EK & & & HYDRO & 22.8 & NA & NA & NA & NA & NA \\
\hline 4504 & 1107010414 & EK & & & Elk River & 35.9 & .01 & .24 & 3.46 & 12.6 & 36.7 \\
\hline 4505 & 1107010236 & EK & WL & & Coon Creek & 12.7 & 0 & .79 & 3.19 & 8.39 & 19.7 \\
\hline 4509 & 1107010438 & EK & & & South Branch Elk River & 11.6 & 0 & 0 & 1.36 & 4.52 & 12.6 \\
\hline 4530 & 1106000136 & EK & & & Wagoner Creek & 4.81 & 0 & 0 & .11 & .68 & 3.01 \\
\hline 4531 & 1107010414 & EK & & & Elk River & 49.3 & .03 & .55 & 4.86 & 17.7 & 51.5 \\
\hline 4536 & 1107010234 & EK & & & Little Indian Creek & 9.79 & 0 & .74 & 2.40 & 5.74 & 13.1 \\
\hline 4540 & 110701045 & EK & & & Painterhood Creek & 6.64 & 0 & .64 & 1.86 & 4.15 & 9.25 \\
\hline 4546 & 110701047 & EK & & & Hitchen Creek & 10.9 & 0 & 0 & .18 & 1.61 & 6.72 \\
\hline 4548 & 1107010414 & EK & & & Elk River & 52.6 & .03 & .59 & 5.14 & 18.9 & 54.9 \\
\hline 4550 & 1107010432 & EK & & & Clear Creek & 13.1 & 0 & 0 & 1.08 & 4.10 & 12.4 \\
\hline 4551 & HYDRO & EK & & & HYDRO & 6.80 & NA & NA & NA & NA & NA \\
\hline 4554 & 110701045 & EK & & & Painterhood Creek & 1.36 & 0 & .10 & .27 & .45 & .91 \\
\hline 4556 & HYDRO & EK & & & HYDRO & 11.7 & NA & NA & NA & NA & NA \\
\hline 4561 & 1107010435 & EK & & & East Hitchen Creek & 3.86 & 0 & 0 & .14 & .55 & 2.45 \\
\hline 4565 & HYDRO & EK & & & HYDRO & 13.0 & NA & NA & NA & NA & NA \\
\hline 4567 & HYDRO & EK & & & HYDRO & 4.03 & NA & NA & NA & NA & NA \\
\hline 4574 & 1107010434 & EK & & & Snake Creek & 23.3 & 0 & 0 & .95 & 4.92 & 17.2 \\
\hline
\end{tabular}


Table 31. Estimated flow-duration values, mean flow values, and peak-discharge frequency values for controlled and uncontrolled flow stream segments on the 1999 Kansas Surface Water Register for Elk County.-Continued

[KSWR, Kansas Surface Water Register; CUSEGA, catalog unit segment number alpha; $\mathrm{mi}^{2}$, square miles; $\mathrm{ft}^{3} / \mathrm{s}$, cubic feet per second; HYDRO, lake or other hydrologic structure; NA, not applicable; NRDitch, irrigation ditch; NRTribal, tribal stream]

\begin{tabular}{|c|c|c|c|c|c|c|c|}
\hline \multirow{2}{*}{$\begin{array}{l}\text { Determi- } \\
\text { nation site } \\
\text { identi- } \\
\text { fication } \\
\text { number } \\
\text { (fig. } 35 \text { ) }\end{array}$} & \multirow{2}{*}{$\begin{array}{c}\text { Estimated mean } \\
\text { flow } \\
\left(\mathrm{ft}^{3} / \mathrm{s}\right)\end{array}$} & \multicolumn{6}{|c|}{ Estimated peak discharge $\left(\mathrm{ft}^{3} / \mathrm{s}\right)$ for indicated peak-discharge frequency } \\
\hline & & 2-year & 5-year & 10-year & 25-year & 50-year & 100-year \\
\hline 4377 & 18.1 & 1,900 & 4,020 & 5,850 & 8,620 & 10,900 & 13,500 \\
\hline 4390 & 9.12 & 1,350 & 2,890 & 4,220 & 6,230 & 7,910 & 9,820 \\
\hline 4391 & NA & NA & NA & NA & NA & NA & NA \\
\hline 4399 & NA & NA & NA & NA & NA & NA & NA \\
\hline 4407 & 446 & 6,570 & 11,400 & 14,600 & 18,300 & 20,800 & 23,100 \\
\hline 4452 & 12.4 & 1,500 & 3,260 & 4,800 & 7,150 & 9,120 & 11,400 \\
\hline 4454 & 10.1 & 1,600 & 3,440 & 5,050 & 7,500 & 9,540 & 11,900 \\
\hline 4462 & NA & NA & NA & NA & NA & NA & NA \\
\hline 4464 & 17.1 & 2,130 & 4,590 & 6,730 & 10,000 & 12,700 & 15,800 \\
\hline 4465 & 38.6 & 3,510 & 7,050 & 10,100 & 14,600 & 18,400 & 22,600 \\
\hline 4477 & 4.86 & 890 & 1,890 & 2,750 & 4,040 & 5,110 & 6,330 \\
\hline 4478 & NA & NA & NA & NA & NA & NA & NA \\
\hline 4480 & 9.69 & 497 & 969 & 1,360 & 1,920 & 2,390 & 2,890 \\
\hline 4484 & 15.2 & 1,960 & 4,250 & 6,260 & 9,330 & 11,900 & 14,900 \\
\hline 4531 & 35.4 & 6,140 & 11,800 & 16,500 & 23,500 & 29,500 & 35,800 \\
\hline 4536 & 8.91 & 1,270 & 2,660 & 3,850 & 5,650 & 7,130 & 8,820 \\
\hline 4540 & 6.31 & 1,030 & 2,130 & 3,060 & 4,460 & 5,620 & 6,930 \\
\hline 4546 & 6.92 & 1,340 & 2,830 & 4,100 & 6,010 & 7,600 & 9,410 \\
\hline 4548 & 37.6 & 5,880 & 11,500 & 16,200 & 23,300 & 29,300 & 35,900 \\
\hline 4550 & 9.83 & 1,400 & 3,010 & 4,410 & 6,550 & 8,340 & 10,400 \\
\hline 4551 & NA & NA & NA & NA & NA & NA & NA \\
\hline 4554 & .95 & 418 & 834 & 1,180 & 1,680 & 2,090 & 2,550 \\
\hline 4556 & $\mathrm{NA}$ & NA & NA & NA & NA & NA & NA \\
\hline 4561 & 2.74 & 747 & 1,530 & 2,190 & 3,180 & 3,990 & 4,900 \\
\hline 4565 & NA & NA & NA & NA & NA & NA & NA \\
\hline 4567 & NA & NA & NA & NA & NA & NA & NA \\
\hline 4574 & 15.1 & 1,030 & 2,300 & 3,440 & 5,250 & 6,830 & 8,660 \\
\hline
\end{tabular}


Table 31. Estimated flow-duration values, mean flow values, and peak-discharge frequency values for controlled and uncontrolled flow stream segments on the 1999 Kansas Surface Water Register for Elk County.-Continued

[KSWR, Kansas Surface Water Register; CUSEGA, catalog unit segment number alpha; $\mathrm{mi}^{2}$, square miles; $\mathrm{ft}^{3} / \mathrm{s}$, cubic feet per second; HYDRO, lake or other hydrologic structure; NA, not applicable; NRDitch, irrigation ditch; NRTribal, tribal stream]

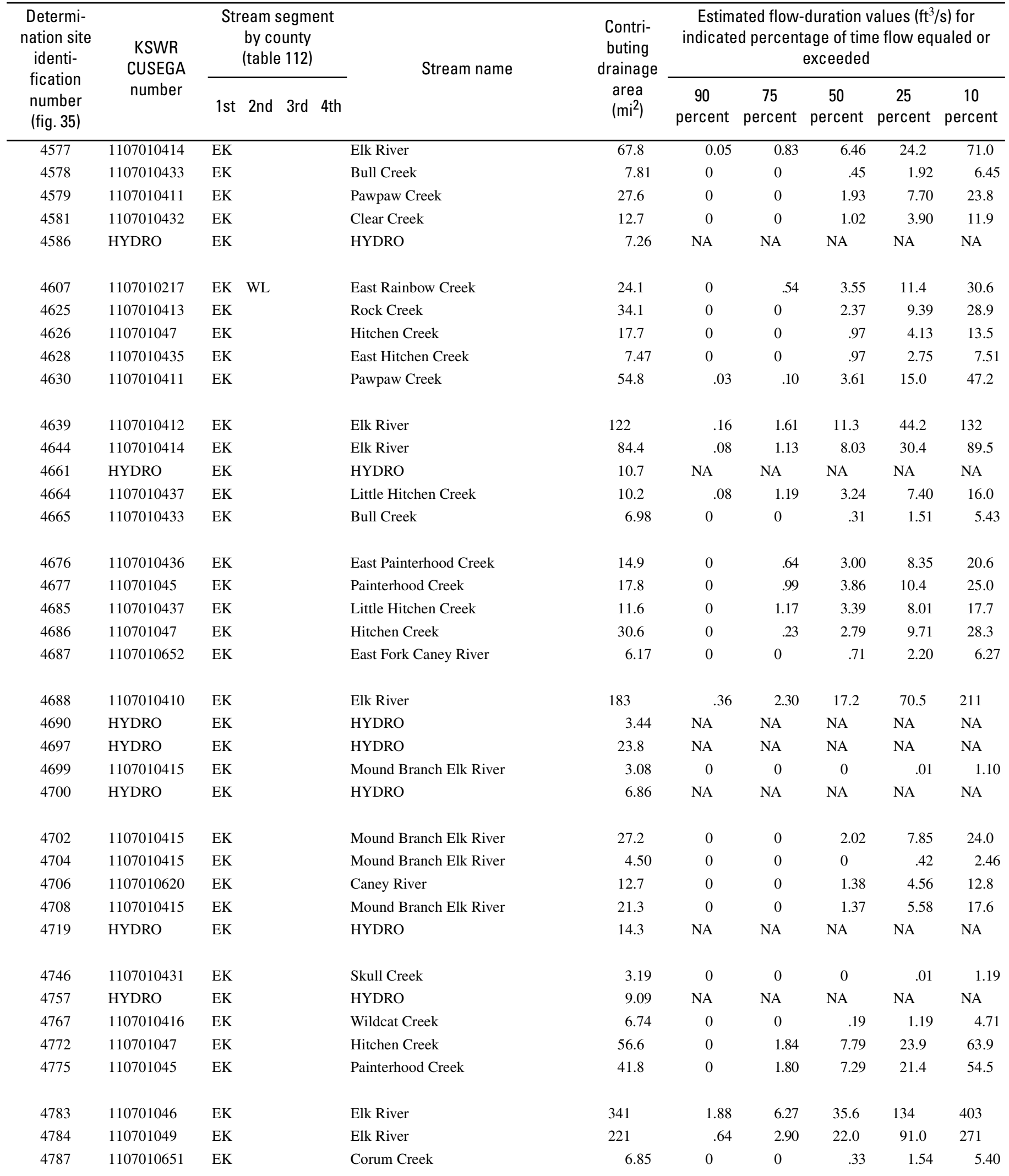


Table 31. Estimated flow-duration values, mean flow values, and peak-discharge frequency values for controlled and uncontrolled flow stream segments on the 1999 Kansas Surface Water Register for Elk County.-Continued

[KSWR, Kansas Surface Water Register; CUSEGA, catalog unit segment number alpha; $\mathrm{mi}^{2}$, square miles; $\mathrm{ft}^{3} / \mathrm{s}$, cubic feet per second; HYDRO, lake or other hydrologic structure; NA, not applicable; NRDitch, irrigation ditch; NRTribal, tribal stream]

\begin{tabular}{|c|c|c|c|c|c|c|c|}
\hline \multirow{2}{*}{$\begin{array}{l}\text { Determi- } \\
\text { nation site } \\
\text { identi- } \\
\text { fication } \\
\text { number } \\
\text { (fig. } 35 \text { ) }\end{array}$} & \multirow{2}{*}{$\begin{array}{c}\text { Estimated mean } \\
\text { flow } \\
\left(\mathrm{ft}^{3} / \mathrm{s}\right)\end{array}$} & \multicolumn{6}{|c|}{ Estimated peak discharge $\left(\mathrm{ft}^{3} / \mathrm{s}\right)$ for indicated peak-discharge frequency } \\
\hline & & 2-year & 5-year & 10-year & 25-year & 50-year & 100-year \\
\hline 4577 & 47.6 & 6,610 & 12,900 & 18,400 & 26,600 & 33,600 & 41,400 \\
\hline 4581 & 9.50 & 1,370 & 2,950 & 4,330 & 6,430 & 8,180 & 10,200 \\
\hline 4586 & NA & NA & NA & NA & NA & NA & NA \\
\hline 4607 & 21.5 & 2,350 & 4,910 & 7,110 & 10,400 & 13,200 & 16,300 \\
\hline 4625 & 22.5 & 3,580 & 7,430 & 10,800 & 15,900 & 20,200 & 25,000 \\
\hline 4639 & 82.7 & 7,140 & 15,100 & 22,400 & 33,800 & 44,100 & 55,800 \\
\hline 4644 & 58.6 & 6,130 & 12,600 & 18,300 & 27,100 & 34,800 & 43,400 \\
\hline 4661 & NA & NA & NA & NA & NA & NA & NA \\
\hline 4664 & 10.2 & 1,350 & 2,810 & 4,050 & 5,920 & 7,460 & 9,210 \\
\hline 4665 & 5.01 & 975 & 2,070 & 3,000 & 4,420 & 5,600 & 6,930 \\
\hline 4676 & 14.0 & 1,710 & 3,570 & 5,160 & 7,550 & 9,530 & 11,800 \\
\hline 4677 & 16.4 & 1,850 & 3,890 & 5,650 & 8,320 & 10,500 & 13,000 \\
\hline 4685 & 11.4 & 1,460 & 3,030 & 4,380 & 6,410 & 8,090 & 9,990 \\
\hline 4686 & 21.8 & 4,940 & 9,190 & 12,700 & 17,600 & 21,800 & 26,000 \\
\hline 4702 & 18.7 & 2,200 & 4,820 & 7,120 & 10,700 & 13,700 & 17,100 \\
\hline 4704 & 2.89 & 752 & 1,580 & 2,290 & 3,350 & 4,230 & 5,230 \\
\hline 4706 & 9.77 & 1,360 & 2,920 & 4,280 & 6,340 & 8,060 & 10,000 \\
\hline 4708 & 14.4 & 1,890 & 4,120 & 6,070 & 9,060 & 11,600 & 14,500 \\
\hline 4719 & NA & NA & NA & NA & NA & NA & NA \\
\hline 4746 & 1.89 & 611 & 1,280 & 1,840 & 2,690 & 3,390 & 4,180 \\
\hline 4757 & NA & NA & NA & NA & NA & NA & NA \\
\hline 4767 & 4.63 & 948 & 2,010 & 2,920 & 4,290 & 5,420 & 6,720 \\
\hline 4772 & 43.1 & 4,750 & 8,990 & 12,500 & 17,600 & 21,900 & 26,400 \\
\hline 4775 & 35.5 & 3,840 & 7,350 & 10,300 & 14,500 & 18,000 & 21,700 \\
\hline 4783 & 229 & 10,200 & 23,400 & 36,200 & 57,400 & 77,100 & 101,000 \\
\hline 4784 & 154 & 9,000 & 21,300 & 33,300 & 53,400 & 72,200 & 94,600 \\
\hline 4787 & 4.90 & 933 & 1,990 & 2,900 & 4,270 & 5,410 & 6,710 \\
\hline
\end{tabular}


Table 31. Estimated flow-duration values, mean flow values, and peak-discharge frequency values for controlled and uncontrolled flow stream segments on the 1999 Kansas Surface Water Register for Elk County.-Continued

[KSWR, Kansas Surface Water Register; CUSEGA, catalog unit segment number alpha; $\mathrm{mi}^{2}$, square miles; $\mathrm{ft}^{3} / \mathrm{s}$, cubic feet per second; HYDRO, lake or other hydrologic structure; NA, not applicable; NRDitch, irrigation ditch; NRTribal, tribal stream]

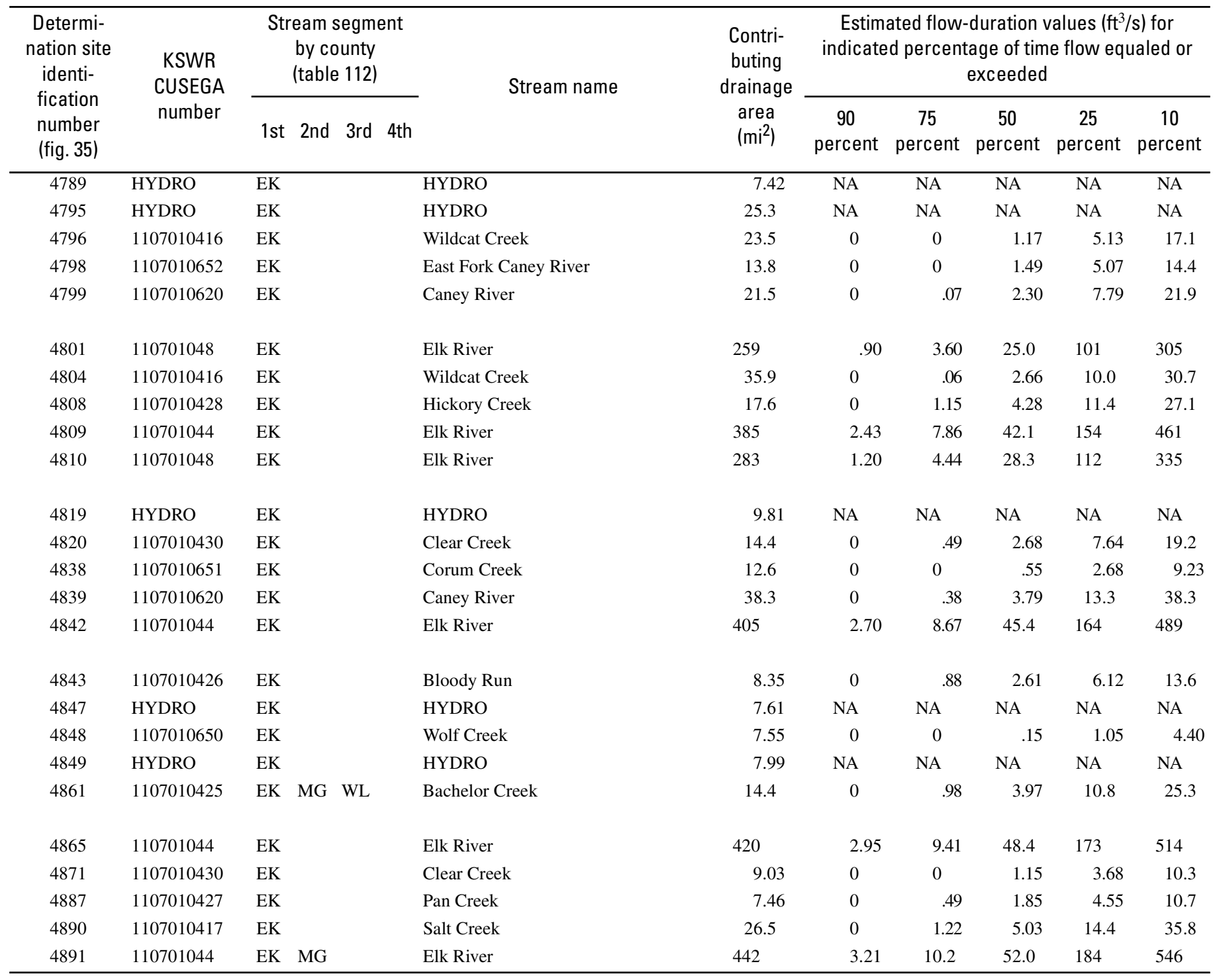


Table 31. Estimated flow-duration values, mean flow values, and peak-discharge frequency values for controlled and uncontrolled flow stream segments on the 1999 Kansas Surface Water Register for Elk County.-Continued

[KSWR, Kansas Surface Water Register; CUSEGA, catalog unit segment number alpha; $\mathrm{mi}^{2}$, square miles; $\mathrm{ft}^{3} / \mathrm{s}$, cubic feet per second; HYDRO, lake or other hydrologic structure; NA, not applicable; NRDitch, irrigation ditch; NRTribal, tribal stream]

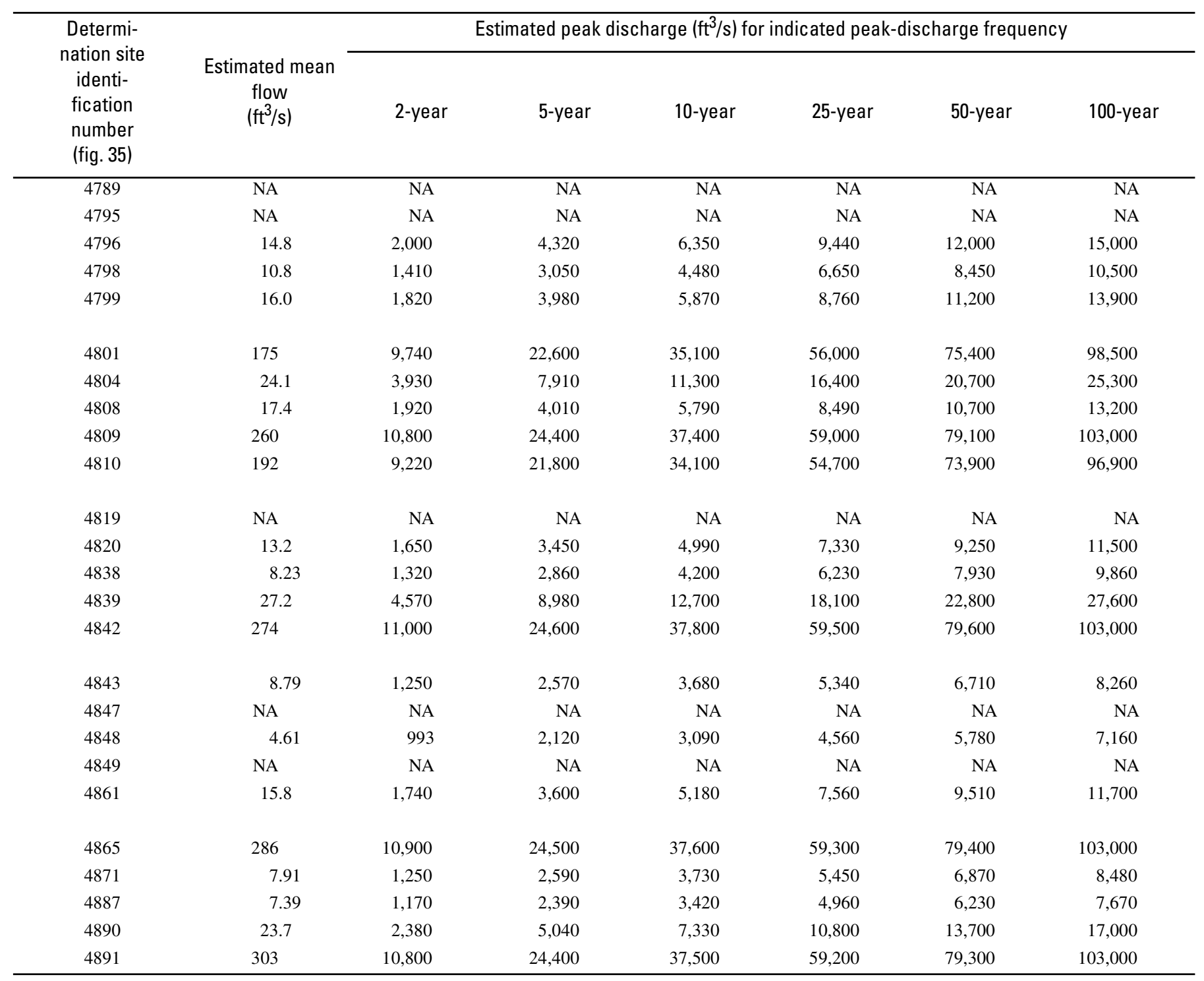




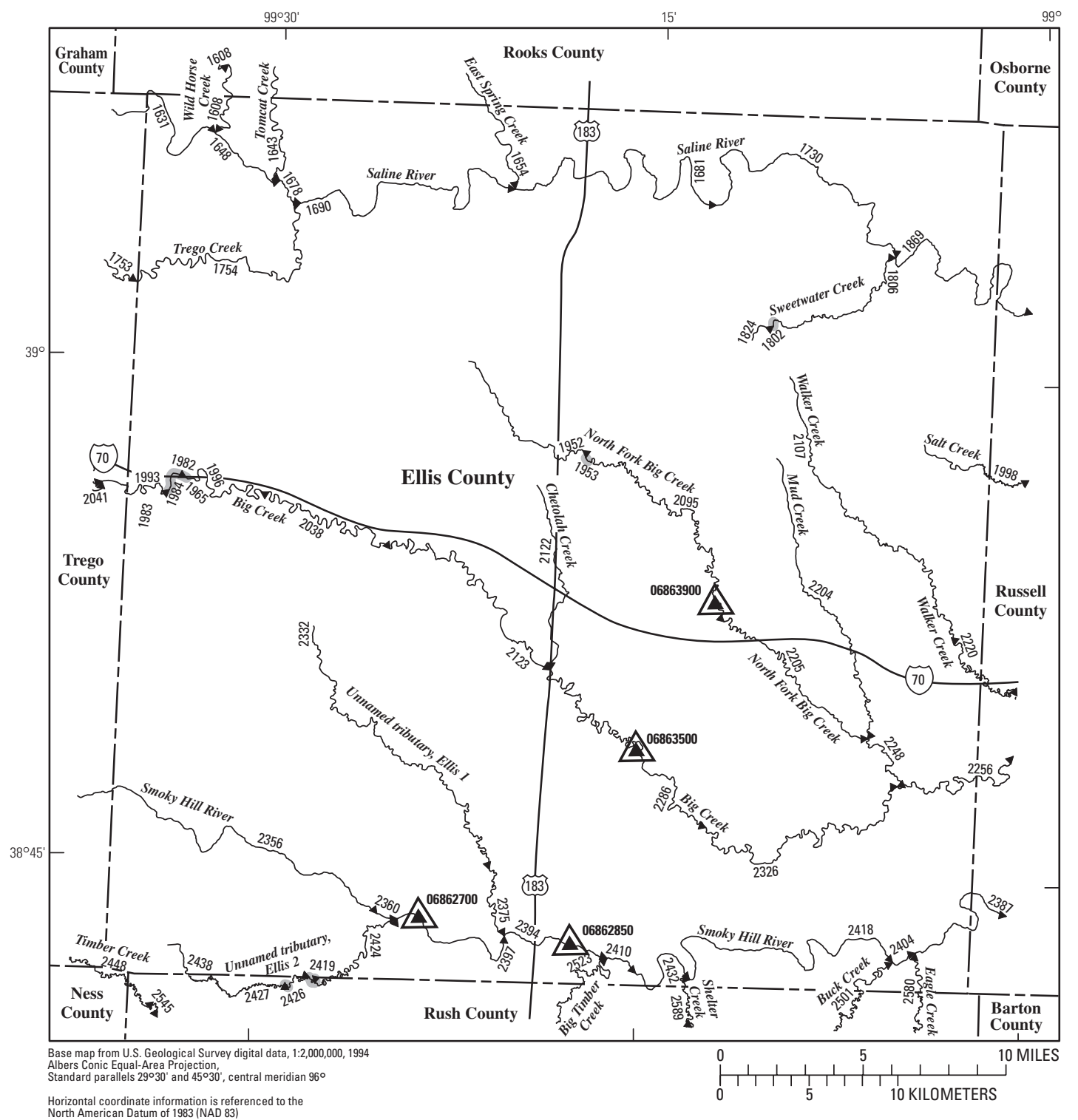

EXPLANATION

2545 Location of streamflow-statistics determination site (small triangle) and associated identification number-small triangle points in downstream direction

06862700

A.S. Geological Survey streamflow-gaging station and number used for estimates of flow duration

${ }^{06862850} \triangle$ U.S. Geological Survey streamflow-gaging station and number used for estimates of peak-discharge frequency values

KANSAS

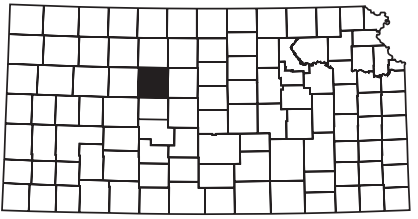

Index map 1984 Lake and determination site identification number

Figure 36. Location of streamflow-statistics determination sites, associated identification numbers, and U.S. Geological Survey streamflow-gaging stations used in the flow-duration and peak-discharge frequency analyses for Ellis County. 
Table 32. Estimated flow-duration values, mean flow values, and peak-discharge frequency values for controlled and uncontrolled flow stream segments on the 1999 Kansas Surface Water Register for Ellis County.

[KSWR, Kansas Surface Water Register; CUSEGA, catalog unit segment number alpha; $\mathrm{mi}^{2}$, square miles; $\mathrm{ft}^{3} / \mathrm{s}$, cubic feet per second; HYDRO, lake or other hydrologic structure; NA, not applicable; NRDitch, irrigation ditch; NRTribal, tribal stream]

\begin{tabular}{|c|c|c|c|c|c|c|c|c|c|c|c|}
\hline \multirow{2}{*}{$\begin{array}{l}\text { Determi- } \\
\text { nation site } \\
\text { identi- } \\
\text { fication } \\
\text { number } \\
\text { (fig. } 36 \text { ) }\end{array}$} & \multirow{2}{*}{$\begin{array}{l}\text { KSWR } \\
\text { CUSEGA } \\
\text { number }\end{array}$} & \multicolumn{3}{|c|}{$\begin{array}{l}\text { Stream segment } \\
\text { by county } \\
\text { (table 112) }\end{array}$} & \multirow{2}{*}{ Stream name } & \multirow{2}{*}{$\begin{array}{l}\text { Contri- } \\
\text { buting } \\
\text { drainage } \\
\text { area } \\
\left(\mathrm{mi}^{2}\right)\end{array}$} & \multicolumn{5}{|c|}{$\begin{array}{c}\text { Estimated flow-duration values }\left(\mathrm{ft}^{3} / \mathrm{s}\right) \text { for } \\
\text { indicated percentage of time flow equaled or } \\
\text { exceeded }\end{array}$} \\
\hline & & $1 \mathrm{st}$ & 2nd & 3rd 4th & & & $\begin{array}{c}90 \\
\text { percent }\end{array}$ & $\begin{array}{c}75 \\
\text { percent }\end{array}$ & $\begin{array}{c}50 \\
\text { percent }\end{array}$ & $\begin{array}{c}25 \\
\text { percent }\end{array}$ & $\begin{array}{c}10 \\
\text { percent }\end{array}$ \\
\hline 1608 & 1026000927 & EL & RO & & Wild Horse Creek & 39.3 & 0 & 0 & 0.01 & 0.02 & 0.05 \\
\hline 1631 & 1026000912 & EL & RO & TR & Saline River & 963 & 1.11 & 3.13 & 9.92 & 27.5 & 59.6 \\
\hline 1643 & 1026000928 & EL & RO & & Tomcat Creek & 32.0 & 0 & 0 & .01 & .02 & .03 \\
\hline 1648 & 1026000912 & EL & & & Saline River & 1,010 & 1.30 & 3.72 & 11.3 & 30.5 & 66.5 \\
\hline 1654 & 1026000910 & EL & RO & & East Spring Creek & 54.2 & 0 & .01 & .02 & .05 & .84 \\
\hline 1678 & 1026000912 & EL & & & Saline River & 1,040 & 1.45 & 4.16 & 12.3 & 32.7 & 71.4 \\
\hline 1681 & 102600099 & EL & & & Saline River & 1,260 & 2.68 & 7.41 & 20.0 & 49.2 & 109 \\
\hline 1690 & 1026000911 & EL & & & Saline River & 1,150 & 1.94 & 5.77 & 16.1 & 40.8 & 90.0 \\
\hline 1730 & 102600099 & EL & & & Saline River & 1,300 & 3.05 & 8.22 & 22.0 & 53.4 & 119 \\
\hline 1754 & 1026000919 & EL & TR & & Trego Creek & 75.9 & .01 & .02 & .04 & .36 & 1.94 \\
\hline 1802 & HYDRO & EL & & & HYDRO & 16.5 & NA & NA & NA & NA & NA \\
\hline 1806 & 1026000929 & EL & & & Sweetwater Creek & 36.3 & 0 & 0 & .01 & .16 & 1.08 \\
\hline 1824 & 1026000929 & EL & & & Sweetwater Creek & 16.5 & 0 & 0 & 0 & 0 & .01 \\
\hline 1869 & 102600099 & EL & RS & & Saline River & 1,400 & 3.84 & 9.90 & 25.9 & 62.0 & 139 \\
\hline 1952 & 102600074 & EL & & & North Fork Big Creek & 50.9 & 0 & 0 & 0 & 0 & 0 \\
\hline 1953 & HYDRO & EL & & & HYDRO & 51.0 & NA & NA & NA & NA & NA \\
\hline 1965 & 102600075 & EL & & & Big Creek & 436 & .99 & 2.00 & 5.17 & 12.2 & 25.7 \\
\hline 1982 & HYDRO & EL & & & HYDRO & 436 & NA & NA & NA & NA & NA \\
\hline 1983 & 102600075 & EL & & & Big Creek & 432 & .97 & 1.97 & 5.11 & 12.1 & 25.4 \\
\hline 1984 & HYDRO & EL & & & HYDRO & 432 & NA & NA & NA & NA & NA \\
\hline 1993 & 102600075 & EL & $\mathrm{TR}$ & & Big Creek & 432 & .97 & 1.97 & 5.11 & 12.1 & 25.4 \\
\hline 1996 & 102600075 & EL & & & Big Creek & 460 & 1.13 & 2.24 & 5.59 & 13.3 & 27.7 \\
\hline 1998 & 1026000920 & EL & $\mathrm{RS}$ & & Salt Creek & 35.1 & 0 & 0 & .01 & .22 & 1.54 \\
\hline 2038 & 102600075 & EL & & & Big Creek & 489 & 1.31 & 2.53 & 6.13 & 14.7 & 30.2 \\
\hline 2095 & 102600074 & EL & & & North Fork Big Creek & 9.3 & 0 & 0 & 0 & 0 & .90 \\
\hline 2107 & 102600072 & EL & & & Walker Creek & 40.4 & 0 & 0 & 0 & 0 & .31 \\
\hline 2122 & 102600078 & EL & & & Chetolah Creek & 23.0 & 0 & 0 & .01 & .02 & .02 \\
\hline 2123 & 102600075 & EL & & & Big Creek & 521 & 1.50 & 2.83 & 6.70 & 16.1 & 32.7 \\
\hline 2204 & 102600079 & EL & & & Mud Creek & 31.6 & 0 & 0 & 0 & 0 & 0 \\
\hline 2205 & 102600074 & EL & & & North Fork Big Creek & 113 & 0 & 0 & .17 & .60 & 2.38 \\
\hline 2220 & 102600072 & EL & RS & & Walker Creek & 59.4 & 0 & 0 & 0 & .34 & 2.17 \\
\hline 2248 & 102600074 & EL & & & North Fork Big Creek & 149 & 0 & 0 & .56 & 1.65 & 4.99 \\
\hline 2256 & 102600073 & EL & RS & & Big Creek & 788 & 1.90 & 4.67 & 10.4 & 25.3 & 53.9 \\
\hline 2286 & 102600075 & EL & & & Big Creek & 589 & 1.90 & 3.50 & 7.90 & 19.0 & 38.0 \\
\hline 2326 & 102600075 & EL & & & Big Creek & 620 & 1.90 & 3.69 & 8.29 & 20.0 & 40.6 \\
\hline 2332 & 1026000620 & EL & & & Unnamed tributary, Ellis 1 & 74.1 & 0 & 0 & 0 & 0 & 0.41 \\
\hline 2356 & 1026000622 & EL & TR & & Smoky Hill River & 5,670 & .24 & 1.77 & 7.32 & 12.1 & 21.3 \\
\hline 2360 & 1026000622 & EL & & & Smoky Hill River & 5,670 & .24 & 1.77 & 7.32 & 12.1 & 21.3 \\
\hline
\end{tabular}


Table 32. Estimated flow-duration values, mean flow values, and peak-discharge frequency values for controlled and uncontrolled flow stream segments on the 1999 Kansas Surface Water Register for Ellis County._Continued

[KSWR, Kansas Surface Water Register; CUSEGA, catalog unit segment number alpha; $\mathrm{mi}^{2}$, square miles; $\mathrm{ft}^{3} / \mathrm{s}$, cubic feet per second; HYDRO, lake or other hydrologic structure; NA, not applicable; NRDitch, irrigation ditch; NRTribal, tribal stream]

\begin{tabular}{|c|c|c|c|c|c|c|c|}
\hline \multirow{2}{*}{$\begin{array}{l}\text { Determi- } \\
\text { nation site } \\
\text { identi- } \\
\text { fication } \\
\text { number } \\
\text { (fig. } 36 \text { ) }\end{array}$} & \multirow{2}{*}{$\begin{array}{c}\text { Estimated mean } \\
\text { flow } \\
\left(\mathrm{ft}^{3} / \mathrm{s}\right)\end{array}$} & \multicolumn{6}{|c|}{ Estimated peak discharge $\left(\mathrm{ft}^{3} / \mathrm{s}\right)$ for indicated peak-discharge frequency } \\
\hline & & 2-year & 5-year & 10-year & 25-year & 50-year & 100-year \\
\hline 1608 & 1.13 & 405 & 1,220 & 2,090 & 3,580 & 5,010 & 6,670 \\
\hline 1648 & 44.7 & 2,490 & 7,510 & 13,100 & 23,200 & 33,400 & 46,000 \\
\hline 1654 & 2.72 & 629 & 1,770 & 2,950 & 4,940 & 6,790 & 8,940 \\
\hline 1678 & 47.4 & 2,480 & 7,450 & 12,900 & 23,000 & 33,100 & 45,700 \\
\hline 1681 & 68.2 & 2,360 & 7,030 & 12,200 & 21,800 & 31,400 & 43,600 \\
\hline 1802 & NA & NA & NA & NA & NA & NA & NA \\
\hline 1806 & 2.53 & 828 & 2,130 & 3,400 & 5,440 & 7,290 & 9,360 \\
\hline 1824 & .23 & 456 & 1,330 & 2,230 & 3,730 & 5,090 & 6,700 \\
\hline 1869 & 84.5 & 2,290 & 6,760 & 11,700 & 21,000 & 30,300 & 42,200 \\
\hline 1952 & 1.52 & 401 & 1,330 & 2,430 & 4,480 & 6,560 & 9,140 \\
\hline 1953 & NA & NA & NA & NA & $\mathrm{NA}$ & NA & NA \\
\hline 1965 & 26.8 & 1,340 & 3,930 & 6,780 & 12,000 & 17,200 & 23,700 \\
\hline 1982 & NA & NA & NA & NA & NA & NA & NA \\
\hline 1983 & 26.7 & 1,340 & 3,940 & 6,820 & 12,100 & 17,300 & 23,900 \\
\hline 2107 & 2.37 & 486 & 1,420 & 2,410 & 4,090 & 5,680 & 7,530 \\
\hline 2122 & .02 & 487 & 1,480 & 2,510 & 4,270 & 5,880 & 7,800 \\
\hline 2123 & 30.5 & 1,310 & 3,540 & 5,850 & 9,810 & 13,600 & 18,200 \\
\hline 2204 & 1.00 & 417 & 1,240 & 2,120 & 3,630 & 5,070 & 6,740 \\
\hline 2205 & 4.47 & 326 & 1,430 & 2,950 & 6,100 & 9,520 & 14,000 \\
\hline 2220 & 4.07 & 543 & 1,600 & 2,720 & 4,660 & 6,490 & 8,660 \\
\hline 2248 & 6.77 & 482 & 1,840 & 3,610 & 7,190 & 11,000 & 16,000 \\
\hline 2256 & 45.0 & 1,610 & 4,060 & 6,430 & 10,200 & 13,700 & 17,700 \\
\hline 2286 & 33.3 & 1,280 & 3,210 & 5,050 & 7,990 & 10,600 & 13,600 \\
\hline 2326 & 35.2 & 1,320 & 3,320 & 5,240 & 8,300 & 11,000 & 14,200 \\
\hline 2332 & 2.82 & 625 & 1,850 & 3,160 & 5,430 & 7,600 & 10,100 \\
\hline 2356 & 18.3 & 982 & 4,650 & 9,080 & 16,700 & 23,700 & 31,200 \\
\hline 2360 & 18.3 & 982 & 4,650 & 9,080 & 16,700 & 23,700 & 31,200 \\
\hline
\end{tabular}


Table 32. Estimated flow-duration values, mean flow values, and peak-discharge frequency values for controlled and uncontrolled flow stream segments on the 1999 Kansas Surface Water Register for Ellis County.

[KSWR, Kansas Surface Water Register; CUSEGA, catalog unit segment number alpha; $\mathrm{mi}^{2}$, square miles; $\mathrm{ft}^{3} / \mathrm{s}$, cubic feet per second; HYDRO, lake or other hydrologic structure; NA, not applicable; NRDitch, irrigation ditch; NRTribal, tribal stream]

\begin{tabular}{|c|c|c|c|c|c|c|c|c|c|c|c|c|}
\hline $\begin{array}{l}\text { Determi- } \\
\text { nation site } \\
\text { identi- } \\
\text { fication } \\
\text { number } \\
\text { (fig. } 36 \text { ) }\end{array}$ & $\begin{array}{l}\text { KSWR } \\
\text { CUSEGA } \\
\text { number }\end{array}$ & \multicolumn{4}{|c|}{$\begin{array}{l}\text { Stream segment } \\
\text { by county } \\
\text { (table 112) }\end{array}$} & Stream name & $\begin{array}{l}\text { Contri- } \\
\text { buting } \\
\text { drainage } \\
\text { area } \\
\left(\mathrm{mi}^{2}\right)\end{array}$ & \multicolumn{5}{|c|}{$\begin{array}{c}\text { Estimated flow-duration values }\left(\mathrm{ft}^{3} / \mathrm{s}\right) \text { for } \\
\text { indicated percentage of time flow equaled or } \\
\text { exceeded }\end{array}$} \\
\hline 2375 & 1026000620 & $\overline{E L}$ & & & & Unnamed tributary, Ellis 1 & 82.4 & 0 & 0 & 0 & 0 & $\overline{0.93}$ \\
\hline 2397 & 1026000621 & EL & & & & Smoky Hill River & 5,730 & .37 & 2.70 & 11.0 & 18.0 & 29.0 \\
\hline 2404 & 1026000617 & EL & & & & Smoky Hill River & 6,150 & 4.06 & 5.58 & 10.2 & 27.2 & 70.9 \\
\hline 2410 & 1026000618 & EL & & & & Smoky Hill River & 6,040 & 2.69 & 3.70 & 7.39 & 21.8 & 55.5 \\
\hline 2418 & 1026000618 & EL & & & & Smoky Hill River & 6,110 & 3.59 & 4.94 & 9.25 & 25.4 & 65.6 \\
\hline 2432 & 1026000618 & EL & & & & Smoky Hill River & 6,050 & 2.75 & 3.79 & 7.52 & 22.0 & 56.2 \\
\hline 2438 & 1026000623 & EL & $\mathrm{RH}$ & & & Unnamed tributary, Ellis 2 & 24.2 & 0 & 0 & 0 & 0 & 0 \\
\hline 2448 & 1026000626 & EL & NS & RH & TR & Timber Creek & 39.9 & 0 & 0 & 0 & 0 & 0 \\
\hline 2501 & 1026000629 & EL & RH & & & Buck Creek & 36.5 & 0 & 0 & 0 & 0 & 0 \\
\hline 2523 & 1026000624 & EL & $\mathrm{RH}$ & & & Big Timber Creek & 216 & 0 & 0 & .61 & 2.33 & 7.36 \\
\hline 2580 & 1026000630 & EL & RH & & & Eagle Creek & 53.8 & 0 & 0 & 0 & 0 & 1.26 \\
\hline 2589 & 1026000643 & EL & RH & & & Shelter Creek & 40.5 & 0 & 0 & 0 & 0 & 0 \\
\hline
\end{tabular}


Table 32. Estimated flow-duration values, mean flow values, and peak-discharge frequency values for controlled and uncontrolled flow stream segments on the 1999 Kansas Surface Water Register for Ellis County.-Continued

[KSWR, Kansas Surface Water Register; CUSEGA, catalog unit segment number alpha; $\mathrm{mi}^{2}$, square miles; $\mathrm{ft}^{3} / \mathrm{s}$, cubic feet per second; HYDRO, lake or other hydrologic structure; NA, not applicable; NRDitch, irrigation ditch; NRTribal, tribal stream]

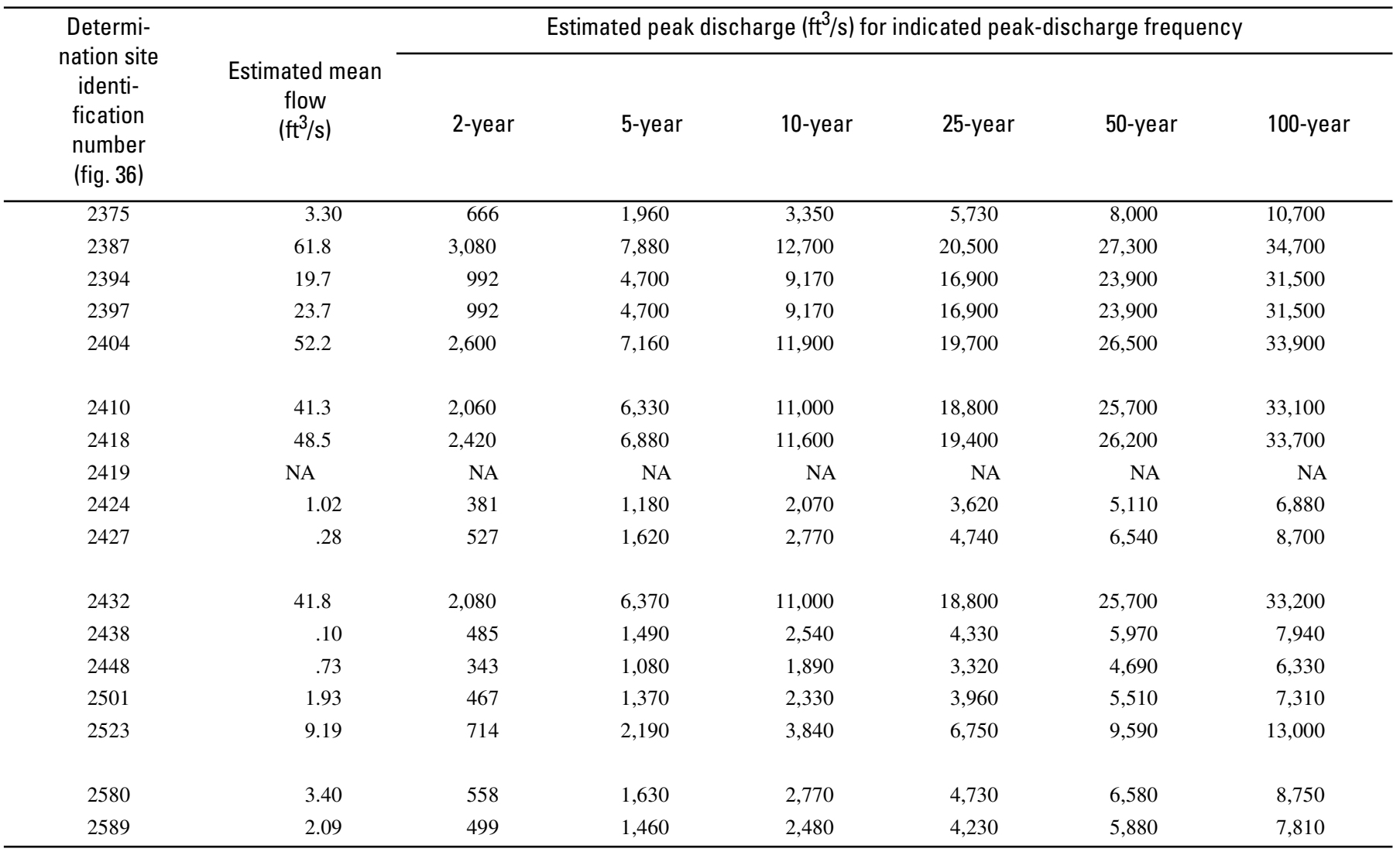




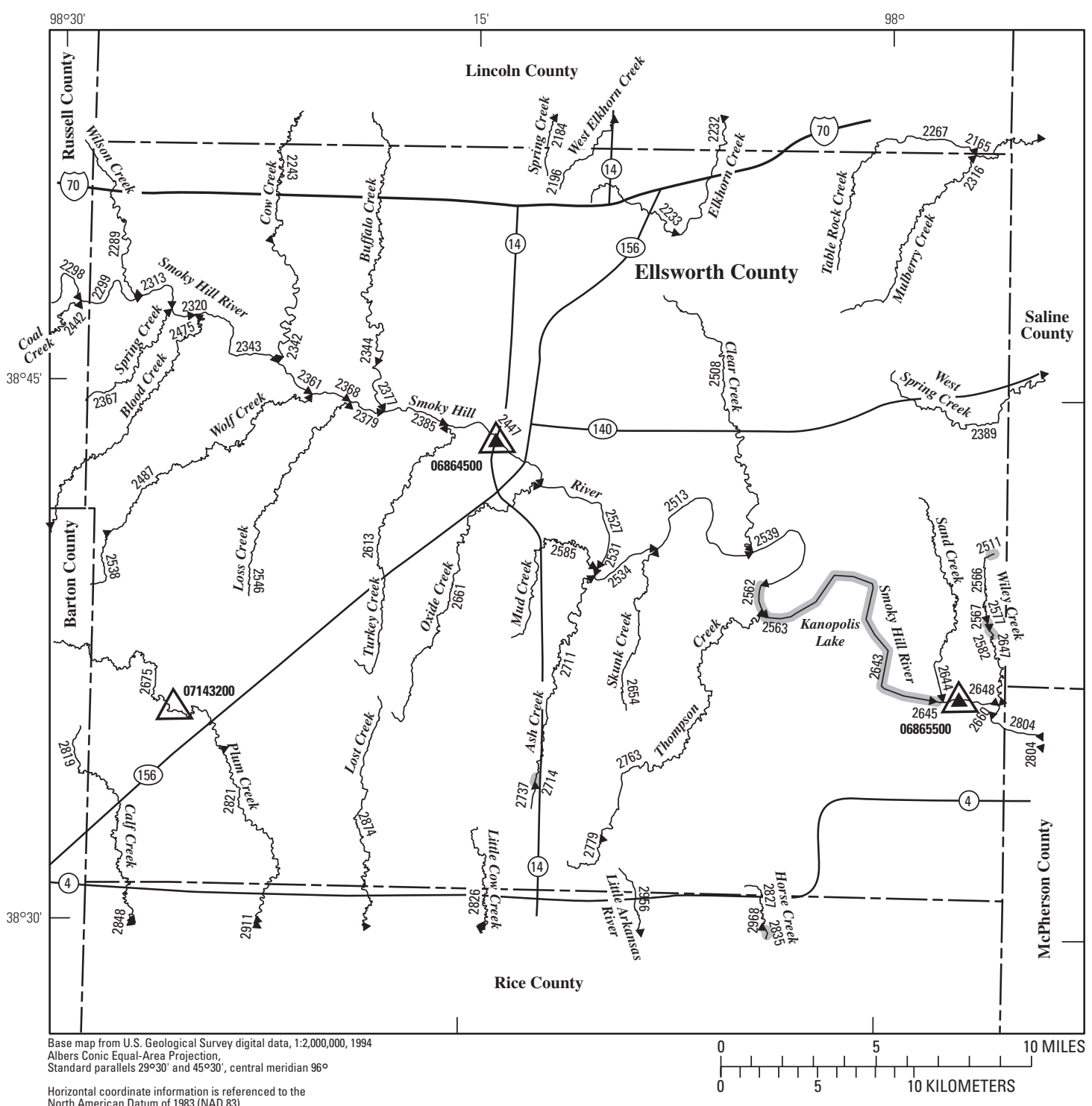

EXPLANATION

2911 Location of streamflow-statistics determination site (small triangle) and associated identification number-small triangle points in downstream direction

06865500 U.S. Geological Survey streamflow-gaging station and number used for estimates of flow duration

${ }^{06864500} \triangle$ U.S. Geological Survey streamflow-gaging station and number used for estimates of peak-discharge frequency values

KANSAS

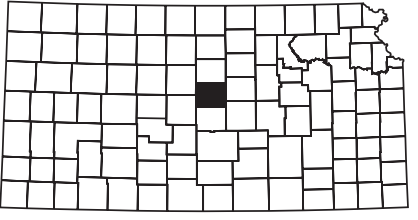

Index map

2643 Lake and determination site identification number

Figure 37. Location of streamflow-statistics determination sites, associated identification numbers, and U.S. Geological Survey streamflow-gaging stations used in the flow-duration and peak-discharge frequency analyses for Ellsworth County. 
Table 33. Estimated flow-duration values, mean flow values, and peak-discharge frequency values for controlled and uncontrolled flow stream segments on the 1999 Kansas Surface Water Register for Ellsworth County.

[KSWR, Kansas Surface Water Register; CUSEGA, catalog unit segment number alpha; $\mathrm{mi}^{2}$, square miles; $\mathrm{ft}^{3} / \mathrm{s}$, cubic feet per second; HYDRO, lake or other hydrologic structure; NA, not applicable; NRDitch, irrigation ditch; NRTribal, tribal stream]

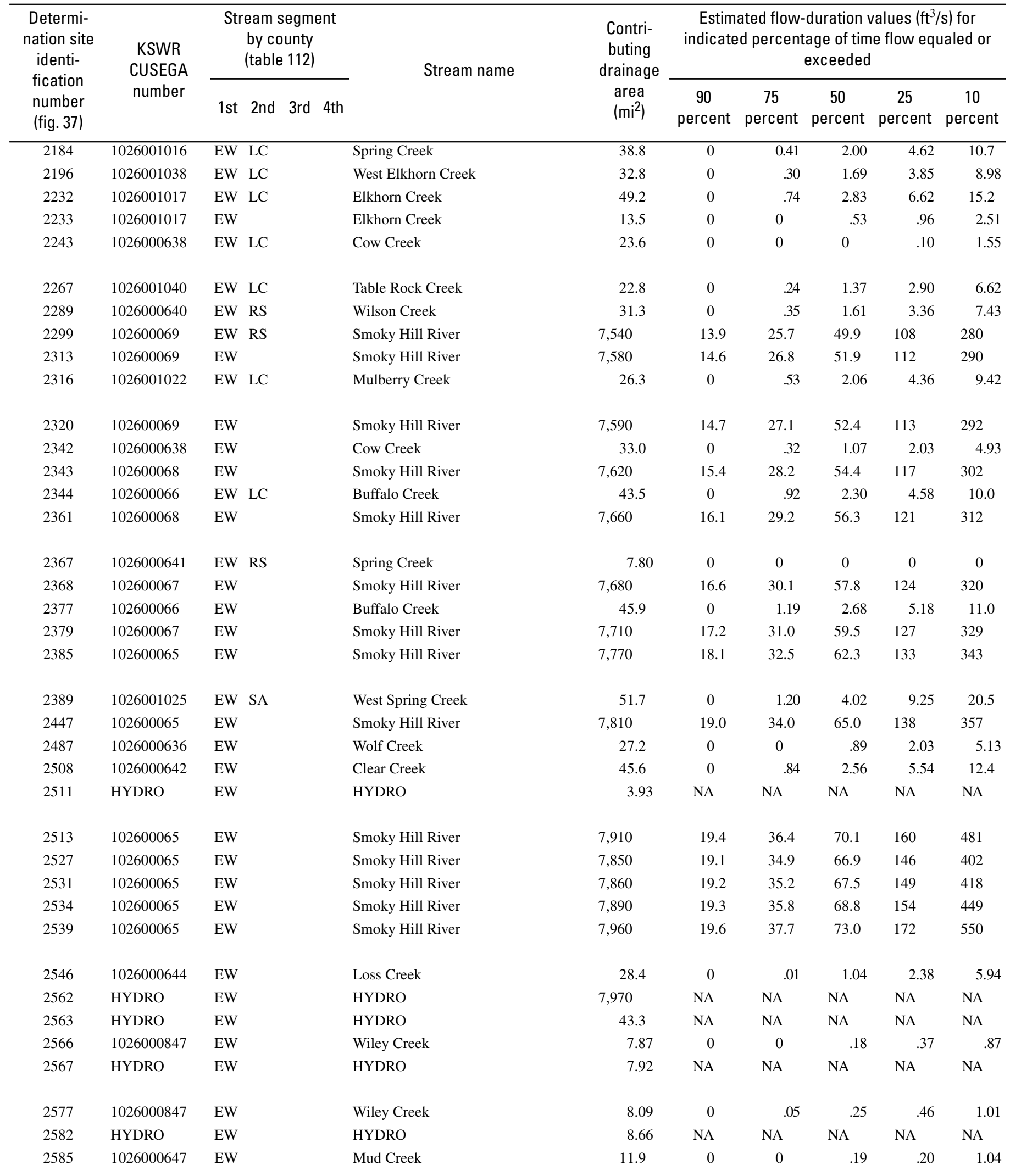


Table 33. Estimated flow-duration values, mean flow values, and peak-discharge frequency values for controlled and uncontrolled flow stream segments on the 1999 Kansas Surface Water Register for Ellsworth County.-Continued

[KSWR, Kansas Surface Water Register; CUSEGA, catalog unit segment number alpha; $\mathrm{mi}^{2}$, square miles; $\mathrm{ft}^{3} / \mathrm{s}$, cubic feet per second; HYDRO, lake or other hydrologic structure; NA, not applicable; NRDitch, irrigation ditch; NRTribal, tribal stream]

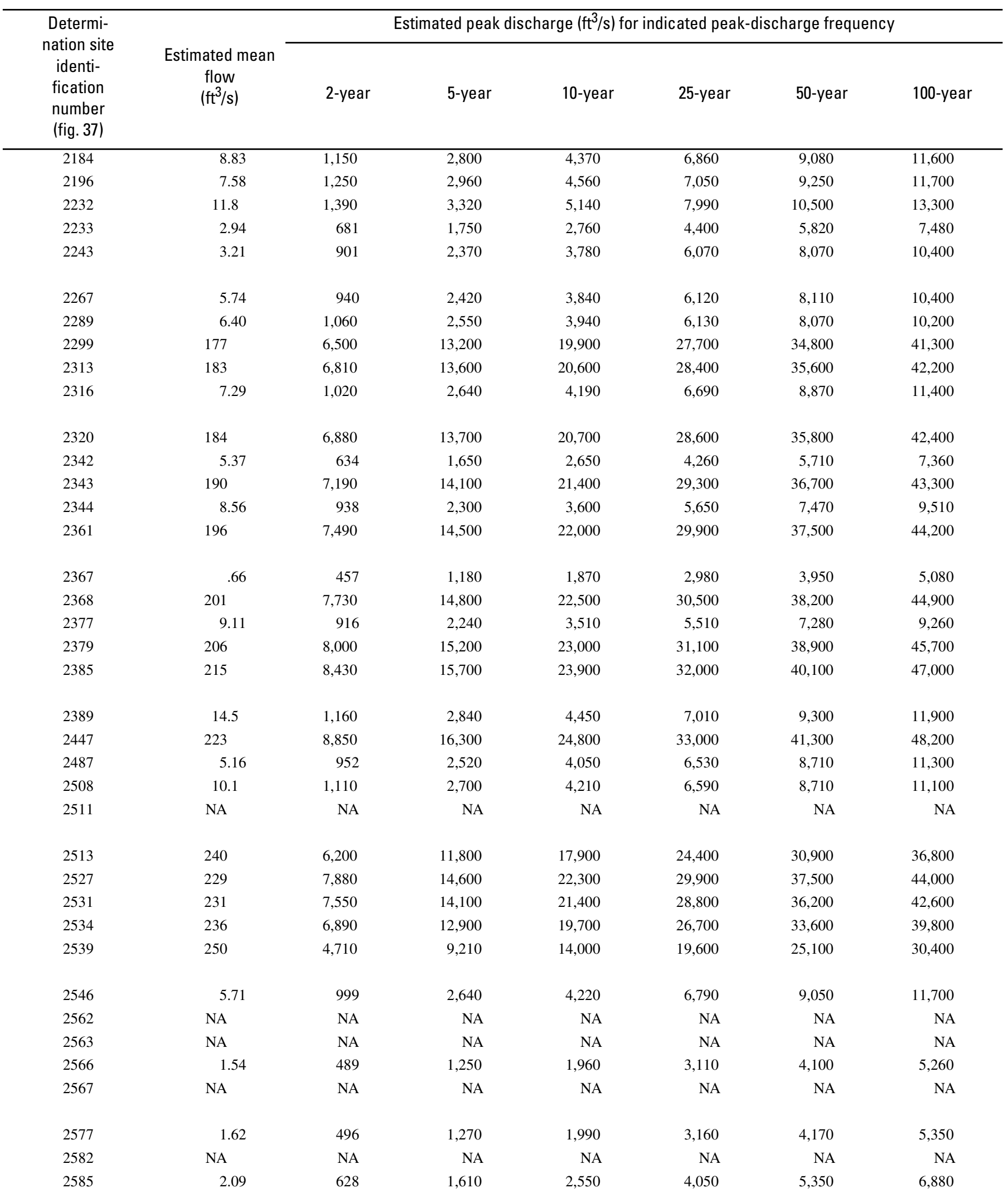


Table 33. Estimated flow-duration values, mean flow values, and peak-discharge frequency values for controlled and uncontrolled flow stream segments on the 1999 Kansas Surface Water Register for Ellsworth County.-Continued

[KSWR, Kansas Surface Water Register; CUSEGA, catalog unit segment number alpha; $\mathrm{mi}^{2}$, square miles; $\mathrm{ft}^{3} / \mathrm{s}$, cubic feet per second; HYDRO, lake or other hydrologic structure; NA, not applicable; NRDitch, irrigation ditch; NRTribal, tribal stream]

\begin{tabular}{|c|c|c|c|c|c|c|c|c|c|c|c|c|}
\hline \multirow{2}{*}{$\begin{array}{l}\text { Determi- } \\
\text { nation site } \\
\text { identi- } \\
\text { fication } \\
\text { number } \\
\text { (fig. } 37 \text { ) }\end{array}$} & \multirow{2}{*}{$\begin{array}{l}\text { KSWR } \\
\text { CUSEGA } \\
\text { number }\end{array}$} & \multicolumn{4}{|c|}{$\begin{array}{l}\text { Stream segment } \\
\text { by county } \\
\text { (table 112) }\end{array}$} & \multirow{2}{*}{ Stream name } & \multirow{2}{*}{$\begin{array}{l}\text { Contri- } \\
\text { buting } \\
\text { drainage } \\
\text { area } \\
\left(\mathrm{mi}^{2}\right)\end{array}$} & \multicolumn{5}{|c|}{$\begin{array}{c}\text { Estimated flow-duration values }\left(\mathrm{ft}^{3} / \mathrm{s}\right) \text { for } \\
\text { indicated percentage of time flow equaled or } \\
\text { exceeded }\end{array}$} \\
\hline & & $1 s t$ & 2nd & $3 r d$ & 4th & & & $\begin{array}{c}90 \\
\text { percent }\end{array}$ & $\begin{array}{c}75 \\
\text { percent }\end{array}$ & $\begin{array}{c}50 \\
\text { percent }\end{array}$ & $\begin{array}{c}25 \\
\text { percent }\end{array}$ & $\begin{array}{c}10 \\
\text { percent }\end{array}$ \\
\hline 2613 & 1026000646 & EW & & & & Turkey Creek & 28.8 & 0 & 0.09 & 1.16 & 2.61 & 6.37 \\
\hline 2643 & HYDRO & EW & & & & HYDRO & 8,040 & NA & NA & NA & NA & NA \\
\hline 2644 & 1026000846 & EW & & & & Sand Creek & 19.0 & 0 & 1.54 & 2.66 & 3.96 & 6.58 \\
\hline 2645 & 1026000815 & EW & & & & Smoky Hill River & 8,040 & 19.9 & 39.5 & 76.9 & 189 & 646 \\
\hline 2647 & 1026000847 & EW & & & & Wiley Creek & 13.3 & 0 & .34 & 1.07 & 1.63 & 3.14 \\
\hline 2648 & 1026000815 & EW & & & & Smoky Hill River & 8,060 & 20.0 & 40.0 & 78.0 & 194 & 672 \\
\hline 2654 & 1026000648 & EW & & & & Skunk Creek & 13.0 & 0 & 0 & .19 & .24 & 1.17 \\
\hline 2660 & 1026000815 & EW & & & & Smoky Hill River & 8,070 & 20.6 & 40.9 & 79.6 & 197 & 679 \\
\hline 2661 & 1026000645 & EW & & & & Oxide Creek & 25.2 & 0 & .15 & 1.24 & 2.67 & 6.26 \\
\hline 2711 & 102600061190 & EW & & & & Ash Creek & 22.3 & 0 & .01 & .92 & 1.96 & 4.81 \\
\hline 2714 & HYDRO & EW & & & & HYDRO & 4.48 & NA & NA & NA & NA & NA \\
\hline 2737 & 102600061190 & EW & & & & Ash Creek & 4.30 & 0 & 0 & 0 & 0 & 0 \\
\hline 2763 & 1026000637 & EW & & & & Thompson Creek & 43.3 & 0 & .37 & 1.86 & 4.37 & 10.4 \\
\hline 2779 & 1026000637 & EW & & & & Thompson Creek & 7.24 & 0 & 0 & 0 & 0 & 0 \\
\hline 2804 & 1026000815 & EW & MP & & & Smoky Hill River & 8,140 & 23.7 & 45.6 & 87.9 & 215 & 720 \\
\hline 2821 & 110300114 & EW & $\mathrm{RC}$ & & & Plum Creek & 53.3 & 0 & 0 & .48 & 1.56 & 5.59 \\
\hline 2826 & 110300112 & EW & $\mathrm{RC}$ & & & Little Cow Creek & 15.6 & 0 & 0 & 0 & 0 & .02 \\
\hline 2827 & 1103001219 & EW & $\mathrm{RC}$ & & & Horse Creek & 22.8 & 0 & 0 & .37 & .80 & 2.59 \\
\hline 2874 & 1103001117 & EW & $\mathrm{RC}$ & & & Lost Creek & 32.6 & 0 & 0 & 0 & 0 & 1.54 \\
\hline 2956 & 1103001214 & EW & $\mathrm{RC}$ & & & Little Arkansas River & 40.0 & 0 & 0 & .40 & 1.21 & 4.32 \\
\hline
\end{tabular}


Table 33. Estimated flow-duration values, mean flow values, and peak-discharge frequency values for controlled and uncontrolled flow stream segments on the 1999 Kansas Surface Water Register for Ellsworth County-Continued.

[KSWR, Kansas Surface Water Register; CUSEGA, catalog unit segment number alpha; $\mathrm{mi}^{2}$, square miles; $\mathrm{ft}^{3} / \mathrm{s}$, cubic feet per second; HYDRO, lake or other hydrologic structure; NA, not applicable; NRDitch, irrigation ditch; NRTribal, tribal stream]

\begin{tabular}{|c|c|c|c|c|c|c|c|}
\hline \multirow{2}{*}{$\begin{array}{l}\text { Determi- } \\
\text { nation site } \\
\text { identi- } \\
\text { fication } \\
\text { number } \\
\text { (fig. } 37 \text { ) }\end{array}$} & \multirow{2}{*}{$\begin{array}{c}\text { Estimated mean } \\
\text { flow } \\
\left(\mathrm{ft}^{3} / \mathrm{s}\right)\end{array}$} & \multicolumn{6}{|c|}{ Estimated peak discharge $\left(\mathrm{ft}^{3} / \mathrm{s}\right)$ for indicated peak-discharge frequency } \\
\hline & & 2-year & 5 -year & 10-year & 25-year & 50-year & 100-year \\
\hline 2613 & 5.99 & 1,030 & 2,700 & 4,310 & 6,930 & 9,230 & 11,900 \\
\hline 2645 & 264 & 2,670 & 5,710 & 8,670 & 13,000 & 17,100 & 21,600 \\
\hline 2647 & 3.00 & 644 & 1,670 & 2,660 & 4,240 & 5,630 & 7,240 \\
\hline 2648 & 267 & 2,120 & 4,760 & 7,230 & 11,200 & 14,900 & 19,200 \\
\hline 2654 & 2.24 & 655 & 1,690 & 2,670 & 4,250 & 5,630 & 7,240 \\
\hline 2714 & NA & NA & NA & NA & NA & NA & NA \\
\hline 2737 & 0 & 343 & 865 & 1,350 & 2,130 & 2,800 & 3,580 \\
\hline 2763 & 8.96 & 1,160 & 2,850 & 4,450 & 6,990 & 9,270 & 11,800 \\
\hline 2779 & .25 & 464 & 1,180 & 1,860 & 2,940 & 3,880 & 4,980 \\
\hline 2804 & 287 & 2,510 & 5,300 & 7,500 & 11,900 & 16,100 & 21,400 \\
\hline 2821 & 6.73 & 811 & 1,640 & 2,370 & 3,510 & 4,520 & 5,670 \\
\hline 2826 & 1.39 & 719 & 1,870 & 2,960 & 4,730 & 6,270 & 8,080 \\
\hline 2827 & 3.60 & 879 & 2,270 & 3,590 & 5,740 & 7,610 & 9,800 \\
\hline 2874 & 3.62 & 632 & 1,730 & 2,850 & 4,700 & 6,420 & 8,400 \\
\hline
\end{tabular}




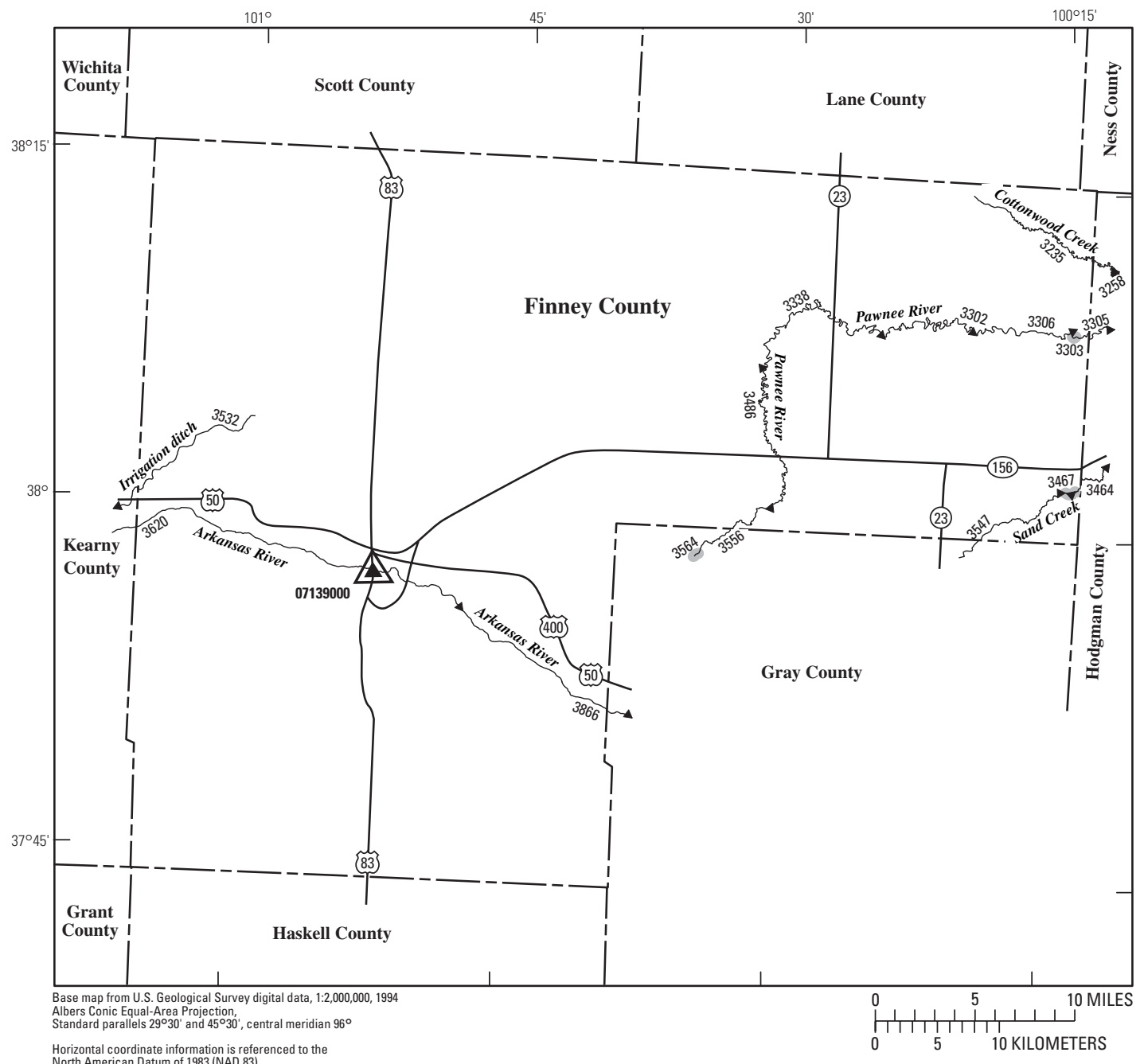

EXPLANATION

\$620 Location of streamflow-statistics determination site (small triangle) and associated identification number-small triangle points in downstream direction

07139000

U.S. Geological Survey streamflow-gaging station and number used for estimates of flow duration

07139000 U.S. Geological Survey streamflow-gaging station and number used for estimates of peak-discharge frequency values

KANSAS

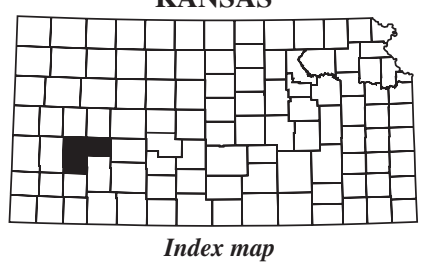

Index map 3564

Lake and determination site identification number

Figure 38. Location of streamflow-statistics determination sites, associated identification numbers, and U.S. Geological Survey streamflow-gaging stations used in the flow-duration and peak-discharge frequency analyses for Finney County. 
Table 34. Estimated flow-duration values, mean flow values, and peak-discharge frequency values for controlled and uncontrolled flow stream segments on the 1999 Kansas Surface Water Register for Finney County.

[KSWR, Kansas Surface Water Register; CUSEGA, catalog unit segment number alpha; $\mathrm{mi}^{2}$, square miles; $\mathrm{ft}^{3} / \mathrm{s}$, cubic feet per second; HYDRO, lake or other hydrologic structure; NA, not applicable; NRDitch, irrigation ditch; NRTribal, tribal stream]

\begin{tabular}{|c|c|c|c|c|c|c|c|c|c|c|c|}
\hline \multirow{2}{*}{$\begin{array}{l}\text { Determi- } \\
\text { nation site } \\
\text { identi- } \\
\text { fication } \\
\text { number } \\
\text { (fig. 38) }\end{array}$} & \multirow{2}{*}{$\begin{array}{l}\text { KSWR } \\
\text { CUSEGA } \\
\text { number }\end{array}$} & \multicolumn{3}{|c|}{$\begin{array}{l}\text { Stream segment } \\
\text { by county } \\
\text { (table 112) }\end{array}$} & \multirow{2}{*}{ Stream name } & \multirow{2}{*}{$\begin{array}{l}\text { Contri- } \\
\text { buting } \\
\text { drainage } \\
\text { area } \\
\left(\mathrm{mi}^{2}\right)\end{array}$} & \multicolumn{5}{|c|}{$\begin{array}{l}\text { Estimated flow-duration values }\left(\mathrm{ft}^{3} / \mathrm{s}\right) \text { for } \\
\text { indicated percentage of time flow equaled or } \\
\text { exceeded }\end{array}$} \\
\hline & & $1 s t$ & 2nd & 3rd 4th & & & $\begin{array}{c}90 \\
\text { percent }\end{array}$ & $\begin{array}{c}75 \\
\text { percent }\end{array}$ & $\begin{array}{c}50 \\
\text { percent }\end{array}$ & $\begin{array}{c}25 \\
\text { percent }\end{array}$ & $\begin{array}{c}10 \\
\text { percent }\end{array}$ \\
\hline 3235 & 110300058 & FI & $\mathrm{HG}$ & & Cottonwood Creek & 56.0 & 0 & 0 & 0 & 0 & 0 \\
\hline 3302 & 110300055 & FI & & & Pawnee River & 401 & 0 & 0 & 0 & 0 & .76 \\
\hline 3303 & HYDRO & FI & & & HYDRO & 434 & NA & NA & NA & NA & NA \\
\hline 3305 & 110300055 & FI & HG & & Pawnee River & 447 & 0 & 0 & 0 & 0 & 1.46 \\
\hline 3306 & 110300055 & FI & & & Pawnee River & 433 & 0 & 0 & 0 & 0 & 1.25 \\
\hline 3338 & 110300055 & FI & & & Pawnee River & 356 & 0 & 0 & 0 & 0 & .01 \\
\hline 3464 & 110300059 & FI & $\mathrm{HG}$ & & Sand Creek & 85.0 & 0 & 0 & 0 & 0 & 0 \\
\hline 3467 & HYDRO & FI & & & HYDRO & 57.7 & NA & NA & NA & NA & NA \\
\hline 3486 & 110300055 & FI & & & Pawnee River & 203 & 0 & 0 & 0 & 0 & 0 \\
\hline 3532 & NRDitch & FI & $\mathrm{KE}$ & & NRDitch & 185 & 0 & 0 & 0 & 0 & 0 \\
\hline 3547 & 110300059 & FI & GY & & Sand Creek & 54.8 & 0 & 0 & 0 & 0 & 0 \\
\hline 3556 & 110300055 & FI & GY & & Pawnee River & 105 & 0 & 0 & 0 & 0 & 0 \\
\hline 3620 & 110300011 & FI & $\mathrm{KE}$ & & Arkansas River & 26,900 & 0 & 1.10 & 32.0 & 161 & 314 \\
\hline 3866 & 110300031 & FI & GY & & Arkansas River & 27,300 & 0 & .34 & 15.8 & 98.4 & 221 \\
\hline
\end{tabular}


Table 34. Estimated flow-duration values, mean flow values, and peak-discharge frequency values for controlled and uncontrolled flow stream segments on the 1999 Kansas Surface Water Register for Finney County.-Continued

[KSWR, Kansas Surface Water Register; CUSEGA, catalog unit segment number alpha; $\mathrm{mi}^{2}$, square miles; $\mathrm{ft}^{3} / \mathrm{s}$, cubic feet per second; HYDRO, lake or other hydrologic structure; NA, not applicable; NRDitch, irrigation ditch; NRTribal, tribal stream]

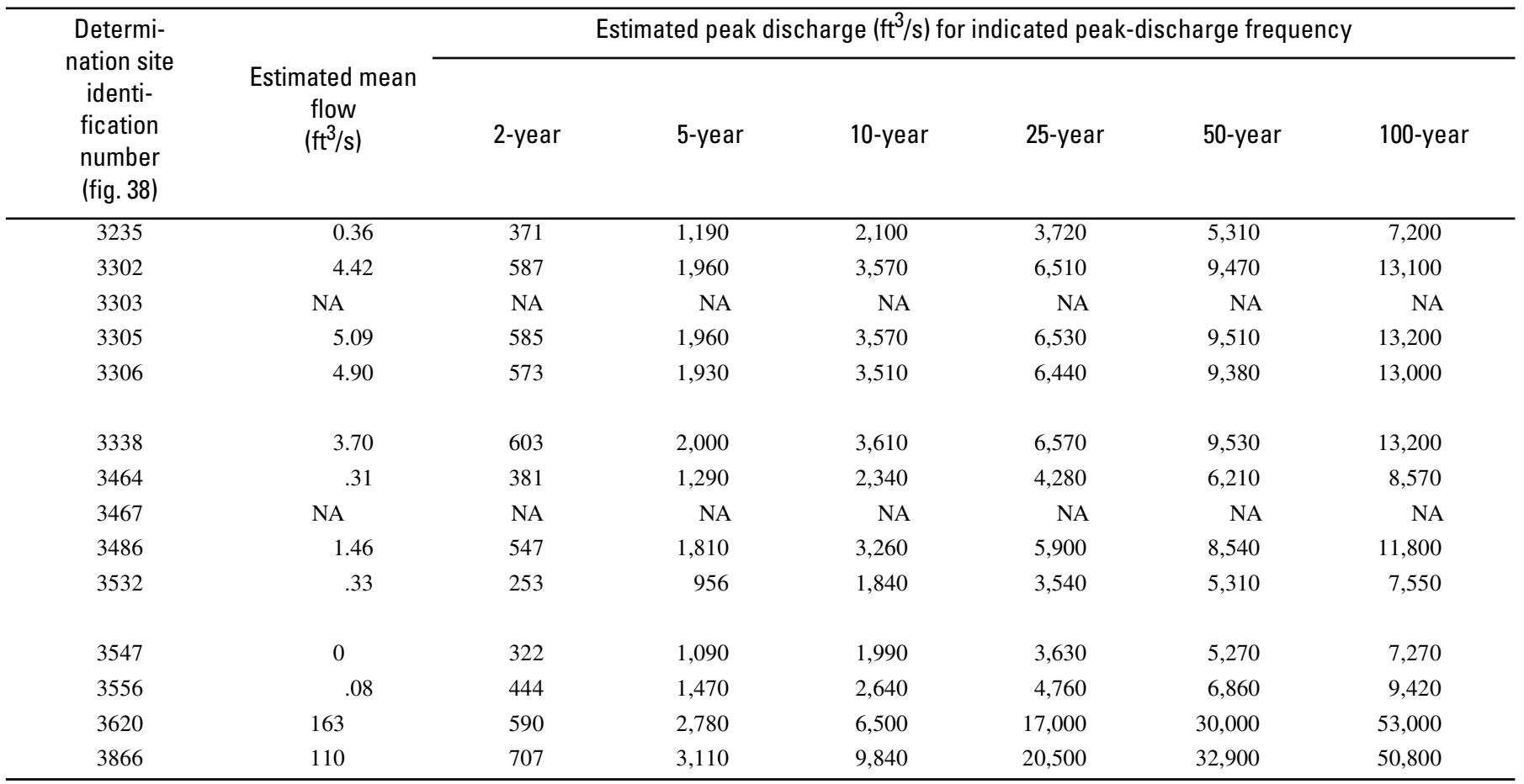




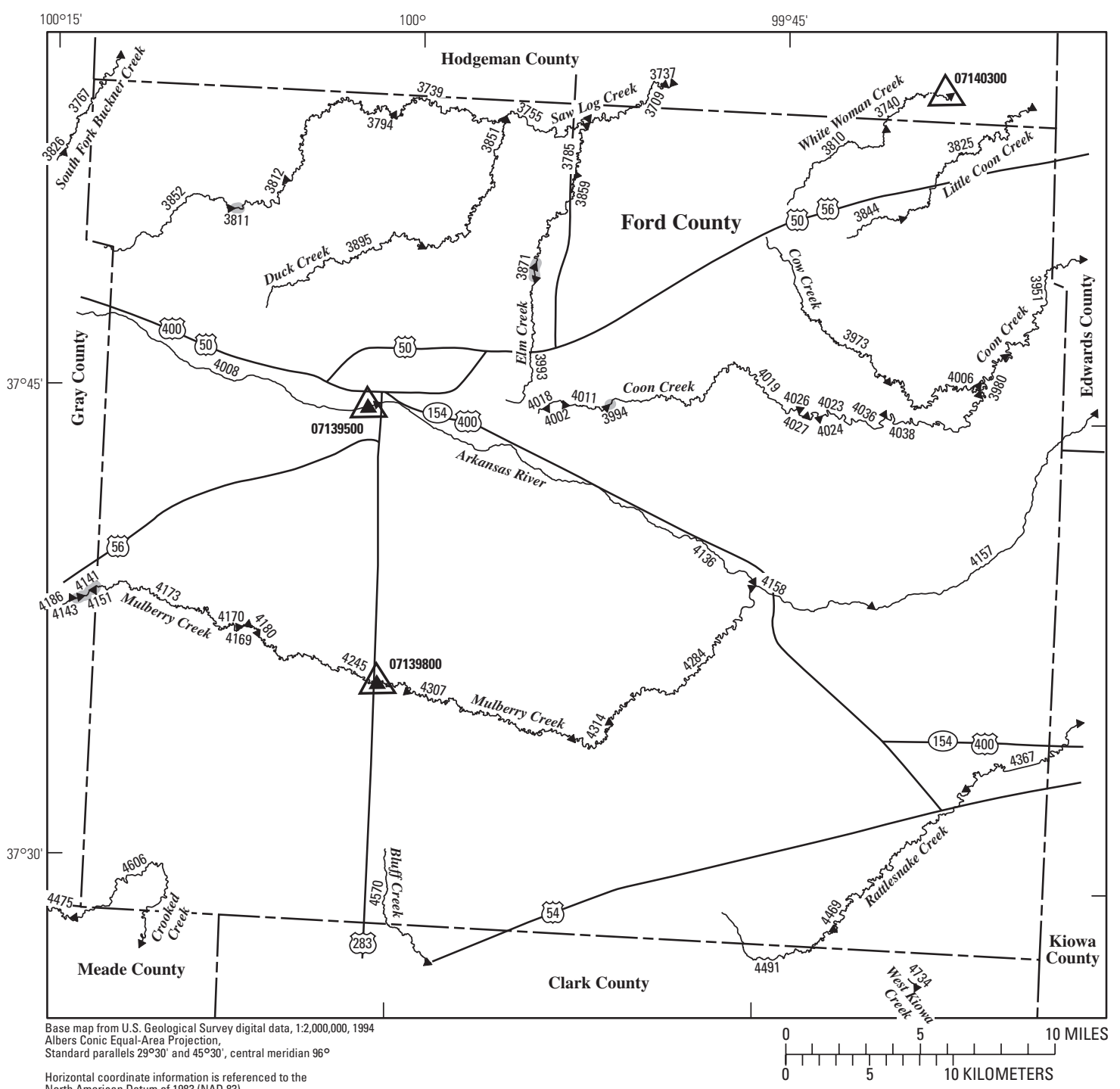

EXPLANATION

4606 Location of streamflow-statistics determination site (small triangle) and associated identification number-small triangle points in downstream direction

07139500

U.S. Geological Survey streamflow-gaging station and number used for estimates of flow duration

07139800 U.S. Geological Survey streamflow-gaging station and number used for estimates of peak-discharge frequency values

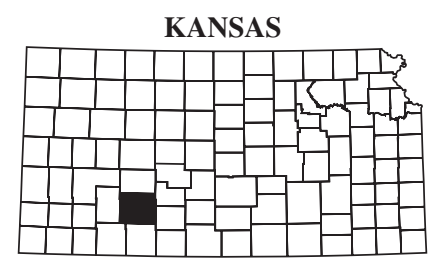

Index map

3994 Lake and determination site identification number

Figure 39. Location of streamflow-statistics determination sites, associated identification numbers, and U.S. Geological Survey streamflow-gaging stations used in the flow-duration and peak-discharge frequency analyses for Ford County. 
Table 35. Estimated flow-duration values, mean flow values, and peak-discharge frequency values for controlled and uncontrolled flow stream segments on the 1999 Kansas Surface Water Register for Ford County.

[KSWR, Kansas Surface Water Register; CUSEGA, catalog unit segment number alpha; $\mathrm{mi}^{2}$, square miles; $\mathrm{ft}^{3} / \mathrm{s}$, cubic feet per second; HYDRO, lake or other hydrologic structure; NA, not applicable; NRDitch, irrigation ditch; NRTribal, tribal stream]

\begin{tabular}{|c|c|c|c|c|c|c|c|c|c|c|c|}
\hline \multirow{2}{*}{$\begin{array}{c}\text { Determi- } \\
\text { nation site } \\
\text { identi- } \\
\text { fication } \\
\text { number } \\
\text { (fig. } 39 \text { ) }\end{array}$} & \multirow{2}{*}{$\begin{array}{l}\text { KSWR } \\
\text { CUSEGA } \\
\text { number }\end{array}$} & \multicolumn{3}{|c|}{$\begin{array}{l}\text { Stream segment } \\
\text { by county } \\
\text { (table 112) }\end{array}$} & \multirow{2}{*}{ Stream name } & \multirow{2}{*}{$\begin{array}{l}\text { Contri- } \\
\text { buting } \\
\text { drainage } \\
\text { area } \\
\left(\mathrm{mi}^{2}\right)\end{array}$} & \multicolumn{5}{|c|}{$\begin{array}{l}\text { Estimated flow-duration values }\left(\mathrm{ft}^{3} / \mathrm{s}\right) \text { for } \\
\text { indicated percentage of time flow equaled or } \\
\text { exceeded }\end{array}$} \\
\hline & & $1 \mathrm{st}$ & 2 nd & 3rd 4th & & & $\begin{array}{c}90 \\
\text { percent }\end{array}$ & $\begin{array}{c}75 \\
\text { percent }\end{array}$ & $\begin{array}{c}50 \\
\text { percent }\end{array}$ & $\begin{array}{c}25 \\
\text { percent }\end{array}$ & $\begin{array}{c}10 \\
\text { percent }\end{array}$ \\
\hline 3737 & 110300063 & FO & $\mathrm{HG}$ & & Saw Log Creek & 223 & 0 & 0 & 0.28 & 1.59 & 5.88 \\
\hline 3739 & 110300064 & FO & HG & & Saw Log Creek & 86.8 & 0 & 0 & 0 & 0 & 0 \\
\hline 3740 & 1103000415 & FO & HG & & White Woman Creek & 69.1 & 0 & 0 & 0 & 0 & 0 \\
\hline 3755 & 110300064 & $\mathrm{FO}$ & & & Saw Log Creek & 157 & 0 & 0 & 0 & .21 & 2.50 \\
\hline 3767 & 110300066 & $\mathrm{FO}$ & GY & HG & South Fork Buckner Creek & 55.5 & 0 & 0 & 0 & 0 & 0 \\
\hline 3785 & 110300065 & FO & & & Elm Creek & 49.4 & 0 & 0 & 0 & 0 & 0 \\
\hline 3794 & 110300064 & FO & & & Saw Log Creek & 67.7 & 0 & 0 & 0 & 0 & 0 \\
\hline 3810 & 1103000415 & FO & & & White Woman Creek & 26.3 & 0 & 0 & 0 & 0 & 0 \\
\hline 3811 & HYDRO & FO & & & HYDRO & 30.1 & NA & NA & NA & NA & NA \\
\hline 3812 & 110300064 & FO & & & Saw Log Creek & 37.7 & 0 & 0 & 0 & 0 & 0 \\
\hline 3825 & 110300048 & FO & HG & & Little Coon Creek & 38.3 & 0 & 0 & 0 & 0 & 0 \\
\hline 3844 & 110300048 & FO & & & Little Coon Creek & 13.8 & 0 & 0 & 0 & 0 & 0 \\
\hline 3851 & 110300068 & FO & & & Duck Creek & 54.5 & 0 & 0 & 0 & 0 & 0 \\
\hline 3852 & 110300064 & FO & & & Saw Log Creek & 29.9 & 0 & 0 & 0 & 0 & 0 \\
\hline 3859 & 110300065 & FO & & & Elm Creek & 45.6 & 0 & 0 & 0 & 0 & 0 \\
\hline 3871 & HYDRO & FO & & & HYDRO & 25.3 & NA & NA & NA & NA & NA \\
\hline 3895 & 110300068 & FO & & & Duck Creek & 35.8 & 0 & 0 & 0 & 0 & 0 \\
\hline 3973 & 1103000414 & FO & & & Cow Creek & 37.1 & 0 & 0 & 0 & 0 & 0 \\
\hline 3980 & 110300049 & FO & & & Coon Creek & 132 & 0 & 0 & .25 & .92 & 3.52 \\
\hline 3993 & 110300065 & FO & & & Elm Creek & 24.2 & 0 & 0 & 0 & 0 & 0 \\
\hline 3994 & HYDRO & FO & & & HYDRO & 8.20 & NA & NA & NA & NA & NA \\
\hline 4002 & 110300049 & FO & & & Coon Creek & 3.61 & 0 & 0 & 0 & 0 & 0 \\
\hline 4006 & 1103000414 & FO & & & Cow Creek & 45.1 & 0 & 0 & 0 & 0 & 0 \\
\hline 4008 & 110300031 & FO & GY & & Arkansas River & 27,400 & 0 & 0 & 8.70 & 71.0 & 180 \\
\hline 4011 & 110300049 & FO & & & Coon Creek & 8.20 & 0 & 0 & 0 & 0 & 0 \\
\hline 4018 & 110300049 & FO & & & Coon Creek & 1.67 & 0 & 0 & 0 & 0 & 0 \\
\hline 4019 & 110300049 & FO & & & Coon Creek & 51.1 & 0 & 0 & 0 & 0 & 0 \\
\hline 4023 & 110300049 & FO & & & Coon Creek & 56.5 & 0 & 0 & 0 & 0 & 0 \\
\hline 4024 & 110300049 & FO & & & Coon Creek & 56.6 & 0 & 0 & 0 & 0 & 0 \\
\hline 4026 & 110300049 & FO & & & Coon Creek & 56.5 & 0 & 0 & 0 & 0 & 0 \\
\hline 4027 & 110300049 & FO & & & Coon Creek & 53.9 & 0 & 0 & 0 & 0 & 0 \\
\hline 4036 & 110300049 & FO & & & Coon Creek & 68.8 & 0 & 0 & 0 & 0 & 0 \\
\hline 4038 & 110300049 & FO & & & Coon Creek & 82.9 & 0 & 0 & 0 & 0 & .91 \\
\hline 4136 & 1103000411 & FO & & & Arkansas River & 27,500 & .09 & .33 & 11.6 & 75.1 & 183 \\
\hline 4158 & 1103000410 & FO & & & Arkansas River & 27,900 & .45 & 1.65 & 23.5 & 91.9 & 194 \\
\hline 4169 & 1103000412 & FO & & & Mulberry Creek & 162 & 0 & 0 & 0 & 0 & 0 \\
\hline 4170 & 1103000412 & FO & & & Mulberry Creek & 154 & 0 & 0 & 0 & 0 & 0 \\
\hline 4173 & 1103000412 & FO & GY & & Mulberry Creek & 154 & 0 & 0 & 0 & 0 & 0 \\
\hline
\end{tabular}


Table 35. Estimated flow-duration values, mean flow values, and peak-discharge frequency values for controlled and uncontrolled flow stream segments on the 1999 Kansas Surface Water Register for Ford County.-Continued

[KSWR, Kansas Surface Water Register; CUSEGA, catalog unit segment number alpha; $\mathrm{mi}^{2}$, square miles; $\mathrm{ft}^{3} / \mathrm{s}$, cubic feet per second; HYDRO, lake or other hydrologic structure; NA, not applicable; NRDitch, irrigation ditch; NRTribal, tribal stream]

\begin{tabular}{|c|c|c|c|c|c|c|c|}
\hline \multirow{2}{*}{$\begin{array}{l}\text { Determi- } \\
\text { nation site } \\
\text { identi- } \\
\text { fication } \\
\text { number } \\
\text { (fig. 39) }\end{array}$} & \multirow{2}{*}{$\begin{array}{c}\text { Estimated mean } \\
\text { flow } \\
\left(\mathrm{ft}^{3} / \mathrm{s}\right)\end{array}$} & \multicolumn{6}{|c|}{ Estimated peak discharge $\left(\mathrm{ft}^{3} / \mathrm{s}\right)$ for indicated peak-discharge frequency } \\
\hline & & 2-year & 5-year & 10 -year & 25-year & 50-year & 100 -year \\
\hline 3737 & 8.29 & 831 & 2,470 & 4,270 & 7,410 & 10,400 & 14,100 \\
\hline 3755 & 5.25 & 673 & 2,060 & 3,590 & 6,290 & 8,910 & 12,100 \\
\hline 3767 & 0.63 & 433 & 1,410 & 2,510 & 4,500 & 6,460 & 8,820 \\
\hline 3785 & 1.18 & 379 & 1,180 & 2,060 & 3,600 & 5,090 & 6,850 \\
\hline 3794 & 1.37 & 434 & 1,390 & 2,470 & 4,390 & 6,280 & 8,560 \\
\hline 3825 & .97 & 381 & 1,180 & 2,060 & 3,590 & 5,060 & 6,820 \\
\hline 3844 & 0 & 408 & 1,190 & 1,990 & 3,330 & 4,530 & 5,960 \\
\hline 3851 & 1.15 & 365 & 1,170 & 2,060 & 3,650 & 5,210 & 7,070 \\
\hline 3852 & 0 & 545 & 1,680 & 2,870 & 4,930 & 6,800 & 9,060 \\
\hline 3859 & .94 & 373 & 1,160 & 2,030 & 3,540 & 5,000 & 6,730 \\
\hline 3871 & NA & NA & NA & NA & NA & NA & NA \\
\hline 3895 & .22 & 303 & 978 & 1,740 & 3,090 & 4,400 & 5,980 \\
\hline 3973 & .69 & 381 & 1,180 & 2,050 & 3,570 & 5,040 & 6,780 \\
\hline 3980 & 5.81 & 578 & 1,720 & 2,950 & 5,070 & 7,110 & 9,530 \\
\hline 4018 & 0 & 104 & 301 & 499 & 826 & 1,120 & 1,460 \\
\hline 4019 & 1.24 & 395 & 1,210 & 2,110 & 3,670 & 5,170 & 6,950 \\
\hline 4023 & 1.58 & 413 & 1,260 & 2,180 & 3,780 & 5,310 & 7,130 \\
\hline 4024 & 1.58 & 412 & 1,260 & 2,180 & 3,780 & 5,310 & 7,130 \\
\hline 4026 & 1.57 & 414 & 1,260 & 2,190 & 3,790 & 5,320 & 7,140 \\
\hline 4027 & 1.41 & 406 & 1,240 & 2,150 & 3,730 & 5,250 & 7,050 \\
\hline 4036 & 2.38 & 449 & 1,350 & 2,340 & 4,030 & 5,650 & 7,570 \\
\hline 4038 & 3.32 & 443 & 1,330 & 2,290 & 3,960 & 5,550 & 7,430 \\
\hline 4136 & 88.3 & 740 & 3,240 & 10,900 & 21,200 & 33,100 & 48,500 \\
\hline 4158 & 95.4 & 662 & 3,150 & 9,530 & 18,200 & 28,700 & 43,000 \\
\hline 4169 & 1.11 & 481 & 1,380 & 2,330 & 3,930 & 5,440 & 7,220 \\
\hline 4170 & 1.18 & 489 & 1,420 & 2,390 & 4,060 & 5,630 & 7,480 \\
\hline 4173 & 1.15 & 497 & 1,440 & 2,420 & 4,100 & 5,690 & 7,560 \\
\hline
\end{tabular}


Table 35. Estimated flow-duration values, mean flow values, and peak-discharge frequency values for controlled and uncontrolled flow stream segments on the 1999 Kansas Surface Water Register for Ford County.-Continued

[KSWR, Kansas Surface Water Register; CUSEGA, catalog unit segment number alpha; $\mathrm{mi}^{2}$, square miles; $\mathrm{ft}^{3} / \mathrm{s}$, cubic feet per second; HYDRO, lake or other hydrologic structure; NA, not applicable; NRDitch, irrigation ditch; NRTribal, tribal stream]

\begin{tabular}{|c|c|c|c|c|c|c|c|c|c|c|c|}
\hline $\begin{array}{l}\text { Determi- } \\
\text { nation site } \\
\text { identi- } \\
\text { fication } \\
\text { number } \\
\text { (fig. 39) }\end{array}$ & $\begin{array}{l}\text { KSWR } \\
\text { CUSEGA } \\
\text { number }\end{array}$ & \multicolumn{3}{|c|}{$\begin{array}{l}\text { Stream segment } \\
\text { by county } \\
\text { (table 112) }\end{array}$} & Stream name & $\begin{array}{l}\text { Contri- } \\
\text { buting } \\
\text { drainage } \\
\text { area } \\
\left(\mathrm{mi}^{2}\right)\end{array}$ & \multicolumn{5}{|c|}{$\begin{array}{l}\text { Estimated flow-duration values }\left(\mathrm{ft}^{3} / \mathrm{s}\right) \text { for } \\
\text { indicated percentage of time flow equaled or } \\
\text { exceeded }\end{array}$} \\
\hline 4180 & 1103000412 & FO & & & Mulberry Creek & 164 & 0 & 0 & 0 & 0 & 0 \\
\hline 4307 & 1103000412 & FO & & & Mulberry Creek & 263 & 0 & 0 & .54 & 1.33 & 3.04 \\
\hline 4314 & 1103000412 & FO & & & Mulberry Creek & 286 & 0 & 0 & .90 & 2.22 & 5.08 \\
\hline 4367 & 110300094 & FO & KW & & Rattlesnake Creek & 164 & 0 & .49 & 2.14 & 5.34 & 12.3 \\
\hline 4469 & 110300094 & $\mathrm{FO}$ & & & Rattlesnake Creek & 102 & 0 & 0 & .35 & 1.62 & 5.37 \\
\hline
\end{tabular}


Table 35. Estimated flow-duration values, mean flow values, and peak-discharge frequency values for controlled and uncontrolled flow stream segments on the 1999 Kansas Surface Water Register for Ford County.-Continued

[KSWR, Kansas Surface Water Register; CUSEGA, catalog unit segment number alpha; $\mathrm{mi}^{2}$, square miles; $\mathrm{ft}^{3} / \mathrm{s}$, cubic feet per second; HYDRO, lake or other hydrologic structure; NA, not applicable; NRDitch, irrigation ditch; NRTribal, tribal stream]

\begin{tabular}{|c|c|c|c|c|c|c|c|}
\hline \multirow{2}{*}{$\begin{array}{l}\text { Determi- } \\
\text { nation site } \\
\text { identi- } \\
\text { fication } \\
\text { number } \\
\text { (fig. } 39 \text { ) }\end{array}$} & \multirow{2}{*}{$\begin{array}{c}\text { Estimated mean } \\
\text { flow } \\
\left(\mathrm{ft}^{3} / \mathrm{s}\right)\end{array}$} & \multicolumn{6}{|c|}{ Estimated peak discharge $\left(\mathrm{ft}^{3} / \mathrm{s}\right)$ for indicated peak-discharge frequency } \\
\hline & & 2-year & 5-year & 10-year & 25-year & 50-year & 100-year \\
\hline 4180 & 1.10 & 470 & 1,350 & 2,270 & 3,840 & 5,320 & 7,060 \\
\hline 4307 & 3.14 & 286 & 774 & 1,260 & 2,050 & 2,770 & 3,600 \\
\hline 4314 & 4.74 & 312 & 846 & 1,380 & 2,260 & 3,060 & 3,990 \\
\hline 4367 & 12.2 & 689 & 1,990 & 3,390 & 5,790 & 8,080 & 10,800 \\
\hline 4469 & 7.23 & 600 & 1,820 & 3,150 & 5,480 & 7,720 & 10,400 \\
\hline
\end{tabular}




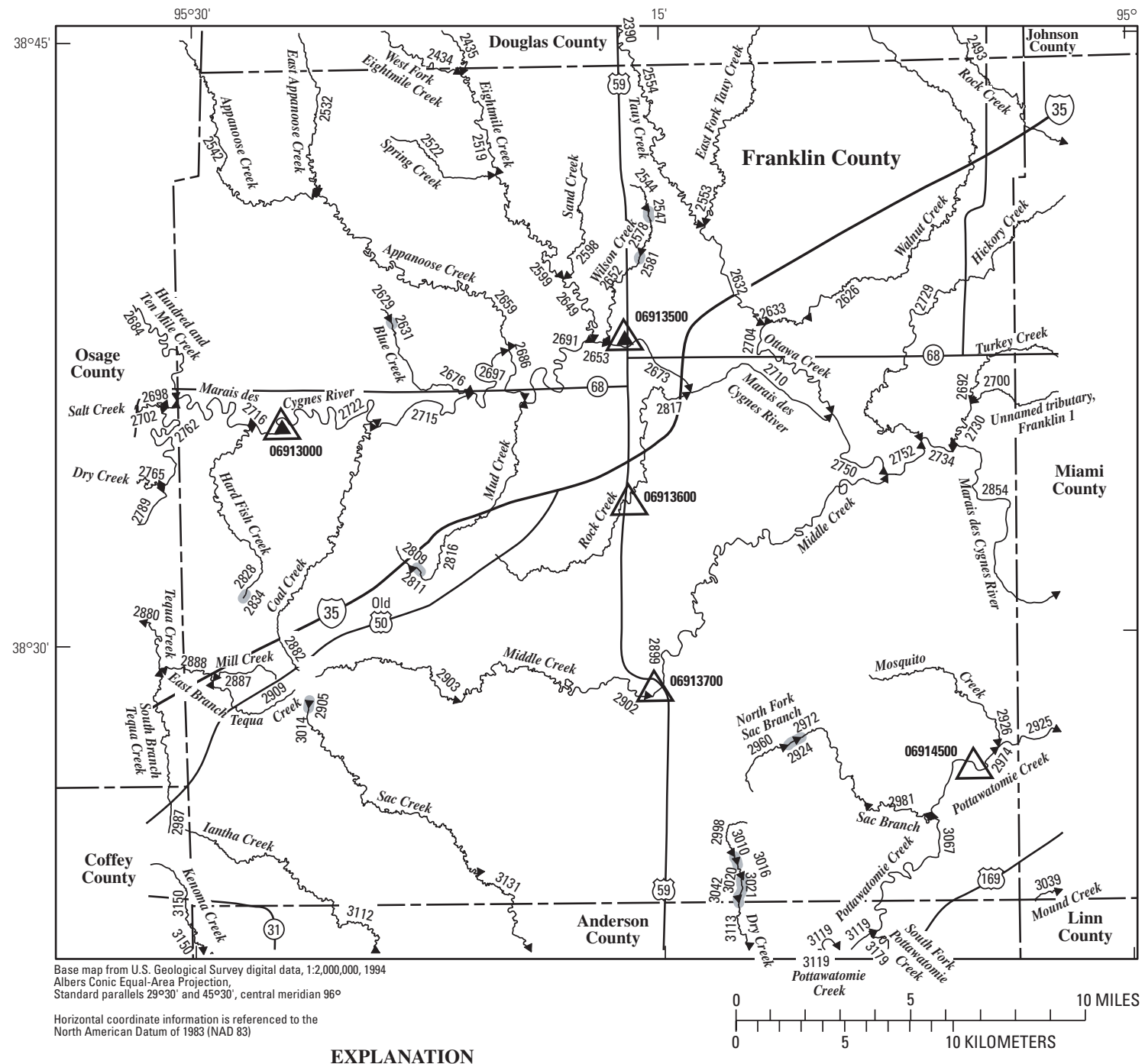

\section{EXPLANATION}

KANSAS

$\$ 3150$ Location of streamflow-statistics determination site (small triangle) and associated identification number-small triangle points in downstream direction

${ }^{06913500} \Delta$ U.S. Geological Survey streamflow-gaging station and number used for estimates of flow duration

${ }^{06913700} \triangle$ U.S. Geological Survey streamflow-gaging station and number used for estimates of peak-discharge frequency values

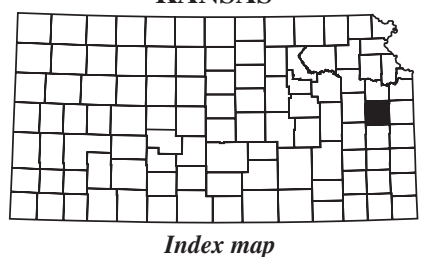

2905 Lake and determination site identification number

Figure 40. Location of streamflow-statistics determination sites, associated identification numbers, and U.S. Geological Survey streamflow-gaging stations used in the flow-duration and peak-discharge frequency analyses for Franklin County. 
Table 36. Estimated flow-duration values, mean flow values, and peak-discharge frequency values for controlled and uncontrolled flow stream segments on the 1999 Kansas Surface Water Register for Franklin County.

[KSWR, Kansas Surface Water Register; CUSEGA, catalog unit segment number alpha; $\mathrm{mi}^{2}$, square miles; $\mathrm{ft}^{3} / \mathrm{s}$, cubic feet per second; HYDRO, lake or other hydrologic structure; NA, not applicable; NRDitch, irrigation ditch; NRTribal, tribal stream]

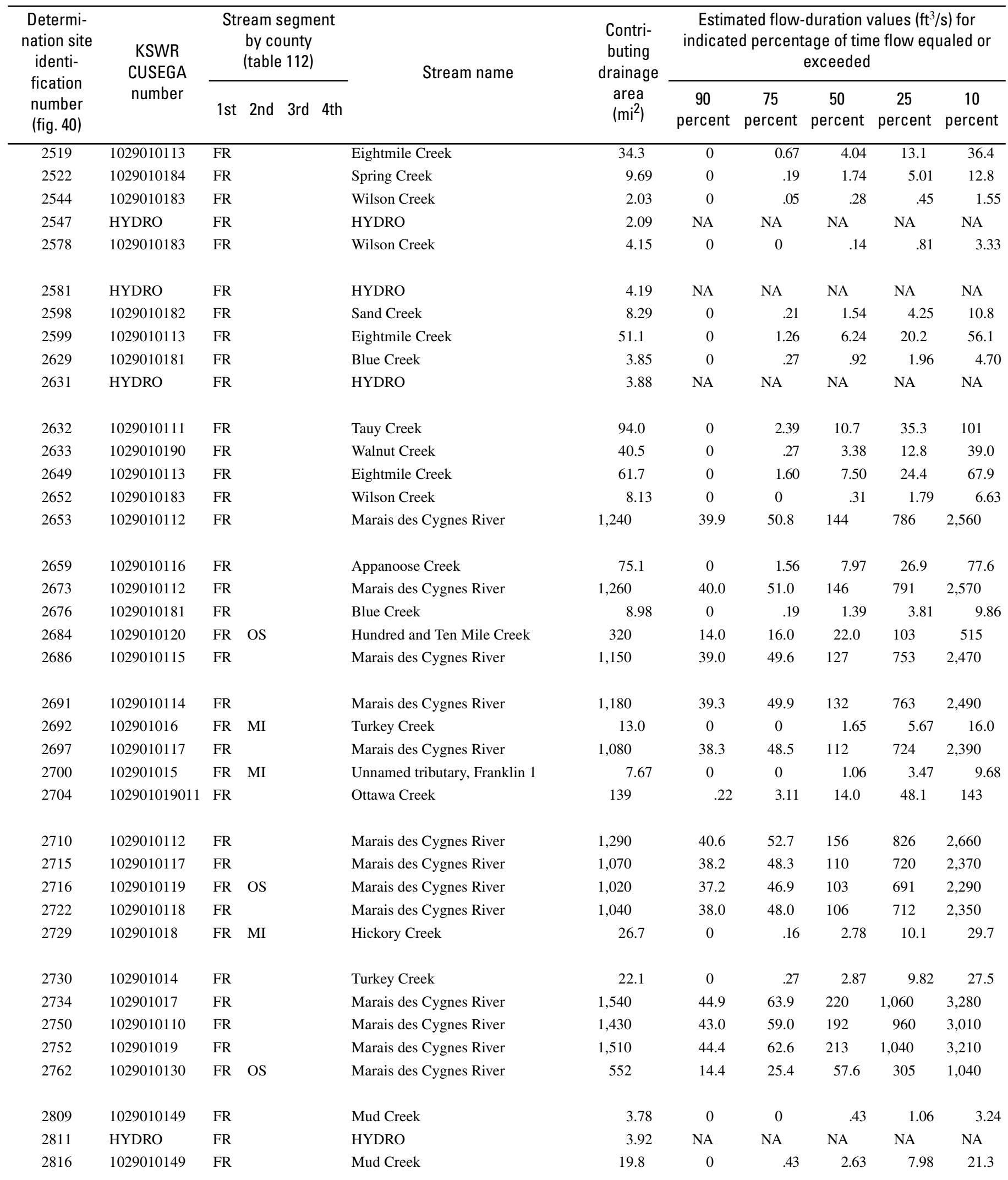


Table 36. Estimated flow-duration values, mean flow values, and peak-discharge frequency values for controlled and uncontrolled flow stream segments on the 1999 Kansas Surface Water Register for Franklin County.-Continued

[KSWR, Kansas Surface Water Register; CUSEGA, catalog unit segment number alpha; $\mathrm{mi}^{2}$, square miles; $\mathrm{ft}^{3} / \mathrm{s}$, cubic feet per second; HYDRO, lake or other hydrologic structure; NA, not applicable; NRDitch, irrigation ditch; NRTribal, tribal stream]

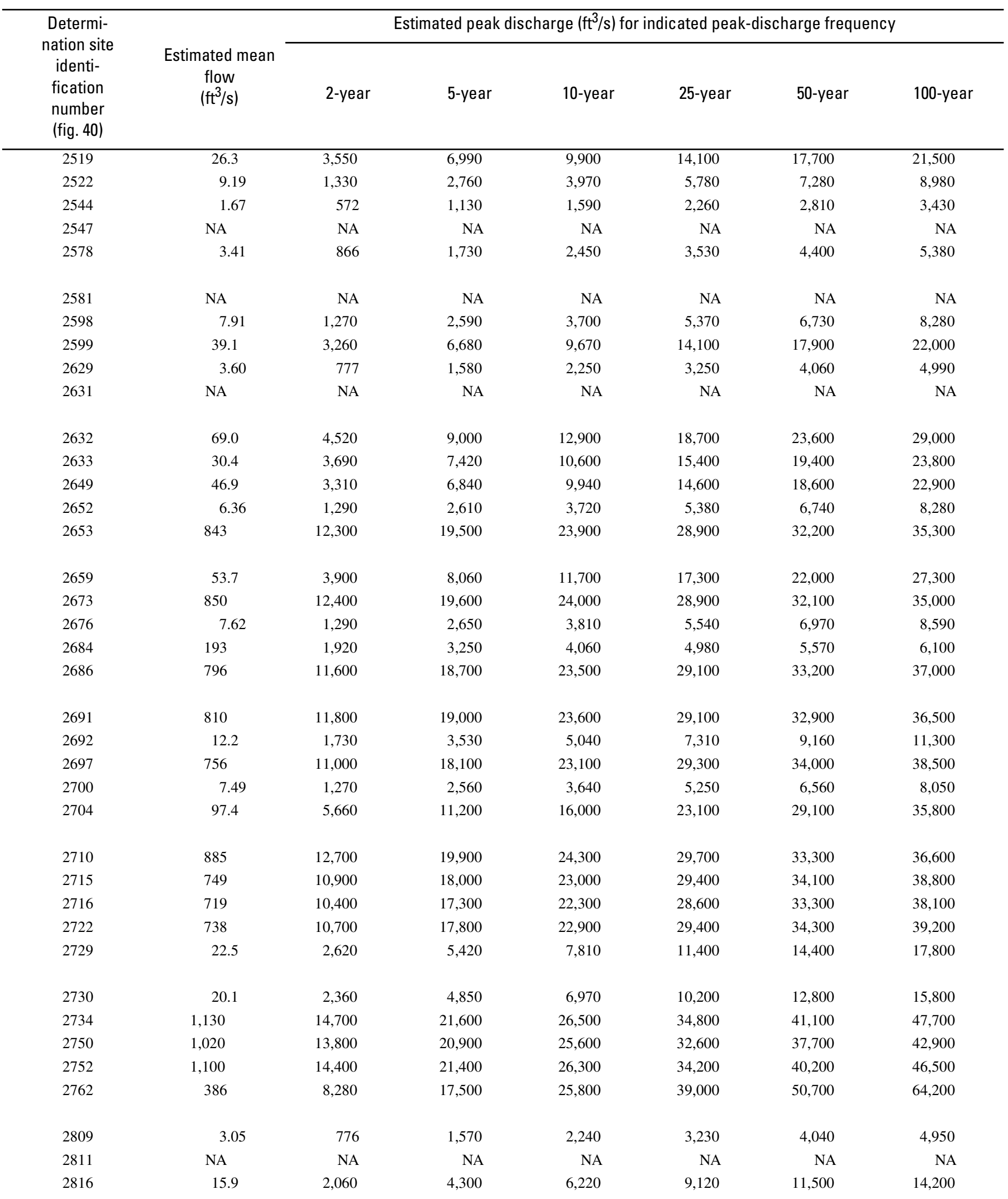


Table 36. Estimated flow-duration values, mean flow values, and peak-discharge frequency values for controlled and uncontrolled flow stream segments on the 1999 Kansas Surface Water Register for Franklin County.-Continued

[KSWR, Kansas Surface Water Register; CUSEGA, catalog unit segment number alpha; $\mathrm{mi}^{2}$, square miles; $\mathrm{ft}^{3} / \mathrm{s}$, cubic feet per second; HYDRO, lake or other hydrologic structure; NA, not applicable; NRDitch, irrigation ditch; NRTribal, tribal stream]

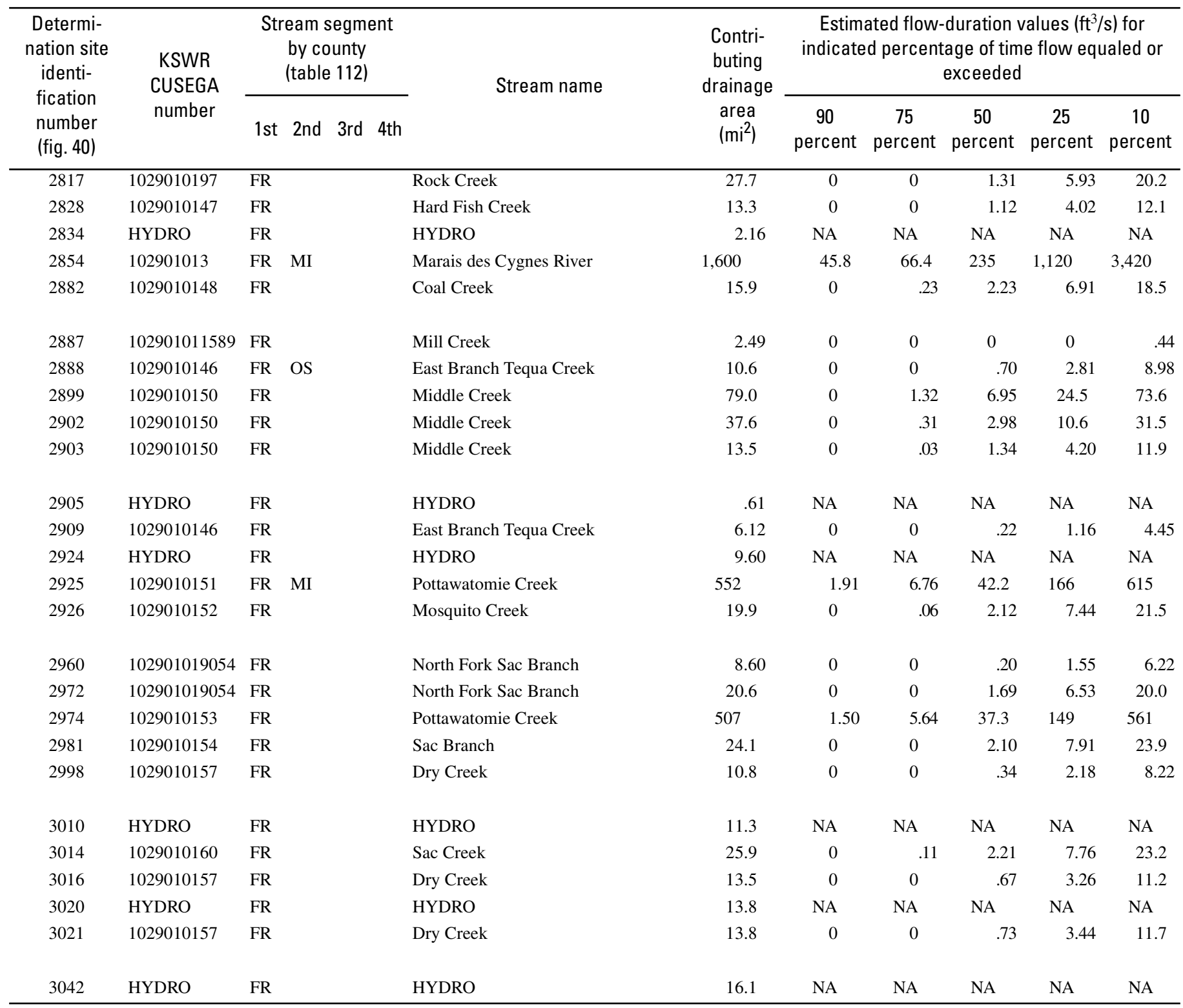


Table 36. Estimated flow-duration values, mean flow values, and peak-discharge frequency values for controlled and uncontrolled flow stream segments on the 1999 Kansas Surface Water Register for Franklin County.-Continued

[KSWR, Kansas Surface Water Register; CUSEGA, catalog unit segment number alpha; $\mathrm{mi}^{2}$, square miles; $\mathrm{ft}^{3} / \mathrm{s}$, cubic feet per second; HYDRO, lake or other hydrologic structure; NA, not applicable; NRDitch, irrigation ditch; NRTribal, tribal stream]

\begin{tabular}{|c|c|c|c|c|c|c|c|}
\hline \multirow{2}{*}{$\begin{array}{l}\text { Determi- } \\
\text { nation site } \\
\text { identi- } \\
\text { fication } \\
\text { number } \\
\text { (fig. 40) }\end{array}$} & \multirow{2}{*}{$\begin{array}{c}\text { Estimated mean } \\
\text { flow } \\
\left(\mathrm{ft}^{3} / \mathrm{s}\right)\end{array}$} & \multicolumn{6}{|c|}{ Estimated peak discharge $\left(\mathrm{ft}^{3} / \mathrm{s}\right)$ for indicated peak-discharge frequency } \\
\hline & & 2-year & 5 -year & 10 -year & 25-year & 50 -year & 100-year \\
\hline 2817 & $\begin{array}{l}18.1 \\
\end{array}$ & 597 & 1,300 & 1,960 & 3,060 & 4,090 & 5,310 \\
\hline 2828 & 9.91 & 1,510 & 3,180 & 4,620 & 6,800 & 8,600 & 10,700 \\
\hline 2834 & NA & NA & NA & NA & NA & NA & NA \\
\hline 2854 & 1,180 & 15,100 & 22,000 & 27,000 & 35,900 & 42,800 & 50,200 \\
\hline 2882 & 13.5 & 1,750 & 3,660 & 5,310 & 7,790 & 9,850 & 12,200 \\
\hline 2887 & 1.40 & 579 & 1,180 & 1,680 & 2,420 & 3,030 & 3,720 \\
\hline 2888 & 7.84 & 1,340 & 2,810 & 4,070 & 5,960 & 7,520 & 9,310 \\
\hline 2899 & 54.4 & 3,300 & 5,310 & 6,840 & 8,970 & 10,700 & 12,600 \\
\hline 2902 & 25.3 & 2,930 & 5,650 & 7,940 & 11,300 & 14,100 & 17,200 \\
\hline 2903 & 9.84 & 1,590 & 3,270 & 4,690 & 6,840 & 8,600 & 10,600 \\
\hline 2905 & NA & NA & NA & NA & NA & NA & NA \\
\hline 2909 & 4.43 & 979 & 2,030 & 2,910 & 4,240 & 5,340 & 6,580 \\
\hline 2924 & NA & NA & NA & NA & NA & NA & NA \\
\hline 2925 & 385 & 13,200 & 25,300 & 35,900 & 52,100 & 66,700 & 83,200 \\
\hline 2926 & 16.6 & 2,170 & 4,480 & 6,450 & 9,420 & 11,900 & 14,600 \\
\hline 2960 & 6.34 & 1,350 & 2,730 & 3,890 & 5,610 & 7,030 & 8,640 \\
\hline 2972 & 16.3 & 2,240 & 4,620 & 6,650 & 9,700 & 12,200 & 15,100 \\
\hline 2974 & 355 & 13,400 & 25,500 & 36,000 & 52,100 & 66,500 & 82,800 \\
\hline 2981 & 19.1 & 2,460 & 5,080 & 7,310 & 10,700 & 13,500 & 16,600 \\
\hline 2998 & 8.02 & 1,550 & 3,140 & 4,480 & 6,490 & 8,140 & 10,000 \\
\hline 3010 & NA & NA & NA & NA & NA & NA & NA \\
\hline 3014 & 18.5 & 2,370 & 5,000 & 7,250 & 10,700 & 13,500 & 16,700 \\
\hline 3016 & 10.2 & 1,760 & 3,590 & 5,130 & 7,450 & 9,350 & 11,500 \\
\hline 3020 & NA & NA & NA & NA & NA & NA & NA \\
\hline 3021 & 10.5 & 1,790 & 3,650 & 5,220 & 7,580 & 9,520 & 11,700 \\
\hline 3042 & NA & NA & NA & NA & NA & NA & NA \\
\hline
\end{tabular}


238 Estimates of Flow Duration, Mean Flow, and Peak-Discharge Frequency Values for Kansas Stream Locations 


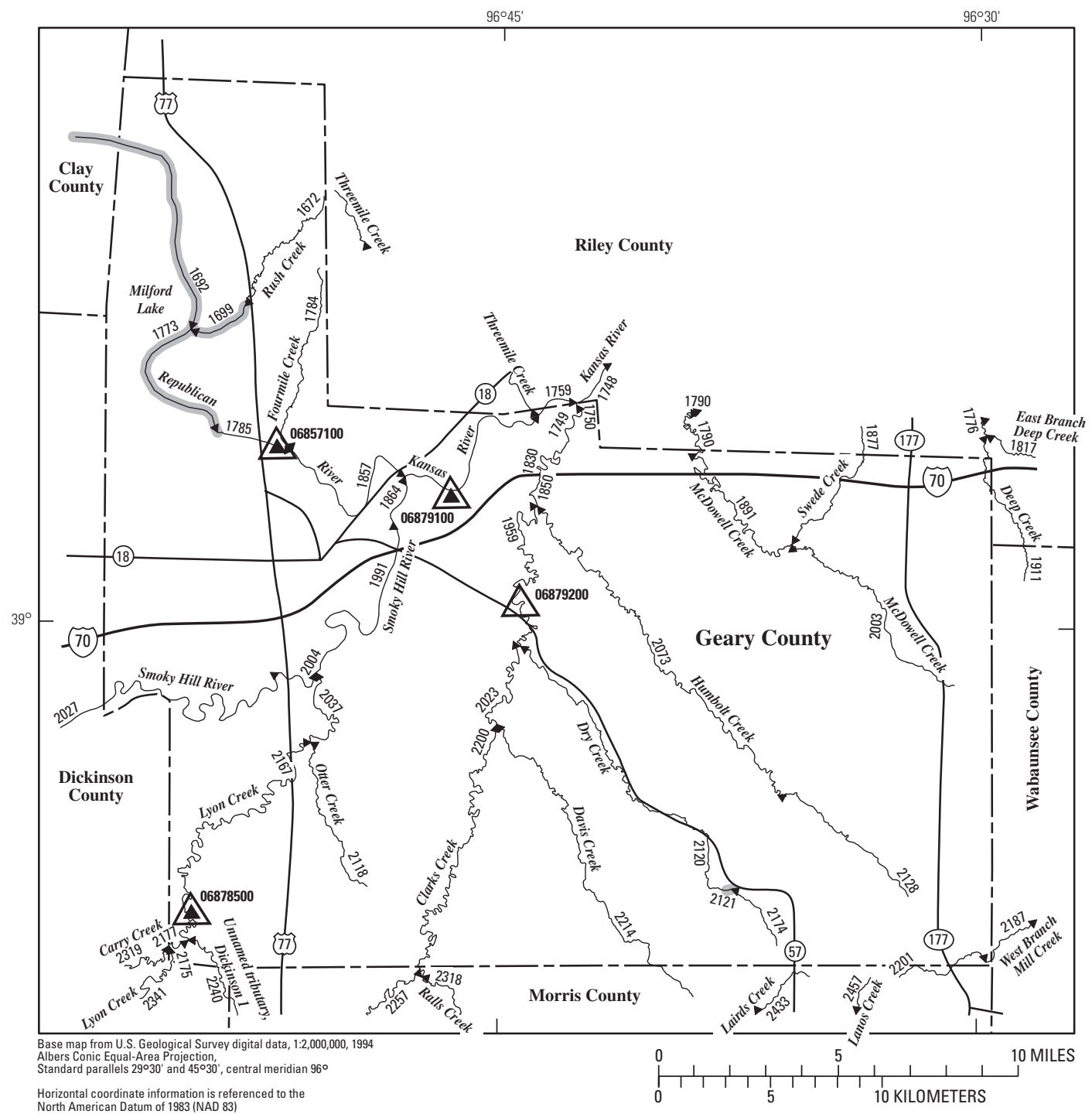

EXPLANATION

2341 Location of streamflow-statistics determination site (small triangle) and associated identification number-small triangle points in downstream direction

${ }^{06878500} \Delta$ U.S. Geological Survey streamflow-gaging station and number used for estimates of flow duration

${ }^{06879200} \triangle$ U.S. Geological Survey streamflow-gaging station and number used for estimates of peak-discharge frequency values

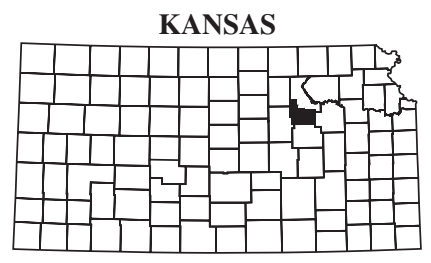

Index map 2121 Lake and determination site identification number

Figure 41. Location of streamflow-statistics determination sites, associated identification numbers, and U.S. Geological Survey streamflow-gaging stations used in the flow-duration and peak-discharge frequency analyses for Geary County. 
Table 37. Estimated flow-duration values, mean flow values, and peak-discharge frequency values for controlled and uncontrolled flow stream segments on the 1999 Kansas Surface Water Register for Geary County.

[KSWR, Kansas Surface Water Register; CUSEGA, catalog unit segment number alpha; $\mathrm{mi}^{2}$, square miles; $\mathrm{ft}^{3} / \mathrm{s}$, cubic feet per second; HYDRO, lake or other hydrologic structure; NA, not applicable; NRDitch, irrigation ditch; NRTribal, tribal stream]

\begin{tabular}{|c|c|c|c|c|c|c|c|c|c|c|c|}
\hline $\begin{array}{l}\text { Determi- } \\
\text { nation site } \\
\text { identi- } \\
\text { fication } \\
\text { number } \\
\text { (fig. 41) }\end{array}$ & $\begin{array}{l}\text { KSWR } \\
\text { CUSEGA } \\
\text { number }\end{array}$ & \multicolumn{3}{|c|}{$\begin{array}{l}\text { Stream segment } \\
\text { by county } \\
\text { (table 112) }\end{array}$} & Stream name & $\begin{array}{l}\text { Contri- } \\
\text { buting } \\
\text { drainage } \\
\text { area } \\
\left(\mathrm{mi}^{2}\right)\end{array}$ & \multicolumn{5}{|c|}{$\begin{array}{c}\text { Estimated flow-duration values }\left(\mathrm{ft}^{3} / \mathrm{s}\right) \text { for } \\
\text { indicated percentage of time flow equaled or } \\
\text { exceeded }\end{array}$} \\
\hline 1672 & 102500171477 & GE & & & Rush Creek & 10.9 & 0 & 0 & 0.51 & 1.67 & 5.32 \\
\hline 1784 & 1025001767 & GE & & & Fourmile Creek & 13.8 & 0 & .11 & 1.41 & 3.68 & 9.38 \\
\hline 1785 & 102500171 & GE & & & Republican River & 23,100 & 57.0 & 126 & 357 & 1,020 & 2,400 \\
\hline 1830 & 102701017 & GE & RL & & Kansas River & 43,500 & 408 & 637 & 1,350 & 3,310 & 7,450 \\
\hline 1850 & 102701018 & GE & RL & & Clarks Creek & 260 & .36 & 4.29 & 18.7 & 62.2 & 189 \\
\hline 1891 & 1027010111 & GE & RL & & McDowell Creek & 69.5 & 0 & 1.00 & 6.09 & 20.4 & 58.6 \\
\hline 1911 & 1027010226 & GE & RL & WB & Deep Creek & 22.7 & 0 & .01 & 2.13 & 7.10 & 20.0 \\
\hline 1959 & 102701019 & GE & & & Clarks Creek & 195 & 0 & 2.89 & 12.9 & 43.5 & 132 \\
\hline 1991 & 102600081 & GE & & & Smoky Hill River & 20,300 & 196 & 309 & 618 & 1,530 & 4,040 \\
\hline 2003 & 1027010111 & GE & & & McDowell Creek & 35.5 & 0 & .20 & 2.99 & 10.3 & 29.6 \\
\hline 2004 & 102600081 & GE & & & Smoky Hill River & 20,000 & 193 & 305 & 608 & 1,500 & 3,990 \\
\hline 2023 & 102701019 & GE & & & Clarks Creek & 155 & 0 & 2.09 & 9.70 & 32.8 & 99.3 \\
\hline 2037 & 1026000831 & GE & & & Lyon Creek & 312 & 3.52 & 17.4 & 34.6 & 70.3 & 152 \\
\hline 2073 & 1027010110 & GE & & & Humbolt Creek & 57.1 & 0 & .69 & 4.89 & 16.6 & 47.8 \\
\hline 2187 & 1027010229 & GE & WB & & West Branch Mill Creek & 64.3 & .16 & 1.53 & 7.01 & 22.2 & 58.6 \\
\hline 2200 & 102701019 & GE & MR & & Clarks Creek & 119 & 0 & 1.41 & 6.99 & 23.7 & 71.8 \\
\hline 2201 & 1027010229 & GE & MR & & West Branch Mill Creek & 10.2 & 0 & .01 & .04 & .94 & 4.55 \\
\hline 2214 & 1027010118 & GE & MR & & Davis Creek & 28.7 & 0 & 0 & 2.07 & 7.17 & 20.9 \\
\hline
\end{tabular}


Table 37. Estimated flow-duration values, mean flow values, and peak-discharge frequency values for controlled and uncontrolled flow stream segments on the 1999 Kansas Surface Water Register for Geary County.-Continued

[KSWR, Kansas Surface Water Register; CUSEGA, catalog unit segment number alpha; $\mathrm{mi}^{2}$, square miles; $\mathrm{ft}^{3} / \mathrm{s}$, cubic feet per second; HYDRO, lake or other hydrologic structure; NA, not applicable; NRDitch, irrigation ditch; NRTribal, tribal stream]

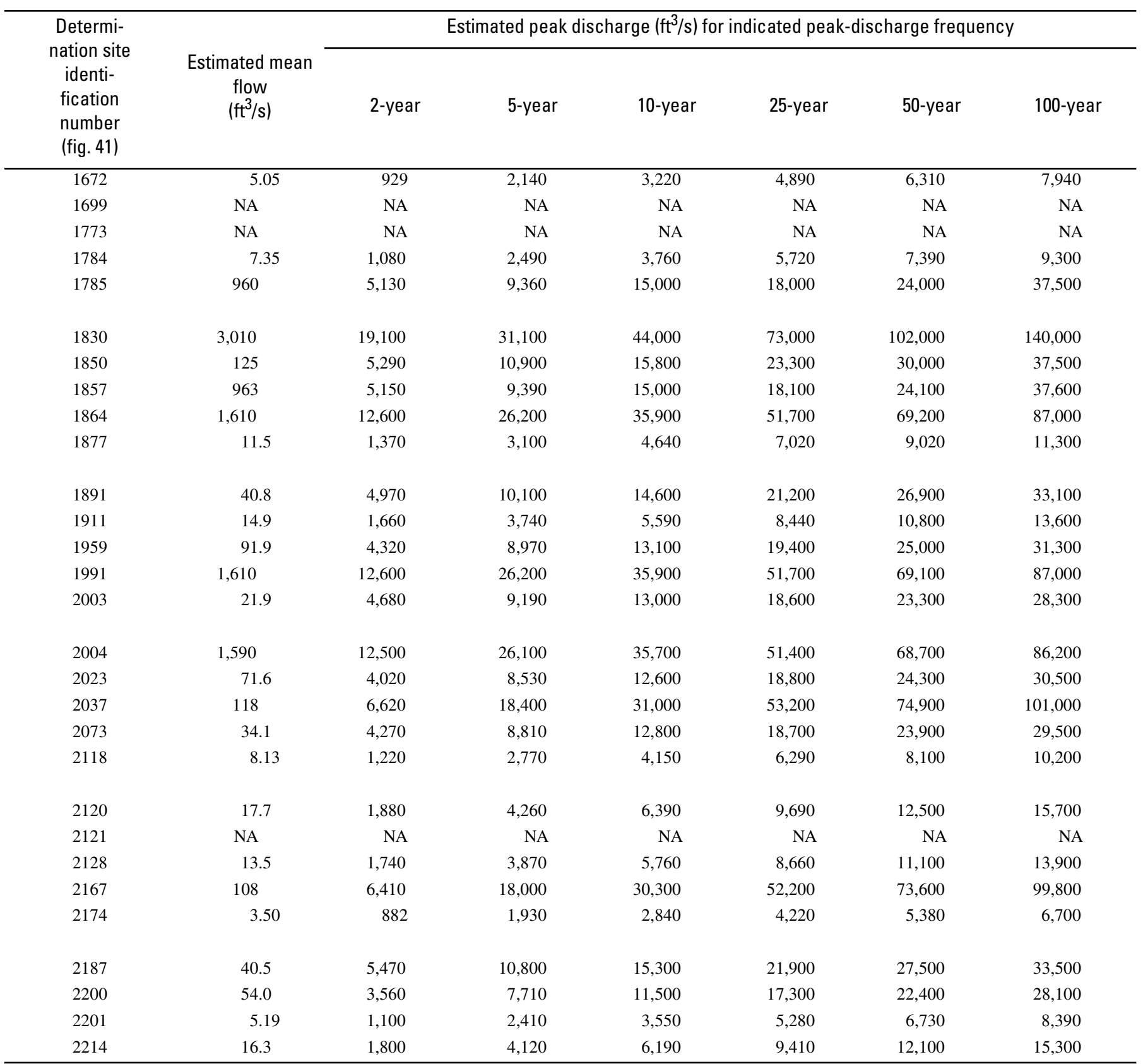




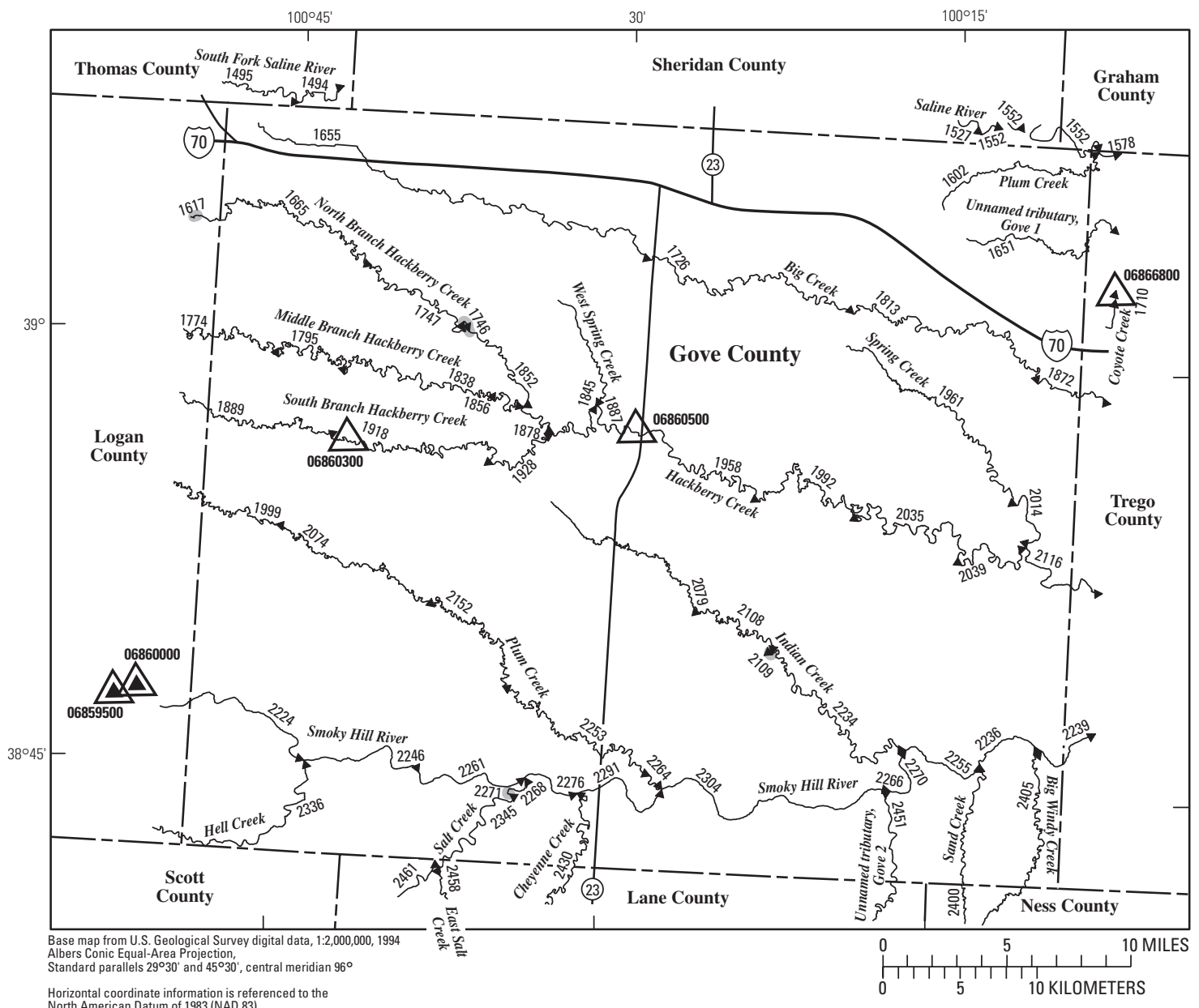

EXPLANATION

\footnotetext{
\$224 Location of streamflow-statistics determination site (small triangle) and associated identification number-small triangle points in downstream direction

${ }^{07139500} \Delta$ U.S. Geological Survey streamflow-gaging station and number used for estimates of flow duration

07139800

U.S. Geological Survey streamflow-gaging station and number used for estimates of peak-discharge frequency values

2109 Lake and determination site identification number
}

KANSAS

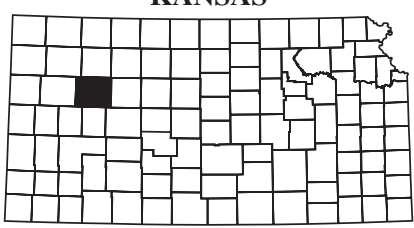

Index map

Figure 42. Location of streamflow-statistics determination sites, associated identification numbers, and U.S. Geological Survey streamflow-gaging stations used in the flow-duration and peak-discharge frequency analyses for Gove County. 
Table 38. Estimated flow-duration values, mean flow values, and peak-discharge frequency values for controlled and uncontrolled flow stream segments on the 1999 Kansas Surface Water Register for Gove County.

[KSWR, Kansas Surface Water Register; CUSEGA, catalog unit segment number alpha; $\mathrm{mi}^{2}$, square miles; $\mathrm{ft}^{3} / \mathrm{s}$, cubic feet per second; HYDRO, lake or other hydrologic structure; NA, not applicable; NRDitch, irrigation ditch; NRTribal, tribal stream]

\begin{tabular}{|c|c|c|c|c|c|c|c|c|c|c|c|}
\hline \multirow{2}{*}{$\begin{array}{l}\text { Determi- } \\
\text { nation site } \\
\text { identi- } \\
\text { fication } \\
\text { number } \\
\text { (fig. 42) }\end{array}$} & \multirow{2}{*}{$\begin{array}{l}\text { KSWR } \\
\text { CUSEGA } \\
\text { number }\end{array}$} & \multicolumn{3}{|c|}{$\begin{array}{l}\text { Stream segment } \\
\text { by county } \\
\text { (table 112) }\end{array}$} & \multirow{2}{*}{ Stream name } & \multirow{2}{*}{$\begin{array}{l}\text { Contri- } \\
\text { buting } \\
\text { drainage } \\
\text { area } \\
\left(\mathrm{mi}^{2}\right)\end{array}$} & \multicolumn{5}{|c|}{$\begin{array}{l}\text { Estimated flow-duration values }\left(\mathrm{ft}^{3} / \mathrm{s}\right) \text { for } \\
\text { indicated percentage of time flow equaled or } \\
\text { exceeded }\end{array}$} \\
\hline & & $1 \mathrm{st}$ & 2nd $3 r d$ & 4th & & & $\begin{array}{c}90 \\
\text { percent }\end{array}$ & $\begin{array}{c}75 \\
\text { percent }\end{array}$ & $\begin{array}{c}50 \\
\text { percent }\end{array}$ & $\begin{array}{c}25 \\
\text { percent }\end{array}$ & $\begin{array}{c}10 \\
\text { percent }\end{array}$ \\
\hline 1602 & 1026000922 & $\overline{\mathrm{GO}}$ & $\begin{array}{ll}\mathrm{GH} & \mathrm{TR}\end{array}$ & & Plum Creek & 26.3 & 0 & 0 & 0 & 0.01 & $\overline{0.01}$ \\
\hline 1651 & 102600091061 & $\mathrm{GO}$ & $\mathrm{TR}$ & & Unnamed tributary, Gove 1 & 24.2 & 0 & 0 & 0 & .01 & .01 \\
\hline 1655 & 102600077 & GO & & & Big Creek & 73.9 & .01 & .07 & .14 & .25 & .49 \\
\hline 1665 & 102600055 & GO & LG & & North Branch Hackberry Creek & 81.4 & 0 & 0 & 0 & 0 & 0 \\
\hline 1726 & 102600077 & GO & & & Big Creek & 130 & .04 & .22 & .42 & .76 & 1.52 \\
\hline 1746 & HYDRO & GO & & & HYDRO & 101 & NA & NA & NA & NA & NA \\
\hline 1747 & 102600055 & GO & & & North Branch Hackberry Creek & 98.8 & 0 & 0 & 0 & 0 & 0 \\
\hline 1774 & 102600056 & GO & LG & & Middle Branch Hackberry Creek & 146 & 0 & 0 & 0 & 0 & 0 \\
\hline 1795 & 102600056 & GO & & & Middle Branch Hackberry Creek & 157 & 0 & 0 & 0 & 0 & 0 \\
\hline 1813 & 102600077 & GO & & & Big Creek & 164 & .06 & .35 & .67 & 1.22 & 3.83 \\
\hline 1838 & 102600056 & GO & & & Middle Branch Hackberry Creek & 175 & 0 & 0 & 0 & 0 & 0 \\
\hline 1845 & 102600058 & GO & & & West Spring Creek & 23.0 & 0 & 0 & 0 & 0 & 0 \\
\hline 1852 & 102600055 & GO & & & North Branch Hackberry Creek & 111 & 0 & 0 & 0 & 0 & 0 \\
\hline 1856 & 102600056 & GO & & & Middle Branch Hackberry Creek & 176 & 0 & 0 & 0 & 0 & 0 \\
\hline 1872 & 102600077 & GO & TR & & Big Creek & 186 & .08 & .45 & .86 & 1.69 & 5.26 \\
\hline 1878 & 102600054 & GO & & & Middle Branch Hackberry Creek & 289 & 0 & 0 & 0 & 0 & .82 \\
\hline 1887 & 102600053 & GO & & & Hackberry Creek & 389 & 0 & 0 & 0 & .38 & 2.98 \\
\hline 1889 & 102600057 & GO & LG & & South Branch Hackberry Creek & 59.5 & 0 & 0 & 0 & 0 & 0 \\
\hline 1918 & 102600057 & GO & & & South Branch Hackberry Creek & 87.6 & 0 & 0 & 0 & 0 & 0 \\
\hline 1928 & 102600057 & GO & & & South Branch Hackberry Creek & 94.3 & 0 & 0 & 0 & 0 & 0 \\
\hline 1958 & 102600053 & GO & & & Hackberry Creek & 446 & 0 & 0 & 0 & 1.13 & 4.83 \\
\hline 1961 & 102600052 & GO & & & Spring Creek & 40.2 & 0 & 0 & 0 & 0 & 0 \\
\hline 1992 & 102600053 & GO & & & Hackberry Creek & 477 & 0 & 0 & .16 & 1.60 & 5.96 \\
\hline 1999 & 1026000318 & GO & LG & & Plum Creek & 83.1 & 0 & 0 & 0 & 0 & 0 \\
\hline 2014 & 102600052 & GO & & & Spring Creek & 52.0 & 0 & 0 & 0 & 0 & 0 \\
\hline 2035 & 102600053 & GO & & & Hackberry Creek & 510 & 0 & 0 & .35 & 2.12 & 7.25 \\
\hline 2039 & 102600053 & GO & & & Hackberry Creek & 520 & 0 & 0 & .41 & 2.30 & 7.66 \\
\hline 2074 & 1026000318 & GO & & & Plum Creek & 123 & 0 & 0 & 0 & 0 & 0 \\
\hline 2079 & 1026000315 & GO & & & Indian Creek & 47.7 & 0 & 0 & 0 & 0 & 0 \\
\hline 2108 & 1026000315 & GO & & & Indian Creek & 66.5 & 0 & 0 & 0 & 0 & 0 \\
\hline 2109 & HYDRO & GO & & & HYDRO & 68.3 & NA & NA & NA & NA & NA \\
\hline 2116 & 102600051 & GO & TR & & Hackberry Creek & 622 & 0 & 0 & .95 & 3.83 & 11.5 \\
\hline 2152 & 1026000318 & GO & & & Plum Creek & 156 & 0 & 0 & 0 & 0 & .14 \\
\hline 2224 & 1026000321 & GO & LG & & Smoky Hill River & 3,770 & 0 & .11 & 1.50 & 6.00 & 22.0 \\
\hline 2234 & 1026000315 & GO & & & Indian Creek & 105 & 0 & 0 & 0 & 0 & 0 \\
\hline 2236 & 1026000314 & GO & & & Smoky Hill River & 4,470 & 0 & .05 & 1.73 & 10.1 & 33.2 \\
\hline 2239 & 1026000314 & GO & $\mathrm{TR}$ & & Smoky Hill River & 4,500 & 0 & .08 & 1.81 & 10.4 & 34.1 \\
\hline 2246 & 1026000320 & GO & & & Smoky Hill River & 3,850 & 0 & .09 & 1.50 & 6.41 & 23.1 \\
\hline
\end{tabular}


Table 38. Estimated flow-duration values, mean flow values, and peak-discharge frequency values for controlled and uncontrolled flow stream segments on the 1999 Kansas Surface Water Register for Gove County.-Continued

[KSWR, Kansas Surface Water Register; CUSEGA, catalog unit segment number alpha; $\mathrm{mi}^{2}$, square miles; $\mathrm{ft}^{3} / \mathrm{s}$, cubic feet per second; HYDRO, lake or other hydrologic structure; NA, not applicable; NRDitch, irrigation ditch; NRTribal, tribal stream]

\begin{tabular}{|c|c|c|c|c|c|c|c|}
\hline \multirow{2}{*}{$\begin{array}{l}\text { Determi- } \\
\text { nation site } \\
\text { identi- } \\
\text { fication } \\
\text { number } \\
\text { (fig. 42) }\end{array}$} & \multirow{2}{*}{$\begin{array}{c}\text { Estimated mean } \\
\text { flow } \\
\left(\mathrm{ft}^{3} / \mathrm{s}\right)\end{array}$} & \multicolumn{6}{|c|}{ Estimated peak discharge $\left(\mathrm{ft}^{3} / \mathrm{s}\right)$ for indicated peak-discharge frequency } \\
\hline & & 2-year & 5-year & 10-year & 25-year & 50-year & 100-year \\
\hline 1602 & 0.61 & 568 & 1,700 & 2,870 & 4,860 & 6,660 & 8,820 \\
\hline 1665 & .23 & 294 & 1,030 & 1,910 & 3,570 & 5,280 & 7,420 \\
\hline 1726 & 4.74 & 571 & 1,880 & 3,400 & 6,220 & 9,090 & 12,600 \\
\hline 1746 & NA & NA & NA & NA & NA & NA & NA \\
\hline 1747 & .73 & 326 & 1,140 & 2,120 & 3,980 & 5,910 & 8,360 \\
\hline 1838 & 1.63 & 332 & 1,270 & 2,490 & 4,950 & 7,660 & 11,300 \\
\hline 1845 & 0 & 403 & 1,280 & 2,220 & 3,860 & 5,380 & 7,210 \\
\hline 1852 & 1.11 & 354 & 1,230 & 2,300 & 4,310 & 6,410 & 9,090 \\
\hline 1856 & 1.68 & 334 & 1,280 & 2,510 & 4,990 & 7,720 & 11,400 \\
\hline 1872 & 9.43 & 811 & 2,650 & 4,790 & 8,790 & 12,900 & 18,100 \\
\hline 1878 & 3.98 & 433 & 1,760 & 3,580 & 7,500 & 12,000 & 18,300 \\
\hline 1887 & 6.12 & 476 & 2,130 & 4,590 & 10,200 & 17,000 & 26,900 \\
\hline 1889 & 0 & 269 & 868 & 1,550 & 2,800 & 4,050 & 5,580 \\
\hline 1918 & .63 & 397 & 1,190 & 2,050 & 3,590 & 5,110 & 6,970 \\
\hline 2035 & 9.95 & 578 & 2,550 & 5,450 & 12,100 & 20,200 & 31,800 \\
\hline 2039 & 10.3 & 582 & 2,560 & 5,490 & 12,200 & 20,200 & 31,900 \\
\hline 2074 & 1.82 & 403 & 1,320 & 2,380 & 4,290 & 6,180 & 8,480 \\
\hline 2079 & .44 & 380 & 1,180 & 2,060 & 3,580 & 5,060 & 6,800 \\
\hline 2108 & 1.04 & 400 & 1,260 & 2,220 & 3,900 & 5,540 & 7,500 \\
\hline 2109 & NA & NA & NA & NA & NA & NA & NA \\
\hline 2116 & 13.6 & 715 & 2,940 & 6,120 & 13,200 & 21,700 & 33,900 \\
\hline 2152 & 2.80 & 464 & 1,500 & 2,690 & 4,810 & 6,910 & 9,460 \\
\hline 2224 & 23.9 & 1,510 & 6,400 & 13,000 & 26,900 & 42,200 & 62,500 \\
\hline 2234 & 2.37 & 484 & 1,520 & 2,670 & 4,700 & 6,680 & 9,060 \\
\hline 2236 & 33.4 & 1,870 & 7,030 & 13,700 & 27,400 & 42,300 & 62,100 \\
\hline 2239 & 34.0 & 1,880 & 7,050 & 13,700 & 27,400 & 42,300 & 62,000 \\
\hline 2246 & 24.9 & 1,550 & 6,470 & 13,100 & 27,000 & 42,200 & 62,400 \\
\hline
\end{tabular}


Table 38. Estimated flow-duration values, mean flow values, and peak-discharge frequency values for controlled and uncontrolled flow stream segments on the 1999 Kansas Surface Water Register for Gove County.-Continued

[KSWR, Kansas Surface Water Register; CUSEGA, catalog unit segment number alpha; $\mathrm{mi}^{2}$, square miles; $\mathrm{ft}^{3} / \mathrm{s}$, cubic feet per second; HYDRO, lake or other hydrologic structure; NA, not applicable; NRDitch, irrigation ditch; NRTribal, tribal stream]

\begin{tabular}{|c|c|c|c|c|c|c|c|c|c|c|c|}
\hline $\begin{array}{l}\text { Determi- } \\
\text { nation site } \\
\text { identi- } \\
\text { fication } \\
\text { number } \\
\text { (fig. 42) }\end{array}$ & $\begin{array}{l}\text { KSWR } \\
\text { CUSEGA } \\
\text { number }\end{array}$ & \multicolumn{3}{|c|}{$\begin{array}{l}\text { Stream segment } \\
\text { by county } \\
\text { (table 112) }\end{array}$} & Stream name & $\begin{array}{l}\text { Contri- } \\
\text { buting } \\
\text { drainage } \\
\text { area } \\
\left(\mathrm{mi}^{2}\right)\end{array}$ & \multicolumn{5}{|c|}{$\begin{array}{c}\text { Estimated flow-duration values }\left(\mathrm{ft}^{3} / \mathrm{s}\right) \text { for } \\
\text { indicated percentage of time flow equaled or } \\
\text { exceeded }\end{array}$} \\
\hline 2253 & 1026000318 & $\mathrm{GO}$ & & & Plum Creek & 185 & 0 & 0 & 0 & 0 & 1.08 \\
\hline 2264 & 1026000318 & GO & & & Plum Creek & 186 & 0 & 0 & 0 & 0 & 1.12 \\
\hline 2266 & 1026000316 & GO & & & Smoky Hill River & 4,290 & 0 & 0 & 1.46 & 8.54 & 29.1 \\
\hline 2268 & 1026000326 & GO & & & Salt Creek & 121 & 0 & 0 & 0 & 0 & 0 \\
\hline 2270 & 1026000316 & GO & & & Smoky Hill River & 4,320 & 0 & 0 & 1.55 & 8.95 & 30.2 \\
\hline 2304 & 1026000317 & GO & & & Smoky Hill River & 4,290 & 0 & 0 & 1.46 & 8.54 & 29.1 \\
\hline 2336 & 1026000325 & GO & LG & & Hell Creek & 54.7 & 0 & 0 & 0 & 0 & 0 \\
\hline 2345 & 1026000326 & GO & & & Salt Creek & 120 & 0 & 0 & 0 & 0 & 0 \\
\hline 2400 & 1026000337 & GO & NS & & Sand Creek & 16.0 & 0 & 0 & 0 & 0 & 0 \\
\hline 2405 & 1026000338 & GO & NS & & Big Windy Creek & 20.1 & 0 & 0 & 0 & 0 & 0 \\
\hline 2430 & 1026000336 & GO & LE & & Cheyenne Creek & 50.8 & 0 & 0 & 0 & 0 & 0 \\
\hline 2451 & 1026000327 & GO & LE & & Unnamed tributary, Gove 2 & 34.2 & 0 & 0 & 0 & 0 & 0 \\
\hline 2458 & 1026000335 & GO & LE & & East Salt Creek & 20.1 & 0 & 0 & 0 & 0 & 0 \\
\hline 2461 & 1026000326 & GO & LE & $\mathrm{SC}$ & Salt Creek & 87.3 & 0 & 0 & 0 & 0 & 0 \\
\hline
\end{tabular}


Table 38. Estimated flow-duration values, mean flow values, and peak-discharge frequency values for controlled and uncontrolled flow stream segments on the 1999 Kansas Surface Water Register for Gove County.-Continued

[KSWR, Kansas Surface Water Register; CUSEGA, catalog unit segment number alpha; $\mathrm{mi}^{2}$, square miles; $\mathrm{ft}^{3} / \mathrm{s}$, cubic feet per second; HYDRO, lake or other hydrologic structure; NA, not applicable; NRDitch, irrigation ditch; NRTribal, tribal stream]

\begin{tabular}{|c|c|c|c|c|c|c|c|}
\hline \multirow{2}{*}{$\begin{array}{l}\text { Determi- } \\
\text { nation site } \\
\text { identi- } \\
\text { fication } \\
\text { number } \\
\text { (fig. 42) }\end{array}$} & \multirow{2}{*}{$\begin{array}{c}\text { Estimated mean } \\
\text { flow } \\
\left(\mathrm{ft}^{3} / \mathrm{s}\right)\end{array}$} & \multicolumn{6}{|c|}{ Estimated peak discharge $\left(\mathrm{ft}^{3} / \mathrm{s}\right)$ for indicated peak-discharge frequency } \\
\hline & & 2-year & 5-year & 10-year & 25-year & 50-year & 100-year \\
\hline 2253 & 3.70 & 522 & 1,670 & 2,970 & 5,290 & 7,580 & 10,400 \\
\hline 2255 & 32.8 & 1,850 & 7,010 & 13,700 & 27,400 & 42,300 & 62,100 \\
\hline 2261 & 25.0 & 1,560 & 6,490 & 13,100 & 27,000 & 42,200 & 62,500 \\
\hline 2264 & 3.73 & 522 & 1,670 & 2,970 & 5,290 & 7,580 & 10,400 \\
\hline 2266 & 30.2 & 1,770 & 6,850 & 13,500 & 27,200 & 42,300 & 62,200 \\
\hline 2268 & 2.02 & 592 & 1,800 & 3,120 & 5,420 & 7,650 & 10,300 \\
\hline 2270 & 31.0 & 1,780 & 6,880 & 13,500 & 27,300 & 42,300 & 62,100 \\
\hline 2271 & NA & NA & NA & NA & NA & NA & NA \\
\hline 2276 & 26.4 & 1,630 & 6,620 & 13,300 & 27,100 & 42,300 & 62,500 \\
\hline 2291 & 27.3 & 1,660 & 6,670 & 13,300 & 27,200 & 42,300 & 62,400 \\
\hline 2304 & 30.2 & 1,770 & 6,860 & 13,500 & 27,300 & 42,300 & 62,200 \\
\hline 2336 & .53 & 408 & 1,250 & 2,170 & 3,760 & 5,300 & 7,100 \\
\hline 2345 & 1.98 & 589 & 1,790 & 3,110 & 5,410 & 7,630 & 10,300 \\
\hline 2400 & 0 & 359 & 1,110 & 1,890 & 3,240 & 4,470 & 5,940 \\
\hline 2405 & 0 & 409 & 1,270 & 2,170 & 3,730 & 5,150 & 6,860 \\
\hline 2430 & .50 & 357 & 1,120 & 1,950 & 3,410 & 4,830 & 6,500 \\
\hline 2451 & .56 & 369 & 1,100 & 1,880 & 3,200 & 4,460 & 5,920 \\
\hline 2458 & 0 & 353 & 1,140 & 1,980 & 3,440 & 4,790 & 6,420 \\
\hline 2461 & .96 & 499 & 1,540 & 2,690 & 4,690 & 6,640 & 8,950 \\
\hline
\end{tabular}




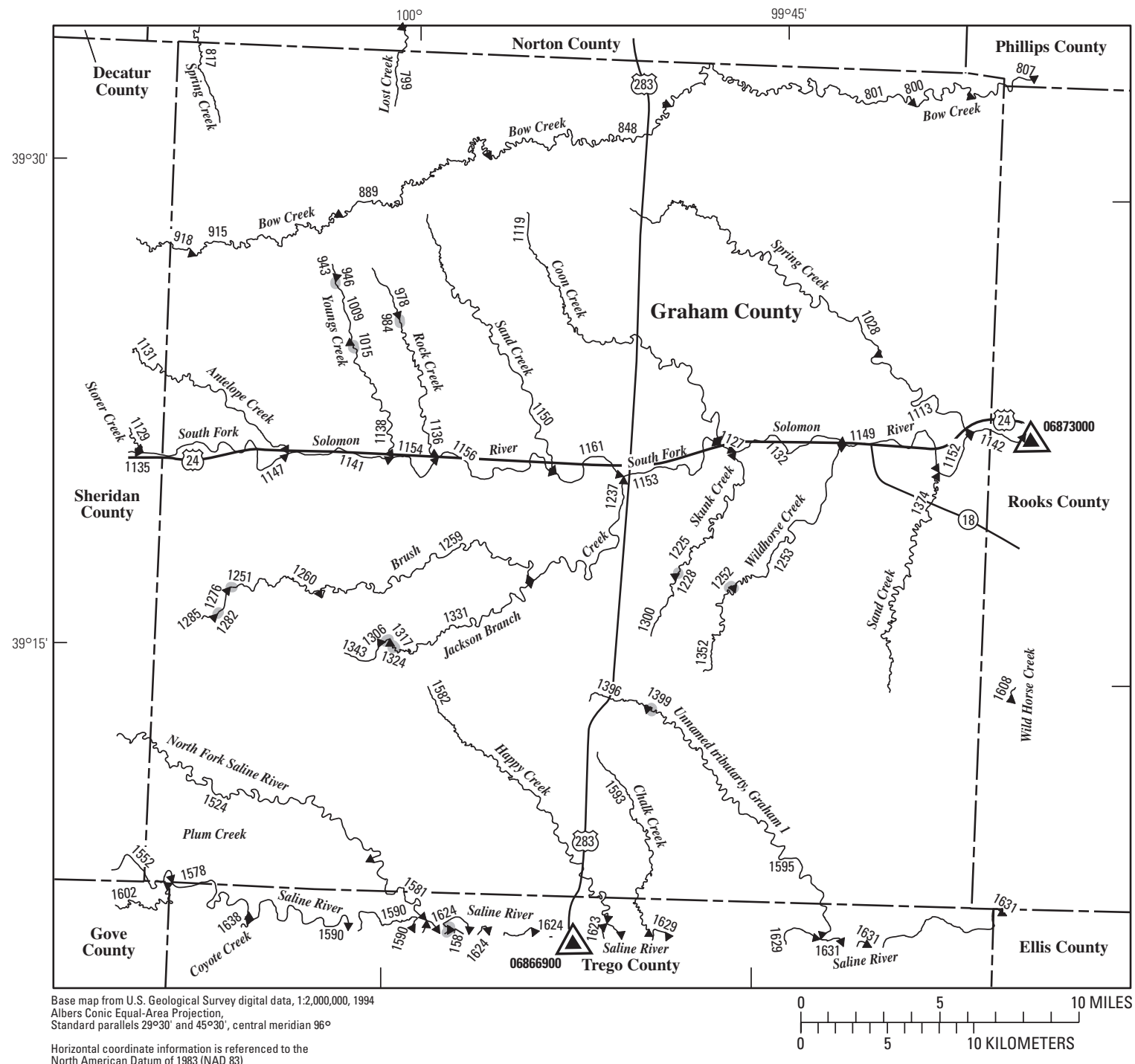

EXPLANATION

$\$ 1578$ Location of streamflow-statistics determination site (small triangle) and associated identification number-small triangle points in downstream direction

$06866900 \Delta$ U.S. Geological Survey streamflow-gaging station and number used for estimates of flow duration

${ }^{06873000} \triangle$ U.S. Geological Survey streamflow-gaging station and number used for estimates of peak-discharge frequency values

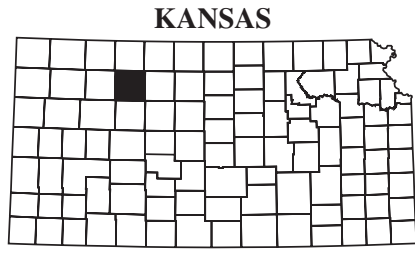

Index map 1399

Lake and determination site identification number

Figure 43. Location of streamflow-statistics determination sites, associated identification numbers, and U.S. Geological Survey streamflow-gaging stations used in the flow-duration and peak-discharge frequency analyses for Graham County. 
Table 39. Estimated flow-duration values, mean flow values, and peak-discharge frequency values for controlled and uncontrolled flow stream segments on the 1999 Kansas Surface Water Register for Graham County.

[KSWR, Kansas Surface Water Register; CUSEGA, catalog unit segment number alpha; $\mathrm{mi}^{2}$, square miles; $\mathrm{ft}^{3} / \mathrm{s}$, cubic feet per second; HYDRO, lake or other hydrologic structure; NA, not applicable; NRDitch, irrigation ditch; NRTribal, tribal stream]

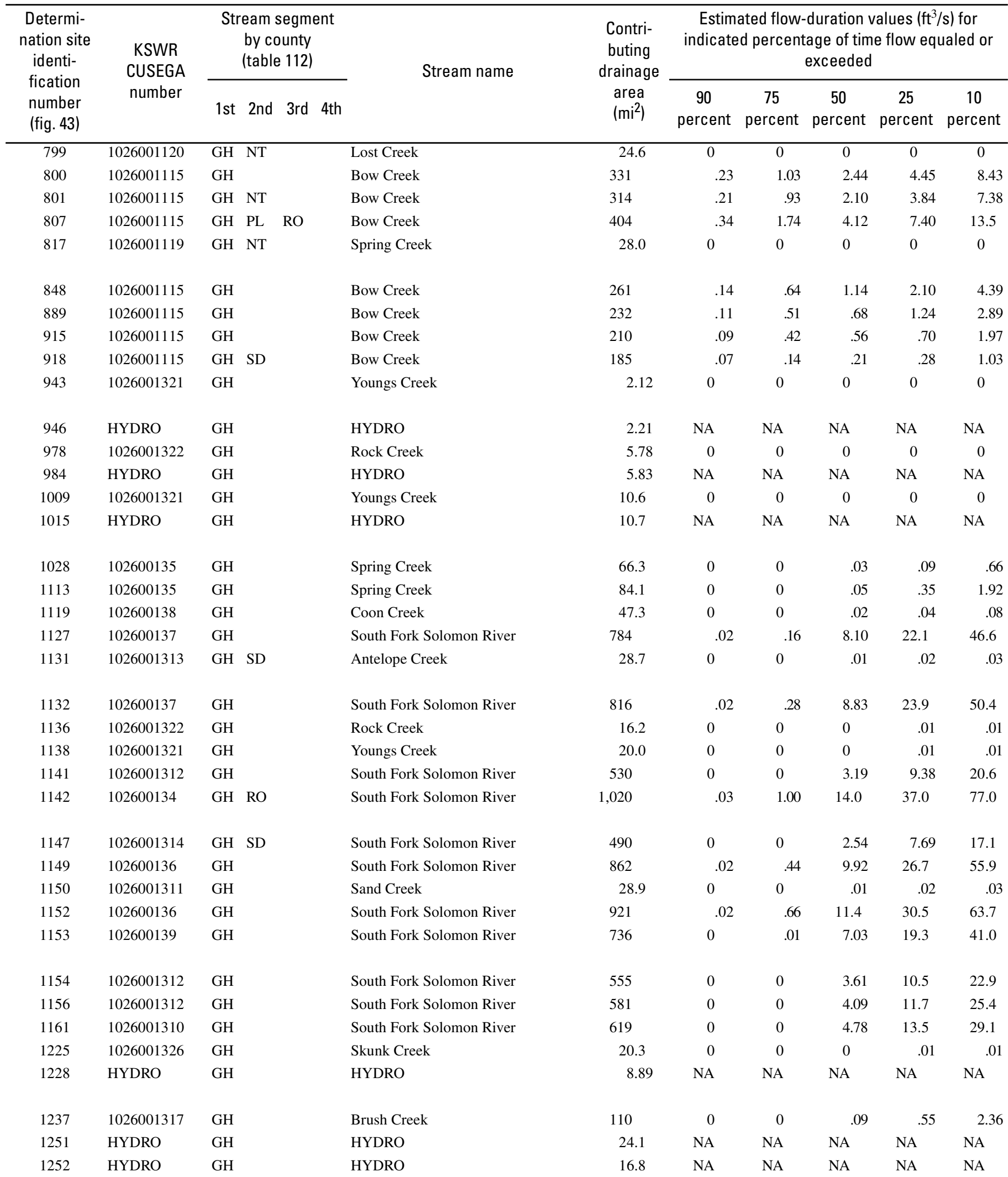


Table 39. Estimated flow-duration values, mean flow values, and peak-discharge frequency values for controlled and uncontrolled flow stream segments on the 1999 Kansas Surface Water Register for Graham County.-Continued

[KSWR, Kansas Surface Water Register; CUSEGA, catalog unit segment number alpha; $\mathrm{mi}^{2}$, square miles; $\mathrm{ft}^{3} / \mathrm{s}$, cubic feet per second; HYDRO, lake or other hydrologic structure; NA, not applicable; NRDitch, irrigation ditch; NRTribal, tribal stream]

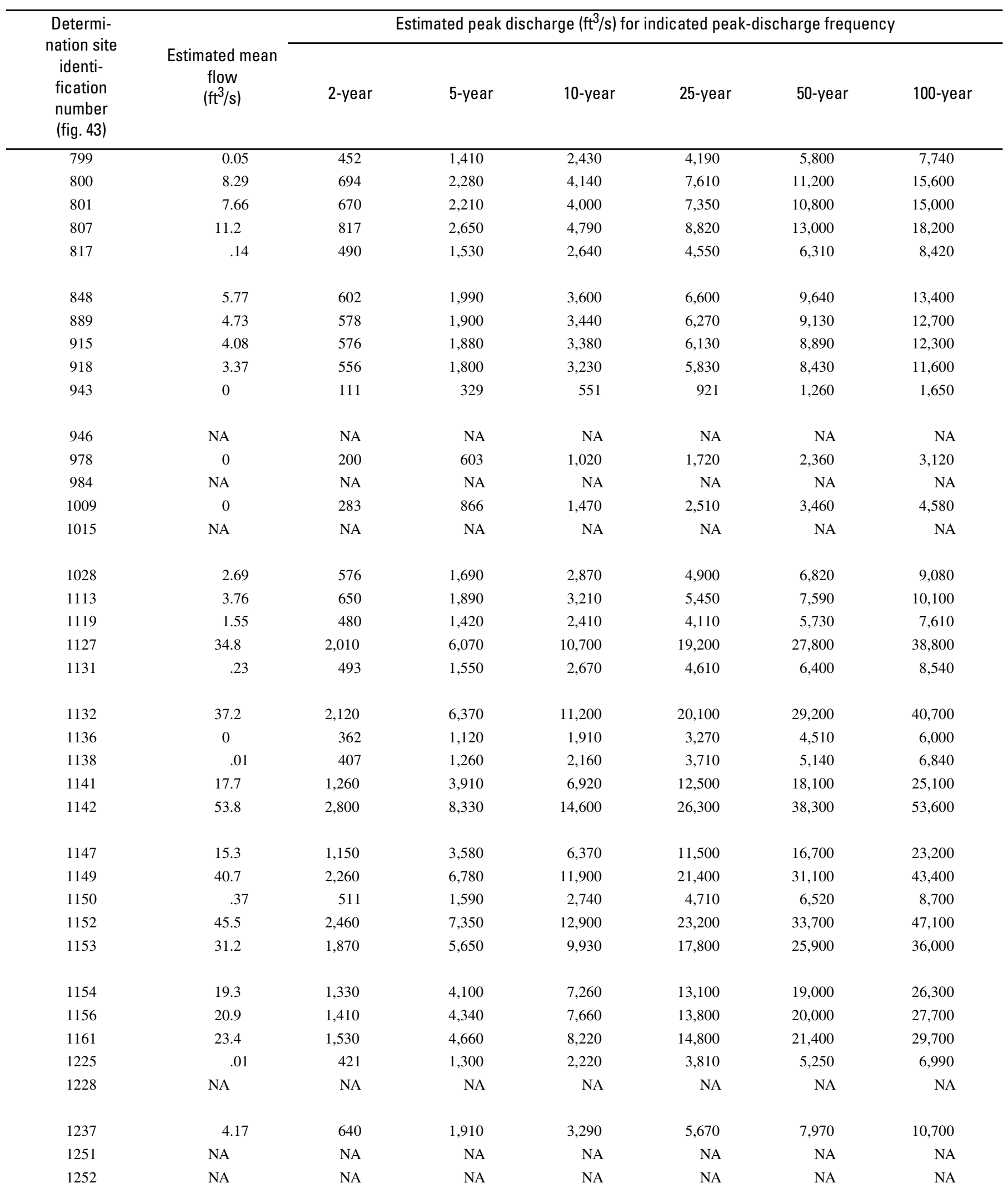


Table 39. Estimated flow-duration values, mean flow values, and peak-discharge frequency values for controlled and uncontrolled flow stream segments on the 1999 Kansas Surface Water Register for Graham County.-Continued

[KSWR, Kansas Surface Water Register; CUSEGA, catalog unit segment number alpha; $\mathrm{mi}^{2}$, square miles; $\mathrm{ft}^{3} / \mathrm{s}$, cubic feet per second; HYDRO, lake or other hydrologic structure; NA, not applicable; NRDitch, irrigation ditch; NRTribal, tribal stream]

\begin{tabular}{|c|c|c|c|c|c|c|c|c|c|c|c|}
\hline $\begin{array}{l}\text { Determi- } \\
\text { nation site } \\
\text { identi- } \\
\text { fication } \\
\text { number } \\
\text { (fig. 43) }\end{array}$ & $\begin{array}{l}\text { KSWR } \\
\text { CUSEGA } \\
\text { number }\end{array}$ & \multicolumn{3}{|c|}{$\begin{array}{l}\text { Stream segment } \\
\text { by county } \\
\text { (table 112) }\end{array}$} & Stream name & $\begin{array}{l}\text { Contri- } \\
\text { buting } \\
\text { drainage } \\
\text { area } \\
\left(\mathrm{mi}^{2}\right)\end{array}$ & \multicolumn{5}{|c|}{$\begin{array}{c}\text { Estimated flow-duration values }\left(\mathrm{ft}^{3} / \mathrm{s}\right) \text { for } \\
\text { indicated percentage of time flow equaled or } \\
\text { exceeded }\end{array}$} \\
\hline 1253 & 1026001318 & GH & & & Wildhorse Creek & 37.8 & 0 & 0 & 0.01 & 0.03 & 0.05 \\
\hline 1276 & 1026001317 & GH & & & Brush Creek & 24.0 & 0 & 0 & 0 & .01 & .02 \\
\hline 1282 & HYDRO & $\mathrm{GH}$ & & & HYDRO & 17.8 & NA & NA & NA & NA & NA \\
\hline 1285 & 1026001317 & $\mathrm{GH}$ & & & Brush Creek & 17.5 & 0 & 0 & 0 & .01 & .01 \\
\hline 1300 & 1026001326 & GH & & & Skunk Creek & 8.89 & 0 & 0 & 0 & 0 & 0 \\
\hline 1331 & 1026001324 & GH & & & Jackson Branch & 32.0 & 0 & 0 & .01 & .02 & .04 \\
\hline 1343 & 1026001324 & GH & & & Jackson Branch & 13.8 & 0 & 0 & 0 & 0 & .01 \\
\hline 1352 & 1026001318 & GH & & & Wildhorse Creek & 16.4 & 0 & 0 & 0 & .01 & .01 \\
\hline 1374 & 1026001327 & GH & & & Sand Creek & 54.5 & 0 & 0 & .02 & .06 & .10 \\
\hline 1396 & 1026000913 & GH & & & Unnamed tributary, Graham 1 & 12.9 & 0 & 0 & 0 & 0 & .01 \\
\hline 1399 & HYDRO & GH & & & HYDRO & 12.9 & NA & NA & NA & NA & NA \\
\hline 1524 & 1026000915 & GH & SD & & North Fork Saline River & 122 & 0 & 0 & .01 & .18 & 1.71 \\
\hline 1552 & 1026000916 & GH & SD & & Saline River & 456 & 0 & 0 & .42 & 3.83 & 8.32 \\
\hline 1578 & 1026000916 & GH & TR & & Saline River & 490 & 0 & 0 & .73 & 4.92 & 10.5 \\
\hline
\end{tabular}


Table 39. Estimated flow-duration values, mean flow values, and peak-discharge frequency values for controlled and uncontrolled flow stream segments on the 1999 Kansas Surface Water Register for Graham County.-Continued

[KSWR, Kansas Surface Water Register; CUSEGA, catalog unit segment number alpha; $\mathrm{mi}^{2}$, square miles; $\mathrm{ft}^{3} / \mathrm{s}$, cubic feet per second; HYDRO, lake or other hydrologic structure; NA, not applicable; NRDitch, irrigation ditch; NRTribal, tribal stream]

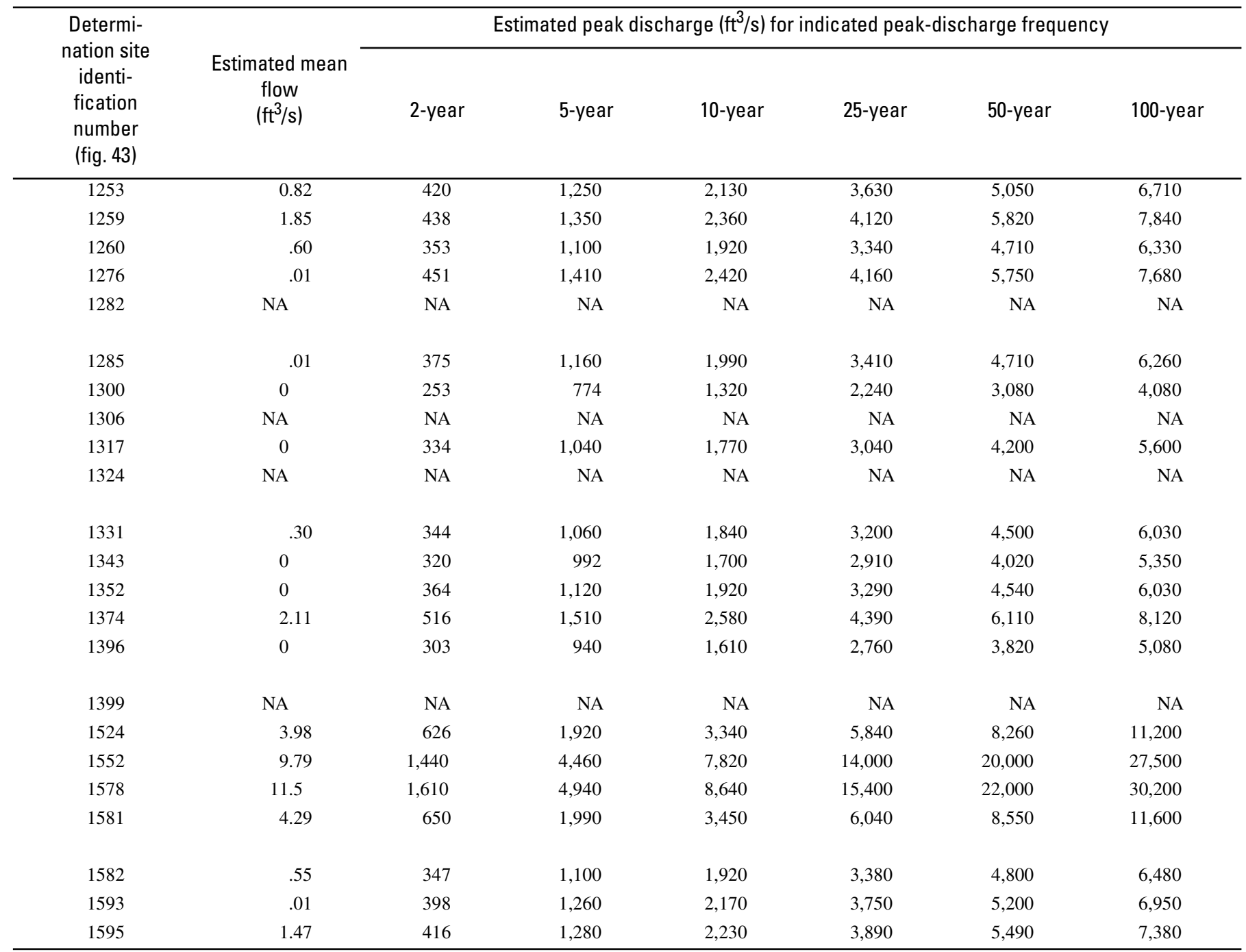




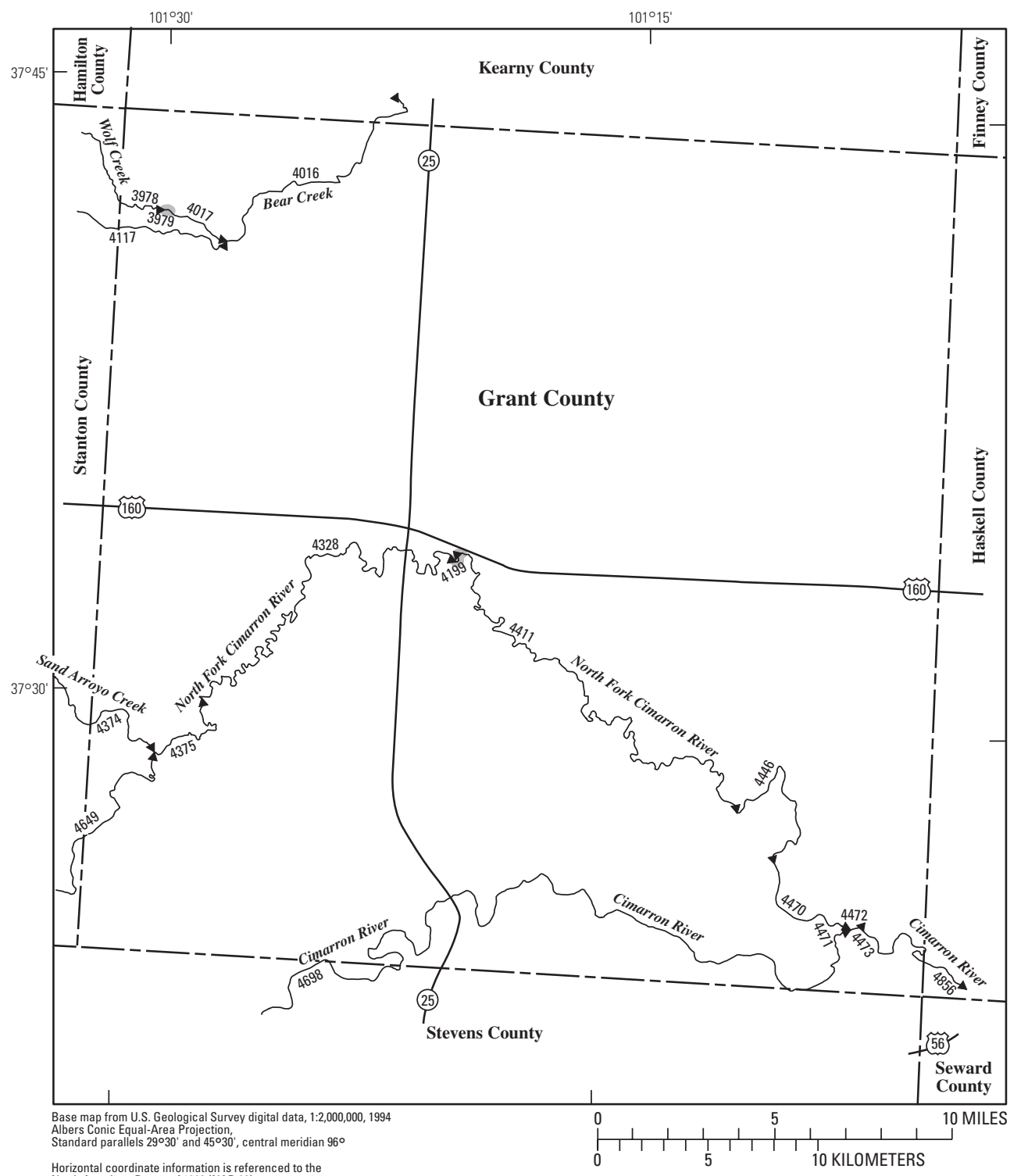

EXPLANATION

Location of streamflow-statistics determination site (small triangle) and associated identification number-small triangle points in downstream direction

$07139500 \Delta$ U.S. Geological Survey streamflow-gaging station and number used for estimates of flow duration

${ }^{07139800} \triangle$ U.S. Geological Survey streamflow-gaging station and number used for estimates of peak-discharge frequency values

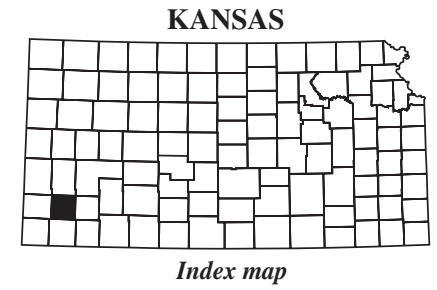

4199 Lake and determination site identification number

Figure 44. Location of streamflow-statistics determination sites, associated identification numbers, and U.S. Geological Survey streamflow-gaging stations used in the flow-duration and peak-discharge frequency analyses for Grant County. 
Table 40. Estimated flow-duration values, mean flow values, and peak-discharge frequency values for controlled and uncontrolled flow stream segments on the 1999 Kansas Surface Water Register for Grant County.

[KSWR, Kansas Surface Water Register; CUSEGA, catalog unit segment number alpha; $\mathrm{mi}^{2}$, square miles; $\mathrm{ft}^{3} / \mathrm{s}$, cubic feet per second; HYDRO, lake or other hydrologic structure; NA, not applicable; NRDitch, irrigation ditch; NRTribal, tribal stream]

\begin{tabular}{|c|c|c|c|c|c|c|c|c|c|c|c|c|}
\hline $\begin{array}{l}\text { Determi- } \\
\text { nation site } \\
\text { identi- } \\
\text { fication } \\
\text { number } \\
\text { (fig. 44) }\end{array}$ & $\begin{array}{l}\text { KSWR } \\
\text { CUSEGA } \\
\text { number }\end{array}$ & \multicolumn{4}{|c|}{$\begin{array}{l}\text { Stream segment } \\
\text { by county } \\
\text { (table 112) }\end{array}$} & Stream name & $\begin{array}{l}\text { Contri- } \\
\text { buting } \\
\text { drainage } \\
\text { area } \\
\left(\mathrm{mi}^{2}\right)\end{array}$ & \multicolumn{5}{|c|}{$\begin{array}{c}\text { Estimated flow-duration values }\left(\mathrm{ft}^{3} / \mathrm{s}\right) \text { for } \\
\text { indicated percentage of time flow equaled or } \\
\text { exceeded }\end{array}$} \\
\hline 3978 & 110400052 & GT & HM & ST & & Wolf Creek & 87.0 & 0 & 0 & 0 & 0 & 0 \\
\hline 4017 & 110400052 & GT & & & & Wolf Creek & 90.5 & 0 & 0 & 0 & 0 & 0 \\
\hline 4117 & 110400051 & GT & ST & & & Bear Creek & 1,090 & 0 & 0 & 0 & 0 & 0 \\
\hline 4199 & HYDRO & GT & & & & HYDRO & 1,720 & NA & NA & NA & NA & NA \\
\hline 4328 & 110400031 & GT & & & & North Fork Cimarron River & 1,710 & 3.96 & 4.71 & 5.65 & 6.10 & 6.39 \\
\hline 4446 & 110400031 & GT & & & & North Fork Cimarron River & 2,020 & 5.21 & 6.21 & 7.44 & 8.74 & 9.45 \\
\hline 4470 & 110400031 & GT & & & & North Fork Cimarron River & 2,030 & 5.24 & 6.24 & 7.48 & 8.82 & 9.56 \\
\hline 4471 & 110400031 & GT & & & & North Fork Cimarron River & 2,030 & 5.24 & 6.24 & 7.48 & 8.82 & 9.56 \\
\hline 4472 & 110400062 & GT & & & & Cimarron River & 5,750 & 16.9 & 21.8 & 27.6 & 36.1 & 43.9 \\
\hline 4473 & 110400062 & GT & & & & Cimarron River & 5,750 & 16.9 & 21.9 & 27.7 & 36.1 & 43.9 \\
\hline 4649 & 110400032 & GT & MT & ST & SV & North Fork Cimarron River & 768 & 1.08 & 1.29 & 1.54 & 1.67 & 3.34 \\
\hline 4698 & 110400021 & GT & SV & & & Cimarron River & 3,720 & 2.20 & 2.97 & 3.80 & 5.13 & 7.88 \\
\hline 4856 & 110400062 & GT & HS & SW & & Cimarron River & 6,260 & 21.2 & 27.4 & 34.9 & 46.6 & 58.5 \\
\hline
\end{tabular}


Table 40. Estimated flow-duration values, mean flow values, and peak-discharge frequency values for controlled and uncontrolled flow stream segments on the 1999 Kansas Surface Water Register for Grant County.-Continued

[KSWR, Kansas Surface Water Register; CUSEGA, catalog unit segment number alpha; $\mathrm{mi}^{2}$, square miles; $\mathrm{ft}^{3} / \mathrm{s}$, cubic feet per second; HYDRO, lake or other hydrologic structure; NA, not applicable; NRDitch, irrigation ditch; NRTribal, tribal stream]

\begin{tabular}{|c|c|c|c|c|c|c|c|}
\hline \multirow{2}{*}{$\begin{array}{l}\text { Determi- } \\
\text { nation site } \\
\text { identi- } \\
\text { fication } \\
\text { number } \\
\text { (fig. 44) }\end{array}$} & \multirow[b]{2}{*}{$\begin{array}{c}\text { Estimated mean } \\
\text { flow } \\
\left(\mathrm{ft}^{3} / \mathrm{s}\right)\end{array}$} & \multicolumn{6}{|c|}{ Estimated peak discharge $\left(\mathrm{ft}^{3} / \mathrm{s}\right)$ for indicated peak-discharge frequency } \\
\hline & & 2-year & 5-year & 10-year & 25-year & 50-year & 100-year \\
\hline 3978 & 0 & 216 & 802 & 1,520 & 2,880 & 4,280 & 6,020 \\
\hline 4017 & 0 & 199 & 753 & 1,440 & 2,750 & 4,110 & 5,810 \\
\hline 4117 & 3.35 & 724 & 3,020 & 5,940 & 11,600 & 17,300 & 24,400 \\
\hline 4199 & NA & NA & NA & NA & NA & NA & NA \\
\hline 4328 & 6.39 & 511 & 1,680 & 3,020 & 5,480 & 7,930 & 10,900 \\
\hline 4446 & 9.16 & 619 & 2,000 & 3,590 & 6,490 & 9,380 & 12,900 \\
\hline 4470 & 9.25 & 613 & 1,990 & 3,570 & 6,450 & 9,320 & 12,900 \\
\hline 4471 & 9.25 & 613 & 1,990 & 3,560 & 6,450 & 9,310 & 12,900 \\
\hline 4472 & 35.3 & 1,850 & 5,480 & 9,340 & 16,000 & 22,300 & 29,900 \\
\hline 4473 & 35.3 & 1,850 & 5,480 & 9,340 & 16,000 & 22,300 & 29,900 \\
\hline 4649 & 7.16 & 976 & 4,030 & 8,040 & 16,200 & 24,800 & 36,100 \\
\hline 4698 & 14.6 & 1,350 & 4,080 & 6,940 & 11,900 & 16,400 & 21,800 \\
\hline 4856 & 44.2 & 1,980 & 5,810 & 9,860 & 16,900 & 23,400 & 31,400 \\
\hline
\end{tabular}


258 Estimates of Flow Duration, Mean Flow, and Peak-Discharge Frequency Values for Kansas Stream Locations 


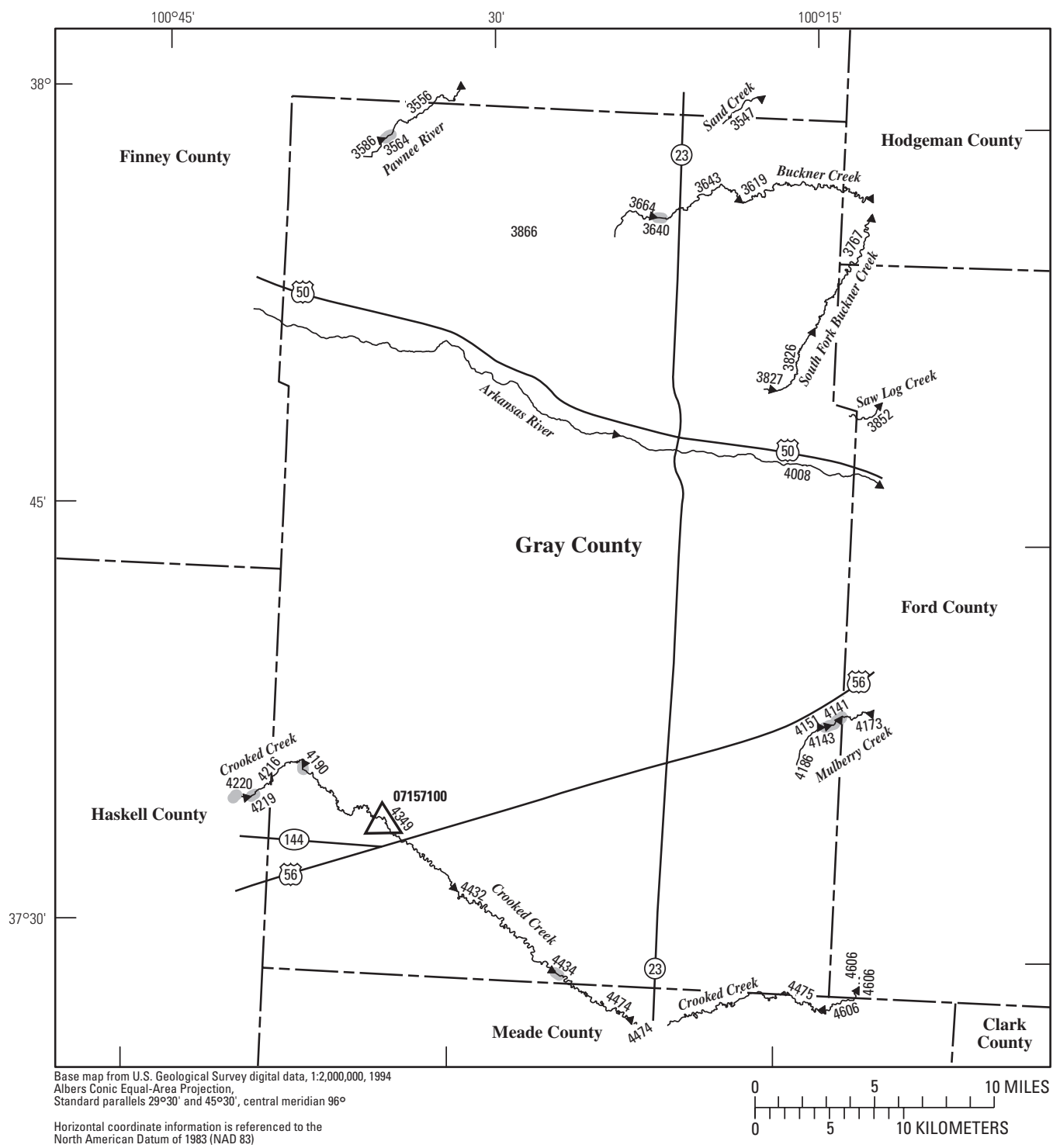

EXPLANATION

4432 Location of streamflow-statistics determination site (small triangle) and associated identification number-small triangle points in downstream direction

${ }^{07139500} \Delta$ U.S. Geological Survey streamflow-gaging station and number used for estimates of flow duration

${ }^{07157100} \triangle$ U.S. Geological Survey streamflow-gaging station and number used for estimates of peak-discharge frequency values

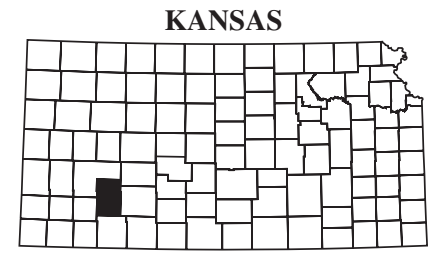

Index map 4434

Lake and determination site identification number

Figure 45. Location of streamflow-statistics determination sites, associated identification numbers, and U.S. Geological Survey streamflow-gaging stations used in the flow-duration and peak-discharge frequency analyses for Gray County. 
Table 41. Estimated flow-duration values, mean flow values, and peak-discharge frequency values for controlled and uncontrolled flow stream segments on the 1999 Kansas Surface Water Register for Gray County.

[KSWR, Kansas Surface Water Register; CUSEGA, catalog unit segment number alpha; $\mathrm{mi}^{2}$, square miles; $\mathrm{ft}^{3} / \mathrm{s}$, cubic feet per second; HYDRO, lake or other hydrologic structure; NA, not applicable; NRDitch, irrigation ditch; NRTribal, tribal stream]

\begin{tabular}{|c|c|c|c|c|c|c|c|c|c|c|c|}
\hline $\begin{array}{l}\text { Determi- } \\
\text { nation site } \\
\text { identi- } \\
\text { fication } \\
\text { number } \\
\text { (fig. } 45 \text { ) }\end{array}$ & $\begin{array}{l}\text { KSWR } \\
\text { CUSEGA } \\
\text { number }\end{array}$ & \multicolumn{3}{|c|}{$\begin{array}{l}\text { Stream segment } \\
\text { by county } \\
\text { (table 112) }\end{array}$} & Stream name & $\begin{array}{l}\text { Contri- } \\
\text { buting } \\
\text { drainage } \\
\text { area } \\
\left(\mathrm{mi}^{2}\right)\end{array}$ & \multicolumn{5}{|c|}{$\begin{array}{c}\text { Estimated flow-duration values }\left(\mathrm{ft}^{3} / \mathrm{s}\right) \text { for } \\
\text { indicated percentage of time flow equaled or } \\
\text { exceeded }\end{array}$} \\
\hline 3564 & HYDRO & $\overline{G Y}$ & & & HYDRO & 54.8 & NA & $\overline{\mathrm{NA}}$ & NA & NA & NA \\
\hline 3640 & HYDRO & GY & & & HYDRO & 48.5 & NA & NA & NA & NA & NA \\
\hline 3643 & 110300062 & GY & & & Buckner Creek & 71.5 & 0 & 0 & 0 & 0 & 0 \\
\hline 3664 & 110300062 & GY & & & Buckner Creek & 47.8 & 0 & 0 & 0 & 0 & 0 \\
\hline 3826 & 110300066 & GY & & & South Fork Buckner Creek & 27.2 & 0 & 0 & 0 & 0 & 0 \\
\hline 4151 & HYDRO & GY & & & HYDRO & 95.8 & NA & NA & NA & NA & NA \\
\hline 4186 & 1103000412 & GY & & & Mulberry Creek & 94.8 & 0 & 0 & 0 & 0 & 0 \\
\hline 4190 & HYDRO & GY & & & HYDRO & 378 & NA & NA & NA & NA & NA \\
\hline 4216 & 110400072 & GY & HS & & Crooked Creek & 378 & 0 & 10 & 20 & 29 & 58 \\
\hline 4349 & 110400072 & GY & & & Crooked Creek & 489 & 24 & 58 & 66 & 74 & 2.41 \\
\hline 4432 & 110400072 & GY & & & Crooked Creek & 562 & 32 & 77 & 1.11 & 1.62 & 4.16 \\
\hline 4434 & HYDRO & GY & & & HYDRO & 562 & NA & NA & NA & NA & NA \\
\hline 4474 & 110400072 & GY & ME & & Crooked Creek & 606 & 37 & 89 & 1.46 & 2.22 & 5.37 \\
\hline 4475 & 110400072 & GY & ME & & Crooked Creek & 672 & 46 & 1.10 & 2.02 & 3.13 & 7.15 \\
\hline
\end{tabular}


Table 41. Estimated flow-duration values, mean flow values, and peak-discharge frequency values for controlled and uncontrolled flow stream segments on the 1999 Kansas Surface Water Register for Gray County.-Continued

[KSWR, Kansas Surface Water Register; CUSEGA, catalog unit segment number alpha; $\mathrm{mi}^{2}$, square miles; $\mathrm{ft}^{3} / \mathrm{s}$, cubic feet per second; HYDRO, lake or other hydrologic structure; NA, not applicable; NRDitch, irrigation ditch; NRTribal, tribal stream]

\begin{tabular}{|c|c|c|c|c|c|c|c|}
\hline \multirow{2}{*}{$\begin{array}{l}\text { Determi- } \\
\text { nation site } \\
\text { identi- } \\
\text { fication } \\
\text { number } \\
\text { (fig. 45) }\end{array}$} & \multirow{2}{*}{$\begin{array}{l}\text { Estimated mean } \\
\text { flow } \\
\left(\mathrm{ft}^{3} / \mathrm{s}\right)\end{array}$} & \multicolumn{6}{|c|}{ Estimated peak discharge $\left(\mathrm{ft}^{3} / \mathrm{s}\right)$ for indicated peak-discharge frequency } \\
\hline & & 2-year & 5-year & 10-year & 25-year & 50-year & 100-year \\
\hline 3564 & NA & NA & NA & NA & NA & NA & NA \\
\hline 3640 & NA & NA & NA & NA & NA & NA & NA \\
\hline 3643 & .35 & 460 & 1,510 & 2,720 & 4,900 & 7,070 & 9,700 \\
\hline 3664 & 0 & 310 & 1,070 & 1,970 & 3,640 & 5,310 & 7,360 \\
\hline 3826 & 0 & 545 & 1,650 & 2,810 & 4,780 & 6,580 & 8,740 \\
\hline 4151 & NA & NA & NA & NA & NA & NA & NA \\
\hline 4186 & .86 & 647 & 1,850 & 3,100 & 5,230 & 7,230 & 9,560 \\
\hline 4190 & NA & NA & NA & NA & NA & NA & NA \\
\hline 4216 & 3.72 & 802 & 2,390 & 4,060 & 6,880 & 9,520 & 12,500 \\
\hline 4349 & 6.17 & 488 & 1,630 & 2,860 & 4,960 & 6,900 & 9,120 \\
\hline 4432 & 7.93 & 458 & 1,640 & 2,960 & 5,290 & 7,500 & 10,100 \\
\hline 4434 & NA & NA & NA & NA & NA & NA & NA \\
\hline 4474 & 9.11 & 432 & 1,620 & 2,990 & 5,430 & 7,780 & 10,600 \\
\hline 4475 & 10.8 & 447 & 1,730 & 3,220 & 5,920 & 8,560 & 11,700 \\
\hline
\end{tabular}


262 Estimates of Flow Duration, Mean Flow, and Peak-Discharge Frequency Values for Kansas Stream Locations 


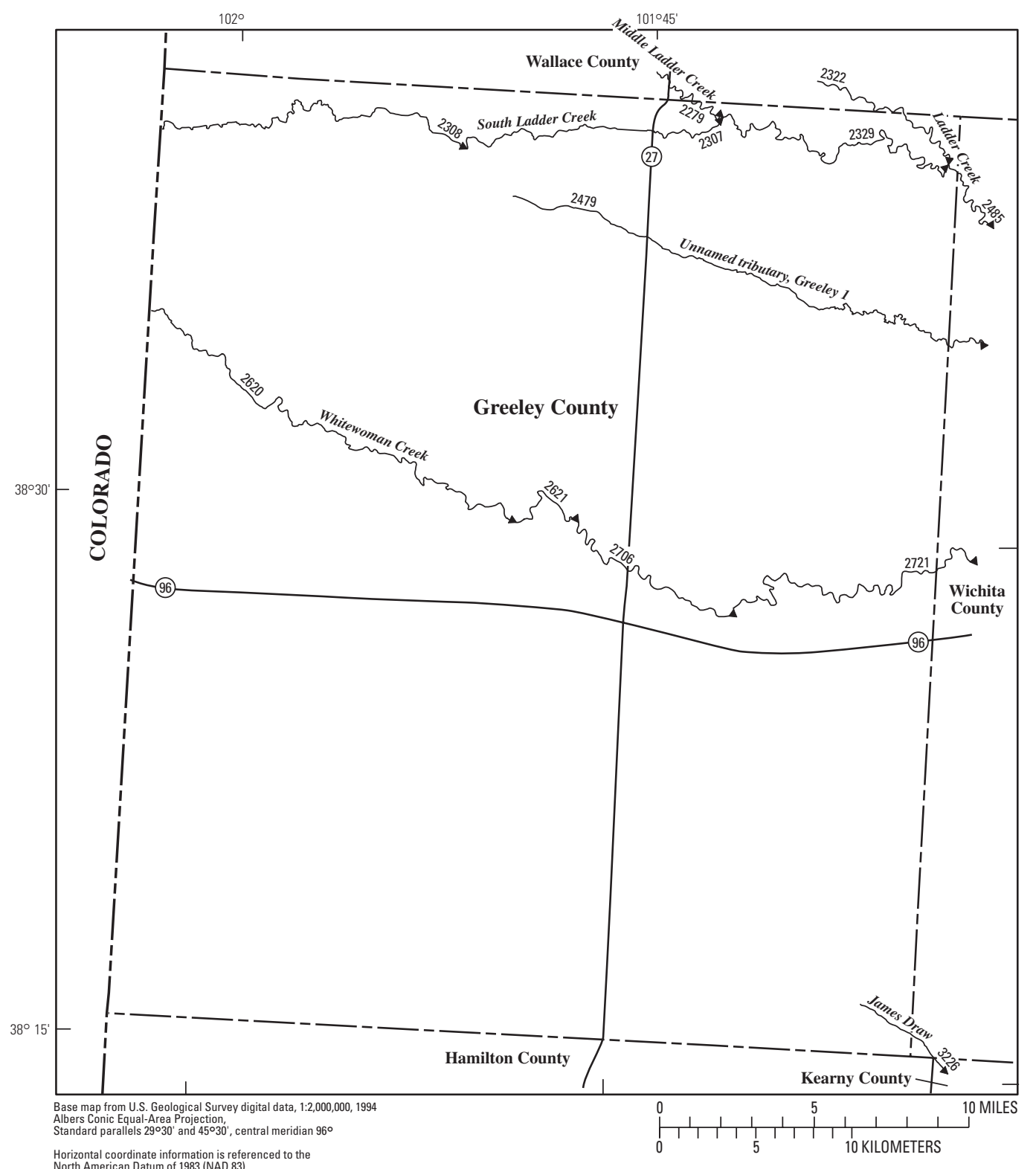

EXPLANATION

$\$ 2341$ Location of streamflow-statistics determination site (small triangle) and associated identification number-small triangle points in downstream direction

06878500 U.S. Geological Survey streamflow-gaging station and number used for estimates of flow duration

$06879200 \bigwedge$ U.S. Geological Survey streamflow-gaging station and number used for estimates of peak-discharge frequency values

KANSAS

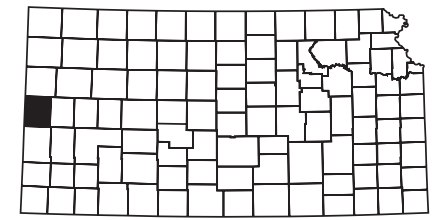

Index map 2121

Lake and determination site identification number

Figure 46. Location of streamflow-statistics determination sites, associated identification numbers, and U.S. Geological Survey streamflow-gaging stations used in the flow-duration and peak-discharge frequency analyses for Greeley County. 
Table 42. Estimated flow-duration values, mean flow values, and peak-discharge frequency values for controlled and uncontrolled flow stream segments on the 1999 Kansas Surface Water Register for Greeley County.

[KSWR, Kansas Surface Water Register; CUSEGA, catalog unit segment number alpha; $\mathrm{mi}^{2}$, square miles; $\mathrm{ft}^{3} / \mathrm{s}$, cubic feet per second; HYDRO, lake or other hydrologic structure; NA, not applicable; NRDitch, irrigation ditch; NRTribal, tribal stream]

\begin{tabular}{|c|c|c|c|c|c|c|c|c|c|c|c|c|}
\hline \multirow{2}{*}{$\begin{array}{c}\text { Determi- } \\
\text { nation site } \\
\text { identi- } \\
\text { fication } \\
\text { number } \\
\text { (fig. 46) }\end{array}$} & \multirow{2}{*}{$\begin{array}{l}\text { KSWR } \\
\text { CUSEGA } \\
\text { number }\end{array}$} & \multicolumn{4}{|c|}{$\begin{array}{l}\text { Stream segment by } \\
\text { county } \\
\text { (table 112) }\end{array}$} & \multirow{2}{*}{ Stream name } & \multirow{2}{*}{$\begin{array}{l}\text { Contri- } \\
\text { buting } \\
\text { drainage } \\
\text { area } \\
\left(\mathrm{mi}^{2}\right)\end{array}$} & \multicolumn{5}{|c|}{$\begin{array}{l}\text { Estimated flow-duration values }\left(\mathrm{ft}^{3} / \mathrm{s}\right) \text { for } \\
\text { indicated percentage of time flow equaled or } \\
\text { exceeded }\end{array}$} \\
\hline & & $1 \mathrm{st}$ & 2nd & $3 r d$ & 4th & & & $\begin{array}{c}90 \\
\text { percent }\end{array}$ & $\begin{array}{c}75 \\
\text { percent }\end{array}$ & $\begin{array}{c}50 \\
\text { percent }\end{array}$ & $\begin{array}{c}25 \\
\text { percent }\end{array}$ & $\begin{array}{c}10 \\
\text { percent }\end{array}$ \\
\hline 2279 & 1026000413 & GL & WA & & & Middle Ladder Creek & 82.2 & 0 & 0 & 0 & 0 & 0 \\
\hline 2322 & 102600049 & GL & WA & & & Ladder Creek & 269 & 0 & .01 & .04 & .04 & .04 \\
\hline 2329 & 1026000412 & GL & WA & & & South Ladder Creek & 350 & 0 & .02 & .07 & .08 & .16 \\
\hline 2479 & 1026000415 & GL & WH & & & Unnamed tributary, Greeley 1 & 122 & 0 & 0 & .01 & .01 & .01 \\
\hline 2485 & 102600048 & GL & WH & & & Ladder Creek & 658 & 0 & .06 & .26 & .27 & .54 \\
\hline 2721 & 110300022 & GL & WH & & & Whitewoman Creek & 759 & 0 & 0 & 0 & 0 & 0 \\
\hline 2307 & 1026000414 & GL & & & & South Ladder Creek & 244 & 0 & .01 & .04 & .08 & .16 \\
\hline 2308 & 1026000414 & GL & & & & South Ladder Creek & 220 & 0 & .01 & .03 & .03 & .03 \\
\hline 2620 & 110300022 & GL & & & & Whitewoman Creek & 517 & 0 & 0 & 0 & 0 & 0 \\
\hline 2621 & 110300022 & GL & & & & Whitewoman Creek & 543 & 0 & 0 & 0 & 0 & 0 \\
\hline 2706 & 110300022 & GL & & & & Whitewoman Creek & 647 & 0 & 0 & 0 & 0 & 0 \\
\hline 3226 & 1103000110 & GL & HM & $\mathrm{KE}$ & WH & James Draw & 217 & 0 & 0 & 0 & 0 & 0 \\
\hline
\end{tabular}


Table 42. Estimated flow-duration values, mean flow values, and peak-discharge frequency values for controlled and uncontrolled flow stream segments on the 1999 Kansas Surface Water Register for Greeley County.-Continued

[KSWR, Kansas Surface Water Register; CUSEGA, catalog unit segment number alpha; $\mathrm{mi}^{2}$, square miles; $\mathrm{ft}^{3} / \mathrm{s}$, cubic feet per second; HYDRO, lake or other hydrologic structure; NA, not applicable; NRDitch, irrigation ditch; NRTribal, tribal stream]

\begin{tabular}{|c|c|c|c|c|c|c|c|}
\hline \multirow{2}{*}{$\begin{array}{l}\text { Determi- } \\
\text { nation site } \\
\text { identi- } \\
\text { fication } \\
\text { number } \\
\text { (fig. 46) }\end{array}$} & \multirow{2}{*}{$\begin{array}{c}\text { Estimated mean } \\
\text { flow } \\
\left(\mathrm{ft}^{3} / \mathrm{s}\right)\end{array}$} & \multicolumn{6}{|c|}{ Estimated peak discharge $\left(\mathrm{ft}^{3} / \mathrm{s}\right)$ for indicated peak-discharge frequency } \\
\hline & & 2-year & 5 -year & 10-year & 25-year & 50-year & 100-year \\
\hline 2279 & 0 & 185 & 702 & 1,350 & 2,590 & 3,870 & 5,470 \\
\hline 2322 & 0 & 312 & 1,180 & 2,270 & 4,370 & 6,590 & 9,410 \\
\hline 2329 & .16 & 388 & 1,440 & 2,740 & 5,250 & 7,880 & 11,200 \\
\hline 2479 & 0 & 244 & 917 & 1,750 & 3,360 & 5,020 & 7,110 \\
\hline 2485 & .80 & 472 & 1,790 & 3,460 & 6,790 & 10,400 & 15,100 \\
\hline 2721 & 1.00 & 232 & 1,400 & 3,310 & 7,870 & 13,400 & 21,100 \\
\hline 3226 & 0 & 305 & 1,140 & 2,180 & 4,180 & 6,260 & 8,880 \\
\hline
\end{tabular}




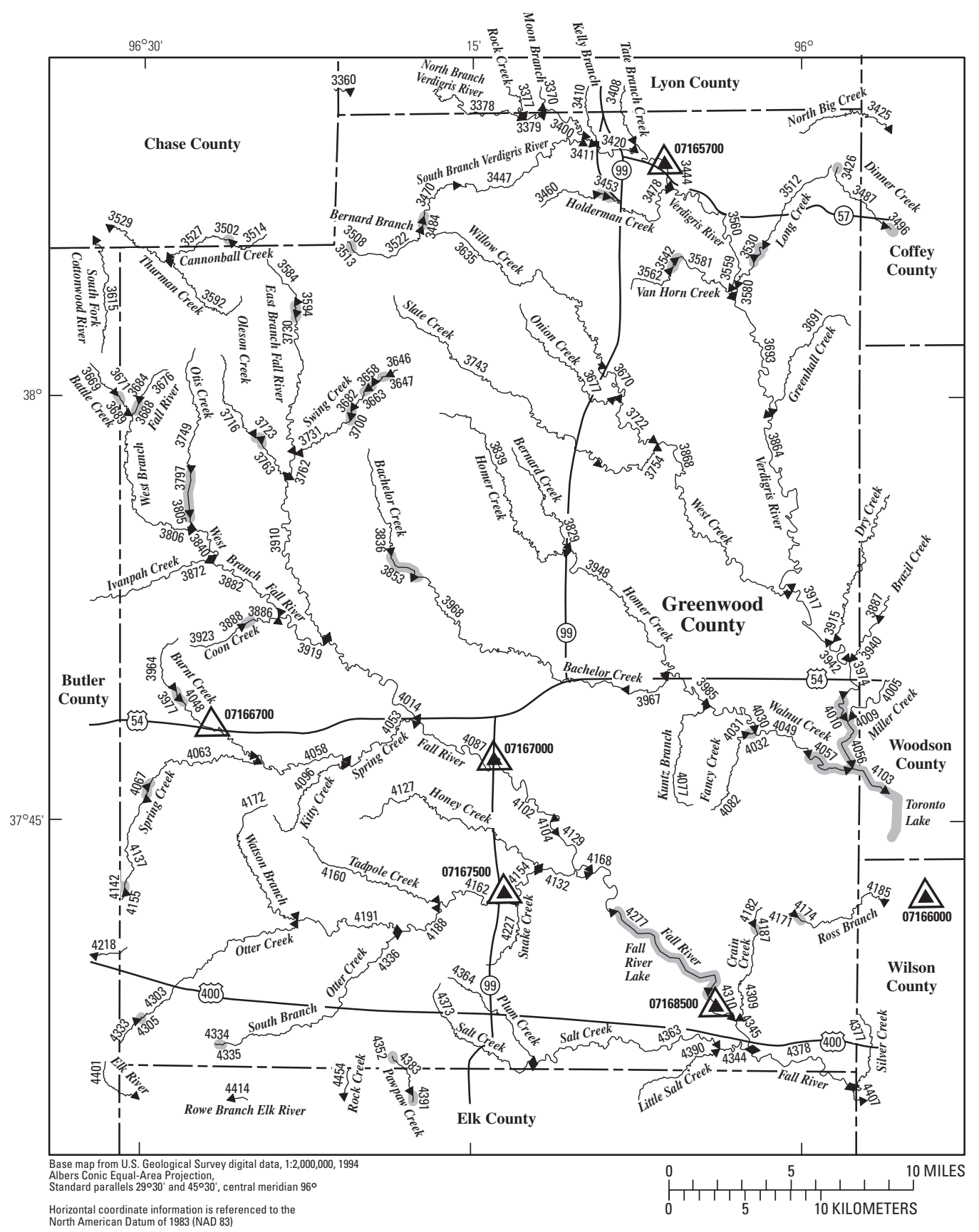

EXPLANATION

4414 Location of streamflow-statistics determination site (small triangle) and associated identification number-small triangle points in downstream direction

${ }^{07167500} \Delta$ U.S. Geological Survey streamflow-gaging station and number used for estimates of flow duration

${ }^{07166700} \triangle$ U.S. Geological Survey streamflow-gaging station and number used for estimates of peak-discharge frequency values

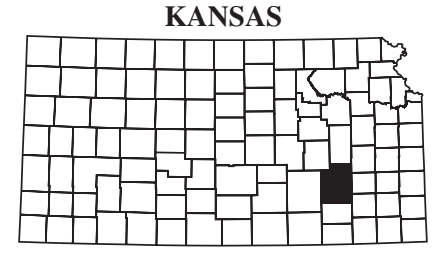

Index map 4391

Lake and determination site identification number

Figure 47. Location of streamflow-statistics determination sites, associated identification numbers, and U.S. Geological Survey streamflow-gaging stations used in the flow-duration and peak-discharge frequency analyses for Greenwood County. 
Table 43. Estimated flow-duration values, mean flow values, and peak-discharge frequency values for controlled and uncontrolled flow stream segments on the 1999 Kansas Surface Water Register for Greenwood County.

[KSWR, Kansas Surface Water Register; CUSEGA, catalog unit segment number alpha; $\mathrm{mi}^{2}$, square miles; $\mathrm{ft}^{3} / \mathrm{s}$, cubic feet per second; HYDRO, lake or other hydrologic structure; NA, not applicable; NRDitch, irrigation ditch; NRTribal, tribal stream]

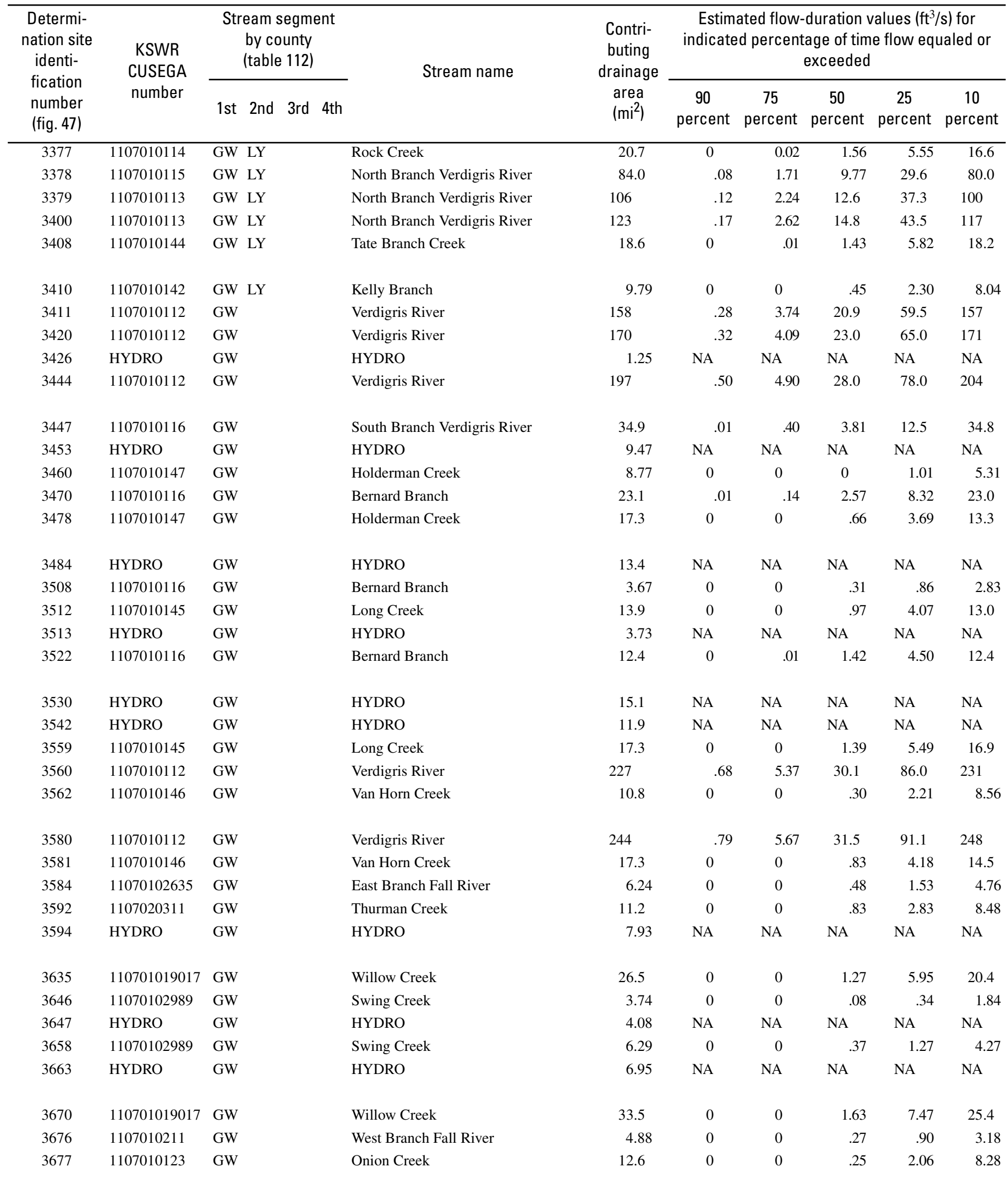


Table 43. Estimated flow-duration values, mean flow values, and peak-discharge frequency values for controlled and uncontrolled flow stream segments on the 1999 Kansas Surface Water Register for Greenwood County.-Continued

[KSWR, Kansas Surface Water Register; CUSEGA, catalog unit segment number alpha; $\mathrm{mi}^{2}$, square miles; $\mathrm{ft}^{3} / \mathrm{s}$, cubic feet per second; HYDRO, lake or other hydrologic structure; NA, not applicable; NRDitch, irrigation ditch; NRTribal, tribal stream]

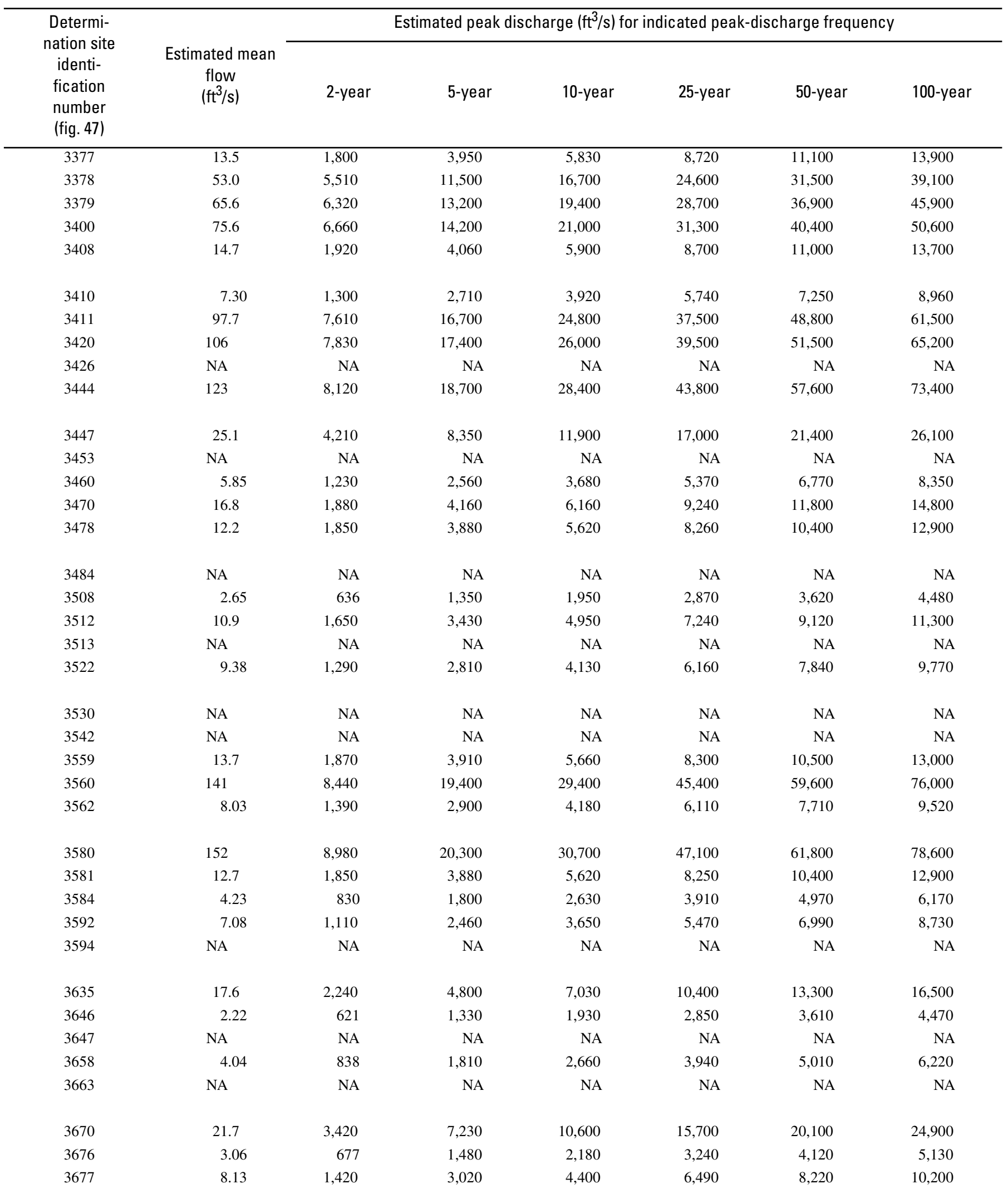


Table 43. Estimated flow-duration values, mean flow values, and peak-discharge frequency values for controlled and uncontrolled flow stream segments on the 1999 Kansas Surface Water Register for Greenwood County.-Continued

[KSWR, Kansas Surface Water Register; CUSEGA, catalog unit segment number alpha; $\mathrm{mi}^{2}$, square miles; $\mathrm{ft}^{3} / \mathrm{s}$, cubic feet per second; HYDRO, lake or other hydrologic structure; NA, not applicable; NRDitch, irrigation ditch; NRTribal, tribal stream]

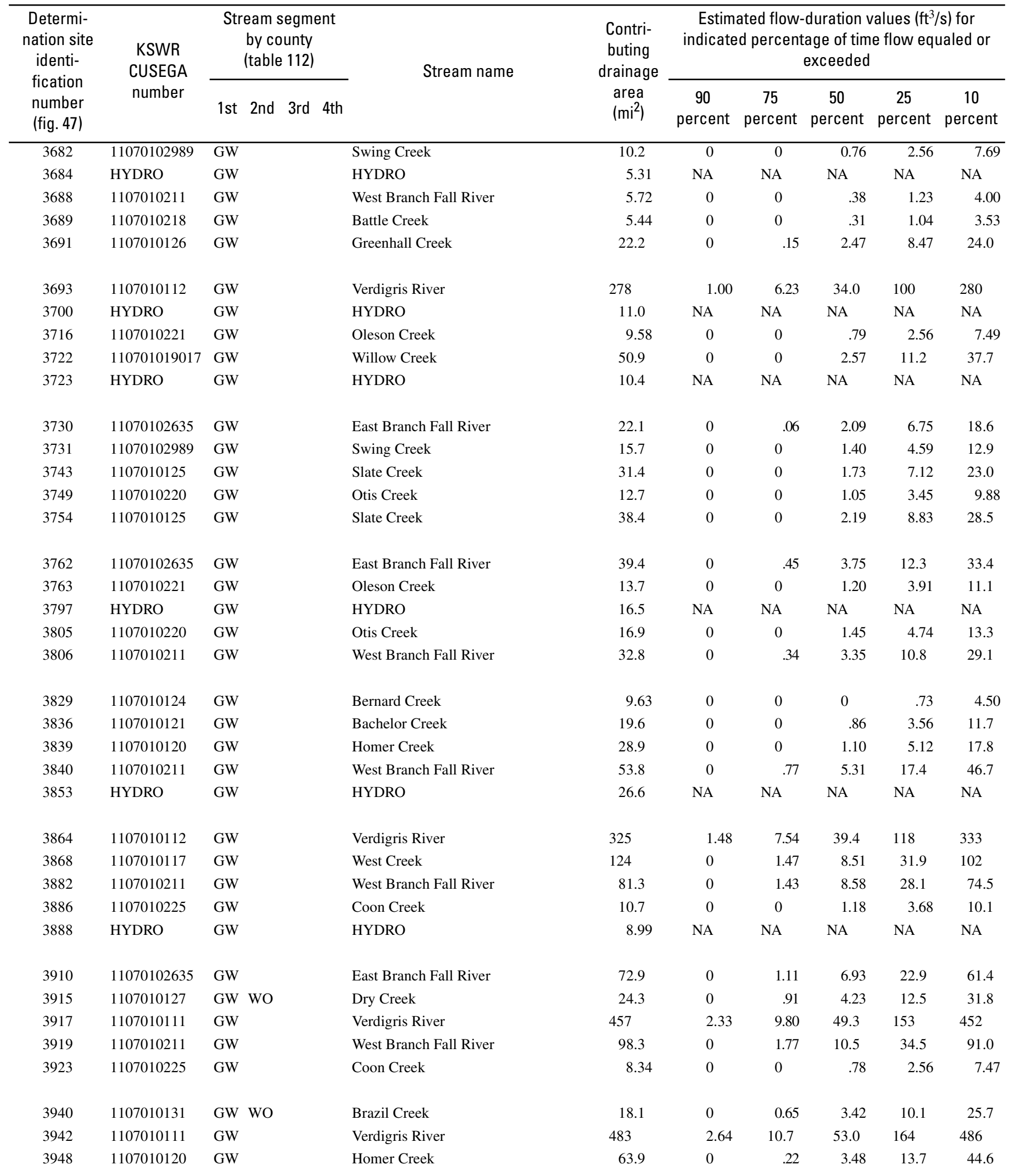


Table 43. Estimated flow-duration values, mean flow values, and peak-discharge frequency values for controlled and uncontrolled flow stream segments on the 1999 Kansas Surface Water Register for Greenwood County.-Continued

[KSWR, Kansas Surface Water Register; CUSEGA, catalog unit segment number alpha; $\mathrm{mi}^{2}$, square miles; $\mathrm{ft}^{3} / \mathrm{s}$, cubic feet per second; HYDRO, lake or other hydrologic structure; NA, not applicable; NRDitch, irrigation ditch; NRTribal, tribal stream]

\begin{tabular}{|c|c|c|c|c|c|c|c|}
\hline \multirow{2}{*}{$\begin{array}{l}\text { Determi- } \\
\text { nation site } \\
\text { identi- } \\
\text { fication } \\
\text { number } \\
\text { (fig. } 47 \text { ) }\end{array}$} & \multirow{2}{*}{$\begin{array}{c}\text { Estimated mean } \\
\text { flow } \\
\left(\mathrm{ft}^{3} / \mathrm{s}\right)\end{array}$} & \multicolumn{6}{|c|}{ Estimated peak discharge $\left(\mathrm{ft}^{3} / \mathrm{s}\right)$ for indicated peak-discharge frequency } \\
\hline & & 2-year & 5-year & 10 -year & 25-year & 50-year & 100 -year \\
\hline 3682 & 6.57 & 1,100 & 2,420 & 3,570 & 5,330 & 6,800 & 8,490 \\
\hline 3689 & 3.34 & 706 & 1,550 & 2,290 & 3,420 & 4,370 & 5,450 \\
\hline 3691 & 18.0 & 2,140 & 4,520 & 6,560 & 9,660 & 12,200 & 15,200 \\
\hline 3693 & 171 & 9,240 & 20,900 & 31,600 & 48,500 & 63,600 & 80,900 \\
\hline 3700 & NA & NA & NA & NA & NA & NA & NA \\
\hline 3730 & 14.2 & 1,720 & 3,860 & 5,770 & 8,720 & 11,200 & 14,100 \\
\hline 3731 & 10.2 & 1,400 & 3,120 & 4,640 & 6,980 & 8,950 & 11,200 \\
\hline 3743 & 19.2 & 3,540 & 7,330 & 10,600 & 15,600 & 19,800 & 24,400 \\
\hline 3749 & 8.02 & 1,200 & 2,670 & 3,970 & 5,980 & 7,660 & 9,590 \\
\hline 3754 & 23.4 & 3,690 & 7,680 & 11,200 & 16,400 & 21,000 & 25,900 \\
\hline 3762 & 24.3 & 3,620 & 7,520 & 10,900 & 16,100 & 20,500 & 25,300 \\
\hline 3763 & 8.82 & 1,270 & 2,830 & 4,210 & 6,330 & 8,110 & 10,200 \\
\hline 3797 & NA & NA & NA & NA & NA & NA & NA \\
\hline 3805 & 10.5 & 1,420 & 3,190 & 4,760 & 7,190 & 9,230 & 11,600 \\
\hline 3840 & 32.7 & 4,540 & 9,350 & 13,600 & 19,900 & 25,500 & 31,500 \\
\hline 3853 & NA & NA & NA & NA & NA & NA & NA \\
\hline 3864 & 201 & 9,110 & 20,800 & 31,500 & 48,500 & 63,800 & 81,300 \\
\hline 3868 & 75.0 & 5,970 & 12,100 & 17,600 & 25,700 & 32,800 & 40,500 \\
\hline 3882 & 49.9 & 5,390 & 11,300 & 16,600 & 24,700 & 31,900 & 39,800 \\
\hline 3886 & 7.89 & 1,180 & 2,570 & 3,780 & 5,630 & 7,170 & 8,940 \\
\hline 3888 & NA & NA & NA & NA & NA & NA & NA \\
\hline 3910 & 42.9 & 4,340 & 9,380 & 13,900 & 21,000 & 27,300 & 34,300 \\
\hline 3915 & 21.6 & 2,280 & 4,810 & 6,980 & 10,300 & 13,000 & 16,100 \\
\hline 3917 & 273 & 11,400 & 24,800 & 37,100 & 56,400 & 73,700 & 93,400 \\
\hline 3919 & 60.2 & 5,710 & 12,200 & 18,100 & 27,300 & 35,400 & 44,600 \\
\hline 3923 & 6.11 & 1,020 & 2,210 & 3,240 & 4,800 & 6,100 & 7,590 \\
\hline 3940 & 17.4 & 1,980 & 4,120 & 5,950 & 8,700 & 11,000 & 13,600 \\
\hline 3942 & 291 & 11,500 & 25,000 & 37,400 & 56,800 & 74,100 & 94,000 \\
\hline 3948 & 36.2 & 4,430 & 9,190 & 13,400 & 19,700 & 25,200 & 31,200 \\
\hline
\end{tabular}


Table 43. Estimated flow-duration values, mean flow values, and peak-discharge frequency values for controlled and uncontrolled flow stream segments on the 1999 Kansas Surface Water Register for Greenwood County.-Continued

[KSWR, Kansas Surface Water Register; CUSEGA, catalog unit segment number alpha; $\mathrm{mi}^{2}$, square miles; $\mathrm{ft}^{3} / \mathrm{s}$, cubic feet per second; HYDRO, lake or other hydrologic structure; NA, not applicable; NRDitch, irrigation ditch; NRTribal, tribal stream]

\begin{tabular}{|c|c|c|c|c|c|c|c|c|c|c|c|}
\hline \multirow{2}{*}{$\begin{array}{l}\text { Determi- } \\
\text { nation site } \\
\text { identi- } \\
\text { fication } \\
\text { number } \\
\text { (fig. } 47 \text { ) }\end{array}$} & \multirow{2}{*}{$\begin{array}{l}\text { KSWR } \\
\text { CUSEGA } \\
\text { number }\end{array}$} & \multicolumn{3}{|c|}{$\begin{array}{l}\text { Stream segment } \\
\text { by county } \\
\text { (table 112) }\end{array}$} & \multirow{2}{*}{ Stream name } & \multirow{2}{*}{$\begin{array}{l}\text { Contri- } \\
\text { buting } \\
\text { drainage } \\
\text { area } \\
\left(\mathrm{mi}^{2}\right)\end{array}$} & \multicolumn{5}{|c|}{$\begin{array}{l}\text { Estimated flow-duration values }\left(\mathrm{ft}^{3} / \mathrm{s}\right) \text { for } \\
\text { indicated percentage of time flow equaled or } \\
\text { exceeded }\end{array}$} \\
\hline & & $1 \mathrm{st}$ & 2nd 3rd & 4th & & & $\begin{array}{c}90 \\
\text { percent }\end{array}$ & $\begin{array}{c}75 \\
\text { percent }\end{array}$ & $\begin{array}{c}50 \\
\text { percent }\end{array}$ & $\begin{array}{c}25 \\
\text { percent }\end{array}$ & $\begin{array}{c}10 \\
\text { percent }\end{array}$ \\
\hline 3964 & 1107010224 & GW & & & Burnt Creek & 12.1 & 0 & 0 & 1.26 & 4.05 & 11.2 \\
\hline 3967 & 1107010121 & GW & & & Bachelor Creek & 74.6 & 0 & .36 & 4.09 & 16.1 & 52.1 \\
\hline 3968 & 1107010121 & GW & & & Bachelor Creek & 71.2 & 0 & .26 & 3.73 & 14.8 & 48.4 \\
\hline 3974 & 1107010111 & GW & & & Verdigris River & 504 & 2.87 & 11.3 & 55.8 & 173 & 514 \\
\hline 3977 & HYDRO & GW & & & HYDRO & 13.7 & NA & NA & NA & NA & NA \\
\hline 3985 & 1107010119 & GW & & & Walnut Creek & 143 & 0 & 1.41 & 8.16 & 31.0 & 101 \\
\hline 4005 & 1107010130 & GW & WO & & Miller Creek & 21.1 & 0 & .16 & 2.30 & 7.73 & 21.9 \\
\hline 4009 & HYDRO & GW & & & HYDRO & 21.1 & NA & NA & NA & NA & NA \\
\hline 4010 & HYDRO & GW & & & HYDRO & 506 & NA & NA & NA & NA & NA \\
\hline 4014 & 110701029 & $\mathrm{GW}$ & & & Fall River & 188 & .05 & 3.34 & 21.5 & 71.5 & 181 \\
\hline 4030 & 1107010119 & GW & & & Walnut Creek & 170 & 0 & 2.12 & 10.9 & 40.2 & 129 \\
\hline 4031 & 1107010128 & $\mathrm{GW}$ & & & Fancy Creek & 15.3 & 0 & .44 & 2.53 & 7.24 & 18.4 \\
\hline 4032 & HYDRO & $\mathrm{GW}$ & & & HYDRO & 15.3 & NA & NA & $\mathrm{NA}$ & NA & NA \\
\hline 4048 & 1107010224 & $\mathrm{GW}$ & & & Burnt Creek & 23.2 & 0 & 0 & 2.12 & 7.28 & 20.6 \\
\hline 4049 & 1107010119 & $\mathrm{GW}$ & & & Walnut Creek & 193 & .04 & 2.87 & 13.7 & 49.0 & 155 \\
\hline 4053 & 1107010212 & GW & & & Spring Creek & 85.3 & 0 & 1.42 & 8.99 & 30.6 & 83.1 \\
\hline 4056 & HYDRO & GW & & & HYDRO & 530 & NA & NA & NA & NA & NA \\
\hline 4057 & HYDRO & $\mathrm{GW}$ & & & HYDRO & 200 & NA & NA & NA & NA & NA \\
\hline 4058 & 1107010212 & $\mathrm{GW}$ & & & Spring Creek & 71.2 & 0 & 1.14 & 7.41 & 25.1 & 68.4 \\
\hline 4063 & 1107010212 & $\mathrm{GW}$ & & & Spring Creek & 37.5 & 0 & .54 & 4.27 & 14.1 & 38.2 \\
\hline 4067 & HYDRO & GW & & & HYDRO & 23.7 & NA & NA & NA & NA & NA \\
\hline 4077 & 1107010129 & GW & & & Kuntz Branch & 19.3 & 0 & .02 & 2.11 & 7.25 & 20.6 \\
\hline 4082 & 1107010128 & $\mathrm{GW}$ & & & Fancy Creek & 15.3 & 0 & .44 & 2.52 & 7.21 & 18.3 \\
\hline 4087 & 110701028 & $\mathrm{GW}$ & & & Fall River & 301 & .70 & 5.20 & 40.0 & 134 & 324 \\
\hline 4096 & 1107010227 & $\mathrm{GW}$ & & & Kitty Creek & 7.33 & 0 & 0 & .31 & 1.58 & 5.69 \\
\hline 4102 & 110701028 & GW & & & Fall River & 302 & .70 & 5.22 & 40.1 & 134 & 325 \\
\hline 4103 & HYDRO & $\mathrm{GW}$ & WO & & HYDRO & 745 & NA & NA & NA & NA & NA \\
\hline 4104 & 110701028 & $\mathrm{GW}$ & & & Fall River & 302 & .70 & 5.22 & 40.1 & 134 & 325 \\
\hline 4127 & 1107010226 & $\mathrm{GW}$ & & & Honey Creek & 22.9 & 0 & 0 & 1.01 & 4.96 & 17.3 \\
\hline 4129 & 110701028 & $\mathrm{GW}$ & & & Fall River & 306 & .75 & 5.33 & 40.5 & 136 & 329 \\
\hline 4132 & 1107010213 & GW & & & Otter Creek & 153 & 0 & 1.52 & 12.3 & 53.1 & 152 \\
\hline 4137 & 1107010212 & $\mathrm{GW}$ & & & Spring Creek & 21.4 & 0 & .13 & 2.44 & 7.96 & 21.7 \\
\hline 4142 & HYDRO & GW & & & HYDRO & 4.73 & NA & NA & NA & NA & NA \\
\hline 4154 & 1107010213 & $\mathrm{GW}$ & & & Otter Creek & 127 & 0 & 1.19 & 10.8 & 47.2 & 132 \\
\hline 4155 & 1107010212 & GW & & & Spring Creek & 4.62 & 0 & 0 & .42 & 1.28 & 3.98 \\
\hline 4160 & 1107010229 & GW & & & Tadpole Creek & 14.6 & 0 & 0 & .44 & 2.82 & 10.3 \\
\hline 4162 & 1107010213 & $\mathrm{GW}$ & & & Otter Creek & 113 & 0 & 1.00 & 9.95 & 44.0 & 121 \\
\hline 4168 & 110701027 & GW & & & Fall River & 471 & 1.81 & 8.34 & 54.0 & 183 & 486 \\
\hline
\end{tabular}


Table 43. Estimated flow-duration values, mean flow values, and peak-discharge frequency values for controlled and uncontrolled flow stream segments on the 1999 Kansas Surface Water Register for Greenwood County.-Continued

[KSWR, Kansas Surface Water Register; CUSEGA, catalog unit segment number alpha; $\mathrm{mi}^{2}$, square miles; $\mathrm{ft}^{3} / \mathrm{s}$, cubic feet per second; HYDRO, lake or other hydrologic structure; NA, not applicable; NRDitch, irrigation ditch; NRTribal, tribal stream]

\begin{tabular}{|c|c|c|c|c|c|c|c|}
\hline \multirow{2}{*}{$\begin{array}{l}\text { Determi- } \\
\text { nation site } \\
\text { identi- } \\
\text { fication } \\
\text { number } \\
\text { (fig. 47) }\end{array}$} & \multirow{2}{*}{$\begin{array}{c}\text { Estimated mean } \\
\text { flow } \\
\left(\mathrm{ft}^{3} / \mathrm{s}\right)\end{array}$} & \multicolumn{6}{|c|}{ Estimated peak discharge $\left(\mathrm{ft}^{3} / \mathrm{s}\right)$ for indicated peak-discharge frequency } \\
\hline & & 2-year & 5-year & 10 -year & 25-year & 50-year & 100 -year \\
\hline 3964 & 8.69 & 1,180 & 2,720 & 4,140 & 6,430 & 8,470 & 10,900 \\
\hline 3974 & 305 & 11,500 & 25,100 & 37,500 & 57,000 & 74,300 & 94,300 \\
\hline 3977 & NA & NA & NA & NA & NA & NA & NA \\
\hline 3985 & 76.7 & 7,460 & 14,800 & 21,100 & 30,600 & 38,800 & 47,800 \\
\hline 4005 & 16.8 & 2,150 & 4,490 & 6,500 & 9,530 & 12,000 & 14,900 \\
\hline 4030 & 94.1 & 7,970 & 15,700 & 22,300 & 32,300 & 40,800 & 50,100 \\
\hline 4031 & 13.0 & 1,640 & 3,480 & 5,060 & 7,460 & 9,440 & 11,700 \\
\hline 4032 & NA & NA & NA & NA & NA & NA & NA \\
\hline 4048 & 15.7 & 1,630 & 4,100 & 6,570 & 10,800 & 14,800 & 19,600 \\
\hline 4049 & 109 & 8,290 & 16,100 & 23,000 & 33,100 & 41,800 & 51,300 \\
\hline 4053 & 56.4 & 6,230 & 14,100 & 21,700 & 34,100 & 45,500 & 58,900 \\
\hline 4056 & NA & NA & NA & NA & NA & NA & NA \\
\hline 4057 & NA & NA & NA & NA & NA & NA & NA \\
\hline 4058 & 47.0 & 5,780 & 12,900 & 19,700 & 30,700 & 40,900 & 52,800 \\
\hline 4102 & 191 & 11,600 & 29,800 & 48,000 & 79,000 & 108,000 & 143,000 \\
\hline 4103 & NA & NA & NA & NA & NA & NA & NA \\
\hline 4104 & 191 & 11,600 & 29,800 & 48,000 & 79,000 & 108,000 & 143,000 \\
\hline 4127 & 15.3 & 2,080 & 4,450 & 6,490 & 9,600 & 12,200 & 15,100 \\
\hline 4129 & 193 & 11,500 & 29,600 & 47,700 & 78,700 & 107,000 & 142,000 \\
\hline 4132 & 104 & 8,270 & 19,600 & 29,700 & 45,300 & 58,700 & 73,300 \\
\hline 4137 & 15.8 & 1,780 & 3,940 & 5,850 & 8,790 & 11,300 & 14,100 \\
\hline 4142 & NA & NA & NA & NA & NA & NA & NA \\
\hline 4154 & 89.5 & 7,640 & 18,400 & 28,000 & 42,800 & 55,500 & 69,300 \\
\hline 4155 & 3.49 & 735 & 1,560 & 2,270 & 3,330 & 4,220 & 5,220 \\
\hline 4160 & 9.61 & 1,560 & 3,380 & 4,960 & 7,360 & 9,360 & 11,600 \\
\hline 4162 & 82.0 & 7,480 & 18,000 & 27,400 & 41,800 & 54,200 & 67,600 \\
\hline 4168 & 285 & 14,600 & 35,200 & 55,400 & 89,300 & 121,000 & 159,000 \\
\hline
\end{tabular}


Table 43. Estimated flow-duration values, mean flow values, and peak-discharge frequency values for controlled and uncontrolled flow stream segments on the 1999 Kansas Surface Water Register for Greenwood County.-Continued

[KSWR, Kansas Surface Water Register; CUSEGA, catalog unit segment number alpha; $\mathrm{mi}^{2}$, square miles; $\mathrm{ft}^{3} / \mathrm{s}$, cubic feet per second; HYDRO, lake or other hydrologic structure; NA, not applicable; NRDitch, irrigation ditch; NRTribal, tribal stream]

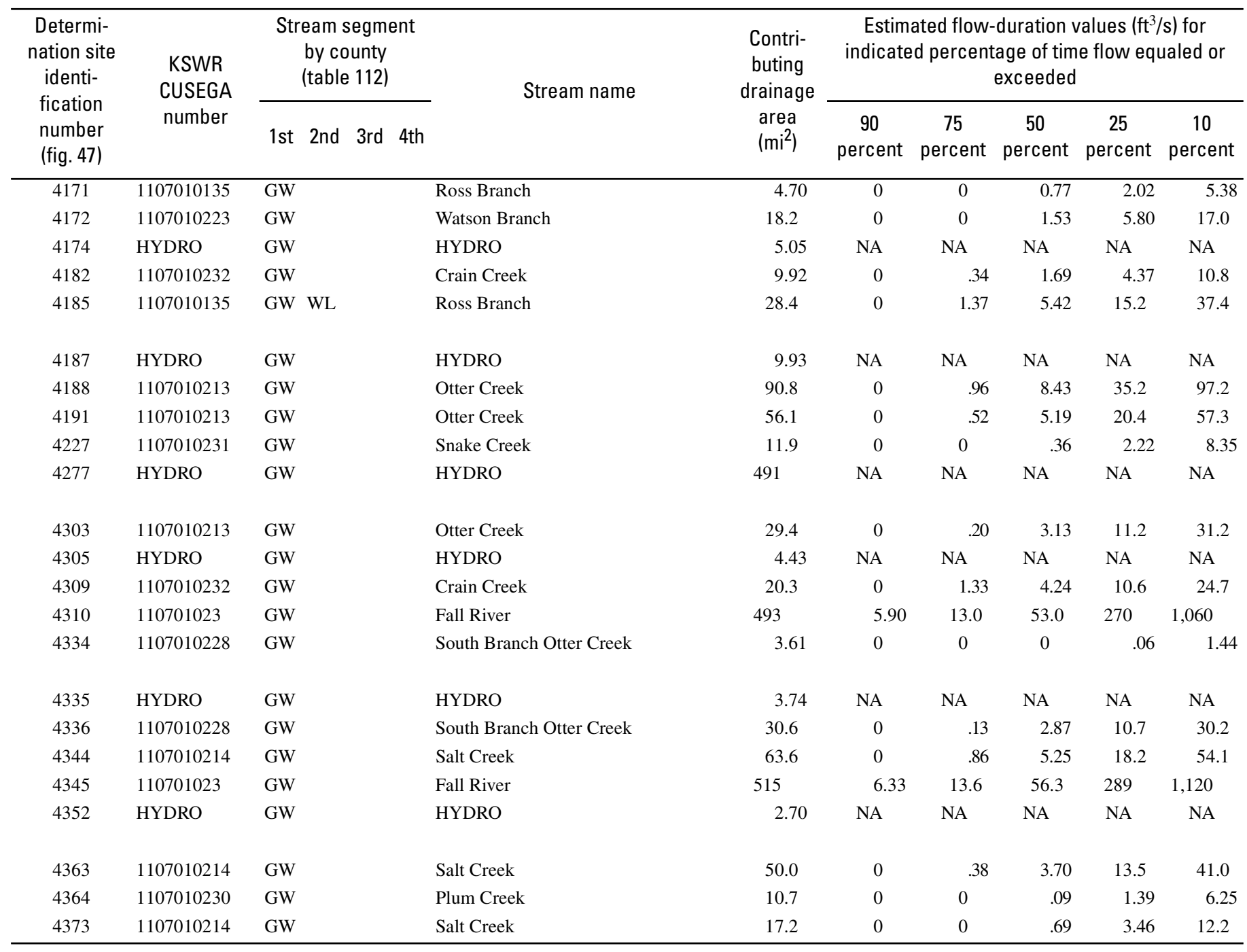


Table 43. Estimated flow-duration values, mean flow values, and peak-discharge frequency values for controlled and uncontrolled flow stream segments on the 1999 Kansas Surface Water Register for Greenwood County.-Continued

[KSWR, Kansas Surface Water Register; CUSEGA, catalog unit segment number alpha; $\mathrm{mi}^{2}$, square miles; $\mathrm{ft}^{3} / \mathrm{s}$, cubic feet per second; HYDRO, lake or other hydrologic structure; NA, not applicable; NRDitch, irrigation ditch; NRTribal, tribal stream]

\begin{tabular}{|c|c|c|c|c|c|c|c|}
\hline \multirow{2}{*}{$\begin{array}{l}\text { Determi- } \\
\text { nation site } \\
\text { identi- } \\
\text { fication } \\
\text { number } \\
\text { (fig. 47) }\end{array}$} & \multirow{2}{*}{$\begin{array}{c}\text { Estimated mean } \\
\text { flow } \\
\left(\mathrm{ft}^{3} / \mathrm{s}\right)\end{array}$} & \multicolumn{6}{|c|}{ Estimated peak discharge $\left(\mathrm{ft}^{3} / \mathrm{s}\right)$ for indicated peak-discharge frequency } \\
\hline & & 2-year & 5 -year & 10-year & 25-year & 50-year & 100-year \\
\hline 4171 & 4.20 & 821 & 1,700 & 2,440 & 3,550 & 4,470 & 5,510 \\
\hline 4172 & 13.3 & 1,700 & 3,760 & 5,560 & 8,320 & 10,600 & 13,300 \\
\hline 4174 & NA & NA & NA & NA & NA & NA & NA \\
\hline 4182 & 7.99 & 1,240 & 2,610 & 3,790 & 5,580 & 7,060 & 8,750 \\
\hline 4185 & 24.4 & 2,360 & 5,050 & 7,390 & 11,000 & 13,900 & 17,300 \\
\hline 4187 & NA & NA & NA & NA & NA & NA & NA \\
\hline 4188 & 66.2 & 7,300 & 16,200 & 24,000 & 35,800 & 45,900 & 56,700 \\
\hline 4191 & 40.5 & 5,860 & 12,100 & 17,500 & 25,600 & 32,400 & 39,700 \\
\hline 4227 & 7.93 & 1,370 & 2,920 & 4,240 & 6,250 & 7,920 & 9,820 \\
\hline 4277 & NA & NA & NA & NA & NA & NA & NA \\
\hline 4303 & 22.4 & 2,210 & 5,080 & 7,620 & 11,500 & 14,800 & 18,600 \\
\hline 4305 & NA & NA & NA & NA & NA & NA & NA \\
\hline 4309 & 16.4 & 1,840 & 3,970 & 5,820 & 8,640 & 11,000 & 13,700 \\
\hline 4310 & 361 & 3,980 & 6,910 & 8,730 & 10,800 & 12,100 & 13,300 \\
\hline 4334 & 2.12 & 659 & 1,380 & 2,000 & 2,920 & 3,680 & 4,550 \\
\hline 4335 & NA & NA & NA & NA & NA & NA & NA \\
\hline 4336 & 22.5 & 4,110 & 8,320 & 11,900 & 17,200 & 21,600 & 26,400 \\
\hline 4344 & 40.2 & 4,660 & 9,310 & 13,300 & 19,300 & 24,300 & 29,800 \\
\hline 4345 & 377 & 4,470 & 7,760 & 9,830 & 12,200 & 13,700 & 15,100 \\
\hline 4352 & NA & NA & NA & NA & NA & NA & NA \\
\hline 4363 & 31.7 & 4,170 & 8,460 & 12,200 & 17,700 & 22,400 & 27,500 \\
\hline 4364 & 6.58 & 1,290 & 2,720 & 3,960 & 5,830 & 7,380 & 9,150 \\
\hline 4373 & 11.1 & 1,710 & 3,650 & 5,330 & 7,880 & 10,000 & 12,400 \\
\hline
\end{tabular}




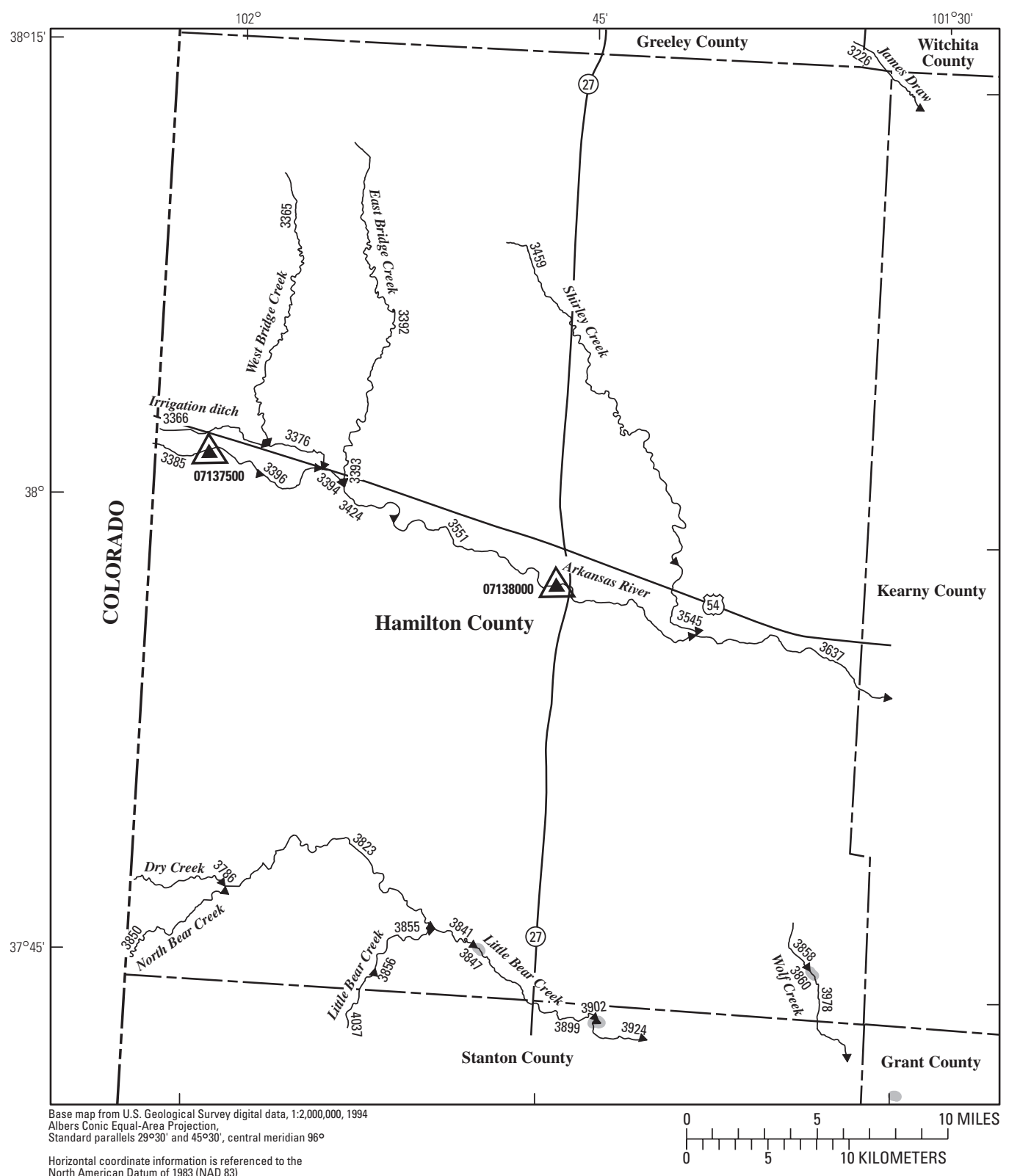

EXPLANATION

$\$ 3850$ Location of streamflow-statistics determination site (small triangle) and associated identification number-small triangle points in downstream direction

$07137500 \Delta$ U.S. Geological Survey streamflow-gaging station and number used for estimates of flow duration

${ }^{07138000} \triangle$ U.S. Geological Survey streamflow-gaging station and number used for estimates of peak-discharge frequency values

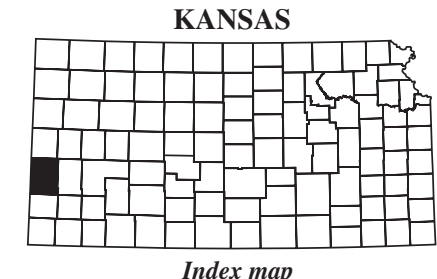
3847

Lake and determination site identification number

Figure 48. Location of streamflow-statistics determination sites, associated identification numbers, and U.S. Geological Survey streamflow-gaging stations used in the flow-duration and peak-discharge frequency analyses for Hamilton County. 
Table 44. Estimated flow-duration values, mean flow values, and peak-discharge frequency values for controlled and uncontrolled flow stream segments on the 1999 Kansas Surface Water Register for Hamilton County.

[KSWR, Kansas Surface Water Register; CUSEGA, catalog unit segment number alpha; $\mathrm{mi}^{2}$, square miles; $\mathrm{ft}^{3} / \mathrm{s}$, cubic feet per second; HYDRO, lake or other hydrologic structure; NA, not applicable; NRDitch, irrigation ditch; NRTribal, tribal stream]

\begin{tabular}{|c|c|c|c|c|c|c|c|c|c|c|c|}
\hline $\begin{array}{l}\text { Determi- } \\
\text { nation site } \\
\text { identi- } \\
\text { fication } \\
\text { number } \\
\text { (fig. 48) }\end{array}$ & $\begin{array}{l}\text { KSWR } \\
\text { CUSEGA } \\
\text { number }\end{array}$ & \multicolumn{3}{|c|}{$\begin{array}{l}\text { Stream segment } \\
\text { by county } \\
\text { (table 112) }\end{array}$} & Stream name & $\begin{array}{l}\text { Contri- } \\
\text { buting } \\
\text { drainage } \\
\text { area } \\
\left(\mathrm{mi}^{2}\right)\end{array}$ & \multicolumn{5}{|c|}{$\begin{array}{c}\text { Estimated flow-duration values }\left(\mathrm{ft}^{3} / \mathrm{s}\right) \text { for } \\
\text { indicated percentage of time flow equaled or } \\
\text { exceeded }\end{array}$} \\
\hline 3365 & 110300018 & HM & & & West Bridge Creek & 197 & 0 & 0 & 0 & 0 & 0 \\
\hline 3385 & 110300019 & HM & & & Arkansas River & 25,000 & 9.30 & 47.0 & 129 & 242 & 482 \\
\hline 3392 & 110300016 & HM & & & East Bridge Creek & 174 & 0 & 0 & 0 & 0 & 0 \\
\hline 3393 & NRDitch & HM & & & NRDitch & 174 & 0 & 0 & 0 & 0 & 0 \\
\hline 3394 & 110300017 & HM & & & Arkansas River & 25,300 & 8.09 & 48.3 & 131 & 245 & 478 \\
\hline 3545 & 110300014 & HM & & & Shirley Creek & 213 & 0 & 0 & 0 & 0 & 0 \\
\hline 3551 & 110300015 & $\mathrm{HM}$ & & & Arkansas River & 25,600 & 6.50 & 50.0 & 133 & 250 & 474 \\
\hline 3637 & 110300013 & HM & $\mathrm{KE}$ & & Arkansas River & 25,900 & 4.96 & 38.4 & 109 & 229 & 436 \\
\hline 3786 & 110400055 & HM & & & Dry Creek & 67.0 & 0 & 0 & 0 & 0 & 0 \\
\hline 3823 & 110400056 & $\mathrm{HM}$ & & & North Bear Creek & 233 & 0 & 0 & 0 & 0 & 0 \\
\hline 3841 & 110400057 & HM & & & Little Bear Creek & 457 & 0 & 0 & 0 & 0 & 0 \\
\hline 3847 & HYDRO & HM & & & HYDRO & 466 & NA & NA & NA & NA & NA \\
\hline 3850 & 110400056 & HM & & & North Bear Creek & 66.4 & 0 & 0 & 0 & 0 & 0 \\
\hline 3855 & 110400057 & HM & & & Little Bear Creek & 208 & 0 & 0 & 0 & 0 & 0 \\
\hline
\end{tabular}


Table 44. Estimated flow-duration values, mean flow values, and peak-discharge frequency values for controlled and uncontrolled flow stream segments on the 1999 Kansas Surface Water Register for Hamilton County.-Continued

[KSWR, Kansas Surface Water Register; CUSEGA, catalog unit segment number alpha; $\mathrm{mi}^{2}$, square miles; $\mathrm{ft}^{3} / \mathrm{s}$, cubic feet per second; HYDRO, lake or other hydrologic structure; NA, not applicable; NRDitch, irrigation ditch; NRTribal, tribal stream]

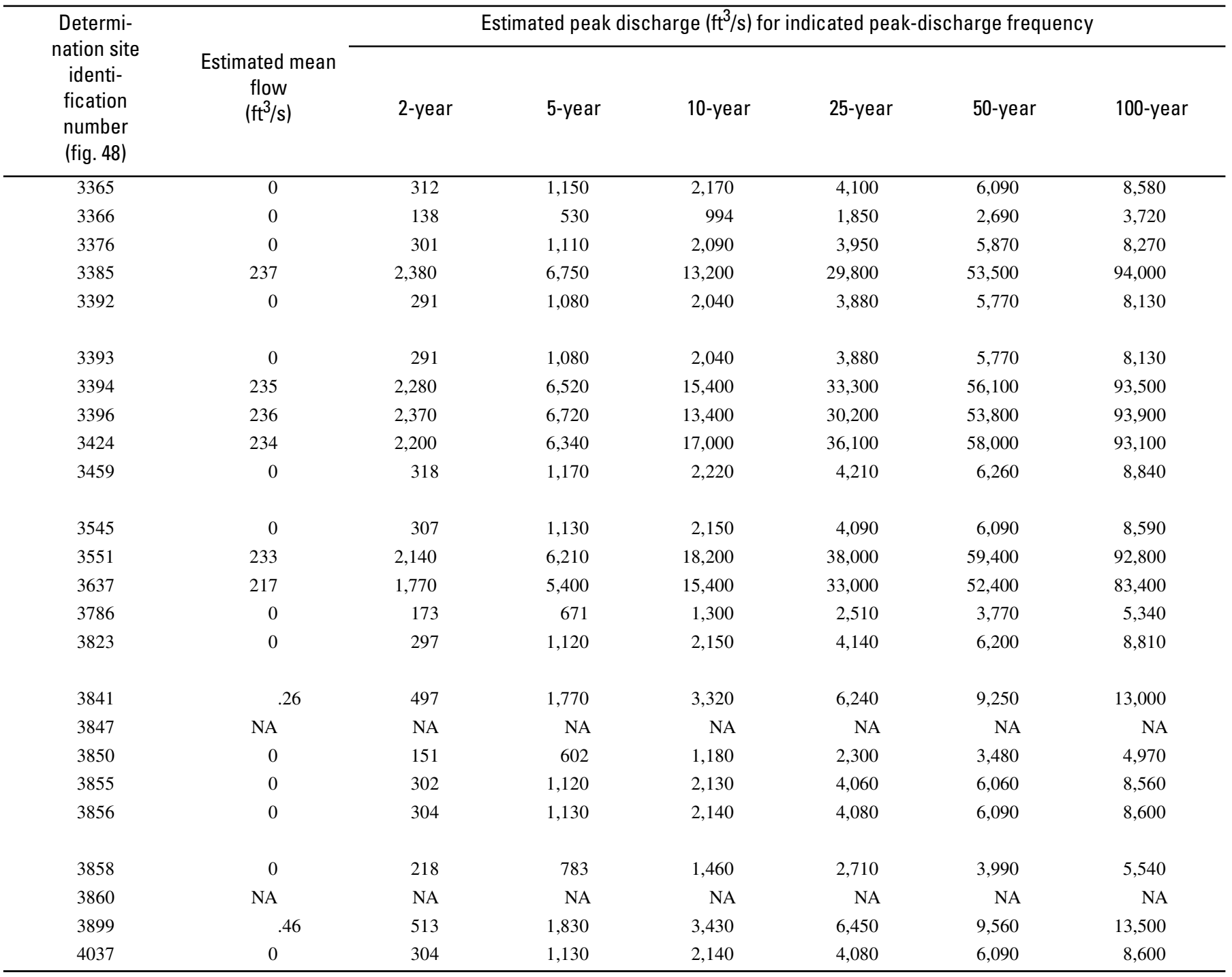




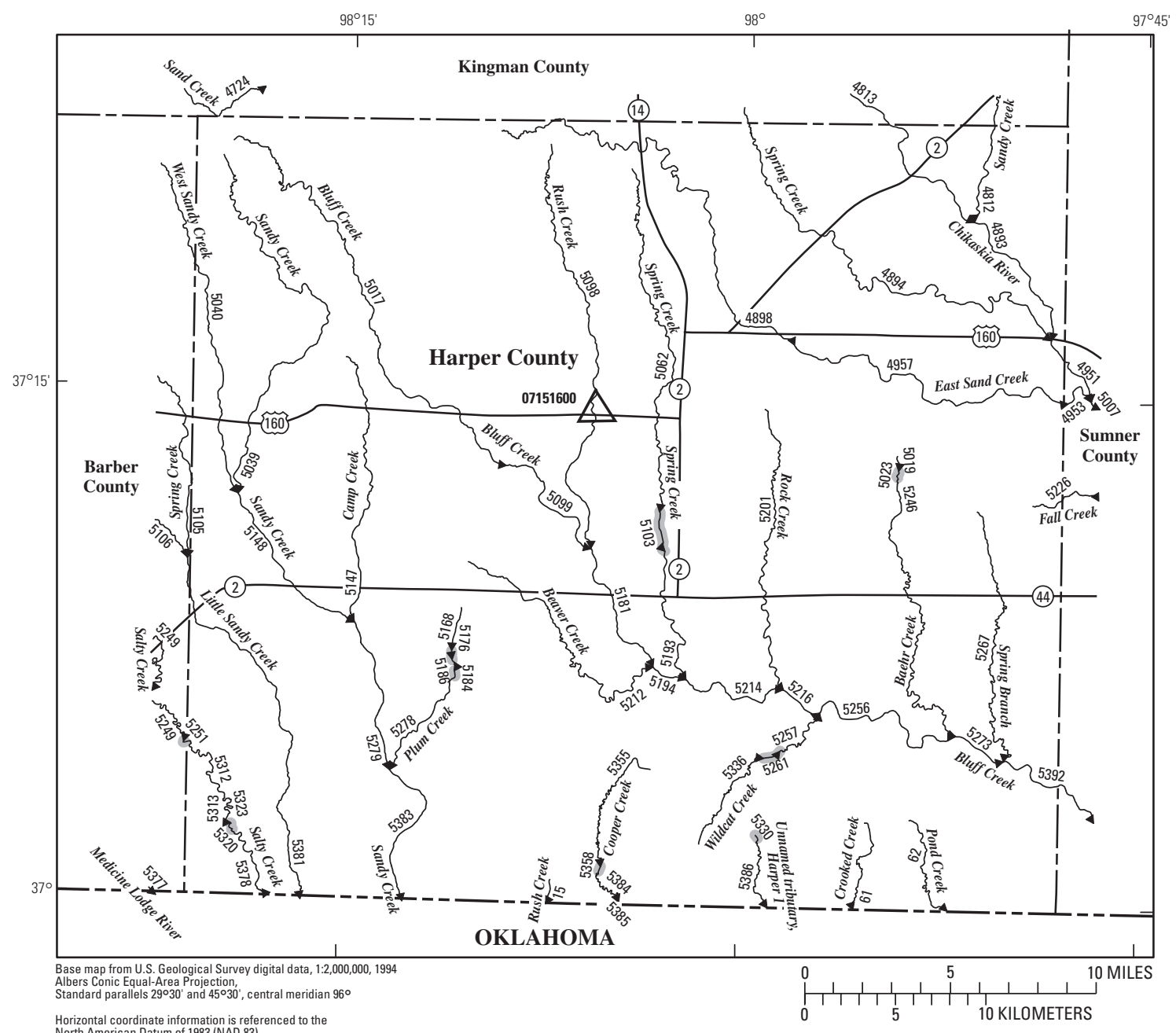

EXPLANATION

45378 Location of streamflow-statistics determination site (small triangle) and associated identification number-small triangle points in downstream direction

07151600 U.S. Geological Survey streamflow-gaging station and number used for estimates of flow duration

${ }^{07151600} \triangle$ U.S. Geological Survey streamflow-gaging station and number used for estimates of peak-discharge frequency values

KANSAS

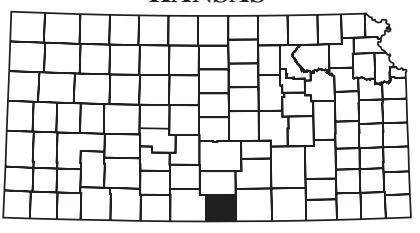

Index map 5320

Lake and determination site identification number

Figure 49. Location of streamflow-statistics determination sites, associated identification numbers, and U.S. Geological Survey streamflow-gaging stations used in the flow-duration and peak-discharge frequency analyses for Harper County. 
Table 45. Estimated flow-duration values, mean flow values, and peak-discharge frequency values for controlled and uncontrolled flow stream segments on the 1999 Kansas Surface Water Register for Harper County.

[KSWR, Kansas Surface Water Register; CUSEGA, catalog unit segment number alpha; $\mathrm{mi}^{2}$, square miles; $\mathrm{ft}^{3} / \mathrm{s}$, cubic feet per second; HYDRO, lake or other hydrologic structure; NA, not applicable; NRDitch, irrigation ditch; NRTribal, tribal stream]

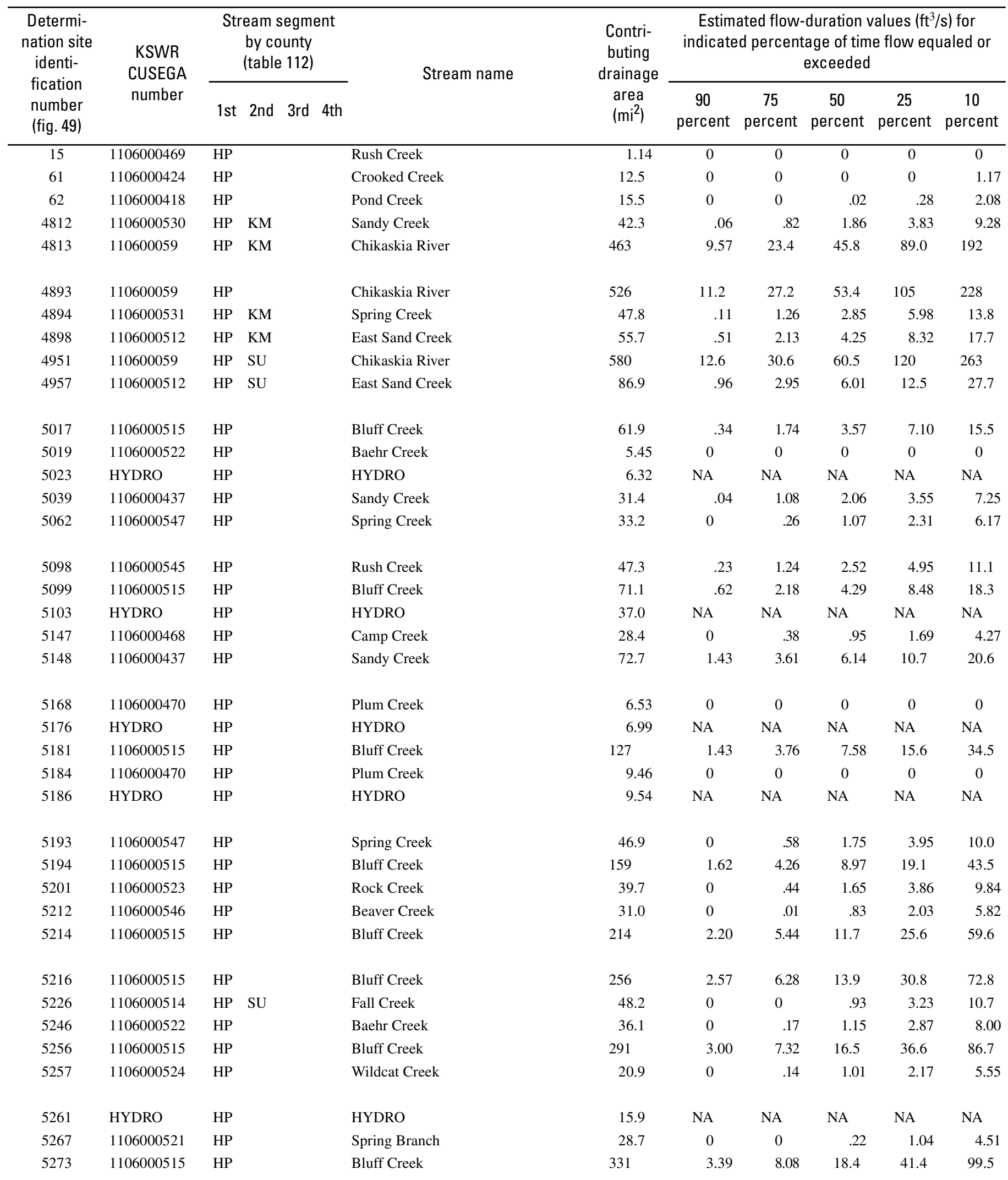


Table 45. Estimated flow-duration values, mean flow values, and peak-discharge frequency values for controlled and uncontrolled flow stream segments on the 1999 Kansas Surface Water Register for Harper County.-Continued

[KSWR, Kansas Surface Water Register; CUSEGA, catalog unit segment number alpha; $\mathrm{mi}^{2}$, square miles; $\mathrm{ft}^{3} / \mathrm{s}$, cubic feet per second; HYDRO, lake or other hydrologic structure; NA, not applicable; NRDitch, irrigation ditch; NRTribal, tribal stream]

\begin{tabular}{|c|c|c|c|c|c|c|c|}
\hline \multirow{2}{*}{$\begin{array}{l}\text { Determi- } \\
\text { nation site } \\
\text { identi- } \\
\text { fication } \\
\text { number } \\
\text { (fig. 49) }\end{array}$} & \multirow{2}{*}{$\begin{array}{c}\text { Estimated mean } \\
\text { flow } \\
\left(\mathrm{ft}^{3} / \mathrm{s}\right)\end{array}$} & \multicolumn{6}{|c|}{ Estimated peak discharge $\left(\mathrm{ft}^{3} / \mathrm{s}\right)$ for indicated peak-discharge frequency } \\
\hline & & 2-year & 5-year & 10-year & 25-year & 50-year & 100-year \\
\hline 15 & 0 & 181 & 430 & 654 & 1,000 & 1,300 & 1,640 \\
\hline 4812 & 8.84 & 1,570 & 3,470 & 5,160 & 7,700 & 9,900 & 12,300 \\
\hline 4813 & 115 & 4,290 & 9,510 & 14,100 & 21,000 & 26,900 & 33,400 \\
\hline 4893 & 136 & 5,030 & 11,100 & 16,300 & 24,200 & 31,000 & 38,300 \\
\hline 4894 & 11.6 & 1,320 & 3,050 & 4,650 & 7,090 & 9,240 & 11,600 \\
\hline 5017 & 12.7 & 1,510 & 3,390 & 5,110 & 7,720 & 9,990 & 12,500 \\
\hline 5019 & .21 & 473 & 1,150 & 1,760 & 2,730 & 3,570 & 4,520 \\
\hline 5023 & NA & NA & NA & NA & NA & NA & NA \\
\hline 5039 & 6.44 & 980 & 2,270 & 3,460 & 5,270 & 6,850 & 8,570 \\
\hline 5062 & 6.61 & 1,080 & 2,570 & 3,950 & 6,100 & 8,000 & 10,100 \\
\hline 5098 & 9.79 & 1,190 & 2,290 & 3,140 & 4,310 & 5,250 & 6,220 \\
\hline 5099 & 14.6 & 1,550 & 3,480 & 5,240 & 7,920 & 10,200 & 12,800 \\
\hline 5103 & NA & NA & NA & NA & NA & NA & NA \\
\hline 5147 & 4.99 & 1,110 & 2,860 & 4,530 & 7,220 & 9,560 & 12,300 \\
\hline 5193 & 9.61 & 1,210 & 2,880 & 4,450 & 6,900 & 9,060 & 11,500 \\
\hline 5194 & 32.2 & 2,370 & 4,750 & 6,740 & 9,650 & 12,100 & 14,800 \\
\hline 5201 & 9.16 & 1,150 & 2,770 & 4,300 & 6,690 & 8,820 & 11,200 \\
\hline 5212 & 6.36 & 855 & 2,160 & 3,420 & 5,440 & 7,260 & 9,310 \\
\hline 5214 & 43.1 & 2,850 & 5,740 & 8,200 & 11,800 & 14,900 & 18,200 \\
\hline 5216 & 51.8 & 3,230 & 6,520 & 9,330 & 13,500 & 17,000 & 20,900 \\
\hline 5226 & 11.2 & 1,350 & 3,350 & 5,300 & 8,420 & 11,300 & 14,500 \\
\hline 5246 & 8.16 & 1,050 & 2,570 & 4,020 & 6,310 & 8,350 & 10,600 \\
\hline 5256 & 60.2 & 3,320 & 6,730 & 9,690 & 14,100 & 17,800 & 22,000 \\
\hline 5257 & 5.44 & 1,020 & 2,550 & 3,980 & 6,270 & 8,240 & 10,500 \\
\hline 5261 & NA & NA & NA & NA & NA & NA & NA \\
\hline 5267 & 6.00 & 1,310 & 3,240 & 5,040 & 7,920 & 10,400 & 13,300 \\
\hline 5273 & 68.6 & 3,630 & 7,370 & 10,600 & 15,400 & 19,600 & 24,200 \\
\hline
\end{tabular}




\section{Estimates of Flow Duration, Mean Flow, and Peak-Discharge Frequency Values for Kansas Stream Locations}

Table 45. Estimated flow-duration values, mean flow values, and peak-discharge frequency values for controlled and uncontrolled flow stream segments on the 1999 Kansas Surface Water Register for Harper County.-Continued

[KSWR, Kansas Surface Water Register; CUSEGA, catalog unit segment number alpha; $\mathrm{mi}^{2}$, square miles; $\mathrm{ft}^{3} / \mathrm{s}$, cubic feet per second; HYDRO, lake or other hydrologic structure; NA, not applicable; NRDitch, irrigation ditch; NRTribal, tribal stream]

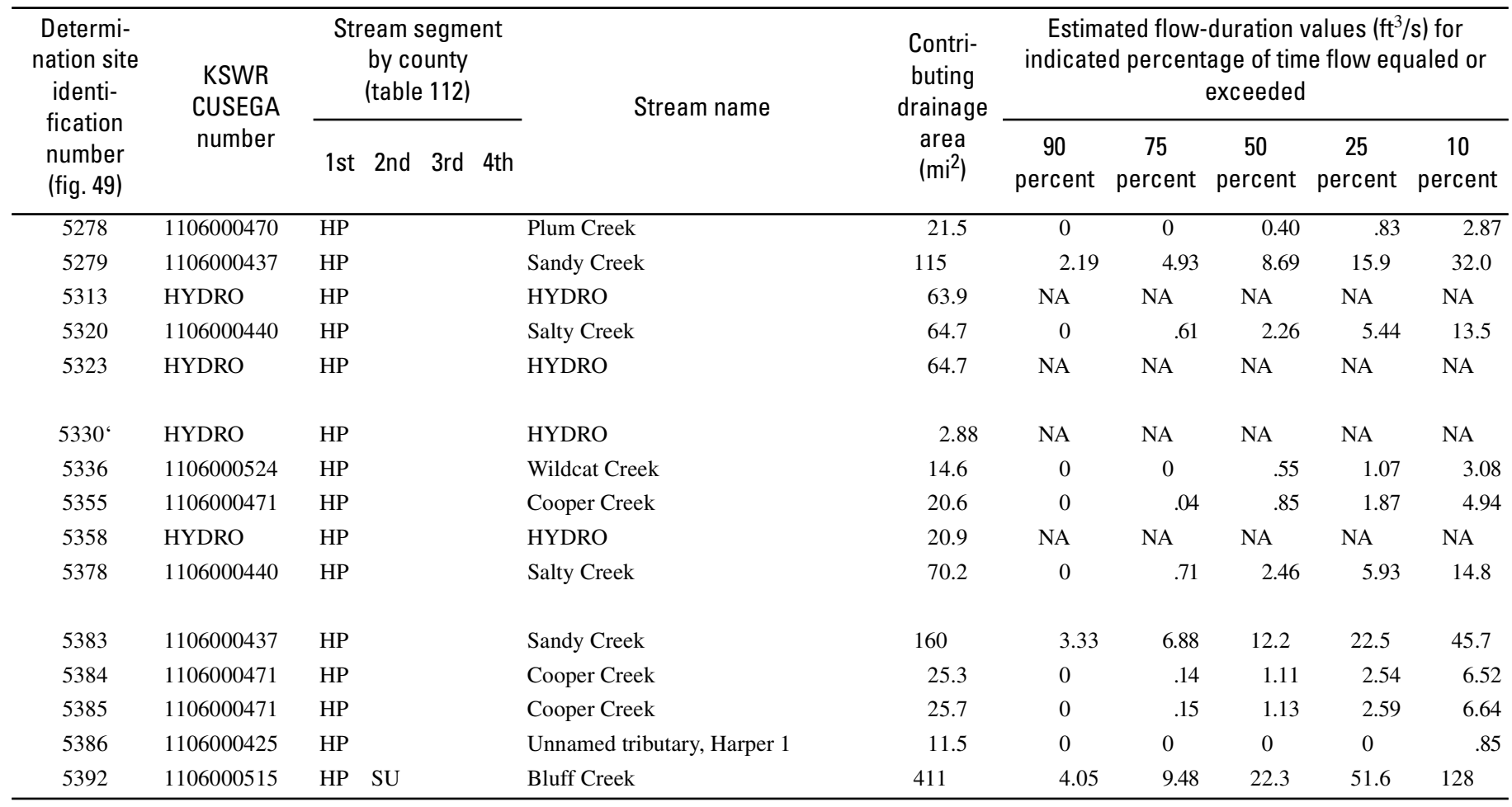


Table 45. Estimated flow-duration values, mean flow values, and peak-discharge frequency values for controlled and uncontrolled flow stream segments on the 1999 Kansas Surface Water Register for Harper County.-Continued

[KSWR, Kansas Surface Water Register; CUSEGA, catalog unit segment number alpha; $\mathrm{mi}^{2}$, square miles; $\mathrm{ft}^{3} / \mathrm{s}$, cubic feet per second; HYDRO, lake or other hydrologic structure; NA, not applicable; NRDitch, irrigation ditch; NRTribal, tribal stream]

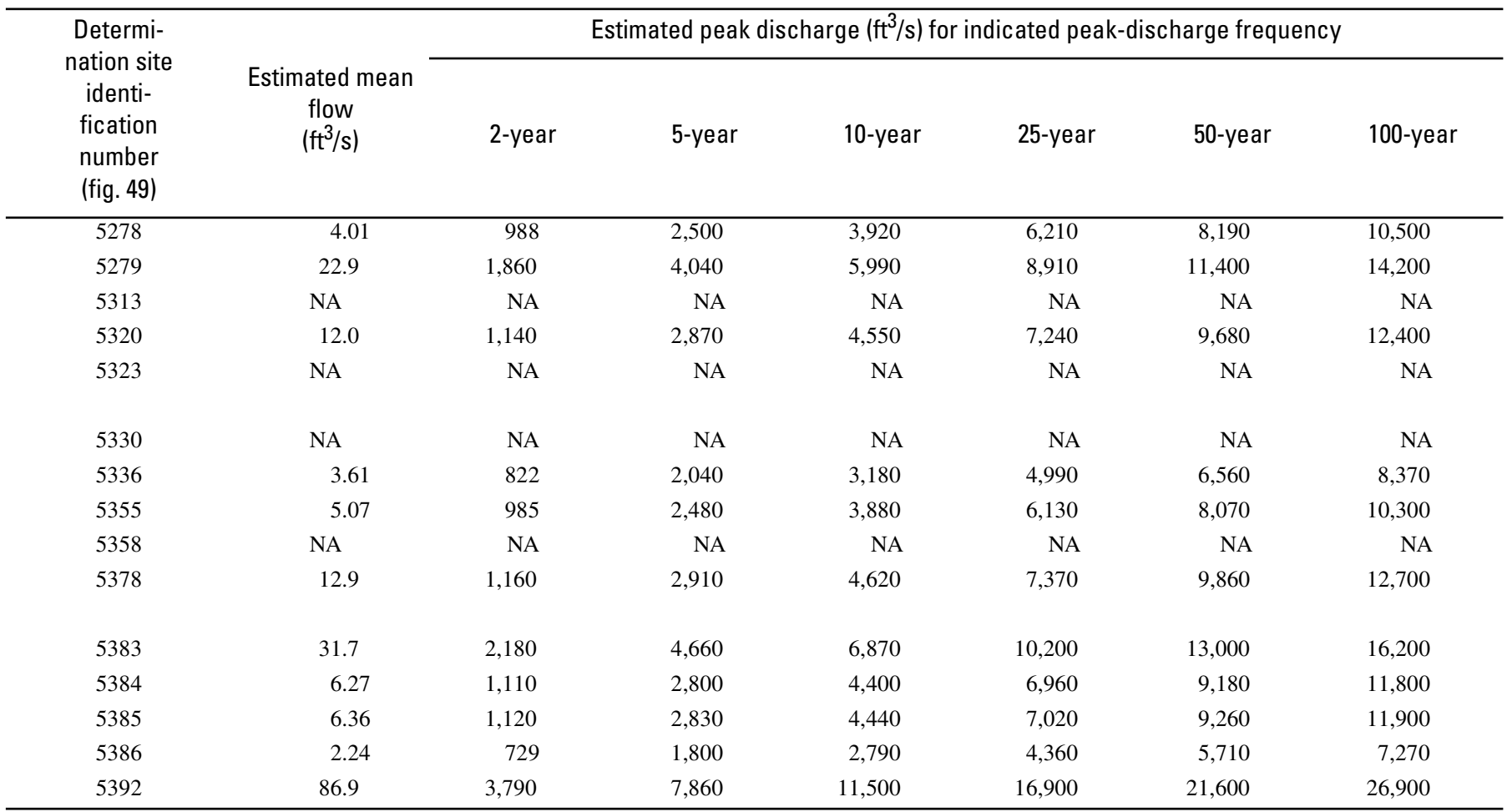




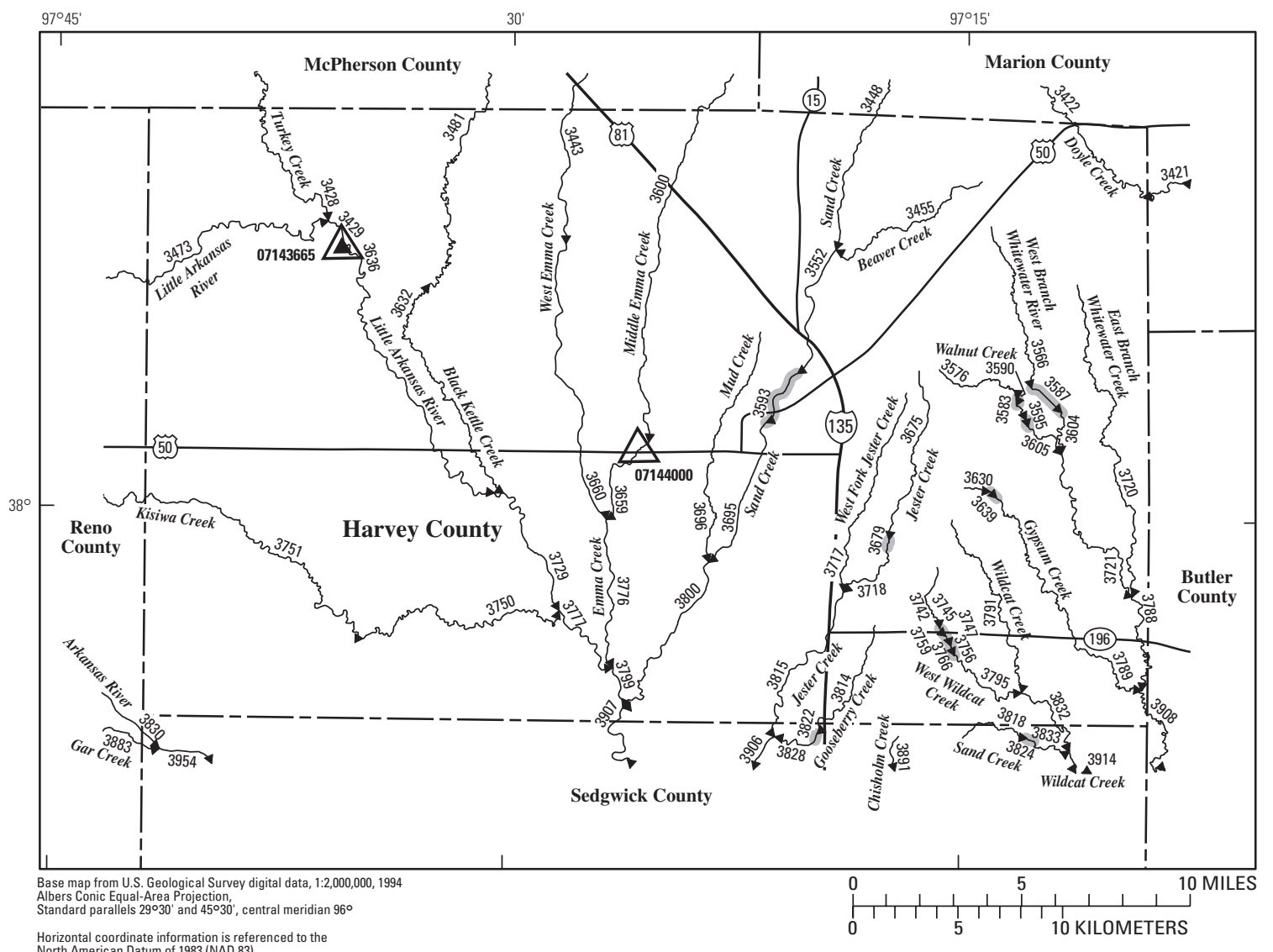

EXPLANATION

«954 Location of streamflow-statistics determination site (small triangle) and associated identification number-small triangle points in downstream direction

$07143665 \Delta$ U.S. Geological Survey streamflow-gaging station and number used for estimates of flow duration

07144000 U.S. Geological Survey streamflow-gaging station and number used for estimates of peak-discharge frequency values

KANSAS

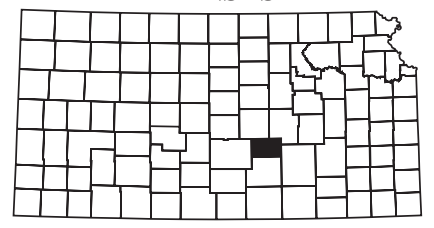

Index map 3824

Lake and determination site identification number

Figure 50. Location of streamflow-statistics determination sites, associated identification numbers, and U.S. Geological Survey streamflow-gaging stations used in the flow-duration and peak-discharge frequency analyses for Harvey County. 
Table 46. Estimated flow-duration values, mean flow values, and peak-discharge frequency values for controlled and uncontrolled flow stream segments on the 1999 Kansas Surface Water Register for Harvey County.

[KSWR, Kansas Surface Water Register; CUSEGA, catalog unit segment number alpha; $\mathrm{mi}^{2}$, square miles; $\mathrm{ft}^{3} / \mathrm{s}$, cubic feet per second; HYDRO, lake or other hydrologic structure; NA, not applicable; NRDitch, irrigation ditch; NRTribal, tribal stream]

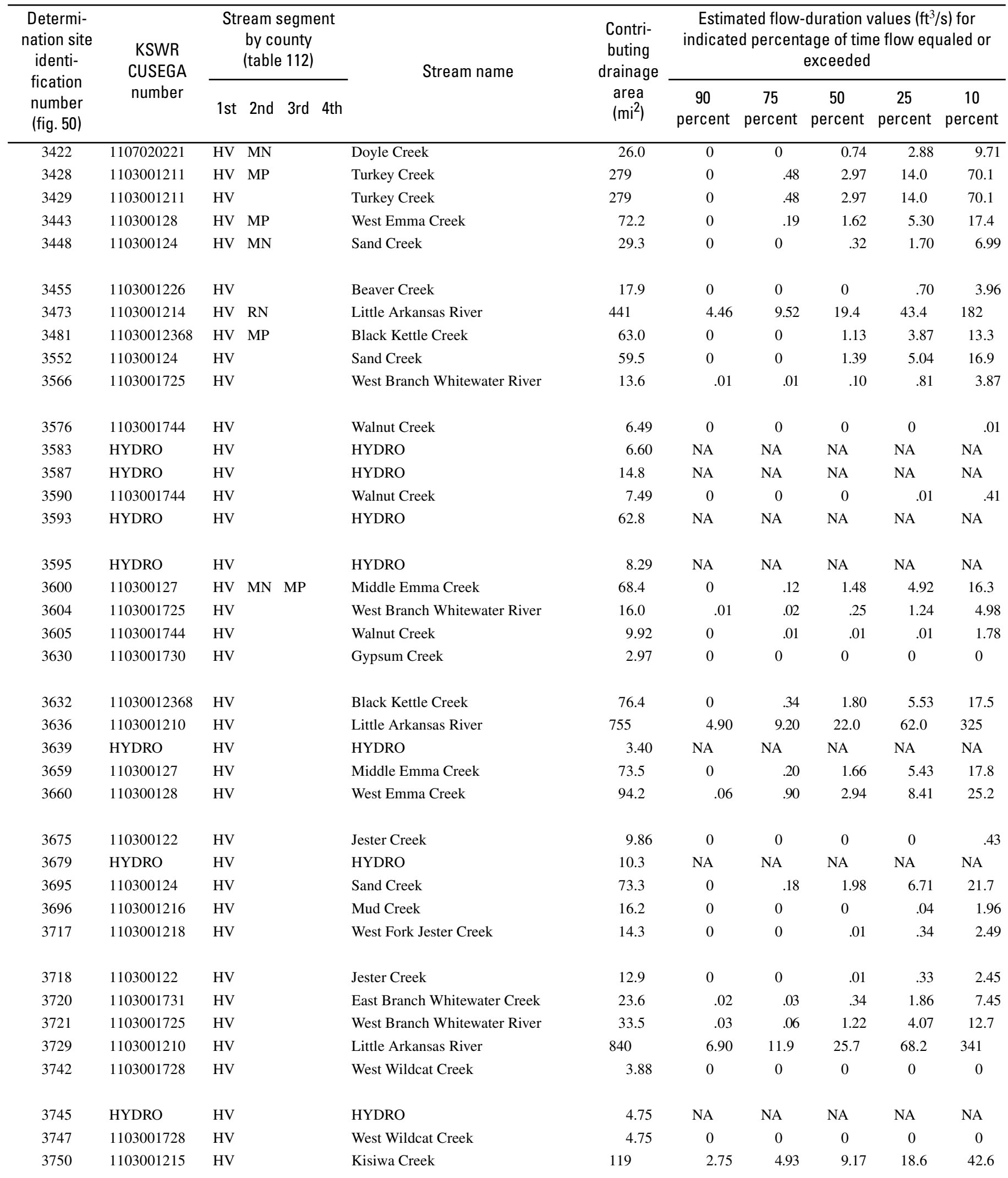


Table 46. Estimated flow-duration values, mean flow values, and peak-discharge frequency values for controlled and uncontrolled flow stream segments on the 1999 Kansas Surface Water Register for Harvey County.-Continued

[KSWR, Kansas Surface Water Register; CUSEGA, catalog unit segment number alpha; $\mathrm{mi}^{2}$, square miles; $\mathrm{ft}^{3} / \mathrm{s}$, cubic feet per second; HYDRO, lake or other hydrologic structure; NA, not applicable; NRDitch, irrigation ditch; NRTribal, tribal stream]

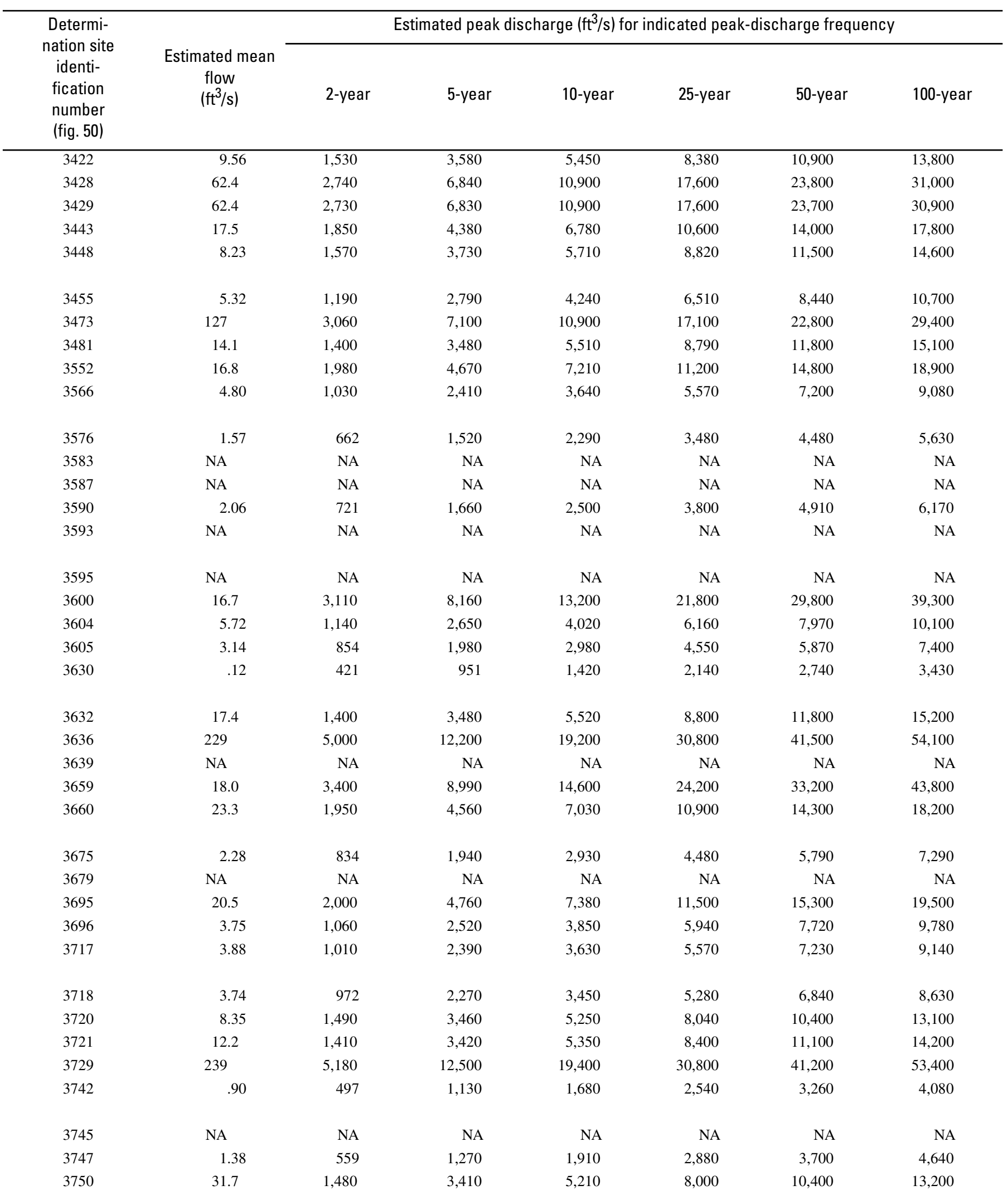


Table 46. Estimated flow-duration values, mean flow values, and peak-discharge frequency values for controlled and uncontrolled flow stream segments on the 1999 Kansas Surface Water Register for Harvey County.-Continued

[KSWR, Kansas Surface Water Register; CUSEGA, catalog unit segment number alpha; $\mathrm{mi}^{2}$, square miles; $\mathrm{ft}^{3} / \mathrm{s}$, cubic feet per second; HYDRO, lake or other hydrologic structure; NA, not applicable; NRDitch, irrigation ditch; NRTribal, tribal stream]

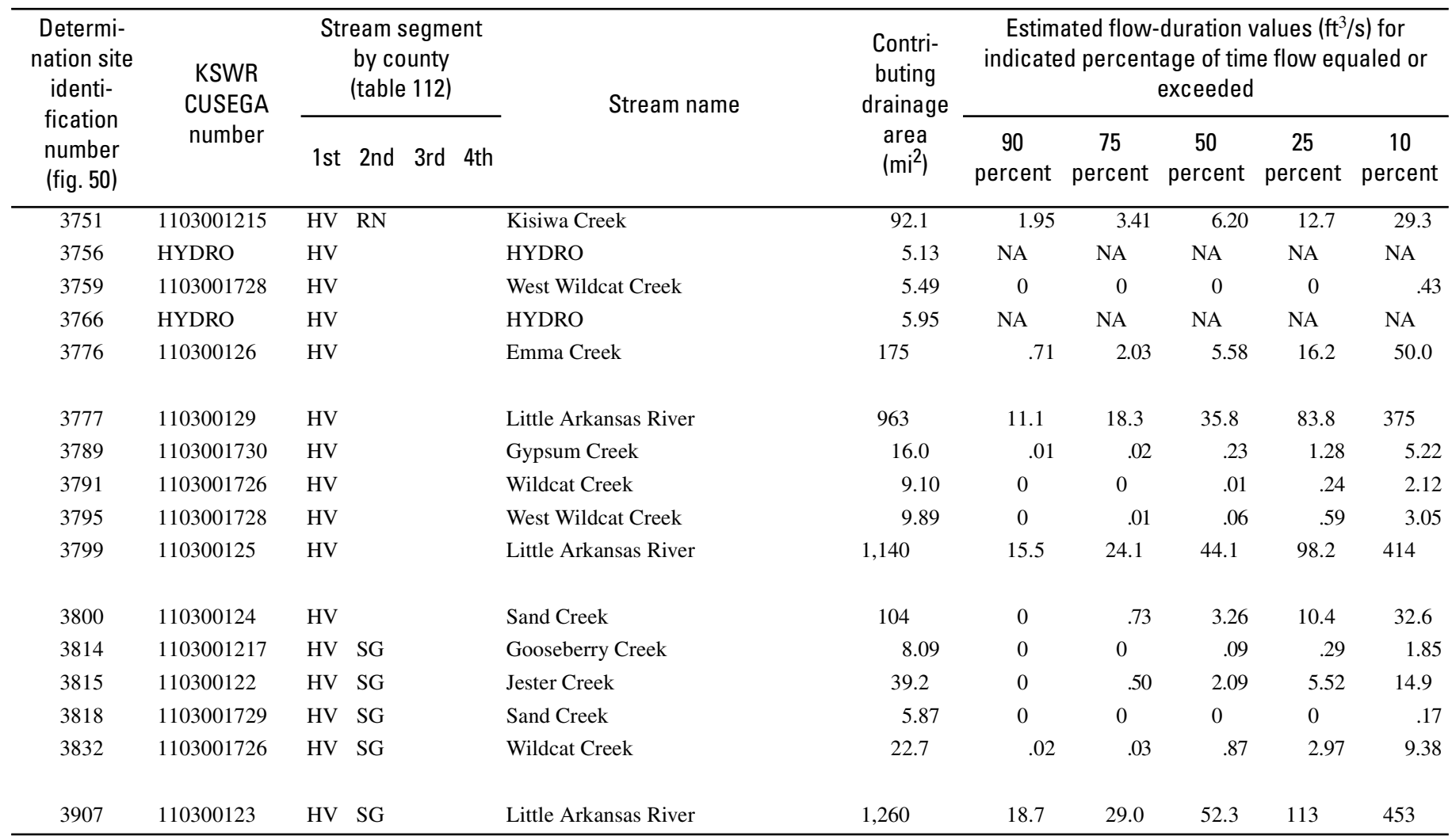


Table 46. Estimated flow-duration values, mean flow values, and peak-discharge frequency values for controlled and uncontrolled flow stream segments on the 1999 Kansas Surface Water Register for Harvey County.-Continued

[KSWR, Kansas Surface Water Register; CUSEGA, catalog unit segment number alpha; $\mathrm{mi}^{2}$, square miles; $\mathrm{ft}^{3} / \mathrm{s}$, cubic feet per second; HYDRO, lake or other hydrologic structure; NA, not applicable; NRDitch, irrigation ditch; NRTribal, tribal stream]

\begin{tabular}{|c|c|c|c|c|c|c|c|}
\hline \multirow{2}{*}{$\begin{array}{l}\text { Determi- } \\
\text { nation site } \\
\text { identi- } \\
\text { fication } \\
\text { number } \\
\text { (fig. 50) }\end{array}$} & \multirow{2}{*}{$\begin{array}{c}\text { Estimated mean } \\
\text { flow } \\
\left(\mathrm{ft}^{3} / \mathrm{s}\right)\end{array}$} & \multicolumn{6}{|c|}{ Estimated peak discharge $\left(\mathrm{ft}^{3} / \mathrm{s}\right)$ for indicated peak-discharge frequency } \\
\hline & & 2-year & 5-year & 10-year & 25-year & 50-year & 100 -year \\
\hline 3751 & 23.1 & 1,270 & 2,980 & 4,580 & 7,080 & 9,280 & 11,700 \\
\hline 3766 & NA & NA & NA & NA & NA & NA & NA \\
\hline 3776 & 43.1 & 4,330 & 10,900 & 17,200 & 27,900 & 37,800 & 49,300 \\
\hline 3777 & 255 & 5,390 & 12,700 & 19,400 & 30,200 & 40,000 & 51,100 \\
\hline 3789 & 5.93 & 1,160 & 2,690 & 4,070 & 6,220 & 8,050 & 10,200 \\
\hline 3800 & 29.0 & 2,370 & 5,540 & 8,550 & 13,300 & 17,600 & 22,400 \\
\hline 3814 & 2.76 & 752 & 1,740 & 2,620 & 3,980 & 5,140 & 6,470 \\
\hline 3815 & 13.1 & 1,630 & 3,700 & 5,600 & 8,520 & 11,100 & 13,900 \\
\hline 3818 & 1.71 & 644 & 1,470 & 2,190 & 3,320 & 4,270 & 5,350 \\
\hline 3832 & 8.95 & 1,400 & 3,300 & 5,010 & 7,700 & 9,980 & 12,600 \\
\hline 3907 & 296 & 6,170 & 13,800 & 20,400 & 30,400 & 39,000 & 48,500 \\
\hline
\end{tabular}




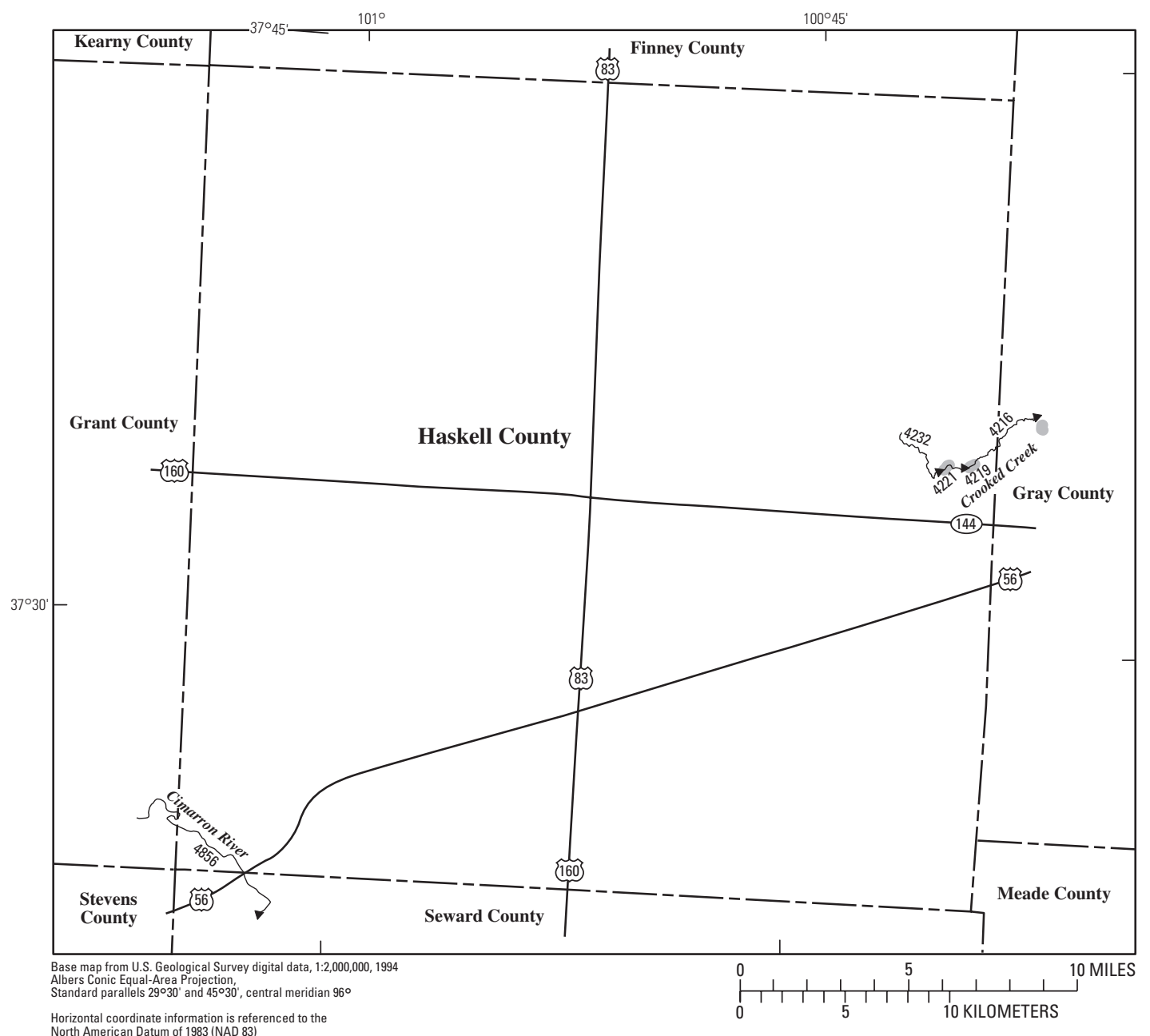

EXPLANATION

4856 Location of streamflow-statistics determination site (small triangle) and associated identification number-small triangle points in downstream direction

${ }^{07139500} \Delta$ U.S. Geological Survey streamflow-gaging station and number used for estimates of flow duration

${ }^{07139800} \triangle$ U.S. Geological Survey streamflow-gaging station and number used for estimates of peak-discharge frequency values

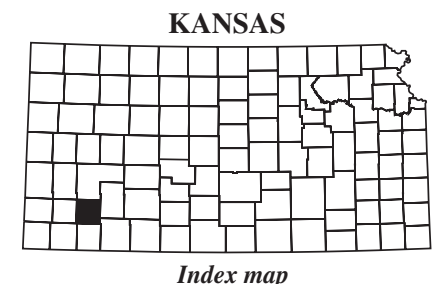

4221 Lake and determination site identification number

Figure 51. Location of streamflow-statistics determination sites, associated identification numbers, and U.S. Geological Survey streamflow-gaging stations used in the flow-duration and peak-discharge frequency analyses for Haskell County. 
Table 47. Estimated flow-duration values, mean flow values, and peak-discharge frequency values for controlled and uncontrolled flow stream segments on the 1999 Kansas Surface Water Register for Haskell County.

[KSWR, Kansas Surface Water Register; CUSEGA, catalog unit segment number alpha; $\mathrm{mi}^{2}$, square miles; $\mathrm{ft}^{3} / \mathrm{s}$, cubic feet per second; HYDRO, lake or other hydrologic structure; NA, not applicable; NRDitch, irrigation ditch;NRTribal, tribal stream]

\begin{tabular}{|c|c|c|c|c|c|c|c|c|c|}
\hline $\begin{array}{l}\text { Determi- } \\
\text { nation site } \\
\text { identi- } \\
\text { fication } \\
\text { number } \\
\text { (fig. 51) }\end{array}$ & $\begin{array}{l}\text { KSWR } \\
\text { CUSEGA } \\
\text { number }\end{array}$ & $\begin{array}{l}\text { Stream segment } \\
\text { by county } \\
\text { (table 112) }\end{array}$ & Stream name & $\begin{array}{l}\text { Contri- } \\
\text { buting } \\
\text { drainage } \\
\text { area } \\
\left(\mathrm{mi}^{2}\right)\end{array}$ & \multicolumn{5}{|c|}{$\begin{array}{l}\text { Estimated flow-duration values }\left(\mathrm{ft}^{3} / \mathrm{s}\right) \text { for } \\
\text { indicated percentage of time flow equaled or } \\
\text { exceeded }\end{array}$} \\
\hline 4219 & HYDRO & $\mathrm{HS}$ & HYDRO & 310 & NA & NA & NA & $\overline{\mathrm{NA}}$ & NA \\
\hline 4232 & 110400072 & HS & Crooked Creek & 302 & 0 & 0 & .10 & .18 & .36 \\
\hline
\end{tabular}


Table 47. Estimated flow-duration values, mean flow values, and peak-discharge frequency values for controlled and uncontrolled flow stream segments on the 1999 Kansas Surface Water Register for Haskell County.-Continued

[KSWR, Kansas Surface Water Register; CUSEGA, catalog unit segment number alpha; $\mathrm{mi}^{2}$, square miles; $\mathrm{ft}^{3} / \mathrm{s}$, cubic feet per second; HYDRO, lake or other hydrologic structure; NA, not applicable; NRDitch, irrigation ditch; NRTribal, tribal stream]

\begin{tabular}{|c|c|c|c|c|c|c|c|}
\hline \multirow{2}{*}{$\begin{array}{l}\text { Determi- } \\
\text { nation site } \\
\text { identi- } \\
\text { fication } \\
\text { number } \\
\text { (fig. 51) }\end{array}$} & \multirow{2}{*}{$\begin{array}{c}\text { Estimated mean } \\
\text { flow } \\
\left(\mathrm{ft}^{3} / \mathrm{s}\right)\end{array}$} & \multicolumn{6}{|c|}{ Estimated peak discharge $\left(\mathrm{ft}^{3} / \mathrm{s}\right)$ for indicated peak-discharge frequency } \\
\hline & & 2-year & 5-year & 10-year & 25-year & 50-year & 100-year \\
\hline 4219 & NA & NA & NA & NA & NA & NA & NA \\
\hline 4232 & 2.33 & 791 & 2,370 & 4,060 & 6,950 & 9,690 & 12,900 \\
\hline
\end{tabular}




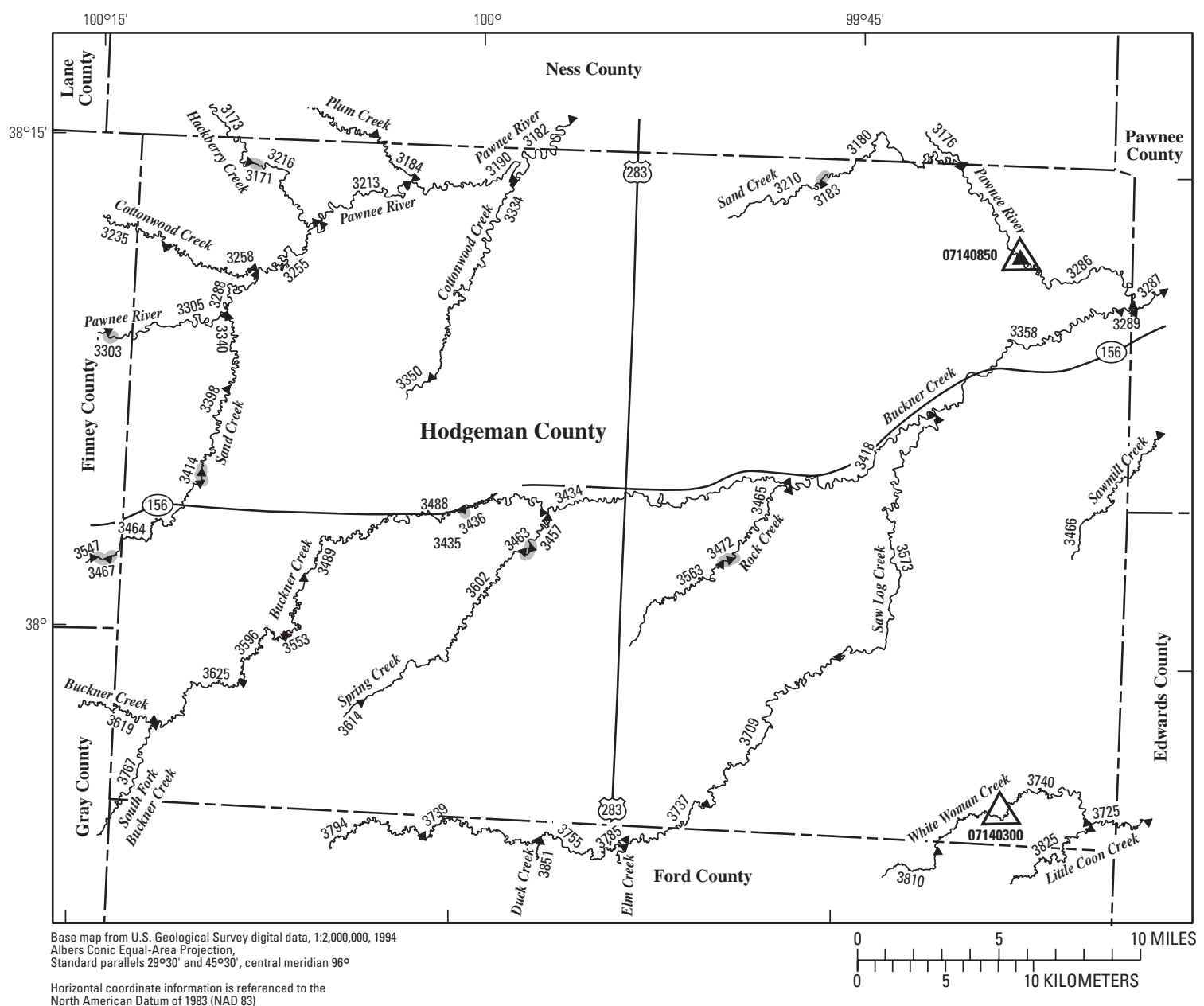

EXPLANATION

\$794 Location of streamflow-statistics determination site (small triangle) and associated identification number — small triangle points in downstream direction

$07140850 \Delta$ U.S. Geological Survey streamflow-gaging station and number used for estimates of flow duration

07140300

$\triangle$ U.S. Geological Survey streamflow-gaging station and number used for estimates of peak-discharge frequency values

KANSAS

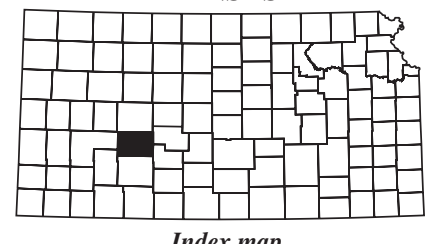

Index map

3436 Lake and determination site identification number

Figure 52. Location of streamflow-statistics determination sites, associated identification numbers, and U.S. Geological Survey streamflow-gaging stations used in the flow-duration and peak-discharge frequency analyses for Hodgeman County. 
Table 48. Estimated flow-duration values, mean flow values, and peak-discharge frequency values for controlled and uncontrolled flow stream segments on the 1999 Kansas Surface Water Register for Hodgeman County.

[KSWR, Kansas Surface Water Register; CUSEGA, catalog unit segment number alpha; $\mathrm{mi}^{2}$, square miles; $\mathrm{ft}^{3} / \mathrm{s}$, cubic feet per second; HYDRO, lake or other hydrologic structure; NA, not applicable; NRDitch, irrigation ditch; NRTribal, tribal stream]

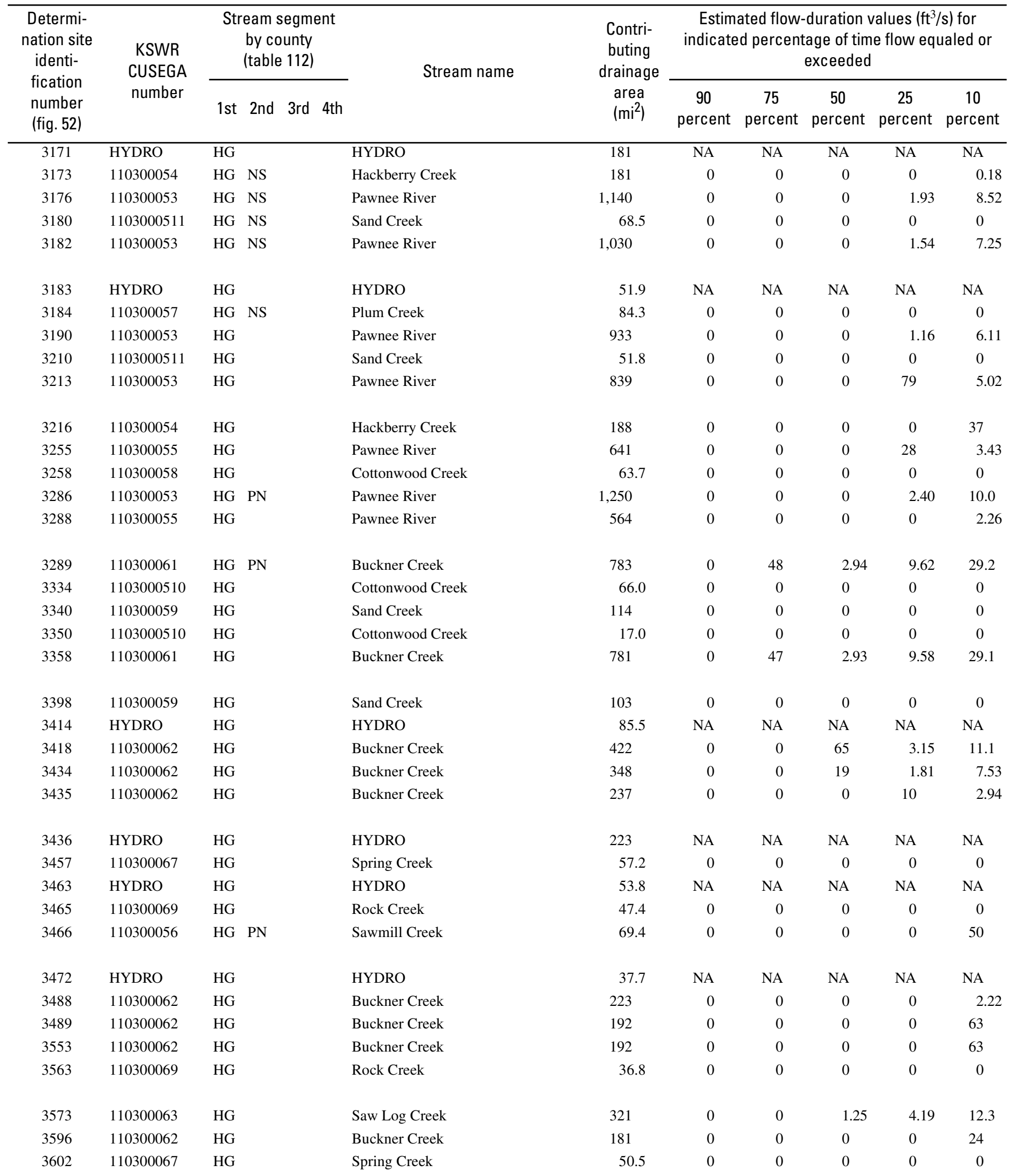


Table 48. Estimated flow-duration values, mean flow values, and peak-discharge frequency values for controlled and uncontrolled flow stream segments on the 1999 Kansas Surface Water Register for Hodgeman County.-Continued

[KSWR, Kansas Surface Water Register; CUSEGA, catalog unit segment number alpha; $\mathrm{mi}^{2}$, square miles; $\mathrm{ft}^{3} / \mathrm{s}$, cubic feet per second; HYDRO, lake or other hydrologic structure; NA, not applicable; NRDitch, irrigation ditch; NRTribal, tribal stream]

\begin{tabular}{|c|c|c|c|c|c|c|c|}
\hline \multirow{2}{*}{$\begin{array}{l}\text { Determi- } \\
\text { nation site } \\
\text { identi- } \\
\text { fication } \\
\text { number } \\
\text { (fig. 52) }\end{array}$} & \multirow{2}{*}{$\begin{array}{c}\text { Estimated mean } \\
\text { flow } \\
\left(\mathrm{ft}^{3} / \mathrm{s}\right)\end{array}$} & \multicolumn{6}{|c|}{ Estimated peak discharge $\left(\mathrm{ft}^{3} / \mathrm{s}\right)$ for indicated peak-discharge frequency } \\
\hline & & 2-year & 5-year & 10-year & 25-year & 50-year & 100 -year \\
\hline 3171 & $\mathrm{NA}$ & NA & $\mathrm{NA}$ & $\mathrm{NA}$ & $\mathrm{NA}$ & NA & $\mathrm{NA}$ \\
\hline 3180 & 1.74 & 426 & 1,340 & 2,350 & 4,150 & 5,890 & 7,970 \\
\hline 3182 & 9.77 & 591 & 1,870 & 3,310 & 5,900 & 8,450 & 11,600 \\
\hline 3183 & NA & NA & NA & NA & NA & NA & NA \\
\hline 3184 & 1.56 & 467 & 1,460 & 2,570 & 4,530 & 6,430 & 8,710 \\
\hline 3216 & 3.37 & 573 & 1,830 & 3,250 & 5,800 & 8,320 & 11,400 \\
\hline 3255 & 7.06 & 646 & 2,130 & 3,850 & 6,990 & 10,100 & 14,000 \\
\hline 3258 & .58 & 361 & 1,170 & 2,090 & 3,730 & 5,340 & 7,270 \\
\hline 3286 & 11.1 & 469 & 1,430 & 2,460 & 4,300 & 6,070 & 8,210 \\
\hline 3288 & 6.07 & 645 & 2,130 & 3,860 & 7,020 & 10,200 & 14,100 \\
\hline 3289 & 29.7 & 1,660 & 4,510 & 7,520 & 12,700 & 17,700 & 23,600 \\
\hline 3334 & 1.23 & 425 & 1,340 & 2,360 & 4,160 & 5,920 & 8,020 \\
\hline 3340 & 1.11 & 468 & 1,540 & 2,770 & 4,990 & 7,200 & 9,890 \\
\hline 3350 & 0 & 349 & 1,100 & 1,890 & 3,250 & 4,500 & 6,000 \\
\hline 3436 & NA & NA & NA & NA & NA & NA & NA \\
\hline 3457 & 1.00 & 468 & 1,450 & 2,530 & 4,420 & 6,250 & 8,430 \\
\hline 3463 & NA & NA & NA & NA & NA & NA & NA \\
\hline 3465 & 1.21 & 460 & 1,390 & 2,400 & 4,130 & 5,800 & 7,750 \\
\hline 3466 & 2.87 & 574 & 1,710 & 2,930 & 5,040 & 7,050 & 9,430 \\
\hline 3472 & NA & NA & NA & $\mathrm{NA}$ & NA & NA & NA \\
\hline 3488 & 5.75 & 763 & 2,390 & 4,240 & 7,540 & 10,800 & 14,800 \\
\hline 3489 & 4.30 & 709 & 2,250 & 4,010 & 7,180 & 10,300 & 14,100 \\
\hline 3553 & 4.30 & 709 & 2,250 & 4,010 & 7,180 & 10,300 & 14,100 \\
\hline 3563 & .61 & 457 & 1,360 & 2,320 & 3,980 & 5,550 & 7,390 \\
\hline 3573 & 13.6 & 962 & 2,820 & 4,840 & 8,370 & 11,800 & 15,900 \\
\hline 3596 & 3.90 & 710 & 2,250 & 4,000 & 7,160 & 10,300 & 14,100 \\
\hline 3602 & .59 & 428 & 1,340 & 2,350 & 4,130 & 5,860 & 7,910 \\
\hline
\end{tabular}


Table 48. Estimated flow-duration values, mean flow values, and peak-discharge frequency values for controlled and uncontrolled flow stream segments on the 1999 Kansas Surface Water Register for Hodgeman County._-Continued

[KSWR, Kansas Surface Water Register; CUSEGA, catalog unit segment number alpha; $\mathrm{mi}^{2}$, square miles; $\mathrm{ft}^{3} / \mathrm{s}$, cubic feet per second; HYDRO, lake or other hydrologic structure; NA, not applicable; NRDitch, irrigation ditch; NRTribal, tribal stream]

\begin{tabular}{|c|c|c|c|c|c|c|c|c|c|}
\hline $\begin{array}{l}\text { Determi- } \\
\text { nation site } \\
\text { identi- } \\
\text { fication } \\
\text { number } \\
\text { (fig. 52) }\end{array}$ & $\begin{array}{l}\text { KSWR } \\
\text { CUSEGA } \\
\text { number }\end{array}$ & $\begin{array}{l}\text { Stream segment } \\
\text { by county } \\
\text { (table 112) }\end{array}$ & Stream name & $\begin{array}{l}\text { Contri- } \\
\text { buting } \\
\text { drainage } \\
\text { area } \\
\left(\mathrm{mi}^{2}\right)\end{array}$ & \multicolumn{5}{|c|}{$\begin{array}{l}\text { Estimated flow-duration values }\left(\mathrm{ft}^{3} / \mathrm{s}\right) \text { for } \\
\text { indicated percentage of time flow equaled or } \\
\text { exceeded }\end{array}$} \\
\hline 3614 & 110300067 & $\mathrm{HG}$ & Spring Creek & 11.2 & 0 & 0 & 0 & 0 & 0 \\
\hline
\end{tabular}


Table 48. Estimated flow-duration values, mean flow values, and peak-discharge frequency values for controlled and uncontrolled flow stream segments on the 1999 Kansas Surface Water Register for Hodgeman County.-Continued

[KSWR, Kansas Surface Water Register; CUSEGA, catalog unit segment number alpha; $\mathrm{mi}^{2}$, square miles; $\mathrm{ft}^{3} / \mathrm{s}$, cubic feet per second; HYDRO, lake or other hydrologic structure; NA, not applicable; NRDitch, irrigation ditch; NRTribal, tribal stream]

\begin{tabular}{|c|c|c|c|c|c|c|c|}
\hline $\begin{array}{l}\text { Determi- } \\
\text { nation site } \\
\text { identi- } \\
\text { fication } \\
\text { number } \\
\text { (fig. 52) }\end{array}$ & $\begin{array}{c}\text { Estimated mean } \\
\text { flow } \\
\left(\mathrm{ft}^{3} / \mathrm{s}\right)\end{array}$ & \multicolumn{6}{|c|}{ Estimated peak discharge $\left(\mathrm{ft}^{3} / \mathrm{s}\right)$ for indicated peak-discharge frequency } \\
\hline 3614 & 0 & 289 & 888 & 1,510 & 2,580 & 3,550 & 4,720 \\
\hline
\end{tabular}




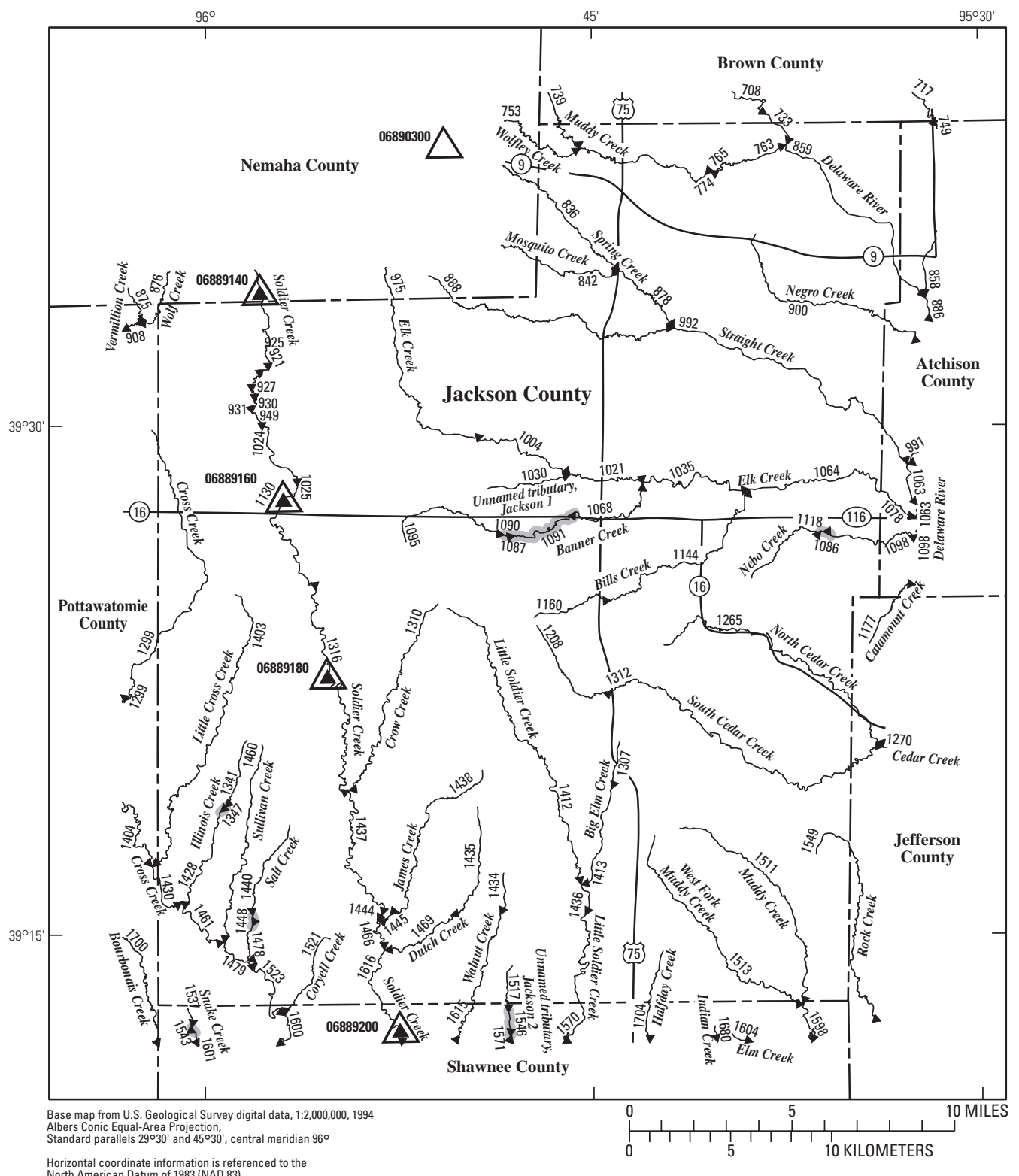

Horizontal coordinate information is referenced to the
orth American Datum of 1983 (NAD 83)

EXPLANATION

$\$ 1537$ Location of streamflow-statistics determination site (small triangle) and associated identification number-small triangle points in downstream direction

${ }^{06889200} \Delta$ U.S. Geological Survey streamflow-gaging station and number used for estimates of flow duration

${ }^{06890300} \triangle$ U.S. Geological Survey streamflow-gaging station and number used for estimates of peak-discharge frequency values

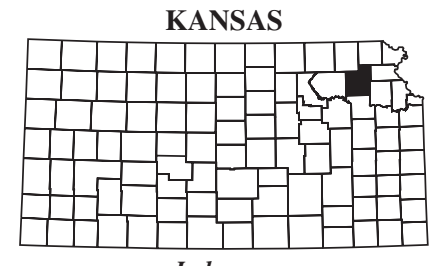

Index map 1543

Lake and determination site identification number

Figure 53. Location of streamflow-statistics determination sites, associated identification numbers, and U.S. Geological Survey streamflow-gaging stations used in the flow-duration and peak-discharge frequency analyses for Jackson County. 
Table 49. Estimated flow-duration values, mean flow values, and peak-discharge frequency values for controlled and uncontrolled flow stream segments on the 1999 Kansas Surface Water Register for Jackson County.

[KSWR, Kansas Surface Water Register; CUSEGA, catalog unit segment number alpha; $\mathrm{mi}^{2}$, square miles; $\mathrm{ft}^{3} / \mathrm{s}$, cubic feet per second; HYDRO, lake or other hydrologic structure; NA, not applicable; NRDitch, irrigation ditch; NRTribal, tribal stream]

\begin{tabular}{|c|c|c|c|c|c|c|c|c|c|c|c|c|}
\hline \multirow{2}{*}{$\begin{array}{l}\text { Determi- } \\
\text { nation site } \\
\text { identi- } \\
\text { fication } \\
\text { number } \\
\text { (fig. 53) }\end{array}$} & \multirow{2}{*}{$\begin{array}{l}\text { KSWR } \\
\text { CUSEGA } \\
\text { number }\end{array}$} & \multicolumn{4}{|c|}{$\begin{array}{l}\text { Stream segment } \\
\text { by county } \\
\text { (table 112) }\end{array}$} & \multirow{2}{*}{ Stream name } & \multirow{2}{*}{$\begin{array}{l}\text { Contri- } \\
\text { buting } \\
\text { drainage } \\
\text { area } \\
\left(\mathrm{mi}^{2}\right)\end{array}$} & \multicolumn{5}{|c|}{$\begin{array}{l}\text { Estimated flow-duration values }\left(\mathrm{ft}^{3} / \mathrm{s}\right) \text { for } \\
\text { indicated percentage of time flow equaled or } \\
\text { exceeded }\end{array}$} \\
\hline & & $1 s t$ & 2nd & 3rd & 4th & & & $\begin{array}{c}90 \\
\text { percent }\end{array}$ & $\begin{array}{c}75 \\
\text { percent }\end{array}$ & $\begin{array}{c}50 \\
\text { percent }\end{array}$ & $\begin{array}{c}25 \\
\text { percent }\end{array}$ & $\begin{array}{c}10 \\
\text { percent }\end{array}$ \\
\hline 753 & 1027010327 & JA & $\overline{\mathrm{NM}}$ & & & Wolfley Creek & 43.7 & 0.04 & 0.37 & 3.79 & 13.4 & 39.6 \\
\hline 763 & 1027010325 & JA & & & & Muddy Creek & 108 & .25 & 1.90 & 9.06 & 31.2 & 95.1 \\
\hline 765 & 1027010325 & JA & & & & Muddy Creek & 103 & .23 & 1.74 & 8.53 & 29.5 & 89.9 \\
\hline 774 & 1027010325 & $\mathrm{JA}$ & & & & Muddy Creek & 102 & .22 & 1.73 & 8.48 & 29.3 & 89.4 \\
\hline 836 & 1027010342 & JA & NM & & & Spring Creek & 32.9 & 0 & .22 & 3.24 & 11.4 & 32.8 \\
\hline 842 & 10270103602 & $\mathrm{JA}$ & NM & & & Mosquito Creek & 18.0 & 0 & 0 & 1.79 & 6.47 & 18.9 \\
\hline 876 & 1027010249 & $\mathrm{JA}$ & NM & PT & & Wolf Creek & 14.5 & 0 & 0 & .84 & 3.44 & 11.0 \\
\hline 878 & 1027010342 & JA & & & & Spring Creek & 58.1 & .01 & .77 & 5.51 & 19.4 & 56.7 \\
\hline 888 & 1027010328 & JA & NM & & & Straight Creek & 28.4 & 0 & 0 & 2.49 & 9.32 & 27.9 \\
\hline 921 & 102701029009 & JA & NM & & & Soldier Creek & 34.6 & .21 & .48 & 1.20 & 3.50 & 10.0 \\
\hline 925 & 102701029009 & JA & & & & Soldier Creek & 34.6 & .21 & .48 & 1.20 & 3.51 & 10.0 \\
\hline 927 & 102701029009 & $\mathrm{JA}$ & & & & Soldier Creek & 34.8 & .21 & .48 & 1.22 & 3.55 & 10.1 \\
\hline 930 & 102701029009 & $\mathrm{JA}$ & & & & Soldier Creek & 34.9 & .21 & .48 & 1.23 & 3.59 & 10.3 \\
\hline 931 & 102701029009 & $\mathrm{JA}$ & & & & Soldier Creek & 35.1 & .21 & .48 & 1.24 & 3.64 & 10.4 \\
\hline 949 & 102701029009 & $\mathrm{JA}$ & & & & Soldier Creek & 37.6 & .27 & .57 & 1.49 & 4.35 & 12.8 \\
\hline 951 & 102701029009 & $\mathrm{JA}$ & & & & Soldier Creek & 37.7 & .27 & .57 & 1.49 & 4.36 & 12.8 \\
\hline 975 & 1027010330 & JA & NM & & & Elk Creek & 39.3 & 0 & .21 & 3.40 & 12.5 & 37.4 \\
\hline 1004 & 1027010330 & $\mathrm{JA}$ & & & & Elk Creek & 49.4 & 0 & .41 & 4.22 & 15.5 & 46.5 \\
\hline 1021 & 1027010329 & $\mathrm{JA}$ & & & & Elk Creek & 60.0 & .01 & .67 & 5.18 & 18.7 & 56.0 \\
\hline 1024 & 102701029009 & JA & & & & Soldier Creek & 49.3 & .50 & 1.20 & 2.84 & 8.16 & 24.5 \\
\hline 1025 & 102701029 & JA & & & & Soldier Creek & 49.3 & .50 & 1.20 & 2.84 & 8.16 & 24.5 \\
\hline 1030 & 1027010331 & $\mathrm{JA}$ & & & & Unnamed tributary, Jackson 1 & 4.50 & 0 & 0 & .19 & .99 & 3.80 \\
\hline 1035 & 1027010329 & JA & & & & Elk Creek & 101 & .02 & 1.44 & 8.30 & 29.9 & 91.0 \\
\hline 1068 & 1027010345 & JA & & & & Banner Creek & 28.6 & 0 & 0 & 2.42 & 9.13 & 27.5 \\
\hline 1086 & HYDRO & $\mathrm{JA}$ & & & & HYDRO & 9.16 & NA & NA & NA & NA & NA \\
\hline 1087 & 1027010345 & JA & & & & Banner Creek & 18.2 & 0 & 0 & 1.59 & 5.98 & 17.9 \\
\hline 1090 & HYDRO & $\mathrm{JA}$ & & & & HYDRO & 18.2 & NA & NA & NA & NA & NA \\
\hline 1091 & HYDRO & JA & & & & HYDRO & 22.9 & NA & NA & NA & NA & NA \\
\hline 1095 & 1027010345 & JA & & & & Banner Creek & 17.1 & 0 & 0 & 1.47 & 5.56 & 16.7 \\
\hline 1118 & 1027010348 & $\mathrm{JA}$ & & & & Nebo Creek & 8.39 & 0 & 0 & .15 & 1.47 & 6.11 \\
\hline 1130 & 102701029009 & $\mathrm{JA}$ & & & & Soldier Creek & 63.1 & .80 & 2.00 & 4.60 & 13.0 & 39.0 \\
\hline 1144 & 1027010347 & $\mathrm{JA}$ & & & & Bills Creek & 20.8 & 0 & 0 & 1.61 & 6.38 & 19.7 \\
\hline 1160 & NRTribal & $\mathrm{JA}$ & & & & Bills Creek & 7.73 & 0 & 0 & .46 & 2.09 & 7.03 \\
\hline 1208 & NRTribal & JA & & & & South Cedar Creek & 8.70 & 0 & 0 & .63 & 2.60 & 8.30 \\
\hline 1265 & 1027010346 & JA & JF & & & North Cedar Creek & 26.7 & 0 & .08 & 2.61 & 9.32 & 27.2 \\
\hline 1299 & 1027010212 & $\mathrm{JA}$ & PT & & & Cross Creek & 46.0 & 0 & .18 & 3.35 & 12.5 & 38.2 \\
\hline 1307 & 1027010290 & JA & & & & Big Elm Creek & 6.39 & 0 & 0 & .52 & 2.02 & 6.38 \\
\hline 1310 & NRTribal & $\mathrm{JA}$ & & & & Crow Creek & 19.2 & .04 & .08 & 1.96 & 6.67 & 19.1 \\
\hline
\end{tabular}


Table 49. Estimated flow-duration values, mean flow values, and peak-discharge frequency values for controlled and uncontrolled flow stream segments on the 1999 Kansas Surface Water Register for Jackson County.-Continued

[KSWR, Kansas Surface Water Register; CUSEGA, catalog unit segment number alpha; $\mathrm{mi}^{2}$, square miles; $\mathrm{ft}^{3} / \mathrm{s}$, cubic feet per second; HYDRO, lake or other hydrologic structure; NA, not applicable; NRDitch, irrigation ditch; NRTribal, tribal stream]

\begin{tabular}{|c|c|c|c|c|c|c|c|}
\hline \multirow{2}{*}{$\begin{array}{l}\text { Determi- } \\
\text { nation site } \\
\text { identi- } \\
\text { fication } \\
\text { number } \\
\text { (fig. 53) }\end{array}$} & \multirow{2}{*}{$\begin{array}{c}\text { Estimated mean } \\
\text { flow } \\
\left(\mathrm{ft}^{3} / \mathrm{s}\right)\end{array}$} & \multicolumn{6}{|c|}{ Estimated peak discharge $\left(\mathrm{ft}^{3} / \mathrm{s}\right)$ for indicated peak-discharge frequency } \\
\hline & & 2-year & 5-year & 10-year & 25-year & 50-year & 100-year \\
\hline 753 & 29.1 & 3,180 & 6,810 & 10,100 & 15,000 & 19,300 & 24,000 \\
\hline 774 & 63.3 & 5,330 & 10,800 & 15,600 & 22,800 & 29,100 & 36,000 \\
\hline 836 & 23.8 & 1,600 & 3,680 & 5,750 & 9,360 & 12,900 & 17,200 \\
\hline 842 & 14.2 & 1,680 & 3,630 & 5,330 & 7,920 & 10,100 & 12,500 \\
\hline 876 & 9.31 & 1,380 & 3,020 & 4,450 & 6,650 & 8,470 & 10,600 \\
\hline 925 & 10.8 & 1,820 & 3,310 & 4,580 & 6,500 & 8,180 & 10,100 \\
\hline 927 & 10.8 & 1,820 & 3,320 & 4,580 & 6,510 & 8,190 & 10,100 \\
\hline 930 & 10.9 & 1,850 & 3,370 & 4,650 & 6,600 & 8,300 & 10,200 \\
\hline 931 & 11.0 & 1,870 & 3,400 & 4,700 & 6,670 & 8,380 & 10,300 \\
\hline 949 & 13.0 & 2,110 & 3,800 & 5,230 & 7,380 & 9,260 & 11,400 \\
\hline 951 & 13.0 & 2,070 & 3,740 & 5,150 & 7,280 & 9,150 & 11,300 \\
\hline 975 & 27.7 & 4,100 & 8,320 & 12,000 & 17,400 & 22,100 & 27,100 \\
\hline 1004 & 34.2 & 4,460 & 9,040 & 13,000 & 18,900 & 24,000 & 29,500 \\
\hline 1021 & 40.8 & 4,720 & 9,540 & 13,700 & 20,000 & 25,300 & 31,100 \\
\hline 1087 & 13.9 & 1,730 & 3,720 & 5,450 & 8,080 & 10,300 & 12,800 \\
\hline 1090 & NA & NA & NA & NA & NA & NA & NA \\
\hline 1091 & NA & NA & NA & NA & NA & NA & NA \\
\hline 1095 & 13.1 & 1,670 & 3,580 & 5,240 & 7,760 & 9,860 & 12,300 \\
\hline 1118 & 5.97 & 1,130 & 2,370 & 3,440 & 5,040 & 6,370 & 7,880 \\
\hline 1130 & 32.4 & 4,030 & 6,850 & 9,140 & 12,500 & 15,400 & 18,700 \\
\hline 1144 & 15.6 & 1,900 & 4,080 & 5,970 & 8,850 & 11,200 & 14,000 \\
\hline 1160 & 6.10 & 1,070 & 2,250 & 3,260 & 4,790 & 6,050 & 7,480 \\
\hline 1208 & 6.93 & 1,140 & 2,400 & 3,480 & 5,120 & 6,470 & 8,020 \\
\hline 1265 & 20.3 & 2,210 & 4,750 & 6,970 & 10,400 & 13,200 & 16,400 \\
\hline 1299 & 29.0 & 4,240 & 8,720 & 12,600 & 18,400 & 23,500 & 28,900 \\
\hline 1307 & 5.28 & 936 & 1,970 & 2,860 & 4,190 & 5,290 & 6,540 \\
\hline 1310 & 14.4 & 1,720 & 3,700 & 5,420 & 8,050 & 10,200 & 12,800 \\
\hline
\end{tabular}


Table 49. Estimated flow-duration values, mean flow values, and peak-discharge frequency values for controlled and uncontrolled flow stream segments on the 1999 Kansas Surface Water Register for Jackson County.—Continued

[KSWR, Kansas Surface Water Register; CUSEGA, catalog unit segment number alpha; $\mathrm{mi}^{2}$, square miles; $\mathrm{ft}^{3} / \mathrm{s}$, cubic feet per second; HYDRO, lake or other hydrologic structure; NA, not applicable; NRDitch, irrigation ditch; NRTribal, tribal stream]

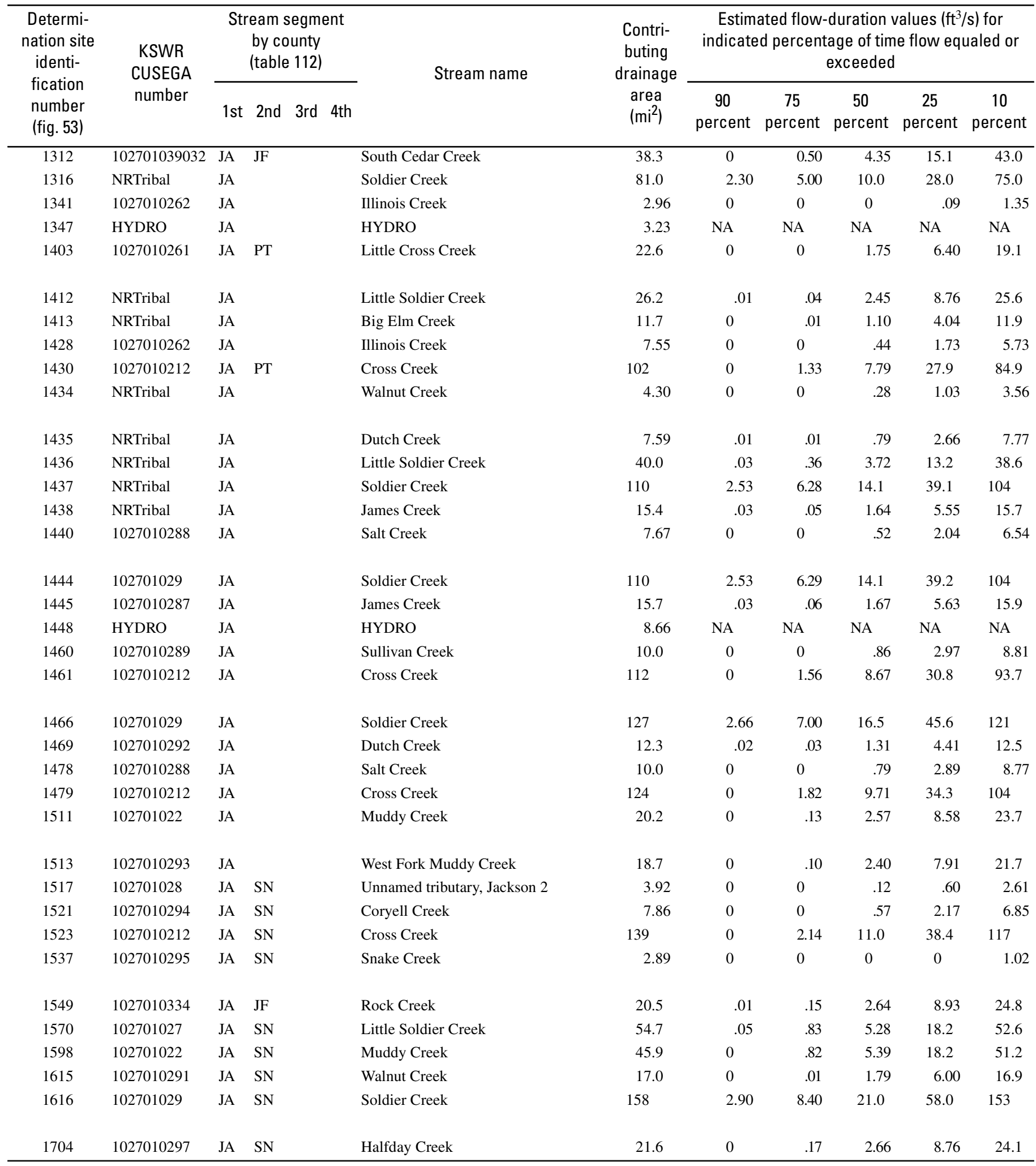


Table 49. Estimated flow-duration values, mean flow values, and peak-discharge frequency values for controlled and uncontrolled flow stream segments on the 1999 Kansas Surface Water Register for Jackson County.-Continued

[KSWR, Kansas Surface Water Register; CUSEGA, catalog unit segment number alpha; $\mathrm{mi}^{2}$, square miles; $\mathrm{ft}^{3} / \mathrm{s}$, cubic feet per second; HYDRO, lake or other hydrologic structure; NA, not applicable; NRDitch, irrigation ditch; NRTribal, tribal stream]

\begin{tabular}{|c|c|c|c|c|c|c|c|}
\hline \multirow{2}{*}{$\begin{array}{l}\text { Determi- } \\
\text { nation site } \\
\text { identi- } \\
\text { fication } \\
\text { number } \\
\text { (fig. 53) }\end{array}$} & \multirow{2}{*}{$\begin{array}{c}\text { Estimated mean } \\
\text { flow } \\
\left(\mathrm{ft}^{3} / \mathrm{s}\right)\end{array}$} & \multicolumn{6}{|c|}{ Estimated peak discharge $\left(\mathrm{ft}^{3} / \mathrm{s}\right)$ for indicated peak-discharge frequency } \\
\hline & & 2-year & 5-year & 10-year & 25-year & 50-year & 100-year \\
\hline 1312 & 30.1 & 3,610 & 7,410 & 10,700 & 15,700 & 19,900 & 24,400 \\
\hline 1347 & NA & NA & NA & NA & NA & NA & NA \\
\hline 1403 & 15.0 & 1,810 & 3,980 & 5,890 & 8,820 & 11,300 & 14,100 \\
\hline 1412 & 19.3 & 2,090 & 4,550 & 6,700 & 9,980 & 12,700 & 15,900 \\
\hline 1413 & 9.35 & 1,320 & 2,820 & 4,110 & 6,070 & 7,700 & 9,560 \\
\hline 1435 & 6.14 & 998 & 2,120 & 3,090 & 4,550 & 5,760 & 7,140 \\
\hline 1436 & 28.5 & 3,630 & 7,490 & 10,900 & 15,900 & 20,200 & 24,900 \\
\hline 1437 & 69.3 & 4,520 & 7,500 & 9,770 & 13,000 & 15,600 & 18,400 \\
\hline 1438 & 11.9 & 1,490 & 3,210 & 4,710 & 6,990 & 8,890 & 11,100 \\
\hline 1440 & 5.58 & 969 & 2,090 & 3,050 & 4,510 & 5,730 & 7,120 \\
\hline 1444 & 69.4 & 4,520 & 7,490 & 9,770 & 13,000 & 15,600 & 18,400 \\
\hline 1445 & 12.0 & 1,500 & 3,230 & 4,740 & 7,040 & 8,960 & 11,200 \\
\hline 1448 & NA & NA & NA & NA & NA & NA & NA \\
\hline 1460 & 7.17 & 1,130 & 2,440 & 3,580 & 5,320 & 6,760 & 8,420 \\
\hline 1513 & 15.4 & 1,710 & 3,700 & 5,430 & 8,080 & 10,300 & 12,800 \\
\hline 1517 & 2.74 & 678 & 1,430 & 2,070 & 3,030 & 3,820 & 4,720 \\
\hline 1521 & 5.77 & 987 & 2,120 & 3,100 & 4,590 & 5,830 & 7,240 \\
\hline 1523 & 80.8 & 6,180 & 12,600 & 18,200 & 26,700 & 34,100 & 42,200 \\
\hline 1537 & 1.66 & 544 & 1,150 & 1,670 & 2,440 & 3,080 & 3,810 \\
\hline 1549 & 17.5 & 1,370 & 2,730 & 3,880 & 5,650 & 7,070 & 8,780 \\
\hline 1570 & 37.8 & 3,690 & 7,720 & 11,300 & 16,600 & 21,200 & 26,300 \\
\hline 1598 & 35.0 & 3,820 & 7,820 & 11,300 & 16,500 & 20,900 & 25,700 \\
\hline 1615 & 12.7 & 1,580 & 3,420 & 5,040 & 7,500 & 9,540 & 11,900 \\
\hline 1616 & 99.5 & 4,510 & 7,650 & 10,200 & 13,900 & 17,100 & 20,600 \\
\hline 1704 & 17.2 & 1,840 & 4,010 & 5,910 & 8,810 & 11,200 & 14,000 \\
\hline
\end{tabular}




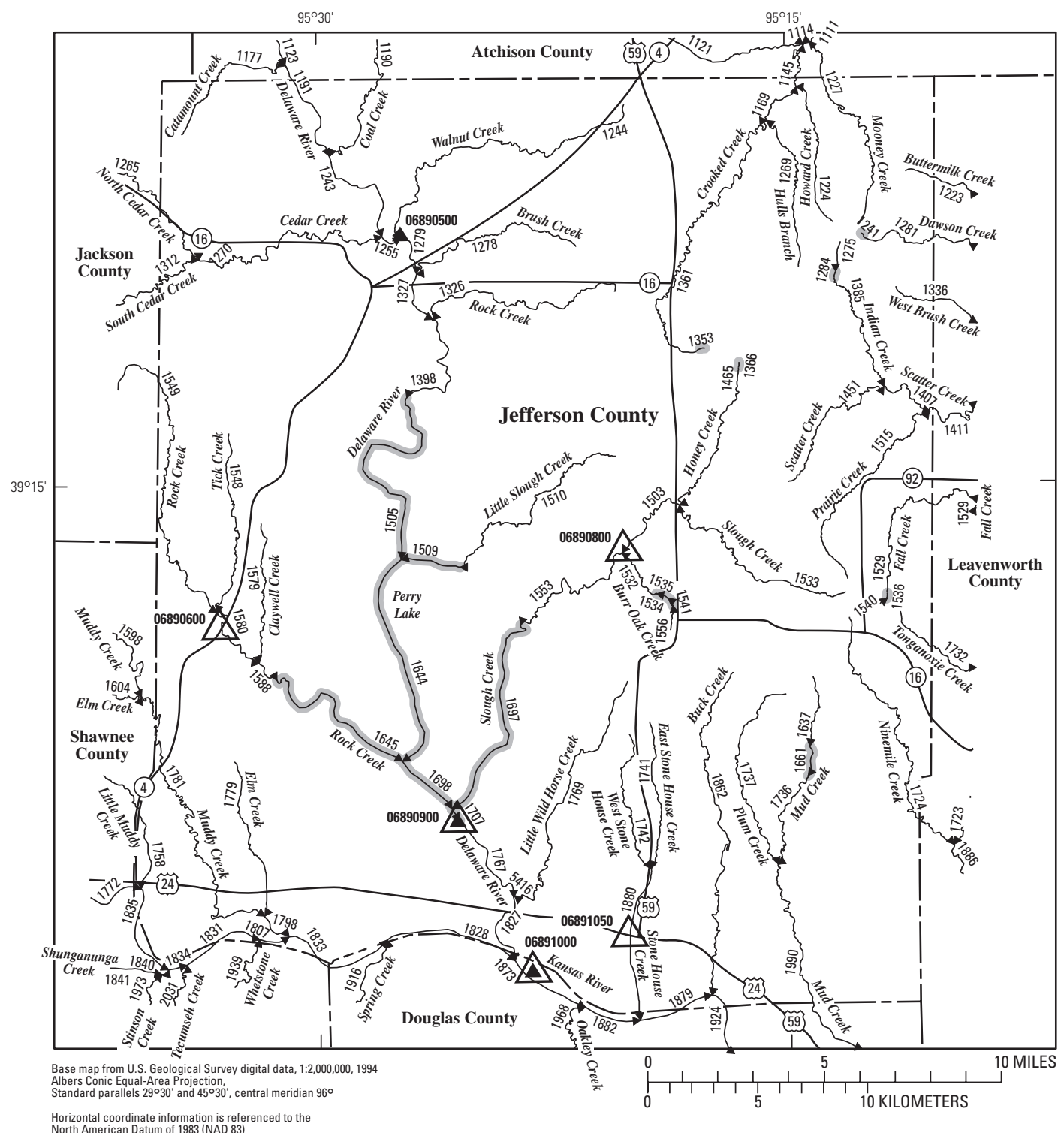

EXPLANATION

$\stackrel{1973}{\longleftarrow}$ Location of streamflow-statistics determination site (small triangle) and associated identification number-small triangle points in downstream direction

${ }^{06891000}$ U.S. Geological Survey streamflow-gaging station and number used for estimates of flow duration

${ }^{06891050} \triangle$ U.S. Geological Survey streamflow-gaging station and number used for estimates of peak-discharge frequency values

KANSAS

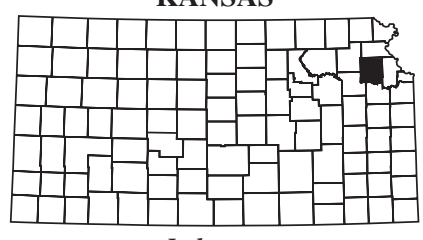

Index map

Lake and determination site identification number

Figure 54. Location of streamflow-statistics determination sites, associated identification numbers, and U.S. Geological Survey streamflow-gaging stations used in the flow-duration and peak-discharge frequency analyses for Jefferson County. 
Table 50. Estimated flow-duration values, mean flow values, and peak-discharge frequency values for controlled and uncontrolled flow stream segments on the 1999 Kansas Surface Water Register for Jefferson County.

[KSWR, Kansas Surface Water Register; CUSEGA, catalog unit segment number alpha; $\mathrm{mi}^{2}$, square miles; $\mathrm{ft}^{3} / \mathrm{s}$, cubic feet per second; HYDRO, lake or other hydrologic structure; NA, not applicable; NRDitch, irrigation ditch; NRTribal, tribal stream]

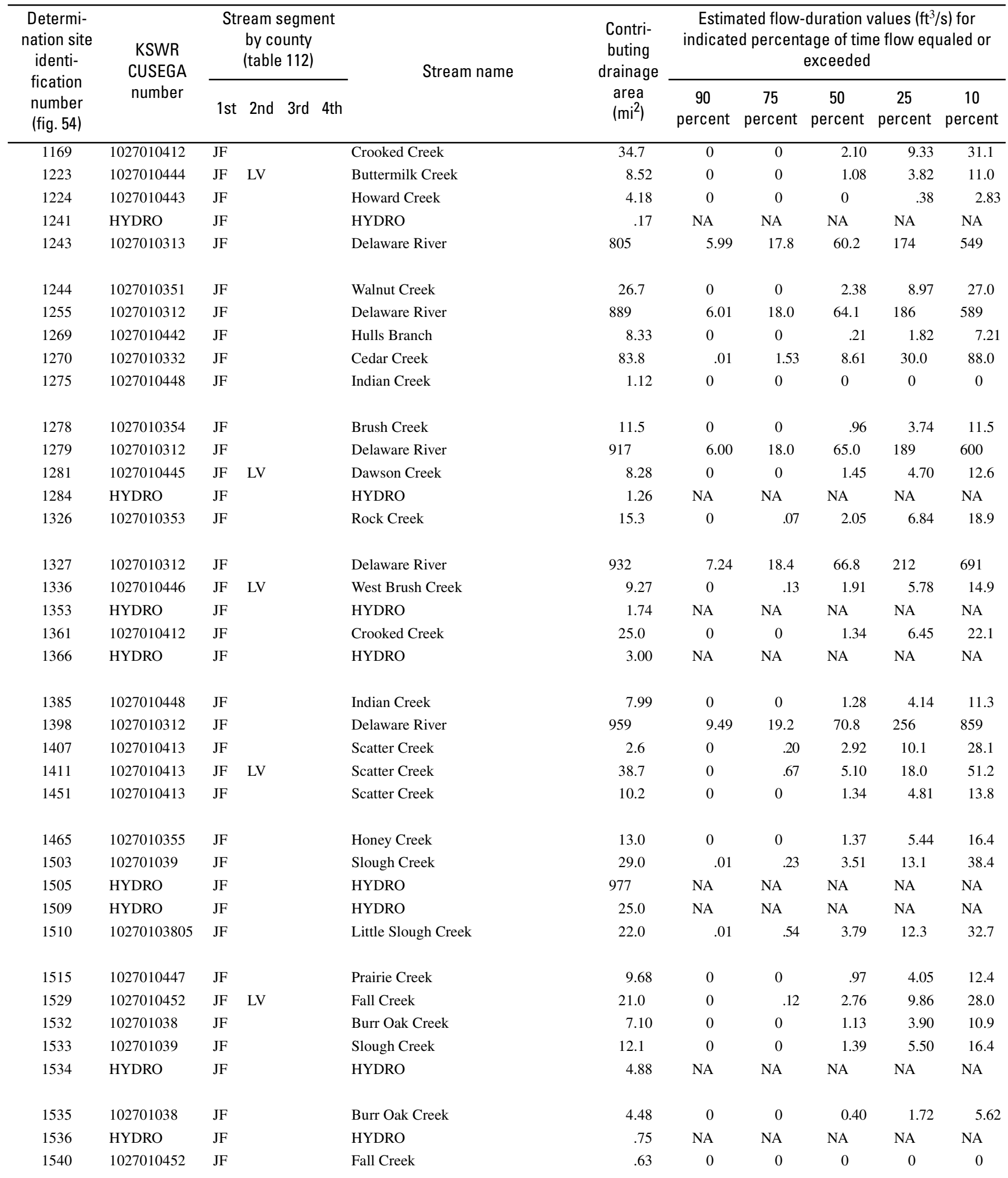


Table 50. Estimated flow-duration values, mean flow values, and peak-discharge frequency values for controlled and uncontrolled flow stream segments on the 1999 Kansas Surface Water Register for Jefferson County.-Continued

[KSWR, Kansas Surface Water Register; CUSEGA, catalog unit segment number alpha; $\mathrm{mi}^{2}$, square miles; $\mathrm{ft}^{3} / \mathrm{s}$, cubic feet per second; HYDRO, lake or other hydrologic structure; NA, not applicable; NRDitch, irrigation ditch; NRTribal, tribal stream]

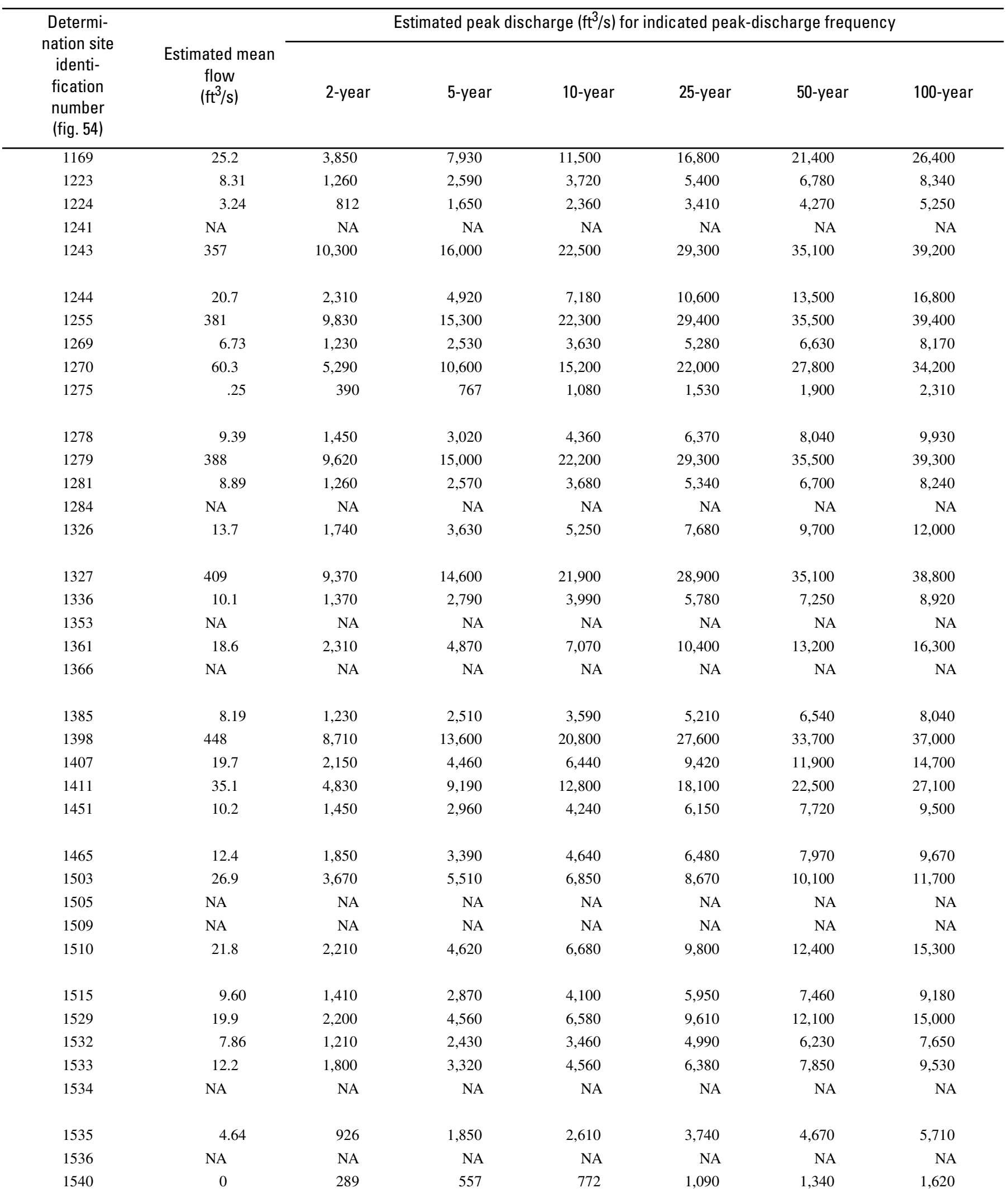


Table 50. Estimated flow-duration values, mean flow values, and peak-discharge frequency values for controlled and uncontrolled flow stream segments on the 1999 Kansas Surface Water Register for Jefferson County.-Continued

[KSWR, Kansas Surface Water Register; CUSEGA, catalog unit segment number alpha; $\mathrm{mi}^{2}$, square miles; $\mathrm{ft}^{3} / \mathrm{s}$, cubic feet per second; HYDRO, lake or other hydrologic structure; NA, not applicable; NRDitch, irrigation ditch; NRTribal, tribal stream]

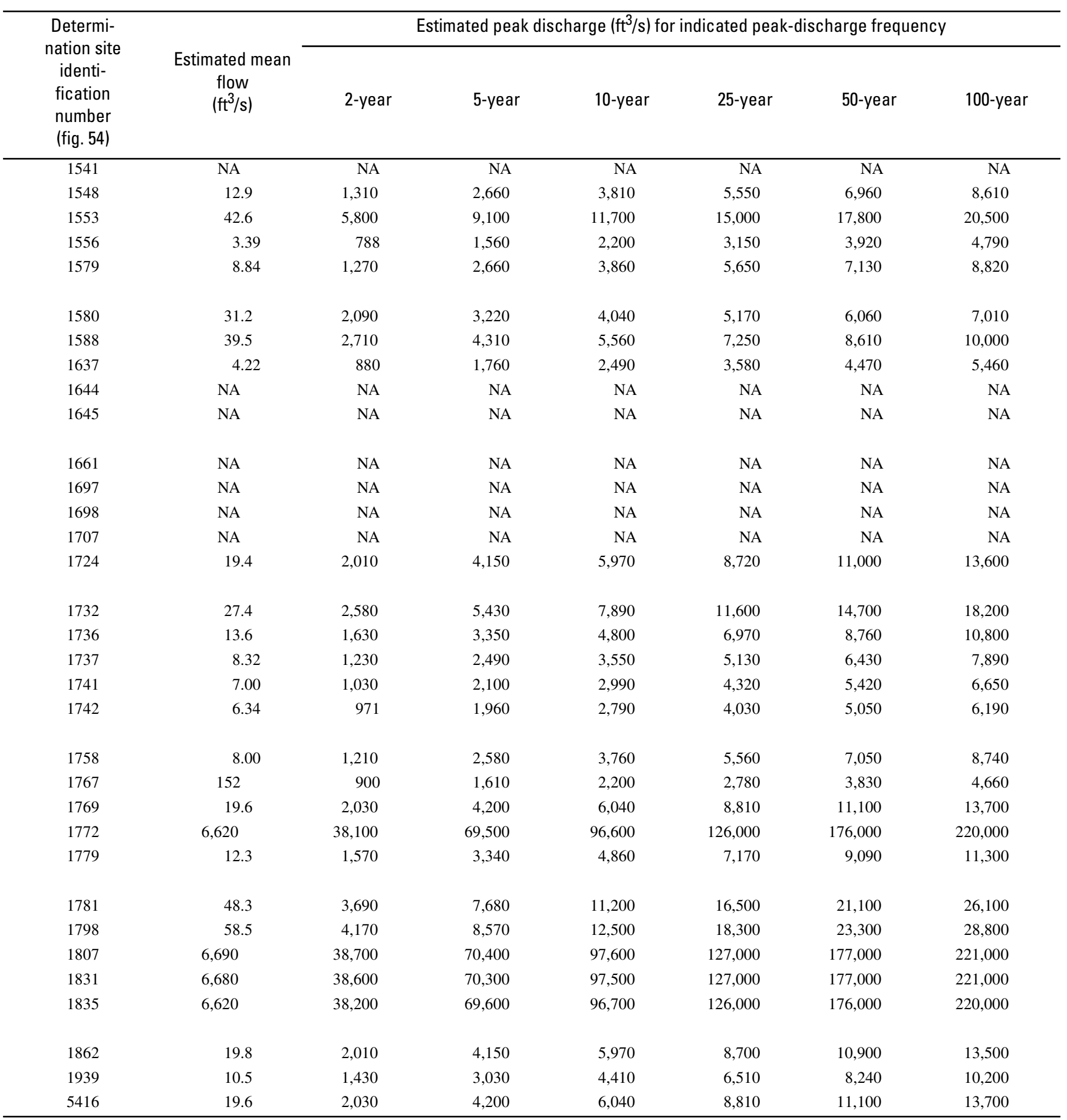


Table 50. Estimated flow-duration values, mean flow values, and peak-discharge frequency values for controlled and uncontrolled flow stream segments on the 1999 Kansas Surface Water Register for Jefferson County.—Continued

[KSWR, Kansas Surface Water Register; CUSEGA, catalog unit segment number alpha; $\mathrm{mi}^{2}$, square miles; $\mathrm{ft}^{3} / \mathrm{s}$, cubic feet per second; HYDRO, lake or other hydrologic structure; NA, not applicable; NRDitch, irrigation ditch; NRTribal, tribal stream]

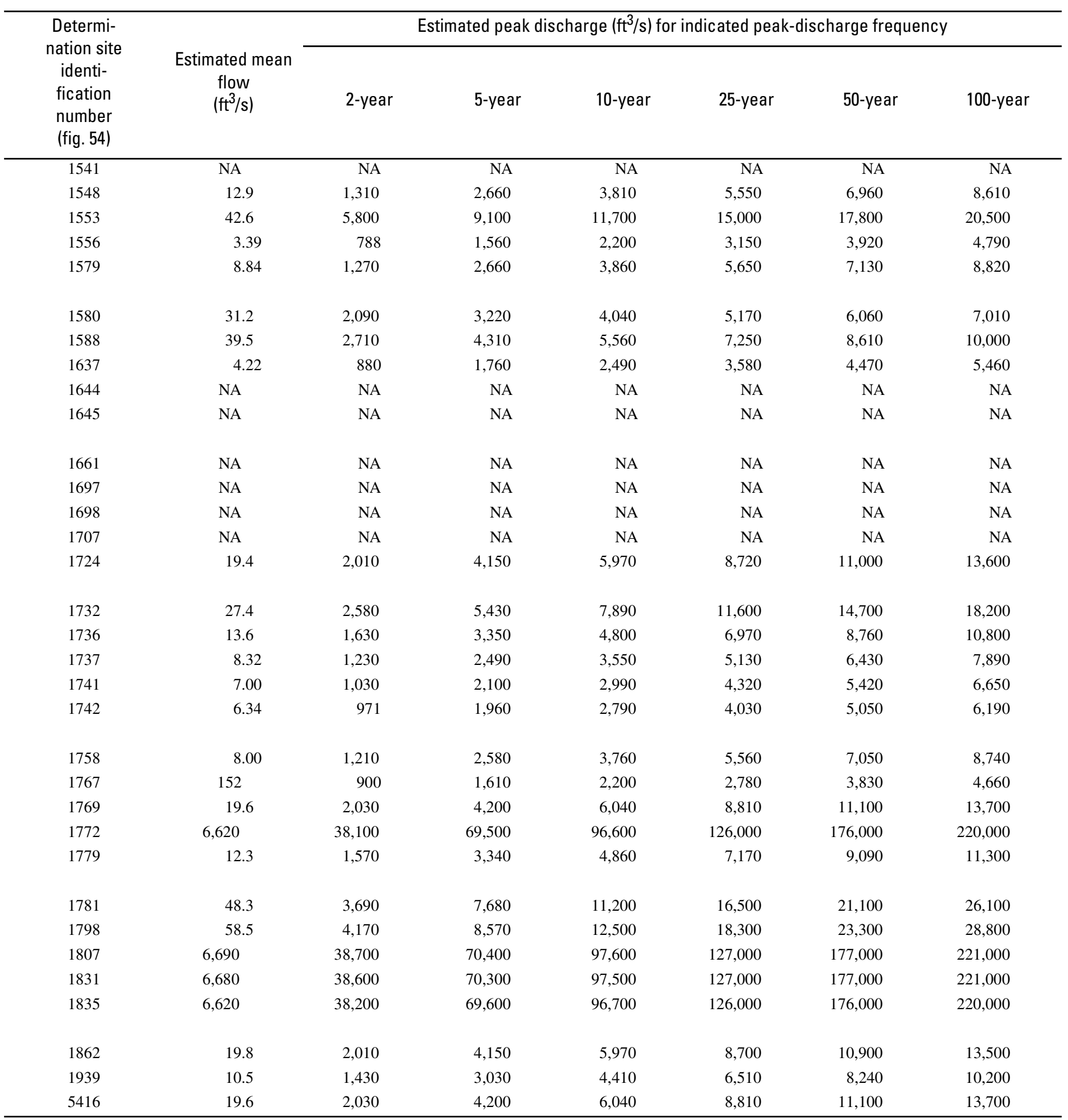




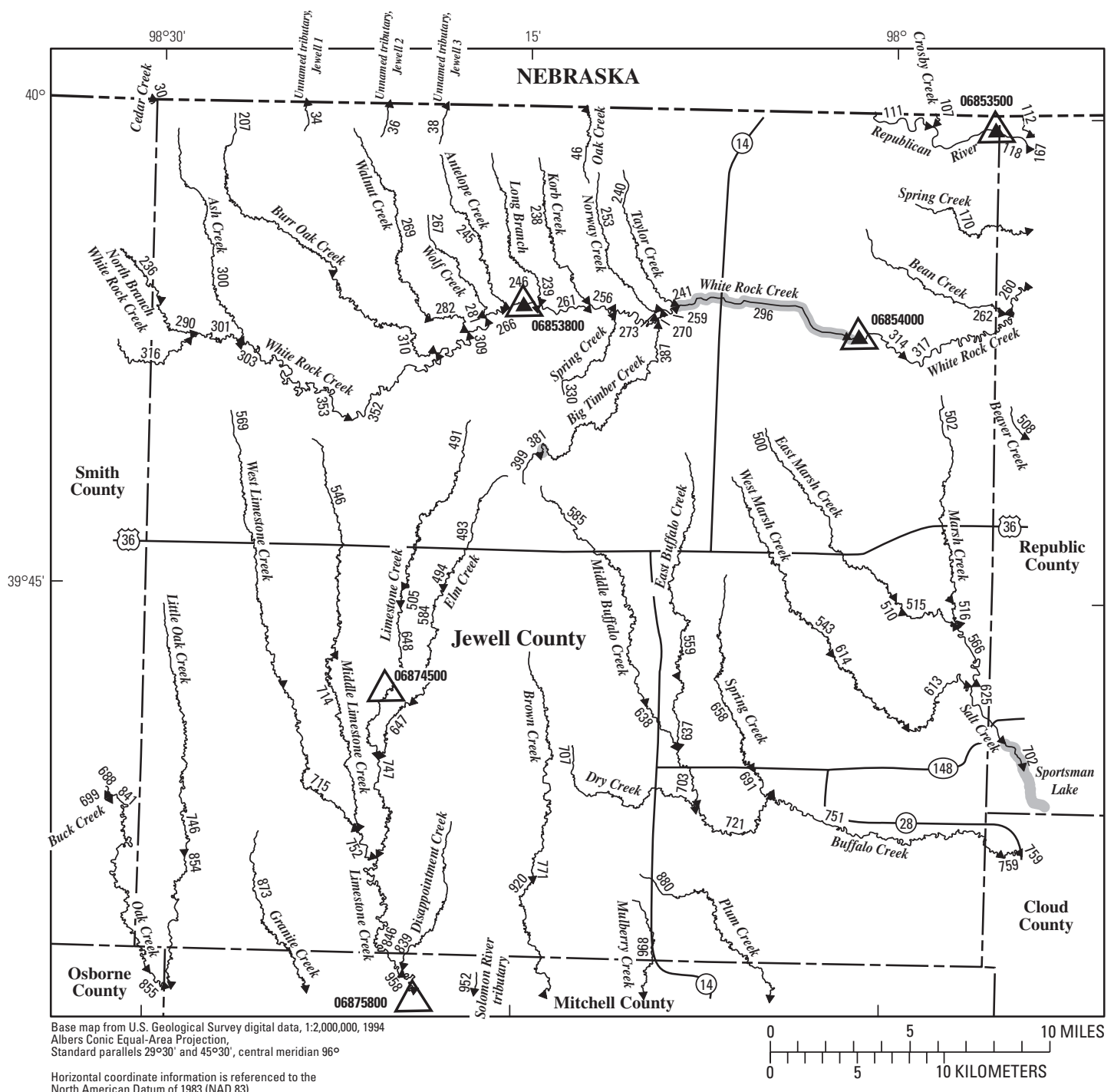

EXPLANATION

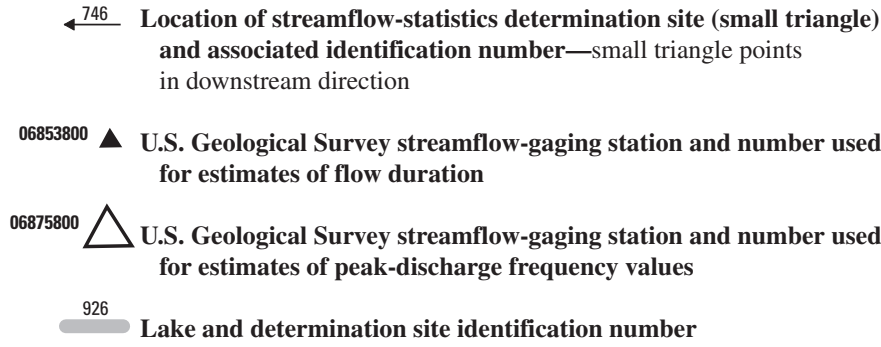

KANSAS

ocation of streamflow-statistics determination site (small triangle) and associated identification number-small triangle points

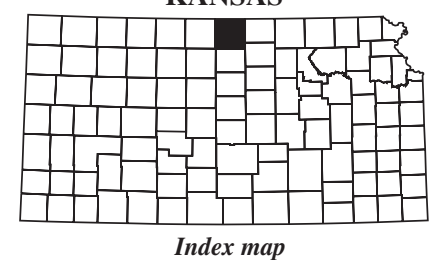

Index map

Figure 55. Location of streamflow-statistics determination sites, associated identification numbers, and U.S. Geological Survey streamflow-gaging stations used in the flow-duration and peak-discharge frequency analyses for Jewell County. 
Table 51. Estimated flow-duration values, mean flow values, and peak-discharge frequency values for controlled and uncontrolled flow stream segments on the 1999 Kansas Surface Water Register for Jewell County.

[KSWR, Kansas Surface Water Register; CUSEGA, catalog unit segment number alpha; $\mathrm{mi}^{2}$, square miles; $\mathrm{ft}^{3} / \mathrm{s}$, cubic feet per second; HYDRO, lake or other hydrologic structure; NA, not applicable; NRDitch, irrigation ditch; NRTribal, tribal stream]

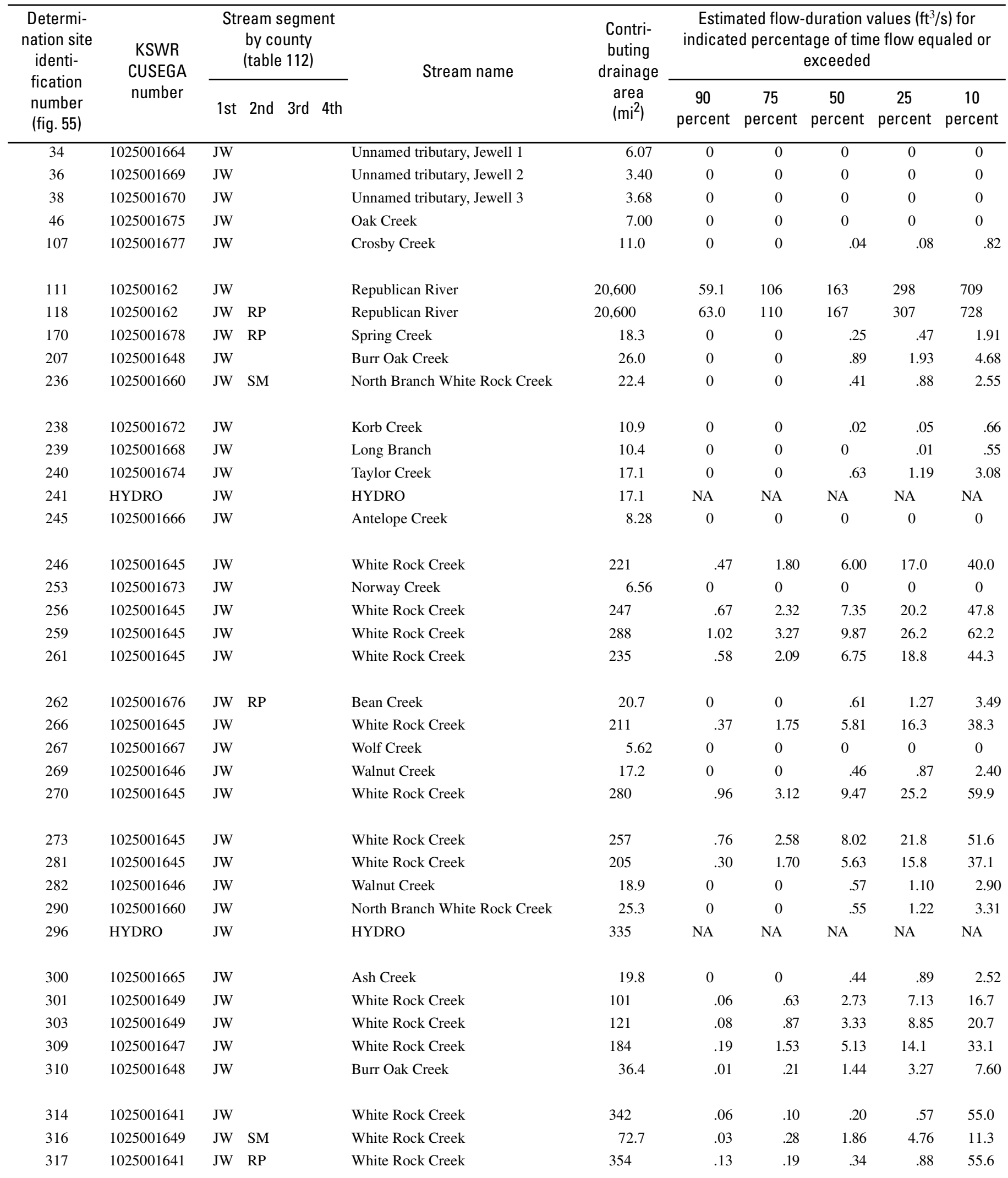


Table 51. Estimated flow-duration values, mean flow values, and peak-discharge frequency values for controlled and uncontrolled flow stream segments on the 1999 Kansas Surface Water Register for Jewell County.-Continued

[KSWR, Kansas Surface Water Register; CUSEGA, catalog unit segment number alpha; $\mathrm{mi}^{2}$, square miles; $\mathrm{ft}^{3} / \mathrm{s}$, cubic feet per second; HYDRO, lake or other hydrologic structure; NA, not applicable; NRDitch, irrigation ditch; NRTribal, tribal stream]

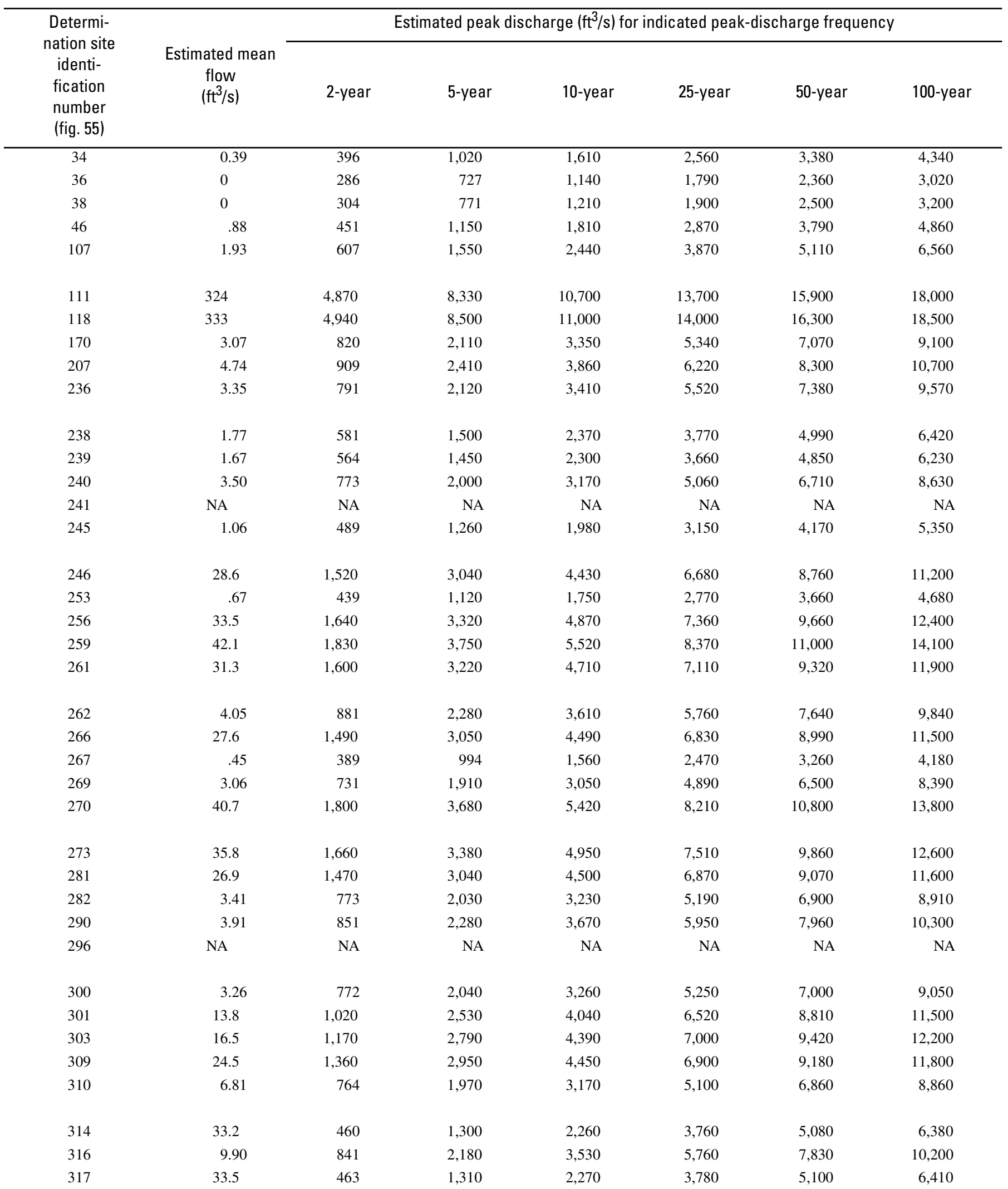


Table 51. Estimated flow-duration values, mean flow values, and peak-discharge frequency values for controlled and uncontrolled flow stream segments on the 1999 Kansas Surface Water Register for Jewell County.-Continued

[KSWR, Kansas Surface Water Register; CUSEGA, catalog unit segment number alpha; $\mathrm{mi}^{2}$, square miles; $\mathrm{ft}^{3} / \mathrm{s}$, cubic feet per second; HYDRO, lake or other hydrologic structure; NA, not applicable; NRDitch, irrigation ditch; NRTribal, tribal stream]

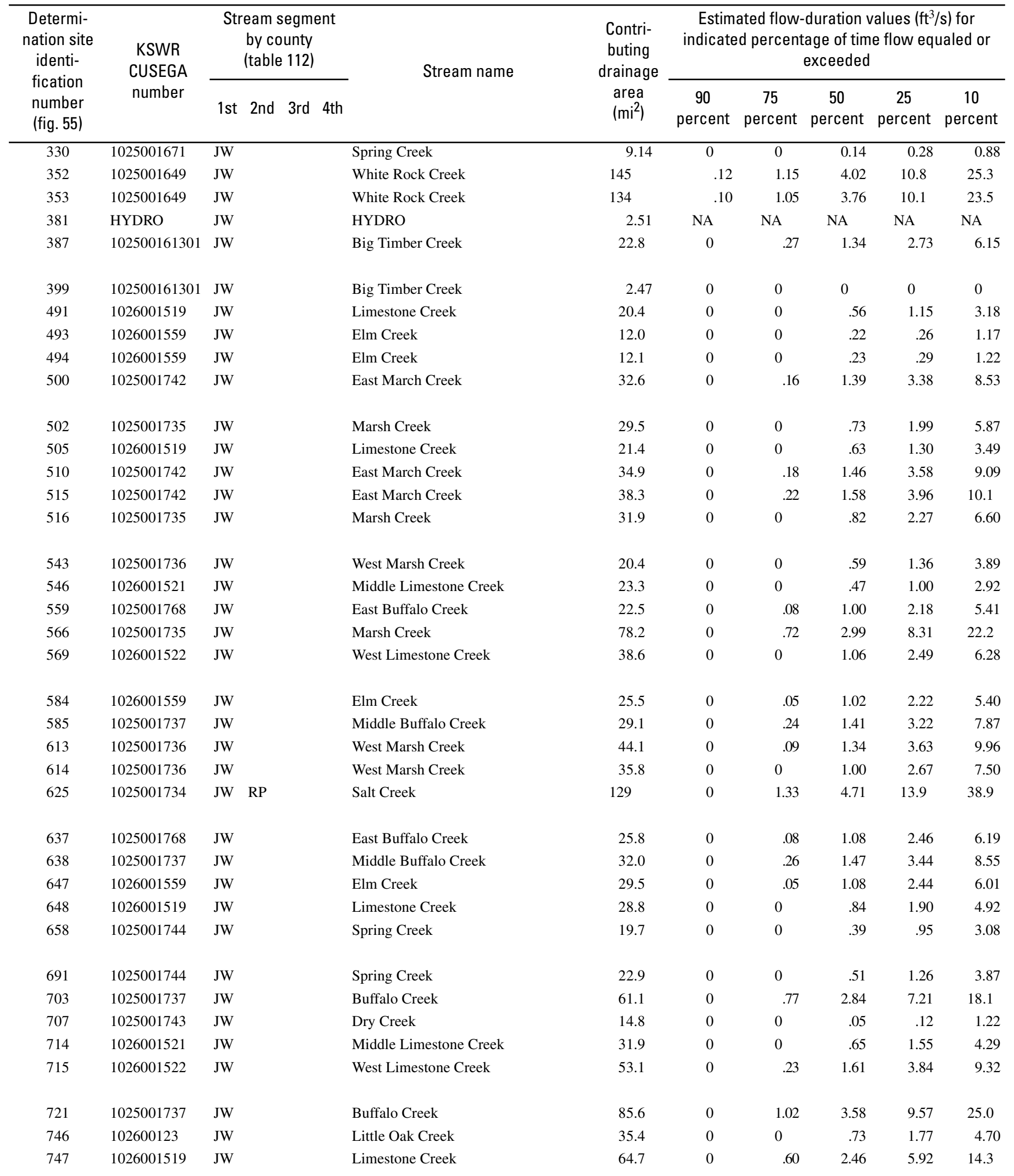


Table 51. Estimated flow-duration values, mean flow values, and peak-discharge frequency values for controlled and uncontrolled flow stream segments on the 1999 Kansas Surface Water Register for Jewell County.-Continued

[KSWR, Kansas Surface Water Register; CUSEGA, catalog unit segment number alpha; $\mathrm{mi}^{2}$, square miles; $\mathrm{ft}^{3} / \mathrm{s}$, cubic feet per second; HYDRO, lake or other hydrologic structure; NA, not applicable; NRDitch, irrigation ditch; NRTribal, tribal stream]

\begin{tabular}{|c|c|c|c|c|c|c|c|}
\hline \multirow{2}{*}{$\begin{array}{l}\text { Determi- } \\
\text { nation site } \\
\text { identi- } \\
\text { fication } \\
\text { number } \\
\text { (fig. } 55 \text { ) }\end{array}$} & \multirow{2}{*}{$\begin{array}{c}\text { Estimated mean } \\
\text { flow } \\
\left(\mathrm{ft}^{3} / \mathrm{s}\right)\end{array}$} & \multicolumn{6}{|c|}{ Estimated peak discharge $\left(\mathrm{ft}^{3} / \mathrm{s}\right)$ for indicated peak-discharge frequency } \\
\hline & & 2-year & 5-year & 10-year & 25-year & 50-year & 100-year \\
\hline 330 & 1.71 & 537 & 1,370 & 2,160 & 3,420 & 4,520 & 5,800 \\
\hline 381 & NA & NA & NA & NA & NA & NA & NA \\
\hline 387 & 5.44 & 931 & 2,410 & 3,830 & 6,110 & 8,110 & 10,500 \\
\hline 399 & 0 & 258 & 638 & 988 & 1,540 & 2,010 & 2,560 \\
\hline 491 & 3.77 & 653 & 1,560 & 2,390 & 3,710 & 4,840 & 6,140 \\
\hline 502 & 5.95 & 1,090 & 2,830 & 4,500 & 7,200 & 9,560 & 12,300 \\
\hline 505 & 3.99 & 657 & 1,560 & 2,380 & 3,680 & 4,800 & 6,090 \\
\hline 510 & 7.81 & 1,220 & 2,920 & 4,530 & 7,030 & 9,260 & 11,700 \\
\hline 515 & 8.53 & 1,130 & 2,760 & 4,320 & 6,790 & 9,010 & 11,500 \\
\hline 516 & 6.49 & 711 & 1,900 & 3,090 & 5,060 & 6,860 & 8,930 \\
\hline 543 & 4.22 & 867 & 2,240 & 3,560 & 5,680 & 7,540 & 9,710 \\
\hline 546 & 3.69 & 830 & 2,210 & 3,540 & 5,710 & 7,620 & 9,860 \\
\hline 559 & 5.08 & 925 & 2,390 & 3,800 & 6,060 & 8,040 & 10,400 \\
\hline 566 & 16.8 & 1,570 & 3,790 & 5,910 & 9,290 & 12,300 & 15,800 \\
\hline 637 & 5.72 & 998 & 2,590 & 4,120 & 6,590 & 8,750 & 11,300 \\
\hline 638 & 7.36 & 993 & 2,440 & 3,830 & 6,010 & 7,980 & 10,200 \\
\hline 647 & 5.79 & 1,000 & 2,640 & 4,210 & 6,770 & 9,020 & 11,700 \\
\hline 648 & 5.16 & 608 & 1,320 & 1,940 & 2,880 & 3,680 & 4,580 \\
\hline 658 & 3.75 & 841 & 2,180 & 3,460 & 5,530 & 7,340 & 9,450 \\
\hline 691 & 4.36 & 913 & 2,380 & 3,780 & 6,050 & 8,030 & 10,400 \\
\hline 703 & 13.8 & 1,380 & 3,320 & 5,170 & 8,090 & 10,700 & 13,700 \\
\hline 707 & 2.40 & 697 & 1,810 & 2,860 & 4,570 & 6,060 & 7,810 \\
\hline 714 & 4.88 & 638 & 1,690 & 2,750 & 4,490 & 6,080 & 7,900 \\
\hline 715 & 8.49 & 913 & 2,290 & 3,640 & 5,820 & 7,790 & 10,000 \\
\hline 721 & 18.3 & 1,480 & 3,590 & 5,610 & 8,850 & 11,800 & 15,100 \\
\hline 746 & 5.11 & 786 & 2,060 & 3,310 & 5,350 & 7,210 & 9,310 \\
\hline 747 & 12.0 & 659 & 1,250 & 1,750 & 2,460 & 3,100 & 3,730 \\
\hline
\end{tabular}


Table 51. Estimated flow-duration values, mean flow values, and peak-discharge frequency values for controlled and uncontrolled flow stream segments on the 1999 Kansas Surface Water Register for Jewell County.-Continued

[KSWR, Kansas Surface Water Register; CUSEGA, catalog unit segment number alpha; $\mathrm{mi}^{2}$, square miles; $\mathrm{ft}^{3} / \mathrm{s}$, cubic feet per second; HYDRO, lake or other hydrologic structure; NA, not applicable; NRDitch, irrigation ditch; NRTribal, tribal stream]

\begin{tabular}{|c|c|c|c|c|c|c|c|c|c|c|c|}
\hline $\begin{array}{l}\text { Determi- } \\
\text { nation site } \\
\text { identi- } \\
\text { fication } \\
\text { number } \\
\text { (fig. } 55 \text { ) }\end{array}$ & $\begin{array}{l}\text { KSWR } \\
\text { CUSEGA } \\
\text { number }\end{array}$ & \multicolumn{3}{|c|}{$\begin{array}{l}\text { Stream segment } \\
\text { by county } \\
\text { (table 112) }\end{array}$} & Stream name & $\begin{array}{l}\text { Contri- } \\
\text { buting } \\
\text { drainage } \\
\text { area } \\
\left(\mathrm{mi}^{2}\right)\end{array}$ & \multicolumn{5}{|c|}{$\begin{array}{c}\text { Estimated flow-duration values }\left(\mathrm{ft}^{3} / \mathrm{s}\right) \text { for } \\
\text { indicated percentage of time flow equaled or } \\
\text { exceeded }\end{array}$} \\
\hline 752 & 1026001520 & JW & & & Limestone Creek & 86.8 & 0 & 0.73 & 2.82 & 6.88 & 16.6 \\
\hline 841 & 102600124 & JW & OB & SM & Oak Creek & 175 & 0 & 1.58 & 4.98 & 12.4 & 30.0 \\
\hline 846 & 1026001518 & JW & $\mathrm{MC}$ & & Limestone Creek & 165 & 0 & 2.00 & 5.93 & 14.6 & 35.4 \\
\hline 854 & 102600123 & JW & MC & & Little Oak Creek & 45.8 & 0 & 0 & .92 & 2.33 & 6.18 \\
\hline 873 & 1026001524 & JW & $\mathrm{MC}$ & & Granite Creek & 28.7 & 0 & 0 & .11 & .44 & 2.10 \\
\hline
\end{tabular}


Table 51. Estimated flow-duration values, mean flow values, and peak-discharge frequency values for controlled and uncontrolled flow stream segments on the 1999 Kansas Surface Water Register for Jewell County.-Continued

[KSWR, Kansas Surface Water Register; CUSEGA, catalog unit segment number alpha; $\mathrm{mi}^{2}$, square miles; $\mathrm{ft}^{3} / \mathrm{s}$, cubic feet per second; HYDRO, lake or other hydrologic structure; NA, not applicable; NRDitch, irrigation ditch; NRTribal, tribal stream]

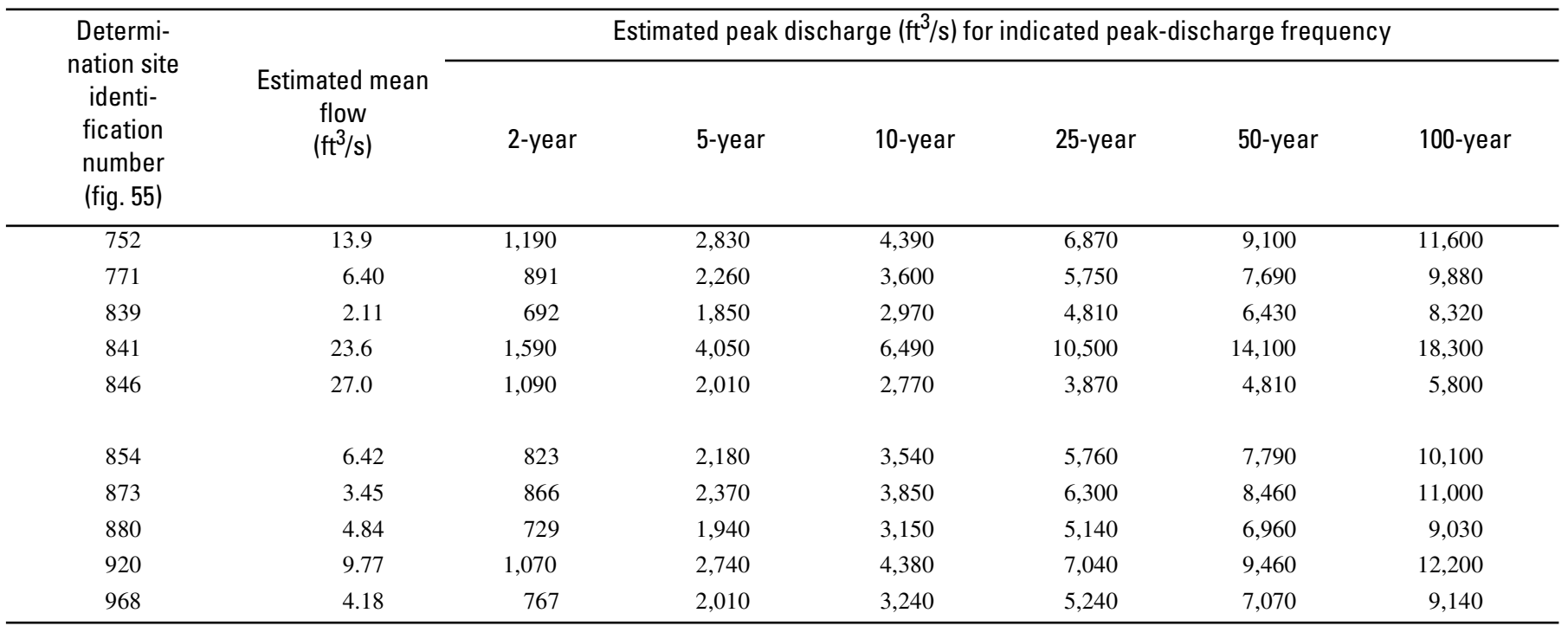




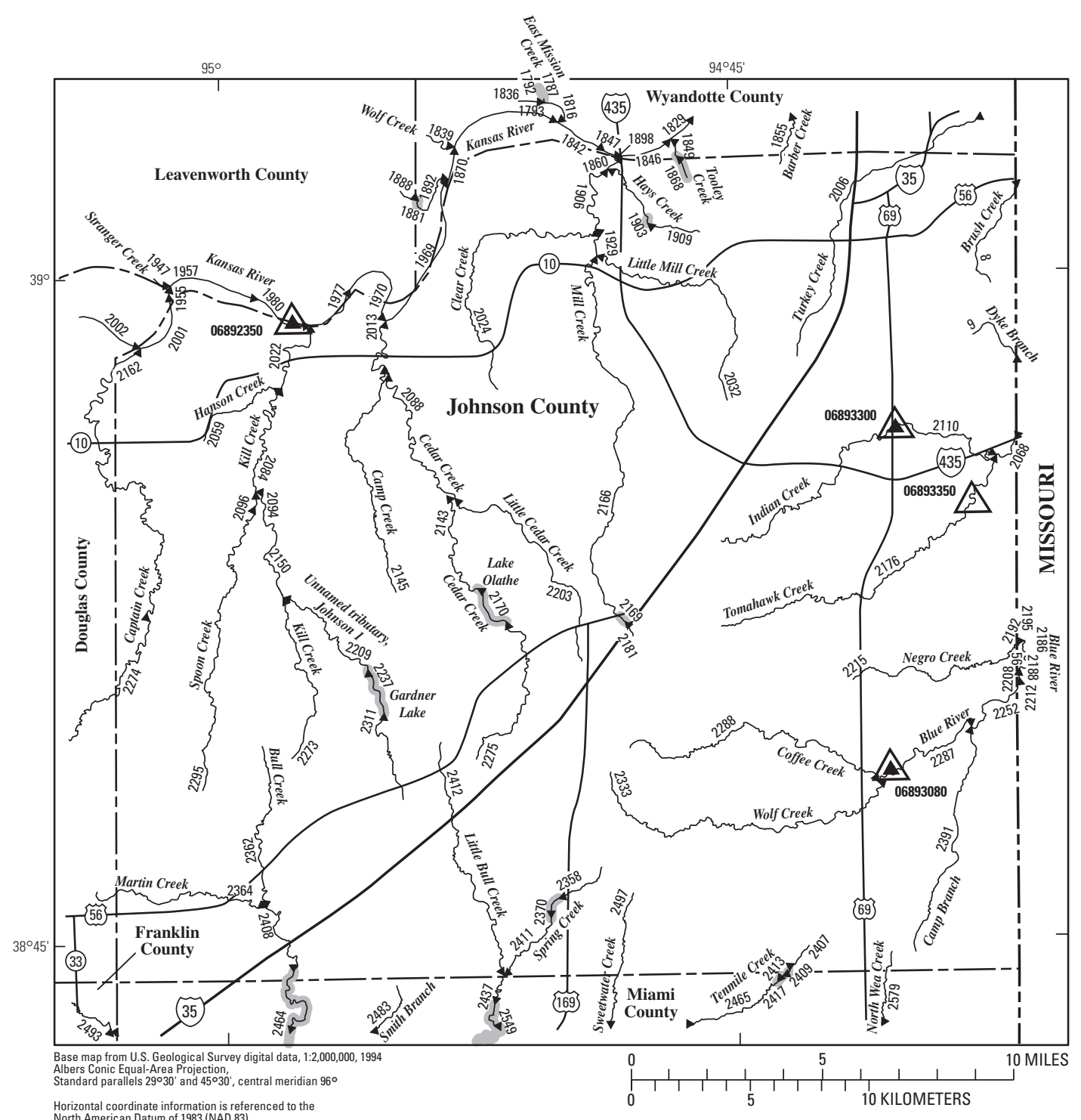

EXPLANATION

«493 Location of streamflow-statistics determination site (small triangle) and associated identification number-small triangle points in downstream direction

${ }^{06893080}$ U.S. Geological Survey streamflow-gaging station and number used for estimates of flow duration

U.S. Geological Survey streamflow-gaging station and number used for estimates of peak-discharge frequency values

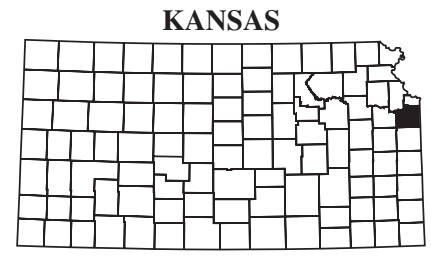

Index map 2483 Lake and determination site identification number

Figure 56. Location of streamflow-statistics determination sites, associated identification numbers, and U.S. Geological Survey streamflow-gaging stations used in the flow-duration and peak-discharge frequency analyses for Johnson County. 
Table 52. Estimated flow-duration values, mean flow values, and peak-discharge frequency values for controlled and uncontrolled flow stream segments on the 1999 Kansas Surface Water Register for Johnson County.

[KSWR, Kansas Surface Water Register; CUSEGA, catalog unit segment number alpha; $\mathrm{mi}^{2}$, square miles; $\mathrm{ft}^{3} / \mathrm{s}$, cubic feet per second; HYDRO, lake or other hydrologic structure; NA, not applicable; NRDitch, irrigation ditch; NRTribal, tribal stream]

\begin{tabular}{|c|c|c|c|c|c|c|c|c|c|c|c|c|}
\hline \multirow{2}{*}{$\begin{array}{c}\text { Determi- } \\
\text { nation site } \\
\text { identi-fication } \\
\text { number } \\
\text { (fig. 56) }\end{array}$} & \multirow{2}{*}{$\begin{array}{l}\text { KSWR } \\
\text { CUSEGA } \\
\text { number }\end{array}$} & \multicolumn{4}{|c|}{$\begin{array}{l}\text { Stream segment by } \\
\text { county } \\
\text { (table 112) }\end{array}$} & \multirow{2}{*}{ Stream name } & \multirow{2}{*}{$\begin{array}{l}\text { Contri- } \\
\text { buting } \\
\text { drainage } \\
\text { area } \\
\left(\mathrm{mi}^{2}\right)\end{array}$} & \multicolumn{5}{|c|}{$\begin{array}{l}\text { Estimated flow-duration values }\left(\mathrm{ft}^{3} / \mathrm{s}\right) \text { for } \\
\text { indicated percentage of time flow equaled or } \\
\text { exceeded }\end{array}$} \\
\hline & & $1 \mathrm{st}$ & 2 nd & $3 r d$ & 4th & & & $\begin{array}{c}90 \\
\text { percent }\end{array}$ & $\begin{array}{c}75 \\
\text { percent }\end{array}$ & $\begin{array}{c}50 \\
\text { percent }\end{array}$ & $\begin{array}{c}25 \\
\text { percent }\end{array}$ & $\begin{array}{c}10 \\
\text { percent }\end{array}$ \\
\hline 8 & 1030010154 & $\mathrm{JO}$ & & & & Brush Creek & 12.0 & 0 & 0.54 & 2.56 & 7.15 & 17.6 \\
\hline 9 & 1030010155 & JO & & & & Dyke Branch & 4.13 & 0 & .05 & .78 & 2.00 & 5.25 \\
\hline 56 & 1030010133 & $\mathrm{JO}$ & & & & Blue River & 83.9 & 3.07 & 11.2 & 31.1 & 88.0 & 234 \\
\hline 1855 & 10270104373 & JO & WY & & & Barber Creek & 10.0 & 0 & 1.33 & 4.03 & 9.66 & 20.8 \\
\hline 1860 & 1027010439 & JO & WY & & & Mill Creek & 74.2 & 0 & 2.29 & 11.0 & 36.6 & 102 \\
\hline 1868 & HYDRO & $\mathrm{JO}$ & WY & & & HYDRO & 2.32 & NA & NA & NA & NA & NA \\
\hline 1898 & 10270104406 & $\mathrm{JO}$ & & & & Hays Creek & 5.90 & 0 & .78 & 2.41 & 5.60 & 12.1 \\
\hline 1903 & HYDRO & $\mathrm{JO}$ & & & & HYDRO & 4.19 & NA & NA & NA & NA & NA \\
\hline 1906 & 1027010439 & JO & & & & Mill Creek & 68.1 & 0 & 1.99 & 9.82 & 32.9 & 91.7 \\
\hline 1909 & 10270104406 & JO & & & & Hays Creek & 4.16 & .04 & .61 & 1.67 & 3.65 & 7.87 \\
\hline 1929 & 1027010439 & $\mathrm{JO}$ & & & & Mill Creek & 47.3 & 0 & 1.38 & 7.29 & 24.3 & 66.8 \\
\hline 1969 & 102701042 & JO & LV & WY & & Kansas River & 58,500 & 1,260 & 2,110 & 4,010 & 9,620 & 21,600 \\
\hline 1970 & 102701042 & $\mathrm{JO}$ & LV & & & Kansas River & 58,400 & 1,250 & 2,110 & 4,010 & 9,610 & 21,600 \\
\hline 2001 & 1027010418 & JO & LV & & & Kansas River & 57,800 & 1,200 & 2,020 & 3,820 & 9,070 & 20,500 \\
\hline 2006 & 1027010477 & JO & WY & & & Turkey Creek & 31.4 & 0 & 1.61 & 6.63 & 19.4 & 48.5 \\
\hline 2013 & 1027010438 & JO & & & & Cedar Creek & 55.4 & 0 & 1.28 & 7.28 & 25.3 & 72.2 \\
\hline 2022 & 1027010437 & JO & LV & & & Kill Creek & 62.1 & 0 & 1.12 & 6.53 & 23.2 & 68.4 \\
\hline 2024 & 10270104383 & $\mathrm{JO}$ & & & & Clear Creek & 18.2 & 0 & .38 & 2.83 & 8.91 & 23.7 \\
\hline 2032 & 1027010478 & $\mathrm{JO}$ & & & & Little Mill Creek & 17.2 & 0 & .70 & 3.66 & 10.9 & 27.4 \\
\hline 2059 & 10270104437 & JO & & & & Hanson Creek & 6.65 & 0 & 0 & .17 & 1.46 & 5.85 \\
\hline 2068 & 1030010132 & JO & & & & Indian Creek & 54.6 & 1.30 & 5.35 & 15.8 & 32.1 & 86.5 \\
\hline 2084 & 1027010437 & $\mathrm{JO}$ & & & & Kill Creek & 52.3 & 0 & .92 & 5.61 & 19.8 & 57.8 \\
\hline 2088 & 1027010438 & $\mathrm{JO}$ & & & & Cedar Creek & 39.0 & 0 & .84 & 5.41 & 18.7 & 52.5 \\
\hline 2094 & 1027010475 & JO & & & & Spoon Creek & 18.3 & 0 & 0 & 1.79 & 6.50 & 19.1 \\
\hline 2096 & 1027010475 & $\mathrm{JO}$ & & & & Spoon Creek & 18.2 & 0 & 0 & 1.77 & 6.45 & 19.0 \\
\hline 2110 & 1030010132 & $\mathrm{JO}$ & & & & Indian Creek & 27.5 & 1.30 & 4.70 & 13.0 & 22.0 & 56.0 \\
\hline 2143 & 1027010438 & JO & & & & Cedar Creek & 19.4 & 0 & .10 & 2.43 & 8.57 & 24.4 \\
\hline 2145 & 1027010474 & $\mathrm{JO}$ & & & & Camp Creek & 13.8 & 0 & 0 & 1.87 & 6.40 & 17.8 \\
\hline 2150 & 1027010437 & JO & & & & Kill Creek & 29.5 & 0 & .38 & 3.41 & 11.9 & 34.0 \\
\hline 2166 & 1027010439 & JO & & & & Mill Creek & 29.8 & 0 & .64 & 4.46 & 15.1 & 41.7 \\
\hline 2169 & HYDRO & $\mathrm{JO}$ & & & & HYDRO & 4.33 & NA & NA & NA & NA & NA \\
\hline 2170 & HYDRO & $\mathrm{JO}$ & & & & HYDRO & 15.0 & NA & NA & NA & NA & NA \\
\hline 2176 & 1030010153 & JO & & & & Tomahawk Creek & 25.2 & 0 & .24 & 2.99 & 10.6 & 30.4 \\
\hline 2181 & 1027010439 & $\mathrm{JO}$ & & & & Mill Creek & 3.48 & 0 & 0 & 0 & .32 & 2.22 \\
\hline 2186 & 1030010158 & $\mathrm{JO}$ & & & & Negro Creek & 11.6 & .06 & .18 & 1.92 & 6.28 & 17.4 \\
\hline 2188 & 1030010133 & JO & & & & Blue River & 84.7 & 3.15 & 11.5 & 31.8 & 89.8 & 239 \\
\hline 2192 & 1030010133 & JO & & & & Blue River & 84.7 & 3.15 & 11.5 & 31.8 & 89.8 & 239 \\
\hline 2195 & 1030010133 & JO & & & & Blue River & 84.7 & 3.15 & 11.5 & 31.8 & 89.8 & 239 \\
\hline
\end{tabular}


Table 52. Estimated flow-duration values, mean flow values, and peak-discharge frequency values for controlled and uncontrolled flow stream segments on the 1999 Kansas Surface Water Register for Johnson County.-Continued

[KSWR, Kansas Surface Water Register; CUSEGA, catalog unit segment number alpha; $\mathrm{mi}^{2}$, square miles; $\mathrm{ft}^{3} / \mathrm{s}$, cubic feet per second; HYDRO, lake or other hydrologic structure; NA, not applicable; NRDitch, irrigation ditch; NRTribal, tribal stream]

\begin{tabular}{|c|c|c|c|c|c|c|c|}
\hline \multirow{2}{*}{$\begin{array}{l}\text { Determi- } \\
\text { nation site } \\
\text { identi- } \\
\text { fication } \\
\text { number } \\
\text { (fig. 56) }\end{array}$} & \multirow{2}{*}{$\begin{array}{c}\text { Estimated mean } \\
\text { flow } \\
\left(\mathrm{ft}^{3} / \mathrm{s}\right)\end{array}$} & \multicolumn{6}{|c|}{ Estimated peak discharge $\left(\mathrm{ft}^{3} / \mathrm{s}\right)$ for indicated peak-discharge frequency } \\
\hline & & 2-year & 5-year & 10-year & 25-year & 50-year & 100-year \\
\hline 8 & 12.2 & 1,650 & 3,360 & 4,800 & 6,950 & 8,720 & 10,700 \\
\hline 1855 & 12.3 & 1,450 & 2,950 & 4,220 & 6,120 & 7,680 & 9,440 \\
\hline 1860 & 65.9 & 8,060 & 14,100 & 19,000 & 25,800 & 31,400 & 37,300 \\
\hline 1868 & NA & NA & NA & $\mathrm{NA}$ & $\mathrm{NA}$ & NA & $\mathrm{NA}$ \\
\hline 1898 & 7.40 & 1,070 & 2,150 & 3,060 & 4,410 & 5,510 & 6,760 \\
\hline 1929 & 44.4 & 6,710 & 11,800 & 15,900 & 21,700 & 26,400 & 31,300 \\
\hline 1969 & 8,470 & 50,100 & 88,900 & 119,000 & 148,000 & 200,000 & 240,000 \\
\hline 1970 & 8,460 & 50,100 & 88,900 & 119,000 & 148,000 & 200,000 & 240,000 \\
\hline 2001 & 8,070 & 47,800 & 85,100 & 115,000 & 144,000 & 196,000 & 236,000 \\
\hline 2006 & 31.1 & 5,020 & 8,850 & 11,900 & 16,100 & 19,500 & 23,100 \\
\hline 2013 & 49.1 & 6,240 & 11,400 & 15,600 & 21,700 & 26,700 & 31,900 \\
\hline 2022 & 49.0 & 6,720 & 12,200 & 16,700 & 23,200 & 28,500 & 34,200 \\
\hline 2024 & 16.9 & 2,030 & 4,200 & 6,050 & 8,840 & 11,100 & 13,700 \\
\hline 2032 & 18.2 & 2,040 & 4,180 & 5,990 & 8,710 & 10,900 & 13,500 \\
\hline 2110 & 34.6 & 4,060 & 6,210 & 7,770 & 9,890 & 11,600 & 13,300 \\
\hline 2143 & 18.0 & 2,210 & 4,520 & 6,480 & 9,430 & 11,800 & 14,600 \\
\hline 2145 & 13.2 & 1,770 & 3,620 & 5,190 & 7,530 & 9,450 & 11,600 \\
\hline 2150 & 25.2 & 2,780 & 5,750 & 8,300 & 12,100 & 15,300 & 18,900 \\
\hline 2166 & 28.7 & 2,830 & 5,850 & 8,430 & 12,300 & 15,500 & 19,200 \\
\hline 2169 & NA & NA & NA & NA & NA & NA & NA \\
\hline 2170 & NA & NA & NA & NA & NA & NA & NA \\
\hline 2176 & 22.6 & 2,630 & 4,890 & 6,760 & 9,520 & 11,900 & 14,500 \\
\hline 2181 & 2.75 & 829 & 1,630 & 2,290 & 3,270 & 4,060 & 4,950 \\
\hline 2186 & 12.8 & 1,650 & 3,340 & 4,760 & 6,870 & 8,610 & 10,600 \\
\hline 2188 & 150 & 6,560 & 11,700 & 15,900 & 22,200 & 27,600 & 33,500 \\
\hline 2192 & 150 & 6,580 & 11,700 & 15,900 & 22,200 & 27,600 & 33,600 \\
\hline 2195 & 150 & 6,590 & 11,700 & 15,900 & 22,200 & 27,600 & 33,600 \\
\hline
\end{tabular}


Table 52. Estimated flow-duration values, mean flow values, and peak-discharge frequency values for controlled and uncontrolled flow stream segments on the 1999 Kansas Surface Water Register for Johnson County.-Continued

[KSWR, Kansas Surface Water Register; CUSEGA, catalog unit segment number alpha; $\mathrm{mi}^{2}$, square miles; $\mathrm{ft}^{3} / \mathrm{s}$, cubic feet per second; HYDRO, lake or other hydrologic structure; NA, not applicable; NRDitch, irrigation ditch; NRTribal, tribal stream]

\begin{tabular}{|c|c|c|c|c|c|c|c|c|c|c|c|c|}
\hline \multirow{2}{*}{$\begin{array}{c}\text { Determi- } \\
\text { nation site } \\
\text { identi-fication } \\
\text { number } \\
\text { (fig. } 56 \text { ) }\end{array}$} & \multirow{2}{*}{$\begin{array}{l}\text { KSWR } \\
\text { CUSEGA } \\
\text { number }\end{array}$} & \multicolumn{4}{|c|}{$\begin{array}{l}\text { Stream segment by } \\
\text { county } \\
\text { (table 112) }\end{array}$} & \multirow{2}{*}{ Stream name } & \multirow{2}{*}{$\begin{array}{l}\text { Contri- } \\
\text { buting } \\
\text { drainage } \\
\text { area } \\
\left(\mathrm{mi}^{2}\right)\end{array}$} & \multicolumn{5}{|c|}{$\begin{array}{l}\text { Estimated flow-duration values }\left(\mathrm{ft}^{3} / \mathrm{s}\right) \text { for } \\
\text { indicated percentage of time flow equaled or } \\
\text { exceeded }\end{array}$} \\
\hline & & $1 \mathrm{st}$ & 2nd & $3 r d$ & 4th & & & $\begin{array}{c}90 \\
\text { percent }\end{array}$ & $\begin{array}{c}75 \\
\text { percent }\end{array}$ & $\begin{array}{c}50 \\
\text { percent }\end{array}$ & $\begin{array}{c}25 \\
\text { percent }\end{array}$ & $\begin{array}{c}10 \\
\text { percent }\end{array}$ \\
\hline 2203 & 1027010476 & $\mathrm{JO}$ & & & & Little Cedar Creek & 12.5 & 0 & 0 & 1.75 & 6.02 & 16.8 \\
\hline 2208 & 1030010133 & JO & & & & Blue River & 80.6 & 2.83 & 10.4 & 29.0 & 82.2 & 219 \\
\hline 2209 & 10270104452 & JO & & & & Unnamed tributary, Johnson 1 & 16.1 & 0 & 0 & 1.57 & 5.84 & 17.3 \\
\hline 2212 & 1030010133 & JO & & & & Blue River & 80.6 & 2.83 & 10.4 & 29.0 & 82.2 & 219 \\
\hline 2215 & 1030010158 & JO & & & & Negro Creek & 11.6 & .06 & .18 & 1.92 & 6.28 & 17.4 \\
\hline 2237 & HYDRO & JO & & & & HYDRO & 10.9 & NA & NA & NA & NA & NA \\
\hline 2252 & 1030010133 & JO & & & & Blue River & 80.4 & 2.75 & 10.1 & 28.4 & 80.7 & 215 \\
\hline 2273 & 1027010437 & JO & & & & Kill Creek & 8.34 & 0 & 0 & 1.04 & 3.39 & 9.57 \\
\hline 2275 & 1027010438 & JO & & & & Cedar Creek & 11.5 & 0 & 0 & .94 & 3.75 & 11.6 \\
\hline 2287 & 1030010133 & JO & & & & Blue River & 46.4 & .07 & .69 & 5.00 & 19.0 & 57.0 \\
\hline 2288 & 1030010157 & $\mathrm{JO}$ & & & & Coffee Creek & 18.2 & 0 & 0 & 1.95 & 7.27 & 21.4 \\
\hline 2295 & 1027010475 & JO & & & & Spoon Creek & 18.2 & 0 & 0 & 1.77 & 6.45 & 19.0 \\
\hline 2311 & 10270104452 & $\mathrm{JO}$ & & & & Unnamed tributary, Johnson 1 & 7.94 & 0 & 0 & .23 & 1.51 & 5.89 \\
\hline 2333 & 103001011102 & $\mathrm{JO}$ & & & & Wolf Creek & 23.9 & .02 & .13 & 2.66 & 9.88 & 29.0 \\
\hline 2358 & 1029010250 & $\mathrm{JO}$ & & & & Spring Creek & 4.71 & 0 & 0 & .42 & 1.60 & 5.13 \\
\hline 2362 & 1029010226 & JO & & & & Bull Creek & 9.70 & 0 & 0 & .98 & 3.42 & 10.1 \\
\hline 2370 & HYDRO & JO & & & & HYDRO & 5.69 & NA & NA & NA & NA & NA \\
\hline 2391 & 1030010156 & JO & & & & Camp Branch & 28.3 & .32 & 1.32 & 5.65 & 17.7 & 48.4 \\
\hline 2407 & 1029010225 & JO & & & & Tenmile Creek & 6.01 & 0 & 0 & .35 & 1.63 & 5.64 \\
\hline 2408 & 1029010226 & JO & & & & Bull Creek & 33.0 & 0 & .42 & 3.48 & 12.2 & 35.3 \\
\hline 2409 & HYDRO & JO & & & & HYDRO & 6.30 & NA & NA & NA & NA & NA \\
\hline 2411 & 1029010250 & JO & & & & Spring Creek & 9.26 & 0 & 0 & 1.25 & 4.26 & 12.0 \\
\hline 2412 & 1029010251 & JO & & & & Little Bull Creek & 16.6 & 0 & 0 & 1.78 & 6.33 & 18.3 \\
\hline 2413 & 1029010225 & $\mathrm{JO}$ & & & & Tenmile Creek & 6.63 & 0 & 0 & .42 & 1.88 & 6.35 \\
\hline 2417 & HYDRO & $\mathrm{JO}$ & MI & & & HYDRO & 7.10 & NA & NA & NA & NA & NA \\
\hline 2437 & 1029010250 & JO & MI & & & Spring Creek & 27.7 & 0 & .31 & 3.18 & 11.2 & 32.2 \\
\hline 2464 & HYDRO & JO & MI & & & HYDRO & 39.0 & NA & NA & NA & NA & NA \\
\hline 2497 & 1029010249 & JO & MI & & & Sweetwater Creek & 12.6 & 0 & 0 & 1.36 & 4.97 & 14.6 \\
\hline 2579 & 1029010221 & $\mathrm{JO}$ & MI & & & North Wea Creek & 26.5 & 0 & .12 & 2.74 & 10.2 & 30.2 \\
\hline
\end{tabular}


Table 52. Estimated flow-duration values, mean flow values, and peak-discharge frequency values for controlled and uncontrolled flow stream segments on the 1999 Kansas Surface Water Register for Johnson County.-Continued

[KSWR, Kansas Surface Water Register; CUSEGA, catalog unit segment number alpha; $\mathrm{mi}^{2}$, square miles; $\mathrm{ft}^{3} / \mathrm{s}$, cubic feet per second; HYDRO, lake or other hydrologic structure; NA, not applicable; NRDitch, irrigation ditch; NRTribal, tribal stream]

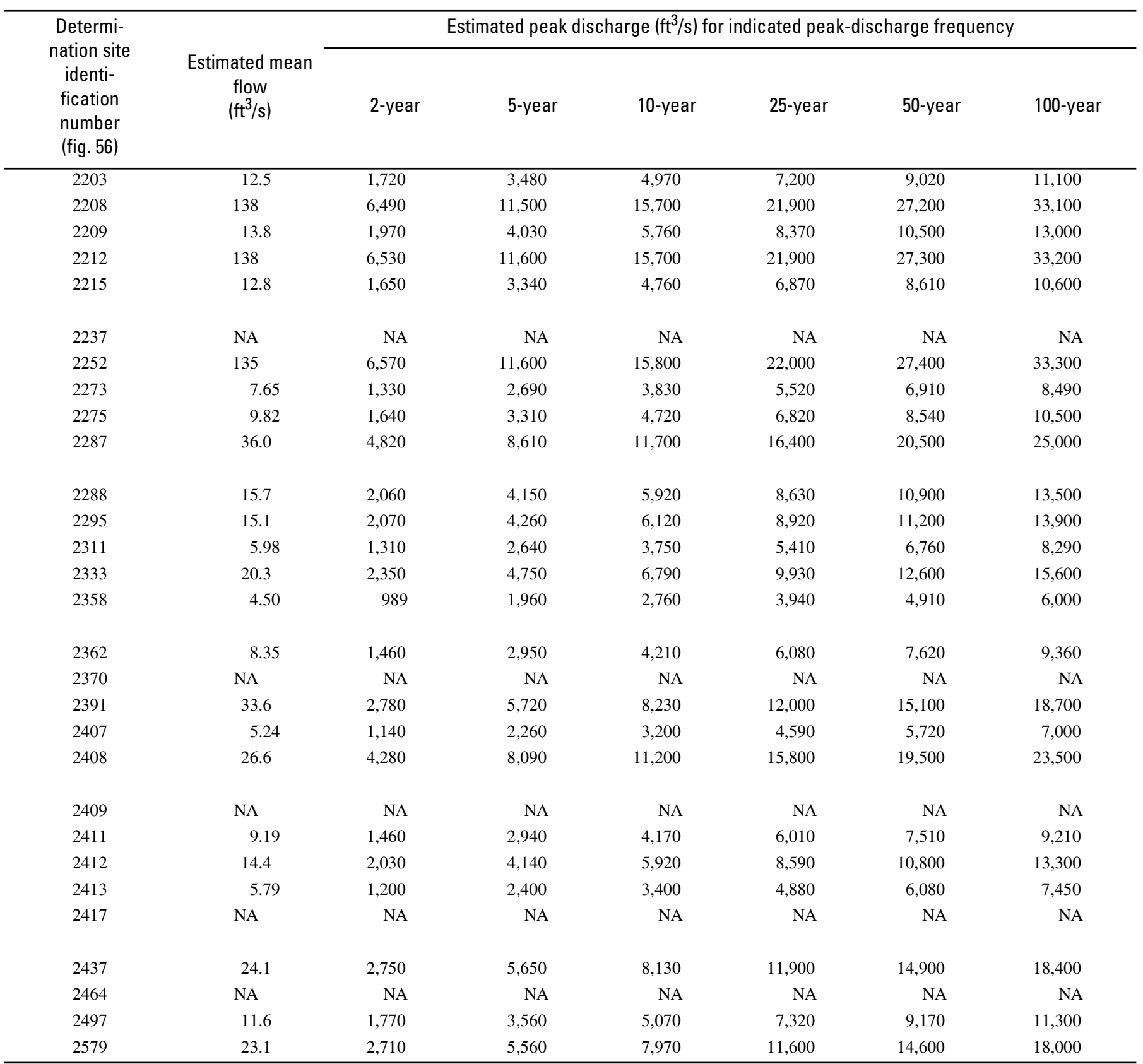




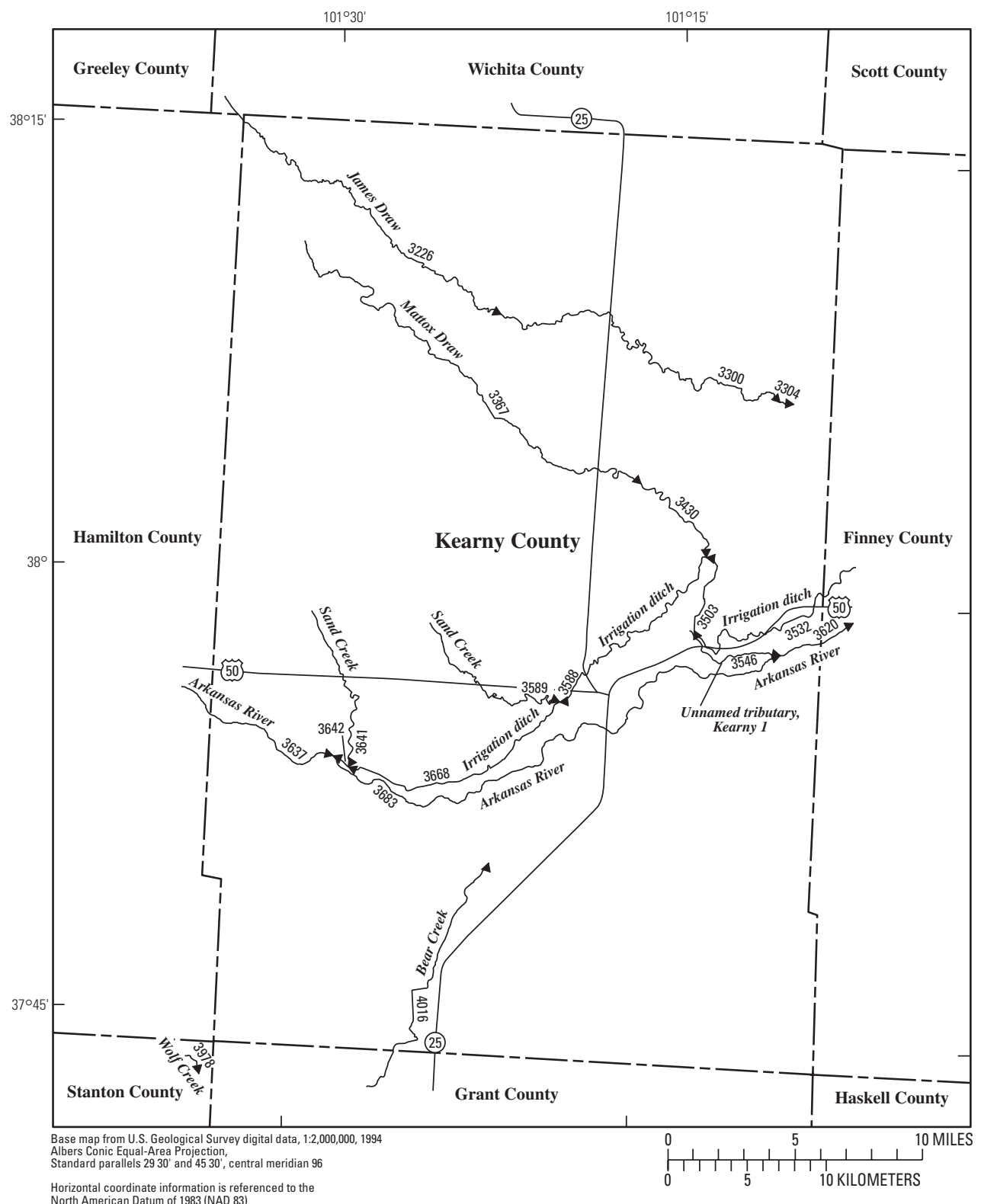

EXPLANATION

4016 Location of streamflow-statistics determination site (small triangle) and associated identification number-small triangle points in downstream direction

$06853800 \Delta$ U.S. Geological Survey streamflow-gaging station and number used for estimates of flow duration

${ }^{06875800} \triangle$ U.S. Geological Survey streamflow-gaging station and number used for estimates of peak-discharge frequency values

KANSAS

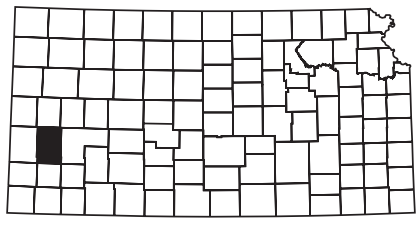

Index map 926

Figure 57. Location of streamflow-statistics determination sites, associated identification numbers, and U.S. Geological Survey streamflow-gaging stations used in the flow-duration and peak-discharge frequency analyses for Kearny County. 
Table 53. Estimated flow-duration values, mean flow values, and peak-discharge frequency values for controlled and uncontrolled flow stream segments on the 1999 Kansas Surface Water Register for Kearny County.

[KSWR, Kansas Surface Water Register; CUSEGA, catalog unit segment number alpha; $\mathrm{mi}^{2}$, square miles; $\mathrm{ft}^{3} / \mathrm{s}$, cubic feet per second; HYDRO, lake or other hydrologic structure; NA, not applicable; NRDitch, irrigation ditch; NRTribal, tribal stream]

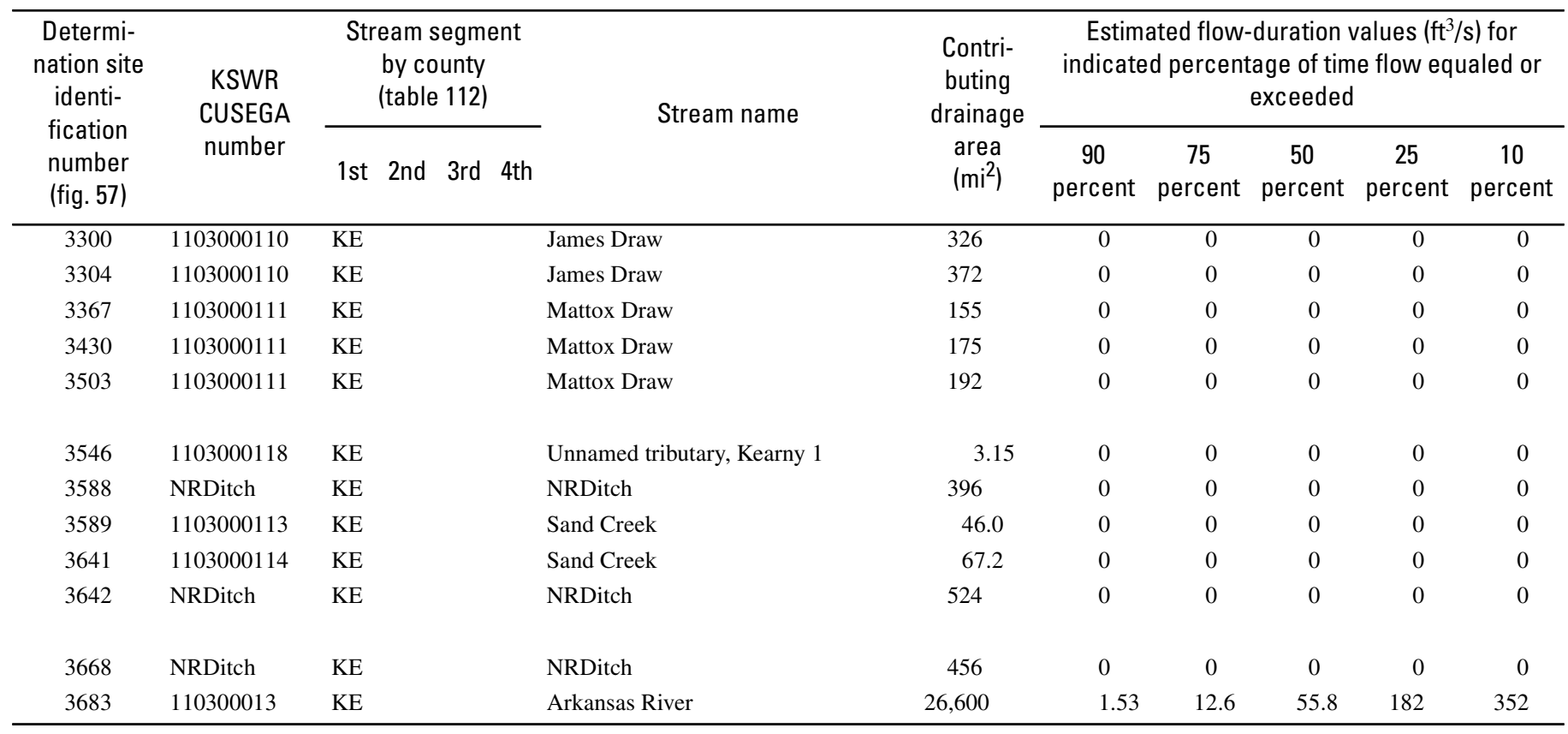


Table 53. Estimated flow-duration values, mean flow values, and peak-discharge frequency values for controlled and uncontrolled flow stream segments on the 1999 Kansas Surface Water Register for Kearny County.-Continued

[KSWR, Kansas Surface Water Register; CUSEGA, catalog unit segment number alpha; $\mathrm{mi}^{2}$, square miles; $\mathrm{ft}^{3} / \mathrm{s}$, cubic feet per second; HYDRO, lake or other hydrologic structure; NA, not applicable; NRDitch, irrigation ditch; NRTribal, tribal stream]

\begin{tabular}{|c|c|c|c|c|c|c|c|}
\hline \multirow{2}{*}{$\begin{array}{l}\text { Determi- } \\
\text { nation site } \\
\text { identi- } \\
\text { fication } \\
\text { number } \\
\text { (fig. 57) }\end{array}$} & \multirow{2}{*}{$\begin{array}{c}\text { Estimated mean } \\
\text { flow } \\
\left(\mathrm{ft}^{3} / \mathrm{s}\right)\end{array}$} & \multicolumn{6}{|c|}{ Estimated peak discharge $\left(\mathrm{ft}^{3} / \mathrm{s}\right)$ for indicated peak-discharge frequency } \\
\hline & & 2-year & 5-year & 10-year & 25-year & 50-year & 100-year \\
\hline 3300 & 0.17 & 416 & 1,510 & 2,840 & 5,380 & 7,990 & 11,300 \\
\hline 3430 & 0 & 297 & 1,100 & 2,090 & 3,960 & 5,900 & 8,340 \\
\hline 3503 & .35 & 275 & 1,020 & 1,960 & 3,740 & 5,600 & 7,940 \\
\hline 3546 & 0 & 76 & 263 & 471 & 840 & 1,190 & 1,610 \\
\hline 3588 & 1.41 & 505 & 1,750 & 3,250 & 6,030 & 8,870 & 12,400 \\
\hline 3668 & 1.88 & 448 & 1,600 & 3,000 & 5,660 & 8,390 & 11,800 \\
\hline 3683 & 180 & 955 & 3,590 & 9,250 & 21,900 & 36,900 & 62,400 \\
\hline
\end{tabular}




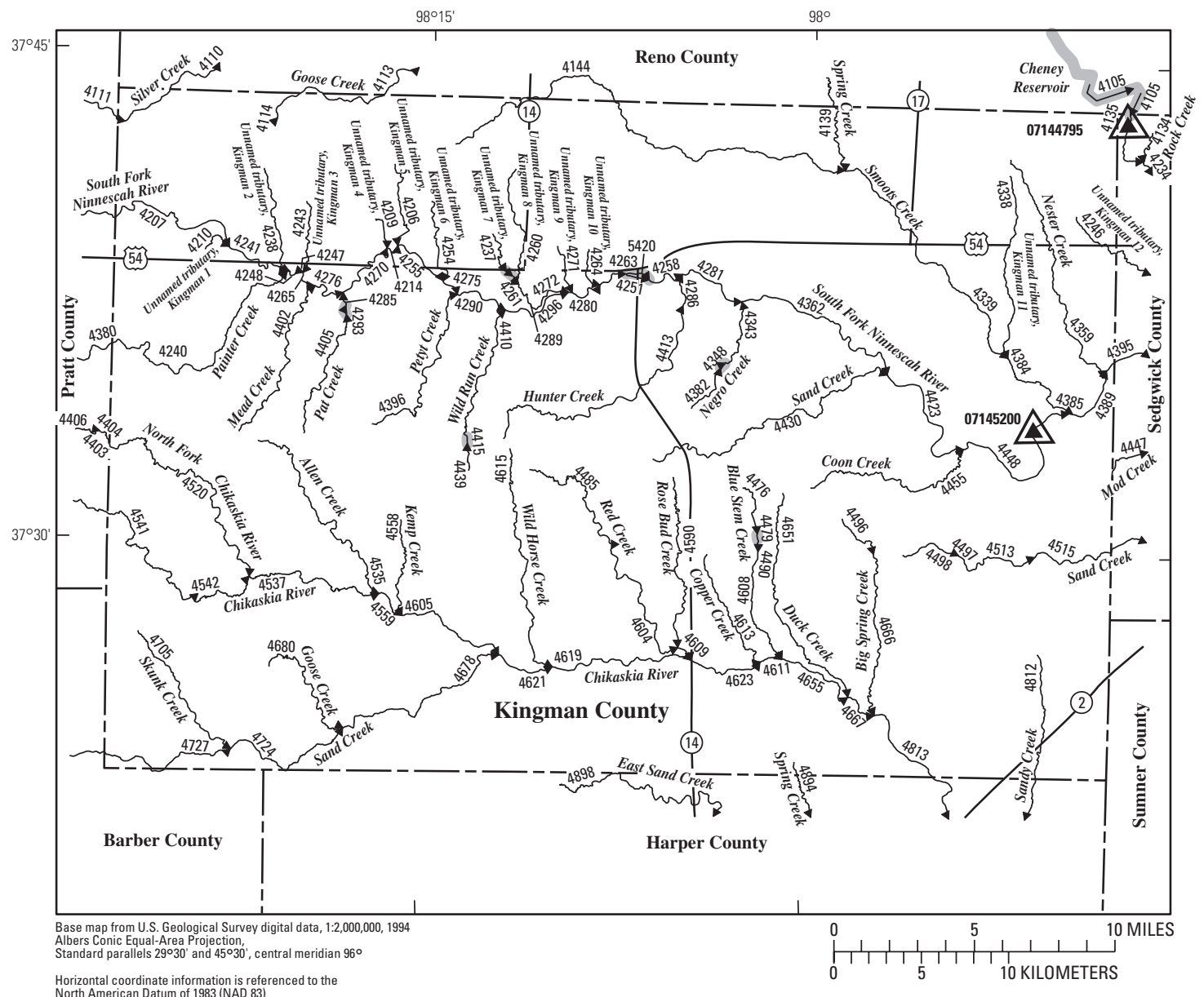

EXPLANATION

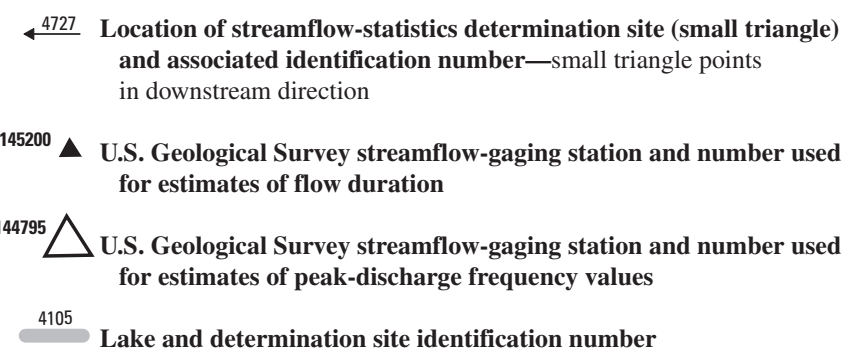

KANSAS

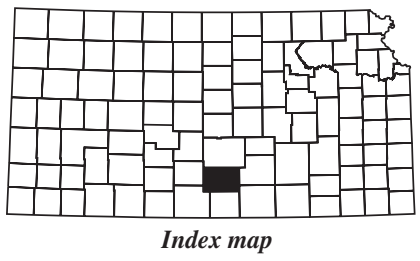

Figure 58. Location of streamflow-statistics determination sites, associated identification numbers, and U.S. Geological Survey streamflow-gaging stations used in the flow-duration and peak-discharge frequency analyses for Kingman County. 
Table 54. Estimated flow-duration values, mean flow values, and peak-discharge frequency values for controlled and uncontrolled flow stream segments on the 1999 Kansas Surface Water Register for Kingman County.

[KSWR, Kansas Surface Water Register; CUSEGA, catalog unit segment number alpha; $\mathrm{mi}^{2}$, square miles; $\mathrm{ft}^{3} / \mathrm{s}$, cubic feet per second; HYDRO, lake or other hydrologic structure; NA, not applicable; NRDitch, irrigation ditch; NRTribal, tribal stream]

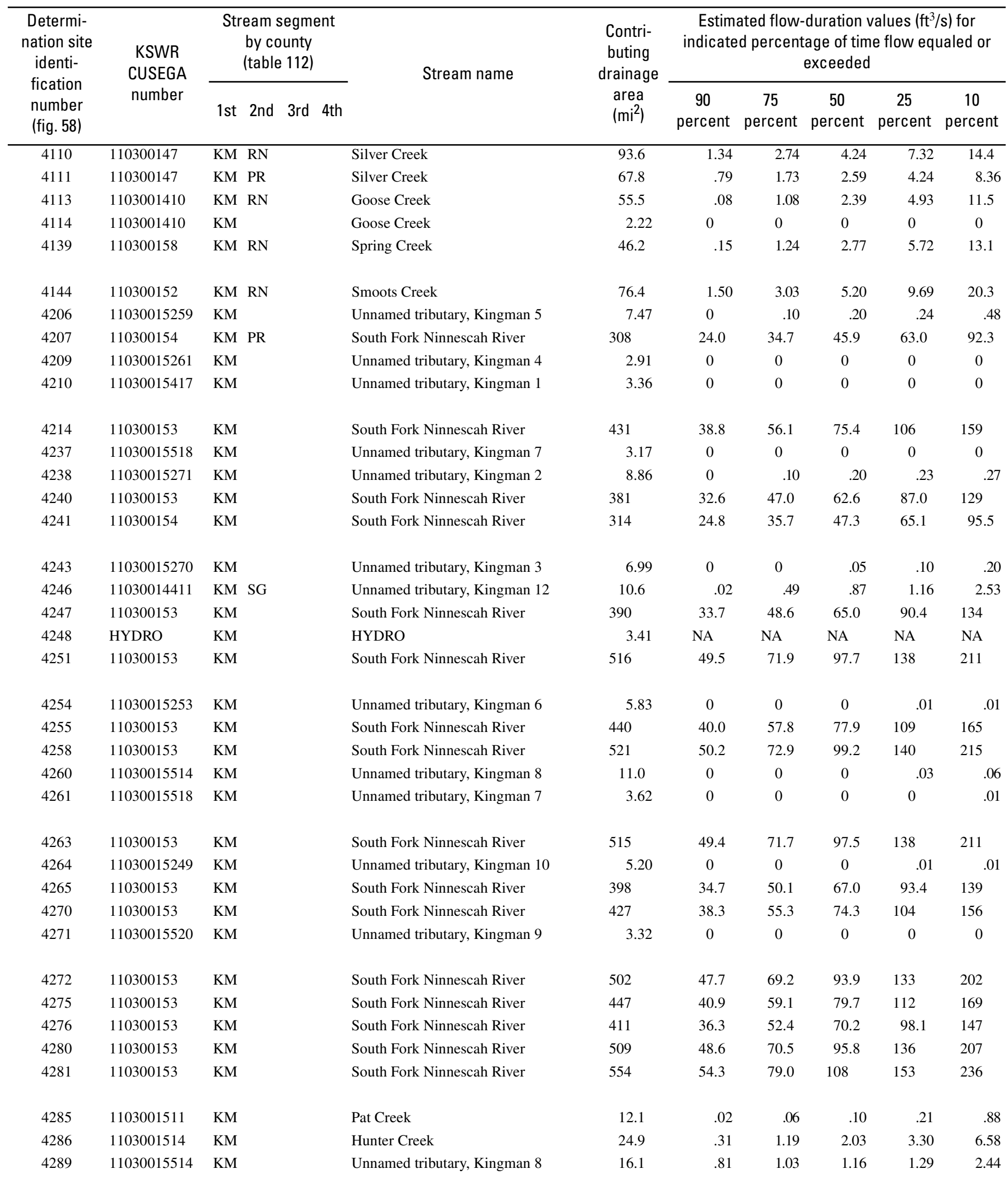


Table 54. Estimated flow-duration values, mean flow values, and peak-discharge frequency values for controlled and uncontrolled flow stream segments on the 1999 Kansas Surface Water Register for Kingman County.-Continued

[KSWR, Kansas Surface Water Register; CUSEGA, catalog unit segment number alpha; $\mathrm{mi}^{2}$, square miles; $\mathrm{ft}^{3} / \mathrm{s}$, cubic feet per second; HYDRO, lake or other hydrologic structure; NA, not applicable; NRDitch, irrigation ditch; NRTribal, tribal stream]

\begin{tabular}{|c|c|c|c|c|c|c|c|}
\hline \multirow{2}{*}{$\begin{array}{l}\text { Determi- } \\
\text { nation site } \\
\text { identi- } \\
\text { fication } \\
\text { number } \\
\text { (fig. 58) }\end{array}$} & \multirow{2}{*}{$\begin{array}{c}\text { Estimated mean } \\
\text { flow } \\
\left(\mathrm{ft}^{3} / \mathrm{s}\right)\end{array}$} & \multicolumn{6}{|c|}{ Estimated peak discharge $\left(\mathrm{ft}^{3} / \mathrm{s}\right)$ for indicated peak-discharge frequency } \\
\hline & & 2-year & 5-year & 10-year & 25-year & 50-year & 100 -year \\
\hline 4110 & 12.7 & 982 & 2,430 & 3,840 & 6,110 & 8,170 & 10,500 \\
\hline 4114 & 0 & 246 & 605 & 935 & 1,450 & 1,900 & 2,410 \\
\hline 4139 & 11.1 & 1,420 & 3,200 & 4,820 & 7,290 & 9,440 & 11,800 \\
\hline 4144 & 16.0 & 1,370 & 3,090 & 4,670 & 7,070 & 9,150 & 11,400 \\
\hline 4206 & 1.15 & 518 & 1,290 & 2,010 & 3,160 & 4,150 & 5,300 \\
\hline 4214 & 116 & 4,030 & 8,700 & 12,600 & 18,500 & 23,400 & 28,700 \\
\hline 4237 & 0 & 322 & 785 & 1,210 & 1,880 & 2,450 & 3,110 \\
\hline 4238 & 1.32 & 548 & 1,390 & 2,170 & 3,430 & 4,520 & 5,790 \\
\hline 4240 & 96.5 & 3,620 & 7,800 & 11,300 & 16,500 & 20,900 & 25,700 \\
\hline 4241 & 73.0 & 3,000 & 6,440 & 9,340 & 13,600 & 17,200 & 21,000 \\
\hline 4243 & 1.02 & 489 & 1,220 & 1,910 & 3,000 & 3,940 & 5,040 \\
\hline 4246 & 2.87 & 724 & 1,760 & 2,720 & 4,230 & 5,530 & 7,030 \\
\hline 4247 & 100 & 3,700 & 7,970 & 11,600 & 16,900 & 21,400 & 26,200 \\
\hline 4248 & NA & NA & NA & NA & NA & NA & NA \\
\hline 4263 & 151 & 4,720 & 10,200 & 14,800 & 21,600 & 27,400 & 33,600 \\
\hline 4264 & .32 & 436 & 1,070 & 1,650 & 2,570 & 3,370 & 4,280 \\
\hline 4265 & 103 & 3,770 & 8,120 & 11,800 & 17,200 & 21,800 & 26,700 \\
\hline 4270 & 115 & 4,000 & 8,640 & 12,600 & 18,300 & 23,200 & 28,500 \\
\hline 4271 & 0 & 335 & 815 & 1,250 & 1,940 & 2,540 & 3,220 \\
\hline 4272 & 145 & 4,640 & 10,000 & 14,500 & 21,200 & 26,900 & 33,000 \\
\hline 4275 & 123 & 4,150 & 8,960 & 13,000 & 19,000 & 24,100 & 29,600 \\
\hline 4276 & 108 & 3,890 & 8,370 & 12,200 & 17,700 & 22,500 & 27,600 \\
\hline 4280 & 148 & 4,670 & 10,100 & 14,700 & 21,400 & 27,100 & 33,300 \\
\hline 4281 & 167 & 5,080 & 10,900 & 15,900 & 23,200 & 29,400 & 36,100 \\
\hline 4285 & 2.06 & 677 & 1,710 & 2,680 & 4,240 & 5,590 & 7,160 \\
\hline 4286 & 5.93 & 1,080 & 2,740 & 4,310 & 6,820 & 9,010 & 11,600 \\
\hline 4289 & 3.09 & 833 & 2,100 & 3,280 & 5,180 & 6,820 & 8,740 \\
\hline
\end{tabular}


Table 54. Estimated flow-duration values, mean flow values, and peak-discharge frequency values for controlled and uncontrolled flow stream segments on the 1999 Kansas Surface Water Register for Kingman County._-Continued

[KSWR, Kansas Surface Water Register; CUSEGA, catalog unit segment number alpha; $\mathrm{mi}^{2}$, square miles; $\mathrm{ft}^{3} / \mathrm{s}$, cubic feet per second; HYDRO, lake or other hydrologic structure; NA, not applicable; NRDitch, irrigation ditch; NRTribal, tribal stream]

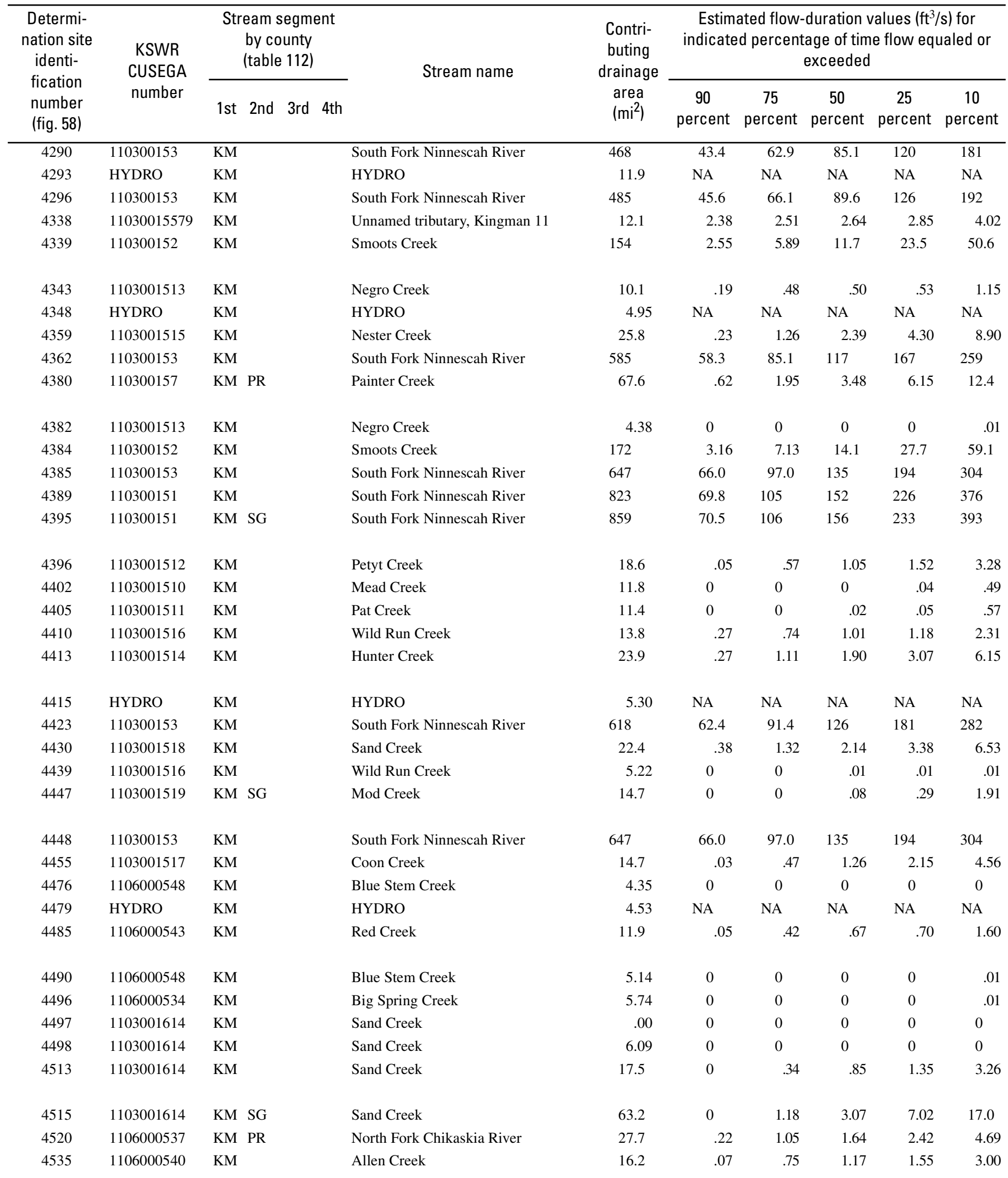


Table 54. Estimated flow-duration values, mean flow values, and peak-discharge frequency values for controlled and uncontrolled flow stream segments on the 1999 Kansas Surface Water Register for Kingman County.-Continued

[KSWR, Kansas Surface Water Register; CUSEGA, catalog unit segment number alpha; $\mathrm{mi}^{2}$, square miles; $\mathrm{ft}^{3} / \mathrm{s}$, cubic feet per second; HYDRO, lake or other hydrologic structure; NA, not applicable; NRDitch, irrigation ditch; NRTribal, tribal stream]

\begin{tabular}{|c|c|c|c|c|c|c|c|}
\hline \multirow{2}{*}{$\begin{array}{l}\text { Determi- } \\
\text { nation site } \\
\text { identi- } \\
\text { fication } \\
\text { number } \\
\text { (fig. 58) }\end{array}$} & \multirow{2}{*}{$\begin{array}{c}\text { Estimated mean } \\
\text { flow } \\
\left(\mathrm{ft}^{3} / \mathrm{s}\right)\end{array}$} & \multicolumn{6}{|c|}{ Estimated peak discharge $\left(\mathrm{ft}^{3} / \mathrm{s}\right)$ for indicated peak-discharge frequency } \\
\hline & & 2-year & 5-year & 10 -year & 25-year & 50-year & 100 -year \\
\hline 4290 & 131 & 4,330 & 9,350 & 13,600 & 19,800 & 25,100 & 30,900 \\
\hline 4338 & 3.33 & 759 & 1,870 & 2,890 & 4,510 & 5,910 & 7,530 \\
\hline 4339 & 35.3 & 2,150 & 4,720 & 7,050 & 10,600 & 13,700 & 17,100 \\
\hline 4343 & 2.02 & 644 & 1,600 & 2,490 & 3,900 & 5,120 & 6,530 \\
\hline 4348 & NA & NA & NA & NA & NA & NA & NA \\
\hline 4382 & 0 & 396 & 968 & 1,490 & 2,320 & 3,030 & 3,850 \\
\hline 4384 & 40.0 & 2,220 & 4,850 & 7,230 & 10,800 & 14,000 & 17,400 \\
\hline 4385 & 209 & 5,850 & 12,600 & 18,300 & 26,700 & 33,800 & 41,500 \\
\hline 4389 & 246 & 6,680 & 14,200 & 20,600 & 29,900 & 37,900 & 46,500 \\
\hline 4395 & 255 & 6,830 & 14,500 & 21,000 & 30,600 & 38,700 & 47,500 \\
\hline 4396 & 3.77 & 880 & 2,240 & 3,520 & 5,580 & 7,370 & 9,450 \\
\hline 4402 & 1.80 & 645 & 1,640 & 2,590 & 4,100 & 5,410 & 6,940 \\
\hline 4405 & 1.84 & 652 & 1,650 & 2,580 & 4,080 & 5,370 & 6,880 \\
\hline 4410 & 2.85 & 750 & 1,890 & 2,960 & 4,670 & 6,150 & 7,880 \\
\hline 4448 & 209 & 5,850 & 12,600 & 18,300 & 26,700 & 33,800 & 41,500 \\
\hline 4455 & 4.19 & 813 & 2,030 & 3,170 & 4,980 & 6,550 & 8,370 \\
\hline 4476 & .09 & 396 & 967 & 1,490 & 2,310 & 3,020 & 3,840 \\
\hline 4479 & NA & NA & NA & NA & NA & NA & NA \\
\hline 4485 & 2.39 & 700 & 1,750 & 2,730 & 4,290 & 5,650 & 7,220 \\
\hline 4490 & .32 & 437 & 1,070 & 1,650 & 2,570 & 3,360 & 4,270 \\
\hline 4496 & 0.90 & 469 & 1,150 & 1,770 & 2,760 & 3,610 & 4,590 \\
\hline 4497 & 0 & 18 & 35 & 49 & 68 & 83 & 100 \\
\hline 4498 & .91 & 484 & 1,190 & 1,840 & 2,860 & 3,740 & 4,760 \\
\hline 4513 & 3.79 & 888 & 2,230 & 3,490 & 5,510 & 7,250 & 9,280 \\
\hline 4515 & 14.4 & 1,530 & 3,550 & 5,430 & 8,330 & 10,900 & 13,700 \\
\hline 4520 & 4.69 & 985 & 2,600 & 4,160 & 6,700 & 8,930 & 11,500 \\
\hline 4535 & 3.29 & 785 & 2,010 & 3,170 & 5,030 & 6,650 & 8,540 \\
\hline
\end{tabular}


Table 54. Estimated flow-duration values, mean flow values, and peak-discharge frequency values for controlled and uncontrolled flow stream segments on the 1999 Kansas Surface Water Register for Kingman County.-Continued

[KSWR, Kansas Surface Water Register; CUSEGA, catalog unit segment number alpha; $\mathrm{mi}^{2}$, square miles; $\mathrm{ft}^{3} / \mathrm{s}$, cubic feet per second; HYDRO, lake or other hydrologic structure; NA, not applicable; NRDitch, irrigation ditch; NRTribal, tribal stream]

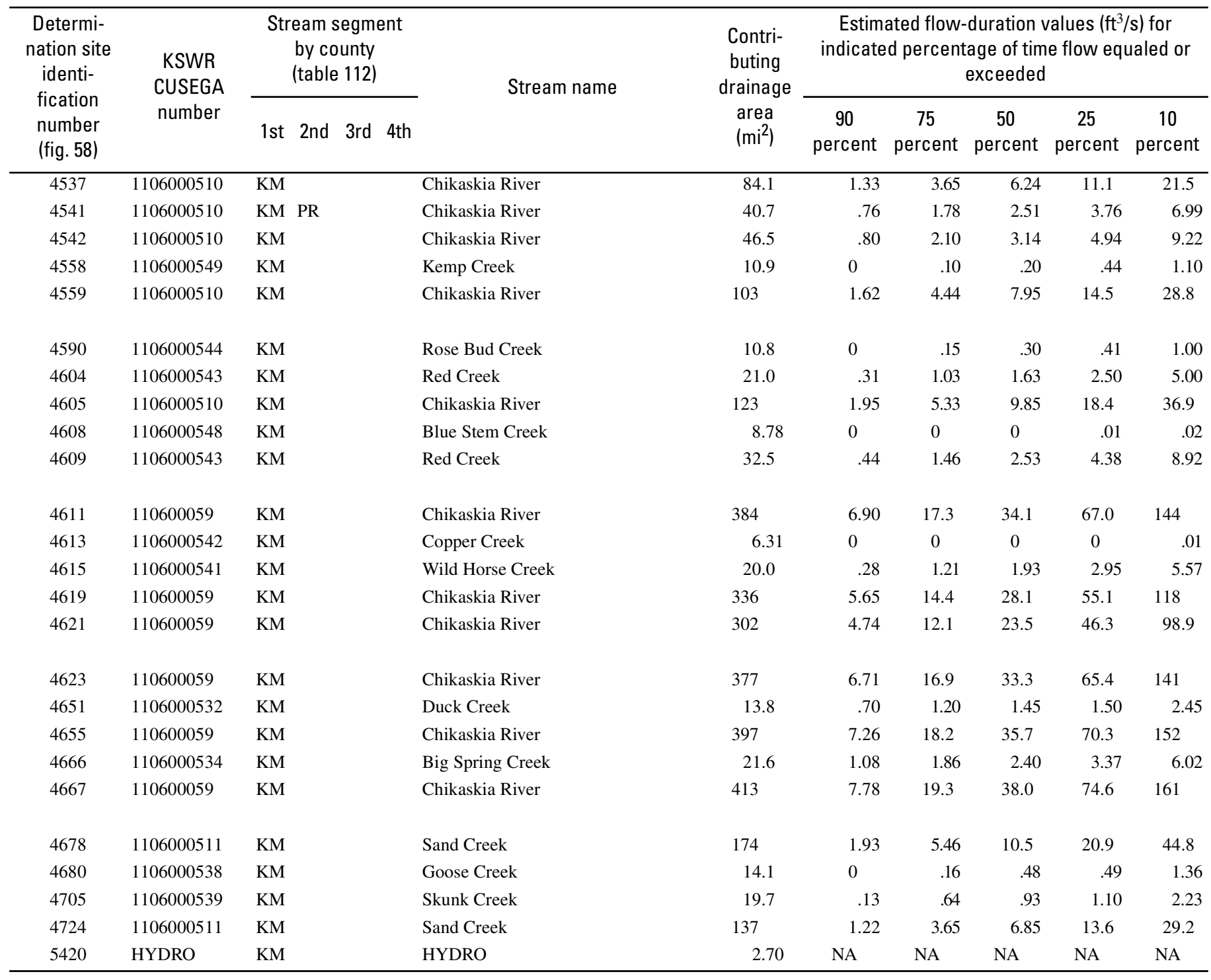


Table 54. Estimated flow-duration values, mean flow values, and peak-discharge frequency values for controlled and uncontrolled flow stream segments on the 1999 Kansas Surface Water Register for Kingman County.-Continued

[KSWR, Kansas Surface Water Register; CUSEGA, catalog unit segment number alpha; $\mathrm{mi}^{2}$, square miles; $\mathrm{ft}^{3} / \mathrm{s}$, cubic feet per second; HYDRO, lake or other hydrologic structure; NA, not applicable; NRDitch, irrigation ditch; NRTribal, tribal stream]

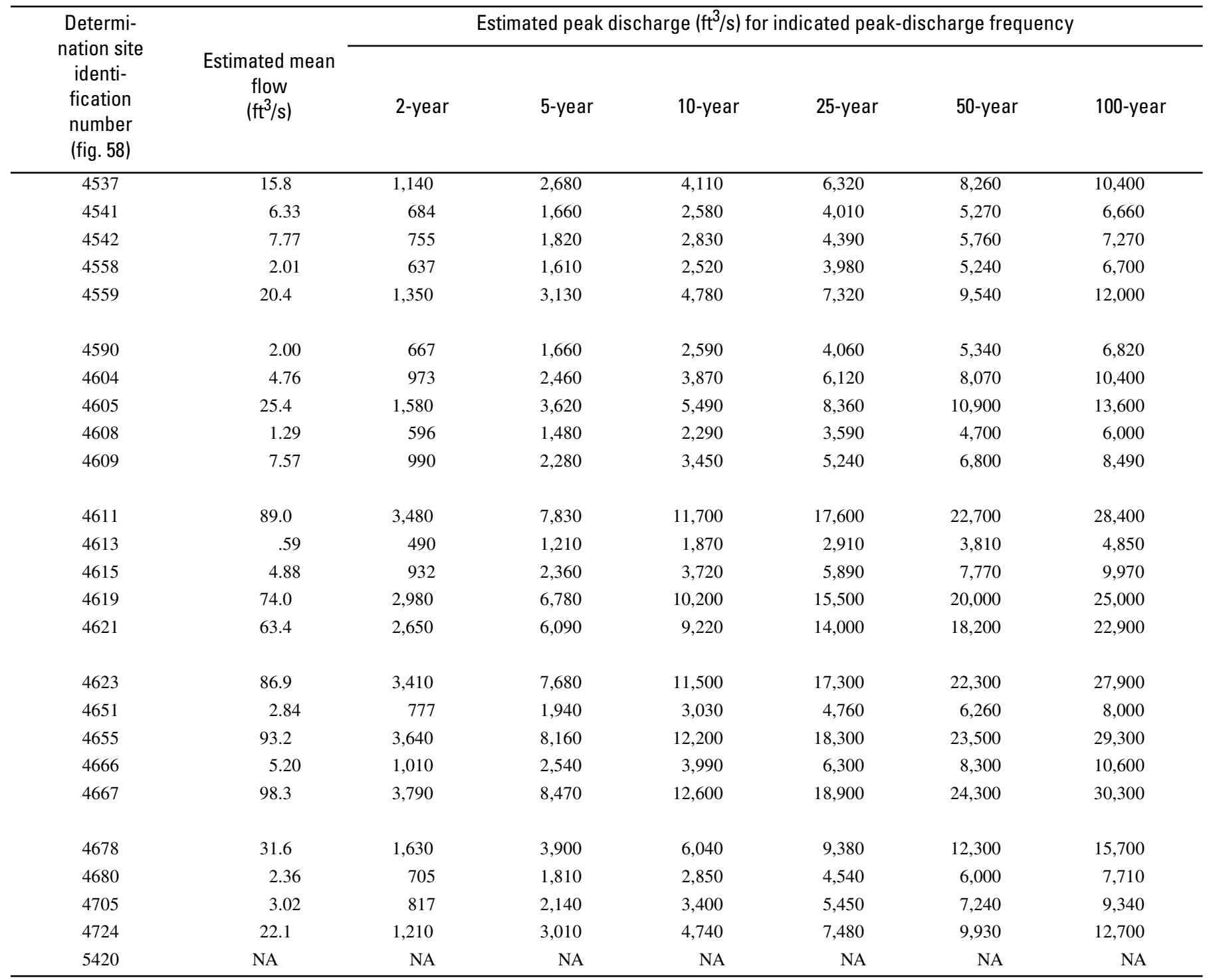




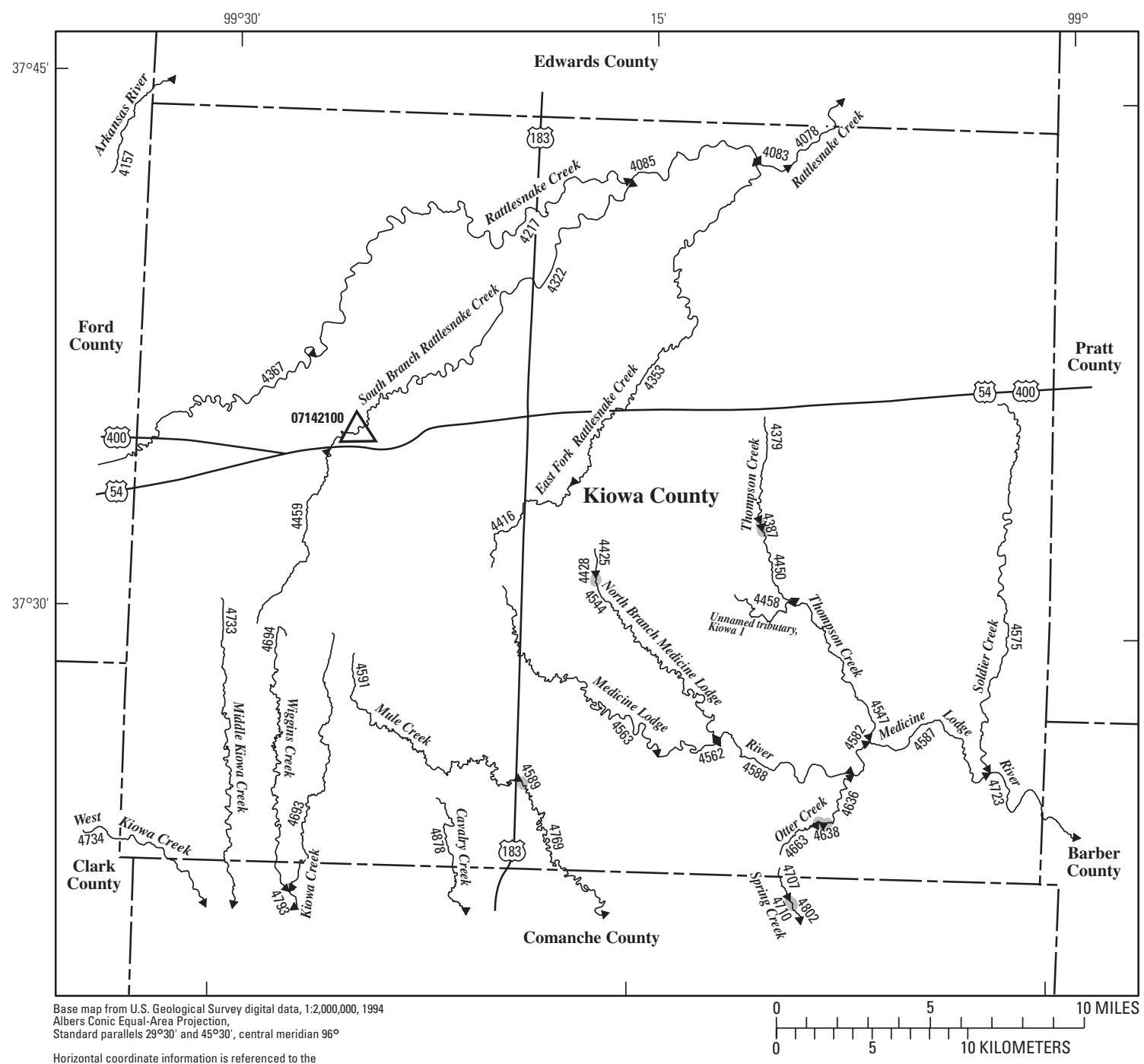

EXPLANATION

$\$ 746$ Location of streamflow-statistics determination site (small triangle) and associated identification number-small triangle points in downstream direction

$06853800 \Delta$ U.S. Geological Survey streamflow-gaging station and number used for estimates of flow duration

${ }^{06875800} \triangle$ U.S. Geological Survey streamflow-gaging station and number used for estimates of peak-discharge frequency values

KANSAS

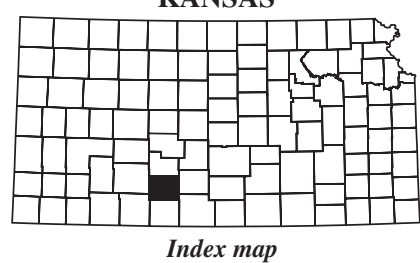

926 Lake and determination site identification number

Figure 59. Location of streamflow-statistics determination sites, associated identification numbers, and U.S. Geological Survey streamflow-gaging stations used in the flow-duration and peak-discharge frequency analyses for Kiowa County. 
Table 55. Estimated flow-duration values, mean flow values, and peak-discharge frequency values for controlled and uncontrolled flow stream segments on the 1999 Kansas Surface Water Register for Kiowa County.

[KSWR, Kansas Surface Water Register; CUSEGA, catalog unit segment number alpha; $\mathrm{mi}^{2}$ square miles; $\mathrm{ft}^{3} / \mathrm{s}$, cubic feet per second; HYDRO, lake or other hydrologic structure; NA, not applicable; NRDitch, irrigation ditch; NRTribal, tribal stream]

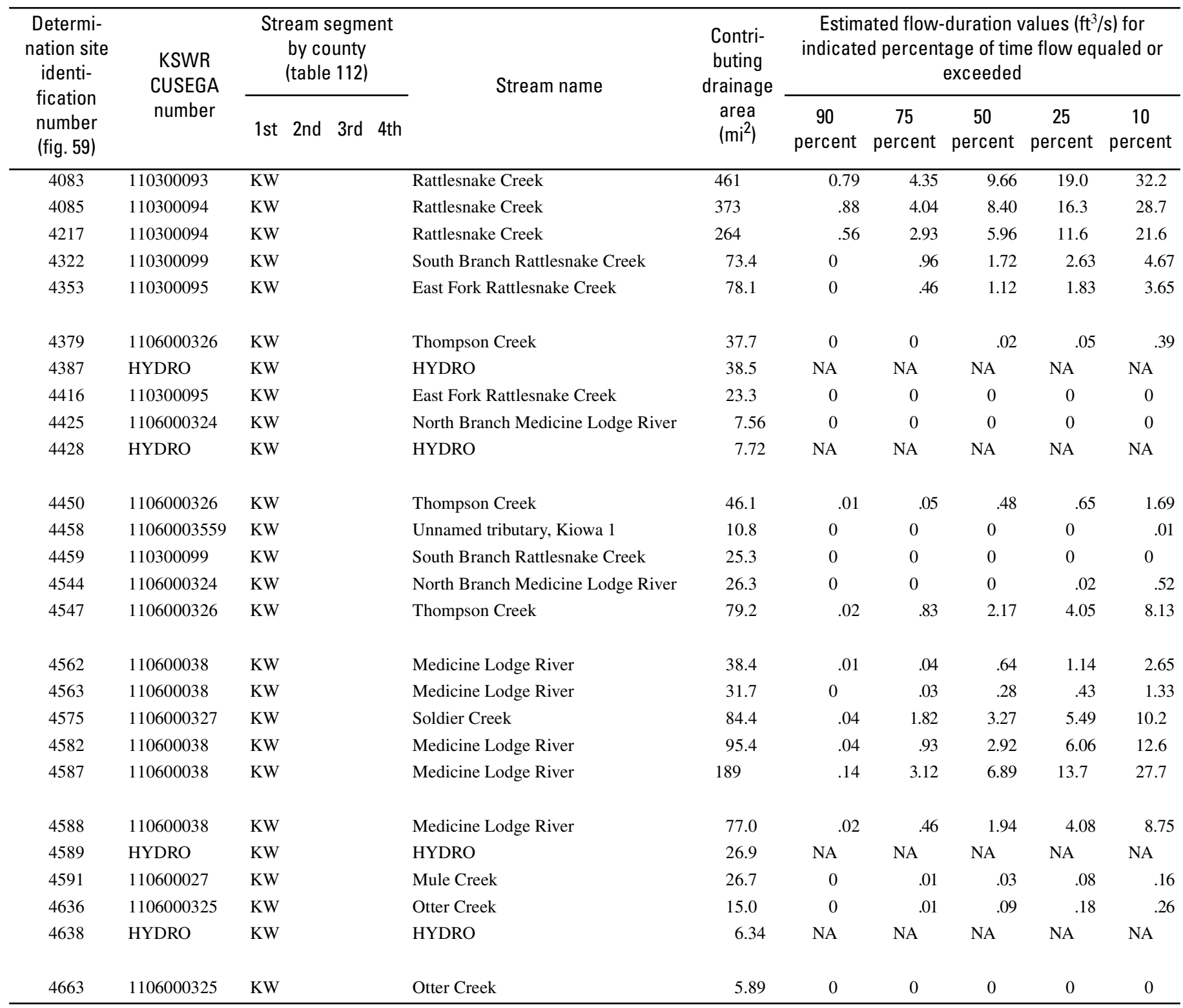


Table 55. Estimated flow-duration values, mean flow values, and peak-discharge frequency values for controlled and uncontrolled flow stream segments on the 1999 Kansas Surface Water Register for Kiowa County.-Continued

[KSWR, Kansas Surface Water Register; CUSEGA, catalog unit segment number alpha; $\mathrm{mi}^{2}$, square miles; $\mathrm{ft}^{3} / \mathrm{s}$, cubic feet per second; HYDRO, lake or other hydrologic structure; NA, not applicable; NRDitch, irrigation ditch; NRTribal, tribal stream]

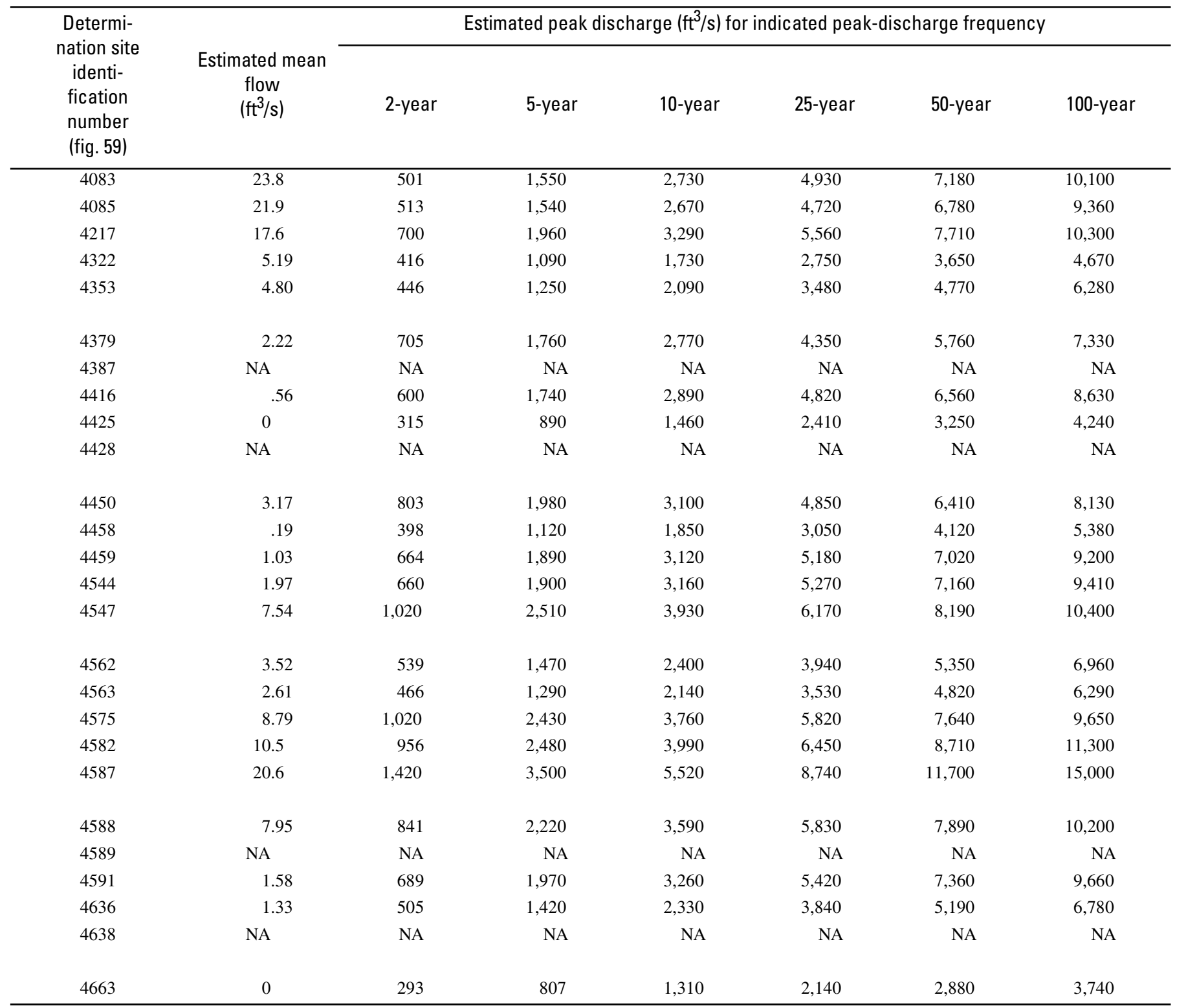




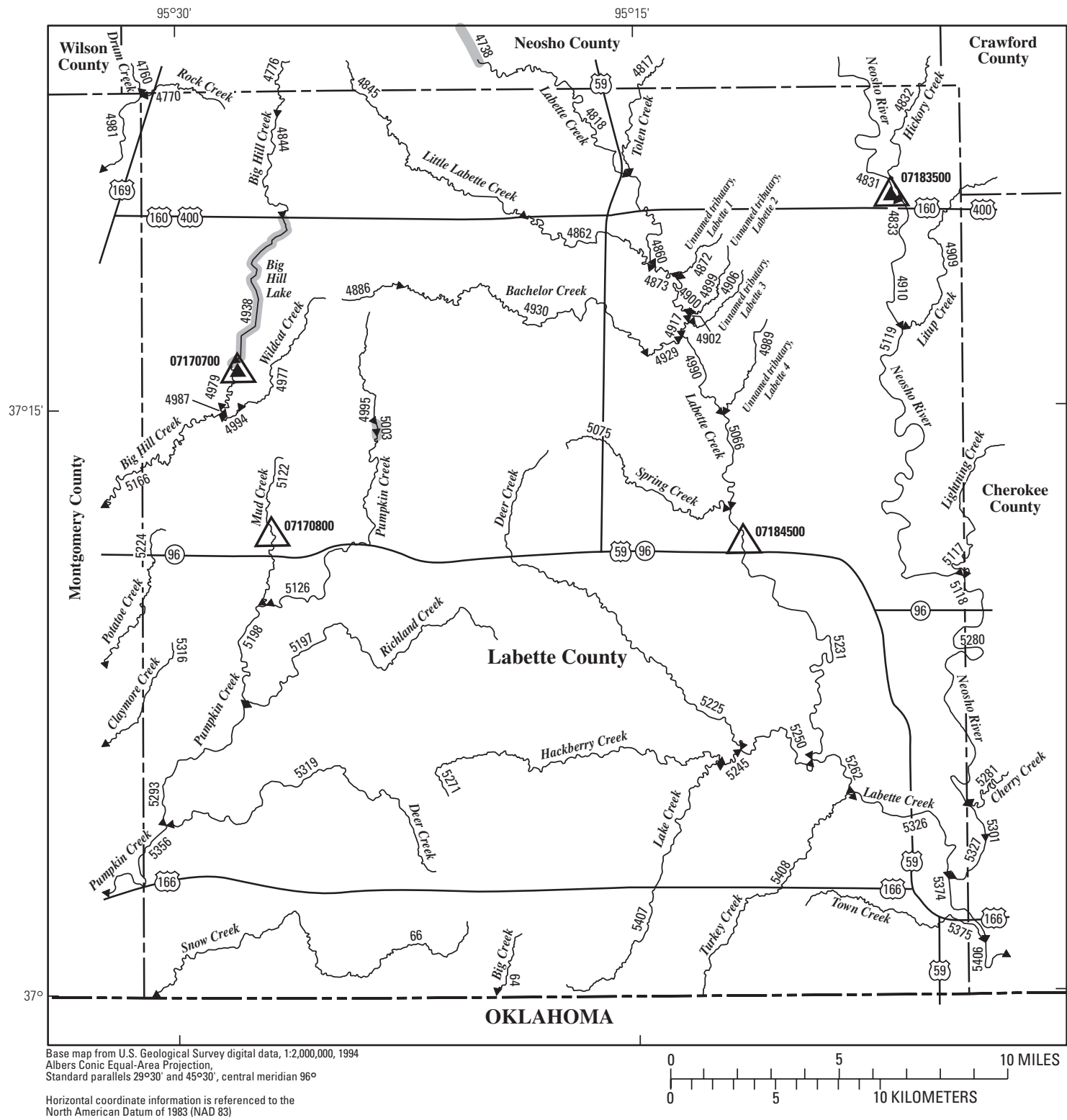

EXPLANATION

\footnotetext{
66 Location of streamflow-statistics determination site (small triangle) and associated identification number-small triangle points in downstream direction

07183500

U.S. Geological Survey streamflow-gaging station and number used for estimates of flow duration

${ }^{07184500} \triangle$ U.S. Geological Survey streamflow-gaging station and number used for estimates of peak-discharge frequency values

Lake and determination site identification number
}

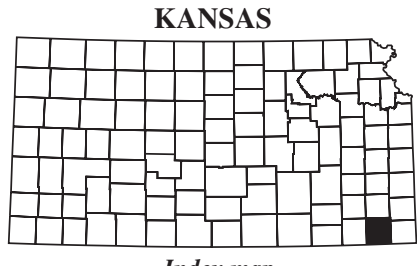

Figure 60. Location of streamflow-statistics determination sites, associated identification numbers, and U.S. Geological Survey streamflow-gaging stations used in the flow-duration and peak-discharge frequency analyses for Labette County. 
Table 56. Estimated flow-duration values, mean flow values, and peak-discharge frequency values for controlled and uncontrolled flow stream segments on the 1999 Kansas Surface Water Register for Labette County.

[KSWR, Kansas Surface Water Register; CUSEGA, catalog unit segment number alpha; $\mathrm{mi}^{2}$, square miles; $\mathrm{ft}^{3} / \mathrm{s}$, cubic feet per second; HYDRO, lake or other hydrologic structure; NA, not applicable; NRDitch, irrigation ditch; NRTribal, tribal stream]

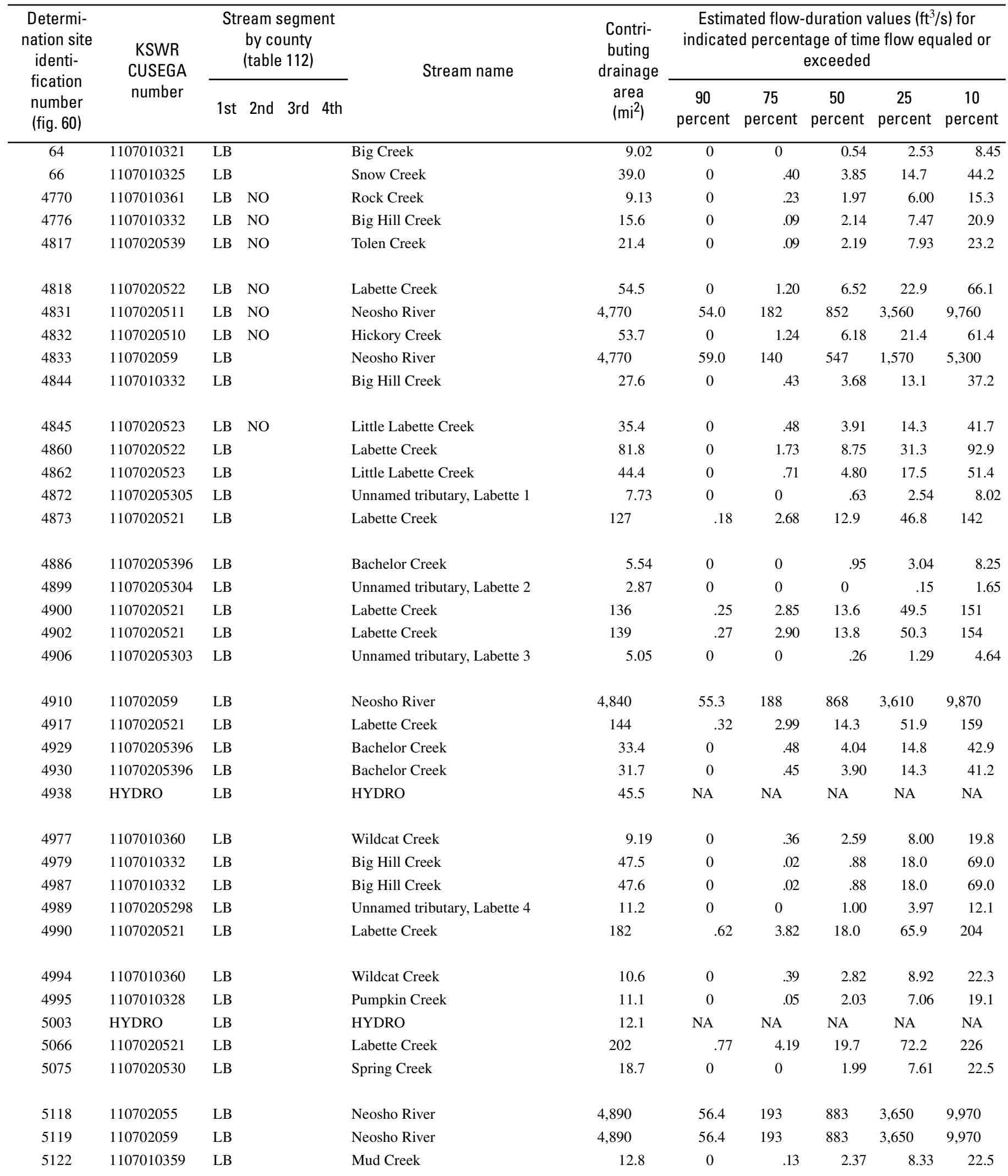


Table 56. Estimated flow-duration values, mean flow values, and peak-discharge frequency values for controlled and uncontrolled flow stream segments on the 1999 Kansas Surface Water Register for Labette County.-Continued

[KSWR, Kansas Surface Water Register; CUSEGA, catalog unit segment number alpha; $\mathrm{mi}^{2}$, square miles; $\mathrm{ft}^{3} / \mathrm{s}$, cubic feet per second; HYDRO, lake or other hydrologic structure; NA, not applicable; NRDitch, irrigation ditch; NRTribal, tribal stream]

\begin{tabular}{|c|c|c|c|c|c|c|c|}
\hline \multirow{2}{*}{$\begin{array}{l}\text { Determi- } \\
\text { nation site } \\
\text { identi- } \\
\text { fication } \\
\text { number } \\
\text { (fig. 60) }\end{array}$} & \multirow{2}{*}{$\begin{array}{c}\text { Estimated mean } \\
\text { flow } \\
\left(\mathrm{ft}^{3} / \mathrm{s}\right)\end{array}$} & \multicolumn{6}{|c|}{ Estimated peak discharge $\left(\mathrm{ft}^{3} / \mathrm{s}\right)$ for indicated peak-discharge frequency } \\
\hline & & 2-year & 5-year & 10 -year & 25-year & 50-year & 100-year \\
\hline 64 & 7.93 & 1,700 & 3,270 & 4,570 & 6,480 & 8,020 & 9,760 \\
\hline 4776 & 15.7 & 2,190 & 4,340 & 6,130 & 8,790 & 11,000 & 13,400 \\
\hline 4817 & 18.5 & 2,590 & 5,170 & 7,330 & 10,500 & 13,200 & 16,200 \\
\hline 4818 & 47.5 & 4,240 & 8,010 & 11,100 & 15,700 & 19,400 & 23,500 \\
\hline 4831 & 3,120 & 29,400 & 42,100 & 50,400 & 60,000 & 86,000 & 121,000 \\
\hline 4845 & 31.4 & 3,460 & 6,700 & 9,420 & 13,400 & 16,700 & 20,200 \\
\hline 4860 & 66.7 & 5,210 & 9,610 & 13,200 & 18,500 & 22,800 & 27,400 \\
\hline 4862 & 38.4 & 3,700 & 7,150 & 10,100 & 14,300 & 17,800 & 21,600 \\
\hline 4872 & 7.11 & 1,460 & 2,840 & 3,980 & 5,670 & 7,030 & 8,580 \\
\hline 4873 & 99.7 & 6,770 & 12,000 & 16,100 & 22,100 & 26,900 & 32,100 \\
\hline 4886 & 6.37 & 1,250 & 2,400 & 3,330 & 4,710 & 5,820 & 7,070 \\
\hline 4899 & 2.32 & 826 & 1,580 & 2,180 & 3,070 & 3,790 & 4,600 \\
\hline 4900 & 106 & 6,870 & 12,100 & 16,300 & 22,200 & 27,000 & 32,200 \\
\hline 4902 & 108 & 6,970 & 12,200 & 16,400 & 22,400 & 27,200 & 32,300 \\
\hline 4977 & 12.9 & 1,770 & 3,390 & 4,720 & 6,670 & 8,250 & 10,000 \\
\hline 4979 & 29.3 & 605 & 1,550 & 2,340 & 3,410 & 4,230 & 5,040 \\
\hline 4987 & 29.3 & 605 & 1,550 & 2,340 & 3,410 & 4,230 & 5,040 \\
\hline 4989 & 10.3 & 1,850 & 3,610 & 5,070 & 7,230 & 8,980 & 11,000 \\
\hline 4990 & 140 & 7,920 & 13,400 & 17,600 & 23,400 & 28,100 & 33,100 \\
\hline 4994 & 14.7 & 1,940 & 3,710 & 5,170 & 7,320 & 9,050 & 11,000 \\
\hline 4995 & 13.6 & 1,950 & 3,760 & 5,250 & 7,450 & 9,230 & 11,200 \\
\hline 5003 & NA & NA & NA & NA & NA & NA & NA \\
\hline 5066 & 154 & 7,830 & 13,100 & 17,000 & 22,500 & 26,800 & 31,400 \\
\hline 5075 & 17.9 & 2,570 & 5,020 & 7,060 & 10,100 & 12,500 & 15,300 \\
\hline 5118 & 3,220 & 30,300 & 43,800 & 52,400 & 64,800 & 91,600 & 127,000 \\
\hline 5119 & 3,220 & 30,300 & 43,800 & 52,400 & 64,800 & 91,600 & 127,000 \\
\hline 5122 & 16.0 & 1,270 & 2,180 & 2,870 & 3,840 & 4,630 & 5,460 \\
\hline
\end{tabular}


Table 56. Estimated flow-duration values, mean flow values, and peak-discharge frequency values for controlled and uncontrolled flow stream segments on the 1999 Kansas Surface Water Register for Labette County.-Continued

[KSWR, Kansas Surface Water Register; CUSEGA, catalog unit segment number alpha; $\mathrm{mi}^{2}$, square miles; $\mathrm{ft}^{3} / \mathrm{s}$, cubic feet per second; HYDRO, lake or other hydrologic structure; NA, not applicable; NRDitch, irrigation ditch; NRTribal, tribal stream]

\begin{tabular}{|c|c|c|c|c|c|c|c|c|c|c|c|}
\hline $\begin{array}{l}\text { Determi- } \\
\text { nation site } \\
\text { identi- } \\
\text { fication } \\
\text { number } \\
\text { (fig. } 60 \text { ) }\end{array}$ & $\begin{array}{l}\text { KSWR } \\
\text { CUSEGA } \\
\text { number }\end{array}$ & \multicolumn{3}{|c|}{$\begin{array}{l}\text { Stream segment } \\
\text { by county } \\
\text { (table 112) }\end{array}$} & Stream name & $\begin{array}{l}\text { Contri- } \\
\text { buting } \\
\text { drainage } \\
\text { area } \\
\left(\mathrm{mi}^{2}\right)\end{array}$ & \multicolumn{5}{|c|}{$\begin{array}{l}\text { Estimated flow-duration values }\left(\mathrm{ft}^{3} / \mathrm{s}\right) \text { for } \\
\text { indicated percentage of time flow equaled or } \\
\text { exceeded }\end{array}$} \\
\hline 5126 & 1107010328 & $\overline{L B}$ & & & Pumpkin Creek & 31.7 & 0 & 0.43 & 4.20 & 16.0 & 46.4 \\
\hline 5198 & 1107010328 & LB & & & Pumpkin Creek & 51.2 & 0 & .95 & 6.54 & 25.0 & 73.6 \\
\hline 5224 & 1107010331 & LB & MG & & Potatoe Creek & 19.8 & 0 & 0 & 2.21 & 8.59 & 25.3 \\
\hline 5225 & 1107020527 & LB & & & Deer Creek & 43.4 & 0 & .50 & 4.41 & 17.1 & 51.5 \\
\hline 5231 & 1107020521 & LB & & & Labette Creek & 248 & 1.16 & 5.15 & 24.0 & 88.4 & 279 \\
\hline 5271 & 11070205460 & LB & & & Hackberry Creek & 32.3 & 0 & .01 & 2.43 & 10.0 & 31.5 \\
\hline 5293 & 1107010328 & LB & & & Pumpkin Creek & 90.3 & 0 & 1.66 & 9.83 & 38.3 & 117 \\
\hline 5316 & 1107010350 & LB & MG & & Claymore Creek & 18.3 & 0 & 0 & 1.12 & 5.03 & 16.4 \\
\hline 5319 & 1107010351 & LB & & & Deer Creek & 31.6 & 0 & .06 & 2.63 & 10.6 & 33.0 \\
\hline 5326 & 1107020520 & LB & & & Labette Creek & 399 & 2.42 & 8.06 & 37.2 & 138 & 447 \\
\hline 5356 & 1107010328 & LB & MG & & Pumpkin Creek & 137 & .03 & 2.49 & 13.4 & 52.3 & 165 \\
\hline 5407 & 1107020524 & LB & & & Lake Creek & 30.1 & 0 & .24 & 2.98 & 11.2 & 33.4 \\
\hline 5408 & 1107020529 & LB & & & Turkey Creek & 26.6 & 0 & .08 & 2.54 & 9.86 & 29.7 \\
\hline
\end{tabular}


Table 56. Estimated flow-duration values, mean flow values, and peak-discharge frequency values for controlled and uncontrolled flow stream segments on the 1999 Kansas Surface Water Register for Labette County.-Continued

[KSWR, Kansas Surface Water Register; CUSEGA, catalog unit segment number alpha; $\mathrm{mi}^{2}$, square miles; $\mathrm{ft}^{3} / \mathrm{s}$, cubic feet per second; HYDRO, lake or other hydrologic structure; NA, not applicable; NRDitch, irrigation ditch; NRTribal, tribal stream]

\begin{tabular}{|c|c|c|c|c|c|c|c|}
\hline \multirow{2}{*}{$\begin{array}{l}\text { Determi- } \\
\text { nation site } \\
\text { identi- } \\
\text { fication } \\
\text { number } \\
\text { (fig. 60) }\end{array}$} & \multirow{2}{*}{$\begin{array}{c}\text { Estimated mean } \\
\text { flow } \\
\left(\mathrm{ft}^{3} / \mathrm{s}\right)\end{array}$} & \multicolumn{6}{|c|}{ Estimated peak discharge $\left(\mathrm{ft}^{3} / \mathrm{s}\right)$ for indicated peak-discharge frequency } \\
\hline & & 2-year & 5-year & 10-year & 25-year & 50-year & 100-year \\
\hline 5126 & 33.6 & 4,010 & 7,490 & 10,400 & 14,500 & 17,900 & 21,500 \\
\hline 5198 & 52.2 & 4,220 & 7,410 & 10,000 & 13,600 & 16,600 & 19,600 \\
\hline 5224 & 19.8 & 2,790 & 5,410 & 7,590 & 10,800 & 13,400 & 16,400 \\
\hline 5225 & 39.2 & 4,820 & 8,910 & 12,300 & 17,100 & 21,100 & 25,300 \\
\hline 5231 & 188 & 8,330 & 13,200 & 16,600 & 21,200 & 24,700 & 28,300 \\
\hline 5271 & 26.5 & 4,090 & 7,640 & 10,600 & 14,800 & 18,200 & 21,900 \\
\hline 5293 & 83.3 & 6,120 & 10,600 & 14,300 & 19,500 & 23,800 & 28,200 \\
\hline 5316 & 14.8 & 2,580 & 5,040 & 7,080 & 10,100 & 12,600 & 15,400 \\
\hline 5319 & 27.0 & 6,330 & 10,800 & 14,400 & 19,300 & 23,200 & 27,300 \\
\hline 5326 & 292 & 11,300 & 18,200 & 23,200 & 30,300 & 35,800 & 41,600 \\
\hline 5356 & 116 & 7,490 & 13,000 & 17,600 & 24,100 & 29,400 & 35,100 \\
\hline 5407 & 26.4 & 4,860 & 8,660 & 11,700 & 16,000 & 19,500 & 23,100 \\
\hline 5408 & 23.8 & 3,200 & 6,300 & 8,890 & 12,700 & 15,900 & 19,400 \\
\hline
\end{tabular}




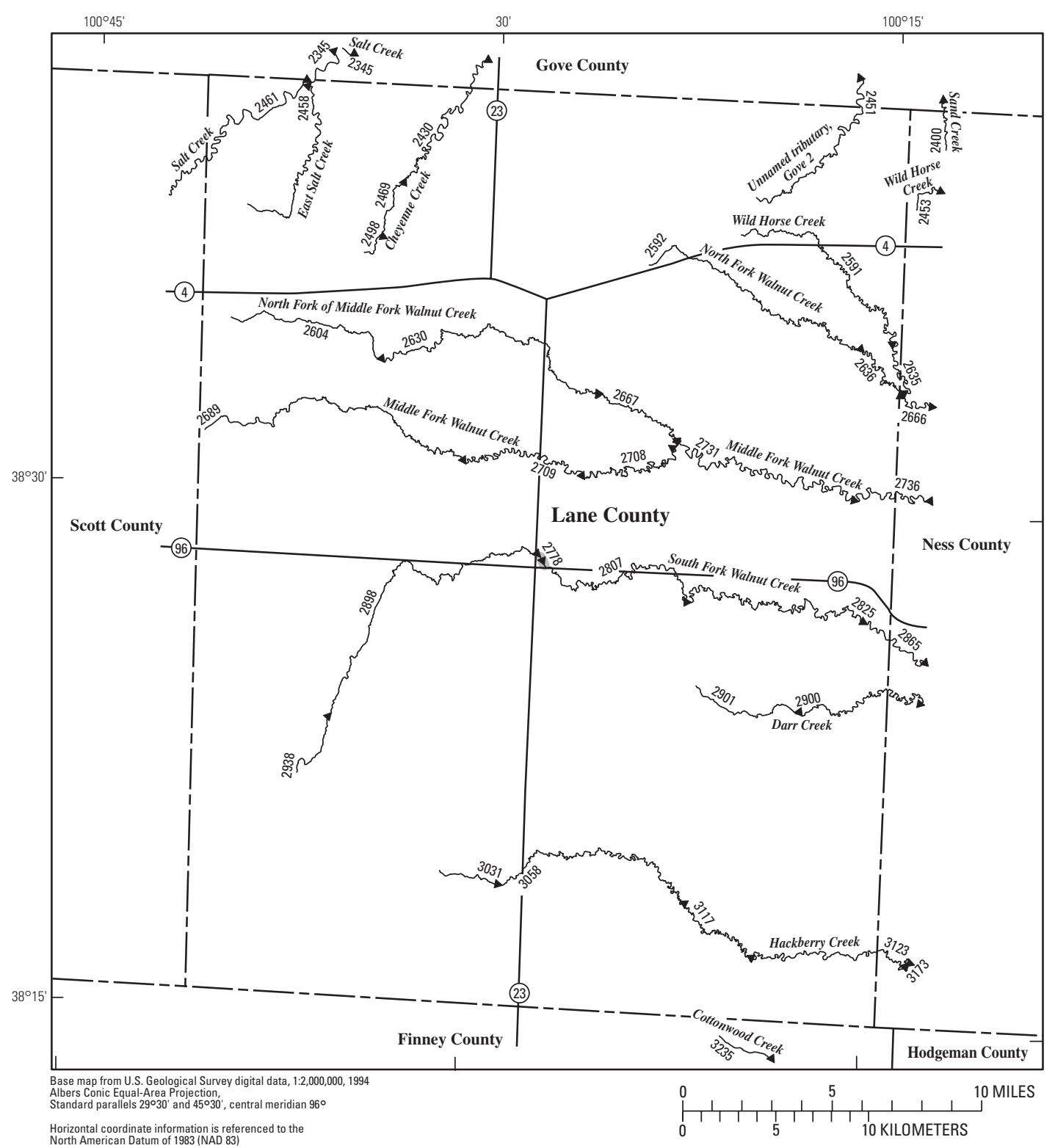

EXPLANATION

3031 Location of streamflow-statistics determination site (small triangle) and associated identification number-small triangle points in downstream direction

06853800

U.S. Geological Survey streamflow-gaging station and number used for estimates of flow duration

${ }^{06875800} \triangle$ U.S. Geological Survey streamflow-gaging station and number used for estimates of peak-discharge frequency values

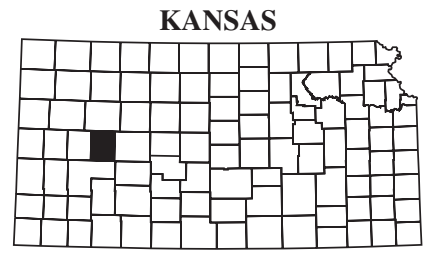

Index map 2778 Lake and determination site identification number

Figure 61. Location of streamflow-statistics determination sites, associated identification numbers, and U.S. Geological Survey streamflow-gaging stations used in the flow-duration and peak-discharge frequency analyses for Lane County. 
Table 57. Estimated flow-duration values, mean flow values, and peak-discharge frequency values for controlled and uncontrolled flow stream segments on the 1999 Kansas Surface Water Register for Lane County.

[KSWR, Kansas Surface Water Register; CUSEGA, catalog unit segment number alpha; $\mathrm{mi}^{2}$, square miles; $\mathrm{ft}^{3} / \mathrm{s}$, cubic feet per second; HYDRO, lake or other hydrologic structure; NA, not applicable; NRDitch, irrigation ditch; NRTribal, tribal stream]

\begin{tabular}{|c|c|c|c|c|c|c|c|c|c|c|c|}
\hline \multirow{2}{*}{$\begin{array}{l}\text { Determi- } \\
\text { nation site } \\
\text { identi- } \\
\text { fication } \\
\text { number } \\
\text { (fig. 61) }\end{array}$} & \multirow{2}{*}{$\begin{array}{l}\text { KSWR } \\
\text { CUSEGA } \\
\text { number }\end{array}$} & \multicolumn{3}{|c|}{$\begin{array}{l}\text { Stream segment } \\
\text { by county } \\
\text { (table 112) }\end{array}$} & \multirow{2}{*}{ Stream name } & \multirow{2}{*}{$\begin{array}{l}\text { Contri- } \\
\text { buting } \\
\text { drainage } \\
\text { area } \\
\left(\mathrm{mi}^{2}\right)\end{array}$} & \multicolumn{5}{|c|}{$\begin{array}{c}\text { Estimated flow-duration values }\left(\mathrm{ft}^{3} / \mathrm{s}\right) \text { for } \\
\text { indicated percentage of time flow equaled or } \\
\text { exceeded }\end{array}$} \\
\hline & & $1 \mathrm{st}$ & 2nd & 3rd 4th & & & $\begin{array}{c}90 \\
\text { percent }\end{array}$ & $\begin{array}{c}75 \\
\text { percent }\end{array}$ & $\begin{array}{c}50 \\
\text { percent }\end{array}$ & $\begin{array}{c}25 \\
\text { percent }\end{array}$ & $\begin{array}{c}10 \\
\text { percent }\end{array}$ \\
\hline 2469 & 1026000336 & LE & & & Cheyenne Creek & 15.3 & 0 & 0 & 0 & 0 & 0 \\
\hline 2498 & 1026000336 & LE & & & Cheyenne Creek & 7.87 & 0 & 0 & 0 & 0 & 0 \\
\hline 2591 & 1103000711 & LE & & & Wild Horse Creek & 23.5 & 0 & 0 & 0 & 0 & 0 \\
\hline 2592 & 110300076 & LE & & & North Fork Walnut Creek & 47.8 & 0 & 0 & 0 & 0 & 0 \\
\hline 2604 & 110300078 & LE & & & North Fork of Middle Fork Walnut Creek & 24.8 & 0 & 0 & 0 & 0 & 0 \\
\hline 2630 & 110300078 & LE & & & North Fork of Middle Fork Walnut Creek & 60.3 & 0 & 0 & 0 & 0 & 0 \\
\hline 2635 & 1103000711 & LE & NS & & Wild Horse Creek & 25.9 & 0 & 0 & 0 & 0 & 0 \\
\hline 2636 & 110300076 & LE & NS & & North Fork Walnut Creek & 52.5 & 0 & 0 & 0 & 0 & 0 \\
\hline 2667 & 110300078 & LE & & & North Fork of Middle Fork Walnut Creek & 73.3 & 0 & 0 & 0 & 0 & 0 \\
\hline 2689 & 110300079 & LE & & & Middle Fork Walnut Creek & 94.6 & 0 & 0 & 0 & 0 & 0 \\
\hline 2708 & 110300079 & LE & & & Middle Fork Walnut Creek & 117 & 0 & 0 & 0 & 0 & 0 \\
\hline 2709 & 110300079 & LE & & & Middle Fork Walnut Creek & 107 & 0 & 0 & 0 & 0 & 0 \\
\hline 2731 & 110300077 & LE & & & Middle Fork Walnut Creek & 217 & 0 & 0 & 0 & 0 & .07 \\
\hline 2736 & 110300077 & LE & NS & & Middle Fork Walnut Creek & 224 & 0 & 0 & 0 & 0 & .42 \\
\hline 2778 & HYDRO & LE & & & HYDRO & 210 & NA & NA & NA & NA & NA \\
\hline 2807 & 1103000710 & $\mathrm{LE}$ & & & South Fork Walnut Creek & 241 & 0 & 0 & 0 & 0 & 0 \\
\hline 2825 & 1103000710 & LE & & & South Fork Walnut Creek & 264 & 0 & 0 & 0 & 0 & .18 \\
\hline 2865 & 1103000710 & LE & NS & & South Fork Walnut Creek & 272 & 0 & 0 & 0 & 0 & .45 \\
\hline 2898 & 1103000710 & LE & & & South Fork Walnut Creek & 209 & 0 & 0 & 0 & 0 & 0 \\
\hline 2900 & 1103000712 & LE & NS & & Darr Creek & 55.8 & 0 & 0 & 0 & 0 & 0 \\
\hline 2901 & 1103000712 & LE & & & Darr Creek & 32.3 & 0 & 0 & 0 & 0 & 0 \\
\hline 2938 & 1103000710 & LE & & & South Fork Walnut Creek & 138 & 0 & 0 & 0 & 0 & 0 \\
\hline 3031 & 110300054 & LE & & & Hackberry Creek & 57.7 & 0 & 0 & 0 & 0 & 0 \\
\hline 3058 & 110300054 & LE & & & Hackberry Creek & 119 & 0 & 0 & 0 & 0 & 0 \\
\hline 3117 & 110300054 & LE & & & Hackberry Creek & 135 & 0 & 0 & 0 & 0 & 0 \\
\hline 3123 & 110300054 & LE & NS & & Hackberry Creek & 163 & 0 & 0 & 0 & 0 & 0 \\
\hline
\end{tabular}


Table 57. Estimated flow-duration values, mean flow values, and peak-discharge frequency values for controlled and uncontrolled flow stream segments on the 1999 Kansas Surface Water Register for Lane County.-Continued

[KSWR, Kansas Surface Water Register; CUSEGA, catalog unit segment number alpha; $\mathrm{mi}^{2}$, square miles; $\mathrm{ft}^{3} / \mathrm{s}$, cubic feet per second; HYDRO, lake or other hydrologic structure; NA, not applicable; NRDitch, irrigation ditch; NRTribal, tribal stream]

\begin{tabular}{|c|c|c|c|c|c|c|c|}
\hline \multirow{2}{*}{$\begin{array}{l}\text { Determi- } \\
\text { nation site } \\
\text { identi- } \\
\text { fication } \\
\text { number } \\
\text { (fig. 61) }\end{array}$} & \multirow{2}{*}{$\begin{array}{c}\text { Estimated mean } \\
\text { flow } \\
\left(\mathrm{ft}^{3} / \mathrm{s}\right)\end{array}$} & \multicolumn{6}{|c|}{ Estimated peak discharge $\left(\mathrm{ft}^{3} / \mathrm{s}\right)$ for indicated peak-discharge frequency } \\
\hline & & 2-year & 5-year & 10-year & 25-year & 50-year & 100-year \\
\hline 2469 & 0 & 305 & 972 & 1,680 & 2,920 & 4,060 & 5,420 \\
\hline 2592 & .62 & 342 & 1,090 & 1,930 & 3,410 & 4,850 & 6,570 \\
\hline 2604 & 0 & 398 & 1,280 & 2,240 & 3,900 & 5,450 & 7,310 \\
\hline 2630 & 0 & 300 & 1,010 & 1,840 & 3,350 & 4,840 & 6,670 \\
\hline 2635 & 0 & 480 & 1,490 & 2,560 & 4,390 & 6,080 & 8,100 \\
\hline 2708 & 1.05 & 383 & 1,290 & 2,350 & 4,280 & 6,200 & 8,570 \\
\hline 2709 & .76 & 387 & 1,290 & 2,340 & 4,260 & 6,160 & 8,490 \\
\hline 2731 & 3.39 & 548 & 1,780 & 3,200 & 5,780 & 8,330 & 11,500 \\
\hline 2736 & 3.70 & 569 & 1,840 & 3,300 & 5,940 & 8,550 & 11,800 \\
\hline 2778 & NA & NA & NA & NA & NA & NA & NA \\
\hline 2807 & 2.82 & 613 & 1,930 & 3,410 & 6,060 & 8,660 & 11,800 \\
\hline 2825 & 3.56 & 637 & 2,000 & 3,540 & 6,280 & 8,970 & 12,200 \\
\hline 2865 & 3.81 & 653 & 2,040 & 3,610 & 6,390 & 9,130 & 12,500 \\
\hline 2898 & 2.03 & 539 & 1,720 & 3,070 & 5,480 & 7,860 & 10,800 \\
\hline 3123 & 2.73 & 567 & 1,800 & 3,190 & 5,660 & 8,100 & 11,000 \\
\hline
\end{tabular}




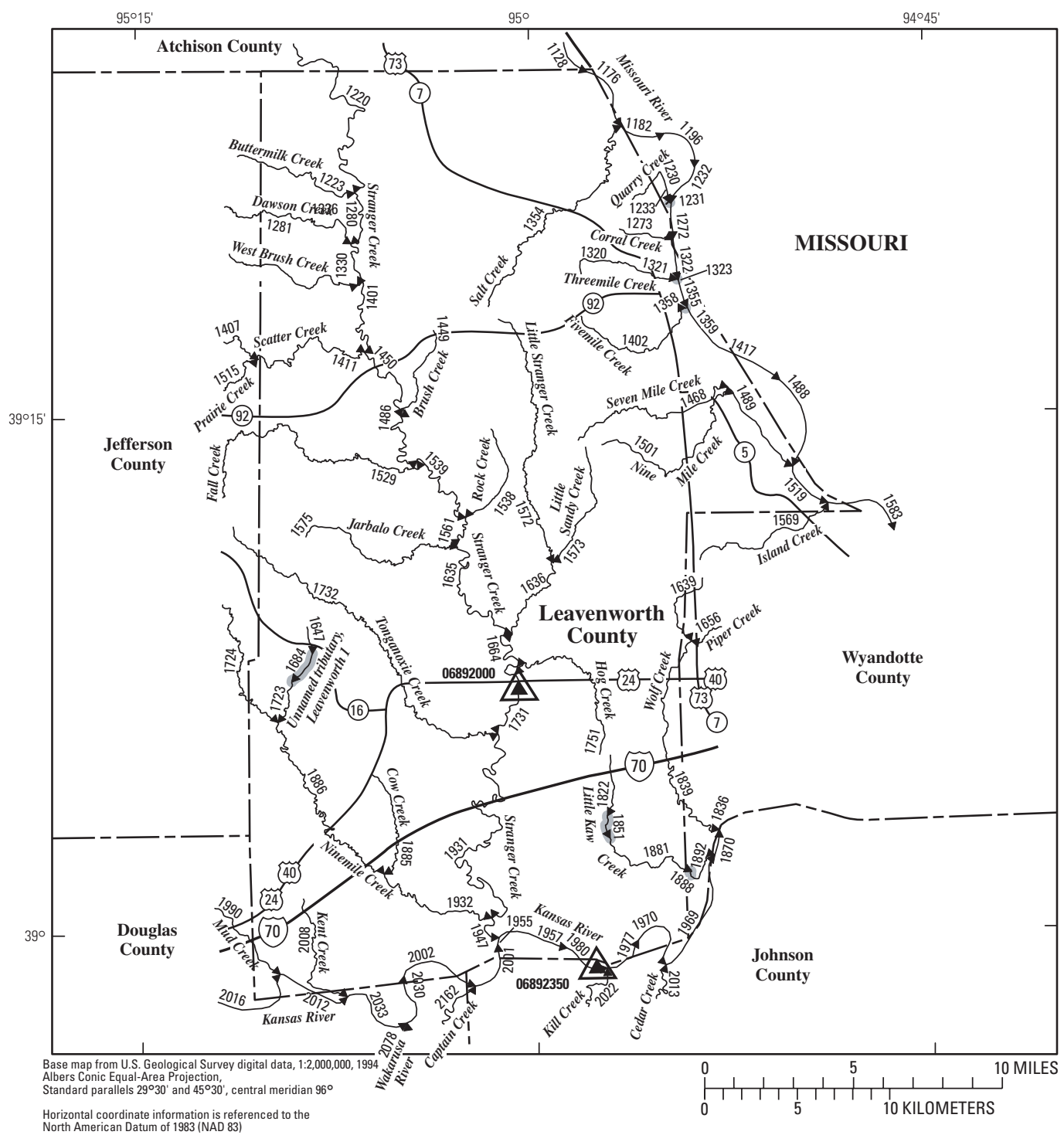

EXPLANATION

¿2016 Location of streamflow-statistics determination site (small triangle) and associated identification number-small triangle points in downstream direction

${ }^{06892000} \Delta$ U.S. Geological Survey streamflow-gaging station and number used for estimates of flow duration

${ }^{06892350} \triangle$

U.S. Geological Survey streamflow-gaging station and number used for estimates of peak-discharge frequency values

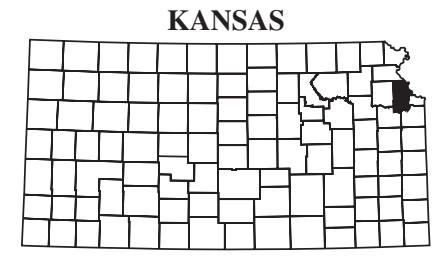

Index map 1851

Lake and determination site identification number

Figure 62. Location of streamflow-statistics determination sites, associated identification numbers, and U.S. Geological Survey streamflow-gaging stations used in the flow-duration and peak-discharge frequency analyses for Leavenworth County. 
Table 58. Estimated flow-duration values, mean flow values, and peak-discharge frequency values for controlled and uncontrolled flow stream segments on the 1999 Kansas Surface Water Register for Leavenworth County.

[KSWR, Kansas Surface Water Register; CUSEGA, catalog unit segment number alpha; $\mathrm{mi}^{2}$, square miles; $\mathrm{ft}^{3} / \mathrm{s}$, cubic feet per second; HYDRO, lake or other hydrologic structure; NA, not applicable; NRDitch, irrigation ditch; NRTribal, tribal stream]

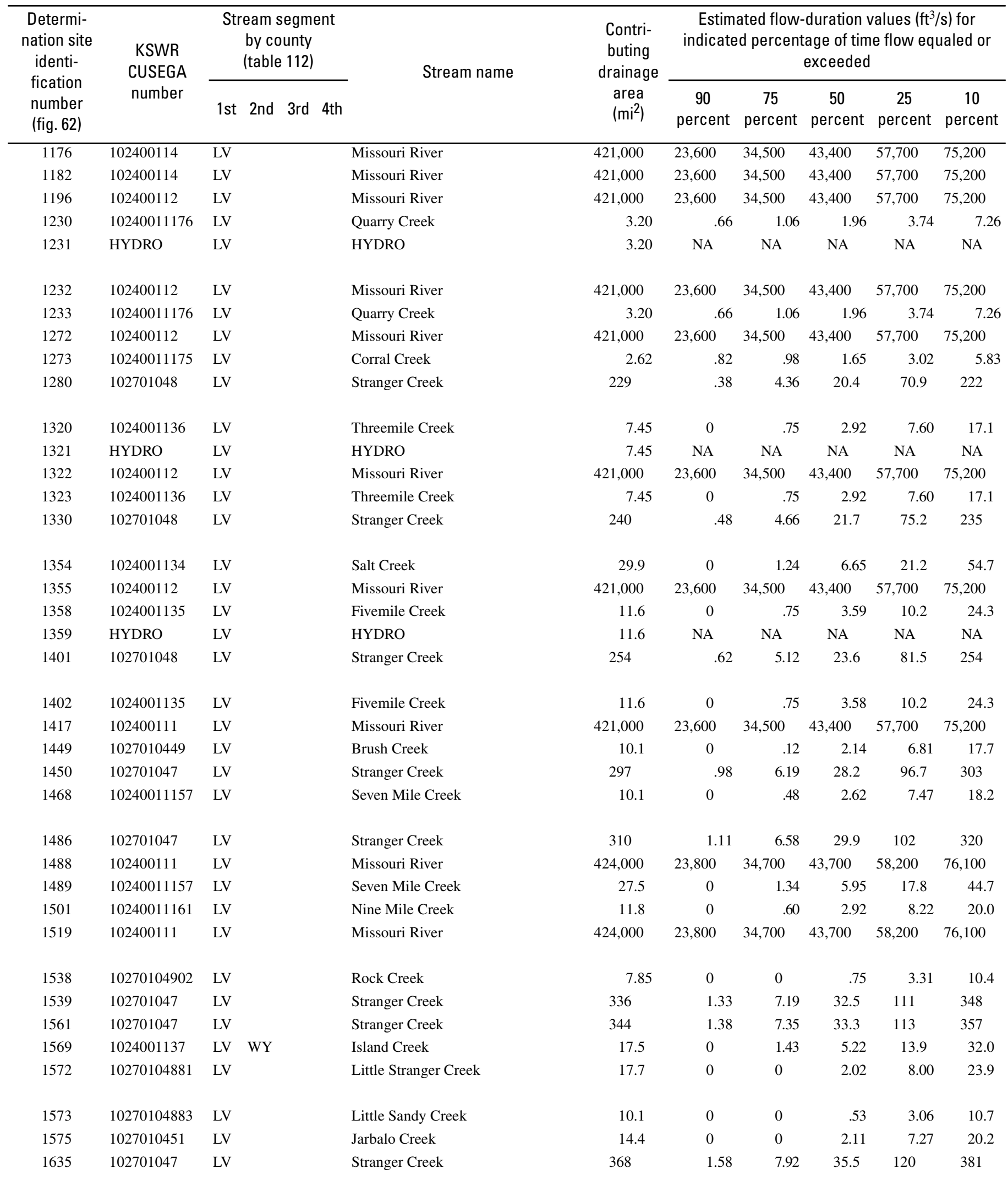


Table 58. Estimated flow-duration values, mean flow values, and peak-discharge frequency values for controlled and uncontrolled flow stream segments on the 1999 Kansas Surface Water Register for Leavenworth County.-Continued

[KSWR, Kansas Surface Water Register; CUSEGA, catalog unit segment number alpha; $\mathrm{mi}^{2}$, square miles; $\mathrm{ft}^{3} / \mathrm{s}$, cubic feet per second; HYDRO, lake or other hydrologic structure; NA, not applicable; NRDitch, irrigation ditch; NRTribal, tribal stream]

\begin{tabular}{|c|c|c|c|c|c|c|c|}
\hline \multirow{2}{*}{$\begin{array}{l}\text { Determi- } \\
\text { nation site } \\
\text { identi- } \\
\text { fication } \\
\text { number } \\
\text { (fig. 62) }\end{array}$} & \multirow{2}{*}{$\begin{array}{c}\text { Estimated mean } \\
\text { flow } \\
\left(\mathrm{ft}^{3} / \mathrm{s}\right)\end{array}$} & \multicolumn{6}{|c|}{ Estimated peak discharge $\left(\mathrm{ft}^{3} / \mathrm{s}\right)$ for indicated peak-discharge frequency } \\
\hline & & 2-year & 5-year & 10-year & 25-year & 50-year & 100-year \\
\hline 1176 & 48,200 & 109,000 & 147,000 & 175,000 & 200,000 & 234,000 & 262,000 \\
\hline 1230 & 4.31 & 797 & 1,560 & 2,190 & 3,120 & 3,870 & 4,710 \\
\hline 1231 & $\mathrm{NA}$ & NA & NA & NA & NA & NA & NA \\
\hline 1232 & 48,200 & 109,000 & 148,000 & 175,000 & 200,000 & 234,000 & 262,000 \\
\hline 1233 & 4.31 & 797 & 1,560 & 2,190 & 3,120 & 3,870 & 4,710 \\
\hline 1320 & 10.4 & 1,350 & 2,670 & 3,770 & 5,390 & 6,710 & 8,200 \\
\hline 1321 & NA & NA & NA & NA & NA & NA & NA \\
\hline 1322 & 48,200 & 109,000 & 148,000 & 175,000 & 200,000 & 234,000 & 263,000 \\
\hline 1323 & 10.4 & 1,350 & 2,670 & 3,770 & 5,390 & 6,710 & 8,200 \\
\hline 1330 & 147 & 5,820 & 11,600 & 16,700 & 24,400 & 31,100 & 38,500 \\
\hline 1354 & 34.0 & 2,870 & 5,900 & 8,500 & 12,400 & 15,600 & 19,300 \\
\hline 1355 & 48,200 & 109,000 & 148,000 & 175,000 & 200,000 & 234,000 & 263,000 \\
\hline 1358 & 15.1 & 1,740 & 3,470 & 4,920 & 7,080 & 8,840 & 10,800 \\
\hline 1359 & NA & NA & NA & NA & NA & NA & NA \\
\hline 1486 & 191 & 6,050 & 11,700 & 16,600 & 24,000 & 30,300 & 37,300 \\
\hline 1488 & 48,600 & 111,000 & 150,000 & 178,000 & 204,000 & 239,000 & 268,000 \\
\hline 1489 & 28.7 & 2,720 & 5,600 & 8,050 & 11,700 & 14,800 & 18,300 \\
\hline 1501 & 13.2 & 1,650 & 3,350 & 4,780 & 6,920 & 8,670 & 10,700 \\
\hline 1519 & 48,600 & 111,000 & 150,000 & 178,000 & 204,000 & 239,000 & 268,000 \\
\hline 1538 & 8.12 & 1,270 & 2,560 & 3,650 & 5,270 & 6,600 & 8,110 \\
\hline 1539 & 205 & 6,140 & 11,800 & 16,600 & 23,700 & 29,900 & 36,700 \\
\hline 1561 & 210 & 6,120 & 11,700 & 16,400 & 23,500 & 29,600 & 36,400 \\
\hline 1569 & 19.4 & 1,970 & 4,080 & 5,870 & 8,580 & 10,800 & 13,300 \\
\hline 1572 & 17.6 & 2,100 & 4,280 & 6,130 & 8,900 & 11,200 & 13,800 \\
\hline 1573 & 9.17 & 1,460 & 2,980 & 4,250 & 6,160 & 7,730 & 9,500 \\
\hline 1575 & 14.3 & 1,740 & 3,600 & 5,180 & 7,550 & 9,500 & 11,700 \\
\hline 1635 & 221 & 6,190 & 11,700 & 16,300 & 23,100 & 29,000 & 35,600 \\
\hline
\end{tabular}


Table 58. Estimated flow-duration values, mean flow values, and peak-discharge frequency values for controlled and uncontrolled flow stream segments on the 1999 Kansas Surface Water Register for Leavenworth County.-Continued

[KSWR, Kansas Surface Water Register; CUSEGA, catalog unit segment number alpha; $\mathrm{mi}^{2}$, square miles; $\mathrm{ft}^{3} / \mathrm{s}$, cubic feet per second; HYDRO, lake or other hydrologic structure; NA, not applicable; NRDitch, irrigation ditch; NRTribal, tribal stream]

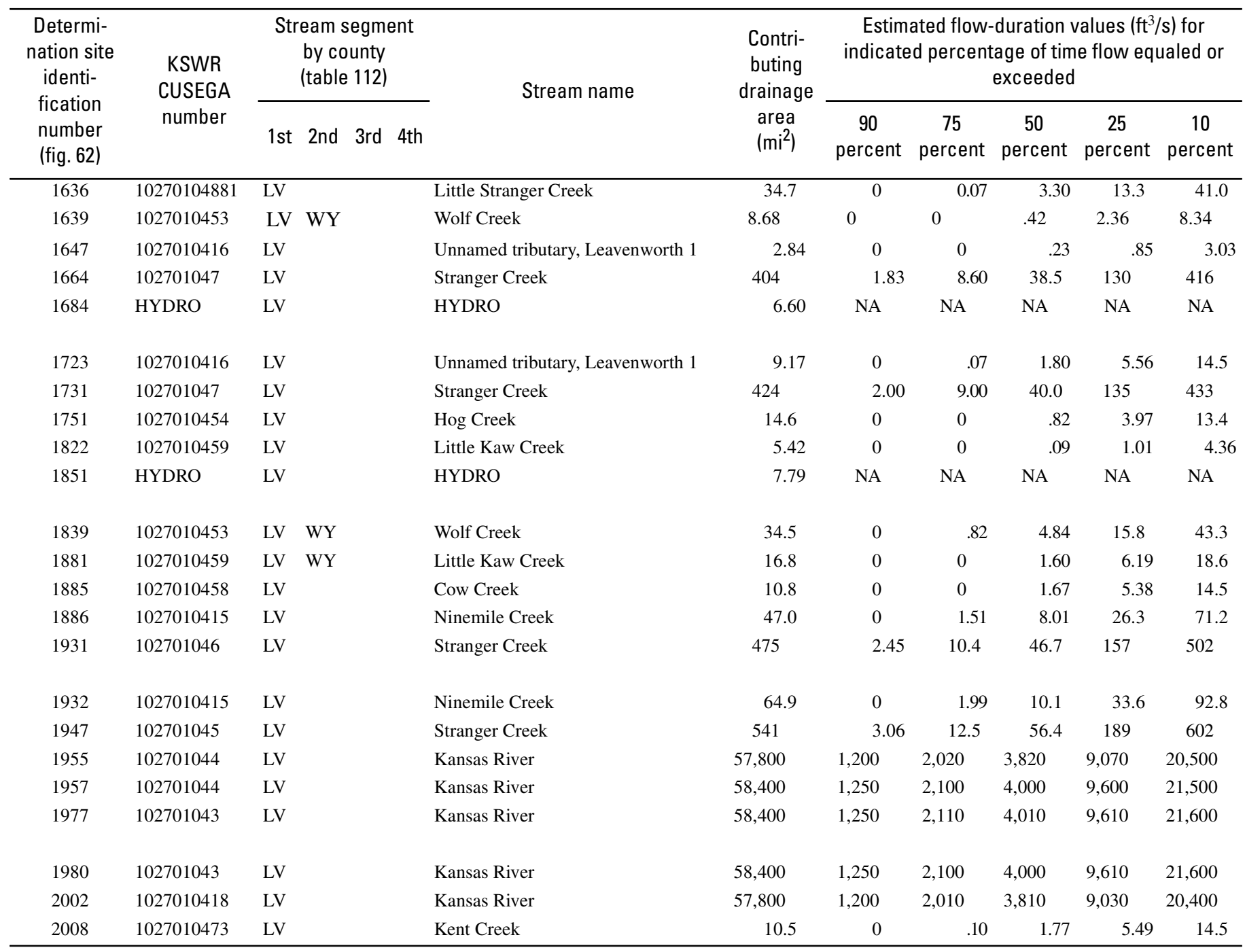


Table 58. Estimated flow-duration values, mean flow values, and peak-discharge frequency values for controlled and uncontrolled flow stream segments on the 1999 Kansas Surface Water Register for Leavenworth County.-Continued

[KSWR, Kansas Surface Water Register; CUSEGA, catalog unit segment number alpha; $\mathrm{mi}^{2}$, square miles; $\mathrm{ft}^{3} / \mathrm{s}$, cubic feet per second; HYDRO, lake or other hydrologic structure; NA, not applicable; NRDitch, irrigation ditch; NRTribal, tribal stream]

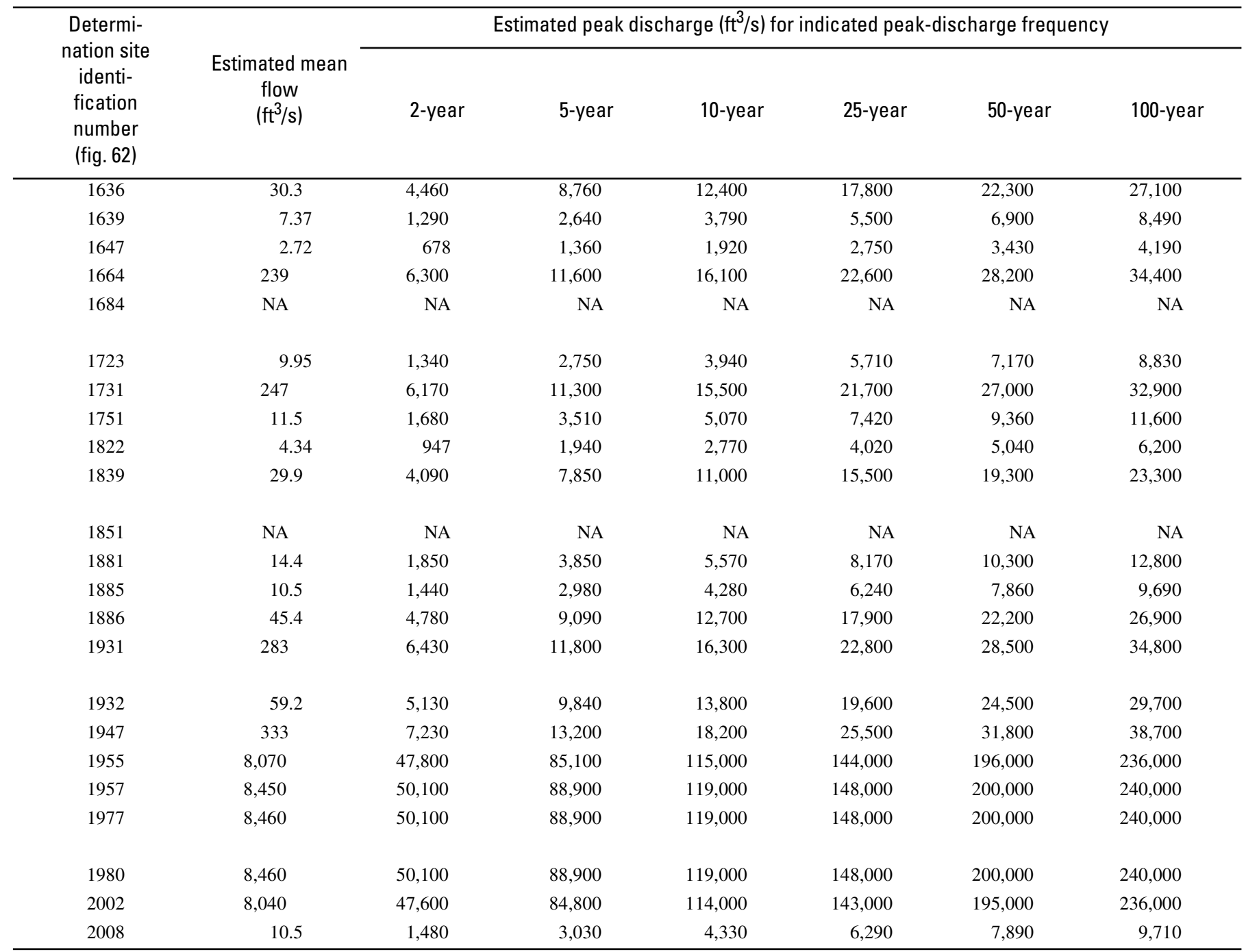




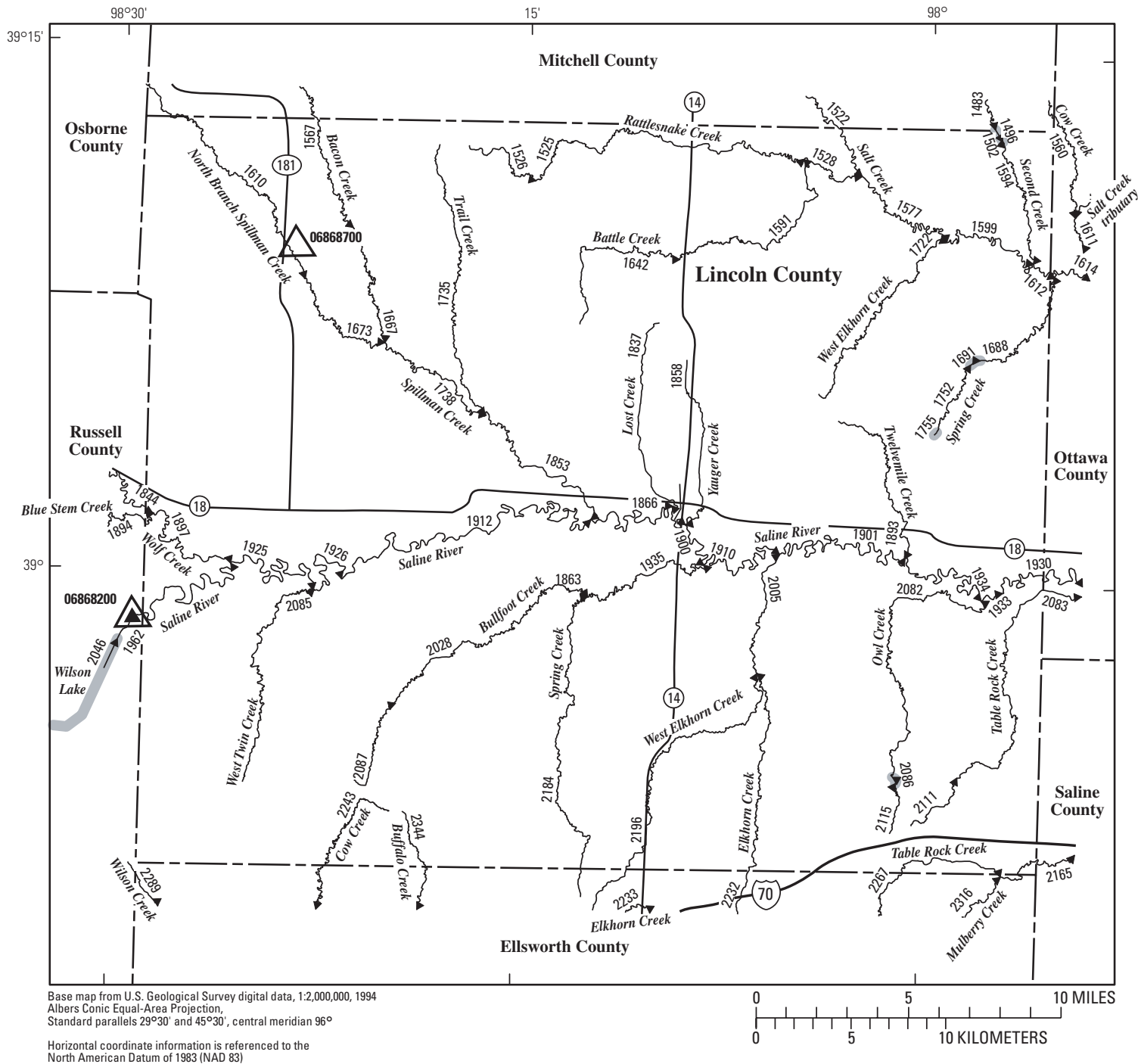

EXPLANATION

\&243 Location of streamflow-statistics determination site (small triangle) and associated identification number-small triangle points in downstream direction

${ }^{06868200} \Delta$ U.S. Geological Survey streamflow-gaging station and number used for estimates of flow duration

U.S. Geological Survey streamflow-gaging station and number used for estimates of peak-discharge frequency values

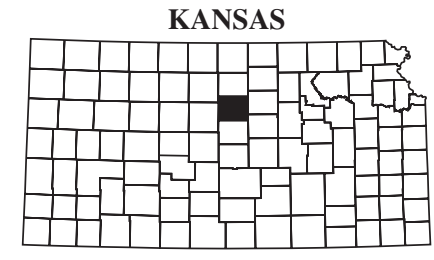

Index map 2046

Lake and determination site identification number

Figure 63. Location of streamflow-statistics determination sites, associated identification numbers, and U.S. Geological Survey streamflow-gaging stations used in the flow-duration and peak-discharge frequency analyses for Lincoln County. 
Table 59. Estimated flow-duration values, mean flow values, and peak-discharge frequency values for controlled and uncontrolled flow stream segments on the 1999 Kansas Surface Water Register for Lincoln County.

[KSWR, Kansas Surface Water Register; CUSEGA, catalog unit segment number alpha; $\mathrm{mi}^{2}$, square miles; $\mathrm{ft}^{3} / \mathrm{s}$, cubic feet per second; HYDRO, lake or other hydrologic structure; NA, not applicable; NRDitch, irrigation ditch; NRTribal, tribal stream]

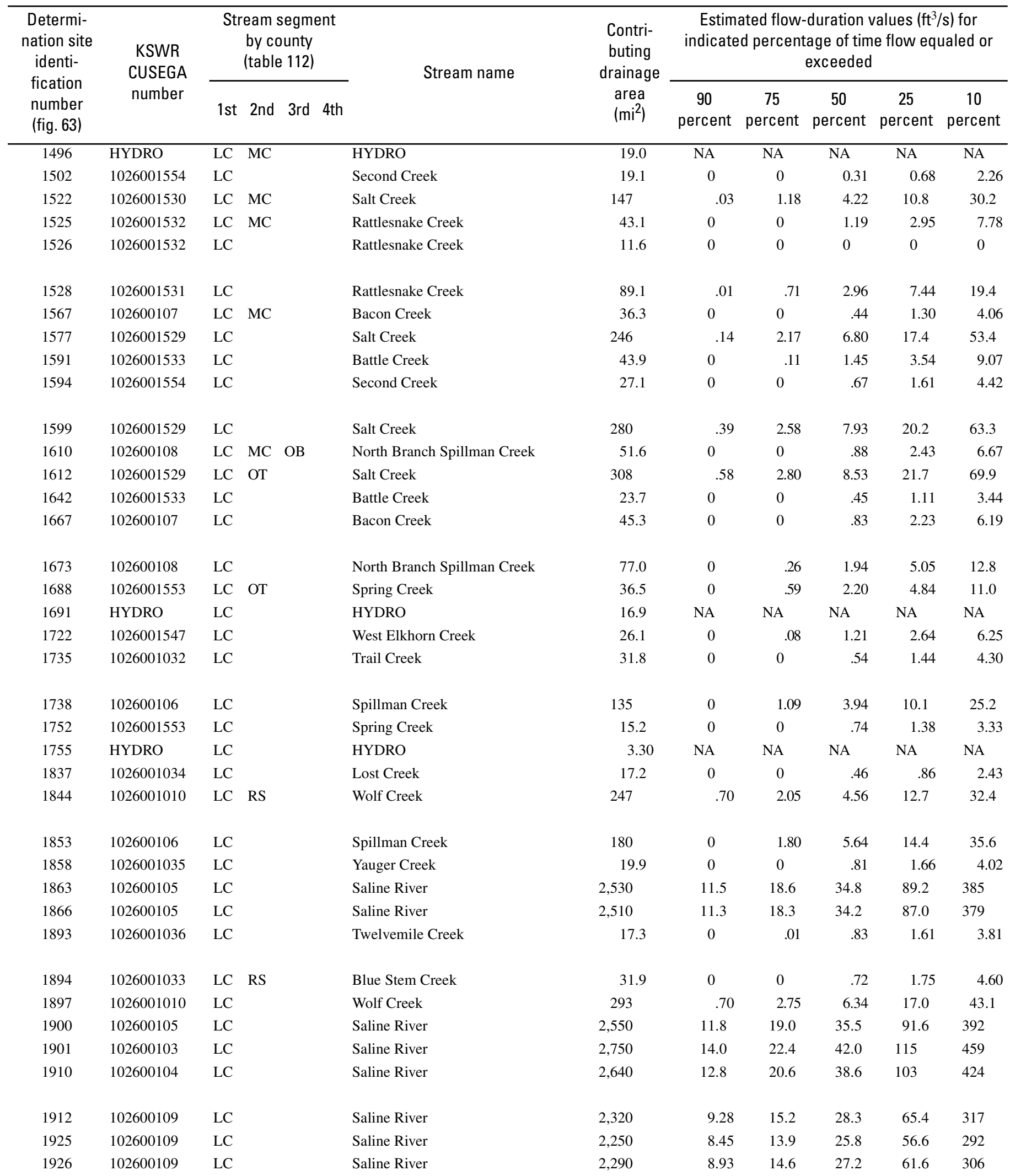


Table 59. Estimated flow-duration values, mean flow values, and peak-discharge frequency values for controlled and uncontrolled flow stream segments on the 1999 Kansas Surface Water Register for Lincoln County.-Continued

[KSWR, Kansas Surface Water Register; CUSEGA, catalog unit segment number alpha; $\mathrm{mi}^{2}$, square miles; $\mathrm{ft}^{3} / \mathrm{s}$, cubic feet per second; HYDRO, lake or other hydrologic structure; NA, not applicable; NRDitch, irrigation ditch; NRTribal, tribal stream]

\begin{tabular}{|c|c|c|c|c|c|c|c|}
\hline \multirow{2}{*}{$\begin{array}{l}\text { Determi- } \\
\text { nation site } \\
\text { identi- } \\
\text { fication } \\
\text { number } \\
\text { (fig. 63) }\end{array}$} & \multirow{2}{*}{$\begin{array}{c}\text { Estimated mean } \\
\text { flow } \\
\left(\mathrm{ft}^{3} / \mathrm{s}\right)\end{array}$} & \multicolumn{6}{|c|}{ Estimated peak discharge $\left(\mathrm{ft}^{3} / \mathrm{s}\right)$ for indicated peak-discharge frequency } \\
\hline & & 2-year & 5-year & 10-year & 25-year & 50-year & 100-year \\
\hline 1496 & NA & NA & NA & NA & NA & NA & NA \\
\hline 1525 & 7.35 & 827 & 2,180 & 3,540 & 5,760 & 7,790 & 10,100 \\
\hline 1526 & .98 & 554 & 1,460 & 2,330 & 3,750 & 4,980 & 6,430 \\
\hline 1528 & 15.7 & 1,240 & 3,170 & 5,100 & 8,230 & 11,100 & 14,400 \\
\hline 1567 & 4.93 & 793 & 2,100 & 3,410 & 5,550 & 7,510 & 9,750 \\
\hline 1599 & 44.9 & 1,610 & 4,270 & 7,000 & 11,600 & 16,000 & 21,200 \\
\hline 1610 & 6.95 & 342 & 1,100 & 1,960 & 3,600 & 5,260 & 7,360 \\
\hline 1612 & 49.1 & 1,630 & 4,350 & 7,130 & 11,900 & 16,400 & 21,800 \\
\hline 1642 & 4.09 & 869 & 2,300 & 3,690 & 5,960 & 7,940 & 10,300 \\
\hline 1667 & 6.54 & 846 & 2,240 & 3,640 & 5,930 & 8,040 & 10,500 \\
\hline 1673 & 11.4 & 569 & 1,650 & 2,810 & 4,950 & 7,050 & 9,670 \\
\hline 1688 & 8.79 & 1,080 & 2,620 & 4,090 & 6,400 & 8,460 & 10,800 \\
\hline 1691 & NA & NA & NA & NA & NA & NA & NA \\
\hline 1722 & 5.60 & 933 & 2,470 & 3,960 & 6,380 & 8,510 & 11,000 \\
\hline 1853 & 27.6 & 1,120 & 2,990 & 4,930 & 8,330 & 11,600 & 15,600 \\
\hline 1858 & 4.09 & 807 & 2,120 & 3,380 & 5,420 & 7,210 & 9,310 \\
\hline 1863 & 170 & 1,900 & 3,830 & 5,180 & 6,820 & 7,960 & 9,060 \\
\hline 1866 & 167 & 1,870 & 3,760 & 5,080 & 6,700 & 7,810 & 8,890 \\
\hline 1893 & 3.84 & 772 & 2,000 & 3,180 & 5,070 & 6,730 & 8,670 \\
\hline 1894 & 4.90 & 751 & 1,980 & 3,200 & 5,180 & 7,000 & 9,060 \\
\hline 1897 & 33.0 & 2,010 & 4,750 & 7,330 & 11,500 & 15,400 & 19,700 \\
\hline 1900 & 173 & 1,950 & 3,910 & 5,280 & 6,960 & 8,130 & 9,250 \\
\hline 1901 & 199 & 2,350 & 4,680 & 6,320 & 8,340 & 9,750 & 11,100 \\
\hline 1910 & 185 & 2,140 & 4,270 & 5,770 & 7,620 & 8,900 & 10,100 \\
\hline 1912 & 142 & 1,500 & 3,060 & 4,130 & 5,430 & 6,310 & 7,160 \\
\hline 1925 & 132 & 1,350 & 2,770 & 3,750 & 4,920 & 5,700 & 6,450 \\
\hline 1926 & 138 & 1,430 & 2,930 & 3,970 & 5,210 & 6,050 & 6,860 \\
\hline
\end{tabular}


Table 59. Estimated flow-duration values, mean flow values, and peak-discharge frequency values for controlled and uncontrolled flow stream segments on the 1999 Kansas Surface Water Register for Lincoln County.-Continued

[KSWR, Kansas Surface Water Register; CUSEGA, catalog unit segment number alpha; $\mathrm{mi}^{2}$, square miles; $\mathrm{ft}^{3} / \mathrm{s}$, cubic feet per second; HYDRO, lake or other hydrologic structure; NA, not applicable; NRDitch, irrigation ditch; NRTribal, tribal stream]

\begin{tabular}{|c|c|c|c|c|c|c|c|c|c|c|c|}
\hline $\begin{array}{l}\text { Determi- } \\
\text { nation site } \\
\text { identi- } \\
\text { fication } \\
\text { number } \\
\text { (fig. 63) }\end{array}$ & $\begin{array}{l}\text { KSWR } \\
\text { CUSEGA } \\
\text { number }\end{array}$ & \multicolumn{3}{|c|}{$\begin{array}{l}\text { Stream segment } \\
\text { by county } \\
\text { (table 112) }\end{array}$} & Stream name & $\begin{array}{l}\text { Contri- } \\
\text { buting } \\
\text { drainage } \\
\text { area } \\
\left(\mathrm{mi}^{2}\right)\end{array}$ & \multicolumn{5}{|c|}{$\begin{array}{l}\text { Estimated flow-duration values }\left(\mathrm{ft}^{3} / \mathrm{s}\right) \text { for } \\
\text { indicated percentage of time flow equaled or } \\
\text { exceeded }\end{array}$} \\
\hline 1930 & 102600103 & $\mathrm{LC}$ & OT & & Saline River & 2,840 & 15.0 & 24.0 & 45.0 & 126 & 490 \\
\hline 1935 & 1026001014 & $\mathrm{LC}$ & & & Bullfoot Creek & 89.1 & 0 & 1.36 & 4.53 & 11.0 & 25.8 \\
\hline 1962 & 1026001013 & $\mathrm{LC}$ & $\mathrm{RS}$ & & Saline River & 1,950 & 5.10 & 8.70 & 16.0 & 21.0 & 191 \\
\hline 2005 & 1026001017 & $\mathrm{LC}$ & & & Elkhorn Creek & 92.9 & 0 & 1.59 & 5.06 & 12.2 & 28.6 \\
\hline 2028 & 1026001015 & $\mathrm{LC}$ & & & Bullfoot Creek & 38.4 & 0 & .32 & 1.82 & 4.21 & 9.86 \\
\hline 2086 & HYDRO & $\mathrm{LC}$ & & & HYDRO & 6.40 & NA & NA & NA & NA & NA \\
\hline 2087 & 1026001015 & $\mathrm{LC}$ & & & Bullfoot Creek & 13.0 & 0 & 0 & .43 & .74 & 2.01 \\
\hline 2111 & 1026001018 & $\mathrm{LC}$ & & & Table Rock Creek & 4.76 & 0 & 0 & 0 & 0 & 0 \\
\hline 2115 & 1026001039 & $\mathrm{LC}$ & & & Owl Creek & 5.88 & 0 & 0 & 0 & 0 & 0 \\
\hline 2165 & 1026001022 & $\mathrm{LC}$ & SA & & Mulberry Creek & 80.1 & 0 & 1.76 & 5.60 & 13.3 & 30.5 \\
\hline
\end{tabular}


Table 59. Estimated flow-duration values, mean flow values, and peak-discharge frequency values for controlled and uncontrolled flow stream segments on the 1999 Kansas Surface Water Register for Lincoln County.-Continued

[KSWR, Kansas Surface Water Register; CUSEGA, catalog unit segment number alpha; $\mathrm{mi}^{2}$, square miles; $\mathrm{ft}^{3} / \mathrm{s}$, cubic feet per second; HYDRO, lake or other hydrologic structure; NA, not applicable; NRDitch, irrigation ditch; NRTribal, tribal stream]

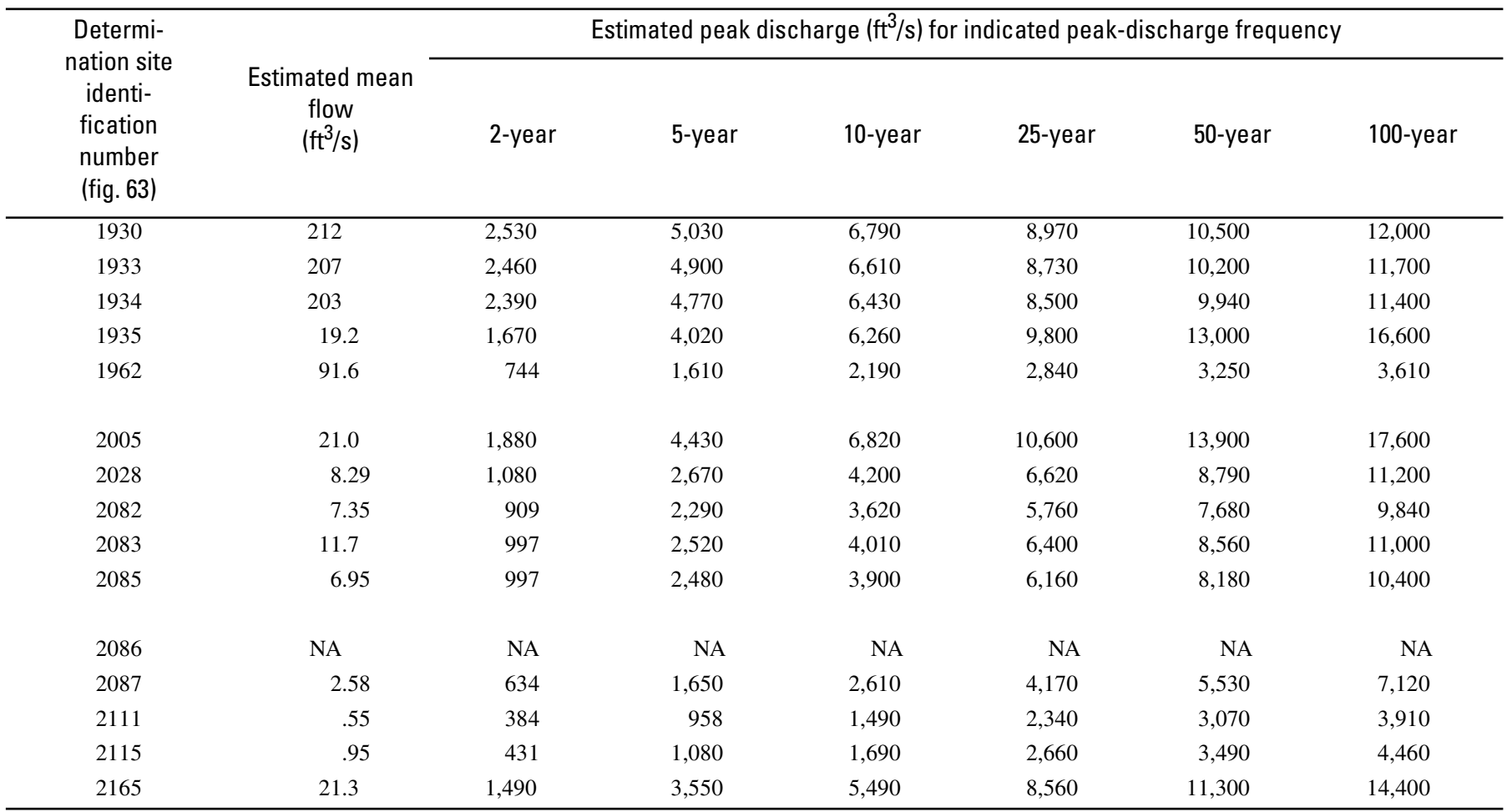




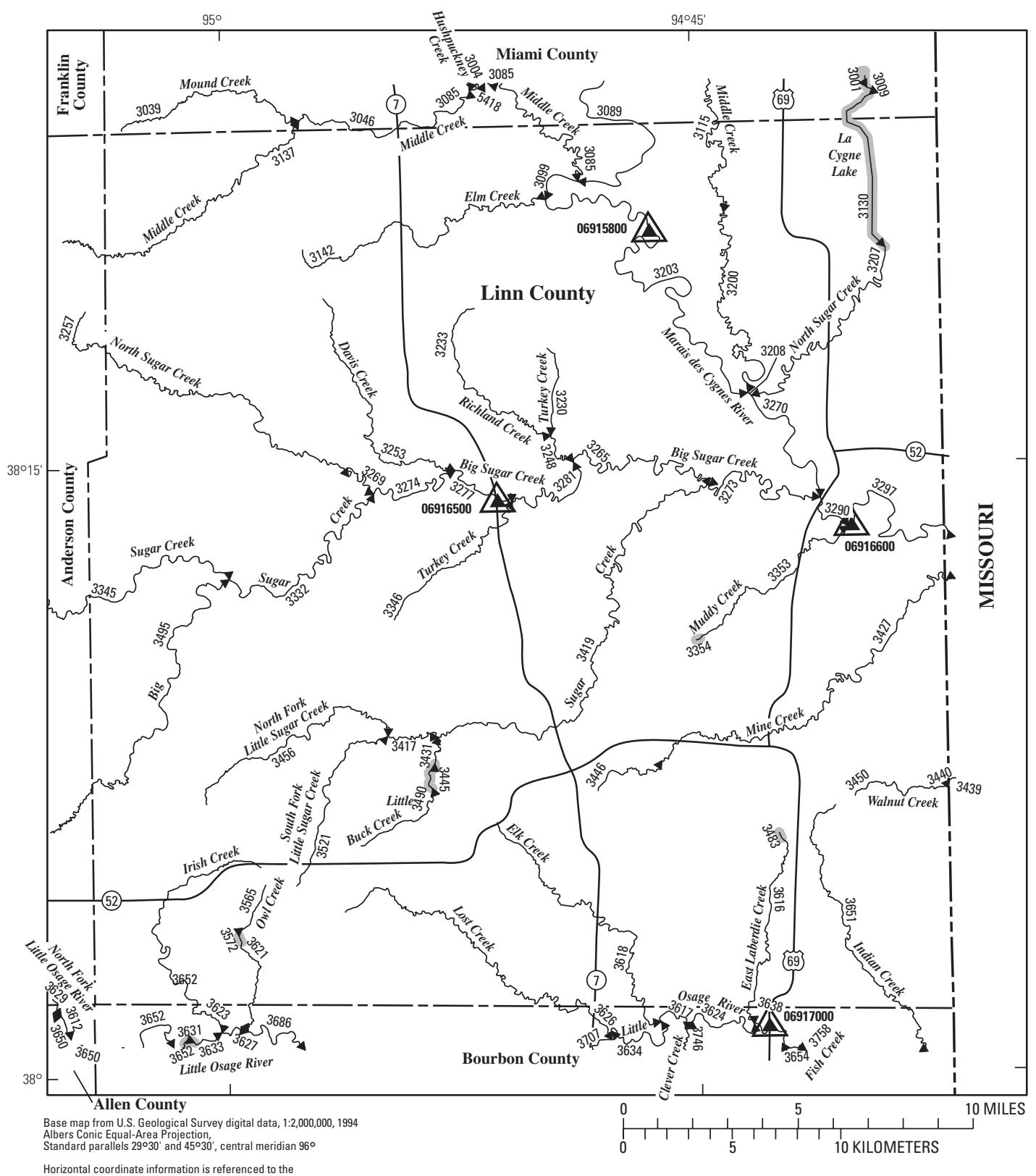

EXPLANATION

Location of streamflow-statistics determination site (small triangle) and associated identification number-small triangle points in downstream direction

06917000

U.S. Geological Survey streamflow-gaging station and number used for estimates of flow duration

${ }^{06916500} \triangle$ U.S. Geological Survey streamflow-gaging station and number used for estimates of peak-discharge frequency values

KANSAS

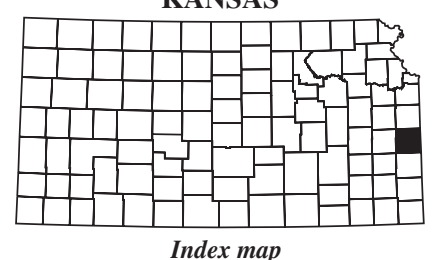

Index map 3445

Lake and determination site identification number

Figure 64. Location of streamflow-statistics determination sites, associated identification numbers, and U.S. Geological Survey streamflow-gaging stations used in the flow-duration and peak-discharge frequency analyses for Linn County. 
Table 60. Estimated flow-duration values, mean flow values, and peak-discharge frequency values for controlled and uncontrolled flow stream segments on the 1999 Kansas Surface Water Register for Linn County.

[KSWR, Kansas Surface Water Register; CUSEGA, catalog unit segment number alpha; $\mathrm{mi}^{2}$, square miles; $\mathrm{ft}^{3} / \mathrm{s}$, cubic feet per second; HYDRO, lake or other hydrologic structure; NA, not applicable; NRDitch, irrigation ditch; NRTribal, tribal stream]

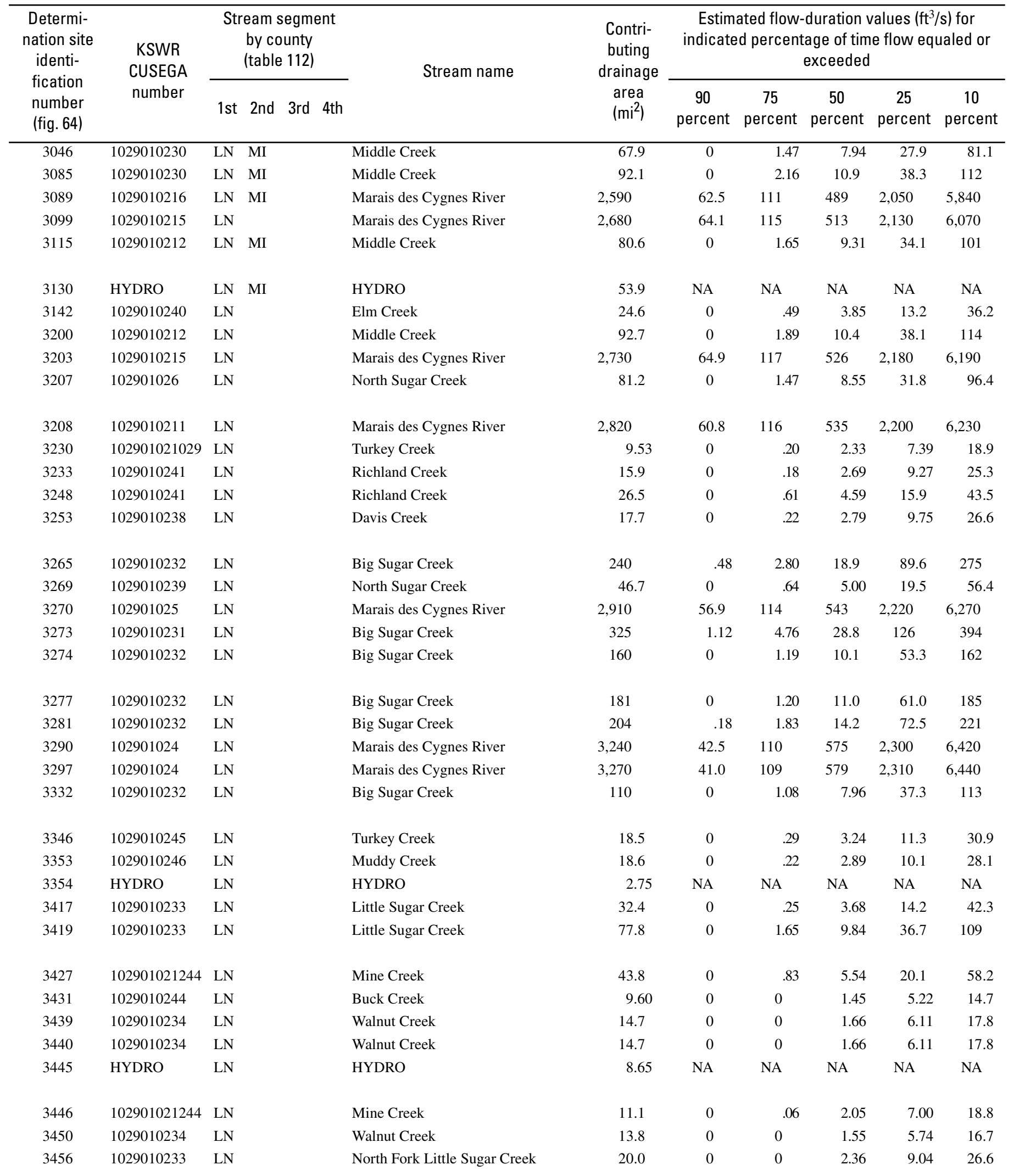


Table 60. Estimated flow-duration values, mean flow values, and peak-discharge frequency values for controlled and uncontrolled flow stream segments on the 1999 Kansas Surface Water Register for Linn County.-Continued

[KSWR, Kansas Surface Water Register; CUSEGA, catalog unit segment number alpha; $\mathrm{mi}^{2}$, square miles; $\mathrm{ft}^{3} / \mathrm{s}$, cubic feet per second; HYDRO, lake or other hydrologic structure; NA, not applicable; NRDitch, irrigation ditch; NRTribal, tribal stream]

\begin{tabular}{|c|c|c|c|c|c|c|c|}
\hline \multirow{2}{*}{$\begin{array}{l}\text { Determi- } \\
\text { nation site } \\
\text { identi- } \\
\text { fication } \\
\text { number } \\
\text { (fig. 64) }\end{array}$} & \multirow{2}{*}{$\begin{array}{c}\text { Estimated mean } \\
\text { flow } \\
\left(\mathrm{ft}^{3} / \mathrm{s}\right)\end{array}$} & \multicolumn{6}{|c|}{ Estimated peak discharge $\left(\mathrm{ft}^{3} / \mathrm{s}\right)$ for indicated peak-discharge frequency } \\
\hline & & 2-year & 5-year & 10 -year & 25-year & 50-year & 100-year \\
\hline 3046 & 56.5 & 6,260 & 11,500 & 15,900 & 22,100 & 27,300 & 32,700 \\
\hline 3099 & 70.0 & 6,540 & 12,100 & 16,700 & 23,400 & 29,000 & 35,000 \\
\hline 3115 & NA & NA & NA & NA & NA & NA & NA \\
\hline 3130 & 2,220 & 23,600 & 29,700 & 36,600 & 58,000 & 76,500 & 97,900 \\
\hline 3142 & 25.1 & 2,660 & 5,410 & 7,740 & 11,200 & 14,100 & 17,400 \\
\hline 3208 & 2,290 & 24,000 & 31,000 & 38,200 & 58,200 & 75,200 & 94,700 \\
\hline 3230 & 12.4 & 1,590 & 3,140 & 4,420 & 6,340 & 7,890 & 9,660 \\
\hline 3233 & 17.7 & 2,120 & 4,250 & 6,030 & 8,700 & 10,900 & 13,300 \\
\hline 3248 & 29.2 & 2,860 & 5,780 & 8,250 & 12,000 & 15,000 & 18,400 \\
\hline 3253 & 18.7 & 2,200 & 4,4504 & 6,340 & 9,180 & 11,500 & 14,200 \\
\hline 3265 & 178 & 6,610 & 13,600 & 20,200 & 30,700 & 40,500 & 52,000 \\
\hline 3269 & 40.6 & 5,440 & 10,100 & 13,900 & 19,500 & 24,200 & 29,100 \\
\hline 3270 & 2,310 & 24,000 & 32,000 & 39,400 & 57,400 & 72,500 & 89,600 \\
\hline 3273 & 246 & 7,960 & 16,000 & 23,500 & 35,400 & 46,300 & 59,100 \\
\hline 3346 & 21.3 & 2,390 & 4,770 & 6,760 & 9,720 & 12,100 & 14,900 \\
\hline 3353 & 20.0 & 2,410 & 4,800 & 6,800 & 9,780 & 12,200 & 15,000 \\
\hline 3354 & NA & NA & NA & NA & NA & NA & NA \\
\hline 3417 & 31.2 & 5,640 & 10,200 & 13,800 & 19,000 & 23,300 & 27,700 \\
\hline 3419 & 73.9 & 7,020 & 12,800 & 17,500 & 24,400 & 30,000 & 36,000 \\
\hline 3427 & 41.4 & 4,140 & 7,930 & 11,100 & 15,800 & 19,600 & 23,700 \\
\hline 3431 & 10.9 & 1,660 & 3,250 & 4,560 & 6,510 & 8,090 & 9,880 \\
\hline 3439 & 14.1 & 2,130 & 4,210 & 5,940 & 8,510 & 10,600 & 13,000 \\
\hline 3440 & 14.1 & 2,130 & 4,210 & 5,940 & 8,510 & 10,600 & 13,000 \\
\hline 3445 & NA & NA & NA & NA & NA & NA & NA \\
\hline 3446 & 13.3 & 1,810 & 3,550 & 5,000 & 7,140 & 8,880 & 10,800 \\
\hline 3450 & 13.3 & 2,060 & 4,060 & 5,720 & 8,190 & 10,200 & 12,500 \\
\hline 3456 & 20.0 & 2,470 & 4,950 & 7,030 & 10,100 & 12,700 & 15,600 \\
\hline
\end{tabular}


Table 60. Estimated flow-duration values, mean flow values, and peak-discharge frequency values for controlled and uncontrolled flow stream segments on the 1999 Kansas Surface Water Register for Linn County.-Continued

[KSWR, Kansas Surface Water Register; CUSEGA, catalog unit segment number alpha; $\mathrm{mi}^{2}$, square miles; $\mathrm{ft}^{3} / \mathrm{s}$, cubic feet per second; HYDRO, lake or other hydrologic structure; NA, not applicable; NRDitch, irrigation ditch; NRTribal, tribal stream]

\begin{tabular}{|c|c|c|c|c|c|c|c|c|c|}
\hline $\begin{array}{l}\text { Determi- } \\
\text { nation site } \\
\text { identi- } \\
\text { fication } \\
\text { number } \\
\text { (fig. 64) }\end{array}$ & $\begin{array}{l}\text { KSWR } \\
\text { CUSEGA } \\
\text { number }\end{array}$ & $\begin{array}{l}\text { Stream segment } \\
\text { by county } \\
\text { (table 112) }\end{array}$ & Stream name & $\begin{array}{l}\text { Contri- } \\
\text { buting } \\
\text { drainage } \\
\text { area } \\
\left(\mathrm{mi}^{2}\right)\end{array}$ & \multicolumn{5}{|c|}{$\begin{array}{l}\text { Estimated flow-duration values }\left(\mathrm{ft}^{3} / \mathrm{s}\right) \text { for } \\
\text { indicated percentage of time flow equaled or } \\
\text { exceeded }\end{array}$} \\
\hline 3483 & HYDRO & $\mathrm{LN}$ & HYDRO & 3.64 & NA & NA & NA & NA & NA \\
\hline 3565 & 102901039 & $\mathrm{LN}$ & Owl Creek & 3.25 & 0 & 0 & .04 & .61 & 2.89 \\
\hline 3572 & HYDRO & $\mathrm{LN}$ & HYDRO & 3.52 & NA & NA & NA & NA & NA \\
\hline
\end{tabular}


Table 60. Estimated flow-duration values, mean flow values, and peak-discharge frequency values for controlled and uncontrolled flow stream segments on the 1999 Kansas Surface Water Register for Linn County.-Continued

[KSWR, Kansas Surface Water Register; CUSEGA, catalog unit segment number alpha; $\mathrm{mi}^{2}$, square miles; $\mathrm{ft}^{3} / \mathrm{s}$, cubic feet per second; HYDRO, lake or other hydrologic structure; NA, not applicable; NRDitch, irrigation ditch; NRTribal, tribal stream]

\begin{tabular}{|c|c|c|c|c|c|c|c|}
\hline $\begin{array}{l}\text { Determi- } \\
\text { nation site } \\
\text { identi- } \\
\text { fication } \\
\text { number } \\
\text { (fig. 64) }\end{array}$ & $\begin{array}{l}\text { Estimated mean } \\
\text { flow } \\
\left(\mathrm{ft}^{3} / \mathrm{s}\right)\end{array}$ & \multicolumn{6}{|c|}{ Estimated peak discharge $\left(\mathrm{ft}^{3} / \mathrm{s}\right)$ for indicated peak-discharge frequency } \\
\hline 3483 & NA & NA & NA & NA & NA & NA & NA \\
\hline 3565 & 3.06 & 864 & 1,660 & 2,320 & 3,270 & 4,050 & 4,920 \\
\hline 3572 & NA & NA & NA & NA & NA & NA & NA \\
\hline
\end{tabular}


372 Estimates of Flow Duration, Mean Flow, and Peak-Discharge Frequency Values for Kansas Stream Locations 


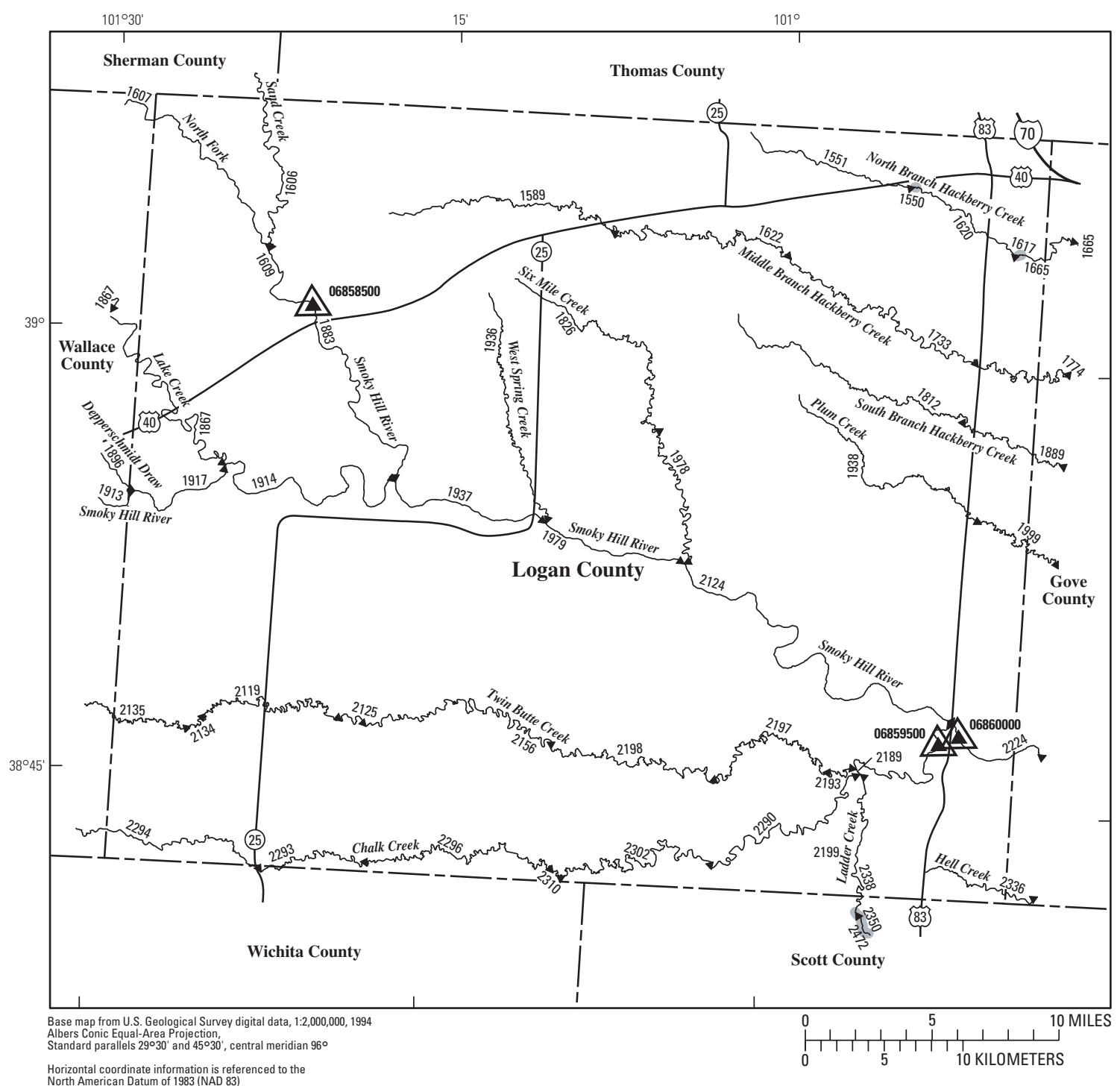

EXPLANATION

2293 Location of streamflow-statistics determination site (small triangle) and associated identification number-small triangle points in downstream direction

06859500 U.S. Geological Survey streamflow-gaging station and number used for estimates of flow duration

06860000

U.S. Geological Survey streamflow-gaging station and number used for estimates of peak-discharge frequency values

KANSAS

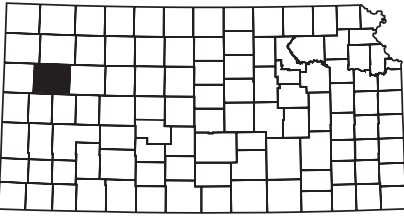

Index map

2350

Lake and determination site identification number

Figure 65. Location of streamflow-statistics determination sites, associated identification numbers, and U.S. Geological Survey streamflow-gaging stations used in the flow-duration and peak-discharge frequency analyses for Logan County. 
Table 61. Estimated flow-duration values, mean flow values, and peak-discharge frequency values for controlled and uncontrolled flow stream segments on the 1999 Kansas Surface Water Register for Logan County.

[KSWR, Kansas Surface Water Register; CUSEGA, catalog unit segment number alpha; $\mathrm{mi}^{2}$, square miles; $\mathrm{ft}^{3} / \mathrm{s}$, cubic feet per second; HYDRO, lake or other hydrologic structure; NA, not applicable; NRDitch, irrigation ditch; NRTribal, tribal stream]

\begin{tabular}{|c|c|c|c|c|c|c|c|c|c|c|c|}
\hline $\begin{array}{l}\text { Determi- } \\
\text { nation site } \\
\text { identi- } \\
\text { fication } \\
\text { number } \\
\text { (fig. 65) }\end{array}$ & $\begin{array}{l}\text { KSWR } \\
\text { CUSEGA } \\
\text { number }\end{array}$ & \multicolumn{3}{|c|}{$\begin{array}{l}\text { Stream segment } \\
\text { by county } \\
\text { (table 112) }\end{array}$} & Stream name & $\begin{array}{l}\text { Contri- } \\
\text { buting } \\
\text { drainage } \\
\text { area } \\
\left(\mathrm{mi}^{2}\right)\end{array}$ & \multicolumn{5}{|c|}{$\begin{array}{l}\text { Estimated flow-duration values }\left(\mathrm{ft}^{3} / \mathrm{s}\right) \text { for } \\
\text { indicated percentage of time flow equaled or } \\
\text { exceeded }\end{array}$} \\
\hline 1550 & HYDRO & $\mathrm{LG}$ & & & HYDRO & 27.5 & NA & NA & NA & NA & NA \\
\hline 1606 & 102600022 & LG & SH & & Sand Creek & 95.8 & 0 & 0 & 0 & .02 & .04 \\
\hline 1607 & 102600023 & LG & SH & WA & North Fork Smoky Hill River & 600 & 0 & 0 & 0 & .64 & 1.50 \\
\hline 1609 & 102600023 & LG & & & North Fork Smoky Hill River & 696 & 0 & 0 & 0 & .86 & 2.02 \\
\hline 1617 & HYDRO & LG & & & HYDRO & 49.7 & NA & NA & NA & NA & NA \\
\hline 1812 & 102600057 & LG & & & South Branch Hackberry Creek & 34.2 & 0 & 0 & 0 & 0 & 0 \\
\hline 1826 & 1026000323 & LG & & & Six Mile Creek & 43.2 & 0 & 0 & 0 & 0 & 0 \\
\hline 1867 & 102600012 & LG & WA & & Lake Creek & 210 & 0 & 0 & 0 & 0 & 0 \\
\hline 1883 & 102600021 & LG & & & North Fork Smoky Hill River & 752 & 0 & 0 & 0 & 1.00 & 3.00 \\
\hline 1896 & 10260001309 & LG & WA & & Depperschmidt Draw & 39.0 & 0 & 0 & 0 & 0 & 0 \\
\hline 1913 & 102600013 & LG & WA & & Smoky Hill River & 788 & 0 & 0 & 0 & 0 & .90 \\
\hline 1914 & 102600011 & LG & & & Smoky Hill River & 1,100 & 0 & 0 & 0 & .95 & 4.62 \\
\hline 1917 & 102600013 & LG & & & Smoky Hill River & 846 & 0 & 0 & 0 & 0 & 1.60 \\
\hline 1936 & 1026000333 & LG & & & West Spring Creek & 42.9 & 0 & 0 & 0 & 0 & 0 \\
\hline 2125 & 102600042 & LG & & & Twin Butte Creek & 99.1 & 0 & 0 & .01 & .01 & .01 \\
\hline 2134 & 102600042 & LG & & & Twin Butte Creek & 50.5 & 0 & 0 & 0 & 0 & 0 \\
\hline 2135 & 102600042 & LG & WA & & Twin Butte Creek & 44.0 & 0 & 0 & 0 & 0 & 0 \\
\hline 2156 & 102600042 & LG & & & Twin Butte Creek & 153 & 0 & 0 & .01 & .01 & .01 \\
\hline 2189 & 102600043 & LG & & & Ladder Creek & 1,420 & 0 & .29 & 1.22 & 2.16 & 6.10 \\
\hline 2193 & 102600042 & LG & & & Twin Butte Creek & 206 & 0 & .01 & .03 & .06 & .12 \\
\hline 2197 & 102600042 & LG & & & Twin Butte Creek & 205 & 0 & .01 & .03 & .06 & .12 \\
\hline 2198 & 102600042 & LG & & & Twin Butte Creek & 186 & 0 & .01 & .02 & .02 & .02 \\
\hline 2199 & 102600041 & LG & & & Ladder Creek & 1,640 & 0 & .39 & 1.90 & 4.00 & 10.0 \\
\hline 2290 & 102600044 & LG & & & Chalk Creek & 210 & 0 & .01 & .03 & .06 & .12 \\
\hline 2293 & 102600044 & LG & & & Chalk Creek & 94.7 & 0 & 0 & .01 & .01 & .01 \\
\hline 2294 & 102600044 & LG & WA & & Chalk Creek & 76.3 & 0 & 0 & 0 & 0 & 0 \\
\hline 2296 & 102600044 & LG & & & Chalk Creek & 136 & 0 & 0 & .01 & .01 & .01 \\
\hline
\end{tabular}


Table 61. Estimated flow-duration values, mean flow values, and peak-discharge frequency values for controlled and uncontrolled flow stream segments on the 1999 Kansas Surface Water Register for Logan County.-Continued

[KSWR, Kansas Surface Water Register; CUSEGA, catalog unit segment number alpha; $\mathrm{mi}^{2}$, square miles; $\mathrm{ft}^{3} / \mathrm{s}$, cubic feet per second; HYDRO, lake or other hydrologic structure; NA, not applicable; NRDitch, irrigation ditch; NRTribal, tribal stream]

\begin{tabular}{|c|c|c|c|c|c|c|c|}
\hline \multirow{2}{*}{$\begin{array}{l}\text { Determi- } \\
\text { nation site } \\
\text { identi- } \\
\text { fication } \\
\text { number } \\
\text { (fig. 65) }\end{array}$} & \multirow{2}{*}{$\begin{array}{c}\text { Estimated mean } \\
\text { flow } \\
\left(\mathrm{ft}^{3} / \mathrm{s}\right)\end{array}$} & \multicolumn{6}{|c|}{ Estimated peak discharge $\left(\mathrm{ft}^{3} / \mathrm{s}\right)$ for indicated peak-discharge frequency } \\
\hline & & 2-year & 5-year & 10 -year & 25-year & 50-year & 100 -year \\
\hline 1550 & NA & NA & NA & NA & NA & NA & NA \\
\hline 1606 & .04 & 311 & 1,080 & 1,980 & 3,660 & 5,370 & 7,470 \\
\hline 1607 & 2.01 & 298 & 1,610 & 3,670 & 8,490 & 14,300 & 22,400 \\
\hline 1609 & 3.05 & 312 & 1,800 & 4,220 & 10,000 & 17,100 & 27,100 \\
\hline 1617 & NA & NA & NA & NA & NA & NA & NA \\
\hline 1812 & 0 & 178 & 618 & 1,140 & 2,100 & 3,070 & 4,260 \\
\hline 1826 & 0 & 231 & 798 & 1,460 & 2,670 & 3,890 & 5,350 \\
\hline 1867 & 1.46 & 437 & 1,470 & 2,660 & 4,850 & 7,040 & 9,720 \\
\hline 1883 & 3.68 & 327 & 1,930 & 4,580 & 11,000 & 18,900 & 30,100 \\
\hline 1896 & 0 & 246 & 822 & 1,480 & 2,660 & 3,830 & 5,220 \\
\hline 1913 & 4.44 & 934 & 3,050 & 5,500 & 10,000 & 14,600 & 20,200 \\
\hline 1914 & 8.03 & 1,190 & 3,830 & 6,870 & 12,500 & 18,100 & 25,100 \\
\hline 1917 & 5.14 & 985 & 3,200 & 5,770 & 10,500 & 15,200 & 21,100 \\
\hline 1936 & 0 & 244 & 832 & 1,510 & 2,740 & 3,960 & 5,430 \\
\hline 2125 & .12 & 276 & 977 & 1,820 & 3,370 & 4,950 & 6,900 \\
\hline 2134 & 0 & 212 & 757 & 1,410 & 2,610 & 3,830 & 5,320 \\
\hline 2135 & 0 & 194 & 697 & 1,300 & 2,420 & 3,550 & 4,930 \\
\hline 2156 & .97 & 329 & 1,140 & 2,110 & 3,900 & 5,710 & 7,950 \\
\hline 2189 & 5.89 & 632 & 2,490 & 5,010 & 10,400 & 16,500 & 25,000 \\
\hline 2193 & 1.93 & 375 & 1,280 & 2,350 & 4,320 & 6,320 & 8,790 \\
\hline 2197 & 1.91 & 377 & 1,290 & 2,360 & 4,340 & 6,350 & 8,830 \\
\hline 2198 & 1.53 & 369 & 1,260 & 2,320 & 4,260 & 6,230 & 8,660 \\
\hline 2199 & 8.00 & 646 & 2,590 & 5,270 & 11,100 & 17,900 & 27,500 \\
\hline 2290 & 1.49 & 454 & 1,520 & 2,770 & 5,040 & 7,330 & 10,200 \\
\hline 2293 & 0 & 291 & 1,020 & 1,890 & 3,500 & 5,130 & 7,140 \\
\hline 2294 & 0 & 255 & 906 & 1,680 & 3,120 & 4,590 & 6,380 \\
\hline 2296 & .14 & 351 & 1,220 & 2,250 & 4,150 & 6,080 & 8,470 \\
\hline
\end{tabular}


Table 61. Estimated flow-duration values, mean flow values, and peak-discharge frequency values for controlled and uncontrolled flow stream segments on the 1999 Kansas Surface Water Register for Logan County.—Continued

[KSWR, Kansas Surface Water Register; CUSEGA, catalog unit segment number alpha; $\mathrm{mi}^{2}$, square miles; $\mathrm{ft}^{3} / \mathrm{s}$, cubic feet per second; HYDRO, lake or other hydrologic structure; NA, not applicable; NRDitch, irrigation ditch; NRTribal, tribal stream]

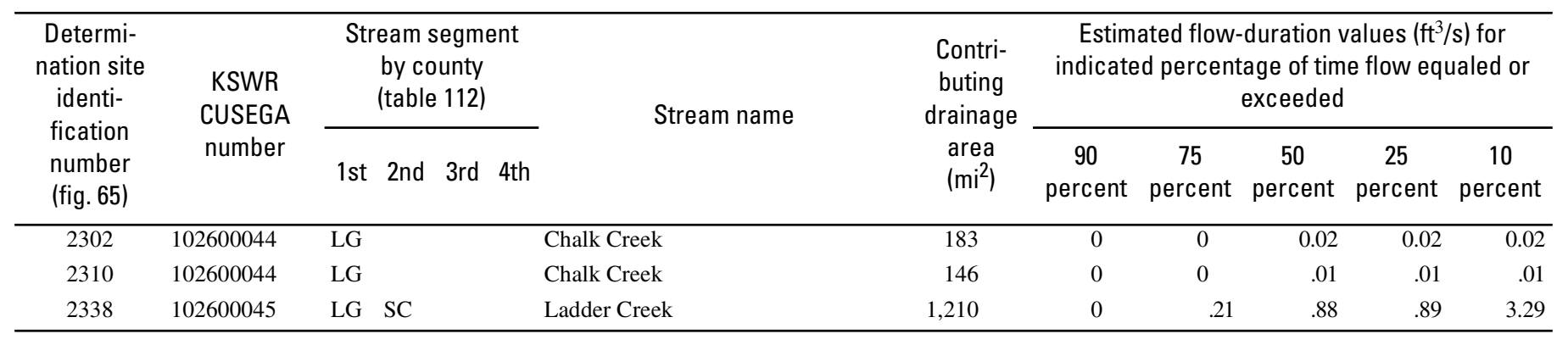


Table 61. Estimated flow-duration values, mean flow values, and peak-discharge frequency values for controlled and uncontrolled flow stream segments on the 1999 Kansas Surface Water Register for Logan County.-Continued

[KSWR, Kansas Surface Water Register; CUSEGA, catalog unit segment number alpha; $\mathrm{mi}^{2}$, square miles; $\mathrm{ft}^{3} / \mathrm{s}$, cubic feet per second; HYDRO, lake or other hydrologic structure; NA, not applicable; NRDitch, irrigation ditch; NRTribal, tribal stream]

\begin{tabular}{|c|c|c|c|c|c|c|c|}
\hline \multirow{2}{*}{$\begin{array}{l}\text { Determi- } \\
\text { nation site } \\
\text { identi- } \\
\text { fication } \\
\text { number } \\
\text { (fig. } 65 \text { ) }\end{array}$} & \multirow{2}{*}{$\begin{array}{c}\text { Estimated mean } \\
\text { flow } \\
\left(\mathrm{ft}^{3} / \mathrm{s}\right)\end{array}$} & \multicolumn{6}{|c|}{ Estimated peak discharge $\left(\mathrm{ft}^{3} / \mathrm{s}\right)$ for indicated peak-discharge frequency } \\
\hline & & 2-year & 5-year & 10-year & 25-year & 50-year & 100-year \\
\hline 2302 & 0.90 & 409 & 1,390 & 2,560 & 4,700 & 6,860 & 9,530 \\
\hline 2310 & .26 & 366 & 1,270 & 2,340 & 4,320 & 6,320 & 8,800 \\
\hline 2338 & 4.28 & 594 & 2,320 & 4,630 & 9,430 & 14,800 & 22,200 \\
\hline
\end{tabular}




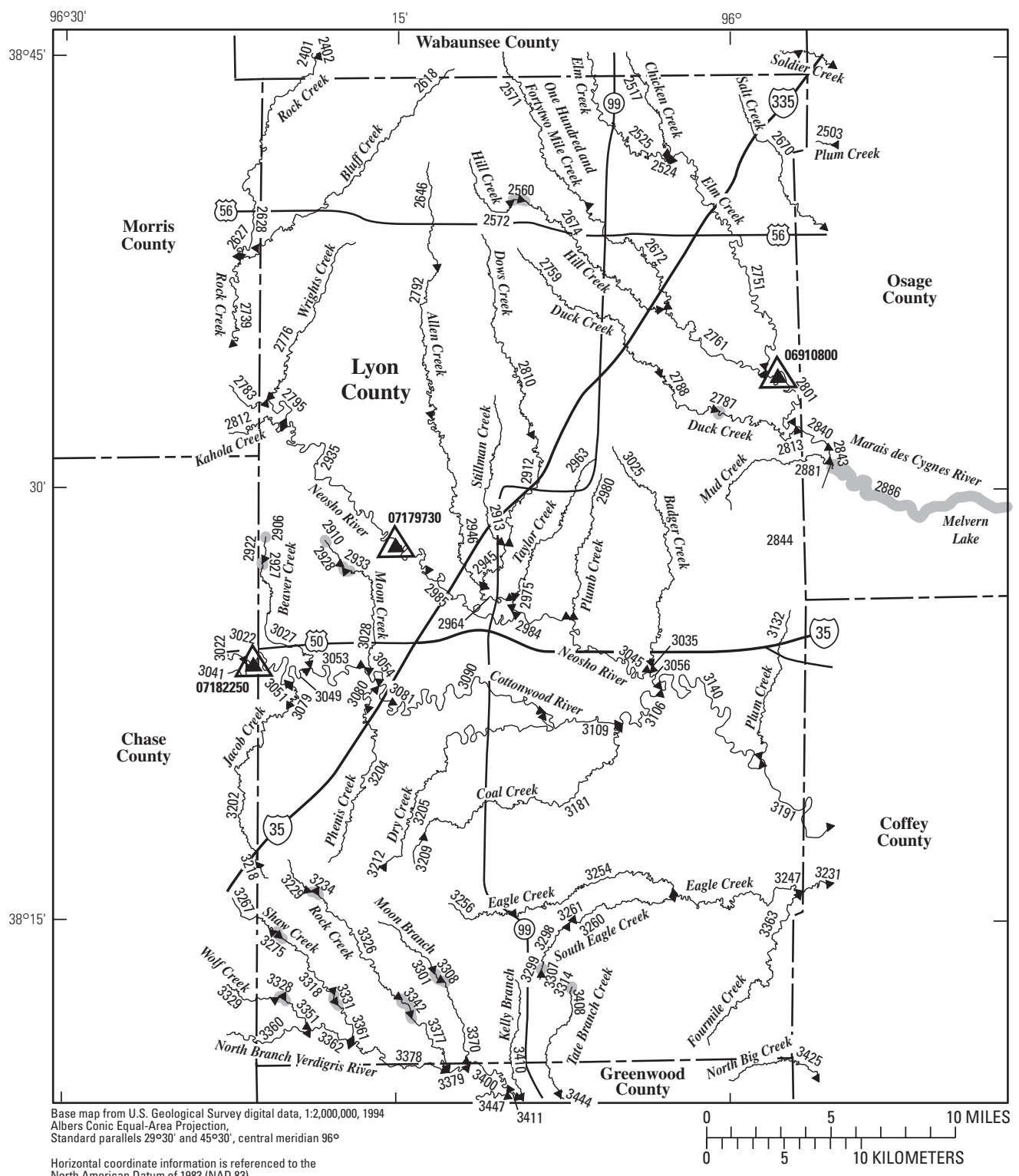

Horizontal coordinate information is referenced to the
North American Datum of 1983 (NAD 83)

EXPLANATION

${ }^{3330}$ Location of streamflow-statistics determination site (small triangle) and associated identification number - small triangle points in downstream direction

07179730 U.S. Geological Survey streamflow-gaging station and number used for estimates of flow duration

${ }^{07182250} \bigwedge$ U.S. Geological Survey streamflow-gaging station and number used for estimates of peak-discharge frequency values

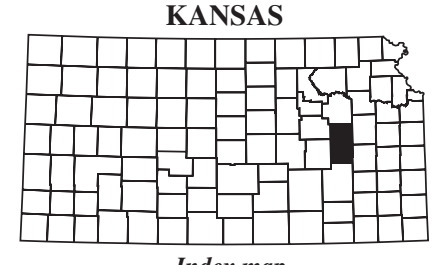

Index map

3342 Lake and determination site identification number

Figure 66. Location of streamflow-statistics determination sites, associated identification numbers, and U.S. Geological Survey streamflow-gaging stations used in the flow-duration and peak-discharge frequency analyses for Lyon County. 
Table 62. Estimated flow-duration values, mean flow values, and peak-discharge frequency values for controlled and uncontrolled flow stream segments on the 1999 Kansas Surface Water Register for Lyon County.

[KSWR, Kansas Surface Water Register; CUSEGA, catalog unit segment number alpha; $\mathrm{mi}^{2}$, square miles; $\mathrm{ft}^{3} / \mathrm{s}$, cubic feet per second; HYDRO, lake or other hydrologic structure; NA, not applicable; NRDitch, irrigation ditch; NRTribal, tribal stream]

\begin{tabular}{|c|c|c|c|c|c|c|c|c|c|c|c|c|}
\hline \multirow{2}{*}{$\begin{array}{l}\text { Determi- } \\
\text { nation site } \\
\text { identi- } \\
\text { fication } \\
\text { number } \\
\text { (fig. 66) }\end{array}$} & \multirow{2}{*}{$\begin{array}{l}\text { KSWR } \\
\text { CUSEGA } \\
\text { number }\end{array}$} & \multicolumn{4}{|c|}{$\begin{array}{l}\text { Stream segment } \\
\text { by county } \\
\text { (table 112) }\end{array}$} & \multirow{2}{*}{ Stream name } & \multirow{2}{*}{$\begin{array}{l}\text { Contri- } \\
\text { buting } \\
\text { drainage } \\
\text { area } \\
\left(\mathrm{mi}^{2}\right)\end{array}$} & \multicolumn{5}{|c|}{$\begin{array}{c}\text { Estimated flow-duration values }\left(\mathrm{ft}^{3} / \mathrm{s}\right) \text { for } \\
\text { indicated percentage of time flow equaled or } \\
\text { exceeded }\end{array}$} \\
\hline & & $1 s t$ & 2nd & $3 r d$ & 4th & & & $\begin{array}{c}90 \\
\text { percent }\end{array}$ & $\begin{array}{c}75 \\
\text { percent }\end{array}$ & $\begin{array}{c}50 \\
\text { percent }\end{array}$ & $\begin{array}{c}25 \\
\text { percent }\end{array}$ & $\begin{array}{c}10 \\
\text { percent }\end{array}$ \\
\hline 2517 & 1029010170 & $\overline{\mathrm{LY}}$ & WB & & & Chicken Creek & 21.7 & 0 & 0 & 1.45 & 5.77 & 18.1 \\
\hline 2524 & 1029010139 & LY & & & & Elm Creek & 42.4 & .01 & .09 & 3.12 & 11.8 & 36.0 \\
\hline 2525 & 1029010139 & LY & WB & & & Elm Creek & 42.1 & .01 & .09 & 3.10 & 11.7 & 35.8 \\
\hline 2560 & HYDRO & LY & & & & HYDRO & 10.4 & NA & NA & NA & NA & NA \\
\hline 2571 & 1029010140 & LY & WB & & & One Hundred and Fortytwo Mile Creek & 24.0 & 0 & 0 & 1.25 & 5.19 & 16.9 \\
\hline 2572 & 1029010171 & LY & & & & Hill Creek & 9.26 & 0 & 0 & .24 & 1.47 & 5.76 \\
\hline 2618 & 110702018 & LY & MR & WB & & Bluff Creek & 33.9 & 0 & .09 & 2.54 & 8.95 & 26.5 \\
\hline 2628 & 110702019 & LY & MR & WB & & Rock Creek & 78.3 & 0 & .97 & 5.63 & 19.1 & 56.1 \\
\hline 2646 & 110702015 & LY & & & & Allen Creek & 12.5 & 0 & 0 & .57 & 2.54 & 8.65 \\
\hline 2670 & 1029010129 & LY & OS & & & Salt Creek & 43.3 & 0 & 0 & 2.44 & 9.86 & 31.2 \\
\hline 2672 & 1029010140 & LY & & & & One Hundred and Fortytwo Mile Creek & 36.7 & .01 & .01 & 2.19 & 8.72 & 27.7 \\
\hline 2674 & 1029010171 & LY & & & & Hill Creek & 21.4 & 0 & 0 & 1.12 & 4.78 & 15.7 \\
\hline 2751 & 1029010139 & LY & & & & Elm Creek & 94.0 & .06 & 1.02 & 7.59 & 28.3 & 87.3 \\
\hline 2759 & 1029010141 & LY & & & & Duck Creek & 21.4 & 0 & 0 & .73 & 3.65 & 13.0 \\
\hline 2761 & 1029010140 & LY & & & & One Hundred and Fortytwo Mile Creek & 68.5 & .03 & .38 & 4.60 & 17.7 & 55.6 \\
\hline 2776 & 1107020138 & LY & & & & Wrights Creek & 26.0 & 0 & 0 & 1.64 & 6.10 & 18.7 \\
\hline 2783 & 110702016 & LY & MR & & & Neosho River & 546 & 10.0 & 17.7 & 57.2 & 202 & 807 \\
\hline 2787 & HYDRO & LY & & & & HYDRO & 30.7 & NA & NA & NA & NA & NA \\
\hline 2788 & 1029010141 & LY & & & & Duck Creek & 30.7 & 0 & 0 & 1.30 & 5.78 & 19.7 \\
\hline 2792 & 110702015 & LY & & & & Allen Creek & 34.9 & 0 & 0 & 1.72 & 7.01 & 22.8 \\
\hline 2795 & 110702016 & LY & & & & Neosho River & 574 & 10.7 & 18.8 & 61.4 & 215 & 850 \\
\hline 2801 & 1029010138 & LY & & & & Marais des Cygnes River & 170 & .18 & 2.30 & 15.0 & 56.0 & 174 \\
\hline 2810 & 110702014 & LY & & & & Dows Creek & 23.0 & 0 & 0 & .80 & 3.89 & 13.7 \\
\hline 2812 & 1107020143 & LY & MR & & & Kahola Creek & 28.3 & 0 & .09 & 2.27 & 7.59 & 21.8 \\
\hline 2813 & 1029010141 & LY & & & & Duck Creek & 36.4 & 0 & 0 & 1.84 & 7.66 & 25.2 \\
\hline 2840 & 1029010137 & LY & OS & & & Marais des Cygnes River & 212 & .18 & 2.91 & 17.5 & 65.2 & 205 \\
\hline 2881 & 1029010191 & LY & OS & & & Mud Creek & 20.2 & 0 & 0 & 1.16 & 4.98 & 16.3 \\
\hline 2906 & HYDRO & LY & & & & HYDRO & 4.76 & NA & NA & NA & NA & NA \\
\hline 2910 & HYDRO & LY & & & & HYDRO & 2.58 & NA & NA & NA & NA & NA \\
\hline 2912 & 110702014 & LY & & & & Dows Creek & 31.0 & 0 & 0 & 1.20 & 5.41 & 18.6 \\
\hline 2913 & 1107020144 & LY & & & & Stillman Creek & 10.5 & 0 & 0 & 0 & .89 & 4.80 \\
\hline 2922 & 1107020329 & LY & & & & Beaver Creek & 8.52 & 0 & 0 & 0 & .70 & 3.78 \\
\hline 2927 & HYDRO & LY & & & & HYDRO & 8.72 & NA & NA & NA & NA & NA \\
\hline 2928 & 1107020331 & LY & & & & Moon Creek & 5.10 & 0 & 0 & 0 & 0 & 1.08 \\
\hline 2933 & HYDRO & LY & & & & HYDRO & 6.84 & NA & NA & NA & NA & NA \\
\hline 2935 & 110702016 & LY & & & & Neosho River & 631 & 12.0 & 21.0 & 70.0 & 240 & 937 \\
\hline 2945 & 110702014 & LY & & & & Dows Creek & 43.6 & 0 & 0 & 1.83 & 7.69 & 25.9 \\
\hline 2946 & 110702015 & LY & & & & Allen Creek & 51.4 & 0 & .06 & 2.65 & 10.3 & 32.9 \\
\hline
\end{tabular}


Table 62. Estimated flow-duration values, mean flow values, and peak-discharge frequency values for controlled and uncontrolled flow stream segments on the 1999 Kansas Surface Water Register for Lyon County.-Continued

[KSWR, Kansas Surface Water Register; CUSEGA, catalog unit segment number alpha; $\mathrm{mi}^{2}$, square miles; $\mathrm{ft}^{3} / \mathrm{s}$, cubic feet per second; HYDRO, lake or other hydrologic structure; NA, not applicable; NRDitch, irrigation ditch; NRTribal, tribal stream]

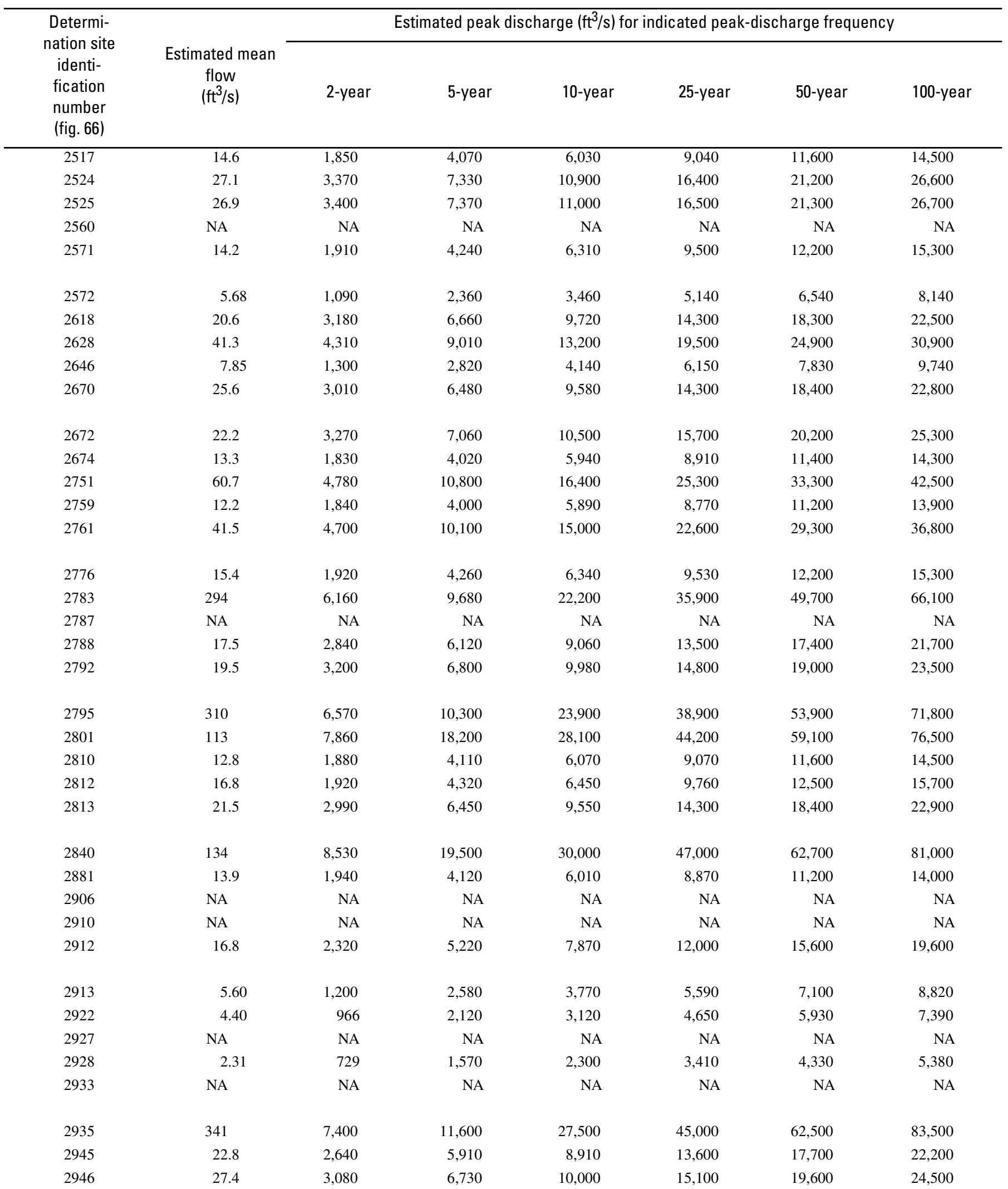


Table 62. Estimated flow-duration values, mean flow values, and peak-discharge frequency values for controlled and uncontrolled flow stream segments on the 1999 Kansas Surface Water Register for Lyon County.-Continued

[KSWR, Kansas Surface Water Register; CUSEGA, catalog unit segment number alpha; $\mathrm{mi}^{2}$, square miles; $\mathrm{ft}^{3} / \mathrm{s}$, cubic feet per second; HYDRO, lake or other hydrologic structure; NA, not applicable; NRDitch, irrigation ditch; NRTribal, tribal stream]

\begin{tabular}{|c|c|c|c|c|c|c|c|c|c|}
\hline \multirow{2}{*}{$\begin{array}{l}\text { Determi- } \\
\text { nation site } \\
\text { identi- } \\
\text { fication } \\
\text { number } \\
\text { (fig. 66) }\end{array}$} & \multirow{2}{*}{$\begin{array}{l}\text { KSWR } \\
\text { CUSEGA } \\
\text { number }\end{array}$} & $\begin{array}{l}\text { Stream segment } \\
\text { by county } \\
\text { (table 112) }\end{array}$ & \multirow{2}{*}{ Stream name } & \multirow{2}{*}{$\begin{array}{l}\text { Contri- } \\
\text { buting } \\
\text { drainage } \\
\text { area } \\
\left(\mathrm{mi}^{2}\right)\end{array}$} & \multicolumn{5}{|c|}{$\begin{array}{l}\text { Estimated flow-duration values }\left(\mathrm{ft}^{3} / \mathrm{s}\right) \text { for } \\
\text { indicated percentage of time flow equaled or } \\
\text { exceeded }\end{array}$} \\
\hline & & 1st 2nd 3rd & & & $\begin{array}{c}90 \\
\text { percent }\end{array}$ & $\begin{array}{c}75 \\
\text { percent }\end{array}$ & $\begin{array}{c}50 \\
\text { percent }\end{array}$ & $\begin{array}{c}25 \\
\text { percent }\end{array}$ & $\begin{array}{c}10 \\
\text { percent }\end{array}$ \\
\hline 2963 & 1107020146 & LY & Taylor Creek & 10.4 & 0 & 0 & 0.08 & 1.19 & 5.47 \\
\hline 2964 & 110702013 & LY & Allen Creek & 96.0 & 0 & .62 & 4.77 & 18.3 & 59.6 \\
\hline 2975 & 110702013 & LY & Allen Creek & 106 & 0 & .76 & 5.29 & 20.2 & 66.2 \\
\hline 2980 & 1107020149 & LY & Plumb Creek & 12.6 & 0 & 0 & .33 & 2.20 & 8.49 \\
\hline 2984 & 110702012 & LY & Neosho River & 751 & 12.8 & 23.4 & 86.9 & 311 & 1,170 \\
\hline 2985 & 110702016 & LY & Neosho River & 641 & 12.1 & 21.2 & 71.4 & 246 & 956 \\
\hline 3025 & 1107020145 & LY & Badger Creek & 33.6 & 0 & 0 & 1.77 & 7.69 & 25.6 \\
\hline 3027 & 1107020329 & LY & Beaver Creek & 20.4 & 0 & 0 & .62 & 2.89 & 10.2 \\
\hline 3028 & 1107020331 & LY & Moon Creek & 17.2 & 0 & 0 & .40 & 2.25 & 8.60 \\
\hline 3035 & 1107020145 & LY & Badger Creek & 33.7 & 0 & 0 & 1.78 & 7.73 & 25.8 \\
\hline 3045 & 110702012 & LY & Neosho River & 774 & 13.0 & 23.8 & 90.1 & 325 & 1,210 \\
\hline 3049 & 110702031 & LY & Cottonwood River & 1,710 & 47.6 & 104 & 300 & 812 & 2,150 \\
\hline 3053 & 110702031 & LY & Cottonwood River & 1,730 & 47.2 & 103 & 302 & 828 & 2,220 \\
\hline 3054 & 110702031 & LY & Cottonwood River & 1,750 & 46.9 & 103 & 304 & 840 & 2,270 \\
\hline 3056 & 110702012 & LY & Neosho River & 808 & 13.2 & 24.5 & 94.9 & 345 & 1,280 \\
\hline 3079 & 1107020328 & LY & Jacob Creek & 26.8 & 0 & 0 & 1.64 & 5.91 & 17.9 \\
\hline 3080 & 1107020330 & LY & Phenis Creek & 21.0 & 0 & 0 & 1.17 & 4.51 & 14.3 \\
\hline 3081 & 110702031 & LY & Cottonwood River & 1,780 & 46.5 & 102 & 305 & 854 & 2,330 \\
\hline 3090 & 110702031 & LY & Cottonwood River & 1,790 & 46.3 & 102 & 307 & 865 & 2,370 \\
\hline 3106 & 110702031 & LY & Cottonwood River & 1,860 & 45.2 & 99.6 & 312 & 910 & 2,550 \\
\hline 3109 & 110702031 & LY & Cottonwood River & 1,820 & 45.8 & 101 & 309 & 883 & 2,440 \\
\hline 3132 & 1107020150 & LY & Plum Creek & 34.4 & 0 & 0 & 1.89 & 8.26 & 27.6 \\
\hline 3140 & 1107020126 & LY & Neosho River & 2,690 & 32.2 & 75.0 & 376 & 1,450 & 4,720 \\
\hline 3181 & 1107020343 & LY & Coal Creek & 35.3 & 0 & 0 & 1.49 & 6.71 & 23.1 \\
\hline 3204 & 1107020330 & LY & Phenis Creek & 20.1 & 0 & 0 & 1.10 & 4.31 & 13.7 \\
\hline 3205 & 1107020342 & LY & Dry Creek & 22.0 & 0 & 0 & 1.09 & 4.54 & 14.9 \\
\hline 3209 & 1107020343 & LY & Coal Creek & 3.36 & 0 & 0 & 0 & 0 & 0 \\
\hline 3212 & 1107020342 & LY & Dry Creek & 2.42 & 0 & 0 & 0 & 0 & 0 \\
\hline 3229 & 1107010114 & LY & Rock Creek & 4.95 & 0 & 0 & .01 & .23 & 1.98 \\
\hline 3234 & HYDRO & LY & HYDRO & 5.33 & NA & NA & NA & NA & NA \\
\hline 3247 & 1107020125 & LY & Eagle Creek & 67.5 & 0 & .16 & 3.33 & 13.7 & 46.1 \\
\hline 3254 & 1107020125 & LY & Eagle Creek & 33.5 & 0 & 0 & 1.37 & 6.34 & 22.0 \\
\hline 3256 & 1107020125 & LY & Eagle Creek & 11.1 & 0 & 0 & .06 & 1.29 & 5.98 \\
\hline 3260 & 1107020147 & LY & South Eagle Creek & 17.1 & 0 & 0 & .61 & 3.29 & 11.9 \\
\hline 3261 & 1107020147 & LY & South Eagle Creek & 5.53 & 0 & 0 & 0 & .08 & 2.30 \\
\hline 3275 & HYDRO & LY & HYDRO & 7.37 & NA & NA & NA & NA & NA \\
\hline 3298 & 1107020147 & LY & South Eagle Creek & 5.23 & 0 & 0 & 0 & 0 & 2.07 \\
\hline 3299 & HYDRO & LY & HYDRO & 1.41 & NA & NA & NA & NA & NA \\
\hline
\end{tabular}


Table 62. Estimated flow-duration values, mean flow values, and peak-discharge frequency values for controlled and uncontrolled flow stream segments on the 1999 Kansas Surface Water Register for Lyon County.-Continued

[KSWR, Kansas Surface Water Register; CUSEGA, catalog unit segment number alpha; $\mathrm{mi}^{2}$, square miles; $\mathrm{ft}^{3} / \mathrm{s}$, cubic feet per second; HYDRO, lake or other hydrologic structure; NA, not applicable; NRDitch, irrigation ditch; NRTribal, tribal stream]

\begin{tabular}{|c|c|c|c|c|c|c|c|}
\hline \multirow{2}{*}{$\begin{array}{l}\text { Determi- } \\
\text { nation site } \\
\text { identi- } \\
\text { fication } \\
\text { number } \\
\text { (fig. 66) }\end{array}$} & \multirow{2}{*}{$\begin{array}{c}\text { Estimated mean } \\
\text { flow } \\
\left(\mathrm{ft}^{3} / \mathrm{s}\right)\end{array}$} & \multicolumn{6}{|c|}{ Estimated peak discharge $\left(\mathrm{ft}^{3} / \mathrm{s}\right)$ for indicated peak-discharge frequency } \\
\hline & & 2-year & 5-year & 10 -year & 25-year & 50-year & 100 -year \\
\hline 2963 & 6.00 & 1,250 & 2,650 & 3,860 & 5,680 & 7,200 & 8,930 \\
\hline 2980 & 8.28 & 1,470 & 3,100 & 4,500 & 6,620 & 8,370 & 10,400 \\
\hline 2984 & 410 & 7,580 & 11,700 & 26,900 & 44,700 & 62,900 & 85,600 \\
\hline 2985 & 347 & 7,420 & 11,600 & 27,500 & 45,000 & 62,500 & 83,700 \\
\hline 3025 & 21.8 & 3,460 & 7,190 & 10,500 & 15,400 & 19,600 & 24,200 \\
\hline 3045 & 423 & 7,620 & 11,800 & 26,800 & 44,700 & 63,000 & 86,100 \\
\hline 3049 & 965 & 14,400 & 25,200 & 33,100 & 52,700 & 74,900 & 105,000 \\
\hline 3053 & 979 & 14,400 & 24,900 & 32,800 & 52,500 & 74,800 & 106,000 \\
\hline 3054 & 990 & 14,300 & 24,800 & 32,500 & 52,300 & 74,800 & 106,000 \\
\hline 3056 & 442 & 7,670 & 11,800 & 26,600 & 44,600 & 63,100 & 86,700 \\
\hline 3079 & 14.9 & 1,890 & 4,230 & 6,300 & 9,510 & 12,200 & 15,300 \\
\hline 3080 & 12.3 & 1,740 & 3,830 & 5,660 & 8,470 & 10,800 & 13,500 \\
\hline 3081 & 1,000 & 14,200 & 24,600 & 32,200 & 52,000 & 74,700 & 106,000 \\
\hline 3090 & 1,010 & 14,200 & 24,400 & 32,000 & 51,900 & 74,700 & 107,000 \\
\hline 3205 & 13.2 & 1,910 & 4,130 & 6,070 & 9,030 & 11,500 & 14,300 \\
\hline 3209 & 1.37 & 645 & 1,340 & 1,930 & 2,810 & 3,540 & 4,360 \\
\hline 3212 & .83 & 524 & 1,090 & 1,560 & 2,270 & 2,850 & 3,510 \\
\hline 3229 & 2.66 & 739 & 1,580 & 2,310 & 3,410 & 4,330 & 5,370 \\
\hline 3234 & NA & NA & NA & NA & NA & NA & NA \\
\hline 3247 & 38.7 & 3,770 & 8,040 & 11,900 & 17,800 & 22,900 & 28,500 \\
\hline 3254 & 19.9 & 2,750 & 5,980 & 8,890 & 13,300 & 17,200 & 21,500 \\
\hline 3256 & 6.53 & 1,320 & 2,800 & 4,070 & 6,000 & 7,590 & 9,410 \\
\hline 3260 & 11.2 & 1,810 & 3,800 & 5,520 & 8,120 & 10,300 & 12,700 \\
\hline 3261 & 3.32 & 921 & 1,900 & 2,730 & 3,980 & 5,000 & 6,160 \\
\hline 3275 & NA & NA & NA & NA & NA & NA & NA \\
\hline 3298 & 3.12 & 892 & 1,840 & 2,640 & 3,840 & 4,830 & 5,950 \\
\hline 3299 & NA & NA & NA & NA & NA & NA & NA \\
\hline
\end{tabular}


Table 62. Estimated flow-duration values, mean flow values, and peak-discharge frequency values for controlled and uncontrolled flow stream segments on the 1999 Kansas Surface Water Register for Lyon County.-Continued

[KSWR, Kansas Surface Water Register; CUSEGA, catalog unit segment number alpha; $\mathrm{mi}^{2}$, square miles; $\mathrm{ft}^{3} / \mathrm{s}$, cubic feet per second; HYDRO, lake or other hydrologic structure; NA, not applicable; NRDitch, irrigation ditch; NRTribal, tribal stream]

\begin{tabular}{|c|c|c|c|c|c|c|c|c|c|}
\hline \multirow{2}{*}{$\begin{array}{l}\text { Determi- } \\
\text { nation site } \\
\text { identi- } \\
\text { fication } \\
\text { number } \\
\text { (fig. 66) }\end{array}$} & \multirow{2}{*}{$\begin{array}{l}\text { KSWR } \\
\text { CUSEGA } \\
\text { number }\end{array}$} & $\begin{array}{l}\text { Stream segment } \\
\text { by county } \\
\text { (table 112) }\end{array}$ & \multirow{2}{*}{ Stream name } & \multirow{2}{*}{$\begin{array}{l}\text { Contri- } \\
\text { buting } \\
\text { drainage } \\
\text { area } \\
\left(\mathrm{mi}^{2}\right)\end{array}$} & \multicolumn{5}{|c|}{$\begin{array}{l}\text { Estimated flow-duration values }\left(\mathrm{ft}^{3} / \mathrm{s}\right) \text { for } \\
\text { indicated percentage of time flow equaled or } \\
\text { exceeded }\end{array}$} \\
\hline & & 1st 2nd 3rd & & & $\begin{array}{c}90 \\
\text { percent }\end{array}$ & $\begin{array}{c}75 \\
\text { percent }\end{array}$ & $\begin{array}{c}50 \\
\text { percent }\end{array}$ & $\begin{array}{c}25 \\
\text { percent }\end{array}$ & $\begin{array}{c}10 \\
\text { percent }\end{array}$ \\
\hline 3301 & 1107010143 & $\overline{\mathrm{LY}}$ & Moon Branch & 8.00 & 0 & 0 & 0.02 & 0.90 & 4.43 \\
\hline 3307 & 1107020147 & LY & South Eagle Creek & 1.11 & 0 & 0 & 0 & 0 & 0 \\
\hline 3308 & HYDRO & LY & HYDRO & 9.66 & NA & NA & NA & NA & NA \\
\hline 3314 & HYDRO & LY & HYDRO & 3.99 & NA & NA & NA & NA & NA \\
\hline 3318 & 1107010140 & LY & Shaw Creek & 13.5 & 0 & .01 & .96 & 3.31 & 9.98 \\
\hline 3326 & 1107010114 & LY & Rock Creek & 15.8 & 0 & .01 & 1.02 & 3.76 & 11.6 \\
\hline 3328 & HYDRO & LY & HYDRO & 13.8 & NA & NA & NA & NA & NA \\
\hline 3331 & HYDRO & LY & HYDRO & 14.0 & NA & NA & NA & NA & NA \\
\hline 3342 & HYDRO & LY & HYDRO & 17.6 & NA & NA & NA & NA & NA \\
\hline 3351 & 1107010141 & LY & Wolf Creek & 15.3 & 0 & .01 & 1.33 & 4.37 & 12.5 \\
\hline 3361 & 1107010140 & LY & Shaw Creek & 16.1 & 0 & .01 & 1.26 & 4.28 & 12.6 \\
\hline 3362 & 1107010115 & LY & North Branch Verdigris River & 57.8 & .04 & 1.06 & 6.63 & 20.5 & 55.4 \\
\hline 3363 & 1107020148 & LY & Fourmile Creek & 36.6 & 0 & 0 & 1.93 & 8.34 & 27.9 \\
\hline 3370 & 1107010143 & LY & Moon Branch & 15.2 & 0 & .01 & .77 & 3.47 & 11.7 \\
\hline
\end{tabular}


Table 62. Estimated flow-duration values, mean flow values, and peak-discharge frequency values for controlled and uncontrolled flow stream segments on the 1999 Kansas Surface Water Register for Lyon County.-Continued

[KSWR, Kansas Surface Water Register; CUSEGA, catalog unit segment number alpha; $\mathrm{mi}^{2}$, square miles; $\mathrm{ft}^{3} / \mathrm{s}$, cubic feet per second; HYDRO, lake or other hydrologic structure; NA, not applicable; NRDitch, irrigation ditch; NRTribal, tribal stream]

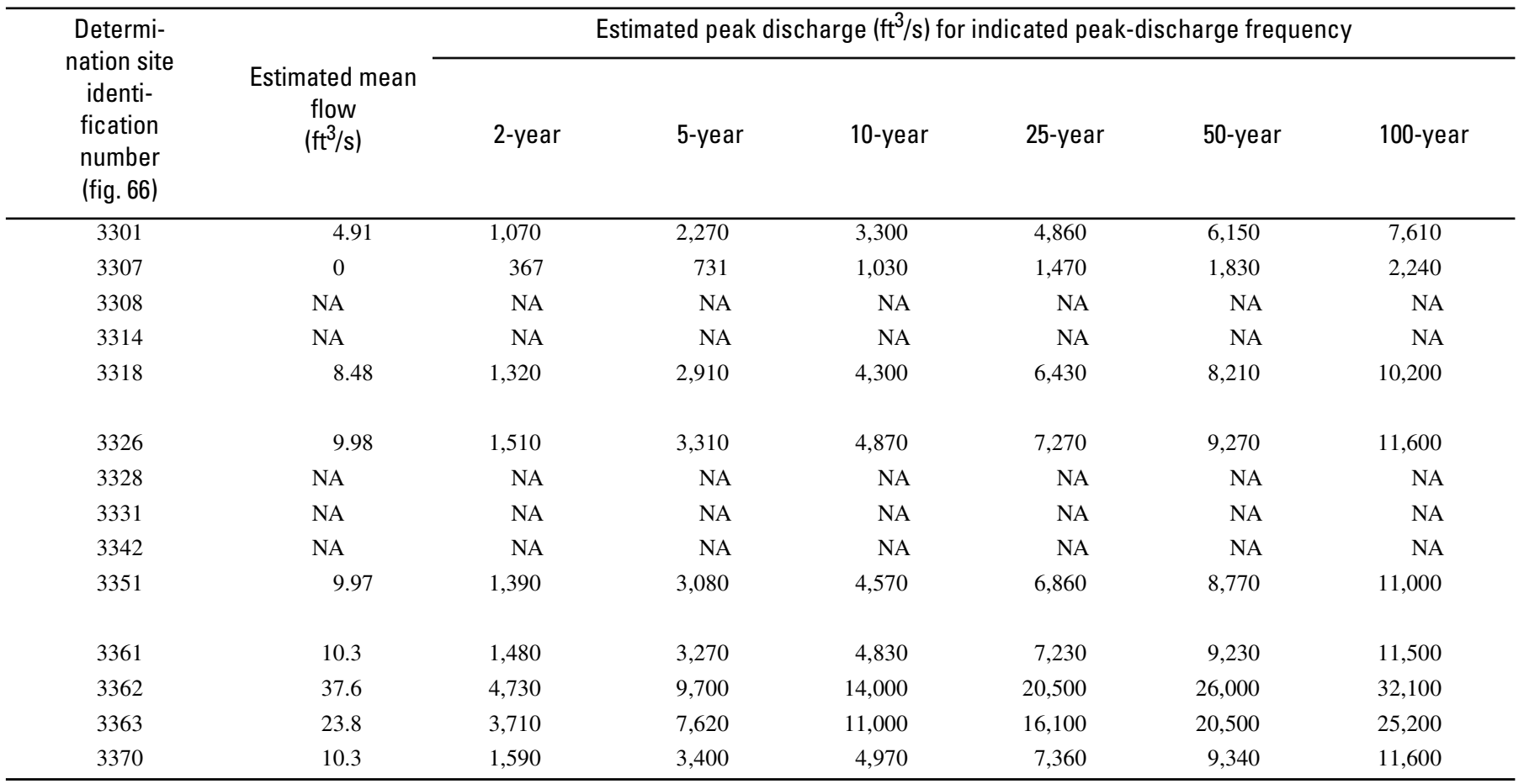




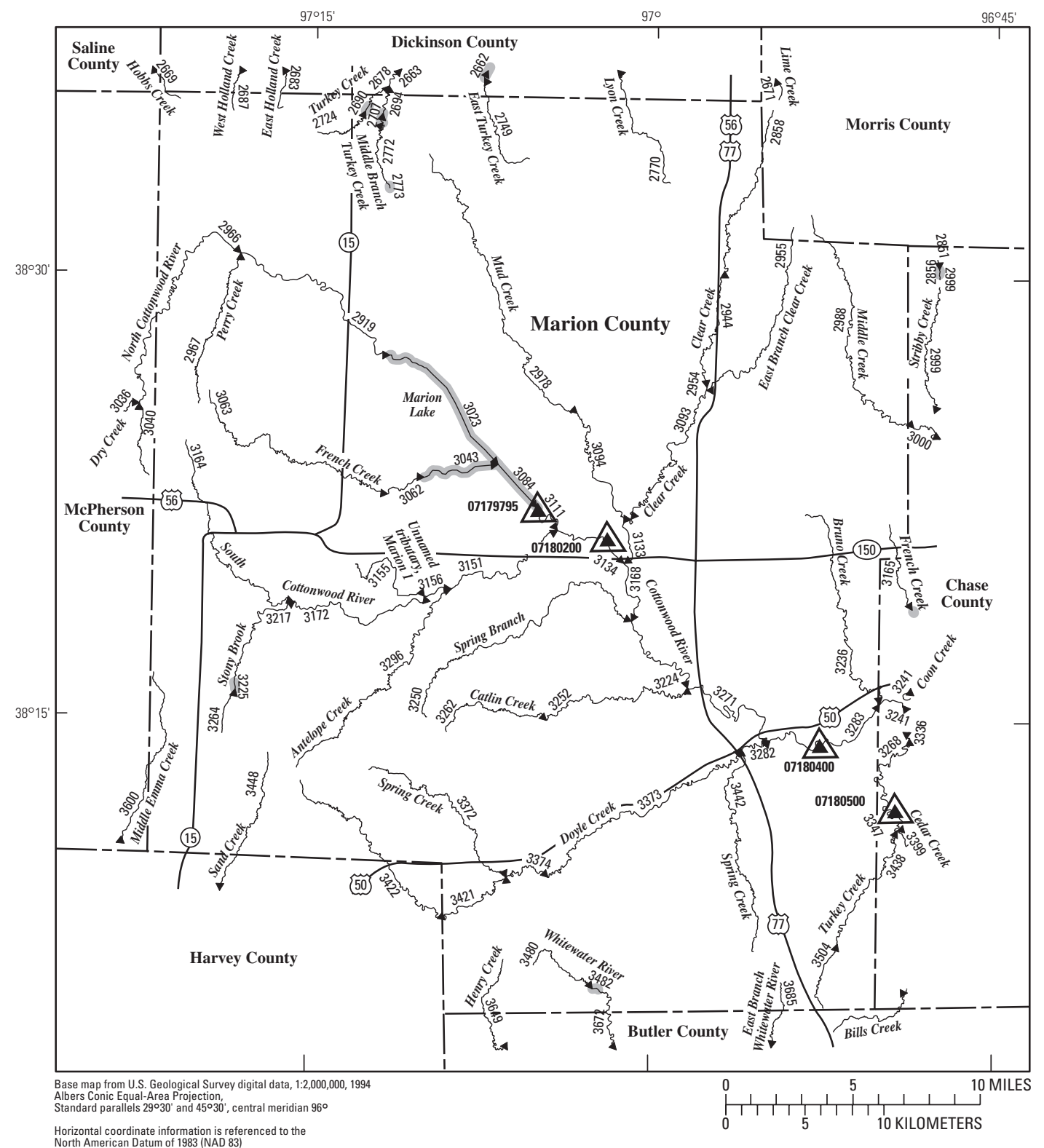

EXPLANATION

$\stackrel{3422}{\longleftarrow}$ Location of streamflow-statistics determination site (small triangle) and associated identification number-small triangle points in downstream direction

07180400

U.S. Geological Survey streamflow-gaging station and number used for estimates of flow duration

07180500

U.S. Geological Survey streamflow-gaging station and number used for estimates of peak-discharge frequency values

KANSAS

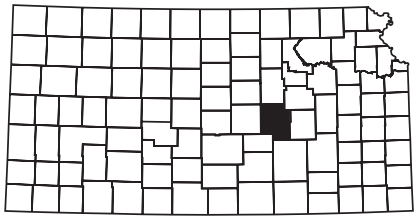

Index map

3043

Lake and determination site identification number

Figure 67. Location of streamflow-statistics determination sites, associated identification numbers, and U.S. Geological Survey streamflow-gaging stations used in the flow-duration and peak-discharge frequency analyses for Marion County. 
Table 63. Estimated flow-duration values, mean flow values, and peak-discharge frequency values for controlled and uncontrolled flow stream segments on the 1999 Kansas Surface Water Register for Marion County.

[KSWR, Kansas Surface Water Register; CUSEGA, catalog unit segment number alpha; $\mathrm{mi}^{2}$, square miles; $\mathrm{ft}^{3} / \mathrm{s}$, cubic feet per second; HYDRO, lake or other hydrologic structure; NA, not applicable; NRDitch, irrigation ditch; NRTribal, tribal stream]

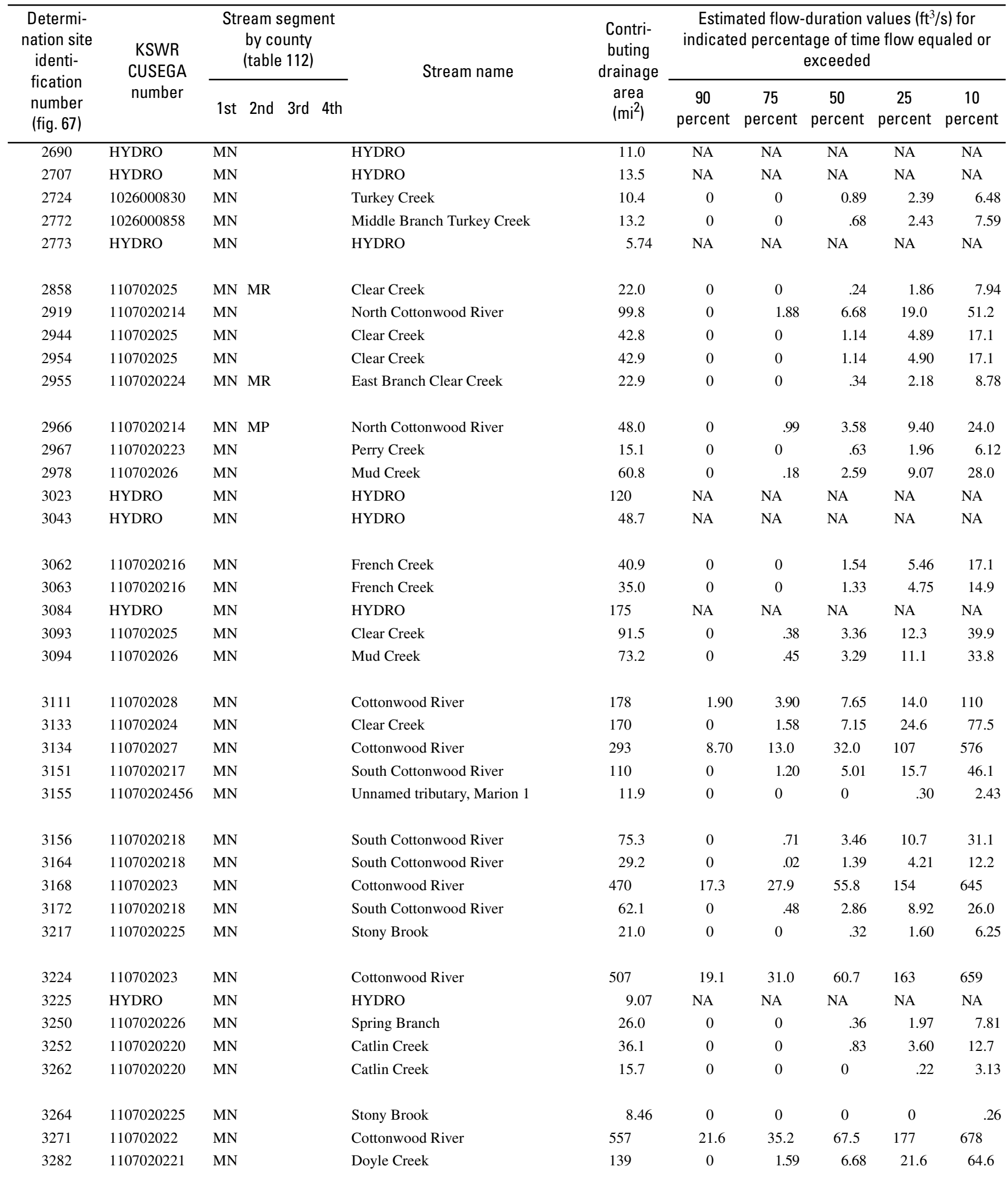


Table 63. Estimated flow-duration values, mean flow values, and peak-discharge frequency values for controlled and uncontrolled flow stream segments on the 1999 Kansas Surface Water Register for Marion County.-Continued

[KSWR, Kansas Surface Water Register; CUSEGA, catalog unit segment number alpha; $\mathrm{mi}^{2}$, square miles; $\mathrm{ft}^{3} / \mathrm{s}$, cubic feet per second; HYDRO, lake or other hydrologic structure; NA, not applicable; NRDitch, irrigation ditch; NRTribal, tribal stream]

\begin{tabular}{|c|c|c|c|c|c|c|c|}
\hline \multirow{2}{*}{$\begin{array}{l}\text { Determi- } \\
\text { nation site } \\
\text { identi- } \\
\text { fication } \\
\text { number } \\
\text { (fig. 67) }\end{array}$} & \multirow{2}{*}{$\begin{array}{c}\text { Estimated mean } \\
\text { flow } \\
\left(\mathrm{ft}^{3} / \mathrm{s}\right)\end{array}$} & \multicolumn{6}{|c|}{ Estimated peak discharge $\left(\mathrm{ft}^{3} / \mathrm{s}\right)$ for indicated peak-discharge frequency } \\
\hline & & 2-year & 5-year & 10 -year & 25-year & 50-year & 100 -year \\
\hline 2690 & NA & NA & NA & NA & NA & NA & NA \\
\hline 2772 & 6.70 & 978 & 2,200 & 3,280 & 4,950 & 6,340 & 7,940 \\
\hline 2773 & NA & NA & NA & NA & NA & NA & NA \\
\hline 2858 & 8.76 & 1,570 & 3,560 & 5,340 & 8,090 & 10,400 & 13,100 \\
\hline 2919 & 37.9 & 2,510 & 5,710 & 8,680 & 13,300 & 17,400 & 22,000 \\
\hline 2966 & 18.5 & 1,690 & 3,890 & 5,930 & 9,100 & 11,900 & 15,000 \\
\hline 2967 & 6.01 & 1,100 & 2,550 & 3,870 & 5,920 & 7,670 & 9,680 \\
\hline 2978 & 23.8 & 1,930 & 4,660 & 7,290 & 11,500 & 15,200 & 19,500 \\
\hline 3023 & NA & NA & NA & NA & NA & NA & NA \\
\hline 3043 & NA & NA & NA & NA & NA & NA & NA \\
\hline 3062 & 15.4 & 2,130 & 4,870 & 7,410 & 11,400 & 14,900 & 18,700 \\
\hline 3063 & 13.5 & 2,060 & 4,700 & 7,130 & 10,900 & 14,200 & 17,900 \\
\hline 3084 & NA & NA & NA & NA & NA & NA & NA \\
\hline 3093 & 34.4 & 2,940 & 6,750 & 10,300 & 16,000 & 21,000 & 26,700 \\
\hline 3156 & 26.0 & 2,600 & 5,830 & 8,810 & 13,400 & 17,500 & 22,000 \\
\hline 3164 & 11.0 & 1,620 & 3,820 & 5,820 & 8,970 & 11,600 & 14,800 \\
\hline 3168 & 271 & 7,790 & 16,600 & 25,900 & 41,800 & 58,700 & 80,400 \\
\hline 3172 & 21.9 & 2,400 & 5,410 & 8,180 & 12,500 & 16,200 & 20,400 \\
\hline 3217 & 7.00 & 1,330 & 3,110 & 4,730 & 7,260 & 9,420 & 11,900 \\
\hline 3224 & 283 & 7,910 & 16,800 & 26,300 & 42,700 & 60,100 & 82,500 \\
\hline 3225 & NA & NA & NA & NA & NA & NA & NA \\
\hline 3250 & 8.67 & 1,560 & 3,640 & 5,530 & 8,480 & 11,000 & 13,900 \\
\hline 3252 & 12.7 & 1,870 & 4,420 & 6,810 & 10,600 & 13,900 & 17,700 \\
\hline 3262 & 4.85 & 1,170 & 2,690 & 4,070 & 6,200 & 8,010 & 10,100 \\
\hline 3264 & 2.06 & 773 & 1,790 & 2,690 & 4,100 & 5,290 & 6,660 \\
\hline 3271 & 299 & 8,090 & 17,200 & 26,800 & 43,900 & 61,900 & 85,400 \\
\hline 3282 & 50.4 & 3,330 & 7,550 & 11,500 & 17,700 & 23,200 & 29,400 \\
\hline
\end{tabular}


Table 63. Estimated flow-duration values, mean flow values, and peak-discharge frequency values for controlled and uncontrolled flow stream segments on the 1999 Kansas Surface Water Register for Marion County.-Continued

[KSWR, Kansas Surface Water Register; CUSEGA, catalog unit segment number alpha; $\mathrm{mi}^{2}$, square miles; $\mathrm{ft}^{3} / \mathrm{s}$, cubic feet per second; HYDRO, lake or other hydrologic structure; NA, not applicable; NRDitch, irrigation ditch; NRTribal, tribal stream]

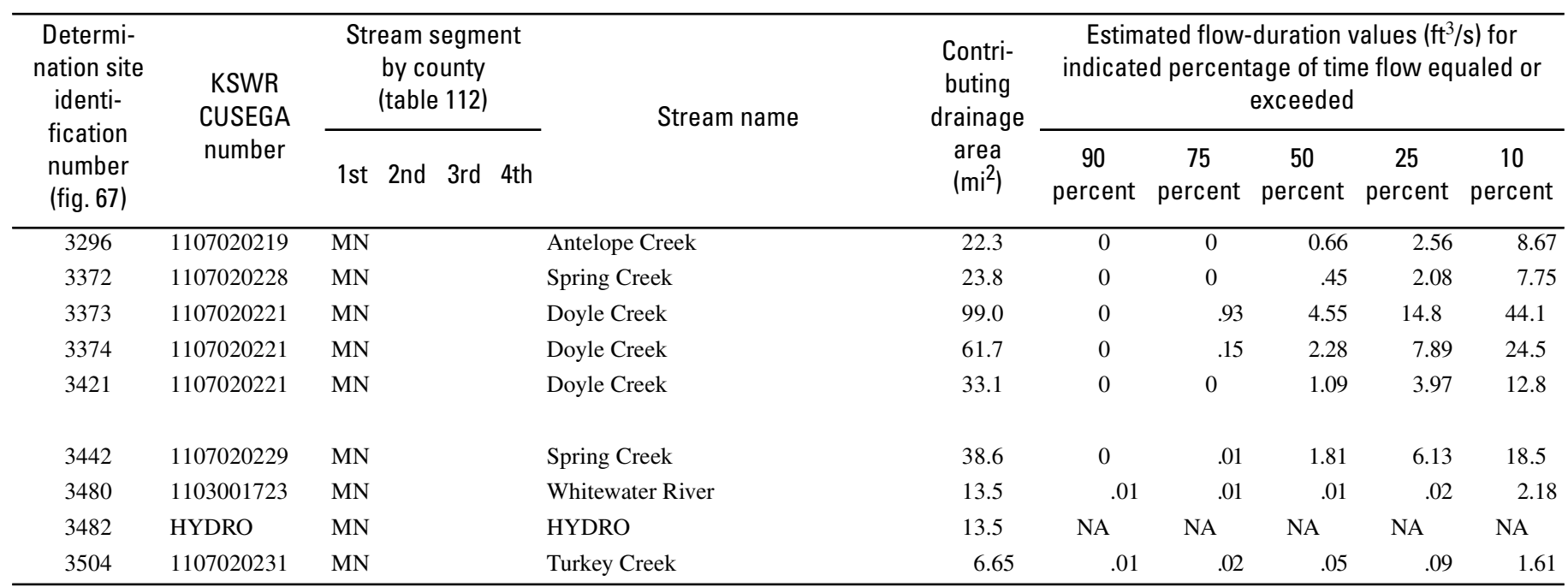


Table 63. Estimated flow-duration values, mean flow values, and peak-discharge frequency values for controlled and uncontrolled flow stream segments on the 1999 Kansas Surface Water Register for Marion County.-Continued

[KSWR, Kansas Surface Water Register; CUSEGA, catalog unit segment number alpha; $\mathrm{mi}^{2}$, square miles; $\mathrm{ft}^{3} / \mathrm{s}$, cubic feet per second; HYDRO, lake or other hydrologic structure; NA, not applicable; NRDitch, irrigation ditch; NRTribal, tribal stream]

\begin{tabular}{|c|c|c|c|c|c|c|c|}
\hline $\begin{array}{l}\text { Determi- } \\
\text { nation site } \\
\text { identi- } \\
\text { fication } \\
\text { number } \\
\text { (fig. } 67 \text { ) }\end{array}$ & $\begin{array}{c}\text { Estimated mean } \\
\text { flow } \\
\left(\mathrm{ft}^{3} / \mathrm{s}\right)\end{array}$ & \multicolumn{6}{|c|}{ Estimated peak discharge $\left(\mathrm{ft}^{3} / \mathrm{s}\right)$ for indicated peak-discharge frequency } \\
\hline 3296 & 8.56 & 1,410 & 3,300 & 5,000 & 7,670 & 9,930 & 12,500 \\
\hline 3374 & 21.6 & 2,240 & 5,210 & 8,010 & 12,400 & 16,300 & 20,700 \\
\hline 3421 & 12.1 & 1,560 & 3,730 & 5,790 & 9,040 & 11,900 & 15,200 \\
\hline 3442 & 16.0 & 2,490 & 5,480 & 8,160 & 12,300 & 15,800 & 19,700 \\
\hline 3480 & 4.13 & 1,100 & 2,510 & 3,770 & 5,730 & 7,380 & 9,280 \\
\hline
\end{tabular}




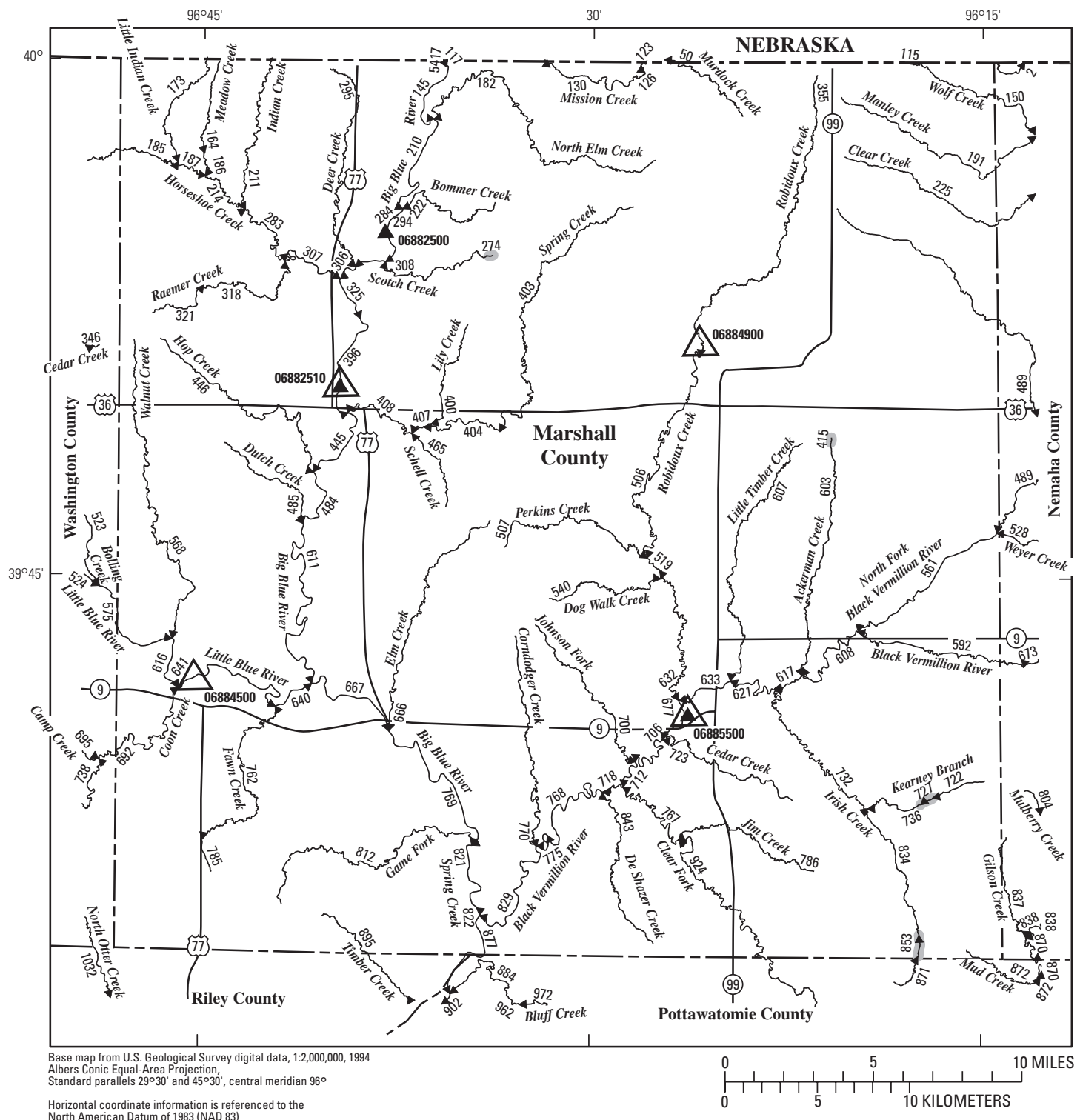

EXPLANATION

Location of streamflow-statistics determination site (small triangle) and associated identification number-small triangle points in downstream direction

${ }^{06885500} \Delta$ U.S. Geological Survey streamflow-gaging station and number used for estimates of flow duration

${ }^{06884500} \triangle$ U.S. Geological Survey streamflow-gaging station and number used for estimates of peak-discharge frequency values

KANSAS

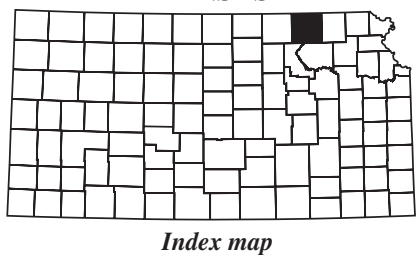

Index map 727

Lake and determination site identification number

Figure 68. Location of streamflow-statistics determination sites, associated identification numbers, and U.S. Geological Survey streamflow-gaging stations used in the flow-duration and peak-discharge frequency analyses for Marshall County. 
Table 64. Estimated flow-duration values, mean flow values, and peak-discharge frequency values for controlled and uncontrolled flow stream segments on the 1999 Kansas Surface Water Register for Marshall County.

[KSWR, Kansas Surface Water Register; CUSEGA, catalog unit segment number alpha; $\mathrm{mi}^{2}$, square miles; $\mathrm{ft}^{3} / \mathrm{s}$, cubic feet per second; HYDRO, lake or other hydrologic structure; NA, not applicable; NRDitch, irrigation ditch; NRTribal, tribal stream]

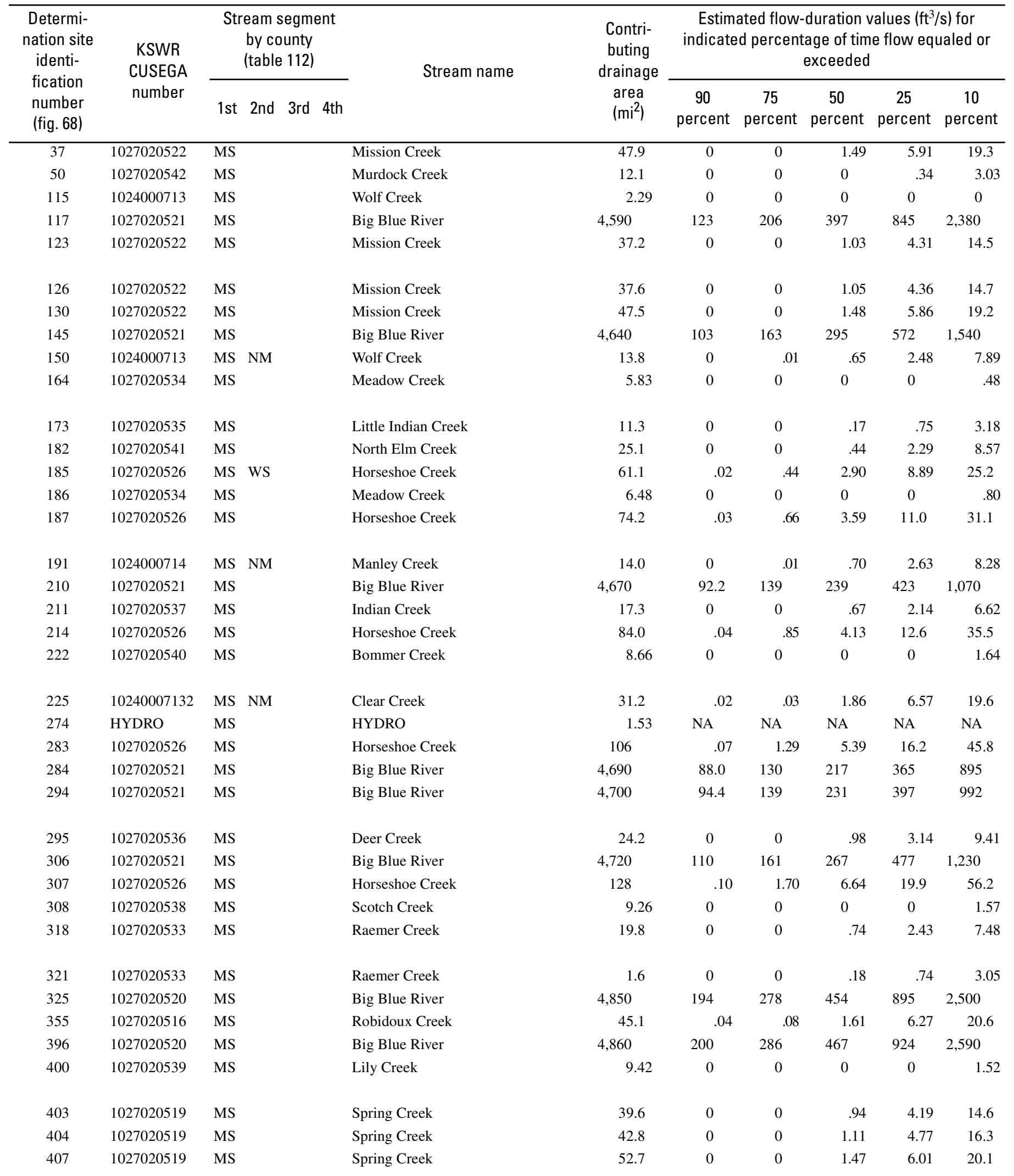


Table 64. Estimated flow-duration values, mean flow values, and peak-discharge frequency values for controlled and uncontrolled flow stream segments on the 1999 Kansas Surface Water Register for Marshall County.-Continued

[KSWR, Kansas Surface Water Register; CUSEGA, catalog unit segment number alpha; $\mathrm{mi}^{2}$, square miles; $\mathrm{ft}^{3} / \mathrm{s}$, cubic feet per second; HYDRO, lake or other hydrologic structure; NA, not applicable; NRDitch, irrigation ditch; NRTribal, tribal stream]

\begin{tabular}{|c|c|c|c|c|c|c|c|}
\hline \multirow{2}{*}{$\begin{array}{l}\text { Determi- } \\
\text { nation site } \\
\text { identi- } \\
\text { fication } \\
\text { number } \\
\text { (fig. 68) }\end{array}$} & \multirow{2}{*}{$\begin{array}{c}\text { Estimated mean } \\
\text { flow } \\
\left(\mathrm{ft}^{3} / \mathrm{s}\right)\end{array}$} & \multicolumn{6}{|c|}{ Estimated peak discharge $\left(\mathrm{ft}^{3} / \mathrm{s}\right)$ for indicated peak-discharge frequency } \\
\hline & & 2-year & 5-year & 10-year & 25-year & 50 -year & 100-year \\
\hline 37 & 17.3 & 2,790 & 6,320 & 9,580 & 14,600 & 19,100 & 24,100 \\
\hline 117 & 1,190 & 17,900 & 31,300 & 41,400 & 55,500 & 66,700 & 78,700 \\
\hline 123 & 13.5 & 3,120 & 6,790 & 10,100 & 15,100 & 19,400 & 24,200 \\
\hline 126 & 13.7 & 3,110 & 6,780 & 10,100 & 15,100 & 19,400 & 24,200 \\
\hline 130 & 17.2 & 2,810 & 6,350 & 9,610 & 14,700 & 19,200 & 24,100 \\
\hline 173 & 3.81 & 839 & 1,990 & 3,040 & 4,680 & 6,080 & 7,690 \\
\hline 182 & 8.75 & 1,390 & 3,330 & 5,100 & 7,880 & 10,300 & 13,000 \\
\hline 185 & 20.4 & 2,390 & 5,450 & 8,290 & 12,700 & 16,600 & 21,000 \\
\hline 186 & 1.93 & 613 & 1,440 & 2,180 & 3,330 & 4,310 & 5,430 \\
\hline 187 & 24.7 & 2,640 & 6,000 & 9,100 & 13,900 & 18,200 & 23,000 \\
\hline 191 & 7.17 & 1,080 & 2,500 & 3,780 & 5,760 & 7,430 & 9,370 \\
\hline 210 & 557 & 18,300 & 31,900 & 42,000 & 56,100 & 67,200 & 79,000 \\
\hline 211 & 6.33 & 1,090 & 2,590 & 3,970 & 6,130 & 7,980 & 10,100 \\
\hline 214 & 27.9 & 2,720 & 6,190 & 9,410 & 14,400 & 18,900 & 23,900 \\
\hline 295 & 8.64 & 1,320 & 3,180 & 4,880 & 7,570 & 9,870 & 12,500 \\
\hline 306 & 613 & 18,500 & 32,000 & 42,100 & 56,100 & 67,100 & 78,800 \\
\hline 307 & 42.1 & 3,170 & 7,210 & 11,000 & 16,900 & 22,100 & 28,000 \\
\hline 308 & 2.91 & 766 & 1,800 & 2,730 & 4,190 & 5,430 & 6,860 \\
\hline 318 & 7.05 & 1,150 & 2,770 & 4,260 & 6,600 & 8,610 & 10,900 \\
\hline 321 & 3.60 & 795 & 1,890 & 2,890 & 4,450 & 5,790 & 7,330 \\
\hline 325 & 1,150 & 19,100 & 32,800 & 43,000 & 56,800 & 67,700 & 79,000 \\
\hline 355 & 18.1 & 1,850 & 3,930 & 5,780 & 8,700 & 11,300 & 14,300 \\
\hline 396 & 1,190 & 19,100 & 32,800 & 42,900 & 56,700 & 67,500 & 78,800 \\
\hline 400 & 2.90 & 772 & 1,820 & 2,760 & 4,230 & 5,480 & 6,930 \\
\hline 403 & 13.8 & 1,950 & 4,690 & 7,310 & 11,500 & 15,200 & 19,500 \\
\hline 404 & 15.1 & 2,090 & 4,990 & 7,750 & 12,100 & 16,100 & 20,500 \\
\hline 407 & 18.2 & 2,370 & 5,610 & 8,690 & 13,600 & 17,900 & 22,900 \\
\hline
\end{tabular}


Table 64. Estimated flow-duration values, mean flow values, and peak-discharge frequency values for controlled and uncontrolled flow stream segments on the 1999 Kansas Surface Water Register for Marshall County._-Continued

[KSWR, Kansas Surface Water Register; CUSEGA, catalog unit segment number alpha; $\mathrm{mi}^{2}$, square miles; $\mathrm{ft}^{3} / \mathrm{s}$, cubic feet per second; HYDRO, lake or other hydrologic structure; NA, not applicable; NRDitch, irrigation ditch; NRTribal, tribal stream]

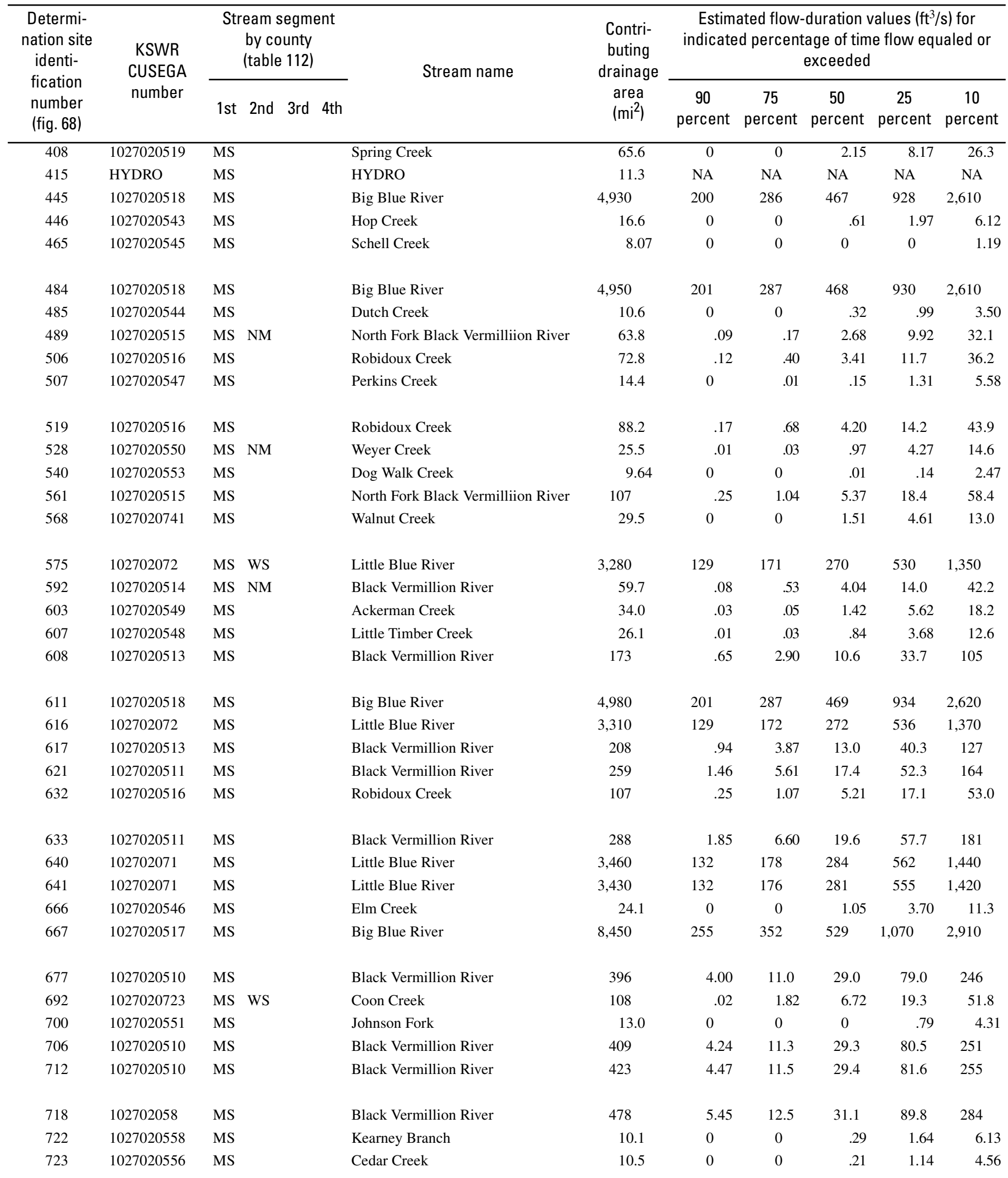


Table 64. Estimated flow-duration values, mean flow values, and peak-discharge frequency values for controlled and uncontrolled flow stream segments on the 1999 Kansas Surface Water Register for Marshall County.-Continued

[KSWR, Kansas Surface Water Register; CUSEGA, catalog unit segment number alpha; $\mathrm{mi}^{2}$, square miles; $\mathrm{ft}^{3} / \mathrm{s}$, cubic feet per second; HYDRO, lake or other hydrologic structure; NA, not applicable; NRDitch, irrigation ditch; NRTribal, tribal stream]

\begin{tabular}{|c|c|c|c|c|c|c|c|}
\hline \multirow{2}{*}{$\begin{array}{l}\text { Determi- } \\
\text { nation site } \\
\text { identi- } \\
\text { fication } \\
\text { number } \\
\text { (fig. 68) }\end{array}$} & \multirow{2}{*}{$\begin{array}{c}\text { Estimated mean } \\
\text { flow } \\
\left(\mathrm{ft}^{3} / \mathrm{s}\right)\end{array}$} & \multicolumn{6}{|c|}{ Estimated peak discharge $\left(\mathrm{ft}^{3} / \mathrm{s}\right)$ for indicated peak-discharge frequency } \\
\hline & & 2-year & 5-year & 10-year & 25-year & 50-year & 100-year \\
\hline 408 & 22.7 & 2,620 & 6,160 & 9,490 & 14,800 & 19,500 & 24,800 \\
\hline 446 & 5.96 & 1,050 & 2,500 & 3,840 & 5,940 & 7,730 & 9,810 \\
\hline 465 & 2.54 & 713 & 1,670 & 2,530 & 3,860 & 5,000 & 6,310 \\
\hline 484 & 1,210 & 19,300 & 33,200 & 43,500 & 57,600 & 68,700 & 80,400 \\
\hline 485 & 3.85 & 819 & 1,930 & 2,950 & 4,530 & 5,880 & 7,430 \\
\hline 519 & 35.3 & 2,460 & 5,490 & 8,310 & 12,900 & 17,100 & 22,000 \\
\hline 528 & 13.1 & 1,750 & 3,970 & 5,940 & 9,010 & 11,600 & 14,600 \\
\hline 540 & 3.62 & 858 & 1,970 & 2,970 & 4,520 & 5,830 & 7,340 \\
\hline 561 & 46.5 & 4,180 & 9,320 & 14,100 & 21,500 & 28,200 & 35,700 \\
\hline 568 & 11.0 & 1,460 & 3,540 & 5,460 & 8,500 & 11,100 & 14,100 \\
\hline 575 & 711 & 12,800 & 21,600 & 28,500 & 38,300 & 46,500 & 55,400 \\
\hline 592 & 32.3 & 2,820 & 6,380 & 9,690 & 14,900 & 19,500 & 24,600 \\
\hline 603 & 15.6 & 2,530 & 5,740 & 8,690 & 13,300 & 17,300 & 21,800 \\
\hline 607 & 11.5 & 1,560 & 3,650 & 5,550 & 8,510 & 11,000 & 14,000 \\
\hline 633 & 130 & 5,520 & 12,600 & 19,400 & 30,600 & 41,000 & 53,300 \\
\hline 640 & 755 & 11,700 & 24,200 & 35,700 & 54,100 & 71,100 & 90,900 \\
\hline 641 & 746 & 11,600 & 24,000 & 35,400 & 53,700 & 70,600 & 90,300 \\
\hline 666 & 9.92 & 1,390 & 3,300 & 5,040 & 7,780 & 10,100 & 12,800 \\
\hline 667 & 1,480 & 23,400 & 40,900 & 54,500 & 73,500 & 88,900 & 105,000 \\
\hline 677 & 174 & 7,030 & 15,700 & 24,100 & 38,000 & 51,200 & 66,900 \\
\hline 692 & 38.1 & 2,540 & 5,850 & 8,960 & 13,800 & 18,200 & 23,100 \\
\hline 700 & 5.14 & 1,040 & 2,390 & 3,600 & 5,480 & 7,080 & 8,910 \\
\hline 706 & 178 & 6,830 & 15,400 & 23,700 & 37,600 & 50,700 & 66,400 \\
\hline 712 & 182 & 6,870 & 15,500 & 23,900 & 37,900 & 51,200 & 67,100 \\
\hline 718 & 203 & 7,570 & 16,800 & 25,800 & 40,700 & 54,700 & 71,500 \\
\hline 722 & 5.82 & 1,020 & 2,260 & 3,360 & 5,040 & 6,450 & 8,060 \\
\hline 723 & 4.83 & 959 & 2,180 & 3,260 & 4,930 & 6,350 & 7,970 \\
\hline
\end{tabular}


Table 64. Estimated flow-duration values, mean flow values, and peak-discharge frequency values for controlled and uncontrolled flow stream segments on the 1999 Kansas Surface Water Register for Marshall County._-Continued

[KSWR, Kansas Surface Water Register; CUSEGA, catalog unit segment number alpha; $\mathrm{mi}^{2}$, square miles; $\mathrm{ft}^{3} / \mathrm{s}$, cubic feet per second; HYDRO, lake or other hydrologic structure; NA, not applicable; NRDitch, irrigation ditch; NRTribal, tribal stream]

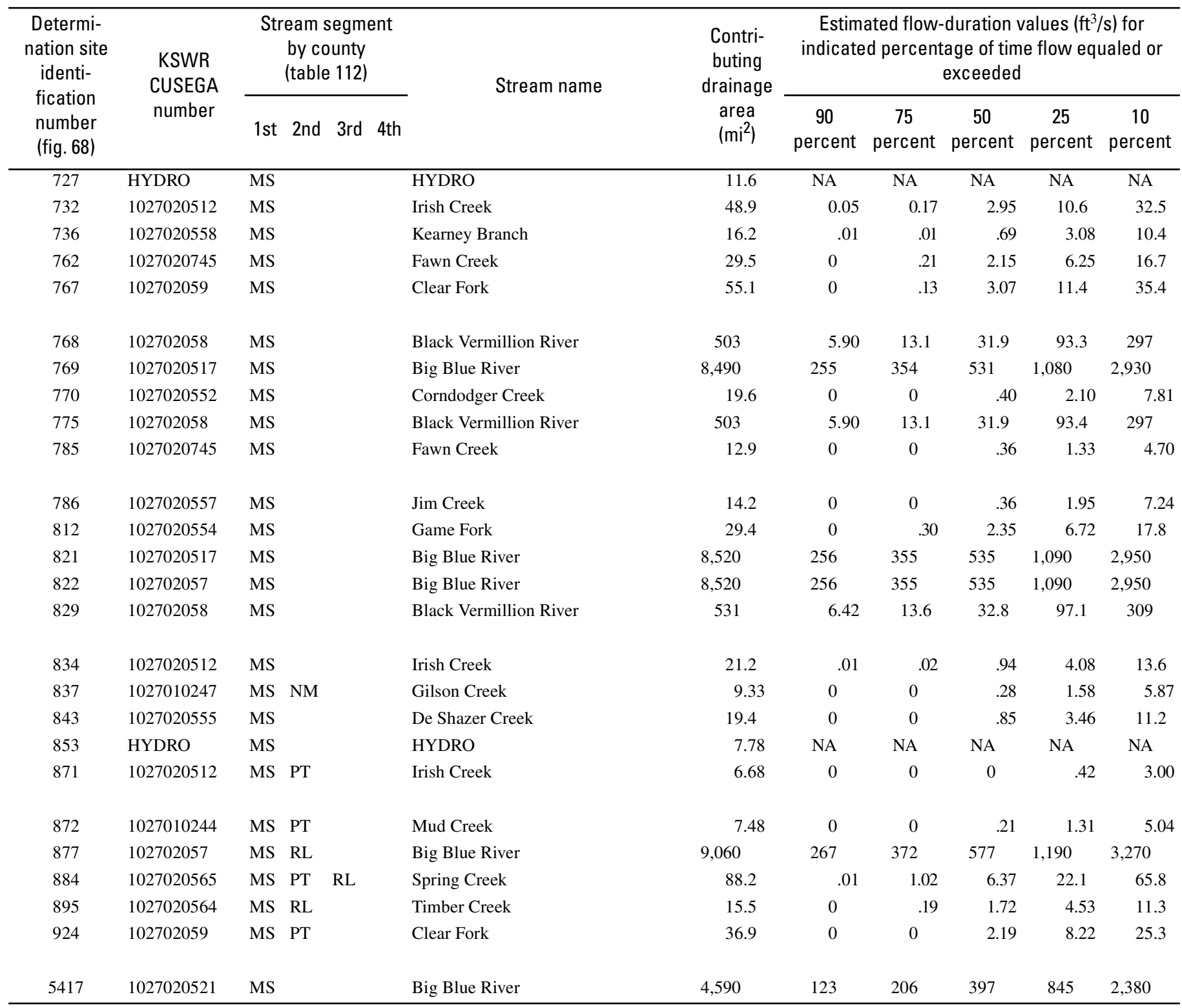


Table 64. Estimated flow-duration values, mean flow values, and peak-discharge frequency values for controlled and uncontrolled flow stream segments on the 1999 Kansas Surface Water Register for Marshall County.-Continued

[KSWR, Kansas Surface Water Register; CUSEGA, catalog unit segment number alpha; $\mathrm{mi}^{2}$, square miles; $\mathrm{ft}^{3} / \mathrm{s}$, cubic feet per second; HYDRO, lake or other hydrologic structure; NA, not applicable; NRDitch, irrigation ditch; NRTribal, tribal stream]

\begin{tabular}{|c|c|c|c|c|c|c|c|}
\hline \multirow{2}{*}{$\begin{array}{l}\text { Determi- } \\
\text { nation site } \\
\text { identi- } \\
\text { fication } \\
\text { number } \\
\text { (fig. 68) }\end{array}$} & \multirow{2}{*}{$\begin{array}{c}\text { Estimated mean } \\
\text { flow } \\
\left(\mathrm{ft}^{3} / \mathrm{s}\right)\end{array}$} & \multicolumn{6}{|c|}{ Estimated peak discharge $\left(\mathrm{ft}^{3} / \mathrm{s}\right)$ for indicated peak-discharge frequency } \\
\hline & & 2-year & 5-year & 10-year & 25-year & 50-year & 100-year \\
\hline 727 & NA & NA & NA & NA & NA & NA & NA \\
\hline 762 & 13.1 & 1,570 & 3,740 & 5,720 & 8,850 & 11,500 & 14,600 \\
\hline 767 & 27.9 & 3,250 & 7,190 & 10,800 & 16,300 & 21,200 & 26,600 \\
\hline 768 & 211 & 7,200 & 16,300 & 25,200 & 39,900 & 53,900 & 70,700 \\
\hline 769 & 1,490 & 23,300 & 40,800 & 54,300 & 73,300 & 88,700 & 105,000 \\
\hline 786 & 7.03 & 1,180 & 2,680 & 4,010 & 6,060 & 7,790 & 9,780 \\
\hline 812 & 13.7 & 1,610 & 3,800 & 5,810 & 8,950 & 11,600 & 14,700 \\
\hline 821 & 1,490 & 23,300 & 40,800 & 54,400 & 73,400 & 88,800 & 106,000 \\
\hline 822 & 1,490 & 23,300 & 40,800 & 54,400 & 73,400 & 88,800 & 106,000 \\
\hline 829 & 220 & 6,870 & 15,800 & 24,600 & 39,200 & 53,200 & 69,900 \\
\hline 834 & 11.8 & 1,570 & 3,540 & 5,300 & 8,010 & 10,300 & 12,900 \\
\hline 837 & 5.56 & 1,010 & 2,210 & 3,270 & 4,880 & 6,230 & 7,770 \\
\hline 843 & 9.84 & 1,400 & 3,200 & 4,800 & 7,300 & 9,410 & 11,800 \\
\hline 853 & NA & NA & NA & NA & NA & NA & NA \\
\hline 5417 & 1,190 & 17,900 & 31,300 & 41,400 & 55,500 & 66,800 & 78,700 \\
\hline
\end{tabular}




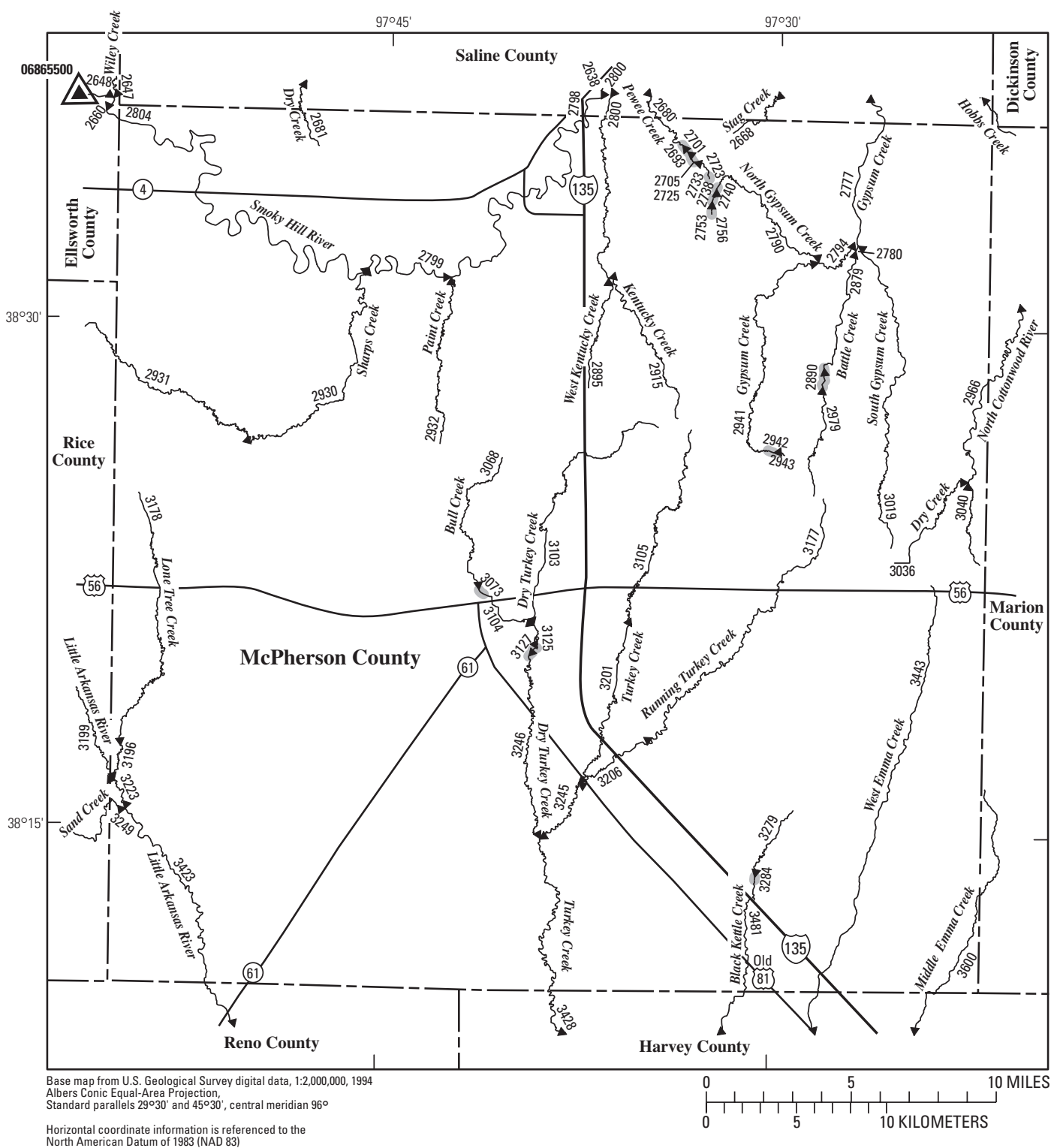

EXPLANATION

$\stackrel{3423}{ }$ Location of streamflow-statistics determination site (small triangle) and associated identification number-small triangle points in downstream direction

06865500 U.S. Geological Survey streamflow-gaging station and number used for estimates of flow duration

${ }^{06865500} \triangle$ U.S. Geological Survey streamflow-gaging station and number used for estimates of peak-discharge frequency values

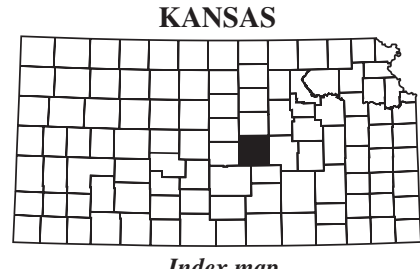

Index map

Lake and determination site identification number

Figure 69. Location of streamflow-statistics determination sites, associated identification numbers, and U.S. Geological Survey streamflow-gaging stations used in the flow-duration and peak-discharge frequency analyses for McPherson County. 
Table 65. Estimated flow-duration values, mean flow values, and peak-discharge frequency values for controlled and uncontrolled flow stream segments on the 1999 Kansas Surface Water Register for McPherson County.

[KSWR, Kansas Surface Water Register; CUSEGA, catalog unit segment number alpha; $\mathrm{mi}^{2}$, square miles; $\mathrm{ft}^{3} / \mathrm{s}$, cubic feet per second; HYDRO, lake or other hydrologic structure; NA, not applicable; NRDitch, irrigation ditch; NRTribal, tribal stream]

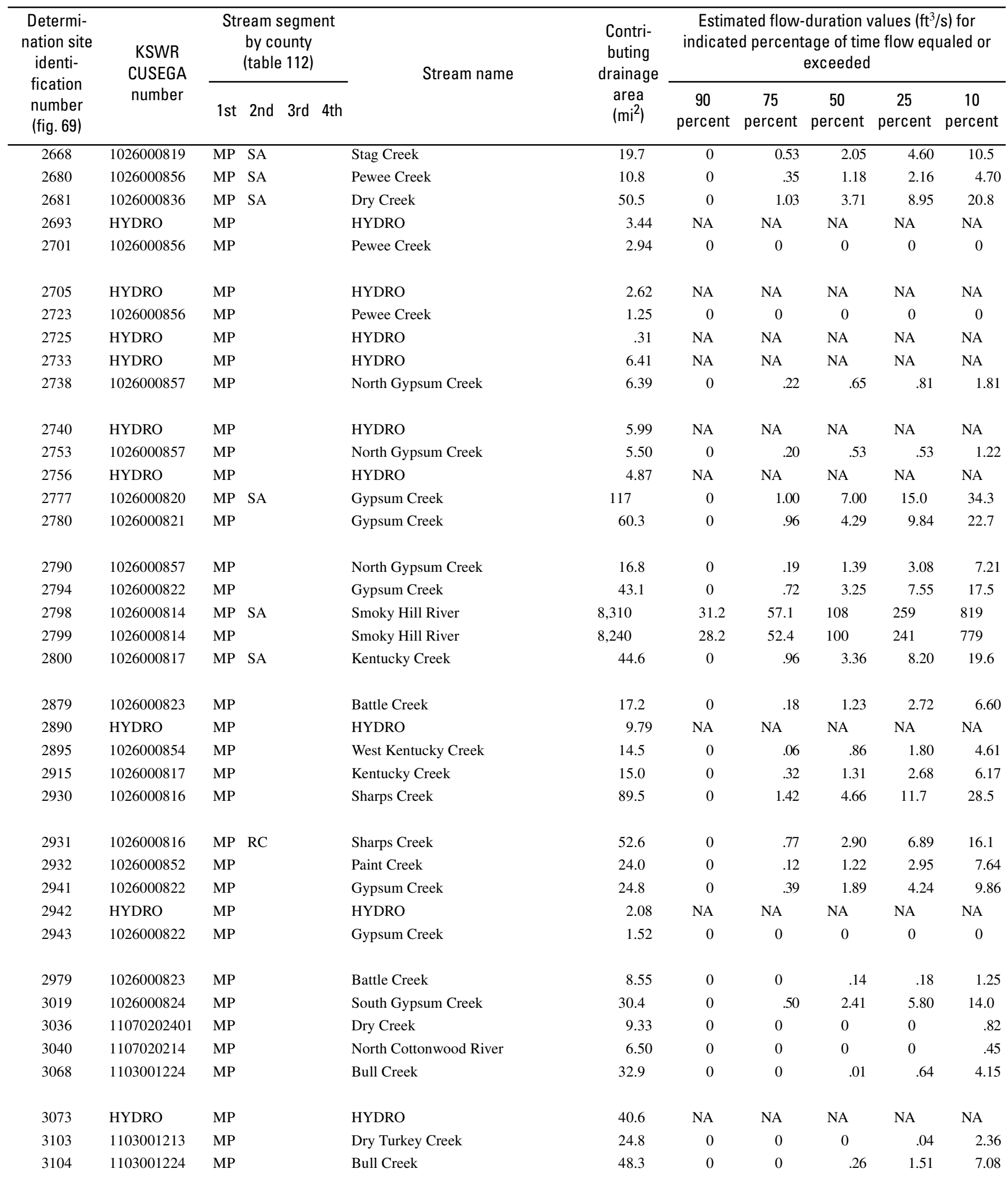


Table 65. Estimated flow-duration values, mean flow values, and peak-discharge frequency values for controlled and uncontrolled flow stream segments on the 1999 Kansas Surface Water Register for McPherson County.-Continued

[KSWR, Kansas Surface Water Register; CUSEGA, catalog unit segment number alpha; $\mathrm{mi}^{2}$, square miles; $\mathrm{ft}^{3} / \mathrm{s}$, cubic feet per second; HYDRO, lake or other hydrologic structure; NA, not applicable; NRDitch, irrigation ditch; NRTribal, tribal stream]

\begin{tabular}{|c|c|c|c|c|c|c|c|}
\hline \multirow{2}{*}{$\begin{array}{l}\text { Determi- } \\
\text { nation site } \\
\text { identi- } \\
\text { fication } \\
\text { number } \\
\text { (fig. 69) }\end{array}$} & \multirow{2}{*}{$\begin{array}{c}\text { Estimated mean } \\
\text { flow } \\
\left(\mathrm{ft}^{3} / \mathrm{s}\right)\end{array}$} & \multicolumn{6}{|c|}{ Estimated peak discharge $\left(\mathrm{ft}^{3} / \mathrm{s}\right)$ for indicated peak-discharge frequency } \\
\hline & & 2-year & 5-year & 10-year & 25-year & 50-year & 100-year \\
\hline 2668 & 7.99 & 1,110 & 2,690 & 4,160 & 6,460 & 8,440 & 10,700 \\
\hline 2693 & NA & NA & NA & NA & NA & NA & NA \\
\hline 2701 & .51 & 352 & 830 & 1,260 & 1,930 & 2,490 & 3,140 \\
\hline 2705 & NA & NA & NA & NA & NA & NA & NA \\
\hline 2723 & 0 & 215 & 498 & 748 & 1,130 & 1,460 & 1,830 \\
\hline 2740 & NA & NA & NA & NA & NA & NA & NA \\
\hline 2753 & 1.66 & 506 & 1,210 & 1,840 & 2,830 & 3,680 & 4,660 \\
\hline 2756 & NA & NA & NA & NA & NA & NA & NA \\
\hline 2777 & 25.9 & 2,290 & 4,310 & 5,960 & 8,400 & 10,500 & 12,700 \\
\hline 2780 & 17.1 & 2,120 & 4,490 & 6,570 & 9,720 & 12,500 & 15,400 \\
\hline 2790 & 5.97 & 966 & 2,340 & 3,600 & 5,600 & 7,320 & 9,310 \\
\hline 2794 & 13.3 & 1,730 & 3,820 & 5,700 & 8,570 & 11,100 & 13,800 \\
\hline 2798 & 328 & 3,320 & 6,430 & 8,070 & 13,200 & 18,700 & 26,000 \\
\hline 2799 & 311 & 2,990 & 5,980 & 7,840 & 12,700 & 17,700 & 24,200 \\
\hline 2915 & 5.24 & 902 & 2,200 & 3,400 & 5,300 & 6,930 & 8,820 \\
\hline 2930 & 21.6 & 1,810 & 4,290 & 6,650 & 10,400 & 13,700 & 17,400 \\
\hline 2931 & 12.6 & 1,410 & 3,380 & 5,240 & 8,160 & 10,800 & 13,700 \\
\hline 2932 & 6.95 & 1,140 & 2,840 & 4,440 & 6,970 & 9,160 & 11,700 \\
\hline 2941 & 8.08 & 1,210 & 2,930 & 4,530 & 7,060 & 9,230 & 11,800 \\
\hline 2942 & NA & NA & NA & NA & NA & NA & NA \\
\hline 2943 & 0 & 248 & 572 & 859 & 1,300 & 1,670 & 2,100 \\
\hline 2979 & 2.26 & 678 & 1,610 & 2,460 & 3,800 & 4,930 & 6,240 \\
\hline 3019 & 11.2 & 1,780 & 3,870 & 5,730 & 8,540 & 11,000 & 13,600 \\
\hline 3036 & 2.35 & 789 & 1,840 & 2,790 & 4,270 & 5,530 & 6,970 \\
\hline 3040 & 1.77 & 658 & 1,520 & 2,280 & 3,460 & 4,470 & 5,610 \\
\hline 3068 & 6.09 & 721 & 1,960 & 3,240 & 5,350 & 7,310 & 9,580 \\
\hline 3073 & NA & NA & NA & NA & NA & NA & NA \\
\hline 3103 & 4.46 & 1,190 & 2,950 & 4,590 & 7,200 & 9,450 & 12,100 \\
\hline 3104 & 8.96 & 858 & 2,320 & 3,830 & 6,320 & 8,640 & 11,300 \\
\hline
\end{tabular}


Table 65. Estimated flow-duration values, mean flow values, and peak-discharge frequency values for controlled and uncontrolled flow stream segments on the 1999 Kansas Surface Water Register for McPherson County.-Continued

[KSWR, Kansas Surface Water Register; CUSEGA, catalog unit segment number alpha; $\mathrm{mi}^{2}$, square miles; $\mathrm{ft}^{3} / \mathrm{s}$, cubic feet per second; HYDRO, lake or other hydrologic structure; NA, not applicable; NRDitch, irrigation ditch; NRTribal, tribal stream]

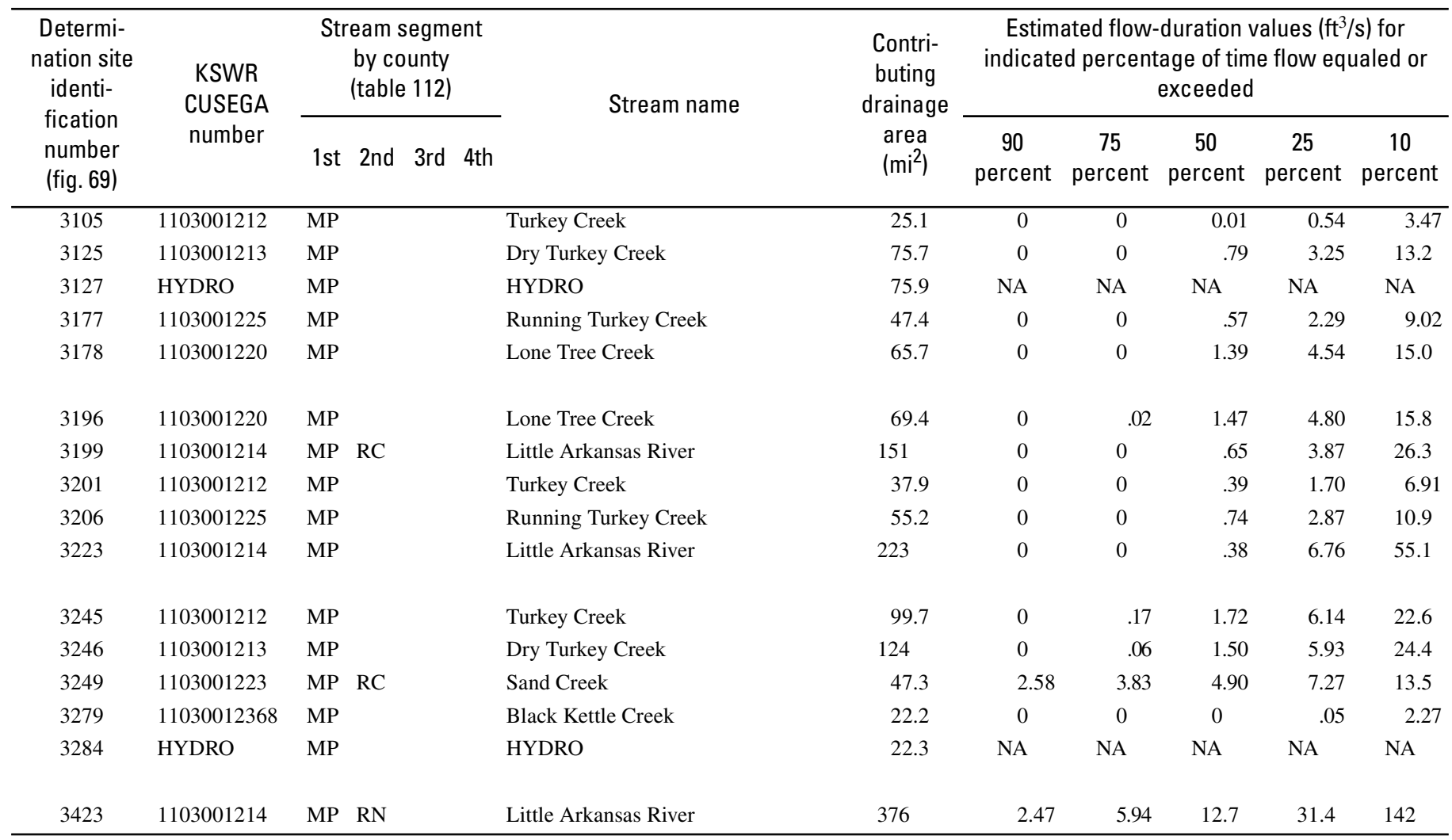


Table 65. Estimated flow-duration values, mean flow values, and peak-discharge frequency values for controlled and uncontrolled flow stream segments on the 1999 Kansas Surface Water Register for McPherson County.-Continued

[KSWR, Kansas Surface Water Register; CUSEGA, catalog unit segment number alpha; $\mathrm{mi}^{2}$, square miles; $\mathrm{ft}^{3} / \mathrm{s}$, cubic feet per second; HYDRO, lake or other hydrologic structure; NA, not applicable; NRDitch, irrigation ditch; NRTribal, tribal stream]

\begin{tabular}{|c|c|c|c|c|c|c|c|}
\hline \multirow{2}{*}{$\begin{array}{c}\text { Determi- } \\
\text { nation site } \\
\text { identi- } \\
\text { fication } \\
\text { number } \\
\text { (fig. 69) }\end{array}$} & \multirow{2}{*}{$\begin{array}{c}\text { Estimated mean } \\
\text { flow } \\
\left(\mathrm{ft}^{3} / \mathrm{s}\right)\end{array}$} & \multicolumn{6}{|c|}{ Estimated peak discharge $\left(\mathrm{ft}^{3} / \mathrm{s}\right)$ for indicated peak-discharge frequency } \\
\hline & & 2-year & 5-year & 10-year & 25 -year & 50-year & 100 -year \\
\hline 3105 & 5.19 & 1,220 & 3,020 & 4,680 & 7,340 & 9,630 & 12,300 \\
\hline 3125 & 14.5 & 1,350 & 3,460 & 5,560 & 8,980 & 12,100 & 15,700 \\
\hline 3127 & NA & NA & NA & NA & NA & NA & NA \\
\hline 3177 & 10.3 & 1,220 & 3,070 & 4,870 & 7,770 & 10,400 & 13,400 \\
\hline 3178 & 14.4 & 1,610 & 3,950 & 6,200 & 9,790 & 13,000 & 16,700 \\
\hline 3196 & 15.1 & 1,610 & 3,970 & 6,240 & 9,890 & 13,200 & 16,900 \\
\hline 3199 & 25.1 & 1,640 & 3,530 & 5,270 & 8,060 & 10,600 & 13,500 \\
\hline 3201 & 8.23 & 835 & 2,230 & 3,640 & 5,970 & 8,110 & 10,600 \\
\hline 3206 & 12.1 & 1,300 & 3,260 & 5,170 & 8,260 & 11,100 & 14,300 \\
\hline 3223 & 48.1 & 2,370 & 5,390 & 8,240 & 12,900 & 17,100 & 22,000 \\
\hline 3245 & 22.1 & 1,550 & 3,940 & 6,310 & 10,200 & 13,700 & 17,800 \\
\hline 3246 & 24.4 & 1,570 & 4,060 & 6,570 & 10,700 & 14,500 & 19,000 \\
\hline 3249 & 10.4 & 958 & 2,140 & 3,200 & 4,790 & 6,150 & 7,630 \\
\hline 3279 & 4.37 & 1,210 & 2,930 & 4,520 & 7,030 & 9,180 & 11,700 \\
\hline 3284 & NA & NA & NA & NA & NA & NA & NA \\
\hline 3423 & 103 & 2,920 & 6,650 & 10,100 & 15,800 & 20,900 & 26,800 \\
\hline
\end{tabular}




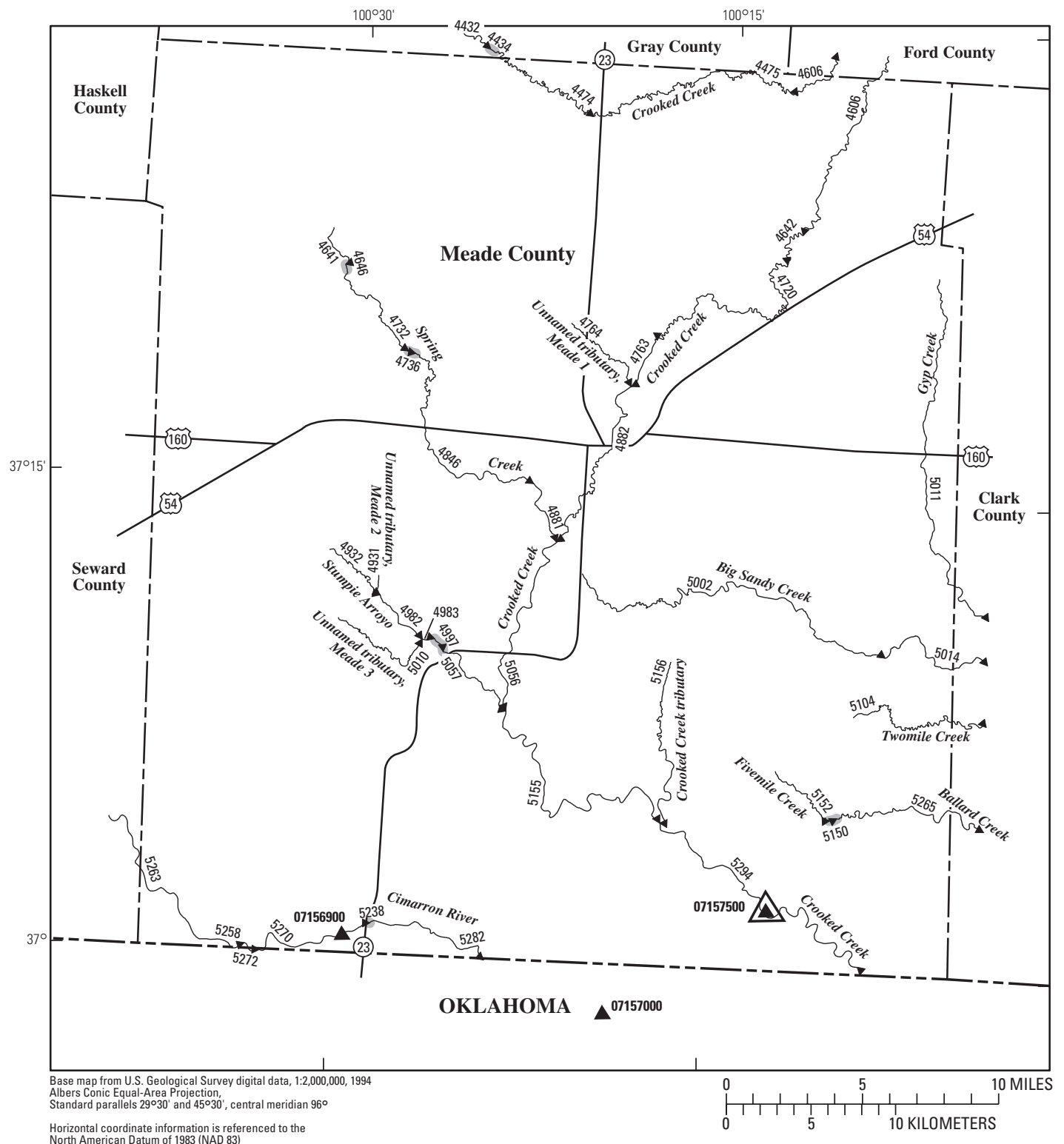

EXPLANATION

5258 Location of streamflow-statistics determination site (small triangle) and associated identification number-small triangle points in downstream direction

07157000

A U.S. Geological Survey streamflow-gaging station and number used for estimates of flow duration

07157500 U.S. Geological Survey streamflow-gaging station and number used for estimates of peak-discharge frequency values

KANSAS

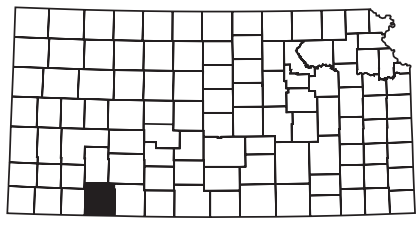

Index map 5238

Lake and determination site identification number

Figure 70. Location of streamflow-statistics determination sites, associated identification numbers, and U.S. Geological Survey streamflow-gaging stations used in the flow-duration and peak-discharge frequency analyses for Meade County. 
Table 66. Estimated flow-duration values, mean flow values, and peak-discharge frequency values for controlled and uncontrolled flow stream segments on the 1999 Kansas Surface Water Register for Meade County.

[KSWR, Kansas Surface Water Register; CUSEGA, catalog unit segment number alpha; $\mathrm{mi}^{2}$, square miles; $\mathrm{ft}^{3} / \mathrm{s}$, cubic feet per second; HYDRO, lake or other hydrologic structure; NA, not applicable; NRDitch, irrigation ditch; NRTribal, tribal stream]

\begin{tabular}{|c|c|c|c|c|c|c|c|c|c|c|c|}
\hline $\begin{array}{l}\text { Determi- } \\
\text { nation site } \\
\text { identi- } \\
\text { fication } \\
\text { number } \\
\text { (fig. 70) }\end{array}$ & $\begin{array}{l}\text { KSWR } \\
\text { CUSEGA } \\
\text { number }\end{array}$ & \multicolumn{3}{|c|}{$\begin{array}{l}\text { Stream segment } \\
\text { by county } \\
\text { (table 112) }\end{array}$} & Stream name & $\begin{array}{l}\text { Contri- } \\
\text { buting } \\
\text { drainage } \\
\text { area } \\
\left(\mathrm{mi}^{2}\right)\end{array}$ & \multicolumn{5}{|c|}{$\begin{array}{c}\text { Estimated flow-duration values }\left(\mathrm{ft}^{3} / \mathrm{s}\right) \text { for } \\
\text { indicated percentage of time flow equaled or } \\
\text { exceeded }\end{array}$} \\
\hline 4641 & 110400073 & $\mathrm{ME}$ & & & Spring Creek & 117 & 0 & 0 & 0 & 0.03 & 0.06 \\
\hline 4720 & 110400072 & ME & & & Crooked Creek & 814 & 67 & 1.64 & 3.44 & 5.28 & 11.0 \\
\hline 4732 & 110400073 & ME & & & Spring Creek & 168 & 0 & 0 & .05 & .06 & 12 \\
\hline 4736 & HYDRO & ME & & & HYDRO & 169 & NA & NA & NA & NA & NA \\
\hline 4763 & 110400072 & ME & & & Crooked Creek & 821 & 68 & 1.68 & 3.51 & 5.38 & 11.1 \\
\hline 4882 & 110400072 & ME & & & Crooked Creek & 880 & .78 & 2.15 & 4.26 & 6.66 & 13.6 \\
\hline 4931 & 110400071253 & ME & & & Unnamed tributary, Meade 2 & 5.03 & 0 & 0 & 0 & 0 & 0 \\
\hline 4932 & 110400071247 & ME & & & Stumpie Arroyo & 59.2 & 0 & .01 & .02 & .04 & .08 \\
\hline 4982 & 110400071247 & ME & & & Stumpie Arroyo & 68.7 & 0 & 0 & 0 & .01 & .02 \\
\hline 4983 & 110400071247 & ME & & & Stumpie Arroyo & 123 & 0 & 0 & 0 & .03 & .06 \\
\hline 4997 & HYDRO & ME & & & HYDRO & 124 & NA & NA & NA & NA & NA \\
\hline 5002 & 110400089 & ME & & & Big Sandy Creek & 66.8 & 0 & 0 & 0 & .01 & .21 \\
\hline 5010 & 110400071259 & ME & & & Unnamed tributary, Meade 3 & 52.0 & 0 & .01 & .02 & .04 & .08 \\
\hline 5056 & 110400071 & ME & & & Crooked Creek & 1,120 & 1.27 & 3.85 & 6.87 & 10.2 & 19.6 \\
\hline 5258 & 110400061 & ME & & & Cimarron River & 6,750 & 25.6 & 33.2 & 42.5 & 58.1 & 75.9 \\
\hline 5263 & 110400062 & ME & SW & & Cimarron River & 6,750 & 25.6 & 33.2 & 42.5 & 58.1 & 75.9 \\
\hline 5270 & 110400061 & ME & & & Cimarron River & 6,890 & 27.0 & 35.0 & 45.0 & 62.0 & 82.0 \\
\hline 5272 & 110400061 & ME & & & Cimarron River & 6,750 & 25.6 & 33.2 & 42.6 & 58.2 & 75.9 \\
\hline 5282 & 110400061 & ME & & & Cimarron River & 6,930 & 27.5 & 38.0 & 51.0 & 73.7 & 109 \\
\hline 5294 & 110400071 & ME & & & Crooked Creek & 1,370 & 2.40 & 7.10 & 12.0 & 18.0 & 33.0 \\
\hline
\end{tabular}


Table 66. Estimated flow-duration values, mean flow values, and peak-discharge frequency values for controlled and uncontrolled flow stream segments on the 1999 Kansas Surface Water Register for Meade County.-Continued

[KSWR, Kansas Surface Water Register; CUSEGA, catalog unit segment number alpha; $\mathrm{mi}^{2}$, square miles; $\mathrm{ft}^{3} / \mathrm{s}$, cubic feet per second; HYDRO, lake or other hydrologic structure; NA, not applicable; NRDitch, irrigation ditch; NRTribal, tribal stream]

\begin{tabular}{|c|c|c|c|c|c|c|c|}
\hline \multirow{2}{*}{$\begin{array}{l}\text { Determi- } \\
\text { nation site } \\
\text { identi- } \\
\text { fication } \\
\text { number } \\
\text { (fig. } 70 \text { ) }\end{array}$} & \multirow{2}{*}{$\begin{array}{c}\text { Estimated mean } \\
\text { flow } \\
\left(\mathrm{ft}^{3} / \mathrm{s}\right)\end{array}$} & \multicolumn{6}{|c|}{ Estimated peak discharge $\left(\mathrm{ft}^{3} / \mathrm{s}\right)$ for indicated peak-discharge frequency } \\
\hline & & 2-year & 5-year & 10-year & 25-year & 50-year & 100-year \\
\hline 4641 & 0.50 & 318 & 1,170 & 2,210 & 4,190 & 6,240 & 8,810 \\
\hline 4720 & 14.1 & 474 & 1,900 & 3,590 & 6,690 & 9,780 & 13,500 \\
\hline 4732 & 1.51 & 664 & 2,160 & 3,870 & 6,970 & 10,100 & 13,800 \\
\hline 4736 & NA & NA & NA & NA & NA & NA & NA \\
\hline 4763 & 14.3 & 470 & 1,900 & 3,600 & 6,710 & 9,810 & 13,500 \\
\hline 4882 & 16.3 & 501 & 2,030 & 3,860 & 7,220 & 10,600 & 14,600 \\
\hline 4931 & 0 & 182 & 549 & 928 & 1,570 & 2,150 & 2,840 \\
\hline 4932 & .08 & 711 & 2,070 & 3,510 & 5,970 & 8,310 & 11,000 \\
\hline 4982 & .58 & 742 & 2,130 & 3,590 & 6,060 & 8,410 & 11,100 \\
\hline 4983 & 2.51 & 919 & 2,440 & 3,950 & 6,430 & 8,720 & 11,300 \\
\hline 4997 & NA & NA & NA & NA & NA & NA & NA \\
\hline 5002 & 2.30 & 577 & 1,660 & 2,790 & 4,710 & 6,510 & 8,610 \\
\hline 5010 & .55 & 499 & 1,340 & 2,160 & 3,510 & 4,730 & 6,100 \\
\hline 5056 & 21.6 & 697 & 2,750 & 5,210 & 9,770 & 14,400 & 19,900 \\
\hline 5258 & 54.7 & 2,100 & 6,100 & 10,300 & 17,600 & 24,400 & 32,600 \\
\hline 5263 & 54.7 & 2,100 & 6,100 & 10,300 & 17,600 & 24,400 & 32,600 \\
\hline 5270 & 58.4 & 2,170 & 6,260 & 10,600 & 18,000 & 24,900 & 33,300 \\
\hline 5272 & 54.7 & 2,100 & 6,100 & 10,300 & 17,600 & 24,400 & 32,600 \\
\hline 5282 & 80.2 & 2,170 & 6,270 & 10,600 & 18,000 & 24,900 & 33,300 \\
\hline 5294 & 30.5 & 992 & 3,600 & 6,650 & 12,200 & 17,800 & 24,400 \\
\hline
\end{tabular}




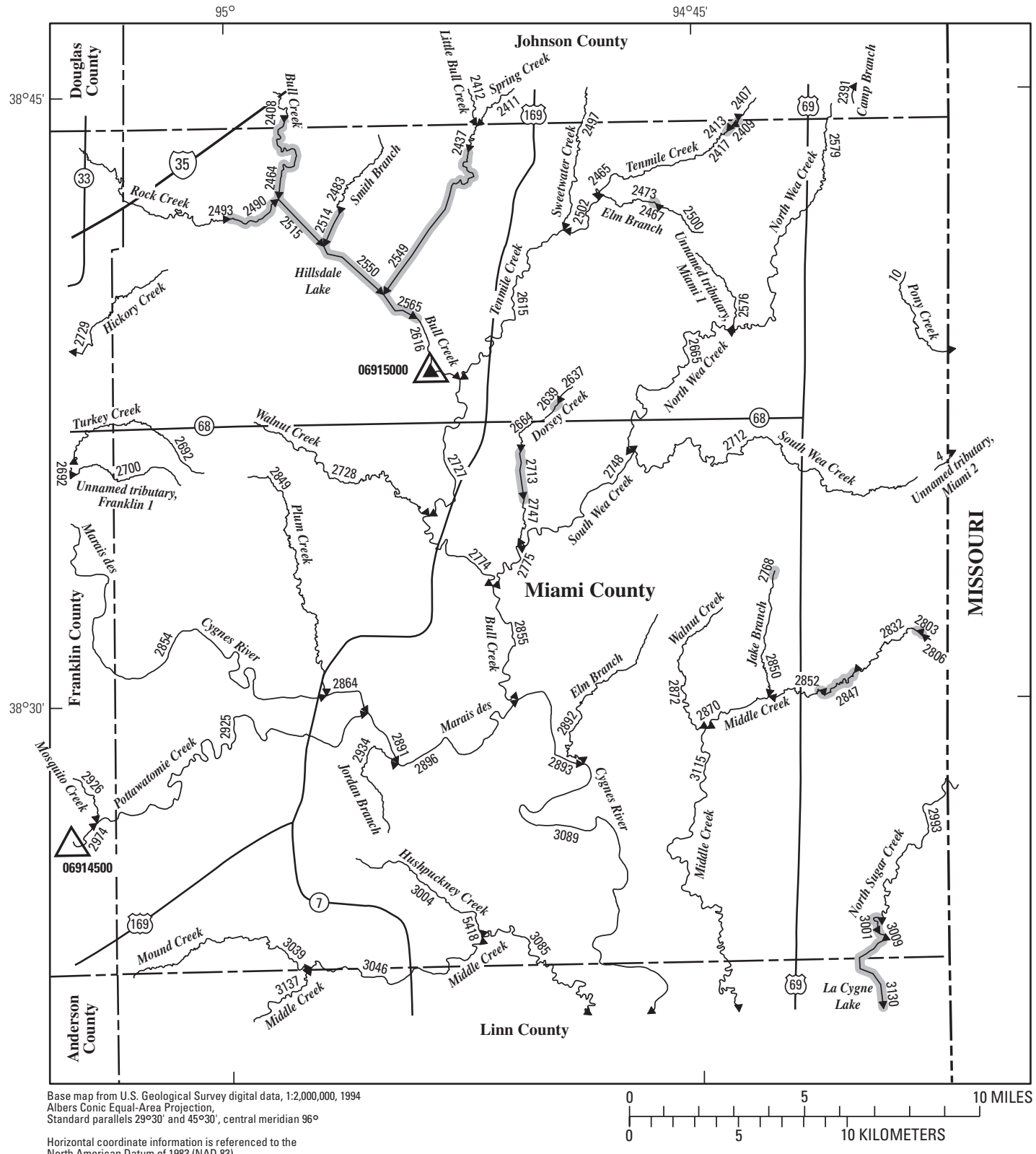

tion is referenced to the

EXPLANATION

3039 Location of streamflow-statistics determination site (small triangle) and associated identification number-small triangle points in downstream direction

$06915000 \Delta$ U.S. Geological Survey streamflow-gaging station and number used for estimates of flow duration

06914500

U.S. Geological Survey streamflow-gaging station and number used for estimates of peak-discharge frequency values

KANSAS

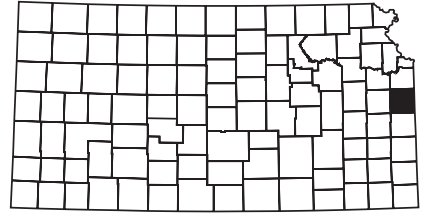

Index map 3130

Lake and determination site identification number

Figure 71. Location of streamflow-statistics determination sites, associated identification numbers, and U.S. Geological Survey streamflow-gaging stations used in the flow-duration and peak-discharge frequency analyses for Miami County. 
Table 67. Estimated flow-duration values, mean flow values, and peak-discharge frequency values for controlled and uncontrolled flow stream segments on the 1999 Kansas Surface Water Register for Miami County.

[KSWR, Kansas Surface Water Register; CUSEGA, catalog unit segment number alpha; $\mathrm{mi}^{2}$, square miles; $\mathrm{ft}^{3} / \mathrm{s}$, cubic feet per second; HYDRO, lake or other hydrologic structure; NA, not applicable; NRDitch, irrigation ditch; NRTribal, tribal stream]

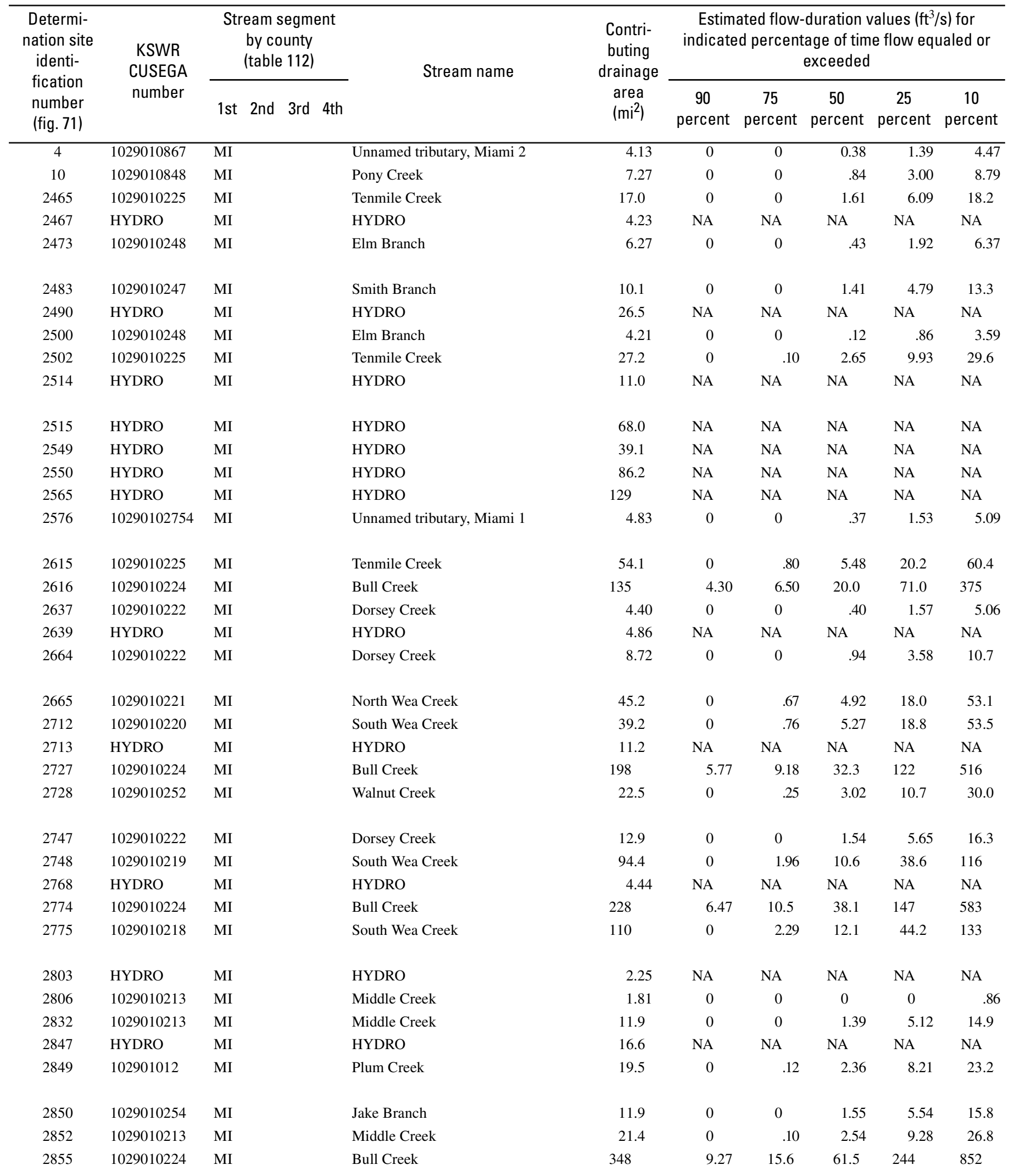


Table 67. Estimated flow-duration values, mean flow values, and peak-discharge frequency values for controlled and uncontrolled flow stream segments on the 1999 Kansas Surface Water Register for Miami County.-Continued

[KSWR, Kansas Surface Water Register; CUSEGA, catalog unit segment number alpha; $\mathrm{mi}^{2}$, square miles; $\mathrm{ft}^{3} / \mathrm{s}$, cubic feet per second; HYDRO, lake or other hydrologic structure; NA, not applicable; NRDitch, irrigation ditch; NRTribal, tribal stream]

\begin{tabular}{|c|c|c|c|c|c|c|c|}
\hline \multirow{2}{*}{$\begin{array}{l}\text { Determi- } \\
\text { nation site } \\
\text { identi- } \\
\text { fication } \\
\text { number } \\
\text { (fig. 71) }\end{array}$} & \multirow{2}{*}{$\begin{array}{c}\text { Estimated mean } \\
\text { flow } \\
\left(\mathrm{ft}^{3} / \mathrm{s}\right)\end{array}$} & \multicolumn{6}{|c|}{ Estimated peak discharge $\left(\mathrm{ft}^{3} / \mathrm{s}\right)$ for indicated peak-discharge frequency } \\
\hline & & 2-year & 5-year & 10-year & 25-year & 50-year & 100-year \\
\hline 4 & 3.98 & 927 & 1,820 & 2,560 & 3,660 & 4,550 & 5,550 \\
\hline 2467 & NA & NA & NA & NA & NA & NA & NA \\
\hline 2473 & 5.70 & 1,180 & 2,350 & 3,310 & 4,750 & 5,910 & 7,230 \\
\hline 2483 & 10.1 & 1,540 & 3,100 & 4,410 & 6,360 & 7,950 & 9,750 \\
\hline 2490 & NA & NA & NA & NA & NA & NA & NA \\
\hline 2515 & NA & NA & NA & NA & NA & NA & NA \\
\hline 2549 & NA & NA & NA & NA & NA & NA & NA \\
\hline 2550 & NA & NA & NA & NA & NA & NA & NA \\
\hline 2565 & NA & NA & NA & NA & NA & NA & NA \\
\hline 2576 & 4.57 & 1,020 & 2,020 & 2,840 & 4,050 & 5,040 & 6,150 \\
\hline 2615 & 44.4 & 6,010 & 11,000 & 15,200 & 21,100 & 26,100 & 31,300 \\
\hline 2616 & 116 & 986 & 2,140 & 3,040 & 4,230 & 5,130 & 6,020 \\
\hline 2637 & 4.44 & 1,010 & 1,970 & 2,750 & 3,910 & 4,850 & 5,910 \\
\hline 2639 & NA & NA & NA & NA & NA & NA & NA \\
\hline 2747 & 12.7 & 1,900 & 3,770 & 5,340 & 7,670 & 9,560 & 11,700 \\
\hline 2748 & 79.4 & 7,320 & 13,400 & 18,500 & 25,800 & 31,900 & 38,300 \\
\hline 2768 & NA & NA & NA & NA & NA & NA & NA \\
\hline 2774 & 193 & 1,810 & 3,140 & 4,260 & 6,190 & 7,740 & 9,390 \\
\hline 2775 & 91.1 & 7,810 & 14,300 & 19,700 & 27,400 & 33,900 & 40,800 \\
\hline 2803 & NA & NA & NA & NA & NA & NA & NA \\
\hline 2806 & 1.42 & 592 & 1,140 & 1,580 & 2,230 & 2,750 & 3,330 \\
\hline 2832 & 11.6 & 1,760 & 3,520 & 5,000 & 7,190 & 8,980 & 11,000 \\
\hline 2847 & NA & NA & NA & NA & NA & NA & NA \\
\hline 2849 & 17.5 & 2,200 & 4,510 & 6,470 & 9,420 & 11,800 & 14,600 \\
\hline 2850 & 12.0 & 1,760 & 3,520 & 4,990 & 7,170 & 8,960 & 11,000 \\
\hline 2852 & 20.2 & 2,480 & 5,020 & 7,160 & 10,400 & 13,000 & 16,000 \\
\hline 2855 & 293 & 2,880 & 4,430 & 5,830 & 8,730 & 11,100 & 13,700 \\
\hline
\end{tabular}


Table 67. Estimated flow-duration values, mean flow values, and peak-discharge frequency values for controlled and uncontrolled flow stream segments on the 1999 Kansas Surface Water Register for Miami County.-Continued

[KSWR, Kansas Surface Water Register; CUSEGA, catalog unit segment number alpha; $\mathrm{mi}^{2}$, square miles; $\mathrm{ft}^{3} / \mathrm{s}$, cubic feet per second; HYDRO, lake or other hydrologic structure; NA, not applicable; NRDitch, irrigation ditch; NRTribal, tribal stream]

\begin{tabular}{|c|c|c|c|c|c|c|c|c|c|c|}
\hline $\begin{array}{l}\text { Determi- } \\
\text { nation site } \\
\text { identi- } \\
\text { fication } \\
\text { number } \\
\text { (fig. 71) }\end{array}$ & $\begin{array}{l}\text { KSWR } \\
\text { CUSEGA } \\
\text { number }\end{array}$ & \multicolumn{2}{|c|}{$\begin{array}{l}\text { Stream segment } \\
\text { by county } \\
\text { (table 112) }\end{array}$} & Stream name & $\begin{array}{l}\text { Contri- } \\
\text { buting } \\
\text { drainage } \\
\text { area } \\
\left(\mathrm{mi}^{2}\right)\end{array}$ & \multicolumn{5}{|c|}{$\begin{array}{c}\text { Estimated flow-duration values }\left(\mathrm{ft}^{3} / \mathrm{s}\right) \text { for } \\
\text { indicated percentage of time flow equaled or } \\
\text { exceeded }\end{array}$} \\
\hline 2864 & 102901011 & MI & & Marais des Cygnes River & 1,620 & 46.2 & 67.5 & 241 & 1,140 & 3,480 \\
\hline 2891 & 1029010229 & MI & & Marais des Cygnes River & 2,180 & 55.6 & 92.3 & 383 & 1,660 & 4,840 \\
\hline 2892 & 1029010253 & MI & & Elm Branch & 14.2 & 0 & .07 & 2.10 & 7.21 & 19.9 \\
\hline 2893 & 1029010216 & MI & & Marais des Cygnes River & 2,550 & 61.8 & 109 & 478 & 2,010 & 5,740 \\
\hline 2896 & 1029010216 & MI & & Marais des Cygnes River & 2,190 & 55.9 & 93.0 & 388 & 1,680 & 4,880 \\
\hline 3004 & 1029010237 & MI & & Hushpuckney Creek & 15.5 & 0 & .23 & 2.45 & 7.96 & 21.4 \\
\hline 3009 & 1029010210 & MI & & North Sugar Creek & 33.5 & 0 & .48 & 4.18 & 15.2 & 43.8 \\
\hline 3039 & 1029010235 & MI & & Mound Creek & 23.5 & 0 & .28 & 2.92 & 10.0 & 28.2 \\
\hline 5418 & 1029010230 & MI & & Middle Creek & 83.4 & 0 & 1.89 & 9.72 & 34.2 & 100.0 \\
\hline
\end{tabular}


Table 67. Estimated flow-duration values, mean flow values, and peak-discharge frequency values for controlled and uncontrolled flow stream segments on the 1999 Kansas Surface Water Register for Miami County.-Continued

[KSWR, Kansas Surface Water Register; CUSEGA, catalog unit segment number alpha; $\mathrm{mi}^{2}$, square miles; $\mathrm{ft}^{3} / \mathrm{s}$, cubic feet per second; HYDRO, lake or other hydrologic structure; NA, not applicable; NRDitch, irrigation ditch; NRTribal, tribal stream]

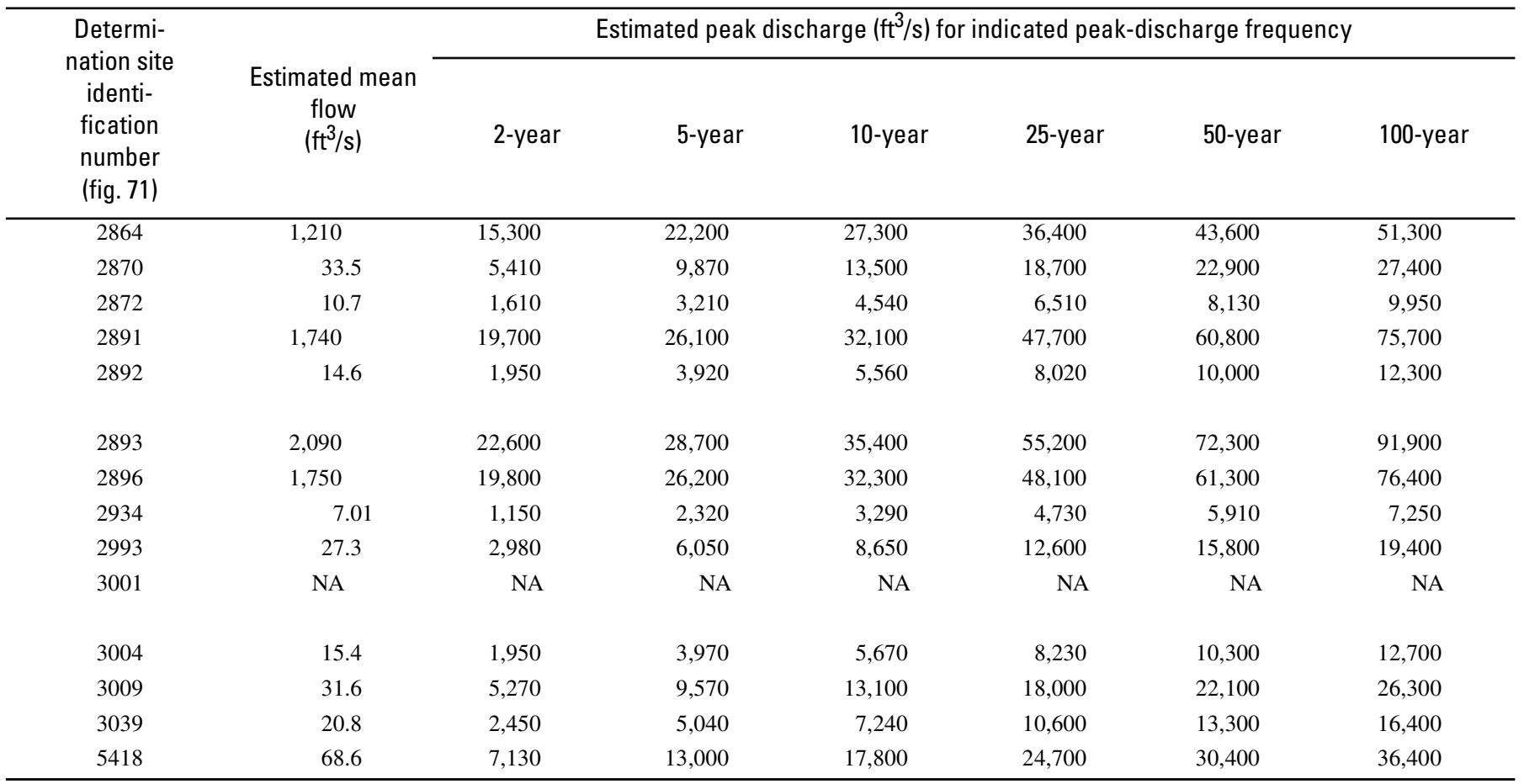




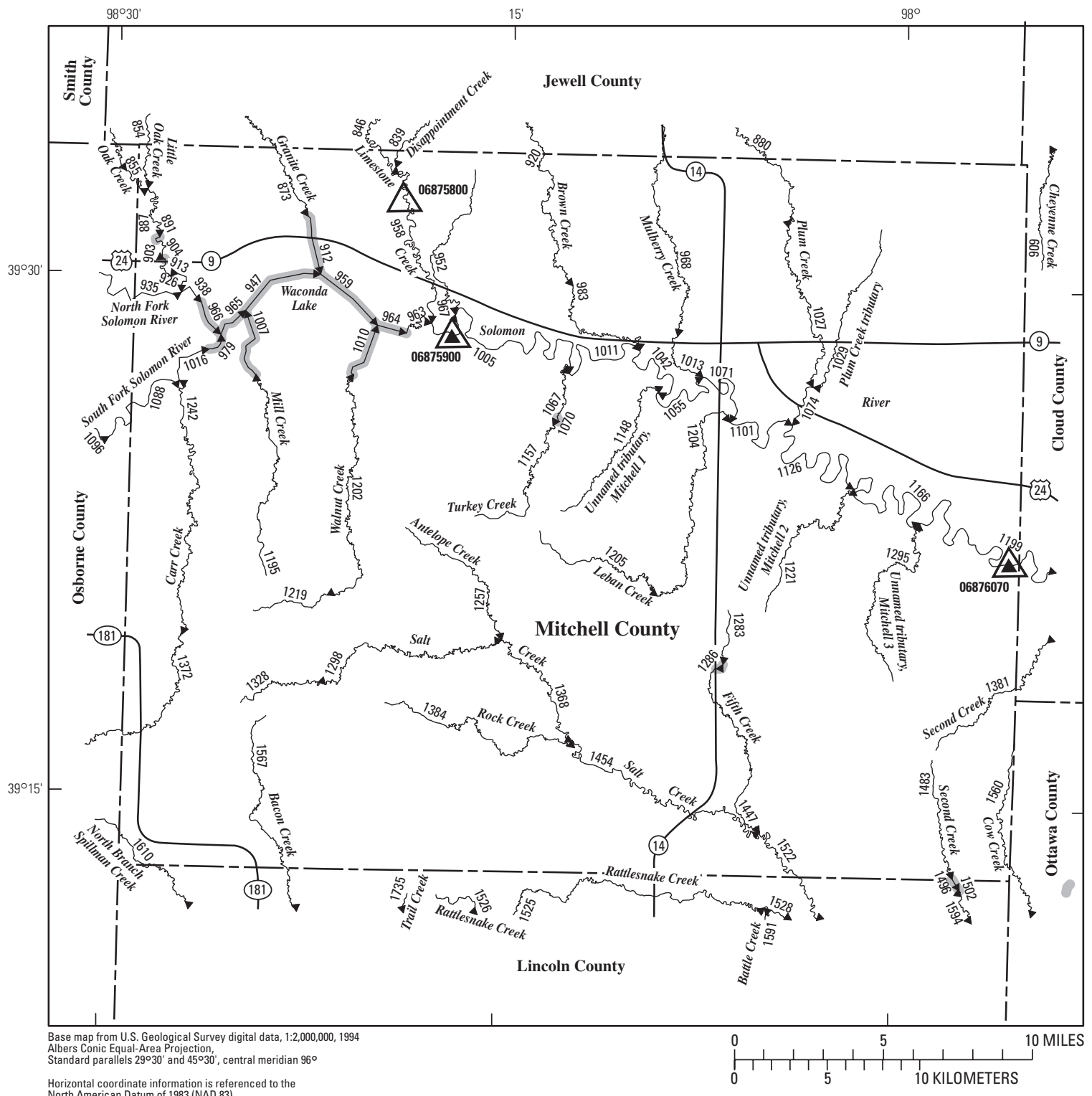

EXPLANATION

$₫ 1610$ Location of streamflow-statistics determination site (small triangle) and associated identification number-small triangle points in downstream direction

06875900 U.S. Geological Survey streamflow-gaging station and number used for estimates of flow duration

$06875800 \bigwedge$ U.S. Geological Survey streamflow-gaging station and number used for estimates of peak-discharge frequency values

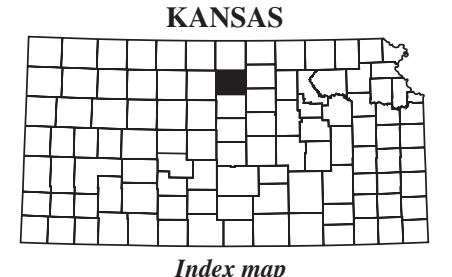

1286

Lake and determination site identification number

Figure 72. Location of streamflow-statistics determination sites, associated identification numbers, and U.S. Geological Survey streamflow-gaging stations used in the flow-duration and peak-discharge frequency analyses for Mitchell County. 
Table 68. Estimated flow-duration values, mean flow values, and peak-discharge frequency values for controlled and uncontrolled flow stream segments on the 1999 Kansas Surface Water Register for Mitchell County.

[KSWR, Kansas Surface Water Register; CUSEGA, catalog unit segment number alpha; $\mathrm{mi}^{2}$, square miles; $\mathrm{ft}^{3} / \mathrm{s}$, cubic feet per second; HYDRO, lake or other hydrologic structure; NA, not applicable; NRDitch, irrigation ditch; NRTribal, tribal stream]

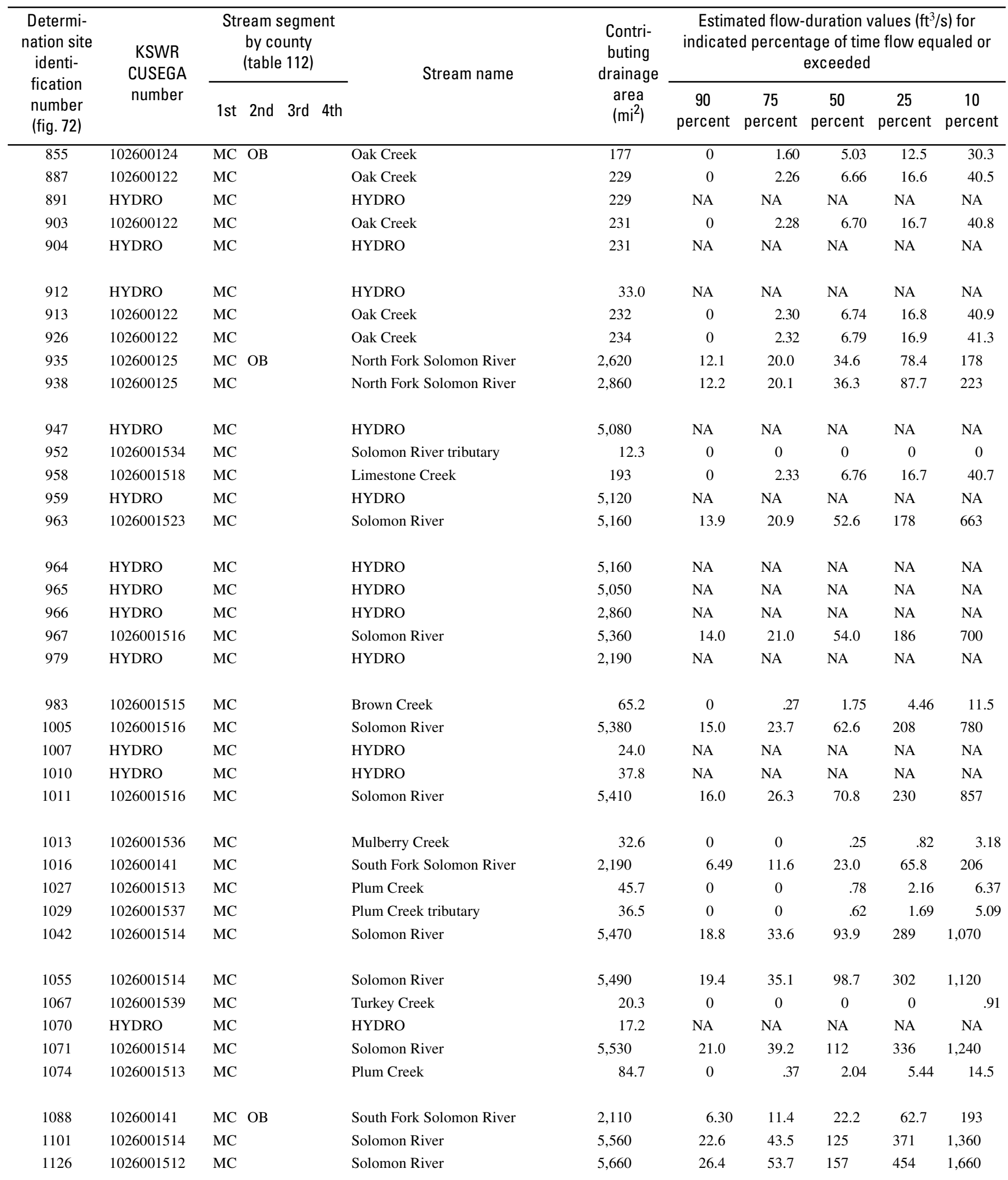


Table 68. Estimated flow-duration values, mean flow values, and peak-discharge frequency values for controlled and uncontrolled flow stream segments on the 1999 Kansas Surface Water Register for Mitchell County.-Continued

[KSWR, Kansas Surface Water Register; CUSEGA, catalog unit segment number alpha; $\mathrm{mi}^{2}$, square miles; $\mathrm{ft}^{3} / \mathrm{s}$, cubic feet per second; HYDRO, lake or other hydrologic structure; NA, not applicable; NRDitch, irrigation ditch; NRTribal, tribal stream]

\begin{tabular}{|c|c|c|c|c|c|c|c|}
\hline \multirow{2}{*}{$\begin{array}{l}\text { Determi- } \\
\text { nation site } \\
\text { identi- } \\
\text { fication } \\
\text { number } \\
\text { (fig. 72) }\end{array}$} & \multirow{2}{*}{$\begin{array}{c}\text { Estimated mean } \\
\text { flow } \\
\left(\mathrm{ft}^{3} / \mathrm{s}\right)\end{array}$} & \multicolumn{6}{|c|}{ Estimated peak discharge $\left(\mathrm{ft}^{3} / \mathrm{s}\right)$ for indicated peak-discharge frequency } \\
\hline & & 2-year & 5-year & 10-year & 25-year & 50-year & 100-year \\
\hline 855 & 23.9 & 1,570 & 4,000 & 6,430 & 10,400 & 14,000 & 18,200 \\
\hline 903 & 31.1 & 1,810 & 4,580 & 7,340 & 11,800 & 16,000 & 20,700 \\
\hline 904 & NA & NA & NA & NA & NA & NA & NA \\
\hline 912 & NA & NA & NA & NA & NA & NA & NA \\
\hline 913 & 31.2 & 1,790 & 4,550 & 7,290 & 11,800 & 15,900 & 20,600 \\
\hline 947 & NA & NA & NA & NA & $\mathrm{NA}$ & NA & NA \\
\hline 952 & 1.00 & 553 & 1,470 & 2,360 & 3,810 & 5,080 & 6,570 \\
\hline 958 & 30.7 & 1,060 & 1,910 & 2,600 & 3,590 & 4,430 & 5,340 \\
\hline 959 & NA & NA & NA & NA & NA & NA & NA \\
\hline 963 & 243 & 1,800 & 3,820 & 5,480 & 7,870 & 9,800 & 11,900 \\
\hline 964 & NA & NA & NA & NA & NA & NA & NA \\
\hline 965 & NA & NA & NA & NA & NA & NA & NA \\
\hline 966 & NA & NA & NA & NA & NA & NA & NA \\
\hline 967 & 254 & 1,720 & 3,630 & 5,170 & 7,350 & 9,080 & 10,900 \\
\hline 1013 & 4.50 & 769 & 2,030 & 3,280 & 5,320 & 7,180 & 9,300 \\
\hline 1016 & 101 & 1,420 & 4,780 & 9,060 & 18,000 & 28,300 & 42,300 \\
\hline 1027 & 7.01 & 789 & 2,120 & 3,460 & 5,670 & 7,720 & 10,100 \\
\hline 1029 & 5.82 & 1,210 & 2,950 & 4,580 & 7,150 & 9,450 & 12,000 \\
\hline 1042 & 346 & 2,130 & 4,360 & 6,020 & 8,230 & 9,900 & 11,600 \\
\hline 1055 & 357 & 2,180 & 4,440 & 6,120 & 8,340 & 9,990 & 11,600 \\
\hline 1067 & 2.45 & 743 & 2,000 & 3,220 & 5,210 & 6,970 & 9,040 \\
\hline 1070 & NA & $\mathrm{NA}$ & NA & NA & NA & NA & NA \\
\hline 1071 & 387 & 2,320 & 4,680 & 6,400 & 8,630 & 10,300 & 11,900 \\
\hline 1074 & 13.4 & 1,160 & 3,020 & 4,880 & 7,910 & 10,700 & 13,900 \\
\hline 1088 & 97.3 & 1,410 & 4,800 & 9,160 & 18,300 & 28,700 & 43,100 \\
\hline 1101 & 418 & 2,460 & 4,920 & 6,680 & 8,920 & 10,500 & 12,100 \\
\hline 1126 & 492 & 2,790 & 5,510 & 7,370 & 9,640 & 11,200 & 12,600 \\
\hline
\end{tabular}


Table 68. Estimated flow-duration values, mean flow values, and peak-discharge frequency values for controlled and uncontrolled flow stream segments on the 1999 Kansas Surface Water Register for Mitchell County.-Continued

[KSWR, Kansas Surface Water Register; CUSEGA, catalog unit segment number alpha; $\mathrm{mi}^{2}$, square miles; $\mathrm{ft}^{3} / \mathrm{s}$, cubic feet per second; HYDRO, lake or other hydrologic structure; NA, not applicable; NRDitch, irrigation ditch; NRTribal, tribal stream]

\begin{tabular}{|c|c|c|c|c|c|c|c|c|c|c|c|}
\hline $\begin{array}{l}\text { Determi- } \\
\text { nation site } \\
\text { identi- } \\
\text { fication } \\
\text { number } \\
\text { (fig. 72) }\end{array}$ & $\begin{array}{l}\text { KSWR } \\
\text { CUSEGA } \\
\text { number }\end{array}$ & \multicolumn{3}{|c|}{$\begin{array}{l}\text { Stream segment } \\
\text { by county } \\
\text { (table 112) }\end{array}$} & Stream name & $\begin{array}{l}\text { Contri- } \\
\text { buting } \\
\text { drainage } \\
\text { area } \\
\left(\mathrm{mi}^{2}\right)\end{array}$ & \multicolumn{5}{|c|}{$\begin{array}{c}\text { Estimated flow-duration values }\left(\mathrm{ft}^{3} / \mathrm{s}\right) \text { for } \\
\text { indicated percentage of time flow equaled or } \\
\text { exceeded }\end{array}$} \\
\hline 1148 & 1026001540 & $\mathrm{MC}$ & & & Unnamed tributary, Mitchell 1 & 12.4 & 0 & 0 & 0 & 0 & 0 \\
\hline 1195 & 1026001538 & MC & & & Mill Creek & 19.4 & 0 & 0 & 0 & 0 & .28 \\
\hline 1202 & 1026001526 & MC & & & Walnut Creek & 33.1 & 0 & 0 & .33 & .99 & 3.32 \\
\hline 1204 & 1026001541 & MC & & & Leban Creek & 34.8 & 0 & 0 & .26 & .89 & 3.38 \\
\hline 1205 & 1026001541 & MC & & & Leban Creek & 16.7 & 0 & 0 & 0 & 0 & 0 \\
\hline 1257 & 1026001543 & MC & & & Antelope Creek & 15.5 & 0 & 0 & 0 & 0 & .12 \\
\hline 1283 & 1026001545 & MC & & & Fifth Creek & 5.35 & 0 & 0 & 0 & 0 & 0 \\
\hline 1286 & HYDRO & MC & & & HYDRO & 6.86 & NA & NA & NA & NA & NA \\
\hline 1295 & 1026001546 & MC & & & Unnamed tributary, Mitchell 3 & 26.9 & 0 & 0 & .49 & 1.24 & 3.72 \\
\hline 1298 & 1026001530 & MC & & & Salt Creek & 24.4 & 0 & 0 & .44 & 1.02 & 2.99 \\
\hline 1328 & 1026001530 & MC & & & Salt Creek & 9.50 & 0 & 0 & 0 & 0 & 0 \\
\hline 1368 & 1026001530 & MC & & & Salt Creek & 53.0 & 0 & .02 & 1.33 & 3.39 & 9.04 \\
\hline 1372 & 1026001421 & MC & OB & & Carr Creek & 49.8 & 0 & 0 & .93 & 2.38 & 6.18 \\
\hline 1384 & 1026001544 & MC & & & Rock Creek & 28.1 & 0 & 0 & .40 & 1.04 & 3.38 \\
\hline
\end{tabular}


Table 68. Estimated flow-duration values, mean flow values, and peak-discharge frequency values for controlled and uncontrolled flow stream segments on the 1999 Kansas Surface Water Register for Mitchell County.-Continued

[KSWR, Kansas Surface Water Register; CUSEGA, catalog unit segment number alpha; $\mathrm{mi}^{2}$, square miles; $\mathrm{ft}^{3} / \mathrm{s}$, cubic feet per second; HYDRO, lake or other hydrologic structure; NA, not applicable; NRDitch, irrigation ditch; NRTribal, tribal stream]

\begin{tabular}{|c|c|c|c|c|c|c|c|}
\hline \multirow{2}{*}{$\begin{array}{l}\text { Determi- } \\
\text { nation site } \\
\text { identi- } \\
\text { fication } \\
\text { number } \\
\text { (fig. 72) }\end{array}$} & \multirow{2}{*}{$\begin{array}{c}\text { Estimated mean } \\
\text { flow } \\
\left(\mathrm{ft}^{3} / \mathrm{s}\right)\end{array}$} & \multicolumn{6}{|c|}{ Estimated peak discharge $\left(\mathrm{ft}^{3} / \mathrm{s}\right)$ for indicated peak-discharge frequency } \\
\hline & & 2-year & 5-year & 10-year & 25-year & 50-year & 100-year \\
\hline 1148 & 1.18 & 575 & 1,520 & 2,430 & 3,900 & 5,190 & 6,700 \\
\hline 1157 & 1.86 & 671 & 1,800 & 2,890 & 4,680 & 6,250 & 8,100 \\
\hline 1166 & 513 & 2,890 & 5,670 & 7,560 & 9,850 & 11,400 & 12,800 \\
\hline 1195 & 1.97 & 684 & 1,860 & 3,010 & 4,910 & 6,580 & 8,560 \\
\hline 1202 & 4.36 & 673 & 1,830 & 2,990 & 4,920 & 6,700 & 8,730 \\
\hline 1204 & 4.68 & 621 & 1,720 & 2,850 & 4,720 & 6,470 & 8,480 \\
\hline 1205 & 1.70 & 682 & 1,810 & 2,910 & 4,690 & 6,250 & 8,090 \\
\hline 1219 & 52 & 425 & 1,130 & 1,820 & 2,940 & 3,920 & 5,060 \\
\hline 1221 & 2.18 & 702 & 1,860 & 2,970 & 4,780 & 6,370 & 8,240 \\
\hline 1242 & 9.90 & 872 & 2,360 & 3,880 & 6,400 & 8,740 & 11,400 \\
\hline 1257 & 1.74 & 630 & 1,690 & 2,710 & 4,380 & 5,860 & 7,590 \\
\hline 1283 & 0 & 357 & 924 & 1,460 & 2,320 & 3,080 & 3,950 \\
\hline 1286 & NA & NA & NA & NA & NA & NA & NA \\
\hline 1295 & 4.42 & 926 & 2,470 & 3,960 & 6,410 & 8,560 & 11,100 \\
\hline 1298 & 3.65 & 800 & 2,180 & 3,520 & 5,740 & 7,700 & 10,000 \\
\hline 1328 & 92 & 455 & 1,220 & 1,960 & 3,160 & 4,220 & 5,460 \\
\hline 1368 & 8.38 & 1,090 & 2,760 & 4,410 & 7,060 & 9,470 & 12,200 \\
\hline 1372 & 6.36 & 840 & 2,230 & 3,630 & 5,910 & 8,010 & 10,400 \\
\hline 1384 & 4.18 & 896 & 2,420 & 3,920 & 6,370 & 8,540 & 11,100 \\
\hline 1447 & 4.80 & 778 & 2,030 & 3,270 & 5,280 & 7,110 & 9,180 \\
\hline 1454 & 16.7 & 1,280 & 3,310 & 5,330 & 8,640 & 11,700 & 15,200 \\
\hline 1483 & 2.96 & 729 & 1,940 & 3,110 & 5,020 & 6,690 & 8,660 \\
\hline 1560 & 3.15 & 724 & 1,890 & 3,020 & 4,830 & 6,420 & 8,280 \\
\hline
\end{tabular}




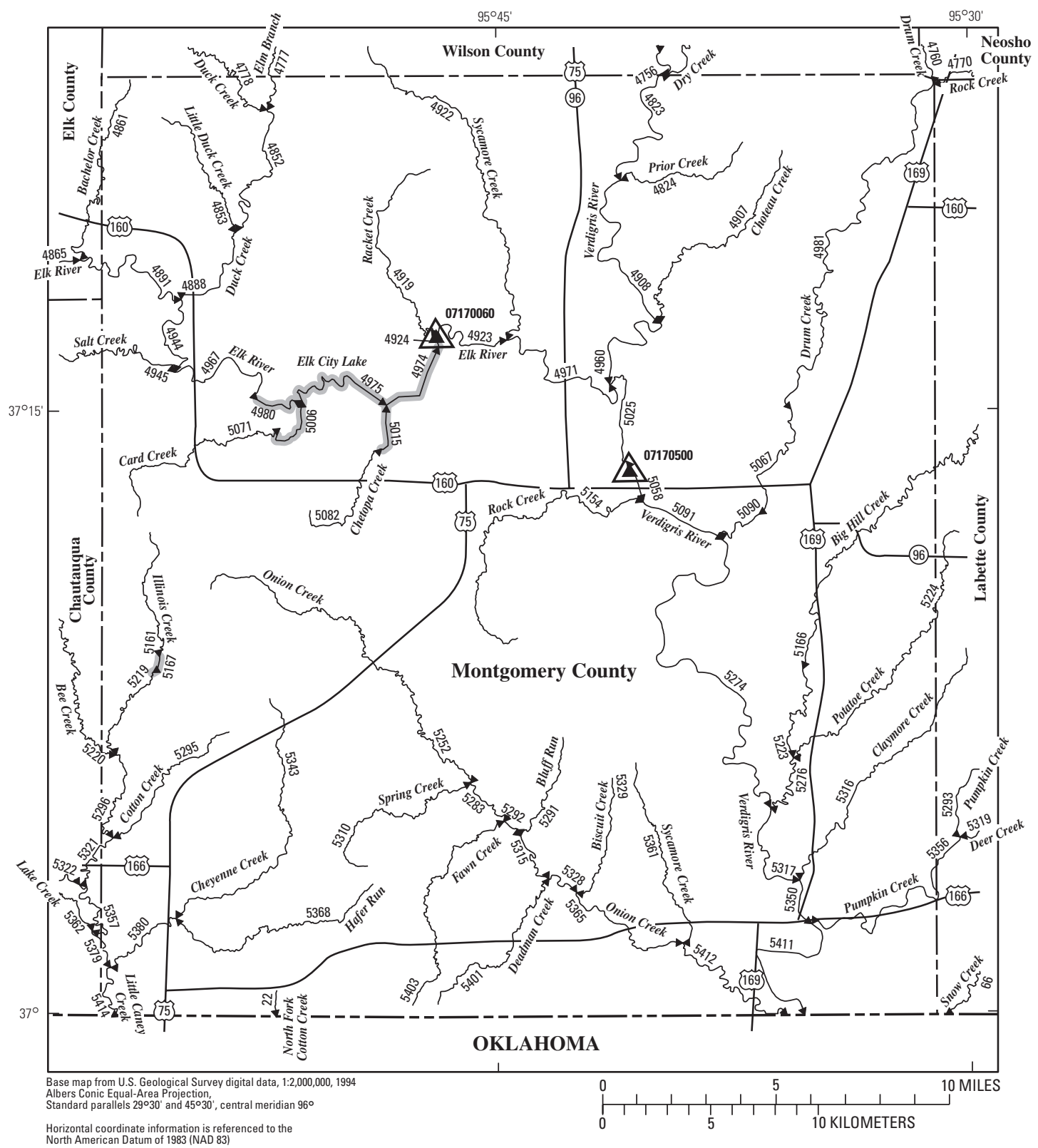

EXPLANATION

«368 Location of streamflow-statistics determination site (small triangle) and associated identification number-small triangle points in downstream direction

07170500 U.S. Geological Survey streamflow-gaging station and number used for estimates of flow duration

07170060 U.S. Geological Survey streamflow-gaging station and number used for estimates of peak-discharge frequency values

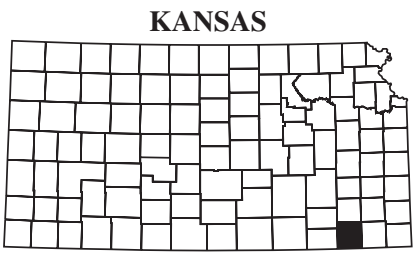

Index map 5167

Lake and determination site identification number

Figure 73. Location of streamflow-statistics determination sites, associated identification numbers, and U.S. Geological Survey streamflow-gaging stations used in the flow-duration and peak-discharge frequency analyses for Montgomery County. 
Table 69. Estimated flow-duration values, mean flow values, and peak-discharge frequency values for controlled and uncontrolled flow stream segments on the 1999 Kansas Surface Water Register for Montgomery County.

[KSWR, Kansas Surface Water Register; CUSEGA, catalog unit segment number alpha; $\mathrm{mi}^{2}$, square miles; $\mathrm{ft}^{3} / \mathrm{s}$, cubic feet per second; HYDRO, lake or other hydrologic structure; NA, not applicable; NRDitch, irrigation ditch; NRTribal, tribal stream]

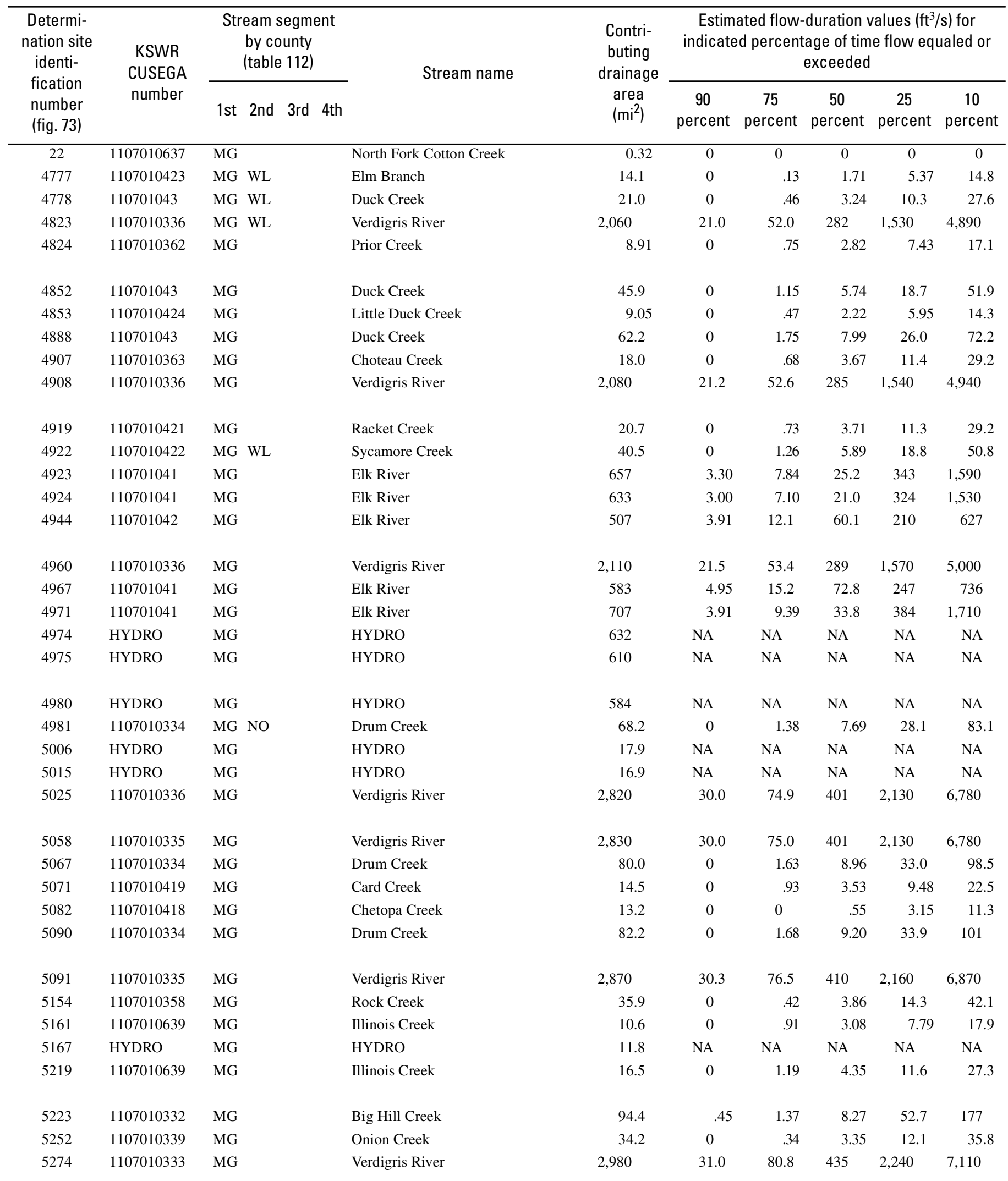


Table 69. Estimated flow-duration values, mean flow values, and peak-discharge frequency values for controlled and uncontrolled flow stream segments on the 1999 Kansas Surface Water Register for Montgomery County.-Continued

[KSWR, Kansas Surface Water Register; CUSEGA, catalog unit segment number alpha; $\mathrm{mi}^{2}$, square miles; $\mathrm{ft}^{3} / \mathrm{s}$, cubic feet per second; HYDRO, lake or other hydrologic structure; NA, not applicable; NRDitch, irrigation ditch; NRTribal, tribal stream]

\begin{tabular}{|c|c|c|c|c|c|c|c|}
\hline \multirow{2}{*}{$\begin{array}{l}\text { Determi- } \\
\text { nation site } \\
\text { identi- } \\
\text { fication } \\
\text { number } \\
\text { (fig. 73) }\end{array}$} & \multirow{2}{*}{$\begin{array}{c}\text { Estimated mean } \\
\text { flow } \\
\left(\mathrm{ft}^{3} / \mathrm{s}\right)\end{array}$} & \multicolumn{6}{|c|}{ Estimated peak discharge $\left(\mathrm{ft}^{3} / \mathrm{s}\right)$ for indicated peak-discharge frequency } \\
\hline & & 2-year & 5-year & 10-year & 25-year & 50-year & 100-year \\
\hline 22 & 0 & 188 & 361 & 499 & 699 & 862 & 1,040 \\
\hline 4823 & 1,510 & 17,900 & 24,200 & 30,300 & 41,100 & 49,600 & 55,200 \\
\hline 4824 & 11.0 & 1,530 & 3,020 & 4,250 & 6,090 & 7,580 & 9,260 \\
\hline 4852 & 36.8 & 4,160 & 7,970 & 11,200 & 15,800 & 19,700 & 23,800 \\
\hline 4853 & 9.64 & 1,360 & 2,770 & 3,960 & 5,730 & 7,190 & 8,840 \\
\hline 4919 & 20.3 & 2,370 & 4,810 & 6,880 & 9,990 & 12,500 & 15,400 \\
\hline 4922 & 35.5 & 4,930 & 8,900 & 12,100 & 16,700 & 20,400 & 24,300 \\
\hline 4923 & 478 & 4,940 & 7,400 & 8,890 & 10,600 & 11,800 & 12,800 \\
\hline 4924 & 460 & 4,740 & 7,170 & 8,600 & 10,200 & 11,200 & 12,100 \\
\hline 4944 & 346 & 11,700 & 25,900 & 39,500 & 61,900 & 82,600 & 107,000 \\
\hline 4960 & 1,540 & 18,200 & 24,400 & 30,600 & 41,700 & 50,500 & 56,200 \\
\hline 4967 & 400 & 12,500 & 27,100 & 41,100 & 64,000 & 85,100 & 110,000 \\
\hline 4971 & 515 & 5,350 & 7,870 & 9,510 & 11,600 & 13,000 & 14,100 \\
\hline 4974 & NA & NA & NA & NA & NA & NA & NA \\
\hline 5058 & 2,080 & 22,900 & 28,000 & 35,500 & 51,000 & 64,000 & 72,000 \\
\hline 5067 & 69.6 & 7,110 & 12,700 & 17,300 & 23,900 & 29,300 & 35,000 \\
\hline 5071 & 14.9 & 1,820 & 3,730 & 5,340 & 7,770 & 9,760 & 12,000 \\
\hline 5082 & 10.5 & 1,800 & 3,630 & 5,180 & 7,490 & 9,380 & 11,500 \\
\hline 5090 & 71.5 & 7,010 & 12,600 & 17,200 & 23,800 & 29,200 & 34,900 \\
\hline 5091 & 2,120 & 23,300 & 29,000 & 36,800 & 52,400 & 65,400 & 73,600 \\
\hline 5154 & 31.5 & 3,360 & 6,680 & 9,510 & 13,700 & 17,200 & 20,900 \\
\hline 5161 & 11.5 & 1,490 & 3,040 & 4,350 & 6,310 & 7,920 & 9,760 \\
\hline 5167 & NA & NA & NA & NA & NA & NA & NA \\
\hline 5219 & 17.4 & 1,910 & 3,940 & 5,670 & 8,280 & 10,400 & 12,900 \\
\hline 5223 & 63.6 & 983 & 2,150 & 3,100 & 4,370 & 5,350 & 6,310 \\
\hline 5252 & 27.4 & 3,320 & 6,620 & 9,440 & 13,600 & 17,100 & 20,900 \\
\hline 5274 & 2,200 & 24,300 & 32,000 & 40,600 & 56,300 & 69,300 & 78,200 \\
\hline
\end{tabular}


Table 69. Estimated flow-duration values, mean flow values, and peak-discharge frequency values for controlled and uncontrolled flow stream segments on the 1999 Kansas Surface Water Register for Montgomery County.-Continued

[KSWR, Kansas Surface Water Register; CUSEGA, catalog unit segment number alpha; $\mathrm{mi}^{2}$, square miles; $\mathrm{ft}^{3} / \mathrm{s}$, cubic feet per second; HYDRO, lake or other hydrologic structure; NA, not applicable; NRDitch, irrigation ditch; NRTribal, tribal stream]

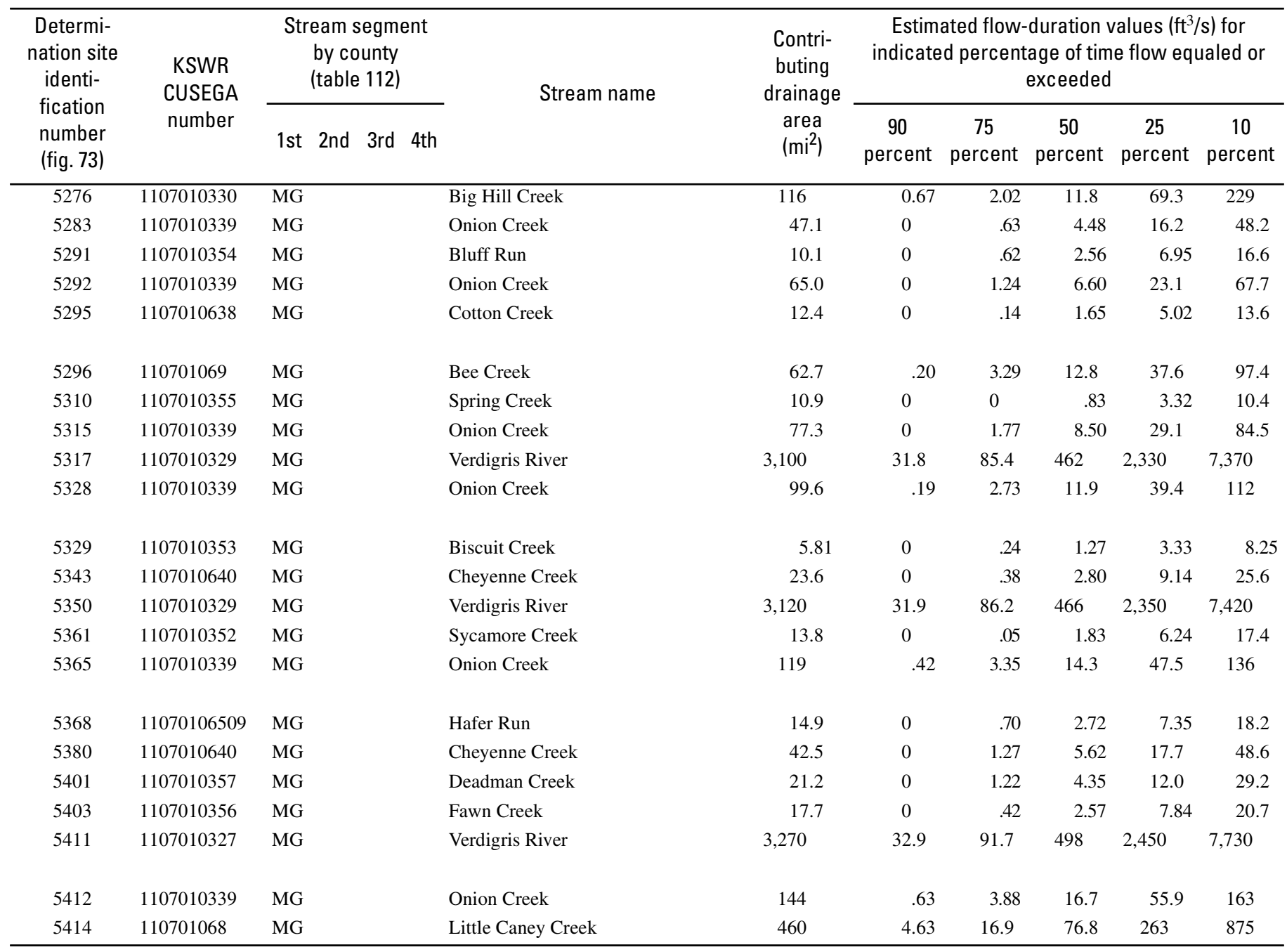


Table 69. Estimated flow-duration values, mean flow values, and peak-discharge frequency values for controlled and uncontrolled flow stream segments on the 1999 Kansas Surface Water Register for Montgomery County.-Continued

[KSWR, Kansas Surface Water Register; CUSEGA, catalog unit segment number alpha; $\mathrm{mi}^{2}$, square miles; $\mathrm{ft}^{3} / \mathrm{s}$, cubic feet per second; HYDRO, lake or other hydrologic structure; NA, not applicable; NRDitch, irrigation ditch; NRTribal, tribal stream]

\begin{tabular}{|c|c|c|c|c|c|c|c|}
\hline \multirow{2}{*}{$\begin{array}{l}\text { Determi- } \\
\text { nation site } \\
\text { identi- } \\
\text { fication } \\
\text { number } \\
\text { (fig. 73) }\end{array}$} & \multirow{2}{*}{$\begin{array}{c}\text { Estimated mean } \\
\text { flow } \\
\left(\mathrm{ft}^{3} / \mathrm{s}\right)\end{array}$} & \multicolumn{6}{|c|}{ Estimated peak discharge $\left(\mathrm{ft}^{3} / \mathrm{s}\right)$ for indicated peak-discharge frequency } \\
\hline & & 2-year & 5-year & 10-year & 25-year & 50-year & 100-year \\
\hline 5276 & 80.1 & 1,160 & 2,440 & 3,460 & 4,840 & 5,880 & 6,920 \\
\hline 5283 & 36.4 & 3,760 & 7,480 & 10,700 & 15,400 & 19,400 & 23,700 \\
\hline 5291 & 11.3 & 1,620 & 3,210 & 4,540 & 6,520 & 8,130 & 9,950 \\
\hline 5292 & 49.4 & 4,470 & 8,720 & 12,400 & 17,700 & 22,200 & 27,000 \\
\hline 5295 & 10.5 & 1,590 & 3,280 & 4,720 & 6,880 & 8,650 & 10,700 \\
\hline 5296 & 60.2 & 3,920 & 7,580 & 10,700 & 15,200 & 18,900 & 23,000 \\
\hline 5310 & 9.00 & 1,560 & 3,170 & 4,520 & 6,540 & 8,200 & 10,100 \\
\hline 5315 & 59.8 & 4,890 & 9,430 & 13,300 & 18,900 & 23,700 & 28,800 \\
\hline 5317 & 2,300 & 25,300 & 35,200 & 44,600 & 60,600 & 73,500 & 83,200 \\
\hline 5328 & 76.7 & 5,510 & 10,400 & 14,600 & 20,700 & 25,800 & 31,200 \\
\hline 5329 & 6.15 & 1,170 & 2,290 & 3,230 & 4,600 & 5,720 & 6,990 \\
\hline 5343 & 19.4 & 2,340 & 4,880 & 7,050 & 10,300 & 13,000 & 16,100 \\
\hline 5350 & 2,310 & 25,500 & 35,700 & 45,300 & 61,300 & 74,200 & 84,000 \\
\hline 5361 & 13.4 & 1,980 & 3,940 & 5,580 & 8,020 & 10,000 & 12,300 \\
\hline 5365 & 91.5 & 5,890 & 11,100 & 15,600 & 22,100 & 27,500 & 33,300 \\
\hline 5368 & 13.1 & 1,760 & 3,650 & 5,250 & 7,670 & 9,670 & 11,900 \\
\hline 5380 & 34.6 & 3,930 & 7,490 & 10,500 & 14,700 & 18,300 & 22,100 \\
\hline 5401 & 19.9 & 2,350 & 4,810 & 6,890 & 10,000 & 12,600 & 15,500 \\
\hline 5403 & 15.4 & 2,050 & 4,210 & 6,040 & 8,800 & 11,100 & 13,600 \\
\hline 5411 & 2,430 & 26,800 & 39,500 & 50,100 & 66,300 & 79,200 & 89,800 \\
\hline 5412 & 109 & 6,500 & 12,200 & 17,100 & 24,100 & 30,000 & 36,400 \\
\hline 5414 & 538 & 11,800 & 21,400 & 29,500 & 41,300 & 51,200 & 62,000 \\
\hline
\end{tabular}




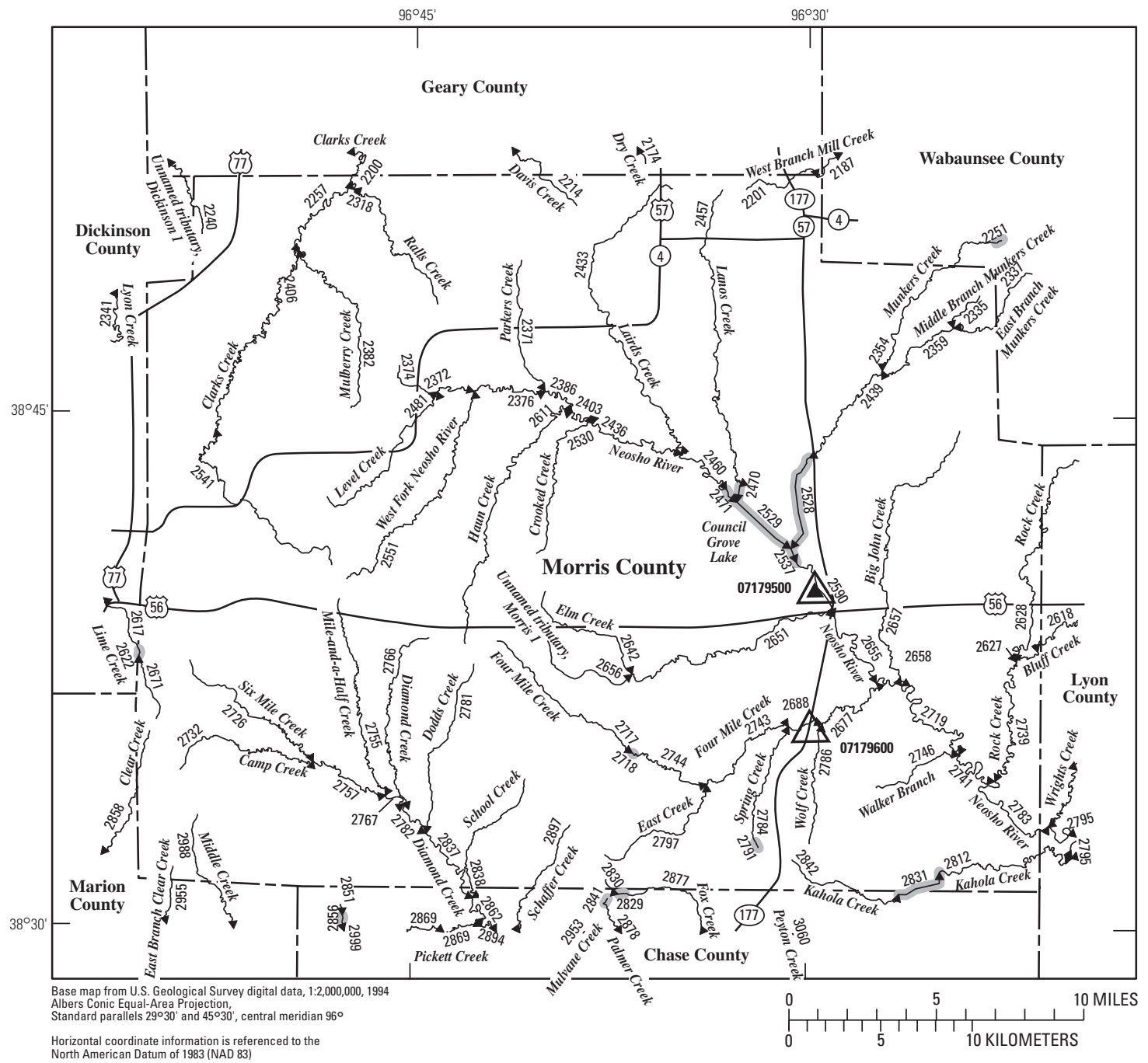

EXPLANATION

\2955 Location of streamflow-statistics determination site (small triangle) and associated identification number-small triangle points in downstream direction

07179500 U.S. Geological Survey streamflow-gaging station and number used for estimates of flow duration

${ }^{07179600} \triangle$ U.S. Geological Survey streamflow-gaging station and number used for estimates of peak-discharge frequency values

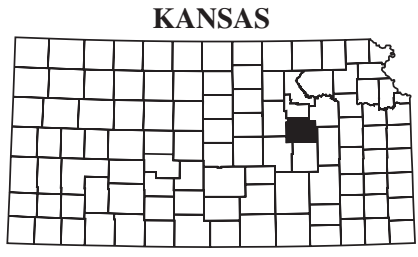

Index map

Lake and determination site identification number

Figure 74. Location of streamflow-statistics determination sites, associated identification numbers, and U.S. Geological Survey streamflow-gaging stations used in the flow-duration and peak-discharge frequency analyses for Morris County. 
Table 70. Estimated flow-duration values, mean flow values, and peak-discharge frequency values for controlled and uncontrolled flow stream segments on the 1999 Kansas Surface Water Register for Morris County.

[KSWR, Kansas Surface Water Register; CUSEGA, catalog unit segment number alpha; $\mathrm{ft}^{3} / \mathrm{s}$, cubic feet per second; HYDRO, lake or other hydrologic structure; NRTribal, tribal stream; NA, not applicable; NRDitch, irrigation ditch]

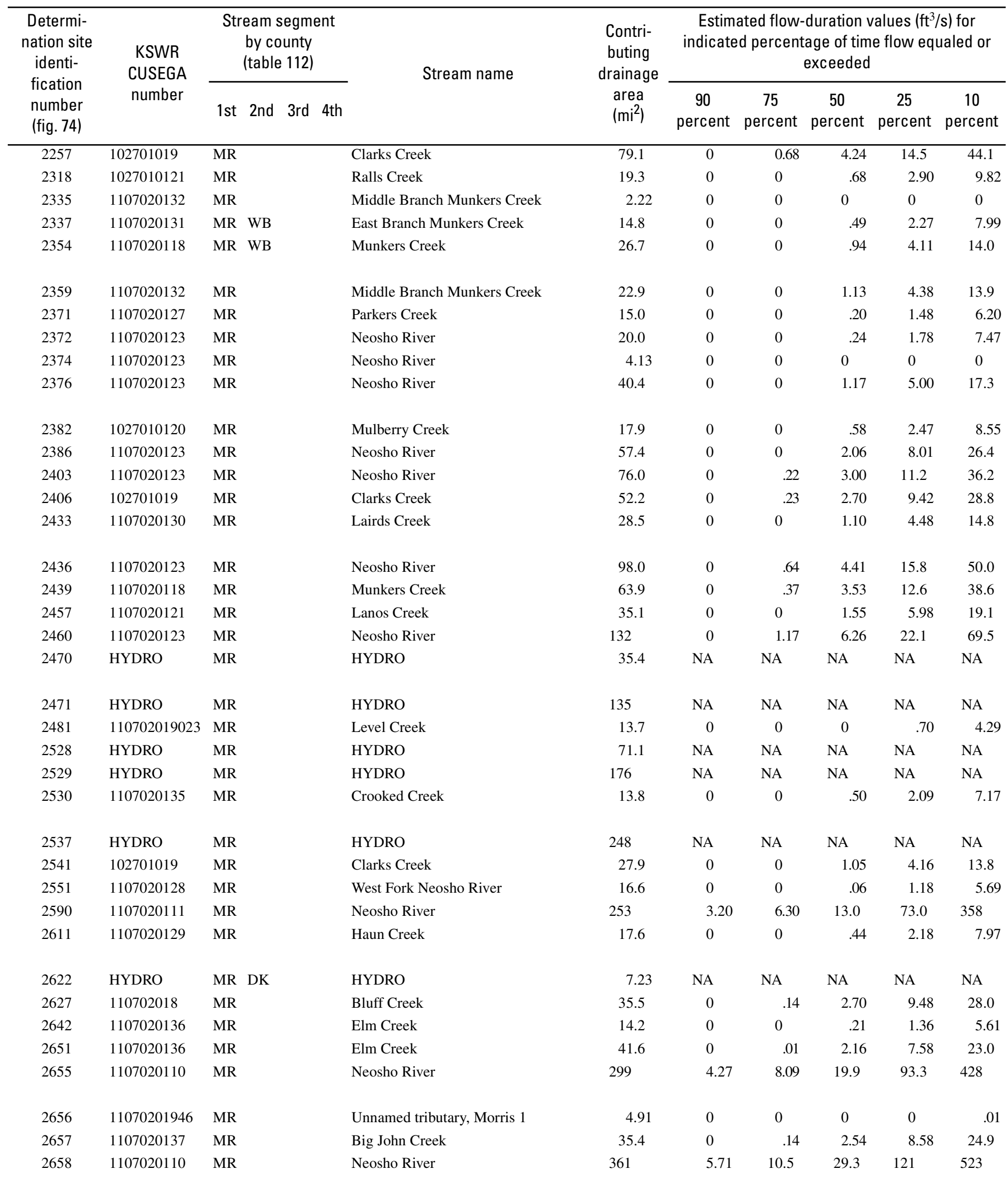


Table 70. Estimated flow-duration values, mean flow values, and peak-discharge frequency values for controlled and uncontrolled flow stream segments on the 1999 Kansas Surface Water Register for Morris County.-Continued

[KSWR, Kansas Surface Water Register; CUSEGA, catalog unit segment number alpha; $\mathrm{mi}^{2}$, square miles; $\mathrm{ft}^{3} / \mathrm{s}$, cubic feet per second; HYDRO, lake or other hydrologic structure; NA, not applicable; NRDitch, irrigation ditch; NRTribal, tribal stream]

\begin{tabular}{|c|c|c|c|c|c|c|c|}
\hline \multirow{2}{*}{$\begin{array}{l}\text { Determi- } \\
\text { nation site } \\
\text { identi- } \\
\text { fication } \\
\text { number } \\
\text { (fig. 74) }\end{array}$} & \multirow{2}{*}{$\begin{array}{c}\text { Estimated mean } \\
\text { flow } \\
\left(\mathrm{ft}^{3} / \mathrm{s}\right)\end{array}$} & \multicolumn{6}{|c|}{ Estimated peak discharge $\left(\mathrm{ft}^{3} / \mathrm{s}\right)$ for indicated peak-discharge frequency } \\
\hline & & 2-year & 5-year & 10-year & 25-year & 50-year & 100-year \\
\hline 2257 & 35.3 & 2,920 & 6,450 & 9,680 & 14,700 & 19,100 & 24,000 \\
\hline 2337 & 7.61 & 1,270 & 2,850 & 4,240 & 6,390 & 8,200 & 10,300 \\
\hline 2354 & 13.0 & 1,850 & 4,160 & 6,220 & 9,390 & 12,100 & 15,200 \\
\hline 2359 & 12.0 & 1,640 & 3,710 & 5,550 & 8,400 & 10,800 & 13,600 \\
\hline 2371 & 6.67 & 1,240 & 2,810 & 4,190 & 6,340 & 8,150 & 10,200 \\
\hline 2382 & 8.30 & 1,380 & 3,120 & 4,670 & 7,060 & 9,070 & 11,400 \\
\hline 2386 & 23.4 & 2,940 & 6,580 & 9,920 & 15,100 & 19,700 & 24,800 \\
\hline 2403 & 30.8 & 3,420 & 7,570 & 11,400 & 17,300 & 22,500 & 28,300 \\
\hline 2406 & 24.0 & 2,300 & 5,210 & 7,900 & 12,100 & 15,800 & 19,900 \\
\hline 2433 & 13.4 & 1,840 & 4,180 & 6,280 & 9,540 & 12,300 & 15,500 \\
\hline 2436 & 40.5 & 3,840 & 8,450 & 12,700 & 19,200 & 24,900 & 31,400 \\
\hline 2439 & 30.8 & 4,150 & 8,680 & 12,700 & 18,700 & 24,000 & 29,700 \\
\hline 2457 & 16.7 & 3,210 & 6,790 & 9,960 & 14,700 & 18,800 & 23,300 \\
\hline 2460 & 54.4 & 4,410 & 9,630 & 14,400 & 21,800 & 28,300 & 35,600 \\
\hline 2537 & NA & NA & NA & NA & NA & NA & NA \\
\hline 2541 & 12.8 & 1,870 & 4,200 & 6,280 & 9,500 & 12,200 & 15,300 \\
\hline 2551 & 6.63 & 1,300 & 2,950 & 4,430 & 6,700 & 8,620 & 10,800 \\
\hline 2590 & 132 & 1,870 & 3,060 & 3,820 & 4,740 & 5,370 & 5,960 \\
\hline 2611 & 7.91 & 1,330 & 3,040 & 4,560 & 6,910 & 8,900 & 11,200 \\
\hline 2622 & NA & NA & NA & NA & NA & NA & NA \\
\hline 2627 & 21.6 & 3,130 & 6,600 & 9,660 & 14,300 & 18,200 & 22,500 \\
\hline 2642 & 6.07 & 1,160 & 2,640 & 3,960 & 6,000 & 7,730 & 9,710 \\
\hline 2651 & 19.0 & 2,990 & 6,470 & 9,580 & 14,300 & 18,400 & 22,900 \\
\hline 2655 & 158 & 2,540 & 4,100 & 6,700 & 9,640 & 12,300 & 15,400 \\
\hline 2656 & 1.56 & 625 & 1,390 & 2,060 & 3,090 & 3,960 & 4,940 \\
\hline 2657 & 19.3 & 3,150 & 6,640 & 9,700 & 14,300 & 18,300 & 22,600 \\
\hline 2658 & 192 & 3,450 & 5,500 & 10,600 & 16,200 & 21,700 & 28,100 \\
\hline
\end{tabular}


Table 70. Estimated flow-duration values, mean flow values, and peak-discharge frequency values for controlled and uncontrolled flow stream segments on the 1999 Kansas Surface Water Register for Morris County.-Continued

[KSWR, Kansas Surface Water Register; CUSEGA, catalog unit segment number alpha; $\mathrm{ft}^{3} / \mathrm{s}$, cubic feet per second; HYDRO, lake or other hydrologic structure; NRTribal, tribal stream; NA, not applicable; NRDitch, irrigation ditch]

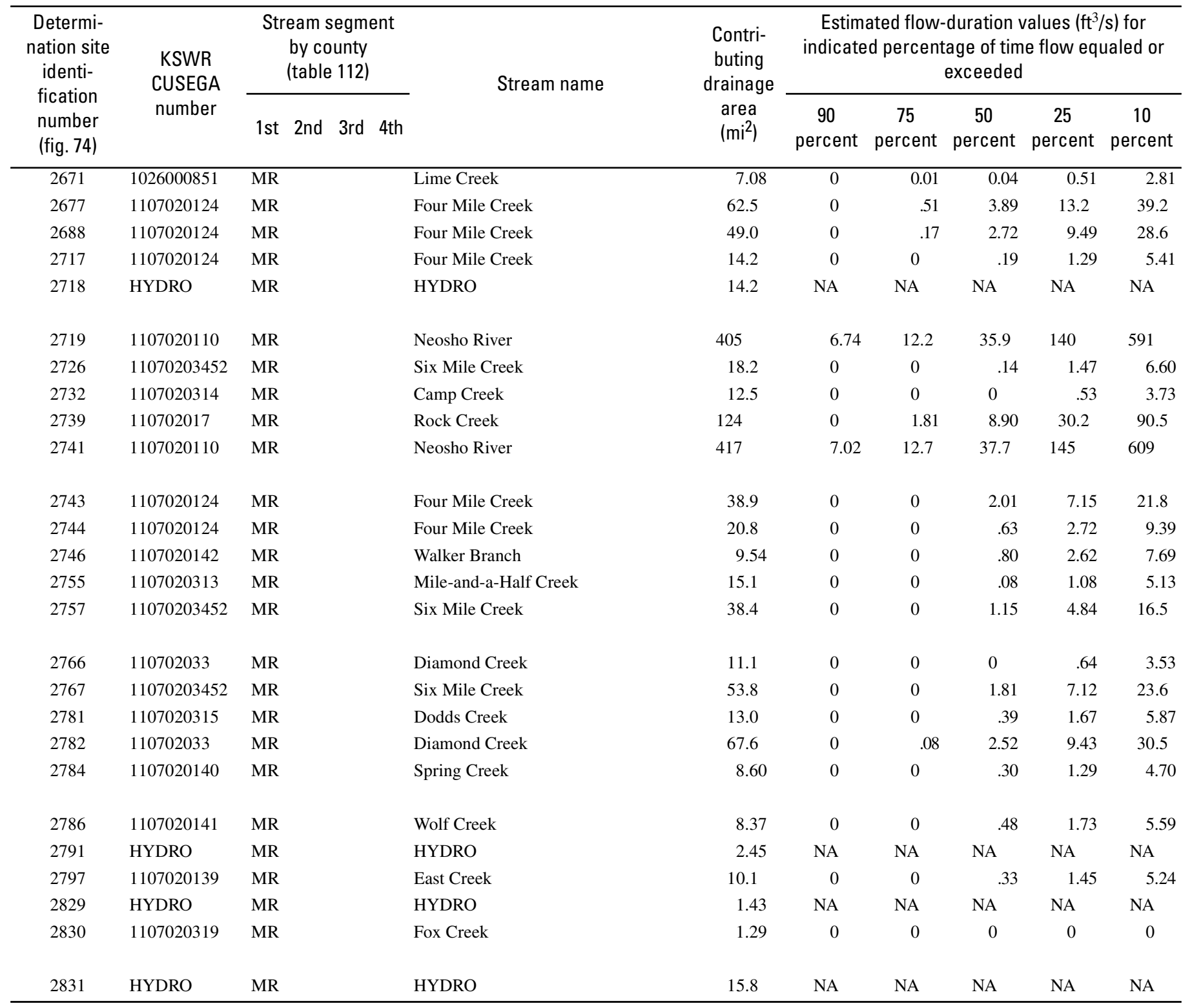


Table 70. Estimated flow-duration values, mean flow values, and peak-discharge frequency values for controlled and uncontrolled flow stream segments on the 1999 Kansas Surface Water Register for Morris County.-Continued

[KSWR, Kansas Surface Water Register; CUSEGA, catalog unit segment number alpha; $\mathrm{mi}^{2}$, square miles; $\mathrm{ft}^{3} / \mathrm{s}$, cubic feet per second; HYDRO, lake or other hydrologic structure; NA, not applicable; NRDitch, irrigation ditch; NRTribal, tribal stream]

\begin{tabular}{|c|c|c|c|c|c|c|c|}
\hline \multirow{2}{*}{$\begin{array}{l}\text { Determi- } \\
\text { nation site } \\
\text { identi- } \\
\text { fication } \\
\text { number } \\
\text { (fig. 74) }\end{array}$} & \multirow[b]{2}{*}{$\begin{array}{c}\text { Estimated mean } \\
\text { flow } \\
\left(\mathrm{ft}^{3} / \mathrm{s}\right)\end{array}$} & \multicolumn{6}{|c|}{ Estimated peak discharge $\left(\mathrm{ft}^{3} / \mathrm{s}\right)$ for indicated peak-discharge frequency } \\
\hline & & 2-year & 5-year & 10-year & 25-year & 50-year & 100-year \\
\hline 2671 & 3.49 & 851 & 1,870 & 2,760 & 4,110 & 5,250 & 6,540 \\
\hline 2717 & 5.91 & 1,300 & 2,940 & 4,410 & 6,730 & 8,720 & 11,000 \\
\hline 2718 & NA & NA & NA & NA & NA & NA & NA \\
\hline 2719 & 216 & 4,090 & 6,490 & 13,300 & 20,900 & 28,300 & 37,100 \\
\hline 2726 & 7.46 & 1,410 & 3,190 & 4,770 & 7,210 & 9,270 & 11,600 \\
\hline 2743 & 18.1 & 4,160 & 8,870 & 13,200 & 20,000 & 26,200 & 33,300 \\
\hline 2744 & 9.04 & 1,820 & 4,080 & 6,130 & 9,390 & 12,200 & 15,600 \\
\hline 2746 & 6.33 & 1,020 & 2,250 & 3,320 & 4,970 & 6,340 & 7,910 \\
\hline 2755 & 5.97 & 1,190 & 2,720 & 4,090 & 6,200 & 7,990 & 10,000 \\
\hline 2757 & 15.5 & 2,390 & 5,430 & 8,220 & 12,600 & 16,400 & 20,700 \\
\hline 2766 & 4.35 & 952 & 2,180 & 3,280 & 4,990 & 6,430 & 8,090 \\
\hline 2767 & 21.2 & 2,880 & 6,460 & 9,740 & 14,800 & 19,300 & 24,300 \\
\hline 2781 & 5.78 & 1,030 & 2,380 & 3,590 & 5,480 & 7,070 & 8,910 \\
\hline 2782 & 26.4 & 3,150 & 7,040 & 10,600 & 16,200 & 21,100 & 26,500 \\
\hline 2831 & NA & NA & NA & NA & NA & NA & NA \\
\hline
\end{tabular}




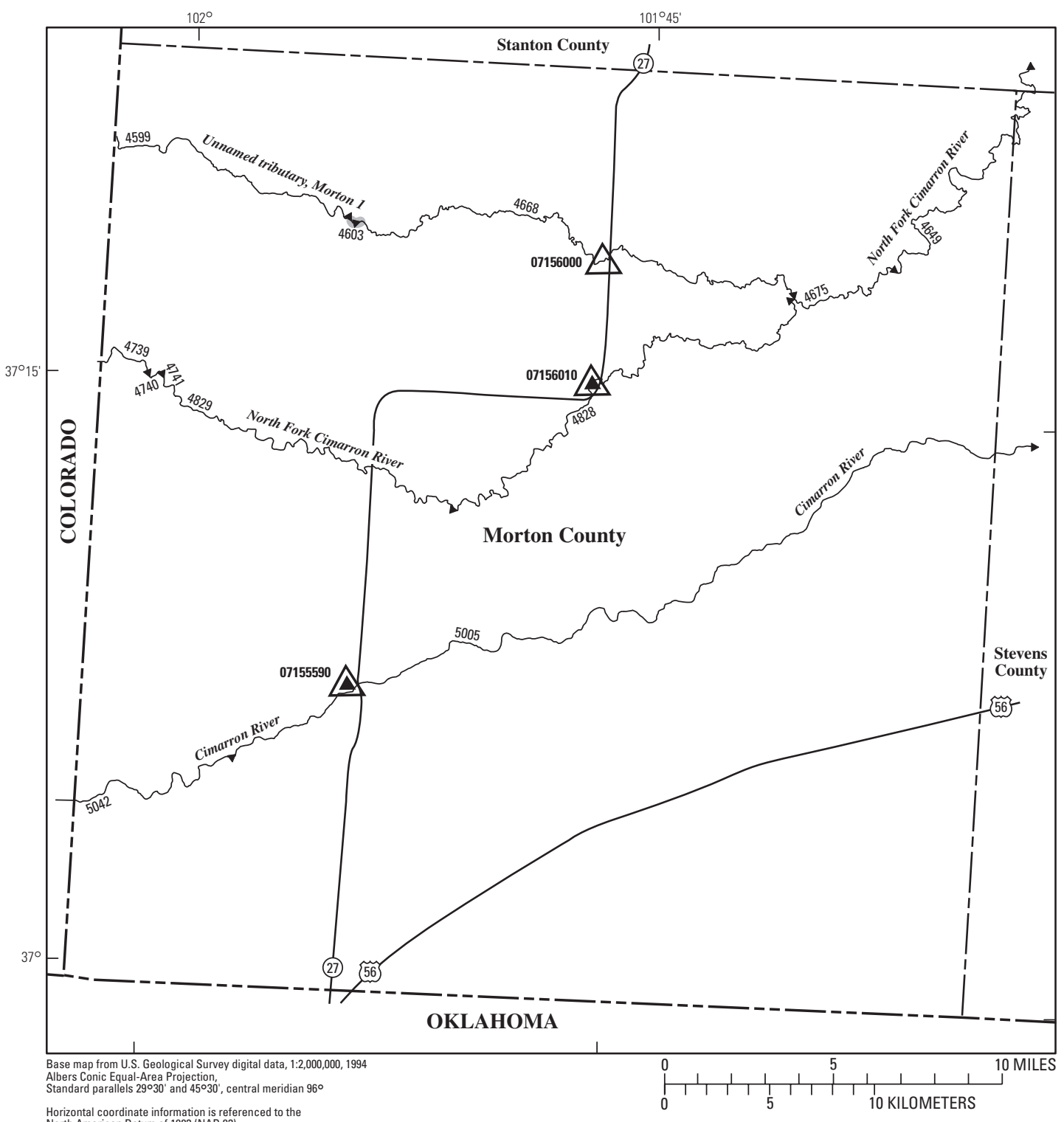

EXPLANATION

$\underset{5042}{\longleftarrow}$ Location of streamflow-statistics determination site (small triangle) and associated identification number-small triangle points in downstream direction

07155590 U.S. Geological Survey streamflow-gaging station and number used for estimates of flow duration

07156000 U.S. Geological Survey streamflow-gaging station and number used for estimates of peak-discharge frequency values

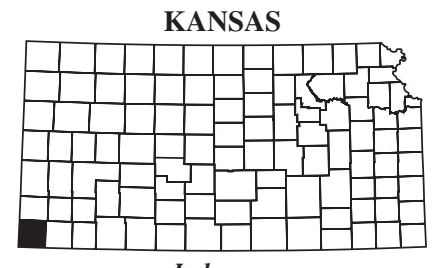

Index map 4603

Lake and determination site identification number

Figure 75. Location of streamflow-statistics determination sites, associated identification numbers, and U.S. Geological Survey streamflow-gaging stations used in the flow-duration and peak-discharge frequency analyses for Morton County. 
Table 71. Estimated flow-duration values, mean flow values, and peak-discharge frequency values for controlled and uncontrolled flow stream segments on the 1999 Kansas Surface Water Register for Morton County.

[KSWR, Kansas Surface Water Register; CUSEGA, catalog unit segment number alpha; $\mathrm{mi}^{2}$, square miles; $\mathrm{ft}^{3} / \mathrm{s}$, cubic feet per second; HYDRO, lake or other hydrologic structure; NA, not applicable; NRDitch, irrigation ditch; NRTribal, tribal stream]

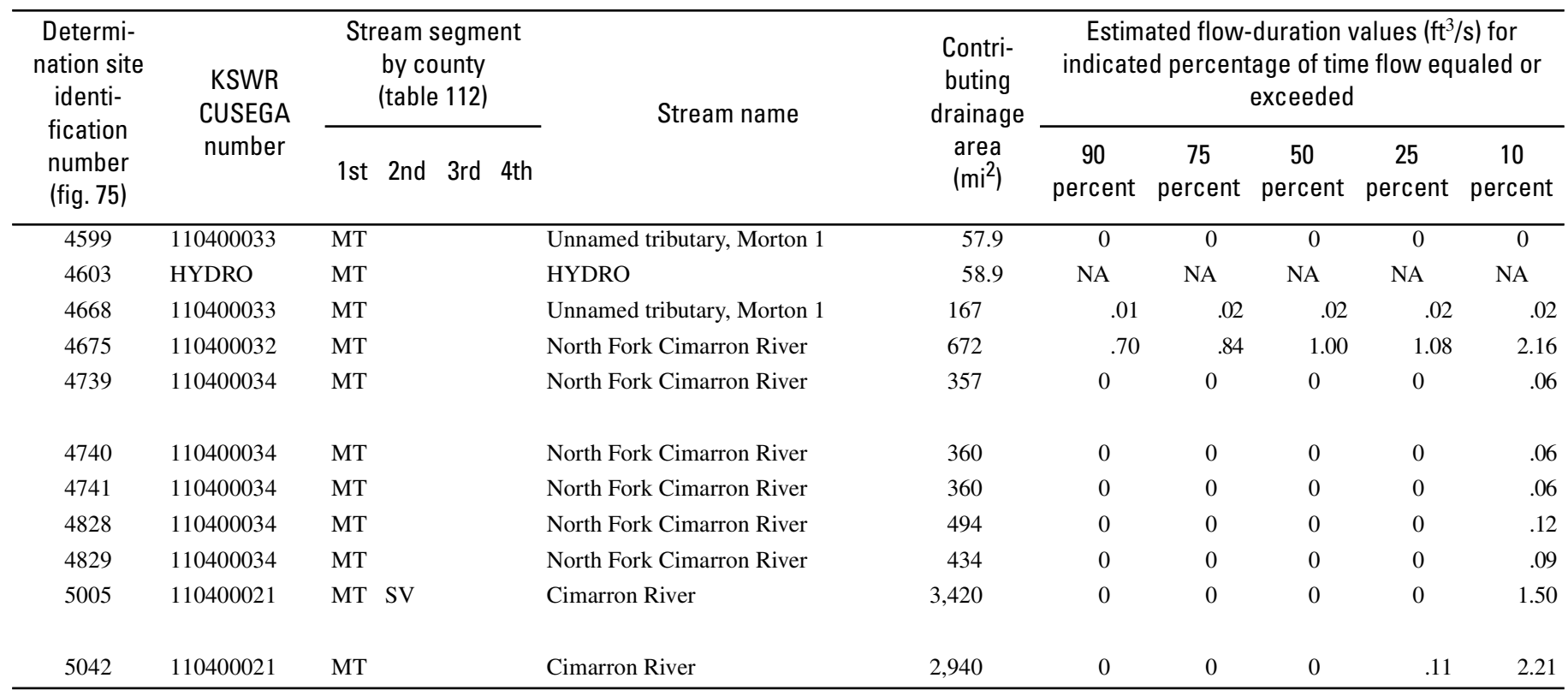


Table 71. Estimated flow-duration values, mean flow values, and peak-discharge frequency values for controlled and uncontrolled flow stream segments on the 1999 Kansas Surface Water Register for Morton County.-Continued

[KSWR, Kansas Surface Water Register; CUSEGA, catalog unit segment number alpha; $\mathrm{mi}^{2}$, square miles; $\mathrm{ft}^{3} / \mathrm{s}$, cubic feet per second; HYDRO, lake or other hydrologic structure; NA, not applicable; NRDitch, irrigation ditch; NRTribal, tribal stream]

\begin{tabular}{|c|c|c|c|c|c|c|c|}
\hline \multirow{2}{*}{$\begin{array}{l}\text { Determi- } \\
\text { nation site } \\
\text { identi- } \\
\text { fication } \\
\text { number } \\
\text { (fig. 75) }\end{array}$} & \multirow{2}{*}{$\begin{array}{l}\text { Estimated mean } \\
\text { flow } \\
\left(\mathrm{ft}^{3} / \mathrm{s}\right)\end{array}$} & \multicolumn{6}{|c|}{ Estimated peak discharge $\left(\mathrm{ft}^{3} / \mathrm{s}\right)$ for indicated peak-discharge frequency } \\
\hline & & 2-year & 5-year & 10-year & 25-year & 50-year & 100-year \\
\hline 4599 & 0 & 206 & 728 & 1,340 & 2,470 & 3,610 & 5,000 \\
\hline 4675 & 6.69 & 981 & 4,010 & 7,980 & 16,000 & 24,500 & 35,600 \\
\hline 4739 & 2.56 & 604 & 2,400 & 4,710 & 9,310 & 14,200 & 20,400 \\
\hline 4740 & 2.61 & 612 & 2,430 & 4,770 & 9,430 & 14,400 & 20,700 \\
\hline 4741 & 2.61 & 611 & 2,430 & 4,770 & 9,430 & 14,400 & 20,700 \\
\hline 5042 & 12.5 & 1,310 & 4,010 & 6,910 & 12,000 & 16,700 & 22,500 \\
\hline
\end{tabular}




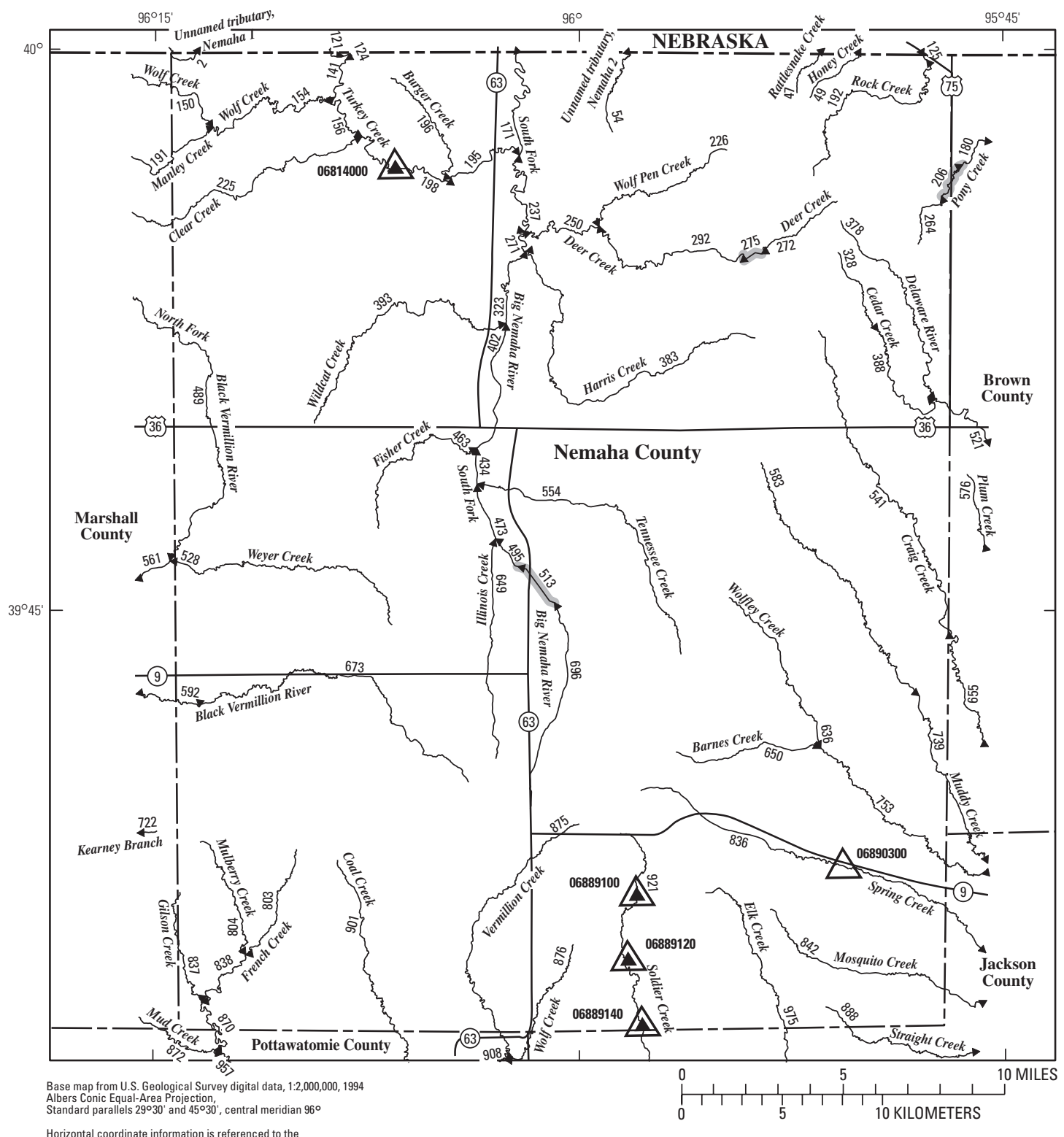

Horizontal coordinate information is referenced to the

EXPLANATION
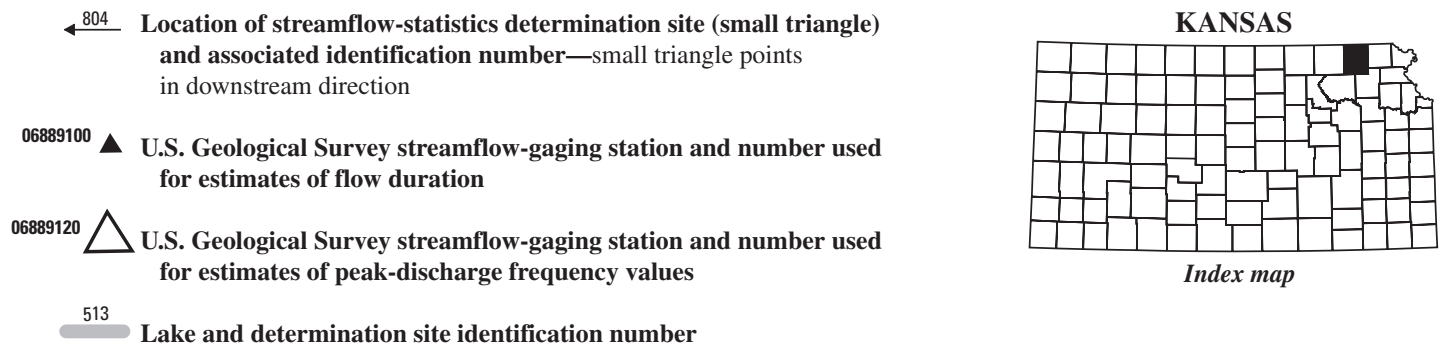

Figure 76. Location of streamflow-statistics determination sites, associated identification numbers, and U.S. Geological Survey streamflow-gaging stations used in the flow-duration and peak-discharge frequency analyses for Nemaha County. 
Table 72. Estimated flow-duration values, mean flow values, and peak-discharge frequency values for controlled and uncontrolled flow stream segments on the 1999 Kansas Surface Water Register for Nemaha County.

[KSWR, Kansas Surface Water Register; CUSEGA, catalog unit segment number alpha; $\mathrm{mi}^{2}$, square miles; $\mathrm{ft}^{3} / \mathrm{s}$, cubic feet per second; HYDRO, lake or other hydrologic structure; NA, not applicable; NRDitch, irrigation ditch; NRTribal, tribal stream]

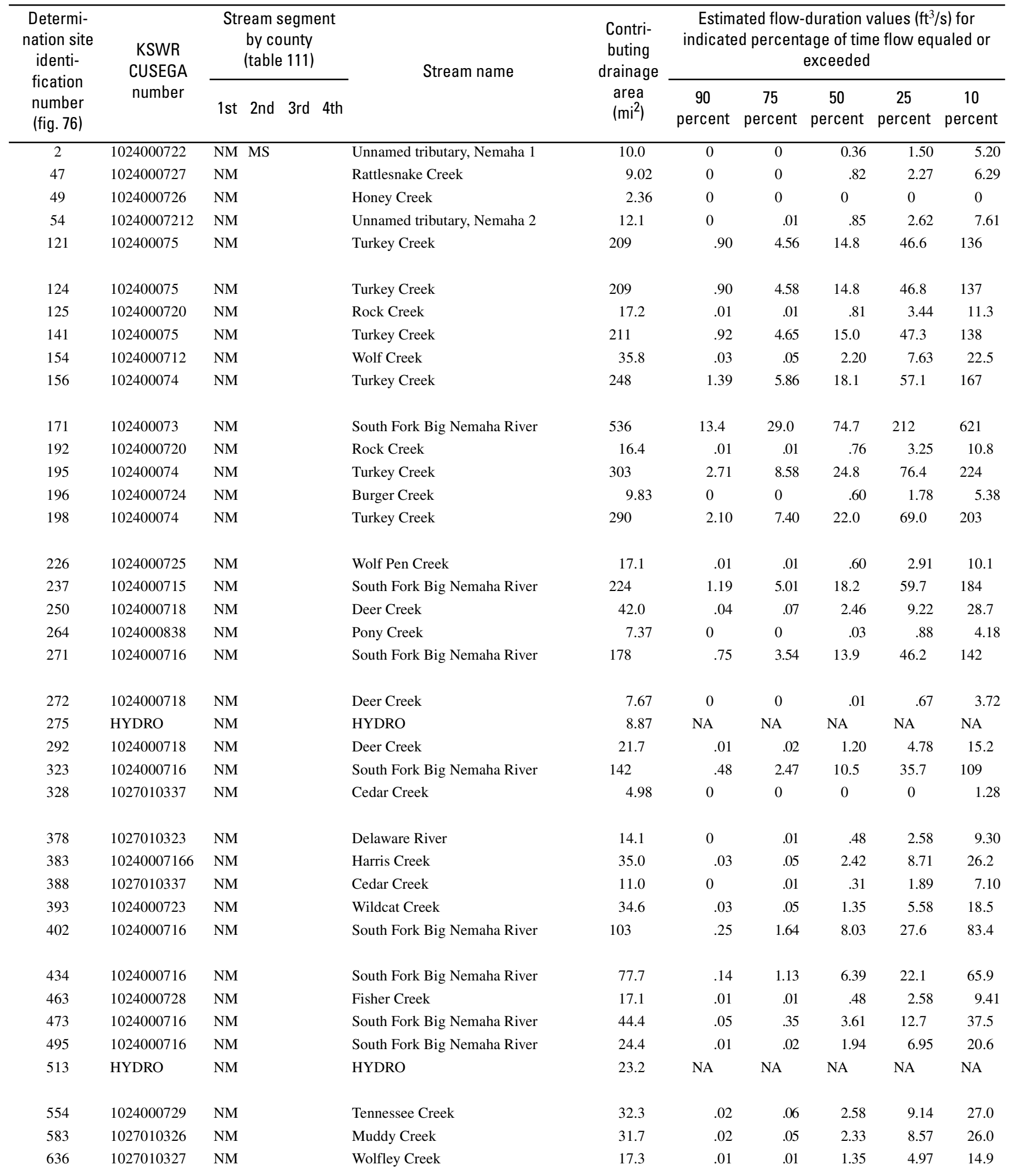


Table 72. Estimated flow-duration values, mean flow values, and peak-discharge frequency values for controlled and uncontrolled flow stream segments on the 1999 Kansas Surface Water Register for Nemaha County.-Continued

[KSWR, Kansas Surface Water Register; CUSEGA, catalog unit segment number alpha; $\mathrm{mi}^{2}$, square miles; $\mathrm{ft}^{3} / \mathrm{s}$, cubic feet per second; HYDRO, lake or other hydrologic structure; NA, not applicable; NRDitch, irrigation ditch; NRTribal, tribal stream]

\begin{tabular}{|c|c|c|c|c|c|c|c|}
\hline \multirow{2}{*}{$\begin{array}{l}\text { Determi- } \\
\text { nation site } \\
\text { identi- } \\
\text { fication } \\
\text { number } \\
\text { (fig. 76) }\end{array}$} & \multirow{2}{*}{$\begin{array}{c}\text { Estimated mean } \\
\text { flow } \\
\left(\mathrm{ft}^{3} / \mathrm{s}\right)\end{array}$} & \multicolumn{6}{|c|}{ Estimated peak discharge $\left(\mathrm{ft}^{3} / \mathrm{s}\right)$ for indicated peak-discharge frequency } \\
\hline & & 2-year & 5-year & 10 -year & 25-year & 50-year & 100 -year \\
\hline 2 & 4.94 & 888 & 2,040 & 3,070 & 4,660 & 6,000 & 7,550 \\
\hline 54 & 6.52 & 1,060 & 2,400 & 3,590 & 5,430 & 6,990 & 8,770 \\
\hline 121 & 90.5 & 5,090 & 11,000 & 16,300 & 24,600 & 32,000 & 40,200 \\
\hline 124 & 90.8 & 5,070 & 11,000 & 16,300 & 24,600 & 31,900 & 40,200 \\
\hline 125 & 9.82 & 1,390 & 3,130 & 4,660 & 7,040 & 9,030 & 11,300 \\
\hline 171 & 379 & 9,040 & 18,700 & 27,300 & 40,700 & 52,500 & 65,800 \\
\hline 192 & 9.38 & 1,350 & 3,040 & 4,530 & 6,830 & 8,760 & 11,000 \\
\hline 195 & 142 & 5,900 & 12,700 & 18,700 & 28,100 & 36,500 & 45,900 \\
\hline 196 & 4.91 & 898 & 2,050 & 3,070 & 4,660 & 6,000 & 7,540 \\
\hline 198 & 129 & 5,820 & 12,500 & 18,400 & 27,700 & 36,000 & 45,300 \\
\hline 226 & 9.17 & 1,350 & 3,050 & 4,570 & 6,910 & 8,880 & 11,100 \\
\hline 237 & 125 & 6,900 & 14,400 & 21,200 & 31,600 & 40,800 & 51,000 \\
\hline 250 & 22.8 & 3,350 & 7,240 & 10,700 & 16,000 & 20,700 & 25,800 \\
\hline 264 & 4.39 & 892 & 1,950 & 2,860 & 4,260 & 5,420 & 6,750 \\
\hline 378 & 8.51 & 1,330 & 2,920 & 4,320 & 6,450 & 8,230 & 10,300 \\
\hline 383 & 20.4 & 2,900 & 6,300 & 9,350 & 14,000 & 18,000 & 22,500 \\
\hline 388 & 6.70 & 1,140 & 2,500 & 3,680 & 5,490 & 7,010 & 8,740 \\
\hline 393 & 16.1 & 2,470 & 5,630 & 8,550 & 13,100 & 17,100 & 21,600 \\
\hline 402 & 59.5 & 5,220 & 10,900 & 15,900 & 23,500 & 30,200 & 37,400 \\
\hline 434 & 47.2 & 4,840 & 10,000 & 14,600 & 21,500 & 27,400 & 33,900 \\
\hline 463 & 8.87 & 1,370 & 3,090 & 4,610 & 6,970 & 8,950 & 11,200 \\
\hline 473 & 27.8 & 3,490 & 7,400 & 10,900 & 16,100 & 20,700 & 25,700 \\
\hline 495 & 15.9 & 1,800 & 4,020 & 5,990 & 9,020 & 11,600 & 14,500 \\
\hline 513 & NA & NA & NA & NA & NA & NA & NA \\
\hline 554 & 20.5 & 3,300 & 6,930 & 10,100 & 14,900 & 19,000 & 23,500 \\
\hline 583 & 20.2 & 2,720 & 5,920 & 8,790 & 13,200 & 17,000 & 21,200 \\
\hline 636 & 11.8 & 1,530 & 3,350 & 4,940 & 7,380 & 9,430 & 11,800 \\
\hline
\end{tabular}


Table 72. Estimated flow-duration values, mean flow values, and peak-discharge frequency values for controlled and uncontrolled flow stream segments on the 1999 Kansas Surface Water Register for Nemaha County.-Continued

[KSWR, Kansas Surface Water Register; CUSEGA, catalog unit segment number alpha; $\mathrm{mi}^{2}$, square miles; $\mathrm{ft}^{3} / \mathrm{s}$, cubic feet per second; HYDRO, lake or other hydrologic structure; NA, not applicable; NRDitch, irrigation ditch; NRTribal, tribal stream]

\begin{tabular}{|c|c|c|c|c|c|c|c|c|c|c|c|}
\hline $\begin{array}{l}\text { Determi- } \\
\text { nation site } \\
\text { identi- } \\
\text { fication } \\
\text { number } \\
\text { (fig. } 76 \text { ) }\end{array}$ & $\begin{array}{l}\text { KSWR } \\
\text { CUSEGA } \\
\text { number }\end{array}$ & \multicolumn{3}{|c|}{$\begin{array}{l}\text { Stream segment } \\
\text { by county } \\
\text { (table 111) }\end{array}$} & Stream name & $\begin{array}{l}\text { Contri- } \\
\text { buting } \\
\text { drainage } \\
\text { area } \\
\left(\mathrm{mi}^{2}\right)\end{array}$ & \multicolumn{5}{|c|}{$\begin{array}{c}\text { Estimated flow-duration values }\left(\mathrm{ft}^{3} / \mathrm{s}\right) \text { for } \\
\text { indicated percentage of time flow equaled or } \\
\text { exceeded }\end{array}$} \\
\hline 649 & 1024000730 & NM & & & Illinois Creek & 16.4 & 0.01 & 0.01 & 1.26 & 4.60 & 13.8 \\
\hline 696 & 1024000716 & NM & & & South Fork Big Nemaha River & 19.3 & .01 & .02 & 1.55 & 5.59 & 16.6 \\
\hline 803 & 1027010219 & NM & & & French Creek & 13.4 & 0 & 0 & .80 & 3.13 & 9.79 \\
\hline 804 & 1027010242 & NM & & & Mulberry Creek & 10.6 & 0 & 0 & .44 & 1.99 & 6.83 \\
\hline 838 & 1027010219 & NM & & & French Creek & 27.9 & 0 & 0 & 1.83 & 6.70 & 20.1 \\
\hline
\end{tabular}


Table 72. Estimated flow-duration values, mean flow values, and peak-discharge frequency values for controlled and uncontrolled flow stream segments on the 1999 Kansas Surface Water Register for Nemaha County.-Continued

[KSWR, Kansas Surface Water Register; CUSEGA, catalog unit segment number alpha; $\mathrm{mi}^{2}$, square miles; $\mathrm{ft}^{3} / \mathrm{s}$, cubic feet per second; HYDRO, lake or other hydrologic structure; NA, not applicable; NRDitch, irrigation ditch; NRTribal, tribal stream]

\begin{tabular}{|c|c|c|c|c|c|c|c|}
\hline \multirow{2}{*}{$\begin{array}{l}\text { Determi- } \\
\text { nation site } \\
\text { identi- } \\
\text { fication } \\
\text { number } \\
\text { (fig. 76) }\end{array}$} & \multirow{2}{*}{$\begin{array}{c}\text { Estimated mean } \\
\text { flow } \\
\left(\mathrm{ft}^{3} / \mathrm{s}\right)\end{array}$} & \multicolumn{6}{|c|}{ Estimated peak discharge $\left(\mathrm{ft}^{3} / \mathrm{s}\right)$ for indicated peak-discharge frequency } \\
\hline & & 2-year & 5-year & 10-year & 25-year & 50-year & 100-year \\
\hline 649 & 11.0 & 1,430 & 3,160 & 4,690 & 7,030 & 9,000 & 11,300 \\
\hline 696 & 12.9 & 1,580 & 3,500 & 5,200 & 7,800 & 9,990 & 12,500 \\
\hline 803 & 8.29 & 1,260 & 2,770 & 4,110 & 6,150 & 7,860 & 9,820 \\
\hline 804 & 6.26 & 1,080 & 2,390 & 3,540 & 5,290 & 6,760 & 8,440 \\
\hline 838 & 16.0 & 1,910 & 4,290 & 6,390 & 9,650 & 12,400 & 15,500 \\
\hline
\end{tabular}




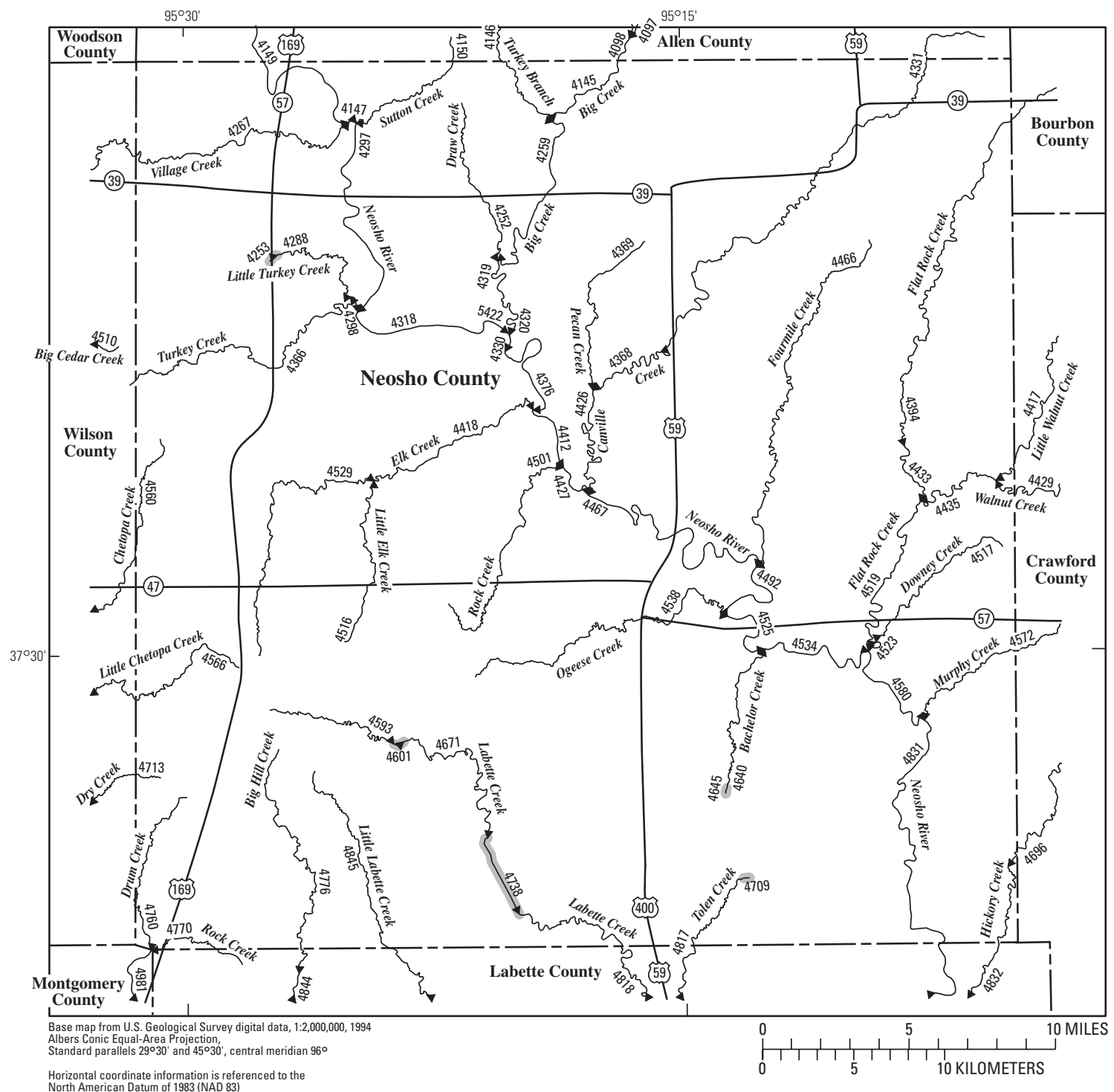

EXPLANATION

4981 Location of streamflow-statistics determination site (small triangle) and associated identification number-small triangle points in downstream direction

${ }^{06875900}$ U.S. Geological Survey streamflow-gaging station and number used for estimates of flow duration

${ }^{06875800} \triangle$ U.S. Geological Survey streamflow-gaging station and number used for estimates of peak-discharge frequency values

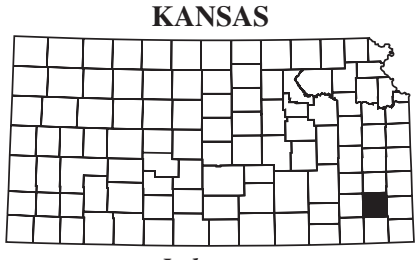

Index map 4738

Lake and determination site identification number

Figure 77. Location of streamflow-statistics determination sites, associated identification numbers, and U.S. Geological Survey streamflow-gaging stations used in the flow-duration and peak-discharge frequency analyses for Neosho County. 
Table 73. Estimated flow-duration values, mean flow values, and peak-discharge frequency values for controlled and uncontrolled flow stream segments on the 1999 Kansas Surface Water Register for Neosho County.

[KSWR, Kansas Surface Water Register; CUSEGA, catalog unit segment number alpha; $\mathrm{mi}^{2}$, square miles; $\mathrm{ft}^{3} / \mathrm{s}$, cubic feet per second; HYDRO, lake or other hydrologic structure; NA, not applicable; NRDitch, irrigation ditch; NRTribal, tribal stream]

\begin{tabular}{|c|c|c|c|c|c|c|c|c|c|c|c|}
\hline \multirow{2}{*}{$\begin{array}{l}\text { Determi- } \\
\text { nation site } \\
\text { identi- } \\
\text { fication } \\
\text { number } \\
\text { (fig. 77) }\end{array}$} & \multirow{2}{*}{$\begin{array}{l}\text { KSWR } \\
\text { CUSEGA } \\
\text { number }\end{array}$} & \multicolumn{3}{|c|}{$\begin{array}{l}\text { Stream segment } \\
\text { by county } \\
\text { (table 112) }\end{array}$} & \multirow{2}{*}{ Stream name } & \multirow{2}{*}{$\begin{array}{l}\text { Contri- } \\
\text { buting } \\
\text { drainage } \\
\text { area } \\
\left(\mathrm{mi}^{2}\right)\end{array}$} & \multicolumn{5}{|c|}{$\begin{array}{l}\text { Estimated flow-duration values }\left(\mathrm{ft}^{3} / \mathrm{s}\right) \text { for } \\
\text { indicated percentage of time flow equaled or } \\
\text { exceeded }\end{array}$} \\
\hline & & $1 \mathrm{st}$ & 2nd & 3rd 4th & & & $\begin{array}{c}90 \\
\text { percent }\end{array}$ & $\begin{array}{c}75 \\
\text { percent }\end{array}$ & $\begin{array}{c}50 \\
\text { percent }\end{array}$ & $\begin{array}{c}25 \\
\text { percent }\end{array}$ & $\begin{array}{c}10 \\
\text { percent }\end{array}$ \\
\hline 4147 & 110702043 & $\mathrm{NO}$ & & & Neosho River & 4,140 & 46.4 & 142 & 679 & 2,830 & 8,190 \\
\hline 4252 & 1107020434 & NO & & & Draw Creek & 10.5 & 0 & .17 & 1.89 & 6.01 & 15.8 \\
\hline 4253 & HYDRO & NO & & & HYDRO & 5.28 & NA & NA & NA & NA & NA \\
\hline 4259 & 110702042 & NO & & & Big Creek & 114 & 0 & 2.12 & 11.0 & 41.3 & 127 \\
\hline 4267 & 1107020433 & NO & WL & & Village Creek & 49.2 & 0 & .32 & 4.09 & 16.7 & 52.9 \\
\hline 4288 & 11070204397 & NO & & & Little Turkey Creek & 9.54 & 0 & 0 & .56 & 3.13 & 10.7 \\
\hline 4297 & 110702043 & NO & & & Neosho River & 4,160 & 46.6 & 143 & 685 & 2,850 & 8,250 \\
\hline 4298 & 1107020432 & NO & & & Turkey Creek & 30.4 & 0 & 0 & 2.62 & 11.3 & 35.7 \\
\hline 4318 & 110702043 & NO & & & Neosho River & 4,200 & 47.1 & 146 & 696 & 2,900 & 8,350 \\
\hline 4319 & 110702042 & NO & & & Big Creek & 128 & .05 & 2.52 & 12.7 & 47.3 & 146 \\
\hline 4320 & 110702041 & NO & & & Neosho River & 4,200 & 47.1 & 146 & 696 & 2,900 & 8,350 \\
\hline 4330 & 110702041 & NO & & & Neosho River & 4,330 & 48.6 & 154 & 731 & 3,050 & 8,660 \\
\hline 4366 & 1107020432 & NO & WL & & Turkey Creek & 20.8 & 0 & 0 & 1.89 & 8.14 & 25.5 \\
\hline 4368 & 1107020516 & NO & & & Canville Creek & 55.4 & 0 & .71 & 5.54 & 21.6 & 66.0 \\
\hline 4369 & 1107020545 & NO & & & Pecan Creek & 13.4 & 0 & .06 & 2.07 & 7.24 & 20.0 \\
\hline 4376 & 1107020517 & NO & & & Neosho River & 4,330 & 48.7 & 154 & 732 & 3,050 & 8,670 \\
\hline 4412 & 1107020517 & NO & & & Neosho River & 4,380 & 49.3 & 157 & 745 & 3,110 & 8,790 \\
\hline 4418 & 1107020519 & NO & & & Elk Creek & 47.8 & 0 & .92 & 5.77 & 20.9 & 60.7 \\
\hline 4426 & 1107020516 & NO & & & Canville Creek & 73.7 & 0 & 1.20 & 7.56 & 29.0 & 88.6 \\
\hline 4427 & 1107020517 & NO & & & Neosho River & 4,400 & 49.5 & 158 & 750 & 3,130 & 8,840 \\
\hline 4433 & 1107020514 & NO & & & Flat Rock Creek & 45.2 & 0 & .58 & 4.58 & 17.4 & 52.4 \\
\hline 4435 & 1107020513 & NO & & & Walnut Creek & 75.4 & 0 & .80 & 6.13 & 25.1 & 80.4 \\
\hline 4466 & 1107020549 & NO & & & Fourmile Creek & 31.2 & 0 & .03 & 2.62 & 10.7 & 33.2 \\
\hline 4467 & 1107020515 & NO & & & Neosho River & 4,490 & 50.5 & 164 & 774 & 3,230 & 9,050 \\
\hline 4492 & 1107020515 & NO & & & Neosho River & 4,520 & 51.0 & 166 & 783 & 3,270 & 9,130 \\
\hline 4501 & 1107020548 & NO & & & Rock Creek & 16.7 & 0 & .15 & 2.37 & 8.31 & 23.1 \\
\hline 4516 & 1107020547 & NO & & & Little Elk Creek & 13.6 & 0 & .22 & 2.21 & 7.23 & 19.3 \\
\hline 4517 & 11070205731 & NO & & & Downey Creek & 9.78 & 0 & 0 & .91 & 3.70 & 11.3 \\
\hline 4519 & 1107020512 & NO & & & Flat Rock Creek & 129 & 0 & 1.93 & 10.8 & 43.0 & 138 \\
\hline 4523 & 1107020512 & NO & & & Flat Rock Creek & 139 & 0 & 2.13 & 11.7 & 46.2 & 149 \\
\hline 4525 & 1107020515 & NO & & & Neosho River & 4,550 & 51.3 & 168 & 790 & 3,300 & 9,200 \\
\hline 4529 & 1107020519 & NO & & & Elk Creek & 21.4 & 0 & .10 & 2.49 & 9.25 & 26.9 \\
\hline 4534 & 1107020515 & NO & & & Neosho River & 4,580 & 51.6 & 169 & 798 & 3,330 & 9,270 \\
\hline 4538 & 1107020538 & NO & & & Ogeese Creek & 25.7 & 0 & .44 & 3.68 & 13.0 & 36.4 \\
\hline 4560 & 1107010122 & NO & WL & & Chetopa Creek & 28.8 & 0 & 0 & 2.00 & 9.00 & 29.5 \\
\hline 4566 & 11070101471 & NO & WL & & Little Chetopa Creek & 16.0 & 0 & .25 & 2.57 & 8.58 & 23.2 \\
\hline 4580 & 1107020511 & NO & & & Neosho River & 4,720 & 53.3 & 179 & 837 & 3,500 & 9,620 \\
\hline 4593 & 1107020522 & NO & & & Labette Creek & 8.73 & 0 & .01 & 1.39 & 4.52 & 12.2 \\
\hline
\end{tabular}


Table 73. Estimated flow-duration values, mean flow values, and peak-discharge frequency values for controlled and uncontrolled flow stream segments on the 1999 Kansas Surface Water Register for Neosho County.-Continued

[KSWR, Kansas Surface Water Register; CUSEGA, catalog unit segment number alpha; $\mathrm{mi}^{2}$, square miles; $\mathrm{ft}^{3} / \mathrm{s}$, cubic feet per second; HYDRO, lake or other hydrologic structure; NA, not applicable; NRDitch, irrigation ditch; NRTribal, tribal stream]

\begin{tabular}{|c|c|c|c|c|c|c|c|}
\hline \multirow{2}{*}{$\begin{array}{l}\text { Determi- } \\
\text { nation site } \\
\text { identi- } \\
\text { fication } \\
\text { number } \\
\text { (fig. } 77 \text { ) }\end{array}$} & \multirow{2}{*}{$\begin{array}{c}\text { Estimated mean } \\
\text { flow } \\
\left(\mathrm{ft}^{3} / \mathrm{s}\right)\end{array}$} & \multicolumn{6}{|c|}{ Estimated peak discharge $\left(\mathrm{ft}^{3} / \mathrm{s}\right)$ for indicated peak-discharge frequency } \\
\hline & & 2-year & 5-year & 10 -year & 25-year & 50-year & 100 -year \\
\hline 4147 & 2,540 & 24,700 & 35,200 & 39,800 & 59,700 & 86,000 & 122,000 \\
\hline 4259 & 90.9 & 7,380 & 13,500 & 18,600 & 26,000 & 32,200 & 38,800 \\
\hline 4267 & 41.4 & 4,060 & 8,020 & 11,400 & 16,400 & 20,600 & 25,200 \\
\hline 4288 & 9.44 & 1,740 & 3,360 & 4,700 & 6,670 & 8,270 & 10,100 \\
\hline 4297 & 2,560 & 24,800 & 35,500 & 40,200 & 59,700 & 86,000 & 122,000 \\
\hline 4320 & 2,600 & 25,100 & 35,900 & 40,900 & 59,700 & 86,000 & 122,000 \\
\hline 4330 & 2,720 & 26,100 & 37,300 & 43,000 & 59,800 & 86,000 & 122,000 \\
\hline 4366 & 20.3 & 2,710 & 5,340 & 7,530 & 10,800 & 13,400 & 16,500 \\
\hline 4368 & 49.1 & 5,220 & 9,790 & 13,600 & 19,100 & 23,700 & 28,600 \\
\hline 4369 & 14.7 & 2,070 & 4,050 & 5,690 & 8,130 & 10,100 & 12,300 \\
\hline 4376 & 2,720 & 26,100 & 37,300 & 43,100 & 59,800 & 86,000 & 122,000 \\
\hline 4412 & 2,760 & 26,500 & 37,900 & 43,900 & 59,800 & 86,000 & 122,000 \\
\hline 4418 & 43.9 & 4,840 & 8,970 & 12,400 & 17,300 & 21,300 & 25,600 \\
\hline 4426 & 64.3 & 5,510 & 10,400 & 14,500 & 20,500 & 25,500 & 30,800 \\
\hline 4501 & 17.2 & 2,340 & 4,610 & 6,500 & 9,310 & 11,600 & 14,200 \\
\hline 4516 & 14.3 & 2,060 & 4,050 & 5,700 & 8,150 & 10,100 & 12,400 \\
\hline 4517 & 9.61 & 1,790 & 3,450 & 4,820 & 6,830 & 8,470 & 10,300 \\
\hline 4519 & 102 & 9,530 & 16,800 & 22,700 & 31,100 & 38,100 & 45,500 \\
\hline 4523 & 109 & 9,790 & 17,200 & 23,300 & 32,000 & 39,100 & 46,800 \\
\hline 4525 & 2,910 & 27,700 & 39,600 & 46,600 & 59,900 & 86,000 & 121,000 \\
\hline 4529 & 20.6 & 2,700 & 5,340 & 7,560 & 10,900 & 13,500 & 16,600 \\
\hline 4534 & 2,940 & 27,900 & 39,900 & 47,100 & 59,900 & 86,000 & 121,000 \\
\hline 4538 & 26.2 & 2,990 & 5,940 & 8,430 & 12,100 & 15,200 & 18,600 \\
\hline 4560 & 24.5 & 3,130 & 6,270 & 8,920 & 12,900 & 16,100 & 19,800 \\
\hline 4566 & 16.8 & 2,210 & 4,380 & 6,200 & 8,900 & 11,100 & 13,600 \\
\hline 4580 & 3,070 & 29,000 & 41,500 & 49,500 & 60,000 & 86,000 & 121,000 \\
\hline 4593 & 9.32 & 1,580 & 3,080 & 4,320 & 6,150 & 7,630 & 9,310 \\
\hline
\end{tabular}


Table 73. Estimated flow-duration values, mean flow values, and peak-discharge frequency values for controlled and uncontrolled flow stream segments on the 1999 Kansas Surface Water Register for Neosho County.-Continued

[KSWR, Kansas Surface Water Register; CUSEGA, catalog unit segment number alpha; $\mathrm{mi}^{2}$, square miles; $\mathrm{ft}^{3} / \mathrm{s}$, cubic feet per second; HYDRO, lake or other hydrologic structure; NA, not applicable; NRDitch, irrigation ditch; NRTribal, tribal stream]

\begin{tabular}{|c|c|c|c|c|c|c|c|c|c|c|c|}
\hline $\begin{array}{l}\text { Determi- } \\
\text { nation site } \\
\text { identi- } \\
\text { fication } \\
\text { number } \\
\text { (fig. } 77 \text { ) }\end{array}$ & $\begin{array}{l}\text { KSWR } \\
\text { CUSEGA } \\
\text { number }\end{array}$ & \multicolumn{3}{|c|}{$\begin{array}{l}\text { Stream segment } \\
\text { by county } \\
\text { (table 112) }\end{array}$} & Stream name & $\begin{array}{l}\text { Contri- } \\
\text { buting } \\
\text { drainage } \\
\text { area } \\
\left(\mathrm{mi}^{2}\right)\end{array}$ & \multicolumn{5}{|c|}{$\begin{array}{c}\text { Estimated flow-duration values }\left(\mathrm{ft}^{3} / \mathrm{s}\right) \text { for } \\
\text { indicated percentage of time flow equaled or } \\
\text { exceeded }\end{array}$} \\
\hline 4601 & HYDRO & $\mathrm{NO}$ & & & HYDRO & 9.53 & NA & NA & NA & NA & NA \\
\hline 4671 & 1107020522 & NO & & & Labette Creek & 26.2 & 0 & .48 & 3.58 & 12.4 & 34.6 \\
\hline 4709 & HYDRO & NO & & & HYDRO & 6.10 & NA & NA & NA & NA & NA \\
\hline 4713 & 1107010337 & NO & WL & & Dry Creek & 24.1 & 0 & .76 & 4.01 & 12.8 & 33.7 \\
\hline 4738 & HYDRO & NO & & & HYDRO & 34.0 & NA & NA & NA & NA & NA \\
\hline
\end{tabular}


Table 73. Estimated flow-duration values, mean flow values, and peak-discharge frequency values for controlled and uncontrolled flow stream segments on the 1999 Kansas Surface Water Register for Neosho County.-Continued

[KSWR, Kansas Surface Water Register; CUSEGA, catalog unit segment number alpha; $\mathrm{mi}^{2}$, square miles; $\mathrm{ft}^{3} / \mathrm{s}$, cubic feet per second; HYDRO, lake or other hydrologic structure; NA, not applicable; NRDitch, irrigation ditch; NRTribal, tribal stream]

\begin{tabular}{|c|c|c|c|c|c|c|c|}
\hline \multirow{2}{*}{$\begin{array}{l}\text { Determi- } \\
\text { nation site } \\
\text { identi- } \\
\text { fication } \\
\text { number } \\
\text { (fig. 77) }\end{array}$} & \multirow{2}{*}{$\begin{array}{c}\text { Estimated mean } \\
\text { flow } \\
\left(\mathrm{ft}^{3} / \mathrm{s}\right)\end{array}$} & \multicolumn{6}{|c|}{ Estimated peak discharge $\left(\mathrm{ft}^{3} / \mathrm{s}\right)$ for indicated peak-discharge frequency } \\
\hline & & 2-year & 5-year & 10-year & 25-year & 50-year & 100-year \\
\hline 4601 & NA & NA & NA & NA & $\mathrm{NA}$ & NA & NA \\
\hline 4671 & 25.3 & 2,970 & 5,900 & 8,370 & 12,000 & 15,000 & 18,500 \\
\hline 4709 & NA & NA & NA & NA & NA & NA & NA \\
\hline 4713 & 23.8 & 2,760 & 5,540 & 7,890 & 11,400 & 14,300 & 17,500 \\
\hline 4738 & NA & NA & NA & NA & NA & NA & NA \\
\hline
\end{tabular}




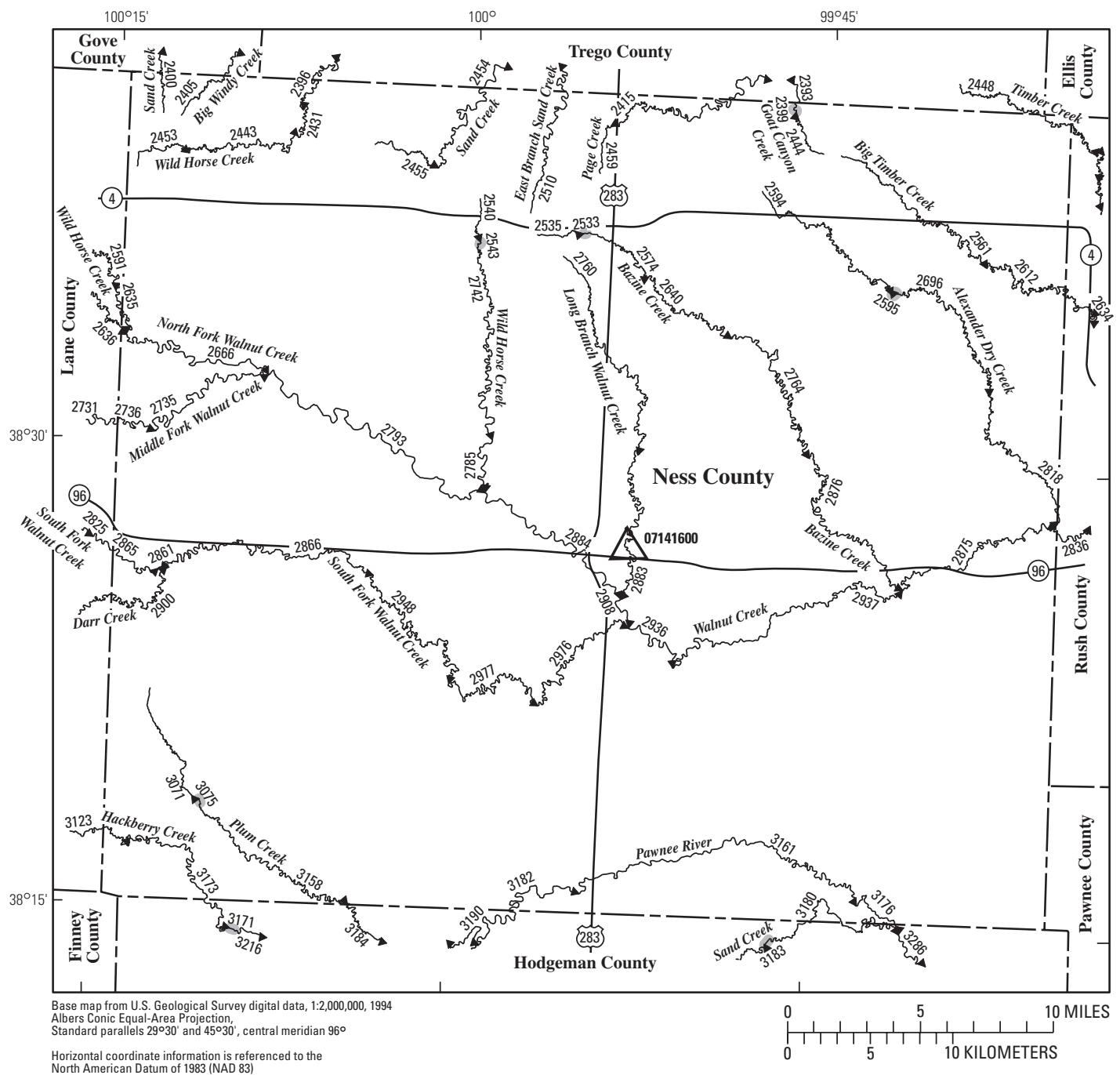

EXPLANATION

$\$ 116$ Location of streamflow-statistics determination site (small triangle) and associated identification number-small triangle points in downstream direction

07141600 U.S. Geological Survey streamflow-gaging station and number used for estimates of flow duration

U.S. Geological Survey streamflow-gaging station and number used for estimates of peak-discharge frequency values

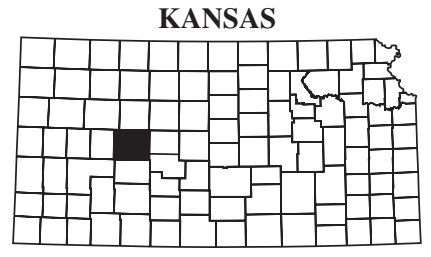

Index map 3171

Lake and determination site identification number

Figure 78. Location of streamflow-statistics determination sites, associated identification numbers, and U.S. Geological Survey streamflow-gaging stations used in the flow-duration and peak-discharge frequency analyses for Ness County. 
Table 74. Estimated flow-duration values, mean flow values, and peak-discharge frequency values for controlled and uncontrolled flow stream segments on the 1999 Kansas Surface Water Register for Ness County.

[KSWR, Kansas Surface Water Register; CUSEGA, catalog unit segment number alpha; $\mathrm{mi}^{2}$, square miles; $\mathrm{ft}^{3} / \mathrm{s}$, cubic feet per second; HYDRO, lake or other hydrologic structure; NA, not applicable; NRDitch, irrigation ditch; NRTribal, tribal stream]

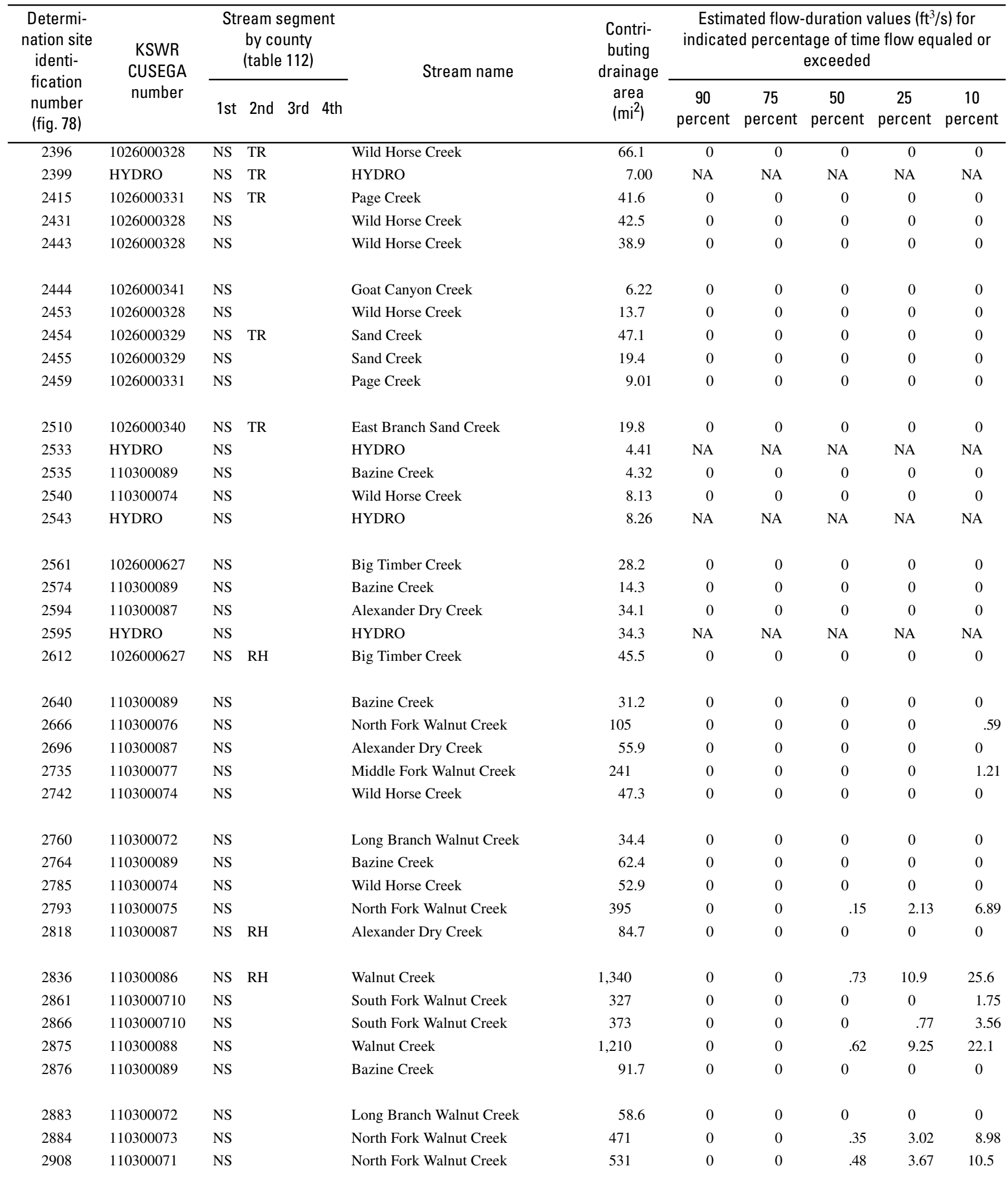


Table 74. Estimated flow-duration values, mean flow values, and peak-discharge frequency values for controlled and uncontrolled flow stream segments on the 1999 Kansas Surface Water Register for Ness County.-Continued

[KSWR, Kansas Surface Water Register; CUSEGA, catalog unit segment number alpha; $\mathrm{mi}^{2}$, square miles; $\mathrm{ft}^{3} / \mathrm{s}$, cubic feet per second; HYDRO, lake or other hydrologic structure; NA, not applicable; NRDitch, irrigation ditch; NRTribal, tribal stream]

\begin{tabular}{|c|c|c|c|c|c|c|c|}
\hline \multirow{2}{*}{$\begin{array}{l}\text { Determi- } \\
\text { nation site } \\
\text { identi- } \\
\text { fication } \\
\text { number } \\
\text { (fig. 78) }\end{array}$} & \multirow{2}{*}{$\begin{array}{c}\text { Estimated mean } \\
\text { flow } \\
\left(\mathrm{ft}^{3} / \mathrm{s}\right)\end{array}$} & \multicolumn{6}{|c|}{ Estimated peak discharge $\left(\mathrm{ft}^{3} / \mathrm{s}\right)$ for indicated peak-discharge frequency } \\
\hline & & 2-year & 5-year & 10-year & 25-year & 50-year & 100-year \\
\hline 2396 & 1.71 & 446 & 1,380 & 2,410 & 4,220 & 5,970 & 8,050 \\
\hline 2431 & .63 & 334 & 1,060 & 1,880 & 3,330 & 4,730 & 6,410 \\
\hline 2443 & .45 & 328 & 1,040 & 1,840 & 3,250 & 4,620 & 6,250 \\
\hline 2444 & 0 & 198 & 605 & 1,030 & 1,750 & 2,410 & 3,190 \\
\hline 2453 & 0 & 341 & 1,040 & 1,760 & 3,000 & 4,130 & 5,480 \\
\hline 2510 & 0 & 378 & 1,190 & 2,060 & 3,550 & 4,920 & 6,580 \\
\hline 2533 & NA & NA & NA & NA & NA & NA & NA \\
\hline 2535 & 0 & 153 & 470 & 799 & 1,360 & 1,870 & 2,480 \\
\hline 2540 & 0 & 228 & 705 & 1,200 & 2,050 & 2,830 & 3,760 \\
\hline 2543 & NA & NA & NA & NA & NA & NA & NA \\
\hline 2561 & .04 & 503 & 1,570 & 2,690 & 4,630 & 6,410 & 8,550 \\
\hline 2574 & 0 & 308 & 969 & 1,670 & 2,870 & 3,980 & 5,310 \\
\hline 2594 & .11 & 251 & 836 & 1,510 & 2,720 & 3,910 & 5,350 \\
\hline 2595 & NA & NA & NA & NA & NA & NA & NA \\
\hline 2760 & 0 & 185 & 672 & 1,280 & 2,460 & 3,700 & 5,270 \\
\hline 2764 & .89 & 364 & 1,180 & 2,110 & 3,760 & 5,390 & 7,350 \\
\hline 2785 & .64 & 304 & 1,000 & 1,800 & 3,230 & 4,640 & 6,350 \\
\hline 2793 & 9.37 & 885 & 2,680 & 4,660 & 8,160 & 11,600 & 15,700 \\
\hline 2818 & 2.22 & 462 & 1,450 & 2,560 & 4,530 & 6,450 & 8,750 \\
\hline 2836 & 22.5 & 993 & 2,430 & 3,820 & 6,100 & 8,190 & 10,600 \\
\hline 2861 & 5.10 & 747 & 2,300 & 4,030 & 7,100 & 10,100 & 13,800 \\
\hline 2866 & 6.65 & 820 & 2,490 & 4,340 & 7,610 & 10,800 & 14,700 \\
\hline 2875 & 20.5 & 952 & 2,390 & 3,820 & 6,210 & 8,450 & 11,100 \\
\hline 2876 & 1.94 & 426 & 1,370 & 2,450 & 4,370 & 6,270 & 8,560 \\
\hline 2883 & .71 & 75 & 433 & 1,000 & 2,310 & 3,840 & 5,950 \\
\hline 2884 & 11.2 & 973 & 2,900 & 5,010 & 8,730 & 12,300 & 16,700 \\
\hline 2908 & 12.4 & 679 & 1,970 & 3,420 & 6,100 & 8,820 & 12,200 \\
\hline
\end{tabular}


Table 74. Estimated flow-duration values, mean flow values, and peak-discharge frequency values for controlled and uncontrolled flow stream segments on the 1999 Kansas Surface Water Register for Ness County.-Continued

[KSWR, Kansas Surface Water Register; CUSEGA, catalog unit segment number alpha; $\mathrm{mi}^{2}$, square miles; $\mathrm{ft}^{3} / \mathrm{s}$, cubic feet per second; HYDRO, lake or other hydrologic structure; NA, not applicable; NRDitch, irrigation ditch; NRTribal, tribal stream]

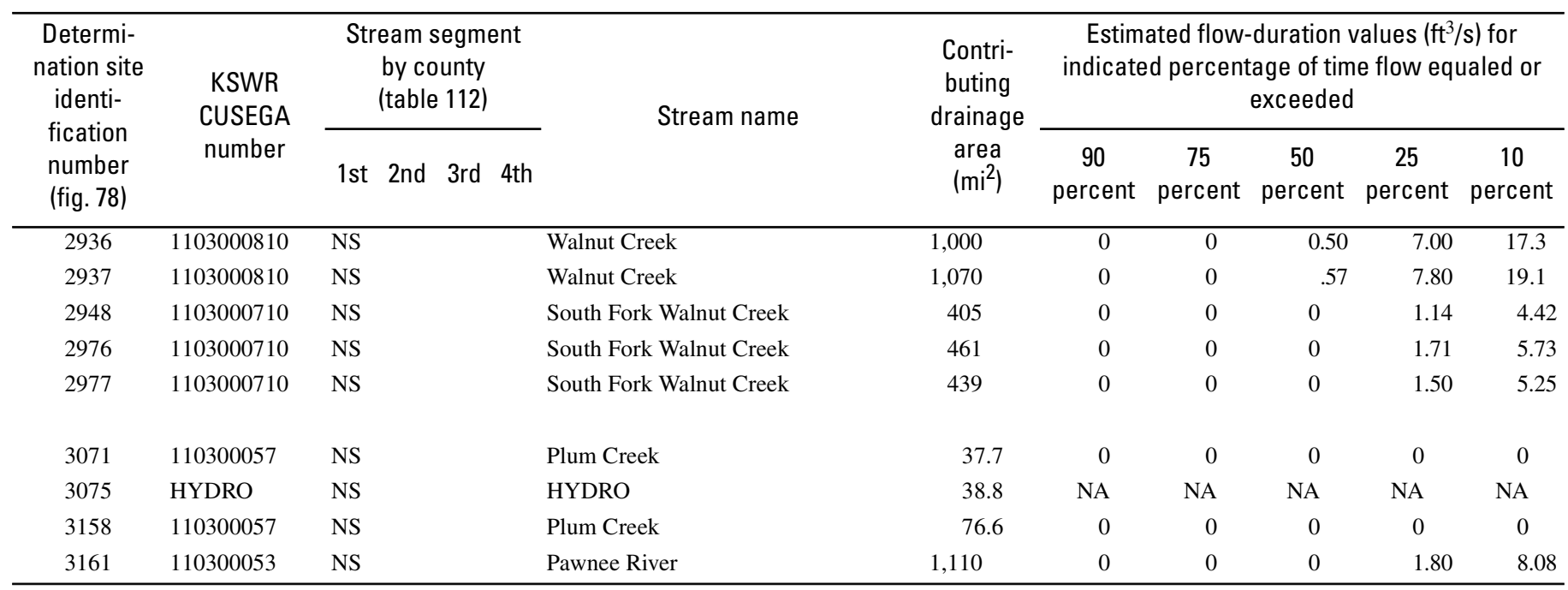


Table 74. Estimated flow-duration values, mean flow values, and peak-discharge frequency values for controlled and uncontrolled flow stream segments on the 1999 Kansas Surface Water Register for Ness County.-Continued

[KSWR, Kansas Surface Water Register; CUSEGA, catalog unit segment number alpha; $\mathrm{mi}^{2}$, square miles; $\mathrm{ft}^{3} / \mathrm{s}$, cubic feet per second; HYDRO, lake or other hydrologic structure; NA, not applicable; NRDitch, irrigation ditch; NRTribal, tribal stream]

\begin{tabular}{|c|c|c|c|c|c|c|c|}
\hline \multirow{2}{*}{$\begin{array}{l}\text { Determi- } \\
\text { nation site } \\
\text { identi- } \\
\text { fication } \\
\text { number } \\
\text { (fig. 78) }\end{array}$} & \multirow[b]{2}{*}{$\begin{array}{c}\text { Estimated mean } \\
\text { flow } \\
\left(\mathrm{ft}^{3} / \mathrm{s}\right)\end{array}$} & \multicolumn{6}{|c|}{ Estimated peak discharge $\left(\mathrm{ft}^{3} / \mathrm{s}\right)$ for indicated peak-discharge frequency } \\
\hline & & 2-year & 5-year & 10-year & 25-year & 50-year & 100-year \\
\hline 2936 & 17.6 & 929 & 2,410 & 3,930 & 6,540 & 9,050 & 12,100 \\
\hline 2976 & 8.65 & 859 & 2,600 & 4,530 & 7,940 & 11,300 & 15,300 \\
\hline 2977 & 8.20 & 861 & 2,600 & 4,530 & 7,940 & 11,300 & 15,300 \\
\hline 3071 & .13 & 406 & 1,240 & 2,130 & 3,680 & 5,170 & 6,910 \\
\hline 3075 & NA & NA & NA & NA & NA & NA & NA \\
\hline
\end{tabular}




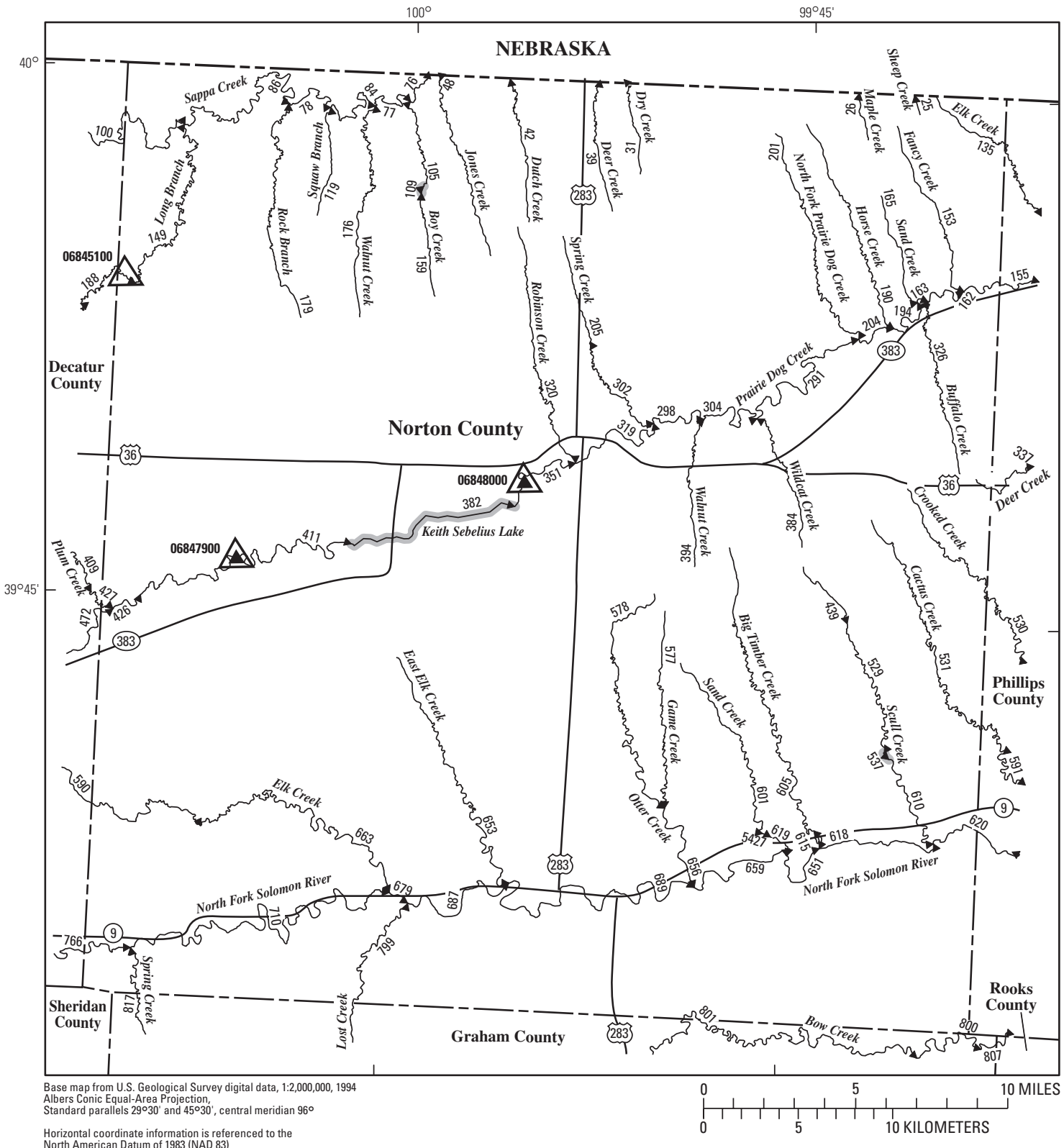

EXPLANATION

466 Location of streamflow-statistics determination site (small triangle) and associated identification number-small triangle points in downstream direction

06847900 U.S. Geological Survey streamflow-gaging station and number used for estimates of flow duration

$06845100 \bigwedge$ U.S. Geological Survey streamflow-gaging station and number used for estimates of peak-discharge frequency values

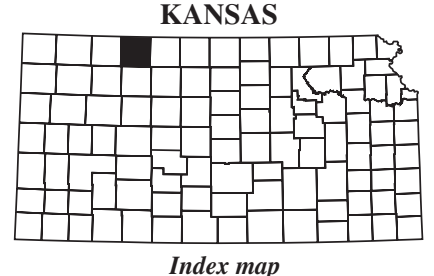
382

Lake and determination site identification number

Figure 79. Location of streamflow-statistics determination sites, associated identification numbers, and U.S. Geological Survey streamflow-gaging stations used in the flow-duration and peak-discharge frequency analyses for Norton County. 
Table 75. Estimated flow-duration values, mean flow values, and peak-discharge frequency values for controlled and uncontrolled flow stream segments on the 1999 Kansas Surface Water Register for Norton County.

[KSWR, Kansas Surface Water Register; CUSEGA, catalog unit segment number alpha; $\mathrm{mi}^{2}$, square miles; $\mathrm{ft}^{3} / \mathrm{s}$, cubic feet per second; HYDRO, lake or other hydrologic structure; NA, not applicable; NRDitch, irrigation ditch; NRTribal, tribal stream]

\begin{tabular}{|c|c|c|c|c|c|c|c|c|c|c|c|}
\hline \multirow{2}{*}{$\begin{array}{l}\text { Determi- } \\
\text { nation site } \\
\text { identi- } \\
\text { fication } \\
\text { number } \\
\text { (fig. 79) }\end{array}$} & \multirow{2}{*}{$\begin{array}{l}\text { KSWR } \\
\text { CUSEGA } \\
\text { number }\end{array}$} & \multicolumn{3}{|c|}{$\begin{array}{l}\text { Stream segment } \\
\text { by county } \\
\text { (table 112) }\end{array}$} & \multirow{2}{*}{ Stream name } & \multirow{2}{*}{$\begin{array}{l}\text { Contri- } \\
\text { buting } \\
\text { drainage } \\
\text { area } \\
\left(\mathrm{mi}^{2}\right)\end{array}$} & \multicolumn{5}{|c|}{$\begin{array}{l}\text { Estimated flow-duration values }\left(\mathrm{ft}^{3} / \mathrm{s}\right) \text { for } \\
\text { indicated percentage of time flow equaled or } \\
\text { exceeded }\end{array}$} \\
\hline & & $1 \mathrm{st}$ & 2nd & 3rd 4th & & & $\begin{array}{c}90 \\
\text { percent }\end{array}$ & $\begin{array}{c}75 \\
\text { percent }\end{array}$ & $\begin{array}{c}50 \\
\text { percent }\end{array}$ & $\begin{array}{c}25 \\
\text { percent }\end{array}$ & $\begin{array}{c}10 \\
\text { percent }\end{array}$ \\
\hline 25 & 1025001119 & NT & & & Sheep Creek & 1.36 & $\overline{0}$ & 0 & 0 & 0 & 0 \\
\hline 26 & 1025001118 & NT & & & Maple Creek & 3.86 & 0 & 0 & 0 & 0 & 0 \\
\hline 31 & 102500118 & NT & & & Dry Creek & 14.8 & 0 & 0 & 0 & 0 & 0 \\
\hline 39 & 102500117 & NT & & & Deer Creek & 15.1 & 0 & 0 & 0 & 0 & 0 \\
\hline 42 & 1025001116 & NT & & & Dutch Creek & 10.8 & 0 & 0 & 0 & 0 & 0 \\
\hline 48 & 1025001117 & NT & & & Jones Creek & 11.8 & 0 & 0 & 0 & 0 & 0 \\
\hline 76 & 102500113 & NT & & & Sappa Creek & 1,490 & 0 & 3.55 & 11.3 & 34.1 & 101 \\
\hline 77 & 102500113 & NT & & & Sappa Creek & 1,480 & 0 & 3.35 & 10.7 & 32.5 & 96.6 \\
\hline 78 & 102500113 & NT & & & Sappa Creek & 1,440 & 0 & 2.98 & 9.56 & 29.5 & 88.1 \\
\hline 84 & 102500113 & NT & & & Sappa Creek & 1,450 & 0 & 3.09 & 9.89 & 30.4 & 90.6 \\
\hline 86 & 102500113 & NT & & & Sappa Creek & 1,410 & 0 & 2.61 & 8.46 & 26.5 & 79.5 \\
\hline 105 & 1025001113 & NT & & & Boy Creek & 17.6 & 0 & 0 & 0 & 0 & .01 \\
\hline 109 & HYDRO & NT & & & HYDRO & 14.0 & NA & NA & NA & NA & NA \\
\hline 119 & 1025001112 & NT & & & Squaw Branch & 6.02 & 0 & 0 & 0 & 0 & 0 \\
\hline 135 & 102500153 & NT & PL & & Elk Creek & 32.2 & 0 & 0 & 0 & 0 & 0 \\
\hline 149 & 102500115 & NT & & & Long Branch & 77.8 & 0 & 0 & .01 & .11 & 1.53 \\
\hline 153 & 1025001519 & NT & & & Fancy Creek & 11.2 & 0 & 0 & 0 & 0 & 0 \\
\hline 155 & 102500152 & NT & PL & & Prairie Dog Creek & 850 & 0 & .01 & 1.45 & 5.47 & 12.3 \\
\hline 159 & 1025001113 & NT & & & Boy Creek & 13.6 & 0 & 0 & 0 & 0 & 0 \\
\hline 162 & 102500152 & NT & & & Prairie Dog Creek & 824 & 0 & .01 & 1.27 & 4.81 & 11.1 \\
\hline 163 & 102500152 & NT & & & Prairie Dog Creek & 805 & 0 & .01 & 1.14 & 4.33 & 10.2 \\
\hline 165 & 1025001520 & NT & & & Sand Creek & 5.58 & 0 & 0 & 0 & 0 & 0 \\
\hline 176 & 1025001111 & NT & & & Walnut Creek & 22.1 & 0 & 0 & 0 & 0 & .01 \\
\hline 179 & 1025001110 & NT & & & Rock Branch & 32.9 & 0 & 0 & 0 & .01 & .03 \\
\hline 190 & 1025001518 & NT & & & Horse Creek & 12.0 & 0 & 0 & 0 & 0 & 0 \\
\hline 194 & 102500154 & NT & & & Prairie Dog Creek & 799 & 0 & .01 & 1.10 & 4.18 & 9.90 \\
\hline 201 & 1025001517 & NT & & & North Fork Prairie Dog Creek & 30.0 & 0 & 0 & 0 & 0 & 0 \\
\hline 204 & 102500154 & NT & & & Prairie Dog Creek & 786 & 0 & .01 & 1.01 & 3.85 & 9.29 \\
\hline 205 & 1025001515 & NT & & & Spring Creek & 14.0 & 0 & 0 & 0 & 0 & 0 \\
\hline 291 & 102500154 & NT & & & Prairie Dog Creek & 753 & 0 & .01 & .79 & 3.01 & 7.76 \\
\hline 298 & 102500154 & NT & & & Prairie Dog Creek & 708 & 0 & .02 & .48 & 1.87 & 5.66 \\
\hline 302 & 1025001515 & NT & & & Spring Creek & 21.0 & 0 & 0 & 0 & 0 & 0 \\
\hline 304 & 102500154 & NT & & & Prairie Dog Creek & 726 & 0 & .01 & .60 & 2.32 & 6.50 \\
\hline 319 & 102500154 & NT & & & Prairie Dog Creek & 681 & 0 & .02 & .29 & 1.18 & 4.40 \\
\hline 320 & 1025001516 & NT & & & Robinson Creek & 20.2 & 0 & 0 & 0 & 0 & 0 \\
\hline 326 & 1025001521 & NT & & & Buffalo Creek & 17.0 & 0 & 0 & 0 & 0 & 0 \\
\hline 337 & 1026001231 & NT & PL & & Deer Creek & 6.62 & 0 & 0 & .01 & .01 & .02 \\
\hline 351 & 102500154 & NT & & & Prairie Dog Creek & 653 & 0 & .02 & .10 & .47 & 3.10 \\
\hline
\end{tabular}


Table 75. Estimated flow-duration values, mean flow values, and peak-discharge frequency values for controlled and uncontrolled flow stream segments on the 1999 Kansas Surface Water Register for Norton County.-Continued

[KSWR, Kansas Surface Water Register; CUSEGA, catalog unit segment number alpha; $\mathrm{mi}^{2}$, square miles; $\mathrm{ft}^{3} / \mathrm{s}$, cubic feet per second; HYDRO, lake or other hydrologic structure; NA, not applicable; NRDitch, irrigation ditch; NRTribal, tribal stream]

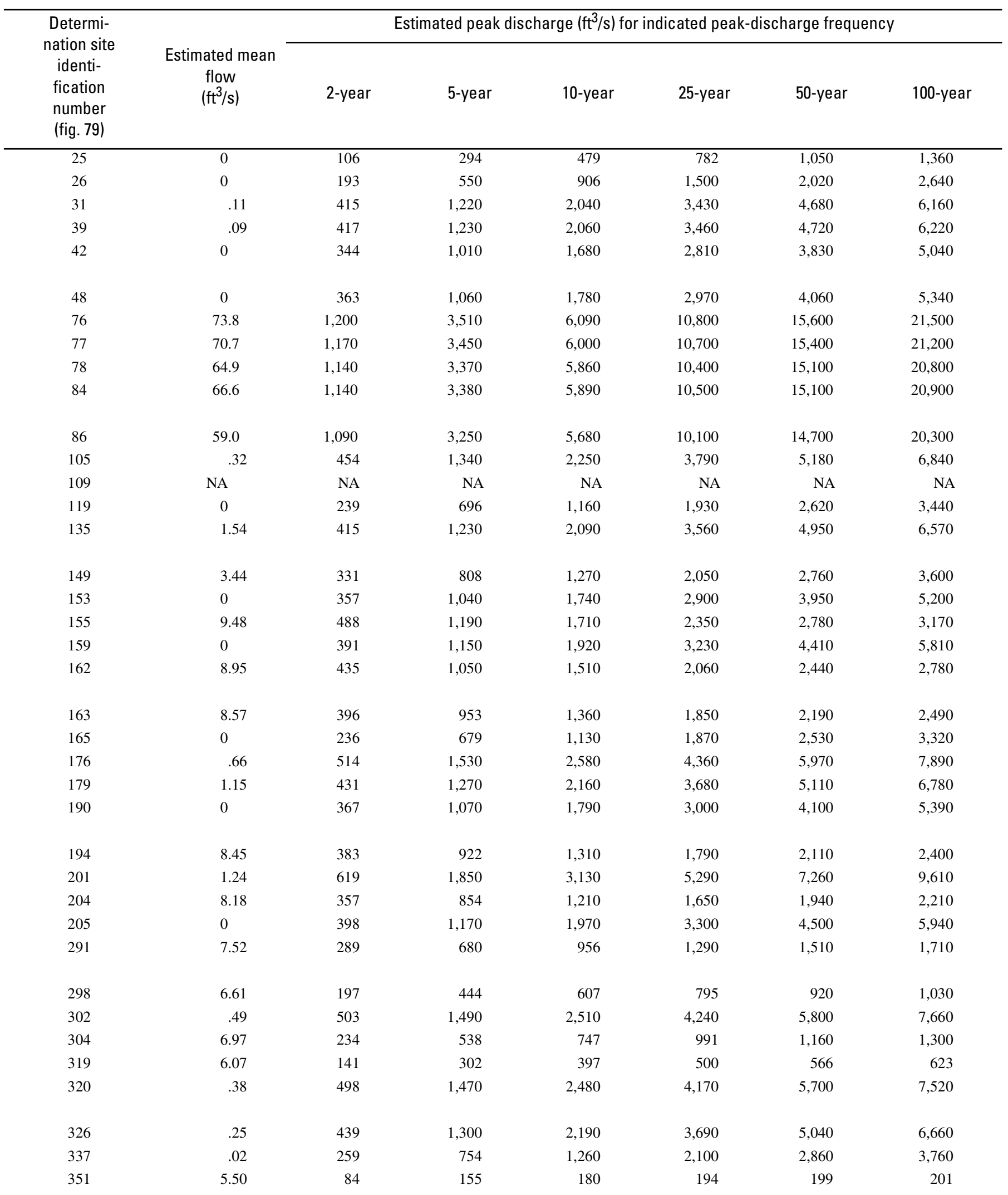


Table 75. Estimated flow-duration values, mean flow values, and peak-discharge frequency values for controlled and uncontrolled flow stream segments on the 1999 Kansas Surface Water Register for Norton County.-Continued

[KSWR, Kansas Surface Water Register; CUSEGA, catalog unit segment number alpha; $\mathrm{mi}^{2}$, square miles; $\mathrm{ft}^{3} / \mathrm{s}$, cubic feet per second; HYDRO, lake or other hydrologic structure; NA, not applicable; NRDitch, irrigation ditch; NRTribal, tribal stream]

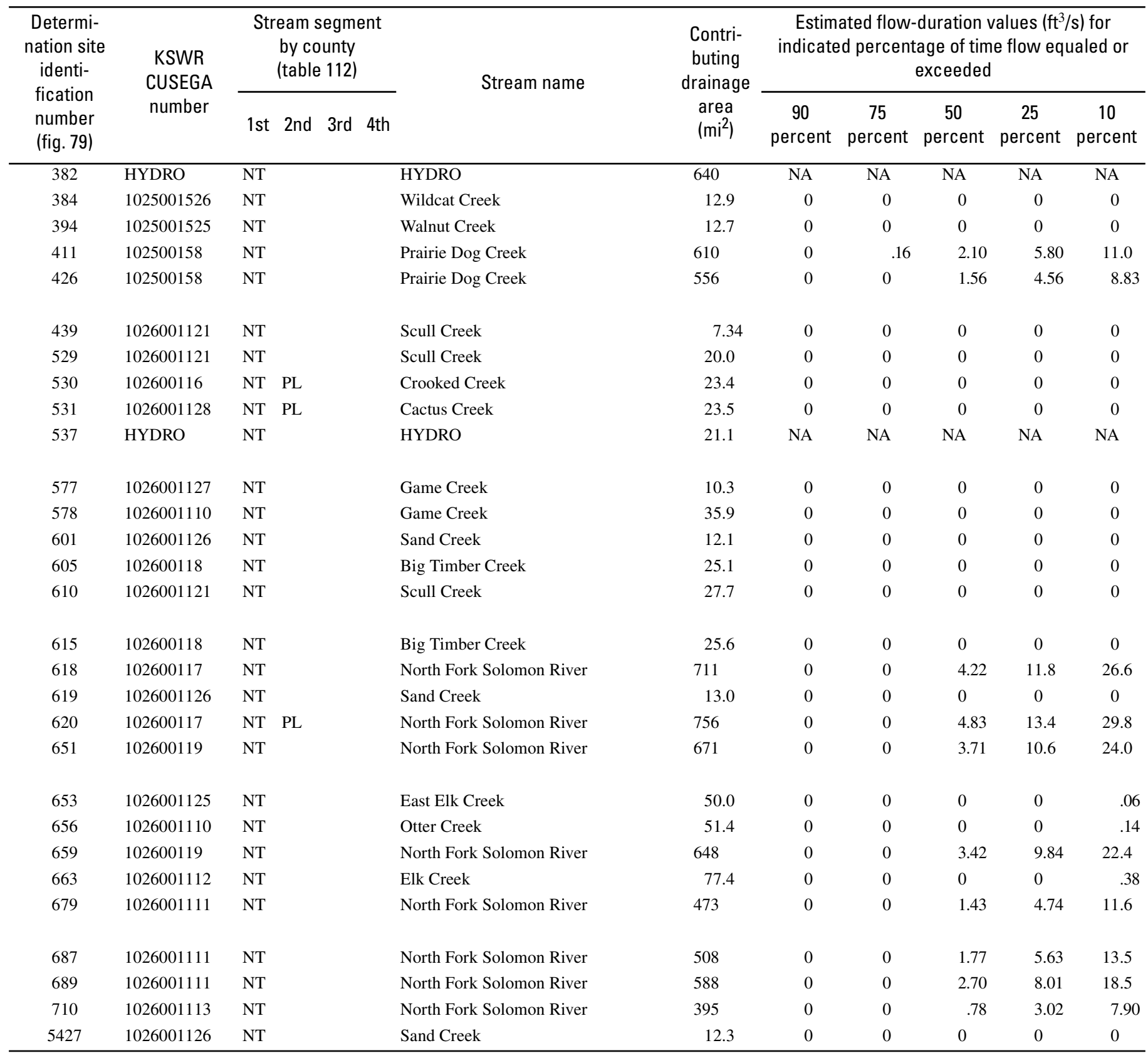


Table 75. Estimated flow-duration values, mean flow values, and peak-discharge frequency values for controlled and uncontrolled flow stream segments on the 1999 Kansas Surface Water Register for Norton County.-Continued

[KSWR, Kansas Surface Water Register; CUSEGA, catalog unit segment number alpha; $\mathrm{mi}^{2}$, square miles; $\mathrm{ft}^{3} / \mathrm{s}$, cubic feet per second; HYDRO, lake or other hydrologic structure; NA, not applicable; NRDitch, irrigation ditch; NRTribal, tribal stream]

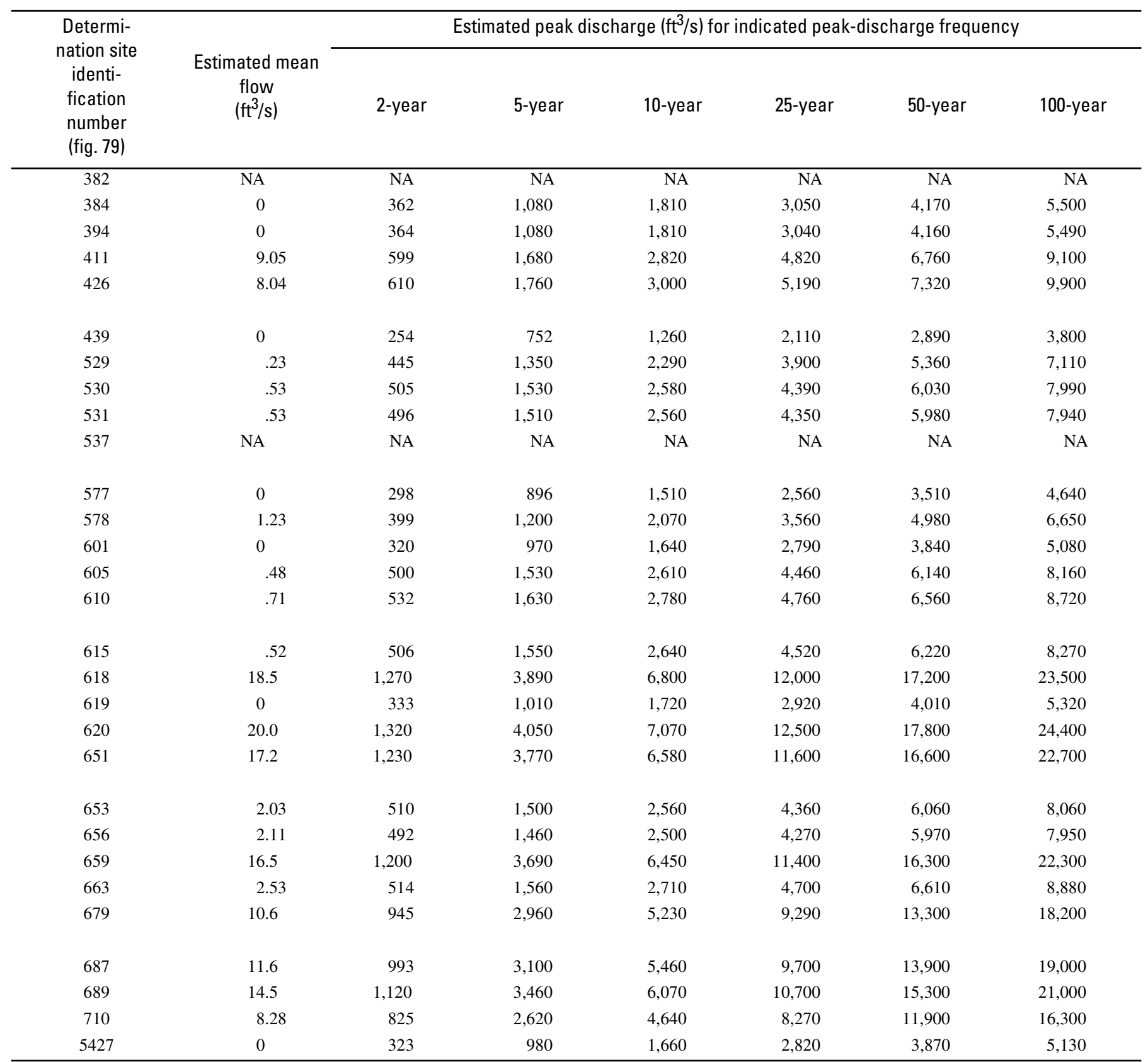




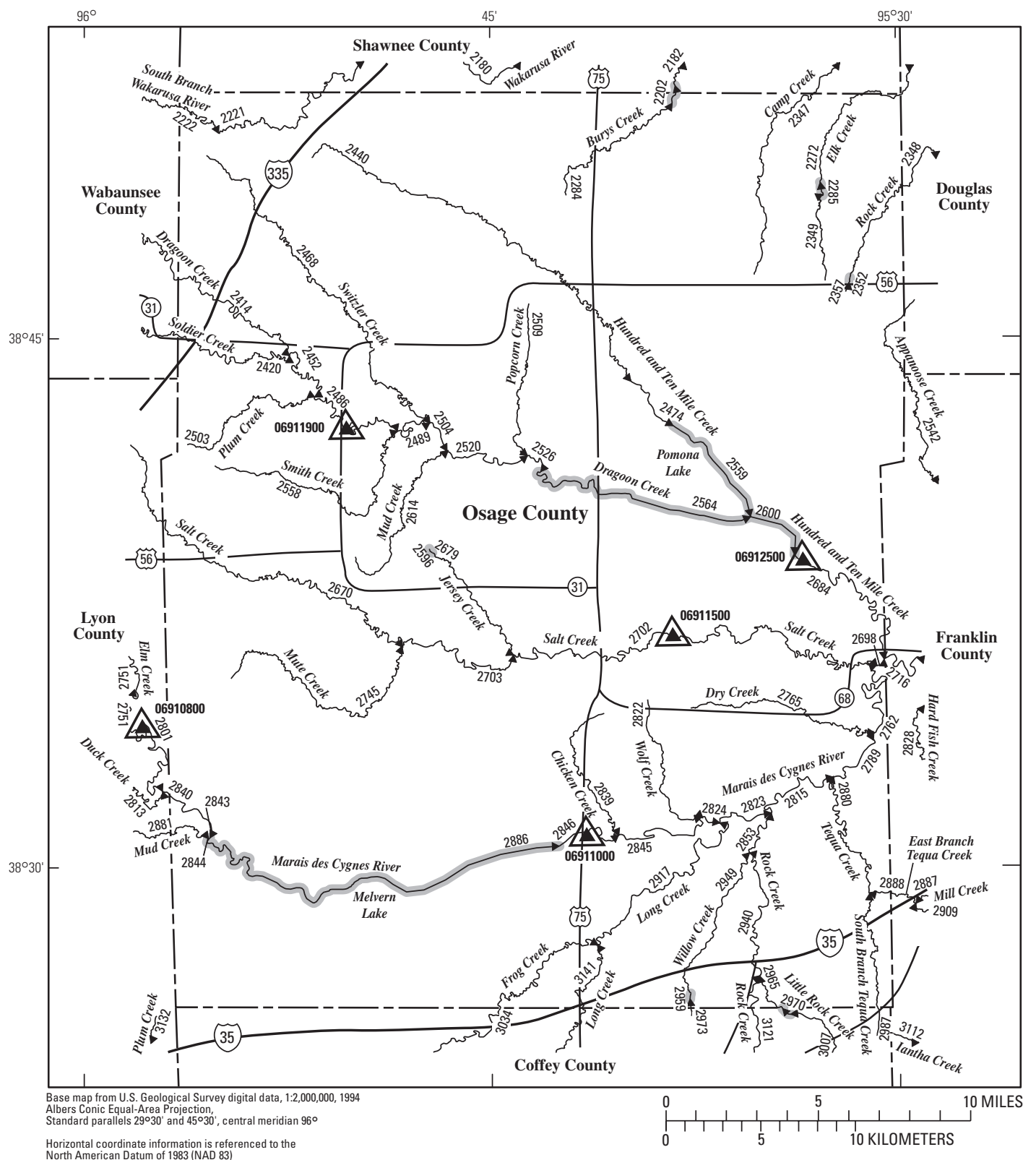

EXPLANATION

3034 Location of streamflow-statistics determination site (small triangle) and associated identification number-small triangle points in downstream direction

06910800

U.S. Geological Survey streamflow-gaging station and number used for estimates of flow duration

${ }^{06911000} \triangle$ U.S. Geological Survey streamflow-gaging station and number used for estimates of peak-discharge frequency values

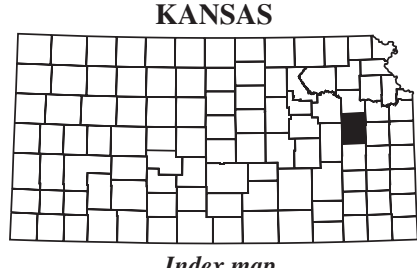

2886

Lake and determination site identification number

Figure 80. Location of streamflow-statistics determination sites, associated identification numbers, and U.S. Geological Survey streamflow-gaging stations used in the flow-duration and peak-discharge frequency analyses for Osage County. 
Table 76. Estimated flow-duration values, mean flow values, and peak-discharge frequency values for controlled and uncontrolled flow stream segments on the 1999 Kansas Surface Water Register for Osage County.

[KSWR, Kansas Surface Water Register; CUSEGA, catalog unit segment number alpha; $\mathrm{mi}^{2}$, square miles; $\mathrm{ft}^{3} / \mathrm{s}$, cubic feet per second; HYDRO, lake or other hydrologic structure; NA, not applicable; NRDitch, irrigation ditch; NRTribal, tribal stream]

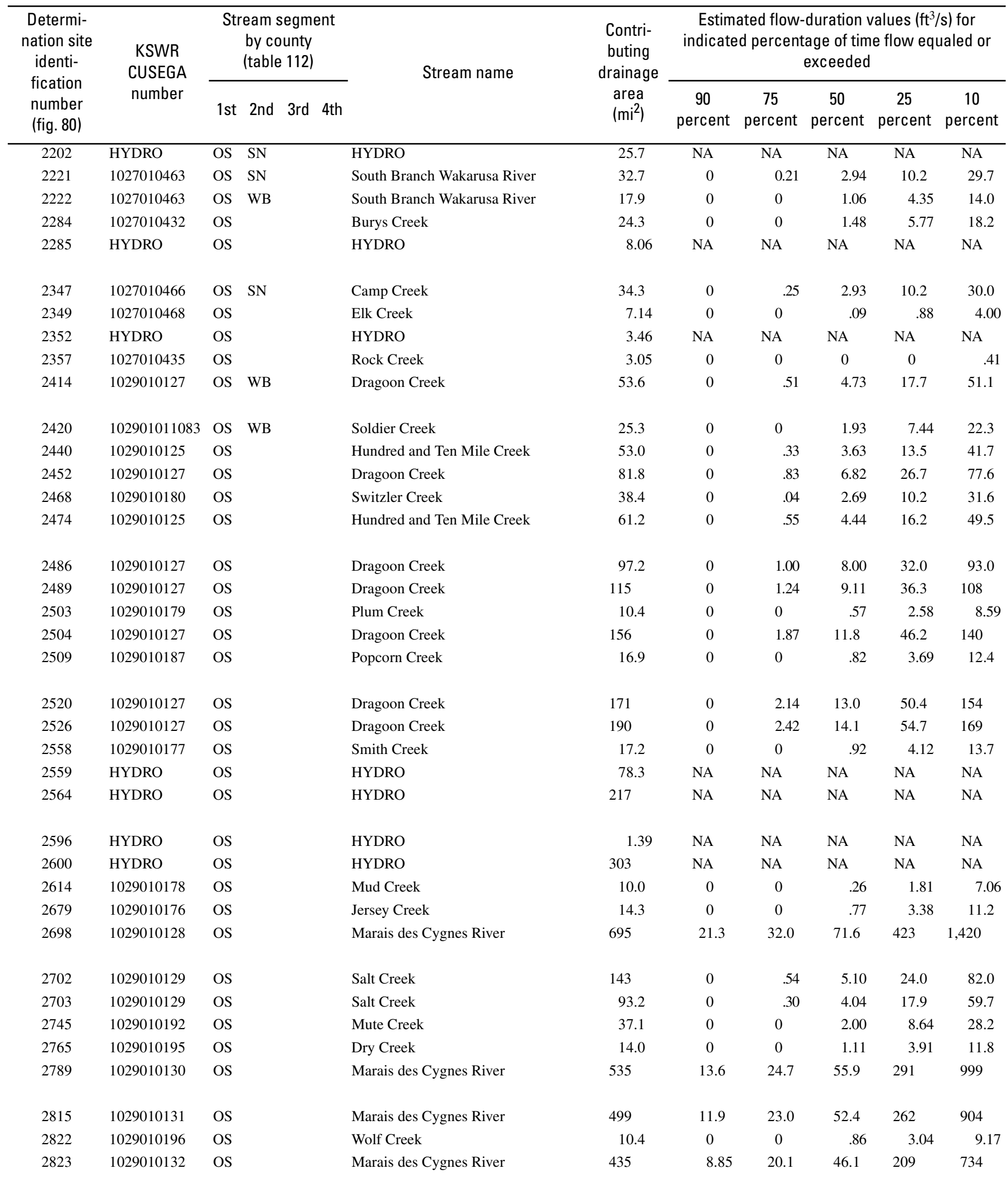


Table 76. Estimated flow-duration values, mean flow values, and peak-discharge frequency values for controlled and uncontrolled flow stream segments on the 1999 Kansas Surface Water Register for Osage County.-Continued

[KSWR, Kansas Surface Water Register; CUSEGA, catalog unit segment number alpha; $\mathrm{mi}^{2}$, square miles; $\mathrm{ft}^{3} / \mathrm{s}$, cubic feet per second; HYDRO, lake or other hydrologic structure; NA, not applicable; NRDitch, irrigation ditch; NRTribal, tribal stream]

\begin{tabular}{|c|c|c|c|c|c|c|c|}
\hline \multirow{2}{*}{$\begin{array}{l}\text { Determi- } \\
\text { nation site } \\
\text { identi- } \\
\text { fication } \\
\text { number } \\
\text { (fig. 80) }\end{array}$} & \multirow{2}{*}{$\begin{array}{c}\text { Estimated mean } \\
\text { flow } \\
\left(\mathrm{ft}^{3} / \mathrm{s}\right)\end{array}$} & \multicolumn{6}{|c|}{ Estimated peak discharge $\left(\mathrm{ft}^{3} / \mathrm{s}\right)$ for indicated peak-discharge frequency } \\
\hline & & 2-year & 5-year & 10-year & 25-year & 50-year & 100-year \\
\hline 2202 & NA & NA & NA & NA & NA & NA & NA \\
\hline 2284 & 15.4 & 2,040 & 4,420 & 6,490 & 9,660 & 12,300 & 15,300 \\
\hline 2285 & NA & NA & NA & NA & NA & NA & NA \\
\hline 2347 & 23.1 & 4,510 & 8,660 & 12,100 & 17,100 & 21,300 & 25,800 \\
\hline 2349 & 4.44 & 1,030 & 2,160 & 3,120 & 4,570 & 5,760 & 7,120 \\
\hline 2420 & 17.7 & 2,030 & 4,350 & 6,360 & 9,420 & 12,000 & 14,900 \\
\hline 2440 & 32.6 & 3,560 & 7,510 & 11,000 & 16,300 & 20,900 & 26,000 \\
\hline 2452 & 57.3 & 4,800 & 9,020 & 12,500 & 17,500 & 21,700 & 26,300 \\
\hline 2468 & 24.9 & 2,890 & 6,250 & 9,280 & 13,900 & 17,900 & 22,300 \\
\hline 2474 & 37.8 & 3,900 & 8,140 & 11,900 & 17,600 & 22,500 & 27,800 \\
\hline 2486 & 68.4 & 4,780 & 8,780 & 12,000 & 16,600 & 20,500 & 24,700 \\
\hline 2489 & 78.6 & 5,180 & 9,590 & 13,200 & 18,400 & 22,800 & 27,600 \\
\hline 2503 & 7.55 & 1,250 & 2,640 & 3,830 & 5,640 & 7,130 & 8,830 \\
\hline 2504 & 101 & 6,170 & 11,500 & 15,900 & 22,300 & 27,700 & 33,700 \\
\hline 2596 & NA & NA & NA & NA & NA & NA & NA \\
\hline 2600 & NA & NA & NA & NA & NA & NA & NA \\
\hline 2614 & 6.83 & 1,260 & 2,650 & 3,840 & 5,650 & 7,140 & 8,840 \\
\hline 2679 & 9.84 & 1,540 & 3,270 & 4,760 & 7,020 & 8,890 & 11,000 \\
\hline 2698 & 488 & 8,980 & 17,600 & 25,000 & 36,200 & 45,900 & 57,000 \\
\hline 2702 & 66.0 & 4,270 & 9,170 & 13,400 & 19,800 & 25,200 & 31,200 \\
\hline 2703 & 48.5 & 4,330 & 9,140 & 13,300 & 19,700 & 25,200 & 31,200 \\
\hline 2745 & 23.4 & 3,920 & 8,010 & 11,600 & 16,800 & 21,300 & 26,200 \\
\hline 2765 & 9.82 & 1,510 & 3,220 & 4,690 & 6,920 & 8,770 & 10,900 \\
\hline 2789 & 374 & 8,190 & 17,500 & 25,900 & 39,300 & 51,300 & 65,100 \\
\hline 2815 & 348 & 8,020 & 17,400 & 26,100 & 40,000 & 52,500 & 66,900 \\
\hline 2822 & 7.73 & 1,290 & 2,720 & 3,950 & 5,810 & 7,340 & 9,090 \\
\hline 2823 & 303 & 7,700 & 17,400 & 26,500 & 41,200 & 54,600 & 70,200 \\
\hline
\end{tabular}


Table 76. Estimated flow-duration values, mean flow values, and peak-discharge frequency values for controlled and uncontrolled flow stream segments on the 1999 Kansas Surface Water Register for Osage County.-Continued

[KSWR, Kansas Surface Water Register; CUSEGA, catalog unit segment number alpha; $\mathrm{mi}^{2}$, square miles; $\mathrm{ft}^{3} / \mathrm{s}$, cubic feet per second; HYDRO, lake or other hydrologic structure; NA, not applicable; NRDitch, irrigation ditch; NRTribal, tribal stream]

\begin{tabular}{|c|c|c|c|c|c|c|c|c|c|c|}
\hline $\begin{array}{l}\text { Determi- } \\
\text { nation site } \\
\text { identi- } \\
\text { fication } \\
\text { number } \\
\text { (fig. 80) }\end{array}$ & $\begin{array}{l}\text { KSWR } \\
\text { CUSEGA } \\
\text { number }\end{array}$ & \multicolumn{2}{|c|}{$\begin{array}{l}\text { Stream segment } \\
\text { by county } \\
\text { (table 112) }\end{array}$} & Stream name & $\begin{array}{l}\text { Contri- } \\
\text { buting } \\
\text { drainage } \\
\text { area } \\
\left(\mathrm{mi}^{2}\right)\end{array}$ & \multicolumn{5}{|c|}{$\begin{array}{l}\text { Estimated flow-duration values }\left(\mathrm{ft}^{3} / \mathrm{s}\right) \text { for } \\
\text { indicated percentage of time flow equaled or } \\
\text { exceeded }\end{array}$} \\
\hline 2824 & 1029010133 & OS & & Marais des Cygnes River & 331 & 3.89 & 15.3 & 35.9 & 123 & 459 \\
\hline 2844 & HYDRO & OS & & HYDRO & 20.2 & NA & NA & NA & NA & NA \\
\hline 2845 & 1029010133 & OS & & Marais des Cygnes River & 320 & 3.36 & 14.8 & 34.8 & 114 & 430 \\
\hline 2846 & 1029010133 & OS & & Marais des Cygnes River & 302 & 2.50 & 14.0 & 33.0 & 99.0 & 382 \\
\hline 2853 & 1029010143 & OS & & Rock Creek & 58.5 & 0 & .51 & 4.04 & 14.8 & 45.5 \\
\hline 2940 & 1029010143 & OS & & Rock Creek & 41.0 & 0 & .16 & 2.74 & 10.1 & 31.3 \\
\hline 2949 & 1029010194 & OS & & Willow Creek & 15.4 & 0 & 0 & .97 & 3.84 & 12.2 \\
\hline 2959 & HYDRO & OS & & HYDRO & 5.67 & NA & NA & NA & NA & NA \\
\hline 2965 & 1029010173 & OS & & Little Rock Creek & 11.1 & 0 & 0 & .37 & 1.92 & 7.08 \\
\hline 2970 & HYDRO & OS & & HYDRO & 9.35 & NA & NA & NA & NA & NA \\
\hline
\end{tabular}


Table 76. Estimated flow-duration values, mean flow values, and peak-discharge frequency values for controlled and uncontrolled flow stream segments on the 1999 Kansas Surface Water Register for Osage County.-Continued

[KSWR, Kansas Surface Water Register; CUSEGA, catalog unit segment number alpha; $\mathrm{mi}^{2}$, square miles; $\mathrm{ft}^{3} / \mathrm{s}$, cubic feet per second; HYDRO, lake or other hydrologic structure; NA, not applicable; NRDitch, irrigation ditch; NRTribal, tribal stream]

\begin{tabular}{|c|c|c|c|c|c|c|c|}
\hline \multirow{2}{*}{$\begin{array}{l}\text { Determi- } \\
\text { nation site } \\
\text { identi- } \\
\text { fication } \\
\text { number } \\
\text { (fig. 80) }\end{array}$} & \multirow{2}{*}{$\begin{array}{l}\text { Estimated mean } \\
\text { flow } \\
\left(\mathrm{ft}^{3} / \mathrm{s}\right)\end{array}$} & \multicolumn{6}{|c|}{ Estimated peak discharge $\left(\mathrm{ft}^{3} / \mathrm{s}\right)$ for indicated peak-discharge frequency } \\
\hline & & 2-year & 5-year & 10-year & 25-year & 50-year & 100-year \\
\hline 2824 & 228 & 7,190 & 17,300 & 27,100 & 43,200 & 58,000 & 75,400 \\
\hline 2844 & NA & NA & NA & NA & NA & NA & NA \\
\hline 2845 & 220 & 7,140 & 17,300 & 27,200 & 43,500 & 58,400 & 76,000 \\
\hline 2846 & 208 & 7,050 & 17,300 & 27,300 & 43,800 & 59,000 & 76,900 \\
\hline 2853 & 35.8 & 5,270 & 10,200 & 14,400 & 20,500 & 25,700 & 31,300 \\
\hline 2940 & 25.4 & 4,360 & 8,550 & 12,100 & 17,300 & 21,700 & 26,400 \\
\hline 2949 & 10.6 & 1,650 & 3,490 & 5,080 & 7,480 & 9,480 & 11,800 \\
\hline 2959 & NA & NA & NA & NA & NA & NA & NA \\
\hline 2965 & 7.02 & 1,360 & 2,860 & 4,140 & 6,090 & 7,700 & 9,530 \\
\hline 2970 & NA & NA & NA & NA & NA & NA & NA \\
\hline
\end{tabular}




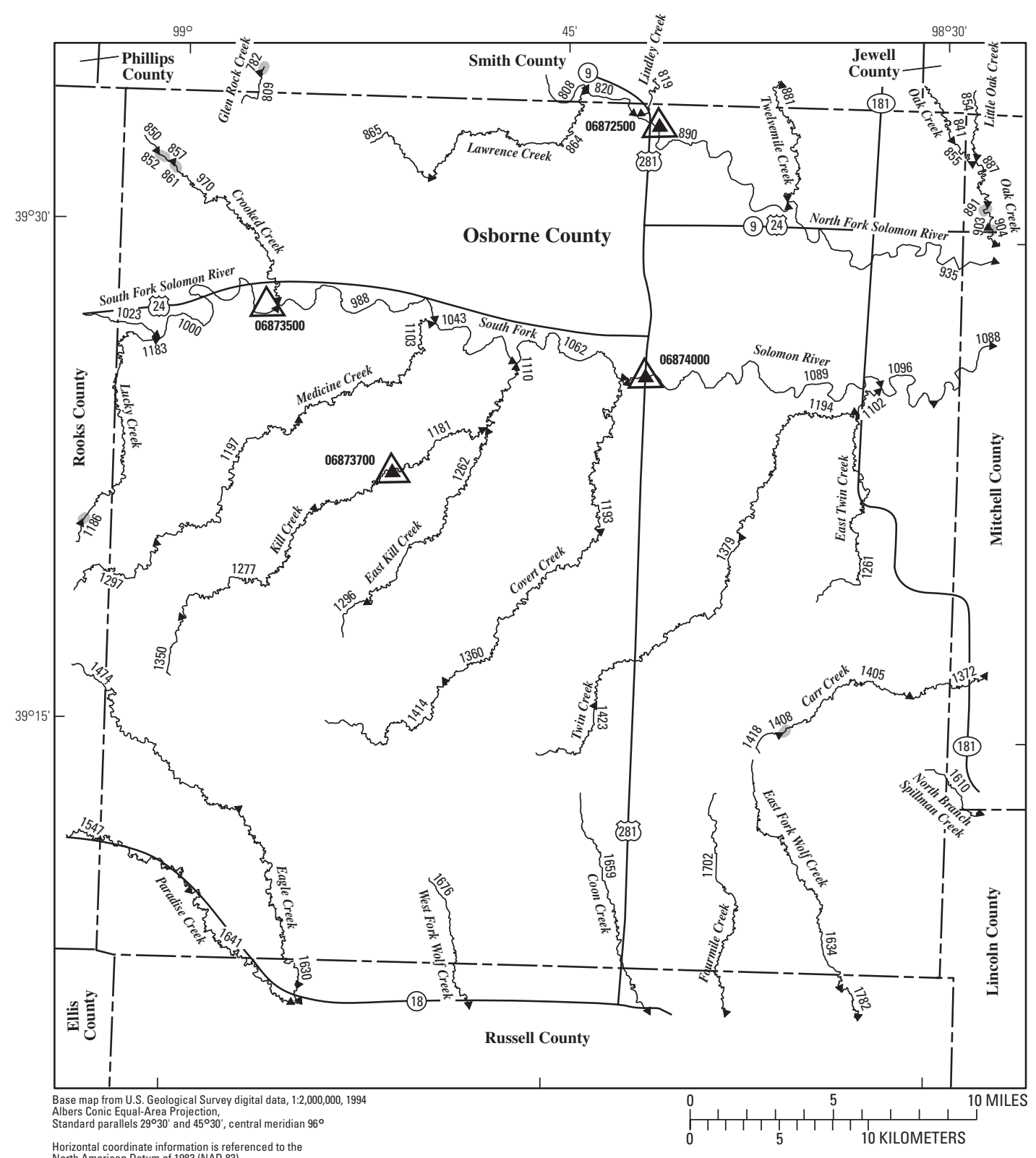

EXPLANATION

1641 Location of streamflow-statistics determination site (small triangle) and associated identification number-small triangle points in downstream direction

06873700 U.S. Geological Survey streamflow-gaging station and number used for estimates of flow duration

06873500 入

S. Geological Survey streamflow-gaging station and number used for estimates of peak-discharge frequency values

1408 Lake and determination site identification number
KANSAS

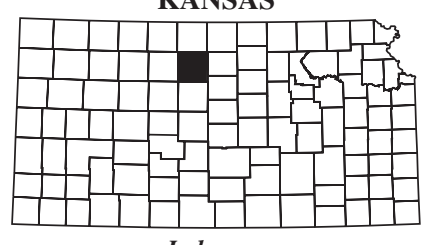

Index map

Figure 81. Location of streamflow-statistics determination sites, associated identification numbers, and U.S. Geological Survey streamflow-gaging stations used in the flow-duration and peak-discharge frequency analyses for Osborne County. 
Table 77. Estimated flow-duration values, mean flow values, and peak-discharge frequency values for controlled and uncontrolled flow stream segments on the 1999 Kansas Surface Water Register for Osborne County.

[KSWR, Kansas Surface Water Register; CUSEGA, catalog unit segment number alpha; $\mathrm{mi}^{2}$, square miles; $\mathrm{ft}^{3} / \mathrm{s}$, cubic feet per second; HYDRO, lake or other hydrologic structure; NA, not applicable; NRDitch, irrigation ditch; NRTribal, tribal stream]

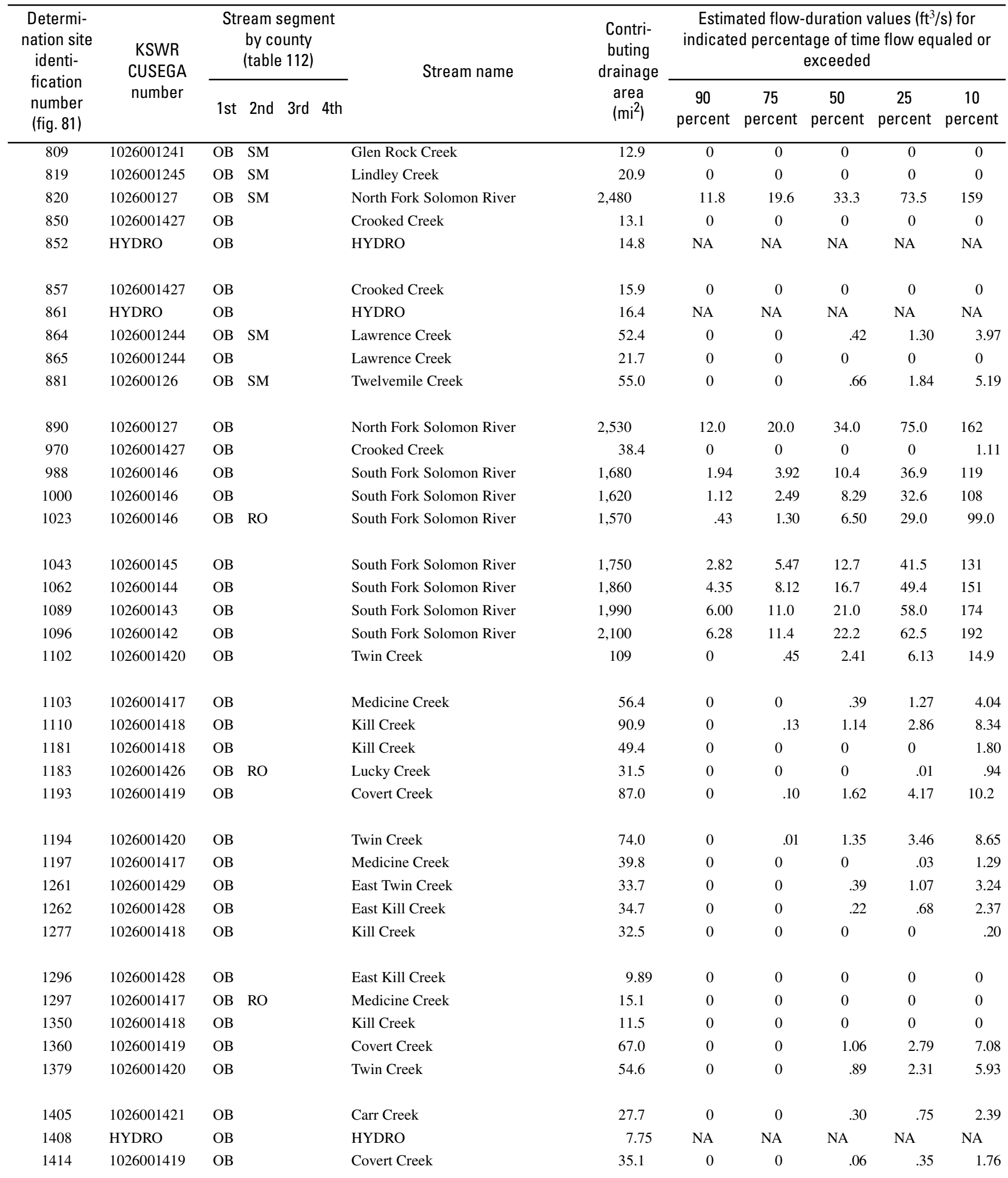


Table 77. Estimated flow-duration values, mean flow values, and peak-discharge frequency values for controlled and uncontrolled flow stream segments on the 1999 Kansas Surface Water Register for Osborne County.-Continued

[KSWR, Kansas Surface Water Register; CUSEGA, catalog unit segment number alpha; $\mathrm{mi}^{2}$, square miles; $\mathrm{ft}^{3} / \mathrm{s}$, cubic feet per second; HYDRO, lake or other hydrologic structure; NA, not applicable; NRDitch, irrigation ditch; NRTribal, tribal stream]

\begin{tabular}{|c|c|c|c|c|c|c|c|}
\hline \multirow{2}{*}{$\begin{array}{l}\text { Determi- } \\
\text { nation site } \\
\text { identi- } \\
\text { fication } \\
\text { number } \\
\text { (fig. 81) }\end{array}$} & \multirow{2}{*}{$\begin{array}{c}\text { Estimated mean } \\
\text { flow } \\
\left(\mathrm{ft}^{3} / \mathrm{s}\right)\end{array}$} & \multicolumn{6}{|c|}{ Estimated peak discharge $\left(\mathrm{ft}^{3} / \mathrm{s}\right)$ for indicated peak-discharge frequency } \\
\hline & & 2-year & 5-year & 10-year & 25-year & 50-year & 100-year \\
\hline 809 & 0.18 & 444 & 1,260 & 2,070 & 3,410 & 4,620 & 6,040 \\
\hline 850 & .05 & 431 & 1,230 & 2,040 & 3,370 & 4,570 & 5,990 \\
\hline 852 & $\mathrm{NA}$ & NA & NA & NA & NA & NA & NA \\
\hline 857 & .35 & 485 & 1,390 & 2,300 & 3,820 & 5,190 & 6,800 \\
\hline 861 & NA & NA & NA & NA & NA & NA & NA \\
\hline 890 & 98.4 & 2,860 & 6,380 & 9,620 & 14,800 & 19,500 & 24,900 \\
\hline 970 & 2.85 & 610 & 1,700 & 2,810 & 4,670 & 6,400 & 8,390 \\
\hline 988 & 62.3 & 3,230 & 11,000 & 21,000 & 41,700 & 64,900 & 96,500 \\
\hline 1000 & 56.4 & 3,600 & 12,300 & 23,400 & 46,300 & 72,000 & 107,000 \\
\hline 1023 & 51.5 & 554 & 2,000 & 3,610 & 6,370 & 8,900 & 11,800 \\
\hline 1043 & 68.6 & 2,830 & 9,690 & 18,500 & 36,600 & 57,100 & 85,100 \\
\hline 1062 & 79.4 & 2,150 & 7,370 & 14,100 & 28,100 & 43,900 & 65,500 \\
\hline 1089 & 91.2 & 1,400 & 4,850 & 9,310 & 18,700 & 29,500 & 44,300 \\
\hline 1096 & 96.9 & 1,410 & 4,810 & 9,170 & 18,300 & 28,800 & 43,100 \\
\hline 1194 & 8.36 & 826 & 2,250 & 3,720 & 6,140 & 8,400 & 11,000 \\
\hline 1197 & 3.07 & 580 & 1,640 & 2,740 & 4,590 & 6,320 & 8,330 \\
\hline 1261 & 4.10 & 832 & 2,160 & 3,470 & 5,590 & 7,530 & 9,700 \\
\hline 1262 & 3.51 & 709 & 1,900 & 3,100 & 5,050 & 6,850 & 8,900 \\
\hline 1277 & 1.47 & 361 & 1,300 & 2,600 & 5,520 & 9,080 & 14,300 \\
\hline 1296 & 0 & 395 & 1,100 & 1,800 & 2,960 & 3,980 & 5,190 \\
\hline 1297 & .36 & 483 & 1,370 & 2,270 & 3,750 & 5,080 & 6,650 \\
\hline 1350 & 0 & 396 & 1,150 & 1,950 & 3,360 & 4,750 & 6,520 \\
\hline 1360 & 7.14 & 856 & 2,310 & 3,790 & 6,230 & 8,490 & 11,100 \\
\hline 1379 & 6.18 & 873 & 2,310 & 3,750 & 6,110 & 8,270 & 10,700 \\
\hline 1405 & 3.33 & 782 & 2,180 & 3,580 & 5,890 & 7,950 & 10,400 \\
\hline 1408 & NA & NA & NA & NA & NA & NA & NA \\
\hline 1414 & 3.14 & 688 & 1,860 & 3,050 & 4,990 & 6,790 & 8,840 \\
\hline
\end{tabular}


Table 77. Estimated flow-duration values, mean flow values, and peak-discharge frequency values for controlled and uncontrolled flow stream segments on the 1999 Kansas Surface Water Register for Osborne County.-Continued

[KSWR, Kansas Surface Water Register; CUSEGA, catalog unit segment number alpha; $\mathrm{mi}^{2}$, square miles; $\mathrm{ft}^{3} / \mathrm{s}$, cubic feet per second; HYDRO, lake or other hydrologic structure; NA, not applicable; NRDitch, irrigation ditch; NRTribal, tribal stream]

\begin{tabular}{|c|c|c|c|c|c|c|c|c|c|c|c|}
\hline \multirow{2}{*}{$\begin{array}{l}\text { Determi- } \\
\text { nation site } \\
\text { identi- } \\
\text { fication } \\
\text { number } \\
\text { (fig. 81) }\end{array}$} & \multirow{2}{*}{$\begin{array}{l}\text { KSWR } \\
\text { CUSEGA } \\
\text { number }\end{array}$} & \multicolumn{3}{|c|}{$\begin{array}{l}\text { Stream segment } \\
\text { by county } \\
\text { (table 112) }\end{array}$} & \multirow{2}{*}{ Stream name } & \multirow{2}{*}{$\begin{array}{l}\text { Contri- } \\
\text { buting } \\
\text { drainage } \\
\text { area } \\
\left(\mathrm{mi}^{2}\right)\end{array}$} & \multicolumn{5}{|c|}{$\begin{array}{c}\text { Estimated flow-duration values }\left(\mathrm{ft}^{3} / \mathrm{s}\right) \text { for } \\
\text { indicated percentage of time flow equaled or } \\
\text { exceeded }\end{array}$} \\
\hline & & $1 \mathrm{st}$ & 2nd & 3rd 4th & & & $\begin{array}{c}90 \\
\text { percent }\end{array}$ & $\begin{array}{c}75 \\
\text { percent }\end{array}$ & $\begin{array}{c}50 \\
\text { percent }\end{array}$ & $\begin{array}{c}25 \\
\text { percent }\end{array}$ & $\begin{array}{c}10 \\
\text { percent }\end{array}$ \\
\hline 1418 & 1026001421 & OB & & & Carr Creek & 7.64 & 0 & 0 & 0 & 0 & 0 \\
\hline 1423 & 1026001420 & OB & & & Twin Creek & 18.8 & 0 & 0 & 0 & 0 & .14 \\
\hline 1474 & 102600096 & OB & RO & & Eagle Creek & 36.7 & 0 & 0 & 0 & .08 & 1.54 \\
\hline 1547 & 102600097 & OB & RO & & Paradise Creek & 122 & 0 & 0 & .37 & 2.14 & 9.85 \\
\hline 1630 & 102600096 & OB & RS & & Eagle Creek & 63.1 & 0 & 0 & .47 & 1.59 & 5.46 \\
\hline 1634 & 1026001011 & OB & RS & & East Fork Wolf Creek & 37.3 & 0 & 0 & .36 & 1.04 & 3.31 \\
\hline 1641 & 102600097 & OB & RS & & Paradise Creek & 148 & 0 & 0 & .49 & 2.84 & 13.3 \\
\hline 1659 & 1026001031 & OB & RS & & Coon Creek & 34.7 & 0 & 0 & 0 & .19 & 1.49 \\
\hline 1676 & 1026001012 & OB & $\mathrm{RS}$ & & West Fork Wolf Creek & 58.3 & 0 & 0 & .46 & 1.49 & 4.45 \\
\hline 1702 & 1026001030 & OB & RS & & Fourmile Creek & 33.5 & 0 & 0 & .22 & .69 & 2.44 \\
\hline
\end{tabular}


Table 77. Estimated flow-duration values, mean flow values, and peak-discharge frequency values for controlled and uncontrolled flow stream segments on the 1999 Kansas Surface Water Register for Osborne County.-Continued

[KSWR, Kansas Surface Water Register; CUSEGA, catalog unit segment number alpha; $\mathrm{mi}^{2}$, square miles; $\mathrm{ft}^{3} / \mathrm{s}$, cubic feet per second; HYDRO, lake or other hydrologic structure; NA, not applicable; NRDitch, irrigation ditch; NRTribal, tribal stream]

\begin{tabular}{|c|c|c|c|c|c|c|c|}
\hline \multirow{2}{*}{$\begin{array}{l}\text { Determi- } \\
\text { nation site } \\
\text { identi- } \\
\text { fication } \\
\text { number } \\
\text { (fig. 81) }\end{array}$} & \multirow[b]{2}{*}{$\begin{array}{c}\text { Estimated mean } \\
\text { flow } \\
\left(\mathrm{ft}^{3} / \mathrm{s}\right)\end{array}$} & \multicolumn{6}{|c|}{ Estimated peak discharge $\left(\mathrm{ft}^{3} / \mathrm{s}\right)$ for indicated peak-discharge frequency } \\
\hline & & 2-year & 5-year & 10-year & 25-year & 50-year & 100-year \\
\hline 1418 & 0.20 & 366 & 997 & 1,610 & 2,620 & 3,510 & 4,560 \\
\hline 1547 & 9.23 & 735 & 2,240 & 3,920 & 6,920 & 9,850 & 13,400 \\
\hline 1630 & 5.89 & 732 & 2,060 & 3,440 & 5,780 & 7,990 & 10,600 \\
\hline 1634 & 4.32 & 829 & 2,170 & 3,500 & 5,650 & 7,620 & 9,850 \\
\hline 1641 & 11.6 & 809 & 2,500 & 4,390 & 7,810 & 11,200 & 15,400 \\
\hline
\end{tabular}




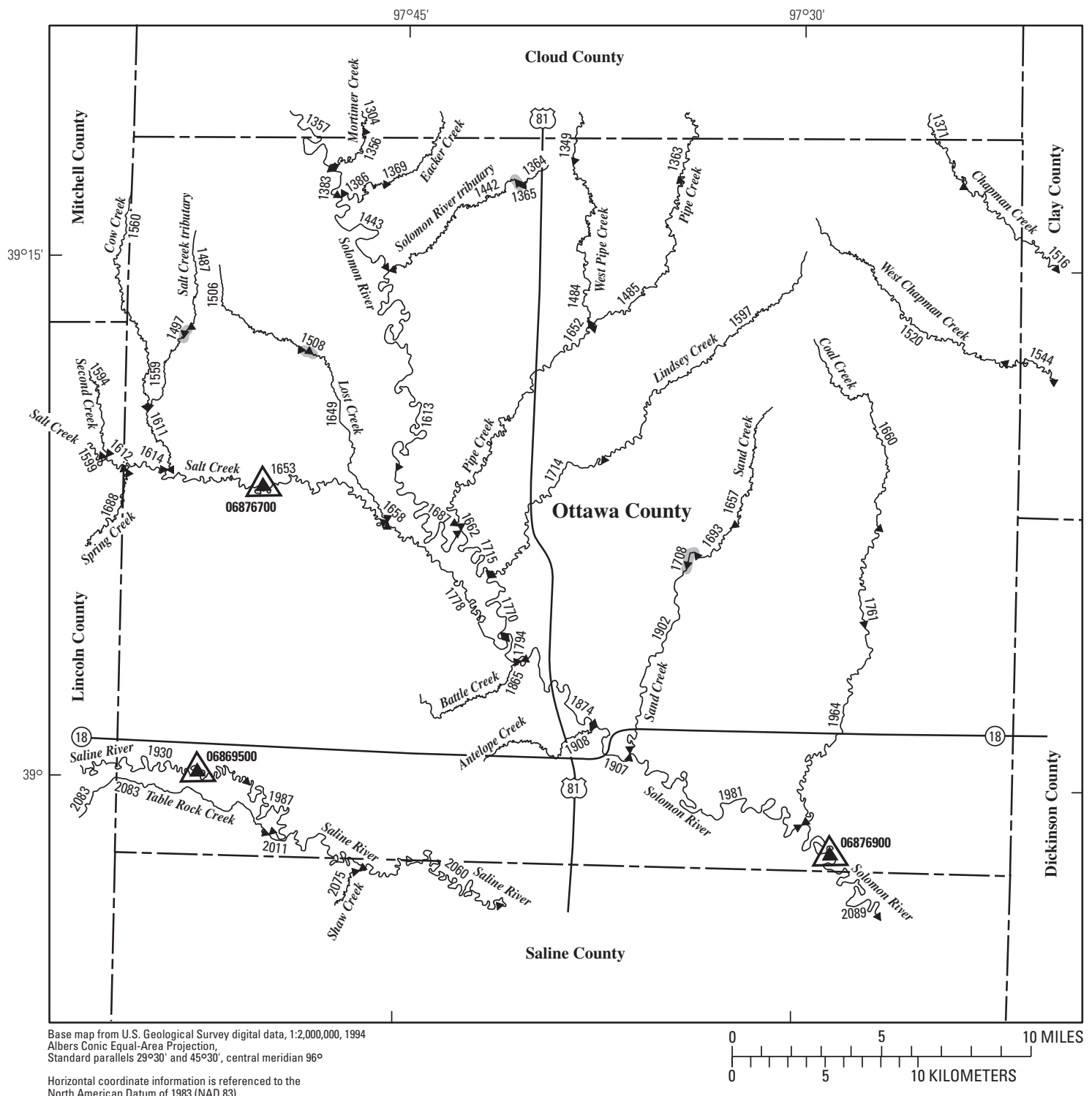

EXPLANATION

\1987 Location of streamflow-statistics determination site (small triangle) and associated identification number-small triangle points in downstream direction

$06869500 \Delta$ U.S. Geological Survey streamflow-gaging station and number used for estimates of flow duration

06876900 for estimates of peak-discharge frequency values

KANSAS

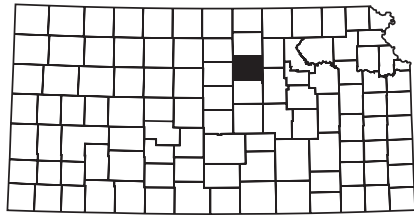

Index map

1708

Lake and determination site identification number

Figure 82. Location of streamflow-statistics determination sites, associated identification numbers, and U.S. Geological Survey streamflow-gaging stations used in the flow-duration and peak-discharge frequency analyses for Ottawa County. 
Table 78. Estimated flow-duration values, mean flow values, and peak-discharge frequency values for controlled and uncontrolled flow stream segments on the 1999 Kansas Surface Water Register for Ottawa County.

[KSWR, Kansas Surface Water Register; CUSEGA, catalog unit segment number alpha; $\mathrm{mi}^{2}$, square miles; $\mathrm{ft}^{3} / \mathrm{s}$, cubic feet per second; HYDRO, lake or other hydrologic structure; NA, not applicable; NRDitch, irrigation ditch; NRTribal, tribal stream]

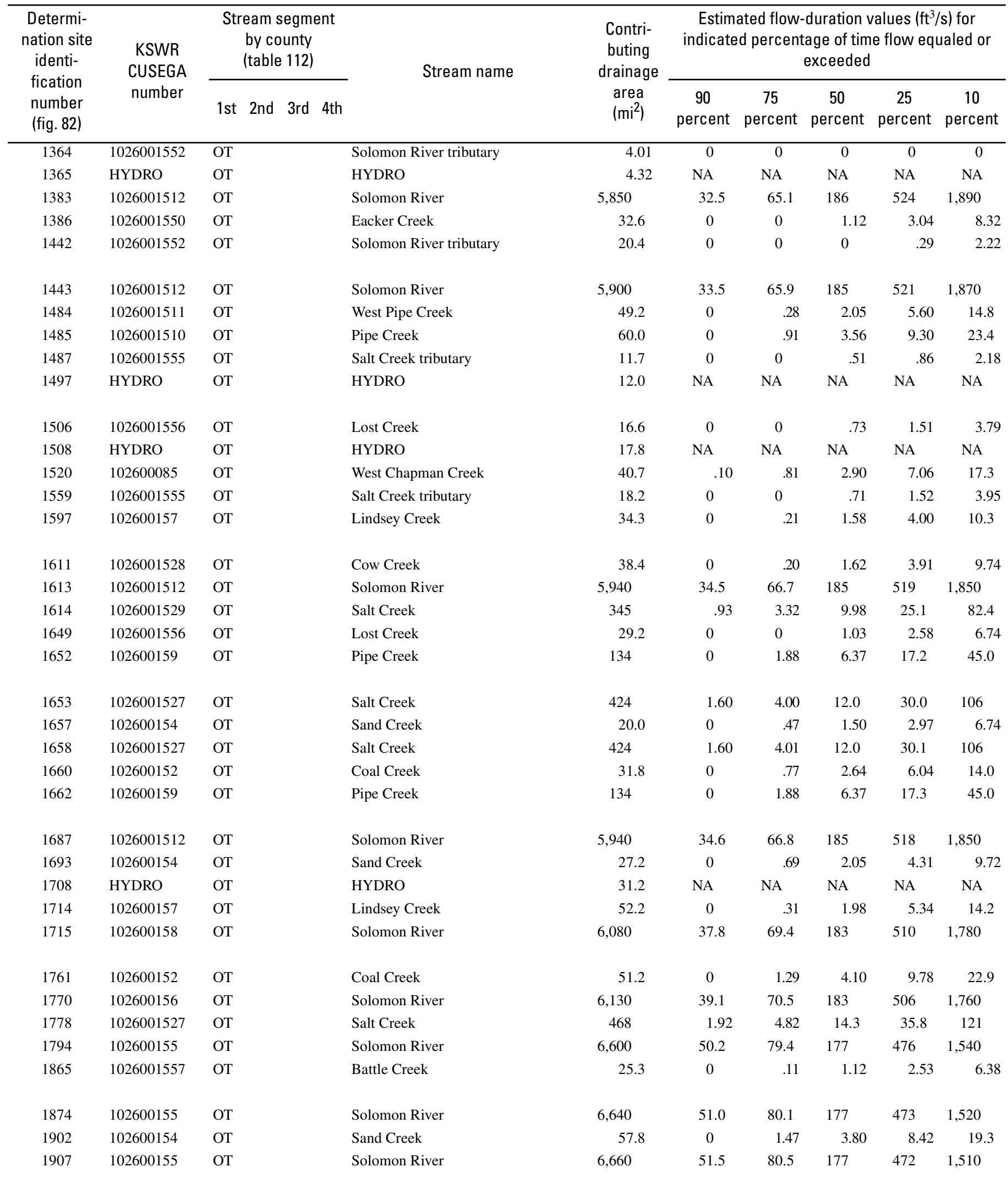


Table 78. Estimated flow-duration values, mean flow values, and peak-discharge frequency values for controlled and uncontrolled flow stream segments on the 1999 Kansas Surface Water Register for Ottawa County.-Continued

[KSWR, Kansas Surface Water Register; CUSEGA, catalog unit segment number alpha; $\mathrm{mi}^{2}$, square miles; $\mathrm{ft}^{3} / \mathrm{s}$, cubic feet per second; HYDRO, lake or other hydrologic structure; NA, not applicable; NRDitch, irrigation ditch; NRTribal, tribal stream]

\begin{tabular}{|c|c|c|c|c|c|c|c|}
\hline \multirow{2}{*}{$\begin{array}{l}\text { Determi- } \\
\text { nation site } \\
\text { identi- } \\
\text { fication } \\
\text { number } \\
\text { (fig. 82) }\end{array}$} & \multirow{2}{*}{$\begin{array}{c}\text { Estimated mean } \\
\text { flow } \\
\left(\mathrm{ft}^{3} / \mathrm{s}\right)\end{array}$} & \multicolumn{6}{|c|}{ Estimated peak discharge $\left(\mathrm{ft}^{3} / \mathrm{s}\right)$ for indicated peak-discharge frequency } \\
\hline & & 2-year & 5-year & 10 -year & 25-year & 50-year & 100 -year \\
\hline 1364 & 0.03 & 374 & 914 & 1,410 & 2,190 & 2,860 & 3,630 \\
\hline 1386 & 7.84 & 1,100 & 2,730 & 4,300 & 6,800 & 9,050 & 11,600 \\
\hline 1442 & 3.68 & 928 & 2,360 & 3,720 & 5,910 & 7,810 & 10,000 \\
\hline 1443 & 561 & 3,370 & 6,630 & 9,340 & 12,600 & 15,300 & 18,000 \\
\hline 1484 & 12.6 & 1,410 & 3,430 & 5,380 & 8,470 & 11,200 & 14,400 \\
\hline 1506 & 3.86 & 775 & 1,990 & 3,150 & 5,020 & 6,650 & 8,550 \\
\hline 1508 & NA & NA & NA & NA & NA & NA & NA \\
\hline 1520 & 13.7 & 1,450 & 3,390 & 5,220 & 8,070 & 10,600 & 13,400 \\
\hline 1559 & 4.05 & 801 & 2,080 & 3,300 & 5,260 & 6,980 & 8,990 \\
\hline 1597 & 9.08 & 993 & 2,500 & 3,960 & 6,310 & 8,420 & 10,800 \\
\hline 1611 & 8.36 & 1,090 & 2,710 & 4,260 & 6,740 & 8,980 & 11,500 \\
\hline 1613 & 561 & 3,450 & 6,800 & 9,730 & 13,300 & 16,300 & 19,400 \\
\hline 1614 & 56.4 & 1,640 & 4,400 & 7,240 & 12,100 & 16,800 & 22,500 \\
\hline 1649 & 6.42 & 1,080 & 2,810 & 4,470 & 7,150 & 9,500 & 12,300 \\
\hline 1687 & 561 & 3,460 & 6,810 & 9,770 & 13,300 & 16,400 & 19,500 \\
\hline 1693 & 7.96 & 1,190 & 3,000 & 4,690 & 7,410 & 9,760 & 12,500 \\
\hline 1708 & NA & NA & NA & NA & NA & NA & NA \\
\hline 1714 & 12.4 & 1,160 & 2,930 & 4,670 & 7,480 & 10,000 & 12,900 \\
\hline 1715 & 561 & 3,710 & 7,340 & 11,000 & 15,400 & 19,600 & 24,000 \\
\hline 1761 & 16.7 & 1,680 & 3,850 & 5,860 & 8,960 & 11,700 & 14,700 \\
\hline 1770 & 561 & 3,810 & 7,560 & 11,500 & 16,300 & 20,900 & 25,800 \\
\hline 1778 & 79.4 & 1,390 & 3,980 & 6,780 & 11,800 & 16,700 & 22,800 \\
\hline 1794 & 562 & 4,660 & 9,370 & 15,700 & 23,500 & 31,800 & 41,200 \\
\hline 1865 & 6.05 & 1,060 & 2,700 & 4,260 & 6,770 & 8,950 & 11,500 \\
\hline 1874 & 562 & 4,730 & 9,510 & 16,000 & 24,100 & 32,700 & 42,400 \\
\hline 1902 & 15.0 & 1,310 & 3,110 & 4,810 & 7,460 & 9,810 & 12,400 \\
\hline 1907 & 562 & 4,760 & 9,590 & 16,200 & 24,400 & 33,100 & 43,000 \\
\hline
\end{tabular}


Table 78. Estimated flow-duration values, mean flow values, and peak-discharge frequency values for controlled and uncontrolled flow stream segments on the 1999 Kansas Surface Water Register for Ottawa County.-Continued

[KSWR, Kansas Surface Water Register; CUSEGA, catalog unit segment number alpha; $\mathrm{mi}^{2}$, square miles; $\mathrm{ft}^{3} / \mathrm{s}$, cubic feet per second; HYDRO, lake or other hydrologic structure; NA, not applicable; NRDitch, irrigation ditch; NRTribal, tribal stream]

\begin{tabular}{|c|c|c|c|c|c|c|c|c|c|c|c|}
\hline $\begin{array}{l}\text { Determi- } \\
\text { nation site } \\
\text { identi- } \\
\text { fication } \\
\text { number } \\
\text { (fig. 82) }\end{array}$ & $\begin{array}{l}\text { KSWR } \\
\text { CUSEGA } \\
\text { number }\end{array}$ & \multicolumn{3}{|c|}{$\begin{array}{l}\text { Stream segment } \\
\text { by county } \\
\text { (table 112) }\end{array}$} & Stream name & $\begin{array}{l}\text { Contri- } \\
\text { buting } \\
\text { drainage } \\
\text { area } \\
\left(\mathrm{mi}^{2}\right)\end{array}$ & \multicolumn{5}{|c|}{$\begin{array}{c}\text { Estimated flow-duration values }\left(\mathrm{ft}^{3} / \mathrm{s}\right) \text { for } \\
\text { indicated percentage of time flow equaled or } \\
\text { exceeded }\end{array}$} \\
\hline 1908 & 1026001558 & OT & & & Antelope Creek & 16.4 & 0 & 0 & 0.74 & 1.59 & $\overline{4.15}$ \\
\hline 1987 & 102600103 & OT & & & Saline River & 2,850 & 15.1 & 24.1 & 45.2 & 126 & 491 \\
\hline 2011 & 102600102 & OT & SA & & Saline River & 2,910 & 15.4 & 24.7 & 46.3 & 129 & 500 \\
\hline 2060 & 102600102 & OT & SA & & Saline River & 2,950 & 15.7 & 25.1 & 47.2 & 131 & 506 \\
\hline 2089 & 102600151 & OT & SA & & Solomon River & 6,890 & 57.0 & 85.0 & 174 & 457 & 1,400 \\
\hline
\end{tabular}


Table 78. Estimated flow-duration values, mean flow values, and peak-discharge frequency values for controlled and uncontrolled flow stream segments on the 1999 Kansas Surface Water Register for Ottawa County.-Continued

[KSWR, Kansas Surface Water Register; CUSEGA, catalog unit segment number alpha; $\mathrm{mi}^{2}$, square miles; $\mathrm{ft}^{3} / \mathrm{s}$, cubic feet per second; HYDRO, lake or other hydrologic structure; NA, not applicable; NRDitch, irrigation ditch; NRTribal, tribal stream]

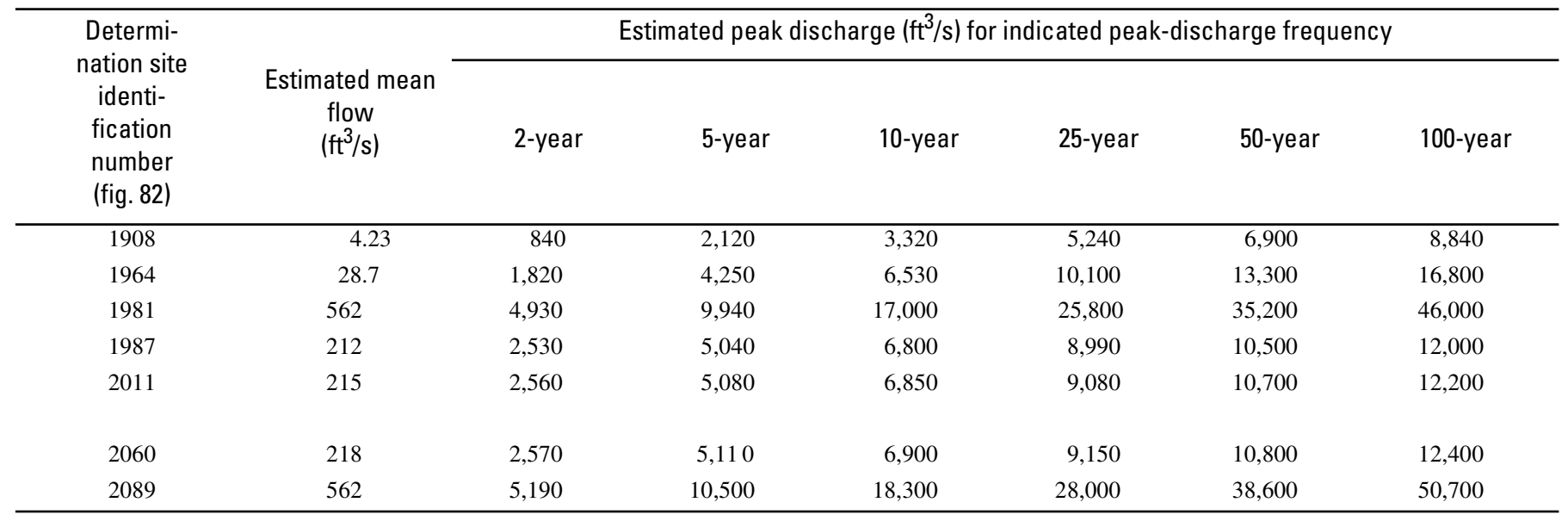




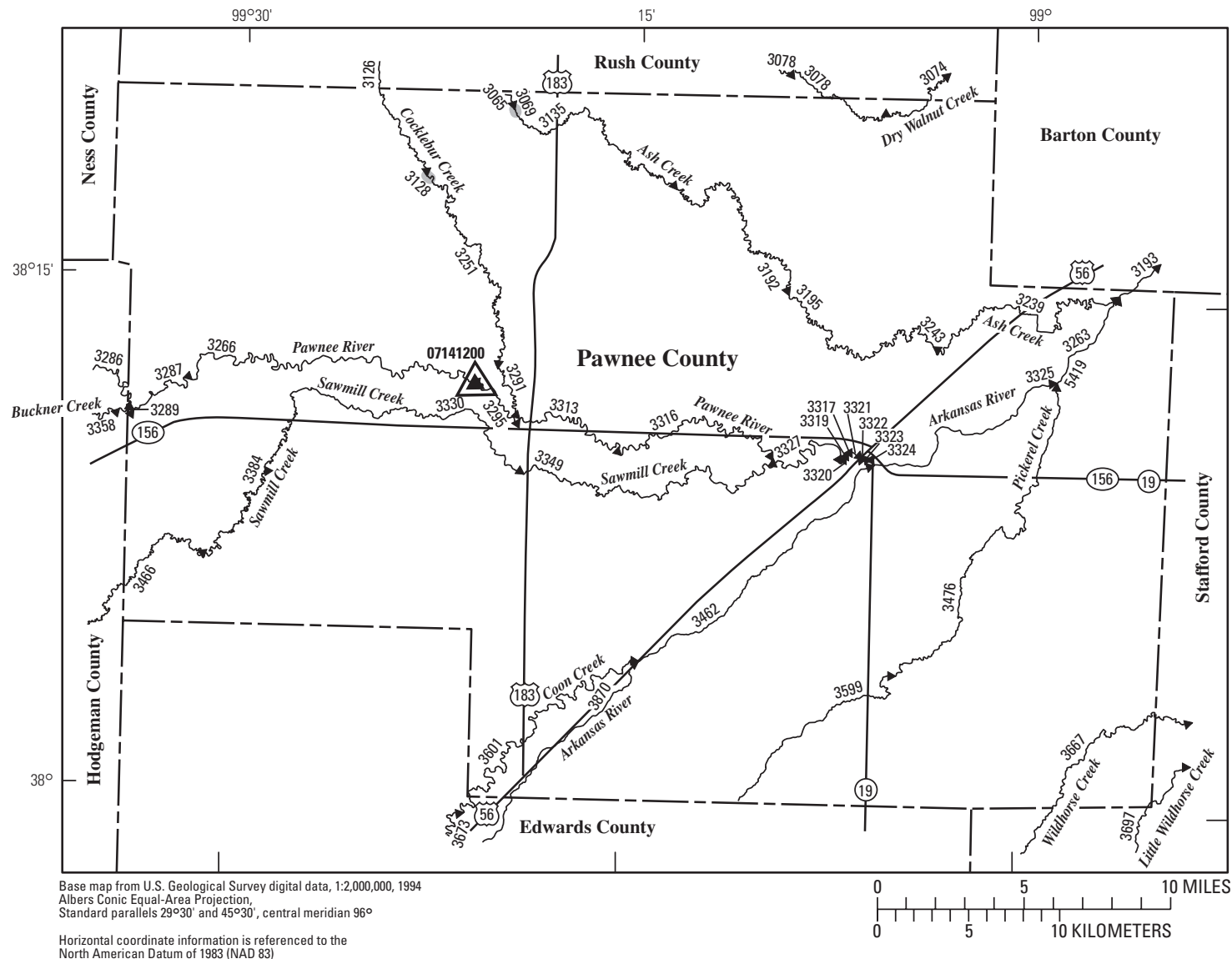

EXPLANATION

$\stackrel{3601}{\longleftarrow}$ Location of streamflow-statistics determination site (small triangle) and associated identification number-small triangle points in downstream direction

$07141200 \Delta$ U.S. Geological Survey streamflow-gaging station and number used for estimates of flow duration

07141200

U.S. Geological Survey streamflow-gaging station and number used for estimates of peak-discharge frequency values
KANSAS

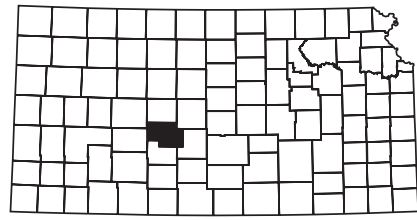

Index map 3069

Lake and determination site identification number

Figure 83. Location of streamflow-statistics determination sites, associated identification numbers, and U.S. Geological Survey streamflow-gaging stations used in the flow-duration and peak-discharge frequency analyses for Pawnee County. 
Table 79. Estimated flow-duration values, mean flow values, and peak-discharge frequency values for controlled and uncontrolled flow stream segments on the 1999 Kansas Surface Water Register for Pawnee County.

[KSWR, Kansas Surface Water Register; CUSEGA, catalog unit segment number alpha; $\mathrm{mi}^{2}$, square miles; $\mathrm{ft}^{3} / \mathrm{s}$, cubic feet per second; HYDRO, lake or other hydrologic structure; NA, not applicable; NRDitch, irrigation ditch; NRTribal, tribal stream]

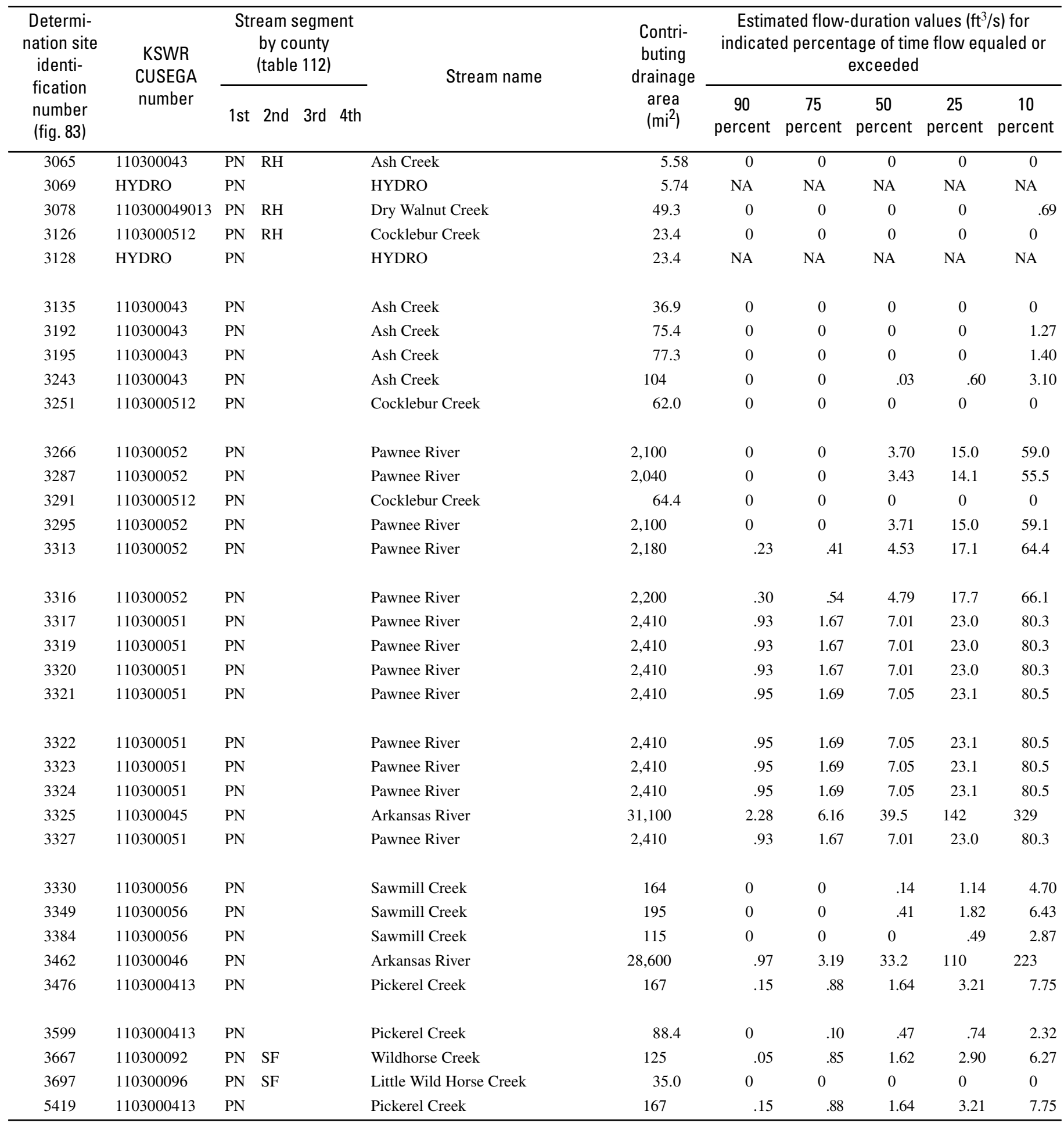


Table 79. Estimated flow-duration values, mean flow values, and peak-discharge frequency values for controlled and uncontrolled flow stream segments on the 1999 Kansas Surface Water Register for Pawnee County.-Continued

[KSWR, Kansas Surface Water Register; CUSEGA, catalog unit segment number alpha; $\mathrm{mi}^{2}$, square miles; $\mathrm{ft}^{3} / \mathrm{s}$, cubic feet per second; HYDRO, lake or other hydrologic structure; NA, not applicable; NRDitch, irrigation ditch; NRTribal, tribal stream]

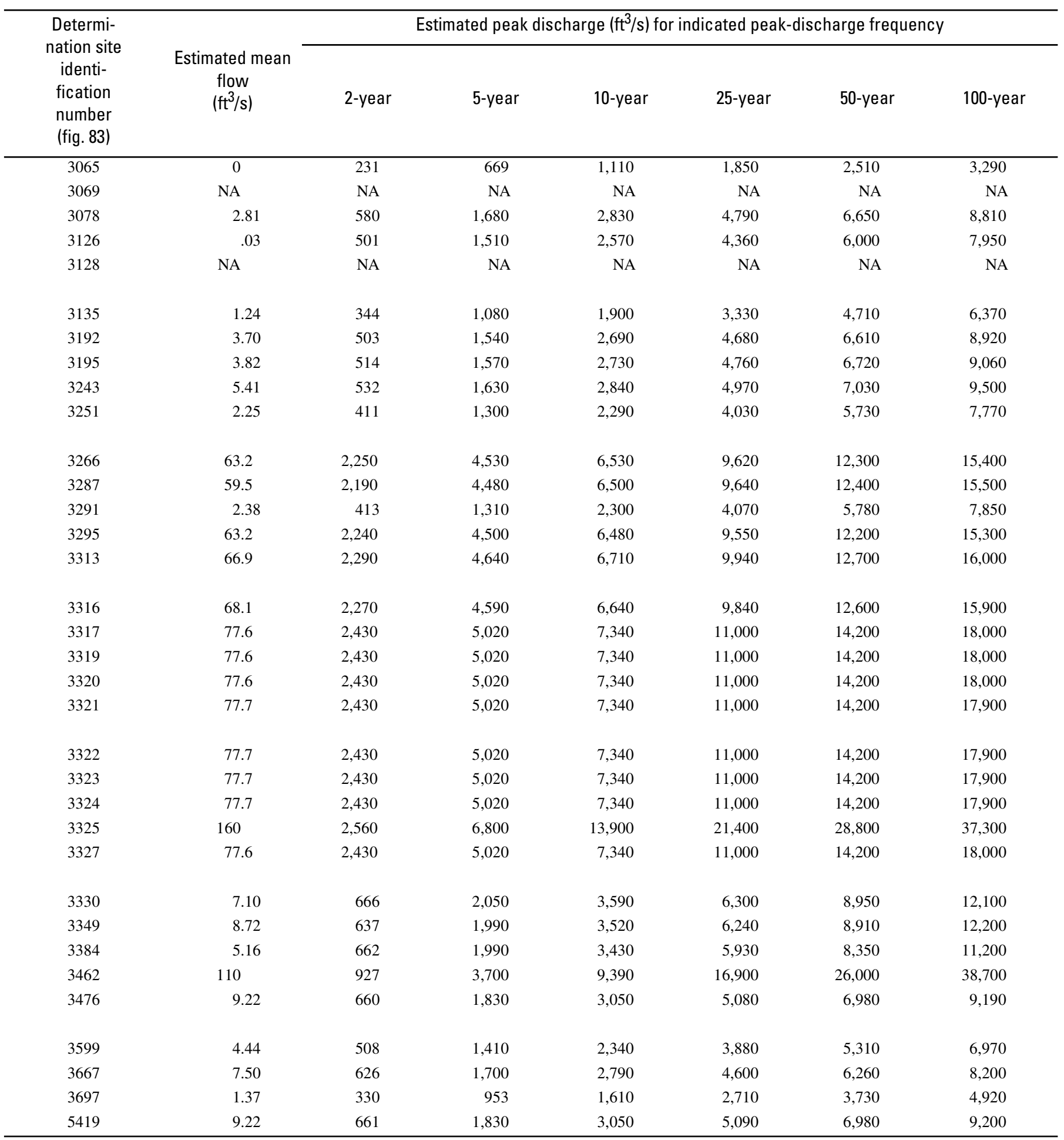




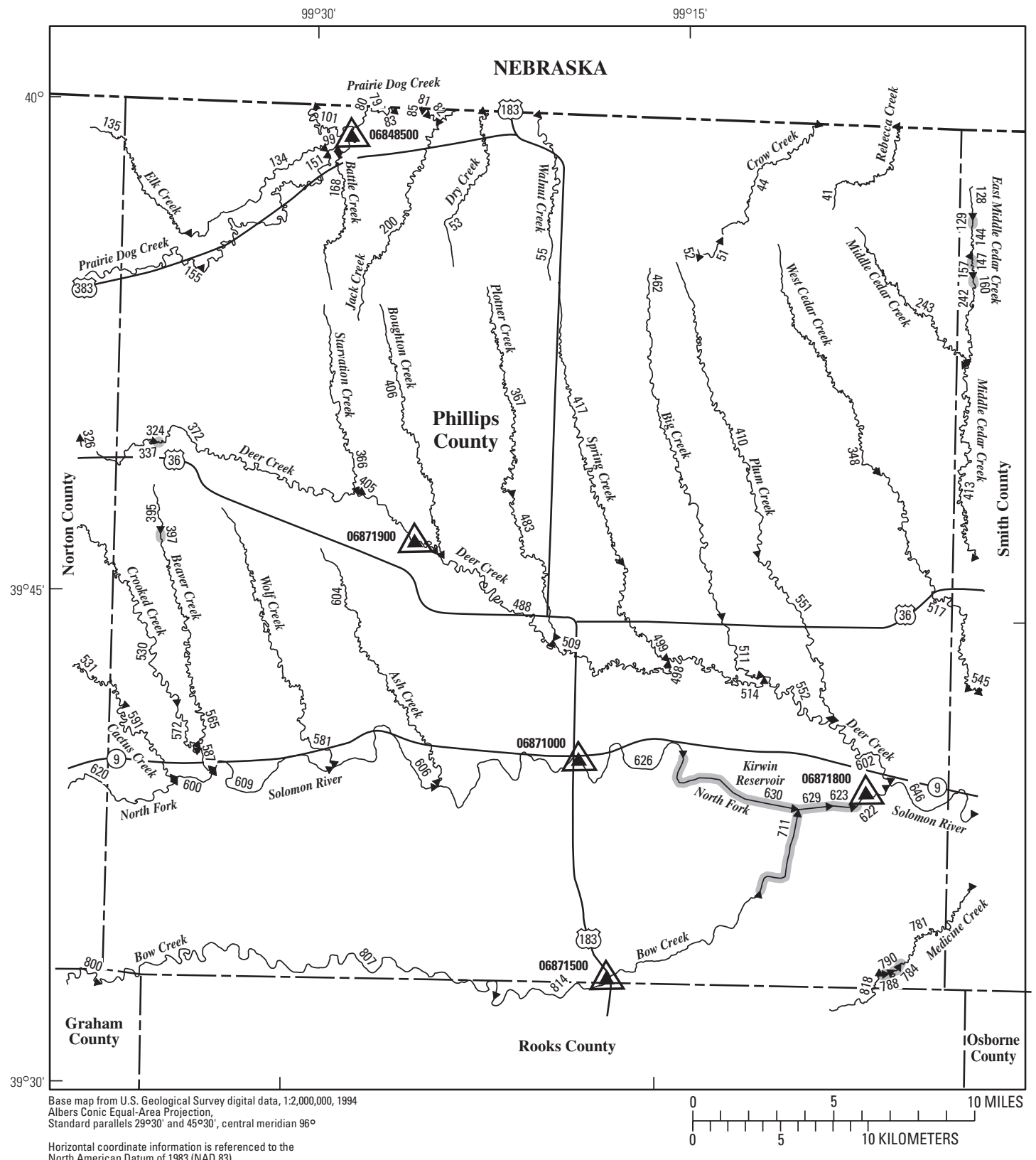

EXPLANATION

$₫ 800$ Location of streamflow-statistics determination site (small triangle) and associated identification number-small triangle points in downstream direction

06871900 U.S. Geological Survey streamflow-gaging station and number used for estimates of flow duration

${ }^{06871000} \bigwedge$ U.S. Geological Survey streamflow-gaging station and number used for estimates of peak-discharge frequency values

630

Lake and determination site identification number
KANSAS

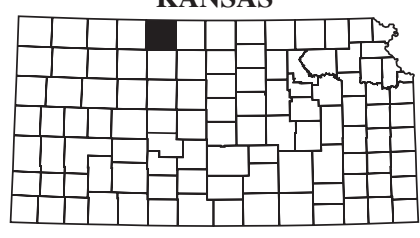

Index map

Figure 84. Location of streamflow-statistics determination sites, associated identification numbers, and U.S. Geological Survey streamflow-gaging stations used in the flow-duration and peak-discharge frequency analyses for Phillips County. 
Table 80. Estimated flow-duration values, mean flow values, and peak-discharge frequency values for controlled and uncontrolled flow stream segments on the 1999 Kansas Surface Water Register for Phillips County.

[KSWR, Kansas Surface Water Register; CUSEGA, catalog unit segment number alpha; $\mathrm{mi}^{2}$, square miles; $\mathrm{ft}^{3} / \mathrm{s}$, cubic feet per second; HYDRO, lake or other hydrologic structure; NA, not applicable; NRDitch, irrigation ditch; NRTribal, tribal stream]

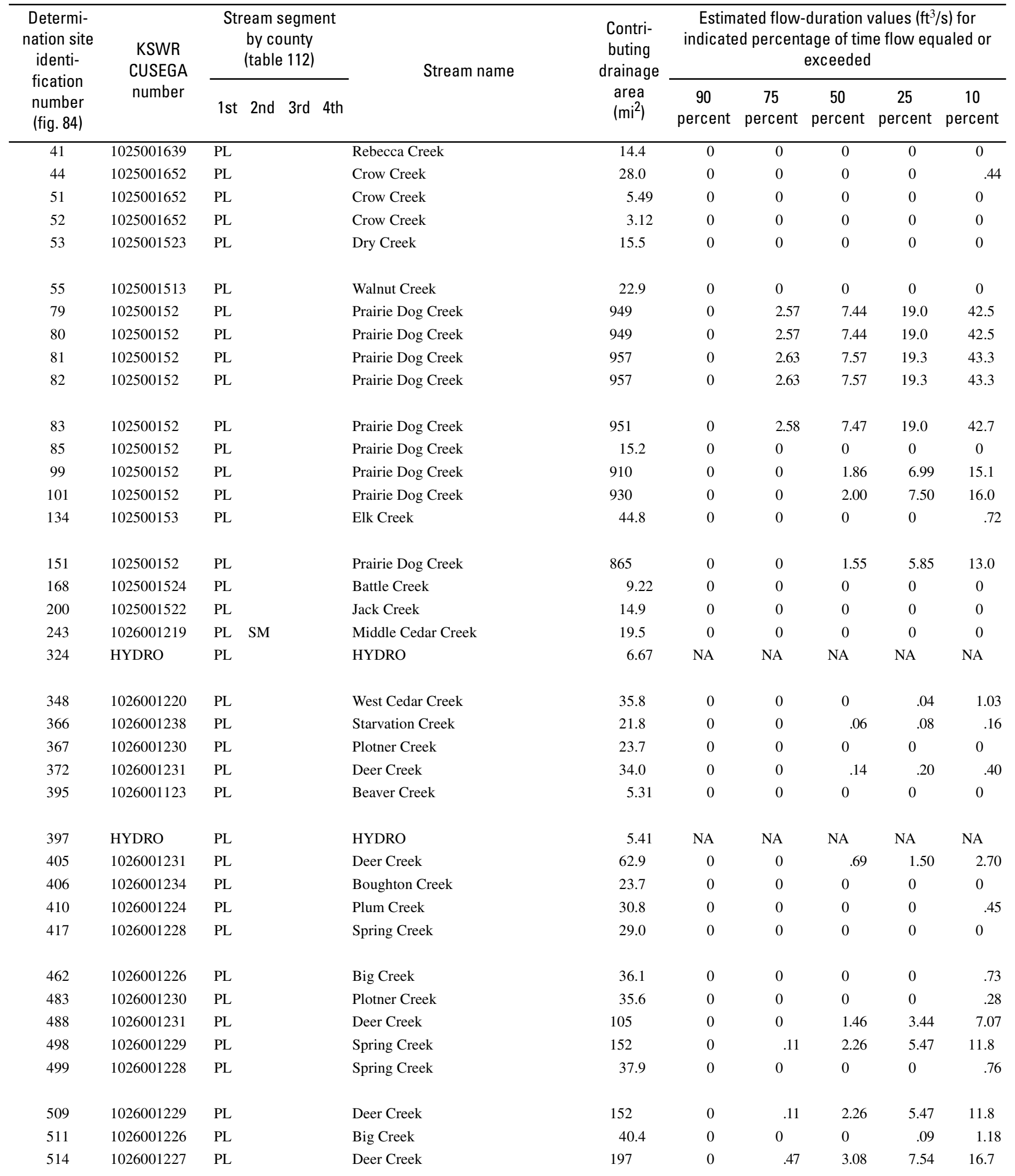


Table 80. Estimated flow-duration values, mean flow values, and peak-discharge frequency values for controlled and uncontrolled flow stream segments on the 1999 Kansas Surface Water Register for Phillips County.-Continued

[KSWR, Kansas Surface Water Register; CUSEGA, catalog unit segment number alpha; $\mathrm{mi}^{2}$, square miles; $\mathrm{ft}^{3} / \mathrm{s}$, cubic feet per second; HYDRO, lake or other hydrologic structure; NA, not applicable; NRDitch, irrigation ditch; NRTribal, tribal stream]

\begin{tabular}{|c|c|c|c|c|c|c|c|}
\hline \multirow{2}{*}{$\begin{array}{l}\text { Determi- } \\
\text { nation site } \\
\text { identi- } \\
\text { fication } \\
\text { number } \\
\text { (fig. 84) }\end{array}$} & \multirow{2}{*}{$\begin{array}{c}\text { Estimated mean } \\
\text { flow } \\
\left(\mathrm{ft}^{3} / \mathrm{s}\right)\end{array}$} & \multicolumn{6}{|c|}{ Estimated peak discharge $\left(\mathrm{ft}^{3} / \mathrm{s}\right)$ for indicated peak-discharge frequency } \\
\hline & & 2-year & 5-year & 10-year & 25-year & 50-year & 100-year \\
\hline 41 & 0.59 & 460 & 1,310 & 2,170 & 3,600 & 4,880 & 6,390 \\
\hline 52 & 0 & 181 & 506 & 826 & 1,350 & 1,820 & 2,370 \\
\hline 53 & .46 & 443 & 1,290 & 2,150 & 3,600 & 4,910 & 6,450 \\
\hline 55 & 1.27 & 562 & 1,650 & 2,760 & 4,630 & 6,310 & 8,320 \\
\hline 79 & 30.7 & 1,110 & 2,980 & 4,920 & 8,260 & 11,400 & 15,300 \\
\hline 83 & 30.8 & 1,110 & 2,980 & 4,920 & 8,260 & 11,400 & 15,300 \\
\hline 85 & .37 & 436 & 1,270 & 2,120 & 3,550 & 4,840 & 6,370 \\
\hline 99 & 10.7 & 611 & 1,500 & 2,170 & 3,000 & 3,570 & 4,080 \\
\hline 101 & 11.1 & 652 & 1,610 & 2,330 & 3,220 & 3,830 & 4,380 \\
\hline 134 & 2.54 & 417 & 1,250 & 2,160 & 3,720 & 5,200 & 6,950 \\
\hline 151 & 9.78 & 519 & 1,270 & 1,830 & 2,510 & 2,980 & 3,400 \\
\hline 168 & 0 & 327 & 943 & 1,570 & 2,600 & 3,540 & 4,640 \\
\hline 200 & .34 & 431 & 1,260 & 2,100 & 3,510 & 4,790 & 6,300 \\
\hline 243 & 1.08 & 543 & 1,540 & 2,540 & 4,210 & 5,710 & 7,490 \\
\hline 397 & NA & NA & NA & NA & NA & NA & NA \\
\hline 405 & 4.04 & 1,210 & 3,430 & 5,760 & 9,790 & 13,600 & 18,300 \\
\hline 406 & 1.09 & 564 & 1,660 & 2,790 & 4,690 & 6,400 & 8,450 \\
\hline 410 & 2.08 & 493 & 1,410 & 2,360 & 3,960 & 5,460 & 7,190 \\
\hline 417 & 1.71 & 650 & 1,910 & 3,210 & 5,390 & 7,360 & 9,710 \\
\hline 462 & 2.41 & 528 & 1,500 & 2,520 & 4,230 & 5,840 & 7,690 \\
\hline 483 & 2.11 & 439 & 1,290 & 2,200 & 3,750 & 5,220 & 6,930 \\
\hline 488 & 7.48 & 1,360 & 3,830 & 6,440 & 10,900 & 15,200 & 20,400 \\
\hline 498 & 11.2 & 1,490 & 4,210 & 7,060 & 12,000 & 16,700 & 22,400 \\
\hline 499 & 2.46 & 474 & 1,380 & 2,340 & 3,970 & 5,500 & 7,290 \\
\hline 509 & 11.2 & 1,490 & 4,210 & 7,060 & 12,000 & 16,700 & 22,400 \\
\hline 511 & 2.78 & 534 & 1,530 & 2,570 & 4,330 & 5,980 & 7,890 \\
\hline 514 & 14.8 & 1,610 & 4,520 & 7,580 & 12,900 & 17,900 & 24,000 \\
\hline
\end{tabular}


Table 80. Estimated flow-duration values, mean flow values, and peak-discharge frequency values for controlled and uncontrolled flow stream segments on the 1999 Kansas Surface Water Register for Phillips County.-Continued

[KSWR, Kansas Surface Water Register; CUSEGA, catalog unit segment number alpha; $\mathrm{mi}^{2}$, square miles; $\mathrm{ft}^{3} / \mathrm{s}$, cubic feet per second; HYDRO, lake or other hydrologic structure; NA, not applicable; NRDitch, irrigation ditch; NRTribal, tribal stream]

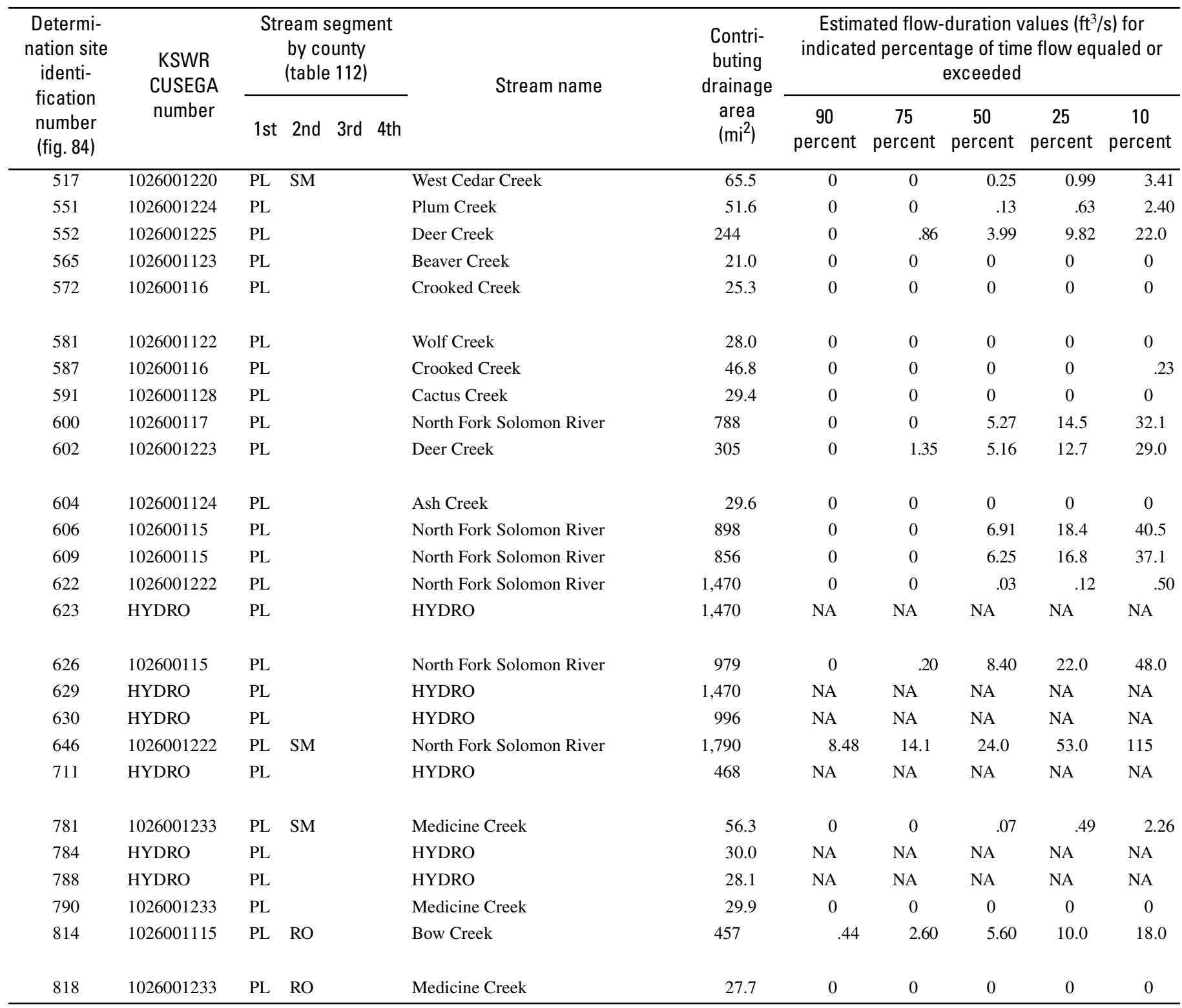


Table 80. Estimated flow-duration values, mean flow values, and peak-discharge frequency values for controlled and uncontrolled flow stream segments on the 1999 Kansas Surface Water Register for Phillips County.-Continued

[KSWR, Kansas Surface Water Register; CUSEGA, catalog unit segment number alpha; $\mathrm{mi}^{2}$, square miles; $\mathrm{ft}^{3} / \mathrm{s}$, cubic feet per second; HYDRO, lake or other hydrologic structure; NA, not applicable; NRDitch, irrigation ditch; NRTribal, tribal stream]

\begin{tabular}{|c|c|c|c|c|c|c|c|}
\hline \multirow{2}{*}{$\begin{array}{c}\text { Determi- } \\
\text { nation site } \\
\text { identi- } \\
\text { fication } \\
\text { number } \\
\text { (fig. 84) }\end{array}$} & \multirow[b]{2}{*}{$\begin{array}{c}\text { Estimated mean } \\
\text { flow } \\
\left(\mathrm{ft}^{3} / \mathrm{s}\right)\end{array}$} & \multicolumn{6}{|c|}{ Estimated peak discharge $\left(\mathrm{ft}^{3} / \mathrm{s}\right)$ for indicated peak-discharge frequency } \\
\hline & & 2-year & 5-year & 10 -year & 25-year & 50 -year & 100-year \\
\hline 517 & 4.81 & 634 & 1,820 & 3,070 & 5,190 & 7,200 & 9,550 \\
\hline 551 & 3.79 & 603 & 1,720 & 2,880 & 4,850 & 6,700 & 8,850 \\
\hline 552 & 18.7 & 1,740 & 4,850 & 8,120 & 13,800 & 19,100 & 25,600 \\
\hline 565 & .35 & 475 & 1,430 & 2,420 & 4,100 & 5,630 & 7,460 \\
\hline 572 & .65 & 527 & 1,600 & 2,710 & 4,600 & 6,330 & 8,390 \\
\hline 581 & .98 & 571 & 1,720 & 2,920 & 4,960 & 6,810 & 9,030 \\
\hline 587 & 2.14 & 478 & 1,420 & 2,420 & 4,130 & 5,760 & 7,670 \\
\hline 591 & .88 & 562 & 1,720 & 2,920 & 4,980 & 6,870 & 9,130 \\
\hline 600 & 21.0 & 1,360 & 4,160 & 7,250 & 12,800 & 18,300 & 25,100 \\
\hline 602 & 23.6 & 1,890 & 5,250 & 8,770 & 14,800 & 20,500 & 27,500 \\
\hline 604 & 1.40 & 625 & 1,860 & 3,140 & 5,300 & 7,260 & 9,610 \\
\hline 606 & 24.6 & 1,490 & 4,520 & 7,870 & 13,900 & 19,900 & 27,200 \\
\hline 609 & 23.2 & 1,440 & 4,390 & 7,640 & 13,500 & 19,300 & 26,400 \\
\hline 622 & 8.76 & 18 & 188 & 606 & 2,010 & 4,240 & 8,180 \\
\hline 623 & NA & NA & NA & NA & NA & NA & NA \\
\hline 626 & 27.9 & 1,580 & 4,790 & 8,320 & 14,700 & 21,000 & 28,800 \\
\hline 629 & NA & NA & NA & NA & NA & NA & NA \\
\hline 630 & NA & NA & NA & NA & NA & NA & NA \\
\hline 646 & 69.5 & 2,020 & 4,510 & 6,800 & 10,500 & 13,800 & 17,600 \\
\hline 711 & NA & NA & NA & NA & NA & NA & NA \\
\hline 781 & 3.88 & 666 & 1,870 & 3,110 & 5,200 & 7,150 & 9,410 \\
\hline 784 & NA & NA & NA & NA & NA & NA & NA \\
\hline 788 & NA & NA & NA & NA & NA & NA & NA \\
\hline 790 & 1.36 & 677 & 1,980 & 3,310 & 5,560 & 7,580 & 10,000 \\
\hline 814 & 13.7 & 926 & 2,960 & 5,320 & 9,790 & 14,400 & 20,200 \\
\hline 818 & 1.18 & 648 & 1,890 & 3,160 & 5,300 & 7,230 & 9,530 \\
\hline
\end{tabular}




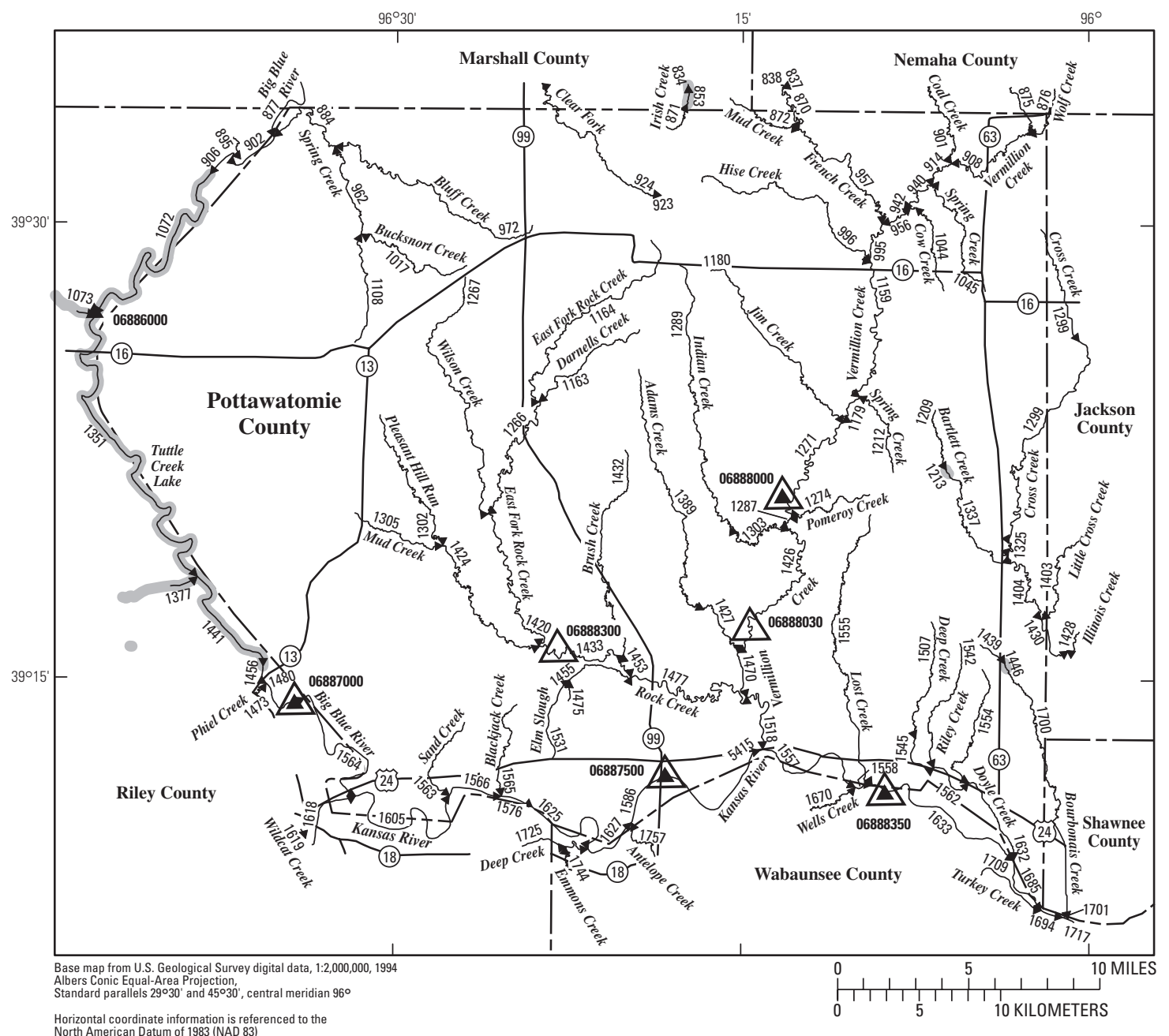

EXPLANATION

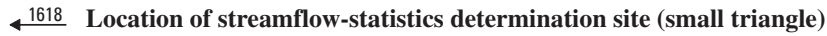
and associated identification number-small triangle points in downstream direction

06887000 U.S. Geological Survey streamflow-gaging station and number used for estimates of flow duration

06888300

$\triangle$ U.S. Geological Survey streamflow-gaging station and number used for estimates of peak-discharge frequency values 1441

Lake and determination site identification number
}

KANSAS

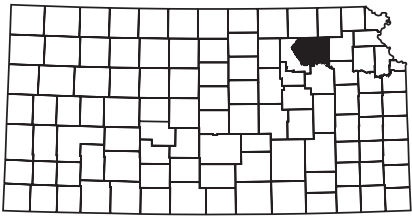

Index map

Figure 85. Location of streamflow-statistics determination sites, associated identification numbers, and U.S. Geological Survey streamflow-gaging stations used in the flow-duration and peak-discharge frequency analyses for Pottawatomie County. 
Table 81. Estimated flow-duration values, mean flow values, and peak-discharge frequency values for controlled and uncontrolled flow stream segments on the 1999 Kansas Surface Water Register for Pottawatomie County.

[KSWR, Kansas Surface Water Register; CUSEGA, catalog unit segment number alpha; $\mathrm{mi}^{2}$, square miles; $\mathrm{ft}^{3} / \mathrm{s}$, cubic feet per second; HYDRO, lake or other hydrologic structure; NA, not applicable; NRDitch, irrigation ditch; NRTribal, tribal stream]

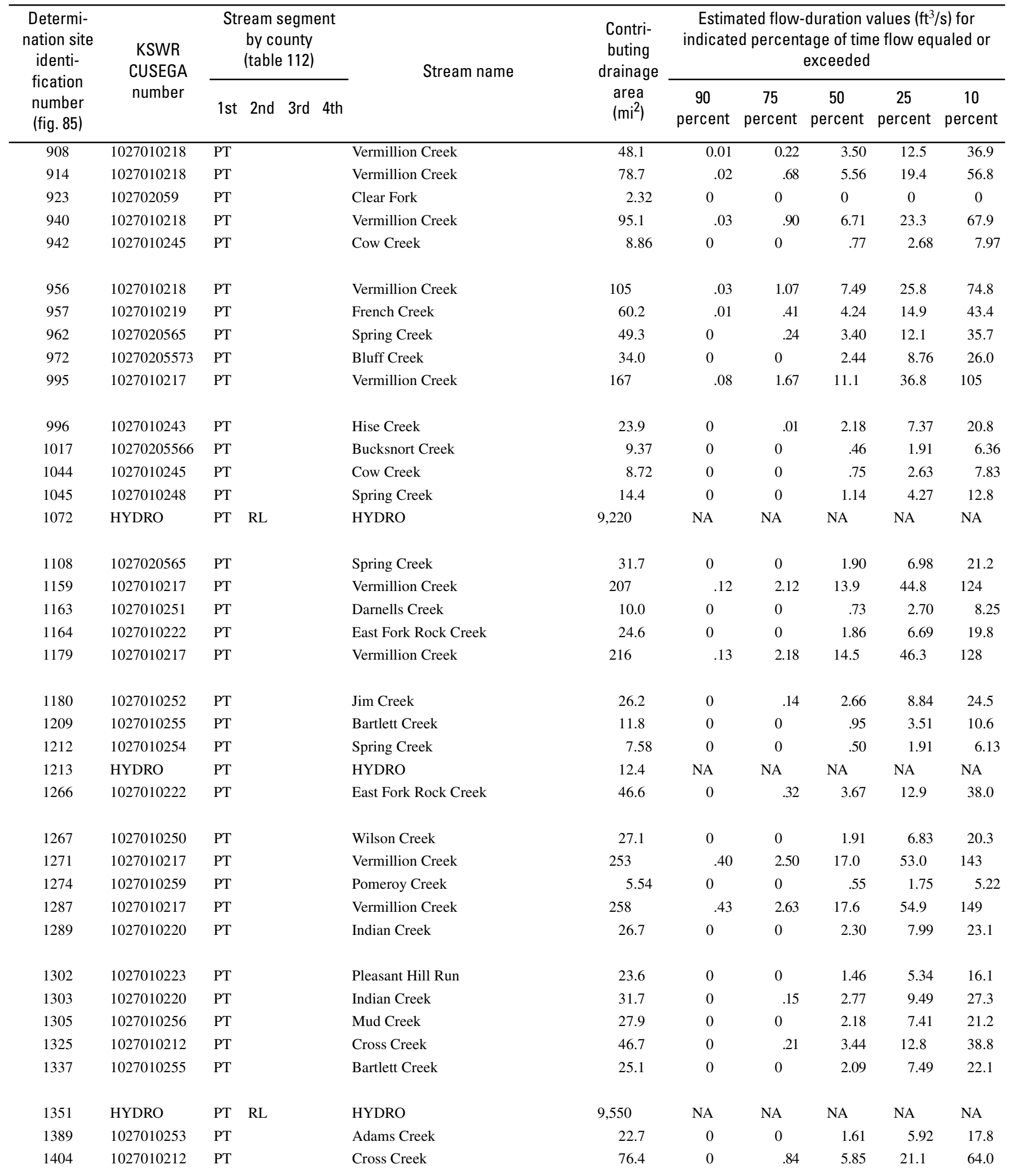


Table 81. Estimated flow-duration values, mean flow values, and peak-discharge frequency values for controlled and uncontrolled flow stream segments on the 1999 Kansas Surface Water Register for Pottawatomie County.-Continued

[KSWR, Kansas Surface Water Register; CUSEGA, catalog unit segment number alpha; $\mathrm{mi}^{2}$, square miles; $\mathrm{ft}^{3} / \mathrm{s}$, cubic feet per second; HYDRO, lake or other hydrologic structure; NA, not applicable; NRDitch, irrigation ditch; NRTribal, tribal stream]

\begin{tabular}{|c|c|c|c|c|c|c|c|}
\hline \multirow{2}{*}{$\begin{array}{l}\text { Determi- } \\
\text { nation site } \\
\text { identi- } \\
\text { fication } \\
\text { number } \\
\text { (fig. 85) }\end{array}$} & \multirow{2}{*}{$\begin{array}{c}\text { Estimated mean } \\
\text { flow } \\
\left(\mathrm{ft}^{3} / \mathrm{s}\right)\end{array}$} & \multicolumn{6}{|c|}{ Estimated peak discharge $\left(\mathrm{ft}^{3} / \mathrm{s}\right)$ for indicated peak-discharge frequency } \\
\hline & & 2-year & 5-year & 10-year & 25-year & 50-year & 100-year \\
\hline 908 & 27.7 & 3,160 & 6,860 & 10,200 & 15,200 & 19,700 & 24,500 \\
\hline 940 & 48.4 & 4,540 & 9,560 & 14,000 & 20,700 & 26,600 & 33,000 \\
\hline 942 & 6.47 & 1,050 & 2,270 & 3,320 & 4,920 & 6,260 & 7,780 \\
\hline 956 & 52.6 & 4,690 & 9,860 & 14,400 & 21,300 & 27,300 & 33,900 \\
\hline 957 & 32.2 & 3,420 & 7,420 & 11,000 & 16,500 & 21,300 & 26,600 \\
\hline 996 & 15.7 & 1,820 & 4,030 & 5,970 & 8,970 & 11,500 & 14,400 \\
\hline 1017 & 5.65 & 963 & 2,140 & 3,180 & 4,780 & 6,110 & 7,640 \\
\hline 1044 & 6.37 & 1,040 & 2,240 & 3,290 & 4,870 & 6,190 & 7,690 \\
\hline 1045 & 10.2 & 1,390 & 3,030 & 4,460 & 6,640 & 8,460 & 10,500 \\
\hline 1072 & NA & NA & NA & NA & NA & NA & NA \\
\hline 1108 & 16.9 & 4,620 & 9,160 & 13,000 & 18,600 & 23,400 & 28,400 \\
\hline 1159 & 80.0 & 6,290 & 12,800 & 18,300 & 26,500 & 33,500 & 41,100 \\
\hline 1163 & 6.75 & 1,040 & 2,280 & 3,370 & 5,050 & 6,440 & 8,040 \\
\hline 1164 & 15.3 & 1,750 & 3,890 & 5,780 & 8,700 & 11,200 & 14,000 \\
\hline 1267 & 15.9 & 1,790 & 4,020 & 6,000 & 9,070 & 11,600 & 14,600 \\
\hline 1271 & 87.4 & 6,190 & 12,600 & 17,900 & 25,800 & 32,400 & 39,700 \\
\hline 1274 & 4.23 & 769 & 1,660 & 2,430 & 3,590 & 4,560 & 5,670 \\
\hline 1287 & 90.7 & 6,250 & 12,300 & 17,300 & 24,700 & 30,900 & 37,700 \\
\hline 1289 & 17.4 & 1,890 & 4,190 & 6,220 & 9,360 & 12,000 & 15,000 \\
\hline 1302 & 13.0 & 1,590 & 3,600 & 5,390 & 8,160 & 10,500 & 13,200 \\
\hline 1303 & 20.3 & 3,190 & 6,630 & 9,630 & 14,100 & 18,000 & 22,100 \\
\hline 1305 & 16.0 & 1,720 & 3,900 & 5,860 & 8,900 & 11,500 & 14,400 \\
\hline 1325 & 29.4 & 4,140 & 8,530 & 12,400 & 18,100 & 23,100 & 28,500 \\
\hline 1337 & 16.9 & 1,890 & 4,190 & 6,220 & 9,340 & 12,000 & 15,000 \\
\hline 1351 & NA & NA & NA & $\mathrm{NA}$ & NA & NA & NA \\
\hline 1389 & 14.0 & 1,670 & 3,750 & 5,590 & 8,450 & 10,800 & 13,600 \\
\hline 1404 & 46.4 & 5,040 & 10,300 & 14,900 & 21,900 & 27,900 & 34,400 \\
\hline
\end{tabular}


Table 81. Estimated flow-duration values, mean flow values, and peak-discharge frequency values for controlled and uncontrolled flow stream segments on the 1999 Kansas Surface Water Register for Pottawatomie County._-Continued

[KSWR, Kansas Surface Water Register; CUSEGA, catalog unit segment number alpha; $\mathrm{mi}^{2}$, square miles; $\mathrm{ft}^{3} / \mathrm{s}$, cubic feet per second; HYDRO, lake or other hydrologic structure; NA, not applicable; NRDitch, irrigation ditch; NRTribal, tribal stream]

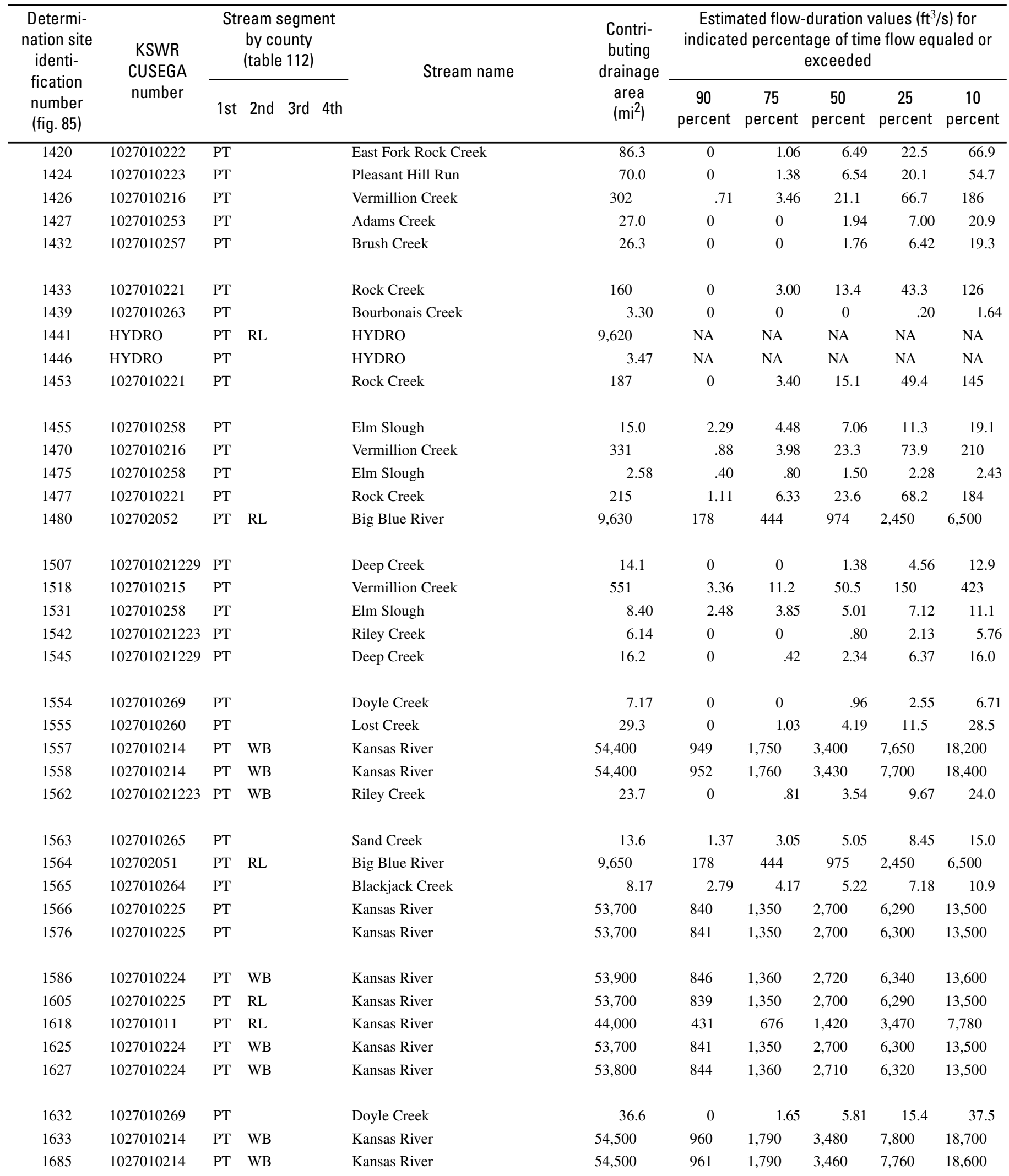


Table 81. Estimated flow-duration values, mean flow values, and peak-discharge frequency values for controlled and uncontrolled flow stream segments on the 1999 Kansas Surface Water Register for Pottawatomie County.-Continued

[KSWR, Kansas Surface Water Register; CUSEGA, catalog unit segment number alpha; $\mathrm{mi}^{2}$, square miles; $\mathrm{ft}^{3} / \mathrm{s}$, cubic feet per second; HYDRO, lake or other hydrologic structure; NA, not applicable; NRDitch, irrigation ditch; NRTribal, tribal stream]

\begin{tabular}{|c|c|c|c|c|c|c|c|}
\hline \multirow{2}{*}{$\begin{array}{l}\text { Determi- } \\
\text { nation site } \\
\text { identi- } \\
\text { fication } \\
\text { number } \\
\text { (fig. 85) }\end{array}$} & \multirow{2}{*}{$\begin{array}{c}\text { Estimated mean } \\
\text { flow } \\
\left(\mathrm{ft}^{3} / \mathrm{s}\right)\end{array}$} & \multicolumn{6}{|c|}{ Estimated peak discharge $\left(\mathrm{ft}^{3} / \mathrm{s}\right)$ for indicated peak-discharge frequency } \\
\hline & & 2-year & 5-year & 10-year & 25-year & 50-year & 100-year \\
\hline 1420 & 47.7 & 4,600 & 9,100 & 13,000 & 18,800 & 23,800 & 29,300 \\
\hline 1427 & 16.3 & 1,840 & 4,160 & 6,220 & 9,420 & 12,100 & 15,200 \\
\hline 1432 & 15.3 & 1,790 & 4,050 & 6,070 & 9,190 & 11,800 & 14,900 \\
\hline 1433 & 83.4 & 5,880 & 10,100 & 13,400 & 18,100 & 21,900 & 26,100 \\
\hline 1439 & 2.05 & 581 & 1,240 & 1,800 & 2,640 & 3,340 & 4,130 \\
\hline 1455 & 10.8 & 1,280 & 2,880 & 4,290 & 6,460 & 8,280 & 10,400 \\
\hline 1470 & 126 & 6,910 & 10,300 & 12,700 & 15,900 & 18,500 & 21,300 \\
\hline 1475 & 1.52 & 463 & 1,000 & 1,460 & 2,160 & 2,740 & 3,400 \\
\hline 1477 & 113 & 5,650 & 9,510 & 12,500 & 16,600 & 19,900 & 23,500 \\
\hline 1480 & 2,490 & 16,600 & 25,500 & 36,300 & 42,000 & 46,800 & 49,600 \\
\hline 1507 & 9.92 & 1,330 & 2,930 & 4,320 & 6,460 & 8,250 & 10,300 \\
\hline 1518 & 235 & 8,560 & 13,200 & 16,600 & 21,400 & 25,200 & 29,300 \\
\hline 1531 & 6.30 & 917 & 2,030 & 3,010 & 4,500 & 5,750 & 7,180 \\
\hline 1542 & 4.58 & 831 & 1,790 & 2,620 & 3,880 & 4,920 & 6,110 \\
\hline 1563 & 9.03 & 1,160 & 2,620 & 3,920 & 5,920 & 7,610 & 9,550 \\
\hline 1564 & 2,490 & 16,600 & 25,500 & 36,300 & 42,000 & 46,900 & 49,700 \\
\hline 1565 & 6.10 & 894 & 1,980 & 2,940 & 4,400 & 5,620 & 7,020 \\
\hline 1566 & 5,620 & 28,500 & 54,200 & 77,900 & 108,000 & 155,000 & 201,000 \\
\hline 1576 & 5,620 & 28,500 & 54,200 & 78,000 & 108,000 & 155,000 & 201,000 \\
\hline 1586 & 5,650 & 28,600 & 54,500 & 78,400 & 108,000 & 156,000 & 202,000 \\
\hline 1605 & 5,610 & 28,500 & 54,200 & 77,900 & 107,000 & 155,000 & 201,000 \\
\hline 1618 & 3,150 & 19,600 & 32,400 & 45,800 & 74,900 & 105,000 & 143,000 \\
\hline 1625 & 5,620 & 28,500 & 54,200 & 78,000 & 108,000 & 155,000 & 201,000 \\
\hline 1627 & 5,640 & 28,600 & 54,400 & 78,300 & 108,000 & 156,000 & 202,000 \\
\hline 1632 & 24.8 & 2,980 & 5,960 & 8,490 & 12,200 & 15,300 & 18,600 \\
\hline 1633 & 7,180 & 33,800 & 59,800 & 82,100 & 117,000 & 148,000 & 183,000 \\
\hline 1685 & 7,140 & 33,900 & 60,100 & 82,600 & 117,000 & 149,000 & 185,000 \\
\hline
\end{tabular}


Table 81. Estimated flow-duration values, mean flow values, and peak-discharge frequency values for controlled and uncontrolled flow stream segments on the 1999 Kansas Surface Water Register for Pottawatomie County.-Continued

[KSWR, Kansas Surface Water Register; CUSEGA, catalog unit segment number alpha; $\mathrm{mi}^{2}$, square miles; $\mathrm{ft}^{3} / \mathrm{s}$, cubic feet per second; HYDRO, lake or other hydrologic structure; NA, not applicable; NRDitch, irrigation ditch; NRTribal, tribal stream]

\begin{tabular}{|c|c|c|c|c|c|c|c|c|c|c|c|}
\hline $\begin{array}{l}\text { Determi- } \\
\text { nation site } \\
\text { identi- } \\
\text { fication } \\
\text { number } \\
\text { (fig. 85) }\end{array}$ & $\begin{array}{l}\text { KSWR } \\
\text { CUSEGA } \\
\text { number }\end{array}$ & \multicolumn{3}{|c|}{$\begin{array}{l}\text { Stream segment } \\
\text { by county } \\
\text { (table 112) }\end{array}$} & Stream name & $\begin{array}{l}\text { Contri- } \\
\text { buting } \\
\text { drainage } \\
\text { area } \\
\left(\mathrm{mi}^{2}\right)\end{array}$ & \multicolumn{5}{|c|}{$\begin{array}{l}\text { Estimated flow-duration values }\left(\mathrm{ft}^{3} / \mathrm{s}\right) \text { for } \\
\text { indicated percentage of time flow equaled or } \\
\text { exceeded }\end{array}$} \\
\hline 1694 & 1027010214 & PT & SN & & Kansas River & 54,500 & 961 & 1,780 & 3,440 & 7,740 & 18,500 \\
\hline 1757 & 1027010267 & PT & WB & & Antelope Creek & 21.8 & 0 & .97 & 3.46 & 8.71 & 20.7 \\
\hline 5415 & 1027010224 & PT & & & Kansas River & 53,900 & 846 & 1,360 & 2,720 & 6,340 & 13,600 \\
\hline
\end{tabular}


Table 81. Estimated flow-duration values, mean flow values, and peak-discharge frequency values for controlled and uncontrolled flow stream segments on the 1999 Kansas Surface Water Register for Pottawatomie County.-Continued

[KSWR, Kansas Surface Water Register; CUSEGA, catalog unit segment number alpha; $\mathrm{mi}^{2}$, square miles; $\mathrm{ft}^{3} / \mathrm{s}$, cubic feet per second; HYDRO, lake or other hydrologic structure; NA, not applicable; NRDitch, irrigation ditch; NRTribal, tribal stream]

\begin{tabular}{|c|c|c|c|c|c|c|c|}
\hline \multirow{2}{*}{$\begin{array}{l}\text { Determi- } \\
\text { nation site } \\
\text { identi- } \\
\text { fication } \\
\text { number } \\
\text { (fig. } 85 \text { ) }\end{array}$} & \multirow{2}{*}{$\begin{array}{l}\text { Estimated mean } \\
\text { flow } \\
\left(\mathrm{ft}^{3} / \mathrm{s}\right)\end{array}$} & \multicolumn{6}{|c|}{ Estimated peak discharge $\left(\mathrm{ft}^{3} / \mathrm{s}\right)$ for indicated peak-discharge frequency } \\
\hline & & 2-year & 5-year & 10-year & 25-year & 50-year & 100-year \\
\hline 1694 & 7,120 & 34,000 & 60,400 & 83,000 & 117,000 & 150,000 & 186,000 \\
\hline 1757 & 14.2 & 1,620 & 3,650 & 5,450 & 8,220 & 10,600 & 13,300 \\
\hline 5415 & 5,650 & 28,600 & 54,500 & 78,400 & 108,000 & 156,000 & 202,000 \\
\hline
\end{tabular}




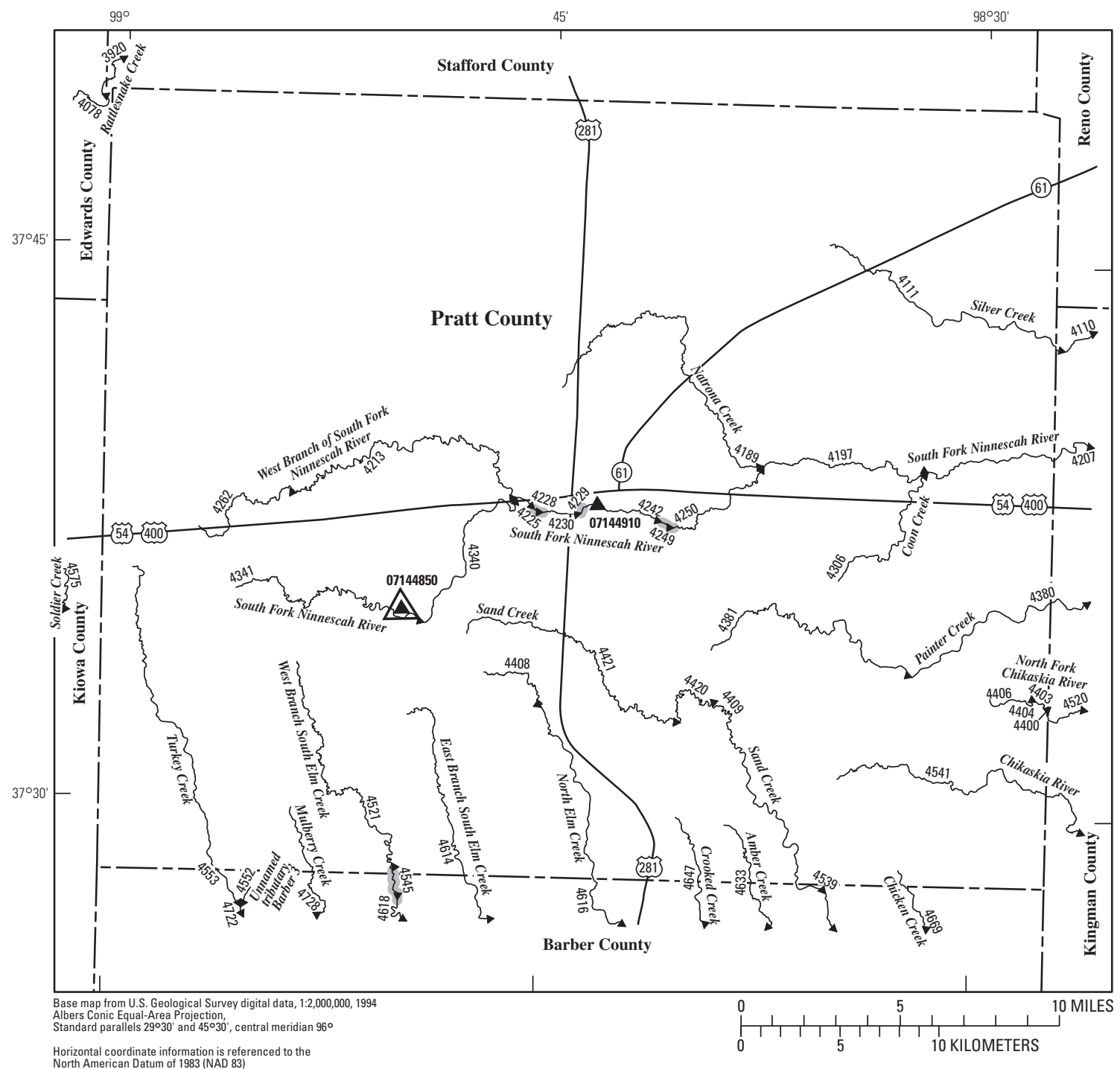

\section{EXPLANATION}

$\$ 4614$ Location of streamflow-statistics determination site (small triangle) and associated identification number-small triangle points in downstream direction

07144910

U.S. Geological Survey streamflow-gaging station and number used for estimates of flow duration

07144850

U.S. Geological Survey streamflow-gaging station and number used for estimates of peak-discharge frequency values

KANSAS

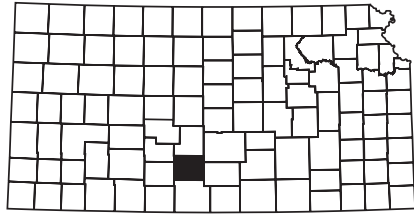

Index map 4545

Figure 86. Location of streamflow-statistics determination sites, associated identification numbers, and U.S. Geological Survey streamflow-gaging stations used in the flow-duration and peak-discharge frequency analyses for Pratt County. 
Table 82. Estimated flow-duration values, mean flow values, and peak-discharge frequency values for controlled and uncontrolled flow stream segments on the 1999 Kansas Surface Water Register for Pratt County.

[KSWR, Kansas Surface Water Register; CUSEGA, catalog unit segment number alpha; $\mathrm{mi}^{2}$, square miles; $\mathrm{ft}^{3} / \mathrm{s}$, cubic feet per second; HYDRO, lake or other hydrologic structure; NA, not applicable; NRDitch, irrigation ditch; NRTribal, tribal stream]

\begin{tabular}{|c|c|c|c|c|c|c|c|c|c|}
\hline \multirow{2}{*}{$\begin{array}{c}\text { Determi- } \\
\text { nation site } \\
\text { identi- } \\
\text { fication } \\
\text { number } \\
\text { (fig. 86) }\end{array}$} & \multirow{2}{*}{$\begin{array}{l}\text { KSWR } \\
\text { CUSEGA } \\
\text { number }\end{array}$} & $\begin{array}{l}\text { Stream segment } \\
\text { by county } \\
\text { (table 112) }\end{array}$ & \multirow{2}{*}{ Stream name } & \multirow{2}{*}{$\begin{array}{l}\text { Contri- } \\
\text { buting } \\
\text { drainage } \\
\text { area } \\
\left(\mathrm{mi}^{2}\right)\end{array}$} & \multicolumn{5}{|c|}{$\begin{array}{c}\text { Estimated flow-duration values }\left(\mathrm{ft}^{3} / \mathrm{s}\right) \text { for } \\
\text { indicated percentage of time flow equaled or } \\
\text { exceeded }\end{array}$} \\
\hline & & 1st 2nd 3rd 4th & & & $\begin{array}{c}90 \\
\text { percent }\end{array}$ & $\begin{array}{c}75 \\
\text { percent }\end{array}$ & $\begin{array}{c}50 \\
\text { percent }\end{array}$ & $\begin{array}{c}25 \\
\text { percent }\end{array}$ & $\begin{array}{c}10 \\
\text { percent }\end{array}$ \\
\hline 4189 & 11030015307 & $\overline{\mathrm{PR}}$ & Natrona Creek & 71.4 & 0.91 & 2.17 & 3.27 & 5.07 & 9.25 \\
\hline 4197 & 110300154 & PR & South Fork Ninnescah River & 261 & 18.3 & 26.2 & 34.3 & 46.5 & 67.3 \\
\hline 4213 & 110300155 & PR & West Branch of South Fork Ninnescah River & 103 & 3.36 & 5.11 & 6.30 & 7.93 & 11.5 \\
\hline 4225 & 110300154 & PR & South Fork Ninnescah River & 141 & 5.37 & 7.67 & 9.64 & 12.1 & 16.9 \\
\hline 4228 & HYDRO & PR & HYDRO & 142 & NA & NA & NA & NA & NA \\
\hline 4229 & HYDRO & PR & HYDRO & 146 & NA & NA & NA & NA & NA \\
\hline 4230 & 110300154 & PR & South Fork Ninnescah River & 145 & 5.53 & 7.90 & 9.98 & 12.6 & 17.7 \\
\hline 4242 & 110300154 & PR & South Fork Ninnescah River & 157 & 6.00 & 8.60 & 11.0 & 14.0 & 20.0 \\
\hline 4249 & HYDRO & PR & HYDRO & 159 & NA & NA & NA & NA & NA \\
\hline 4250 & 110300154 & PR & South Fork Ninnescah River & 170 & 7.54 & 10.9 & 14.1 & 18.4 & 26.5 \\
\hline 4262 & 110300155 & PR & West Branch of South Fork Ninnescah River & 39.7 & .92 & 1.00 & 1.20 & 1.46 & 1.93 \\
\hline 4306 & 110300159 & PR & Coon Creek & 15.3 & .03 & .04 & .05 & .06 & .09 \\
\hline 4340 & 110300156 & PR & South Fork Ninnescah River & 36.5 & .40 & .42 & .95 & 1.12 & 2.28 \\
\hline 4341 & 110300156 & PR & South Fork Ninnescah River & 23.1 & 0 & 0 & 0 & 0 & 0 \\
\hline 4381 & 110300157 & PR & Painter Creek & 27.6 & .10 & .14 & .45 & .51 & 1.52 \\
\hline 4400 & 1106000537 & PR & North Fork Chikaskia River & 6.36 & 0 & 0 & 0 & 0 & .01 \\
\hline 4403 & 1106000537 & PR & North Fork Chikaskia River & 6.38 & 0 & 0 & 0 & 0 & .01 \\
\hline 4404 & 1106000537 & PR & North Fork Chikaskia River & 6.38 & 0 & 0 & 0 & 0 & .01 \\
\hline 4406 & 1106000537 & PR & North Fork Chikaskia River & 6.36 & 0 & 0 & 0 & 0 & .01 \\
\hline 4408 & 110600034 & PR & North Elm Creek & 7.13 & 0 & 0 & 0 & 0 & 0 \\
\hline 4409 & 1106000511 & PR & Sand Creek & 28.4 & .01 & .02 & .04 & .09 & .27 \\
\hline 4420 & 1106000511 & PR & Sand Creek & 28.3 & .01 & .02 & .04 & .09 & .27 \\
\hline 4421 & 1106000511 & PR & Sand Creek & 23.4 & .01 & .02 & .03 & .06 & .13 \\
\hline 4521 & 110600039005 & PR & West Branch South Elm Creek & 25.4 & 0 & .02 & .02 & .02 & .21 \\
\hline
\end{tabular}


Table 82. Estimated flow-duration values, mean flow values, and peak-discharge frequency values for controlled and uncontrolled flow stream segments on the 1999 Kansas Surface Water Register for Pratt County.-Continued

[KSWR, Kansas Surface Water Register; CUSEGA, catalog unit segment number alpha; $\mathrm{mi}^{2}$, square miles; $\mathrm{ft}^{3} / \mathrm{s}$, cubic feet per second; HYDRO, lake or other hydrologic structure; NA, not applicable; NRDitch, irrigation ditch; NRTribal, tribal stream]

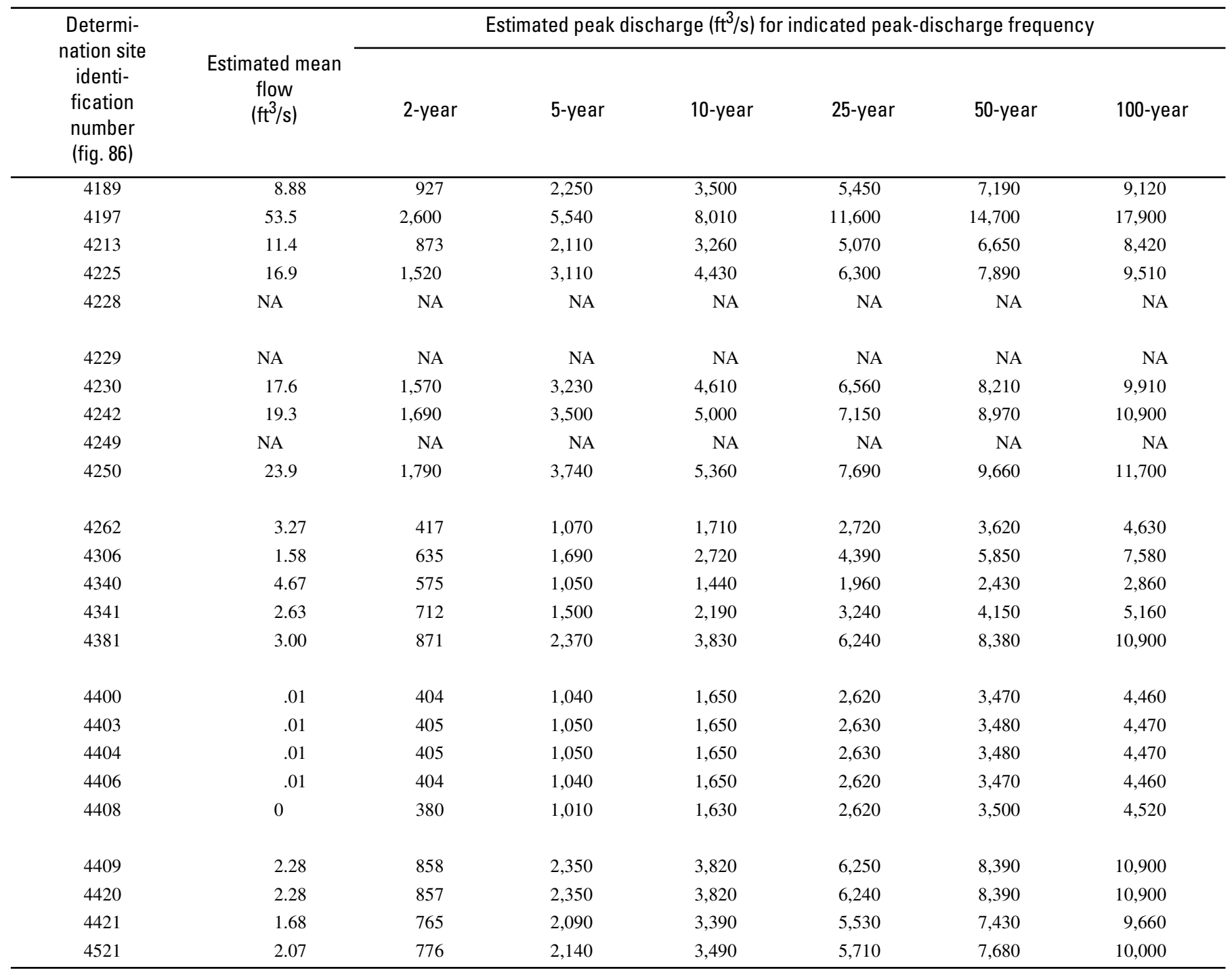




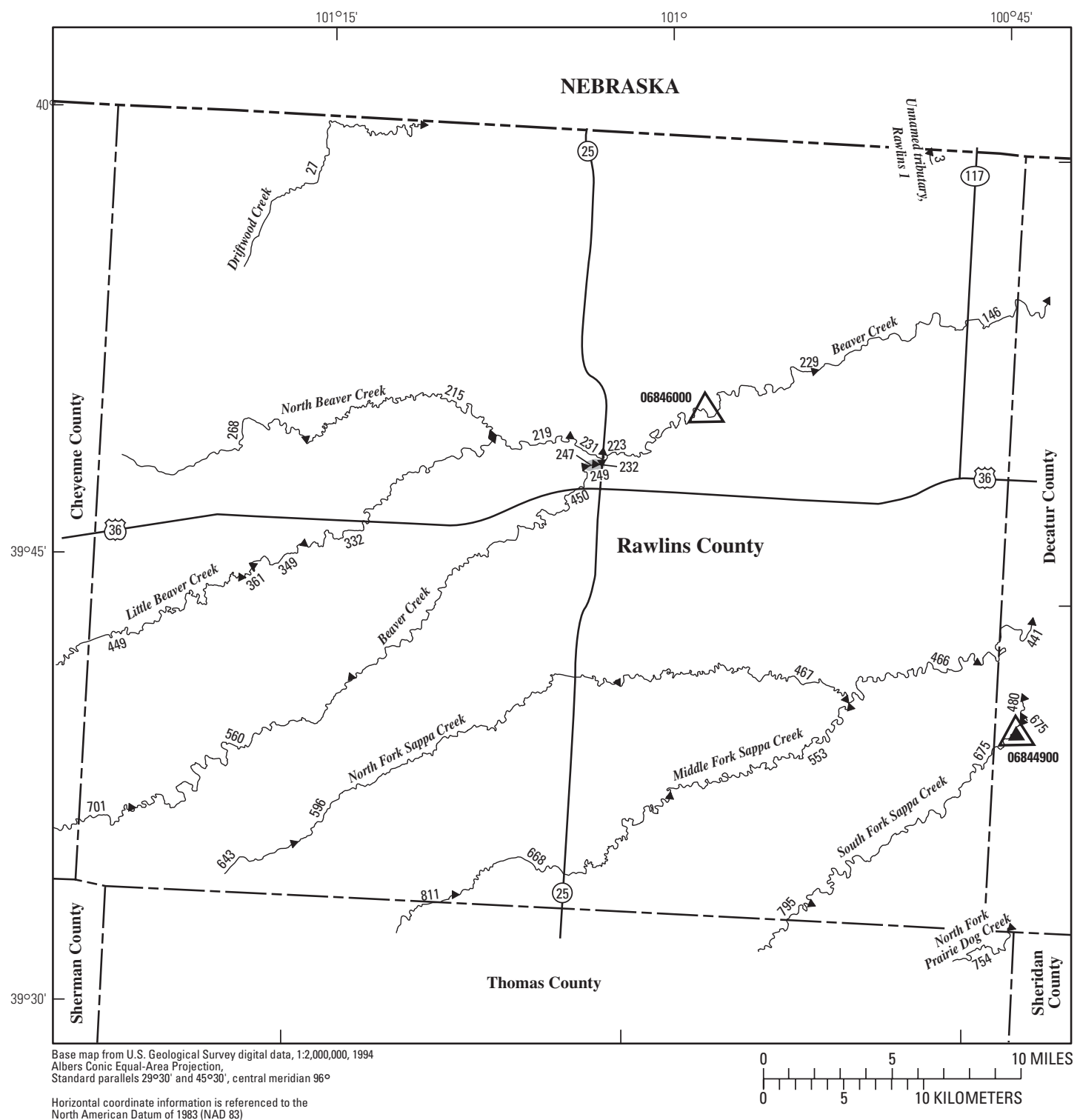

\section{EXPLANATION}

\$701 Location of streamflow-statistics determination site (small triangle) and associated identification number-small triangle points in downstream direction

06844900 U.S. Geological Survey streamflow-gaging station and number used for estimates of flow duration

${ }^{06846000} \triangle$ U.S. Geological Survey streamflow-gaging station and number used for estimates of peak-discharge frequency values

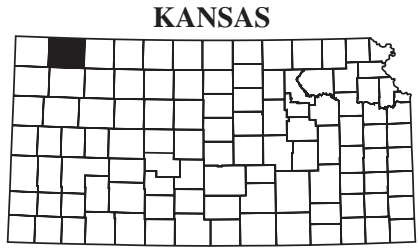

Index map

249 Lake and determination site identification number

Figure 86. Location of streamflow-statistics determination sites, associated identification numbers, and U.S. Geological Survey streamflow-gaging stations used in the flow-duration and peak-discharge frequency analyses for Rawlins County. 
Table 83. Estimated flow-duration values, mean flow values, and peak-discharge frequency values for controlled and uncontrolled flow stream segments on the 1999 Kansas Surface Water Register for Rawlins County.

[KSWR, Kansas Surface Water Register; CUSEGA, catalog unit segment number alpha; $\mathrm{mi}^{2}$, square miles; $\mathrm{ft}^{3} / \mathrm{s}$, cubic feet per second; HYDRO, lake or other hydrologic structure; NA, not applicable; NRDitch, irrigation ditch; NRTribal, tribal stream]

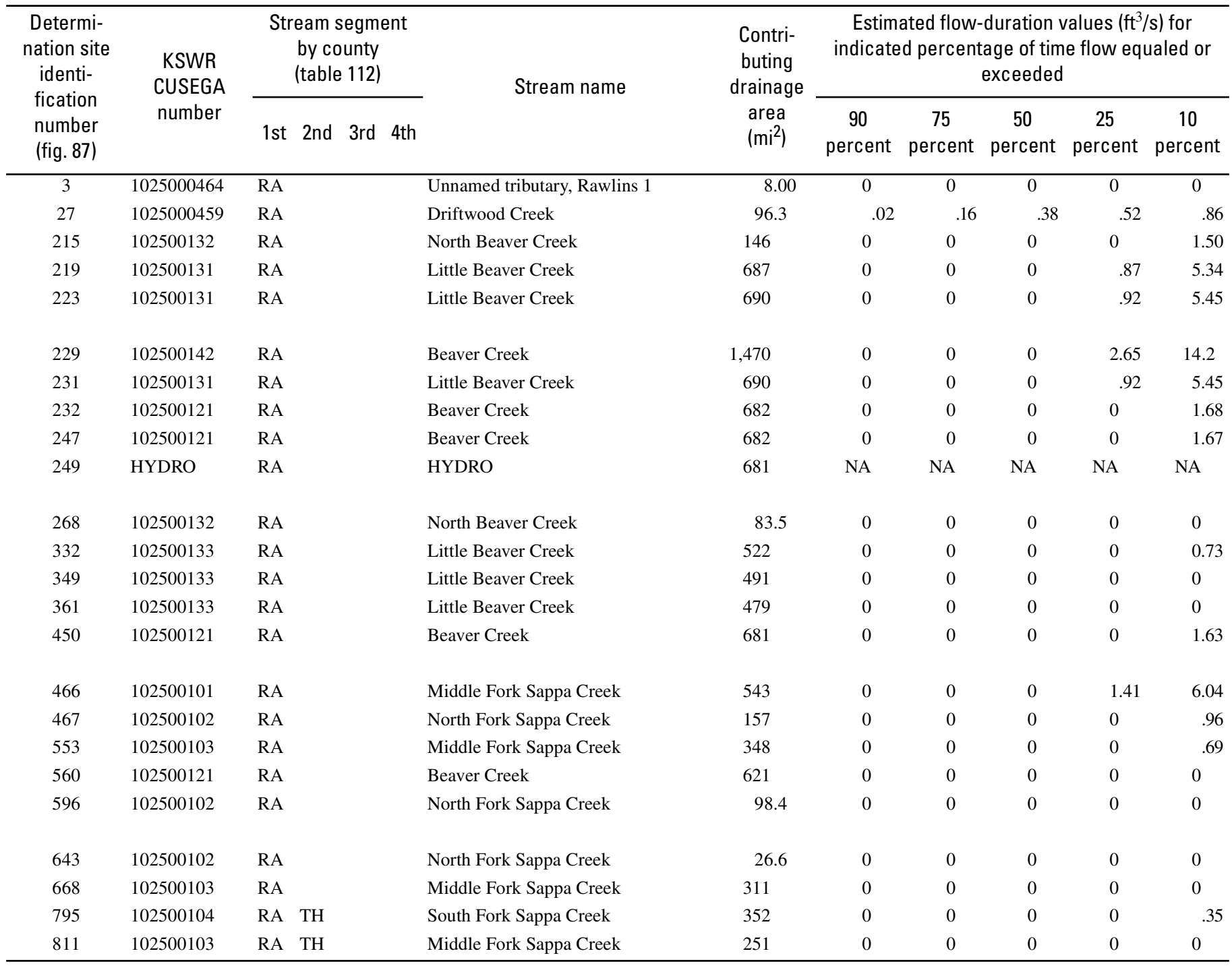


Table 83. Estimated flow-duration values, mean flow values, and peak-discharge frequency values for controlled and uncontrolled flow stream segments on the 1999 Kansas Surface Water Register for Rawlins County.-Continued

[KSWR, Kansas Surface Water Register; CUSEGA, catalog unit segment number alpha; $\mathrm{mi}^{2}$, square miles; $\mathrm{ft}^{3} / \mathrm{s}$, cubic feet per second; HYDRO, lake or other hydrologic structure; NA, not applicable; NRDitch, irrigation ditch; NRTribal, tribal stream]

\begin{tabular}{|c|c|c|c|c|c|c|c|}
\hline \multirow{2}{*}{$\begin{array}{l}\text { Determi- } \\
\text { nation site } \\
\text { identi- } \\
\text { fication } \\
\text { number } \\
\text { (fig. 87) }\end{array}$} & \multirow{2}{*}{$\begin{array}{c}\text { Estimated mean } \\
\text { flow } \\
\left(\mathrm{ft}^{3} / \mathrm{s}\right)\end{array}$} & \multicolumn{6}{|c|}{ Estimated peak discharge $\left(\mathrm{ft}^{3} / \mathrm{s}\right)$ for indicated peak-discharge frequency } \\
\hline & & 2-year & 5-year & 10-year & 25-year & 50-year & 100-year \\
\hline 3 & 0 & 236 & 721 & 1,230 & 2,080 & 2,870 & 3,800 \\
\hline 219 & 6.96 & 775 & 2,390 & 4,210 & 7,470 & 10,700 & 14,600 \\
\hline 223 & 7.04 & 781 & 2,410 & 4,230 & 7,500 & 10,700 & 14,700 \\
\hline 229 & 9.18 & 440 & 1,110 & 1,780 & 2,920 & 4,000 & 5,300 \\
\hline 231 & 7.04 & 781 & 2,410 & 4,230 & 7,500 & 10,700 & 14,700 \\
\hline 268 & 1.30 & 641 & 1,880 & 3,200 & 5,450 & 7,590 & 10,100 \\
\hline 332 & 3.29 & 579 & 1,910 & 3,460 & 6,300 & 9,150 & 12,700 \\
\hline 349 & 2.49 & 519 & 1,750 & 3,210 & 5,890 & 8,620 & 12,000 \\
\hline 361 & 2.20 & 501 & 1,700 & 3,130 & 5,770 & 8,450 & 11,800 \\
\hline 450 & 3.63 & 541 & 1,810 & 3,300 & 6,070 & 8,870 & 12,400 \\
\hline 466 & 8.44 & 804 & 2,540 & 4,520 & 8,110 & 11,700 & 16,100 \\
\hline 467 & 3.58 & 632 & 1,940 & 3,390 & 5,930 & 8,410 & 11,400 \\
\hline 553 & 3.83 & 587 & 1,940 & 3,510 & 6,390 & 9,290 & 12,900 \\
\hline 560 & 2.19 & 476 & 1,650 & 3,050 & 5,690 & 8,380 & 11,800 \\
\hline
\end{tabular}




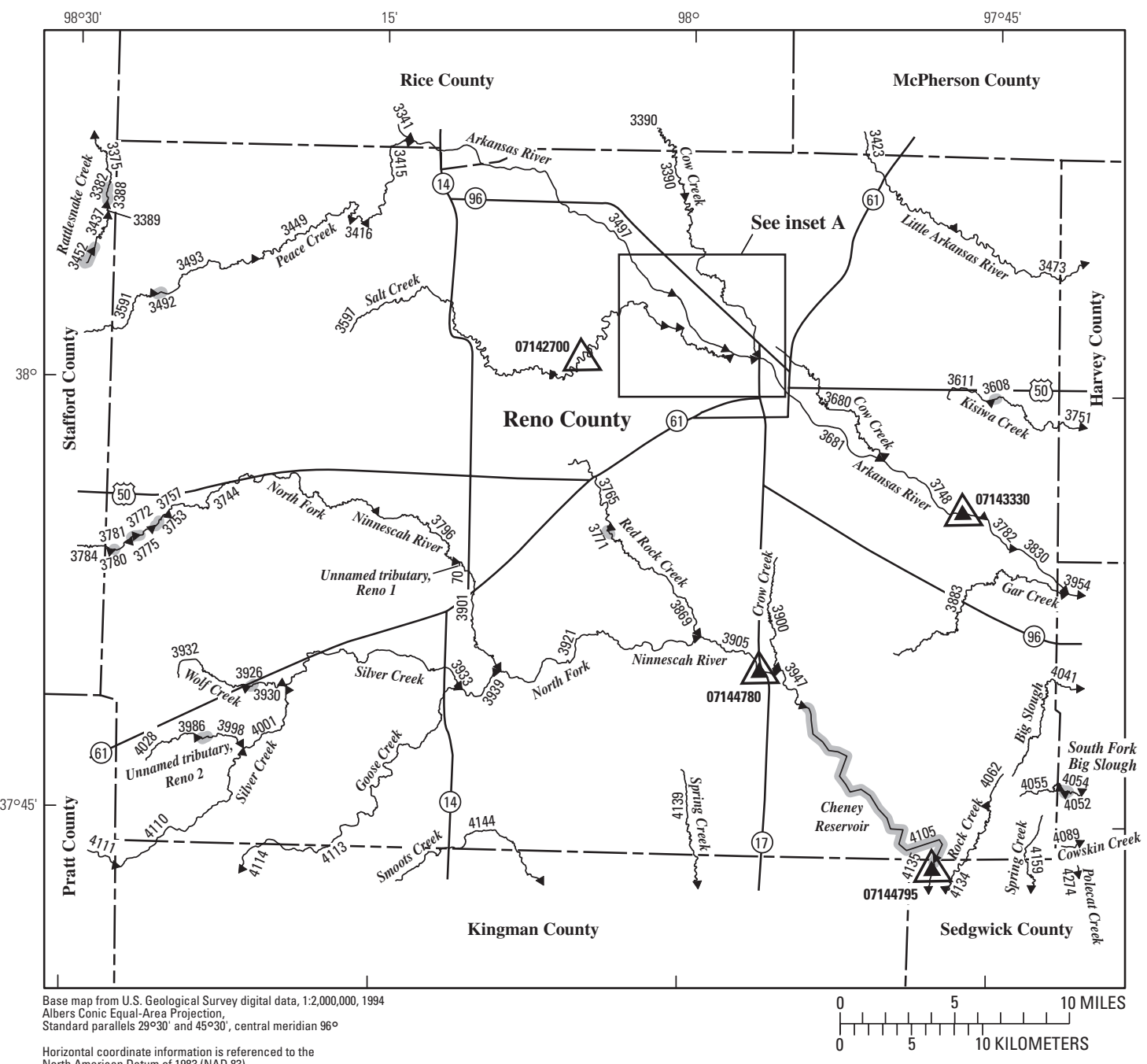

EXPLANATION

4114 Location of streamflow-statistics determination site (small triangle) and associated identification number-small triangle points in downstream direction

07144780 U.S. Geological Survey streamflow-gaging station and number used for estimates of flow duration

07142700

U.S. Geological Survey streamflow-gaging station and number used for estimates of peak-discharge frequency values

KANSAS

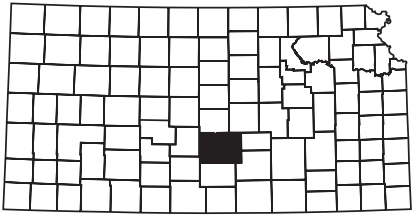

Index map

4105 Lake and determination site identification number

Figure 88. Location of streamflow-statistics determination sites, associated identification numbers, and U.S. Geological Survey streamflow-gaging stations used in the flow-duration and peak-discharge frequency analyses for Reno County. 


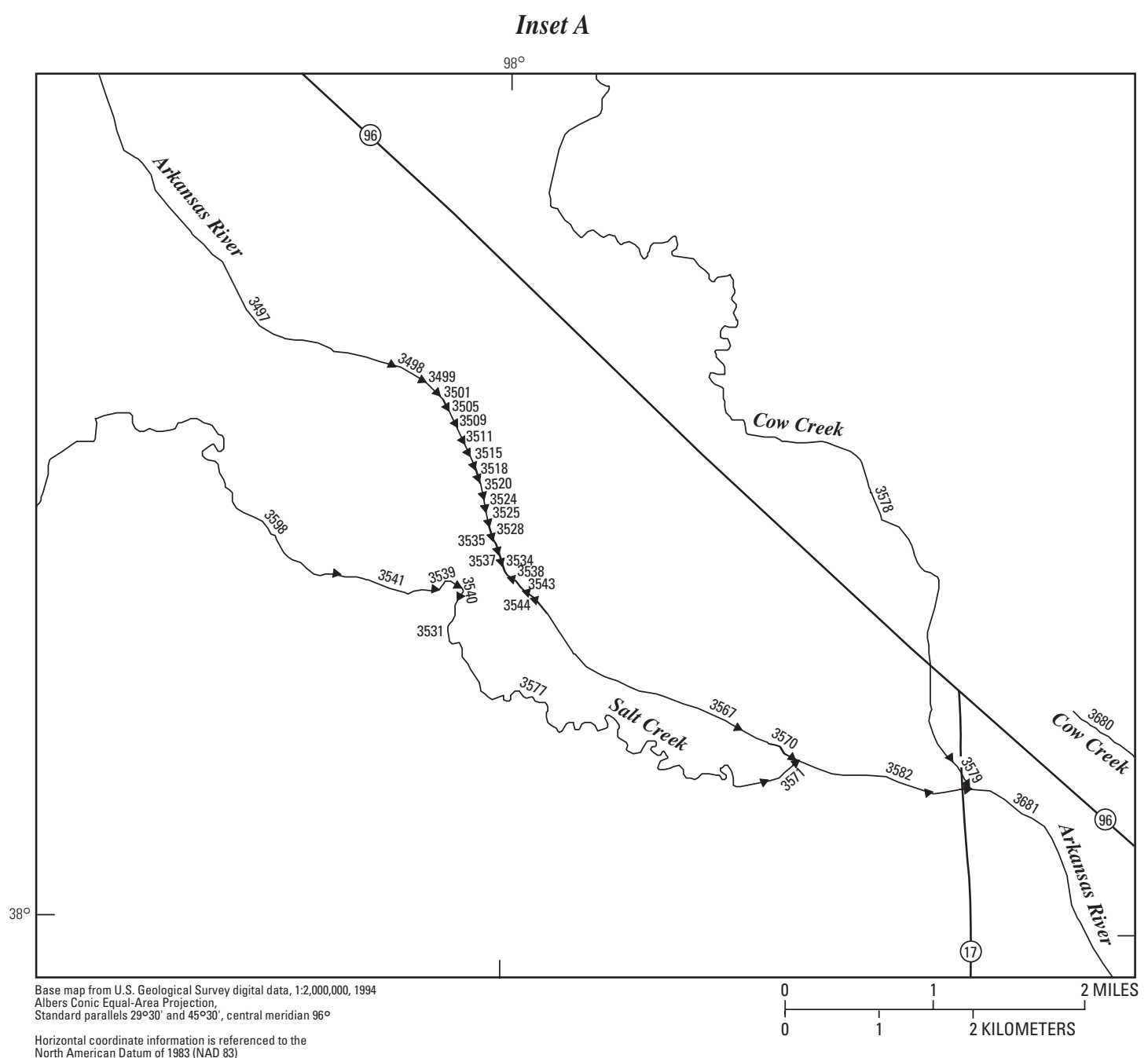

EXPLANATION 3577 Location of streamflow-statistics determination site (small triangle)
and associated identification number-small triangle points in downstream direction

Figure 88. Location of streamflow-statistics determination sites, associated identification numbers, and U.S. Geological Survey streamflow-gaging stations used in the flow-duration and peak-discharge frequency analyses for Reno County.-Continued 
Table 84. Estimated flow-duration values, mean flow values, and peak-discharge frequency values for controlled and uncontrolled flow stream segments on the 1999 Kansas Surface Water Register for Reno County.

[KSWR, Kansas Surface Water Register; CUSEGA, catalog unit segment number alpha; $\mathrm{mi}^{2}$, square miles; $\mathrm{ft}^{3} / \mathrm{s}$, cubic feet per second; HYDRO, lake or other hydrologic structure; NA, not applicable; NRDitch, irrigation ditch; NRTribal, tribal stream]

\begin{tabular}{|c|c|c|c|c|c|c|c|c|c|c|c|}
\hline $\begin{array}{l}\text { Determi- } \\
\text { nation site } \\
\text { identi- } \\
\text { fication } \\
\text { number } \\
\text { (fig. 88) }\end{array}$ & $\begin{array}{l}\text { KSWR } \\
\text { CUSEGA } \\
\text { number }\end{array}$ & \multicolumn{3}{|c|}{$\begin{array}{l}\text { Stream segment } \\
\text { by county } \\
\text { (table 112) }\end{array}$} & Stream name & $\begin{array}{l}\text { Contri- } \\
\text { buting } \\
\text { drainage } \\
\text { area } \\
\left(\mathrm{mi}^{2}\right)\end{array}$ & \multicolumn{5}{|c|}{$\begin{array}{l}\text { Estimated flow-duration values }\left(\mathrm{ft}^{3} / \mathrm{s}\right) \text { for } \\
\text { indicated percentage of time flow equaled or } \\
\text { exceeded }\end{array}$} \\
\hline 70 & 11030014999 & $\mathrm{RN}$ & & & Unnamed tributary, Reno 1 & 0.25 & 0 & 0 & 0 & $\overline{0}$ & 0 \\
\hline 3416 & 110300106 & $\mathrm{RN}$ & & & Peace Creek & 160 & 2.90 & 4.31 & 5.91 & 9.88 & 19.6 \\
\hline 3449 & 110300106 & $\mathrm{RN}$ & & & Peace Creek & 157 & 2.84 & 4.21 & 5.75 & 9.58 & 19.0 \\
\hline 3492 & HYDRO & $\mathrm{RN}$ & & & HYDRO & 93.9 & NA & NA & NA & NA & NA \\
\hline 3493 & 110300106 & $\mathrm{RN}$ & & & Peace Creek & 128 & 1.88 & 2.88 & 3.92 & 6.51 & 13.1 \\
\hline 3501 & 110300104 & $\mathrm{RN}$ & & & Arkansas River & 35,200 & 72.9 & 118 & 219 & 425 & 941 \\
\hline 3505 & 110300104 & $\mathrm{RN}$ & & & Arkansas River & 35,200 & 72.9 & 118 & 219 & 425 & 941 \\
\hline 3509 & 110300104 & $\mathrm{RN}$ & & & Arkansas River & 35,200 & 72.9 & 118 & 219 & 425 & 941 \\
\hline 3511 & 110300104 & $\mathrm{RN}$ & & & Arkansas River & 35,200 & 72.9 & 118 & 219 & 425 & 941 \\
\hline 3515 & 110300104 & $\mathrm{RN}$ & & & Arkansas River & 35,200 & 72.9 & 118 & 219 & 425 & 941 \\
\hline 3518 & 110300104 & $\mathrm{RN}$ & & & Arkansas River & 35,200 & 72.9 & 118 & 219 & 425 & 941 \\
\hline 3520 & 110300104 & $\mathrm{RN}$ & & & Arkansas River & 35,200 & 72.9 & 118 & 219 & 425 & 941 \\
\hline 3524 & 110300104 & $\mathrm{RN}$ & & & Arkansas River & 35,200 & 72.9 & 118 & 219 & 425 & 941 \\
\hline 3525 & 110300104 & RN & & & Arkansas River & 35,200 & 72.9 & 118 & 219 & 425 & 941 \\
\hline 3537 & 110300104 & $\mathrm{RN}$ & & & Arkansas River & 35,200 & 72.9 & 118 & 219 & 425 & 941 \\
\hline 3538 & 110300104 & $\mathrm{RN}$ & & & Arkansas River & 35,200 & 72.9 & 118 & 219 & 425 & 941 \\
\hline 3539 & 110300107 & $\mathrm{RN}$ & & & Salt Creek & 114 & 2.04 & 2.98 & 4.69 & 8.93 & 19.9 \\
\hline 3540 & 110300107 & $\mathrm{RN}$ & & & Salt Creek & 114 & 2.04 & 2.98 & 4.69 & 8.93 & 19.9 \\
\hline 3541 & 110300107 & RN & & & Salt Creek & 114 & 2.04 & 2.98 & 4.69 & 8.93 & 19.9 \\
\hline 3543 & 110300104 & $\mathrm{RN}$ & & & Arkansas River & 35,200 & 72.9 & 118 & 219 & 425 & 941 \\
\hline 3544 & 110300104 & $\mathrm{RN}$ & & & Arkansas River & 35,200 & 72.9 & 118 & 219 & 425 & 941 \\
\hline 3567 & 110300104 & $\mathrm{RN}$ & & & Arkansas River & 35,200 & 73.0 & 118 & 219 & 426 & 941 \\
\hline 3570 & 110300103 & $\mathrm{RN}$ & & & Arkansas River & 35,200 & 73.0 & 118 & 219 & 426 & 941 \\
\hline 3571 & 110300107 & $\mathrm{RN}$ & & & Salt Creek & 130 & 2.24 & 3.34 & 5.41 & 10.5 & 23.6 \\
\hline 3577 & 110300107 & $\mathrm{RN}$ & & & Salt Creek & 130 & 2.24 & 3.34 & 5.41 & 10.5 & 23.6 \\
\hline 3578 & 110300111 & $\mathrm{RN}$ & & & Cow Creek & 886 & 6.67 & 12.5 & 23.5 & 52.7 & 185 \\
\hline 3579 & 110300101 & $\mathrm{RN}$ & & & Arkansas River & 35,300 & 75.6 & 122 & 226 & 436 & 963 \\
\hline 3582 & 110300103 & $\mathrm{RN}$ & & & Arkansas River & 35,300 & 75.6 & 122 & 226 & 436 & 963 \\
\hline 3591 & 110300106 & $\mathrm{RN}$ & SF & & Peace Creek & 93.1 & .69 & 1.34 & 1.95 & 3.24 & 7.00 \\
\hline
\end{tabular}


Table 84. Estimated flow-duration values, mean flow values, and peak-discharge frequency values for controlled and uncontrolled flow stream segments on the 1999 Kansas Surface Water Register for Reno County.-Continued

[KSWR, Kansas Surface Water Register; CUSEGA, catalog unit segment number alpha; $\mathrm{mi}^{2}$, square miles; $\mathrm{ft}^{3} / \mathrm{s}$, cubic feet per second; HYDRO, lake or other hydrologic structure; NA, not applicable; NRDitch, irrigation ditch; NRTribal, tribal stream]

\begin{tabular}{|c|c|c|c|c|c|c|c|}
\hline \multirow{2}{*}{$\begin{array}{l}\text { Determi- } \\
\text { nation site } \\
\text { identi- } \\
\text { fication } \\
\text { number } \\
\text { (fig. 88) }\end{array}$} & \multirow{2}{*}{$\begin{array}{c}\text { Estimated mean } \\
\text { flow } \\
\left(\mathrm{ft}^{3} / \mathrm{s}\right)\end{array}$} & \multicolumn{6}{|c|}{ Estimated peak discharge $\left(\mathrm{ft}^{3} / \mathrm{s}\right)$ for indicated peak-discharge frequency } \\
\hline & & 2-year & 5-year & 10 -year & 25-year & 50-year & 100 -year \\
\hline 70 & 0 & 60 & 145 & 222 & 342 & 445 & 562 \\
\hline 3416 & 16.7 & 854 & 2,140 & 3,400 & 5,410 & 7,210 & 9,270 \\
\hline 3449 & 16.2 & 849 & 2,130 & 3,380 & 5,370 & 7,160 & 9,190 \\
\hline 3492 & NA & NA & NA & NA & NA & NA & NA \\
\hline 3493 & 12.1 & 735 & 1,880 & 3,010 & 4,820 & 6,460 & 8,320 \\
\hline 3501 & 453 & 4,020 & 8,460 & 15,400 & 22,100 & 28,700 & 35,800 \\
\hline 3505 & 453 & 4,020 & 8,460 & 15,400 & 22,100 & 28,700 & 35,800 \\
\hline 3509 & 453 & 4,020 & 8,460 & 15,400 & 22,100 & 28,700 & 35,800 \\
\hline 3511 & 453 & 4,020 & 8,460 & 15,400 & 22,100 & 28,700 & 35,800 \\
\hline 3515 & 453 & 4,020 & 8,460 & 15,400 & 22,100 & 28,700 & 35,800 \\
\hline 3518 & 453 & 4,020 & 8,460 & 15,400 & 22,100 & 28,700 & 35,800 \\
\hline 3520 & 453 & 4,020 & 8,460 & 15,400 & 22,100 & 28,700 & 35,800 \\
\hline 3524 & 453 & 4,020 & 8,460 & 15,400 & 22,100 & 28,700 & 35,800 \\
\hline 3525 & 453 & 4,020 & 8,460 & 15,400 & 22,100 & 28,700 & 35,800 \\
\hline 3539 & 17.5 & 1,150 & 2,170 & 2,960 & 4,090 & 5,000 & 5,970 \\
\hline 3540 & 17.5 & 1,150 & 2,170 & 2,960 & 4,090 & 5,000 & 5,970 \\
\hline 3541 & 17.5 & 1,150 & 2,170 & 2,960 & 4,090 & 5,000 & 5,980 \\
\hline 3543 & 453 & 4,020 & 8,460 & 15,400 & 22,100 & 28,700 & 35,800 \\
\hline 3544 & 453 & 4,020 & 8,460 & 15,400 & 22,100 & 28,700 & 35,800 \\
\hline 3567 & 453 & 4,020 & 8,460 & 15,400 & 22,100 & 28,700 & 35,800 \\
\hline 3570 & 453 & 4,020 & 8,460 & 15,400 & 22,100 & 28,700 & 35,800 \\
\hline 3571 & 20.3 & 1,230 & 2,350 & 3,250 & 4,540 & 5,610 & 6,760 \\
\hline 3577 & 20.3 & 1,230 & 2,350 & 3,250 & 4,540 & 5,610 & 6,760 \\
\hline 3578 & 110 & 2,210 & 5,200 & 8,100 & 12,900 & 17,400 & 22,800 \\
\hline 3579 & 463 & 4,060 & 8,490 & 15,400 & 22,100 & 28,700 & 35,800 \\
\hline 3582 & 463 & 4,060 & 8,490 & 15,400 & 22,100 & 28,700 & 35,800 \\
\hline 3591 & 7.79 & 566 & 1,520 & 2,480 & 4,050 & 5,490 & 7,140 \\
\hline
\end{tabular}


Table 84. Estimated flow-duration values, mean flow values, and peak-discharge frequency values for controlled and uncontrolled flow stream segments on the 1999 Kansas Surface Water Register for Reno County.-Continued

[KSWR, Kansas Surface Water Register; CUSEGA, catalog unit segment number alpha; $\mathrm{mi}^{2}$, square miles; $\mathrm{ft}^{3} / \mathrm{s}$, cubic feet per second; HYDRO, lake or other hydrologic structure; NA, not applicable; NRDitch, irrigation ditch; NRTribal, tribal stream]

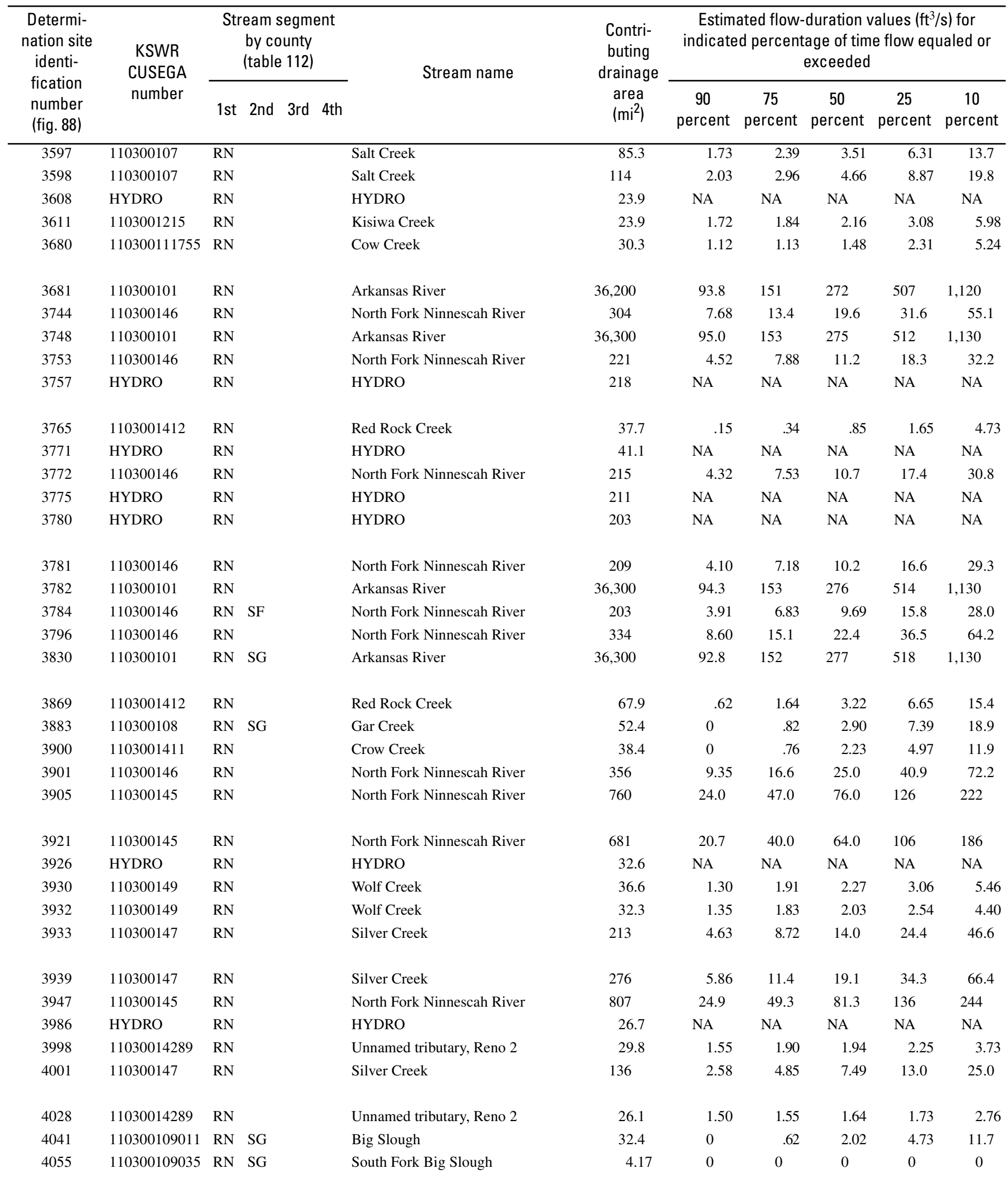


Table 84. Estimated flow-duration values, mean flow values, and peak-discharge frequency values for controlled and uncontrolled flow stream segments on the 1999 Kansas Surface Water Register for Reno County.-Continued

[KSWR, Kansas Surface Water Register; CUSEGA, catalog unit segment number alpha; $\mathrm{mi}^{2}$, square miles; $\mathrm{ft}^{3} / \mathrm{s}$, cubic feet per second; HYDRO, lake or other hydrologic structure; NA, not applicable; NRDitch, irrigation ditch; NRTribal, tribal stream]

\begin{tabular}{|c|c|c|c|c|c|c|c|}
\hline \multirow{2}{*}{$\begin{array}{l}\text { Determi- } \\
\text { nation site } \\
\text { identi- } \\
\text { fication } \\
\text { number } \\
\text { (fig. 88) }\end{array}$} & \multirow{2}{*}{$\begin{array}{c}\text { Estimated mean } \\
\text { flow } \\
\left(\mathrm{ft}^{3} / \mathrm{s}\right)\end{array}$} & \multicolumn{6}{|c|}{ Estimated peak discharge $\left(\mathrm{ft}^{3} / \mathrm{s}\right)$ for indicated peak-discharge frequency } \\
\hline & & 2-year & 5-year & 10 -year & 25-year & 50-year & 100 -year \\
\hline 3597 & 12.7 & 1,080 & 2,220 & 3,190 & 4,620 & 5,830 & 7,130 \\
\hline 3611 & 5.70 & 1,200 & 2,940 & 4,560 & 7,130 & 9,350 & 11,900 \\
\hline 3680 & 5.83 & 496 & 1,270 & 2,030 & 3,240 & 4,320 & 5,540 \\
\hline 3681 & 536 & 4,330 & 8,730 & 15,500 & 22,000 & 28,500 & 35,500 \\
\hline 3744 & 40.5 & 1,690 & 4,460 & 7,350 & 12,400 & 17,400 & 23,500 \\
\hline 3765 & 6.10 & 956 & 2,290 & 3,550 & 5,520 & 7,260 & 9,200 \\
\hline 3771 & NA & NA & NA & NA & NA & NA & NA \\
\hline 3772 & 24.5 & 1,300 & 3,360 & 5,460 & 9,020 & 12,400 & 16,500 \\
\hline 3775 & NA & NA & NA & NA & NA & NA & NA \\
\hline 3780 & NA & NA & NA & NA & NA & NA & NA \\
\hline 3781 & 23.5 & 1,230 & 3,210 & 5,230 & 8,660 & 11,900 & 15,900 \\
\hline 3782 & 546 & 4,440 & 9,030 & 15,700 & 22,300 & 28,900 & 36,000 \\
\hline 3784 & 22.6 & 1,220 & 3,170 & 5,150 & 8,500 & 11,700 & 15,500 \\
\hline 3796 & 46.6 & 1,850 & 4,910 & 8,130 & 13,800 & 19,500 & 26,400 \\
\hline 3921 & 125 & 3,530 & 10,600 & 18,800 & 34,200 & 50,500 & 71,400 \\
\hline 3926 & NA & NA & NA & NA & NA & NA & NA \\
\hline 3930 & 5.47 & 760 & 1,770 & 2,700 & 4,120 & 5,360 & 6,720 \\
\hline 3932 & 4.65 & 704 & 1,640 & 2,500 & 3,800 & 4,940 & 6,180 \\
\hline 3933 & 34.4 & 1,680 & 4,140 & 6,570 & 10,600 & 14,500 & 19,000 \\
\hline 3939 & 47.7 & 2,150 & 5,320 & 8,510 & 13,900 & 19,100 & 25,300 \\
\hline 3947 & 159 & 4,240 & 12,800 & 22,600 & 41,400 & 61,100 & 86,700 \\
\hline 3986 & $\mathrm{NA}$ & NA & NA & NA & NA & NA & NA \\
\hline 3998 & 4.14 & 1,010 & 2,690 & 4,320 & 6,990 & 9,330 & 12,100 \\
\hline 4001 & 19.9 & 1,240 & 3,040 & 4,790 & 7,640 & 10,300 & 13,300 \\
\hline 4028 & 3.43 & 929 & 2,470 & 3,960 & 6,390 & 8,530 & 11,100 \\
\hline 4041 & 10.1 & 1,150 & 2,720 & 4,190 & 6,480 & 8,510 & 10,700 \\
\hline 4055 & .58 & 450 & 1,060 & 1,600 & 2,450 & 3,180 & 4,010 \\
\hline
\end{tabular}




\section{Estimates of Flow Duration, Mean Flow, and Peak-Discharge Frequency Values for Kansas Stream Locations}

Table 84. Estimated flow-duration values, mean flow values, and peak-discharge frequency values for controlled and uncontrolled flow stream segments on the 1999 Kansas Surface Water Register for Reno County.-Continued

[KSWR, Kansas Surface Water Register; CUSEGA, catalog unit segment number alpha; $\mathrm{mi}^{2}$, square miles; $\mathrm{ft}^{3} / \mathrm{s}$, cubic feet per second; HYDRO, lake or other hydrologic structure; NA, not applicable; NRDitch, irrigation ditch; NRTribal, tribal stream]

\begin{tabular}{|c|c|c|c|c|c|c|c|c|c|c|c|}
\hline $\begin{array}{l}\text { Determi- } \\
\text { nation site } \\
\text { identi- } \\
\text { fication } \\
\text { number } \\
\text { (fig. 88) }\end{array}$ & $\begin{array}{l}\text { KSWR } \\
\text { CUSEGA } \\
\text { number }\end{array}$ & \multicolumn{3}{|c|}{$\begin{array}{l}\text { Stream segment } \\
\text { by county } \\
\text { (table 112) }\end{array}$} & Stream name & $\begin{array}{l}\text { Contri- } \\
\text { buting } \\
\text { drainage } \\
\text { area } \\
\left(\mathrm{mi}^{2}\right)\end{array}$ & \multicolumn{5}{|c|}{$\begin{array}{l}\text { Estimated flow-duration values }\left(\mathrm{ft}^{3} / \mathrm{s}\right) \text { for } \\
\text { indicated percentage of time flow equaled or } \\
\text { exceeded }\end{array}$} \\
\hline 4062 & 1103001413 & $\mathrm{RN}$ & & & Rock Creek & 11.2 & 0 & 0 & 0 & 0.25 & 2.15 \\
\hline 4135 & 110300141 & $\mathrm{RN}$ & SG & & North Fork Ninnescah River & 863 & .16 & .24 & .48 & 75.0 & 381 \\
\hline 4159 & 1103001414 & RN & SG & & Spring Creek & 12.1 & 0 & .02 & .55 & 1.01 & 2.88 \\
\hline
\end{tabular}


Table 84. Estimated flow-duration values, mean flow values, and peak-discharge frequency values for controlled and uncontrolled flow stream segments on the 1999 Kansas Surface Water Register for Reno County.-Continued

[KSWR, Kansas Surface Water Register; CUSEGA, catalog unit segment number alpha; $\mathrm{mi}^{2}$, square miles; $\mathrm{ft}^{3} / \mathrm{s}$, cubic feet per second; HYDRO, lake or other hydrologic structure; NA, not applicable; NRDitch, irrigation ditch; NRTribal, tribal stream]

\begin{tabular}{|c|c|c|c|c|c|c|c|}
\hline \multirow{2}{*}{$\begin{array}{l}\text { Determi- } \\
\text { nation site } \\
\text { identi- } \\
\text { fication } \\
\text { number } \\
\text { (fig. 88) }\end{array}$} & \multirow{2}{*}{$\begin{array}{l}\text { Estimated mean } \\
\text { flow } \\
\left(\mathrm{ft}^{3} / \mathrm{s}\right)\end{array}$} & \multicolumn{6}{|c|}{ Estimated peak discharge $\left(\mathrm{ft}^{3} / \mathrm{s}\right)$ for indicated peak-discharge frequency } \\
\hline & & 2-year & 5-year & 10-year & 25-year & 50-year & 100-year \\
\hline 4062 & 3.20 & 786 & 1,890 & 2,910 & 4,500 & 5,860 & 7,440 \\
\hline 4135 & 121 & 1,080 & 2,080 & 2,750 & 3,560 & 4,110 & 4,620 \\
\hline 4159 & 3.40 & 809 & 1,960 & 3,010 & 4,680 & 6,100 & 7,760 \\
\hline
\end{tabular}




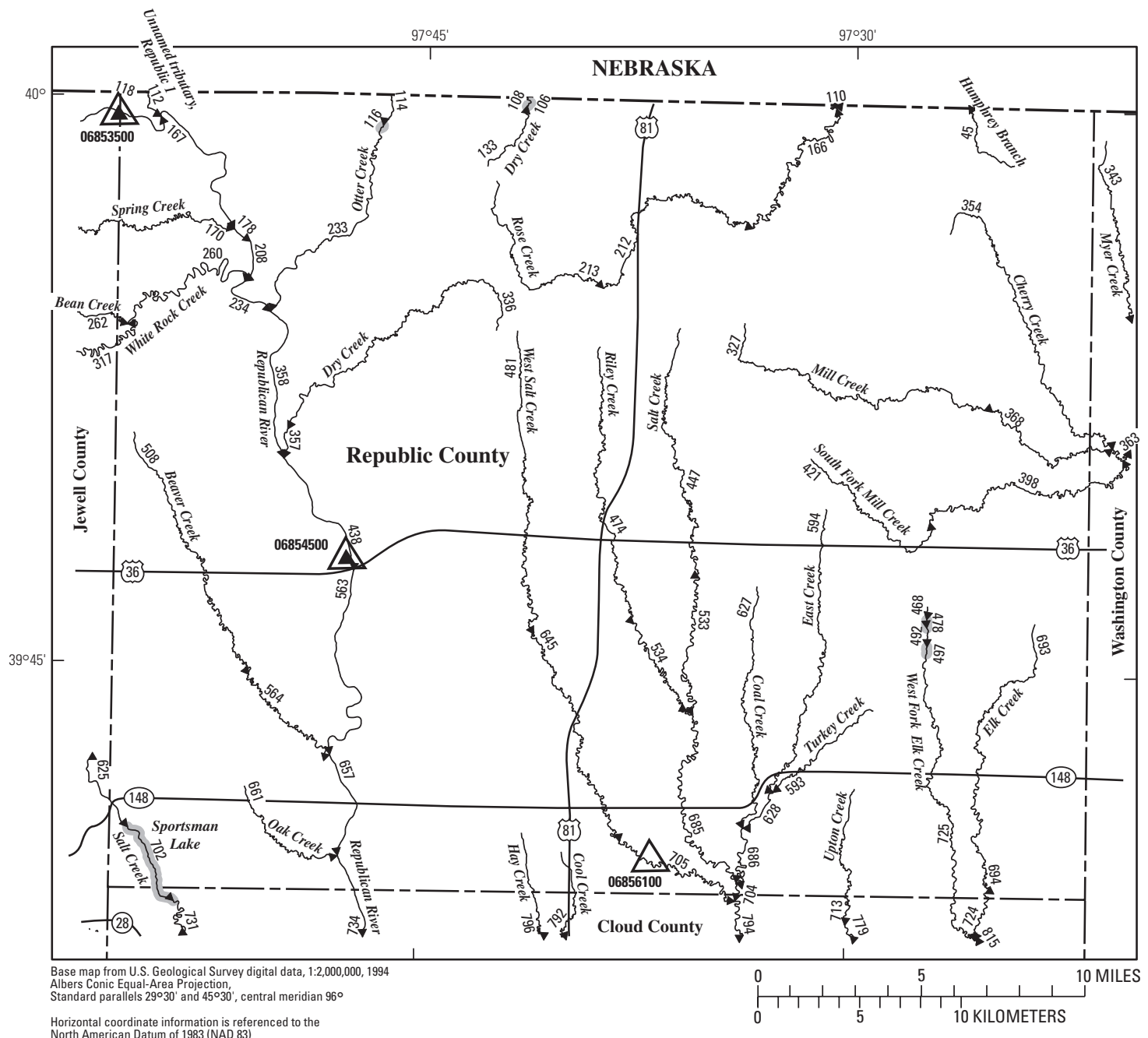

\section{EXPLANATION}

731 Location of streamflow-statistics determination site (small triangle) and associated identification number-small triangle points in downstream direction

06854500

U.S. Geological Survey streamflow-gaging station and number used for estimates of flow duration

${ }^{06856100} \triangle$ U.S. Geological Survey streamflow-gaging station and number used for estimates of peak-discharge frequency values

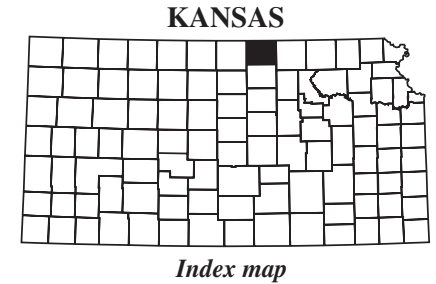
702

Lake and determination site identification number

Figure 89. Location of streamflow-statistics determination sites, associated identification numbers, and U.S. Geological Survey streamflow-gaging stations used in the flow-duration and peak-discharge frequency analyses for Republic County. 
Table 85. Estimated flow-duration values, mean flow values, and peak-discharge frequency values for controlled and uncontrolled flow stream segments on the 1999 Kansas Surface Water Register for Republic County.

[KSWR, Kansas Surface Water Register; CUSEGA, catalog unit segment number alpha; $\mathrm{mi}^{2}$, square miles; $\mathrm{ft}^{3} / \mathrm{s}$, cubic feet per second; HYDRO, lake or other hydrologic structure; NA, not applicable; NRDitch, irrigation ditch; NRTribal, tribal stream]

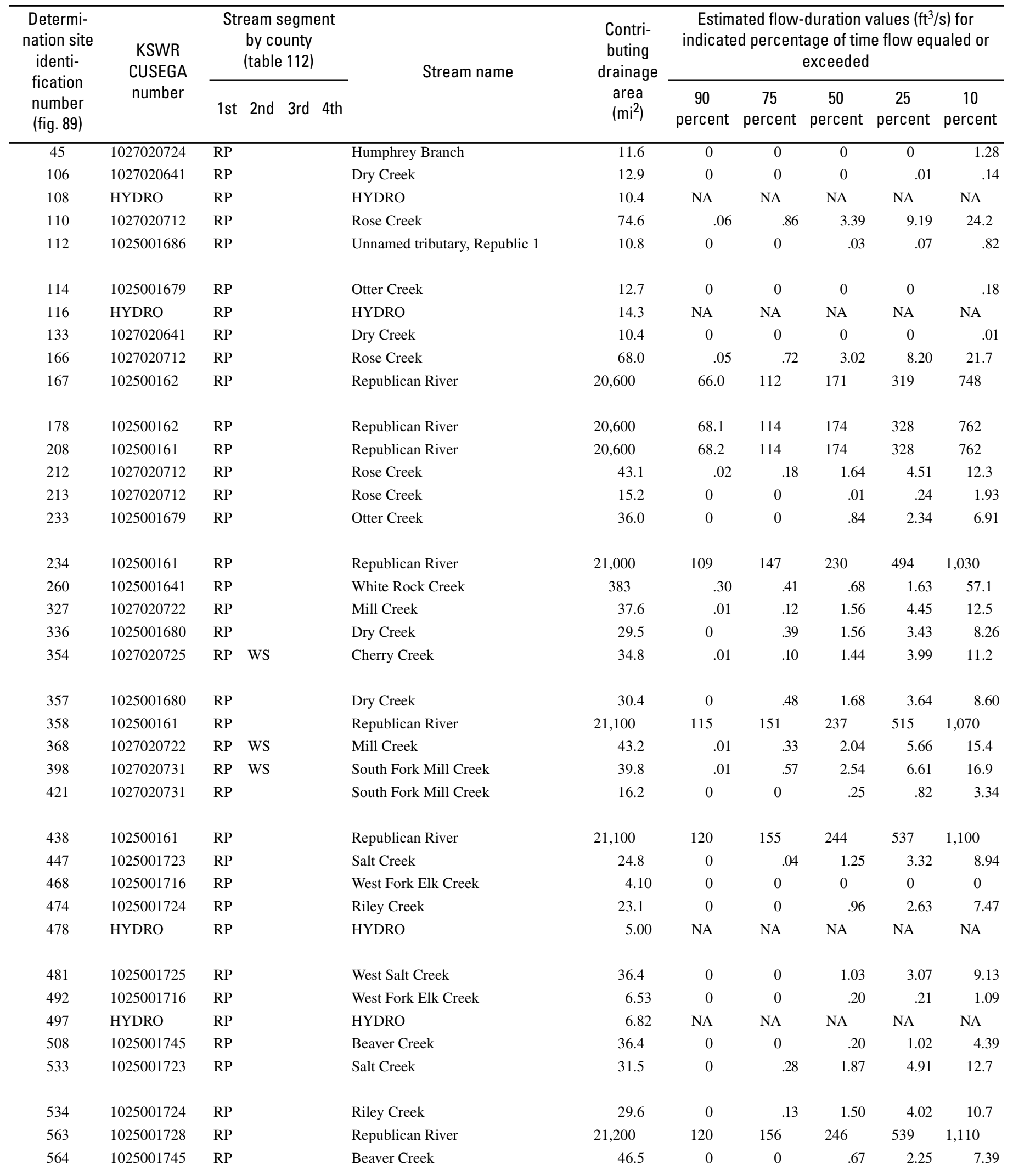


Table 85. Estimated flow-duration values, mean flow values, and peak-discharge frequency values for controlled and uncontrolled flow stream segments on the 1999 Kansas Surface Water Register for Republic County.-Continued

[KSWR, Kansas Surface Water Register; CUSEGA, catalog unit segment number alpha; $\mathrm{mi}^{2}$, square miles; $\mathrm{ft}^{3} / \mathrm{s}$, cubic feet per second; HYDRO, lake or other hydrologic structure; NA, not applicable; NRDitch, irrigation ditch; NRTribal, tribal stream]

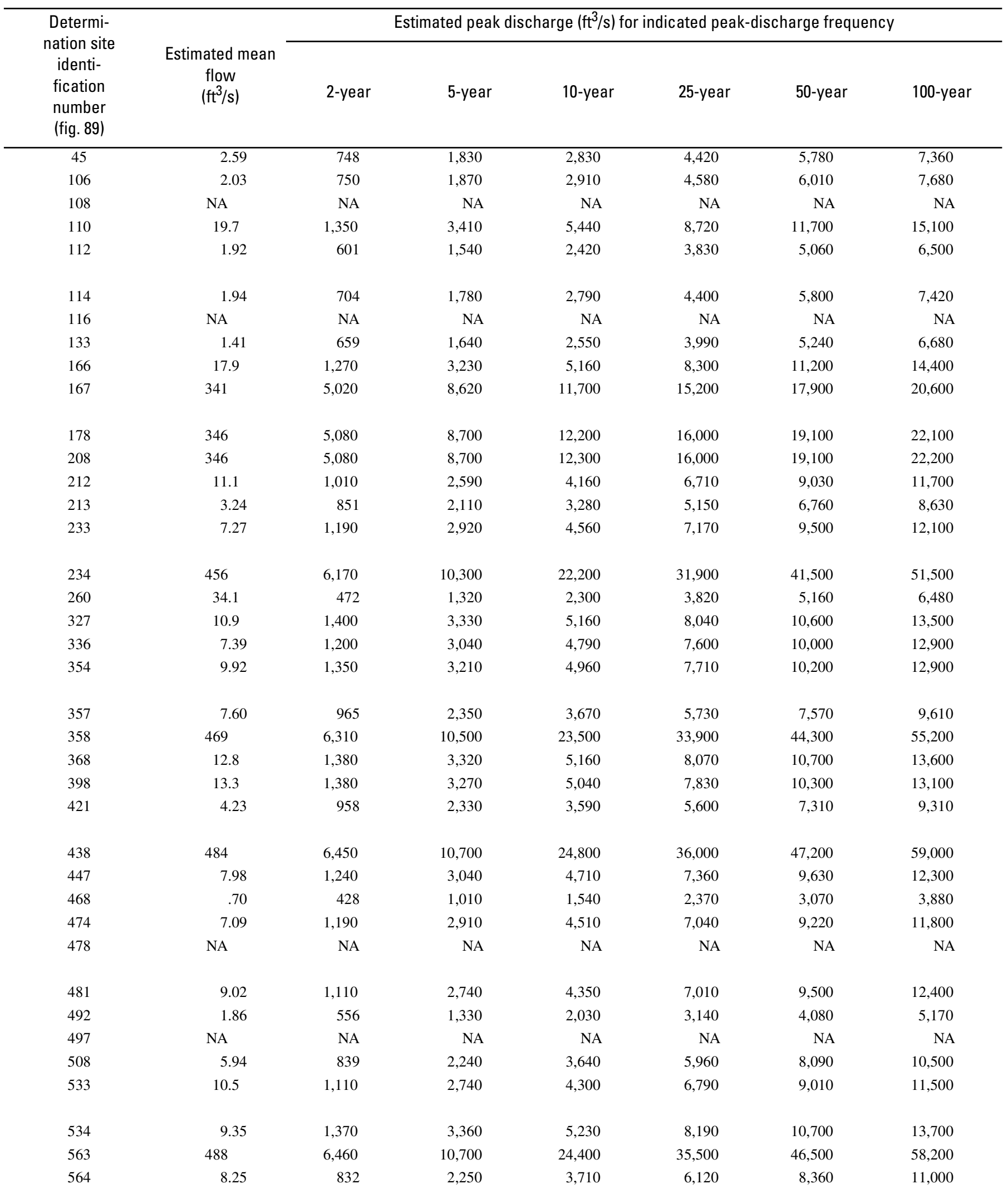




\section{Estimates of Flow Duration, Mean Flow, and Peak-Discharge Frequency Values for Kansas Stream Locations}

Table 85. Estimated flow-duration values, mean flow values, and peak-discharge frequency values for controlled and uncontrolled flow stream segments on the 1999 Kansas Surface Water Register for Republic County.-Continued

[KSWR, Kansas Surface Water Register; CUSEGA, catalog unit segment number alpha; $\mathrm{mi}^{2}$, square miles; $\mathrm{ft}^{3} / \mathrm{s}$, cubic feet per second; HYDRO, lake or other hydrologic structure; NA, not applicable; NRDitch, irrigation ditch; NRTribal, tribal stream]

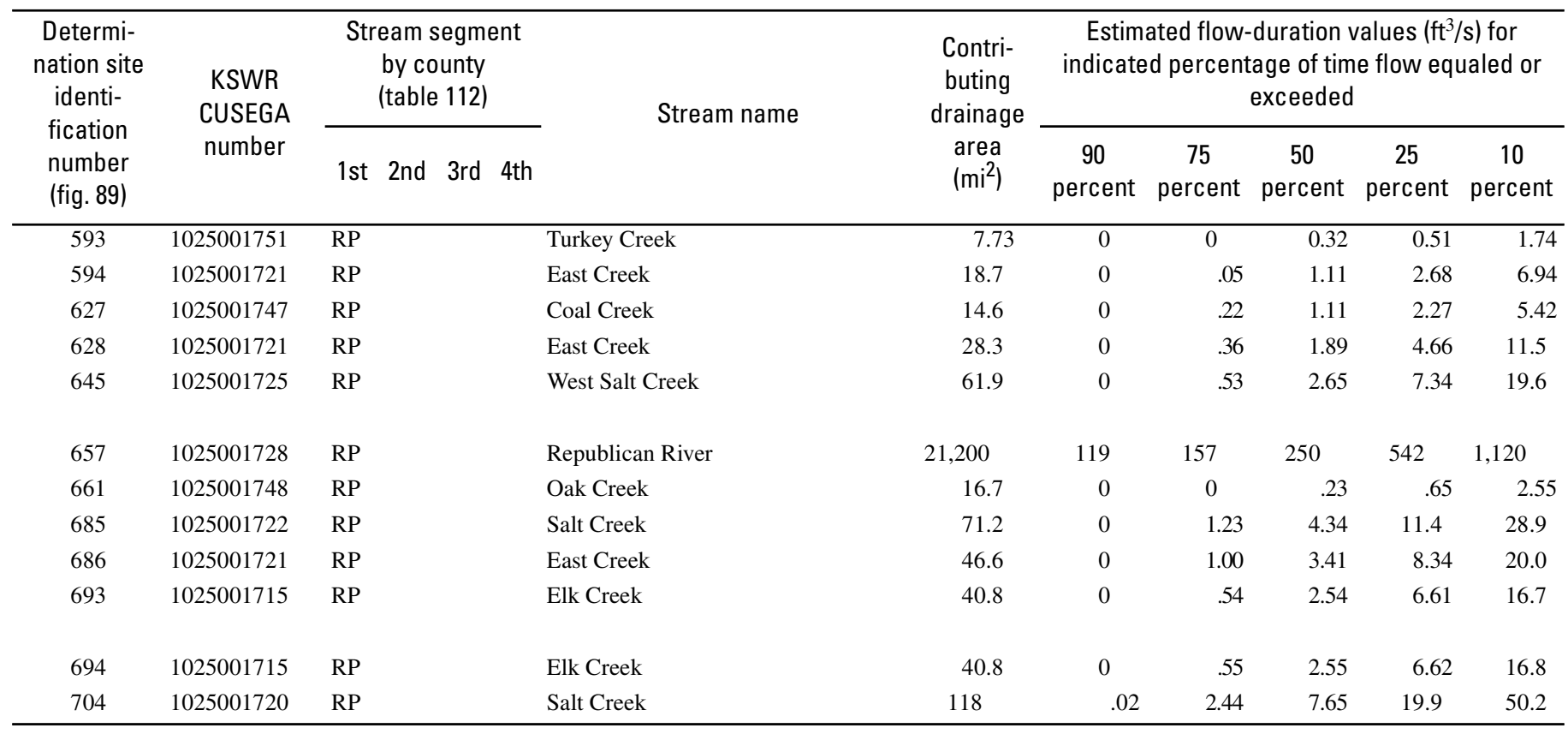


Table 85. Estimated flow-duration values, mean flow values, and peak-discharge frequency values for controlled and uncontrolled flow stream segments on the 1999 Kansas Surface Water Register for Republic County.-Continued

[KSWR, Kansas Surface Water Register; CUSEGA, catalog unit segment number alpha; $\mathrm{mi}^{2}$, square miles; $\mathrm{ft}^{3} / \mathrm{s}$, cubic feet per second; HYDRO, lake or other hydrologic structure; NA, not applicable; NRDitch, irrigation ditch; NRTribal, tribal stream]

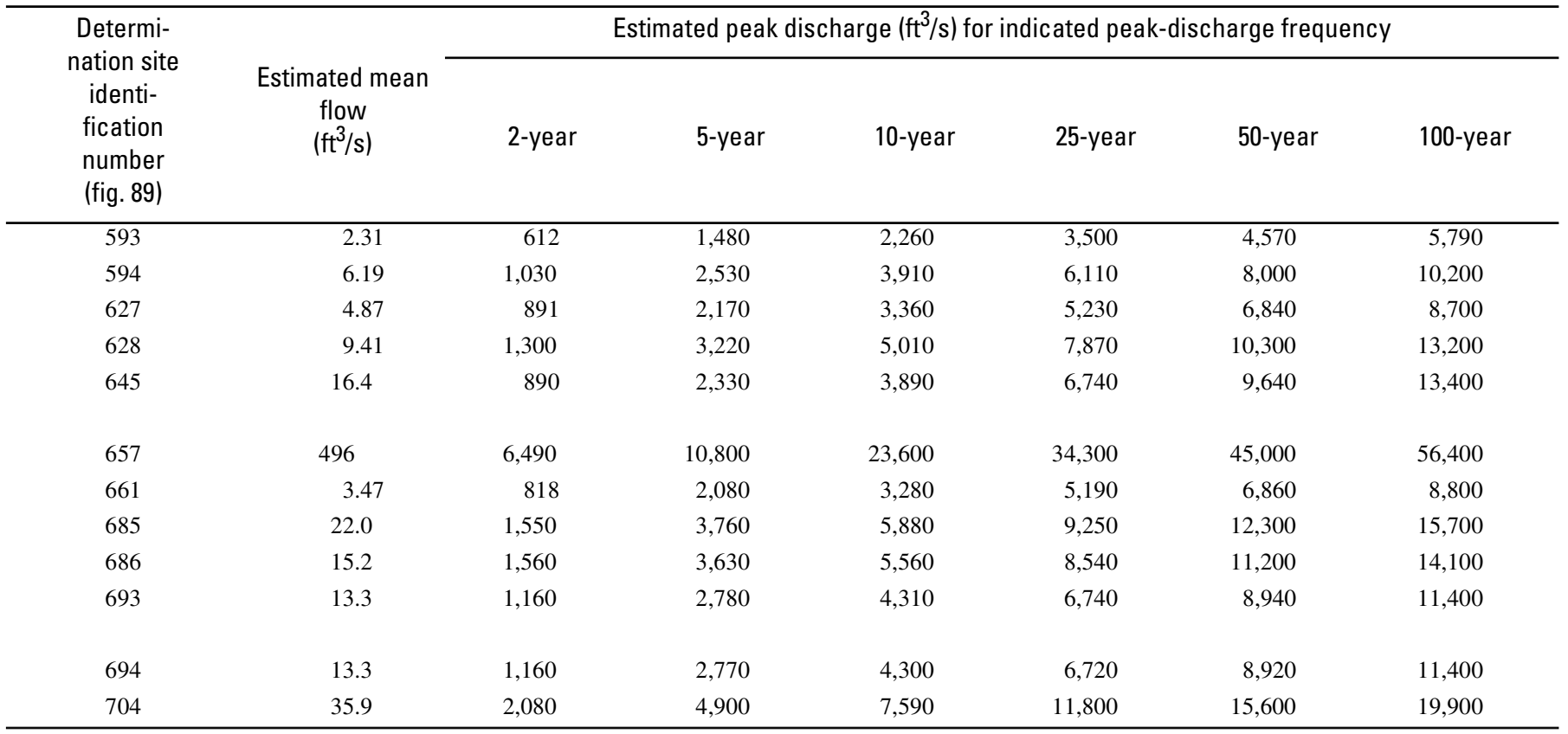




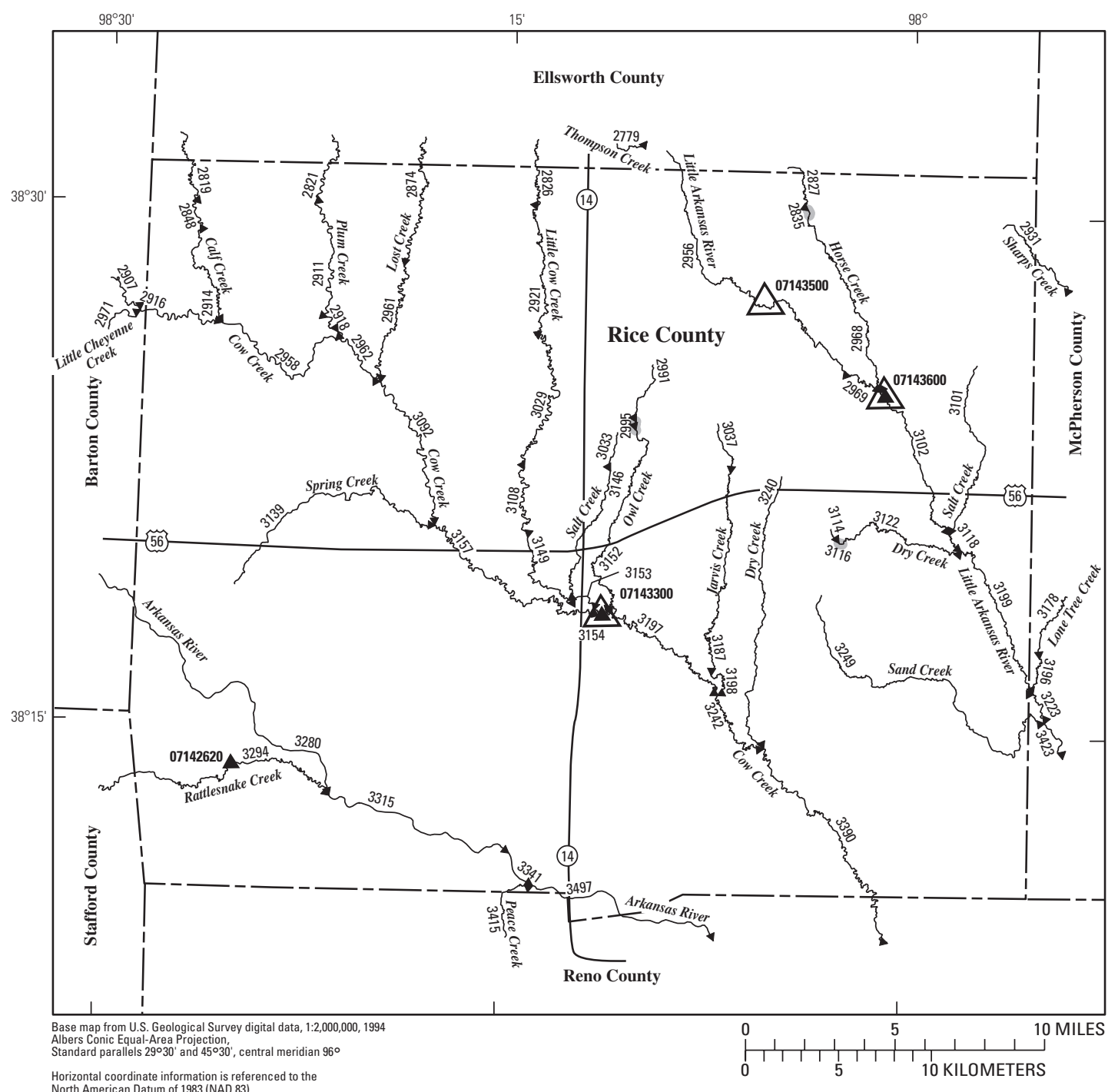

EXPLANATION

\$294 Location of streamflow-statistics determination site (small triangle) and associated identification number-small triangle points in downstream direction

${ }^{07142620} \Delta$ U.S. Geological Survey streamflow-gaging station and number used for estimates of flow duration

${ }^{07143300} \triangle$

U.S. Geological Survey streamflow-gaging station and number used for estimates of peak-discharge frequency values

KANSAS

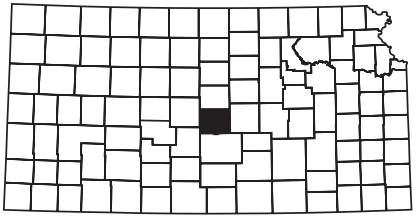

Index map 2995

Lake and determination site identification number

Figure 90. Location of streamflow-statistics determination sites, associated identification numbers, and U.S. Geological Survey streamflow-gaging stations used in the flow-duration and peak-discharge frequency analyses for Rice County. 
Table 86. Estimated flow-duration values, mean flow values, and peak-discharge frequency values for controlled and uncontrolled flow stream segments on the 1999 Kansas Surface Water Register for Rice County.

[KSWR, Kansas Surface Water Register; CUSEGA, catalog unit segment number alpha; $\mathrm{mi}^{2}$, square miles; $\mathrm{ft}^{3} / \mathrm{s}$, cubic feet per second; HYDRO, lake or other hydrologic structure; NA, not applicable; NRDitch, irrigation ditch; NRTribal, tribal stream]

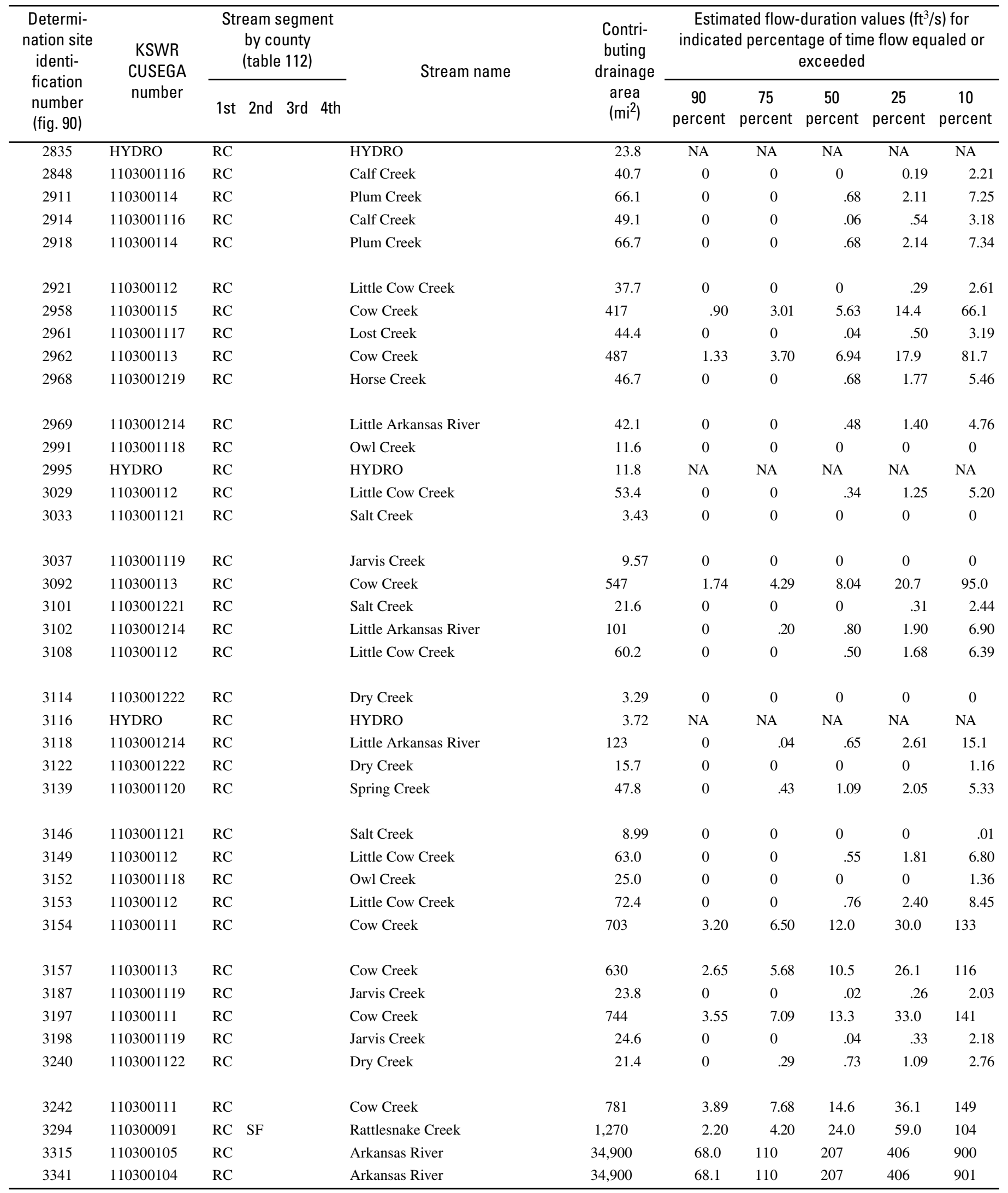


Table 86. Estimated flow-duration values, mean flow values, and peak-discharge frequency values for controlled and uncontrolled flow stream segments on the 1999 Kansas Surface Water Register for Rice County.-Continued

[KSWR, Kansas Surface Water Register; CUSEGA, catalog unit segment number alpha; $\mathrm{mi}^{2}$, square miles; $\mathrm{ft}^{3} / \mathrm{s}$, cubic feet per second; HYDRO, lake or other hydrologic structure; NA, not applicable; NRDitch, irrigation ditch; NRTribal, tribal stream]

\begin{tabular}{|c|c|c|c|c|c|c|c|}
\hline \multirow{2}{*}{$\begin{array}{l}\text { Determi- } \\
\text { nation site } \\
\text { identi- } \\
\text { fication } \\
\text { number } \\
\text { (fig. 90) }\end{array}$} & \multirow{2}{*}{$\begin{array}{c}\text { Estimated mean } \\
\text { flow } \\
\left(\mathrm{ft}^{3} / \mathrm{s}\right)\end{array}$} & \multicolumn{6}{|c|}{ Estimated peak discharge $\left(\mathrm{ft}^{3} / \mathrm{s}\right)$ for indicated peak-discharge frequency } \\
\hline & & 2-year & 5-year & 10-year & 25-year & 50-year & 100-year \\
\hline 2835 & NA & NA & NA & NA & NA & NA & NA \\
\hline 2914 & 4.96 & 646 & 1,820 & 3,030 & 5,080 & 7,000 & 9,240 \\
\hline 2918 & 8.27 & 815 & 1,690 & 2,480 & 3,730 & 4,870 & 6,180 \\
\hline 2921 & 4.59 & 583 & 1,640 & 2,730 & 4,570 & 6,280 & 8,280 \\
\hline 2958 & 42.5 & 1,470 & 3,630 & 5,750 & 9,280 & 12,600 & 16,600 \\
\hline 2969 & 6.04 & 926 & 1,370 & 1,660 & 2,030 & 2,300 & 2,560 \\
\hline 2991 & .72 & 613 & 1,580 & 2,490 & 3,960 & 5,230 & 6,720 \\
\hline 2995 & NA & NA & NA & NA & NA & NA & NA \\
\hline 3029 & 6.83 & 601 & 1,720 & 2,890 & 4,890 & 6,770 & 8,990 \\
\hline 3033 & 0 & 302 & 757 & 1,180 & 1,850 & 2,430 & 3,110 \\
\hline 3037 & .59 & 560 & 1,430 & 2,240 & 3,550 & 4,690 & 6,020 \\
\hline 3092 & 58.9 & 1,690 & 4,160 & 6,620 & 10,700 & 14,700 & 19,400 \\
\hline 3101 & 3.87 & 938 & 2,410 & 3,800 & 6,050 & 8,010 & 10,300 \\
\hline 3102 & 9.46 & 1,200 & 2,360 & 3,360 & 4,900 & 6,240 & 7,760 \\
\hline 3146 & .45 & 529 & 1,350 & 2,130 & 3,380 & 4,470 & 5,730 \\
\hline 3149 & 8.20 & 671 & 1,900 & 3,190 & 5,370 & 7,430 & 9,840 \\
\hline 3152 & 3.27 & 962 & 2,510 & 4,000 & 6,400 & 8,510 & 11,000 \\
\hline 3153 & 9.58 & 745 & 2,080 & 3,480 & 5,850 & 8,070 & 10,700 \\
\hline 3154 & 79.6 & 1,940 & 4,690 & 7,420 & 12,000 & 16,400 & 21,700 \\
\hline 3157 & 69.9 & 1,800 & 4,370 & 6,930 & 11,200 & 15,300 & 20,200 \\
\hline 3187 & 3.62 & 953 & 2,470 & 3,930 & 6,280 & 8,330 & 10,700 \\
\hline 3197 & 85.1 & 1,980 & 4,790 & 7,590 & 12,300 & 16,800 & 22,300 \\
\hline 3198 & 3.76 & 974 & 2,530 & 4,010 & 6,420 & 8,520 & 11,000 \\
\hline 3240 & 3.63 & 915 & 2,360 & 3,730 & 5,950 & 7,880 & 10,100 \\
\hline 3242 & 90.4 & 2,070 & 5,010 & 7,930 & 12,800 & 17,500 & 23,200 \\
\hline 3294 & 49.1 & 631 & 1,850 & 3,350 & 6,440 & 9,950 & 14,800 \\
\hline 3315 & 433 & 3,940 & 8,400 & 15,400 & 22,100 & 28,800 & 35,900 \\
\hline 3341 & 433 & 3,940 & 8,400 & 15,400 & 22,100 & 28,800 & 35,900 \\
\hline
\end{tabular}




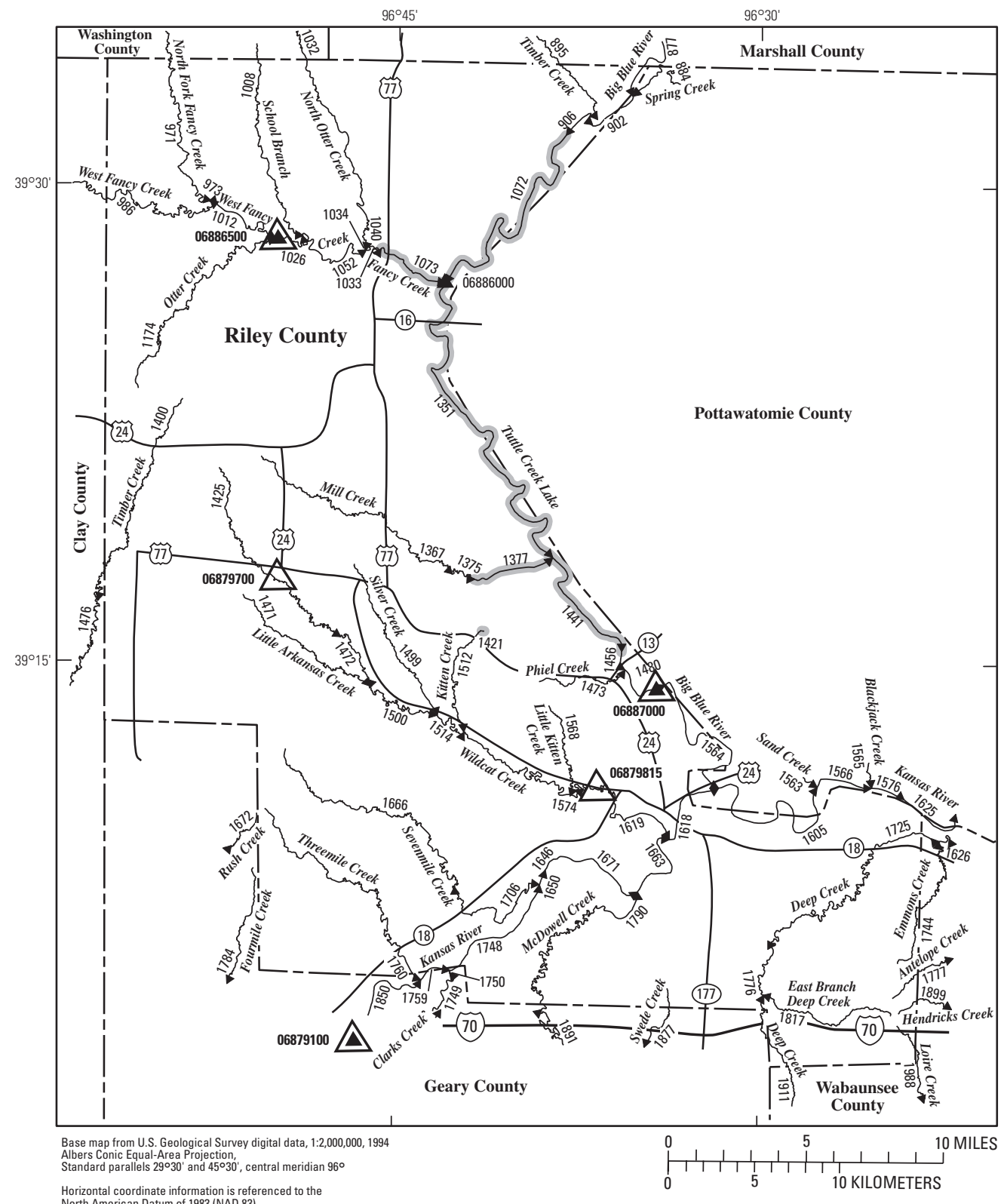

Horizontal coordinate information is referenced to the
North American Datum of 1983 (NAD 83 )

EXPLANATION

$\Perp_{4762}$ Location of streamflow-statistics determination site (small triangle) and associated identification number-small triangle points in downstream direction

06887000 U.S. Geological Survey streamflow-gaging station and number used for estimates of flow duration

${ }^{06879700}$ U.S. Geological Survey streamflow-gaging station and number used for estimates of peak-discharge frequency values

KANSAS

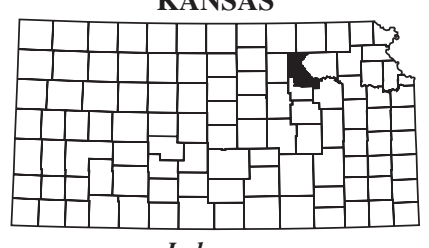

Index map 1441

Lake and determination site identification number

Figure 91. Location of streamflow-statistics determination sites, associated identification numbers, and U.S. Geological Survey streamflow-gaging stations used in the flow-duration and peak-discharge frequency analyses for Riley County. 
Table 87. Estimated flow-duration values, mean flow values, and peak-discharge frequency values for controlled and uncontrolled flow stream segments on the 1999 Kansas Surface Water Register for Riley County.

[KSWR, Kansas Surface Water Register; CUSEGA, catalog unit segment number alpha; $\mathrm{mi}^{2}$, square miles; $\mathrm{ft}^{3} / \mathrm{s}$, cubic feet per second; HYDRO, lake or other hydrologic structure; NA, not applicable; NRDitch, irrigation ditch; NRTribal, tribal stream]

\begin{tabular}{|c|c|c|c|c|c|c|c|c|c|c|c|}
\hline \multirow{2}{*}{$\begin{array}{l}\text { Determi- } \\
\text { nation site } \\
\text { identi- } \\
\text { fication } \\
\text { number } \\
\text { (fig. 91) }\end{array}$} & \multirow{2}{*}{$\begin{array}{l}\text { KSWR } \\
\text { CUSEGA } \\
\text { number }\end{array}$} & \multicolumn{3}{|c|}{$\begin{array}{l}\text { Stream segment } \\
\text { by county } \\
\text { (table 112) }\end{array}$} & \multirow{2}{*}{ Stream name } & \multirow{2}{*}{$\begin{array}{l}\text { Contri- } \\
\text { buting } \\
\text { drainage } \\
\text { area } \\
\left(\mathrm{mi}^{2}\right)\end{array}$} & \multicolumn{5}{|c|}{$\begin{array}{c}\text { Estimated flow-duration values }\left(\mathrm{ft}^{3} / \mathrm{s}\right) \text { for } \\
\text { indicated percentage of time flow equaled or } \\
\text { exceeded }\end{array}$} \\
\hline & & $1 \mathrm{st}$ & 2nd & 3rd 4th & & & $\begin{array}{c}90 \\
\text { percent }\end{array}$ & $\begin{array}{c}75 \\
\text { percent }\end{array}$ & $\begin{array}{c}50 \\
\text { percent }\end{array}$ & $\begin{array}{c}25 \\
\text { percent }\end{array}$ & $\begin{array}{c}10 \\
\text { percent }\end{array}$ \\
\hline 902 & 102702057 & RL & & & Big Blue River & 9,150 & 269 & 376 & 590 & 1,220 & 3,350 \\
\hline 906 & 102702057 & RL & & & Big Blue River & 9,170 & 269 & 377 & 592 & 1,220 & 3,360 \\
\hline 971 & 1027020561 & RL & WS & & North Fork Fancy Creek & 35.2 & 0 & .31 & 2.37 & 6.30 & 16.4 \\
\hline 973 & 102702059029 & RL & & & Fancy Creek & 140 & 0 & 1.74 & 8.40 & 18.6 & 47.4 \\
\hline 1008 & 1027020563 & RL & WS & & School Branch & 20.6 & 0 & .08 & 1.55 & 4.28 & 11.3 \\
\hline 1012 & 102702059029 & RL & & & Fancy Creek & 143 & 0 & 1.78 & 8.65 & 19.2 & 48.8 \\
\hline 1026 & 102702059029 & RL & & & Fancy Creek & 182 & 0 & 2.00 & 11.0 & 23.0 & 59.0 \\
\hline 1032 & 1027020562 & & WS & & North Otter Creek & 39.6 & 0 & .38 & 2.82 & 8.42 & 22.7 \\
\hline 1033 & 1027020529 & RL & & & West Fancy Creek & 256 & .48 & 3.49 & 16.4 & 38.8 & 104 \\
\hline 1034 & 1027020529 & RL & & & West Fancy Creek & 216 & .22 & 2.72 & 13.6 & 30.5 & 80.3 \\
\hline 1040 & 102702059029 & RL & & & Fancy Creek & 257 & .48 & 3.51 & 16.5 & 39.1 & 105 \\
\hline 1052 & 102702059029 & RL & & & Fancy Creek & 216 & .22 & 2.72 & 13.6 & 30.5 & 80.3 \\
\hline 1073 & HYDRO & RL & & & HYDRO & 263 & NA & NA & NA & NA & NA \\
\hline 1174 & 1027020567 & RL & & & Otter Creek & 37.3 & 0 & .15 & 2.29 & 6.65 & 18.2 \\
\hline 1367 & 1027020531 & RL & & & Mill Creek & 40.8 & 0 & .33 & 2.83 & 8.75 & 24.1 \\
\hline 1375 & 1027020531 & RL & & & Mill Creek & 42.8 & 0 & .41 & 3.10 & 9.53 & 26.1 \\
\hline 1377 & HYDRO & RL & & & HYDRO & 48.1 & NA & NA & NA & NA & NA \\
\hline 1421 & HYDRO & RL & & & HYDRO & 2.81 & NA & NA & NA & NA & NA \\
\hline 1425 & 102701012 & RL & & & Wildcat Creek & 21.8 & 0 & 0 & .97 & 3.25 & 9.87 \\
\hline 1456 & 102702052 & RL & & & Big Blue River & 9,620 & 178 & 443 & 973 & 2,450 & 6,490 \\
\hline 1471 & 1027010113 & RL & & & Little Arkansas Creek & 22.9 & 0 & 0 & 1.11 & 3.69 & 11.0 \\
\hline 1472 & 102701012 & RL & & & Wildcat Creek & 24.2 & 0 & 0 & 1.17 & 3.85 & 11.5 \\
\hline 1473 & 1027020568 & RL & & & Phiel Creek & 8.86 & 0 & 0 & 1.06 & 2.64 & 6.64 \\
\hline 1499 & 1027010112 & RL & & & Silver Creek & 12.8 & 0 & 0 & .69 & 2.19 & 6.62 \\
\hline 1500 & 102701012 & RL & & & Wildcat Creek & 54.4 & 0 & .44 & 3.21 & 10.2 & 29.0 \\
\hline 1512 & 1027010114 & RL & & & Kitten Creek & 11.6 & 0 & 0 & 1.12 & 3.03 & 7.97 \\
\hline 1514 & 102701012 & RL & & & Wildcat Creek & 69.7 & 0 & .78 & 4.34 & 13.7 & 38.7 \\
\hline 1568 & 1027010116 & RL & & & Little Kitten Creek & 6.98 & 0 & 0 & .65 & 1.63 & 4.51 \\
\hline 1574 & 102701012 & RL & & & Wildcat Creek & 95.3 & 0 & 1.54 & 6.95 & 21.4 & 59.6 \\
\hline 1619 & 102701012 & RL & & & Wildcat Creek & 111 & 0 & 2.17 & 8.87 & 26.6 & 72.6 \\
\hline 1646 & 102701014 & RL & & & Kansas River & 43,800 & 422 & 660 & 1,390 & 3,410 & 7,650 \\
\hline 1650 & 102701016 & RL & & & Kansas River & 43,800 & 422 & 660 & 1,390 & 3,410 & 7,650 \\
\hline 1663 & 102701013 & RL & & & Kansas River & 43,900 & 427 & 668 & 1,410 & 3,440 & 7,710 \\
\hline 1666 & 102701015 & RL & & & Sevenmile Creek & 37.3 & 0 & .37 & 2.86 & 8.75 & 24.0 \\
\hline 1671 & 102701013 & RL & & & Kansas River & 43,800 & 422 & 661 & 1,400 & 3,410 & 7,650 \\
\hline 1706 & 102701015 & RL & & & Sevenmile Creek & 44.2 & 0 & 1.10 & 4.40 & 12.0 & 30.5 \\
\hline 1725 & 1027010226 & RL & WB & & Deep Creek & 63.9 & 0 & 1.34 & 6.79 & 21.3 & 58.2 \\
\hline 1744 & 1027010266 & RL & WB & & Emmons Creek & 12.0 & 0 & 0 & .97 & 3.26 & 9.50 \\
\hline
\end{tabular}


Table 87. Estimated flow-duration values, mean flow values, and peak-discharge frequency values for controlled and uncontrolled flow stream segments on the 1999 Kansas Surface Water Register for Riley County.-Continued

[KSWR, Kansas Surface Water Register; CUSEGA, catalog unit segment number alpha; $\mathrm{mi}^{2}$, square miles; $\mathrm{ft}^{3} / \mathrm{s}$, cubic feet per second; HYDRO, lake or other hydrologic structure; NA, not applicable; NRDitch, irrigation ditch; NRTribal, tribal stream]

\begin{tabular}{|c|c|c|c|c|c|c|c|}
\hline \multirow{2}{*}{$\begin{array}{l}\text { Determi- } \\
\text { nation site } \\
\text { identi- } \\
\text { fication } \\
\text { number } \\
\text { (fig. 91) }\end{array}$} & \multirow{2}{*}{$\begin{array}{c}\text { Estimated mean } \\
\text { flow } \\
\left(\mathrm{ft}^{3} / \mathrm{s}\right)\end{array}$} & \multicolumn{6}{|c|}{ Estimated peak discharge $\left(\mathrm{ft}^{3} / \mathrm{s}\right)$ for indicated peak-discharge frequency } \\
\hline & & 2-year & 5-year & 10 -year & 25-year & 50-year & 100 -year \\
\hline 902 & 1,660 & 25,100 & 44,000 & 58,900 & 80,000 & 97,100 & 116,000 \\
\hline 973 & 38.1 & 4,270 & 8,360 & 11,800 & 17,100 & 21,500 & 26,400 \\
\hline 1008 & 9.09 & 1,260 & 2,980 & 4,550 & 7,010 & 9,120 & 11,600 \\
\hline 1012 & 39.0 & 4,270 & 8,340 & 11,800 & 17,000 & 21,400 & 26,300 \\
\hline 1026 & 47.4 & 5,690 & 10,600 & 14,600 & 20,500 & 25,400 & 30,700 \\
\hline 1040 & 75.5 & 6,680 & 12,600 & 17,500 & 24,900 & 31,000 & 37,700 \\
\hline 1052 & 60.4 & 6,220 & 11,700 & 16,200 & 22,800 & 28,300 & 34,300 \\
\hline 1073 & NA & NA & NA & NA & NA & NA & NA \\
\hline 1174 & 14.8 & 2,340 & 5,150 & 7,670 & 11,500 & 14,900 & 18,600 \\
\hline 1367 & 18.5 & 2,630 & 5,760 & 8,570 & 12,900 & 16,600 & 20,700 \\
\hline 1375 & 19.6 & 2,660 & 5,830 & 8,680 & 13,000 & 16,800 & 20,900 \\
\hline 1377 & NA & NA & NA & NA & NA & NA & NA \\
\hline 1421 & NA & NA & NA & NA & NA & NA & NA \\
\hline 1425 & 8.90 & 936 & 2,020 & 2,980 & 4,450 & 5,740 & 7,190 \\
\hline 1512 & 6.34 & 963 & 2,200 & 3,310 & 5,020 & 6,470 & 8,130 \\
\hline 1514 & 29.3 & 1,890 & 3,680 & 5,220 & 7,530 & 9,560 & 11,800 \\
\hline 1568 & 3.89 & 740 & 1,670 & 2,490 & 3,750 & 4,810 & 6,030 \\
\hline 1574 & 42.3 & 2,360 & 4,360 & 6,000 & 8,410 & 10,500 & 12,700 \\
\hline 1619 & 50.0 & 2,470 & 4,380 & 5,880 & 8,030 & 9,800 & 11,700 \\
\hline 1646 & 3,100 & 19,400 & 31,900 & 45,100 & 74,100 & 104,000 & 142,000 \\
\hline 1650 & 3,100 & 19,400 & 31,900 & 45,100 & 74,100 & 104,000 & 142,000 \\
\hline 1663 & 3,120 & 19,500 & 32,100 & 45,500 & 74,500 & 104,000 & 143,000 \\
\hline 1666 & 18.2 & 2,680 & 5,770 & 8,530 & 12,700 & 16,300 & 20,200 \\
\hline 1671 & 3,100 & 19,400 & 31,900 & 45,100 & 74,200 & 104,000 & 142,000 \\
\hline 1706 & 21.8 & 2,480 & 5,320 & 7,850 & 11,700 & 15,000 & 18,600 \\
\hline 1725 & 39.2 & 4,090 & 8,430 & 12,200 & 17,900 & 22,800 & 28,100 \\
\hline 1744 & 7.68 & 1,140 & 2,530 & 3,760 & 5,650 & 7,230 & 9,040 \\
\hline
\end{tabular}


Table 87. Estimated flow-duration values, mean flow values, and peak-discharge frequency values for controlled and uncontrolled flow stream segments on the 1999 Kansas Surface Water Register for Riley County.-Continued

[KSWR, Kansas Surface Water Register; CUSEGA, catalog unit segment number alpha; $\mathrm{mi}^{2}$, square miles; $\mathrm{ft}^{3} / \mathrm{s}$, cubic feet per second; HYDRO, lake or other hydrologic structure; NA, not applicable; NRDitch, irrigation ditch; NRTribal, tribal stream]

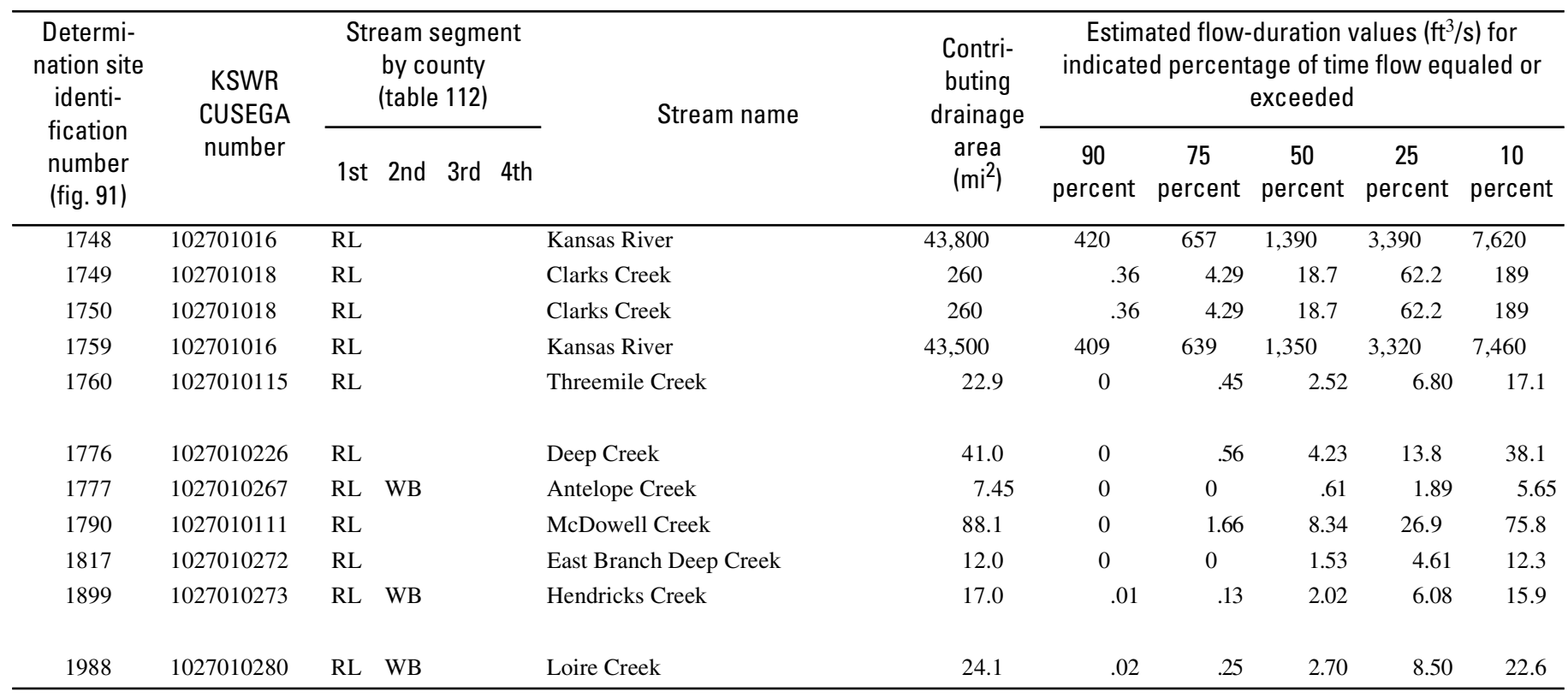


Table 87. Estimated flow-duration values, mean flow values, and peak-discharge frequency values for controlled and uncontrolled flow stream segments on the 1999 Kansas Surface Water Register for Riley County.-Continued

[KSWR, Kansas Surface Water Register; CUSEGA, catalog unit segment number alpha; $\mathrm{mi}^{2}$, square miles; $\mathrm{ft}^{3} / \mathrm{s}$, cubic feet per second; HYDRO, lake or other hydrologic structure; NA, not applicable; NRDitch, irrigation ditch; NRTribal, tribal stream]

\begin{tabular}{|c|c|c|c|c|c|c|c|}
\hline \multirow{2}{*}{$\begin{array}{l}\text { Determi- } \\
\text { nation site } \\
\text { identi- } \\
\text { fication } \\
\text { number } \\
\text { (fig. 91) }\end{array}$} & \multirow[b]{2}{*}{$\begin{array}{l}\text { Estimated mean } \\
\text { flow } \\
\left(\mathrm{ft}^{3} / \mathrm{s}\right)\end{array}$} & \multicolumn{6}{|c|}{ Estimated peak discharge $\left(\mathrm{ft}^{3} / \mathrm{s}\right)$ for indicated peak-discharge frequency } \\
\hline & & 2-year & 5-year & 10-year & 25-year & 50-year & 100-year \\
\hline 1748 & 3,090 & 19,400 & 31,800 & 45,000 & 74,000 & 104,000 & 142,000 \\
\hline 1759 & 3,020 & 19,100 & 31,200 & 44,100 & 73,100 & 102,000 & 140,000 \\
\hline 1760 & 12.5 & 1,480 & 3,440 & 5,200 & 7,940 & 10,300 & 13,000 \\
\hline 1776 & 26.6 & 5,550 & 10,600 & 14,800 & 20,800 & 25,900 & 31,200 \\
\hline 1777 & 4.84 & 876 & 1,920 & 2,840 & 4,230 & 5,400 & 6,730 \\
\hline 1988 & 16.3 & 1,770 & 3,960 & 5,900 & 8,900 & 11,400 & 14,300 \\
\hline
\end{tabular}




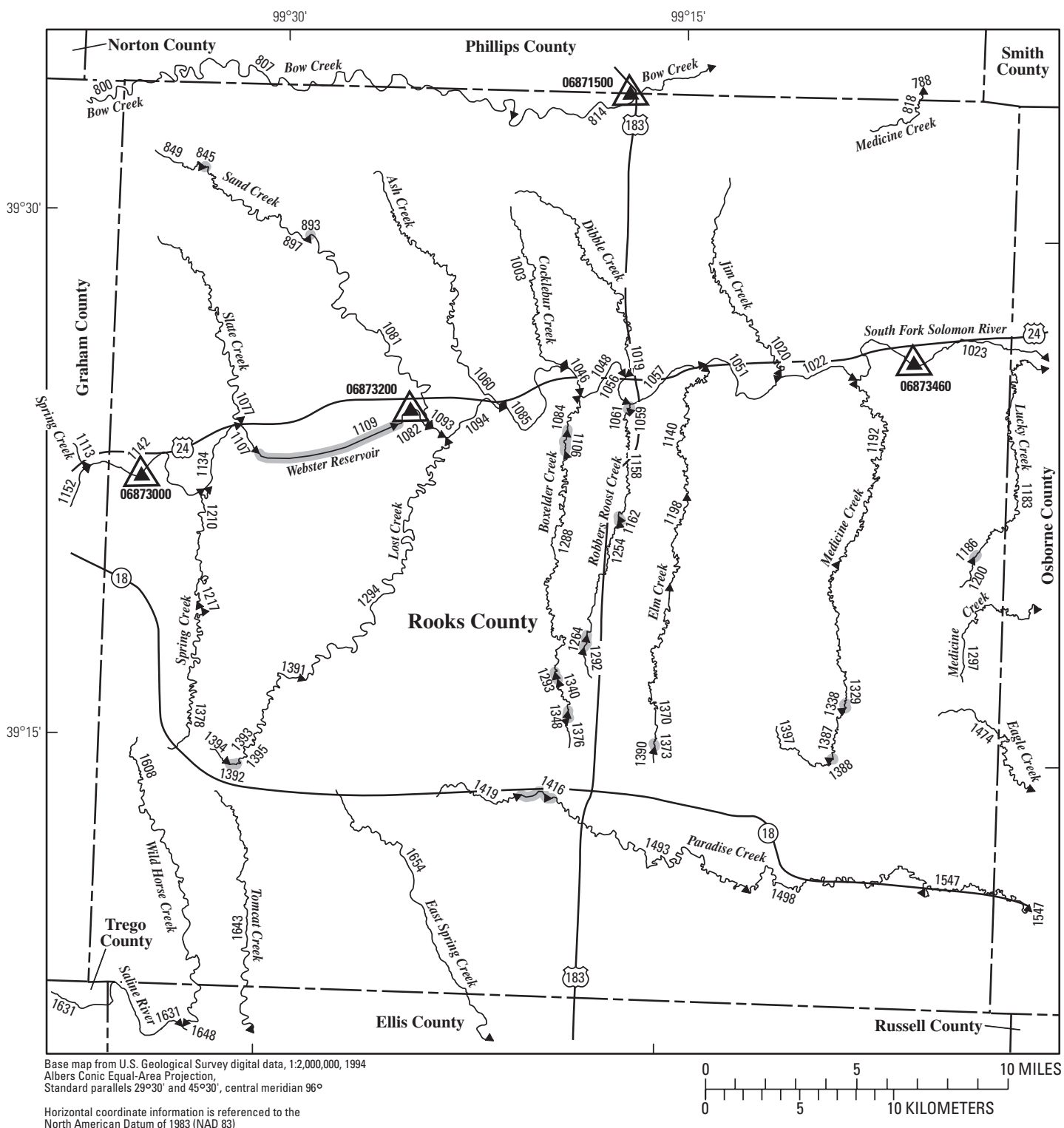

EXPLANATION

$\stackrel{1643}{\longleftarrow}$ Location of streamflow-statistics determination site (small triangle) and associated identification number-small triangle points in downstream direction

06873200

$\Delta$ U.S. Geological Survey streamflow-gaging station and number used for estimates of flow duration

06873460 (

U.S. Geological Survey streamflow-gaging station and number used for estimates of peak-discharge frequency values

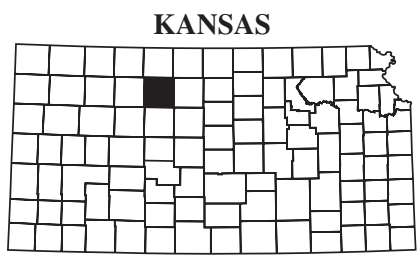

Index map

1416 Lake and determination site identification number

Figure 92. Location of streamflow-statistics determination sites, associated identification numbers, and U.S. Geological Survey streamflow-gaging stations used in the flow-duration and peak-discharge frequency analyses for Rooks County. 
Table 88. Estimated flow-duration values, mean flow values, and peak-discharge frequency values for controlled and uncontrolled flow stream segments on the 1999 Kansas Surface Water Register for Rooks County.

[KSWR, Kansas Surface Water Register; CUSEGA, catalog unit segment number alpha; $\mathrm{mi}^{2}$, square miles; $\mathrm{ft}^{3} / \mathrm{s}$, cubic feet per second; HYDRO, lake or other hydrologic structure; NA, not applicable; NRDitch, irrigation ditch; NRTribal, tribal stream]

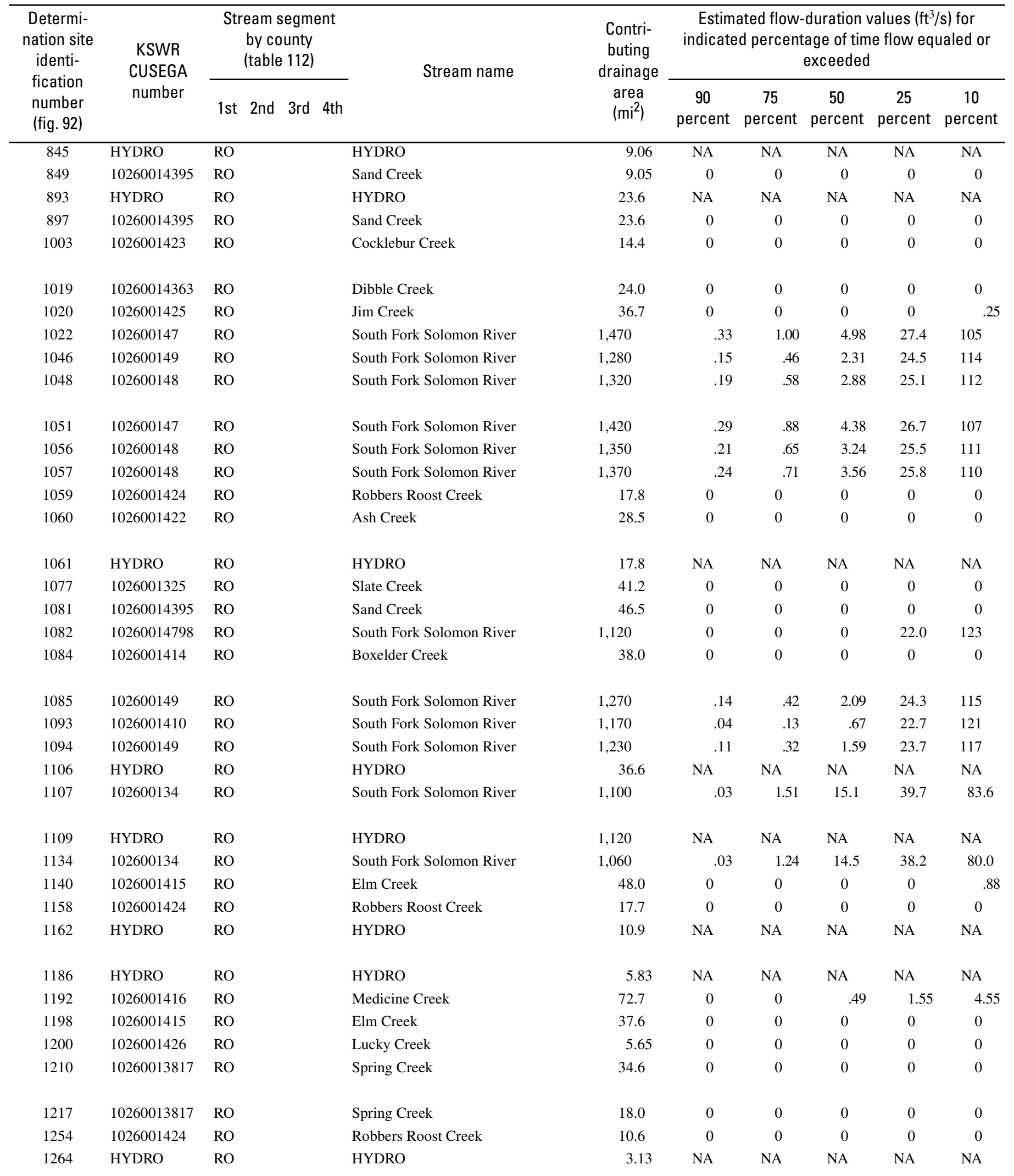


Table 88. Estimated flow-duration values, mean flow values, and peak-discharge frequency values for controlled and uncontrolled flow stream segments on the 1999 Kansas Surface Water Register for Rooks County.-Continued

[KSWR, Kansas Surface Water Register; CUSEGA, catalog unit segment number alpha; $\mathrm{mi}^{2}$, square miles; $\mathrm{ft}^{3} / \mathrm{s}$, cubic feet per second; HYDRO, lake or other hydrologic structure; NA, not applicable; NRDitch, irrigation ditch; NRTribal, tribal stream]

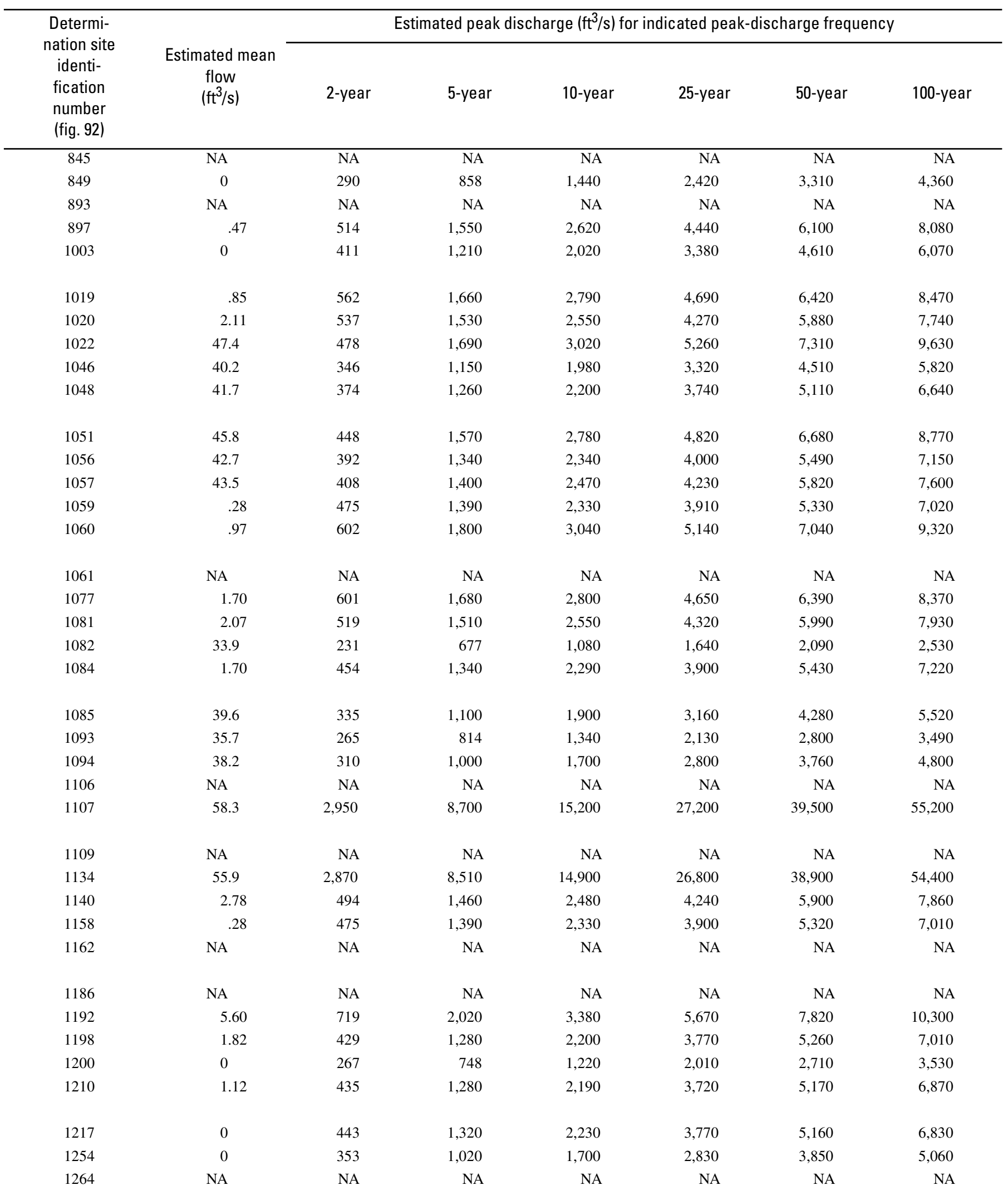


Table 88. Estimated flow-duration values, mean flow values, and peak-discharge frequency values for controlled and uncontrolled flow stream segments on the 1999 Kansas Surface Water Register for Rooks County._Continued

[KSWR, Kansas Surface Water Register; CUSEGA, catalog unit segment number alpha; $\mathrm{mi}^{2}$, square miles; $\mathrm{ft}^{3} / \mathrm{s}$, cubic feet per second; HYDRO, lake or other hydrologic structure; NA, not applicable; NRDitch, irrigation ditch; NRTribal, tribal stream]

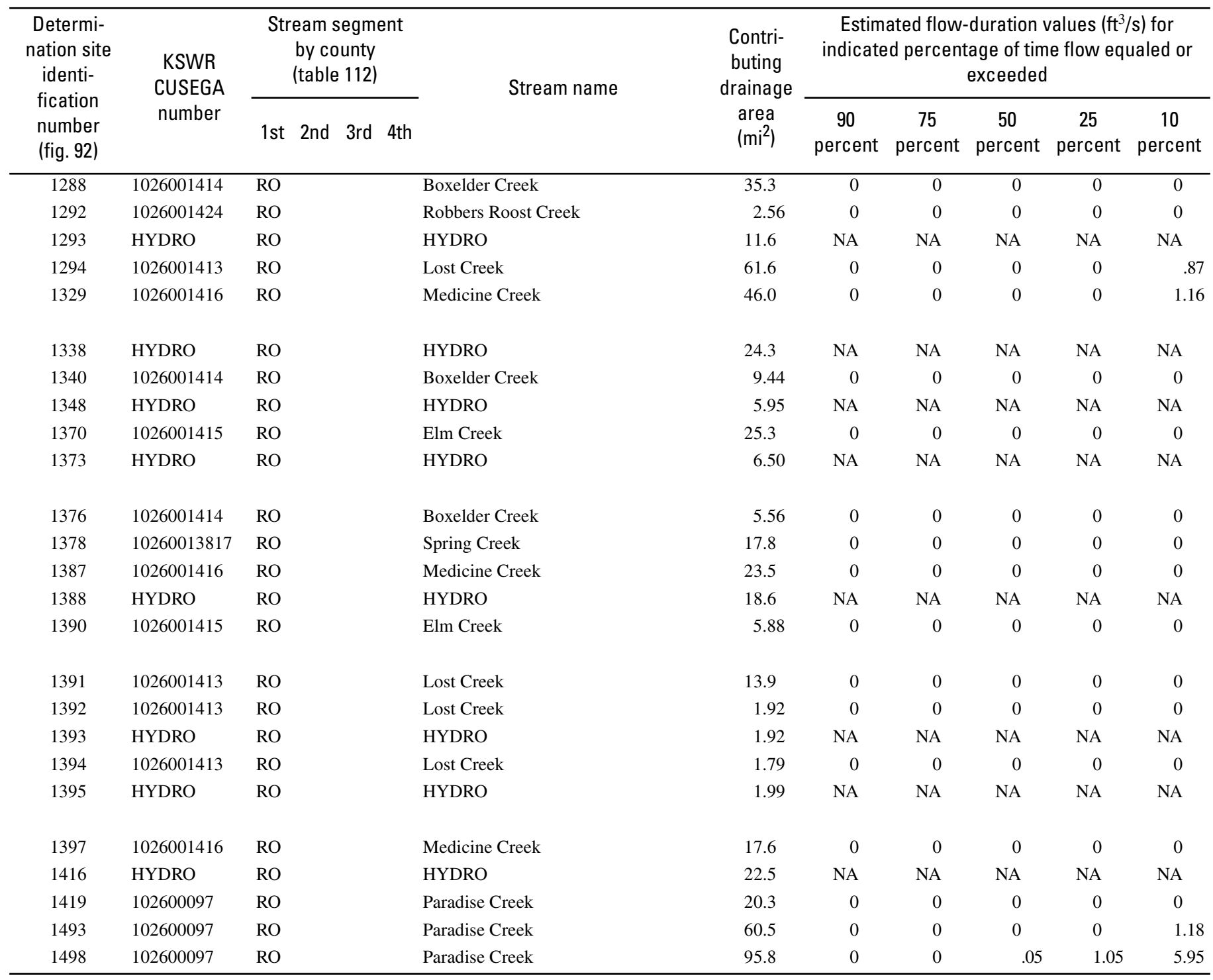


Table 88. Estimated flow-duration values, mean flow values, and peak-discharge frequency values for controlled and uncontrolled flow stream segments on the 1999 Kansas Surface Water Register for Rooks County.-Continued

[KSWR, Kansas Surface Water Register; CUSEGA, catalog unit segment number alpha; $\mathrm{mi}^{2}$, square miles; $\mathrm{ft}^{3} / \mathrm{s}$, cubic feet per second; HYDRO, lake or other hydrologic structure; NA, not applicable; NRDitch, irrigation ditch; NRTribal, tribal stream]

\begin{tabular}{|c|c|c|c|c|c|c|c|}
\hline \multirow{2}{*}{$\begin{array}{l}\text { Determi- } \\
\text { nation site } \\
\text { identi- } \\
\text { fication } \\
\text { number } \\
\text { (fig. 92) }\end{array}$} & \multirow{2}{*}{$\begin{array}{c}\text { Estimated mean } \\
\text { flow } \\
\left(\mathrm{ft}^{3} / \mathrm{s}\right)\end{array}$} & \multicolumn{6}{|c|}{ Estimated peak discharge $\left(\mathrm{ft}^{3} / \mathrm{s}\right)$ for indicated peak-discharge frequency } \\
\hline & & 2-year & 5-year & 10-year & 25-year & 50-year & 100-year \\
\hline 1288 & 1.45 & 431 & 1,280 & 2,190 & 3,750 & 5,230 & 6,960 \\
\hline 1292 & 0 & 155 & 436 & 714 & 1,170 & 1,580 & 2,060 \\
\hline 1293 & NA & NA & NA & NA & NA & NA & NA \\
\hline 1294 & 2.93 & 528 & 1,560 & 2,660 & 4,540 & 6,330 & 8,440 \\
\hline 1329 & 2.98 & 626 & 1,760 & 2,930 & 4,890 & 6,730 & 8,850 \\
\hline 1338 & NA & NA & NA & NA & NA & NA & NA \\
\hline 1340 & 0 & 328 & 950 & 1,580 & 2,630 & 3,570 & 4,690 \\
\hline 1348 & NA & NA & NA & NA & NA & NA & NA \\
\hline 1370 & .81 & 590 & 1,740 & 2,910 & 4,900 & 6,690 & 8,830 \\
\hline 1373 & NA & NA & NA & NA & NA & NA & NA \\
\hline 1376 & 0 & 243 & 694 & 1,150 & 1,900 & 2,570 & 3,360 \\
\hline 1378 & 0 & 440 & 1,310 & 2,210 & 3,740 & 5,130 & 6,780 \\
\hline 1387 & .88 & 592 & 1,720 & 2,870 & 4,800 & 6,530 & 8,600 \\
\hline 1388 & NA & NA & NA & NA & NA & NA & NA \\
\hline 1390 & 0 & 254 & 725 & 1,200 & 1,980 & 2,680 & 3,500 \\
\hline 1391 & 0 & 373 & 1,110 & 1,880 & 3,170 & 4,340 & 5,730 \\
\hline 1392 & 0 & 115 & 332 & 550 & 911 & 1,230 & 1,610 \\
\hline 1393 & NA & NA & NA & NA & NA & NA & NA \\
\hline 1394 & 0 & 111 & 319 & 527 & 872 & 1,180 & 1,540 \\
\hline 1395 & NA & NA & NA & NA & NA & NA & NA \\
\hline 1397 & .30 & 499 & 1,440 & 2,400 & 4,000 & 5,440 & 7,150 \\
\hline 1416 & NA & NA & NA & NA & NA & NA & NA \\
\hline 1419 & .07 & 497 & 1,470 & 2,480 & 4,180 & 5,730 & 7,570 \\
\hline 1493 & 3.13 & 476 & 1,470 & 2,560 & 4,480 & 6,330 & 8,560 \\
\hline 1498 & 6.58 & 652 & 1,980 & 3,430 & 6,010 & 8,520 & 11,500 \\
\hline
\end{tabular}




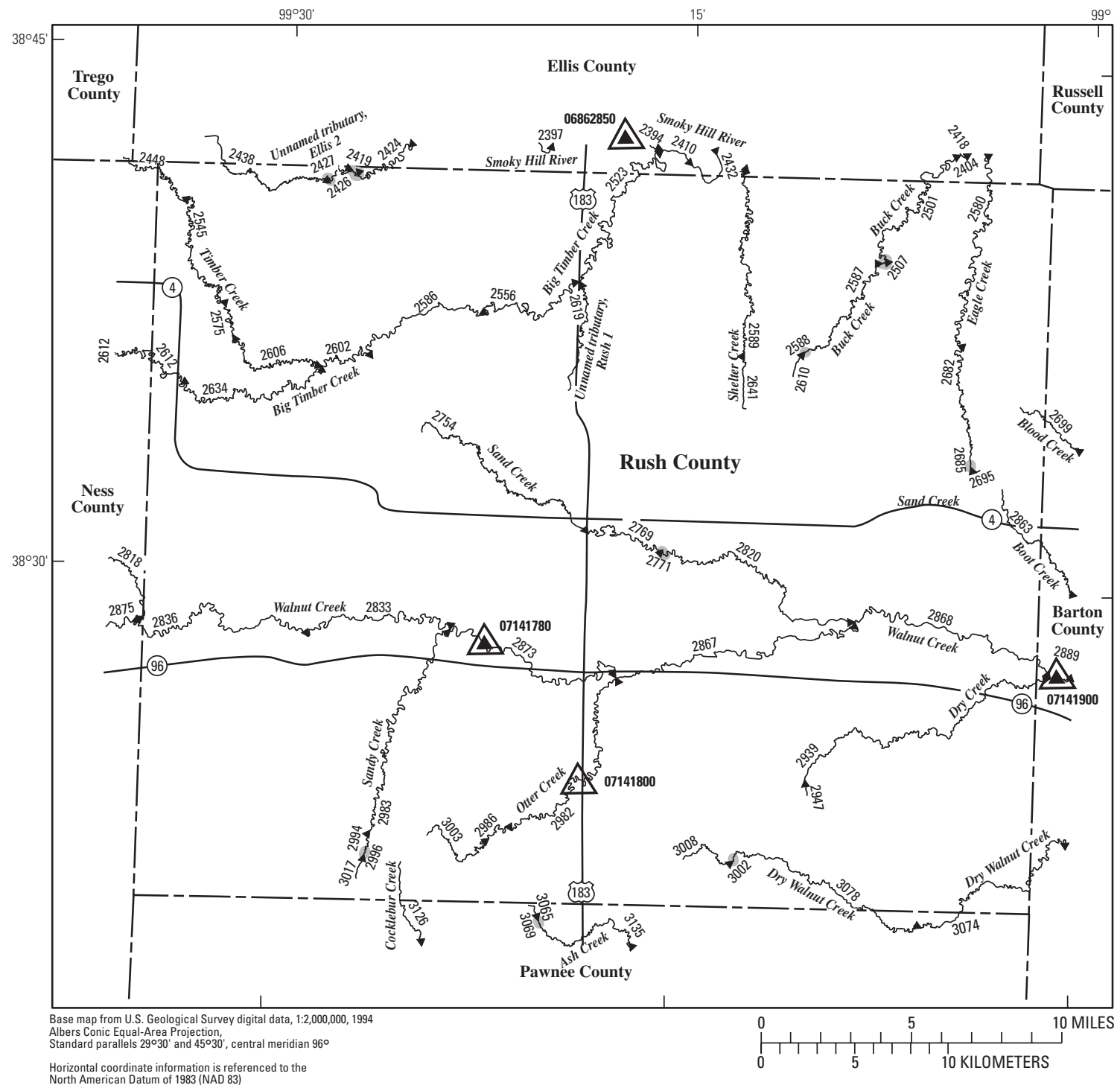

EXPLANATION

$\stackrel{3126}{\longleftarrow}$ Location of streamflow-statistics determination site (small triangle) and associated identification number-small triangle points in downstream direction

$07141900 \Delta$ U.S. Geological Survey streamflow-gaging station and number used for estimates of flow duration

07141800 (

U.S. Geological Survey streamflow-gaging station and number used for estimates of peak-discharge frequency values

KANSAS

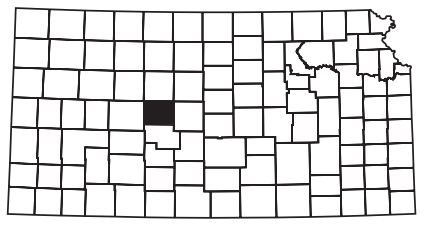

Index map

3002 Lake and determination site identification number

Figure 93. Location of streamflow-statistics determination sites, associated identification numbers, and U.S. Geological Survey streamflow-gaging stations used in the flow-duration and peak-discharge frequency analyses for Rush County. 
Table 89. Estimated flow-duration values, mean flow values, and peak-discharge frequency values for controlled and uncontrolled flow stream segments on the 1999 Kansas Surface Water Register for Rush County.

[KSWR, Kansas Surface Water Register; CUSEGA, catalog unit segment number alpha; $\mathrm{mi}^{2}$, square miles; $\mathrm{ft}^{3} / \mathrm{s}$, cubic feet per second; HYDRO, lake or other hydrologic structure; NA, not applicable; NRDitch, irrigation ditch; NRTribal, tribal stream]

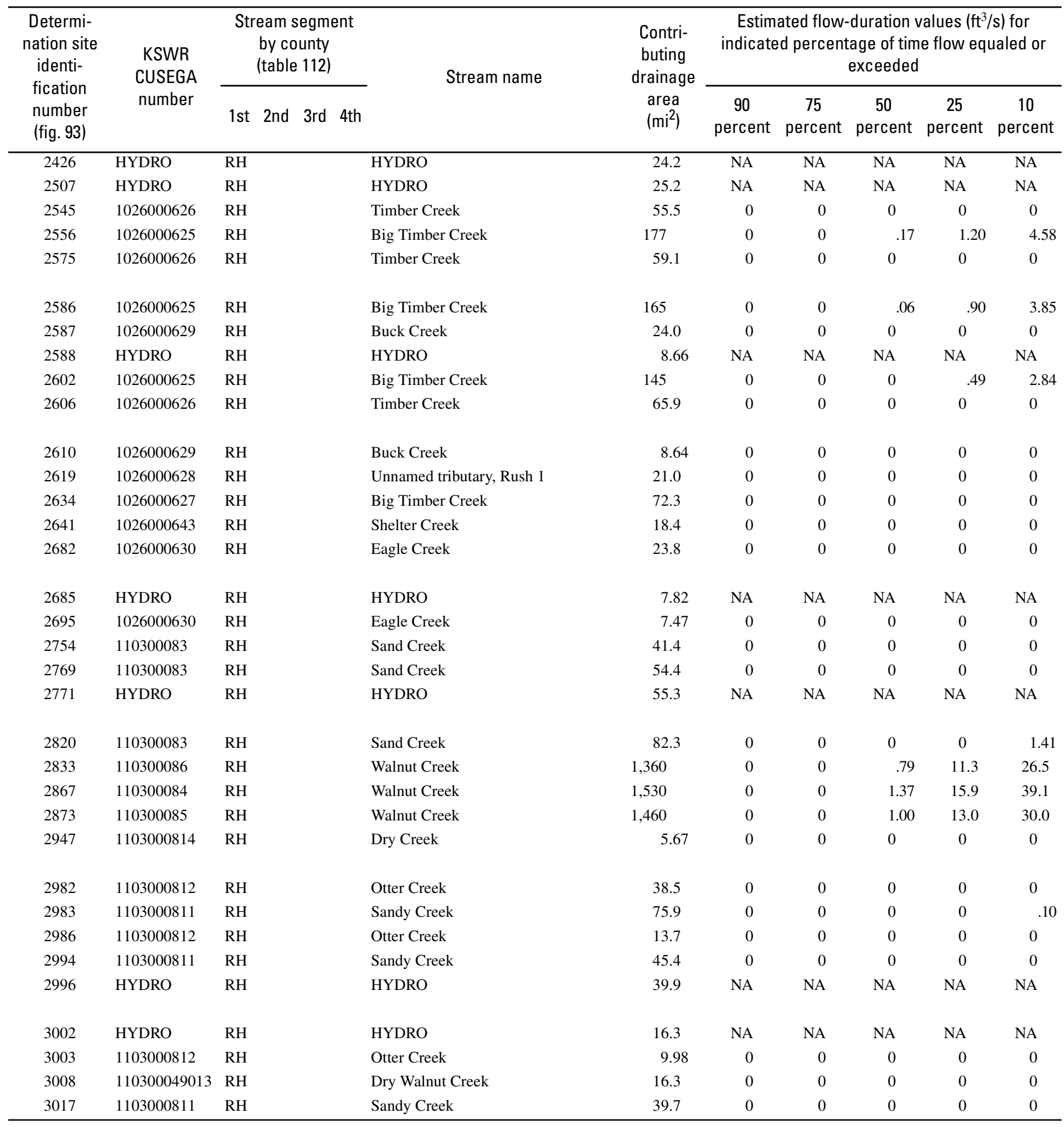


Table 89. Estimated flow-duration values, mean flow values, and peak-discharge frequency values for controlled and uncontrolled flow stream segments on the 1999 Kansas Surface Water Register for Rush County.-Continued

[KSWR, Kansas Surface Water Register; CUSEGA, catalog unit segment number alpha; $\mathrm{mi}^{2}$, square miles; $\mathrm{ft}^{3} / \mathrm{s}$, cubic feet per second; HYDRO, lake or other hydrologic structure; NA, not applicable; NRDitch, irrigation ditch; NRTribal, tribal stream]

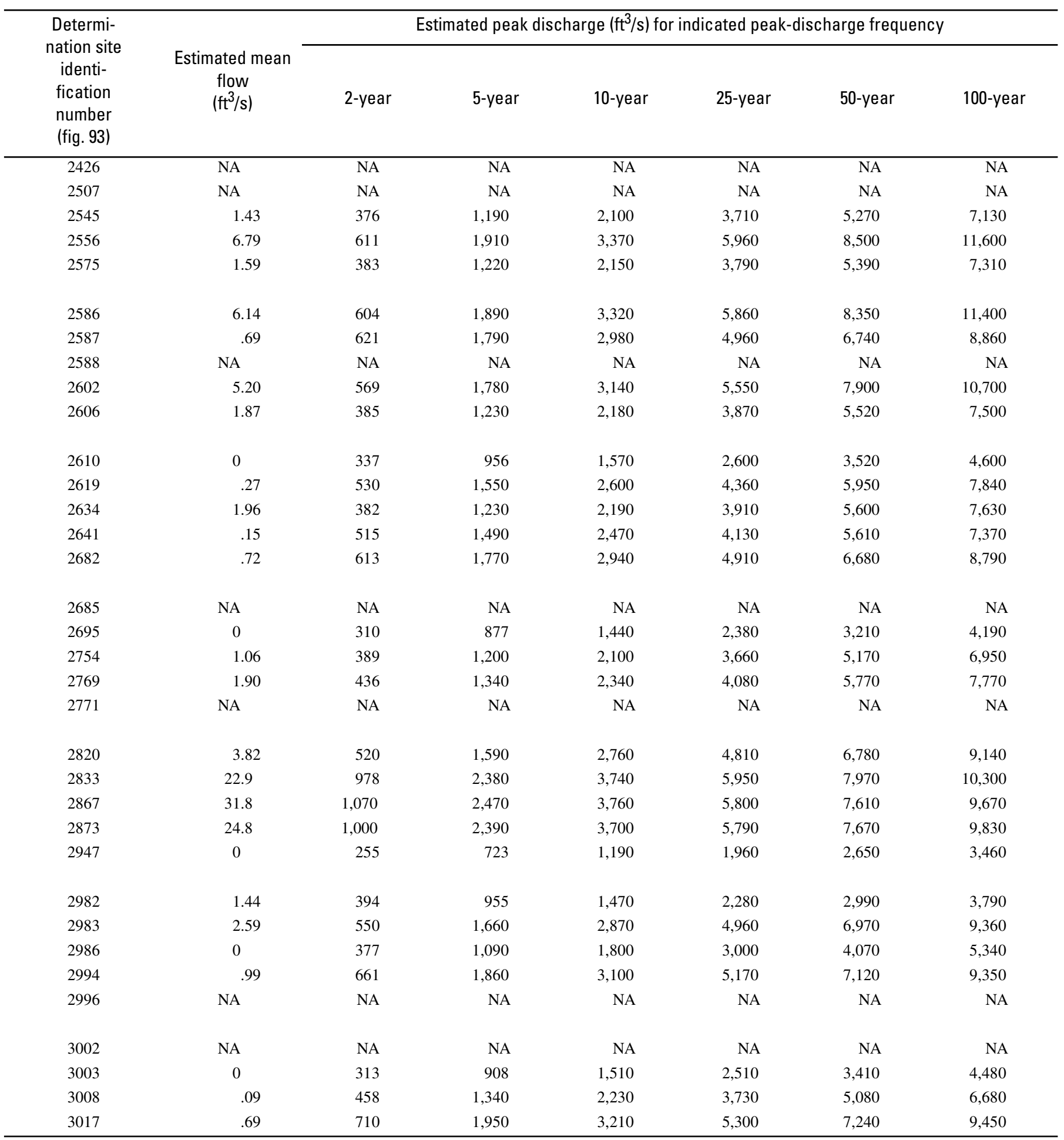




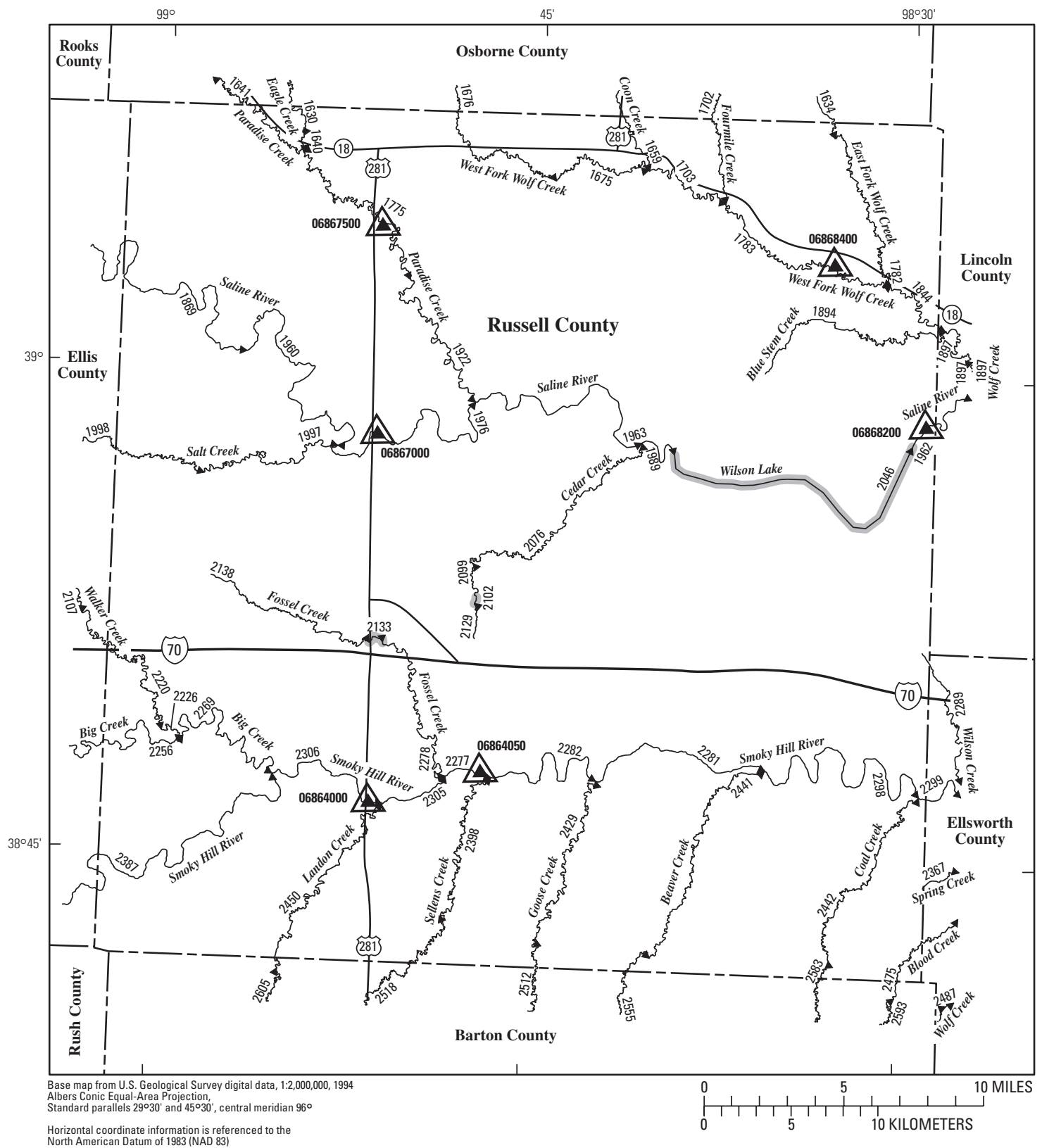

EXPLANATION
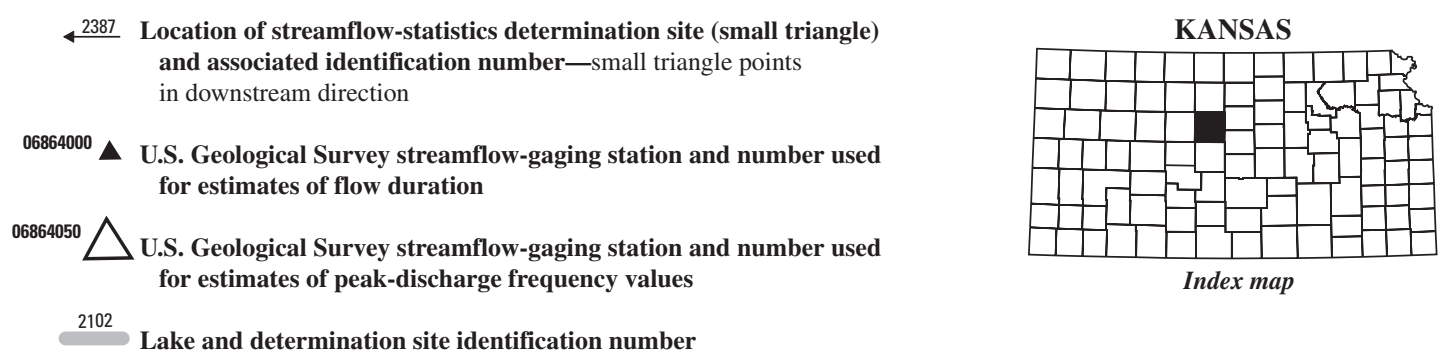

Figure 94. Location of streamflow-statistics determination sites, associated identification numbers, and U.S. Geological Survey streamflow-gaging stations used in the flow-duration and peak-discharge frequency analyses for Russell County. 
Table 90. Estimated flow-duration values, mean flow values, and peak-discharge frequency values for controlled and uncontrolled flow stream segments on the 1999 Kansas Surface Water Register for Russell County.

[KSWR, Kansas Surface Water Register; CUSEGA, catalog unit segment number alpha; $\mathrm{mi}^{2}$, square miles; $\mathrm{ft}^{3} / \mathrm{s}$, cubic feet per second; HYDRO, lake or other hydrologic structure; NA, not applicable; NRDitch, irrigation ditch; NRTribal, tribal stream]

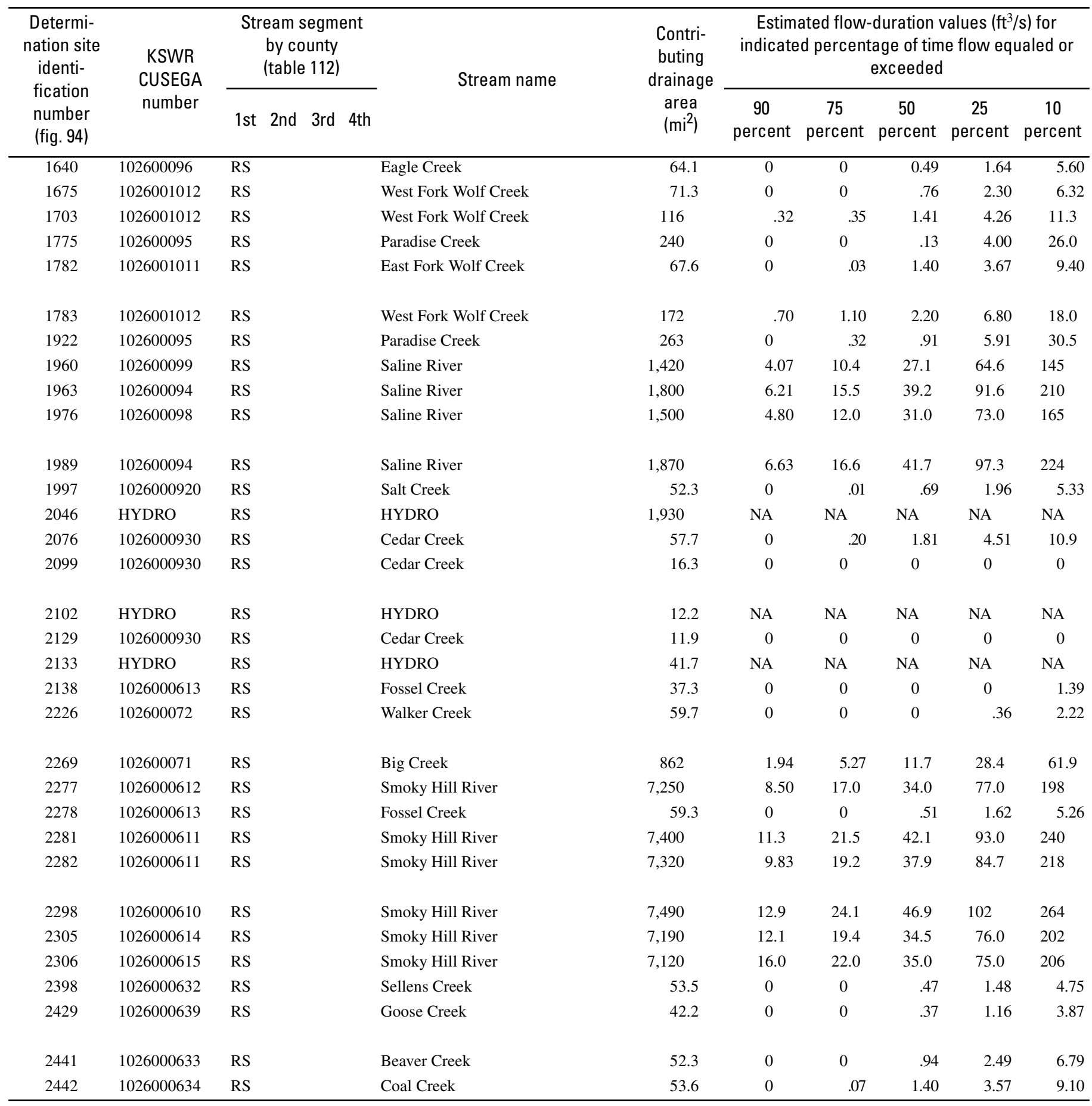


Table 90. Estimated flow-duration values, mean flow values, and peak-discharge frequency values for controlled and uncontrolled flow stream segments on the 1999 Kansas Surface Water Register for Russell County.-Continued

[KSWR, Kansas Surface Water Register; CUSEGA, catalog unit segment number alpha; $\mathrm{mi}^{2}$, square miles; $\mathrm{ft}^{3} / \mathrm{s}$, cubic feet per second; HYDRO, lake or other hydrologic structure; NA, not applicable; NRDitch, irrigation ditch; NRTribal, tribal stream]

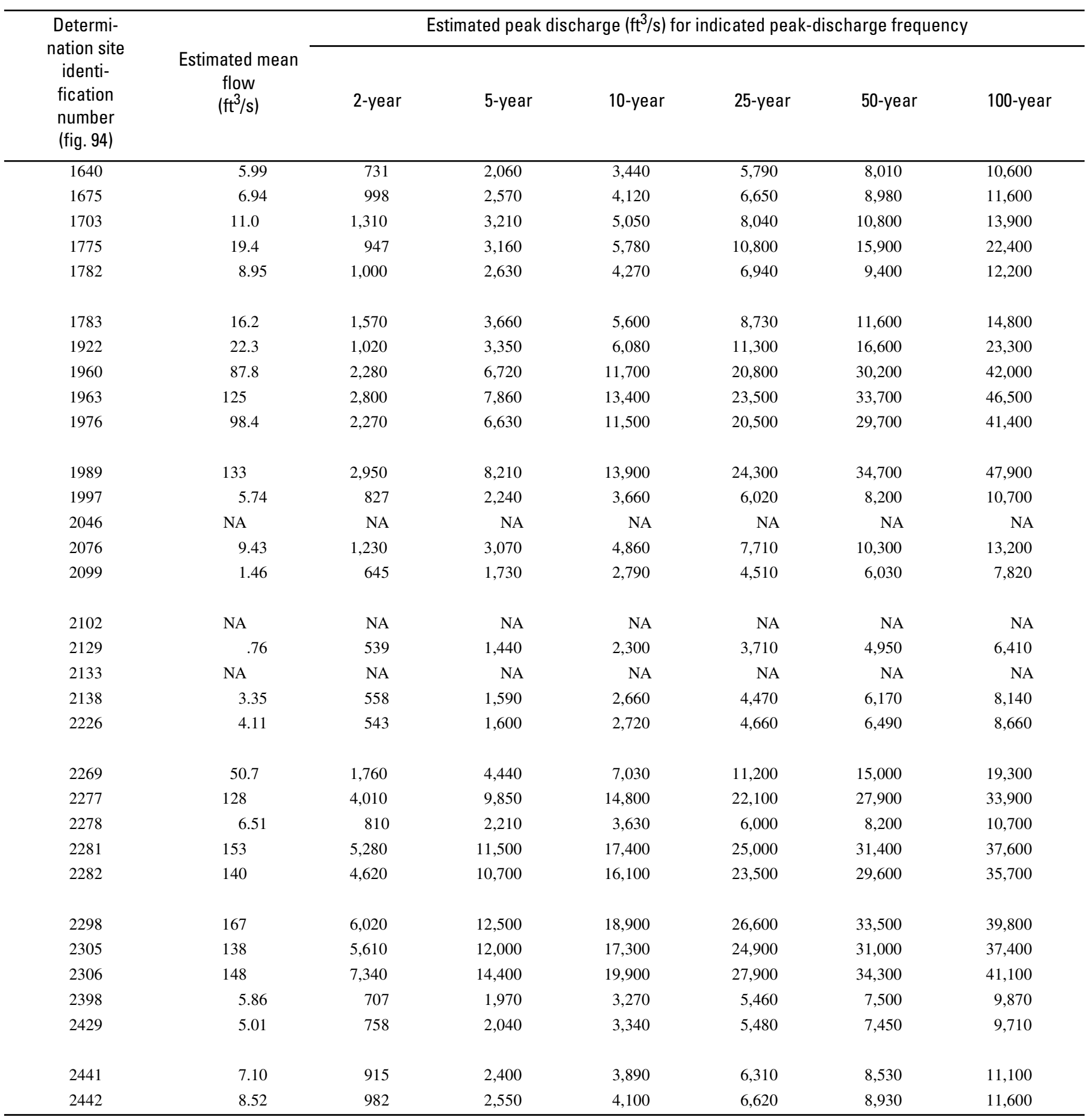




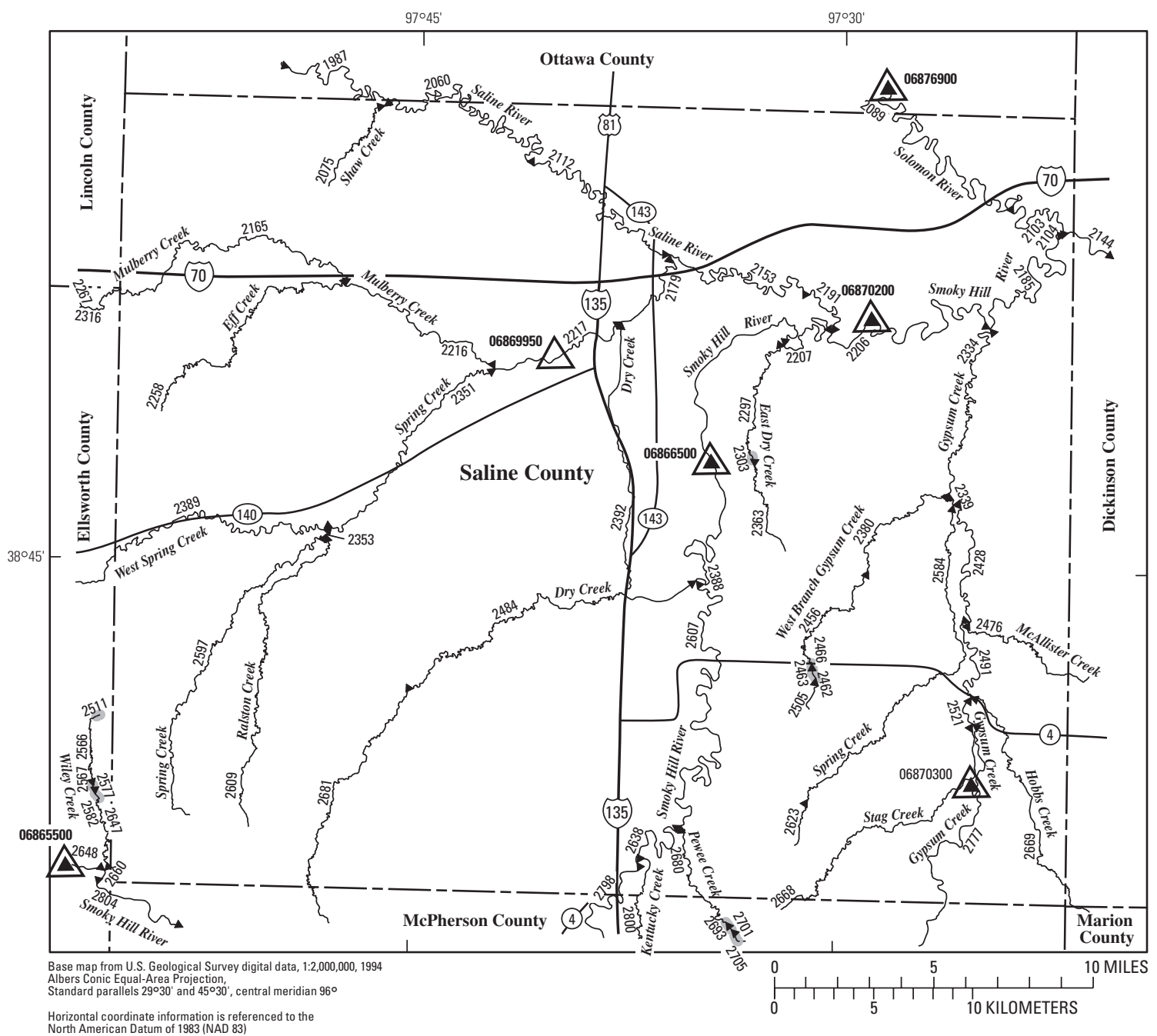

EXPLANATION

2804 Location of streamflow-statistics determination site (small triangle) and associated identification number-small triangle points in downstream direction

06865500 U.S. Geological Survey streamflow-gaging station and number used for estimates of flow duration

${ }^{06869950} \bigwedge$ U.S. Geological Survey streamflow-gaging station and number used for estimates of peak-discharge frequency values

\section{KANSAS}

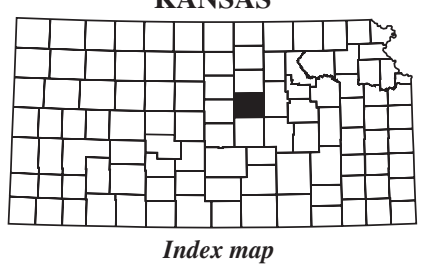

Index map

2701 Lake and determination site identification number

Figure 95. Location of streamflow-statistics determination sites, associated identification numbers, and U.S. Geological Survey streamflow-gaging stations used in the flow-duration and peak-discharge frequency analyses for Saline County. 
Table 91. Estimated flow-duration values, mean flow values, and peak-discharge frequency values for controlled and uncontrolled flow stream segments on the 1999 Kansas Surface Water Register for Saline County.

[KSWR, Kansas Surface Water Register; CUSEGA, catalog unit segment number alpha; $\mathrm{mi}^{2}$, square miles; $\mathrm{ft}^{3} / \mathrm{s}$, cubic feet per second; HYDRO, lake or other hydrologic structure; NA, not applicable; NRDitch, irrigation ditch; NRTribal, tribal stream]

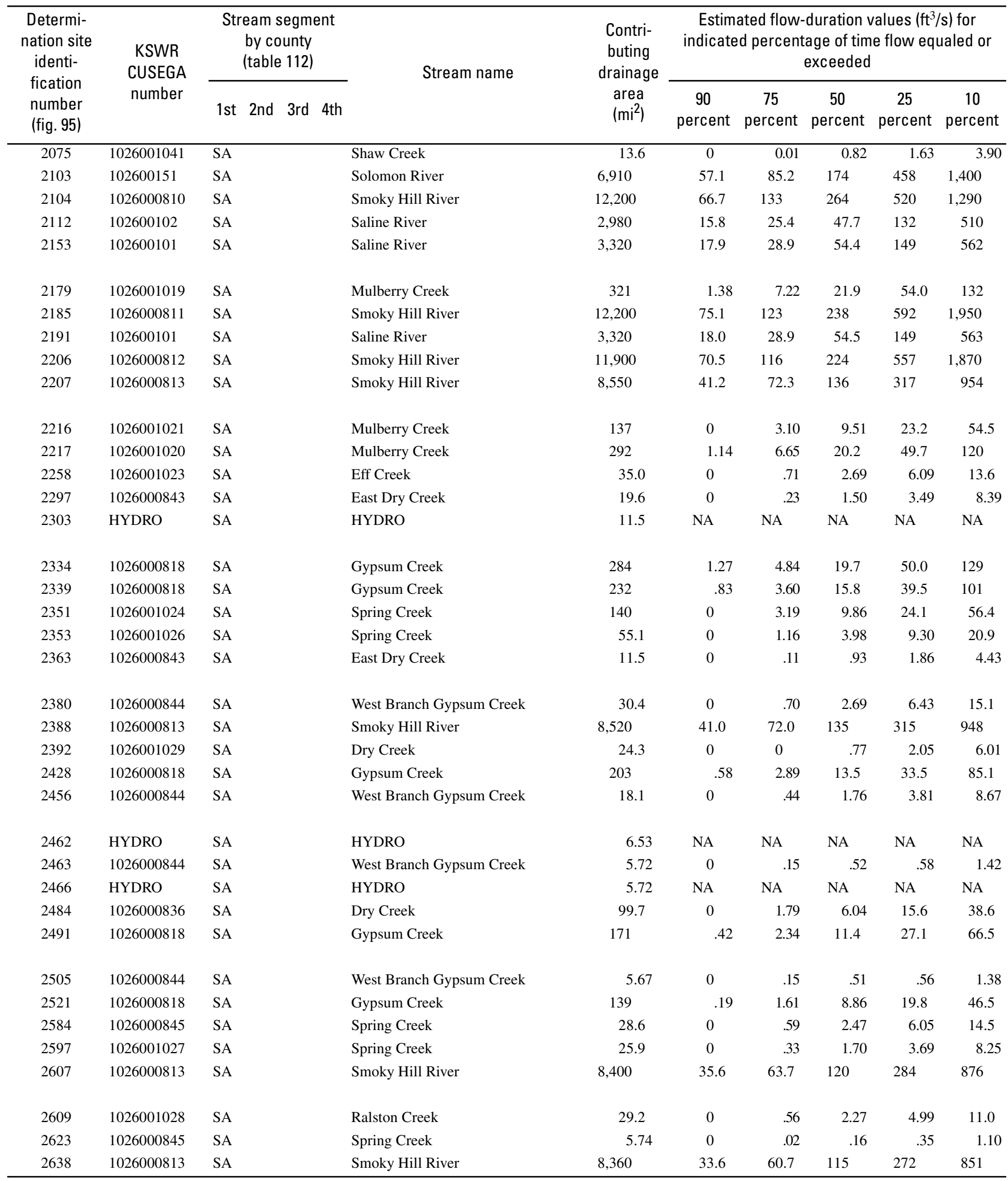


Table 91. Estimated flow-duration values, mean flow values, and peak-discharge frequency values for controlled and uncontrolled flow stream segments on the 1999 Kansas Surface Water Register for Saline County.-Continued

[KSWR, Kansas Surface Water Register; CUSEGA, catalog unit segment number alpha; $\mathrm{mi}^{2}$, square miles; $\mathrm{ft}^{3} / \mathrm{s}$, cubic feet per second; HYDRO, lake or other hydrologic structure; NA, not applicable; NRDitch, irrigation ditch; NRTribal, tribal stream]

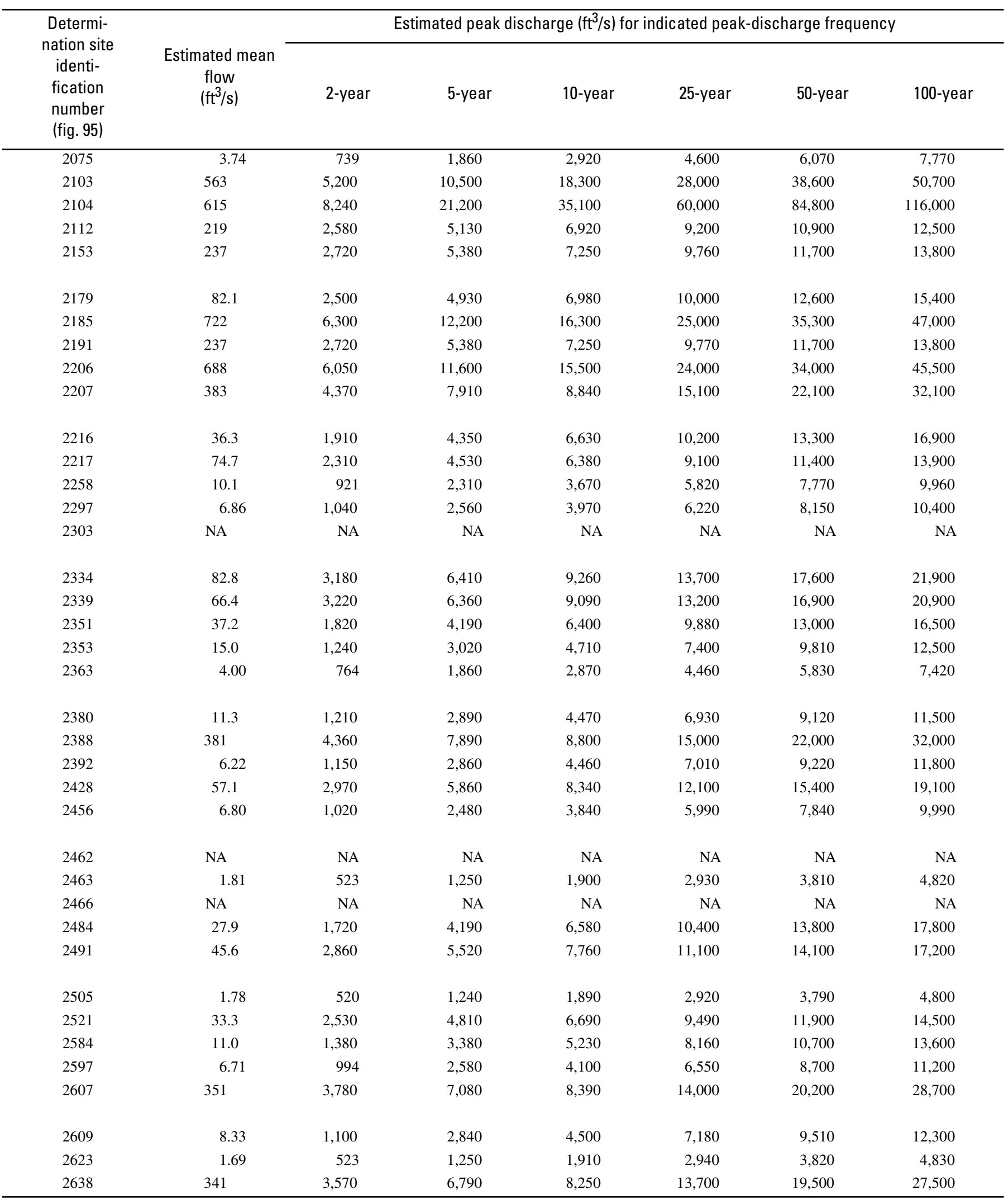




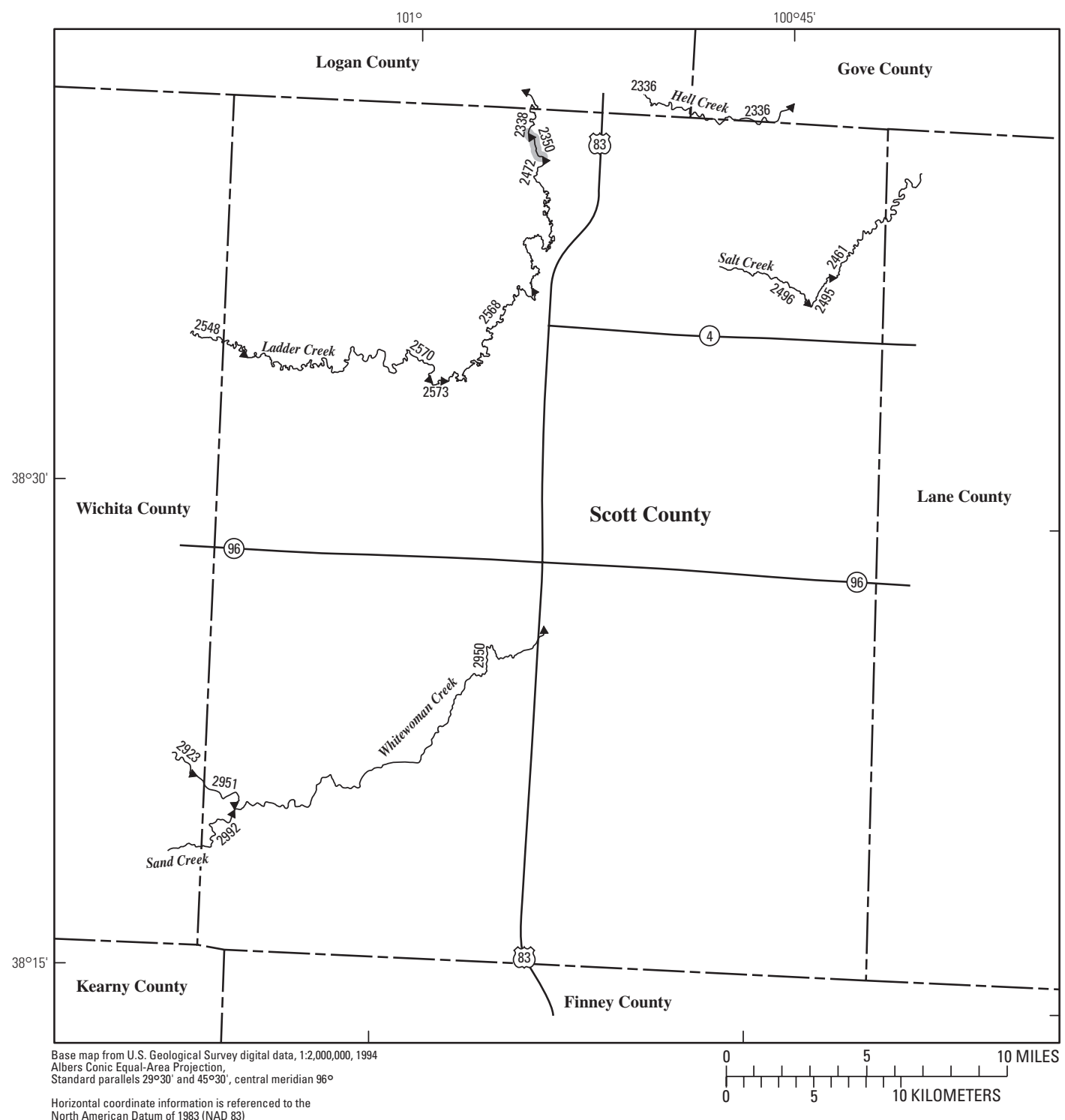

\section{EXPLANATION}

\&2992 Location of streamflow-statistics determination site (small triangle) and associated identification number-small triangle points in downstream direction

$06844900 \Delta$ U.S. Geological Survey streamflow-gaging station and number used for estimates of flow duration

$06846000 \bigwedge$ U.S. Geological Survey streamflow-gaging station and number used for estimates of peak-discharge frequency values

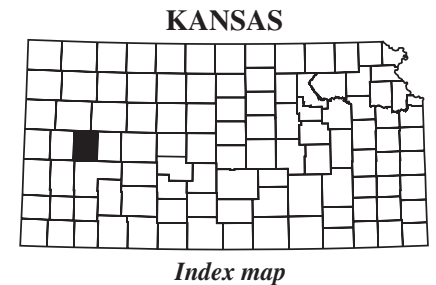

2350 Lake and determination site identification number

Figure 96. Location of streamflow-statistics determination sites, associated identification numbers, and U.S. Geological Survey streamflow-gaging stations used in the flow-duration and peak-discharge frequency analyses for Scott County. 
Table 92. Estimated flow-duration values, mean flow values, and peak-discharge frequency values for controlled and uncontrolled flow stream segments on the 1999 Kansas Surface Water Register for Scott County.

[KSWR, Kansas Surface Water Register; CUSEGA, catalog unit segment number alpha; $\mathrm{mi}^{2}$, square miles; $\mathrm{ft}^{3} / \mathrm{s}$, cubic feet per second; HYDRO, lake or other hydrologic structure; NA, not applicable; NRDitch, irrigation ditch; NRTribal, tribal stream]

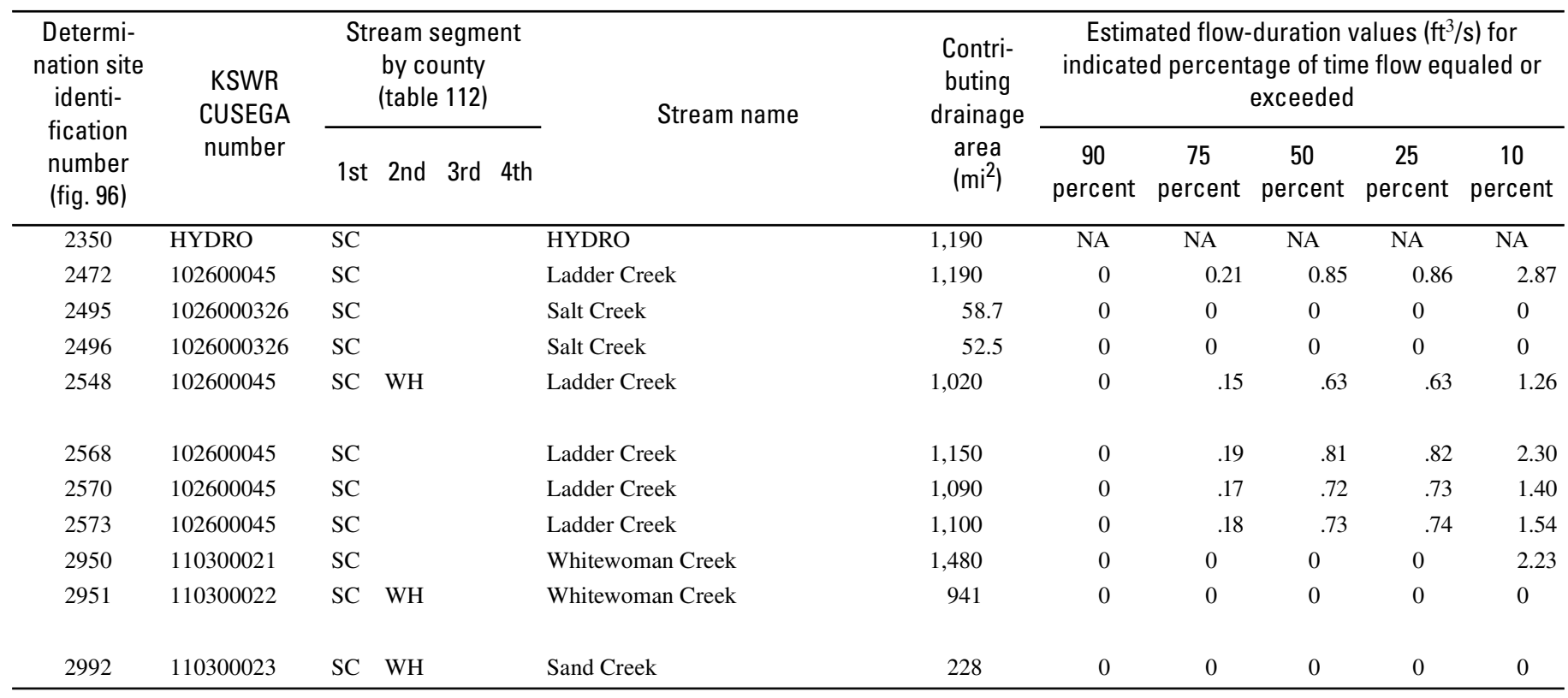


Table 92. Estimated flow-duration values, mean flow values, and peak-discharge frequency values for controlled and uncontrolled flow stream segments on the 1999 Kansas Surface Water Register for Scott County.-Continued

[KSWR, Kansas Surface Water Register; CUSEGA, catalog unit segment number alpha; $\mathrm{mi}^{2}$, square miles; $\mathrm{ft}^{3} / \mathrm{s}$, cubic feet per second; HYDRO, lake or other hydrologic structure; NA, not applicable; NRDitch, irrigation ditch; NRTribal, tribal stream]

\begin{tabular}{|c|c|c|c|c|c|c|c|}
\hline $\begin{array}{l}\text { Determi- } \\
\text { nation site } \\
\text { identi- } \\
\text { fication } \\
\text { number } \\
\text { (fig. 96) }\end{array}$ & $\begin{array}{c}\text { Estimated mean } \\
\text { flow } \\
\left(\mathrm{ft}^{3} / \mathrm{s}\right)\end{array}$ & \multicolumn{6}{|c|}{ Estimated peak discharge $\left(\mathrm{ft}^{3} / \mathrm{s}\right)$ for indicated peak-discharge frequency } \\
\hline 2350 & NA & NA & NA & NA & NA & NA & NA \\
\hline 2496 & 0 & 378 & 1,210 & 2,130 & 3,770 & 5,380 & 7,280 \\
\hline 2548 & 2.27 & 557 & 2,160 & 4,250 & 8,550 & 13,300 & 19,700 \\
\hline 2568 & 3.56 & 592 & 2,300 & 4,560 & 9,240 & 14,500 & 21,600 \\
\hline 2570 & 2.87 & 570 & 2,220 & 4,390 & 8,870 & 13,900 & 20,700 \\
\hline 2992 & .22 & 385 & 1,370 & 2,560 & 4,800 & 7,100 & 9,960 \\
\hline
\end{tabular}




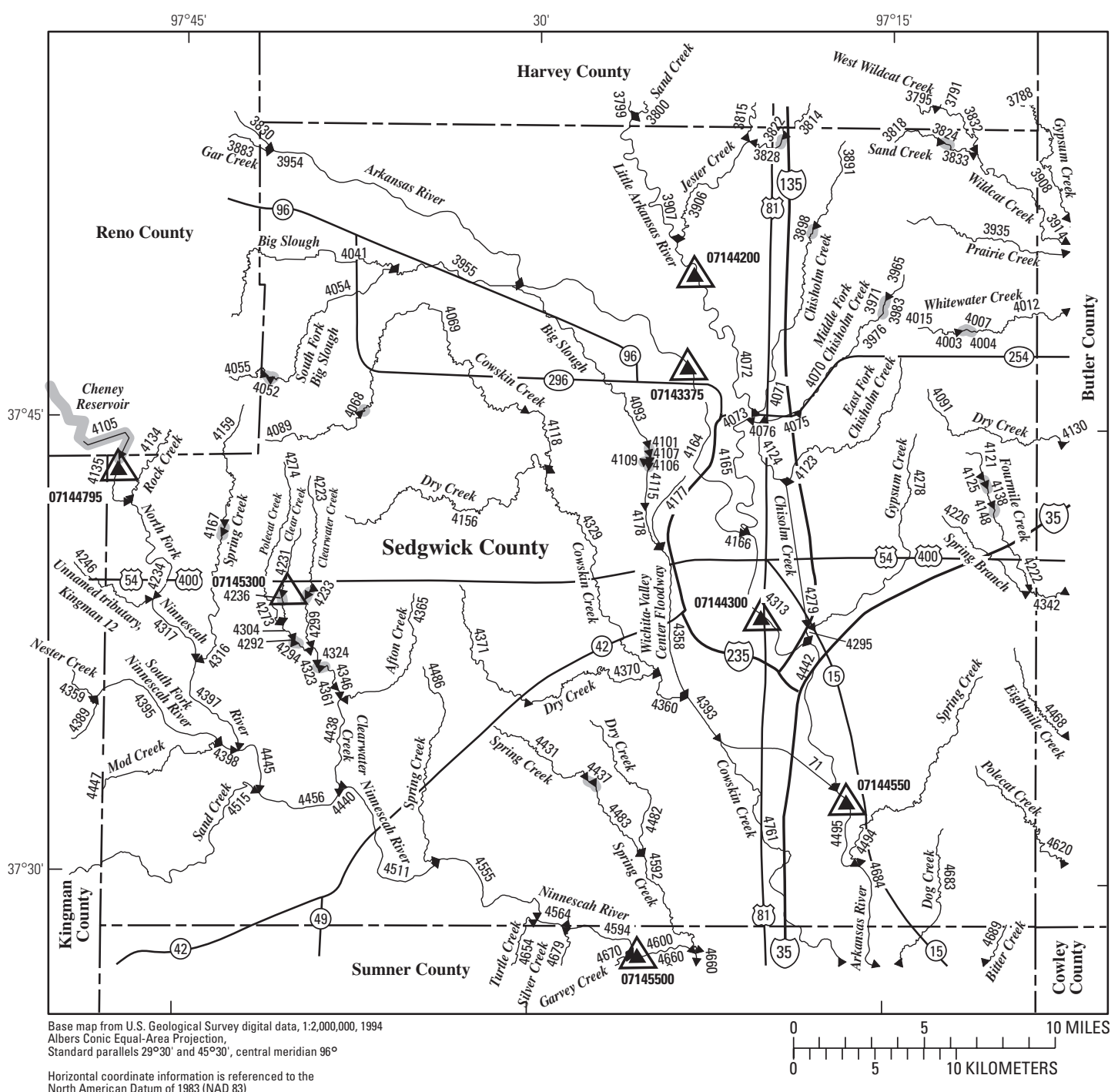

EXPLANATION

4511 Location of streamflow-statistics determination site (small triangle) and associated identification number-small triangle points in downstream direction

07144550

U.S. Geological Survey streamflow-gaging station and number used for estimates of flow duration

07145300 U.S. Geological Survey streamflow-gaging station and number used for estimates of peak-discharge frequency values

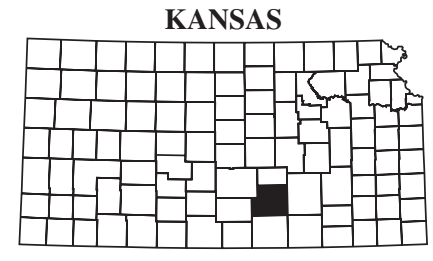

Index map 4437

Lake and determination site identification number

Figure 97. Location of streamflow-statistics determination sites, associated identification numbers, and U.S. Geological Survey streamflow-gaging stations used in the flow-duration and peak-discharge frequency analyses for Sedgwick County. 
Table 93. Estimated flow-duration values, mean flow values, and peak-discharge frequency values for controlled and uncontrolled flow stream segments on the 1999 Kansas Surface Water Register for Sedgwick County.

[KSWR, Kansas Surface Water Register; CUSEGA, catalog unit segment number alpha; $\mathrm{mi}^{2}$, square miles; $\mathrm{ft}^{3} / \mathrm{s}$, cubic feet per second; HYDRO, lake or other hydrologic structure; NA, not applicable; NRDitch, irrigation ditch; NRTribal, tribal stream]

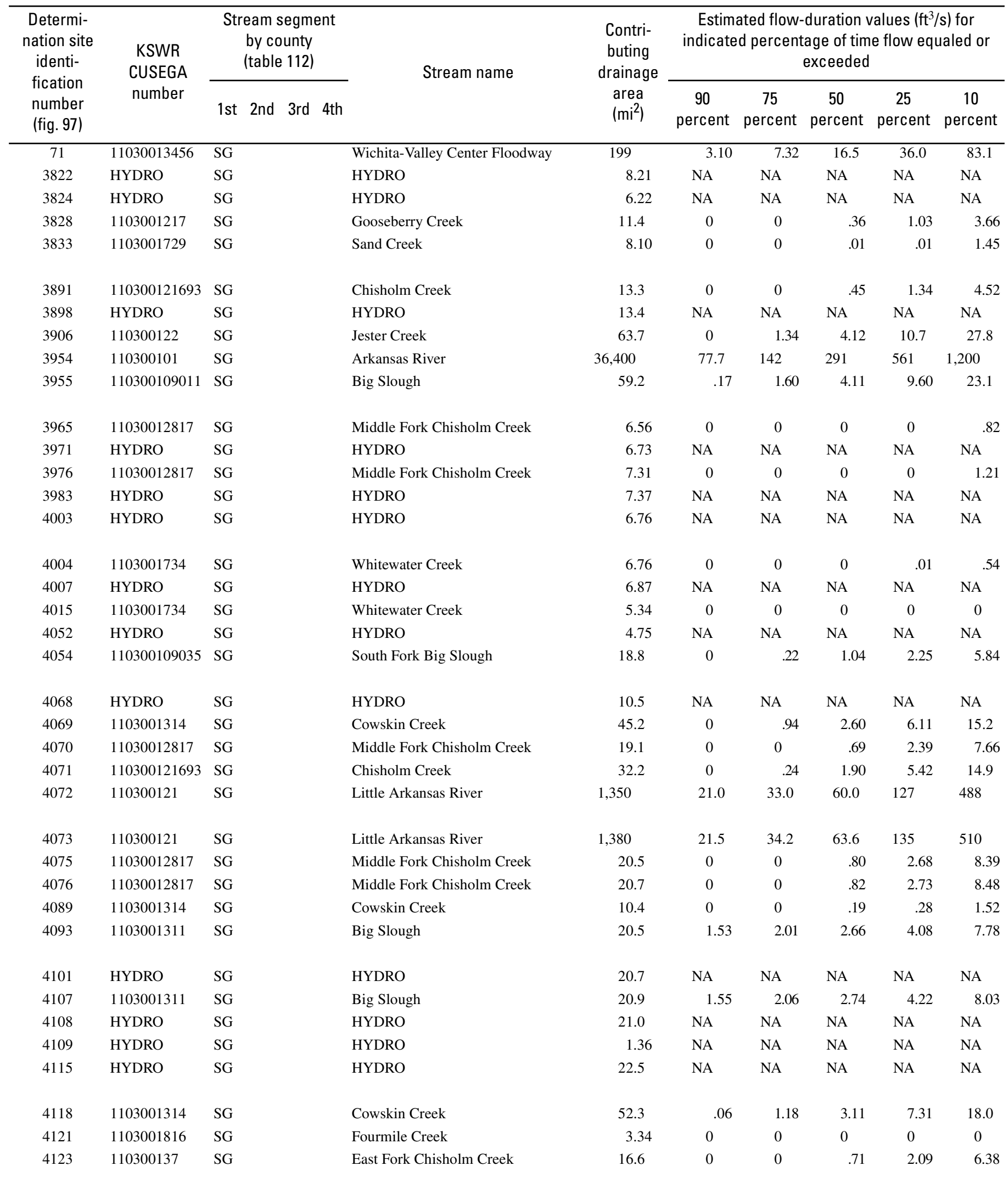


Table 93. Estimated flow-duration values, mean flow values, and peak-discharge frequency values for controlled and uncontrolled flow stream segments on the 1999 Kansas Surface Water Register for Sedgwick County.-Continued

[KSWR, Kansas Surface Water Register; CUSEGA, catalog unit segment number alpha; $\mathrm{mi}^{2}$, square miles; $\mathrm{ft}^{3} / \mathrm{s}$, cubic feet per second; HYDRO, lake or other hydrologic structure; NA, not applicable; NRDitch, irrigation ditch; NRTribal, tribal stream]

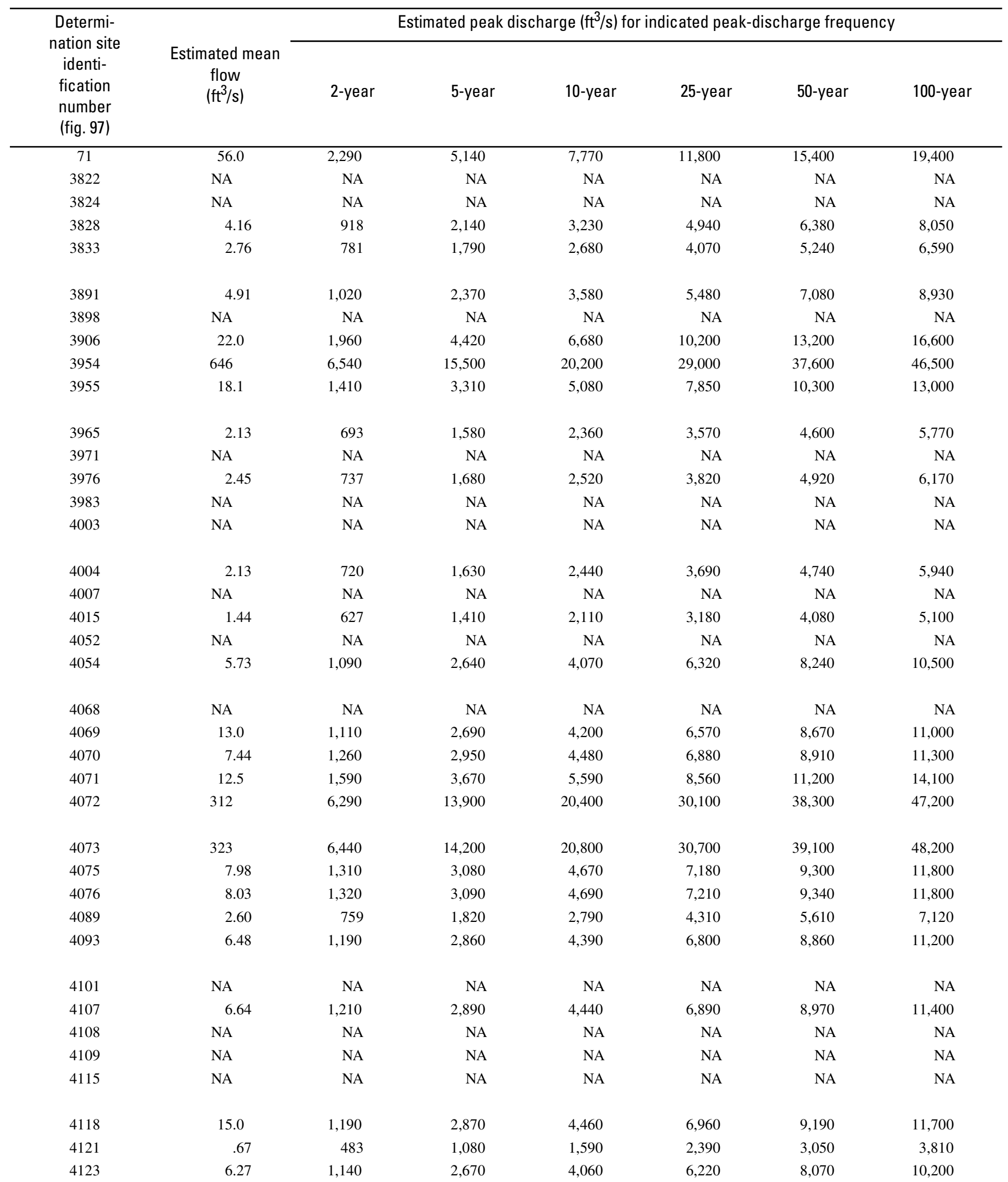


Table 93. Estimated flow-duration values, mean flow values, and peak-discharge frequency values for controlled and uncontrolled flow stream segments on the 1999 Kansas Surface Water Register for Sedgwick County.-Continued

[KSWR, Kansas Surface Water Register; CUSEGA, catalog unit segment number alpha; $\mathrm{mi}^{2}$, square miles; $\mathrm{ft}^{3} / \mathrm{s}$, cubic feet per second; HYDRO, lake or other hydrologic structure; NA, not applicable; NRDitch, irrigation ditch; NRTribal, tribal stream]

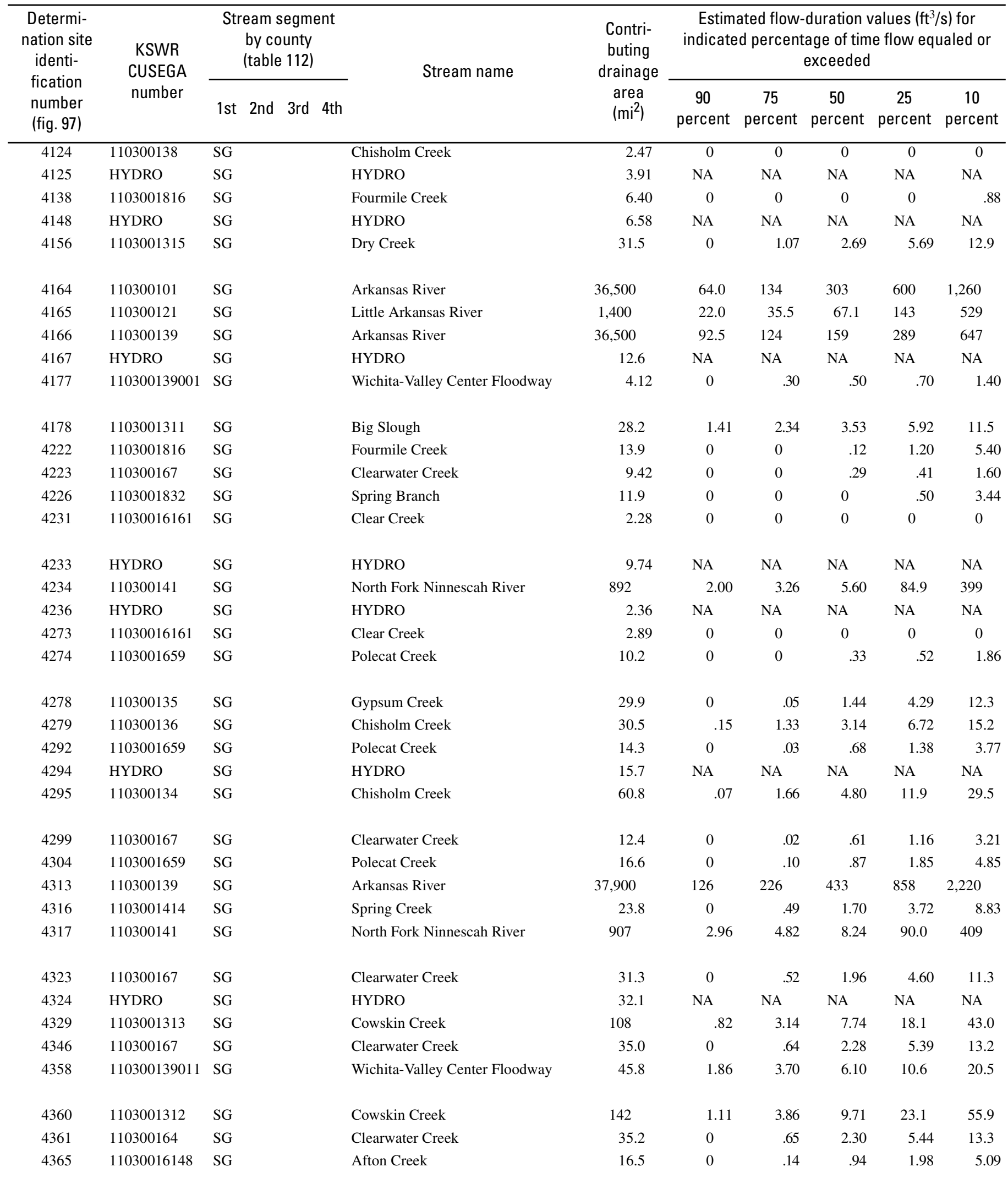


Table 93. Estimated flow-duration values, mean flow values, and peak-discharge frequency values for controlled and uncontrolled flow stream segments on the 1999 Kansas Surface Water Register for Sedgwick County.-Continued

[KSWR, Kansas Surface Water Register; CUSEGA, catalog unit segment number alpha; $\mathrm{mi}^{2}$, square miles; $\mathrm{ft}^{3} / \mathrm{s}$, cubic feet per second; HYDRO, lake or other hydrologic structure; NA, not applicable; NRDitch, irrigation ditch; NRTribal, tribal stream]

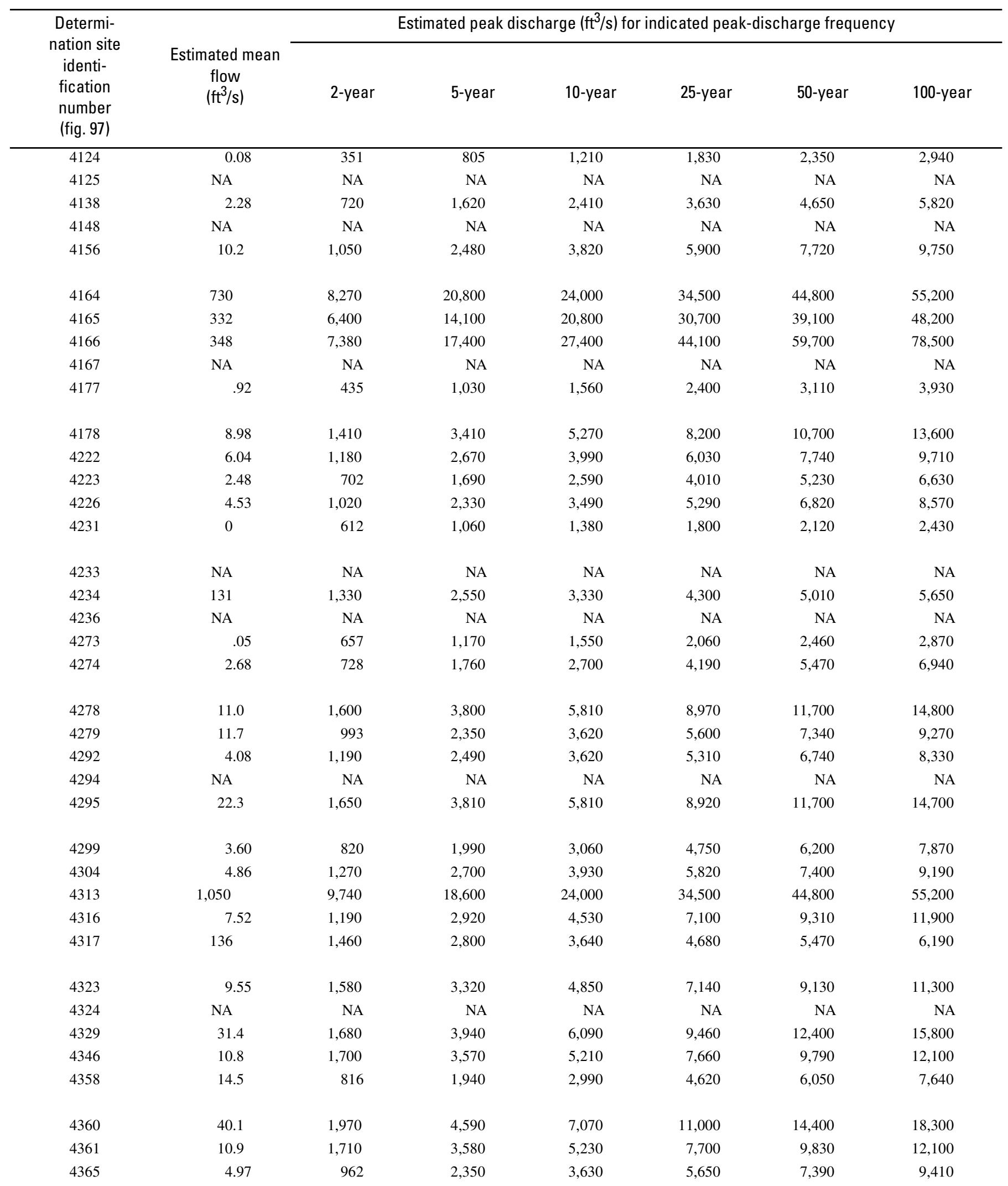


Table 93. Estimated flow-duration values, mean flow values, and peak-discharge frequency values for controlled and uncontrolled flow stream segments on the 1999 Kansas Surface Water Register for Sedgwick County.-Continued

[KSWR, Kansas Surface Water Register; CUSEGA, catalog unit segment number alpha; $\mathrm{mi}^{2}$, square miles; $\mathrm{ft}^{3} / \mathrm{s}$, cubic feet per second; HYDRO, lake or other hydrologic structure; NA, not applicable; NRDitch, irrigation ditch; NRTribal, tribal stream]

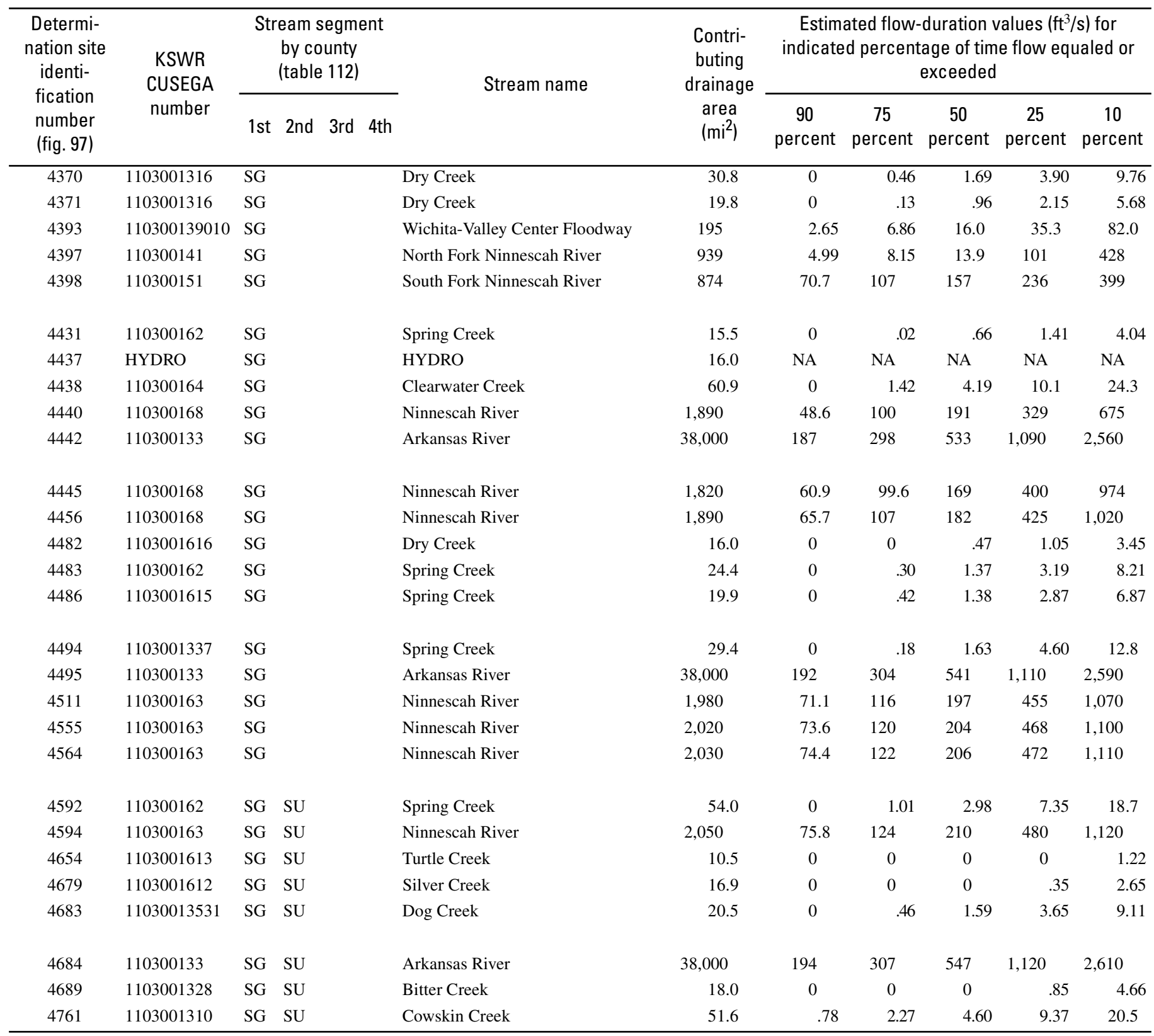


Table 93. Estimated flow-duration values, mean flow values, and peak-discharge frequency values for controlled and uncontrolled flow stream segments on the 1999 Kansas Surface Water Register for Sedgwick County.-Continued

[KSWR, Kansas Surface Water Register; CUSEGA, catalog unit segment number alpha; $\mathrm{mi}^{2}$, square miles; $\mathrm{ft}^{3} / \mathrm{s}$, cubic feet per second; HYDRO, lake or other hydrologic structure; NA, not applicable; NRDitch, irrigation ditch; NRTribal, tribal stream]

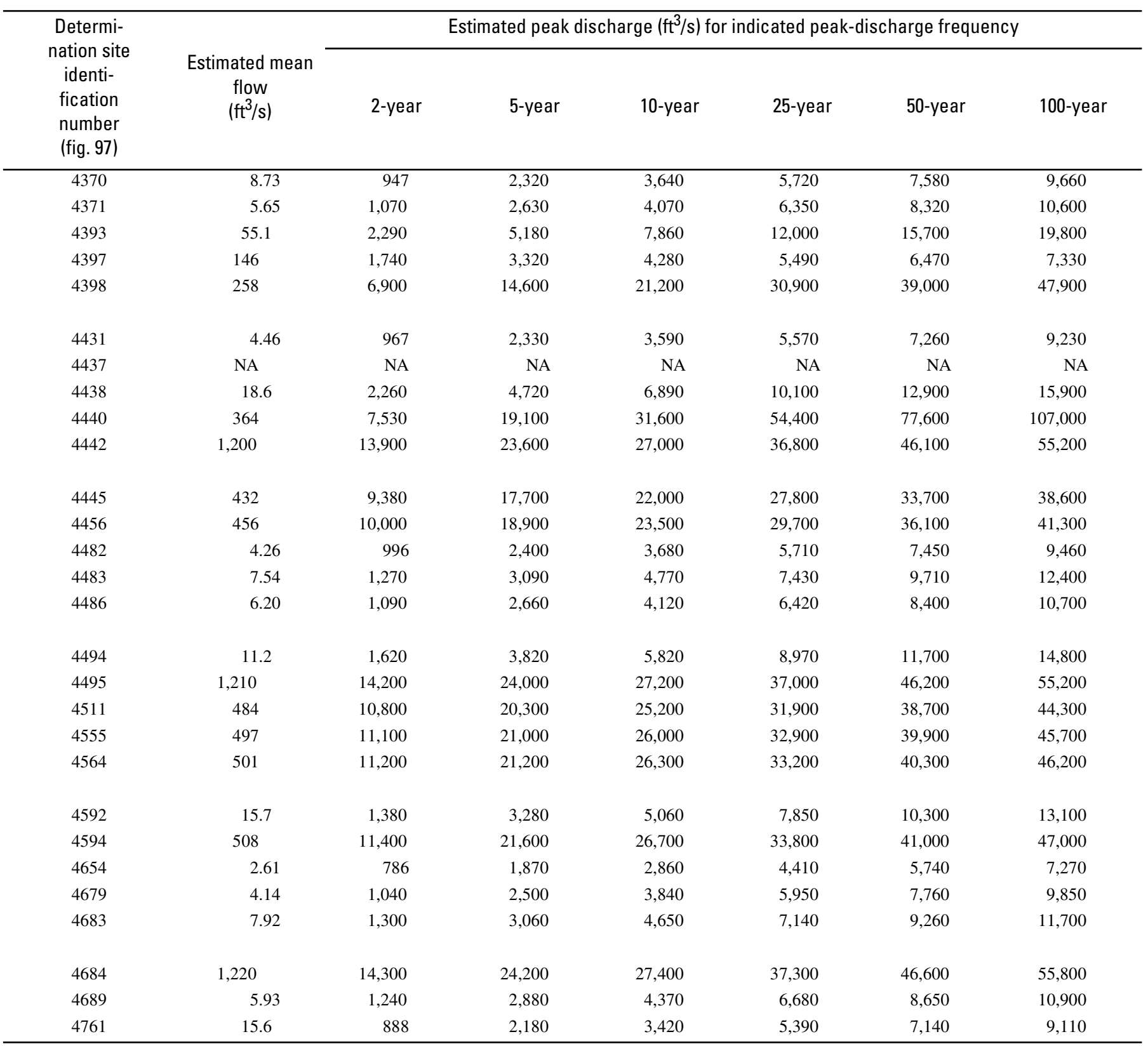




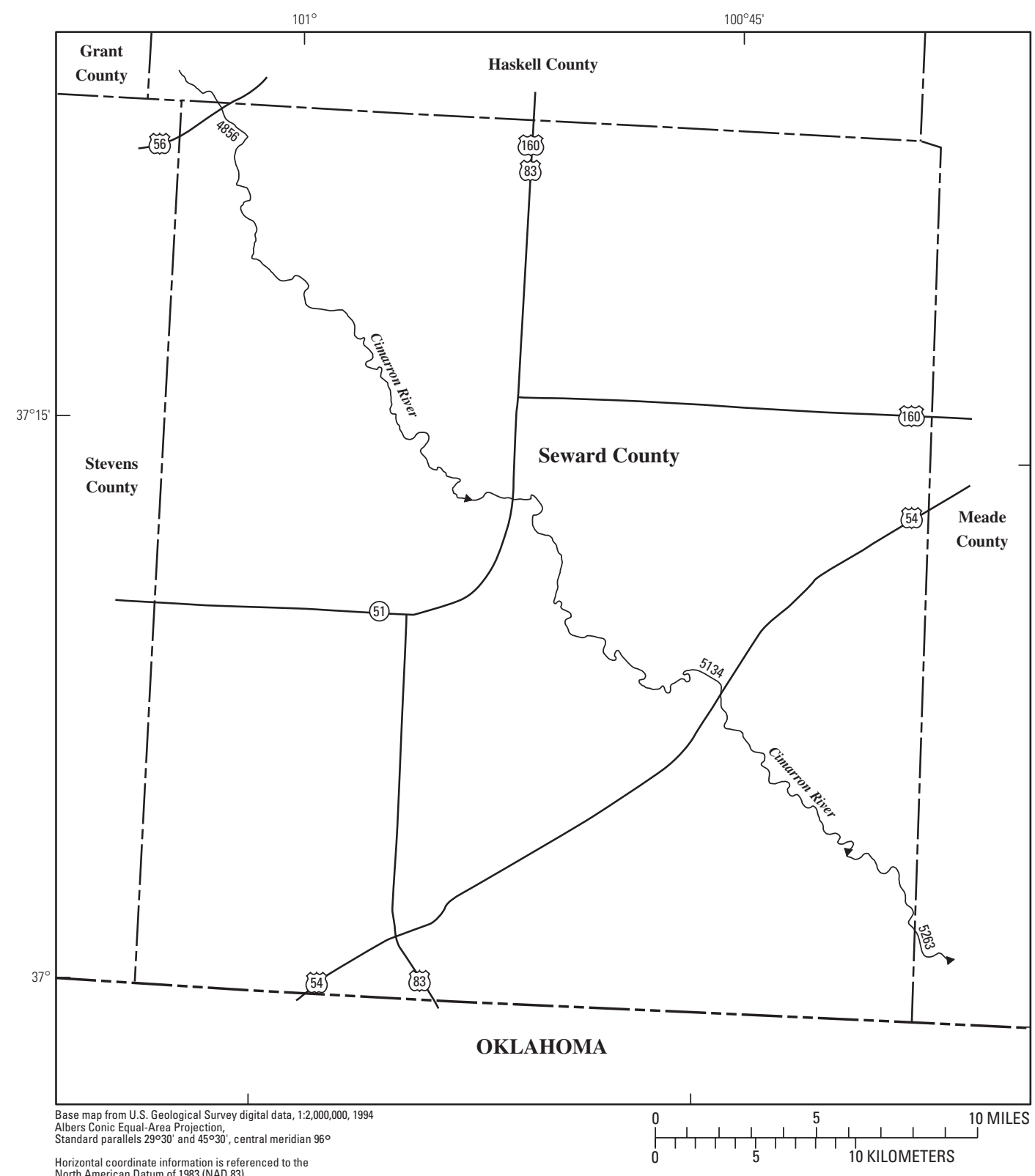

EXPLANATION

$\longleftarrow 5263$ Location of streamflow-statistics determination site (small triangle) and associated identification number-small triangle points in downstream direction

06844900 U.S. Geological Survey streamflow-gaging station and number used for estimates of flow duration

${ }^{06846000} \bigwedge$ U.S. Geological Survey streamflow-gaging station and number used for estimates of peak-discharge frequency values

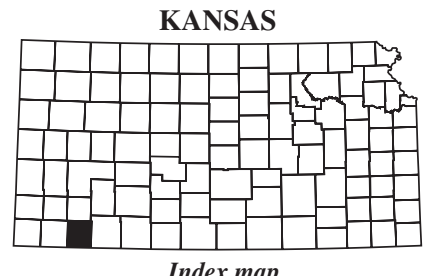

Index map

Lake and determination site identification number

Figure 98. Location of streamflow-statistics determination sites, associated identification numbers, and U.S. Geological Survey streamflow-gaging stations used in the flow-duration and peak-discharge frequency analyses for Seward County. 
Table 94. Estimated flow-duration values, mean flow values, and peak-discharge frequency values for controlled and uncontrolled flow stream segments on the 1999 Kansas Surface Water Register for Seward County.

[KSWR, Kansas Surface Water Register; CUSEGA, catalog unit segment number alpha; $\mathrm{mi}^{2}$, square miles; $\mathrm{ft}^{3} / \mathrm{s}$, cubic feet per second; HYDRO, lake or other hydrologic structure; NA, not applicable; NRDitch, irrigation ditch; NRTribal, tribal stream]

\begin{tabular}{|c|c|c|c|c|c|c|c|c|c|}
\hline $\begin{array}{c}\text { Determi- } \\
\text { nation site } \\
\text { identi- } \\
\text { fication } \\
\text { number } \\
\text { (fig. 98) }\end{array}$ & $\begin{array}{l}\text { KSWR } \\
\text { CUSEGA } \\
\text { number }\end{array}$ & $\begin{array}{l}\text { Stream segment } \\
\text { by county } \\
\text { (table 112) }\end{array}$ & Stream name & $\begin{array}{l}\text { Contri- } \\
\text { buting } \\
\text { drainage } \\
\text { area } \\
\left(\mathrm{mi}^{2}\right)\end{array}$ & \multicolumn{5}{|c|}{$\begin{array}{c}\text { Estimated flow-duration values }\left(\mathrm{ft}^{3} / \mathrm{s}\right) \text { for } \\
\text { indicated percentage of time flow equaled or } \\
\text { exceeded }\end{array}$} \\
\hline 5134 & 110400062 & SW & Cimarron River & 6,660 & 24.8 & 32.0 & 40.9 & 55.6 & 71.9 \\
\hline
\end{tabular}


Table 94. Estimated flow-duration values, mean flow values, and peak-discharge frequency values for controlled and uncontrolled flow stream segments on the 1999 Kansas Surface Water Register for Seward County.-Continued

[KSWR, Kansas Surface Water Register; CUSEGA, catalog unit segment number alpha; $\mathrm{mi}^{2}$, square miles; $\mathrm{ft}^{3} / \mathrm{s}$, cubic feet per second; HYDRO, lake or other hydrologic structure; NA, not applicable; NRDitch, irrigation ditch; NRTribal, tribal stream]

\begin{tabular}{|c|c|c|c|c|c|c|c|}
\hline \multirow{2}{*}{$\begin{array}{l}\text { Determi- } \\
\text { nation site } \\
\text { identi- } \\
\text { fication } \\
\text { number } \\
\text { (fig. 98) }\end{array}$} & \multirow{2}{*}{$\begin{array}{l}\text { Estimated mean } \\
\text { flow } \\
\left(\mathrm{ft}^{3} / \mathrm{s}\right)\end{array}$} & \multicolumn{6}{|c|}{ Estimated peak discharge $\left(\mathrm{ft}^{3} / \mathrm{s}\right)$ for indicated peak-discharge frequency } \\
\hline & & 2-year & 5-year & 10-year & 25-year & 50-year & 100-year \\
\hline 5134 & 52.3 & 2,080 & 6,060 & 10,300 & 17,500 & 24,300 & 32,500 \\
\hline
\end{tabular}




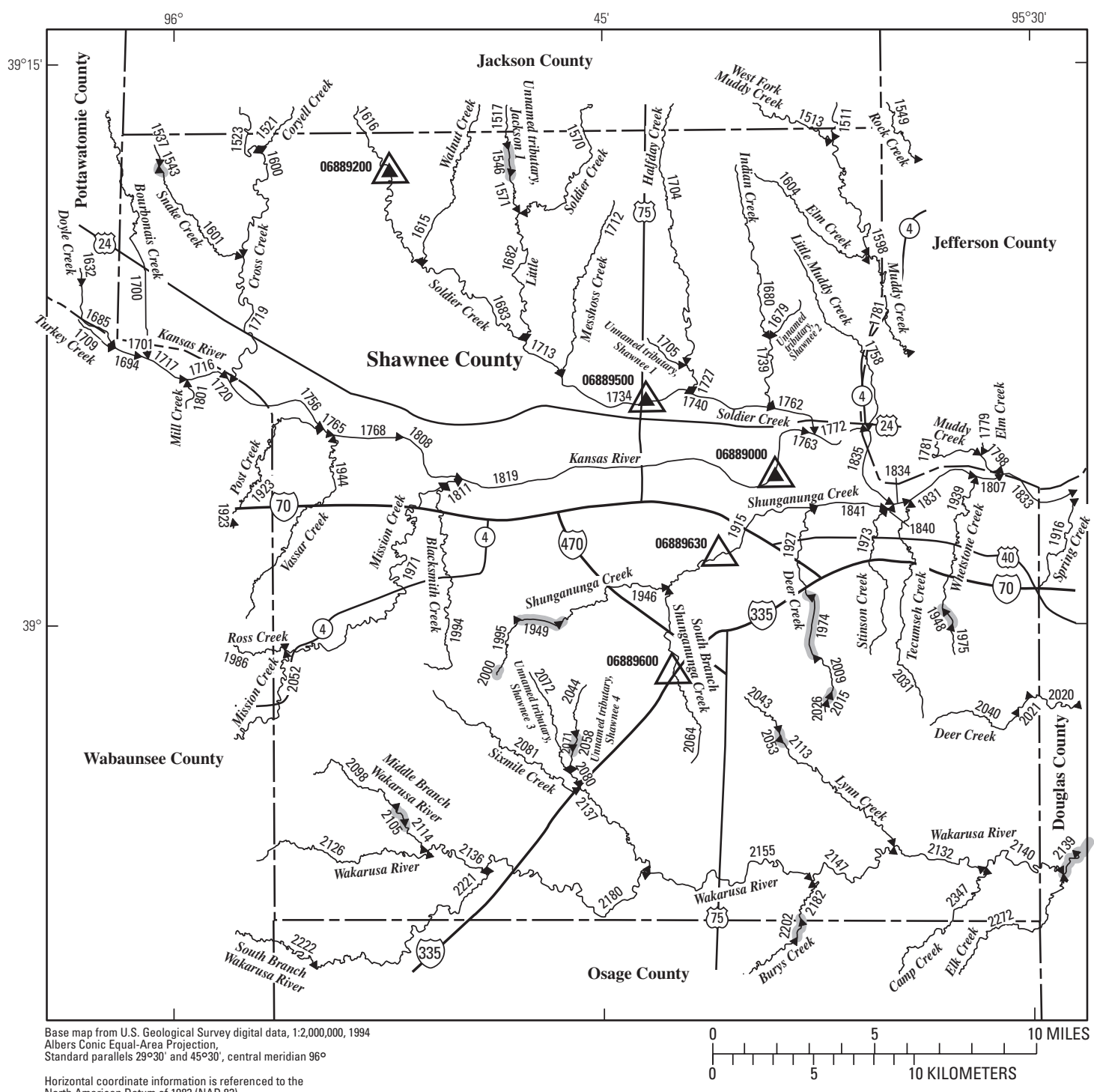

EXPLANATION

ـ222 Location of streamflow-statistics determination site (small triangle) and associated identification number-small triangle points in downstream direction

$06889000 \Delta$ U.S. Geological Survey streamflow-gaging station and number used for estimates of flow duration

$-\infty$

U.S. Geological Survey streamflow-gaging station and number used for estimates of peak-discharge frequency values

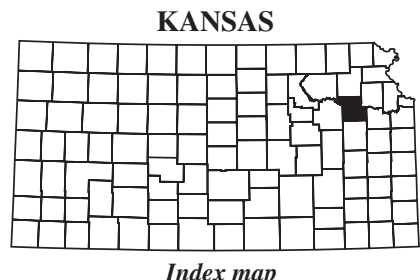

2105 Lake and determination site identification number

Figure 99. Location of streamflow-statistics determination sites, associated identification numbers, and U.S. Geological Survey streamflow-gaging stations used in the flow-duration and peak-discharge frequency analyses for Shawnee County. 
Table 95. Estimated flow-duration values, mean flow values, and peak-discharge frequency values for controlled and uncontrolled flow stream segments on the 1999 Kansas Surface Water Register for Shawnee County.

[KSWR, Kansas Surface Water Register; CUSEGA, catalog unit segment number alpha; $\mathrm{mi}^{2}$, square miles; $\mathrm{ft}^{3} / \mathrm{s}$, cubic feet per second; HYDRO, lake or other hydrologic structure; NA, not applicable; NRDitch, irrigation ditch; NRTribal, tribal stream]

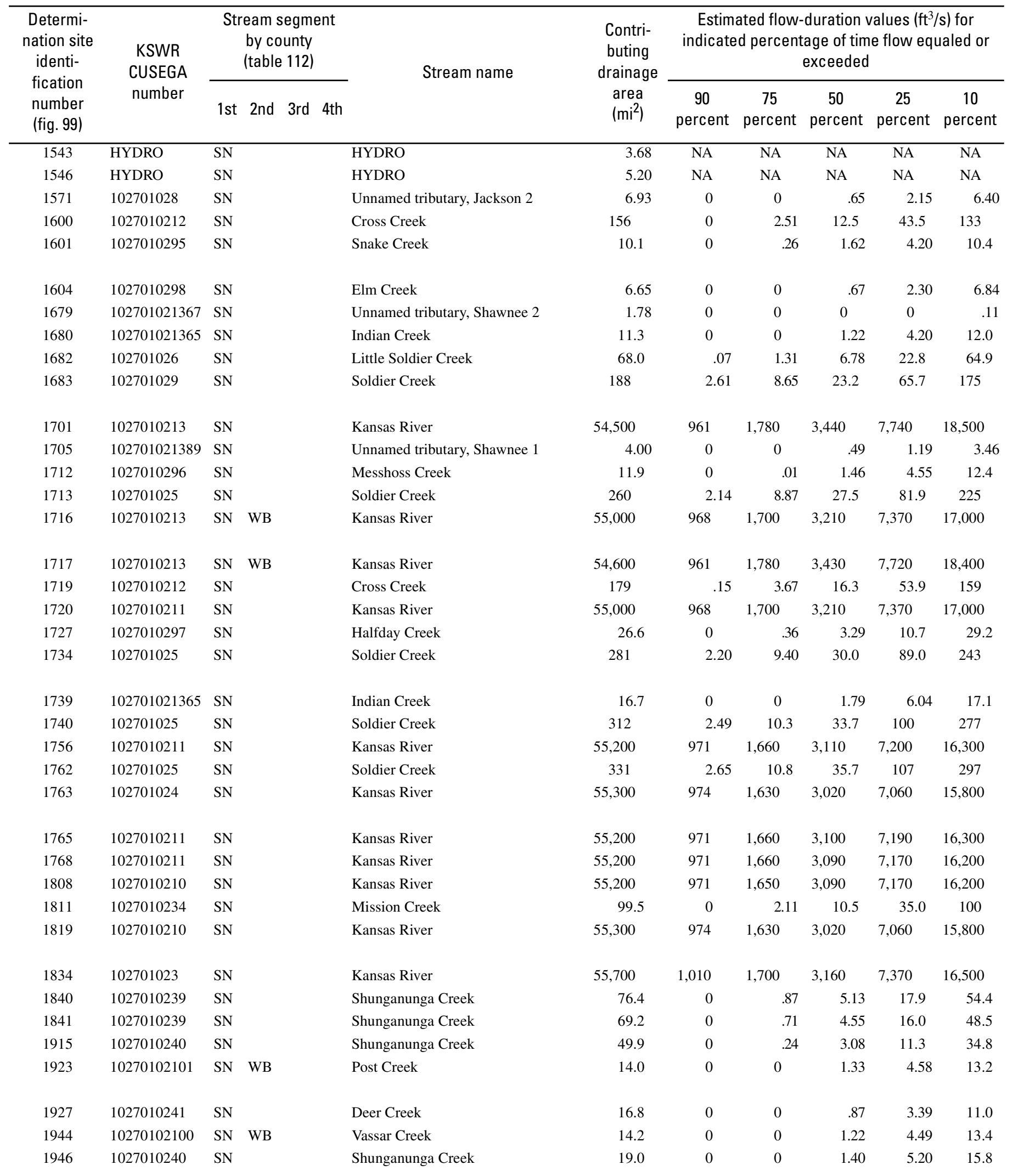


Table 95. Estimated flow-duration values, mean flow values, and peak-discharge frequency values for controlled and uncontrolled flow stream segments on the 1999 Kansas Surface Water Register for Shawnee County.—Continued

[KSWR, Kansas Surface Water Register; CUSEGA, catalog unit segment number alpha; $\mathrm{mi}^{2}$, square miles; $\mathrm{ft}^{3} / \mathrm{s}$, cubic feet per second; HYDRO, lake or other hydrologic structure; NA, not applicable; NRDitch, irrigation ditch; NRTribal, tribal stream]

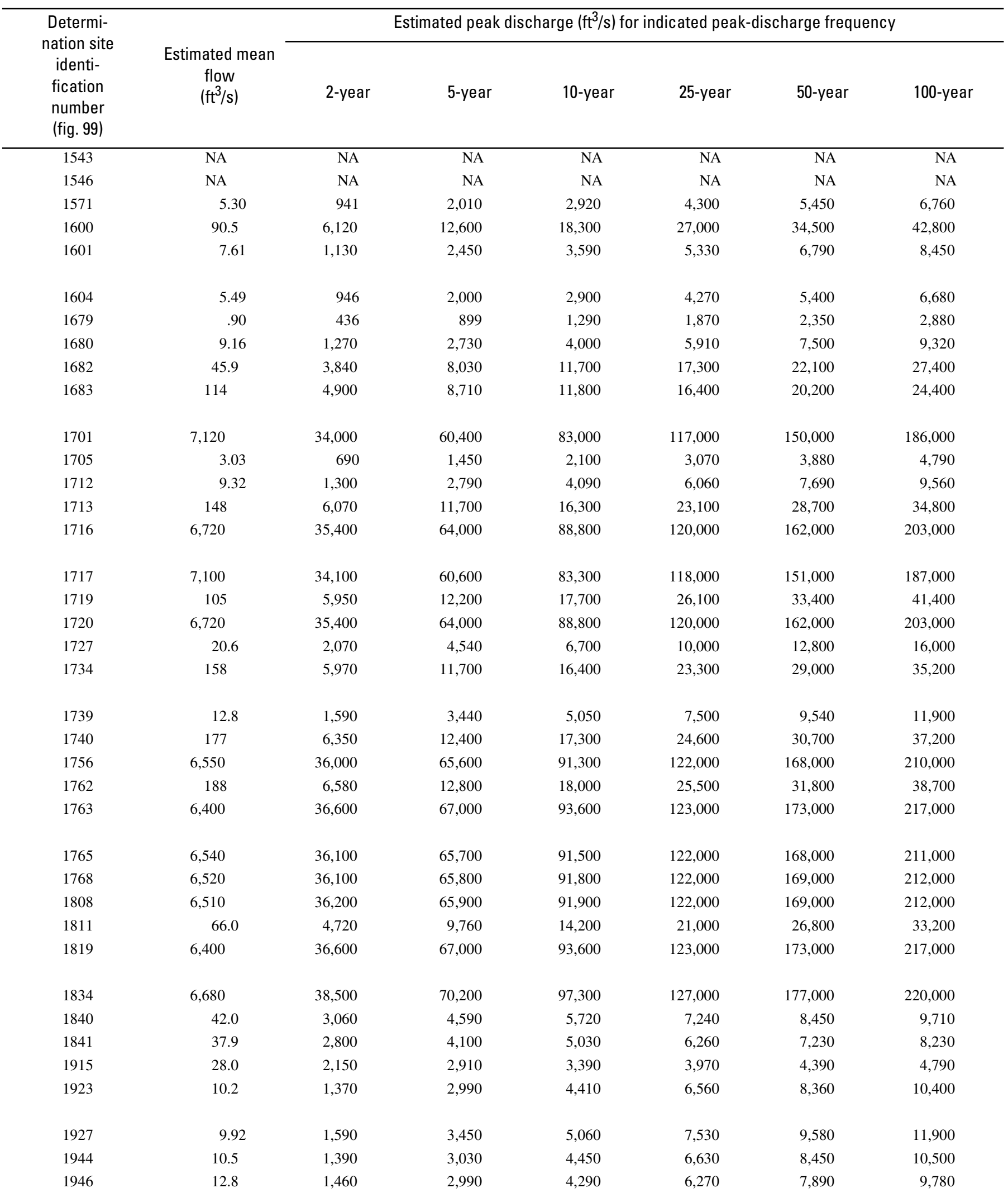


Table 95. Estimated flow-duration values, mean flow values, and peak-discharge frequency values for controlled and uncontrolled flow stream segments on the 1999 Kansas Surface Water Register for Shawnee County.-Continued

[KSWR, Kansas Surface Water Register; CUSEGA, catalog unit segment number alpha; $\mathrm{mi}^{2}$, square miles; $\mathrm{ft}^{3} / \mathrm{s}$, cubic feet per second; HYDRO, lake or other hydrologic structure; NA, not applicable; NRDitch, irrigation ditch; NRTribal, tribal stream]

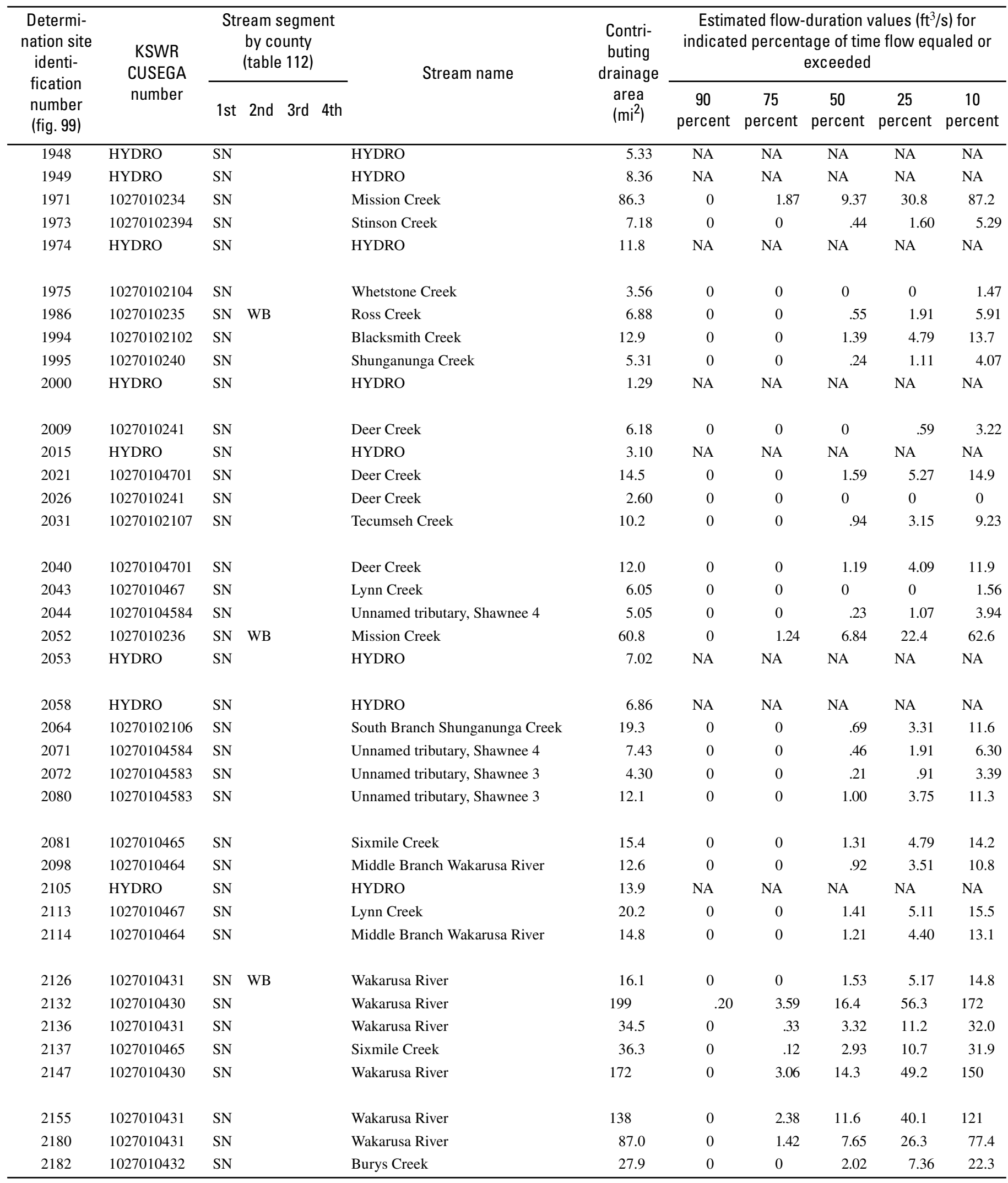


Table 95. Estimated flow-duration values, mean flow values, and peak-discharge frequency values for controlled and uncontrolled flow stream segments on the 1999 Kansas Surface Water Register for Shawnee County.-Continued

[KSWR, Kansas Surface Water Register; CUSEGA, catalog unit segment number alpha; $\mathrm{mi}^{2}$, square miles; $\mathrm{ft}^{3} / \mathrm{s}$, cubic feet per second; HYDRO, lake or other hydrologic structure; NA, not applicable; NRDitch, irrigation ditch; NRTribal, tribal stream]

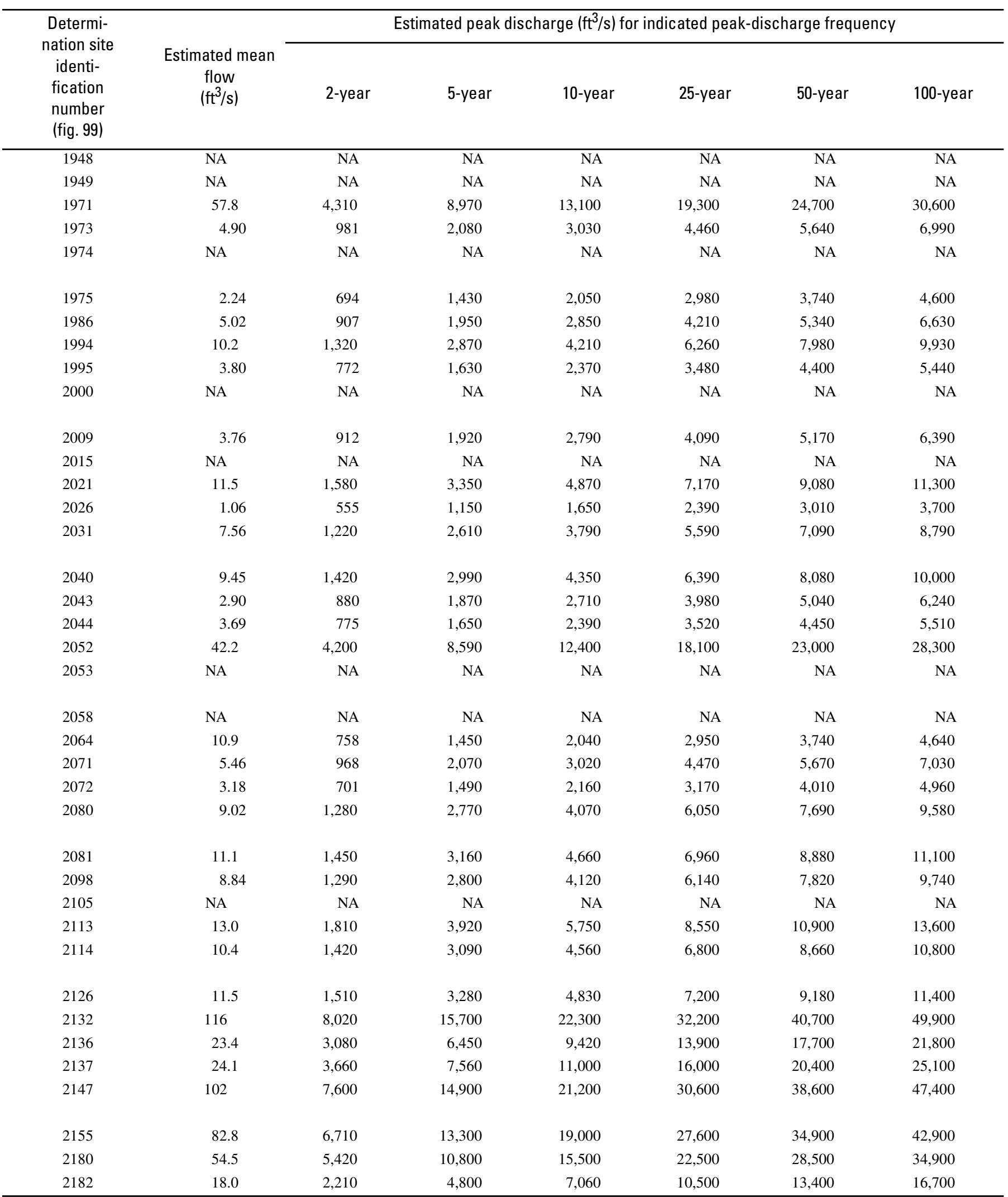




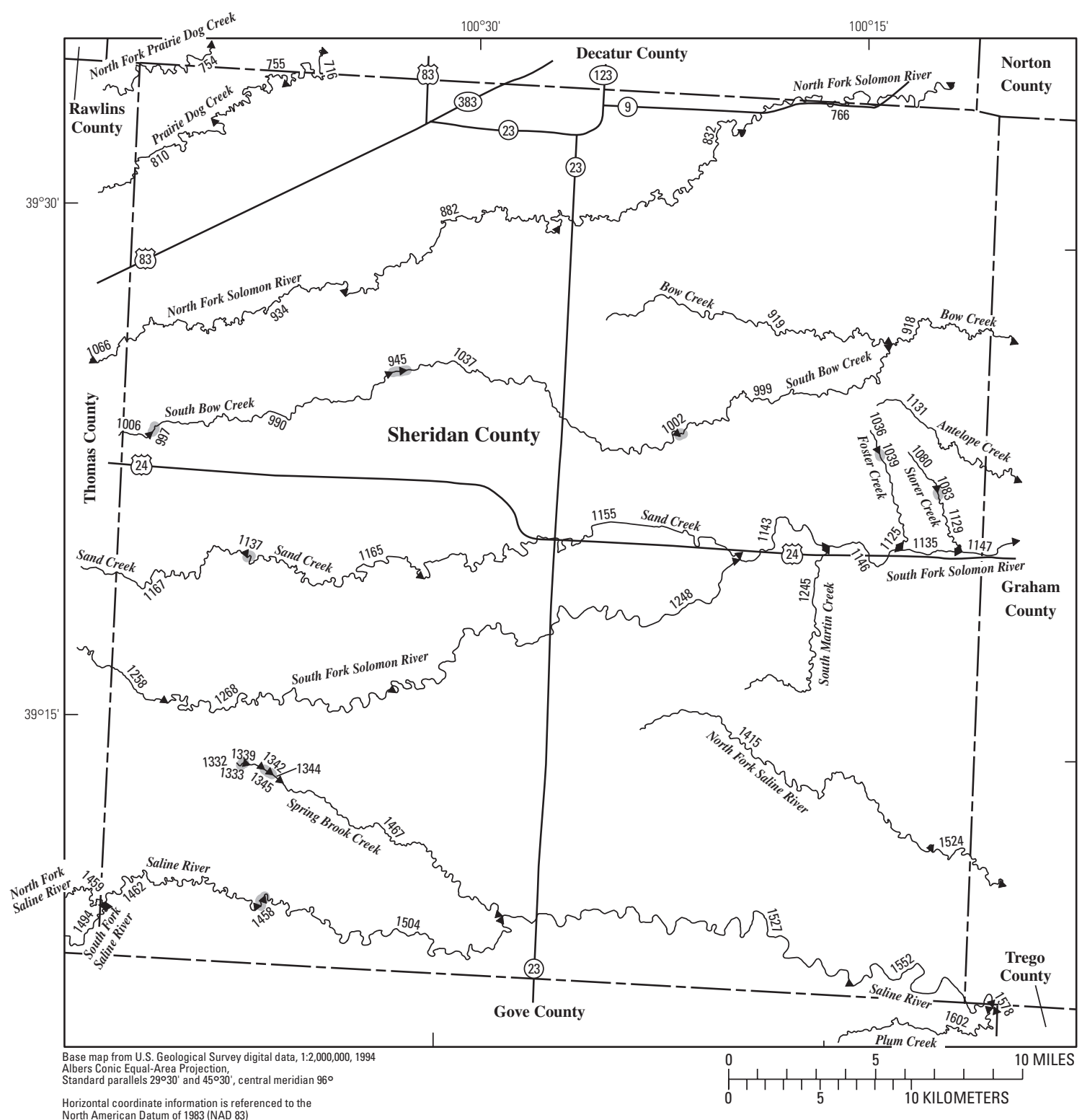

EXPLANATION

1655 Location of streamflow-statistics determination site (small triangle) and associated identification number-small triangle points in downstream direction

${ }_{06844900}^{\Delta}$ U.S. Geological Survey streamflow-gaging station and number used for estimates of flow duration

${ }^{06846000} \triangle$ U.S. Geological Survey streamflow-gaging station and number used for estimates of peak-discharge frequency values

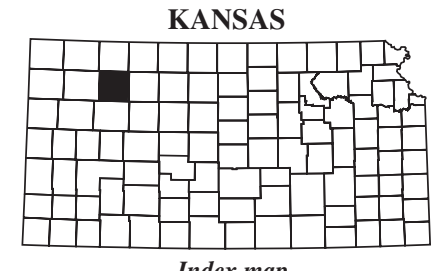

Lake and determination site identification number

Figure 100. Location of streamflow-statistics determination sites, associated identification numbers, and U.S. Geological Survey streamflow-gaging stations used in the flow-duration and peak-discharge frequency analyses for Sheridan County. 
Table 96. Estimated flow-duration values, mean flow values, and peak-discharge frequency values for controlled and uncontrolled flow stream segments on the 1999 Kansas Surface Water Register for Sheridan County.

[KSWR, Kansas Surface Water Register; CUSEGA, catalog unit segment number alpha; $\mathrm{mi}^{2}$, square miles; $\mathrm{ft}^{3} / \mathrm{s}$, cubic feet per second; HYDRO, lake or other hydrologic structure; NA, not applicable; NRDitch, irrigation ditch; NRTribal, tribal stream]

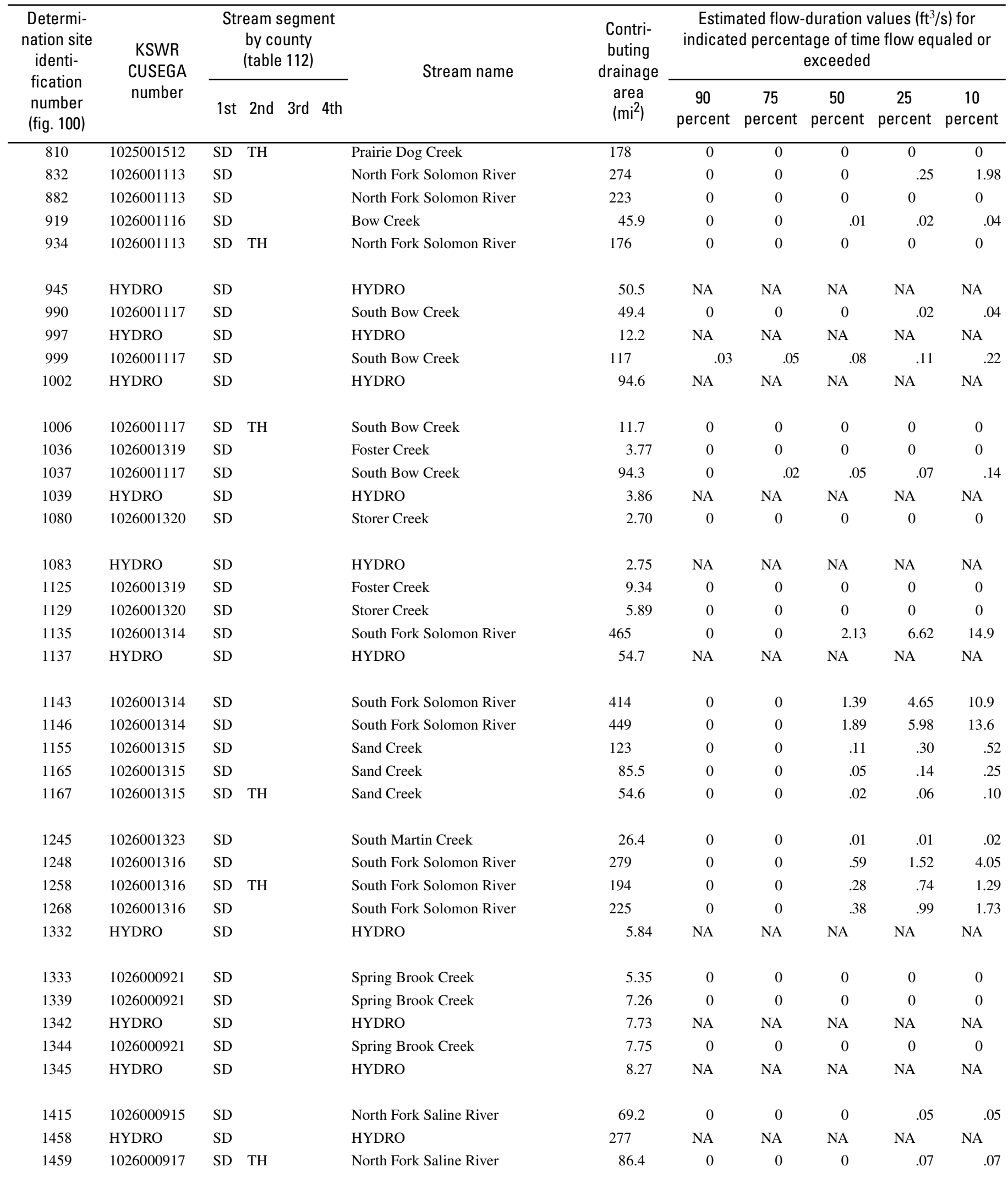


Table 96. Estimated flow-duration values, mean flow values, and peak-discharge frequency values for controlled and uncontrolled flow stream segments on the 1999 Kansas Surface Water Register for Sheridan County.-Continued

[KSWR, Kansas Surface Water Register; CUSEGA, catalog unit segment number alpha; $\mathrm{mi}^{2}$, square miles; $\mathrm{ft}^{3} / \mathrm{s}$, cubic feet per second; HYDRO, lake or other hydrologic structure; NA, not applicable; NRDitch, irrigation ditch; NRTribal, tribal stream]

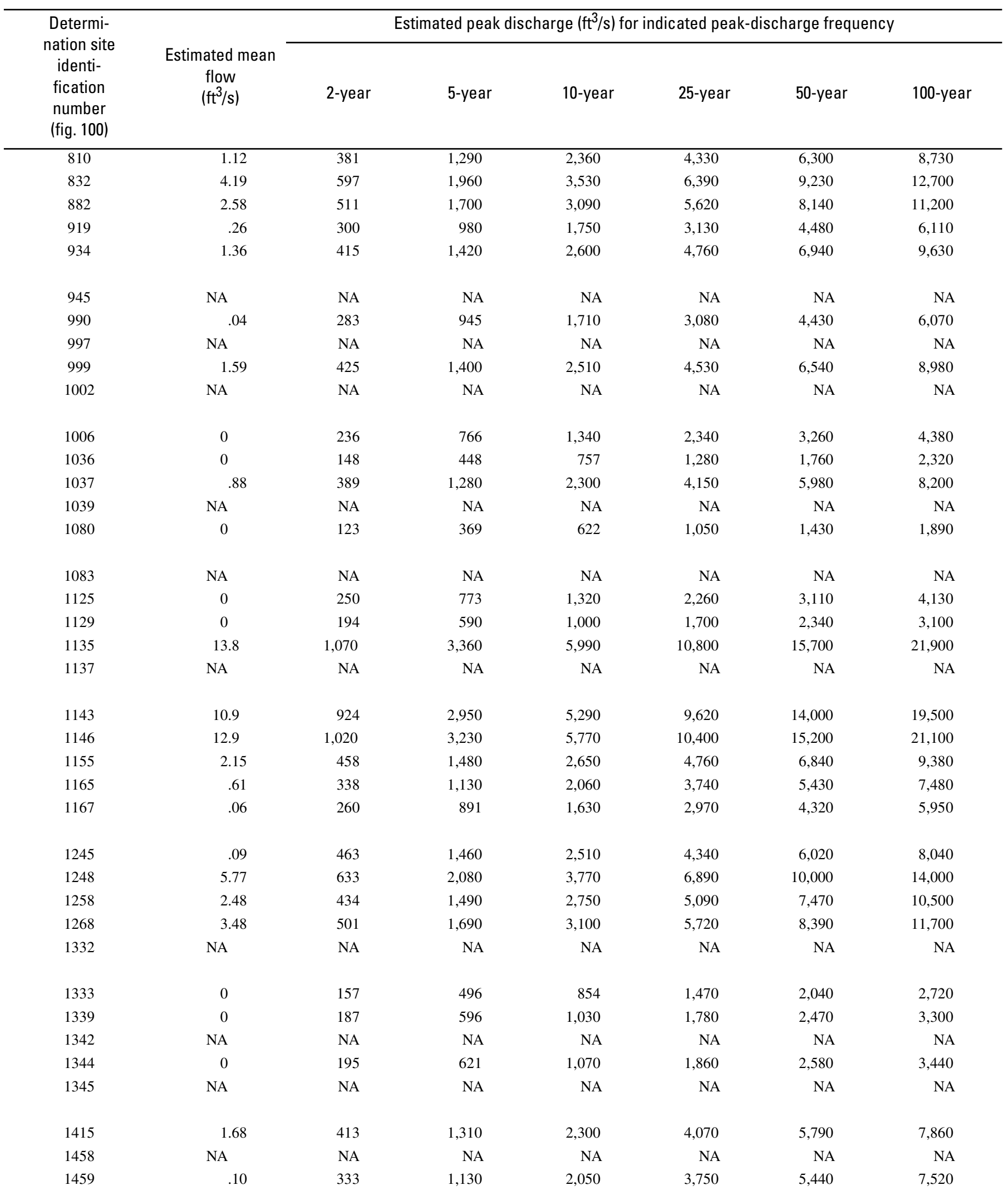




\section{Estimates of Flow Duration, Mean Flow, and Peak-Discharge Frequency Values for Kansas Stream Locations}

Table 96. Estimated flow-duration values, mean flow values, and peak-discharge frequency values for controlled and uncontrolled flow stream segments on the 1999 Kansas Surface Water Register for Sheridan County.-Continued

[KSWR, Kansas Surface Water Register; CUSEGA, catalog unit segment number alpha; $\mathrm{mi}^{2}$, square miles; $\mathrm{ft}^{3} / \mathrm{s}$, cubic feet per second; HYDRO, lake or other hydrologic structure; NA, not applicable; NRDitch, irrigation ditch; NRTribal, tribal stream]

\begin{tabular}{|c|c|c|c|c|c|c|c|c|c|c|c|}
\hline $\begin{array}{l}\text { Determi- } \\
\text { nation site } \\
\text { identi- } \\
\text { fication } \\
\text { number } \\
\text { (fig. 100) }\end{array}$ & $\begin{array}{l}\text { KSWR } \\
\text { CUSEGA } \\
\text { number }\end{array}$ & \multicolumn{3}{|c|}{$\begin{array}{l}\text { Stream segment } \\
\text { by county } \\
\text { (table 112) }\end{array}$} & Stream name & $\begin{array}{l}\text { Contri- } \\
\text { buting } \\
\text { drainage } \\
\text { area } \\
\left(\mathrm{mi}^{2}\right)\end{array}$ & \multicolumn{5}{|c|}{$\begin{array}{c}\text { Estimated flow-duration values }\left(\mathrm{ft}^{3} / \mathrm{s}\right) \text { for } \\
\text { indicated percentage of time flow equaled or } \\
\text { exceeded }\end{array}$} \\
\hline 1462 & 1026000916 & SD & & & Saline River & 277 & 0 & 0 & 0.05 & 0.75 & 1.50 \\
\hline 1504 & 1026000916 & $\mathrm{SD}$ & & & Saline River & 319 & 0 & 0 & .06 & .99 & 2.14 \\
\hline 1527 & 1026000916 & SD & & & Saline River & 439 & 0 & 0 & .27 & 3.30 & 7.28 \\
\hline
\end{tabular}


Table 96. Estimated flow-duration values, mean flow values, and peak-discharge frequency values for controlled and uncontrolled flow stream segments on the 1999 Kansas Surface Water Register for Sheridan County.-Continued

[KSWR, Kansas Surface Water Register; CUSEGA, catalog unit segment number alpha; $\mathrm{mi}^{2}$, square miles; $\mathrm{ft}^{3} / \mathrm{s}$, cubic feet per second; HYDRO, lake or other hydrologic structure; NA, not applicable; NRDitch, irrigation ditch; NRTribal, tribal stream]

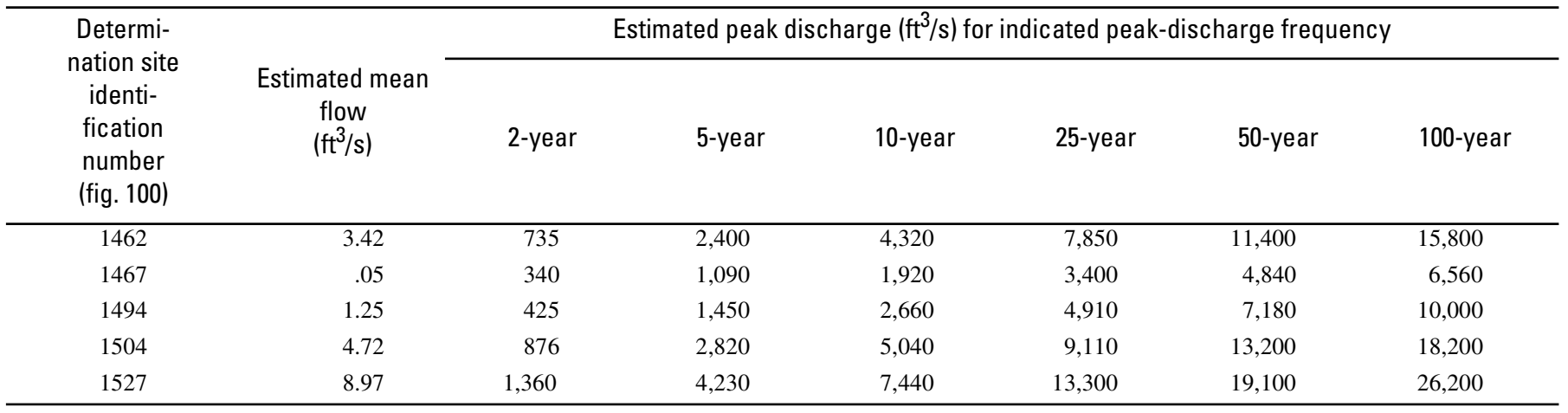




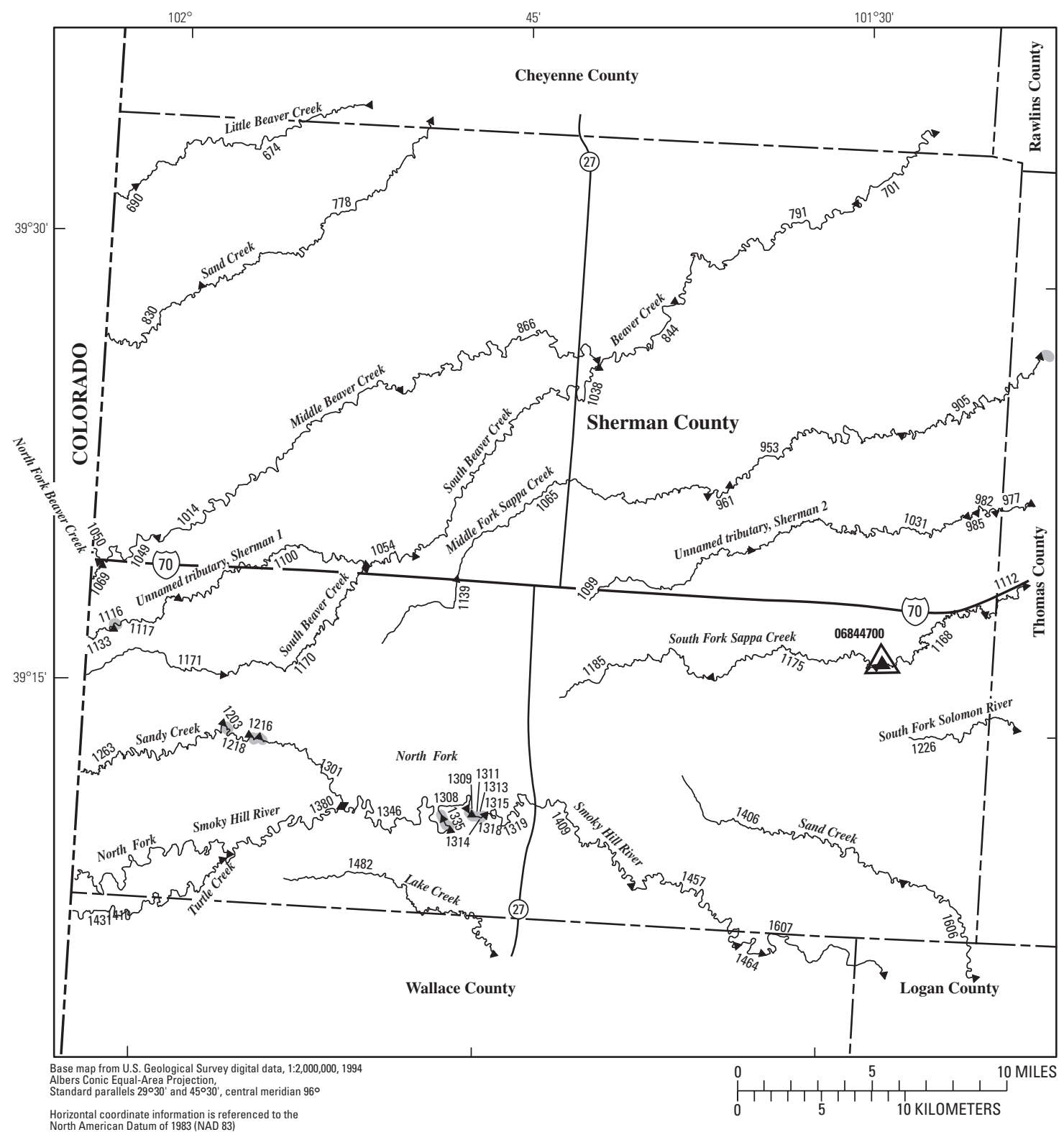

EXPLANATION

«1431 Location of streamflow-statistics determination site (small triangle) and associated identification number-small triangle points in downstream direction

06844700

U.S. Geological Survey streamflow-gaging station and number used for estimates of flow duration

06844700

U.S. Geological Survey streamflow-gaging station and number used for estimates of peak-discharge frequency values

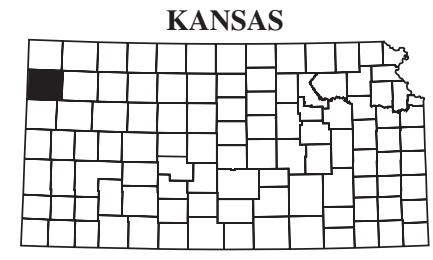

Index map

1216 Lake and determination site identification number

Figure 101. Location of streamflow-statistics determination sites, associated identification numbers, and U.S. Geological Survey streamflow-gaging stations used in the flow-duration and peak-discharge frequency analyses for Sherman County. 
Table 97. Estimated flow-duration values, mean flow values, and peak-discharge frequency values for controlled and uncontrolled flow stream segments on the 1999 Kansas Surface Water Register for Sherman County.

[KSWR, Kansas Surface Water Register; CUSEGA, catalog unit segment number alpha; $\mathrm{mi}^{2}$, square miles; $\mathrm{ft}^{3} / \mathrm{s}$, cubic feet per second; HYDRO, lake or other hydrologic structure; NA, not applicable; NRDitch, irrigation ditch; NRTribal, tribal stream]

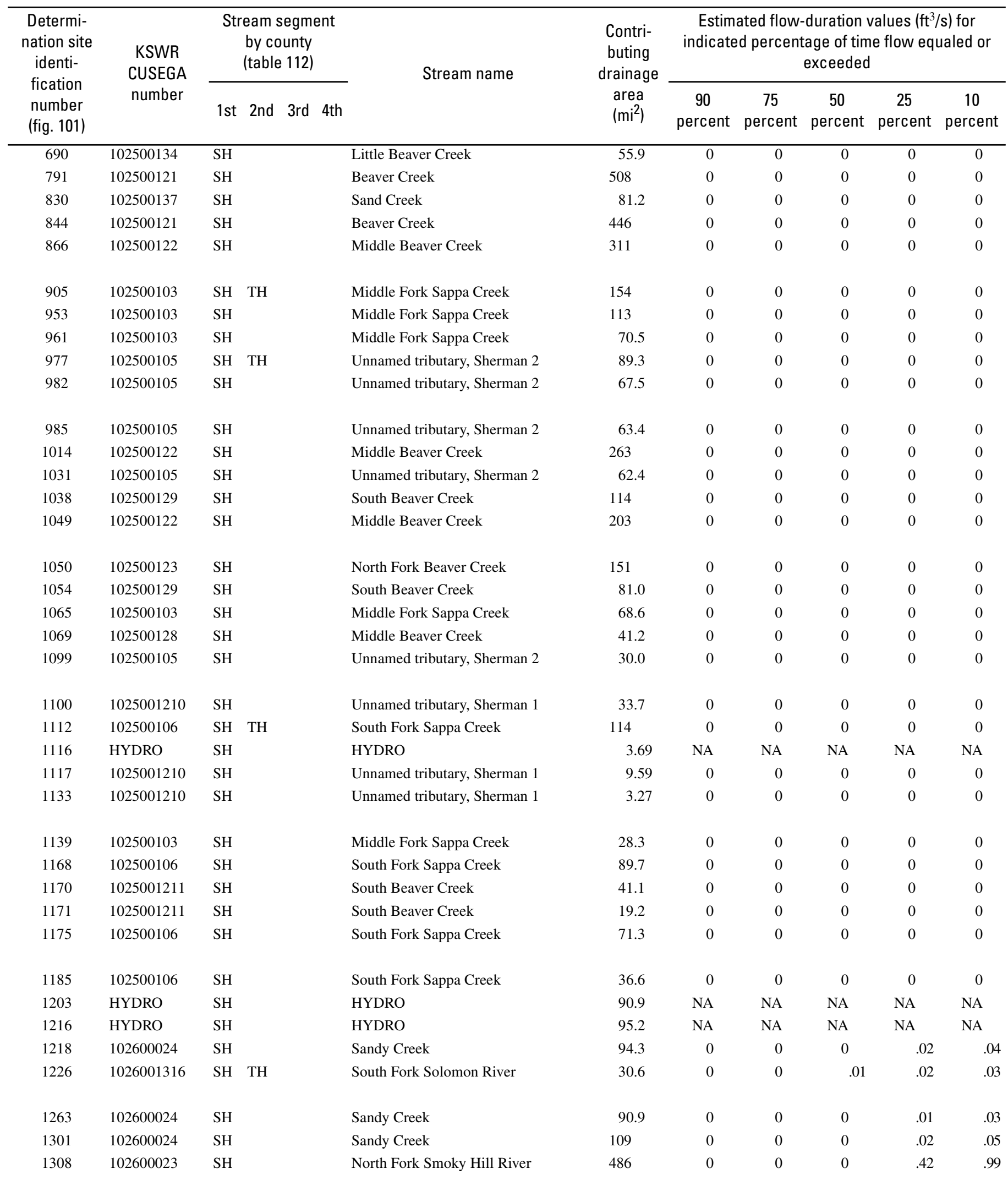


Table 97. Estimated flow-duration values, mean flow values, and peak-discharge frequency values for controlled and uncontrolled flow stream segments on the 1999 Kansas Surface Water Register for Sherman County.-Continued

[KSWR, Kansas Surface Water Register; CUSEGA, catalog unit segment number alpha; $\mathrm{mi}^{2}$, square miles; $\mathrm{ft}^{3} / \mathrm{s}$, cubic feet per second; HYDRO, lake or other hydrologic structure; NA, not applicable; NRDitch, irrigation ditch; NRTribal, tribal stream]

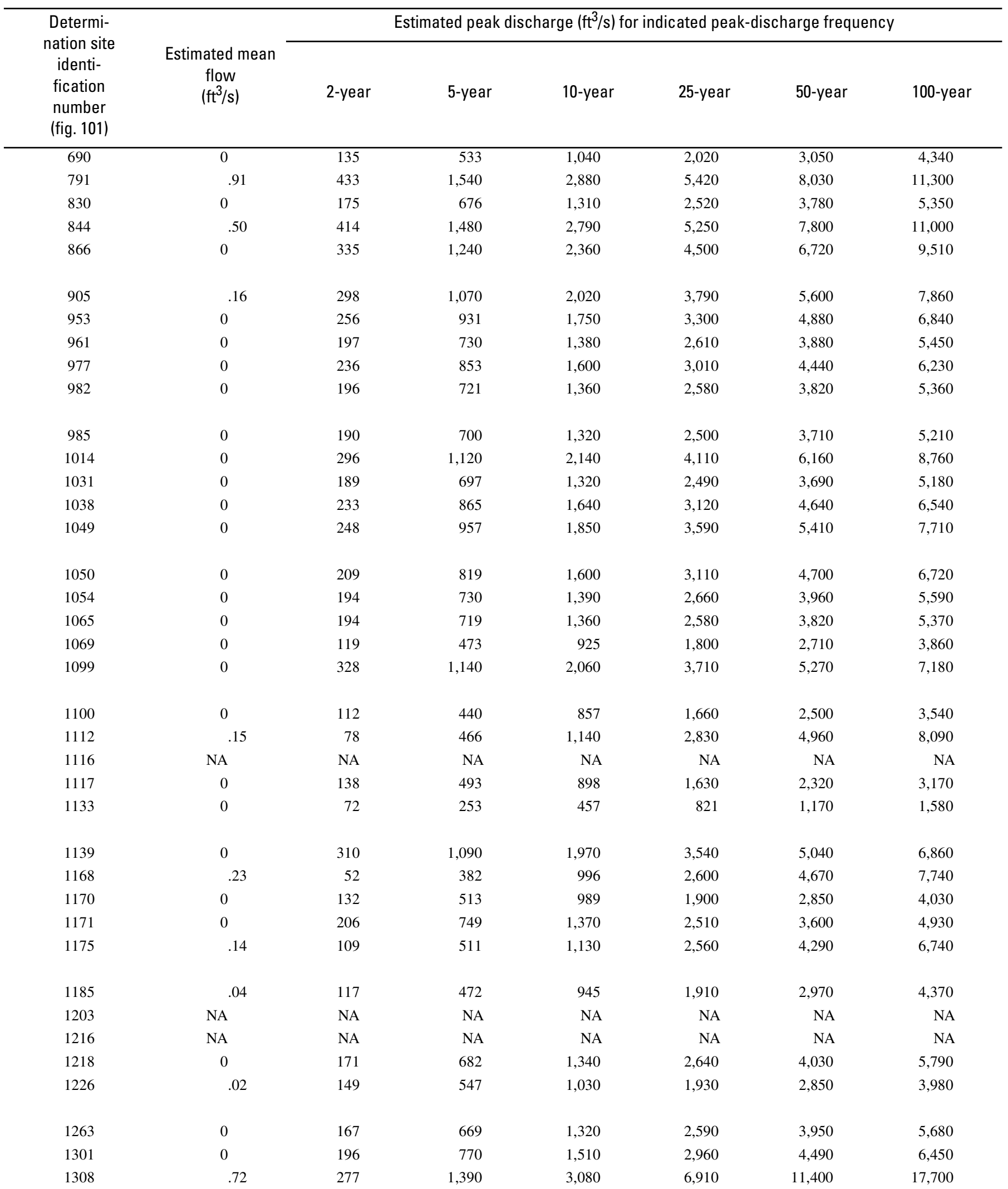


Table 97. Estimated flow-duration values, mean flow values, and peak-discharge frequency values for controlled and uncontrolled flow stream segments on the 1999 Kansas Surface Water Register for Sherman County.-Continued

[KSWR, Kansas Surface Water Register; CUSEGA, catalog unit segment number alpha; $\mathrm{mi}^{2}$, square miles; $\mathrm{ft}^{3} / \mathrm{s}$, cubic feet per second; HYDRO, lake or other hydrologic structure; NA, not applicable; NRDitch, irrigation ditch; NRTribal, tribal stream]

\begin{tabular}{|c|c|c|c|c|c|c|c|c|c|c|c|}
\hline $\begin{array}{l}\text { Determi- } \\
\text { nation site } \\
\text { identi- } \\
\text { fication } \\
\text { number } \\
\text { (fig. 101) }\end{array}$ & $\begin{array}{l}\text { KSWR } \\
\text { CUSEGA } \\
\text { number }\end{array}$ & \multicolumn{3}{|c|}{$\begin{array}{l}\text { Stream segment } \\
\text { by county } \\
\text { (table 112) }\end{array}$} & Stream name & $\begin{array}{l}\text { Contri- } \\
\text { buting } \\
\text { drainage } \\
\text { area } \\
\left(\mathrm{mi}^{2}\right)\end{array}$ & \multicolumn{5}{|c|}{$\begin{array}{c}\text { Estimated flow-duration values }\left(\mathrm{ft}^{3} / \mathrm{s}\right) \text { for } \\
\text { indicated percentage of time flow equaled or } \\
\text { exceeded }\end{array}$} \\
\hline 1309 & HYDRO & SH & & & HYDRO & 486 & NA & NA & NA & NA & NA \\
\hline 1314 & HYDRO & $\mathrm{SH}$ & & & HYDRO & 487 & NA & NA & NA & NA & NA \\
\hline 1315 & HYDRO & $\mathrm{SH}$ & & & HYDRO & 487 & NA & NA & NA & NA & NA \\
\hline 1318 & 102600023 & SH & & & North Fork Smoky Hill River & 487 & 0 & 0 & 0 & .42 & .99 \\
\hline 1319 & HYDRO & $\mathrm{SH}$ & & & HYDRO & 487 & NA & NA & NA & NA & NA \\
\hline 1406 & 102600022 & $\mathrm{SH}$ & & & Sand Creek & 53.0 & 0 & 0 & 0 & 0 & .01 \\
\hline 1409 & 102600023 & $\mathrm{SH}$ & & & North Fork Smoky Hill River & 526 & 0 & 0 & 0 & .49 & 1.15 \\
\hline 1410 & 102600026 & $\mathrm{SH}$ & & & North Fork Smoky Hill River & 281 & 0 & 0 & 0 & .14 & .33 \\
\hline 1431 & 1026000215 & $\mathrm{SH}$ & WA & & Turtle Creek & 55.9 & 0 & 0 & 0 & .01 & .01 \\
\hline 1457 & 102600023 & $\mathrm{SH}$ & WA & & North Fork Smoky Hill River & 549 & 0 & 0 & 0 & .53 & 1.26 \\
\hline 1464 & 102600023 & SH & WA & & North Fork Smoky Hill River & 553 & 0 & 0 & 0 & .54 & 1.28 \\
\hline 1482 & 102600012 & SH & WA & & Lake Creek & 45.2 & 0 & 0 & 0 & 0 & 0 \\
\hline
\end{tabular}


Table 97. Estimated flow-duration values, mean flow values, and peak-discharge frequency values for controlled and uncontrolled flow stream segments on the 1999 Kansas Surface Water Register for Sherman County.-Continued

[KSWR, Kansas Surface Water Register; CUSEGA, catalog unit segment number alpha; $\mathrm{mi}^{2}$, square miles; $\mathrm{ft}^{3} / \mathrm{s}$, cubic feet per second; HYDRO, lake or other hydrologic structure; NA, not applicable; NRDitch, irrigation ditch; NRTribal, tribal stream]

\begin{tabular}{|c|c|c|c|c|c|c|c|}
\hline \multirow{2}{*}{$\begin{array}{l}\text { Determi- } \\
\text { nation site } \\
\text { identi- } \\
\text { fication } \\
\text { number } \\
\text { (fig. 101) }\end{array}$} & \multirow{2}{*}{$\begin{array}{c}\text { Estimated mean } \\
\text { flow } \\
\left(\mathrm{ft}^{3} / \mathrm{s}\right)\end{array}$} & \multicolumn{6}{|c|}{ Estimated peak discharge $\left(\mathrm{ft}^{3} / \mathrm{s}\right)$ for indicated peak-discharge frequency } \\
\hline & & 2-year & 5-year & 10-year & 25-year & 50-year & 100-year \\
\hline 1309 & NA & NA & NA & NA & NA & NA & NA \\
\hline 1311 & 0.72 & 277 & 1,390 & 3,080 & 6,910 & 11,400 & 17,700 \\
\hline 1313 & .72 & 276 & 1,390 & 3,080 & 6,920 & 11,400 & 17,700 \\
\hline 1314 & NA & NA & NA & NA & NA & NA & NA \\
\hline 1315 & NA & NA & NA & NA & NA & NA & NA \\
\hline 1318 & .72 & 276 & 1,390 & 3,080 & 6,920 & 11,500 & 17,700 \\
\hline 1319 & NA & NA & NA & NA & NA & NA & NA \\
\hline 1335 & NA & NA & NA & NA & NA & NA & NA \\
\hline 1346 & .65 & 277 & 1,380 & 3,050 & 6,820 & 11,300 & 17,400 \\
\hline 1380 & 0 & 253 & 1,150 & 2,430 & 5,210 & 8,390 & 12,700 \\
\hline 1406 & 0 & 210 & 753 & 1,410 & 2,620 & 3,860 & 5,390 \\
\hline 1409 & 1.12 & 286 & 1,470 & 3,290 & 7,480 & 12,500 & 19,400 \\
\hline 1410 & 0 & 228 & 1,000 & 2,090 & 4,380 & 6,960 & 10,400 \\
\hline 1431 & 0 & 131 & 522 & 1,020 & 2,000 & 3,020 & 4,310 \\
\hline 1457 & 1.38 & 287 & 1,510 & 3,400 & 7,780 & 13,000 & 20,300 \\
\hline 1464 & 1.43 & 288 & 1,520 & 3,420 & 7,840 & 13,100 & 20,500 \\
\hline 1482 & 0 & 192 & 689 & 1,280 & 2,390 & 3,510 & 4,880 \\
\hline
\end{tabular}




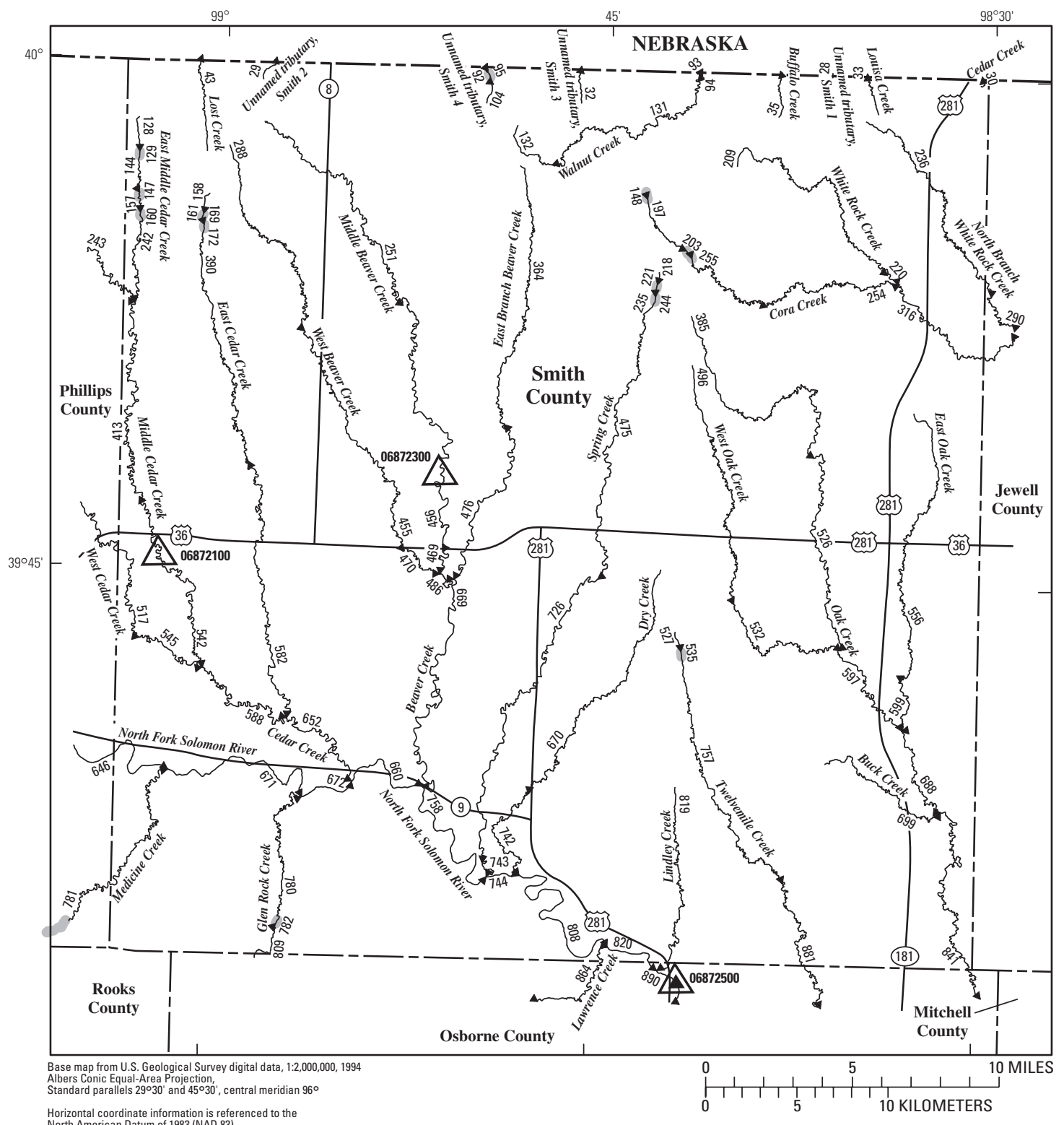

EXPLANATION

$\stackrel{865}{\longleftarrow}$ Location of streamflow-statistics determination site (small triangle) and associated identification number — small triangle points in downstream direction

06872500

$\Delta$ U.S. Geological Survey streamflow-gaging station and number used for estimates of flow duration

06872500 U.S. Geological Survey streamflow-gaging station and number used for estimates of peak-discharge frequency values

Lake and determination site identification number

KANSAS

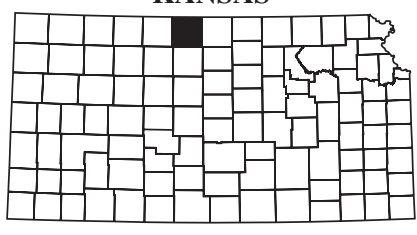

Index map

Figure 102. Location of streamflow-statistics determination sites, associated identification numbers, and U.S. Geological Survey streamflow-gaging stations used in the flow-duration and peak-discharge frequency analyses for Smith County. 
Table 98. Estimated flow-duration values, mean flow values, and peak-discharge frequency values for controlled and uncontrolled flow stream segments on the 1999 Kansas Surface Water Register for Smith County.

[KSWR, Kansas Surface Water Register; CUSEGA, catalog unit segment number alpha; $\mathrm{mi}^{2}$, square miles; $\mathrm{ft}^{3} / \mathrm{s}$, cubic feet per second; HYDRO, lake or other hydrologic structure; NA, not applicable; NRDitch, irrigation ditch; NRTribal, tribal stream]

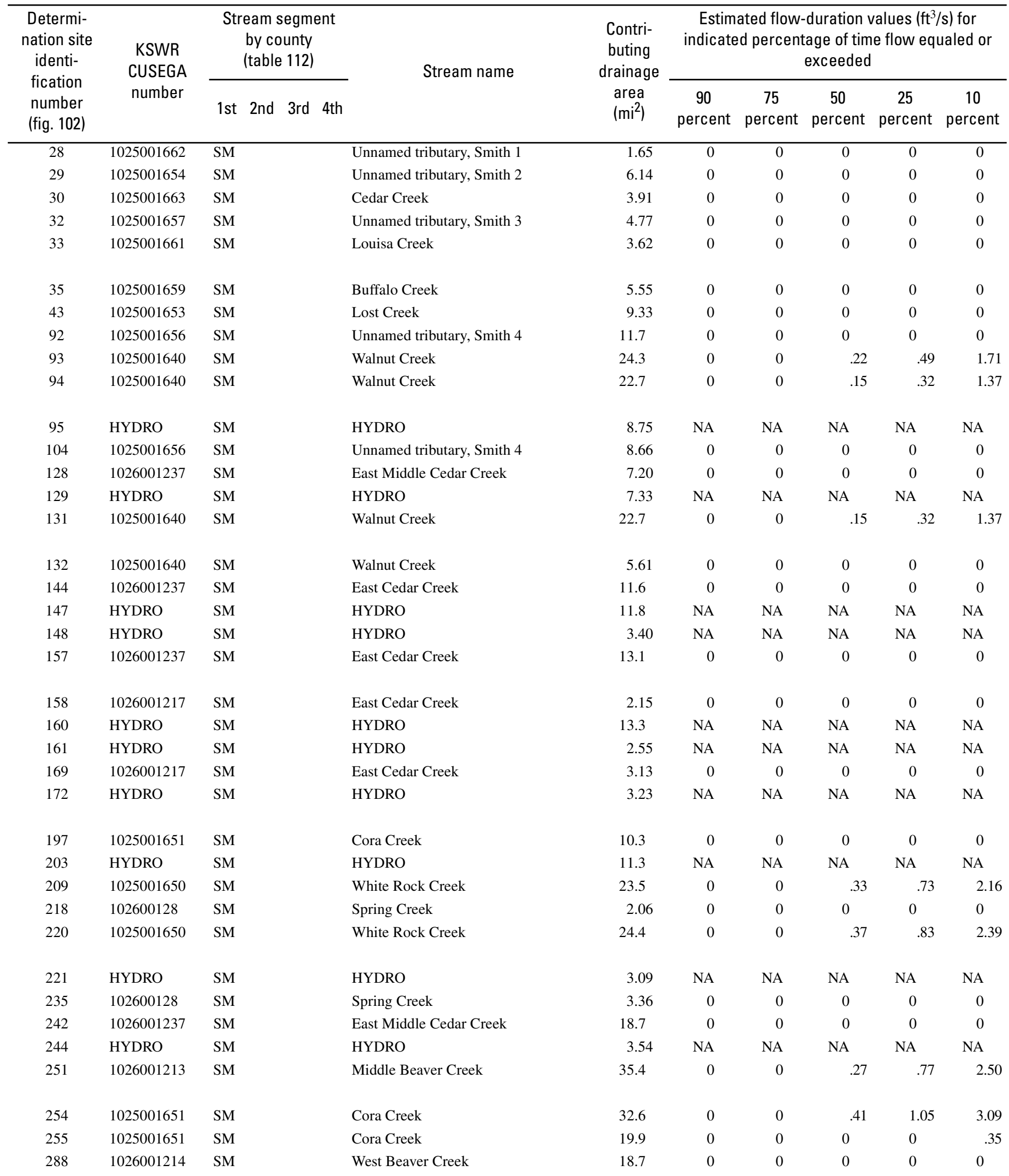


Table 98. Estimated flow-duration values, mean flow values, and peak-discharge frequency values for controlled and uncontrolled flow stream segments on the 1999 Kansas Surface Water Register for Smith County.-Continued

[KSWR, Kansas Surface Water Register; CUSEGA, catalog unit segment number alpha; $\mathrm{mi}^{2}$, square miles; $\mathrm{ft}^{3} / \mathrm{s}$, cubic feet per second; HYDRO, lake or other hydrologic structure; NA, not applicable; NRDitch, irrigation ditch; NRTribal, tribal stream]

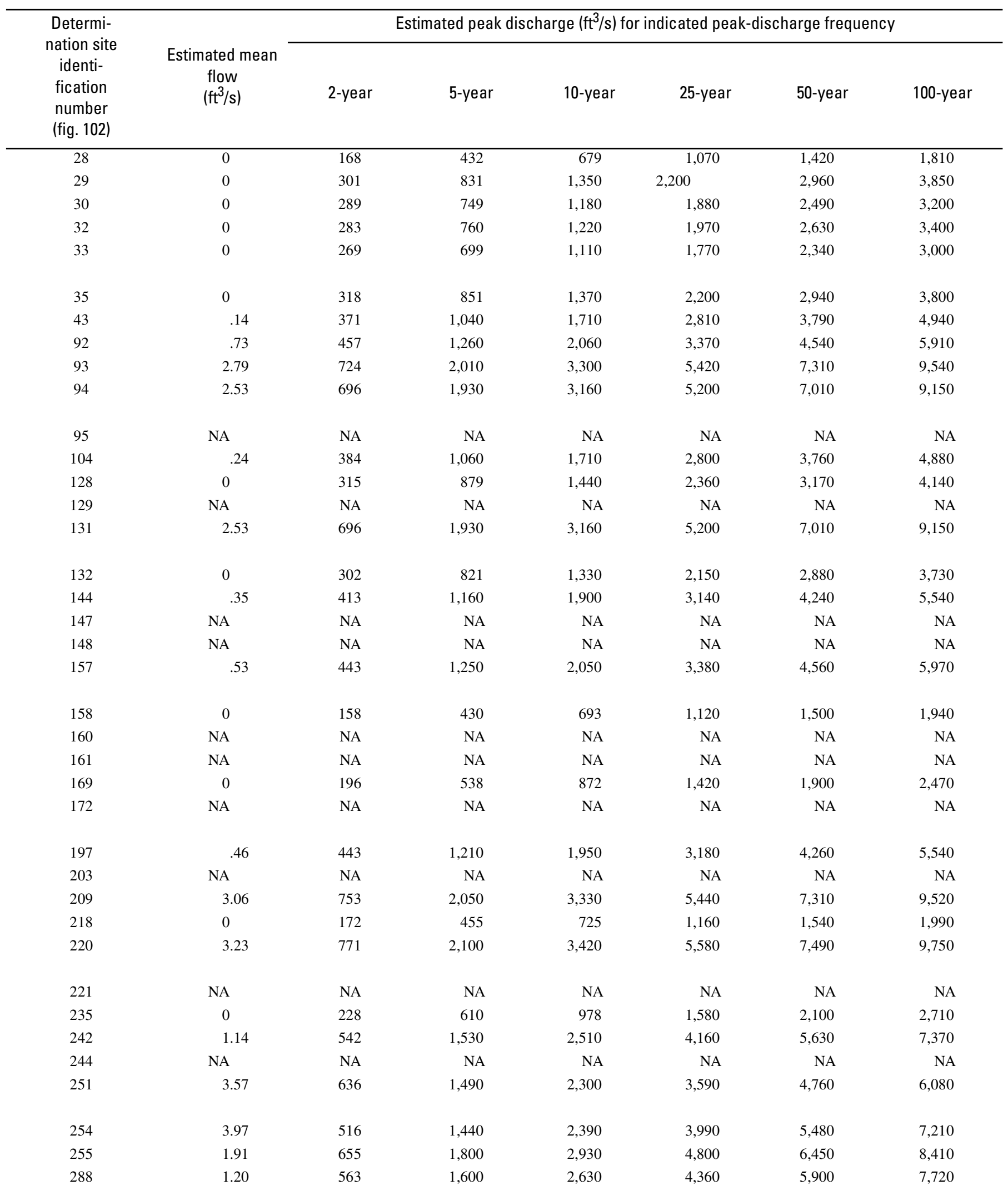


Table 98. Estimated flow-duration values, mean flow values, and peak-discharge frequency values for controlled and uncontrolled flow stream segments on the 1999 Kansas Surface Water Register for Smith County.-Continued

[KSWR, Kansas Surface Water Register; CUSEGA, catalog unit segment number alpha; $\mathrm{mi}^{2}$, square miles; $\mathrm{ft}^{3} / \mathrm{s}$, cubic feet per second; HYDRO, lake or other hydrologic structure; NA, not applicable; NRDitch, irrigation ditch; NRTribal, tribal stream]

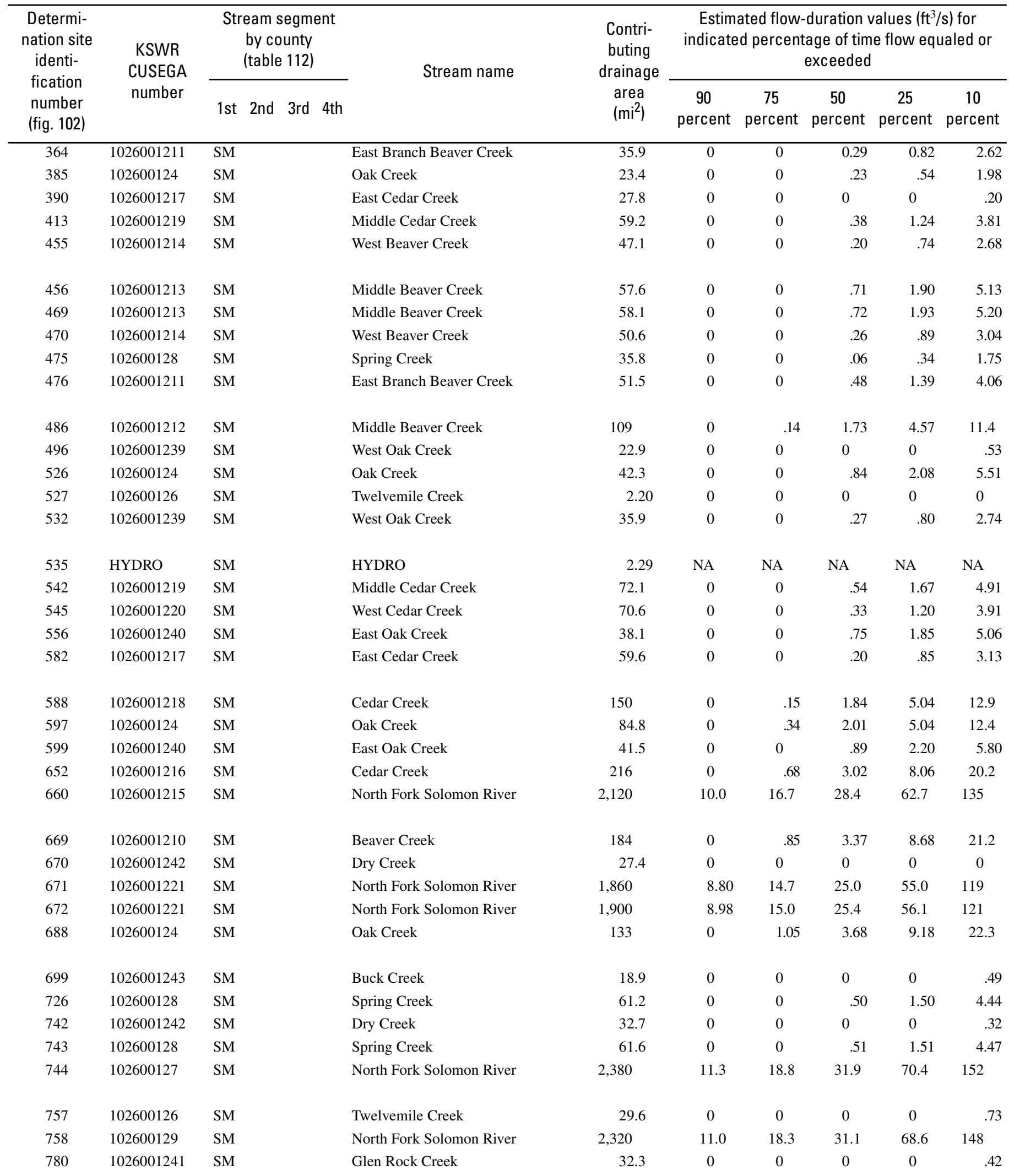


Table 98. Estimated flow-duration values, mean flow values, and peak-discharge frequency values for controlled and uncontrolled flow stream segments on the 1999 Kansas Surface Water Register for Smith County.-Continued

[KSWR, Kansas Surface Water Register; CUSEGA, catalog unit segment number alpha; $\mathrm{mi}^{2}$, square miles; $\mathrm{ft}^{3} / \mathrm{s}$, cubic feet per second; HYDRO, lake or other hydrologic structure; NA, not applicable; NRDitch, irrigation ditch; NRTribal, tribal stream]

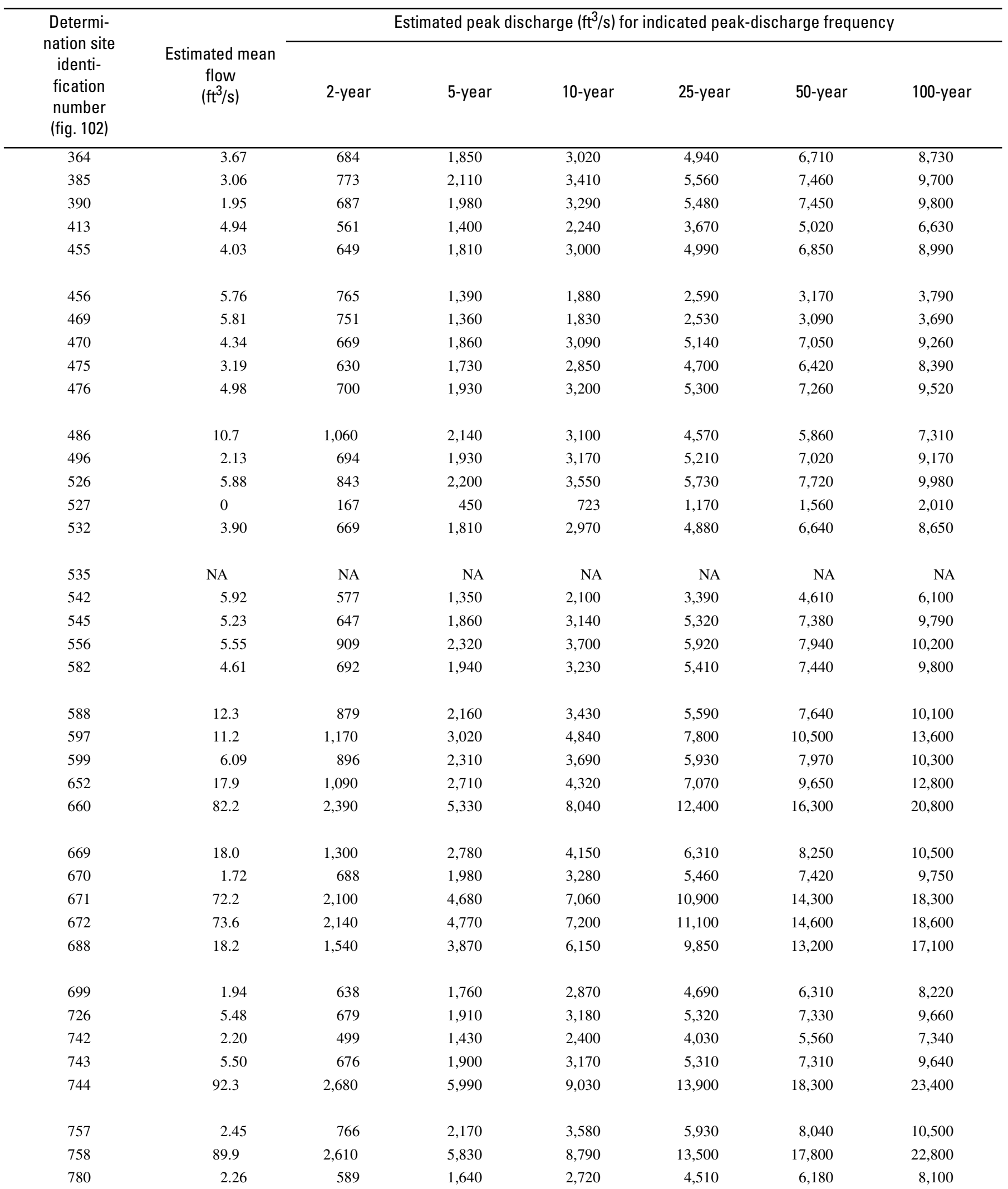


Table 98. Estimated flow-duration values, mean flow values, and peak-discharge frequency values for controlled and uncontrolled flow stream segments on the 1999 Kansas Surface Water Register for Smith County.-Continued

[KSWR, Kansas Surface Water Register; CUSEGA, catalog unit segment number alpha; $\mathrm{mi}^{2}$, square miles; $\mathrm{ft}^{3} / \mathrm{s}$, cubic feet per second; HYDRO, lake or other hydrologic structure; NA, not applicable; NRDitch, irrigation ditch; NRTribal, tribal stream]

\begin{tabular}{|c|c|c|c|c|c|c|c|c|c|}
\hline $\begin{array}{l}\text { Determi- } \\
\text { nation site } \\
\text { identi- } \\
\text { fication } \\
\text { number } \\
\text { (fig. 102) }\end{array}$ & $\begin{array}{l}\text { KSWR } \\
\text { CUSEGA } \\
\text { number }\end{array}$ & $\begin{array}{l}\text { Stream segment } \\
\text { by county } \\
\text { (table 112) }\end{array}$ & Stream name & $\begin{array}{l}\text { Contri- } \\
\text { buting } \\
\text { drainage } \\
\text { area } \\
\left(\mathrm{mi}^{2}\right)\end{array}$ & \multicolumn{5}{|c|}{$\begin{array}{c}\text { Estimated flow-duration values }\left(\mathrm{ft}^{3} / \mathrm{s}\right) \text { for } \\
\text { indicated percentage of time flow equaled or } \\
\text { exceeded }\end{array}$} \\
\hline 782 & HYDRO & SM & HYDRO & 13.1 & NA & NA & NA & NA & NA \\
\hline
\end{tabular}


Table 98. Estimated flow-duration values, mean flow values, and peak-discharge frequency values for controlled and uncontrolled flow stream segments on the 1999 Kansas Surface Water Register for Smith County.-Continued

[KSWR, Kansas Surface Water Register; CUSEGA, catalog unit segment number alpha; $\mathrm{mi}^{2}$, square miles; $\mathrm{ft}^{3} / \mathrm{s}$, cubic feet per second; HYDRO, lake or other hydrologic structure; NA, not applicable; NRDitch, irrigation ditch; NRTribal, tribal stream]

\begin{tabular}{|c|c|c|c|c|c|c|c|}
\hline $\begin{array}{l}\text { Determi- } \\
\text { nation site } \\
\text { identi- } \\
\text { fication } \\
\text { number } \\
\text { (fig. 102) }\end{array}$ & $\begin{array}{l}\text { Estimated mean } \\
\text { flow } \\
\left(\mathrm{ft}^{3} / \mathrm{s}\right)\end{array}$ & \multicolumn{6}{|c|}{ Estimated peak discharge $\left(\mathrm{ft}^{3} / \mathrm{s}\right)$ for indicated peak-discharge frequency } \\
\hline 782 & NA & NA & NA & NA & NA & NA & NA \\
\hline 808 & 94.2 & 2,740 & 6,110 & 9,210 & 14,200 & 18,700 & 23,800 \\
\hline
\end{tabular}




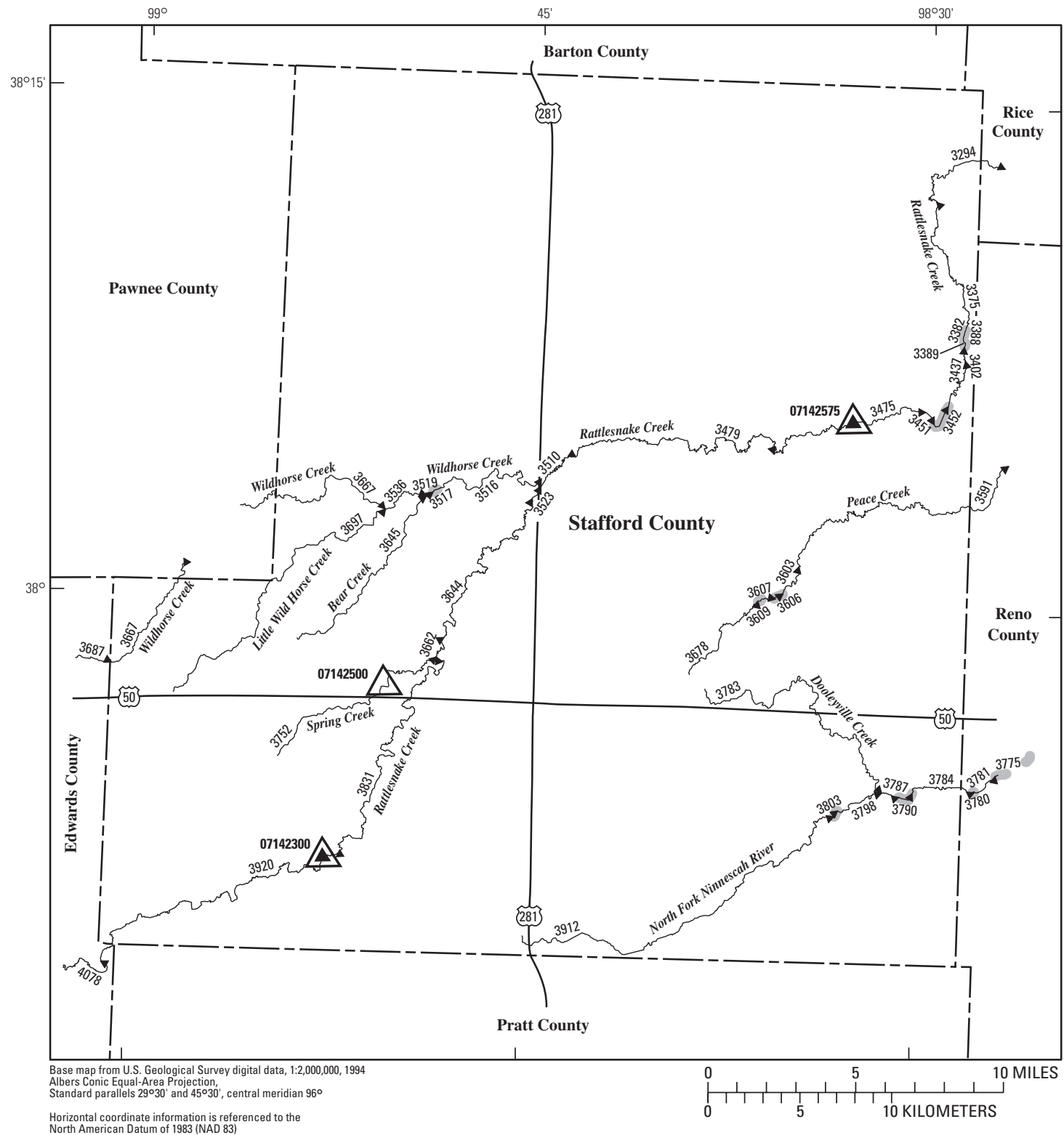

EXPLANATION

\$920 Location of streamflow-statistics determination site (small triangle) and associated identification number-small triangle points in downstream direction

07142300

A.S. Geological Survey streamflow-gaging station and number used for estimates of flow duration

07142500 人

U.S. Geological Survey streamflow-gaging station and number used for estimates of peak-discharge frequency values

KANSAS

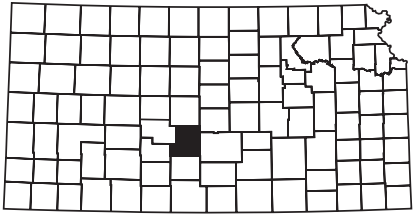

Index map 3803

Lake and determination site identification number

Figure 103. Location of streamflow-statistics determination sites, associated identification numbers, and U.S. Geological Survey streamflow-gaging stations used in the flow-duration and peak-discharge frequency analyses for Stafford County. 
Table 99. Estimated flow-duration values, mean flow values, and peak-discharge frequency values for controlled and uncontrolled flow stream segments on the 1999 Kansas Surface Water Register for Stafford County.

[KSWR, Kansas Surface Water Register; CUSEGA, catalog unit segment number alpha; $\mathrm{mi}^{2}$, square miles; $\mathrm{ft}^{3} / \mathrm{s}$, cubic feet per second; HYDRO, lake or other hydrologic structure; NA, not applicable; NRDitch, irrigation ditch; NRTribal, tribal stream]

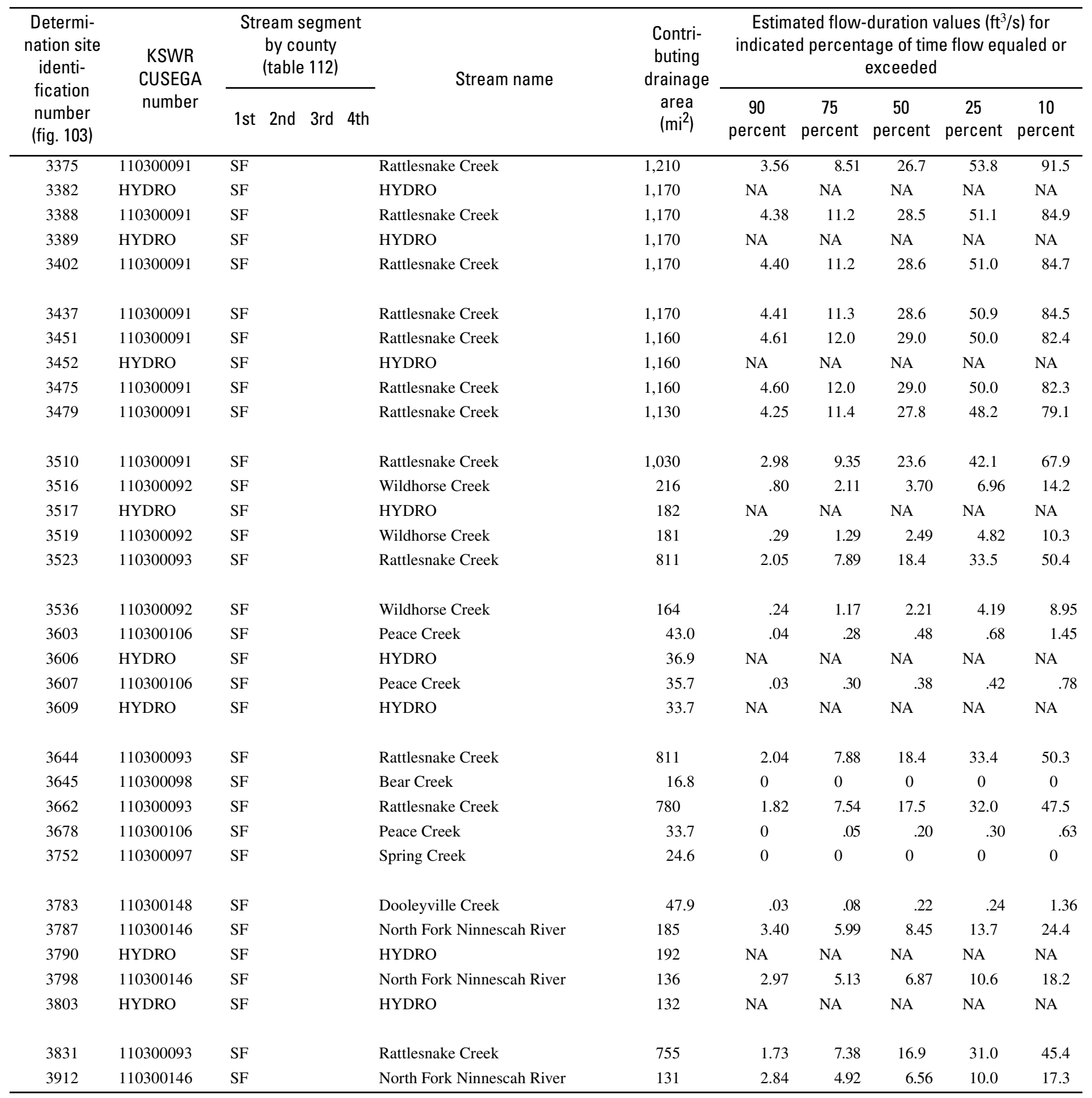


Table 99. Estimated flow-duration values, mean flow values, and peak-discharge frequency values for controlled and uncontrolled flow stream segments on the 1999 Kansas Surface Water Register for Stafford County.-Continued

[KSWR, Kansas Surface Water Register; CUSEGA, catalog unit segment number alpha; $\mathrm{mi}^{2}$, square miles; $\mathrm{ft}^{3} / \mathrm{s}$, cubic feet per second; HYDRO, lake or other hydrologic structure; NA, not applicable; NRDitch, irrigation ditch; NRTribal, tribal stream]

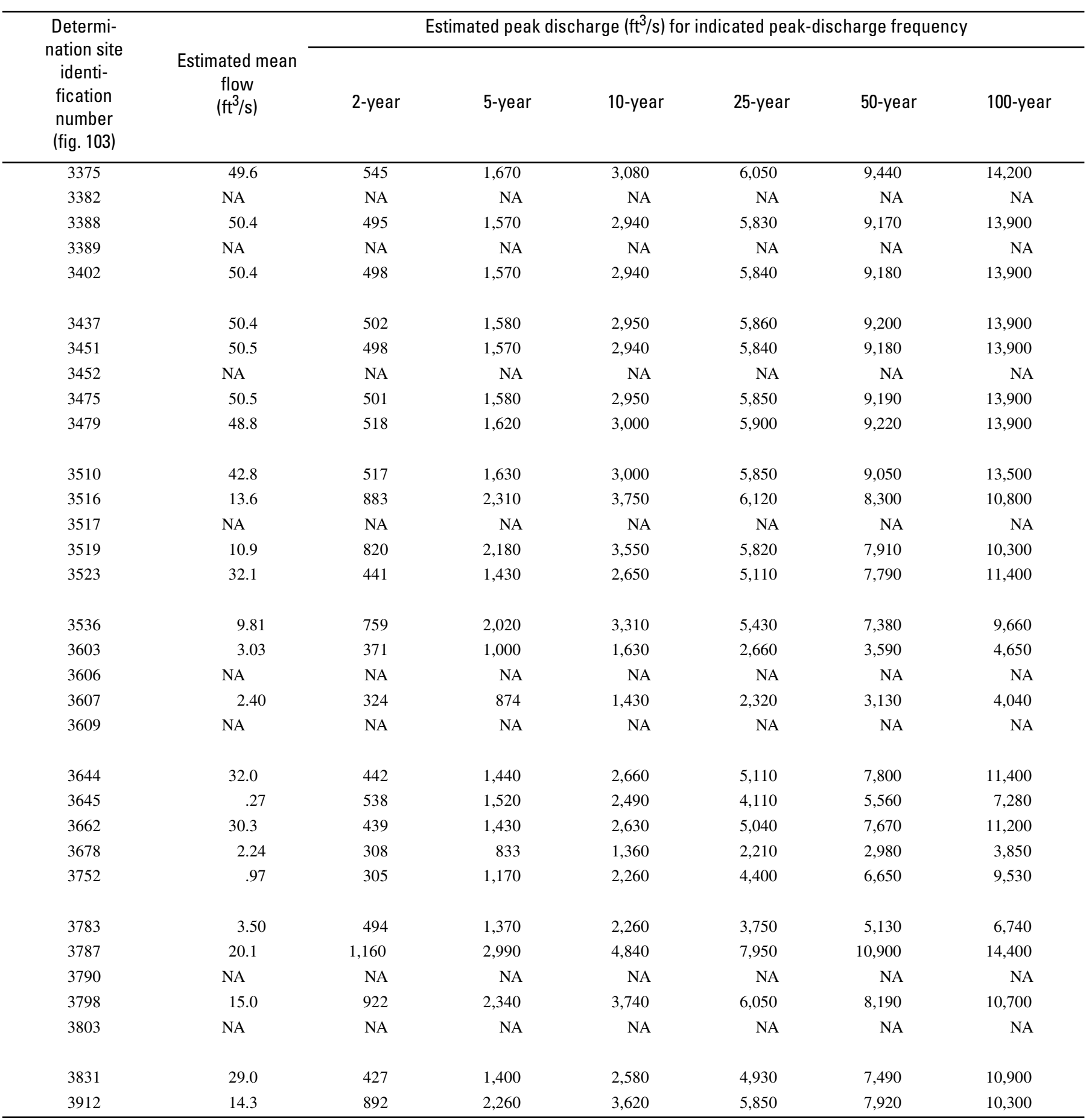




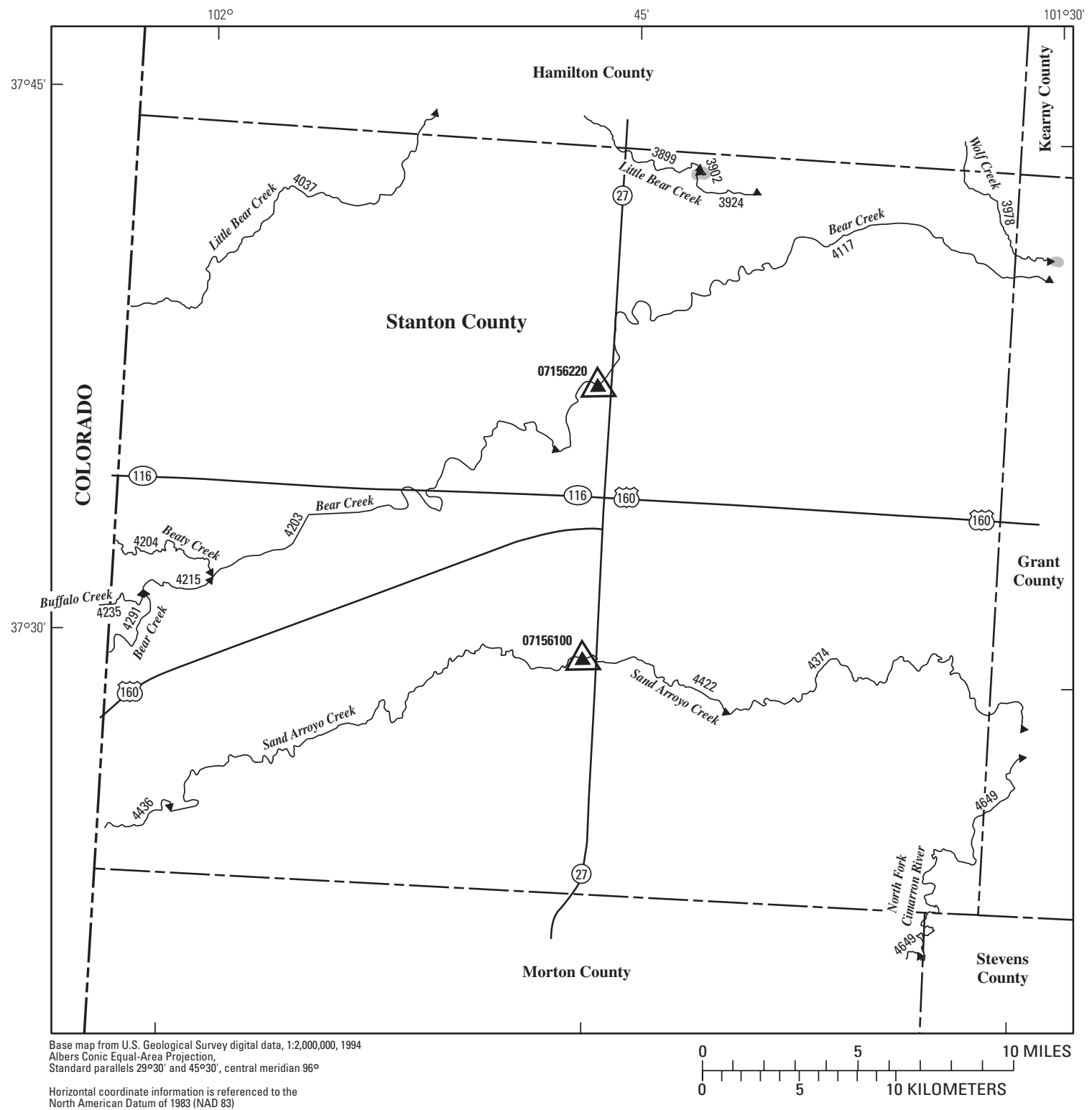

EXPLANATION

\footnotetext{
4436 Location of streamflow-statistics determination site (small triangle) and associated identification number-small triangle points in downstream direction

07156100

$\Delta$ U.S. Geological Survey streamflow-gaging station and number used for estimates of flow duration

${ }^{07156220} \triangle$

U.S. Geological Survey streamflow-gaging station and number used for estimates of peak-discharge frequency values

3902

Lake and determination site identification number
}

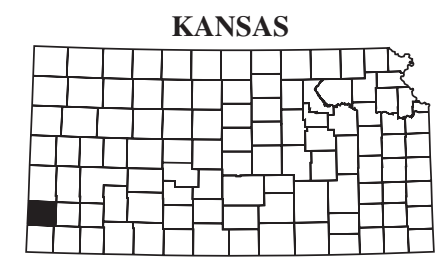

Index map

Figure 104. Location of streamflow-statistics determination sites, associated identification numbers, and U.S. Geological Survey streamflow-gaging stations used in the flow-duration and peak-discharge frequency analyses for Stanton County. 
Table 100. Estimated flow-duration values, mean flow values, and peak-discharge frequency values for controlled and uncontrolled flow stream segments on the 1999 Kansas Surface Water Register for Stanton County.

[KSWR, Kansas Surface Water Register; CUSEGA, catalog unit segment number alpha; $\mathrm{mi}^{2}$, square miles; $\mathrm{ft}^{3} / \mathrm{s}$, cubic feet per second; HYDRO, lake or other hydrologic structure; NA, not applicable; NRDitch, irrigation ditch; NRTribal, tribal stream]

\begin{tabular}{|c|c|c|c|c|c|c|c|c|c|c|}
\hline $\begin{array}{l}\text { Determi- } \\
\text { nation site } \\
\text { identi- } \\
\text { fication } \\
\text { number } \\
\text { (fig. 104) }\end{array}$ & $\begin{array}{l}\text { KSWR } \\
\text { CUSEGA } \\
\text { number }\end{array}$ & \multicolumn{2}{|c|}{$\begin{array}{l}\text { Stream segment } \\
\text { by county } \\
\text { (table 112) }\end{array}$} & Stream name & $\begin{array}{l}\text { Contri- } \\
\text { buting } \\
\text { drainage } \\
\text { area } \\
\left(\mathrm{mi}^{2}\right)\end{array}$ & \multicolumn{5}{|c|}{$\begin{array}{c}\text { Estimated flow-duration values }\left(\mathrm{ft}^{3} / \mathrm{s}\right) \text { for } \\
\text { indicated percentage of time flow equaled or } \\
\text { exceeded }\end{array}$} \\
\hline 3902 & HYDRO & ST & & HYDRO & 511 & NA & NA & NA & NA & NA \\
\hline 4204 & 110400058 & ST & & Beaty Creek & 49.8 & 0 & 0 & 0 & 0 & 0 \\
\hline 4215 & 110400059 & ST & & Bear Creek & 797 & 0 & 0 & 0 & 0 & 0 \\
\hline 4235 & 1104000510 & ST & & Buffalo Creek & 87.0 & 0 & 0 & 0 & 0 & 0 \\
\hline 4291 & 1104000511 & ST & & Bear Creek & 702 & 0 & 0 & 0 & 0 & 0 \\
\hline
\end{tabular}


Table 100. Estimated flow-duration values, mean flow values, and peak-discharge frequency values for controlled and uncontrolled flow stream segments on the 1999 Kansas Surface Water Register for Stanton County.-Continued

[KSWR, Kansas Surface Water Register; CUSEGA, catalog unit segment number alpha; $\mathrm{mi}^{2}$, square miles; $\mathrm{ft}^{3} / \mathrm{s}$, cubic feet per second; HYDRO, lake or other hydrologic structure; NA, not applicable; NRDitch, irrigation ditch; NRTribal, tribal stream]

\begin{tabular}{|c|c|c|c|c|c|c|c|}
\hline \multirow{2}{*}{$\begin{array}{l}\text { Determi- } \\
\text { nation site } \\
\text { identi- } \\
\text { fication } \\
\text { number } \\
\text { (fig. 104) }\end{array}$} & \multirow{2}{*}{$\begin{array}{c}\text { Estimated mean } \\
\text { flow } \\
\left(\mathrm{ft}^{3} / \mathrm{s}\right)\end{array}$} & \multicolumn{6}{|c|}{ Estimated peak discharge $\left(\mathrm{ft}^{3} / \mathrm{s}\right)$ for indicated peak-discharge frequency } \\
\hline & & 2-year & 5-year & 10-year & 25-year & 50-year & 100-year \\
\hline 3902 & NA & NA & NA & NA & NA & NA & NA \\
\hline 3924 & 0.51 & 510 & 1,820 & 3,430 & 6,450 & 9,580 & 13,500 \\
\hline 4203 & 2.64 & 723 & 2,860 & 5,540 & 10,700 & 15,900 & 22,300 \\
\hline 4204 & 0 & 134 & 536 & 1,050 & 2,050 & 3,090 & 4,410 \\
\hline 4215 & 1.98 & 689 & 2,660 & 5,110 & 9,780 & 14,500 & 20,400 \\
\hline 4235 & 0 & 205 & 787 & 1,510 & 2,910 & 4,350 & 6,160 \\
\hline 4291 & 1.50 & 632 & 2,420 & 4,640 & 8,880 & 13,200 & 18,600 \\
\hline 4422 & .25 & 146 & 541 & 1,000 & 1,850 & 2,680 & 3,680 \\
\hline 4436 & .15 & 242 & 835 & 1,520 & 2,770 & 4,000 & 5,490 \\
\hline
\end{tabular}




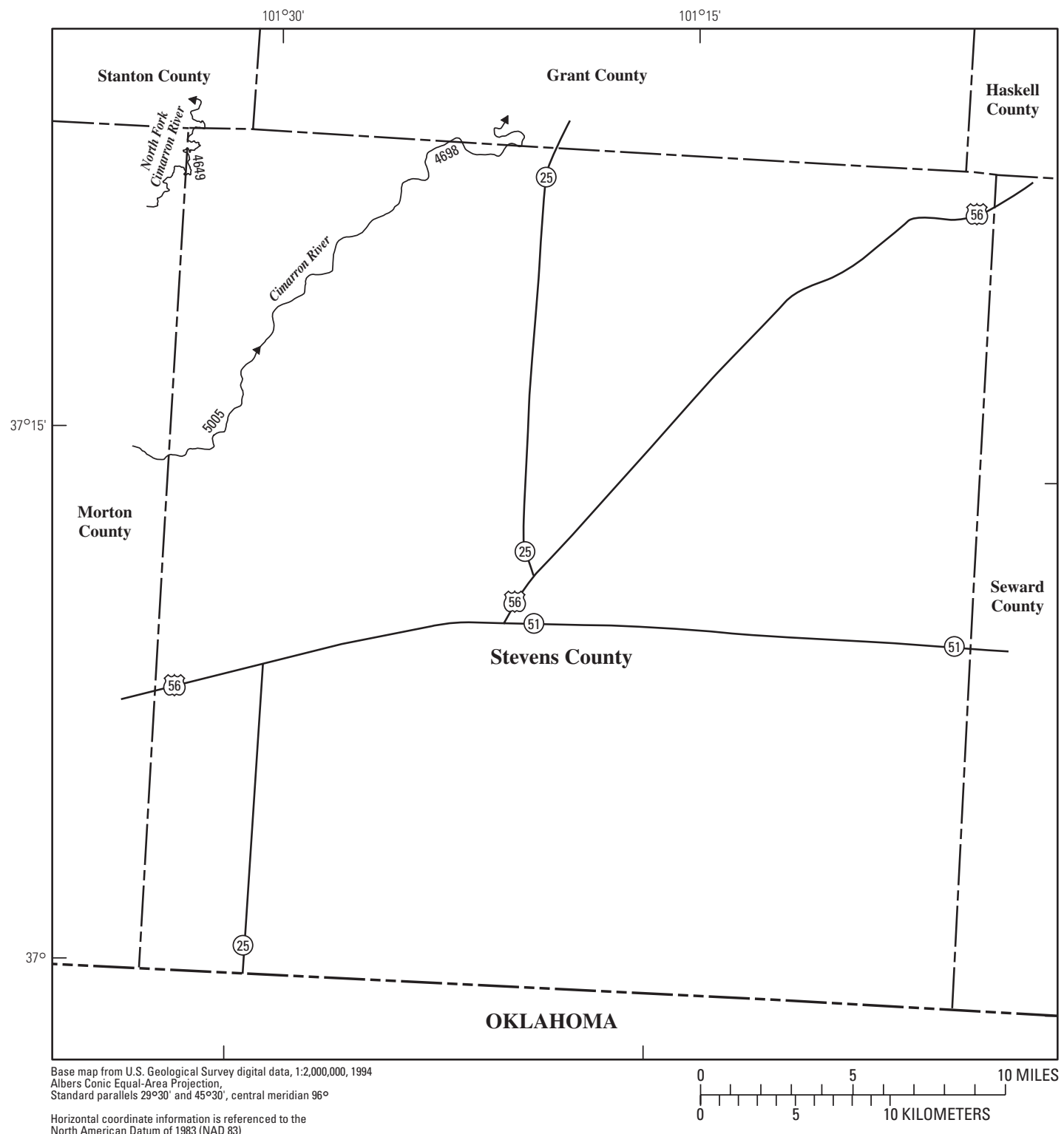

EXPLANATION

$\llbracket 5005$ Location of streamflow-statistics determination site (small triangle) and associated identification number-small triangle points in downstream direction

07156100

U.S. Geological Survey streamflow-gaging station and number used for estimates of flow duration

${ }^{07156220} \triangle$ U.S. Geological Survey streamflow-gaging station and number used for estimates of peak-discharge frequency values

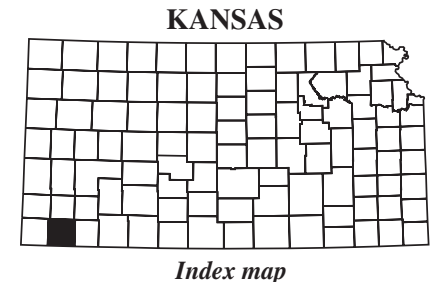

Lake and determination site identification number

Figure 105. Location of streamflow-statistics determination sites, associated identification numbers, and U.S. Geological Survey streamflow-gaging stations used in the flow-duration and peak-discharge frequency analyses for Stevens County. 
Table 101. Estimated flow-duration values, mean flow values, and peak-discharge frequency values for controlled and uncontrolled flow stream segments on the 1999 Kansas Surface Water Register for Stevens County.

[KSWR, Kansas Surface Water Register; CUSEGA, catalog unit segment number alpha; $\mathrm{ft}^{3} / \mathrm{s}$, cubic feet per second; HYDRO, lake or other hydrologic structure; NRTribal, tribal stream; NA, not applicable; NRDitch, irrigation ditch]

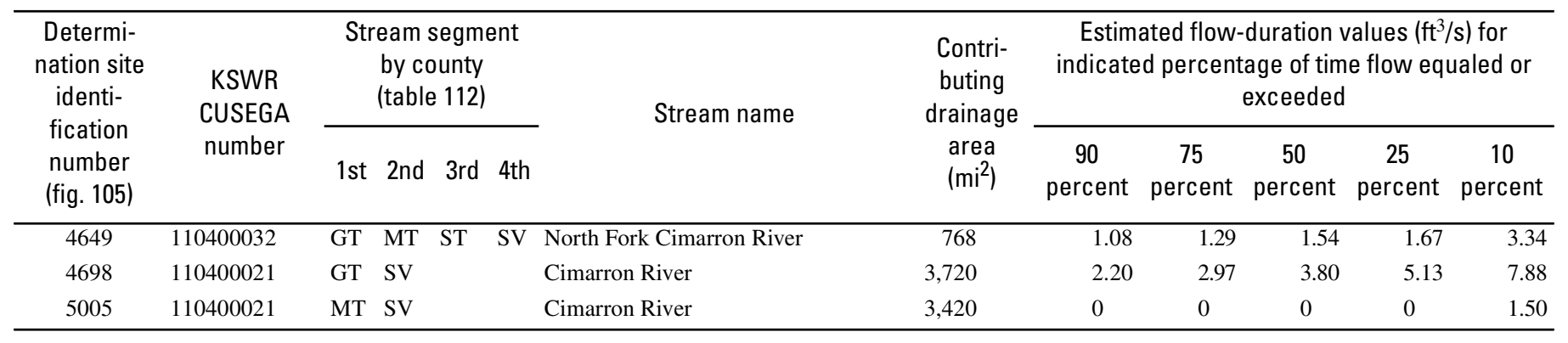


Table 101. Estimated flow-duration values, mean flow values, and peak-discharge frequency values for controlled and uncontrolled flow stream segments on the 1999 Kansas Surface Water Register for Stevens County.-Continued

[KSWR, Kansas Surface Water Register; CUSEGA, catalog unit segment number alpha; $\mathrm{mi}^{2}$, square miles; $\mathrm{ft}^{3} / \mathrm{s}$, cubic feet per second; HYDRO, lake or other hydrologic structure; NA, not applicable; NRDitch, irrigation ditch; NRTribal, tribal stream]

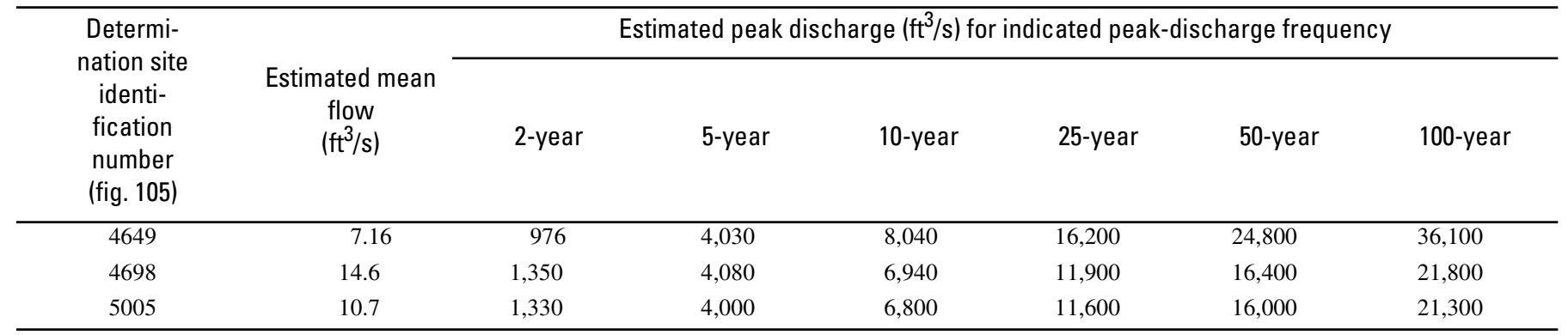




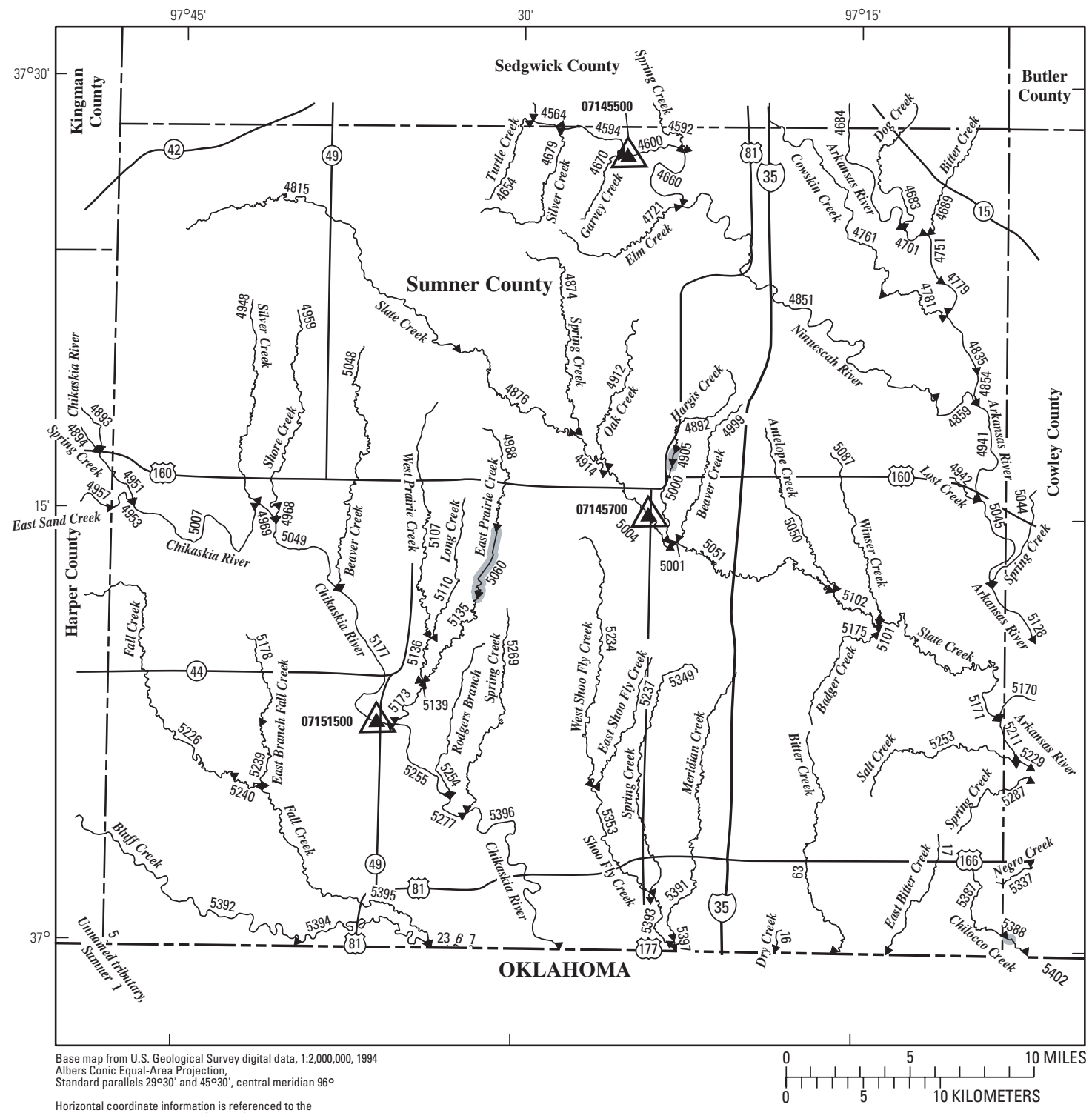

EXPLANATION

^5394 Location of streamflow-statistics determination site (small triangle) and associated identification number-small triangle points in downstream direction

$07145700 \Delta$ U.S. Geological Survey streamflow-gaging station and number used for estimates of flow duration

${ }^{07151500} \triangle$ U.S. Geological Survey streamflow-gaging station and number used for estimates of peak-discharge frequency values

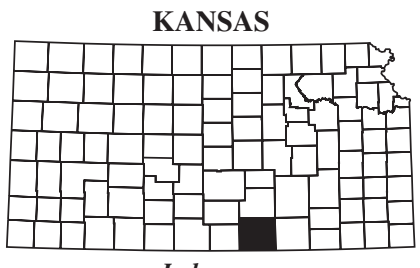
5388 Lake and determination site identification number

Figure 106. Location of streamflow-statistics determination sites, associated identification numbers, and U.S. Geological Survey streamflow-gaging stations used in the flow-duration and peak-discharge frequency analyses for Sumner County. 
Table 102. Estimated flow-duration values, mean flow values, and peak-discharge frequency values for controlled and uncontrolled flow stream segments on the 1999 Kansas Surface Water Register for Sumner County.

[KSWR, Kansas Surface Water Register; CUSEGA, catalog unit segment number alpha; $\mathrm{mi}^{2}$, square miles; $\mathrm{ft}^{3} / \mathrm{s}$, cubic feet per second; HYDRO, lake or other hydrologic structure; NA, not applicable; NRDitch, irrigation ditch; NRTribal, tribal stream]

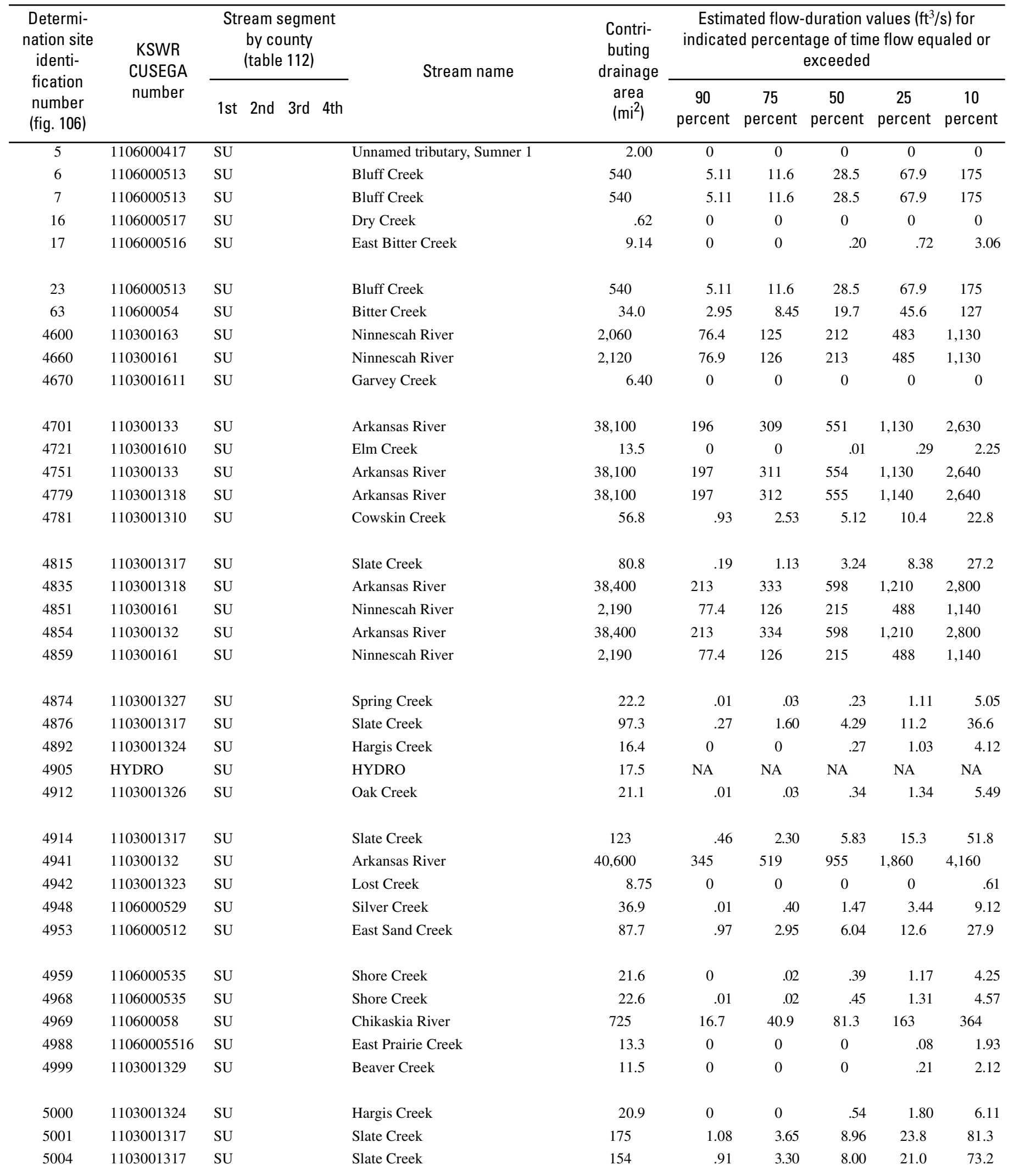


Table 102. Estimated flow-duration values, mean flow values, and peak-discharge frequency values for controlled and uncontrolled flow stream segments on the 1999 Kansas Surface Water Register for Sumner County.-Continued

[KSWR, Kansas Surface Water Register; CUSEGA, catalog unit segment number alpha; $\mathrm{mi}^{2}$, square miles; $\mathrm{ft}^{3} / \mathrm{s}$, cubic feet per second; HYDRO, lake or other hydrologic structure; NA, not applicable; NRDitch, irrigation ditch; NRTribal, tribal stream]

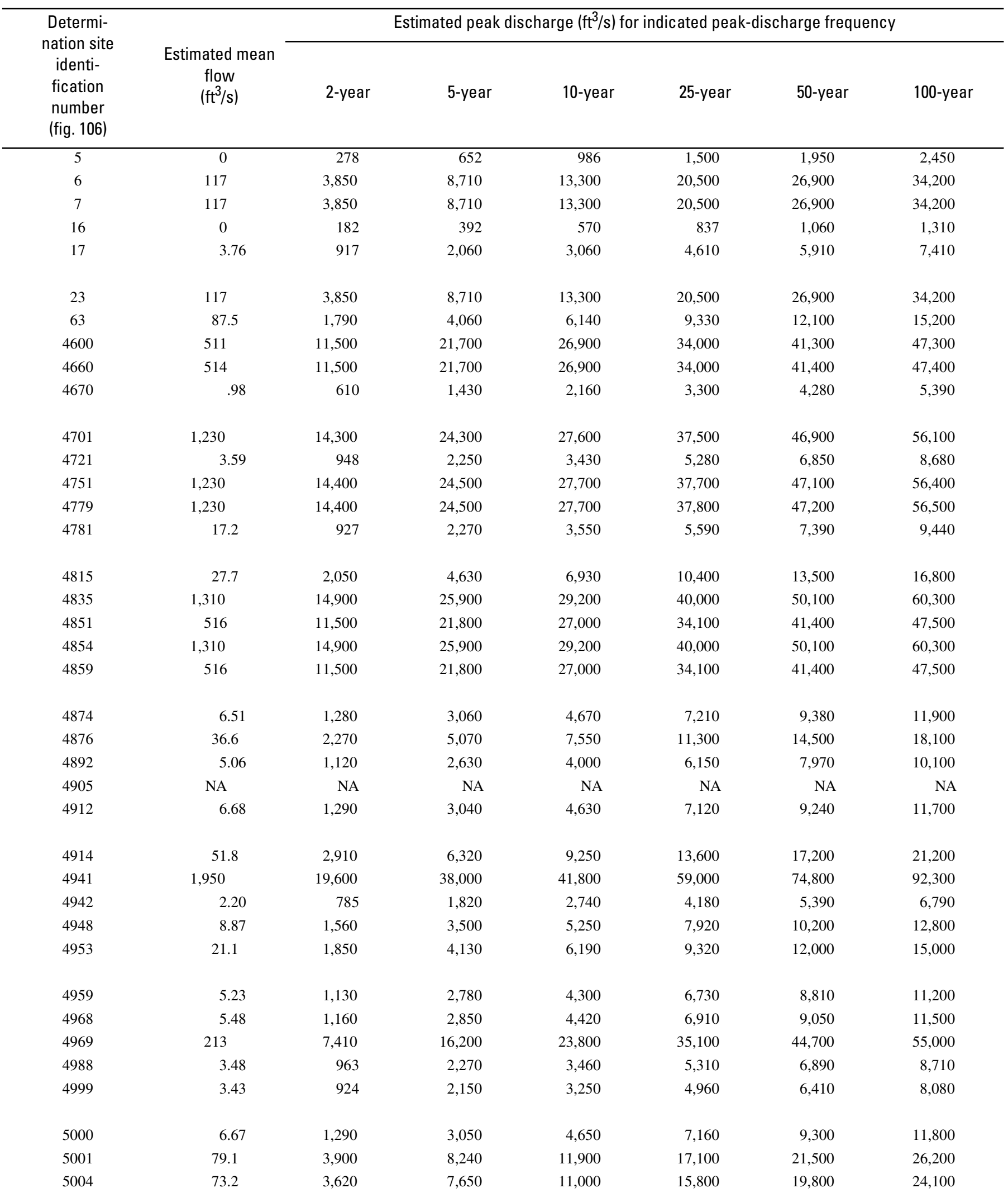


Table 102. Estimated flow-duration values, mean flow values, and peak-discharge frequency values for controlled and uncontrolled flow stream segments on the 1999 Kansas Surface Water Register for Sumner County.-Continued

[KSWR, Kansas Surface Water Register; CUSEGA, catalog unit segment number alpha; $\mathrm{mi}^{2}$, square miles; $\mathrm{ft}^{3} / \mathrm{s}$, cubic feet per second; HYDRO, lake or other hydrologic structure; NA, not applicable; NRDitch, irrigation ditch; NRTribal, tribal stream]

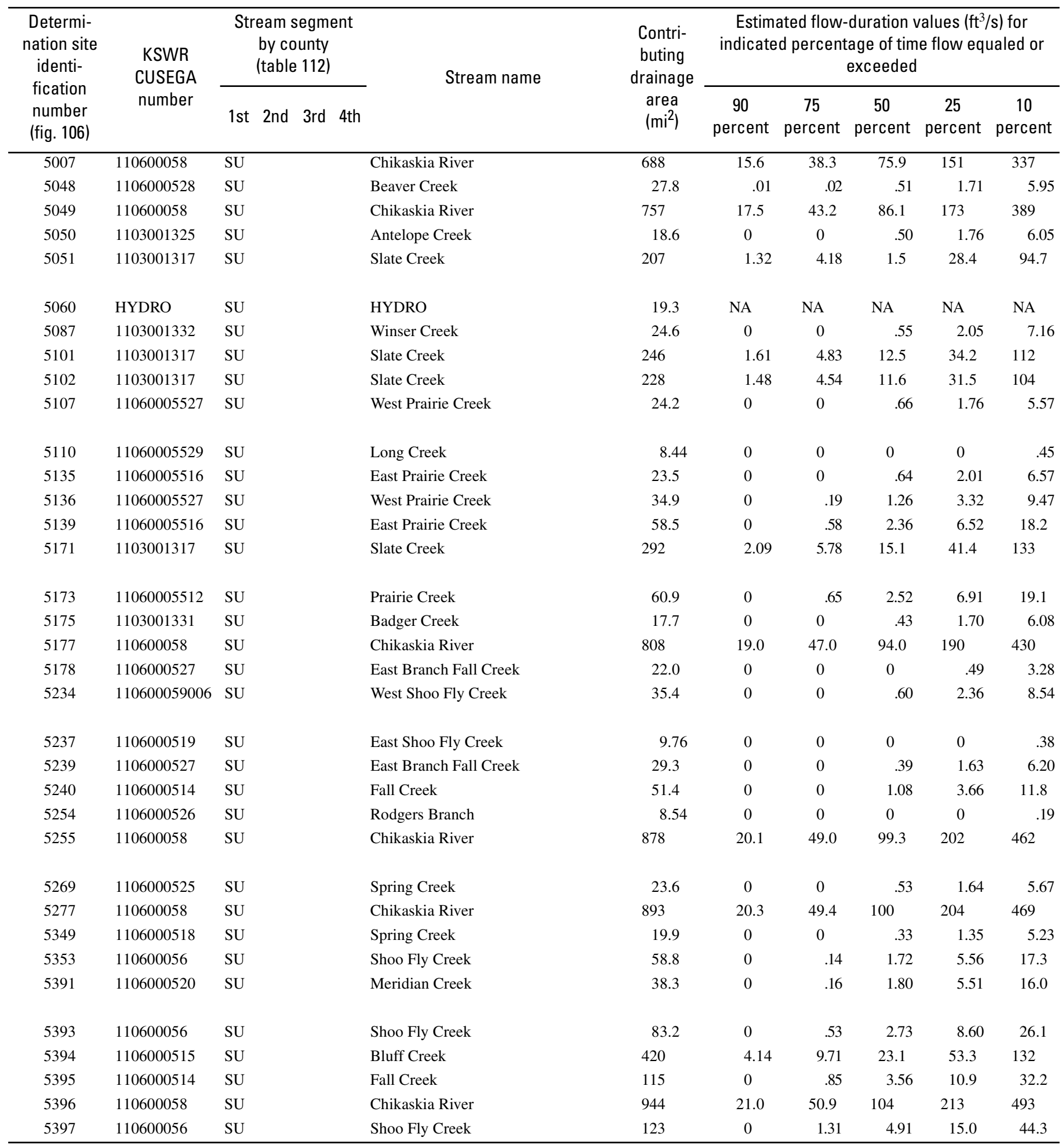


Table 102. Estimated flow-duration values, mean flow values, and peak-discharge frequency values for controlled and uncontrolled flow stream segments on the 1999 Kansas Surface Water Register for Sumner County.-Continued

[KSWR, Kansas Surface Water Register; CUSEGA, catalog unit segment number alpha; $\mathrm{mi}^{2}$, square miles; $\mathrm{ft}^{3} / \mathrm{s}$, cubic feet per second; HYDRO, lake or other hydrologic structure; NA, not applicable; NRDitch, irrigation ditch; NRTribal, tribal stream]

\begin{tabular}{|c|c|c|c|c|c|c|c|}
\hline \multirow{2}{*}{$\begin{array}{l}\text { Determi- } \\
\text { nation site } \\
\text { identi- } \\
\text { fication } \\
\text { number } \\
\text { (fig. 106) }\end{array}$} & \multirow{2}{*}{$\begin{array}{l}\text { Estimated mean } \\
\text { flow } \\
\left(\mathrm{ft}^{3} / \mathrm{s}\right)\end{array}$} & \multicolumn{6}{|c|}{ Estimated peak discharge $\left(\mathrm{ft}^{3} / \mathrm{s}\right)$ for indicated peak-discharge frequency } \\
\hline & & 2-year & 5-year & 10-year & 25-year & 50-year & 100 -year \\
\hline 5007 & 198 & 6,920 & 15,200 & 22,300 & 32,900 & 41,900 & 51,600 \\
\hline 5050 & 6.45 & 1,230 & 2,880 & 4,370 & 6,710 & 8,700 & 11,000 \\
\hline 5051 & 88.7 & 4,100 & 8,710 & 12,600 & 18,200 & 23,000 & 28,200 \\
\hline 5060 & NA & NA & NA & NA & NA & NA & NA \\
\hline 5087 & 7.78 & 1,450 & 3,410 & 5,200 & 8,000 & 10,400 & 13,200 \\
\hline 5110 & 1.96 & 721 & 1,690 & 2,570 & 3,940 & 5,110 & 6,450 \\
\hline 5135 & 7.06 & 1,340 & 3,190 & 4,880 & 7,550 & 9,830 & 12,500 \\
\hline 5136 & 9.40 & 1,230 & 2,920 & 4,510 & 6,990 & 9,190 & 11,600 \\
\hline 5139 & 16.3 & 1,550 & 3,680 & 5,700 & 8,870 & 11,700 & 14,900 \\
\hline 5171 & 115 & 4,590 & 9,790 & 14,300 & 20,800 & 26,400 & 32,600 \\
\hline 5173 & 17.0 & 1,560 & 3,700 & 5,730 & 8,930 & 11,800 & 15,000 \\
\hline 5175 & 6.50 & 1,230 & 2,870 & 4,340 & 6,640 & 8,590 & 10,800 \\
\hline 5177 & 250 & 8,530 & 18,700 & 27,400 & 40,300 & 51,200 & 63,000 \\
\hline 5178 & 4.97 & 1,200 & 2,900 & 4,470 & 6,960 & 9,090 & 11,600 \\
\hline 5269 & 6.57 & 1,350 & 3,220 & 4,930 & 7,610 & 9,900 & 12,600 \\
\hline 5277 & 272 & 9,100 & 19,800 & 28,900 & 42,500 & 54,000 & 66,400 \\
\hline 5349 & 6.16 & 1,270 & 2,990 & 4,560 & 7,000 & 9,080 & 11,500 \\
\hline 5353 & 16.7 & 2,090 & 4,790 & 7,290 & 11,200 & 14,600 & 18,500 \\
\hline 5391 & 14.1 & 1,970 & 4,440 & 6,690 & 10,200 & 13,200 & 16,500 \\
\hline 5393 & 23.7 & 2,590 & 5,830 & 8,820 & 13,500 & 17,600 & 22,100 \\
\hline 5394 & 89.6 & 3,630 & 7,600 & 11,100 & 16,500 & 21,200 & 26,400 \\
\hline 5395 & 28.3 & 2,180 & 5,220 & 8,150 & 12,800 & 17,000 & 21,800 \\
\hline 5396 & 285 & 9,300 & 20,200 & 29,500 & 43,400 & 55,200 & 67,900 \\
\hline 5397 & 37.0 & 3,360 & 7,390 & 11,100 & 16,800 & 21,800 & 27,400 \\
\hline
\end{tabular}




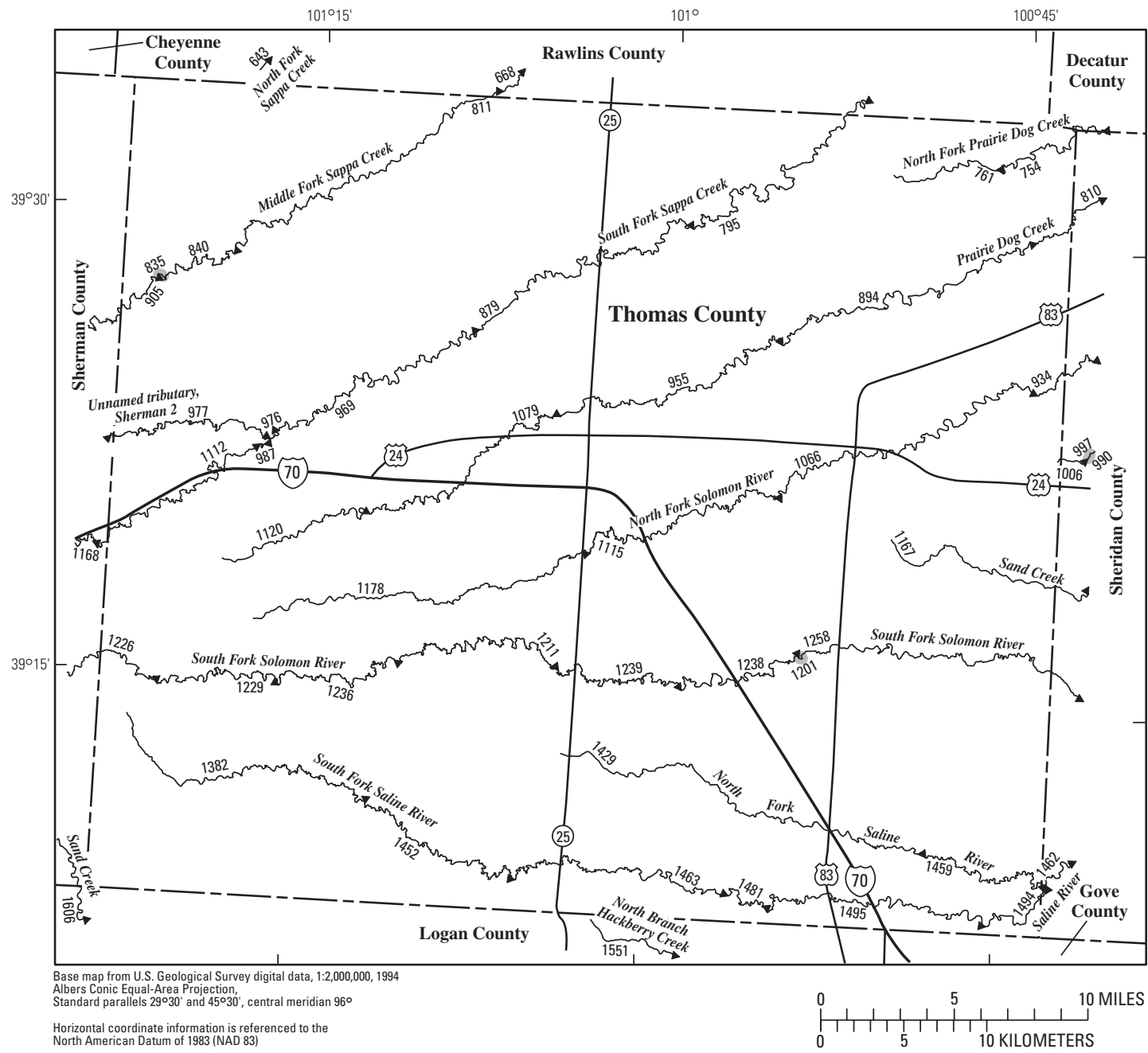

EXPLANATION

1382 Location of streamflow-statistics determination site (small triangle) and associated identification number-small triangle points in downstream direction

07156100 U.S. Geological Survey streamflow-gaging station and number used for estimates of flow duration

${ }^{07156220} \bigwedge$ U.S. Geological Survey streamflow-gaging station and number used for estimates of peak-discharge frequency values

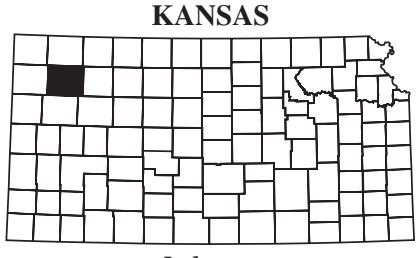

Index map 1201 Lake and determination site identification number

Figure 107. Location of streamflow-statistics determination sites, associated identification numbers, and U.S. Geological Survey streamflow-gaging stations used in the flow-duration and peak-discharge frequency analyses for Thomas County. 
Table 103. Estimated flow-duration values, mean flow values, and peak-discharge frequency values for controlled and uncontrolled flow stream segments on the 1999 Kansas Surface Water Register for Thomas County.

[KSWR, Kansas Surface Water Register; CUSEGA, catalog unit segment number alpha; $\mathrm{mi}^{2}$, square miles; $\mathrm{ft}^{3} / \mathrm{s}$, cubic feet per second; HYDRO, lake or other hydrologic structure; NA, not applicable; NRDitch, irrigation ditch; NRTribal, tribal stream]

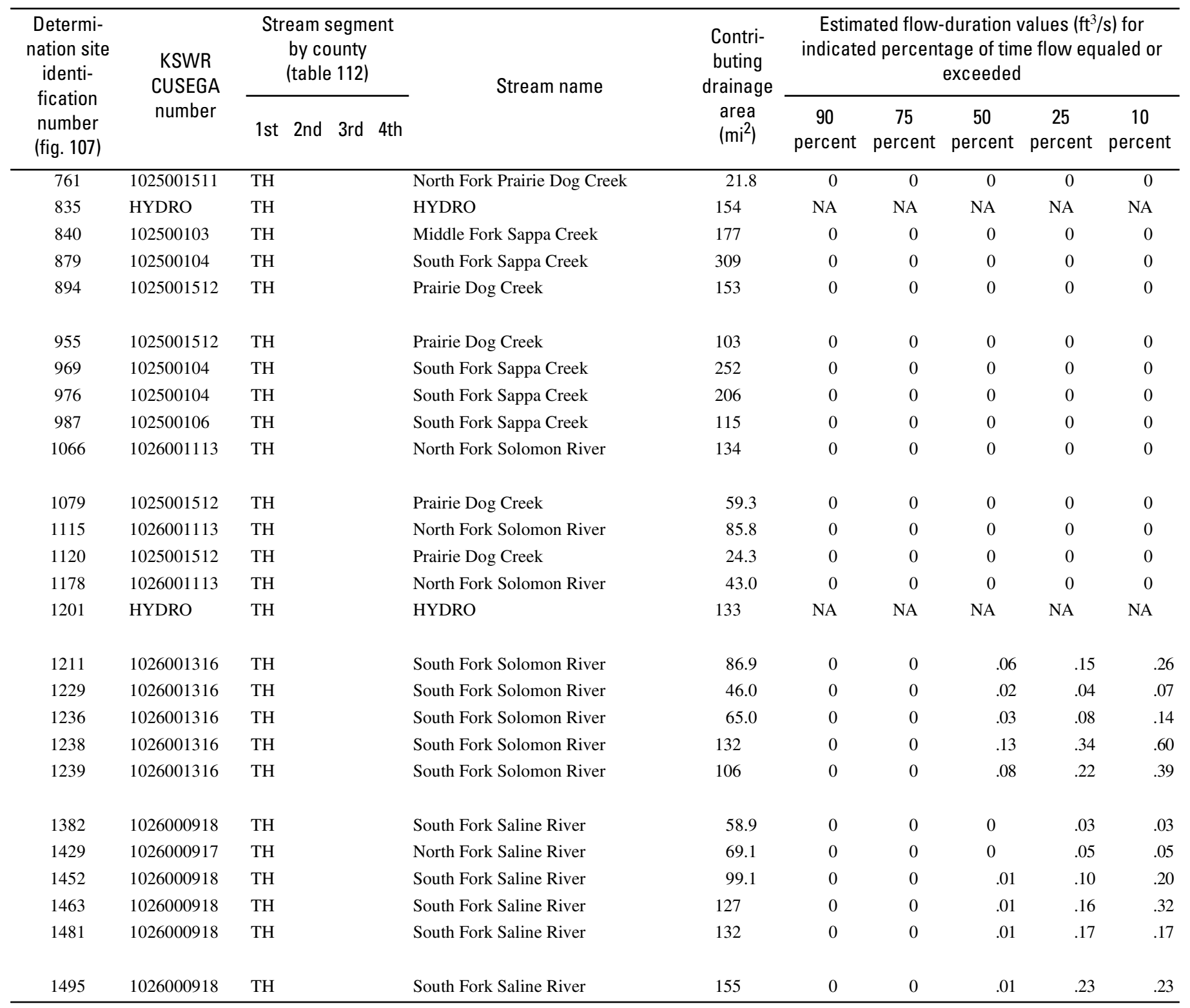


Table 103. Estimated flow-duration values, mean flow values, and peak-discharge frequency values for controlled and uncontrolled flow stream segments on the 1999 Kansas Surface Water Register for Thomas County.-Continued

[KSWR, Kansas Surface Water Register; CUSEGA, catalog unit segment number alpha; $\mathrm{mi}^{2}$, square miles; $\mathrm{ft}^{3} / \mathrm{s}$, cubic feet per second; HYDRO, lake or other hydrologic structure; NA, not applicable; NRDitch, irrigation ditch; NRTribal, tribal stream]

\begin{tabular}{|c|c|c|c|c|c|c|c|}
\hline \multirow{2}{*}{$\begin{array}{l}\text { Determi- } \\
\text { nation site } \\
\text { identi- } \\
\text { fication } \\
\text { number } \\
\text { (fig. 107) }\end{array}$} & \multirow{2}{*}{$\begin{array}{c}\text { Estimated mean } \\
\text { flow } \\
\left(\mathrm{ft}^{3} / \mathrm{s}\right)\end{array}$} & \multicolumn{6}{|c|}{ Estimated peak discharge $\left(\mathrm{ft}^{3} / \mathrm{s}\right)$ for indicated peak-discharge frequency } \\
\hline & & 2-year & 5 -year & 10-year & 25-year & 50-year & 100-year \\
\hline 761 & 0 & 348 & 1,140 & 1,990 & 3,490 & 4,880 & 6,560 \\
\hline 835 & NA & NA & NA & NA & NA & NA & NA \\
\hline 840 & .41 & 325 & 1,160 & 2,180 & 4,080 & 6,020 & 8,440 \\
\hline 879 & 1.37 & 240 & 941 & 1,890 & 3,910 & 6,190 & 9,300 \\
\hline 894 & .78 & 348 & 1,200 & 2,200 & 4,040 & 5,900 & 8,180 \\
\hline 955 & .08 & 271 & 957 & 1,780 & 3,300 & 4,850 & 6,760 \\
\hline 969 & .82 & 197 & 823 & 1,710 & 3,670 & 5,960 & 9,120 \\
\hline 976 & .48 & 165 & 731 & 1,570 & 3,480 & 5,760 & 8,960 \\
\hline 987 & .18 & 81 & 474 & 1,150 & 2,850 & 5,000 & 8,130 \\
\hline 1066 & .55 & 345 & 1,200 & 2,200 & 4,060 & 5,940 & 8,260 \\
\hline 1079 & 0 & 202 & 729 & 1,370 & 2,550 & 3,760 & 5,250 \\
\hline 1115 & 0 & 266 & 939 & 1,740 & 3,230 & 4,730 & 6,590 \\
\hline 1120 & 0 & 327 & 1,100 & 1,960 & 3,480 & 4,910 & 6,640 \\
\hline 1178 & 0 & 186 & 670 & 1,250 & 2,330 & 3,420 & 4,770 \\
\hline 1201 & NA & NA & NA & NA & NA & NA & NA \\
\hline 1211 & .14 & 242 & 872 & 1,640 & 3,070 & 4,540 & 6,360 \\
\hline 1229 & .04 & 181 & 659 & 1,240 & 2,320 & 3,430 & 4,790 \\
\hline 1236 & .08 & 208 & 756 & 1,420 & 2,670 & 3,950 & 5,530 \\
\hline 1238 & 1.01 & 320 & 1,130 & 2,100 & 3,930 & 5,780 & 8,100 \\
\hline 1239 & .49 & 278 & 991 & 1,850 & 3,470 & 5,110 & 7,160 \\
\hline 1382 & .01 & 217 & 778 & 1,450 & 2,710 & 3,980 & 5,560 \\
\hline 1429 & .02 & 293 & 1,000 & 1,830 & 3,340 & 4,850 & 6,700 \\
\hline 1452 & .05 & 280 & 993 & 1,850 & 3,450 & 5,080 & 7,110 \\
\hline 1463 & .52 & 338 & 1,180 & 2,190 & 4,060 & 5,970 & 8,330 \\
\hline 1481 & .61 & 348 & 1,210 & 2,240 & 4,160 & 6,110 & 8,530 \\
\hline 1495 & 1.11 & 408 & 1,400 & 2,570 & 4,750 & 6,950 & 9,680 \\
\hline
\end{tabular}


612 Estimates of Flow Duration, Mean Flow, and Peak-Discharge Frequency Values for Kansas Stream Locations 


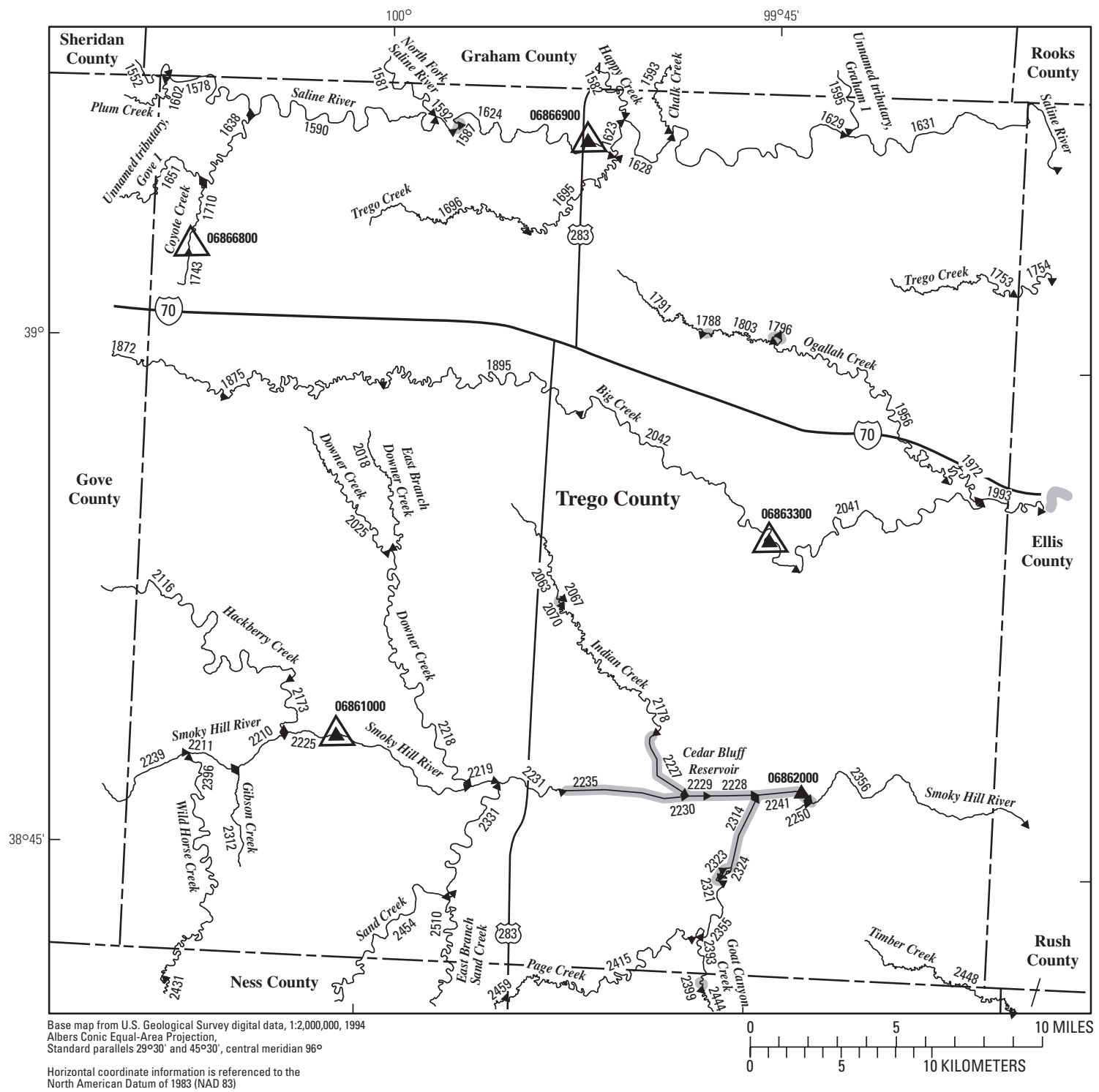

\section{EXPLANATION}

\footnotetext{
2431 Location of streamflow-statistics determination site (small triangle) and associated identification number-small triangle points in downstream direction

${ }^{06862000} \Delta$ U.S. Geological Survey streamflow-gaging station and number used for estimates of flow duration

${ }^{06861000} \triangle$ U.S. Geological Survey streamflow-gaging station and number used for estimates of peak-discharge frequency values

2235 Lake and determination site identification number
}

KANSAS

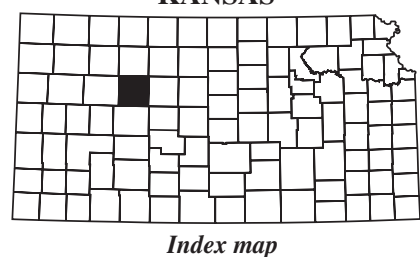

Figure 108. Location of streamflow-statistics determination sites, associated identification numbers, and U.S. Geological Survey streamflow-gaging stations used in the flow-duration and peak-discharge frequency analyses for Trego County. 
Table 104. Estimated flow-duration values, mean flow values, and peak-discharge frequency values for controlled and uncontrolled flow stream segments on the 1999 Kansas Surface Water Register for Trego County.

[KSWR, Kansas Surface Water Register; CUSEGA, catalog unit segment number alpha; $\mathrm{mi}^{2}$, square miles; $\mathrm{ft}^{3} / \mathrm{s}$, cubic feet per second; HYDRO, lake or other hydrologic structure; NA, not applicable; NRDitch, irrigation ditch; NRTribal, tribal stream]

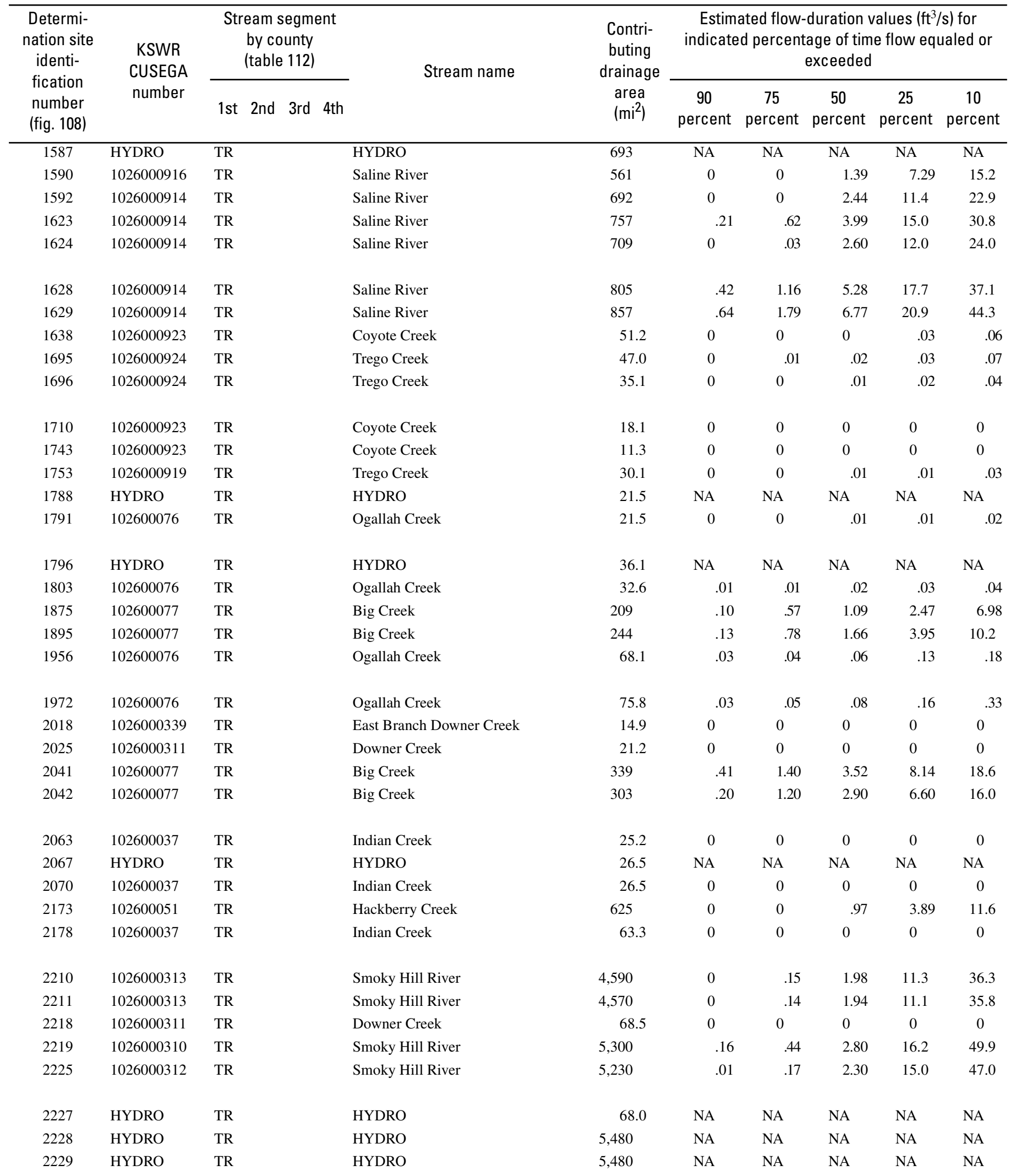


Table 104. Estimated flow-duration values, mean flow values, and peak-discharge frequency values for controlled and uncontrolled flow stream segments on the 1999 Kansas Surface Water Register for Trego County.-Continued

[KSWR, Kansas Surface Water Register; CUSEGA, catalog unit segment number alpha; $\mathrm{mi}^{2}$, square miles; $\mathrm{ft}^{3} / \mathrm{s}$, cubic feet per second; HYDRO, lake or other hydrologic structure; NA, not applicable; NRDitch, irrigation ditch; NRTribal, tribal stream]

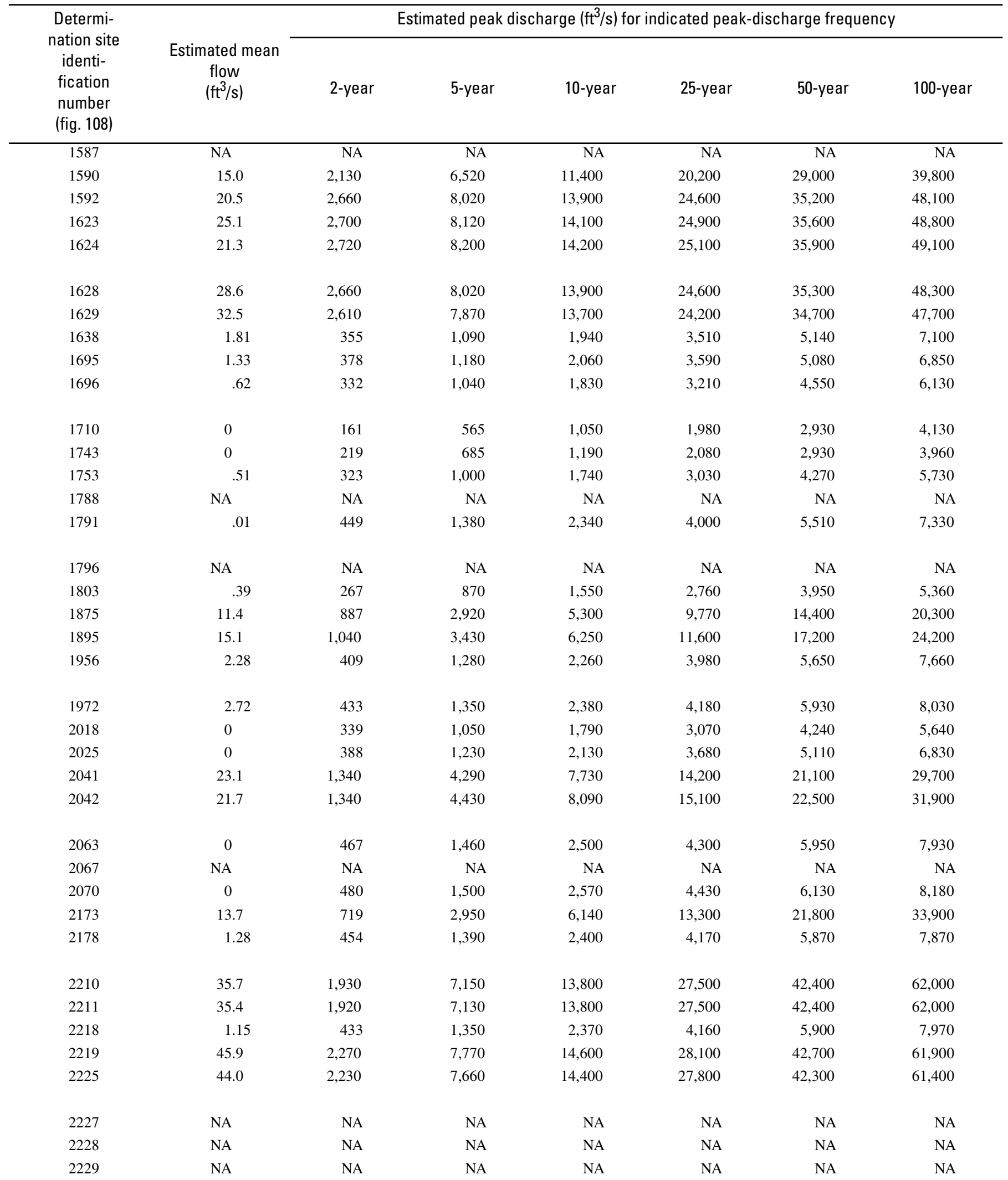


Table 104. Estimated flow-duration values, mean flow values, and peak-discharge frequency values for controlled and uncontrolled flow stream segments on the 1999 Kansas Surface Water Register for Trego County.-Continued

[KSWR, Kansas Surface Water Register; CUSEGA, catalog unit segment number alpha; $\mathrm{mi}^{2}$, square miles; $\mathrm{ft}^{3} / \mathrm{s}$, cubic feet per second; HYDRO, lake or other hydrologic structure; NA, not applicable; NRDitch, irrigation ditch; NRTribal, tribal stream]

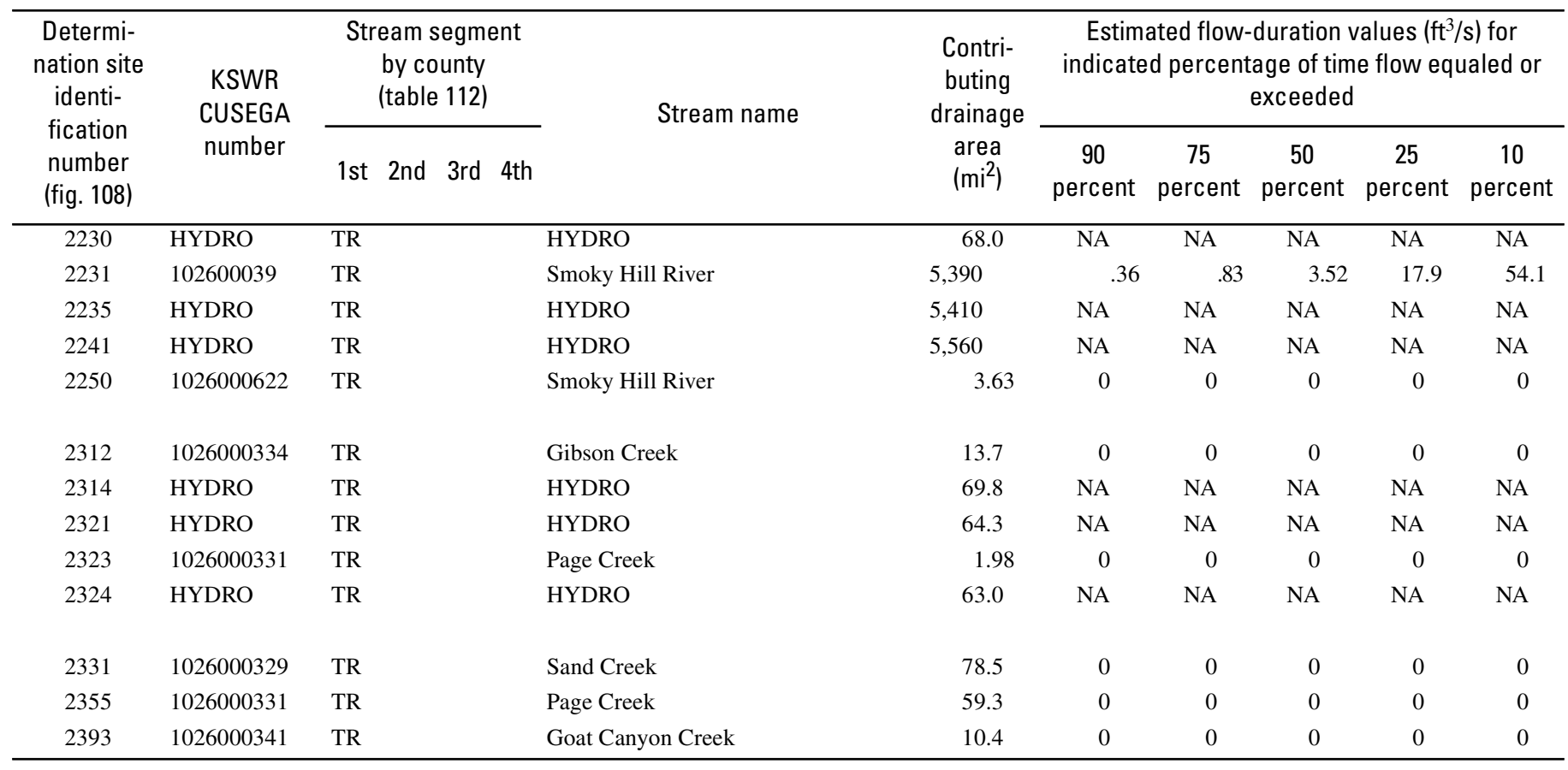


Table 104. Estimated flow-duration values, mean flow values, and peak-discharge frequency values for controlled and uncontrolled flow stream segments on the 1999 Kansas Surface Water Register for Trego County.-Continued

[KSWR, Kansas Surface Water Register; CUSEGA, catalog unit segment number alpha; $\mathrm{mi}^{2}$, square miles; $\mathrm{ft}^{3} / \mathrm{s}$, cubic feet per second; HYDRO, lake or other hydrologic structure; NA, not applicable; NRDitch, irrigation ditch; NRTribal, tribal stream]

\begin{tabular}{|c|c|c|c|c|c|c|c|}
\hline \multirow{2}{*}{$\begin{array}{l}\text { Determi- } \\
\text { nation site } \\
\text { identi- } \\
\text { fication } \\
\text { number } \\
\text { (fig. 108) }\end{array}$} & \multirow{2}{*}{$\begin{array}{c}\text { Estimated mean } \\
\text { flow } \\
\left(\mathrm{ft}^{3} / \mathrm{s}\right)\end{array}$} & \multicolumn{6}{|c|}{ Estimated peak discharge $\left(\mathrm{ft}^{3} / \mathrm{s}\right)$ for indicated peak-discharge frequency } \\
\hline & & 2-year & 5-year & 10-year & 25-year & 50-year & 100-year \\
\hline 2230 & NA & NA & NA & NA & $\mathrm{NA}$ & NA & NA \\
\hline 2241 & NA & NA & NA & NA & NA & NA & NA \\
\hline 2250 & 0 & 140 & 426 & 723 & 1,230 & 1,690 & 2,230 \\
\hline 2312 & 0 & 312 & 971 & 1,670 & 2,860 & 3,950 & 5,260 \\
\hline 2314 & NA & NA & NA & NA & NA & NA & NA \\
\hline 2331 & 1.86 & 515 & 1,560 & 2,700 & 4,680 & 6,580 & 8,830 \\
\hline 2355 & 1.06 & 398 & 1,260 & 2,210 & 3,890 & 5,530 & 7,480 \\
\hline 2393 & 0 & 265 & 821 & 1,400 & 2,400 & 3,320 & 4,410 \\
\hline
\end{tabular}




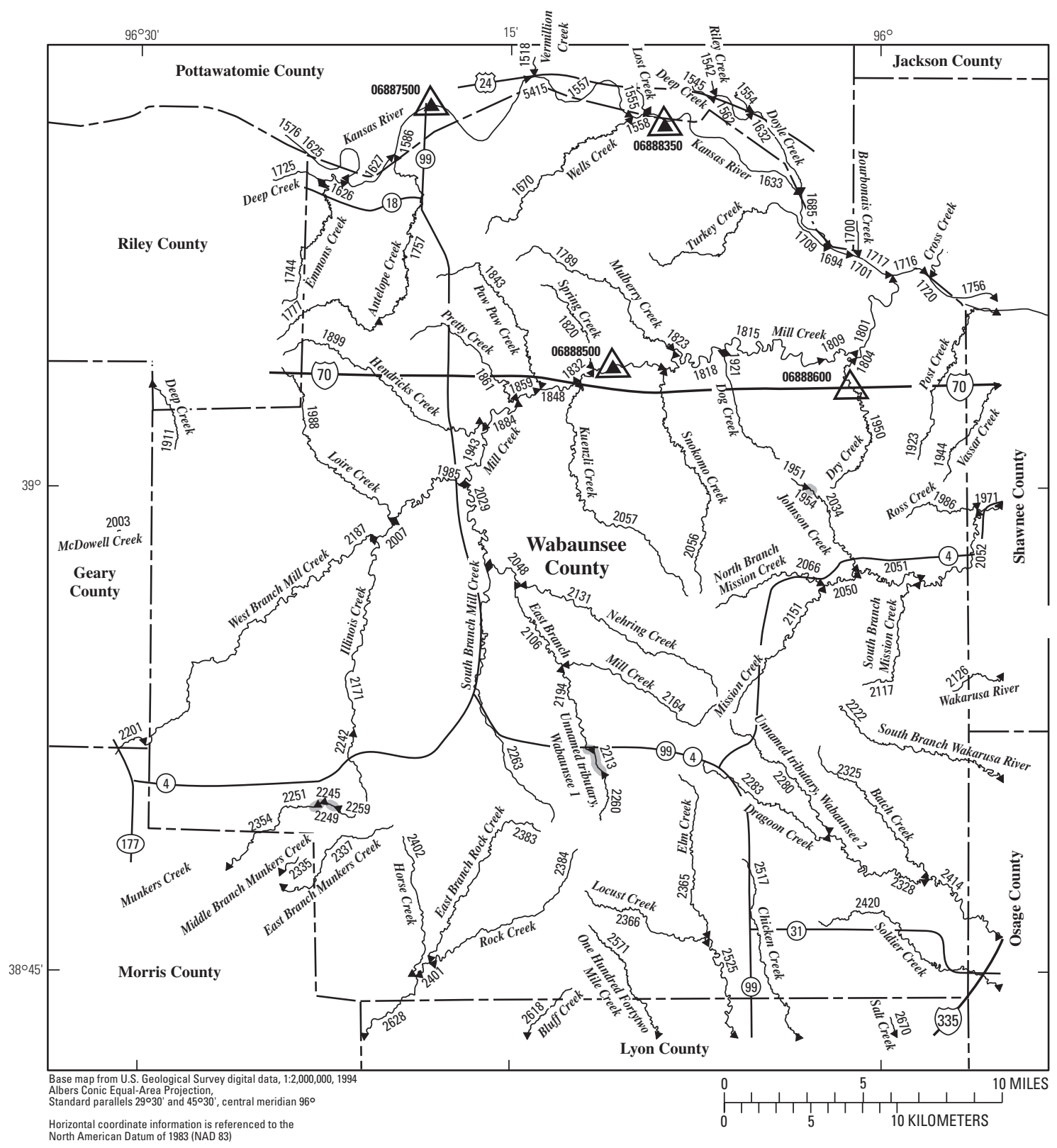

EXPLANATION

2628 Location of streamflow-statistics determination site (small triangle) and associated identification number-small triangle points in downstream direction

06888500 U.S. Geological Survey streamflow-gaging station and number used for estimates of flow duration

06888600 U.S. Geological Survey streamflow-gaging station and number used for estimates of peak-discharge frequency values

KANSAS

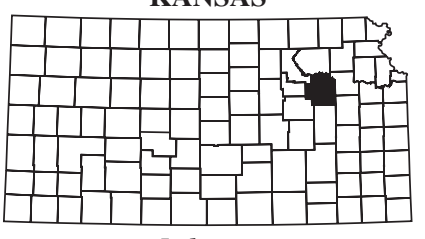

Index map 2213 Lake and determination site identification number

Figure 109. Location of streamflow-statistics determination sites, associated identification numbers, and U.S. Geological Survey streamflow-gaging stations used in the flow-duration and peak-discharge frequency analyses for Wabaunsee County. 
Table 105. Estimated flow-duration values, mean flow values, and peak-discharge frequency values for controlled and uncontrolled flow stream segments on the 1999 Kansas Surface Water Register for Wabaunsee County.

[KSWR, Kansas Surface Water Register; CUSEGA, catalog unit segment number alpha; $\mathrm{mi}^{2}$, square miles; $\mathrm{ft}^{3} / \mathrm{s}$, cubic feet per second; HYDRO, lake or other hydrologic structure; NA, not applicable; NRDitch, irrigation ditch; NRTribal, tribal stream]

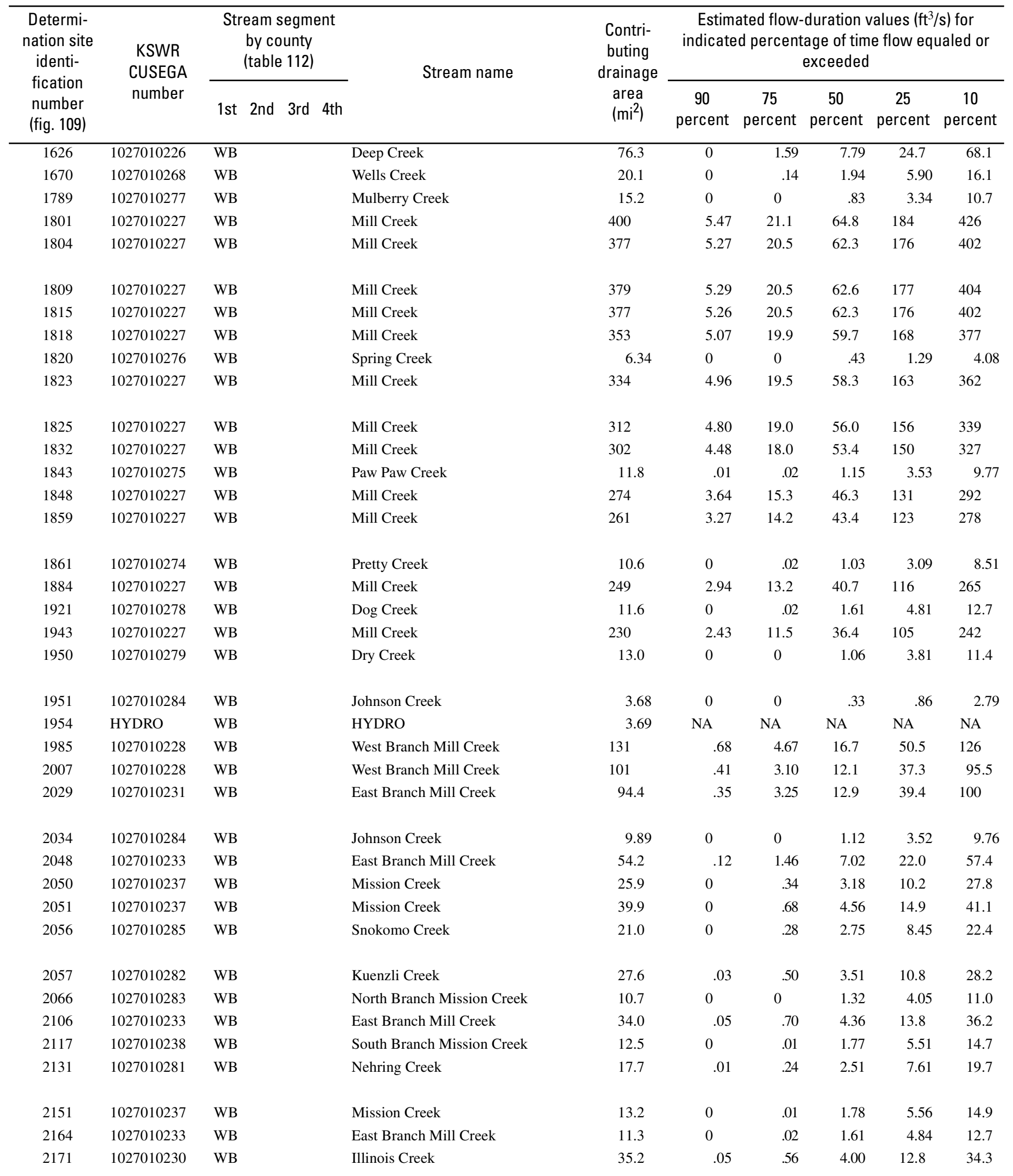


Table 105. Estimated flow-duration values, mean flow values, and peak-discharge frequency values for controlled and uncontrolled flow stream segments on the 1999 Kansas Surface Water Register for Wabaunsee County.-Continued

[KSWR, Kansas Surface Water Register; CUSEGA, catalog unit segment number alpha; $\mathrm{mi}^{2}$, square miles; $\mathrm{ft}^{3} / \mathrm{s}$, cubic feet per second; HYDRO, lake or other hydrologic structure; NA, not applicable; NRDitch, irrigation ditch; NRTribal, tribal stream]

\begin{tabular}{|c|c|c|c|c|c|c|c|}
\hline \multirow{2}{*}{$\begin{array}{l}\text { Determi- } \\
\text { nation site } \\
\text { identi- } \\
\text { fication } \\
\text { number } \\
\text { (fig. 109) }\end{array}$} & \multirow{2}{*}{$\begin{array}{c}\text { Estimated mean } \\
\text { flow } \\
\left(\mathrm{ft}^{3} / \mathrm{s}\right)\end{array}$} & \multicolumn{6}{|c|}{ Estimated peak discharge $\left(\mathrm{ft}^{3} / \mathrm{s}\right)$ for indicated peak-discharge frequency } \\
\hline & & 2-year & 5-year & 10-year & 25-year & 50-year & 100 -year \\
\hline 1626 & 45.7 & 4,400 & 9,050 & 13,100 & 19,200 & 24,500 & 30,200 \\
\hline 1801 & 241 & 10,700 & 21,800 & 31,200 & 45,300 & 57,300 & 70,500 \\
\hline 1804 & 229 & 10,900 & 22,200 & 31,600 & 45,700 & 57,600 & 70,900 \\
\hline 1809 & 230 & 10,600 & 21,700 & 31,000 & 44,900 & 56,700 & 69,800 \\
\hline 1815 & 229 & 10,900 & 22,200 & 31,600 & 45,700 & 57,700 & 70,900 \\
\hline 1825 & 194 & 11,600 & 23,000 & 32,400 & 46,300 & 58,000 & 70,800 \\
\hline 1832 & 188 & 11,900 & 23,400 & 32,900 & 46,800 & 58,500 & 71,300 \\
\hline 1843 & 7.74 & 1,150 & 2,560 & 3,780 & 5,670 & 7,240 & 9,050 \\
\hline 1848 & 170 & 11,200 & 22,000 & 30,900 & 44,000 & 55,100 & 67,200 \\
\hline 1859 & 162 & 11,200 & 21,800 & 30,600 & 43,600 & 54,500 & 66,300 \\
\hline 1861 & 6.86 & 1,080 & 2,390 & 3,530 & 5,280 & 6,750 & 8,420 \\
\hline 1884 & 156 & 11,000 & 21,400 & 30,000 & 42,700 & 53,400 & 65,000 \\
\hline 1921 & 9.11 & 1,200 & 2,620 & 3,860 & 5,760 & 7,340 & 9,150 \\
\hline 1943 & 144 & 10,900 & 21,000 & 29,400 & 41,800 & 52,200 & 63,400 \\
\hline 2034 & 7.49 & 1,110 & 2,410 & 3,540 & 5,250 & 6,690 & 8,320 \\
\hline 2048 & 38.1 & 5,270 & 10,300 & 14,500 & 20,700 & 25,900 & 31,500 \\
\hline 2050 & 19.5 & 1,960 & 4,340 & 6,430 & 9,640 & 12,300 & 15,400 \\
\hline 2051 & 28.5 & 3,890 & 7,840 & 11,200 & 16,300 & 20,600 & 25,200 \\
\hline 2056 & 15.6 & 1,670 & 3,700 & 5,500 & 8,260 & 10,600 & 13,200 \\
\hline 2057 & 19.5 & 1,930 & 4,330 & 6,460 & 9,750 & 12,500 & 15,700 \\
\hline 2066 & 8.23 & 1,160 & 2,530 & 3,720 & 5,520 & 7,030 & 8,750 \\
\hline 2106 & 24.8 & 4,230 & 8,340 & 11,800 & 16,900 & 21,200 & 25,700 \\
\hline 2117 & 10.4 & 1,300 & 2,820 & 4,140 & 6,150 & 7,830 & 9,740 \\
\hline 2131 & 13.7 & 1,540 & 3,390 & 5,010 & 7,500 & 9,590 & 12,000 \\
\hline 2151 & 10.7 & 1,340 & 2,910 & 4,280 & 6,360 & 8,100 & 10,100 \\
\hline 2164 & 9.10 & 1,200 & 2,620 & 3,840 & 5,720 & 7,280 & 9,060 \\
\hline 2171 & 23.9 & 4,230 & 8,430 & 12,000 & 17,200 & 21,700 & 26,400 \\
\hline
\end{tabular}


Table 105. Estimated flow-duration values, mean flow values, and peak-discharge frequency values for controlled and uncontrolled flow stream segments on the 1999 Kansas Surface Water Register for Wabaunsee County.-Continued

[KSWR, Kansas Surface Water Register; CUSEGA, catalog unit segment number alpha; $\mathrm{mi}^{2}$, square miles; $\mathrm{ft}^{3} / \mathrm{s}$, cubic feet per second; HYDRO, lake or other hydrologic structure; NA, not applicable; NRDitch, irrigation ditch; NRTribal, tribal stream]

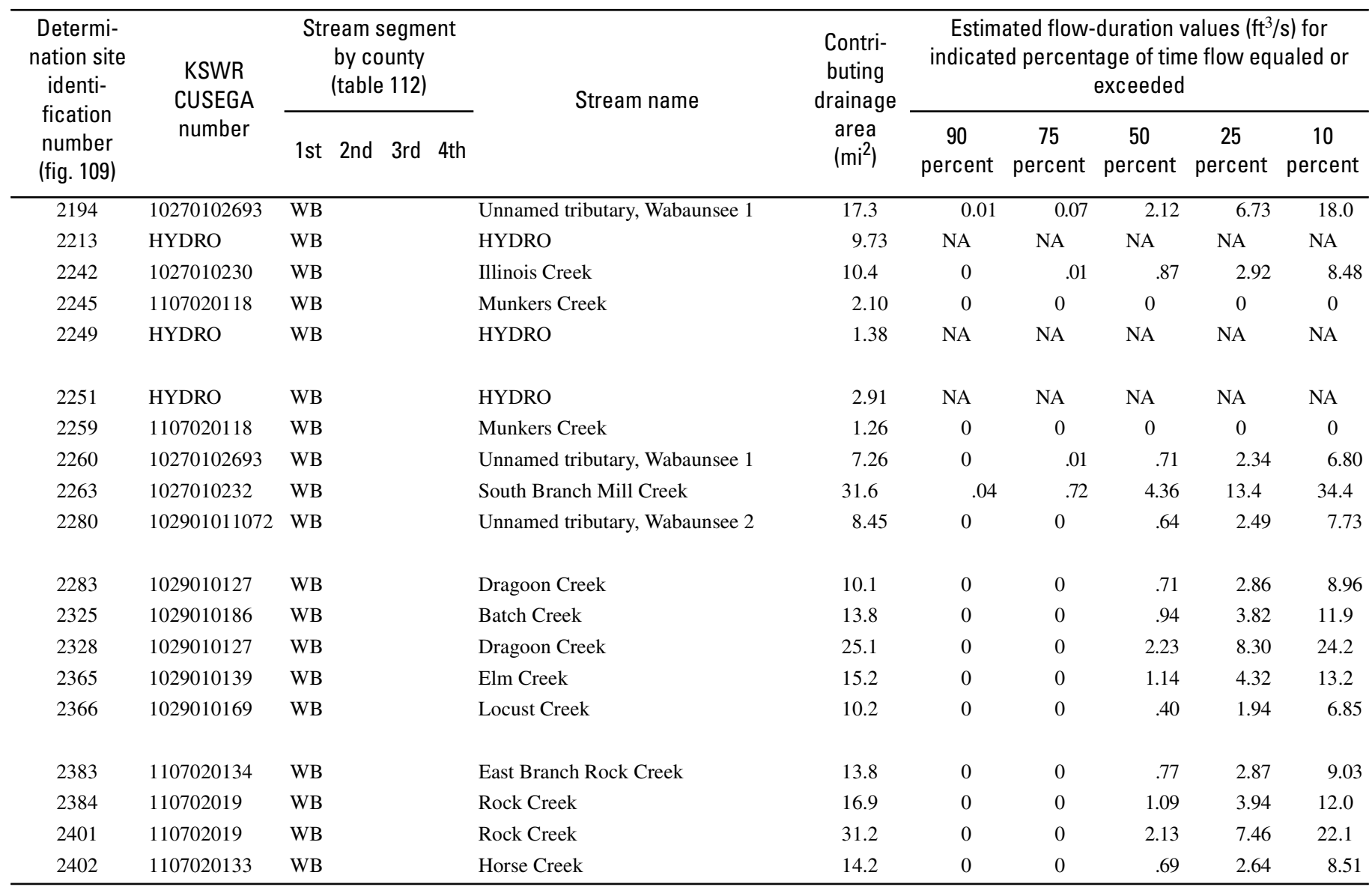


Table 105. Estimated flow-duration values, mean flow values, and peak-discharge frequency values for controlled and uncontrolled flow stream segments on the 1999 Kansas Surface Water Register for Wabaunsee County.-Continued

[KSWR, Kansas Surface Water Register; CUSEGA, catalog unit segment number alpha; $\mathrm{mi}^{2}$, square miles; $\mathrm{ft}^{3} / \mathrm{s}$, cubic feet per second; HYDRO, lake or other hydrologic structure; NA, not applicable; NRDitch, irrigation ditch; NRTribal, tribal stream]

\begin{tabular}{|c|c|c|c|c|c|c|c|}
\hline \multirow{2}{*}{$\begin{array}{l}\text { Determi- } \\
\text { nation site } \\
\text { identi- } \\
\text { fication } \\
\text { number } \\
\text { (fig. 109) }\end{array}$} & \multirow{2}{*}{$\begin{array}{c}\text { Estimated mean } \\
\text { flow } \\
\left(\mathrm{ft}^{3} / \mathrm{s}\right)\end{array}$} & \multicolumn{6}{|c|}{ Estimated peak discharge $\left(\mathrm{ft}^{3} / \mathrm{s}\right)$ for indicated peak-discharge frequency } \\
\hline & & 2-year & 5 -year & 10-year & 25-year & 50-year & 100-year \\
\hline 2194 & 13.0 & 1,510 & 3,340 & 4,940 & 7,390 & 9,450 & 11,800 \\
\hline 2213 & NA & NA & NA & NA & NA & NA & NA \\
\hline 2242 & 6.88 & 1,060 & 2,350 & 3,480 & 5,210 & 6,650 & 8,310 \\
\hline 2245 & .10 & 422 & 901 & 1,310 & 1,930 & 2,440 & 3,020 \\
\hline 2249 & NA & NA & NA & NA & NA & NA & NA \\
\hline 2251 & NA & NA & NA & NA & NA & NA & NA \\
\hline 2259 & 0 & 312 & 660 & 955 & 1,400 & 1,760 & 2,180 \\
\hline 2260 & 5.46 & 916 & 1,980 & 2,900 & 4,300 & 5,470 & 6,800 \\
\hline 2263 & 23.1 & 4,010 & 7,950 & 11,300 & 16,100 & 20,200 & 24,600 \\
\hline 2280 & 6.43 & 1,050 & 2,250 & 3,280 & 4,830 & 6,120 & 7,600 \\
\hline 2283 & 7.48 & 1,160 & 2,490 & 3,640 & 5,380 & 6,820 & 8,470 \\
\hline 2325 & 9.91 & 1,420 & 3,050 & 4,450 & 6,570 & 8,340 & 10,400 \\
\hline 2328 & 18.4 & 1,980 & 4,260 & 6,240 & 9,260 & 11,800 & 14,700 \\
\hline 2365 & 10.6 & 1,460 & 3,190 & 4,710 & 7,040 & 9,000 & 11,200 \\
\hline 2366 & 6.32 & 1,120 & 2,440 & 3,590 & 5,360 & 6,830 & 8,520 \\
\hline 2383 & 7.89 & 1,240 & 2,760 & 4,100 & 6,170 & 7,900 & 9,900 \\
\hline 2384 & 9.99 & 1,420 & 3,160 & 4,690 & 7,060 & 9,050 & 11,300 \\
\hline 2401 & 17.5 & 3,340 & 6,910 & 10,000 & 14,600 & 18,600 & 22,800 \\
\hline 2402 & 7.63 & 1,220 & 2,740 & 4,090 & 6,170 & 7,910 & 9,920 \\
\hline
\end{tabular}




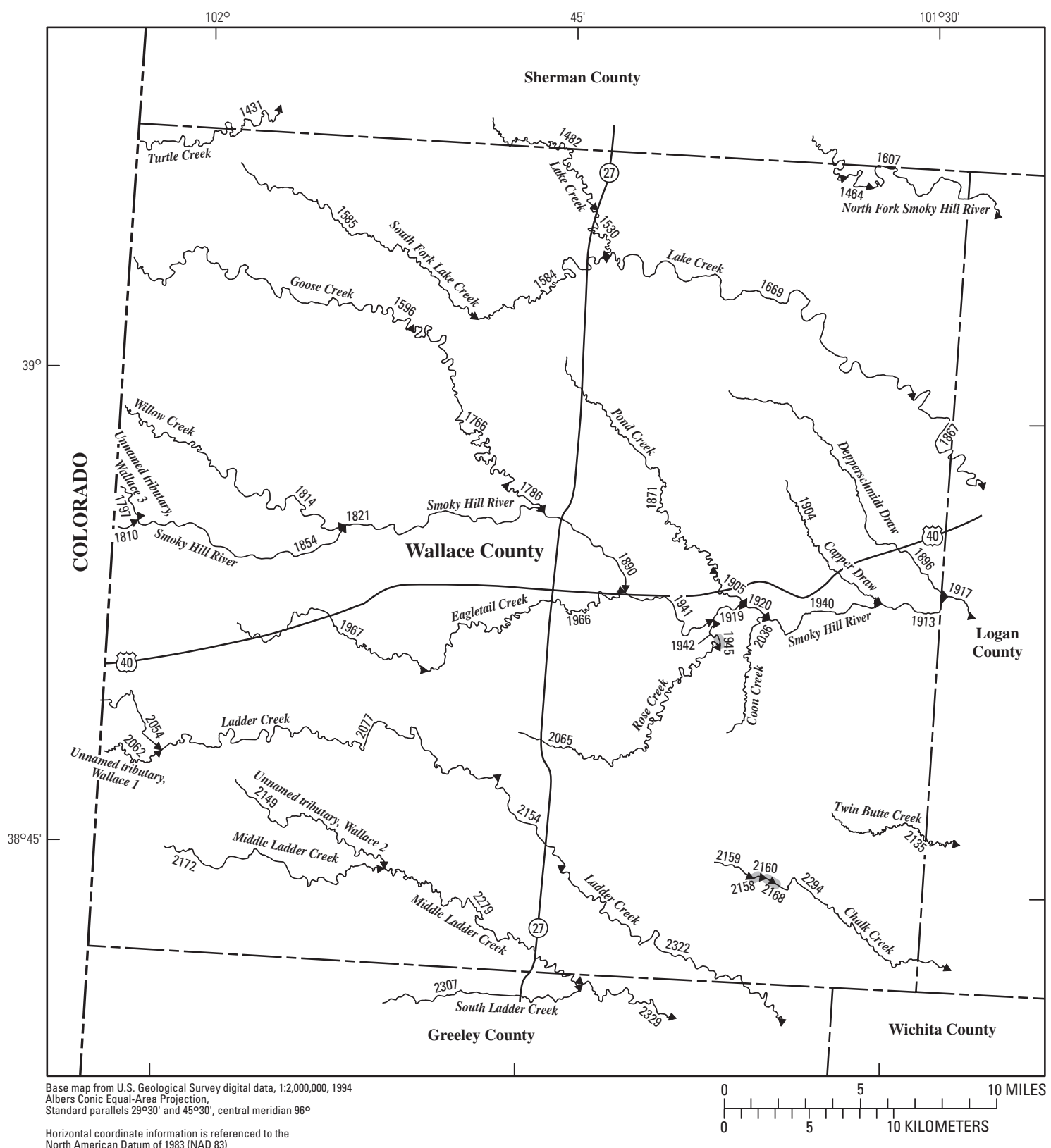

\section{EXPLANATION}

\$2172 Location of streamflow-statistics determination site (small triangle) and associated identification number-small triangle points in downstream direction

${ }^{07156100} \Delta$ U.S. Geological Survey streamflow-gaging station and number used for estimates of flow duration

$07156220 \bigwedge$

U.S. Geological Survey streamflow-gaging station and number used for estimates of peak-discharge frequency values

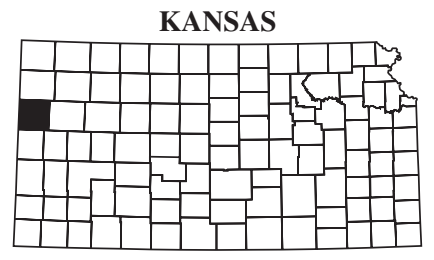

Index map

Lake and determination site identification number

Figure 110. Location of streamflow-statistics determination sites, associated identification numbers, and U.S. Geological Survey streamflow-gaging stations used in the flow-duration and peak-discharge frequency analyses for Wallace County. 
Table 106. Estimated flow-duration values, mean flow values, and peak-discharge frequency values for controlled and uncontrolled flow stream segments on the 1999 Kansas Surface Water Register for Wallace County.

[KSWR, Kansas Surface Water Register; CUSEGA, catalog unit segment number alpha; $\mathrm{mi}^{2}$, square miles; $\mathrm{ft}^{3} / \mathrm{s}$, cubic feet per second; HYDRO, lake or other hydrologic structure; NA, not applicable; NRDitch, irrigation ditch; NRTribal, tribal stream]

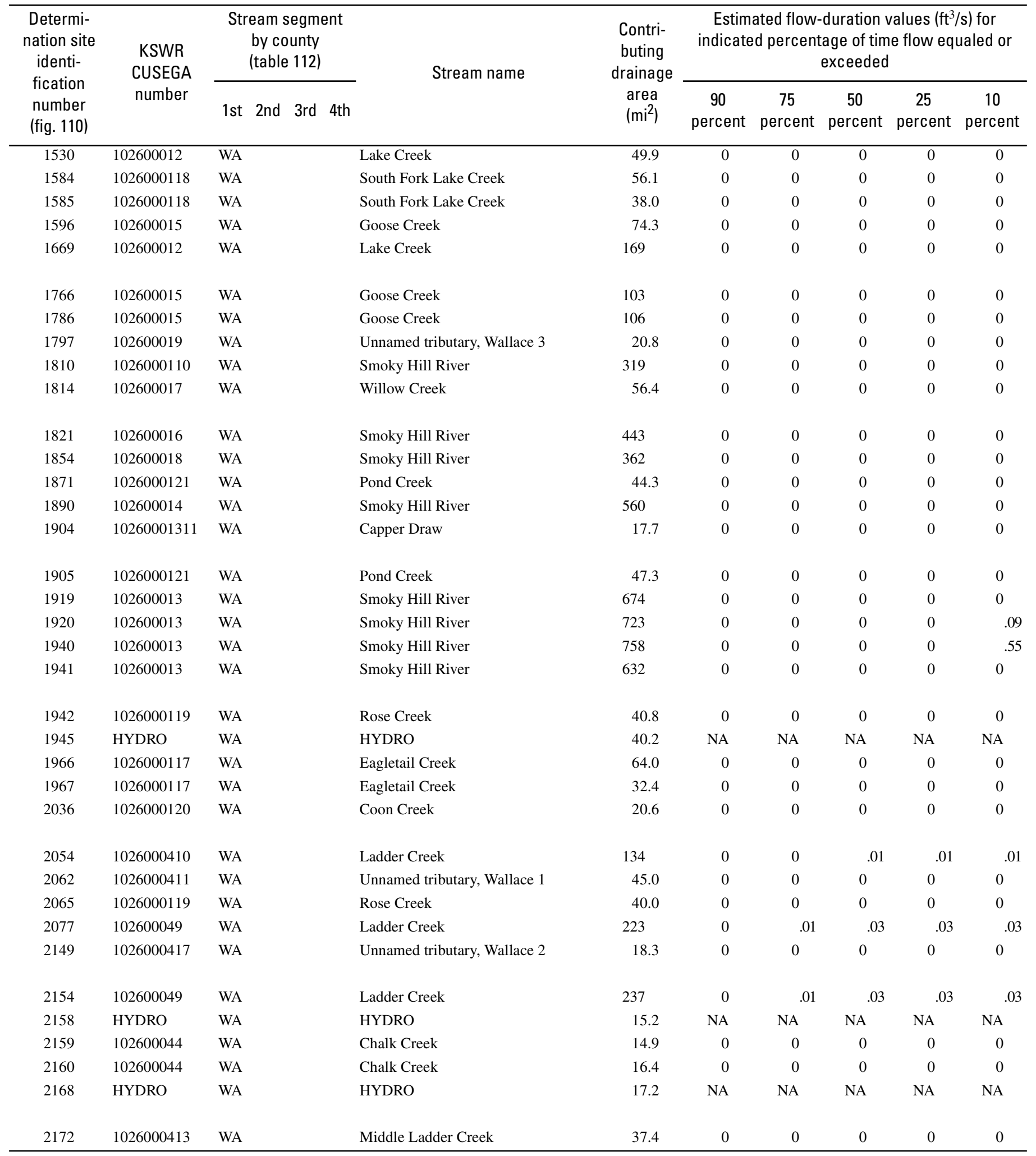


Table 106. Estimated flow-duration values, mean flow values, and peak-discharge frequency values for controlled and uncontrolled flow stream segments on the 1999 Kansas Surface Water Register for Wallace County.-Continued

[KSWR, Kansas Surface Water Register; CUSEGA, catalog unit segment number alpha; $\mathrm{mi}^{2}$, square miles; $\mathrm{ft}^{3} / \mathrm{s}$, cubic feet per second; HYDRO, lake or other hydrologic structure; NA, not applicable; NRDitch, irrigation ditch; NRTribal, tribal stream]

\begin{tabular}{|c|c|c|c|c|c|c|c|}
\hline \multirow{2}{*}{$\begin{array}{l}\text { Determi- } \\
\text { nation site } \\
\text { identi- } \\
\text { fication } \\
\text { number } \\
\text { (fig. 110) }\end{array}$} & \multirow{2}{*}{$\begin{array}{c}\text { Estimated mean } \\
\text { flow } \\
\left(\mathrm{ft}^{3} / \mathrm{s}\right)\end{array}$} & \multicolumn{6}{|c|}{ Estimated peak discharge $\left(\mathrm{ft}^{3} / \mathrm{s}\right)$ for indicated peak-discharge frequency } \\
\hline & & 2-year & 5-year & 10-year & 25-year & 50-year & 100-year \\
\hline 1530 & 0 & 194 & 700 & 1,310 & 2,440 & 3,590 & 5,000 \\
\hline 1596 & 0 & 256 & 907 & 1,680 & 3,120 & 4,580 & 6,370 \\
\hline 1669 & .89 & 416 & 1,400 & 2,550 & 4,650 & 6,750 & 9,320 \\
\hline 1766 & 0 & 275 & 969 & 1,790 & 3,320 & 4,870 & 6,760 \\
\hline 1786 & 0 & 273 & 960 & 1,780 & 3,290 & 4,820 & 6,690 \\
\hline 1821 & .62 & 532 & 1,870 & 3,470 & 6,480 & 9,570 & 13,400 \\
\hline 1854 & 0 & 419 & 1,530 & 2,880 & 5,460 & 8,130 & 11,500 \\
\hline 1871 & 0 & 223 & 757 & 1,370 & 2,490 & 3,600 & 4,930 \\
\hline 1890 & 1.71 & 673 & 2,280 & 4,190 & 7,720 & 11,300 & 15,800 \\
\hline 1904 & 0 & 255 & 869 & 1,550 & 2,760 & 3,890 & 5,270 \\
\hline 1905 & 0 & 210 & 721 & 1,310 & 2,390 & 3,460 & 4,760 \\
\hline 1919 & 3.00 & 818 & 2,710 & 4,930 & 9,000 & 13,100 & 18,200 \\
\hline 1920 & 3.62 & 877 & 2,880 & 5,210 & 9,490 & 13,800 & 19,200 \\
\hline 1940 & 4.08 & 906 & 2,960 & 5,360 & 9,750 & 14,200 & 19,700 \\
\hline 2054 & 0 & 190 & 753 & 1,480 & 2,890 & 4,380 & 6,280 \\
\hline 2062 & 0 & 107 & 438 & 869 & 1,720 & 2,610 & 3,750 \\
\hline 2065 & 0 & 217 & 758 & 1,390 & 2,560 & 3,730 & 5,140 \\
\hline 2077 & 0 & 271 & 1,040 & 2,010 & 3,890 & 5,880 & 8,400 \\
\hline 2149 & 0 & 220 & 780 & 1,420 & 2,560 & 3,650 & 4,990 \\
\hline 2154 & 0 & 285 & 1,090 & 2,100 & 4,060 & 6,120 & 8,740 \\
\hline 2158 & NA & NA & NA & NA & NA & NA & NA \\
\hline 2159 & 0 & 213 & 737 & 1,320 & 2,370 & 3,360 & 4,560 \\
\hline 2160 & 0 & 226 & 783 & 1,410 & 2,520 & 3,570 & 4,860 \\
\hline 2168 & NA & NA & NA & NA & NA & NA & NA \\
\hline 2172 & 0 & 116 & 457 & 890 & 1,720 & 2,590 & 3,680 \\
\hline
\end{tabular}




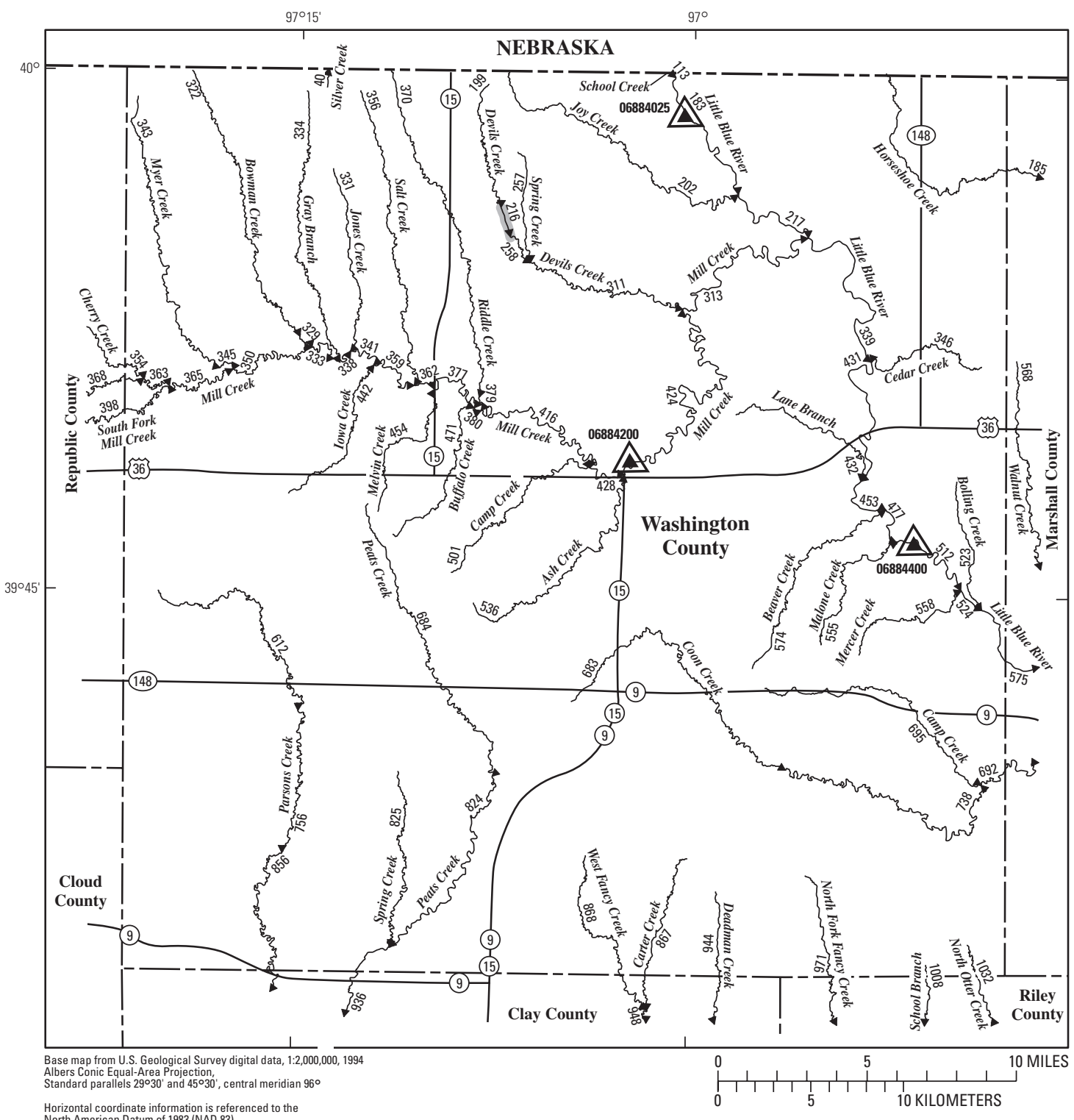

EXPLANATION

$\stackrel{869}{ }$ Location of streamflow-statistics determination site (small triangle) and associated identification number-small triangle points in downstream direction

$06884200 \Delta$ U.S. Geological Survey streamflow-gaging station and number used for estimates of flow duration

06856320

U.S. Geological Survey streamflow-gaging station and number used for estimates of peak-discharge frequency values

KANSAS

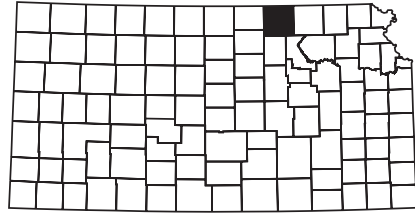

Index map

216 Lake and determination site identification number

Figure 111. Location of streamflow-statistics determination sites, associated identification numbers, and U.S. Geological Survey streamflow-gaging stations used in the flow-duration and peak-discharge frequency analyses for Washington County. 
Table 107. Estimated flow-duration values, mean flow values, and peak-discharge frequency values for controlled and uncontrolled flow stream segments on the 1999 Kansas Surface Water Register for Washington County.

[KSWR, Kansas Surface Water Register; CUSEGA, catalog unit segment number alpha; $\mathrm{mi}^{2}$, square miles; $\mathrm{ft}^{3} / \mathrm{s}$, cubic feet per second; HYDRO, lake or other hydrologic structure; NA, not applicable; NRDitch, irrigation ditch; NRTribal, tribal stream]

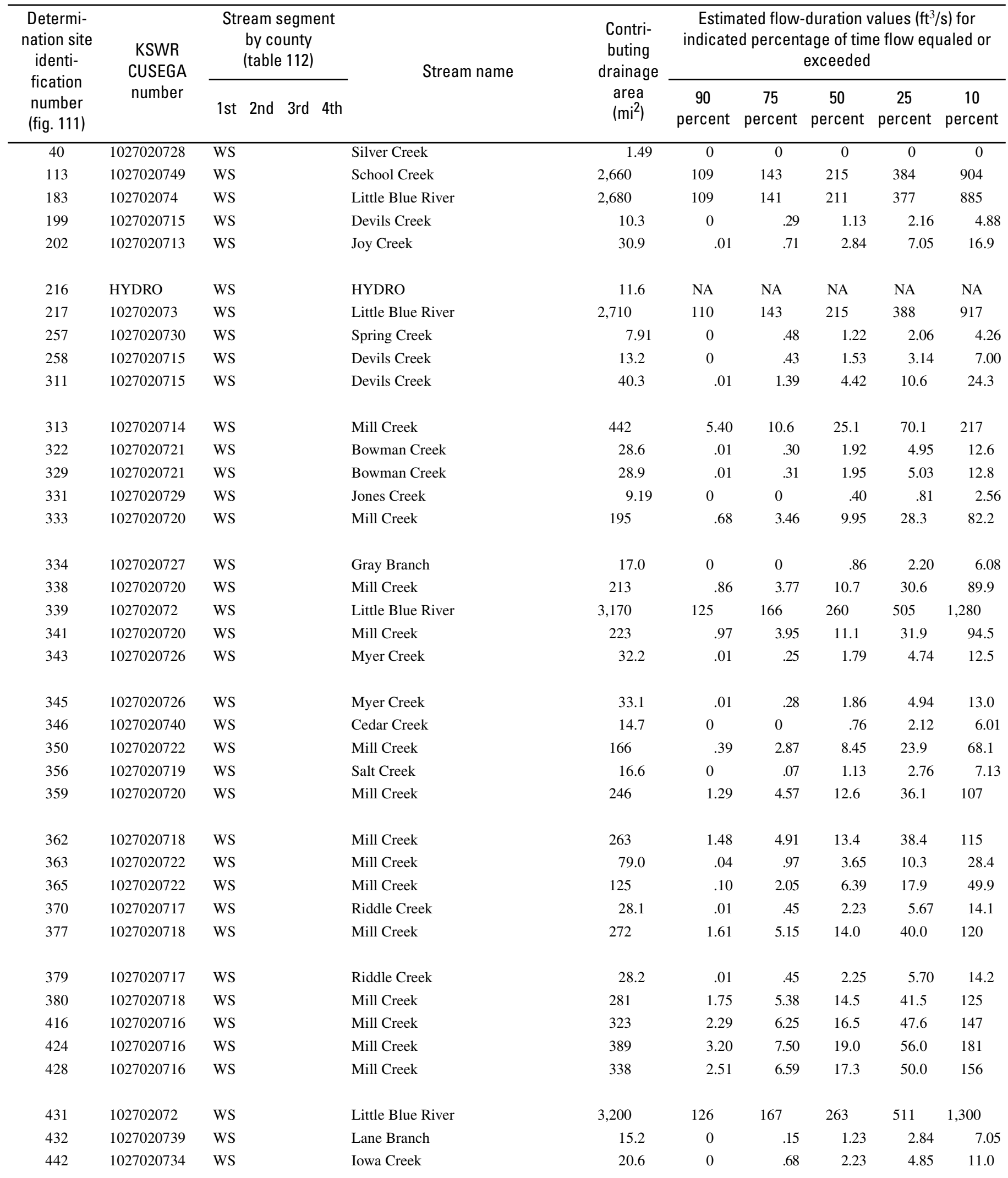


Table 107. Estimated flow-duration values, mean flow values, and peak-discharge frequency values for controlled and uncontrolled flow stream segments on the 1999 Kansas Surface Water Register for Washington County.-Continued

[KSWR, Kansas Surface Water Register; CUSEGA, catalog unit segment number alpha; $\mathrm{mi}^{2}$, square miles; $\mathrm{ft}^{3} / \mathrm{s}$, cubic feet per second; HYDRO, lake or other hydrologic structure; NA, not applicable; NRDitch, irrigation ditch; NRTribal, tribal stream]

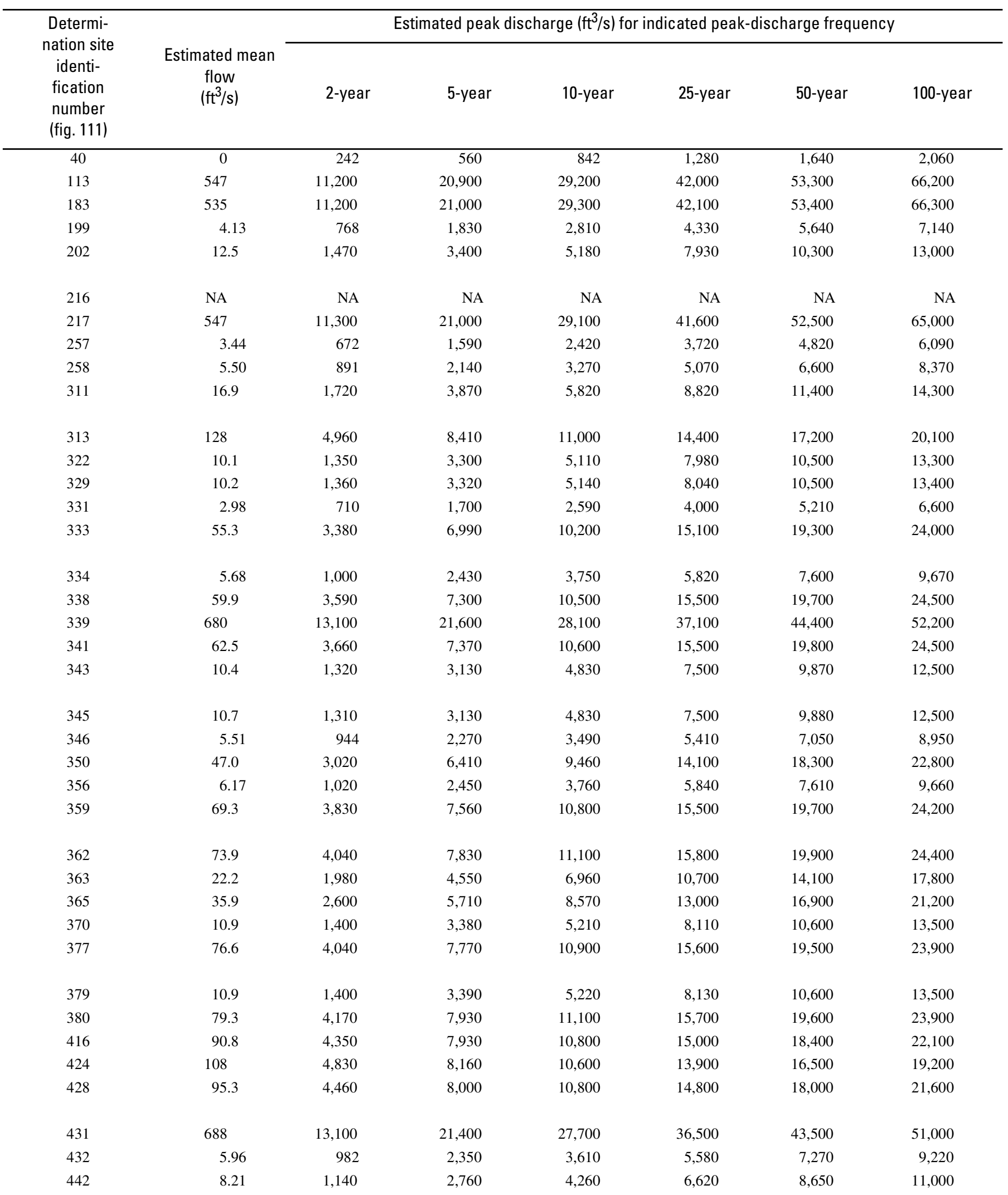


Table 107. Estimated flow-duration values, mean flow values, and peak-discharge frequency values for controlled and uncontrolled flow stream segments on the 1999 Kansas Surface Water Register for Washington County.-Continued

[KSWR, Kansas Surface Water Register; CUSEGA, catalog unit segment number alpha; $\mathrm{mi}^{2}$, square miles; $\mathrm{ft}^{3} / \mathrm{s}$, cubic feet per second; HYDRO, lake or other hydrologic structure; NA, not applicable; NRDitch, irrigation ditch; NRTribal, tribal stream]

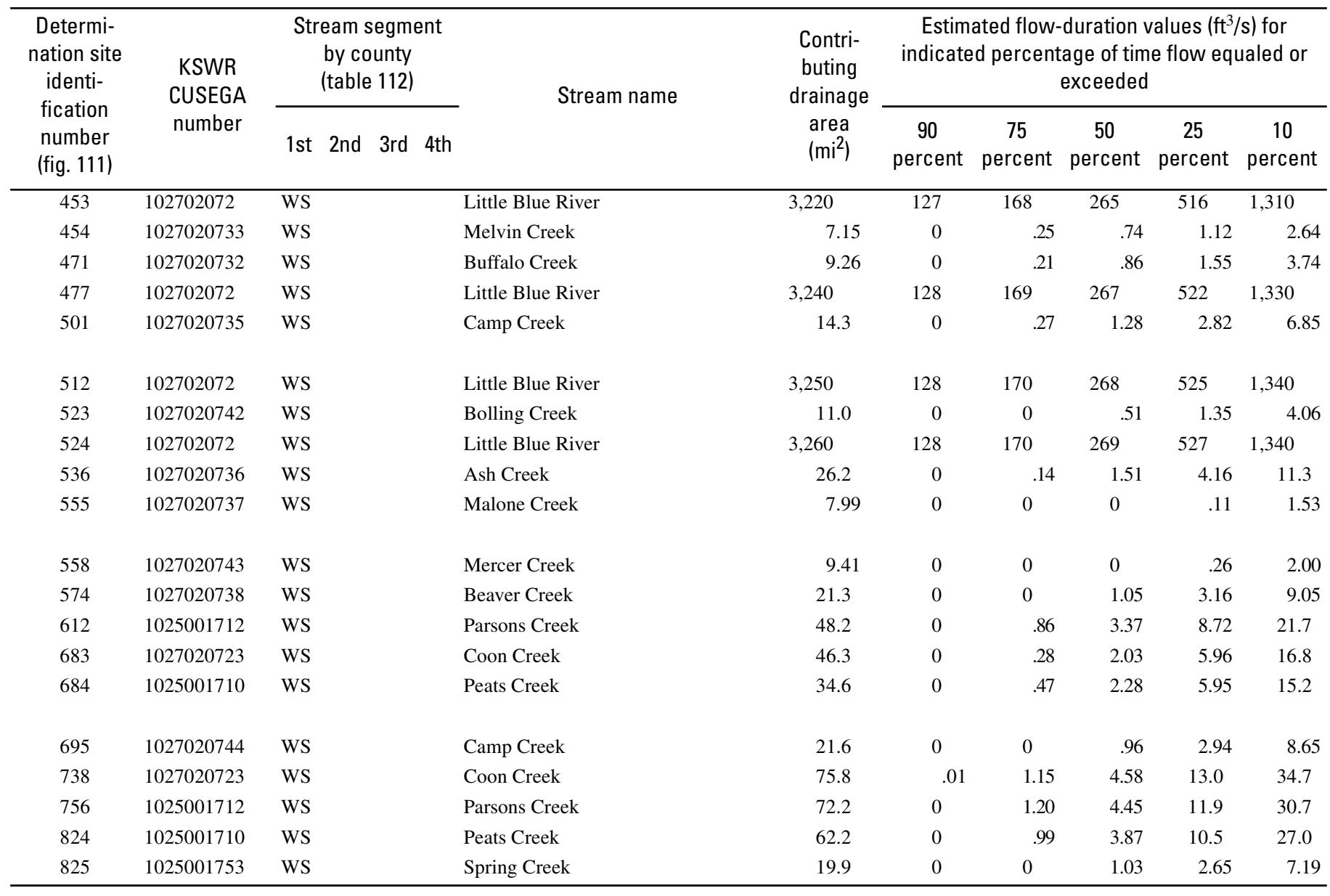


Table 107. Estimated flow-duration values, mean flow values, and peak-discharge frequency values for controlled and uncontrolled flow stream segments on the 1999 Kansas Surface Water Register for Washington County.-Continued

[KSWR, Kansas Surface Water Register; CUSEGA, catalog unit segment number alpha; $\mathrm{mi}^{2}$, square miles; $\mathrm{ft}^{3} / \mathrm{s}$, cubic feet per second; HYDRO, lake or other hydrologic structure; NA, not applicable; NRDitch, irrigation ditch; NRTribal, tribal stream]

\begin{tabular}{|c|c|c|c|c|c|c|c|}
\hline \multirow{2}{*}{$\begin{array}{l}\text { Determi- } \\
\text { nation site } \\
\text { identi- } \\
\text { fication } \\
\text { number } \\
\text { (fig. 111) }\end{array}$} & \multirow{2}{*}{$\begin{array}{c}\text { Estimated mean } \\
\text { flow } \\
\left(\mathrm{ft}^{3} / \mathrm{s}\right)\end{array}$} & \multicolumn{6}{|c|}{ Estimated peak discharge $\left(\mathrm{ft}^{3} / \mathrm{s}\right)$ for indicated peak-discharge frequency } \\
\hline & & 2-year & 5-year & 10-year & 25-year & 50-year & 100-year \\
\hline 453 & 694 & 13,100 & 21,400 & 27,600 & 36,200 & 43,000 & 50,200 \\
\hline 477 & 701 & 13,200 & 21,400 & 27,500 & 35,800 & 42,500 & 49,500 \\
\hline 501 & 5.76 & 980 & 2,320 & 3,540 & 5,460 & 7,090 & 8,970 \\
\hline 512 & 704 & 13,100 & 21,200 & 27,200 & 35,400 & 41,900 & 48,700 \\
\hline 523 & 4.05 & 803 & 1,920 & 2,930 & 4,530 & 5,890 & 7,470 \\
\hline 558 & 2.95 & 752 & 1,780 & 2,710 & 4,170 & 5,410 & 6,840 \\
\hline 574 & 7.99 & 1,210 & 2,910 & 4,470 & 6,930 & 9,040 & 11,500 \\
\hline 612 & 16.6 & 1,810 & 4,140 & 6,290 & 9,620 & 12,600 & 15,800 \\
\hline 683 & 14.6 & 1,740 & 4,070 & 6,240 & 9,620 & 12,600 & 16,000 \\
\hline 684 & 12.2 & 1,300 & 3,110 & 4,830 & 7,510 & 9,900 & 12,600 \\
\hline 695 & 7.91 & 1,240 & 2,970 & 4,560 & 7,060 & 9,200 & 11,700 \\
\hline 738 & 26.5 & 2,050 & 4,800 & 7,380 & 11,500 & 15,100 & 19,100 \\
\hline 756 & 23.2 & 1,890 & 4,440 & 6,840 & 10,600 & 14,000 & 17,700 \\
\hline 824 & 20.8 & 1,570 & 3,790 & 5,910 & 9,270 & 12,300 & 15,700 \\
\hline
\end{tabular}




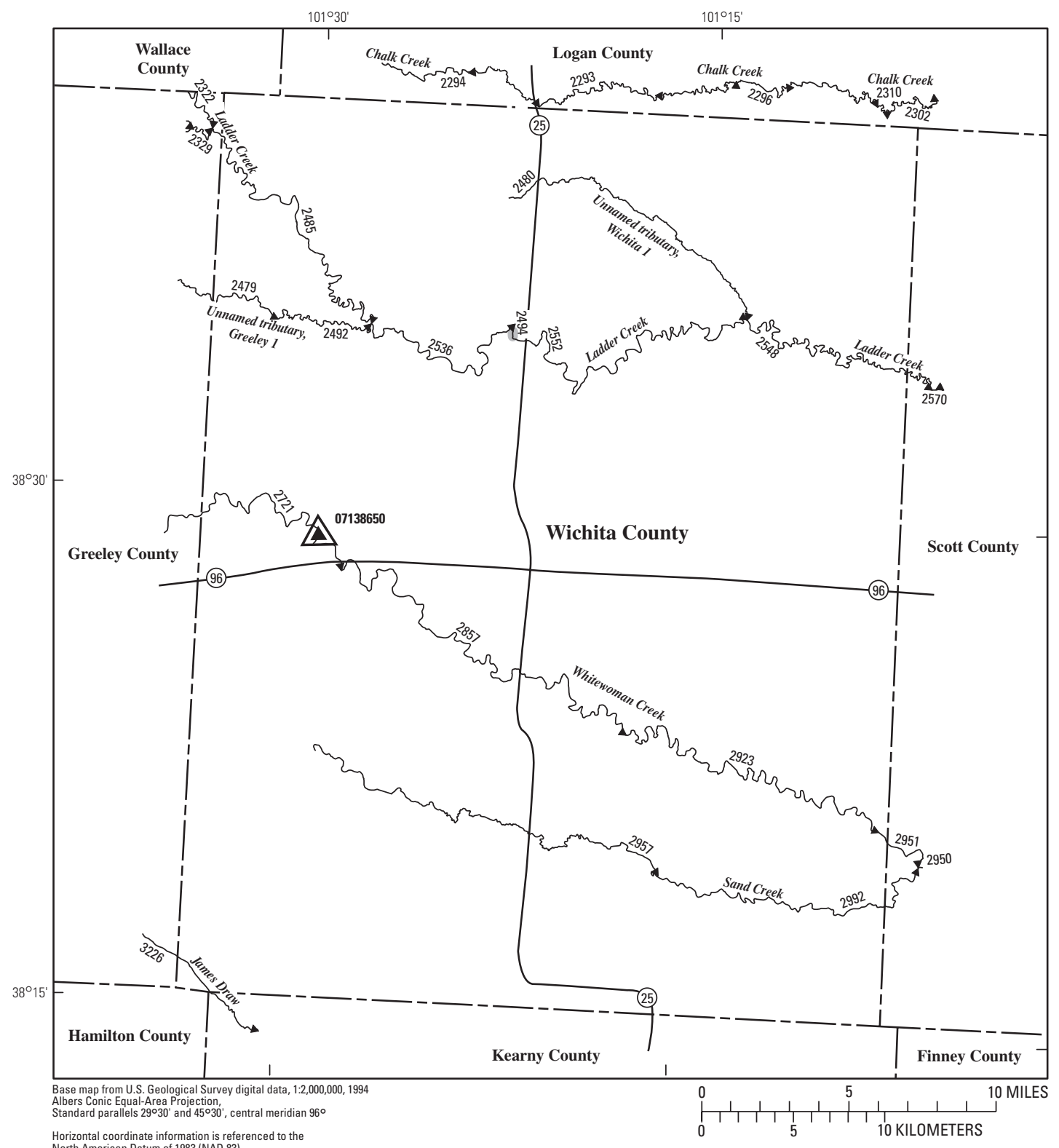

EXPLANATION

\$226 Location of streamflow-statistics determination site (small triangle) and associated identification number-small triangle points in downstream direction

$07138650 \Delta$ U.S. Geological Survey streamflow-gaging station and number used for estimates of flow duration

${ }^{07138650} \triangle$ U.S. Geological Survey streamflow-gaging station and number used for estimates of peak-discharge frequency values

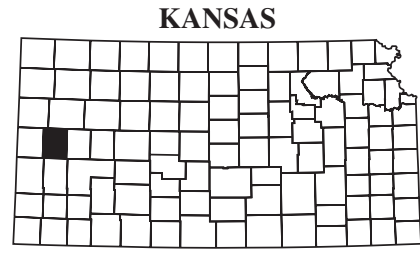

Index map 2494

Lake and determination site identification number

Figure 112. Location of streamflow-statistics determination sites, associated identification numbers, and U.S. Geological Survey streamflow-gaging stations used in the flow-duration and peak-discharge frequency analyses for Wichita County. 
Table 108. Estimated flow-duration values, mean flow values, and peak-discharge frequency values for controlled and uncontrolled flow stream segments on the 1999 Kansas Surface Water Register for Wichita County.

[KSWR, Kansas Surface Water Register; CUSEGA, catalog unit segment number alpha; $\mathrm{mi}^{2}$, square miles; $\mathrm{ft}^{3} / \mathrm{s}$, cubic feet per second; HYDRO, lake or other hydrologic structure; NA, not applicable; NRDitch, irrigation ditch; NRTribal, tribal stream]

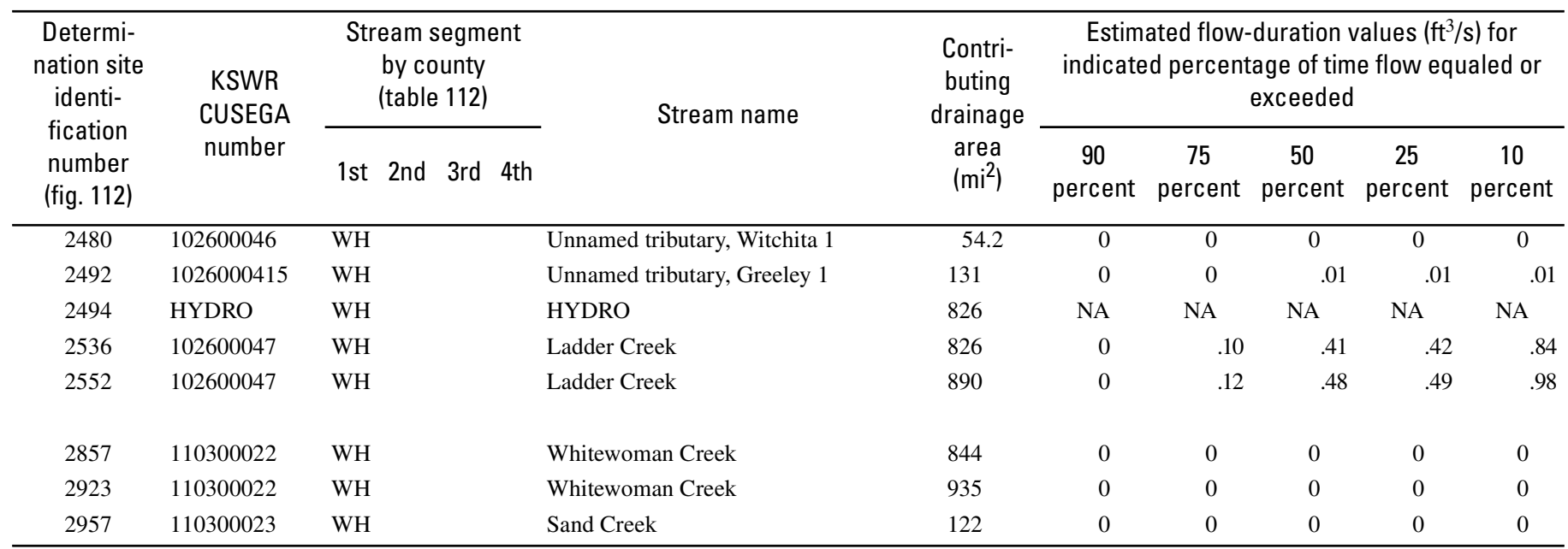


Table 108. Estimated flow-duration values, mean flow values, and peak-discharge frequency values for controlled and uncontrolled flow stream segments on the 1999 Kansas Surface Water Register for Wichita County.-Continued

[KSWR, Kansas Surface Water Register; CUSEGA, catalog unit segment number alpha; $\mathrm{mi}^{2}$, square miles; $\mathrm{ft}^{3} / \mathrm{s}$, cubic feet per second; HYDRO, lake or other hydrologic structure; NA, not applicable; NRDitch, irrigation ditch; NRTribal, tribal stream]

\begin{tabular}{|c|c|c|c|c|c|c|c|}
\hline \multirow{2}{*}{$\begin{array}{l}\text { Determi- } \\
\text { nation site } \\
\text { identi- } \\
\text { fication } \\
\text { number } \\
\text { (fig. 112) }\end{array}$} & \multirow{2}{*}{$\begin{array}{c}\text { Estimated mean } \\
\text { flow } \\
\left(\mathrm{ft}^{3} / \mathrm{s}\right)\end{array}$} & \multicolumn{6}{|c|}{ Estimated peak discharge $\left(\mathrm{ft}^{3} / \mathrm{s}\right)$ for indicated peak-discharge frequency } \\
\hline & & 2-year & 5-year & 10-year & 25-year & 50-year & 100-year \\
\hline 2480 & 0 & 210 & 759 & 1,420 & 2,650 & 3,910 & 5,460 \\
\hline 2492 & 0 & 253 & 951 & 1,820 & 3,470 & 5,200 & 7,360 \\
\hline 2494 & NA & NA & NA & NA & NA & NA & NA \\
\hline 2536 & 1.28 & 523 & 1,990 & 3,890 & 7,700 & 11,900 & 17,400 \\
\hline 2552 & 1.57 & 533 & 2,040 & 4,000 & 7,970 & 12,300 & 18,100 \\
\hline 2857 & 1.46 & 260 & 1,490 & 3,490 & 8,200 & 13,900 & 21,800 \\
\hline 2923 & 2.09 & 308 & 1,650 & 3,760 & 8,690 & 14,600 & 22,800 \\
\hline 2957 & 0 & 250 & 928 & 1,770 & 3,360 & 5,000 & 7,050 \\
\hline
\end{tabular}




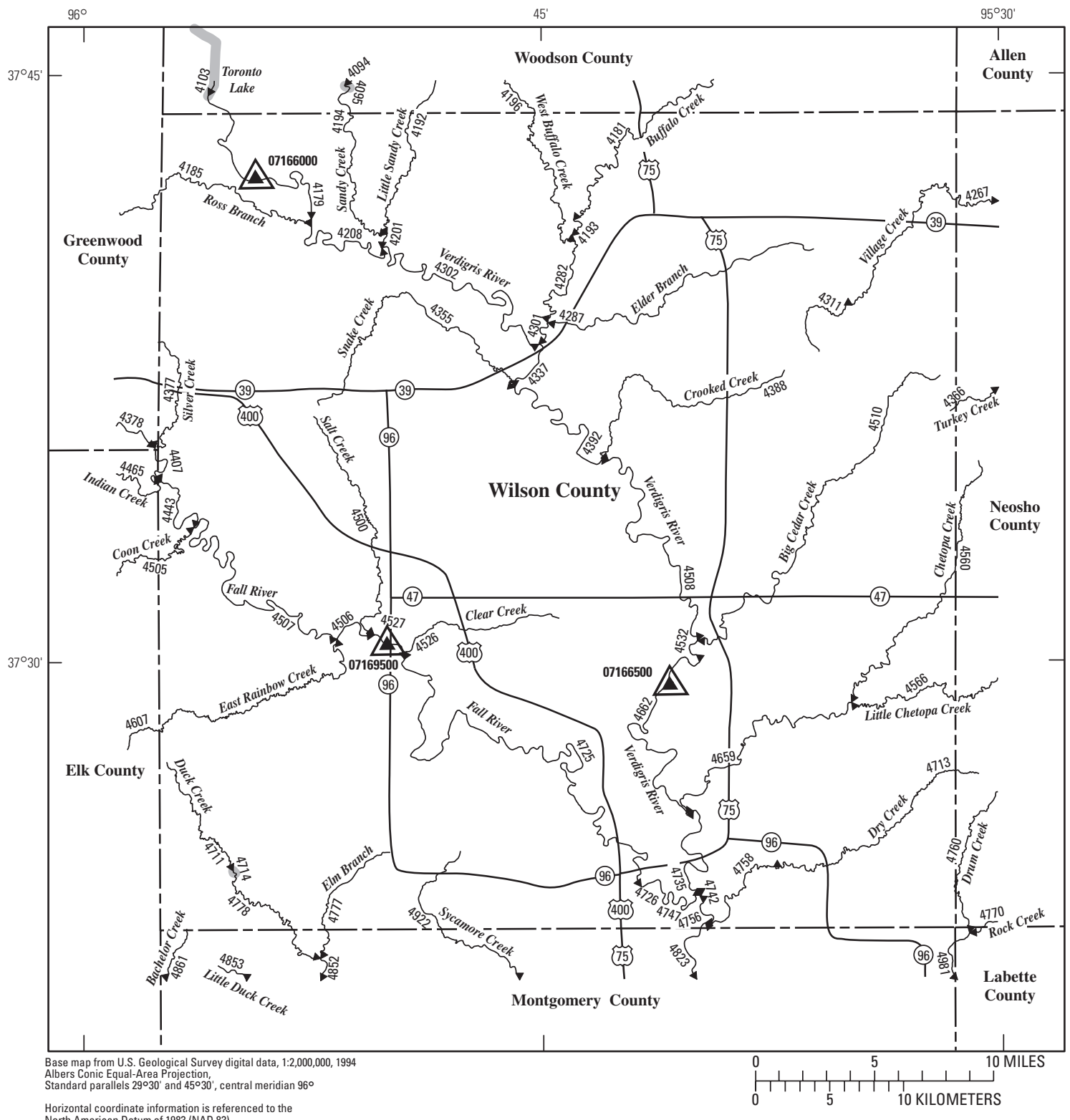

EXPLANATION

$\$ 4853$ Location of streamflow-statistics determination site (small triangle) and associated identification number-small triangle points in downstream direction

$07169500 \Delta$ U.S. Geological Survey streamflow-gaging station and number used for estimates of flow duration

${ }^{07166500} \triangle$ U.S. Geological Survey streamflow-gaging station and number used for estimates of peak-discharge frequency values

KANSAS

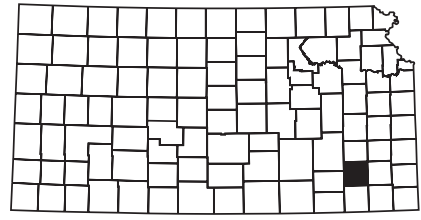

Index map

4103 Lake and determination site identification number

Figure 113. Location of streamflow-statistics determination sites, associated identification numbers, and U.S. Geological Survey streamflow-gaging stations used in the flow-duration and peak-discharge frequency analyses for Wilson County. 
Table 109. Estimated flow-duration values, mean flow values, and peak-discharge frequency values for controlled and uncontrolled flow stream segments on the 1999 Kansas Surface Water Register for Wilson County.

[KSWR, Kansas Surface Water Register; CUSEGA, catalog unit segment number alpha; $\mathrm{mi}^{2}$, square miles; $\mathrm{ft}^{3} / \mathrm{s}$, cubic feet per second; HYDRO, lake or other hydrologic structure; NA, not applicable; NRDitch, irrigation ditch; NRTribal, tribal stream]

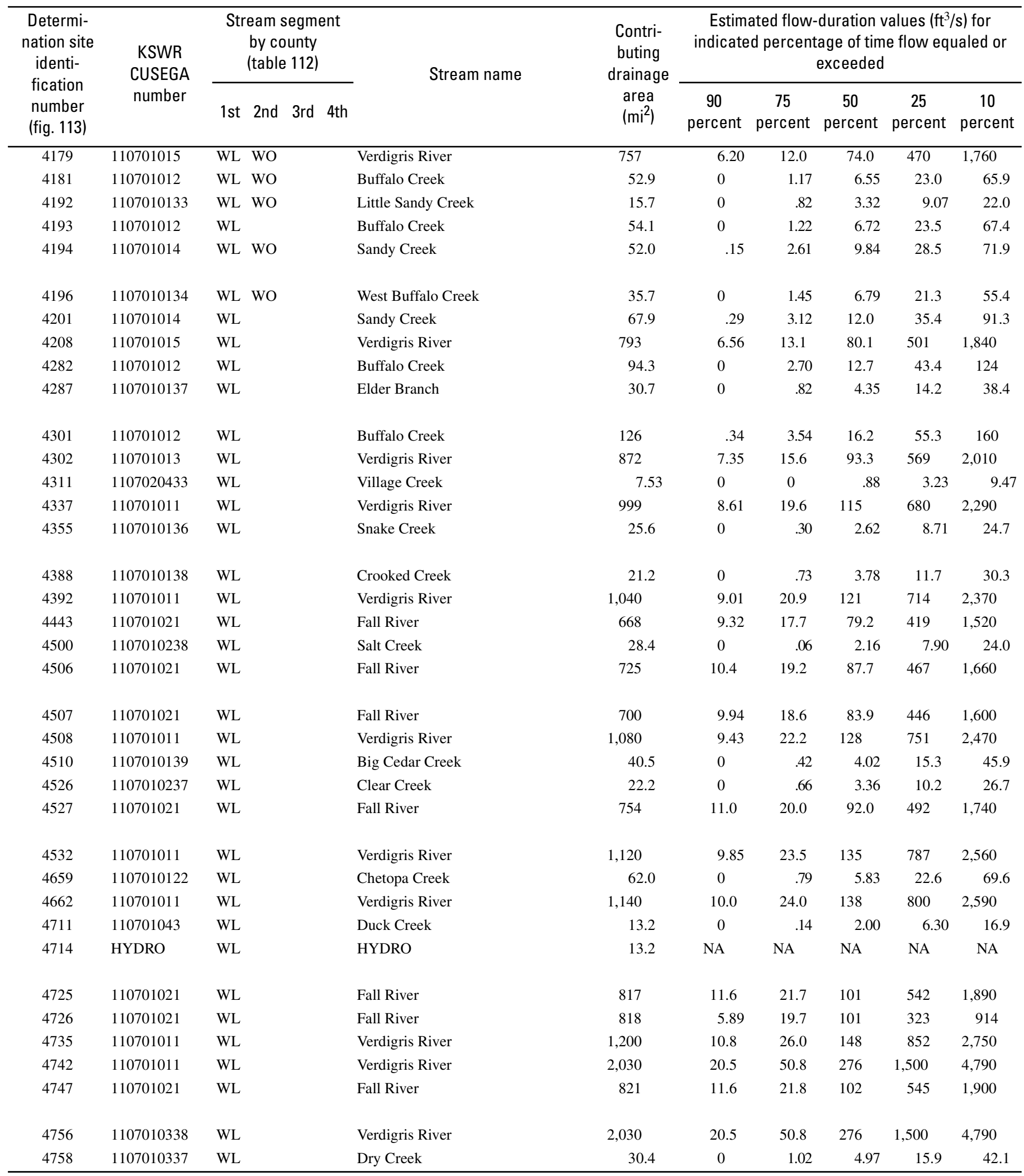


Table 109. Estimated flow-duration values, mean flow values, and peak-discharge frequency values for controlled and uncontrolled flow stream segments on the 1999 Kansas Surface Water Register for Wilson County.-Continued

[KSWR, Kansas Surface Water Register; CUSEGA, catalog unit segment number alpha; $\mathrm{mi}^{2}$, square miles; $\mathrm{ft}^{3} / \mathrm{s}$, cubic feet per second; HYDRO, lake or other hydrologic structure; NA, not applicable; NRDitch, irrigation ditch; NRTribal, tribal stream]

\begin{tabular}{|c|c|c|c|c|c|c|c|}
\hline \multirow{2}{*}{$\begin{array}{l}\text { Determi- } \\
\text { nation site } \\
\text { identi- } \\
\text { fication } \\
\text { number } \\
\text { (fig. 113) }\end{array}$} & \multirow{2}{*}{$\begin{array}{c}\text { Estimated mean } \\
\text { flow } \\
\left(\mathrm{ft}^{3} / \mathrm{s}\right)\end{array}$} & \multicolumn{6}{|c|}{ Estimated peak discharge $\left(\mathrm{ft}^{3} / \mathrm{s}\right)$ for indicated peak-discharge frequency } \\
\hline & & 2-year & 5-year & 10-year & 25-year & 50-year & 100-year \\
\hline 4179 & 520 & 5,290 & 7,490 & 8,500 & 9,400 & 9,860 & 10,200 \\
\hline 4193 & 47.6 & 4,580 & 8,710 & 12,200 & 17,200 & 21,400 & 25,900 \\
\hline 4194 & 45.9 & 4,010 & 7,570 & 10,500 & 14,800 & 18,300 & 22,000 \\
\hline 4196 & 36.1 & 5,020 & 8,980 & 12,200 & 16,600 & 20,300 & 24,100 \\
\hline 4201 & 57.9 & 4,700 & 8,790 & 12,200 & 17,100 & 21,100 & 25,400 \\
\hline 4301 & 105 & 6,890 & 12,700 & 17,600 & 24,700 & 30,600 & 36,900 \\
\hline 4302 & 607 & 7,290 & 11,100 & 13,200 & 15,300 & 16,600 & 17,600 \\
\hline 4311 & 7.72 & 1,440 & 2,810 & 3,940 & 5,600 & 6,950 & 8,480 \\
\hline 4337 & 703 & 9,490 & 15,100 & 18,300 & 21,800 & 24,000 & 25,800 \\
\hline 4355 & 19.2 & 2,420 & 5,070 & 7,340 & 10,800 & 13,600 & 16,900 \\
\hline 4388 & 21.0 & 2,430 & 4,920 & 7,040 & 10,200 & 12,800 & 15,800 \\
\hline 4392 & 733 & 10,200 & 16,400 & 20,000 & 23,900 & 26,300 & 28,400 \\
\hline 4443 & 488 & 7,850 & 13,700 & 17,500 & 22,000 & 25,100 & 28,000 \\
\hline 4500 & 19.8 & 2,600 & 5,440 & 7,880 & 11,600 & 14,600 & 18,100 \\
\hline 4532 & 797 & 11,600 & 19,000 & 23,400 & 28,200 & 31,200 & 33,800 \\
\hline 4659 & 51.8 & 5,470 & 10,300 & 14,400 & 20,300 & 25,300 & 30,600 \\
\hline 4662 & 808 & 11,900 & 19,500 & 24,000 & 29,000 & 32,100 & 34,800 \\
\hline 4711 & 12.3 & 1,660 & 3,430 & 4,930 & 7,170 & 9,020 & 11,100 \\
\hline 4714 & NA & NA & NA & NA & NA & NA & NA \\
\hline 4725 & 597 & 10,100 & 17,300 & 22,200 & 28,200 & 32,500 & 36,300 \\
\hline 4726 & 502 & 15,200 & 36,400 & 57,300 & 92,500 & 125,000 & 164,000 \\
\hline 4735 & 858 & 12,300 & 19,800 & 24,400 & 29,900 & 33,300 & 36,300 \\
\hline 4742 & 1,480 & 17,700 & 24,000 & 30,000 & 40,600 & 48,900 & 54,400 \\
\hline 4747 & 600 & 10,200 & 17,400 & 22,200 & 28,300 & 32,600 & 36,400 \\
\hline 4756 & 1,480 & 17,700 & 24,000 & 30,000 & 40,600 & 48,900 & 54,400 \\
\hline 4758 & 29.3 & 4,280 & 7,740 & 10,500 & 14,500 & 17,700 & 21,000 \\
\hline
\end{tabular}




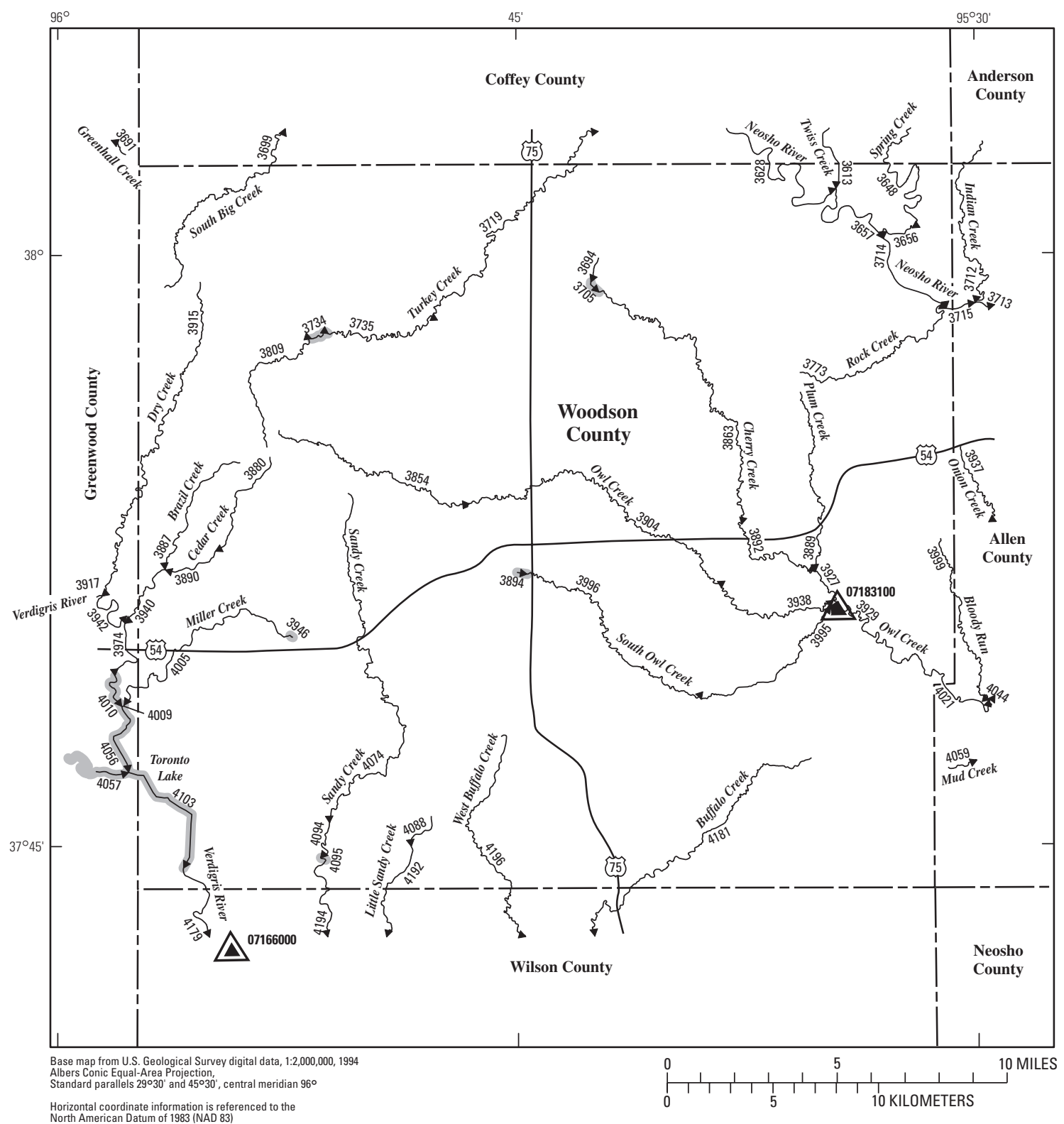

EXPLANATION
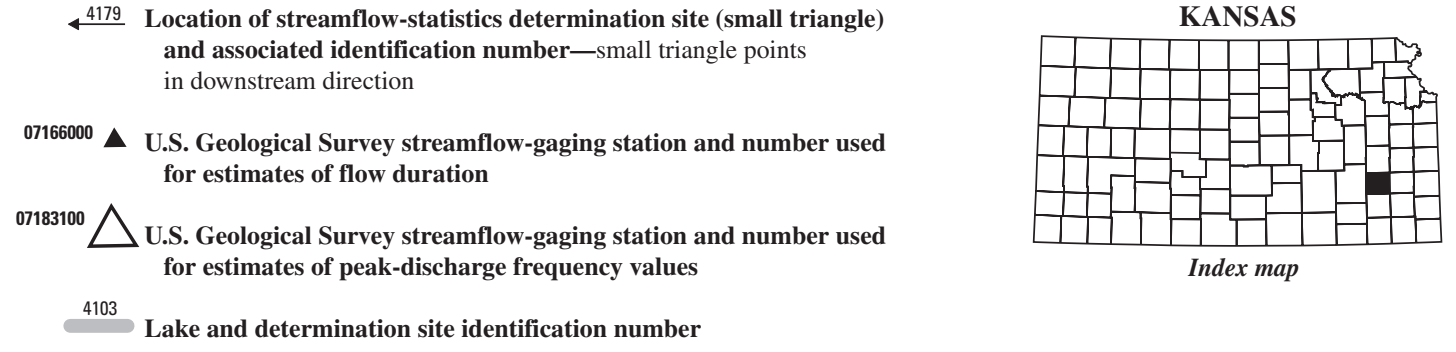

Figure 114. Location of streamflow-statistics determination sites, associated identification numbers, and U.S. Geological Survey streamflow-gaging stations used in the flow-duration and peak-discharge frequency analyses for Woodson County. 
Table 110. Estimated flow-duration values, mean flow values, and peak-discharge frequency values for controlled and uncontrolled flow stream segments on the 1999 Kansas Surface Water Register for Woodson County.

[KSWR, Kansas Surface Water Register; CUSEGA, catalog unit segment number alpha; $\mathrm{mi}^{2}$, square miles; $\mathrm{ft}^{3} / \mathrm{s}$, cubic feet per second; HYDRO, lake or other hydrologic structure; NA, not applicable; NRDitch, irrigation ditch; NRTribal, tribal stream]

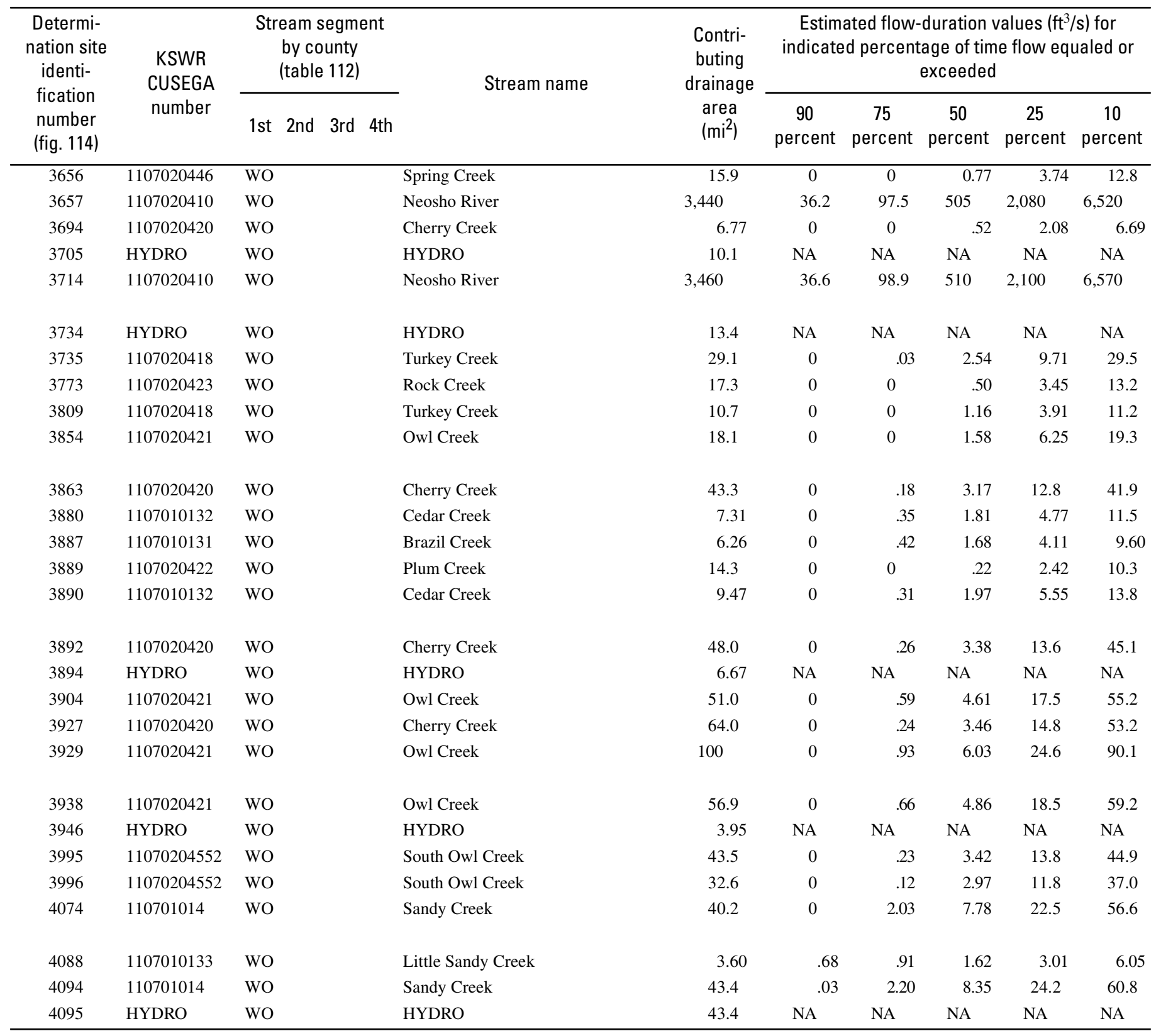


Table 110. Estimated flow-duration values, mean flow values, and peak-discharge frequency values for controlled and uncontrolled flow stream segments on the 1999 Kansas Surface Water Register for Woodson County._-Continued

[KSWR, Kansas Surface Water Register; CUSEGA, catalog unit segment number alpha; $\mathrm{mi}^{2}$, square miles; $\mathrm{ft}^{3} / \mathrm{s}$, cubic feet per second; HYDRO, lake or other hydrologic structure; NA, not applicable; NRDitch, irrigation ditch; NRTribal, tribal stream]

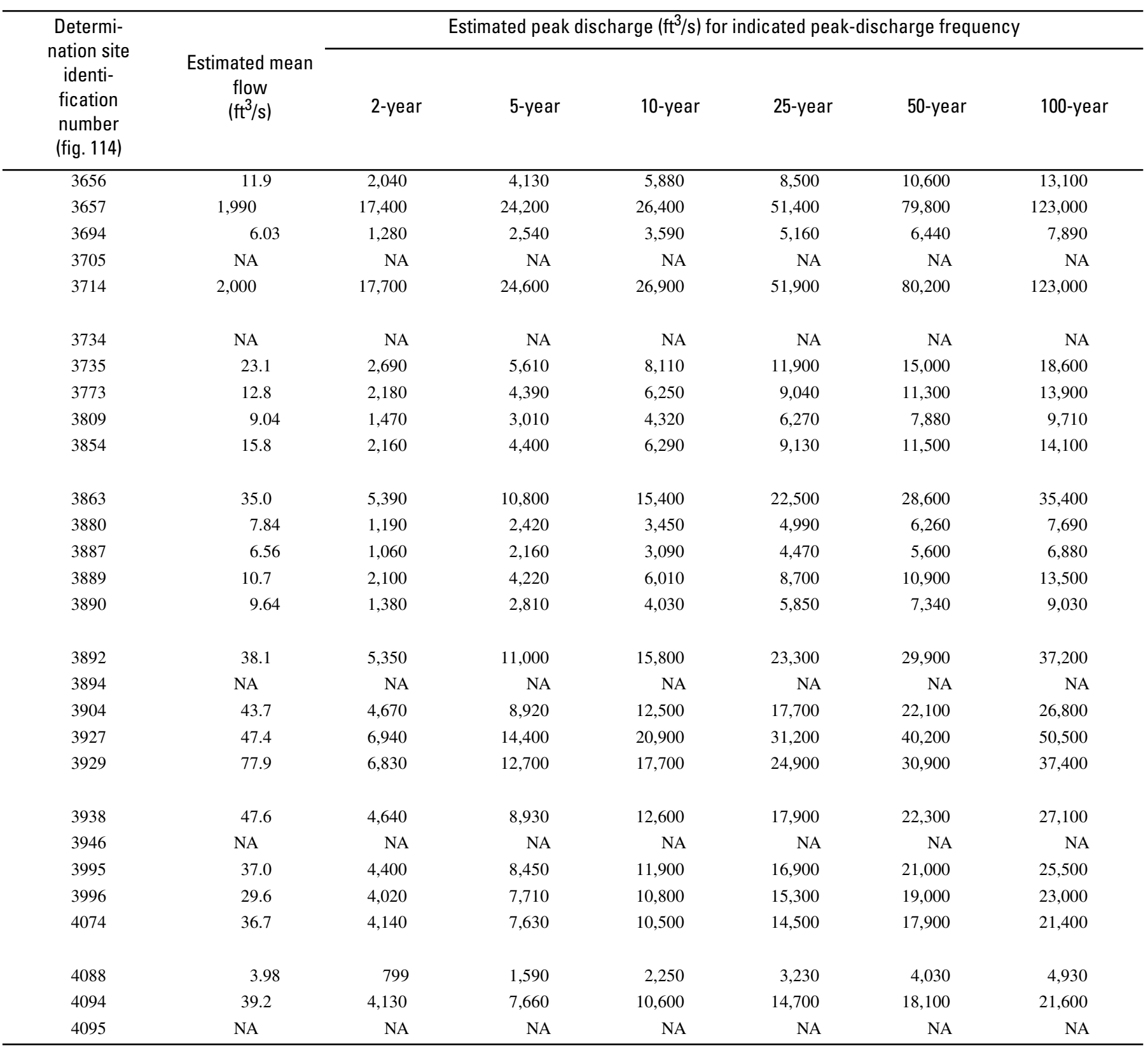


646 Estimates of Flow Duration, Mean Flow, and Peak-Discharge Frequency Values for Kansas Stream Locations 


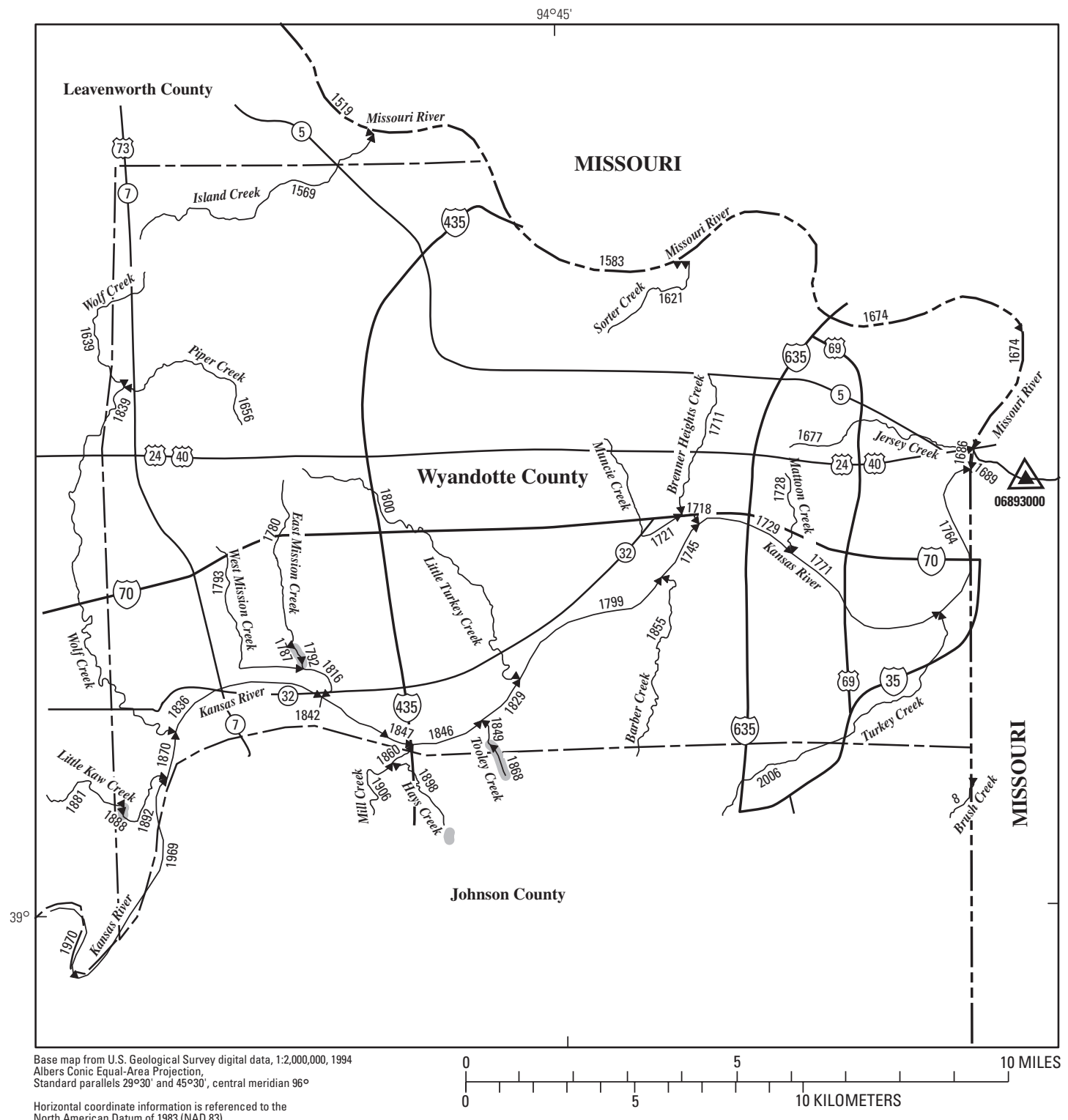

EXPLANATION

$\stackrel{1969}{\longleftarrow}$ Location of streamflow-statistics determination site (small triangle) and associated identification number-small triangle points in downstream direction

06893000 U.S. Geological Survey streamflow-gaging station and number used for estimates of flow duration

${ }^{06893000} \triangle$ U.S. Geological Survey streamflow-gaging station and number used for estimates of peak-discharge frequency values

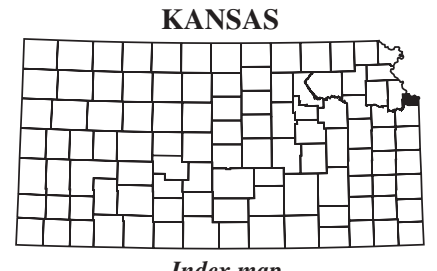
1868

Lake and determination site identification number

Figure 115. Location of streamflow-statistics determination sites, associated identification numbers, and U.S. Geological Survey streamflow-gaging stations used in the flow-duration and peak-discharge frequency analyses for Wyandotte County. 


\section{Estimates of Flow Duration, Mean Flow, and Peak-Discharge Frequency Values for Kansas Stream Locations}

Table 111. Estimated flow-duration values, mean flow values, and peak-discharge frequency values for controlled and uncontrolled flow stream segments on the 1999 Kansas Surface Water Register for Wyandotte County.

[KSWR, Kansas Surface Water Register; CUSEGA, catalog unit segment number alpha; $\mathrm{mi}^{2}$, square miles; $\mathrm{ft}^{3} / \mathrm{s}$, cubic feet per second; HYDRO, lake or other hydrologic structure; NA, not applicable; NRDitch, irrigation ditch; NRTribal, tribal stream]

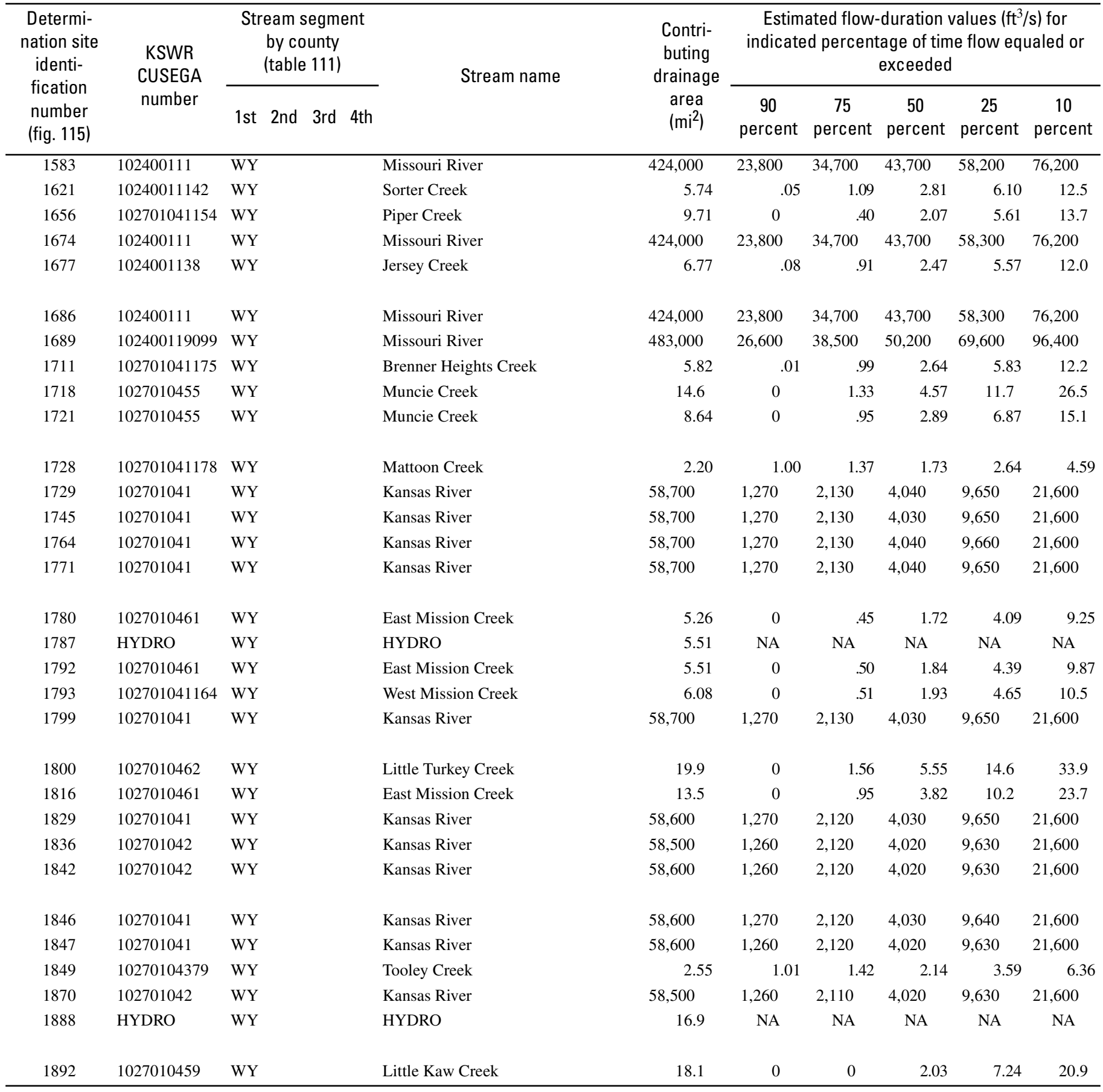


Table 111. Estimated flow-duration values, mean flow values, and peak-discharge frequency values for controlled and uncontrolled flow stream segments on the 1999 Kansas Surface Water Register for Wyandotte County.-Continued

[KSWR, Kansas Surface Water Register; CUSEGA, catalog unit segment number alpha; $\mathrm{mi}^{2}$, square miles; $\mathrm{ft}^{3} / \mathrm{s}$, cubic feet per second; HYDRO, lake or other hydrologic structure; NA, not applicable; NRDitch, irrigation ditch; NRTribal, tribal stream]

\begin{tabular}{|c|c|c|c|c|c|c|c|}
\hline \multirow{2}{*}{$\begin{array}{l}\text { Determi- } \\
\text { nation site } \\
\text { identi- } \\
\text { fication } \\
\text { number } \\
\text { (fig. 115) }\end{array}$} & \multirow{2}{*}{$\begin{array}{c}\text { Estimated mean } \\
\text { flow } \\
\left(\mathrm{ft}^{3} / \mathrm{s}\right)\end{array}$} & \multicolumn{6}{|c|}{ Estimated peak discharge $\left(\mathrm{ft}^{3} / \mathrm{s}\right)$ for indicated peak-discharge frequency } \\
\hline & & 2-year & 5-year & 10-year & 25-year & 50-year & 100-year \\
\hline 1583 & 48,600 & 111,000 & 150,000 & 178,000 & 204,000 & 239,000 & 269,000 \\
\hline 1674 & 48,700 & 111,000 & 150,000 & 178,000 & 204,000 & 240,000 & 269,000 \\
\hline 1677 & 7.63 & 1,150 & 2,320 & 3,300 & 4,770 & 5,980 & 7,340 \\
\hline 1686 & 48,700 & 111,000 & 150,000 & 178,000 & 204,000 & 240,000 & 269,000 \\
\hline 1689 & 57,900 & 142,000 & 201,000 & 245,000 & 289,000 & 351,000 & 401,000 \\
\hline 1728 & 2.68 & 598 & 1,180 & 1,660 & 2,370 & 2,950 & 3,600 \\
\hline 1729 & 8,500 & 50,200 & 89,000 & 119,000 & 148,000 & 200,000 & 240,000 \\
\hline 1745 & 8,490 & 50,200 & 89,000 & 119,000 & 148,000 & 200,000 & 240,000 \\
\hline 1764 & 8,500 & 50,200 & 89,000 & 119,000 & 148,000 & 200,000 & 240,000 \\
\hline 1771 & 8,500 & 50,200 & 89,000 & 119,000 & 148,000 & 200,000 & 240,000 \\
\hline 1780 & 5.99 & 948 & 1,930 & 2,750 & 3,980 & 4,990 & 6,130 \\
\hline 1787 & NA & NA & NA & NA & NA & NA & NA \\
\hline 1792 & 6.33 & 974 & 1,980 & 2,830 & 4,100 & 5,140 & 6,310 \\
\hline 1793 & 6.81 & 1,030 & 2,100 & 3,000 & 4,350 & 5,450 & 6,710 \\
\hline 1846 & 8,490 & 50,200 & 89,000 & 119,000 & 148,000 & 200,000 & 240,000 \\
\hline 1847 & 8,480 & 50,100 & 89,000 & 119,000 & 148,000 & 200,000 & 240,000 \\
\hline 1849 & 3.49 & 655 & 1,300 & 1,830 & 2,610 & 3,250 & 3,960 \\
\hline 1870 & 8,470 & 50,100 & 88,900 & 119,000 & 148,000 & 200,000 & 240,000 \\
\hline 1888 & NA & NA & NA & NA & NA & NA & NA \\
\hline 1892 & 15.6 & 1,930 & 4,030 & 5,840 & 8,560 & 10,800 & 13,400 \\
\hline
\end{tabular}


Table 112. County abbreviations for Kansas.

\begin{tabular}{|c|c|c|c|}
\hline $\begin{array}{l}\text { County abbreviation } \\
\text { used in table } 6\end{array}$ & County name & $\begin{array}{l}\text { County abbreviation } \\
\text { used in table } 6\end{array}$ & County name \\
\hline$\overline{\mathrm{AL}}$ & Allen & HS & Haskell \\
\hline $\mathrm{AN}$ & Anderson & $\mathrm{HV}$ & Harvey \\
\hline AT & Atchison & $\mathrm{JA}$ & Jackson \\
\hline BA & Barber & JF & Jefferson \\
\hline BB & Bourbon & $\mathrm{JO}$ & Johnson \\
\hline BR & Brown & JW & Jewell \\
\hline $\mathrm{BT}$ & Barton & $\mathrm{KE}$ & Kearny \\
\hline BU & Butler & KM & Kingman \\
\hline $\mathrm{CA}$ & Clark & KW & Kiowa \\
\hline $\mathrm{CD}$ & Cloud & LB & Labette \\
\hline $\mathrm{CF}$ & Coffey & $\mathrm{LC}$ & Lincoln \\
\hline $\mathrm{CK}$ & Cherokee & LE & Lane \\
\hline CL & Cowley & LG & Logan \\
\hline $\mathrm{CM}$ & Comanche & $\mathrm{LN}$ & Linn \\
\hline $\mathrm{CN}$ & Cheyenne & LV & Leavenworth \\
\hline CQ & Chautauqua & LY & Lyon \\
\hline $\mathrm{CR}$ & Crawford & MC & Mitchell \\
\hline $\mathrm{CS}$ & Chase & $\mathrm{ME}$ & Meade \\
\hline $\mathrm{CY}$ & Clay & MG & Montgomery \\
\hline DC & Decatur & MI & Miami \\
\hline DG & Douglas & MN & Marion \\
\hline DK & Dickinson & MP & McPherson \\
\hline DP & Doniphan & MR & Morris \\
\hline ED & Edwards & MS & Marshall \\
\hline EK & Elk & MT & Morton \\
\hline EL & Ellis & NM & Nemaha \\
\hline EW & Ellsworth & NO & Neosho \\
\hline FI & Finney & NS & Ness \\
\hline FO & Ford & NT & Norton \\
\hline FR & Franklin & OB & Osborne \\
\hline GE & Geary & OS & Osage \\
\hline GH & Graham & OT & Ottawa \\
\hline GL & Greeley & PL & Phillips \\
\hline GO & Gove & PN & Pawnee \\
\hline GT & Grant & PR & Pratt \\
\hline GW & Greenwood & $\mathrm{PT}$ & Pottawatomie \\
\hline GY & Gray & RA & Rawlins \\
\hline $\mathrm{HG}$ & Hodgeman & $\mathrm{RC}$ & Rice \\
\hline HM & Hamilton & $\mathrm{RH}$ & Rush \\
\hline $\mathrm{HP}$ & Harper & RL & Riley \\
\hline
\end{tabular}


Table 112. County abbreviations for Kansas.-Continued

\begin{tabular}{|c|c|}
\hline $\begin{array}{l}\text { County abbreviation } \\
\text { used in table } 6\end{array}$ & County name \\
\hline$\overline{\mathrm{RN}}$ & Reno \\
\hline RO & Rooks \\
\hline $\mathrm{RP}$ & Republic \\
\hline RS & Russell \\
\hline SA & Saline \\
\hline $\mathrm{SC}$ & Scott \\
\hline SD & Sheridan \\
\hline SF & Stafford \\
\hline SG & Sedgwick \\
\hline SH & Sherman \\
\hline SM & Smith \\
\hline $\mathrm{SN}$ & Shawnee \\
\hline ST & Stanton \\
\hline SU & Sumner \\
\hline SV & Stevens \\
\hline SW & Seward \\
\hline $\mathrm{TH}$ & Thomas \\
\hline TR & Trego \\
\hline WA & Wallace \\
\hline WB & Wabaunsee \\
\hline WH & Wichita \\
\hline WL & Wilson \\
\hline WO & Woodson \\
\hline WS & Washington \\
\hline WY & Wyandotte \\
\hline
\end{tabular}

